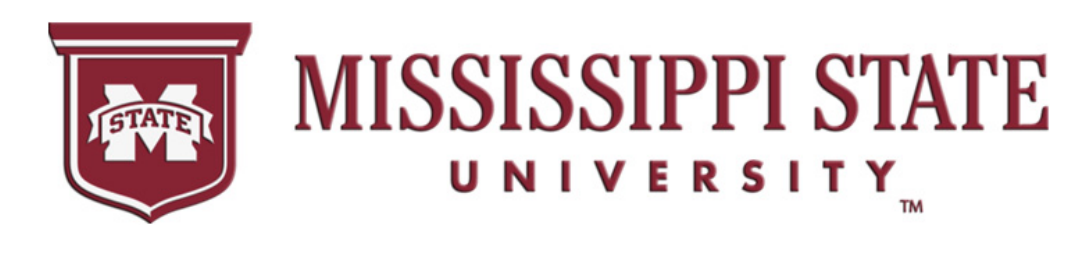

\title{
Southern Regional Center for Lightweight Innovative Design
}

\section{Final Scientific \& Technical Report}

\author{
December 2012
}

For compliance with contract requirements of Award DE-FC26-06NT42755 


\section{DISCLAIMER}

This report was prepared as an account of work sponsored by an agency of the United States Government. Neither the United States Government nor any agency thereof, nor any of their employees, makes any warranty, express or implied, or assumes any legal liability or responsibility for the accuracy, completeness, or usefulness of any information, apparatus, product, or process disclosed, or represents that its use would not infringe privately owned rights. Reference herein to any specific commercial product, process, or service by trade name, trademark, manufacturer, or otherwise does not necessarily constitute or imply its endorsement, recommendation, or favoring by the United States Government or any agency thereof. The views and opinions of authors expressed herein do not necessarily state or reflect those of the United States Government or any agency thereof. 


\section{Southern Regional Center for Lightweight Innovative Design}

Final Scientific and Technical Report

\section{Organization of this Report}

Executive Summary...................................................................................................... 5 page

Task 1: Multiscale material models and design framework for lightweight alloys (magnesium and

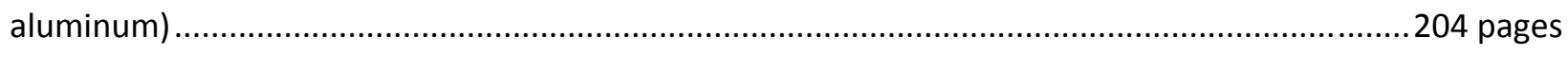

Task 2: Multiscale materials modeling and characterization of steel alloys ..............................40 pages

Task 3: Multiscale material models and processing design for polymeric materials ...................147 pages

ICME demonstration project to support the development of a magnesium front end structure ...91 pages 


\section{SOUTHERn REgIONAL CENTER FOR LIGHTWEIGHT INNOVATIVE DESIGN FINAL REPORT}

Field Technical Monitors: Mark F. Horstemeyer; Paul T. Wang

Center for Advanced Vehicular Systems

Mississippi State University

Box 5405

Mississippi State, MS 39762

(662)325-5449; e-mail: mfhorst@me.msstate.edu

(662)325-2890; e-mail: pwang@cavs.msstate.edu

Technology Area Development Manager: William Joost

U.S. Department of Energy

1000 Independence Ave., S.W., Washington, DC 20585

(202) 287-6020; e-mail: william.joost@ee.doe.gov

Contractor: Mississippi State University (MSST)

Contract No.: DE-FC-26-06NT42755

\section{EXECUTIVE SUMmaRY}

Lightweighting materials are critical to reducing emissions and the United States' reliance on foreign oil. Understanding how lightweighting materials in vehicles under crash scenarios is paramount as well, for passenger safety as well as energy efficiency; these are primary design challenges facing today's automotive industry. The Southern Regional Center for Lightweight Innovative Design (SRCLID) has developed an experimentally validated cradle-to-grave modeling and simulation effort to optimize automotive components in order to decrease weight and cost, yet increase performance and safety in crash scenarios.

SRCLID's "from Atoms to Autos" modeling effort quantified the microstructure-property relations of lightweight materials by evaluating them at various length scales, starting at the atomic level, for each step of the manufacturing process. Using theory development, experimental characterization, and large scale computing, we have developed multiscale physics-based material models that are experimentally validated and account for uncertainty. Our design methodologies then guide the design optimization of components, systems, and materials in engineering practice throughout the southern automotive corridor of the US. Both the new, lightweight materials and the math-based tools developed through SRCLID are being implemented in development of next-generation vehicles, with particular consideration given to their performance under various crash and high-speed impact environments.

In summary, the three major objectives of this project are accomplished:

- To develop experimentally validated cradle-to-grave modeling and simulation tools to optimize automotive and truck components for lightweighting materials (aluminum, steel, and $\mathrm{Mg}$ alloys and polymer-based composites) with consideration of uncertainty to decrease weight and cost, yet increase the performance and safety in impact scenarios; 
- To develop multiscale computational models that quantify microstructure-property relations by evaluating various length scales, from the atomic through component levels, for each step of the manufacturing process for vehicles; and

- To develop an integrated K-12 educational program to educate students on lightweighting designs and impact scenarios.

In this final report, we divided the content into two parts: the first part contains the development of building blocks for the project, including materials and process models, process-structure-property (PSP) relationship, and experimental validation capabilities; the second part presents the demonstration task for Mg front-end work associated with USAMP projects.

In the first part of this SRCLID program, we have generated process-structure-property (PSP) relationships for aluminum, steel and magnesium alloys and developed a physics-based multiscale internal state variable (ISV) model that includes uncertainty. The first part of the SRCLID projects focused on aluminum and steel alloys and then later on magnesium alloys. We developed a material database, ISV material models, and process models for extruded (AM30 and AZ61) and warm formed (AZ31) magnesium alloys. The database includes results from the mechanical and microstructure characterization studies performed using current experimental equipment at CAVS. The material model developed was based on a multiscale modeling approach, where information from crystal plasticity, dislocation dynamics, and molecular dynamics simulations provided insights into the functional form of the ISV's evolution equations and corresponding material parameters. The extrusion and forming process models being constructed are finite element codes that are suitable for problems with large mesh distortions (e.g., extrusion using flat dies). The MultiStage Fatigue (MSF) Model has been correlated with different magnesium alloy strain-life curves and was extended to describe metal joint behavior.

In conjunction with the multiscale internal state variable model, we have initiated optimization methods, including ISV and PSP with finite element analysis and design. With uncertainty under low strain rates, variable temperatures, and high strain rate tension-compression-torsion tests, we characterized several structural materials for lightweighting (i.e., aluminum, steel, and magnesium alloys) throughout their manufacturing and life cycle history. We then tested these materials in crash simulations and safety performance evaluations.

Six of our twelve tasks are directly related to the USAMP Front-End Magnesium Project. In strong support of the Integrated Computational Material Engineering (ICME) program, we established several focused research teams with USAMP members and 10-year draft roadmaps for magnesium, steel and polymers with the DOE.

At the atomic scale, we established a number of atomistic potentials (i.e., $\mathrm{Fe}, \mathrm{C}, \mathrm{Si}, \mathrm{Al}, \mathrm{Mg}$ ) that will be used in development of high strength steel alloys. We showed at the atomic scale that the standard procedure of using just the elastic modulus and cohesive energy is not robust enough to capture the appropriate dislocation behavior. Instead, optimizing both the atomistic potential (MEAM) to the generalized stacking fault (GSF) energy curve and the Peierls stress is the key. In the absence of experimental data, one can use $a b$ initio modeling results to complete the material database. In our research, the improved magnesium MEAM model was correlated with both experiments and $a b$ initio results. Physical properties such as the lattice parameter, the vacancy formation energy, the GSF energy 
curves for basal slip, and mechanical properties such as elastic constants and Peierls stress for basal slip were all well captured.

Our cyberinfrastructure $(\mathrm{Cl})$ integrates our software and experimental information in a Wiki-based format and has garnered high recognition from TMS and USAMP. The $\mathrm{Cl}$ has become the foundation of a virtual organization (VO), known as the Engineering Virtual Organization for Cyber Design (EVOCD at http://icme.hpc.msstate.edu). EVOCD has been developed with the primary goal of accumulating and protecting the intellectual property generated by the participants of the organization. The portal provides powerful passage for accruing and exchanging community knowledge as well as access to repositories of experimental data, material models and computational tools at different length scales, which together exploit the integrative nature of the Integrated Computational Material Engineering (ICME). To achieve this goal, EVOCD is comprised of four primary functional components that are the foundation of the VO: (i) Knowledge Management; (ii) Repository of Codes; (iii) Repository of Data; (iv) Online Calibration Tools. The implementation is primarily based on third-party, open-source software packages (such as Apache's Tomcat, Apache's ServiceMix, View VC, SVN) customized to the particular needs of EVOCD and the principles of the Service-Oriented Architectures (SOA). All of the computational and experimental databases can be found for the MsSt cyberinfrastructure at https://icme.hpc.msstate.edu.

The polymer-based composites effort developed process/product concepts by employing multiscale material models. The natural fiber program is now able to produce good samples for evaluation. The biomechanics program has been advanced to incorporating polymeric ISV models for tissues and evaluating damage phenomena for brain material subjected to high rate impact using finite element analyses. The biomechanics projects are included in order to incorporate the human effects into the multi-objective design of metal and composite materials as objectives.

We have graduated 40 students with $\mathrm{MS}$ and $\mathrm{PhD}$ degrees. For the $\mathrm{K}-12$ program, we have developed crash kits and run K-2 and 3-6 grade "Mission Eggcellence" competitions in multiple counties throughout Mississippi in an effort to inspire their interest in science and engineering.

In our industrial partnership development, we have created strong ties with companies from various sectors (i.e., automotive, software, and local manufacturers), and they have embraced the technology being developed in this program. As the result of extensive interaction with MSC, Alpha Star, SAC, POSCO, and Mitsubishi Motors, we received total $\$ 4,000,000$ in additional cost sharing support from these companies.

Our sunset strategy has been to continue developing the structure-property relationships and multiscale models for magnesium and $3^{\text {rd }}$ generation (3G) steel alloys. We focus on demonstrating the technology through the USAMP Mg Front-End Project sponsored by DOE, and metal making technology of $3 G$ steel alloys. Composite, biomechanics and natural fiber research teams will move forward with various sponsors to develop material-specific multiscale models validated by critical experiments. We will employ these materials in crash simulations with new data generated. We will then update the cyberinfrastructure to expand the national and an international user base.

In the second part of this SRCLID program, we formed a Mg MsSt team to support the USAMP ICME demo project. We documented the results of key enabling technologies being developed for advanced lightweight magnesium processes and alloys to validate and predict mechanical responses of a front-end demo structure, including process-structure-property relationships. The key enabling technologies are 
the following: internal state material plasticity damage model, fatigue model, extrusion process model, sheet forming model, and the integration of the above to validate the performance of demo parts.

The front-end demo structure comprised the top rails made of AZ31 sheet materials, a shock tower of AM 60 and AM90, and a lower rail of AM30 extrusion; those parts were supplied by USAMP partners. In this second part of the SRCLID final report, we demonstrated the development of ICME techniques including key databases via the cyberinfrastructure and the associated materials and process models, by which a zone-mapping method was utilized to show the capability of experimentally validated simulation models at the component level for high-integrity magnesium castings, extrusions, and sheet products. The zone mapping method is to characterize material features at a local level within a given component such that the heterogeneities of the material within the components can be well presented for modeling purposes.

The Mg MsSt team focused its effort into five segments: microstructure-sensitive fatigue modeling for materials and components, extrusion processing and post forming, sheet forming of AZ31 alloys, ICME demo validation via zone mapping, and cyberinfrastructure.

In fatigue modeling, we established the MultiStage Fatigue (MSF) model for Mg alloys (i.e., AM60, AZ91) of three product forms, as-cast, plate, and sheet products. The model was correlated to the experimental strain-life results of the AM60 as-cast, AZ91 as-cast, and the AZ91 T6 magnesium alloys, by using parameters representing microstructural features, such as dendrite cell size, pore size, and cyclic hardening parameters, in order to capture the differences in fatigue behavior of three data sets. The fatigue model showed good correlation to the upper and lower bounds of the strain-life behavior of the AM60 and AZ91 magnesium alloys by employing the maximum and minimum inclusion and dendrite cell sizes. Thus, the inclusion (pore) size had the most influence on the scatter in the fatigue lifetimes. We note that the other material constants in MSF were not changed for the estimation of the upper and lower bounds, regardless of the material processing method.

For the extrusion effort, we demonstrated the ability of performing lab-scale extrusion to construct a database for the extrusion of various Mg alloys. The recorded data captured the effect of processing parameters (ram speed and billet temperature) on processing variables (temperature histories and loaddisplacement curves) and the evolution of material microstructure (texture, grain morphology). We then built robust finite element (FE) models and performed numerical simulations of the lab-scale and industry-scale extrusion processes using HyperXtrude, a commercial Eulerian finite element code, to model the extrusion of complex, industrial profiles. We integrated the VPSC (visco-plastic selfconsistent) crystal plasticity code to predict texture evolution based on streamline information generated by HyperXtrude from an extrusion simulation. The predicted texture patterns agreed well with the trends observed in the experiments. In the post forming stage after extrusion, the same VPSC code using both the computed extrusion texture and the room-temperature material properties captured reasonably well the initial yield strength of the extrudate.

In sheet forming, we established a methodology to quantify the evolution of the yield surface from a mesoscale crystal plasticity code and the bridging to a macroscale internal state variable model. FE simulations of sheet forming as compared to sheet bending experiments conducted at the lab level were established. The potential for predicting texture evolution during sheet forming was demonstrated, similar to the extrusion effort above. Three-point bending was performed at different strain rates at room temperature on an annealed AZ31 Mg sheet material. The results revealed a surprising strain rate 
sensitivity. The bending angle increased as the strain rate increased, sharply different from the asreceived sheet in which the bending angle decreased as the strain rate increased. Preliminary results of curvature and load-displacement showed good agreement between experiments and modeling. In-situ EBSD was performed on AZ31 sheet samples during three-point bending. Unusual, highly localized twin bands were observed in the compression zone. In between the twin bands, no twins were observed. More experiments are needed to understand the mechanism for such localized twin bands.

Our ICME demo validation team focused on using the internal state variable plasticity-damage model and a microstructure-sensitive multiscale fatigue model to predict the monotonic loading and the fatigue behaviors of the AZ91 shock tower. The shock towers provided by USAMP, were examined and tested to provide insight into the mechanical performance and failure mechanisms and validation for the models. When investigating the microstructure of the shock towers, we characterized pores in several locations in order to develop the zone-mapping technique for the shock tower. We worked with the University of Michigan team and Dr. Mei Li from Ford to understand the sample statistics of variation and the porosity correlation with solidification cooling rates, accordingly. The finite element simulations coupled with the MSF model correlated well with the fatigue experimental tests of shock tower. This investigation has shown that fatigue failure of a component must be studied based on an integrated, holistic approach that involves jointly the material properties, geometry, and loading conditions. In general, we observed that the microstructure of the shock tower plays a key role in the material's monotonic loading and fatigue responses. The material mechanical response, along with a large geometry variation could dictate the poor performance of the shock tower component. To increase the shock tower performance, the thickness of cast part must be kept uniform and the material microstructure defects must be substantially reduced.

The scientific understanding in the processing-structure-property relationships of $\mathrm{Mg}$ alloys has been captured in the MsSt ICME Cyberinfrastructure (http://icme.hpc.msstate.edu). This Cyberinfrastructure is being developed with an ambitious objective of providing a platform for the integration of models from different domains to enable development of comprehensive and robust processing-structure-property relationships, which can also be integrated with manufacturing simulation and design. The Cyberinfrastructure can also provide a platform for the optimization of alloys, manufacturing processes, and components. The ICME Cyber program will be continued as a research collaboration between researchers and organizations in Canada, China, and the United States and will serve as an international pilot project for the new Integrated Computational Materials Engineering movement (ICME) within the materials community. 


\section{TABLE OF CONTENTS}

TASK 1: Multiscale Material Models and Design Framework for Lightweight Alloys (Magnesium and Aluminum)

Subtask 1.1: Multiphysics and Multiscale based Internal State Variable Material Model for Simulating Different Manufacturing Processes ................................................................ 5

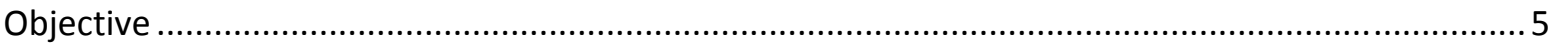

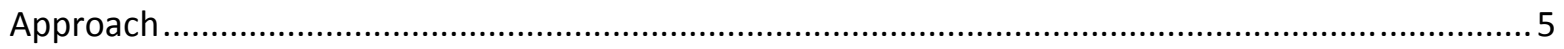

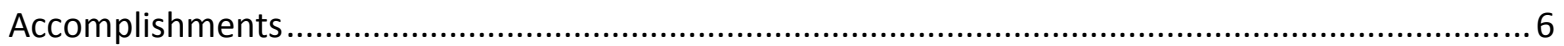

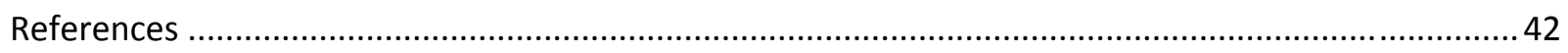

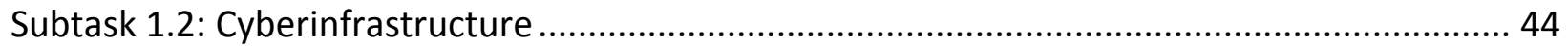

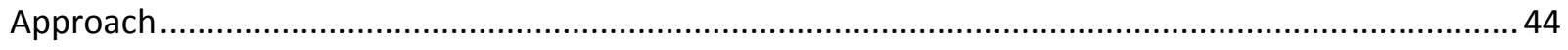

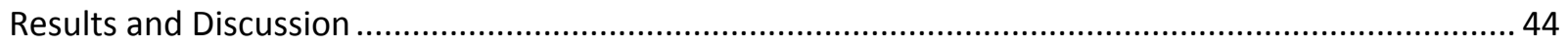

Subtask 1.3: Fatigue Performance of Lightweight Materials ............................................. 46

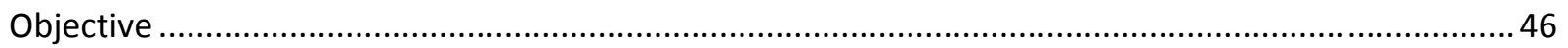

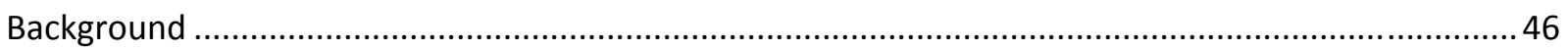

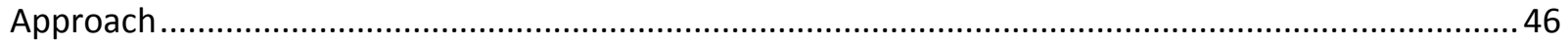

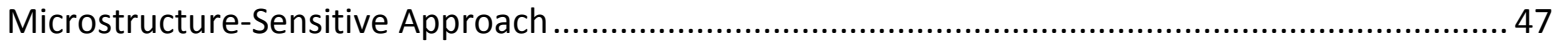

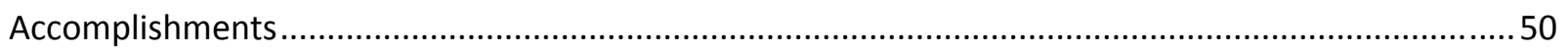

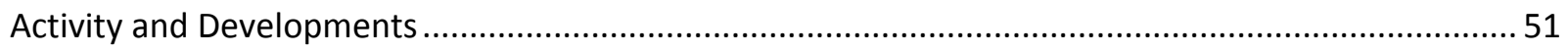

FASTRAN and Fatigue Crack Growth for AZ91 Mg Alloy.............................................................. 51

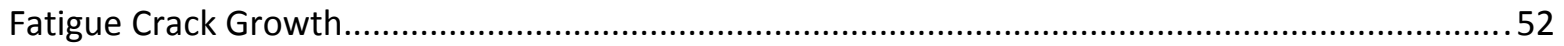

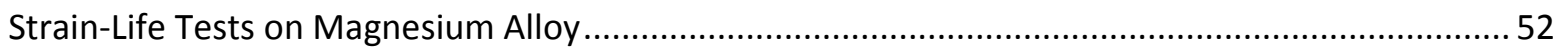

Fatigue Life Calculations on Magnesium Alloy …....................................................................... 53

Structure-property Relations of Fatigue in Mg AM30 Alloy .......................................................... 55

Effect of Twinning, Slip, and Inclusions on the Fatigue Anisotropy of Extrusion-Textured Magnesium

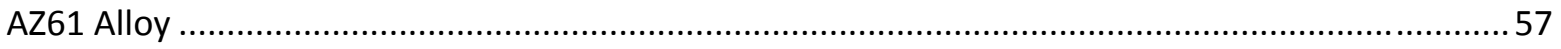

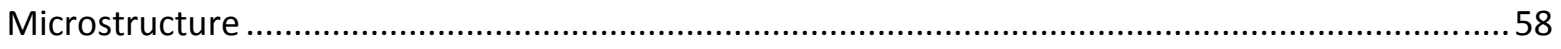

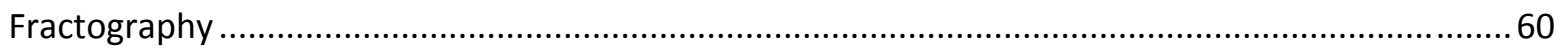

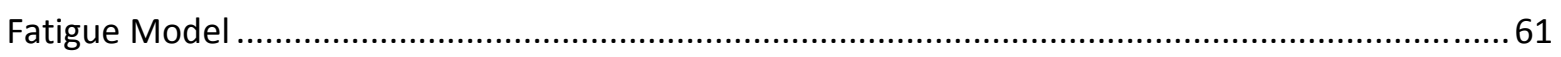

Atomistic Simulations of Fatigue Crack Growth and the Associated Fatigue Crack Tip Stress Evolution in Magnesium Single Crystals ...................................................................................... 62 
Small Fatigue Crack Growth Observations in an Extruded Magnesium Alloy .....

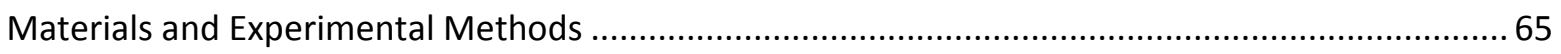

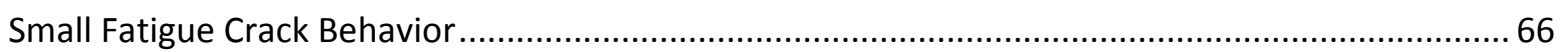

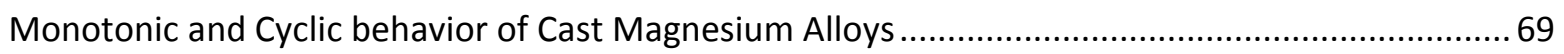

Microstructure and Damage Evolution during Tensile Loading in a Wrought Magnesium Alloy........69

Finite element analysis of the mechanical behavior and local stress intensity factor solutions for

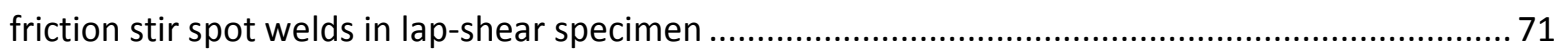

Role of Different Material Processing Methods on the Fatigue Behavior of AZ31 Magnesium Alloy 72

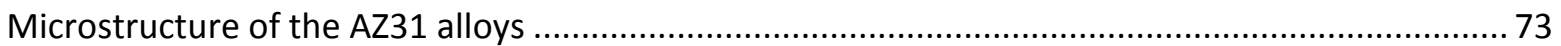

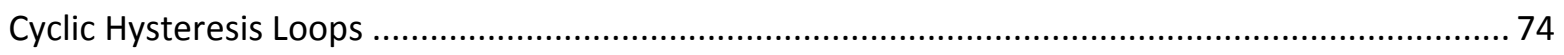

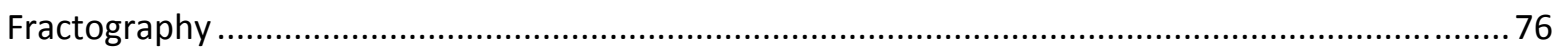

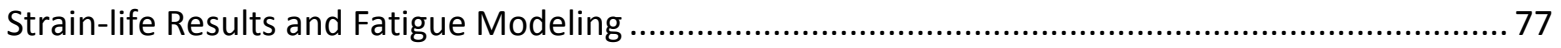

Microstructure-Sensitive Fatigue Modeling of an Extruded AM30 Magnesium Alloy ...................... 80

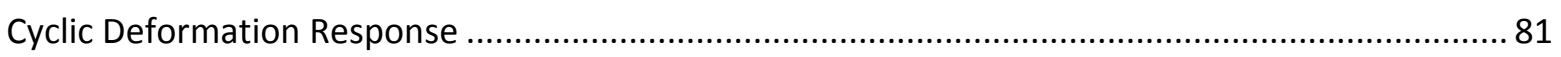

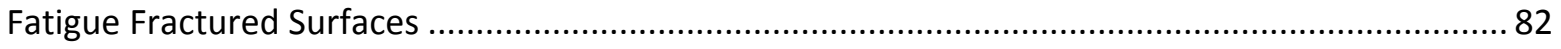

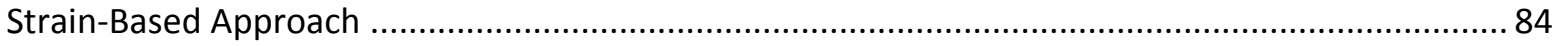

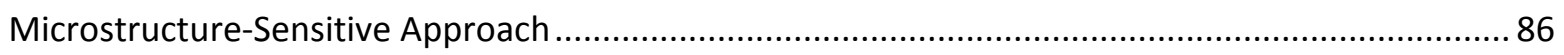

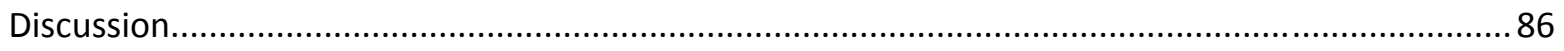

Multiscale Fatigue Modeling of AZ91 and AM60 Magnesium Alloys ................................................ 88

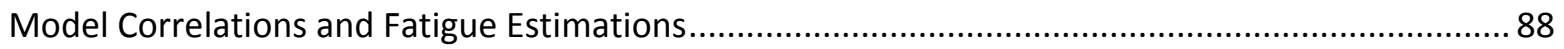

Influence of Structural Integrity on Fatigue Behavior of Friction Stir Spot Welded AZ31 Mg Alloy...91

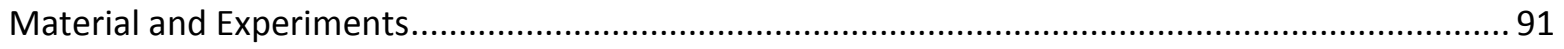

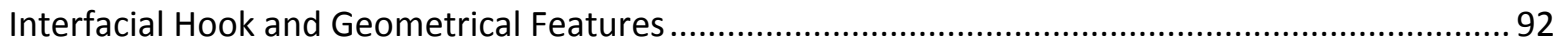

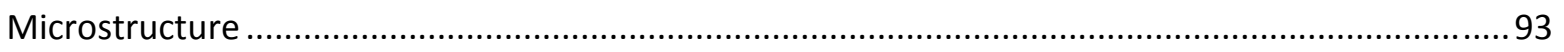

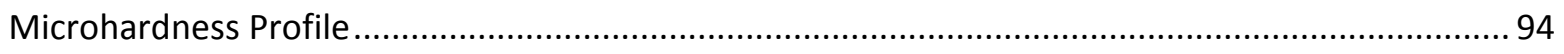

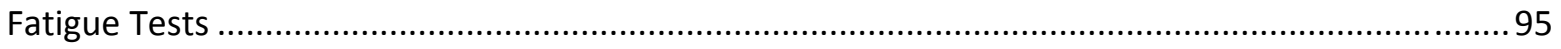

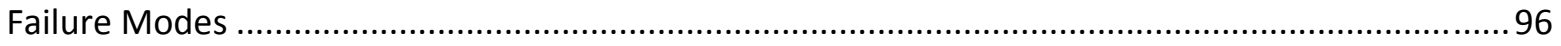

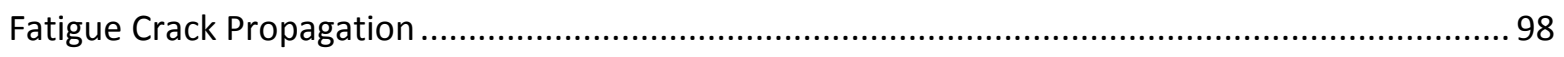

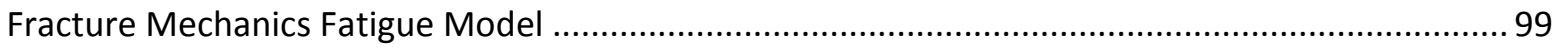

Multi Stage Fatigue Model Adaption for FSSW ..................................................................... 102

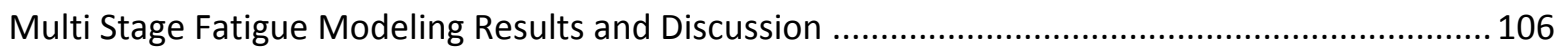

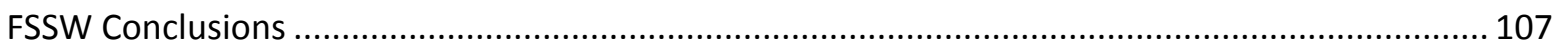




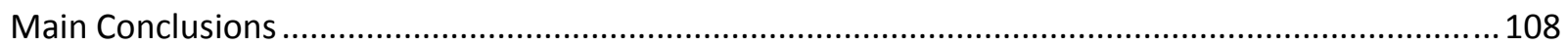

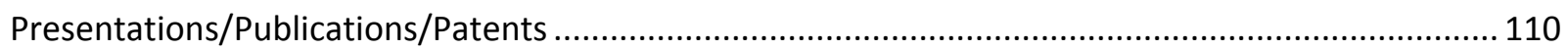

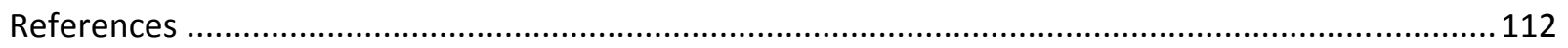

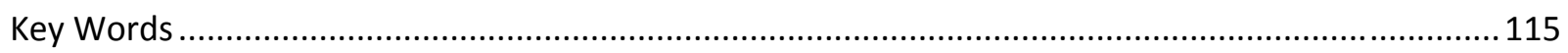

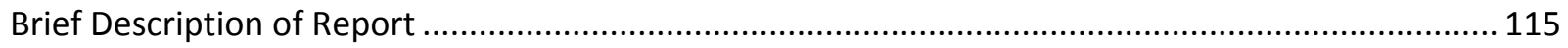

Subtask 1.4: Multiscale Modeling of Corrosion ............................................................. 116

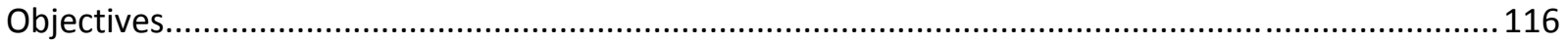

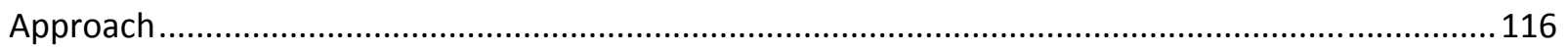

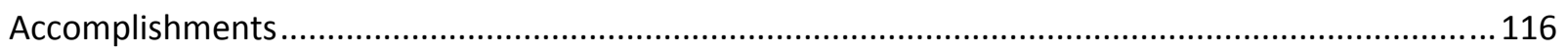

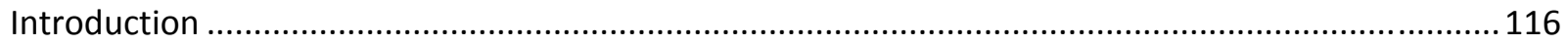

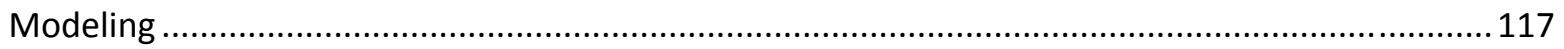

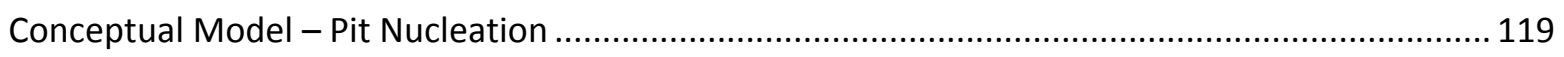

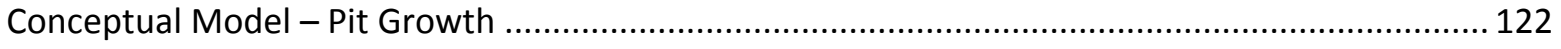

Conceptual Model - Pit Coalescence..................................................................................... 122

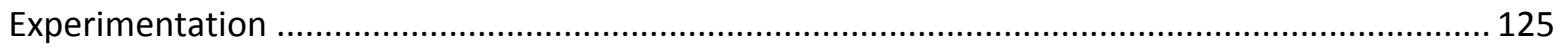

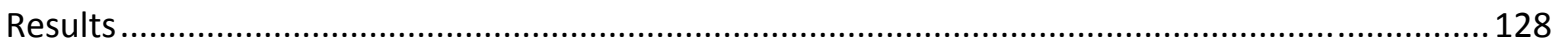

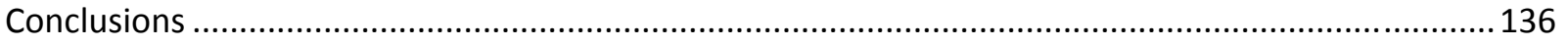

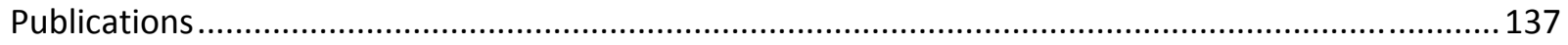

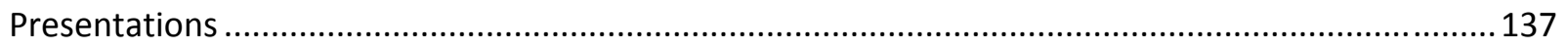

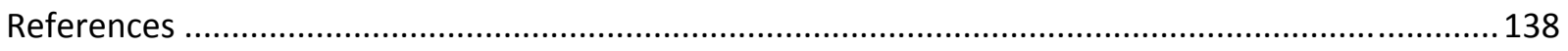

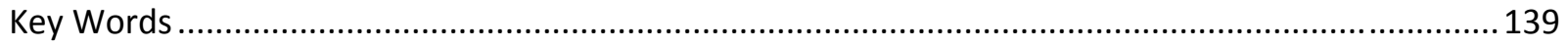

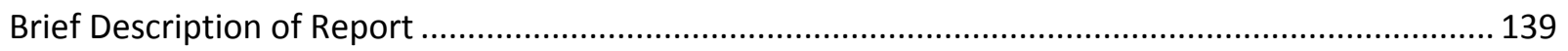

Subtask 1.5: Material Design of Lightweight Mg Alloys ................................................... 140

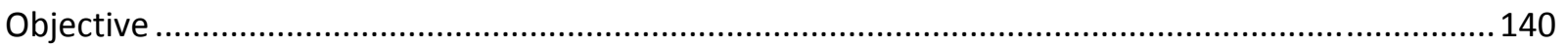

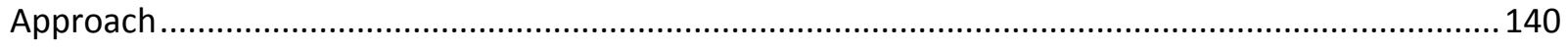

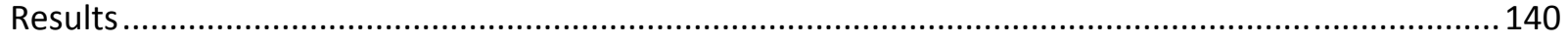

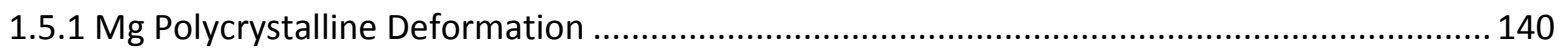

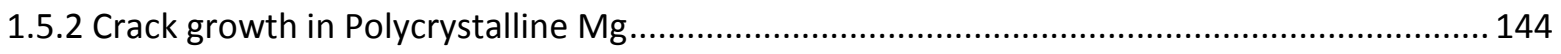

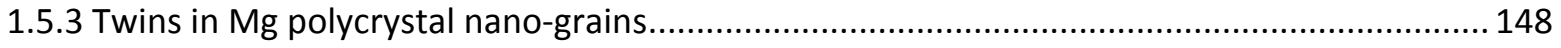

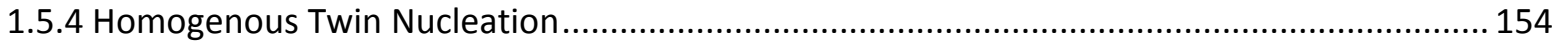

1.5.5 Non-planar $\{1012\}$ Twinning Nucleation in Mg single crystal .......................................... 157 
1.5.6 Temperature dependence on the twin nucleation. 164

References 165

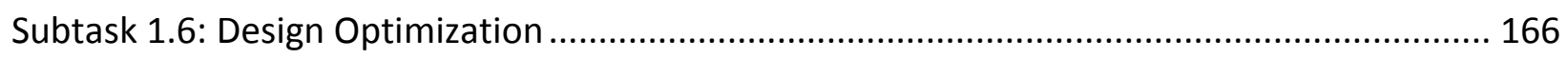

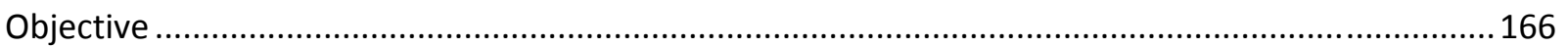

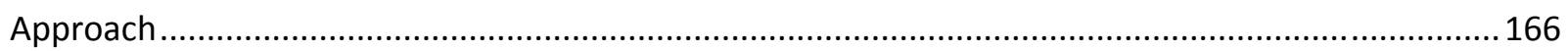

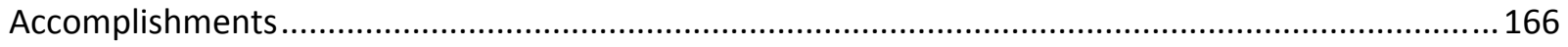

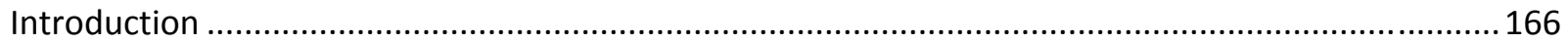

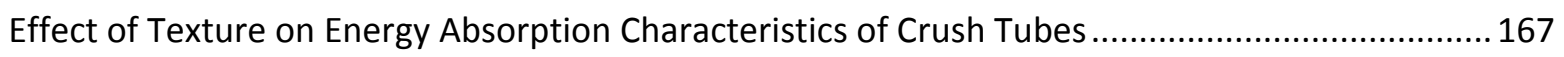

Full Vehicle Structural Optimization Considering Crashworthiness and Vibration Responses ........ 172

Simulation and Optimization of Vehicle Structures Made of Composite Materials......................... 178

Welding Simulation and Process Optimization......................................................................... 183

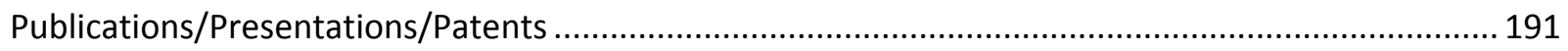

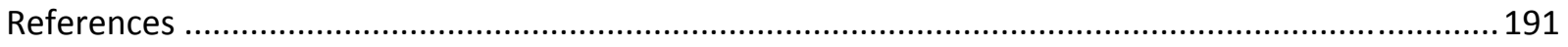

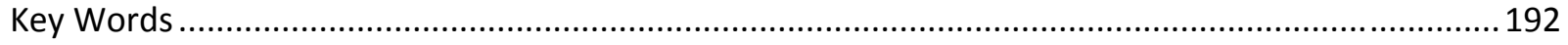

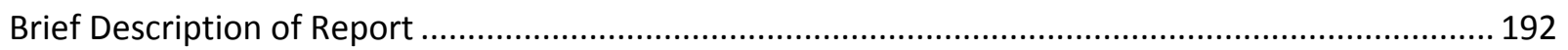

Subtask 1.7: Solidification Microstructure Modeling in Steel and Mg Alloys ........................... 193

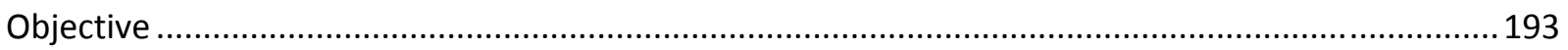

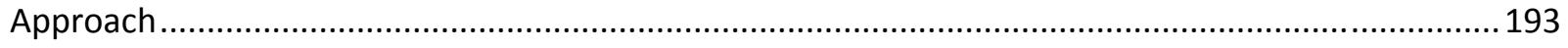

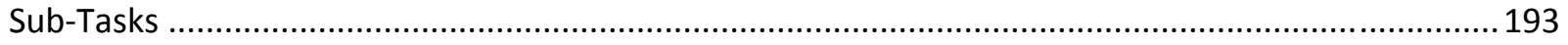

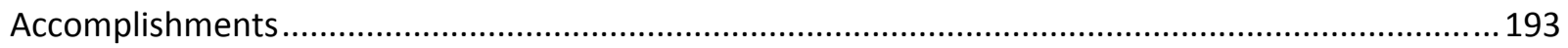

1. Modeling of dendritic microstructures under convection....................................................... 194

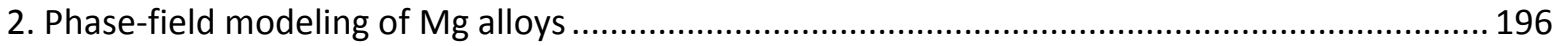

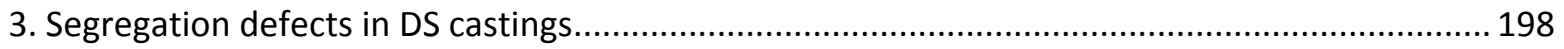

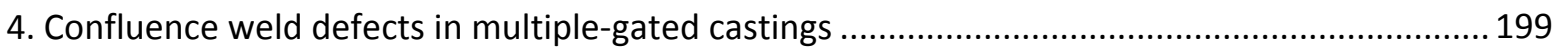

5. Evaluation of four casting processes for magnesium alloy AZ91 ................................................200

6. Modeling and characterization of additively manufactured steel parts ...................................... 201

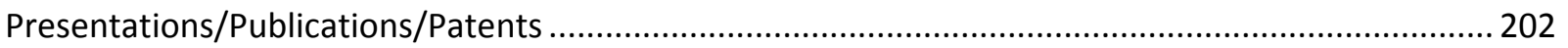

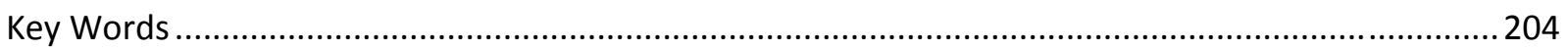

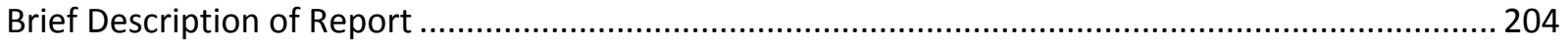

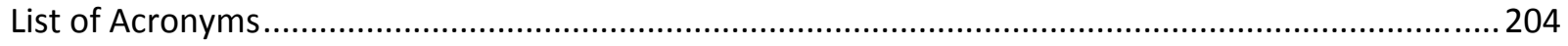




\title{
task 1: Multiscale Material Models and Design framework for Lightweight Alloys (Magnesium and Aluminum)
}

\author{
Principal Investigator: Paul T. Wang \\ Associate Director, CAVS \\ Mississippi State University (MSST) \\ 200 Research Blvd \\ Mississippi State, MS 39762 \\ (662) 325-2890; fax: (662) 325-5433; e-mail:pwang@cavs.msstate.edu
}

Carol Schutte: Technology Area Development Manager

e-mail: carol.schutte@ee.doe.gov

Joseph Renk: Project Officer

email:joseph.renk@netl.doe.gov

\author{
Contractor: $\quad$ Mississippi State University (MSST) \\ Contract No.: $\quad$ DE-FC26-06NT42755
}

\section{Subtask 1.1: Multiphysics and Multiscale based Internal State Variable Material Model for Simulating Different Manufacturing Processes}

Team Members: P. T. Wang, M.F. Horstemeyer, E.B. Marin, D.J. Bammann, , H. El Kadiri, Y. Hammi, C. Bouvard, B. Li, Q. Ma, A. Oppedal

\section{Objective}

To establish a multiscale framework for lightweight materials that accounts for statistical variations from the material microstructure, product geometry, and processing parameters and that can aid in developing new lightweight alloys and design of lightweight structures for automotive applications.

\section{Approach}

We are developing a material database and multiscale internal state variable (ISV) material and process models for lightweight alloys including magnesium, aluminum, and steel alloys. The database includes results from mechanical and microstructural characterization studies performed using advanced experimental techniques. Crystal plasticity finite element method and visco-plastic selfconsistent model (VPSC) [1] and other deformation models are also used to simulate the alloys deformation behaviors at the mesoscale. The ISV material model is formulated by a multiscale modeling approach where information from crystal plasticity, dislocation dynamics and molecular dynamics simulations are utilized to provide insights into the functional forms of the ISV's evolution equations and corresponding material parameters. Finally, a design framework contained in the cyberinfrastructure has been developed to help design structural components of new lightweight alloys. 


\section{Accomplishments}

- we developed physically-based and experimental-validated material models, extrusion and sheet process models, and corresponding design methodologies to enhance the workability of $\mathrm{Mg}$ alloys (extrusion: AM30, AZ61; sheet: AZ31). In particular, the work involved established material anisotropy relationships to understand the effect of material state (microstructure), product geometry and processing parameters on the mechanical properties, and final geometry (distortion) of formed Mg components.

- We established the fundamental knowledge of the extrusion process of $\mathrm{Mg}$ alloys to better understand their processing -structure - property relations. Particular alloys being studied were AM30 and AZ61. The research activities during the phase IV have been focused on the following: (i) performing lab-scale indirect extrusion experiments to characterize the evolution of microstructure during the process, (ii) extending the capability of the extrusion fixture to experimentally study weld seam formation, (iii) enhancing the current constitutive frameworks to increase the fidelity of the predictive response of $\mathrm{Mg}$ alloys with twinning, and (iv) carrying out finite element simulation of the extrusion process using Lagrangian (ABAQUS) and Eulerian (HyperXtrude) finite codes.

- An internal state variable (ISV) material model with mixed hardening laws for Mg AZ31 alloy was established, applicable for warm forming where twinning effect is not yet included.

- An implicit stress integration algorithm was implemented in Abaqus FEM software to solve sheet forming conditions. Sheet forming was simulated by Abaqus FEM software and VPSC model to capture the local texture and stress states.

\section{Publications}

Asle Zaeem, M., El Kadiri H., Horstemeyer, M.F., Wang, P., Mesarovic, S., Effect of the compositional strain on the diffusive interface thickness and on the phase transformation in a phase-field model for binary alloys, Journal of Phase Equilibria and Diffusion, 2011, 1-7.

Asle Zaeem, M., El Kadiri H., Wang, P., Horstemeyer, M.F., Investigating the effects of grain boundary energy anisotropy and second-phase particles on grain growth using a phase filed modle, Computational Materials Science, 50(201) 2488-2492.

Baird, B. Li, S. Parast, S. Horstemeyer, L. Jr. Hector, P. Wang, M.F. Horstemeyer, Localized twin bands in sheet bending of a magnesium alloy, Scripta Materialia, 67 (2012) 471-474.

El Kadiri, H., Oppedal, A.L., A crystal plasticity theory for latent harding by glide twinning through dislocation transmutation and twin accommodation effects, Journal of the Mechanics and Physics of Solids, 58(2010) 613-624.

Li, B., Joshi, S.P., Almagri, O., Ma, Q., Ramesh, K.T., Mukai, T., Rated-dependent hardening due to twinning in an ultrafine-grained magnesium alloy, Acta Materialia, 2012, 60:1818-1826.

Li, B., El Kadiri, H., Horstemeyer, M.F., Extended zonal dislocations mediating \{11-22\} twinning in Titanium, Philosophical Magazine, 92(2012) 1006-1022.

Ma, Q., El Kadiri, H., Oppedal, A.L., Baird, J.C., Horstemeyer, M.F., Cherkaoui, M., Twinning and double twinning upon compression of prismatic textures in an AM30 magnesium alloy, Scripta Materialia, 2011, 64(9): 813-816. 
Ma, Q., Li, B., Oppedal, A.L., Whittington, W.R., Horstemeyer, S.J., Marin, E.B., Wang, P., Horstemeyer, M.F., Strain rate dependence of twinning at $450^{\circ} \mathrm{C}$ and its effect on microstructure of an extruded magnesium alloy, Materials Science and Engineering A, 2013, 559:314-318.

Ma, Q., El Kadiri, H., Oppedal, A.L., Baird, J.C., Li, B., Horstemeyer, M.F., Vogel, S.C., Twinning effects in a rod-textured AM30 magnesium alloy, International Journal of Plasticity, 2012,29: 60-76.

Ma, Q., Li, B., Marin, E.B., Horstemeyer, S.J., Twinning-induced dynamic recrystallization in a magnesium alloy extruded at $450^{\circ} \mathrm{C}$, Scripta Materialia, 2011, 65(9): 823-826.

Oppedal, A.L., El Kadiri, H., Tomé, C.N., Kaschner, G.C., Vogel, S.C., Baird, J.C., Horstemeyer, M.F., Effect of dislocation transmutation on modeling hardening mechanisms by twinning in magnesium, International Journal of Plasticity, 2012,30-31: 41-61.

Zhang, X.Y., Li, B., Wu, X.L., Zhu, Y.T., Ma, Q., Liu, Q., Wang, P., Horstemeyer, M.F., Twin boundaries showing very large deviations from the twinning plane, Scripta Materialia, 2012,67:862-865.

\section{General Framework of Internal State Variable Material Model including Damage and Plasticity}

In order to capture the thinning and initiation of cracks during the sheet metal forming process, the elasto-viscoplastic model [2] has been extended to the coupled elasto-viscoplasticity damage model, named DMG model [3]. The material model was implemented in the user material subroutine VUMAT of Abaqus/Explicit for both 3D and shell elements using a fully implicit integration scheme.

To describe the damage mechanisms, we assume that the process of ductile fracture in magnesium occurs mainly due to the void nucleation, growth and finally coalescence into a microcrack (Figure 1).

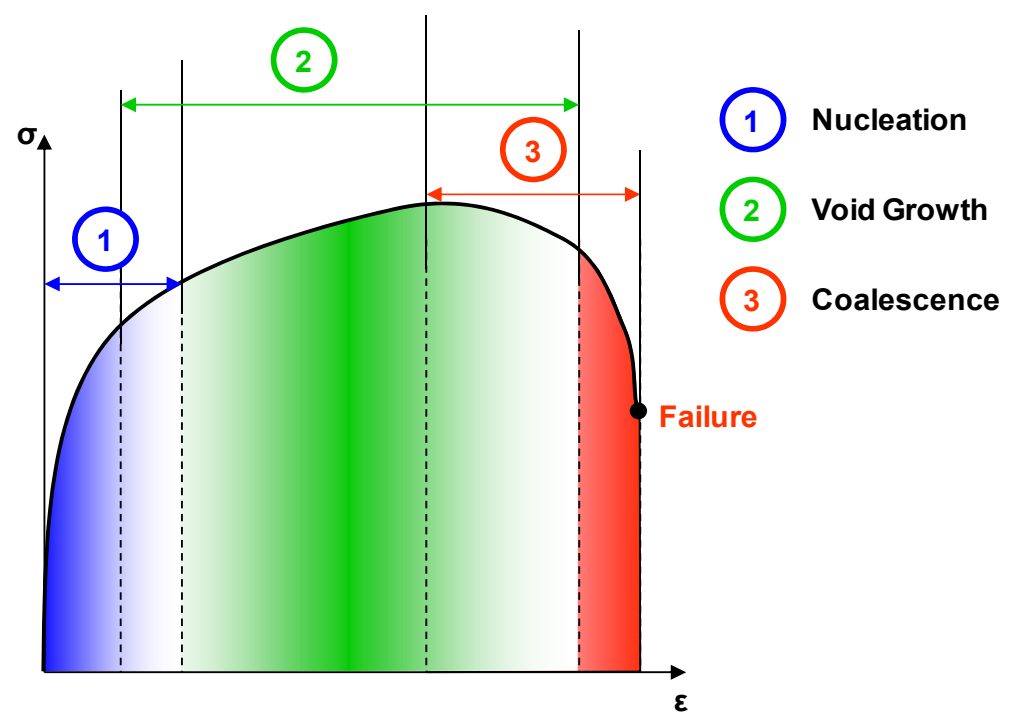

Figure 1: Sequences in a ductile fracture mechanism.

The total damage or void volume fraction is defined by

$$
\phi=C\left(\eta v_{v}+v_{p}\right)
$$


where $\eta$ is the void nucleation, $v_{v}$ the void growth of a spherical void, $v_{p}$ the void growth of large pores, and $C$ the void coalescence [Horstemeyer et al., 2000]. Using the conservation of mass, the damage rate can also be expressed as

$$
\dot{\phi}=(1-\phi) \operatorname{tr}\left(\boldsymbol{D}^{p}\right)
$$

where $\boldsymbol{D}^{p}$ is the plastic strain rate tensor is

$$
\boldsymbol{D}^{p}=\frac{1}{3} \frac{\dot{\gamma}}{\sqrt{1-\phi}} \mathbf{1}+\frac{\dot{\lambda}}{\sqrt{1-\phi}} \boldsymbol{n}
$$

which is composed of a volumetric part parallel to the unit tensor $\mathbf{1}$, and a deviatoric part parallel to the plastic normal tensor $\boldsymbol{n}$. The term $\dot{\gamma}$ is the plastic volumetric strain rate, and $\dot{\lambda}$ the plastic equivalent strain rate defined by the plastic flow rule

$$
\dot{\lambda}=\left\{\begin{array}{c}
f(T) \sinh \left[\frac{|\boldsymbol{s}-\boldsymbol{\alpha}|-\kappa-Y(T)}{(1-\phi) V(T)}\right] \quad \text { if }|\boldsymbol{s}-\boldsymbol{\alpha}|-\kappa-Y(T) \geq 0 \\
0 \quad \text { if }|\boldsymbol{s}-\boldsymbol{\alpha}|-\kappa-Y(T)<0
\end{array},\right.
$$

The plastic flow rule leads to a Mises type yield function defined by

$$
f=|\boldsymbol{s}-\boldsymbol{\alpha}|-\kappa-Y(T)-(1-\phi) V(T) \sinh ^{-1}\left[\frac{\dot{\lambda}_{p}}{f(T)}\right]=0
$$

where $Y(T), f(T)$ and $V(T)$ are temperature dependent functions and are related to yielding with an Arrhenius-type temperature dependence. The isotropic hardening $\kappa$ reflects the effect of the global dislocation density, and is represented by the growth in size of the yield surface while the center remains at a fixed point in stress space. The kinematic hardening $\boldsymbol{\alpha}$, also called Bauschinger effect, reflects the effect of anisotropic dislocation density, and is represented by the translation of the center of the yield surface in stress space, while the size remains fixed.

Material parameters of the ISV model described above for selected alloys (steel, aluminum, magnesium) are all contained in the cyberinfrastructure website for use.

\section{Internal State Variable Constitutive Model for Extrusion Modeling}

The research version of CAVS's macroscopic constitutive framework was used to model the extrusion process using HyperXtrude, once this code has the user interface needed to implement complex material models. Meanwhile, some work has been performed to extend the ISV model to account for recrystallization and grain growth, phenomena typically present during hot extrusion. A summary of the ISV model equations without recrystallization is presented in Figure 2. The same figure presents the experimental data for AZ61 used to fit the material constants of the model. Note that the 
stress softening observed in the experimental data is due to recrystallization. As this version does not account for this phenomenon, the fitting was mainly performed using the hardening part of the curve. This version of the model with the computed constants is being used to model the lab-scale conical die extrusion experiments of AZ61 using ABAQUS and a UMAT that implements the numerical integration of the model.

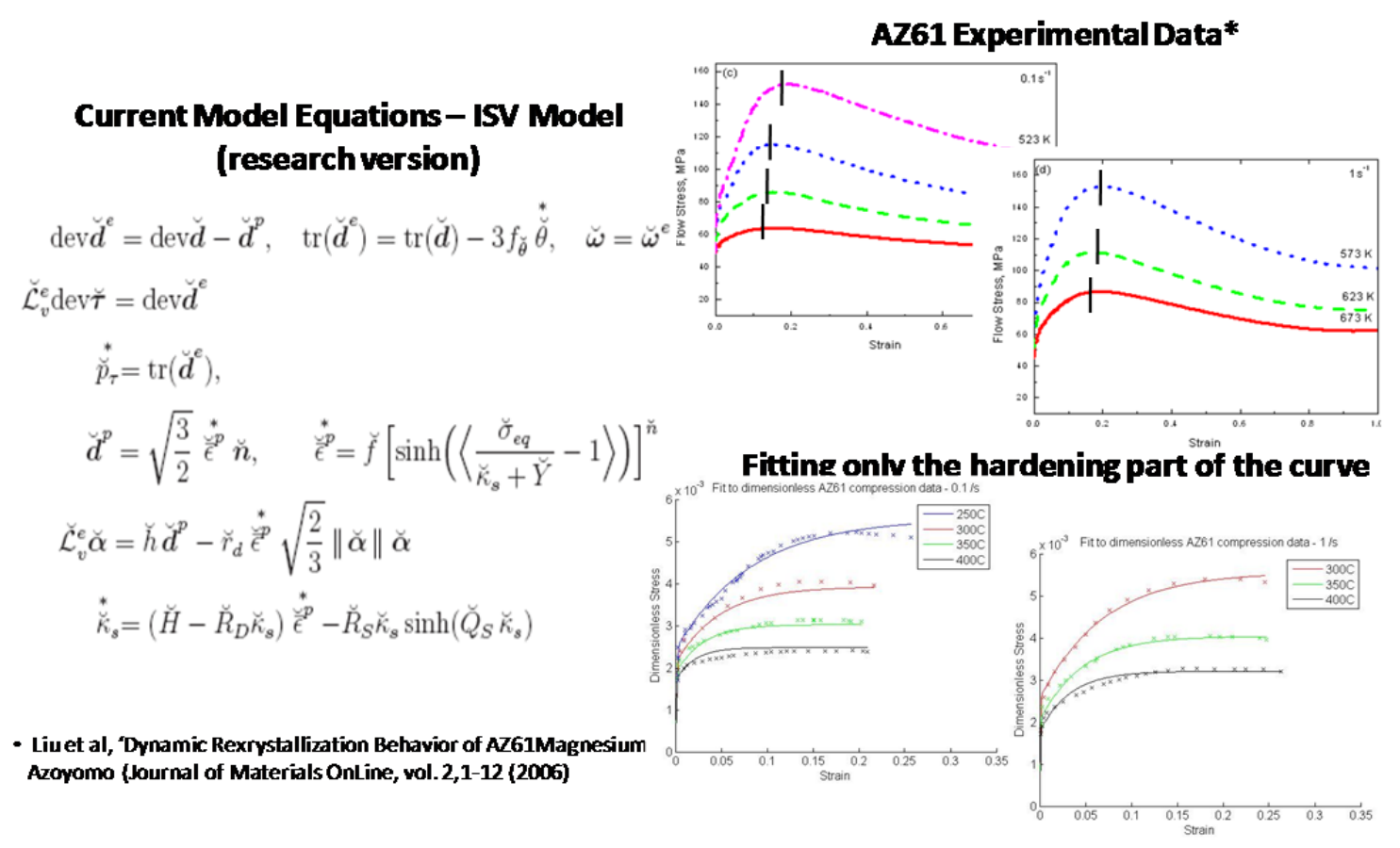

Figure 2: Current research version of ISV material model fitted to AZ61 experimental data. Fitting was limited to the hardening part of the curves.

Recrystallization is a complex inhomogeneous process in which nucleation and growth of new strain-free grains replaced the worked microstructure of a strained material. It is mainly due to the motion of grain and sub-grain boundaries. As the boundaries move, they sweep away the dislocation structure, leaving a strain-free material with a very low dislocation density. When the expanding grain reaches a critical size, it becomes a stable recrystallized grain that can continue growing due to grain growth processes. The driving force for recrystallization is the energy stored in the dislocation structures. In this work we attempt to model recrystallization using the ISV material modeling framework, see Figure 2. For this purpose, we have extended the above constitutive equations with features to capture these phenomena. The specific changes that have been introduced are:

- Add the effect of grain size to both the initial mechanical strength $Y$ and the evolution of the isotropic hardening variable, $\kappa$, a variable that physically represents the evolution of statistically stored dislocation.

- Add a recovery or softening term to the evolution of $\kappa$ whose effect is triggered when the energy stored in the material during deformation reaches a critical value,

- Develop physically-based evolution equations for the fraction of recrystallized grains and grain size, 
- Incorporate an internal state variable for geometrically necessary dislocations through a stress-like variable (mis-orientation variable) that effectively accounts for the stored energy due to misorientations of the deformation-induced dislocation boundaries.

The extended model is presented in Figure 3. The same figure also presents the fitting of the model to AZ61 stress-strain data at $300^{\circ} \mathrm{C}$ and $1.0 \mathrm{~s}^{-1}$. As displayed by the figure, the model is able to capture the stress softening due to recrystallization. Current work is underway to fit the model to other deformation conditions.

\section{Model Equations for Recrystallization Model}

$$
\begin{aligned}
& \operatorname{dev} \breve{\tau}^{\nabla}=\operatorname{dev} \breve{d}^{\boldsymbol{Q}}=\operatorname{dev} \breve{\boldsymbol{d}}-\breve{d}^{p}
\end{aligned}
$$

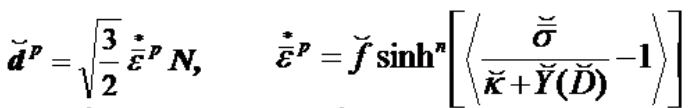

$$
\begin{aligned}
& \dot{\widetilde{\kappa}}_{u}=\left(\breve{H}_{1}+\frac{\breve{H}_{2}}{\breve{\kappa}_{z} \breve{D}^{\prime}}+\frac{\breve{H}_{3} \breve{\zeta}_{u}}{\breve{\kappa}_{u}}\right) \dot{\varepsilon}^{p}-\left(\breve{R}_{d} \dot{\bar{\varepsilon}}^{p}+\breve{R}_{s} \sinh \left(\breve{Q}_{s} \breve{\kappa}_{u}\right)\right) \breve{K}_{u}
\end{aligned}
$$

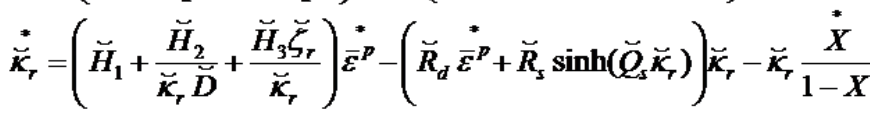

$$
\begin{aligned}
& \dot{\zeta}_{u}=\frac{\breve{H}_{4}}{\breve{D}_{u}} \breve{\zeta}^{1-1 / r} \bar{\varepsilon}^{p} \\
& \dot{\zeta}_{r}=\frac{\breve{H}_{4}}{\breve{D}^{\prime}} \breve{\zeta}_{r}^{1-1 / r} \dot{\bar{\varepsilon}}^{p}-\breve{\zeta}_{r} \frac{\dot{X}}{1-X} \\
& \dot{X}=\frac{\mu(\theta)}{\mu_{0}} \frac{\exp \left(-\breve{Q}_{6} / \breve{\theta}\right)}{\breve{\theta}}\left[1-\exp \left(-\breve{C} \breve{\zeta}_{u}{ }^{m}\right)\right]\left(\breve{C}_{z} \breve{k}_{u}{ }^{2}+\breve{C}_{\zeta} \breve{\zeta}_{u}{ }^{2}\right) X^{\Lambda}(1-X)^{B}
\end{aligned}
$$

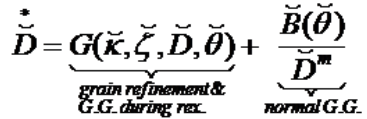

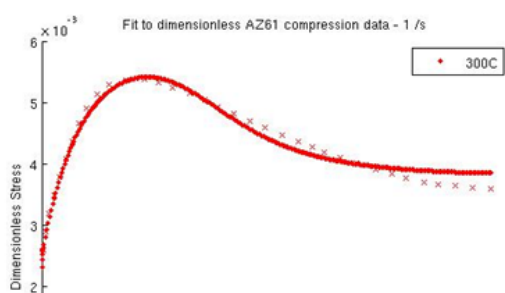

Fitted Stress-Strain Response* AZ61, 300C, $1.0 \mathrm{~s}^{-1}$
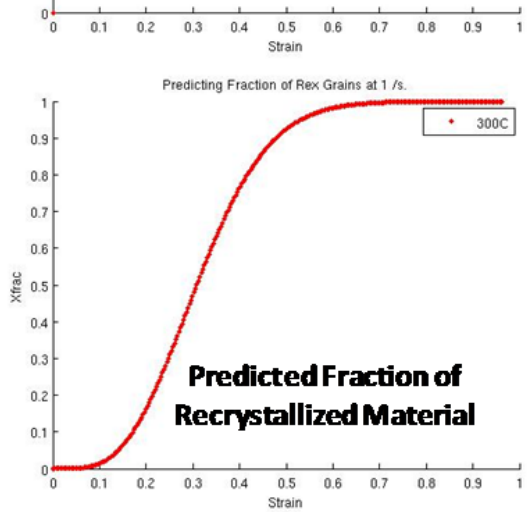

Figure 3: Internal State Variable material model extended to account for recrystallization.

\section{Internal State Variable Constitutive Model for Sheet Forming}

The microstructure-plasticity coupled with damage DMG model (ISV) was used in this work to describe the nonlinear response of the material behavior. A robust fully-implicit stress integration algorithm was developed and implemented in the user material subroutine Vumat of Abaqus/Explicit for 3D, plane strain, and plane stress conditions. Plasticity and hardening parameters were calibrated from experimental stress-stress curves or from the evolution of polycrystal-based yield surface (PCYS). Figure 4 shows the evolution of the two-dimensional projection of PCYS using experimental data on tension and compression performed on samples from a $12 \mathrm{~mm}$ thick AZ31B sheet. 

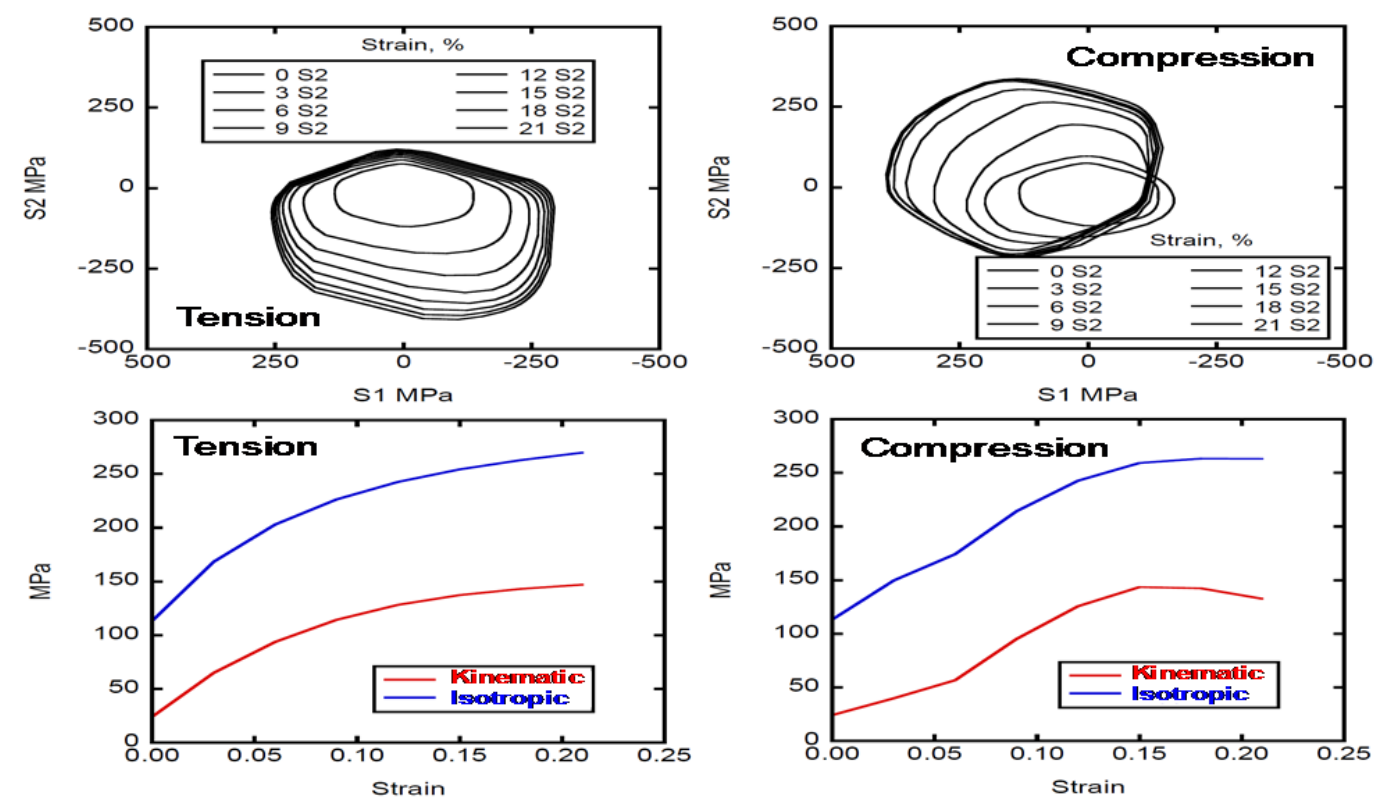

Figure 4. Polycrystal yield surface (PCYS) for tension and compression using the VPSC code and their respective isotropic and kinematic hardening evolution curves.

The PCYS was obtained by incrementally probing it along different strain-paths (i.e., applying different strain-rates $\dot{\varepsilon}_{i j}$ and obtaining the corresponding stress response $\sigma_{i j}$ ) in the VPSC crystal code. By averaging the size of the yield surface and tracking the center of the yield surface, PCYS plots were processed to determine the evolution of isotropic and kinematic hardening for both isotropic and kinematic hardenings. The DMG model with AZ31B plasticity parameters was applied to pan forming and bending simulations (Figure 5).

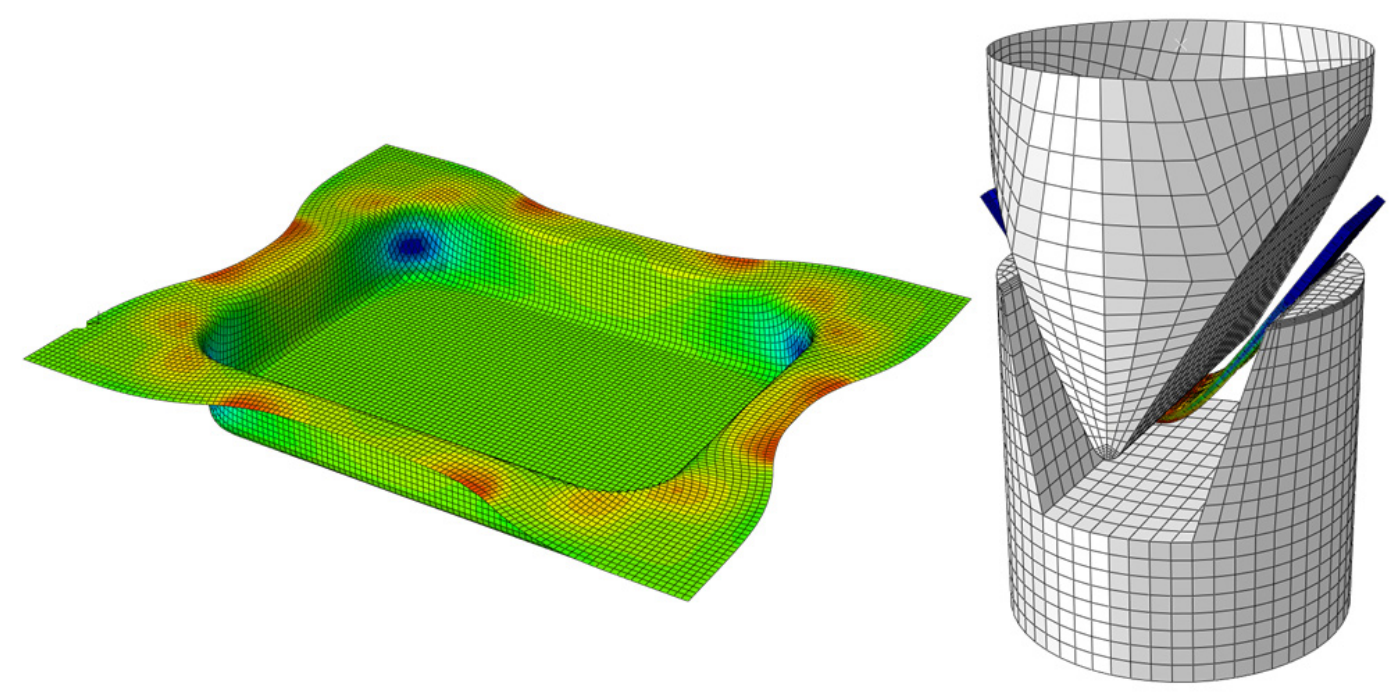

Figure 5. Pan forming and bending simulations using the DMG model. 


\section{Mesoscale Polycrystal Plasticity of Extruded Mg Alloys}

Mesoscale crystal plasticity formulations were developed from a MSU CAVS in-house model and used in Abaqus and a VPSC code developed by Carlos Tome at Los Alamos National Laboratory. The experimental part included evaluation of macro-texture, micro-texture, mechanical behavior and optical microstructure measurements of selected Mg alloys. The modeling part includes simulations of stressstrain curves of $\mathrm{Mg}$ alloys at various temperatures and various strain rates and a dislocation transmutation model development that arose from twinning.

In the experimental part, we conducted simple compression tests at different directions $\left(0^{\circ}\right.$, $30^{\circ}, 60^{\circ}$ and $90^{\circ}$ ) with respect to the extrusion direction of the AM30 billet. In addition, we performed tension and high strain rates compression and reloading compression tests of pure $\mathrm{Mg}$ and $\mathrm{AM} 30$. Optical microstructure of AM30 at intermediate strain levels was also characterized to confirm the presence of primary twins and double twins in AM30. In addition, we performed fine-step EBSD scanning on $\mathrm{AM} 30$ and pure magnesium deformed samples to investigate the distribution of primary $\{10 \overline{1} 1\}$ twins, $\{10 \overline{1} 1\}-\{10 \overline{1} 2\}$ double twins and $\{10 \overline{1} 3\}-\{10 \overline{1} 2\}$ double twins in samples.

In the simulation part, we employed visco-plasticity self-consistent models (VPSC) for magnesium alloys at different temperatures and strain rates. We simulated mechanical behavior of AM30 and AZ61 alloys at $25^{\circ} \mathrm{C}, 190^{\circ} \mathrm{C}, 300^{\circ} \mathrm{C}$ and $400^{\circ} \mathrm{C}$ temperature and at $0.001 / \mathrm{S}, 0.1 / \mathrm{S}$ and $1 / \mathrm{S}$ strain rates based on Voce hardening law within VPSC formulation (Figure 6).

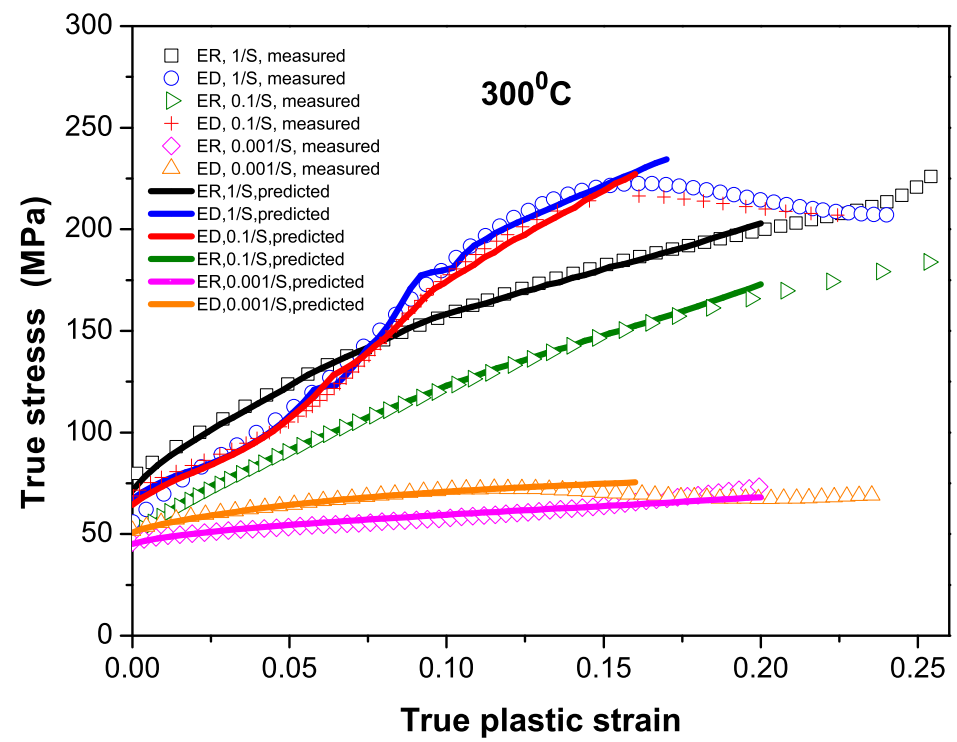

Figure 6. Measured and predicted compresion stress-strain curves of $A M 30$ alloy at $300^{\circ} \mathrm{C}$ and at $1 / \mathrm{S}$, $0.1 / \mathrm{S}, 0.001 / \mathrm{S}$ strain rates along ED and ER directions of the AM30 alloy. (ED is the extrusion direction of the AM30 billet, ER is defined as radial direction of the billet.)

The primary simulation results show that twinning behavior is significantly influenced by temperature and strain rate. However, we observed that the effect of strain rate on mechanical behavior of $\mathrm{Mg}$ was complicated when correlated to twinning. For example, the strain rates of $0.1 / \mathrm{S}$ 
and 1/S appear having similar responses on stress-strain curves for samples deformed along the extrusion direction (ED), but have a very different response for samples deformed along the radial direction of an extrudate (ER) at $300^{\circ} \mathrm{C}$ (Figure 6). A dislocation density model was used to simulate the mechanical behavior of AM30 and AZ61 alloy at $25^{\circ} \mathrm{C}$. The dislocation transmutation upon twinning model was proposed and will be used to simulate the mesoscale plasticity of $\mathrm{Mg}$.

The measured and VPSC predicted stress-strain curves at room temperature and $450{ }^{\circ} \mathrm{C}$ and the corresponding hardening curves are presented in Figures 7 and 8, respectively.
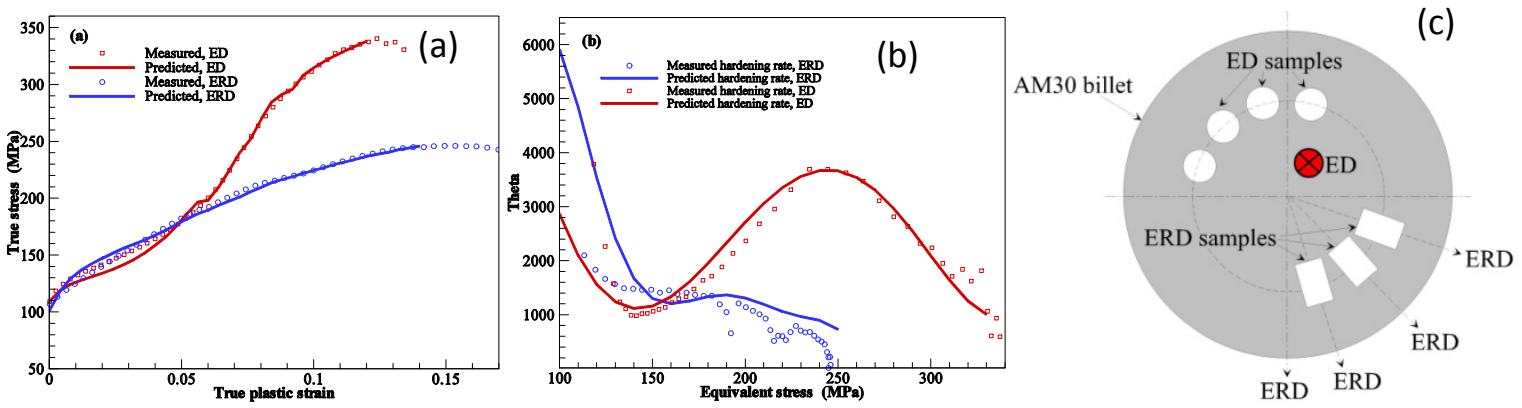

Figure 7. Measured and VPSC predicted mechanical results of AM30 at $25^{\circ} \mathrm{C}$. (a) Measured and VPSC predicted stress-strain curves; (b) measured and VPSC predicted hardening curves; and (c) the extrusion direction (ED) samples and the extrusion radial direction (ERD) samples in the AM30 billet.

At room temperature, the extrusion direction (ED) $\sigma-\varepsilon$ curve shows a typical " $s$ " shape that signifies profuse twinning. Twinning could be observed by the very high hardening rate in the stressstrain curves along the ED. The extrusion radial direction (ERD) $\sigma-\varepsilon$ curve shows the normal plasticity response. At $450^{\circ} \mathrm{C}$, the $\sigma-\varepsilon$ curves are dependent on the strain rate and have no such $\mathrm{S}$ shape curves. For the ER, hardening is weaker than what was observed in the ED. At $450{ }^{\circ} \mathrm{C}$, dynamic recrystallization (DRX) is pervasive and results in the softening of the $\sigma-\varepsilon$ curves.
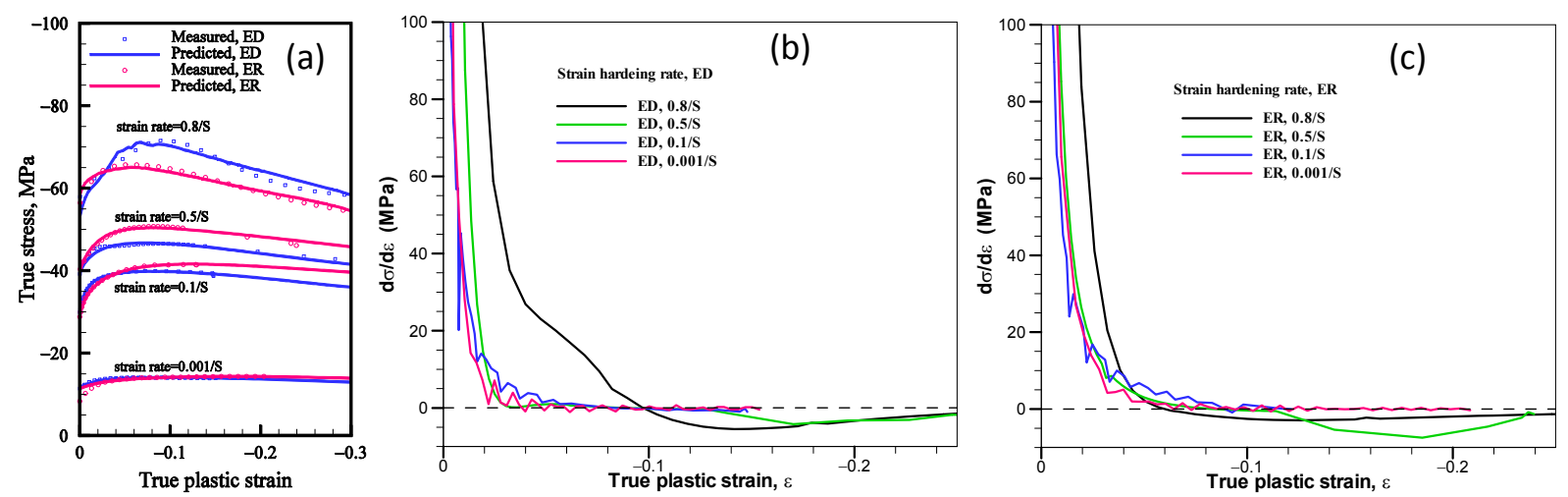

Figure 8. Measured and VPSC predicted mechanical results of AM30 at $450{ }^{\circ} \mathrm{C}$. (a) Stress-strain curves of extrusion direction (ED) and extrusion radial direction (ER); (b) measured hardening curves at the ED, and (c) measured hardening curves at the ER. 


\section{Hardening mechanisms upon profuse twinning in pure magnesium}

Twinning is a common deformation mode in Mg alloys. Twinning plays important hardening roles on mechanical property of $\mathrm{Mg}$ alloys. Textured hexagonal close packed double-lattice structures show stronger anisotropy than textured single-lattice structures. The reason lies behind the necessity to activate both glide twinning and hard slip dislocation modes. Although the mechanisms behind activation of dislocations with non-basal Burgers vectors are still not fundamentally understood, the effect of twinning on hardening presents the most substantial challenge to modelers using polycrystal plasticity simulations.

The origin of the increasing strain hardening rate regime (Regime II) characteristic of the sigmoidal curve upon profuse twinning is still not fundamentally clear. Previous successful attempts to fit the stress-strain behaviors based on a Hall-Petch effect by twin segmentation had systemically led to discrepancies in predicting intermediate textures and/or twin volume fraction evolutions [4].

A recent dislocation-based hardening rule incorporated into the Visco-Plastic Self-Consistent (VPSC) model allows slip and twinning to be physically coupled in the simulations [5]. In this research activity, we investigate hardening mechanisms in pure magnesium and apply a dislocation based formalism to model anisotropy. In contrast to magnesium alloys, we show that pure magnesium under large strains develops substantial multivariant twinning and multifold twinning. These twinning phenomena are accompanied with a marked grain refinement and blunting of former twin boundaries. The blunting of former twin boundaries points to severe accommodation effects in the soft matrix that cause the twin boundary to lose coherency. Thus, multivariant and multifold twinning takes place to accommodate further deformation, but the subsequent twin-twin interactions arise to contribute to

material hardening. The strain path anisotropy related to the saturation stresses revealed major missing links for comprehending hardening by twinning and substantiated an effect related to dislocation transmutation by twinning shear. The simulation results that were compared to experimental counterparts include the stress-strain behavior, volume fraction of twins, and small and large scale texture obtained through Electron Backscattered Diffraction and Neutron Diffraction techniques.

Anisotropy in pure magnesium is characterized by a more complex twinning phenomena compared to magnesium alloys. Substantial multivariant and multifold twinning arose in the matrix and was consistent with severe accommodation effects that could have potentially occurred in the relatively soft matrix. The accommodation effects seemed to have been so severe that the primary twin boundaries lost coherency and triggered other twin variants and modes throughout the matrix so further strain could be accommodated. As a consequence, several load drops appeared in the strain hardening rate - effective stress curves (theta-sigma) characteristic of the lengthwise thickening of the twins. The accommodation effects ultimately led to multivariant and multifold twinning and in turn substantial grain refinement and blunting of former twin boundaries as they lost coherency with the matrix. This radical change in the microstructure and texture of pure magnesium upon profuse twinning was not captured in the crystal plasticity simulations although it could have a noticeable effect on hardening. The grain refinement was found to not be induced by dynamic recrystallization at room temperature as previously observed in the literature for pure magnesium [6]. 
Not utilizing the Hall-Petch effect by twin segmentation allowed us to realize that rotation to hard orientation by twinning is sufficient to cause a Regime II of increasing hardening rate. However, the extent of the hardening rate was substantially underestimated by the model unless a higher hardening rate in the twins was used when compared to the parent. In this case, the other loading orientations where twinning was limited, such as TTC in basal textures, were still satisfactorily predicted. A fitting parameter called the twin storage factor had a dominant effect on comparing the model to the experimental stress-strain behavior when extra hardening in the twins was considered. This is consistent with the transmutation theory put forward by El Kadiri and Oppedal [7]. However, it is emphasized that according to the transmutation theory, the higher hardening in the twins over that in the parent is not due to an increase in the dislocation generation in the twin over that in the parent. Rather, it is due to an increase of latent hardening in the twins by a rapid increase in dislocation multiplicity within the twins.

When considering that the twin regions harden more than the parent, not only were the stressstrain behaviors well predicted but the strain hardening rate - effective stress curve (theta-sigma) was as well. This kind of model correlation to experimental data was not previously reported in the literature. A hardening level within the twin higher than four times than that in the parent was necessary to satisfactorily capture the theta-sigma curve (Figure 9).
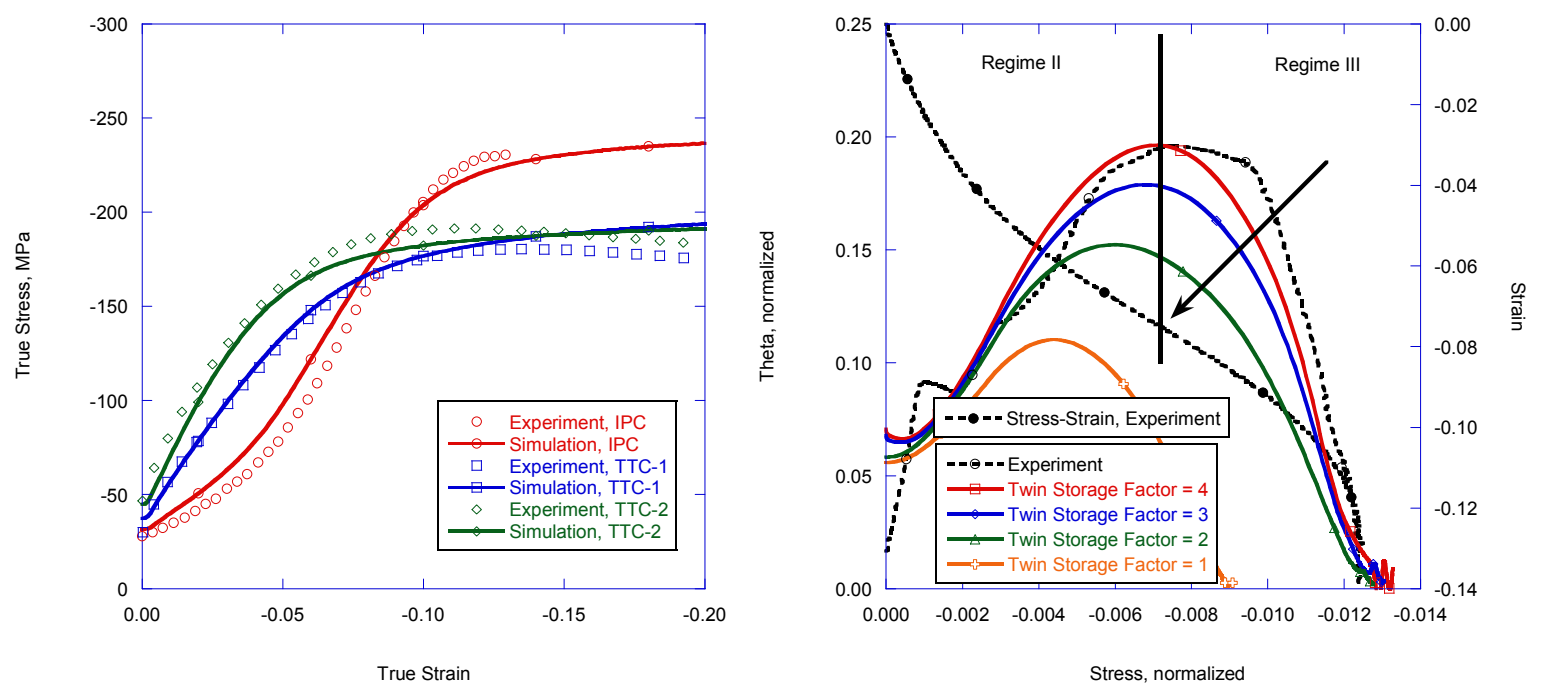

Figure 9. (a) Results of compression tests and simulations for pure magnesium tested along the throughthickness direction (TTC) that corresponds to the normal direction of the sheet, and along the in-plane (IPC) direction that corresponds to the transverse direction of the sheet.(b) Strain hardening rate versus stress $\left(\theta=\frac{d \sigma}{d \varepsilon}, \theta\right.$ vs. $\left.\sigma\right)$ plots from compression tests and simulation results with contractions twins and four values of the twin storage factor, namely unity, two, three and four on a pure magnesium tested along the through-thickness direction (TTC) that corresponds to the normal direction of the sheet, and along the in-plane direction (IPC) that corresponds to the transverse direction of the sheet. Stress values were normalized by $\sigma_{\text {normalized }}=\frac{\left(\sigma-\sigma_{y}\right)}{\mu}$ where $\sigma_{y}$ is yield stress and $\mu$ is shear modulus. 


\section{Modeling the Extrusion Process of Mg Alloys}

MsSt continues to develop the science-based knowledge for modeling the extrusion process of Mg alloys. Particular alloys being studied are AM30 and AZ61. During the last years, the activities have been focused on (i) lab-scale extrusion experiments to generate mechanical and microstructure data for validation of the modeling tools, (ii) numerical simulation of the lab-scale process using both Lagrangian and Eulerian-ALE finite element codes, and (iii) numerical simulation of the industry scale extrusion processing using FEM and VPSC model.

\section{AZ61 magnesium alloy extrusion in lab-scale}

The lab-scale indirect extrusion experiments with flat dies were extended to consider the extrusion of $\mathrm{Mg}$ AZ61 with a $60^{\circ}$ conical (streamlined) die and an orifice diameter of $1 / 2^{\prime \prime}$ (extrusion ratio $=6.25)$. This case was designed with the purpose to use both ABAQUS and HyperXtrude to simulate the process and compared the predictions with the recorded mechanical and microstructure data. Figure 10 shows the microstructure evolution (texture and grain morphology) at particular material points during this process. Processing conditions used were a ram speed of $5 \mathrm{~mm} / \mathrm{s}$ and a billet temperature of $482{ }^{\circ} \mathrm{C}$.

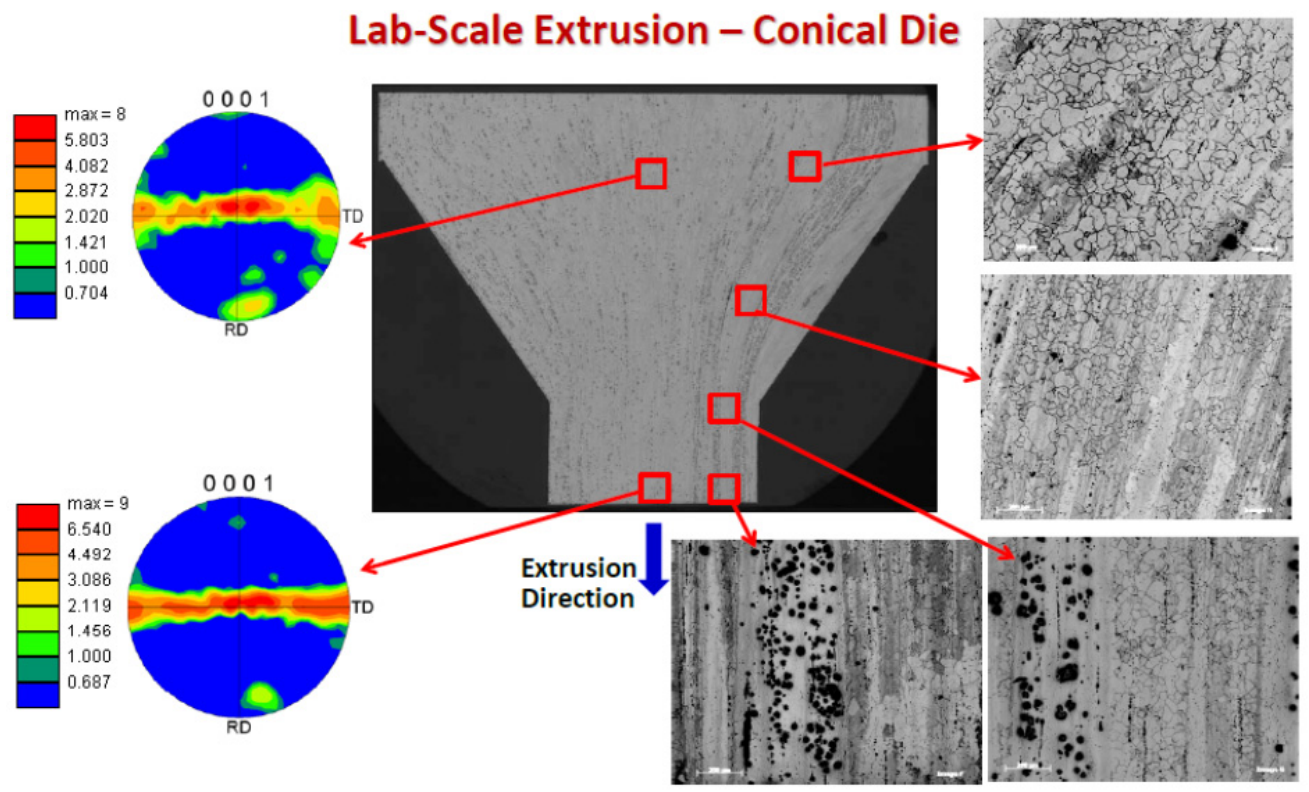

Figure 10: Microstructure characterization study (texture, grain structure) at different material points of extruded AZ61 billet.

HyperXtrude was used to simulate the conical die extrusion process using an axisymmetric model. Due to current limitations of the code to allow user material routines for complex models, texture predictions were performed using a Taylor-based crystal plasticity model in an uncouple approach, where deformation history along streamlines was provided by the code. Figure 11 presents the predicted texture along a streamline close to the workpiece center. As noted, the rod-type predicted texture is similar to the one observed in the experiments (Figure 10). 


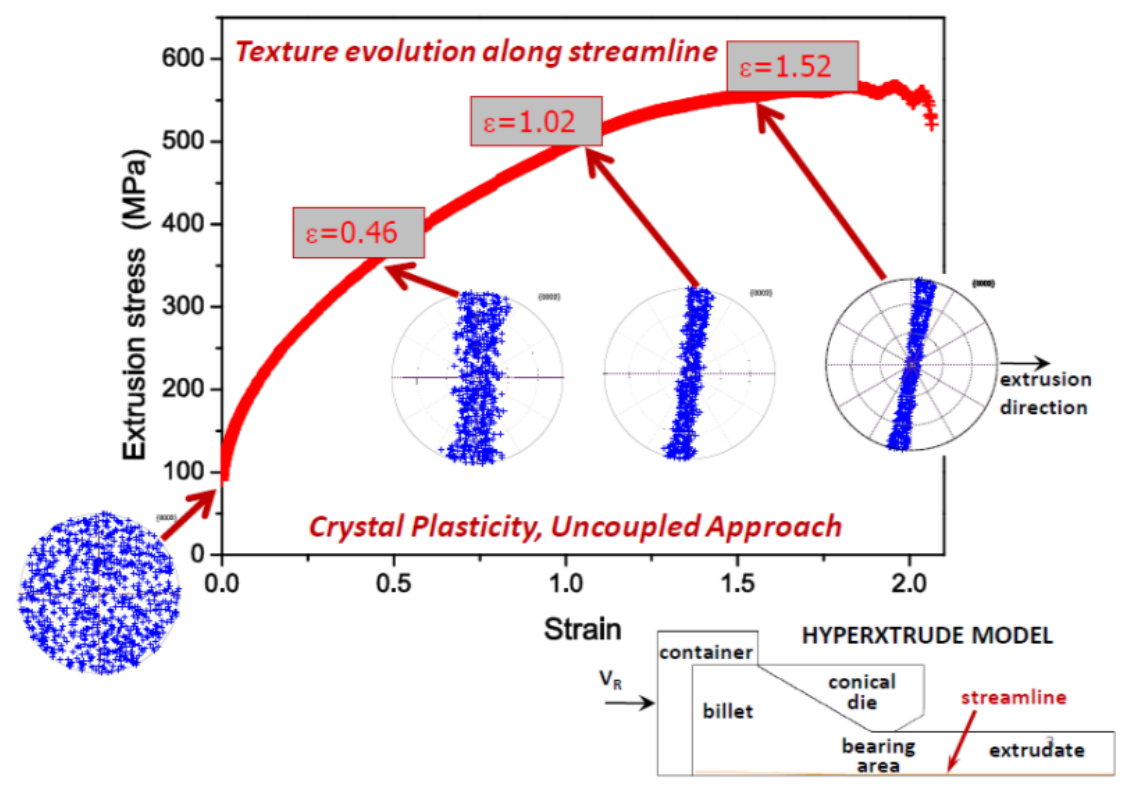

Figure 11: Texture evolution along a streamline predicted by HyperXtrude and crystal plasticity (uncouple approach).

Current efforts in this task are focused on performing lab-scale experiments to study weldseam formation using a two porthole die (splitter), studying recrystallization process using a channel die compression fixture, carrying simulation of the conical die extrusion process using ABAQUS, and employing user defined functions (UDF) in HyperXtrude to couple our own macroscopic constitutive models with the code.

\section{$\underline{\text { Lab-Scale Extrusion Fixture and Extrusion Experiments }}$}

The lab-scale indirect extrusion fixture has been developed to quantify processing-structureproperty relations of extruded Mg AZ61 and AM30. The main goals here are (i) to understand the influence of process parameters on the microstructure and mechanical properties of extruded material, and (ii) to generate experimental data for validation of the modeling tools (finite element and material models). The lab-scale extrusion fixture contains (i) a chamber, (ii) a replaceable die, and (iii) a base. The chamber is made of two parts (top - bottom), each part having an internal sleeve. A clamp holds these two parts together. The designed fixture and its set-up on the Instron machine for the extrusion experiments are shown in Figure 12.

This lab-scale extrusion process is defined by three processing parameters: extrusion ratio (ER), billet temperature, and ram speed. A parametric study of the process is being performed by varying these parameters one-at-a-time to see their effect on (i) the metal flow patterns, (ii) the microstructure evolution, and (iii) the mechanical properties of the Mg alloys. 

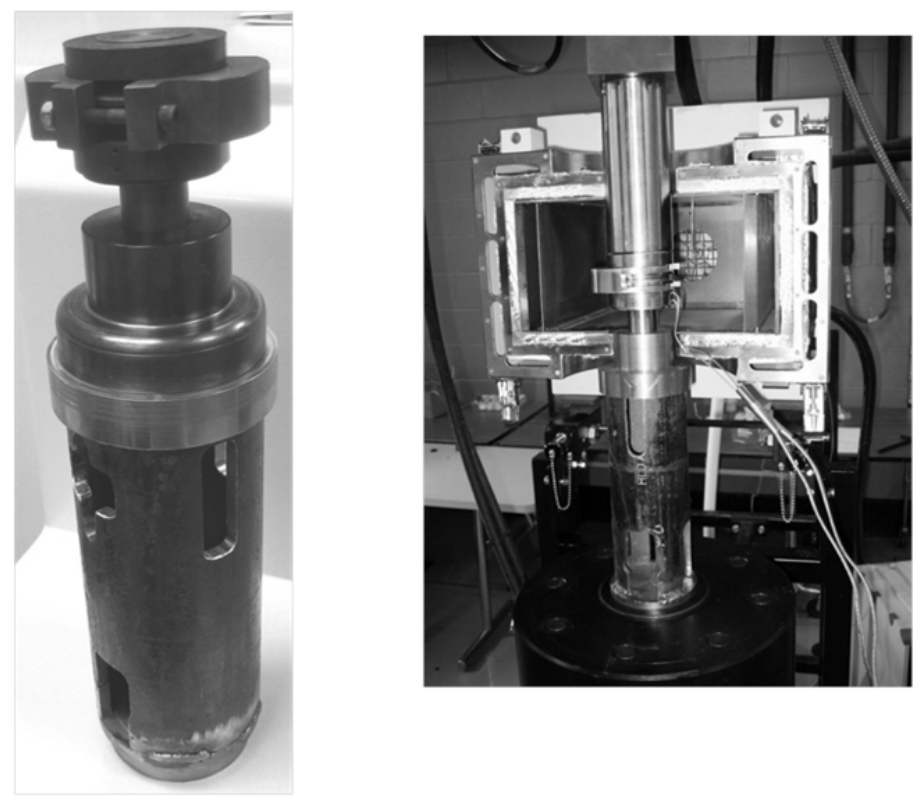

Figure 12. Design of the indirect extrusion fixture and set-up on the Instron machine.

A test matrix is given in Table 1. The sample size is $31.75 \mathrm{~mm}$ diameter $\times 304.8 \mathrm{~mm}$ length, the bearing length is $1.71 \mathrm{~mm}$, and the orifice diameters are $6.35 \mathrm{~mm}(E R=25)$ and $3.1 \mathrm{~mm}$ (ER=100). The $\mathrm{Mg}$ alloy being studied currently is AZ61.

Table 1. Lab-scale Extrusion Parameters

\begin{tabular}{|l|l|l|}
\hline Billet Temp. & Ram Speed & ER \\
\hline $454 \mathrm{C} / 850 \mathrm{~F}$ & $5,10 \mathrm{~mm} / \mathrm{min}$ & 25,100 \\
\hline $482 \mathrm{C} / 900 \mathrm{~F}$ & $5,10 \mathrm{~mm} / \mathrm{min}$ & 25,100 \\
\hline $510 \mathrm{C} / 950 \mathrm{~F}$ & $5,10 \mathrm{~mm} / \mathrm{min}$ & 25,100 \\
\hline
\end{tabular}

Interrupted extrusion experiments for selected cases of Table 1 have been performed to study the flow patterns and microstructure evolution of the material during the extrusion process. Flow patterns for Mg AZ61 samples extruded at $25 \%, 50 \%$, and $75 \%$ of the length have been determined under specified processing conditions (ram speed $=5 \mathrm{~mm} / \mathrm{min}$, billet temperature $=454^{\circ} \mathrm{C}$, and $\mathrm{ER}=$ 25). The microstructure evolution during extrusion has been characterized by performing EBSD studies on the partially extruded billets. In particular, microstructure features at material points along three streamlines have been determined. Figure 13 shows the evolution of texture along one streamline (center) on the $25 \%$ extruded billet. Clearly, at the selected material point, the texture of the preextruded billet evolves towards a rod-type texture typical of extrusion. Shearing effects due to the die wall, which usually tend to weaken this texture, are not predominant at these points because indirect extrusion minimizes the frictional effect as compared to direct extrusion. Figure 14 presents a number of microstructure aspects of a material point at the center of the bearing area. In particular, the grain 
structure is being fully characterized through inverse pole figures and plots for grain orientation spread and grain size. This material database is currently being used to enhance/validate internal state variable and crystal plasticity constitutive models for Mg alloys.

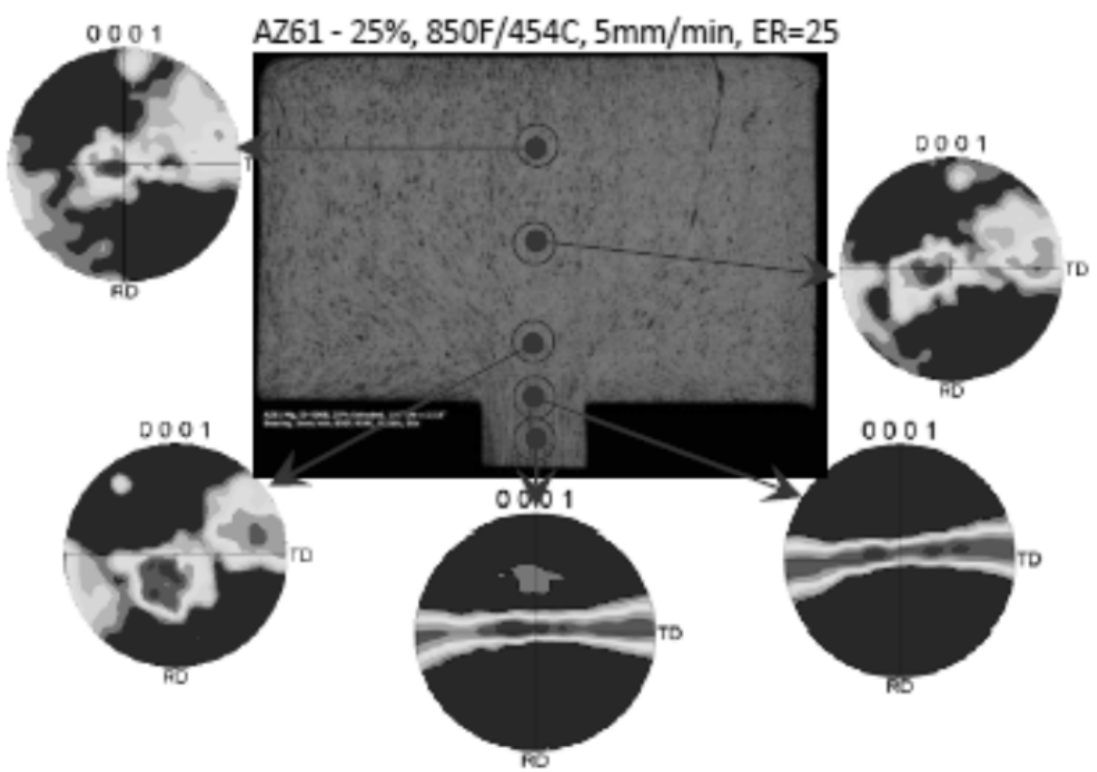

Figure 13. Evolution of texture at material points along a streamline through the center of the $25 \%$ partially extruded sample.

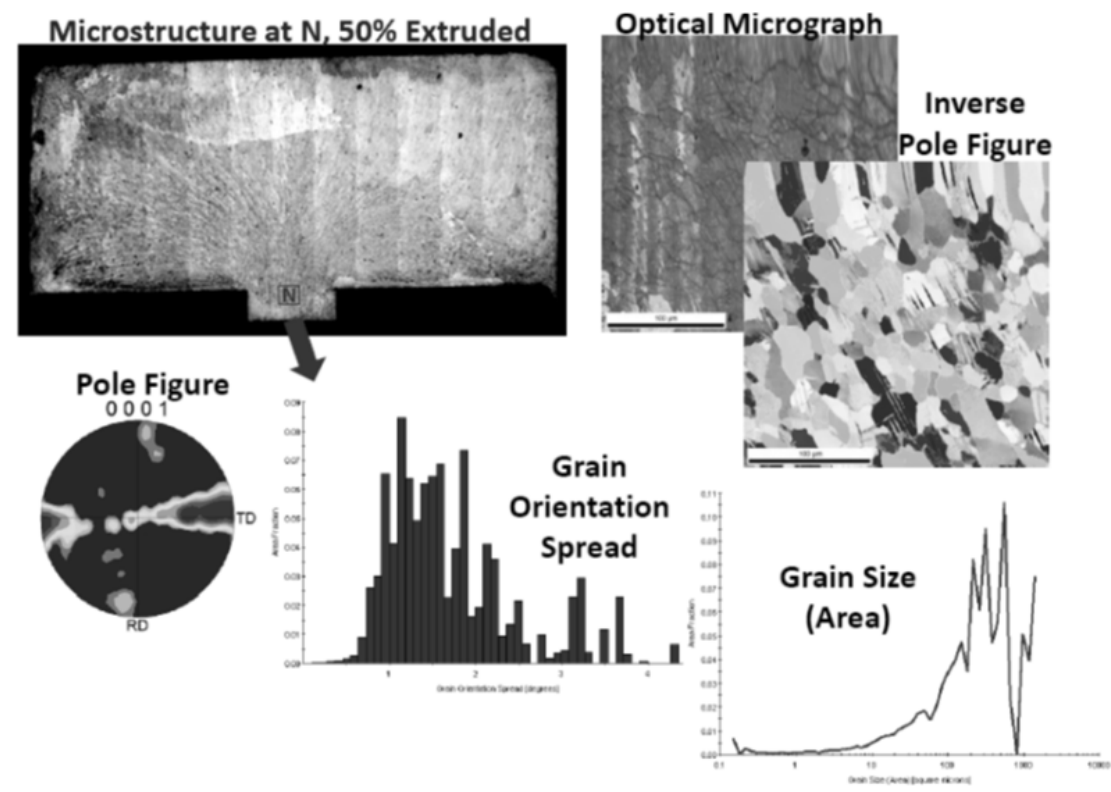

Figure 14: Microstructure characterization study (texture, grain structure) at a material point at the center of the bearing area. 
A new research activity identified in this task is the experimental analysis of longitudinal weld or seam welds that are typically present in extruded hollow profiles. For robust structural applications, the mechanical properties of the extruded product at or near these welds should not be inferior to those of the rest of the extrudate. As such, understanding the formation of weld seams in extruded $\mathrm{Mg}$ alloys and the processing parameters that affect their mechanical properties (strength, ductility) will contribute to the fundamental knowledge of extrusion process for magnesium alloys as well as will enhance the structural applications of $\mathrm{Mg}$ extruded components. Weld seams are usually produced using extrusion dies having webs/hollow channels and weld chambers (porthole dies). The weld seams form as the metal flowing through the die splits over each web and then rejoins and welds together at the rear side of the webs (weld chamber) before passing through the bearing zone. The quality of the solid state bond formed at these seams depends on a number of factors, including processing time, temperature, and pressure.

In this task, the solid-state bonding process that occurs in porthole dies of hollow Mg extrusions will be examined using a "splitter" placed between the chamber and the die. In essence the splitter is a disk with a web that divides the metal flow into two material streams. The material is joined again at the rear of the web forming a solid state bond. The rear end of the web has a butt-ended shape which is apart $1.71 \mathrm{~mm}$ from the bearing area. This very narrow cylindrical space constitutes the weld chamber. The extruded profile obtained in this die will be solid and will contain an extrusion seam in the middle corresponding to the location of the web. Figure 15 presents the top and bottom parts of the preliminary design of the splitter, while Figure 16 displays the set-up of the splitter in the lab-scale extrusion fixture. Preliminary extrusion weld seam experiments using the above splitter have been performed using 1100F Aluminum due to our limited material stock on Mg alloys. Processing conditions were: billet temperature $300^{\circ} \mathrm{C}$, die speed $5 \mathrm{~mm} / \mathrm{min}$, and ER of 25 . Figure 17 a shows the beginning of the extrudate where the two-welded metals streams are clearly observed. The extrudate shows a characteristic groove due to the particular splitter design. Figure 17b presents an optical micrograph at the center of the extrudate. At the magnification used (600X), traces of the weld seam can be observed.
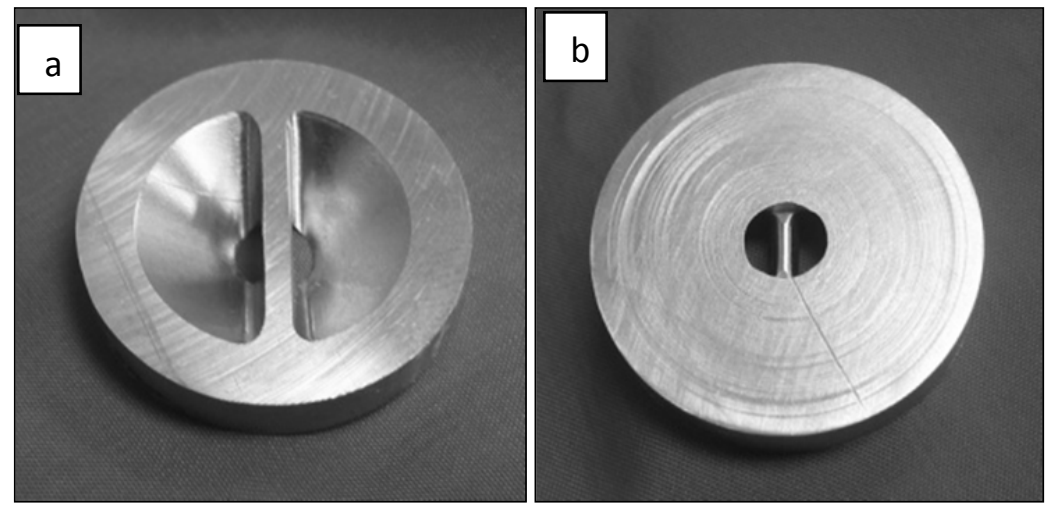

Figure 15. (a) Top and (b) bottom of splitter. 


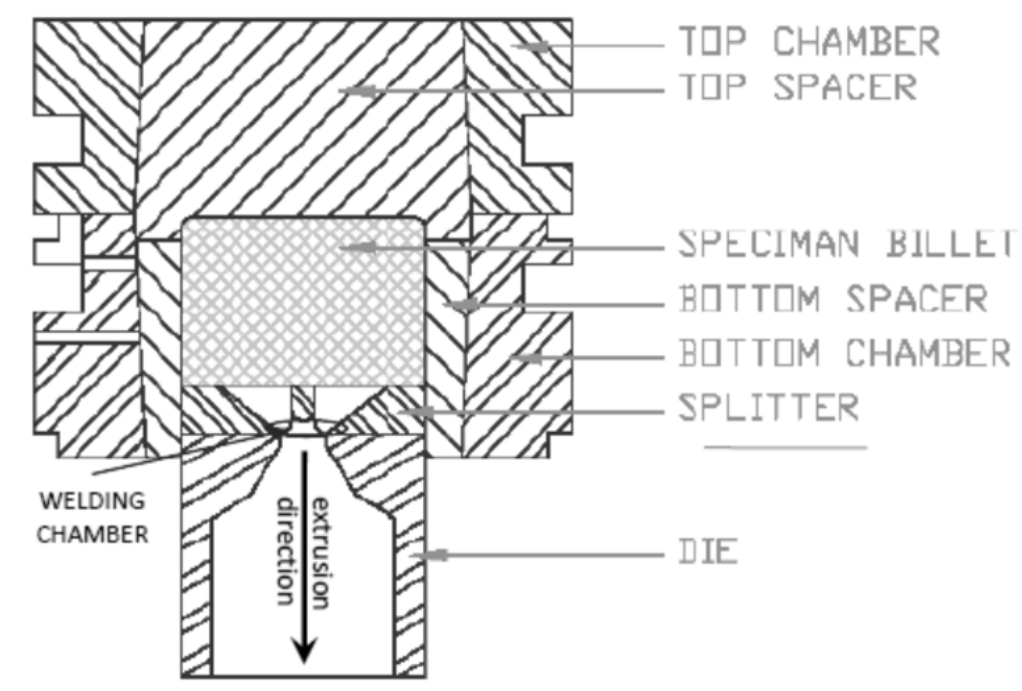

Figure 16. Splitter set-up in extrusion fixture.
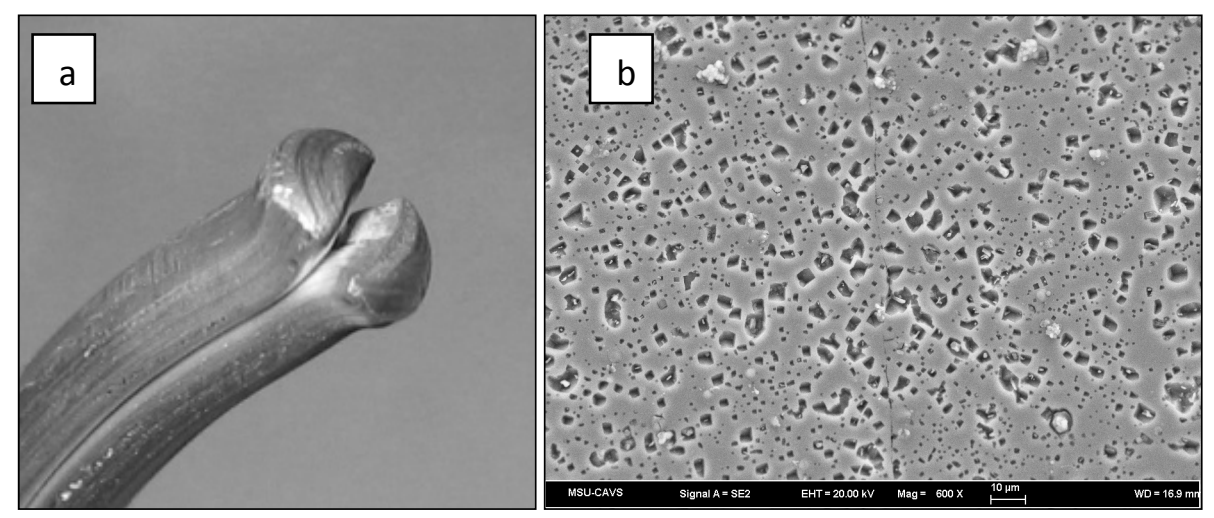

Figure 17. (a) Welded Al extrudate, and (b) weld seam at center of extrudate at $600 x$.

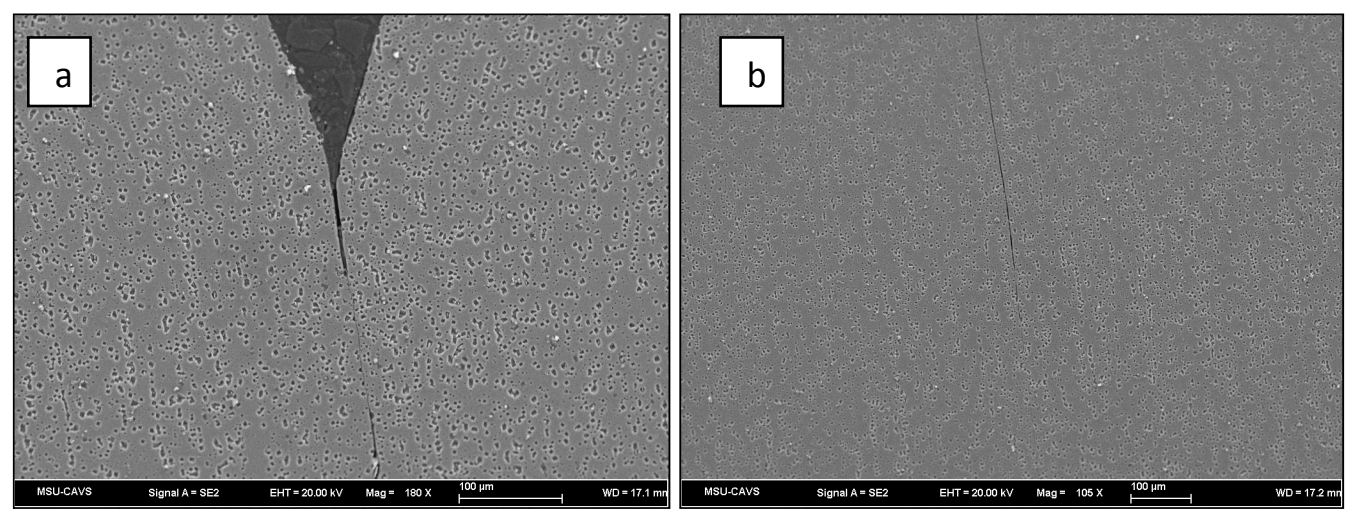

Figure 18. (a) Initiation of weld seam in welding chamber at 180X and (b) weld seam in the bearing area at $105 \mathrm{X}$. 
Figure 18 presents optical micrographs showing (i) the initiation of the solid state bonding just after the rear end of the web (welding chamber) and (ii) the weld in the bearing area. Note that in the welding chamber, just at the rear end of the web, Figure 18a, there is an incomplete filling of metal that induces the formation of a gas pocket. This usually indicates that sliding friction occurs at the rear-end portion of the web due to the small reduction step. Here, the free-surfaces sliding from the web surface will encounter each other at the rear end of the gas pocket, where they are pressed into each other, producing the solid state pressure-welding. Conditions that affect the quality of this weld are surface extension, contact pressure, and temperature. Note that traces of the weld seam are also observed in the bearing area, Figure 18b. Current work on weld seam formation is focused on improving some details of the present splitter design as well as modifying its design to extrude dog-bone profiles for tensile testing to determine the mechanical properties of the weld seam area. Experiments on $\mathrm{Mg}$ alloys will be performed after the preliminary testing on 1100F Aluminum is completed.

\section{Extrusion Simulations using and Eulerian code}

Extrusion simulations aim at reproducing extrusion test that were carried out in CAVS. Following an iterative procedure a final rigid design of the extrusion fixture was achieved which could withstand repeated extrusion runs. Once the fixture design was finalized, a rigorous experiment execution procedure was followed with the aim of achieving repeatability and validity in the testing results. These efforts culminated into the establishment of an extrusion experiment protocol which has helped us to tightly control sources of uncertainty and error in recorded data. Moreover, each experiment was repeated at least thrice to ensure exact replication of the previous trial. The output of the test is load vs. time curve and temperature history of the thermocouples.

Simulations are carried out using a Lagrangian code ABAQUS and an Eulerian code HyperXtrude. By using these two codes, we are able to improve the development of CAVS material model using ABAQUS while setting up boundary conditions for the CFD model using HyperXtrude. The final goal is to implement the ISV model in HyperXtrude to be able to solve complex extrusion processes.

Laboratory-scale indirect extrusion experiments on aluminium were modeled using the commercial code HyperXtrude. A good agreement between thermo-mechanical extrusion simulation results and experimental measurements was achieved by fully considering the real-world physics of laboratory-scale extrusion for flat dies. Figures 19 and 20 present the correlations on aluminium billet for two ram speeds. Figure 21 presents the correlation for a different initial temperature.
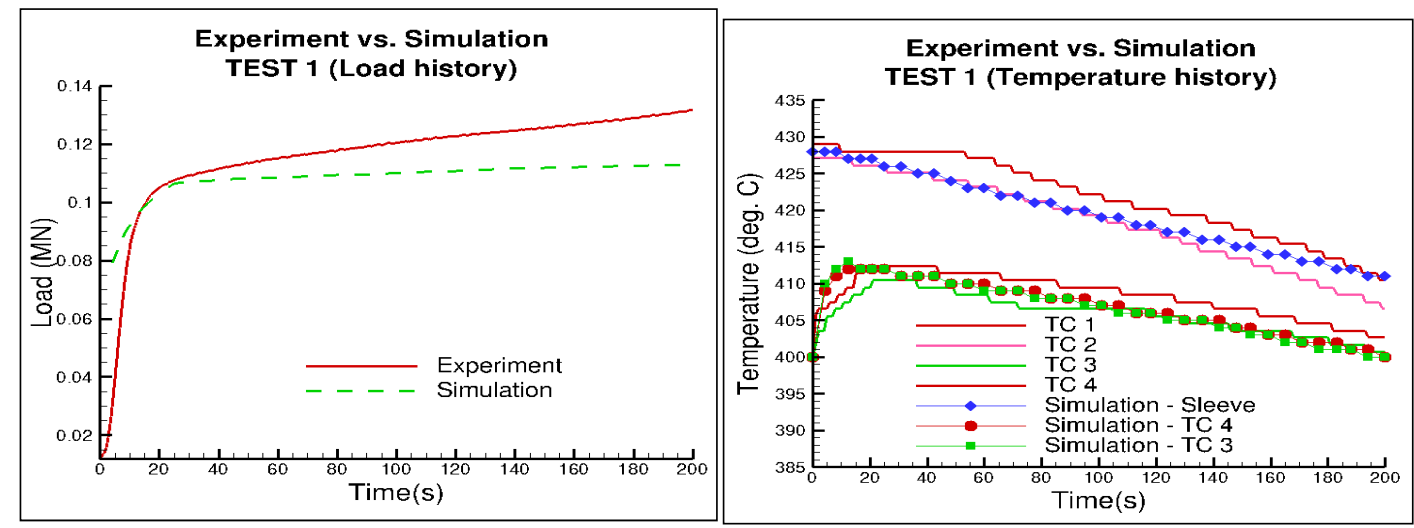
Figure 19: Validation for aluminium billet temperature of $428^{\circ} \mathrm{C}$ and ram speed of $5 \mathrm{~mm} / \mathrm{min}$
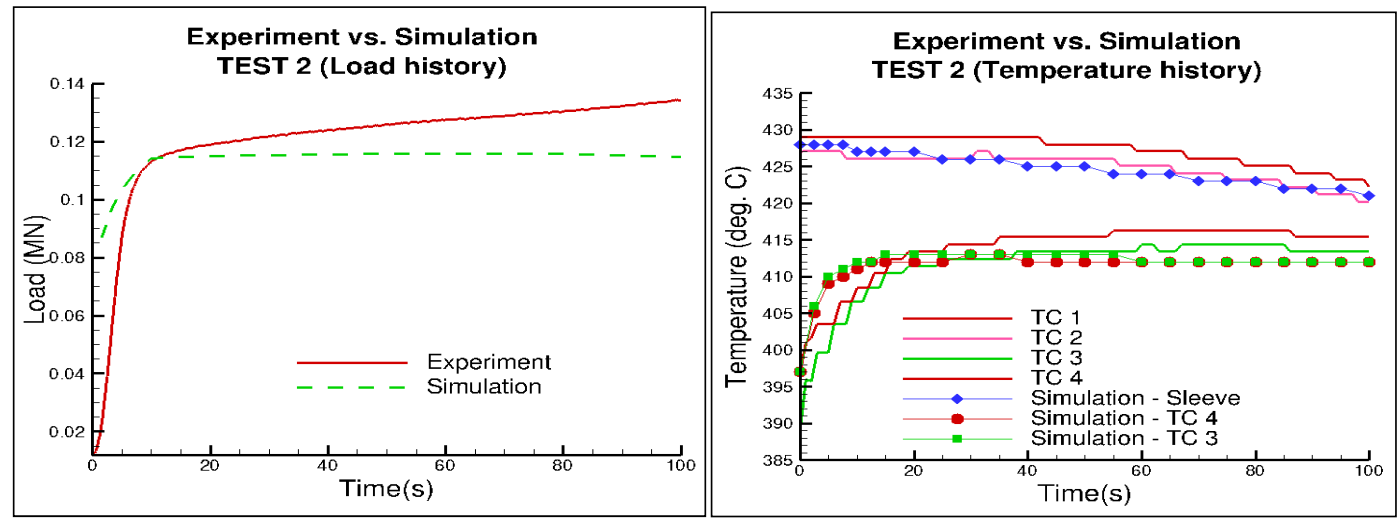

Figure 20: Validation for aluminium billet temperature of $428^{\circ} \mathrm{C}$ and ram speed of $10 \mathrm{~mm} / \mathrm{min}$
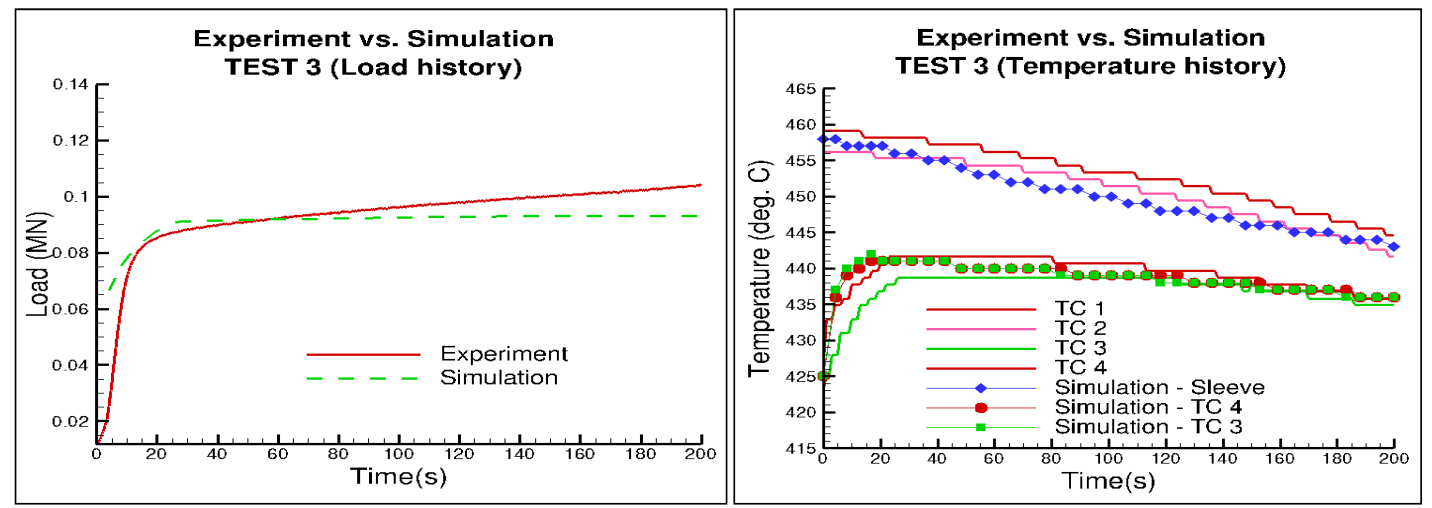

Figure 21: Validation for aluminium billet temperature of $457^{\circ} \mathrm{C}$ and ram speed of $5 \mathrm{~mm} / \mathrm{min}$

Extrusion experiments have been carefully designed and perfected in order to perform credible modelling research and avoid ambiguity associated with boundary conditions. Conical die and use of AZ61 magnesium alloy are presently being used to further validate the coupled experimental-modeling methodology developed in this work. Also, current extensions of HyperXtrude to include Internal State Variable material models will improve the predictive capability of the code. The simulation of AZ61 extrusion through a conical die using HyperXtrude will be presented in the next report.

\section{Simulations of AZ61 extrusion based on crystal plasticity and finite element methods-Couple Approach}

A constitutive elastic-viscoplastic model for hexagonal close-packed magnesium deformation implemented in a UMAT subroutine for ABAQUS has been developed [8]. The single crystal hardening parameters of magnesium AZ61 came from the Visco-plastic self-consistent model (VPSC) stress-strain curves fitting. The extrusion force and the evolving textures can be simulated using these hardening 
parameters and the initial texture of the AZ61 magnesium alloy. Figure 22(a) presents the simulated textures and corresponding measured textures at different locations in the funnel die. In addition, the simulated and the measured curves of the extrusion force-displacement are also presented in Figure 22(b). As shown, the simulation textures and predicted extrusion force-displacement curves are consistent with the measured results.

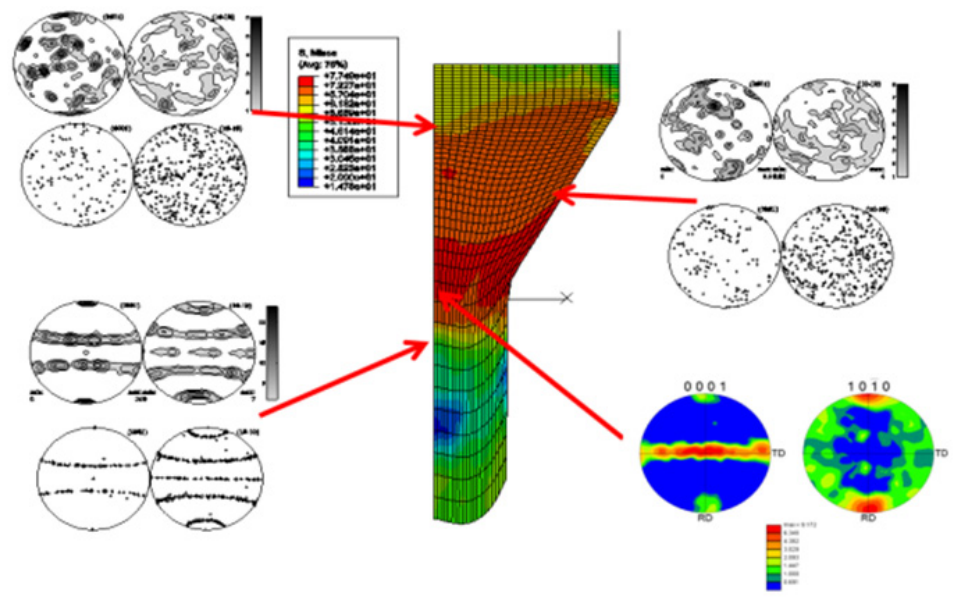

(b)

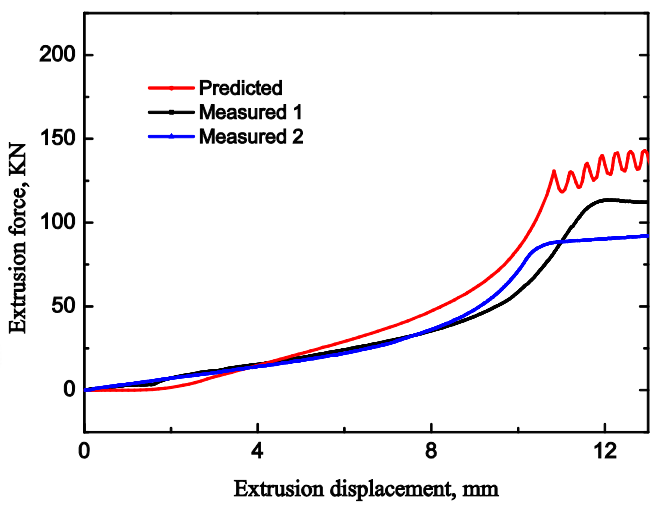

Figure 22: (a) Simulated and measured textures in a funnel die and (b) measured and predicted extrusion force-displacement curves of $\mathrm{AZ61}$ at $380^{\circ} \mathrm{C}$.

Extrusion simulation based on Hyperxtrude and Visco-plastic Self-Consistent (VPSC) models- Uncouple Approach

We have successively simulated and conducted in-house extrusion process of AZ61 at $\sim 400^{\circ} \mathrm{C}$ and various extrusion speeds $(5 \mathrm{~mm} / \mathrm{min}, 10 \mathrm{~mm} / \mathrm{min}$ and $15 \mathrm{~mm} / \mathrm{min}$ ). The streamline data from HyperXtrude (velocity gradient parameters $L_{i j}$ ) can be used as the input data of the VSPC modeling along with the time increments. We can use Hyperxtrude and VPSC to simulate the extrusion of magnesium and get detailed twinning systems, slips activation and their critical resolved shear stress (CRSS) evolution along extrusion. Microtextures in various positions in the extrudate were measured by the electron backscattered diffraction (EBSD) technique. Figure 23 gives the primary simulation textures in the center and middle position of the extrusion AZ61 bar at $420^{\circ} \mathrm{C}$ in comparison with the EBSD experimental microtexture in the identical positions of the flat die extruded billet. As shown in Figure 23, Hyperxtrude wth VPSC simulation textures match well the experimental textures observed using EBSD. The texture configuration shows typical characteristics of the extrusion texture of magnesium in which a strong $\{10 \overline{1} 0\}$ ED (extrusion direction) fiber exhibits in the extruded billet. In this high temperature case, the athermal tensile twinning $\{10 \overline{1} 2\}<10 \overline{1} 1>$ is not as important as the effect of the second-order pyramidal $<\mathrm{c}+\mathrm{a}>$ slip and the prismatic $<\mathrm{a}>$ slip on texture evolution. 
(a)

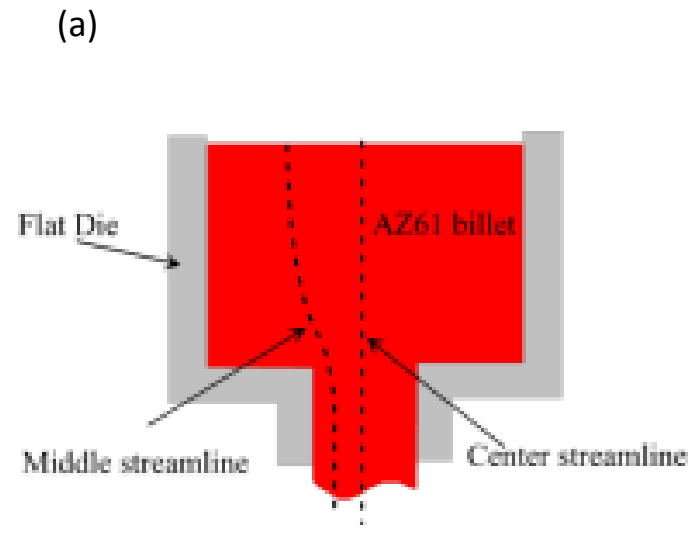

(b)
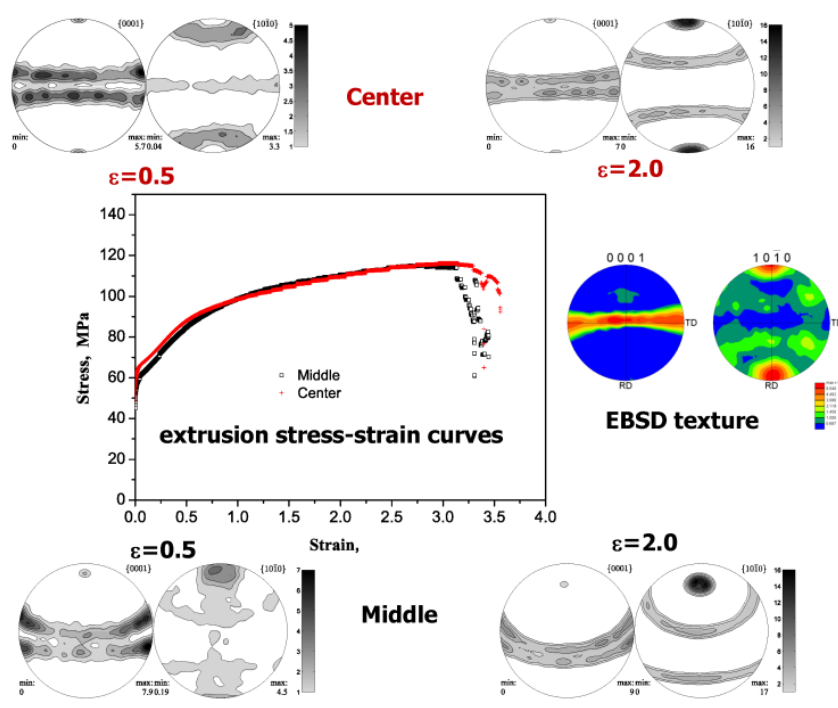

Figure 23: (a) The center and middle streamline in the flat die and (b) the extrusion stress-strain curves and the textures in the flat die based on Hyperxtrude and VPSC simulations.

A post-process numerical technique using the VPSC code to describe the texture and twinning evolutions during extrusion was implemented and compares well with experiments.

Extrusion simulation of magnesium alloys in Lab-scale and industry level Extrusion Process Simulation consideration of temperature and strain rate

The aspect of our extrusion research is summarized in Figure 24. We have completed the AM30 lab-scale flat-die extrusion experiments using different processing parameters: ram speeds of 5, 10, 15, 20,30 and $40 \mathrm{~mm} / \mathrm{min}$, billet temperatures of $454^{\circ} \mathrm{C}$ and $482^{\circ} \mathrm{C}$, and an extrusion ratio (ER) of 25 . Load and temperature profiles together with microstructure information have been recorded for model validation. Current extensions of these experiments are focused on extruding AM30 with various die geometries including conical dies, dies for dog-bone profiles, and porthole dies for circular cross section tubes. 


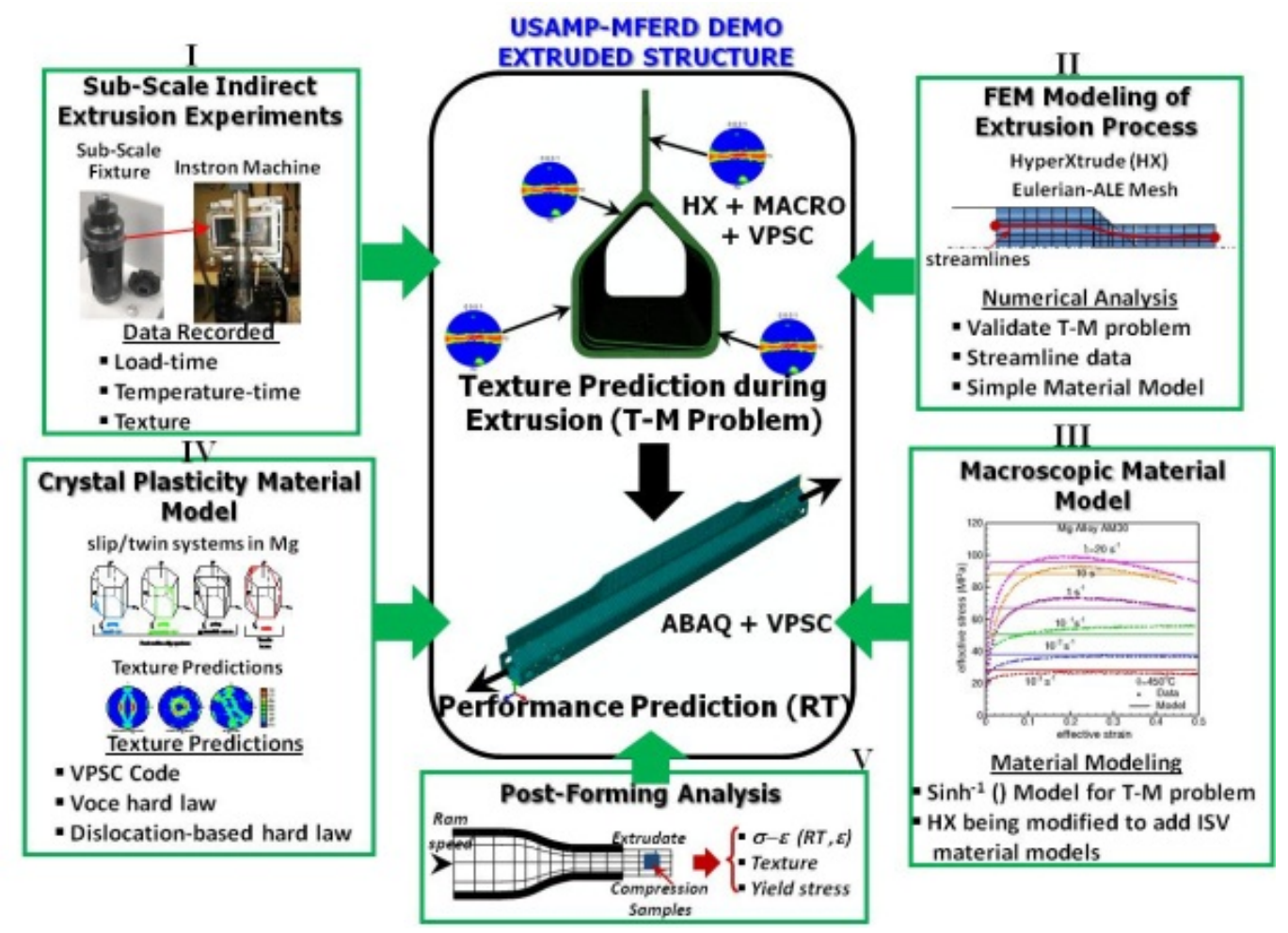

Figure 24. Overview of research activities on extrusion at CAVS as related to the USAMP-MFERD project.

Finite element simulations of the flat-die extrusion experiments have been carried out using $\mathrm{HX}$ and a flow-stress type model (sine hyperbolic inverse law) (Figure 25). The material parameters for AM30 were fitted to experimental stress-strain curves produced at CAVS and from the literature. Predicted results for the load-time and temperature-time curves compared well with the experimental ones. Additional work on extrusion process modeling for other dies' geometries is in progress. Also, $\mathrm{HX}$ developers (from Altair) are working on extending the capabilities of the code to use internal state variable (ISV) material models.

Microstructure (texture and twinning) evolution of material particles during the extrusion process is being modeled using the Visco-Plastic Self Consistence (VPSC) formulation. Deformation histories along streamlines were obtained from the HX post-processor and used in the VPSC code to compute texture and get a detailed account of the slip/twinning activity in the material. A crystal-level dislocation-based model that represents rate- and temperature-dependence response is being used for this purpose [5,9]. This methodology has been applied to predict texture during AM30 flat die extrusion. Computed texture patterns agreed well with experimental (EBSD) ones.

Predicting the microstructure and mechanical properties of the material after extrusion is essential for the post-forming structural analysis of the extruded component. In this respect, mechanical tests and microstructure studies are being performed on specimens cut from flat-die extruded rounded bars obtained with ERs of 6.25 and 25 . Specimens have been subjected to compression at different strain rates and room temperature (RT), with the recorded data being the stress-strain curves, and initial 
and deformed textures (see Figure 26). These data will be used for testing the capability of the developed numerical tools to predict the mechanical properties of the extrudate.
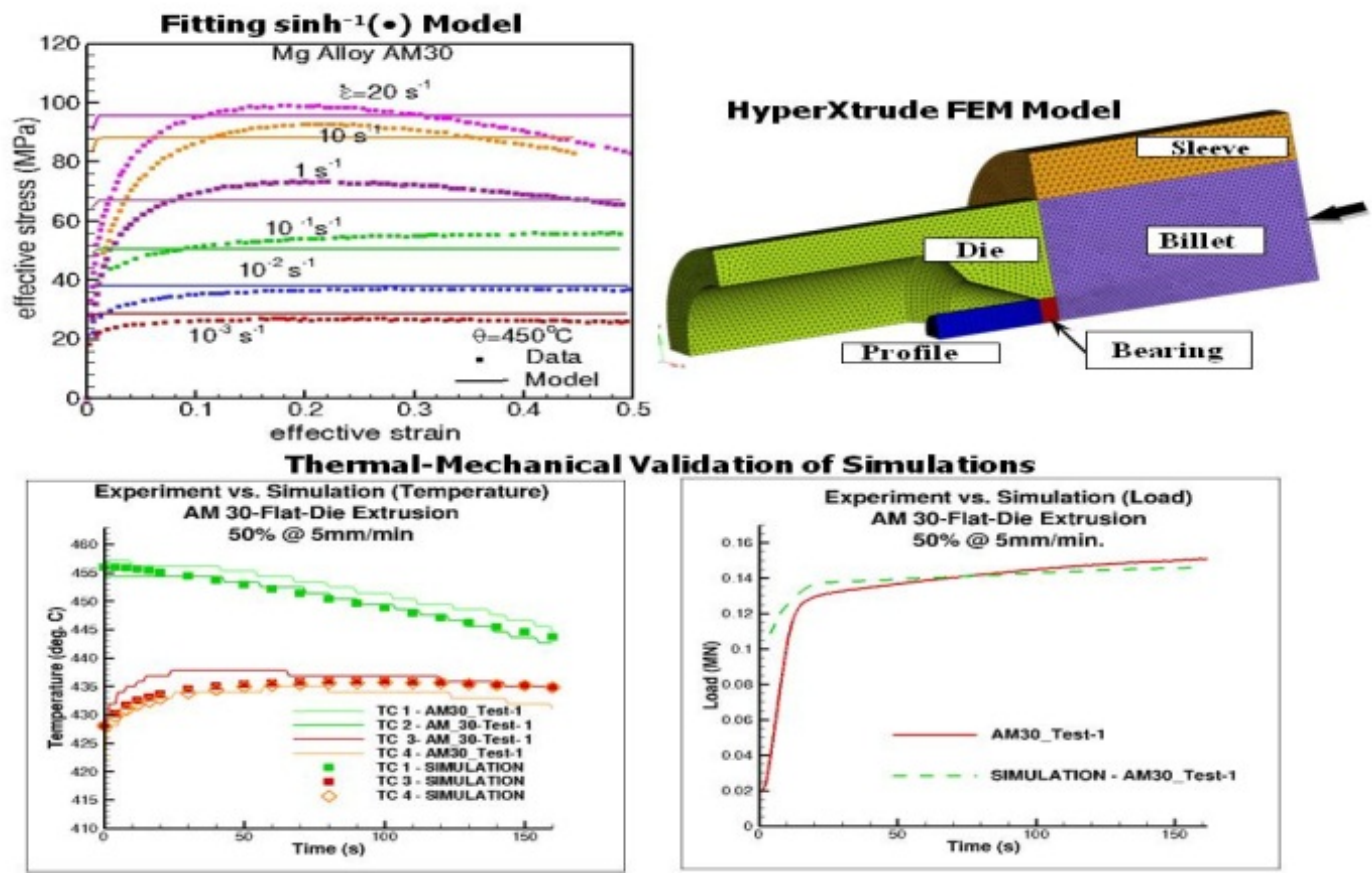

Figure 25. Validation of flat-die extrusion experiments of $A M 30$ using numerical tools: $H X$ and $\operatorname{Sinh}^{-1}(\bullet)$ model
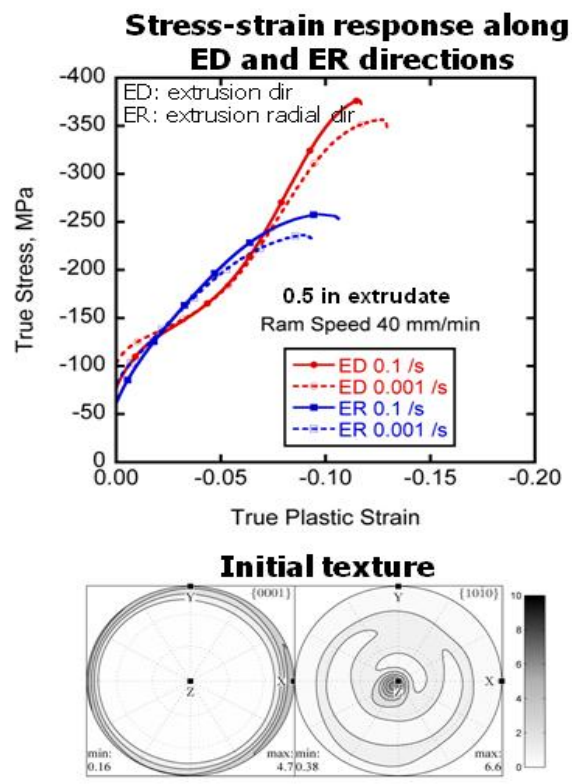

Figure 26. Characterization of compression specimens from flat-die AM30 extruded material 
Prediction of magnesium alloy mechanical properties and texture in lab-scale extrusion

Local texture in the bearing area in the extrusion die is significantly important for $\mathrm{Mg}$ alloys extrusion. On the benefit of the professional specific HyperXtrude software, the velocity gradient data, namely the streamline, along the extrusion lines in the die can be obtained through the extrusion simulation using HyperXtrude software as shown in Figure 27. As shown in Figure 27, the streamlines in different positions in the extrusion die exhibit the different temperature, the extrusion pressure and the strain level along extrusion displacement.
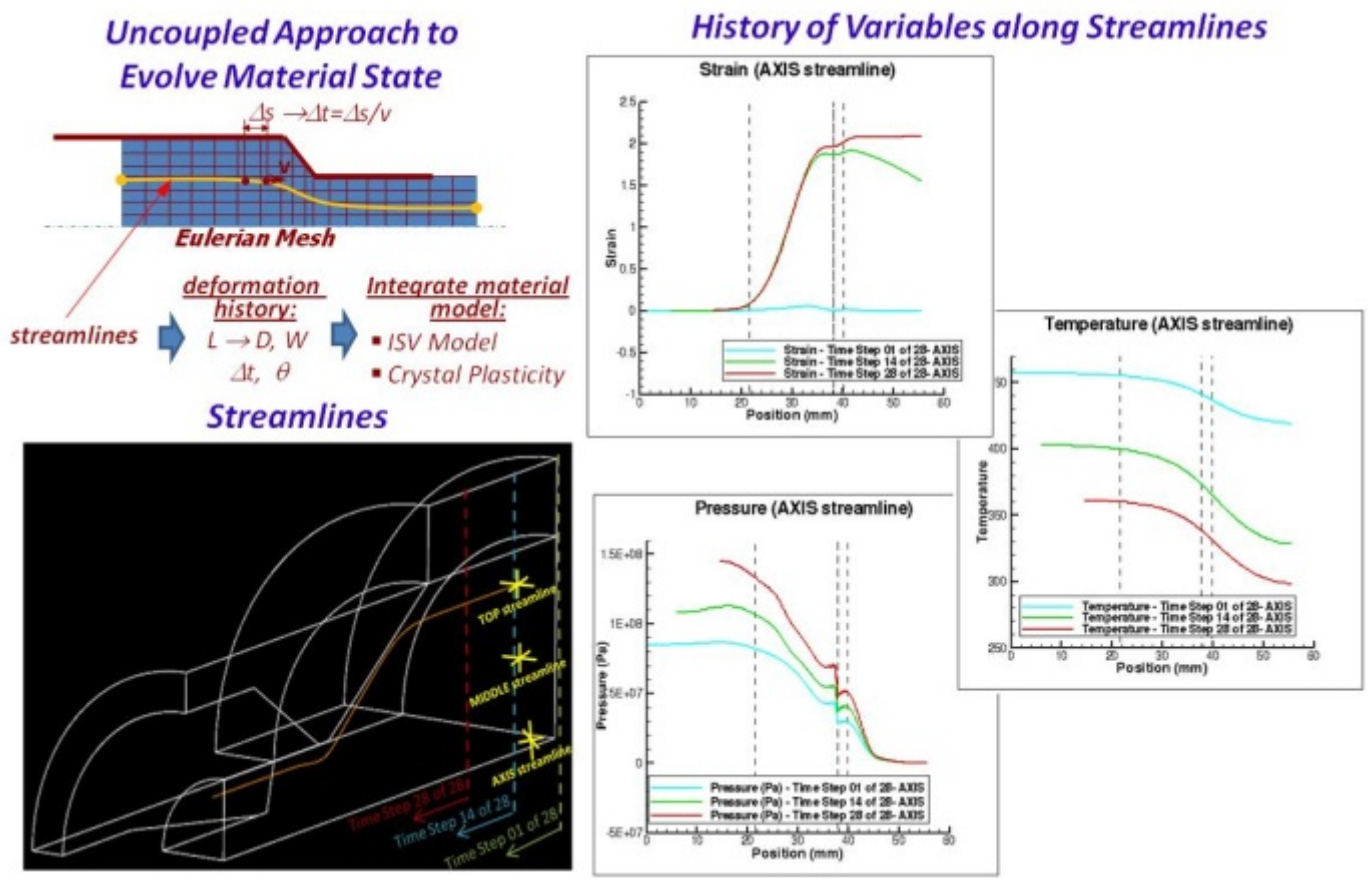

Figure 27. Streamline Information to Compute Texture using VPSC - e.g. Conical Die

The methodology of local texture simulation during Mg alloys extrusion at high temperatures is presented in Figure 28. The philosophy of extrusion local texture prediction in the extrusion die is: using VPSC dislocation density model and the corresponding hardening parameters which come from the stress-strain curves fitting based on the initial texture. Along streamline, local texture and flow stress in any location in the extrusion die can be predicted based on combination of HyperXtrude and VPSC. 


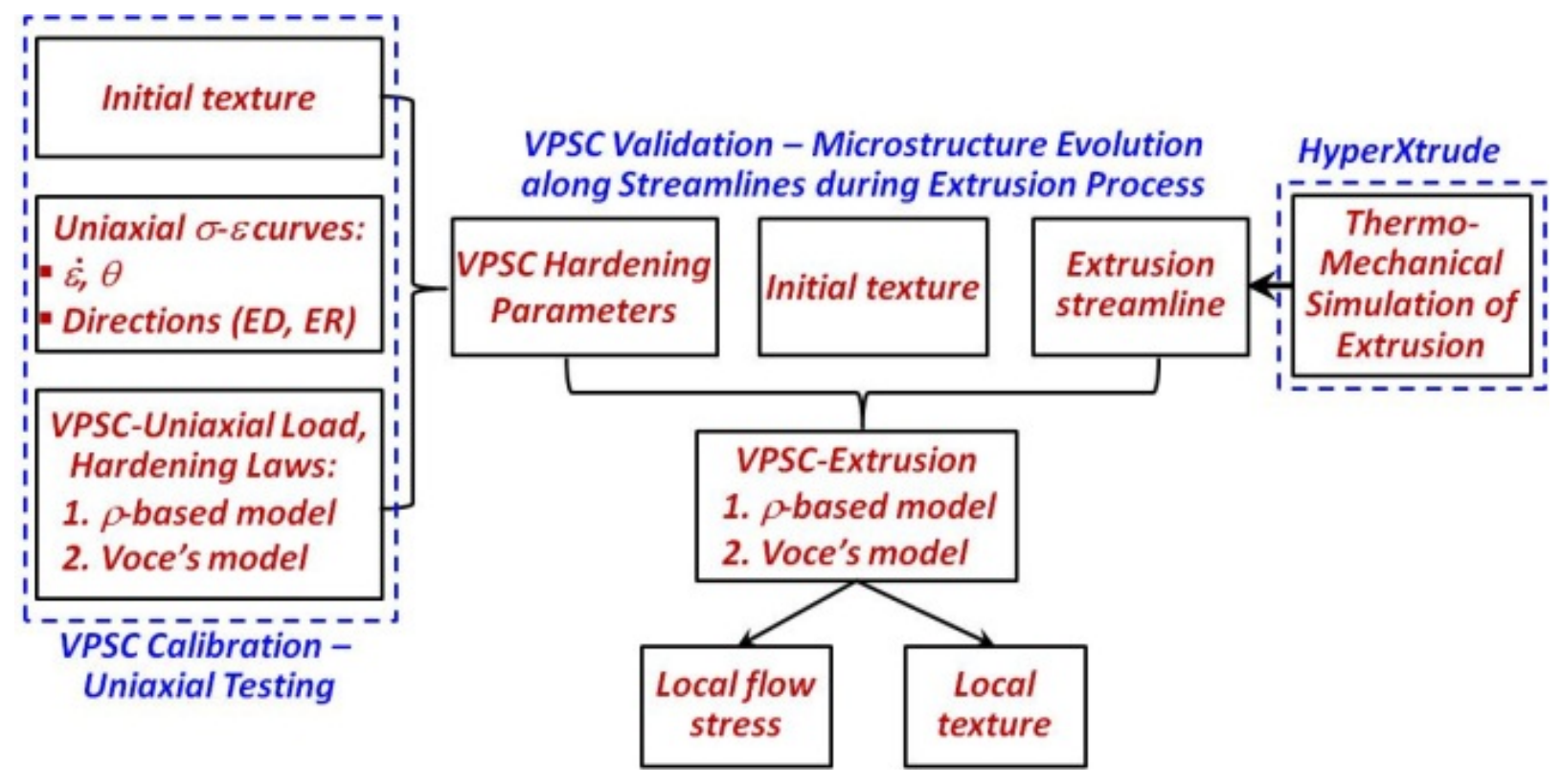

Figure 28. Methodology to predict microstructure (texture) using VPSC

Due to the limited ductility of $\mathrm{Mg}$ and its alloys at low temperature, wrought Mg alloys usually are conducted thermomechanical processing at high temperature, e.g. hot extrusion and hot rolling. As for $\mathrm{Mg}$ extrusion, the hot extrusion temperature usually falls in the rage of $350-450{ }^{\circ} \mathrm{C}$. Therefore, deep understanding of microstructure and texture evolution of $\mathrm{Mg}$ during extrusion at $450{ }^{\circ} \mathrm{C}$ is necessary for $\mathrm{Mg}$ alloys design. On the other hand, the strain rate plays also an important role on $\mathrm{Mg}$ alloys deformation mechanisms, and strain rate is inhomogeneous during hot extrusion. Tt varies in the die from one location to another. As such, any polycrystalline plasticity modeling on $\mathrm{Mg}$ alloys extrusion must incorporate the effects of temperature and strain rate to accurately predict local texture and mechanical property. At present, we focus our study on the VPSC modeling including the effects of temperature and strain rate during $\mathrm{Mg}$ extrusion at high temperature of $450{ }^{\circ} \mathrm{C}$.

First of all, we did simple compression of the pre-extrusion $\mathrm{AM} 30$ at $450{ }^{\circ} \mathrm{C}$ along two perpendicular two directions, the extrusion direction (ED) and the radial direction (ER) of the AM30 extrusion billet at different strain rate, $0.001 \mathrm{~s}^{-1}, 0.1 \mathrm{~s}^{-1}, 0.5 \mathrm{~s}^{-1}$ and $0.8 \mathrm{~s}^{-1}$. Actually, these selected strain rates fall in the lab-scale strain rate $<1 \mathrm{~s}^{-1}$ of $\mathrm{Mg}$ extrusion in our laboratory at CAVS. Then we did stressstrain curves fitting of AM30 at different strain rates based on the VPSC dislocation density model. The initial microtexture, the VPSC hardening parameters at $450^{\circ} \mathrm{C}$, the measured stress-strain curves of the $\mathrm{AM} 30$ at $450{ }^{\circ} \mathrm{C}$ at different strain rates are presented in Figure 24. As shown in Figure 29, other hardening parameters are same at different strain rates, however, only the $\tau_{0}$ of dislocations changed with strain rates at $450{ }^{\circ} \mathrm{C}$. Therefore, we extracted the linear relationship of the $\tau_{0}$ and strain rate as Equations 6-8. The $\tau_{0}$ of dislocation slips by VPSC stress-strain curves fitting and the predicted $\tau_{0}$ using the Equations 6-8 are also shown in Figure 29. Due to twinning being considered an athermal deformation mode in $\mathrm{Mg}$, we fix the critical resolved shear stress (CRSS) and the propagation stress of \{10-12\} twinning as $15 \mathrm{MPa}$ at $450^{\circ} \mathrm{C}$ in this study. 


$$
\begin{gathered}
\tau_{0}=7.0755 \dot{\varepsilon}+9.5218 \mathrm{MPa} \text { (for basal slip) } \\
\tau_{0}=38.0784 \dot{\varepsilon}+10.1613 \mathrm{MPa} \text { (for prismatic slip) } \\
\tau_{0}=36.3559 \dot{\varepsilon}+13.7663 \mathrm{MPa} \text { (for second pyramidal slip) }
\end{gathered}
$$

Compiled these Equations 6-8 into the original VPSC dislocation density model, it then can capture both temperature and strain rate effects. The predicted simple compression stress-strain curves of AM30 at $450{ }^{\circ} \mathrm{C}$ using the $\tau_{0}$ based on the Equations 6-8 and the measured stress-strain results are presented in Figure 29. As shown in Figure 29, the predicted and measured stress-strain curves are in reasonably well agreement with each other.
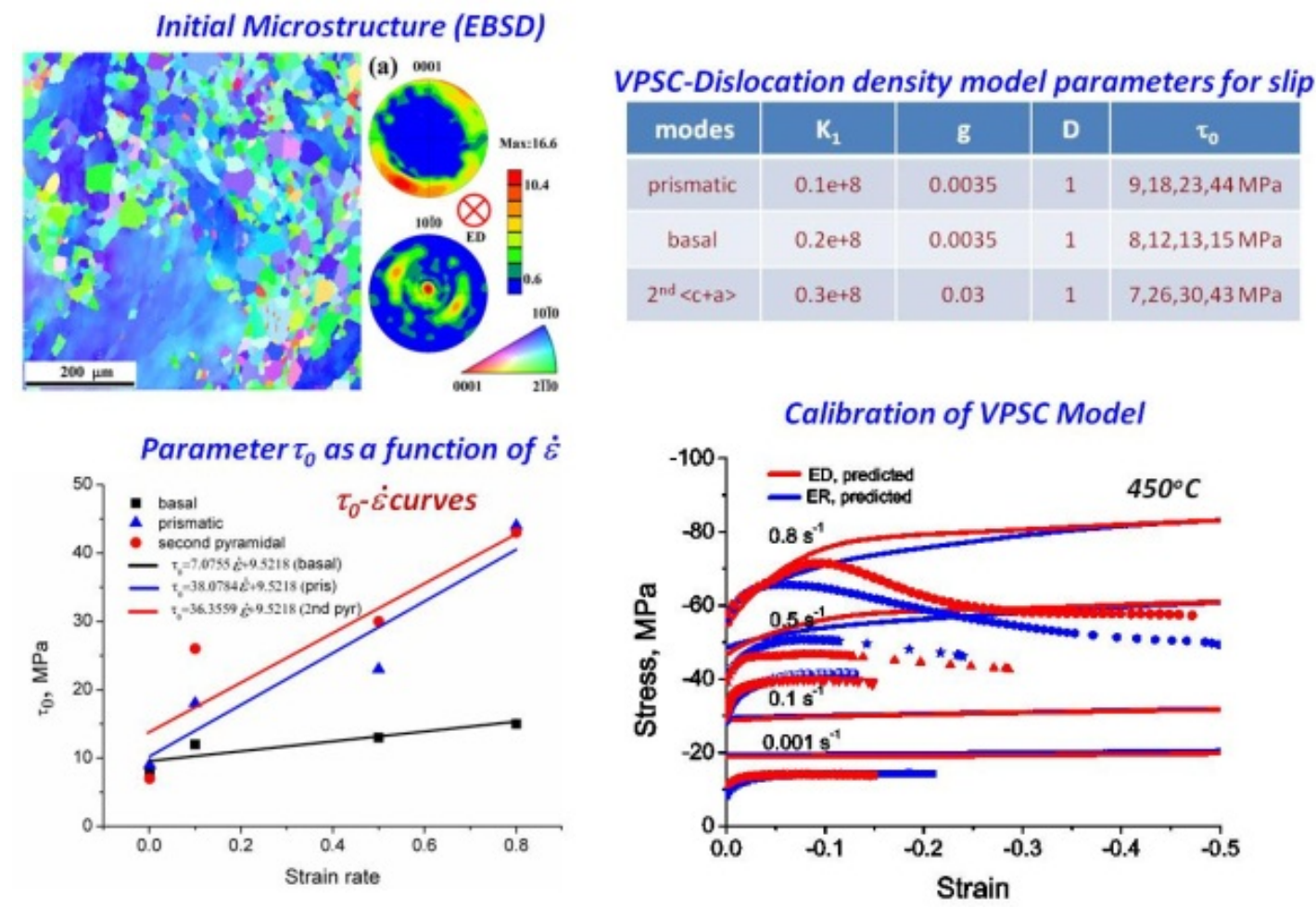

Figure 29. Calibrating the dislocation-based hardening law in VPSC

Using the VPSC dislocation density model parameters and the streamline data output from the HyperXtrude software, we can predict the extrusion stress-strain curve and the local texture of AM30 in flat die and conical die in the extrudate. Figure 30 show the corresponding VPSC results based on the streamline of a flat die of $\mathrm{AM} 30$ extrusion at $450{ }^{\circ} \mathrm{C}$. The results are the corresponding data extrusion along the axis of the flat die at two different extrusion speeds, ram speed of $5 \mathrm{~mm} / \mathrm{minute}$ and 10 $\mathrm{mm} /$ minute. The predicted texture is consistent with the measured EBSD texture (Figure 30). As shown in Figure 30, the extrusion flow stress of $10 \mathrm{~mm} /$ minute is higher than that of $5 \mathrm{~mm} / \mathrm{minute}$ which is reasonable showing that VPSC dislocation density model can capture both temperature and strain rate effects during extrusion in flat die. 


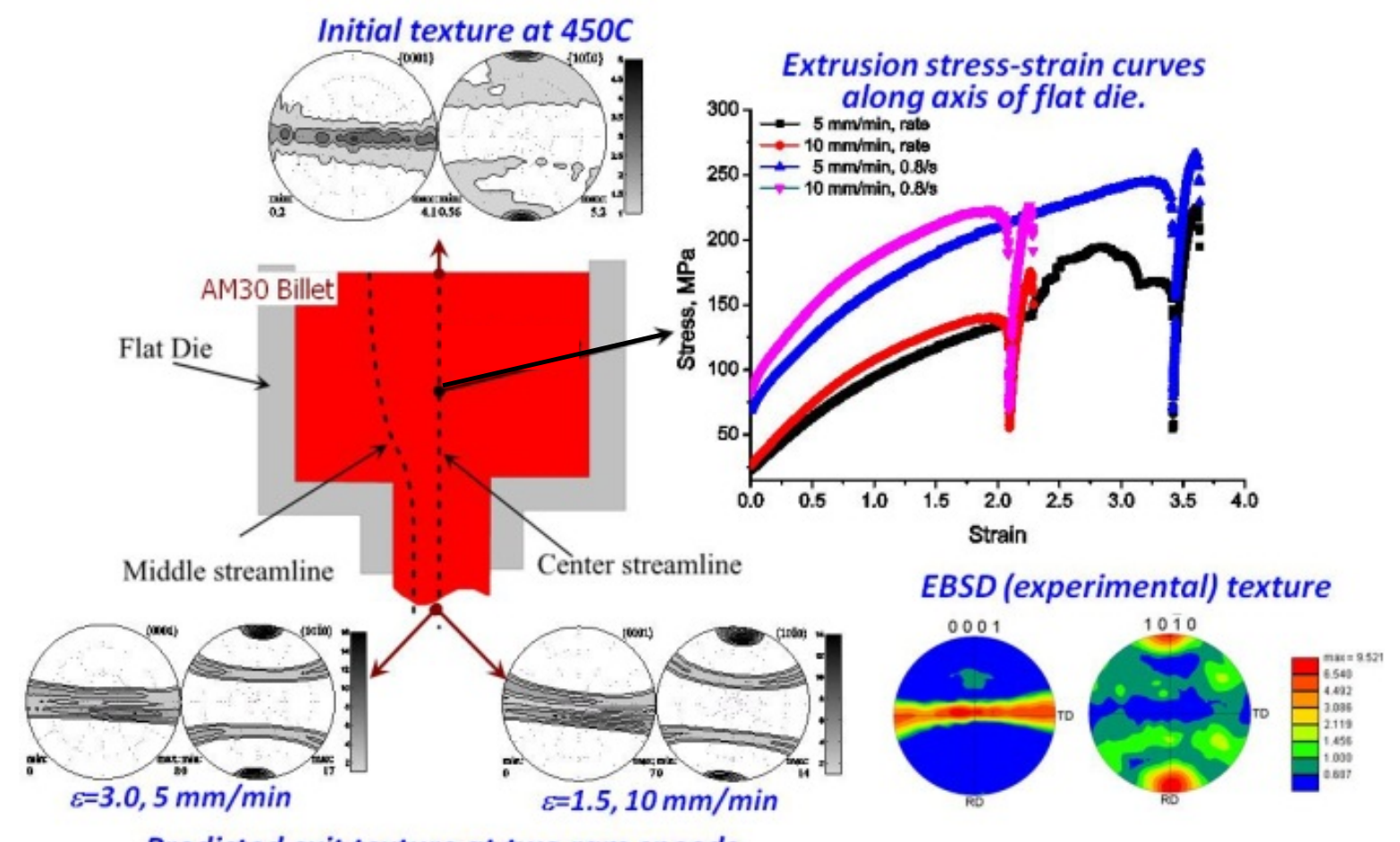

Predicted exit texture at two ram speeds

Figure 30. Predicting texture using VPSC - including temperature and strain rate effects.

\section{Post-Forming Experiments and Modeling}

As for post-forming simulation, we used the VPSC predicted texture of the extrudate as the starting orientations in the VPSC simulation for post-forming. In addition, the room temperature's parameters of the VPSC dislocation density model of the pre-extrusion AM30 came from the room temperature stress-strain curves VPSC fitting. The methodology to predict initial yield stress and texture of the extrudate is presented in Figure 31. The experimental stress strain curves of the extrudate of the AM30 at different ram speeds are presented in Figure 32. 


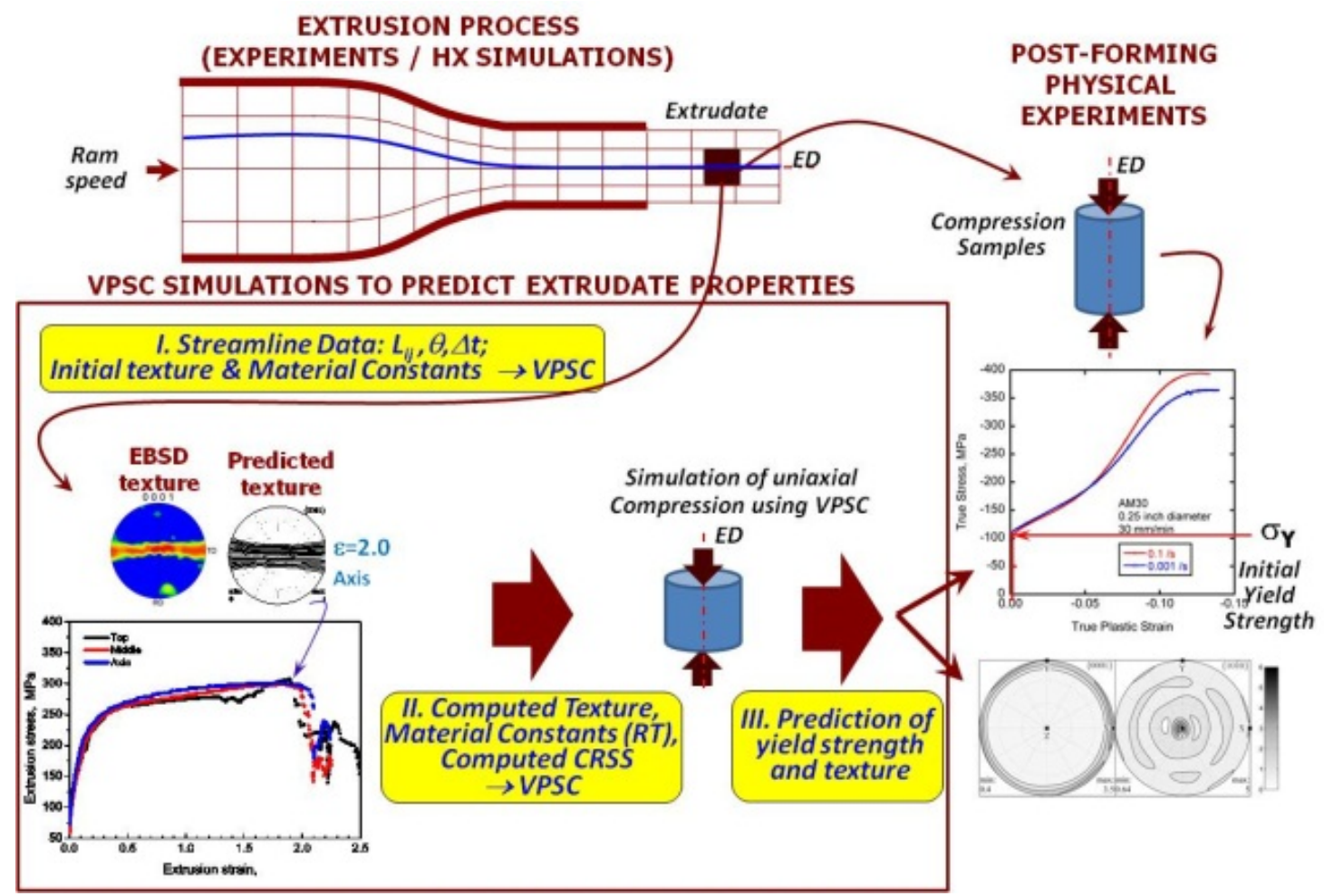

Figure 31. Methodology to Predict Texture \& Initial $\sigma_{Y}$ of Extrudate of the Mg alloy AM30.

Extrudates from Flat Die Extrusion of $A M 30, \theta_{B}=454^{\circ} \mathrm{C}$

$E R=25\left(1 / 4^{\prime \prime}\right.$ extrudate)

$v_{R}=30 \mathrm{~mm} / \mathrm{min}$

$v_{R}=40 \mathrm{~mm} / \mathrm{min}$
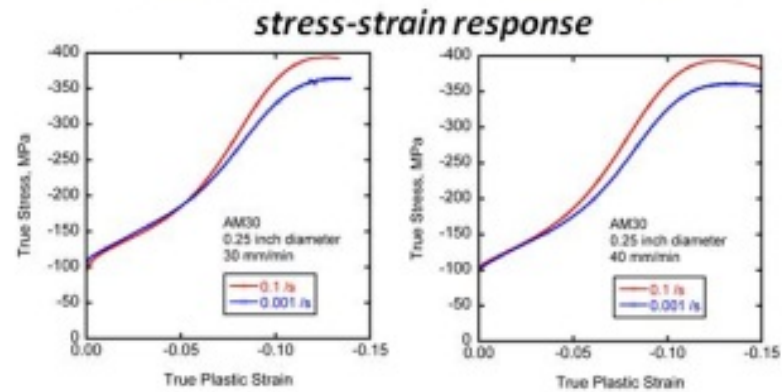

texture at $\varepsilon=0$

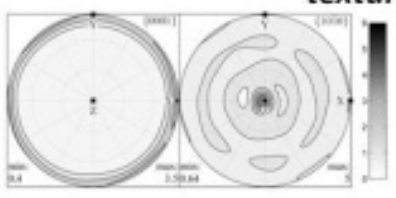

$E R=6.25(1 / 2$ " extrudate) $v_{R}=20 \mathrm{~mm} / \mathrm{min} \quad v_{R}=40 \mathrm{~mm} / \mathrm{min}$

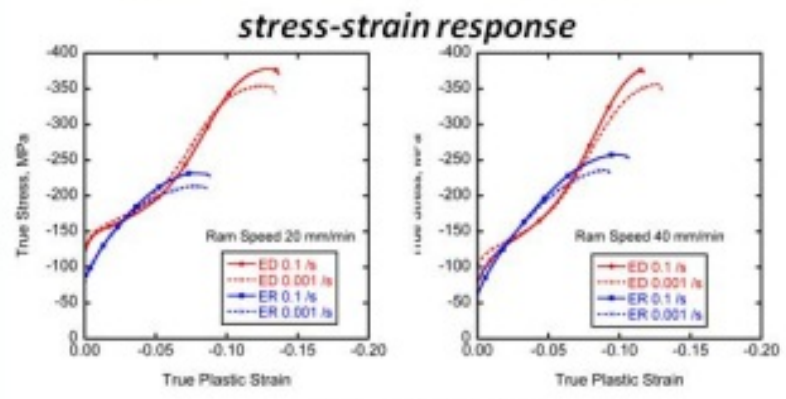

texture at $\varepsilon=0$

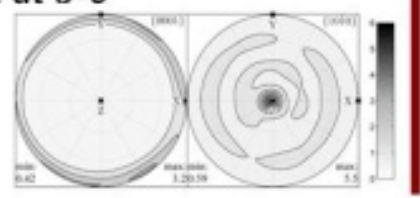

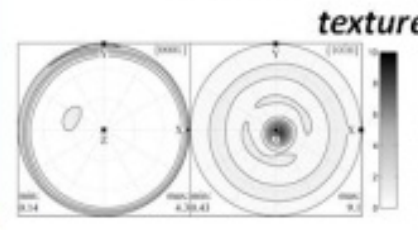

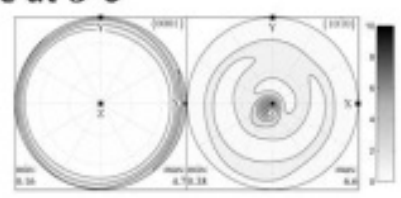

Figure 32. Post-forming experiments on extrudates of $\mathrm{Mg}$ alloy $\mathrm{AM} 30$ from flat-die extrusion

In Figure 33 there is the simulated extrusion stress-strain curve of the AM30 extruded at $450{ }^{\circ} \mathrm{C}$ using the flat die along the die axis and the ram speed is $30 \mathrm{~mm} /$ minute. Using the predicted extrudate 
texture (Figure 33) and the room temperature's VPSC hardening parameters from AM30 stress-strain curves fitting, the post-forming stress-strain curve can be simulated as shown in Figure 33.

As shown in Figure 33, the predicted extrusion flow stress goes up along strain. This is reasonable because the strain rate is higher close the exit of the flat die. The extrudate texture (Figure 33 ) is the typical of extrusion of Mg showing a strong <0001> ED texture.

The VPSC dislocation density model can accurately capture the yield stress of the extrudate (Figure 33, 34). In comparison with experimental results, the modified VPSC dislocation density model can nicely predicted the extrusion stress-strain curve, local texture of the extrudate and post-forming property of the extrudate of AM30 alloy extrusion at $450{ }^{\circ} \mathrm{C}$. A detailed discussion of the model and parameters for the VPSC model is found in Ref [9]. In general, the yield point is accurately predicted and the strain hardening behavior is reasonable.

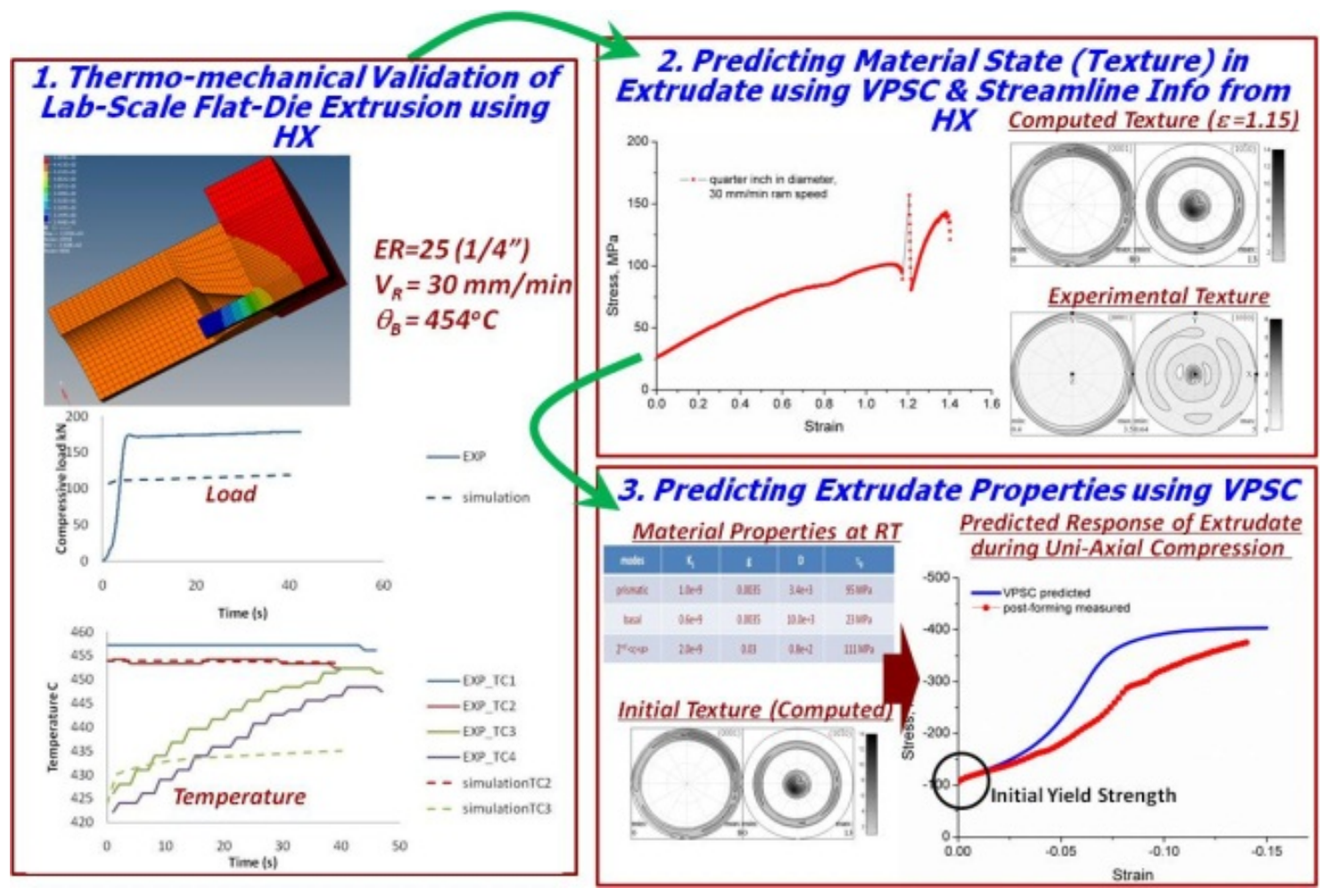

Figure 33. Preliminary Results for Predicting Post-Forming Properties of Extrudate 
Extrudates from Flat Die Extrusion of AM30

Stress-Strain Response, Experiment vs Simulation

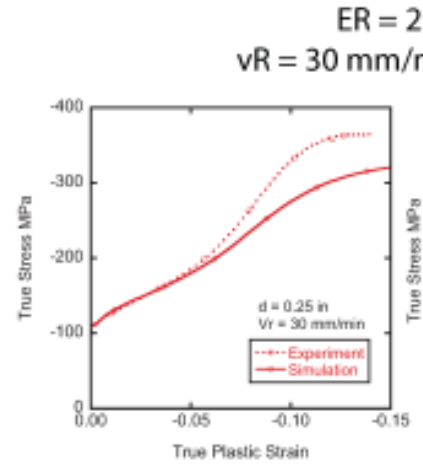

$E R=25\left(0.25^{\prime \prime}\right.$ extrudate $)$

$\mathrm{vR}=40 \mathrm{~mm} / \mathrm{min}$
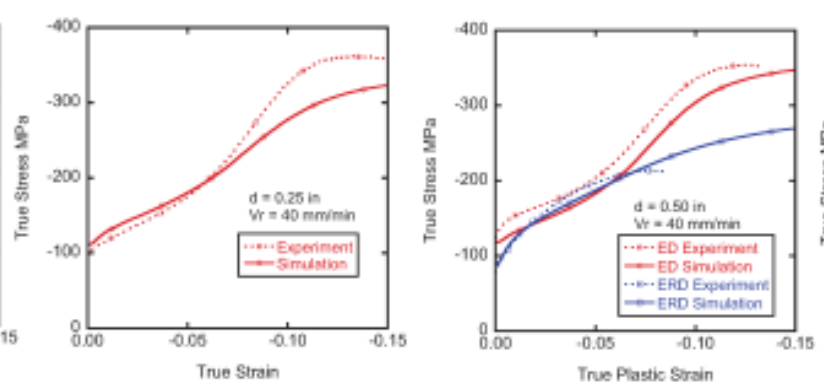

$E R=6.25\left(0.50^{\prime \prime}\right.$ extrudate $)$

$\mathrm{vR}=40 \mathrm{~mm} / \mathrm{min}$

Figure 34. Comparison between experimental and VPSC simulation stress-strain behavior for post-form AM30 laboratory scale extrudate.

\section{Prediction of Mechanical properties and texture in industry level extrusion processes of $\mathbf{M g}$ alloys}

This section describes the industrial application of the methodology presented by the extrusion task team in the previous paragraphs. The purpose of this study is to demonstrate the ability of the methodology to be applied to a real industrial problem. The Timminco die was chosen due to data availability regarding the extrusion process parameters but also the microstructure database along the profile. Figure 35 shows the steps that CAVS have followed to produce accurate simulations of the process. First CAVS team went to visit Timminco plant to take pictures and data of the extrusion tooling and processes. From these, CAD geometry was built and a negative volume was constructed. This volume represents the magnesium alloy "filling" the extrusion tooling during the process, the red part being the undeformed billet ready to be extruded and the light blue part being the profile in the bearing area, just before exiting the tooling. The measured texture and stress-strain data in representative positions in the extruded double hat are presented in Figure 36.

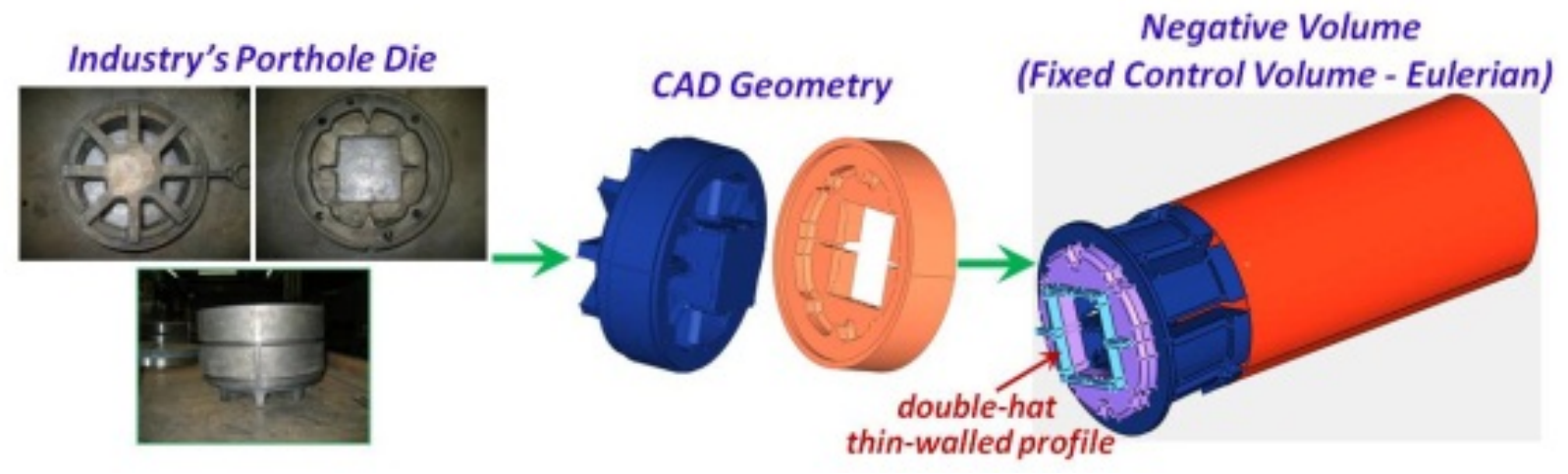

Figure 35. Industry profiles used for extrusion simulations 


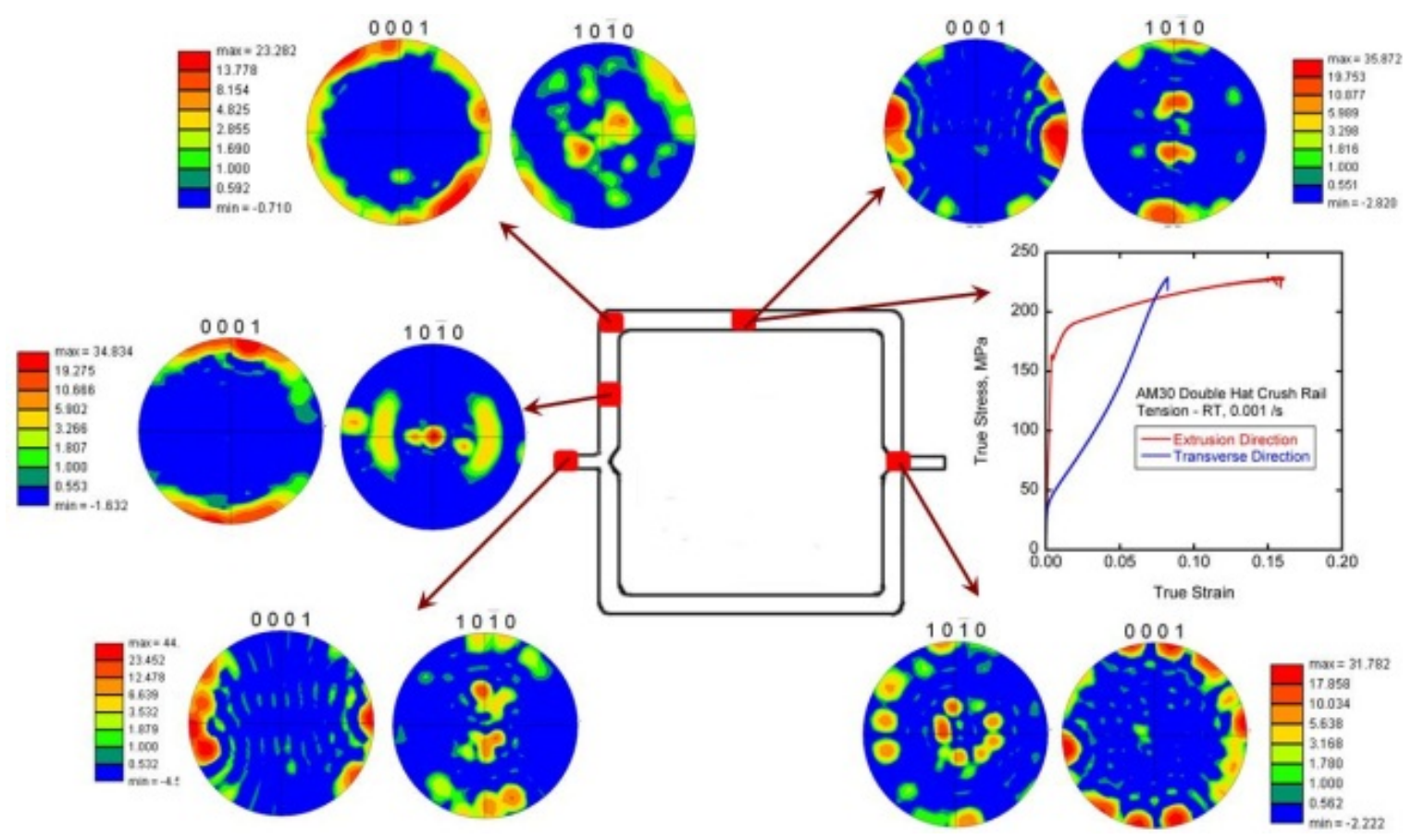

Figure 36. Measured mechanical response and texture for double-hat profile.

Contrary to the simulation of lab scale experiments, the double hat simulations were run in steady state. As no load and temperature histories were available, the assumption was to focus on steady-state results and microstructure prediction.

Figure 37 shows the steady state analysis of this extrusion process at a temperature of $427^{\circ} \mathrm{C}$ and a ram speed of $78 \mathrm{~mm} / \mathrm{min}$ in the left picture.

As information about the die temperature is unknown, it was assumed at $420^{\circ} \mathrm{C}$ which is slightly below the container temperature. This assumption is based on observation from the experimental extrusions ran at CAVS.

The purpose of this simulation is the extraction of material history along several streamlines so texture can be computed in a later step. The right side picture of Figure 37 shows the streamlines that have been selected to analyze the texture. Microstructure observations are already available at these locations. Texture prediction using VPSC are presented in Figure 38. 


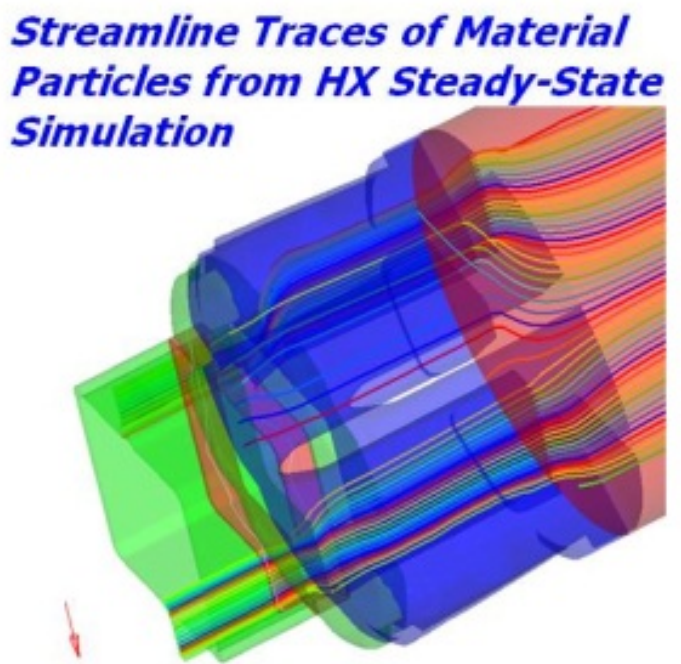

\section{Selected Point Locations on Profile}

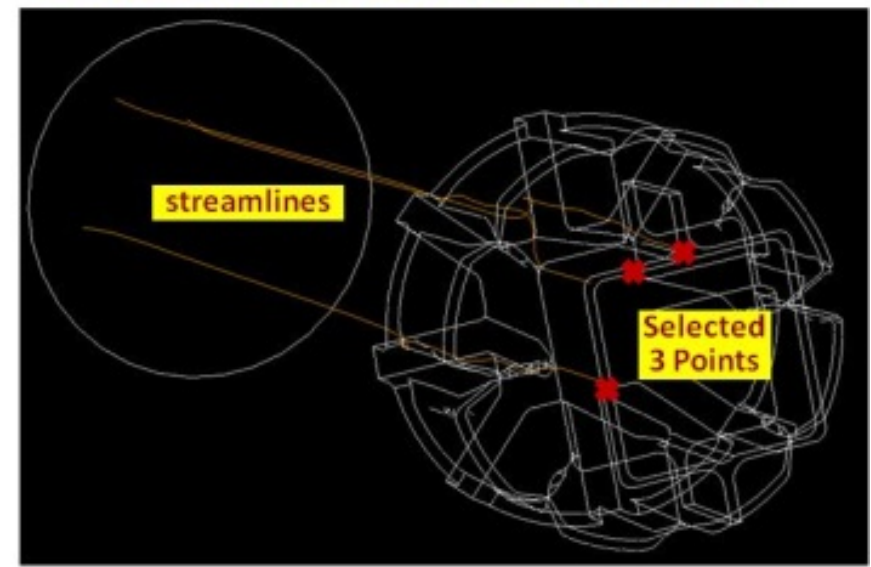

Figure 37. Streamlines in porthole-die thin-walled hollow profile from extrusion simulation using HX.

Using the streamlines in the three representative positions in the double hat and VPSC dislocation density model and the corresponding hardening parameters which were obtained by stressstrain curves fitting can predict the texture and extrusion flow stress during extrusion using the Timminco double hat die as shown in Figure 37. The VPSC predicted texture and EBSD measured texture and the measured compression stress-strain curves in the representative position in the double hat are shown in Figure 38.

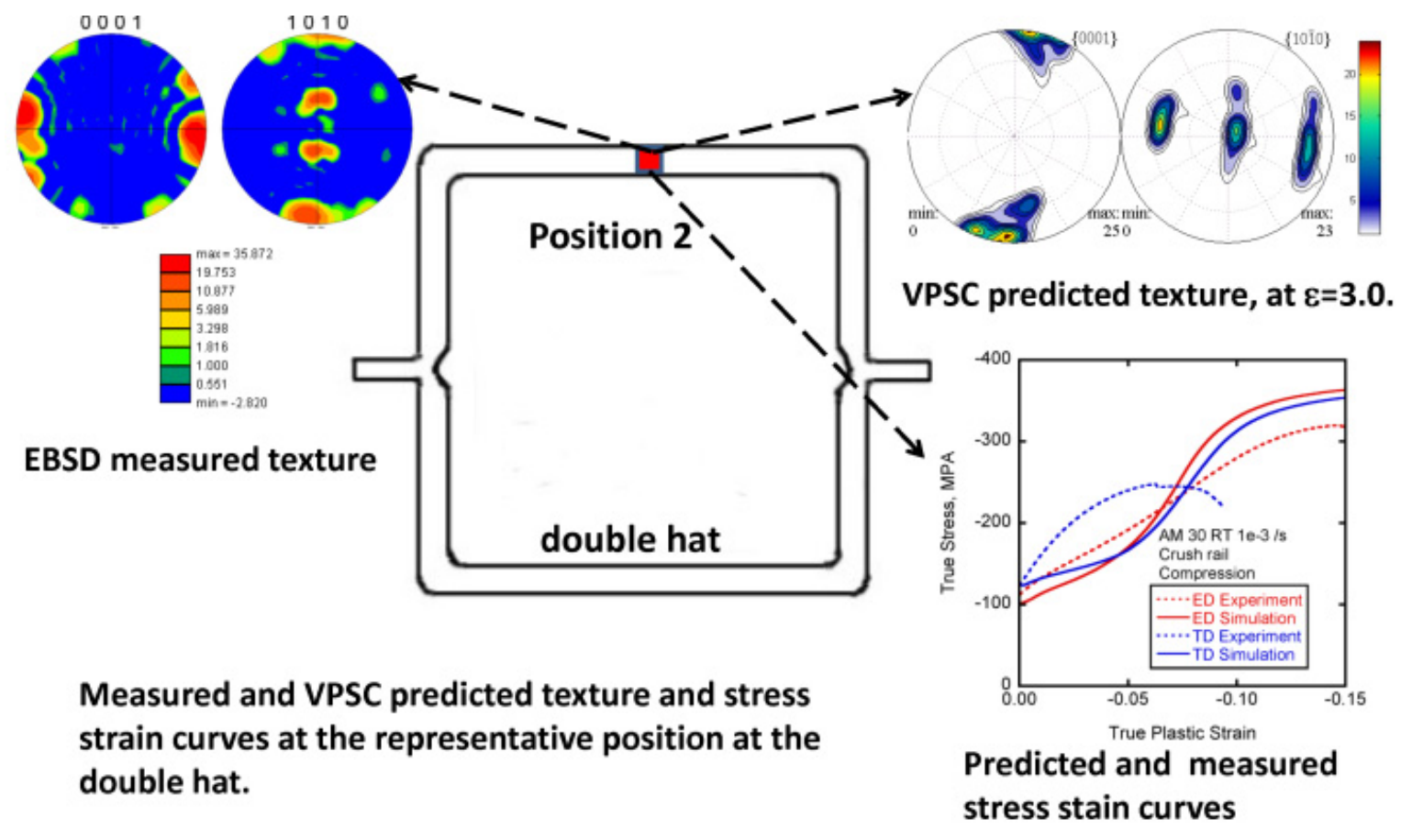

Figure 38: EBSD measured and VPSC predicted texture and predicted and measured stress-strain data in the representative position at the AM30 double hat. 
In Figure 38, event both measured and predicted texture intensity is very strong and due to the severe plasticity during this double hat extrusion, the measured and predicted texture position in the pole figure is different. In contrast to the case of flat die extrusion of AM30 (Figure 30) in which the main predicted texture components by VPSC are in good agreement with EBSD measured texture. The main reason regarding the marked deviation is the non-consideration of the dynamic recrystallization in the present VPSC code even it has taken into the effect of temperature and strain rate. In the simple case of flat die, the effect of dynamic recrystallization may not be significant; however it could play marked roles during severe and more complex deformation, like the double hat extrusion case. Since the difference in texture, the predicted flow stress of ED and TD under compression shows marked deviation from measured values (Figure 38) even the predicted yield stress of ED and TD can be captured by VPSC model and the simulated texture. This should be the future work to solve this problem.

\section{Material Modeling and Sheet Forming Simulations of Magnesium Alloys}

The objective of the sheet forming work is to develop a physically-based material model and an experimental-validated sheet forming model with corresponding design methodologies to enhance the formability of $\mathrm{Mg}$ alloys (AZ31). The current work involves establishing material anisotropy relationships to understand the effects of microstructure state, product geometry and processing parameters on the resulting mechanical properties and distortion of $\mathrm{Mg}$ sheets.

\section{Material Model - DMG Production Version 1.0}

We are developing an appropriate constitutive model which covers critical features including viscoplasticity, anisotropy, damage, strain sensitivity, temperature dependence, grain size and twinning. Currently, an existing MsSt DMG 1.0 microstructure-plasticity model was calibrated with the material

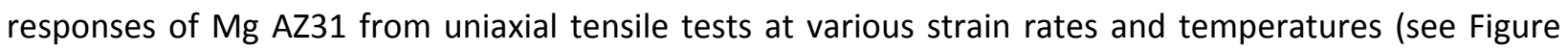
39). This model will be enhanced to include the aforementioned critical features such as twinning. To take advantage of shell elements that have significantly improved numerical run time when compared with 3D elements, the stress integration algorithm was efficiently reformulated in order to take into account of the plane stress condition and implemented in a general finite element code such as ABAQUS.

In this formulation, only the plasticity part of the DMG 1.0 was considered $[3,10]$. The plasticity model is described by the flow rule

$$
\dot{\bar{\varepsilon}}^{p}=f(T) \sinh \left[\frac{|\underline{s}-\underline{\alpha}|-\kappa-Y(T)}{V(T)}\right] \text { and by the kinematic and isotropy hardening evolution equations: }
$$

$\underline{\dot{\beta}}=\underline{\dot{D}}^{p}-\left[r_{d}(T) \dot{\bar{\varepsilon}}^{p}+r_{s}(T)\right]\|\underline{\alpha}\| \underline{\beta} \quad \dot{\varepsilon}_{s s}=\dot{\bar{\varepsilon}}^{p}-\left[R_{d}(T) \dot{\bar{\varepsilon}}^{p}+R_{s}(T)\right] \kappa \varepsilon_{s s}$

$$
\text { with } \quad \underline{\alpha}=\frac{2}{3} h(T) \underline{\beta} \quad \text { with } \quad \kappa=H(T) \varepsilon_{s s}
$$

where $\underline{s}$ is the deviatoric part of the stress tensor, $\underline{\varepsilon}^{p}$ the plastic strain tensor, $\underline{\alpha}$ the kinematic hardening and $\mathrm{k}$ the isotropic hardening, $\underline{B}$ and $\varepsilon_{s s}$ their respective kinematic flux variables, and $f(T)$, 
$V(T), h(T), H(T), r_{d}(T), R_{d}(T), r_{s}(T)$, and $R_{s}(T)$ material parameters. The creep plasticity model used by University of Virginia was also implemented as a special case of the DMG plasticity model (no hardening). In this creep-plasticity model, the Zener-Holloman parameter $Z=\dot{\varepsilon} \exp (Q / R T)$ and the Sellars-Tegart Equation $Z=A[\sinh (\alpha \sigma)]^{n}$ lead to the plastic flow rule $\dot{\bar{\varepsilon}}^{p}=\frac{A}{\exp (Q / R T)}[\sinh \alpha|\underline{S}|]^{n}$

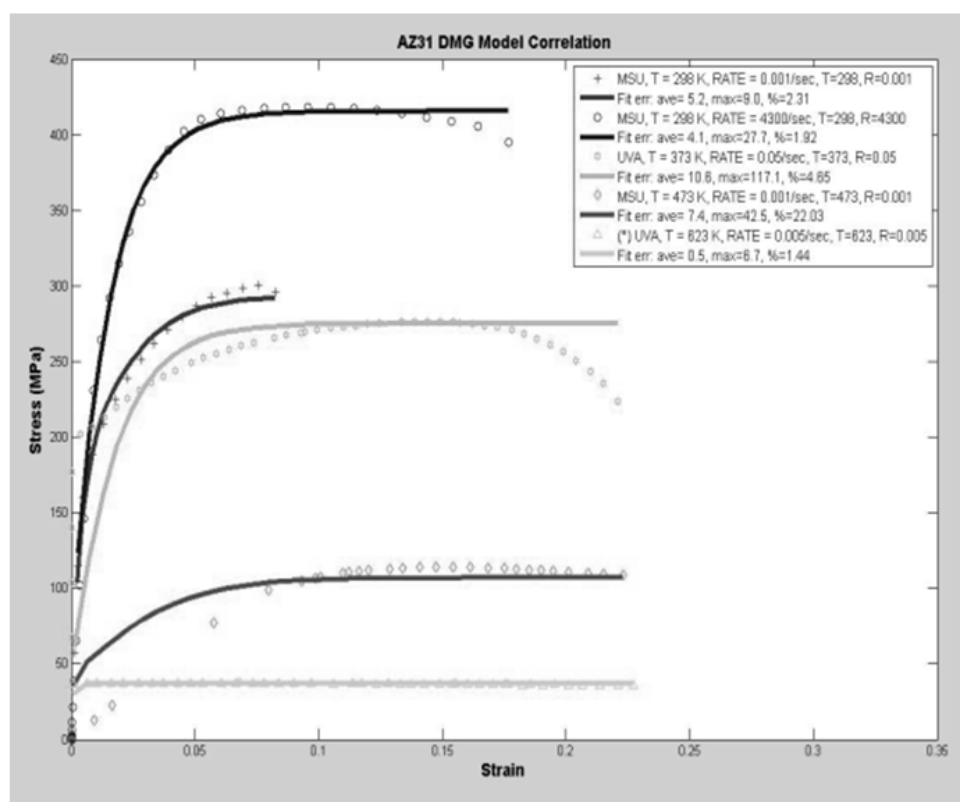

Figure 39: Material fitting of MgAZ31-O with DMG Model.

\section{Sheet Forming Model using DMG 1.0}

A forming limit diagram (FLD) provides the maximum strains of sheet metals that can be sustained prior to the onset of localized necking, which is a common failure mode in metal forming. Generally, this failure process is controlled not only by external loading but also by the state of microstructure. The major mechanical material properties controlling the formability of sheet metals are work hardening, rate sensitivity, and plastic anisotropy. To evaluate the mechanical properties of $\mathrm{Mg}$ AZ31 alloy including formability, warm forming Finite Element (FE) simulations of magnesium sheets (Figure 40a) were performed at various conditions (temperature, tool speed, friction, binding pressure, etc.) using the MsSt DMG 1.0 model. The punch was assumed stationary, while the die moved down after the sheet was clamped with a given binder force, and the sheet was then formed around the stationary punch. The coefficient of friction was set in a range of 0.16 to 0.20 . The sheet and die temperatures were kept to be the same, starting at $250^{\circ} \mathrm{C}$ with an incremental increase of $25^{\circ} \mathrm{C}$ to $350^{\circ} \mathrm{C}$. Regarding the binder pressure, arbitrary values were chosen until actual experimental data will be made available from the forming tests recently conducted by GM. Numerical results will be postprocessed (an example is shown in Figure 40b), and the prediction of forming limit curves (FLCs) and post-formed stress/strain properties will be evaluated. To include the effect of texture, we used experimental data (Jain et al. [10]) in conjunction with the Visco-Plastic Self-Consistent (VPSC) model [1]. 
Figure 36a presents results of AZ31 experimental and simulation stress-strain curves along RD and ND directions based on the Visco-Plastic Self-Consistent (VPSC) model. Using the VPSC code, we first generated the initial and evolving yield surfaces as shown in Figure 41. The next task is to establish a yield surface evolution model from these texture generated yield surface data. The yield surface model will be based on the updated MsSt DMG 1.0 continuum model of anisotropic plasticity.
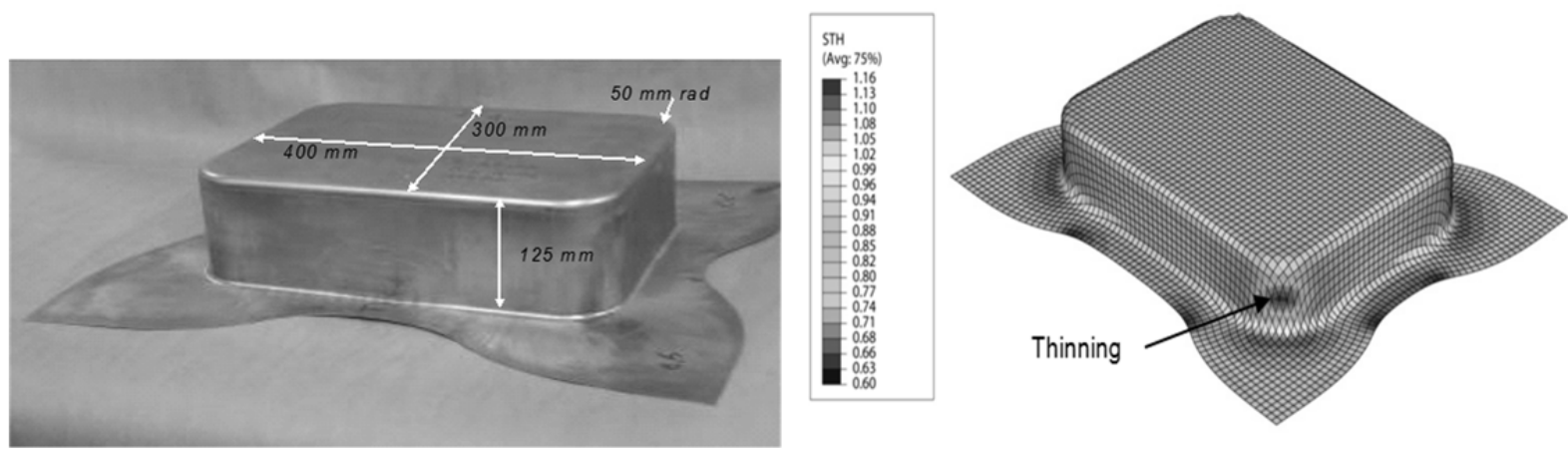

Figure 40: (a) MgAZ31 Warm Forming Pan Geometry for FE Simulations; (b) Thickness distribution after warm forming of a pan box.
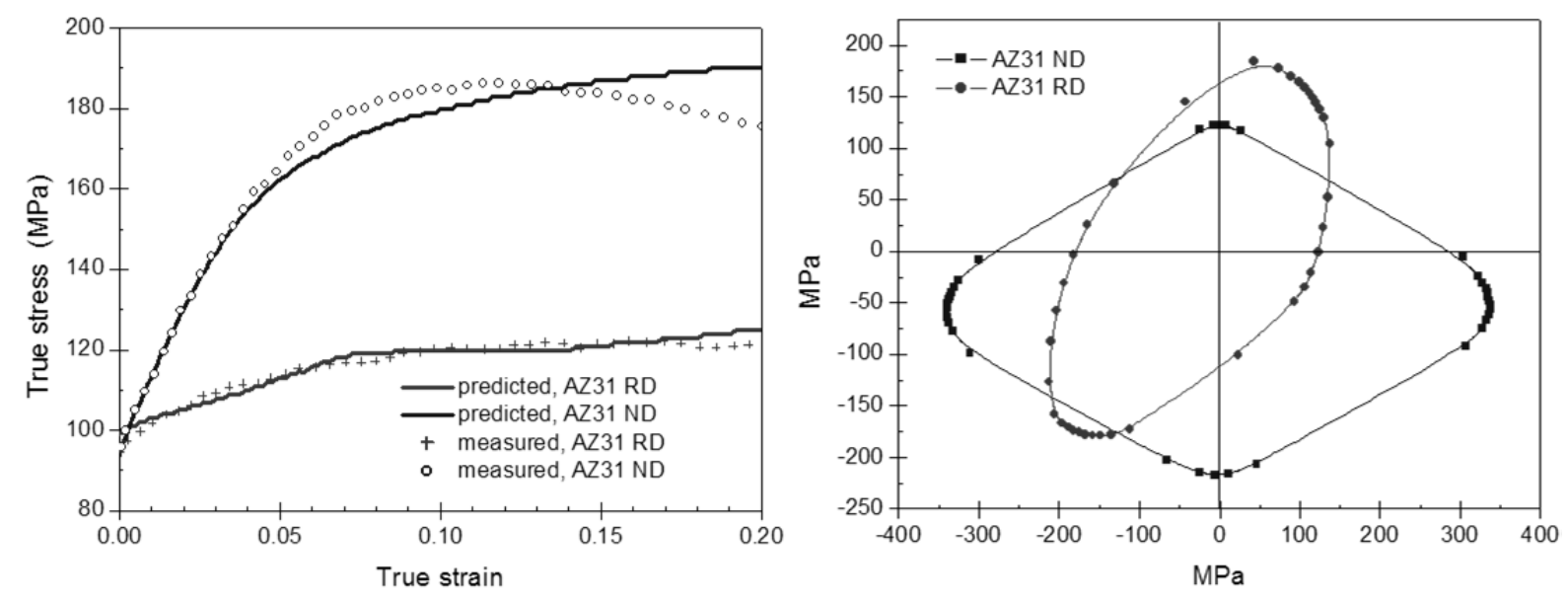

Figure 41: (a)Measured and predicted stress-strain curves of AZ31 sheet compression along RD and ND; (b) Polycrystal yield surface for AZ31 sheet..

Sheet Forming Simulations of Magnesium Alloys: The simulations were performed using the finite element code ABAQUS/Explicit with the DMG material model implemented into the user material subroutine VUMAT. The goal of these simulations was to compare simulation results between viscoplastic material models with and without damage coupling, and to show that the coupling damageplasticity in the DMG material model can obtain a better prediction of the thickness distribution and the onset of localized necking as shown in Figure 42, which is a common failure mode in metal forming. 


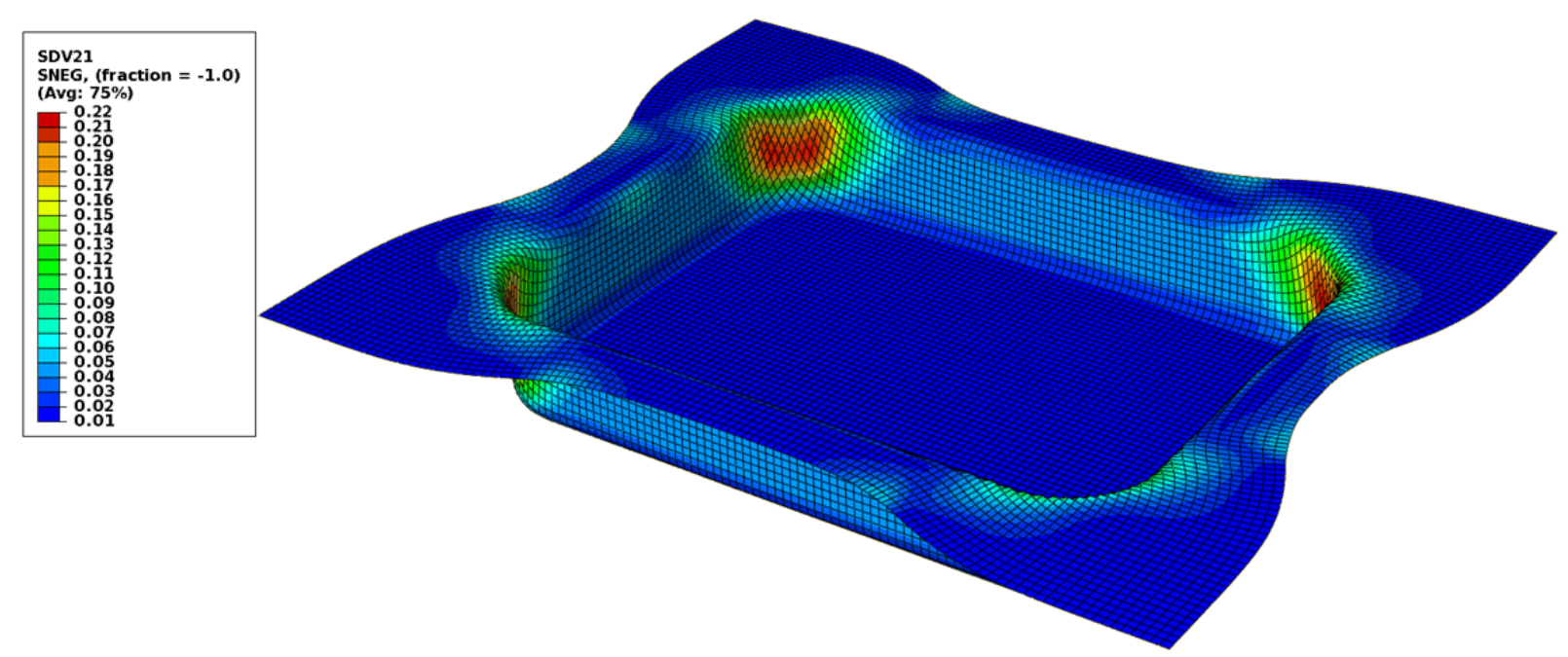

Figure 42. Damage distribution in a pan forming simulation.

We are developing a material database and a multiscale internal state variable (ISV) material and process models for lightweight alloys including magnesium and aluminum. The database will include results from mechanical and microstructural characterization studies performed using advanced experimental techniques. The material model is formulated by a multiscale modeling approach where information from crystal plasticity, dislocation dynamics and molecular dynamics simulations are utilized to provide insights into the functional forms of the ISV's evolution equations and corresponding material parameters. Finally, a design framework contained in the cyberinfrastructure will be developed to design structural components of new lightweight alloys.

\section{Sheet Forming - Experiments and Modeling}

Two sets of bending test fixtures were designed and fabricated for sheet bending experimentation conducted at a FEG-SEM chamber and an Instron machine.

The FEM-based VPSC+UMAT code was implemented on an MSU server and preliminary numerical results look promising, with the capabilities of describing texture/twinning evolution and mechanical responses of the sheet bending process. (Figure 43)

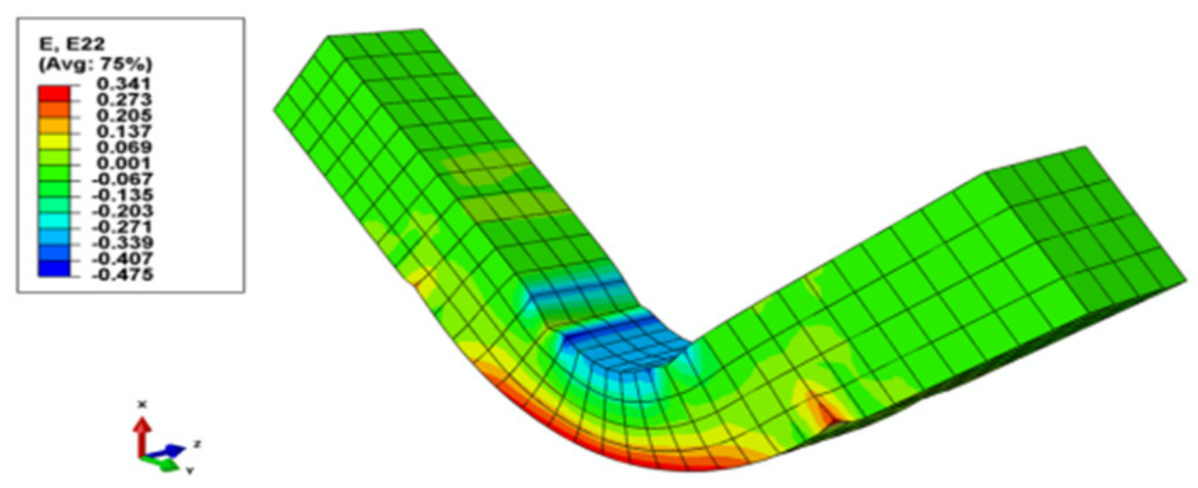


Figure 43. VPSC+UMAT modeling with parallel computing successfully reproduced bending results reported in the literature.

Anomalous twin bands were captured in the in situ electron backscatter diffraction (EBSD) scan. High density twins are localized in alternating bands in the compression zone of the sheet during bending. In between the bands, no twins are present. The nature of these twin bands and how they affect sheet forming, especially at room temperature, requires systematic studies. VPSC+UMAT modeling with parallel computing successfully reproduced bending results reported in the literature. Bending of AZ31 sheet is being modeled with the VPSC+UMAT code.

\section{Conclusions:}

We established the fundamental framework of extrusion processes for $\mathrm{Mg}$ alloys, including lab-scale experimentation to quantify processing -structure - property relations, extrusion modeling to validate texture evolution, the exploration of twinning mechanisms and the associated VPSC models, sheet forming numerical capabilities, and the damaged induced plasticity models with uncertainty. Particular alloys being studied are AM30 extrusion and AZ31 sheets.

For extrusion, we investigated the following: (i) performed lab-scale indirect extrusion experiments to characterize the evolution of microstructure during the process, (ii) extended the capability of the extrusion fixture to experimentally study weld seam formation, (iii) enhanced the current constitutive frameworks to increase the fidelity of the predictive response of $\mathrm{Mg}$ alloys with twinning, (iv) carryed out finite element simulation of the extrusion process using Lagrangian (ABAQUS) and Eulerian (HyperXtrude) finite codes, and ( $v$ ) simulated extrusion of $\mathrm{Mg}$ alloys in both lab-scale and industry scale consideration both temperature and strain rate effects.

For sheet forming work, a material model was implemented in the user material subroutine VUMAT of Abaqus/Explicit for both 3D and shell elements using a fully implicit integration scheme. We continue to investigate the formability of Mg alloys (AZ31) and plan to capture the thinning and initiation of cracks during the sheet metal forming process

In addition, specific research subjects related to this task are listed here: twinning transmutation mechanism in $\mathrm{Mg}$ [7, 9]; twinning effects in rod-textured extruded $\mathrm{Mg}$ [4]; localized twin bands in Mg sheet bending [12]; dislocation mediating \{11-22\} twinning in Titanium [13]; rate-dependent hardening in ultrafine-grained $\mathrm{Mg}$ alloy [14]; twinning induced dynamic recrystallization in Mg alloy [15]; grain growth simulation in binary alloys using PhaseField model [16,17]; extrusion simulation of Al 1100 using HyperXtrude [18]; twinning and double-twinning in prismatic textured $\mathrm{Mg}$ alloy [19]; microstructure and texture evolution during extrusion [20]; grain subdivision and its effect on texture evolution in an annealed Al 
1100 [21]; effects of slip and twinning on texture evolution during dynamic recrystallization in an $\mathrm{Mg}$ alloy [22].

\section{REFERENCES}

[1] R.A. LebensohN, C.N. Tomé, Acta Materialia, 41 (1993) 2611-2624.

[2] D.J. Bammann, M.L. Chiesa, M.F. Horstemeyer, L.I. Weingarten, Failure in Ductile Materials Using Finite Element Methods, Structural Crashworthiness and Failure, eds. T. Wierzbicki and N. Jones, Elsevier Applied Science, The Universities Press (Belfast) Ltd, 1993.

[3] M.F. Horstemeyer, J. Lathrop, A.M. Gokhale, M. Dighe, Modeling stress state dependent damage evolution in a cast Al-Si-Mg aluminum alloy. Theo Appl Frac Mech, 33 (2000) 31-47.

[4] Q. Ma, H. El Kadiri, A.L. Oppedal, et al., Journal of Plasticity 29 (2012) 60-76.

[5] I.J. Beyerlein, C.N. Tomé, International Journal of Plasticity 24 (2008) 867-895.

[6]R. Kaibyshev, O. Sitdikov, Zeitschrift Für Metallkunde 85 (1994) 738-743.

[7] H. El Kadiri, A.L. Oppedal, Journal of the Mechanics and Physics of Solids 58 (2010) 613-624.

[8] E.B. Marin, 2006. Sandia National Laboratories, CA; SAND2006-4170.

[9] A.L. Oppedal, H. El Kadiri, C.N. Tomé, et al. International Journal of Plasticity, 30-31 (2012) 41-61.

[10] Bammann DJ, Chiesa ML, Johnson GC. Modeling large deformation and failure in manufacturing processes. In: Tatsumi T, Watanabe E, Kambe T, eds., Theoretical and applied mechanics. London: Elsevier. 1996, P. 359-76.

[11] A. Jain, S.R. Agnew, Materials Science and Engineering A, 462 (2007) 29-36.

[12] Baird, B. Li, S. Parast, S. Horstemeyer, L. Jr. Hector, P. Wang, M.F. Horstemeyer, Scripta Materialia, 67 (2012) 471-474.

[13] B. Li, H. El Kadiri, M.F. Horstemeyer, Philosophical Magazine, 92(2012) 1006-1022.

[14] B. Li, S.P. Joshi, O. Almagri, Q. Ma, K.T. Ramesh, T. Mukai, Acta Materialia, 60(2012) 1818-1826.

[15] Q. Ma, B. Li, E. Marin, S. Horstemeyer, Scripta Materialia, 65(2011) 823-826.

[16] M. Asle Zaeem, H. El Kadiri, M.F. Horstemeyer, P. Wang, Journal of Phase Equilibria and Diffusion, 2011,1-7.

[17] M. Asle Zaeem, H. El Kadiri, P. Wang, M.F. Horstermyer, Computational Materials Science, 50(2011) 2488-2492. 
[18] A.A. Parkar, C. Bouvard, E. Marin, S. Horstemeyer, P. Wang, M.F. Horstemeyer, Aluminum Alloys: Frabrication, Characterization and Application, 2011 TMS Annual Meeting \& Exhibition.

[19] Q. Ma, H. El Kadiri, A.L. Oppedal, J.C. Baird, M.F. Horstemeyer, M. Cherkaoui, Scripta Materialia, 2011, 64(9): 813-816.

[20] Q. Ma, S.J. Horstemeyer, B. Li, Z. McClelland, P.T. Wang, M.F. Horstemeyer, Magnesium Technology 2013, TMS, accepted.

[21] Q. Ma, W. Mao, B. Li, P.T. Wang, M.F. Horstemeyer, submitted for publication

[22] Q. Ma, B. Li, W.R. Whittington, A.L. Oppedal, E.B. Marin, P.T. Wang, M.F. Horstemeyer, submitted for publication 


\section{SUBTASK 1.2: CYBERINFRASTRUCTURE}

\section{Team Members: T. Haupt, R. Carino, N. Sukhija (student)}

\section{APPROACH}

The objective of this effort has been to design and develop a cyberinfrastructure $(\mathrm{Cl})$ to exploit the recent transformative research in material science involving multiscale physics-based predictive modeling, multiscale experiments, and design. The $\mathrm{Cl}$ has become the foundation of a virtual organization, known as the Engineering Virtual Organization for Cyber Design (EVOCD). EVOCD has been developed with the primary goal of accumulating and protecting the intellectual property generated by the participants of the organization. The portal provides powerful passage for accruing and exchanging community knowledge as well as access to repositories of experimental data, material models and computational tools at different length scales, which together exploit the integrative nature of the Integrated Computational Material Engineering (ICME). To achieve this goal, the EVOCD comprises four primary functional components that are the foundation of the VO: (i) Knowledge Management; (ii) Repository of Codes; (iii) Repository of Data; (iv) Online Calibration Tools. The implementation is primarily based on third-party, open-source software packages (such as Apache's Tomcat, Apache's ServiceMix, View VC, SVN) customized to particular need of EVOCD, and the principles of the ServiceOriented Architectures (SOA).

\section{RESULTS AND DISCUSSION}

The EVOCD portal is up and running (it has been accessed more than 20,000 times by visitors and contributors form over 80 nations around the world since September 1, 2011). The Wiki (http://icme.hpc.msstate.edu), shown in Figure 1, serves as the knowledge management component of EVOCD. It supports the accumulation the knowledge pertaining to ICME. Recently added search and indexing capabilities simplify the navigation through the site contents. In addition, Wiki provides access to all portal components: the repository of the experimental data and material constants, online model calibration tools, and the repository of the open source codes. In the near future it will also provide access to an autonomic runtime environment for composing and executing multiscale workflows directly from the Web Browser. The Wiki are currently populated with information, tutorials, data, and results coming from the other SRCLID tasks. The EVOCD becomes a node of the global ICME cyberinfrastructure - an effort coordinated by the Minerals, Metals, and Materials Society (TMS). The EVOCD portal has been developed during the previous stages of this project. During this year are activities were focused on maintenance of the system and populating it with the data and knowledge. 


\section{Engineering Virtual Organization for CyberDesign}

\section{Welcome!}

Integrated Computational Material Engineering (ICME) is an emerging discipline transforming materials science. Computational engineering accelerates materials development, integrates design and manufacturing, and unifies these with the engineering design optimization process, as well as efficiently employs greater accuracy in simulation-based design. Read more...

Efforts to realize this enormous and complex goal have catalyzed the development of the Engineering Virtual Organization for Cyber Design (EVOCD), which provides a cyberinfrastructure to accumulate and protect the intellectual property pertaining to selected aspects of materials science and engineering that is generated by the participants of the organization, to enforce the quality of that information, and to manage its complexity. The intellectual property includes experimental data, material models and constants, computational tools and software artifacts, and the knowledge pertaining to multiscale physics-based models for selected properties and processes. Read more..

\section{ICME Cyberinfrastructure}

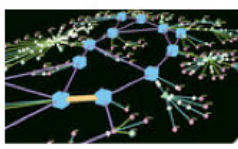

In the vision of the National Materials Advisory Board (NMAB) Es the National Academy of Engineering NAE) committee, the ICME cyberinfrastructure will emerge from individual web sites which offer access to information, data, and tools, each established for specific purposes by different organizations. Linked together, these "constituent" Web Portals will form the ICME cyberinfrastructure. EVOCD is one of the nodes of the emerging cyberinfrastructure. Read more ... 还

The creation of the ICME infrastructure is coordinated by the

\section{The Minerals, Metals, and Materials Society (TMS) 룽.}

\section{Contact}

Tomasz Haupt $\mathrm{E}$ Mark Horstemeyer $\mathrm{E}$

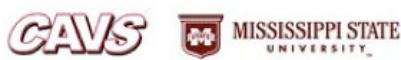

\section{Call for Participation}

The Cyberinfrastructure team created the infrastructure for web-based collaborative efforts. The success of this effort critically depends on the participation of the community towards the generation of the contents that will aid the research in Materials Science and lightweight innovative design. Read more ...

\section{How to contribute}

To learn how to contribute please see our short Tutorial. Please note that contributions (creating new pages or editing existing ones) can be made only be the registered users.

\section{Elements of}

\section{ICME Cyberinfrastructure}

We would also like to invite you to other materials oriented databases: Materials Atlas 色, nanoHub te matweb 居, MatData 层, Granta Design Knowledgebase of Interatomic Potentials (OpenKIM) 타. Korean Materials Design MatForge/MatDL 函, National Institute of Standards and Technology (NIST) Data Gateway 람 See also TMS ICME forum हs

\section{EVOCD components}

- This Wiki

- Repository of Data

- Repository of Codes

- Tools

\section{Site Index}

- Overview Pages

- Research Topics

- Materials

- Material Models

- Multiscale Simulations

- Tutorials

- Input Decks

\section{Our Sponsors}

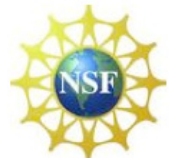

(NSF Award ID: 0742730)

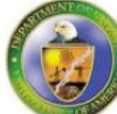

(DOE contract 4000054701)

DOE Southern Regional Center for Lightweight Innovative Design (SRCLID)

Figure 1: Home page of the EVOCD wiki 


\section{Subtask 1.3: Fatigue Performance of Lightweight Materials}

Team Members: M. Lugo, J.B. Jordon, M.F. Horstemeyer, J. Bernard (PhD student)

\section{Objective}

- Develop and experimentally validate prognostic tools for in-service life prediction and fatigue design of lightweight automotive materials and components.

\section{BACKGROUND}

Industry and government have organized research/development/application programs to promote lightweight materials as part of the solution to reduce greenhouse emissions and improve fuel economies in transportation vehicles. This heightened interest in lightweight materials has pushed the materials community to investigate not only cast magnesium but also wrought magnesium products for their high strength-to-weight ratio and better fatigue resistance. While studies of cyclic performance of magnesium alloys exist in the literature most of these are associated with casting products. In fact, wrought magnesium alloys generally have higher mechanical strength and fatigue resistance than cast magnesium alloys due to their ability to limit inclusion sizes and for precipitation hardening after the severe deformation during processing. Thus, a need arises for a predictive tool that can capture the fatigue damage in wrought magnesium alloys with the intent of determining/designing the best alloy for use in a range of automotive applications. Therefore, the overarching goal of this task is the development of a physically-motivated microstructurally-based fatigue model.

In order to reach the goal of using more light weight metals in automobiles, understanding and modeling the fatigue damage of welded joints is of great importance. As with the base materials, research is needed to determine the reliability and performance of potential welding techniques and develop microstructurally-based modeling approaches. While research in the area of fatigue in friction stir spot welds is not unique, recent work has been exclusively focused FCC alloys and not HCP alloys such as magnesium.

Finally, the investigation of fatigue behavior of nanoscale materials has been of significant interest to many researchers due to the development of technologies of nano-materials. As such, understanding how fatigue damage initiates and grows at the nanoscale for magnesium alloys will lead to better modeling approaches at the higher length scales.

\section{APPROACH}

Strain-life fatigue tests of selected magnesium alloys (AZ31, AM30, AZ61, and AZ91) were conducted to investigate the fatigue behavior of magnesium alloys and automotive components. Structure-property analyses were conducted to determine relations between microstructural features and fatigue life. In addition, scanning electron microscope (SEM) experiments were performed to determine fatigue crack incubation and microstructurally/physically small fatigue crack stages. Experiments also were performed to determine the fatigue performance of several different types of joints. 
Fatigue performance of magnesium alloys was characterized, and a structure-property modeling framework for fatigue life using the Multistage Fatigue (MSF) modeling approach was developed. We used a microstructure-sensitive fatigue model which decomposes total fatigue lifetimes into crack incubation, microstructurally small crack (MSC) and physically small crack (PSC), and long crack growth, to correlate the differences in fatigue behavior of magnesium alloys. The model proposed has the capabilities to capture competing structure-property relations, including grain size, inclusions size, and texture, and their consequential impact on fatigue lifetimes.

\section{Microstructure-Sensitive Approach}

The multistage fatigue (MSF) is a microstructure-sensitive model that considers various experimentally observed stages of fatigue damage evolution such as crack incubation, microstructurally small crack (MSC), physically small crack (PSC), and long crack growth. While the model was originally developed for a cast A356 Al alloy [1], it has been modified to extend its application to other alloys and other materials processing methods (e.g. processing of wrought materials) [2]. A brief summary of the MSF model is given below, while further details including the theoretical basis are found elsewhere [1-2]. The total fatigue life $N_{\text {Total }}$ is given by

$$
N_{\text {Total }}=N_{\text {Inc }}+N_{M S C / P S C}+N_{L C}
$$

where $N_{\text {Inc }}$ is the number of cycles to incubate a crack near or at a micro-notch that includes the nucleation of crack-like damage and early crack propagation through the zone of the micronotch's root influence [1]. For purposes of this study, a micro-notch is used interchangeably with the term inclusion, where the term "inclusion" is used to represent any microstructural defect found in a material, such second phase particles, intermetallic particles, and pores, among others. The $N_{M S c}$ term is the number of cycles required for propagation of a microstructurally small crack (MSC); $N_{\text {PSC }}$ is the number of cycles required for propagation of a physically small crack (PSC), and $N_{L C}$ is the number of cycles required for long crack propagation.

Crack incubation involves nucleation plus small crack growth at the micro-notch of the order of $1 / 2 D$, where $D$ is the inclusion size. Small crack growth (MSC) comprises propagation of microstructural cracks with lengths that fall within $a_{i}<a<k M S$, where $M S$ is a characteristic length scale (defined as the smallest grain size) of interaction with microstructural (MS) features, and $k$ is a multiplier where $1 \leq k \leq$ 3 [1-2]. The PSC range consists of crack propagation with a crack length in the interval of $k M S<a<\sim 10$ $M S)$. Depending on the microstructural inclusion morphology and texture of the matrix, the PSC regime may extend from $300 \mu \mathrm{m}$ to $\sim 2-3 \mathrm{~mm}$ in length.

\section{Crack Incubation Regime}

The fatigue damage incubation life, $N_{\text {inc }}$ is characterized by the cyclic damage at the micronotch root. This required implementation of a modified Coffin-Manson law form [1]. at the microscale as follows:

$C_{\mathrm{inc}} N_{\text {inc }}^{\alpha}=\beta=\frac{\Delta \gamma_{\max }{ }^{p *}}{2}(2)$ 
where $\beta$ is the nonlocal damage parameter around an inclusion, and $C_{\mathrm{inc}}$ and $\alpha$ represent the linear coefficient and exponent, respectively in a modified Coffin-Mason approach for the fatigue crack incubation life. The numerical value of the exponent $\alpha$ was selected to be in the range of the macroscopic Coffin-Mason law [1-2]. The coefficient in the Coffin-Manson equation, $C_{\text {inc }}=c_{n}+$ $z\left(c_{m}-c_{n}\right)$, is modified [1] to include a coefficient for nucleation of small cracks at inclusions in the high cycle regime $\left(c_{n}\right)$ and a coefficient for the low cycle regime $\left(c_{m}\right)$. We further note that $z=$ $(1 / 0.7)\left\langle\frac{l}{D}-0.3\right\rangle$ is a localization multiplier [1] that is nonzero below the microplasticity percolation limit. Just beyond the percolation limit, the localization multiplier transitions to unity as the plastic shear strain localizes interdendritically [1]. Above that point, incubation is negligible because of the high strain level that localizes at the inclusion [1]. Note that the ratio $\frac{l}{D}=\sqrt{\frac{A_{\text {plastic zone }}}{A_{\text {particle }}}}$ is defined as the square root of the ratio of the plastic zone over the inclusion area. The $\beta$ parameter is related to the local average maximum plastic shear strain amplitude, $\frac{\Delta \gamma_{\max }{ }^{*}}{2}$, and is estimated by the following relations:

$\beta=\frac{\Delta \gamma_{\max }{ }^{*}}{2}=Y\left[\varepsilon_{a}-\varepsilon_{t h}\right]^{q}, \quad \frac{l}{D}<\eta_{l i m}$,

$\beta=\frac{\Delta \gamma_{\max }{ }^{*}}{2}=Y(1+\zeta z)\left[\varepsilon_{a}-\varepsilon_{t h}\right]^{q}, \quad \frac{l}{D}>\eta_{l i m}$.

Here, ${ }_{a}$ is the remote applied strain amplitude, $\varepsilon_{t h}$ is the microplasticity threshold, and the parameters $q$ and $\zeta$ are determined from micromechanical simulations [1-2]. The limiting ratio, $\eta_{\text {lim }}$, indicates the transition from proportional (constrained) micronotch root plasticity to nonlinear (unconstrained) micronotch root plasticity with respect to the applied strain amplitude, where $\eta_{\text {lim }}=0.3$ has been found to be suitable for Mg alloys [3]. The parameter $Y$ [2] is correlated as $Y=y_{1}+(1+R) y_{2}$, where $R$ is the load ratio, and $y_{1}$ and $y_{2}$ are model constants. For completely reversed loading cases, $Y=y_{1}$. Furthermore, when $\frac{l}{D}$ reaches its limits the parameter $Y$ is revised to include the geometric effects related to the type of inclusion, $\bar{Y}=\left(1+\frac{l}{D}\right) Y$. The debonded particle and pore of the same size may cause a different $Y$ as a factor of three or greater [4]. The correlation of the plastic zone size is calculated using the $\frac{\Delta \gamma_{\max }{ }^{* *}}{2}$ with respect to the remote loading strain amplitude,

$\frac{l}{D}=\eta_{l i m} \frac{\left\langle\varepsilon_{a}-\varepsilon_{t h}\right\rangle}{\varepsilon_{p e r}-\varepsilon_{t h}}, \frac{l}{D} \leq \eta_{l i m}$,

$\frac{l}{D}=1-\left(1-\eta_{\text {lim }}\right)\left(\frac{\varepsilon_{p e r}}{\varepsilon_{a}}\right)^{r}, \frac{l}{D}>\eta_{\text {lim }}$

where $r$, a shape constant for the transition to the limited plasticity [5-6], is determined through micromechanical simulations [2], and $\varepsilon_{\text {per }}$ is the percolation limit [1]. 


\section{Small Crack Growth}

Similar to modeling efforts in wrought aluminum alloys [2], we combine the mathematical expressions for the MSC and PSC regimes into a single mathematical form. Crack growth in the MSC/PSC is governed by the range of crack tip displacement, $\triangle C T D$, which is proportional to the crack length, and the $\mathrm{n}^{\text {th }}$ power of the applied stress amplitude, $\sigma_{a}^{n}$, in the high cycle fatigue (HCF) regime and to the macroscopic plastic shear strain range, $\frac{\Delta \gamma_{\max }^{P}}{2}$, in the low cycle fatigue (LCF), and it is given by the following,

$\left(\frac{d a}{d N}\right)_{M S C}=\chi\left(\Delta C T D-\Delta C T D_{t h}\right), \quad a_{i}=0.625 D$

$\Delta C T D=C_{I I}\left(\frac{G S}{G S_{0}}\right)^{\omega}\left[\frac{U \Delta \widehat{\sigma}}{S_{u t}}\right]^{\zeta} a_{i}+C_{I}\left(\frac{G S}{G S_{0}}\right)^{\omega}\left(\frac{\Delta \gamma_{\max }^{P}}{2}\right)^{2}$

Here, $\chi$ is a constant for a given microstructure, typically less than unity and usually taken as 0.32 for several different alloys [2], and $a_{i}$ is the initial crack length. The $\mathrm{C}_{\mathrm{I}}$ and $\mathrm{C}_{\mathrm{II}}$, and are material dependent parameters which capture the microstructural effects on MSC growth [1-2]. The threshold value for crack tip displacement was defined on the order of the Burger's vector for the Mg-rich matrix [7], $\Delta C T D_{t h}=3.2 * 10^{-4} \mu \mathrm{m}$. The term $\Delta \hat{\sigma}$ is the combination of the uniaxial effective stress amplitude, $\bar{\sigma}_{a}=\sqrt{\frac{3}{2} \frac{\Delta \sigma_{i j}^{\prime}}{2} \frac{\Delta \sigma_{i j}^{\prime}}{2}}$, and the maximum principal stress range, $\Delta \sigma_{1}$, and is given as $\Delta \hat{\sigma}=2 \theta \bar{\sigma}_{a}+(1-$ $\theta) \Delta \sigma_{1}$, with $0 \leq \theta \leq 1$ as the path dependent loading parameter after [8], where $\theta$ is a weighting parameter such that $\theta=0$ gives the von Mises stress state, and $\theta=1$ gives the maximum for principal stress state. The parameter $U$ is employed to capture the load ratio effects and is defined as $U=\frac{1}{1-R}$ [2]. In order to capture the effect of grain size on small crack growth, we employ the ratio of grain size to the reference size $\left(\frac{G S}{G S_{0}}\right)^{\omega}$ for each of the three materials in this study, where $G S_{0}$ is the reference grain size, $G S$ is the specific grain size, and $\omega$ is a material parameter [2].

\section{Long Crack Growth}

The long crack growth stage of the MSF model usually follows classical linear elastic fracture mechanics (LEFM) approaches [1]. We note, however, that the modeling approach presented here is focused on incubation and MSC/PSC regimes similar to Jordon et al. [3,9]. This approach is consistent with the experimental evidence that the formulation for MSC/PSC can characterize fatigue cracks up to several millimeters in length [1]. Thus, the MSF model is essentially used to predict the onset of surface crack initiation in structural components and therefore long crack propagation is generally not considered.

This multistage framework will be evaluated for the prediction of fatigue damage in magnesium alloys and magnesium welded joints. The MSF will also be implemented into commercial FEA codes and validated by solving specific problems concerning the mechanical response and reliability/safety aspects of magnesium alloys used in automotive applications. 


\section{ACCOMPLISHMENTS}

- Performed tensile and compression testing on Mg AZ31 alloy (GM rail).

- Performed strain-life tests for AM30 (GM Rail).

- Performed tensile testing on Mg AZ61 alloy in the extruded and transverse directions.

- Conducted simulations to evaluate the stress field around a crack tip.

- Conduct the finite element analysis for opening stress induced by crack tip plasticity for AM30 wrought $\mathrm{Mg}$ alloy using a developed crystal plasticity model.

- Conducted simulations of crack growth under various crystal orientations.

- Examined and quantified the source of fatigue crack initiation on Mg AM30 (GM rail).

- Determined structure-property relationships between microstructure features and fatigue life for an AM30 Mg extruded alloy.

- Incorporated structure-property relationships into the MultiStage Fatigue (MSF) model to predict the scatter in the fatigue life.

- Conducted strain-life fatigue tests for an extruded AM30 (Ford rail) in the extruded and transverse directions.

- Performed strain-life tests for an extruded AZ31 alloy (GM rail).

- Performed strain-life tests for two different wrought products of AZ31 alloy: plate, and sheet.

- Conducted fractography analysis (SEM) of fracture surfaces of AZ31, AZ61, and AM30 alloys.

- Conducting in-situ SEM experiments of the alloys selected.

- Developed multistage fatigue (MSF) models for AZ31, AM30, AZ61, AM60, and AZ91 alloys.

- Strain-based modeling for AZ31 and AM30 Mg alloys was developed.

- Fatigue experiments for a joint specified by sponsor.

- Developed test matrix and test specimens for Mg friction stir spot weld lap-joint coupon testing.

- Conducted monotonic and cyclic lap-joint testing on Mg friction stir spot welds.

- Characterized fracture mode and path of Mg friction stir spot welds. 
- Developed a first order fatigue model for a joint based on fatigue experiments and joint simulations.

- Implemented crack growth model for predicting fatigue life of lap-joint of $\mathrm{Mg}$ friction stir spot welds.

- We have been developing a microstructure sensitive fatigue model predictions for friction stir welding (FSW) of different welds under various welding conditions and performed fractography analysis to determine failure mechanisms.

- Integrated Multistage Fatigue model with plasticity/damage model (DMG 1.0).

- Identified component for validation purposes.

- Validated ISV/MSF models with components specified by sponsors.

- Conducted finite element simulations for the shock tower to validate the MSF models.

\section{ACtiVITY AND DeVELOPMENTS}

\section{FASTRAN and Fatigue Crack Growth for AZ91 Mg Alloy}

For a number of engineered materials, the fatigue process was shown to be dominated by "crack propagation" from metallurgical features or discontinuities, such as voids, inclusion particle clusters and grain boundary locations [10-12]. Thus, the fatigue life is primarily composed of the cyclic life for microstructurally and physically small cracks and large crack behavior. For these materials, observations of the fatigue process indicated that microcracks are present when the material is processed or they crack very early from microscale plasticity at local discontinuities.

Small crack theory, which assumes an initial flaw size from 1 to $30 \mu \mathrm{m}$ for various materials, has been used with the fatigue crack growth rate properties and a crack-closure model [13] to predict or calculate the stress-life (S-N) curves under both constant and variable amplitude loading. In the small crack theory approach, no distinction is made between microstructurally and physically small cracks. They are both considered "small" cracks and the influences of micro-structure are in the measured crack growth rate data. Small crack growth behavior is achieved by continuum-mechanics analyses using monotonic and cyclic plasticity and the crack-closure transients under the prescribed load histories.

One of the objectives of the project was to determine whether magnesium alloys have similar initial flaw sizes in their microstructure that precipitate fatigue failure from crack propagation alone. In an effort to address the feasibility of using small crack theory to calculate fatigue lives on a magnesium alloy, fatigue $(-\mathrm{N})$ data were recently obtained at MSU for AZ91D. 


\section{Fatigue Crack Growth}

Fatigue crack growth rate data on a AZ91E magnesium alloy has been obtained from Dr. Andy Newman, U.S. Army at NASA Langley Research Center. These data were determined from compact tension specimens under two stress ratios $\left(R=P_{\min } / P_{\max }=0.1\right.$ and 0.7$)$, and these data are shown as symbols in Figure 1. The crack growth rate data was measured from threshold to near fracture, and the fracture toughness was estimated to be about $15 \mathrm{ksi}^{-i \mathrm{n}^{1 / 2}}$. Cracks in the high stress ratio tests have fully open cracks (no crack-closure). Thus, these data are used to establish the effective stress intensity factor against rate ( $\Delta \mathrm{K}_{\text {eff }}$-rate) baseline curve for the material and test conditions (room temperature and lab

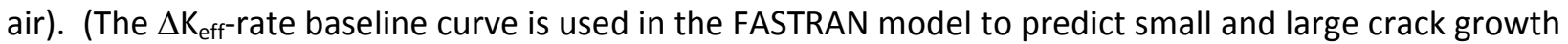
under constant and variable amplitude loading.) Because the high $\mathrm{R}$ tests fracture at lower values of $\Delta \mathrm{K}$ than low $R$ tests, i.e., $\Delta \mathrm{K}_{\mathrm{c}}=\mathrm{K}_{\mathrm{c}}(1-\mathrm{R})$, the low $\mathrm{R}$ test results are also used to estimate the $\Delta \mathrm{K}_{\text {eff }}$-rate curve at high rates. For high rates, an iterative procedure is used because the curves are a function of the crack-front constraint. For these data, the constraint factor, $\alpha$, was found to be 1.55 , which is lower than expected. Increased crack-closure effects due to crack-surface roughness may have caused the lower value. The solid (black) curves are the predicted curves from the FASTRAN model.

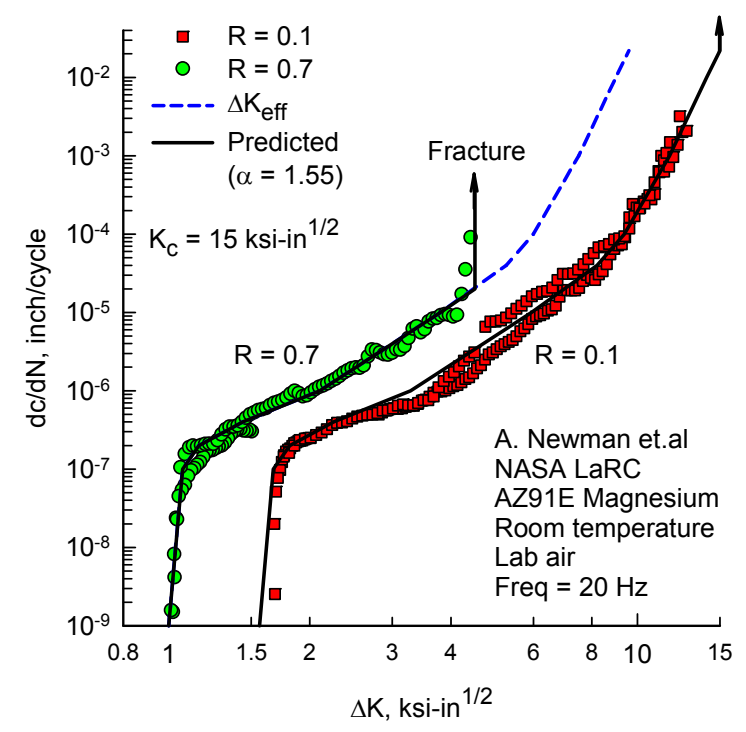

Figure 1. Fatigue crack growth rate data on AZ91E magnesium alloy.

\section{Strain-Life Tests on Magnesium Alloy}

Fatigue test $(\Delta \varepsilon-N)$ data generated on $\mathrm{K}_{\mathrm{T}}=1$ bar specimens under fully reversed loading for the magnesium alloy AZ91D are shown in Figure 2 (diamond symbols). The data also included the full strain and load against cyclic history on eight specimens. The bar specimens were 0.12 inches thick by 0.25 inches wide and tested at three strain levels $(0.002,0.003$ and 0.0035$)$. 


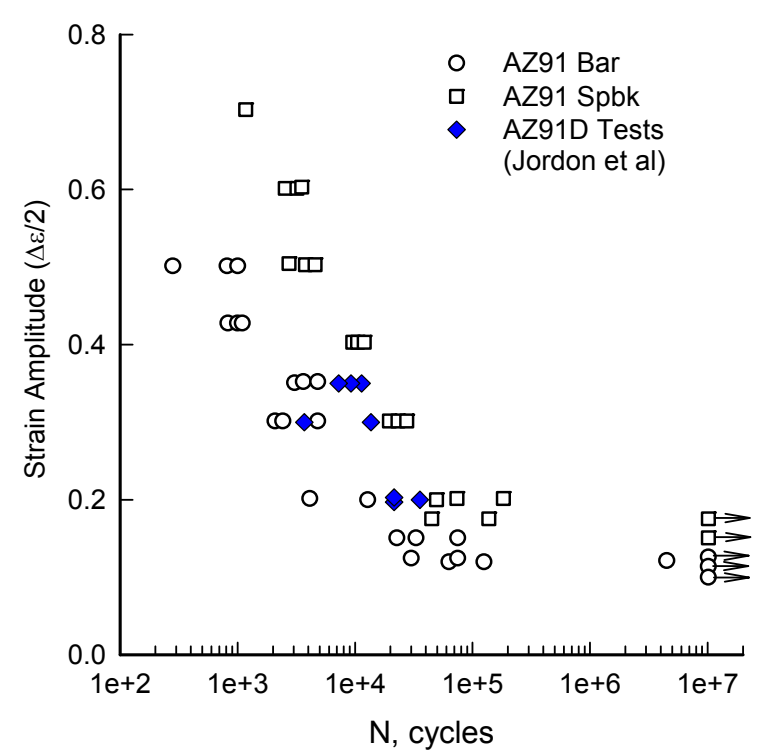

Figure 2. Strain against cycles to failure on AZ91 magnesium alloys.

Figure 3 shows the applied stress (maximum and minimum) for test specimen LCF-3. The maximum applied stress was nearly constant $\left(S_{\max } \sim 20.5 \mathrm{ksi}\right)$ during the duration of the test, but showed a slight reduction near the end of life due to cracking. But the minimum applied stress showed more variation ( -18 to $-22 \mathrm{ksi})$. The reason for this behavior was unknown but could be related to the tensile and compressive stress-strain curves being different, cyclic strain hardening, or caused by variations in the stress-strain response from different sections of the magnesium material.

\section{Fatigue Life Calculations on Magnesium Alloy}

The FASTRAN life-prediction code was used to calculate the fatigue lives on the AZ91D alloy specimens using crack propagation from an equivalent initial flaw size (EIFS). The effective stress-intensity factor against crack growth rate curve had previously been obtained on an AZ91E alloy from NASA Langley.

Since the FASTRAN code is based on the "applied stress" instead of applied strain, either an average stress history or the variations in both minimum and maximum stresses would have to be considered in the fatigue life analyses. For simplicity, an average stress history $\left(S_{\max }, S_{\min }\right)$ was selected for each specimen and these values were used in the fatigue-life analyses. Some typical results are shown in Figure 3, which shows the maximum and minimum stresses applied to LCF-3 as a function of cycles. This test had a stress ratio $R\left(S_{\min } / S_{\max }\right)$ of -0.98 . The rapid (vertical) drop off of stress indicated failure of the specimen at 21,500 cycles. Whereas, the cyclic strains were very accurately maintained during the duration of the test, the stresses showed some variations. The range of stress ratios from the eight tests was from -0.98 to -0.42 . 


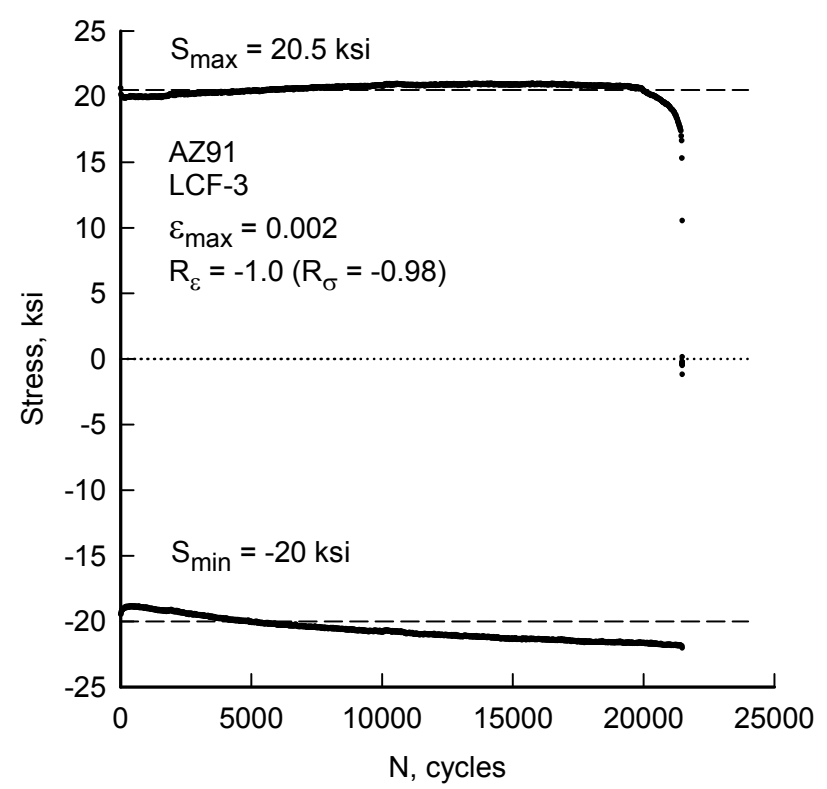

Figure 3. Applied stress against cycles for LCF-3.

The maximum applied stress against cyclic life (S-N) for the AZ91 alloy is shown in Figure 4. As noted, the applied stress ratio $(R)$ varied. Thus, calculations have been made at -1 and -0.4 . A trial-and-error procedure was used to find the equivalent initial flaw size (EIFS) to fit the test data. The EIFS was found to be a 0.012 -inch $(305-\mu \mathrm{m})$ semi-circular surface flaw. The $R=-1$ curve agree well with five of the eight specimens. The $R=-0.4$ curve agree well with specimen LCF-5, which had an applied stress ratio of 0.42. But the results for test specimens LCF-3 and 4 showed that the calculated curves were very conservative.

A typical fatigue surface from the AZ91 alloy is shown in Figure 5. The initiation site appeared to be a large void (620 m deep by $250 \mathrm{~m}$ wide) located near the edge of the specimen. Using Murakami's area rule, a 280- $\mathrm{m}$ semi-circular surface crack would have the same area. Thus, the assumed 305- $\mathrm{m}$ radius surface flaw was very close to the void area.

Most of the calculations fell within $\pm 30 \%$, but calculations made on specimens LCF-3 and 4 were very conservative (factor-of-10) and the calculation on specimen LCF- 6 was un-conservative by over a factorof-2. A determination of the distribution of void sizes in the AZ91 alloy would be useful. Specimens LCF3 and 4 could have had smaller voids that precipitated failure due to the very small specimen tested. FASTRAN could be used with the distribution of voids to make an upper and lower bound estimate for the fatigue scatter. Fastran calculations represented the AZ91 magnesium alloy very well, in that, the fatigue lives on 6 of 8 tests were accurately calculated. However, these tests were basically low-cycle fatigue (LCF) results. Whether small-crack theory will work well on the magnesium alloys for high-cycle fatigue (HCF) is yet to be studied. 


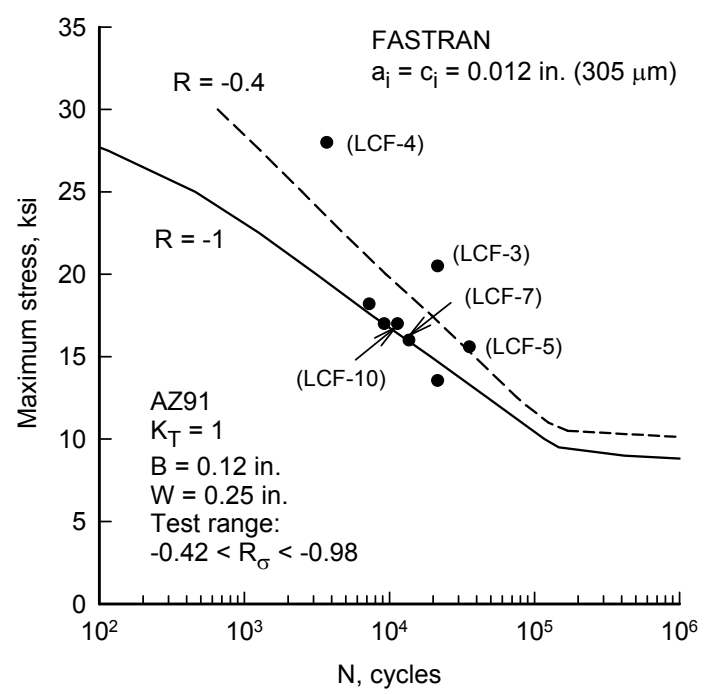

Figure 4. Stress against cycles to failure on AZ91 magnesium alloy.

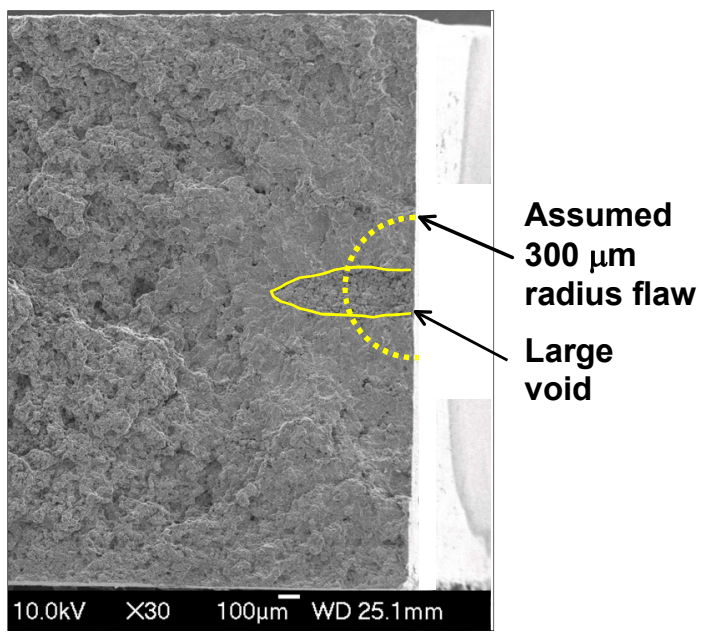

Figure 5. Initiation site at large void and assumed EIFS in AZ91 alloy.

\section{Structure-property Relations of Fatigue in Mg AM30 Alloy}

The fatigue properties of an AM30 magnesium alloy extruded into a crash rail-shaped component were evaluated experimentally and numerically. Fully-reversed, strain control fatigue tests were conducted on specimens extracted from regions having a varying grain size and texture. Using scanning electron microscopy, the fracture surfaces of each failed specimen were analyzed in order to establish the relationship between microstructure and fatigue behavior. Texture and grain size were identified through EBSD analysis. Parallels between microstructural features such as particle size, grain size, Taylor Factor, and the number of cycles to failure were determined.

The microstructure anisotropy associated with the extruded rail contains non-uniform texture and grain sizes as a result of the extrusion process. Due to the speed of the extrusion, limited recrystallization 
occurred and thus microstructural heterogeneities developed. As such, the average grain size and initial Taylor Factor based on electron backscatter diffraction (EBSD) results of the rail were measured and are shown in Fig 6 . The fatigue specimens tested as part of this study were taken from the six regions indicated in Fig 1 and labeled as A, B, C, E, G, and I. Post-mortem fractography was conducted on fatigue specimens tested under fully-reversed constant strain amplitudes of $0.003 \mathrm{~mm} / \mathrm{mm}$ in ambient temperature and humidity. Each of the fracture surfaces were examined under a scanning electron microscope (SEM) with the intent to determine the sources of crack initiation. Figure 7 shows a typcial intermetallic particle that initiated the fatigue crack. Chemical analysis of the intermetallics revealed that the particles were comprosed of Mg-Al-Mn compounds. As such, these types of particles are more brittle than the surrounding matrix and tend to fracture rather than debond. Fracturing of the intermetallic particles was observed on all of the surfaces imaged via the SEM, and no evidence of debonding was observed. Furthermore, all of the particles were found to be located on the surface of the specimen, and no subsurface particles initiated a fatigue crack. Each of the cyclic failures stemmed from only one particle and not multiple particles. Also observed in the SEM images was evidence of twinning on the fatigue fracture surfaces similar to literature [14-15].

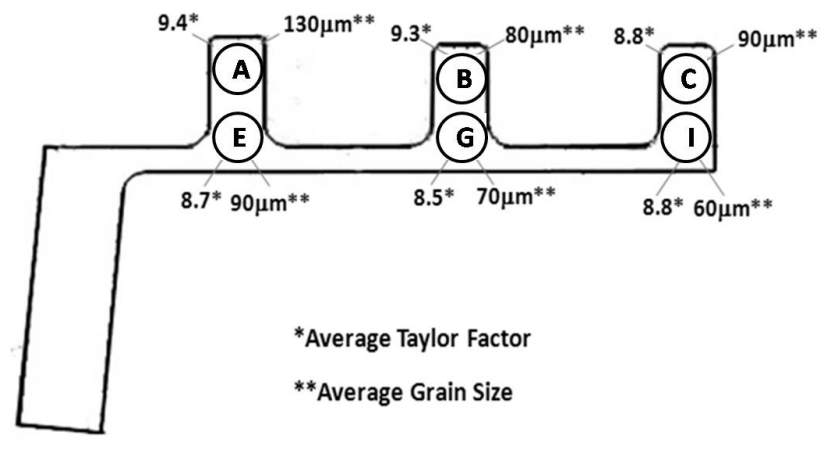

Figure 6. The average grain size and Taylor Factor in the extruded magnesium AM30 obtained using EBSD analysis.

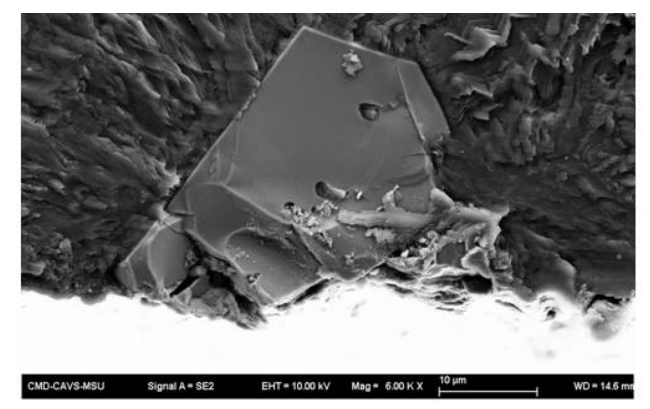

Figure 7. Typical Post-mortem fractograph of magnesium AM30 aluminum alloy that shows the intermetallic particle that initiated the fatigue crack. 
The objective of this study was to correlate structure-property relationships to microstructure features with the intent to predict the scatter that is typically observed in fatigue studies. The square root particle area versus the number of cycles to failure are shown in Fig 8a. The trends of both the square root particle area versus failure cycles show that specimens with smaller particles had greater fatigue resistance compared to specimens with larger particles and specimens with smaller particles had better fatigue life.To further characterize the microstructure of the AM30 alloy in regards to the fatigue behavior, the average grain size and initial Taylor Factors are plotted against the number of cycles to failure. Figure $8 \mathrm{~b}$ displays the number of cycles versus average grain size. While this plot displays significant scatter, the trend suggests that the alloy exhibits greater fatigue resistance for smaller grains compared to specimens with larger grains. This conclusion is not unexpected considering grain boundary blocking can slow the rate of crack growth.

Likewise, Fig 8c shows that the smaller Taylor Factor values resulted in generally higher fatigue lives. While Fig $3 c$ also displays some level of scatter, the trend suggests that the alloy exhibits greater fatigue resistance for smaller initial Taylor Factors compared to specimens with larger Taylor Factors. Taylor Factor, which is calculated by taking the summation of the amount of shear of the active slip system and then normalizing by the deformation step [16], represents basically how susceptible the slip shear systems are to shear deformation. Thus, higher initial Taylor Factors mean more local shear compared to lower Taylor Factors. Since crack initiation is dependent on local shear strain, higher Taylor Factor values suggest that crack initiation will occur earlier compared to lower Taylor Factors.

\section{Effect of Twinning, Slip, and Inclusions on the Fatigue Anisotropy of Extrusion-Textured Magnesium AZ61 Alloy}

Using a MultiStage Fatigue (MSF) model, experiments were conducted with extruded magnesium AZ61 alloy to quantify structure-property relations with respect to fatigue. These experiments were conducted in the extruded and transverse directions under low and high cycle strain control fatigue conditions. Structure-property relations were quantified by examining the fracture surfaces of the fatigued specimens using a scanning electron microscope (SEM). In terms of crack incubation, fatigue cracks were found to initiate from intermetallic particles (inclusions) that were typically larger than the mean size. Quantified sources of fatigue crack incubation, microstructurally small cracks, and cyclic stress-strain behavior were correlated to the MSF model. Based on the specific material parameters, the MSF model was able to predict the difference in the strain-life results of the magnesium AZ61alloy in the extruded (ED) and extruded transverse directions (ETD), including the scatter of the experimental results. Finally, the MSF revealed that the inclusion size was more important in determining the fatigue life than the anisotropic effects from the texture, yield, and work hardening. 

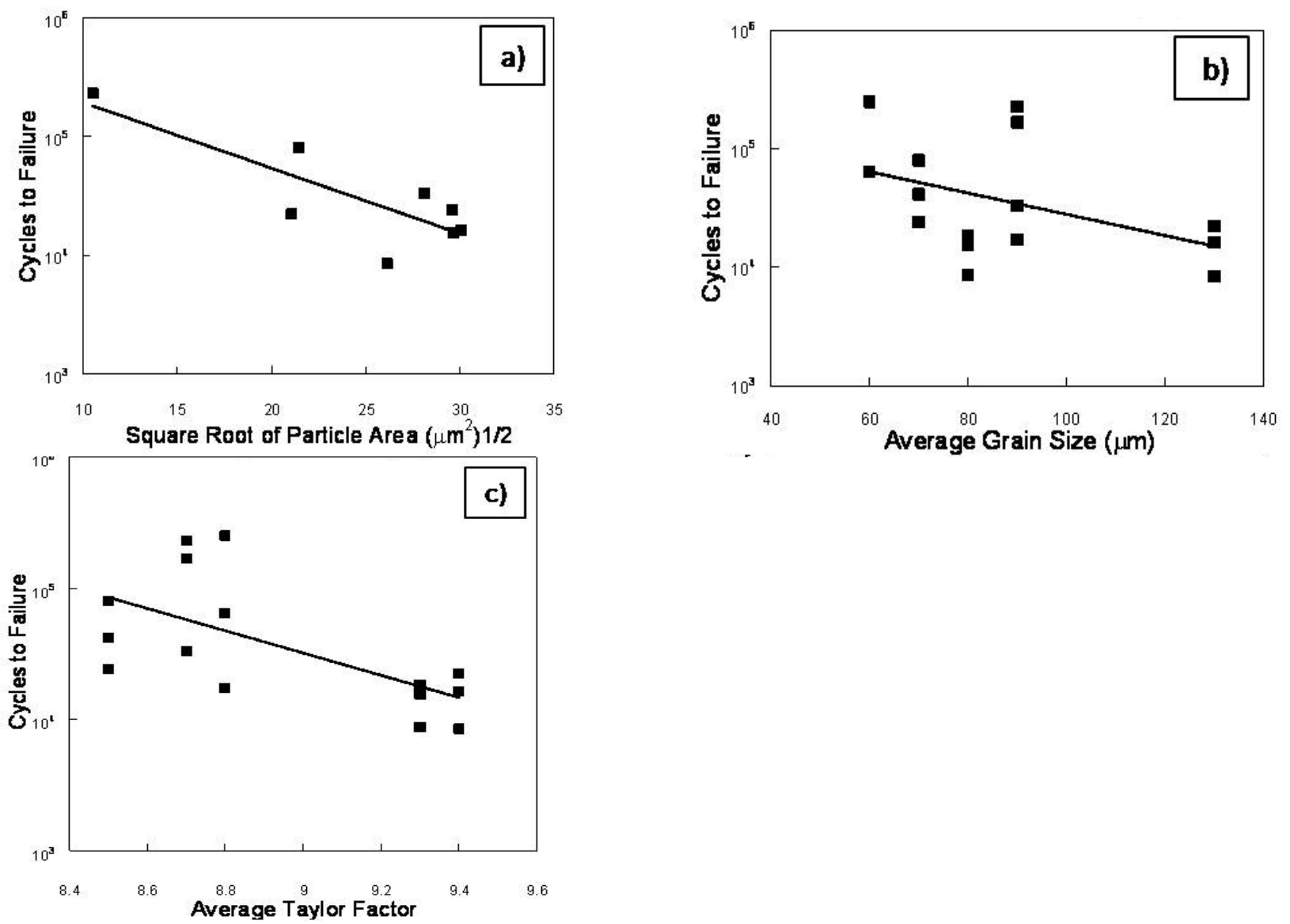

Figure 8. Number of cycles to failure versus the square root of the particle area (a), average grain size (b), and average Taylor Factor (c).

\section{Microstructure}

The material used in the study was an extruded AZ61 magnesium alloy. The as-received air-quenched extruded alloy is in the form of an automotive crash rail with the cross-section shown in Figure 9a. The crash rail was extruded at an average temperature of $500 \stackrel{\circ}{\circ}$ and an extrusion exit speed of $4 \mathrm{ft} / \mathrm{min}$. The as-received material was examined with an optical microscope to quantify the initial microstructure in both a transverse material orientation and an extrusion or longitudinal orientation. X-ray diffraction (XRD) was also performed to quantify texture of the as-received material.

The longitudinal orientation was found to have equiaxed grains averaging $37.3 \mu \mathrm{m}$, an average particle size of $8.4 \mu \mathrm{m}$, an average nearest neighbor distance of $88.5 \mu \mathrm{m}$, and a particle area fraction of 0.038 (Figure 10a). The transverse orientation was found to also have equiaxed grains that averaged $48.4 \mu \mathrm{m}$, particles that averaged $9.4 \mu \mathrm{m}$, a nearest neighbor distance of $104.7 \mu \mathrm{m}$, and a particle area fraction of 0.034 (Figure 10b). EDAX chemical analysis of the particles indicates that these particles are Al-Mn based. Pole figures of the longitudinal and transverse orientation from XRD results are shown in Figure 11. The results shown in Figure 11 suggest that the AZ61 magnesium alloys are not strongly textured; however, the basal plane is aligned with the longitudinal direction. 


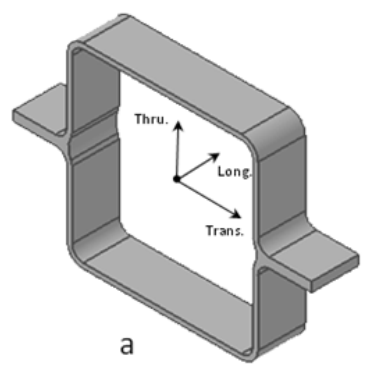

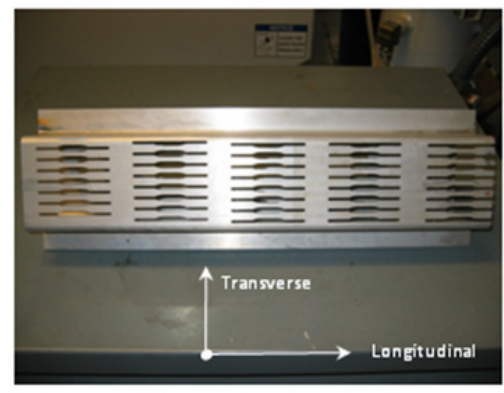

b

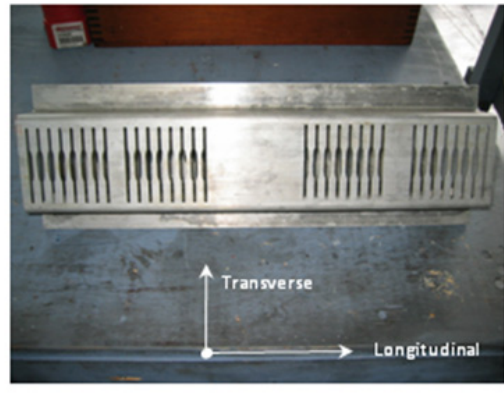

C

Figure 9 (a) Geometry of the as-received, extruded AZ61 magnesium alloy crash rail. (b) Longitudinal and (c) transverse specimen layout on the crash rail.

For further analysis, the comparison of the calculated average Taylor factor for both orientations were made, where the longitudinal direction had an average calculated Taylor value of 2.07 and the transverse direction had an average calculated Taylor value of 2.04. The slight difference in grain size and Taylor factor of both orientations are attributed to the slow extrusion rate that accommodates recrystallization.

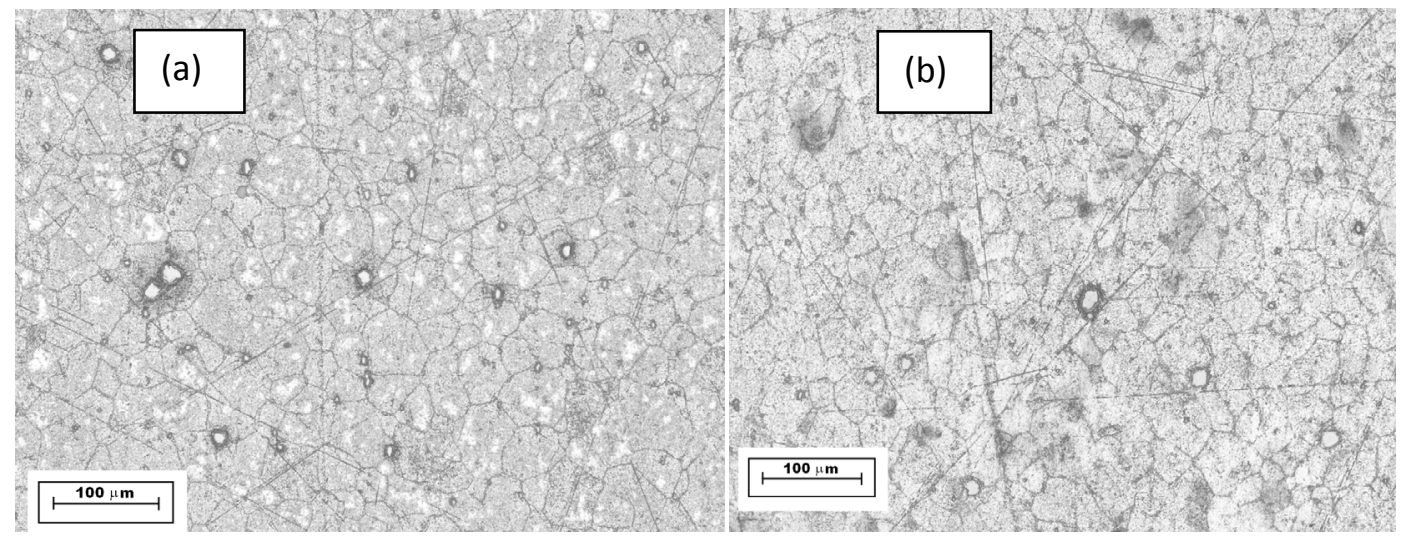

Figure 10. Optical micrographs of as-received extruded AZ61 magnesium alloy polished and etched: (a) longitudinal material orientation with a $37.3 \mu \mathrm{m}$ average grain size (GS), a $8.4 \mu \mathrm{m}$ average particle size (PS), a 0.038 particle area fraction, and a $88.5 \mu \mathrm{m}$ average nearest neighbor distance (NND). (b) Transverse material orientation with a $48.4 \mu \mathrm{m} \mathrm{GS}$, a $9.4 \mu \mathrm{m}$ PS, a 0.034 

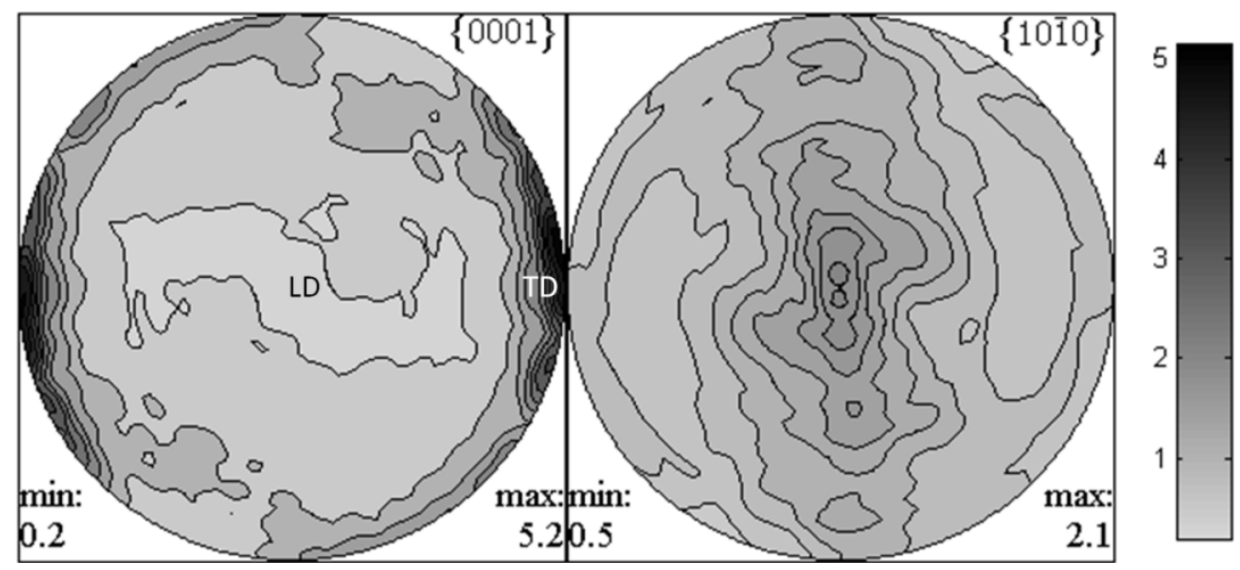

Figure 11. Pole figures of the longitudinal orientation calculated from X-ray diffraction for an AZ61 magnesium alloy. The calculated Taylor factors are 2.04 for the transverse orientation and 2.07 for the longitudinal orientation.

\section{Fractography}

SEM fractography conducted in this study (typical SEM images shown in Figure 12) resulted in the characterization of the different stages of fatigue damage: incubation, microstructurally small crack (MSC)/ physically small crack (PSC), and long crack (LC). For low cycle fatigue, the first few hundred cycles of fatigue loading resulted in particle fracture, and incubation of the fatigue crack in the magnesium arose shortly afterward, indicating that approximately $30 \%$ of the life was incubation. SEM analysis of the fracture surfaces showed that intermetallic particles near the surface fractured without significant debonding.

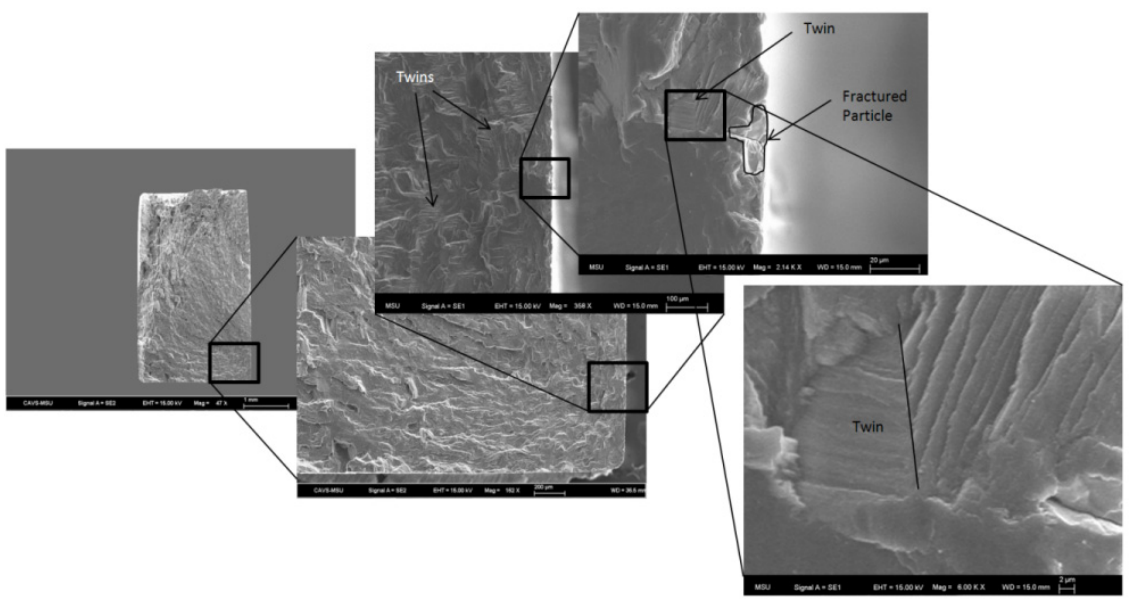

Figure 12. Fracture surface of Mg AZ61 alloy in the extrusion direction (ED). The scale bar magnitudes from left to right are $1 \mathrm{~mm}, 200 \mu \mathrm{m}, 100 \mu \mathrm{m}, 20 \mu \mathrm{m}$, and $2 \mu \mathrm{m}$. Note the twinning on the surface and 
the intermetallic particle that initiated the fatigue crack $(90 \mu \mathrm{m})$. This specimen was fatigue tested at $0.3 \%$ strain amplitude.

\section{Fatigue Model}

While the MSF model has been correlated to magnesium alloys prior to this study, this modeling effort was focused on wrought magnesium whereas past work focused on cast alloys. As such, the driving forces in cast alloys are primarily the casting pores. However, the material of interest is an extruded magnesium alloy that does not contain casting pores or oxide films. Thus, based on experimental results in this study, the primary source of fatigue damage is intermetallic particles. Figure 13 shows the correlations of the MSF model to experimental data for both extruded direction (ED) and the extruded transverse direction (ETD).

For validation purposes, components were made by cutting through the cross-section of the extruded Ford rail. These components were $25.4 \mathrm{~mm}$ wide and were the same magnesium AZ61 alloy presented earlier. These components were tested under fully-reversed displacement control at a frequency of $3 \mathrm{~Hz}$. A total of four components were tested at two different displacement amplitudes: $2 \mathrm{~mm} ; 3 \mathrm{~mm}$. The specimens tested at $2 \mathrm{~mm}$ were switched from displacement control mode to load control mode at 10,000 cycles and continued until failure at $30 \mathrm{~Hz}$.

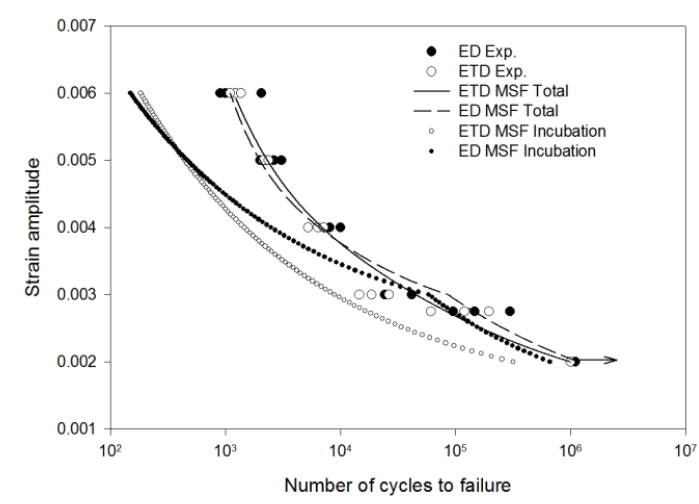

Figure 13. A comparison of the Multistage Fatigue (MSF) model of total and incubation life with experimental data for extruded AZ61 magnesium alloy in both the extrusion direction and the extrusion transverse direction.

In order to make MSF predictions of the component, finite element simulations were performed to determine the local stress/strain state due to the presence of stress concentrations. The material model employed in this present study is the internal state variable (ISV) plasticity/damage model. Threedimensional finite element simulations were performed with the aforementioned constitutive model and a mesh refinement study was employed to determine the appropriate element size. The maximum principal strain amplitude from the finite element results was used as an equivalent uniaxial strain amplitude for the input to the MSF model. Figure $14 \mathrm{~b}$ shows the comparison of the component fatigue specimens to the MSF prediction. The MSF showed good correlation to the fatigue life at the lower 
amplitude but showed a more conservative prediction for the higher amplitude. Also shown in the Figure $14 \mathrm{~b}$ is the incubation and MSC/PSC crack growth predictions. The slightly conservative predictions are likely due to the lack of a long crack growth model, especially for the higher amplitude. At higher amplitudes, the long crack growth will dominate the fatigue damage at a greater percentage than at lower amplitudes where MSC/PSC is more dominant.

a)

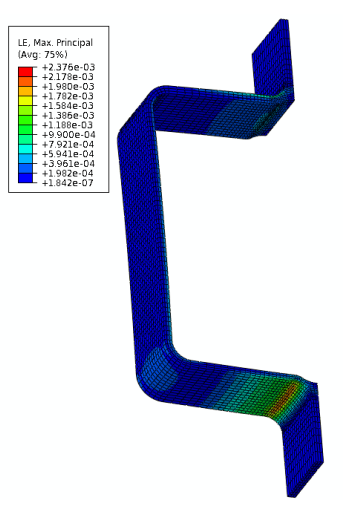

b)

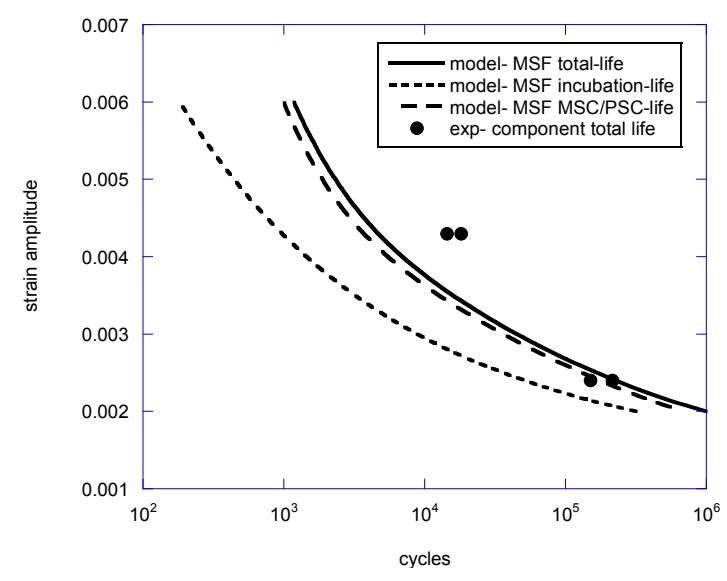

Figure 14. (a) Maximum principal strain contour plot of the finite element analysis of the Mg AZ61 components. Analysis of the half-plane symmetry of the component was performed based on a displacement of $2 \mathrm{~mm}$ and $3 \mathrm{~mm}$ applied at the boundaries. The material model employed was an internal state variable plasticity model for Mg AZ61 alloy in the transverse directions. (b) Comparison of the MSF model to the experimental fatigue results of the AZ61 component test. The components tests were conducted under displacement amplitudes of $2 \mathrm{~mm}$ and $3 \mathrm{~mm}$. Using an internal state variable plasticity finite element analysis, the local multi-axial strain amplitude was estimated. Maximum Principle strain theory was used as input to the MultiStage Fatigue model.

\section{Atomistic Simulations of Fatigue Crack Growth and the Associated Fatigue Crack Tip Stress Evolution in Magnesium Single Crystals}

Using Large-scale Atomic Molecular Massively Parallel Simulator (LAMMPS), a classical molecular dynamics code, atomistic simulations were performed to investigate the fatigue crack growth rate and the evolution of the associated atomic stress fields near the crack tip during fatigue crack growth in magnesium single crystals. The interatomic bonds of atoms were described using the EAM potential. The specimens with initial edge cracks were subjected to uniaxial Mode I cyclic loading. For the sake of revealing the influence of the initial cracks' crystal orientations, three different orientations were considered. The fatigue growth rate can be expressed by da/dN $=\mathrm{c} \triangle \mathrm{CTOD}$, where the values of constant $\mathrm{c}$ are determined by the atomistic simulations. Notably, the values of the constant $\mathrm{c}$ are much larger for magnesium single crystals than for FCC single crystals and vary widely from one orientation to another. 
The simulation results show that the evolution of atomic stress fields was highly dependent on the crystal orientations due to anisotropy and magnesium single crystals' HCP structure. Interestingly, the von Mises stress or normal stress around the crack tip controlled the fatigue crack growth behaviors.

Fig. 15 illustrates the edge crack specimen adopted for the present study. The initial crack was introduced by removing the atoms from the perfect crystal.
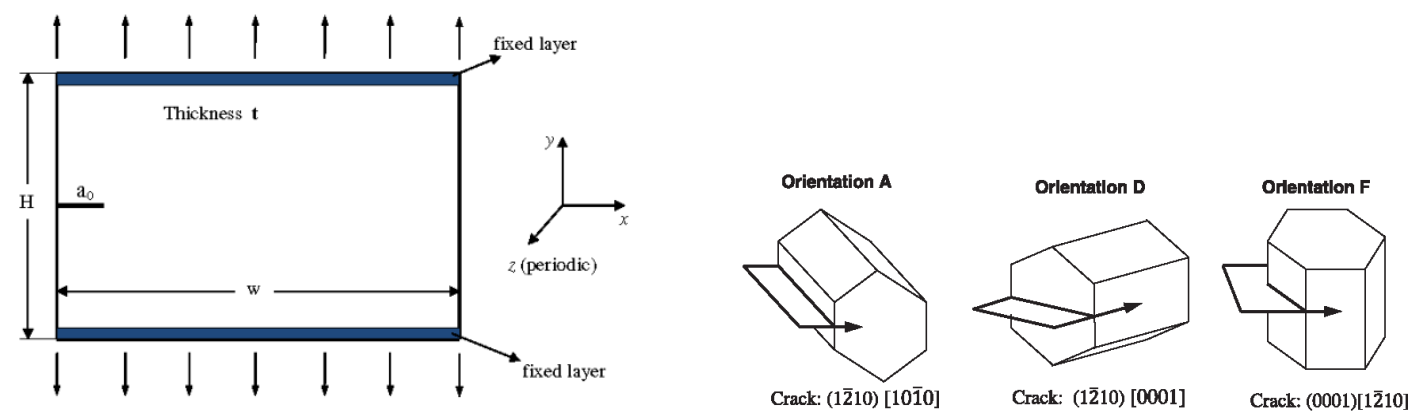

Figure 15. Edge crack specimen used for the simulation of fatigue crack growth and crystallographic orientations of the initial cracks

Results shown, that fatigue crack tip stress evolution is strongly dependent on the crystal orientations. The most substantial differences in fatigue crack growth behaviors of different crystal orientations are essentially caused by the detailed atomic stress evolution around the crack tip due to the Schmid Factor. Figure 16 presents the contour plots of the stress component $\sigma_{y y}$ for Orientation A (1210)[10 $\left.\overline{1} 0\right]$. Stress evolution around the crack tip is shown in Figure 17.

\section{Small Fatigue Crack Growth Observations in an Extruded Magnesium Alloy}

The objective of this work was to make small fatigue crack growth observations of an extruded AZ61 magnesium alloy. Fully reversed and interrupted load-controlled tests were conducted on notched specimens that were taken from the material in the longitudinal and transverse orientations with respect to the extrusion direction. In order to measure crack growth, replicas of the notch surface were made using a two-part silicon-rubber compound at periodic cyclic intervals. By using microscopicanalysis of the replica surfaces, subsequent crack initiation sites from numerous locations along with the crack growth rates were determined. A marked acceleration/deceleration was observed to occur in cracks of smaller length scales. This was consistent with prior observations of small fatigue crack interaction with the native microstructure and texture. Lastly, a multistage fatigue model based upon the microstructure of a material was employed here in order to estimate crack growth behavior with respect to the microstructure, notably grain orientation. 


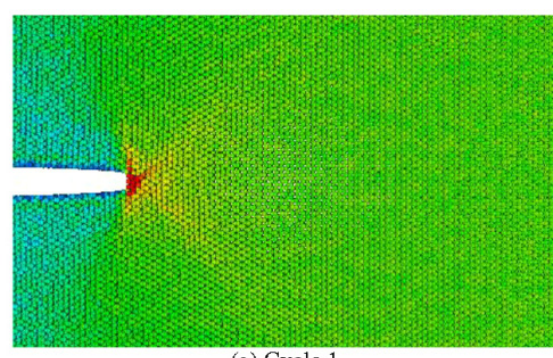

(a) Cycle 1

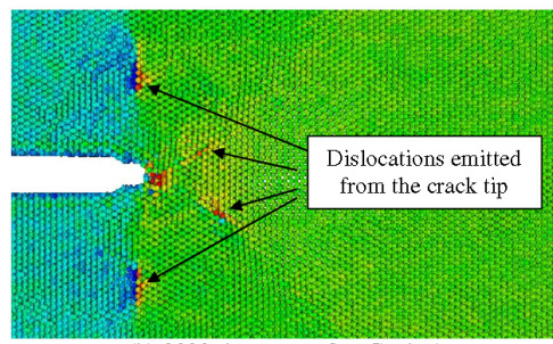

(b) 2000 timesteps after Cycle 1

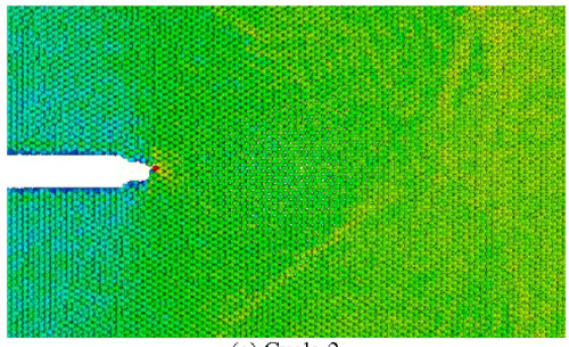

(c) Cycle 2
GPa

$1.979 \mathrm{e}+002$

$1.279 \mathrm{e}+002$

$5.796 \mathrm{e}+001$

$-1.206 \mathrm{e}+001$

GPa

$1.979 \mathrm{e}+002$

$1.279 \mathrm{e}+002$

$5.796 \mathbf{e}+001$

$-1.206 \mathrm{e}+001$

$-8.207 e+001$

GPa

$1.979 \mathrm{e}+002$

$1.279 \mathrm{e}+002$

$5.796 \mathbf{e}+001$

$-1.206 \mathrm{e}+001$

$-8.207 \mathrm{e}+001$

Figure 16. Contour plots of stress component ${ }_{y y}$ of Orientation $A(1 \overline{2} 10)[10 \overline{1} 0]$ at (a) the maximum loading of Cycle 1; (b) 2000 time steps after the maximum loading of Cycle 1; (c) the maximum loading of Cycle 2.
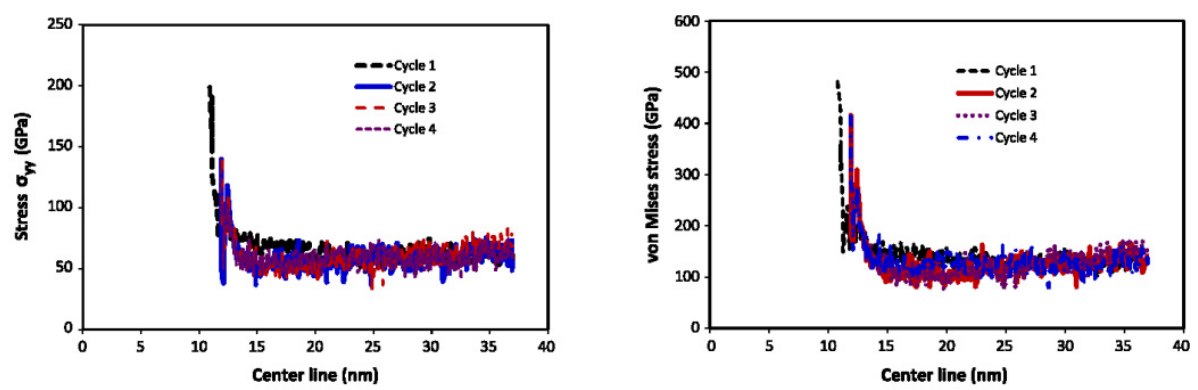

Figure 17. Variations of stress component $s_{y y}$ and von Mises stress along the crack line in Orientation $A$ $(1 \overline{2} 10)[10 \overline{1} 0]$ at the maximum strain points of Cycles $1-4$. 


\section{Materials and Experimental Methods}

The material used in this study was an AZ61 magnesium alloy extruded into an automotive crash rail, as shown in Figure 1. Note in Figure $1 \mathrm{~b}$ the layout of the transverse specimens.
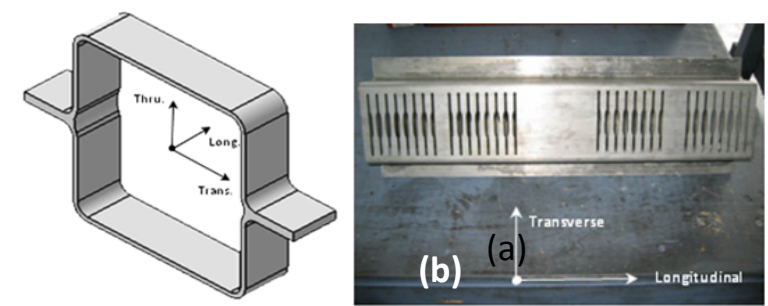

Figure 18.(a) Cross-sectional geometry of the as received, extruded AZ61 magnesium alloy crash rail and (b) transverse specimen layout.

In order to conduct fatigue tests upon the material, flat dog-bone specimens were machined from the rail in the transverse (as shown in Figure 18b) and longitudinal orientations. Since a location favorable for crack growth was required for consistent scrutiny, a notch with a radius of $35 \mathrm{~mm}$ was machined into the face of the gage to a depth of $300 \mu \mathrm{m}$. The specimen used was similar to that found in literature [17] and is shown in Figure 19. In order to reduce surface contaminants and premature failure due to specimen machining, the surfaces of the shoulder, gage, and notch of each specimen were hand ground along the loading direction using 800grit sandpaper.

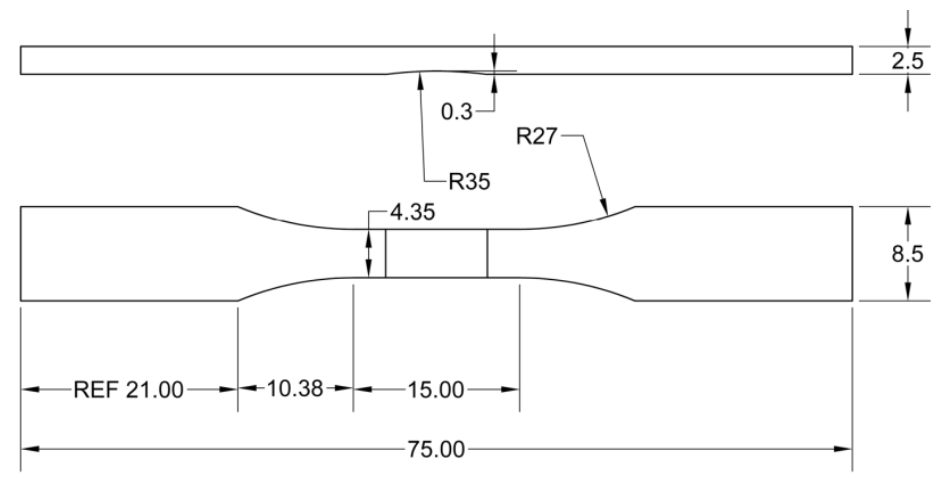

Figure 19. Fatigue specimen geometry with the crack-initiating notch. All dimensions are in millimeters. 


\section{Small Fatigue Crack Behavior}

Using SEM and three-dimensional profiling microscope imaging of the replicas, the locations of fatigue crack initiation and subsequent growth on the notch surfaces were identified. Figure 20 illustrates the fatigue crack outline on a replica image of a specimen prepared with 800 grit sandpaper, hence the vertical texture on the replica surface. This specimen was tested at a stress amplitude of $105 \mathrm{MPa}(\mathrm{R}=-1)$ with replicas taken at intervals of 1,000 cycles until 20,000 cycles, when replicas were taken every 2,000 cycles. From 30,000 cycles until the final fracture of 37,070 cycles, replicas were taken every 5,000 cycles. Points A, B, and C indicate observed crack tip locations at cyclic intervals of 13,000, 19,000, and 24,000 cycles, respectively. Note that only the left side of the mixed-mode crack is observed and that crack growth occurred to the right of the initiation site as well.

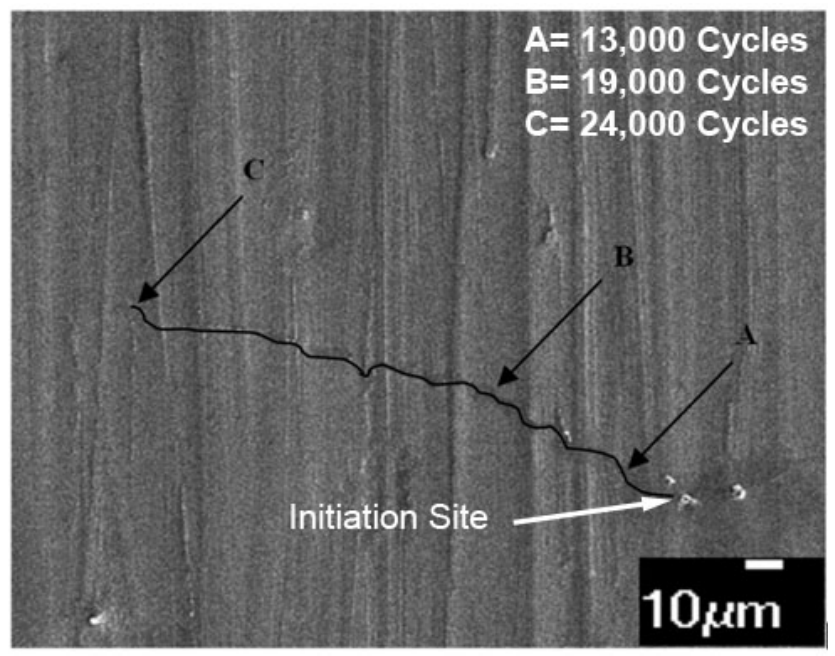

Figure 20. Replica image illustrating the path of the dominant failure-inducing crack.

After identifying the crack initiation site illustrated in Figure 20, crack lengths were measured starting with the last replica prior to failure then progressing backwards until the crack was no longer visible. Figure 21 shows the crack length as a function of cycles for the above crack, while Figure 22 displays the crack growth rate versus crack length. Crack growth rate was calculated utilizing a point-by-point calculation previously utilized by Jordon et al. [18]. 


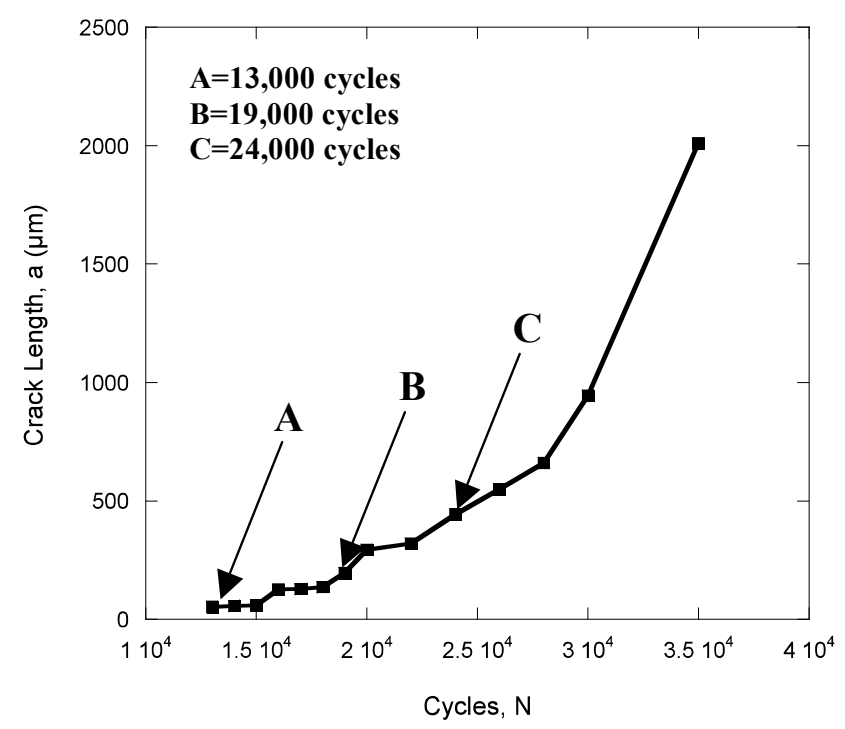

Figure 21. Crack length versus cycles for a stress amplitude of $105 \mathrm{MPa}$.

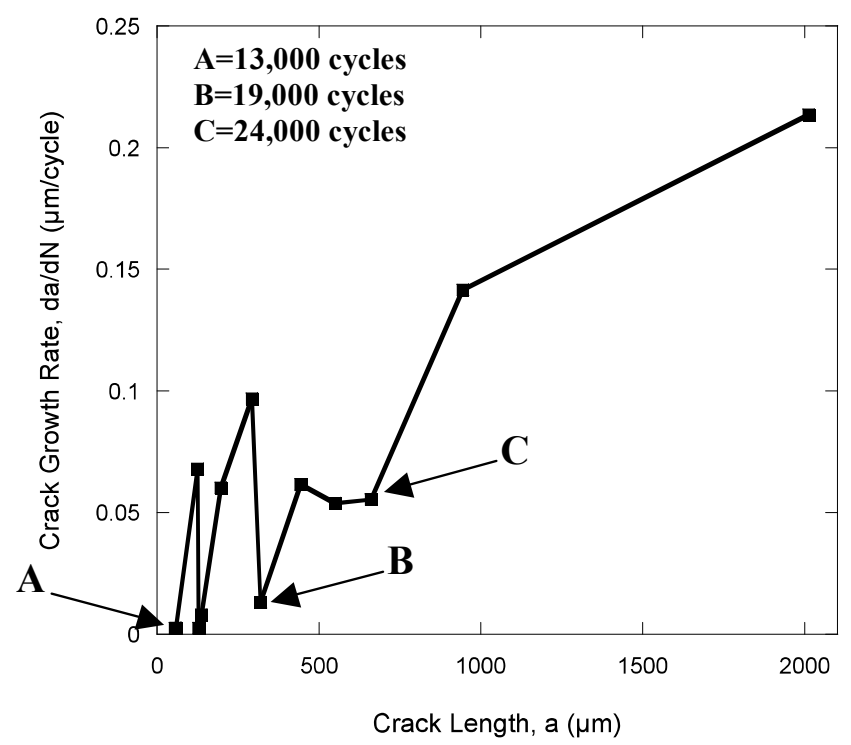

Figure 22. Crack growth rate as a function of crack length for a stress amplitude of $105 \mathrm{MPa}$.

The location of the dominant crack (pictured in Figure 20) was located on the fracture surface using SEM imaging. Since the experimentally observed crack was expected to grow in a symmetric semi-circular fashion, the measured surface crack length is assumed to be twice the length of the crack depth into the thickness of the specimen. Since the earliest recorded crack length as indicated by replica observation stated an initial length of $53.2 \mu \mathrm{m}$, the minimum distance from the notch surface to the beginning of fatigue crack evidence into the specimen should be half of $53.2 \mu \mathrm{m}$, or $26.6 \mu \mathrm{m}$. However, fatigue striations became evident approximately $10 \mu \mathrm{m}$ from the notch surface as indicated in Figure 23a, thus suggesting that, provided the symmetrical semi-circle approximation held true, the initial small crack 
length was about $20 \mu \mathrm{m}$. This would indicate that the end of the incubation region was over-estimated and the beginning of the small crack region was under-estimated. This discrepancy is due to replica iterations being too great, and it is evident that shorter cyclic phases are required between replicating interruptions in order to accurately grasp the transition between the incubation and small crack regimes.

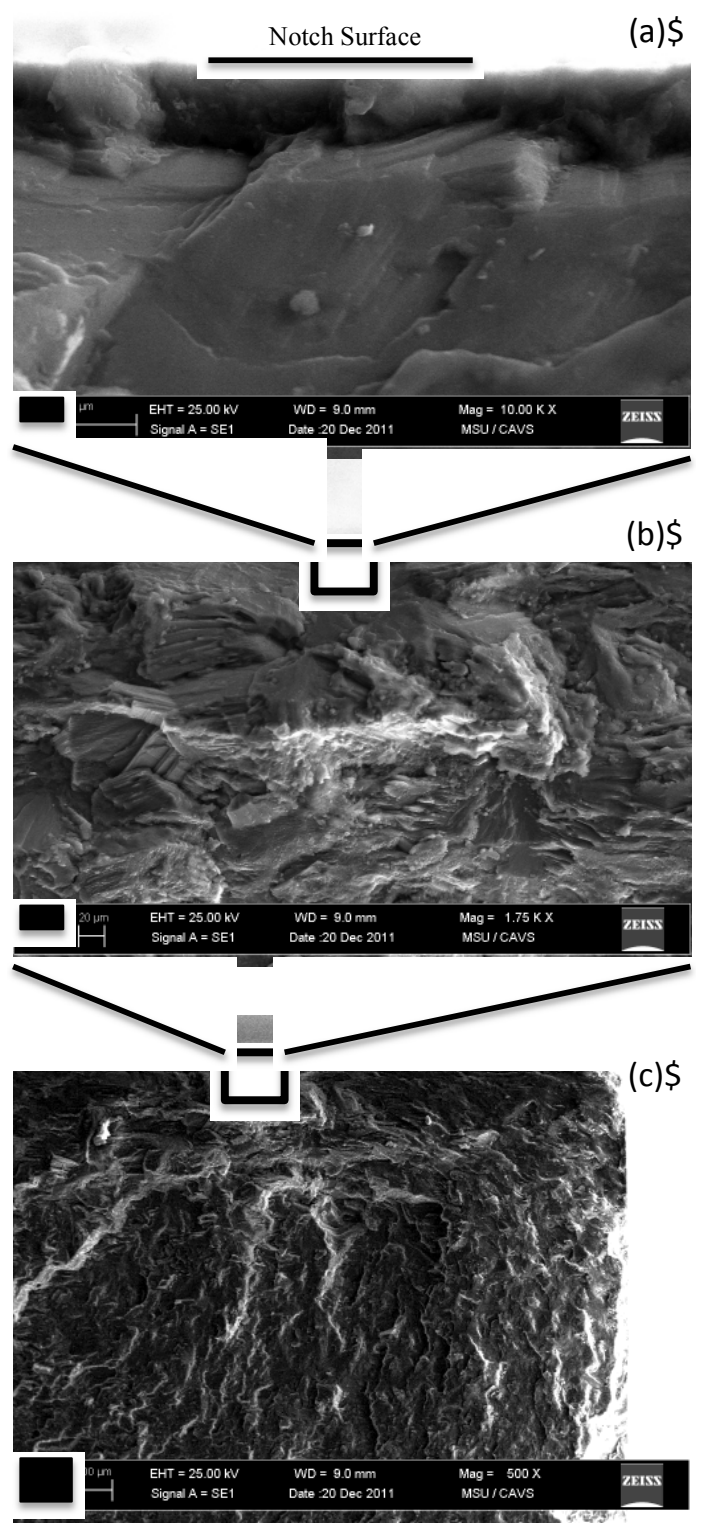

Figure 23. Scanning electron microscope results showing the overall appearance of a typical fracture surface (8c), along with higher magnification images of the initiation location (23a and 23b). As apparent in Figure 23a, subsurface striations became apparent approximately $10 \mu \mathrm{m}$ from the notch surface. 
After further observation, the particular crack under consideration was shown to have leveled off from the mixed mode cracking evident in Figure 20 to a singular Mode I fatigue crack, indicative of long crack fatigue. This transition became apparent on the replica indicated as C in Figures 21 and 22, which corresponded to 24,000 cycles and a measured crack length of $661.2 \mu \mathrm{m}$. Previous studies have suggested a transitional length from small crack to long crack as being approximately $400 \mu \mathrm{m}$ [19], six to ten times the grain size (or approximately $300 \mu \mathrm{m}-500 \mu \mathrm{m}$ in this case) [3, 20], or $0.7 \mathrm{~mm}-1 \mathrm{~mm}$ [1]. Therefore, the value indicated here falls between the suggestions from literature.

\section{Monotonic and Cyclic behavior of Cast Magnesium Alloys}

Monotonic and cyclic testing was performed on five different casting processes: LPPM, Ablation, Squeese Cast, T-Mag, and LPPM (EM-P). In addition, inclusion quantification was performed on the fracture surfaces on the failed specimens. Figure 24 displays the strain-life results for the five casting groups for all of the fatigue tests and the arrow represents test run-outs (all tests were stopped at $10^{6}$ cycles).

The scanning electron microscopy (SEM) analysis of the fracture surfaces confirmed that, compared to the LPPM and Squeeze Cast processes, the Ablation and T-mag processes contained very few fatigue failures resulting from large casting defects. SEM analysis of the fatigue fracture surfaces revealed that the cracked, debonded particles and small casting pores were the source of fatigue crack initiation for the Ablation process. For the least fatigue-resistant process, SEM analysis of the LPPM fracture surfaces revealed that the main source of fatigue crack initiation was due to large pore shrinkage clusters. Some of the pore shrinkage clusters were observed to be as large as the cross-section of the specimen. The Squeeze cast specimens also experienced fatigue crack initiation from pore shrinkage clusters. However, these pore shrinkage clusters were typically smaller than those generated during the LPPM process. In the Squeese Cast process the fatigue cracks were also observed to initiate from oxide films. Several of the T-Mag specimens had fatigue cracks that initiated from large pores and oxide films on the order of a couple hundred microns. The large oxide films and pores greatly reduced the fatigue-life of the T-Mag process specimens as compared to the Ablation process specimens.

\section{Microstructure and Damage Evolution during Tensile Loading in a Wrought Magnesium Alloy}

The damage evolution in a wrought magnesium alloy under uniaxial tensile deformation was investigated. Sectioned specimens subjected to interrupted tensile deformation were examined under optical microscopy to quantify the number density of cracked intermetallic particles as a function of applied strain. Digital image analysis of the optical images was employed to quantify damage by separating cracked from non-cracked particles. Finally, an internal state variable damage model was shown to adequately capture the experimentally observed damage progression due to the intermetallics. 


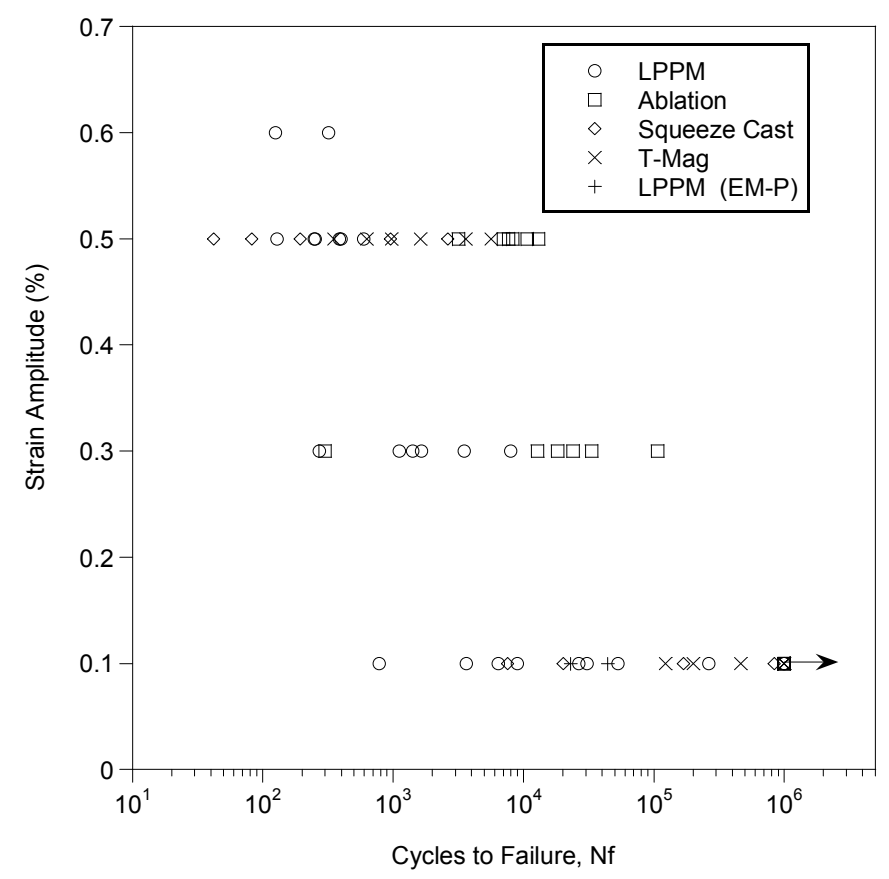

Figure 24. Applied strain amplitude ( $R=-1)$ versus the number of cycles to failure for the LPPM, Ablation, Squeeze Cast, T-Mag, and LPPM electromagnetic (EM-P) process. Arrow indicates run-out.

Horstemeyer and Gokhale [21] proposed a model capable of capturing in ductile materials damage nucleation associated with inclusions or second phase particles. The void nucleation parameters in this model are two main microstructural quantities: length scale parameter $d$, which oftentimes represents the particle size, and particle volume fraction $f$. They developed the following equation for the number of voids per area:

$$
\eta=C_{\text {coeff }} \exp \left(\frac{\varepsilon(t) d^{1 / 2}}{K_{I C} f^{1 / 3}}\left\{a\left[\frac{4}{27}-\frac{J_{3}^{2}}{J_{2}^{3}}\right]+b \frac{J_{3}}{J_{2}^{3 / 2}}+c\left|\frac{I_{1}}{\sqrt{J_{2}}}\right|\right\}\right)
$$

Figure 25 shows the number of cracked particles per volume versus applied tensile strain. Damage in this type of alloy is due mainly to slip and twinning occurring in the matrix magnesium adjacent to the particles. However, Figure 25 shows a strong correlation between progression of cracking particles and damage. The constants used in (Equation 9) are the following: $a=0, b=55000, c=50000, C_{\text {coeff }}=1.9$, $f=0.004, d=2.4 \mu \mathrm{m}$, and $K_{I C}=20 \mathrm{MPa} \cdot \mathrm{m}^{1 / 2}$. Constants $C_{\text {coeff, }} a, b$, and $c$ were determined from the interrupted tension tests using a best correlation method. The damage nucleation model predicted results that are in good agreement with the observed damage progression of the AZ61 magnesium alloy 


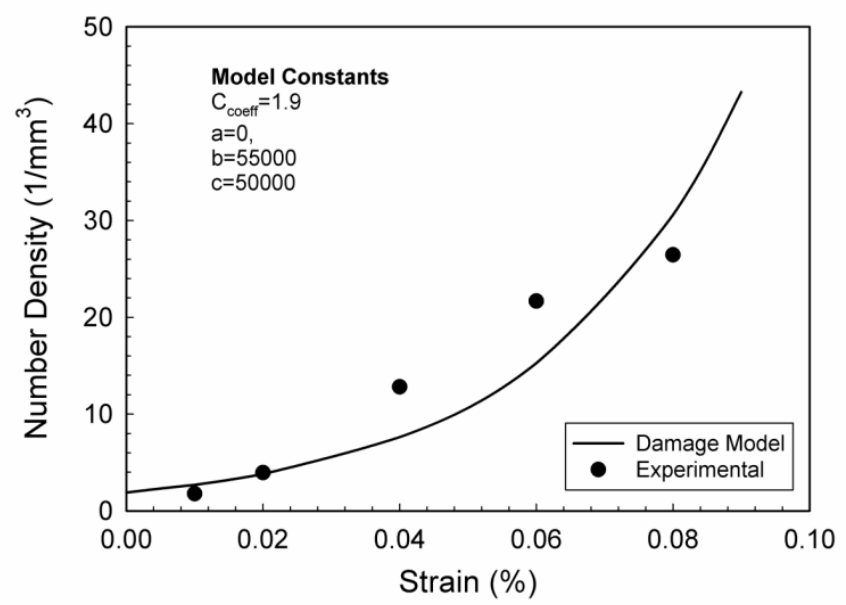

Figure 25. Number density versus strain of an AZ61 magnesium alloy showing a comparison of the Horstemeyer-Gokhale [21] void nucleation model with experimental data.

In regards to the microstructural properties, the following can be concluded: (1) under uniaxial tension the area fraction of cracked particles composed of (Mn-Al) and (Mg-Al) varies in an exponential pattern as function of strain, (2) the number density $\left(1 / \mathrm{mm}^{2}\right)$ of cracks follows a shallow nonlinear pattern, (3) three types of particles were identified: Oxide/intermetallic, Mn-Al, and Mg-Al phases, and (4) cracks were observed in the (Mn-Al) and (Mg-Al) based particles.

\section{Finite element analysis of the mechanical behavior and local stress intensity factor solutions for friction stir spot welds in lap-shear specimen}

The purpose of this study was to investigate the mechanical behavior and to calculate the local stress intensity factors for friction stir spot welds (FSSW) of Mg AZ31 alloy in lap-shear specimens using finite element method. Three dimensional finite element models were constructed to thoroughly analyze the stress field in spot welds and the distribution of local stress intensity factors. For the local stress intensity factors, two cases were considered, namely an infinitesimal kinked crack and a finite length kinked crack. The local stress intensity factors at the critical points of the infinitesimal length kinked crack were determined using the global stress intensity factors and analytical expressions of Cotterell and Rice [22].

A detailed investigation of the stress field in FSSW on lap-shear specimens was performed using three dimensional finite element models in order to understand their influences on the structural performance and failure behavior of Mg AZ31(see Figure 26). The following conclusions were obtained from this study: a) for the specimen with no kinked crack, the asymmetrical geometry of the weld nugget has strong effects on the stress state in the base plate close to the weld nugget, but its influence diminishes as distance from the weld nugget increases, b) because the stress concentration occurs in a very small region around the interception of the nugget boundary with the faying interface in the specimen with no kinked crack, the step hole in the weld nugget should not influence the values of global stress intensity factor (SIF) solutions. However, the asymmetrical geometry of the weld nugget 
due to the step hole will cause the distribution of global SIF to deviate from the distributions of the model having a solid nugget. The local SIF solutions obtained via Cotterell and Rice relations demonstrate that the values of local $k_{l}$ and $k_{l /}$ at the critical point are the highest, which implies that the fatigue crack will initiate from this point.

a)

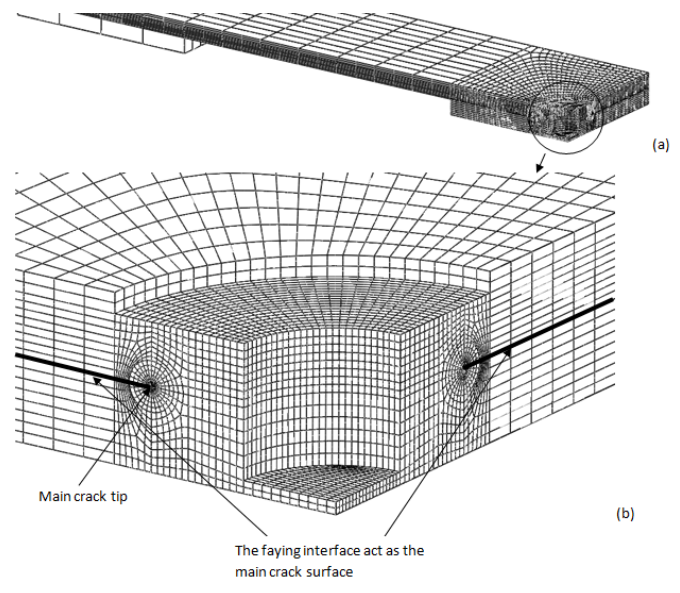

b)

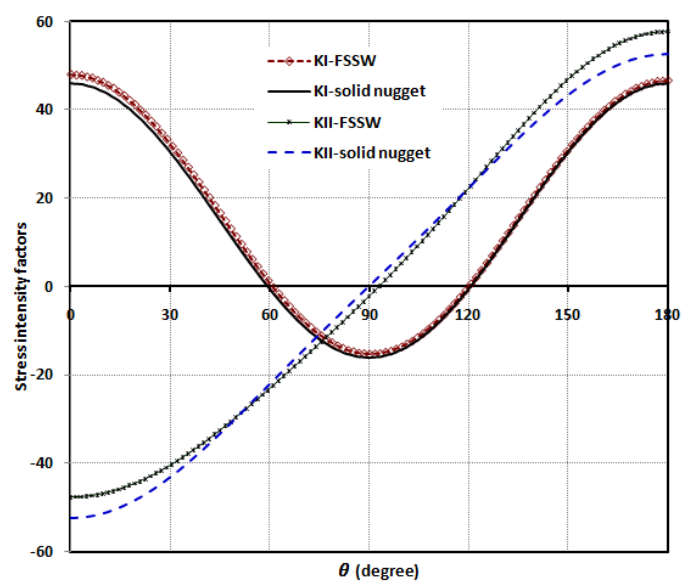

Figure 26. (a) A mesh for the left half finite element model with a close-up view of the mesh near the main crack tip. (b) The global SIF $K_{I}$ and $K_{\|}$of FSSW and the model with solid nugget as functions of the angle $\theta$.

\section{Role of Different Material Processiı.o Methods on the Fatigue Behavior of AZ31 Magnesium Alloy}

A critical design factor for automotive structures is fatigue damage. At present, the effect of material performance on structures fabricated from magnesium (Mg) alloys, the lightest structural metal available, subject to different processing steps, has not been carefully quantified. The present research takes a first step towards this end. The use of magnesium in automotive structural applications is part of our overall vehicle lightweighting strategy. The material used in this study was a Mg AZ31 alloy received in different processed forms: extrusion, plate, and sheet as shown in Figure 27. Using bench scale tests with Mg AZ31 specimens having simple geometries, the effects these specific processing steps on fatigue behavior were explored. Two paths were followed. The first is the more common strainbased approach that does not account for microstructure. The second approach employs a microstructure-sensitive fatigue model that comprises both crack incubation and growth stages. The model describes the effect of processing on the microstructure by incorporating specific mechanical properties and microstructural features, such as grain and inclusion sizes. As such, the model successfully captures the experimentally observed differences in fatigue lifetimes of the $\mathrm{Mg}$ alloy due to the induced in-plane constraint effects resulting from different material processing methods. Unlike the strain-life approach, which relies on curve-fitting, the microstructural approach provides greater insights 
into how the material microstructure might be manipulated to enhance fatigue resistance. Quantitative prediction of cumulative damage due to cyclic loading is described in detail.

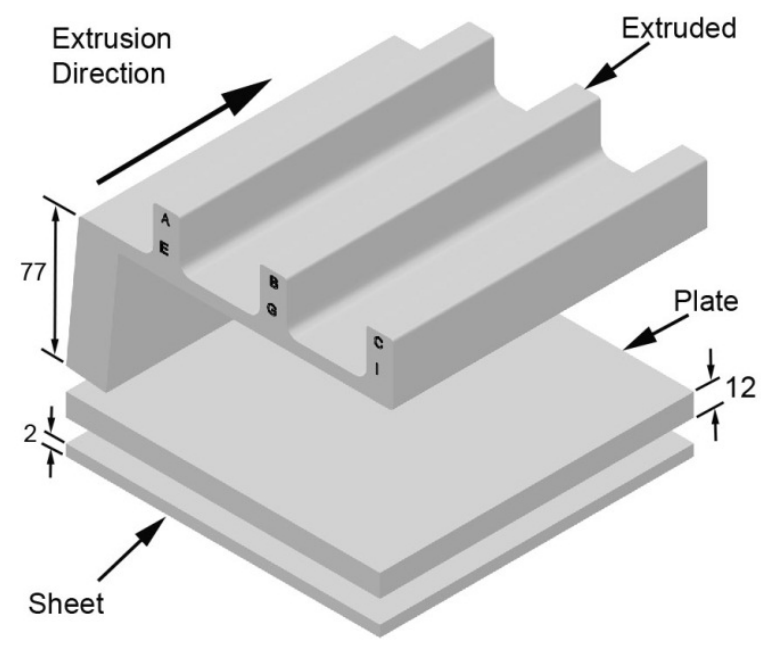

Figure 27. The as-received AZ31 magnesium alloy in the extruded, plate, and sheet geometries. Dimensions are in millimeters.

\section{Microstructure of the AZ31 alloys}

The microstructural features of each material were quantified in order to provide understanding of the anisotropic behavior associated with fatigue. Figure 28 exhibits the grain morphologies and sizes of the AZ31alloys. For the extrusion, sectioned material parallel to the extrusion direction was found to have grains averaging $28.8 \mu \mathrm{m}$ (Figure 28a), however, grains as large as $140 \mu \mathrm{m}$ were seen. Regarding the intermetallic particles present in the material, the particles had an average particle size of $5.77 \mu \mathrm{m}$, an average nearest neighbor distance of $34 \mu \mathrm{m}$, nonetheless, particles of sizes around $40 \mu \mathrm{m}$ were observed. For the plate material, the section parallel to the rolling direction was found to have equiaxed grains and a finer size of grain than the extruded specimen that averaged $10.7 \mu \mathrm{m}$. The intermetallic particles were found to average $3.25 \mu \mathrm{m}$, had a nearest neighbor distance of $22.82 \mu \mathrm{m}$. However, for the sheet material, the section parallel to the rolling direction was found to have elongated grains that were smaller than found in the extrusion and plate and averaged $5.7 \mu \mathrm{m}$. The intermetallic particles were found to average $3.71 \mu \mathrm{m}$, had a nearest neighbor distance of $35.45 \mu \mathrm{m}$. 


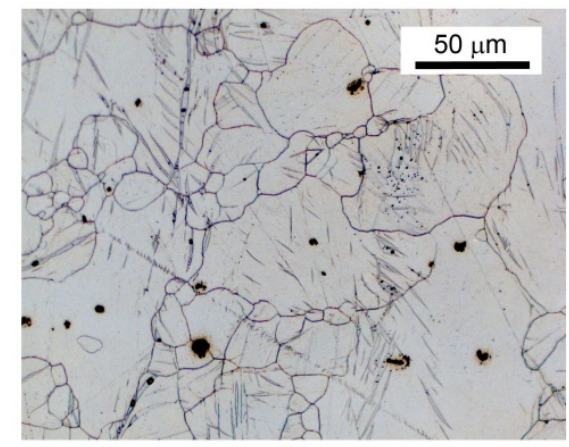

a)

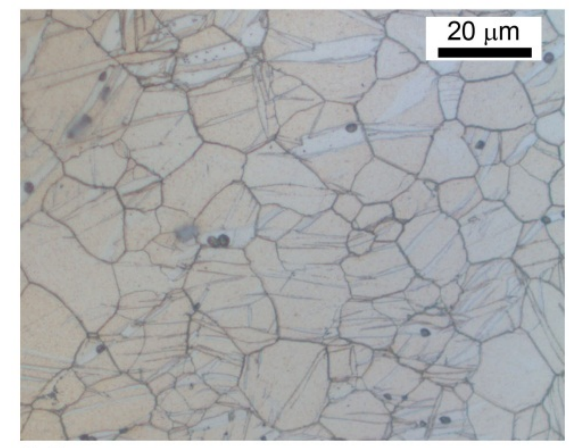

b)

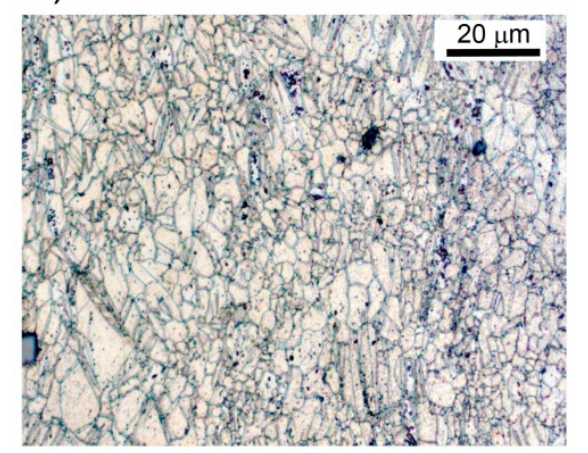

c)

Figure 28. Etched microstructure images of the AZ31 Mg materials: a) extruded, b) plate, and c)

\section{Cyclic Hysteresis Loops}

Figure 29 shows the engineering stress-strain hysteresis loops for the three materials at strain amplitudes of $0.6 \%, 0.5 \%$, and $0.3 \%$. Figure 29 a shows the first cycle of the extruded and plate materials at a strain amplitude of $0.6 \%$ and sheet material at a strain amplitude of $0.5 \%$. An asymmetric pattern was observed in the cyclic hysteresis loops for both the plate and extruded materials while the sheet material showed a more symmetric shape. The cyclic stress-strain asymmetry observed in Mg AZ31 is due to twinning activation during unloading in compression, and detwinning during loading in tension. A typical plateau is observed in the stress-strain response in the plate and extruded specimens during compression loading. It is noted that the asymmetry from tension to compression increased as the strain amplitude increased for the extruded and plate materials. The mid-life hysteresis loops remained relatively asymmetric for both plate and extruded materials while no significant change was noted for 
the sheet material (see Fig. 29b). The observed symmetric cyclic behavior of the sheet material is likely due to the aforementioned limited deformation mechanisms (basel slip and twin) not being activated at these range of strain amplitudes.

Cyclic stress-strain hysteresis loops for the first cycle and mid-life at a strain amplitude of $0.3 \%$ are shown in Figs. 29c and 29d. These loops show that a small amount of asymmetry still existed on the stress amplitude for the three material processing methods, with a slight compression plateau exhibited by the plate and extruded materials. Again, the sheet specimens did not show the characteristic plateau typical of $\mathrm{Mg}$ alloys and almost no change is noted between the first cycle and the mid-life cycle. Texture favors the activation of twining deformation in $\mathrm{Mg}$ and therefore initial texture could explain this difference in the hysteresis curve between the extruded, plate, and sheet materials. We note that the compression plateaus observed on the hysteresis loops are a clear indication of deformation twinning. This feature was observed for both the extruded and plate materials and correlates with the lower number of cycles to failure compared to the sheet material. In addition, both plate and extruded specimens behave nonlinearly during compression unloading that is typical of twinning. As such, deformation twinning is likely a significant contributor in the observed difference in the number of cycles to failure.

a)

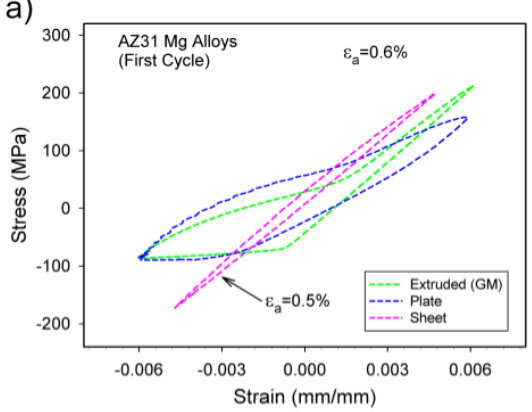

c)

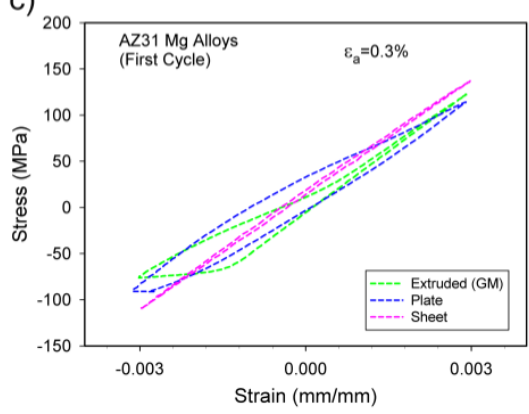

b)

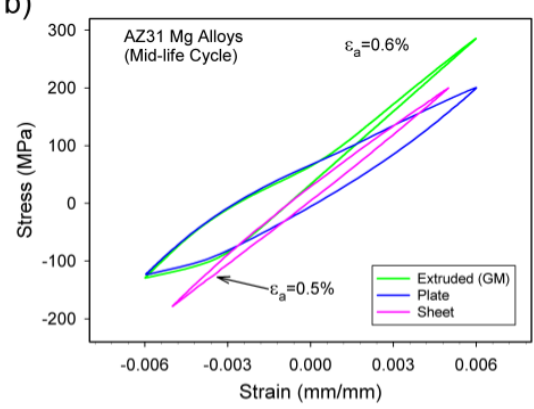

d)

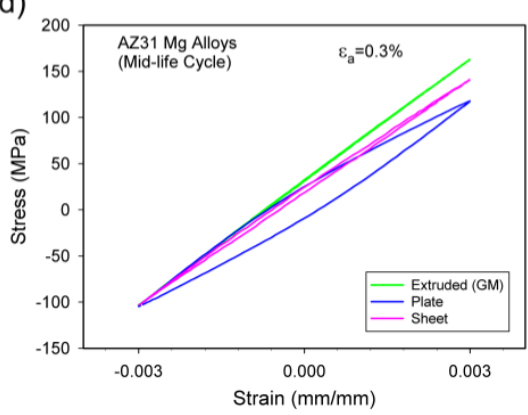

Figure 5. Hysteresis loops of AZ31 magnesium alloy at the strain amplitude of $0.6 \%$ and $0.3 \%$ from the extruded, plate, and sheet materials. 


\section{Fractography}

Extensive fractography was conducted on failed fatigue specimens with the intent to determine the sources of fatigue crack initiation and propagation mechanisms in the extruded, plate, and sheet materials. In this study, fracture surfaces of failed strain-life specimens for the three Mg AZ31 alloys were imaged in an SEM. All fracture surfaces displayed typical indications of fatigue crack propagation, with classical river marks leading back to the general location of crack initiation.

For comparison purposes, Fig. 30 shows representive fractographs of all three materials tested at a strain amplitude of $0.3 \%$. Fatigue cracks predominantly initiated at cracked particles for the three different materials. This observation suggests that a significant incubator for fatigue damage in wrought Mg alloys is the brittle intermetallic particles. This is an important point to consider since the three materials have markedly different mechanical properties and exhibited dissimilar cyclic stress-strain responses. The presence of these hard phases can act as an equalizer in terms of final number of cycles despite anisotropic material characteristics as displayed by the extruded, plate, and sheet material of this study. We note that while the final number of cycles can be empirically correlated to tensile yield/ultimate strengths of each material, the mechanism of fatigue crack incubation cannot be ignored, especially in the high cycle fatigue regime where the incubation life can be a significant percentage of the total life.

Regarding factors influencing total number of cycles to failure, we briefly comment on the twinning observed on the fracture surfaces of each of the materials. We note that profuse twinning was observed through SEM in the extruded material. This in fact corresponds to the shorter low cycle fatigue life observed in the extruded material compared to the sheet material (particularly considering that the particle size was greater for the sheet material on the fracture surface). As such, the presence of the twinning observed on the fracture surface was observed for low cycle specimens but not for high cycle specimens. 

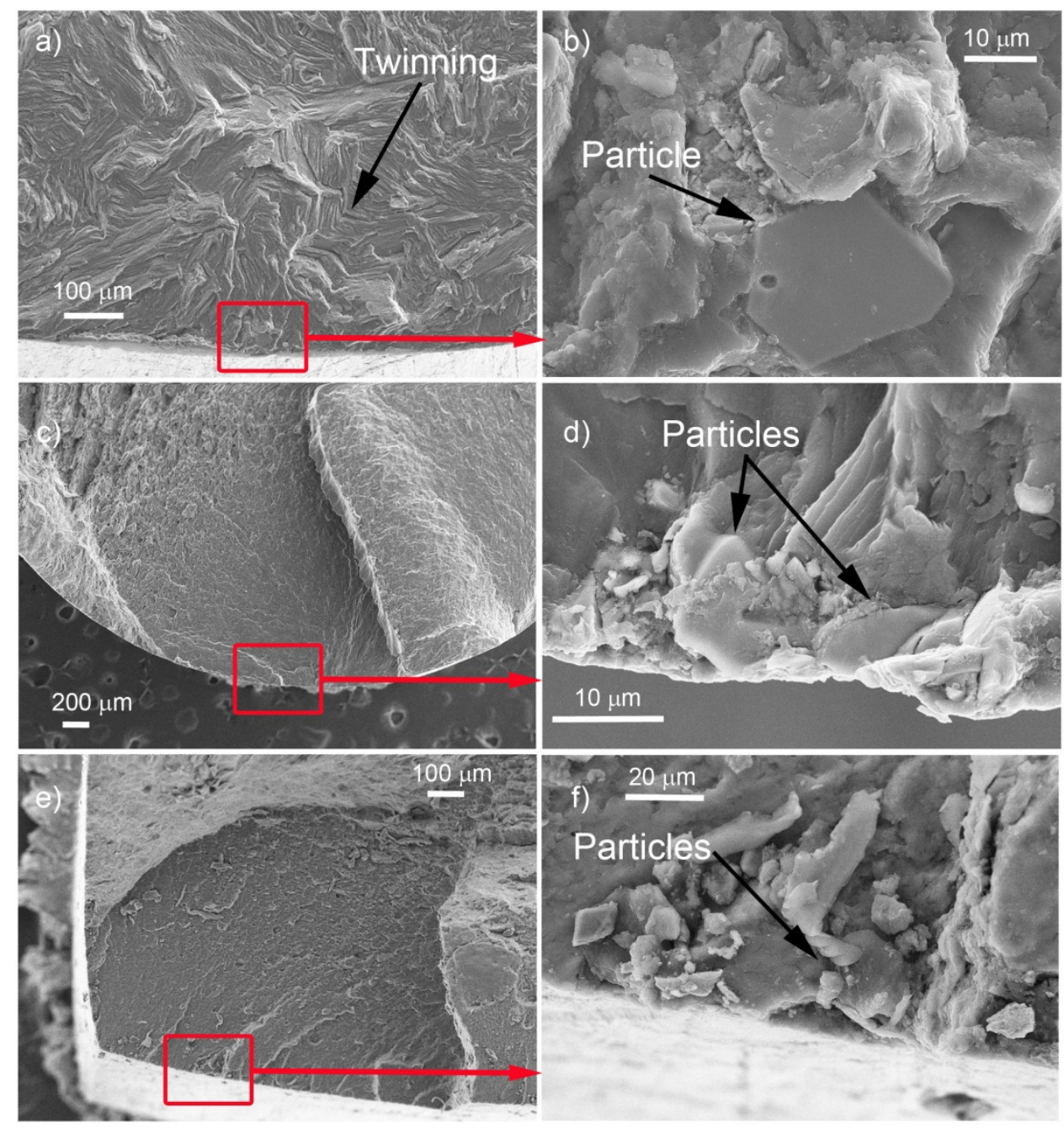

Figure 30. Typical overview of fracture surfaces for AZ31 magnesium materials at $0.3 \%$ of strain amplitude in the (a) extruded, (c), plate, and (e) sheet forms. A magnified view of the areas enclosed by the red boxes indicate that fatigue cracks were found to initiate from intermetallic particles at the surface in the (b) extruded, (d), plate, and (f) sheet materials.

\section{Strain-life Results and Fatigue Modeling}

Figure 31 displays the engineering strain amplitude $(\mathrm{mm} / \mathrm{mm})$ against number of reversals to failure (1 cycle $=2$ reversals) for the constant strain amplitude and completely reversed loading fatigue experiments on the extruded, plate, and sheet materials. For comparison purposes, Fig. 31d displays the fatigue strain-based results for each of the three materials. In general, the sheet material displayed better fatigue resistance, with fatigue lives of the order of two- to three-times greater than those of the other two materials in low cycle regime. Figure $30 \mathrm{~d}$ also includes fatigue data for a high strength 7075T651 aluminum alloy [2]. It is observed that for the low cycle fatigue regime, the aluminum alloy behavior is superior to the Mg alloy, with fatigue lives of the order of four times greater compared with the $\mathrm{Mg}$ alloy in this study. However, the Mg alloys compared more closely to the aluminum alloy in the high cycle regime. 

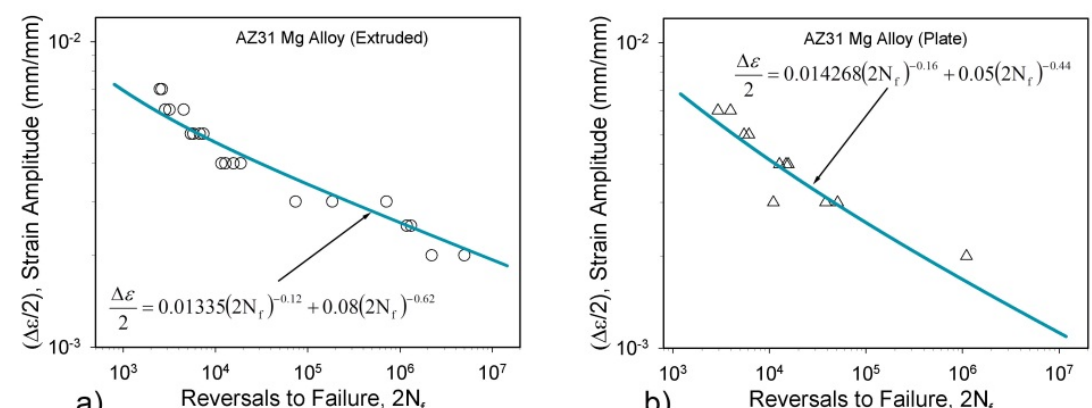

a)
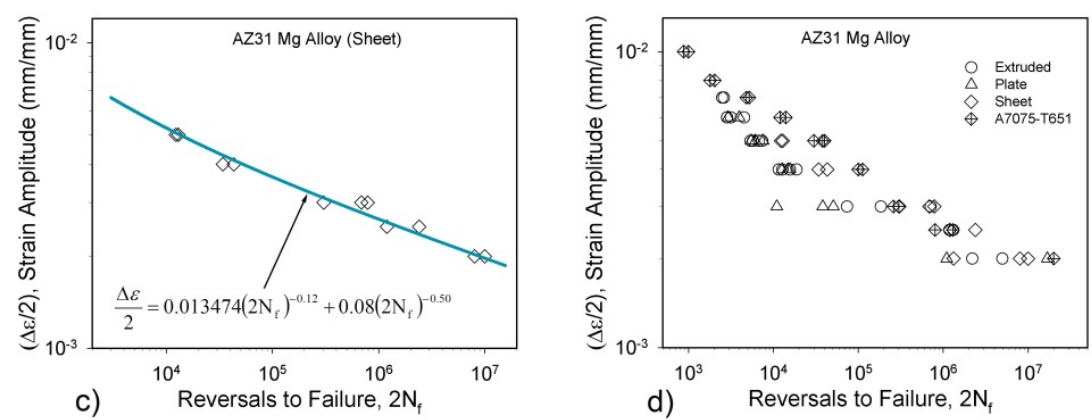

Figure 31. Experimental strain-life results for the AZ31 mg alloy in the (a) extruded, (b) plate, and (c) sheet forms. Strain-based equations are represented by the solid blue curve in each figure (d) Comparison of the strain-life results of the extruded, plate, and sheet materials to the 7075-T651 aluminum alloy [2].

Figure 32 shows the correlations of the MSF model to the experimental strain-life results for the Mg AZ31 alloy in the extruded, plate, and sheet forms. Table 1 lists the model constants used to fit the model to fully-reversed uniaxial strain life results. Note that the basic model constants were identical, but the specific microstructural features due to processing of the three materials were the only parameters that were different.

The primary inputs to the MSF model include the tensile yield and ultimate strengths, the cyclic stressstrain curve, mean inclusion size (particle diameter), and microstructure information such as grain size. The remaining constants are based on micromechanical simulations and other accepted values pertaining to microstructure used in modeling fatigue [1-2, 4]. As observed in Fig. 32, the model is correlated to the experimental data and can distinguish the fatigue lives of the three Mg AZ31 materials based upon the microstructural content and associated mechanical properties that are affected by the microstructural content. This is a significant point since the results from the modeling can be used to help elucidate the effect of a particular microstructure or material property on fatigue lifetimes. As a result of the MSF model correlation process on the three Mg AZ31 materials, we list the microstructure/material property model parameters in order of most significant effect on fatigue lifetimes: (1) yield/ultimate tensile strength; (2) particle size; (3) grain size. This is not necessarily surprising considering the established knowledge regarding fatigue damage. However, since the MSF model can capture the distinct differences in fatigue life based primarily on a change of variables related to microstructure/material properties, the model can be used in situations where anisotropic 
mechanical properties are present or employed to develop new Mg alloys for optimized fatigue properties.

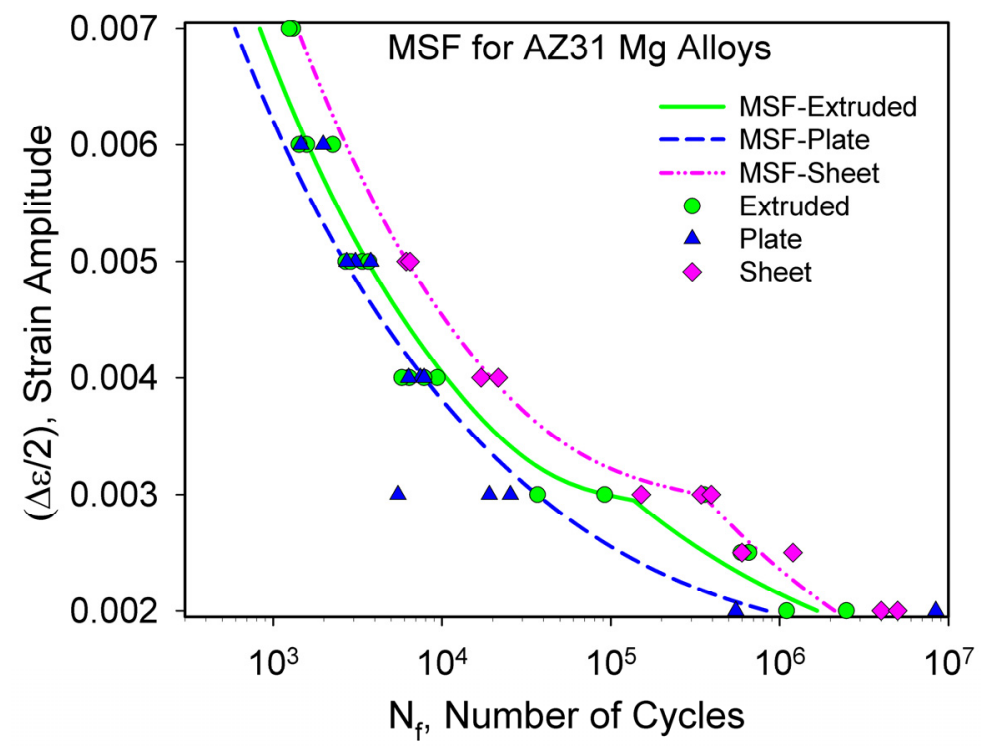

Figure 32. Comparison of the microstructure-sensitive fatigue model to the experimental strain-life results of the AZ31 Mg materials in the extruded, plate, and sheet forms.

Table 1. Microstructure-sensitive fatigue modeling parameters for AZ31 magnesium alloy.

\begin{tabular}{|c|c|c|c|c|c|}
\hline & Constant & Extrusion & Plate & Sheet & Description \\
\hline & $C_{m}$ & 0.95 & 0.95 & 0.95 & LCF coefficient in Modified Coffin Manson Law (Eq. 8) \\
\hline & $C_{n}$ & 0.55 & 0.55 & 0.55 & HCF coefficient in Modified Coffin Manson Law (Eq. 8) \\
\hline & $\alpha$ & -0.635 & -0.635 & -0.635 & Ductility exponent in Modified Coffin Manson Law (Eq. 8) \\
\hline & $q$ & 2.4 & 2.4 & 2.4 & $\begin{array}{l}\text { Exponent in remote strain to local plastic shear strain (Eq. } \\
9 \& 10)\end{array}$ \\
\hline $\begin{array}{l}\frac{0}{\pi} \\
\frac{0}{\pi} \\
\frac{0}{3} \\
\text { 뜨 }\end{array}$ & $y_{1}$ & 170 & 170 & 170 & $\begin{array}{l}\text { Constant in remote strain to local plastic shear strain (Eq. } \\
9 \& 10)\end{array}$ \\
\hline 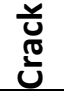 & $y_{2}$ & 0 & 0 & 0 & Linear constant in remote strain to local plastic shear \\
\hline
\end{tabular}




\begin{tabular}{|c|c|c|c|c|c|}
\hline & & & & & strain (Eq. 9 \& 10) \\
\hline & $\xi$ & 4 & 4 & 4 & Geometric factor in micromechanics study (Eq. 10) \\
\hline & $r$ & 0.2 & 0.2 & 0.2 & Exponent in micromechanics study (Eq. 12) \\
\hline & $\omega$ & 0.15 & 0.15 & 0.15 & Omega (Eq. 14) \\
\hline & $\theta$ & 0.135 & 0.135 & 0.135 & $\begin{array}{l}\text { Load path dependent and loading combination parameter } \\
\text { (Eq. 14) }\end{array}$ \\
\hline ত্ & $\zeta$ & 1.2 & 1.2 & 1.2 & Exponent in Small crack growth (Eq. 14) \\
\hline 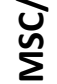 & $C_{l}$ & 300000 & 300000 & 300000 & HCF constant in small crack growth (Eq. 14) \\
\hline 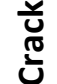 & $C_{11}$ & 0.0001 & 0.0001 & 0.0001 & LCF constant in small crack growth (Eq. 14) \\
\hline$\overline{\overline{\bar{\sigma}}}$ & $\chi$ & 0.32 & 0.32 & 0.32 & Crack growth rate constant (Eq. 13) \\
\hline
\end{tabular}

\section{Microstructure-Sensitive Fatigue Modeling of an Extruded AM30 Magnesium Alloy}

In this research the fatigue performance of an AM30 Mg alloy was investigated. Firstly, the strain-based approach was employed to determine low cycle fatigue parameters. Secondly, a microstructuresensitive fatigue model that decomposes the total fatigue lifetime in three stages: crack incubation, small crack growth, and long crack growth was calibrated. Experimental results for AM30 Mg Alloys have been presented previously and are not included here. The material employed was an extruded AM30 magnesium alloy. The material was provided in the form of an automotive crash rail profile with a thickness of approximately $3.2 \mathrm{~mm}$. Samples for this study were taken in the extrusion direction (ED) and in the transverse direction (ETD) as shown in Figure 33. Fatigue specimens were designed in conformity with ASTM standard E-647 with a gage length of $15 \mathrm{~mm}$. Fatigue tests were conducted on a servo-controlled electro-hydraulic system. These tests were performed under a strain control mode with a constant strain amplitude and were fully reversed. Tests were conducted at room temperature, and a sinusoidal cyclic loading was applied with a frequency of $5 \mathrm{~Hz}$. A $50 \%$ drop of the peak stress load was used to determine failure of the sample. The amplitude levels tested ranged from 0.2 to $0.6 \%$ of strain. 

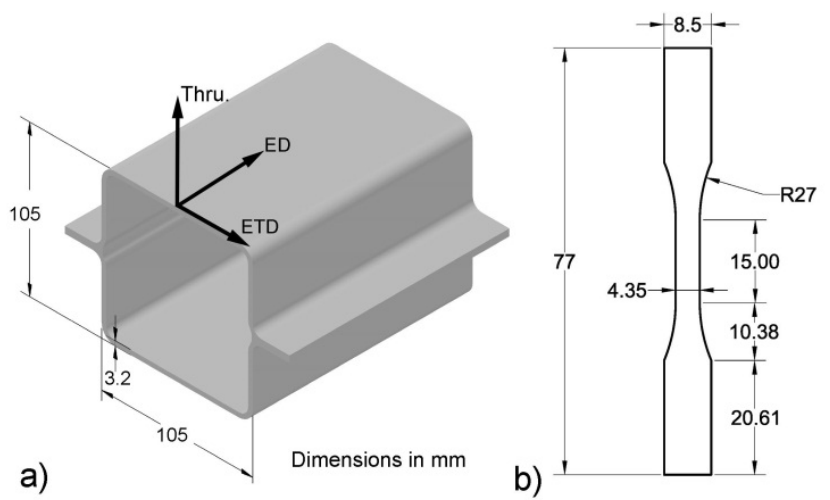

Figure 33. a) Extruded profile of the material under study and location and orientation of samples, b) Dimension of fatigue specimens.

\section{Cyclic Deformation Response}

Figure 34 shows the stress-strain hysteresis loops for the ED at strain amplitudes of 0.6 and 0.3 percent. An asymmetric pattern in the cyclic deformation is observed for the first cycle for all the strain amplitudes. This asymmetry was more pronounced at higher strain levels and for the first cycle. The hysteresis loops showed that hardening takes place at lower strain amplitudes under tensile loading. For lower strain amplitudes the tensile and compressive stresses were almost the same; however, the asymmetry from tension to compression was larger as the strain amplitude increased. A typical plateau is observed in the stress-strain response during compression loading and is observed in other magnesium alloys as reported elsewhere $[3,6,14]$.

Cyclic stress-strain hysteresis curves for the ETD at strain amplitudes of 0.5 and 0.3 percent are shown in Figure 35. The first cycle shows a slightly asymmetric behavior for all strain amplitudes. Similarly for the case of the ED, the hysteresis loops revealed that hardening occurred at lower strain amplitudes under tensile loading. A very tenuous plateau is observed during compression loading for the first cycle markedly at high strain amplitudes, which is a clear indication of twinning. In addition, it is observed that the hysteresis loop began with twinning in tension, and pseudo-elasticity during unloading, while reloading in compression marked an inflection point, which is an indication of detwinning. 

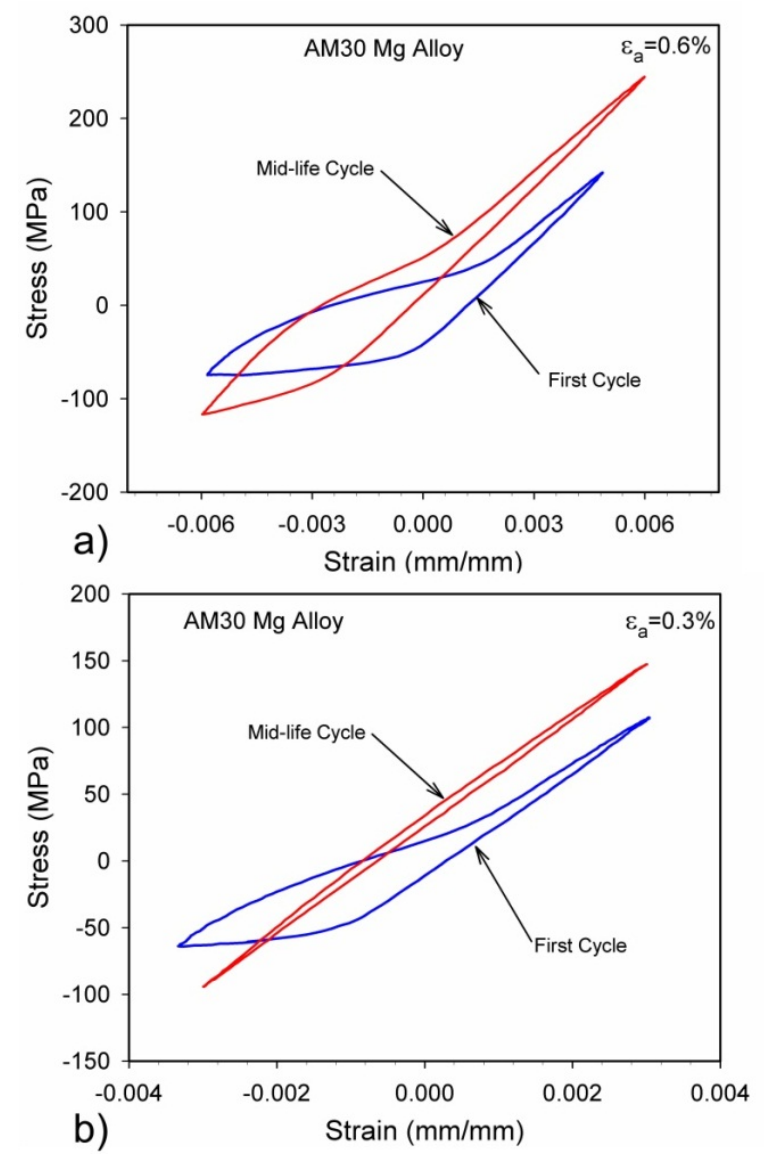

Figure 34. Hysteresis curves of $\mathrm{AM} 30$ extruded $\mathrm{Mg}$ alloy in the extruded direction at the strain amplitudes of a) $0.6 \%$, and b) $0.3 \%$.

\section{Fatigue Fractured Surfaces}

A study of fracture surfaces of failed strain-life specimens for the AM30 Mg alloys was conducted. In this research, fracture surfaces for both loading directions were imaged in an SEM. River marks flowing outward from a single location at the surface were observed on all specimens. Figures 36 and 37 show representative fractographs for both the ED and ETD directions. Figure 35 shows a typical fracture surface in the ED direction. The crack initiated near the surface of the specimen and at an inclusion particle. However, the presence of twinning next to the initial crack initiation site was identified. 

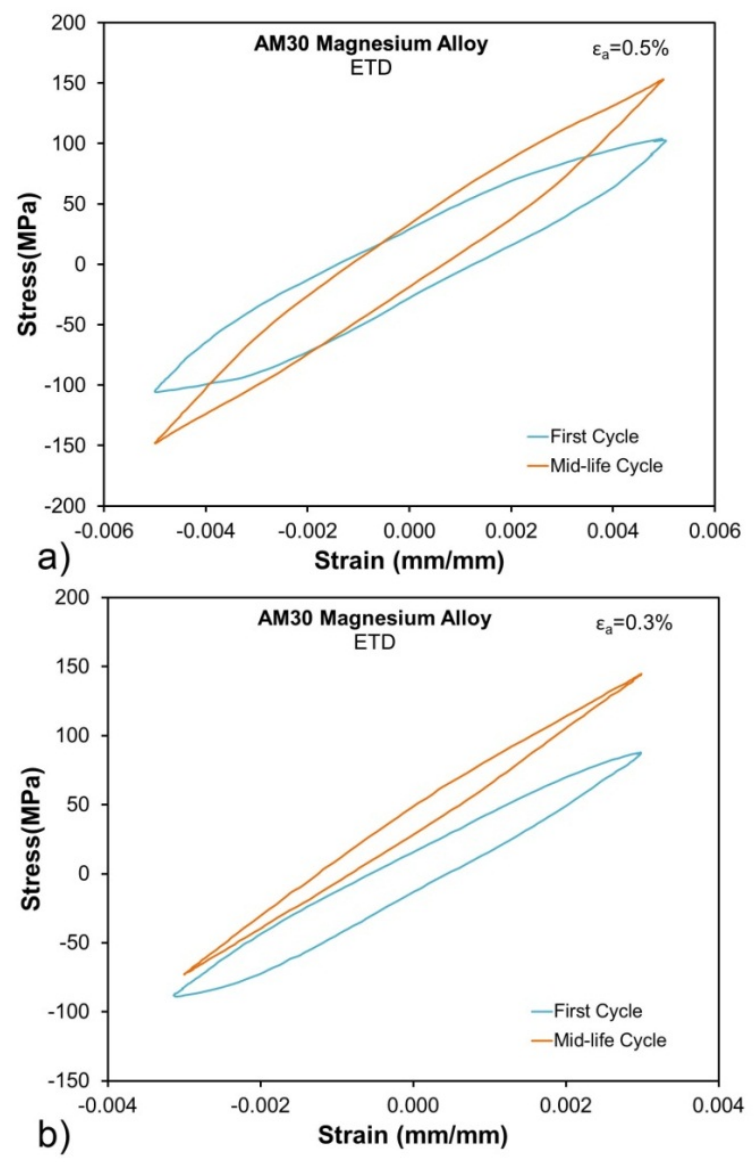

Figure 35. Hysteresis curves of $\mathrm{AM} 30$ extruded $\mathrm{Mg}$ alloy in the transverse direction at the strain amplitudes of a) $0.5 \%$, and b) $0.3 \%$.

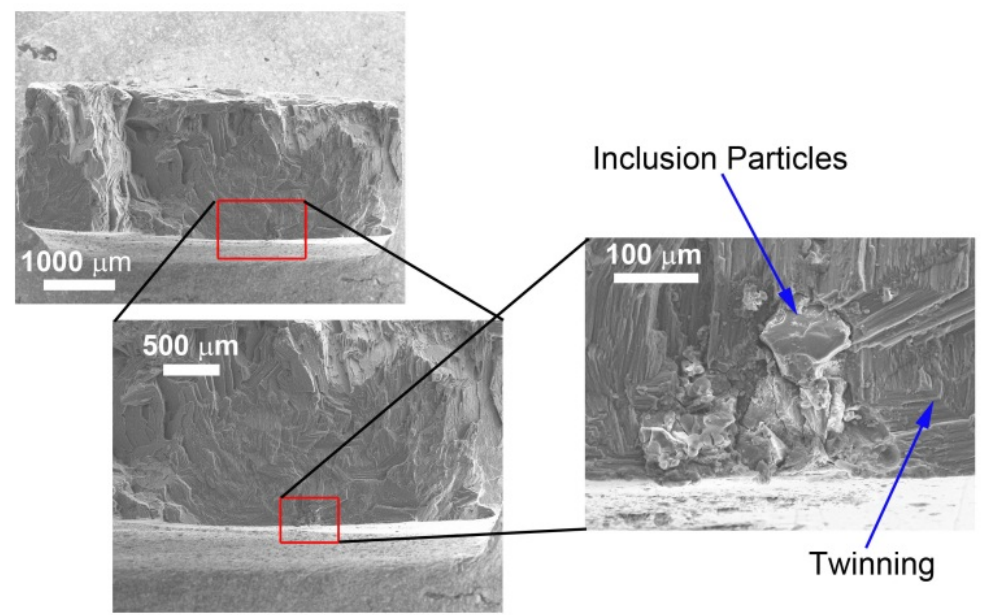

Figure 36. Typical fractured surface for an AM30 magnesium Alloy in the ED direction. The crack developed at a small inclusion. In addition, twinning is observed near the crack site. 


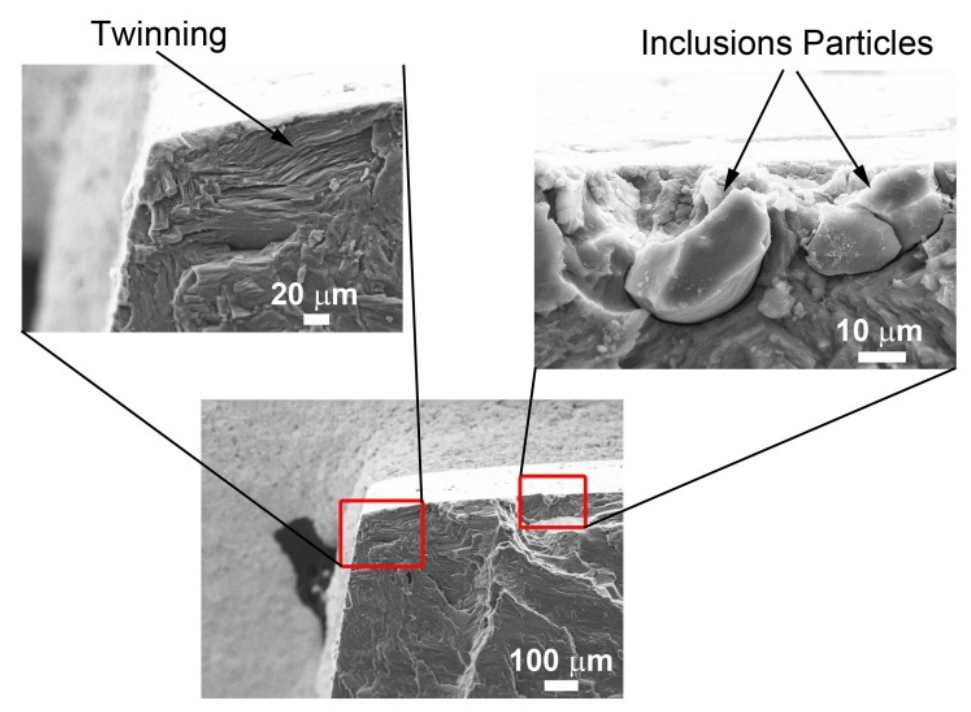

Figure 37. Typical fractured surface for an AM30 magnesium Alloy in the ETD direction.

\section{Strain-Based Approach}

The strain-life method for modeling fatigue damage is used extensively in the ground vehicle industry for designing against fatigue failure. This method is based on the fact that nominal stresses in structures produce plastic deformation in the vicinity of stress concentrations, where stresses and strains in the plastic zone are limited by the elastic displacement of the surrounding elastic stress field [23-25]. These conditions are prevalent in the so called low cycle fatigue (LCF) regime, where a structure undergoes high stresses or strains. Here, the LCF parameters are developed.

The total strain-life amplitude as a function of the number of cycles is given by the following equation.

$\frac{\Delta \varepsilon}{2}=\sigma_{f}^{\prime}\left(2 N_{f}\right)^{b}+\varepsilon_{f}^{\prime}\left(2 N_{f}\right)^{c}$

Figure 38 exhibits the strain-based model correlations along with Eq. (10) and the corresponding parameters, and Table 2 summarizes the LCF parameters for the AM30 Mg alloy. 


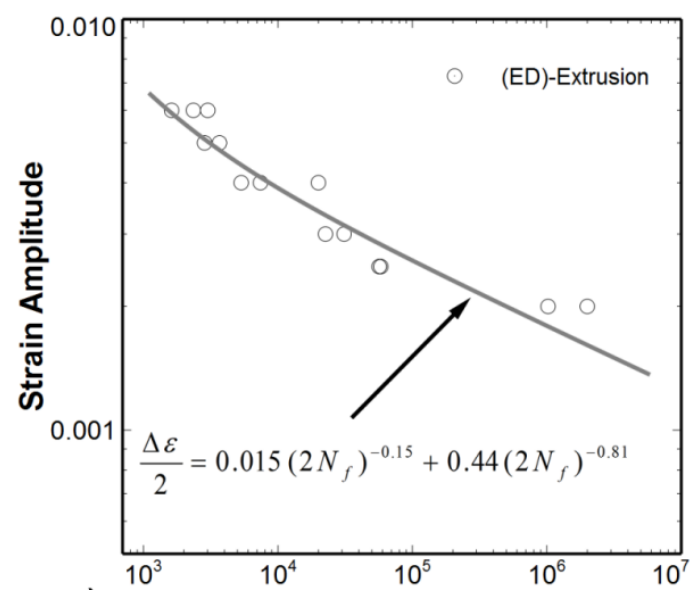

a)

$2 \mathrm{~N}_{\mathrm{f}}$, Reversals to Failure

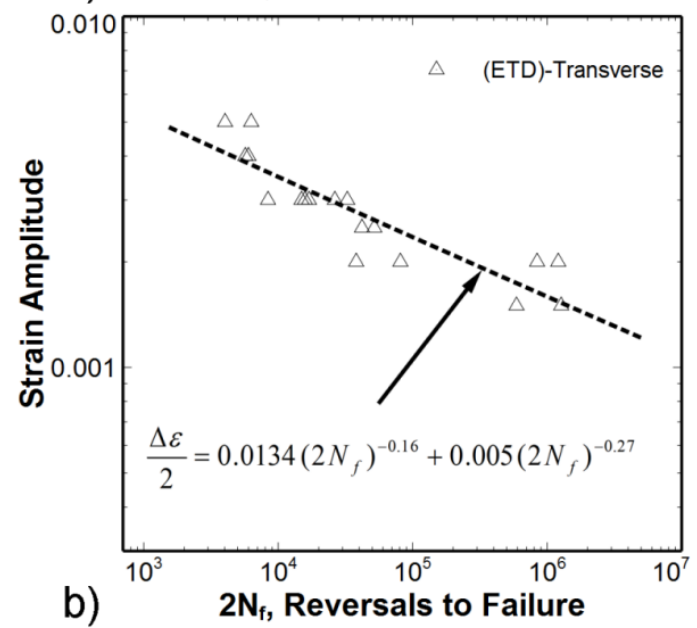

Figure 38. Low cycle fatigue parameters and strain-life results for the AM30 Mg alloy in the (a) (ED), and (b) (ETD) loading directions.

Table 2. Strain-life fatigue parameters for an AM30 Mg Alloy.

\begin{tabular}{|l|l|l|l|}
\hline Parameters & Symbol & Extruded & Transverse \\
\hline Cyclic strain hardening exponent & $n^{\prime}$ & 0.19 & 0.41 \\
\hline $\begin{array}{l}\text { Cyclic strain strength coefficient, ( } \\
\mathrm{MPa})\end{array}$ & $K^{\prime}$ & 657 & 583 \\
\hline Fatigue strength Coefficient, (MPa) & $\sigma_{f}^{\prime}$ & 674 & 602 \\
\hline Fatigue strength exponent & $b$ & -0.15 & -0.16 \\
\hline Fatigue ductility coefficient & $\varepsilon_{f}^{\prime}$ & 0.44 & 0.005 \\
\hline Fatigue ductility exponent & $c$ & -0.81 & -0.27 \\
\hline
\end{tabular}




\section{Microstructure-Sensitive Approach}

Figure 39 shows the correlations of the MSF model to experimental data for both ED and ETD, and Table 3 lists the model constants used to correlate the model to fully-reversed uniaxial strain life results for the extruded AM30 magnesium alloy.

\section{Discussion}

The computation of the SLF parameters is straightforward, if experimental strain-life data is provided along with plastic deformations. The other only monotonic mechanical property needed is the elasticity modulus of the material. These parameters can be immediately implemented in commercial fatigue codes.

The strain-life curve in magnesium alloys shows a transition point where the change of slope is noticeable, this observation applies also for the AM30 Mg alloy as observed in Figure 5 . In the strainbased correlations determined here were observed differences in the SLF parameters with respect to the observed variation in fatigue lifetimes. While a slope is observed at low cycle fatigue a distinct slope is shown at high cycle fatigue. This strong "bilinear" behavior could be explained the fairly observed correlation to the Coffin-Manson relationship.

For the microstructure-sensitive approach we use the MSF model, which decomposes the total fatigue damage in the three distinct stages of fatigue damage: incubation, microstructurally small crack growth, and long crack growth. The long crack regime was not included in this work, and thus only the incubation and small crack regimes were employed. Note that the basic model constants were identical, but the specific microstructural/mechanical features due to the load direction relative to the crystallographic orientation were the only parameters that were different.

The major inputs to the model include the tensile yield and ultimate strengths, the cyclic stress-strain curve, mean inclusion size (particle diameter), and microstructure information such as grain size. As observed in Fig. 39, the correlates very well to the experimental data and can distinguish the fatigue lives of the AM30 Mg alloy for both loading directions ED and ETD. Since the MSF model can capture the differences in fatigue life based mainly on changes of microstructure/material properties, the model can be used in situations where anisotropic mechanical properties are present. 
Table 3. MSF Parameters for extruded AM30 magnesium alloy.

\begin{tabular}{|l|l|l|}
\hline Constant & ED & ETD \\
\hline$C_{n}$ & 0.95 & 0.95 \\
\hline$C_{\text {inc }}$ & 0.55 & 0.55 \\
\hline$\alpha$ & -0.56 & -0.56 \\
\hline$q$ & 2.27 & 2.27 \\
\hline$y_{1}$ & 140 & 140 \\
\hline$y_{2}$ & 0 & 0 \\
\hline$r$ & 0.2 & 0.2 \\
\hline$\omega$ & 0.0 & 0.0 \\
\hline$a i$ & 0.625 & 0.625 \\
\hline$\theta$ & 0.0 & 0.0 \\
\hline$\zeta$ & 3.25 & 3.25 \\
\hline$C_{l}$ & 120000 & 120000 \\
\hline$C_{I I}$ & 0.01 & 0.01 \\
\hline$\chi$ & 0.32 & 0.32 \\
\hline CTDH & 0.00032 & 0.00032 \\
\hline & & \\
\hline & & \\
\hline & & \\
\hline & & \\
\hline & & \\
\hline & & \\
\hline & & \\
\hline & & \\
\hline & & \\
\hline
\end{tabular}




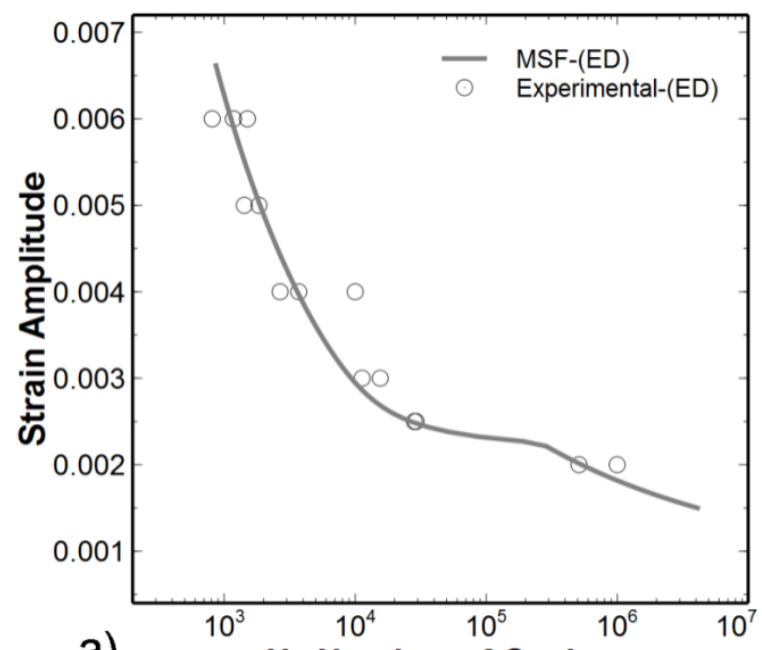

a) $\quad \mathrm{N}_{\mathrm{f}}$, Number of Cycles

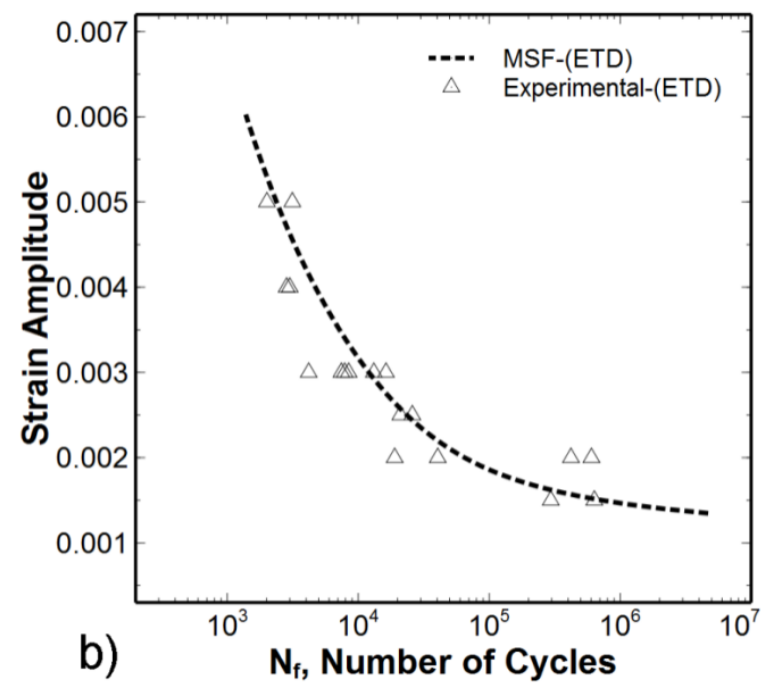

Figure 39. Total fatigue life predicted by the (MSF) model and strain-life results for an AM30 alloy in the a) Extrusion (ED), and b) Transverse (ETD) directions.

\section{Multiscale Fatigue Modeling of AZ91 and AM60 Magnesium Alloys}

In order to construct physically-motivated and microstructurally-sensitive fatigue estimations of the AZ91 and AM60 cast magnesium alloys presented in this study, the Multistage Fatigue (MSF) model was employed.

\section{Model Correlations and Fatigue Estimations}

Figures 40a-c show the MSF model correlations for the AZ91 as-cast, AZ91-T6, and AM60 as-cast alloys, respectively. The incubation life in cast alloys are typically a larger percentage of total fatigue life compare to wrought alloys. For the mean life prediction, an incubation life of $36 \%, 43 \%$, and $48 \%$ for total life was found suitable for the AM60 as-cast, AZ91 as-cast, and AZ91-T6 alloys, respectively. In addition, the mean fit for each of the fatigue data sets were based on mean microstructure values along with the corresponding cyclic mechanical properties. The mean pore size responsible for incubating the 
fatigue crack determined through the 3-D fractograghy analysis were used in the mean fits for each of the data sets.

To show the robustness of the MSF model and to capture the effect of microstructure on fatigue lifetimes, estimations for the upper and lower bounds of the strain-life data sets are also presented. These model predictions were determined by using the extreme pores sizes and DCS's for each data set. The model showed good correlation to both the upper and lower bounds as compared to the experimental results as shown in Fig. 40. As such, the MSF model was generally able to bound the scatter of each fatigue data set considering only the microstructure variation observed between locations L1 and L2. The model showed more sensitivity to pore size as compared to DCS. This is not unexpected considering there was not much variation of the DCS from locations L1 and L2. As such, the defect size was the primary factor determining number of cycles to failure for both the AM60 and the AZ91 alloys.

a)

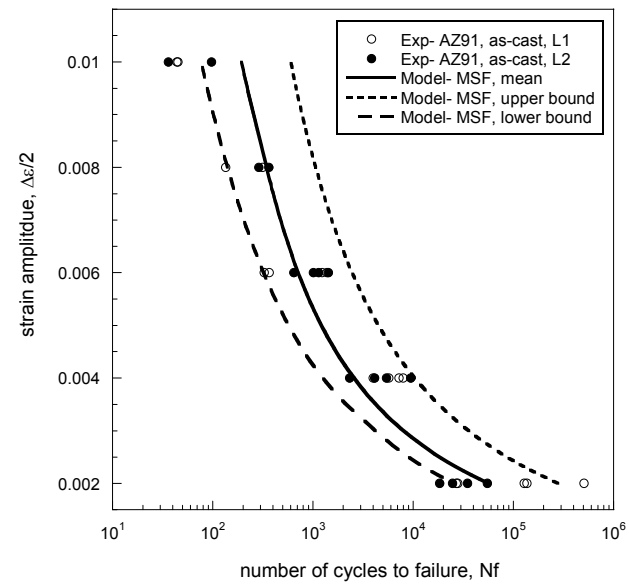

b)

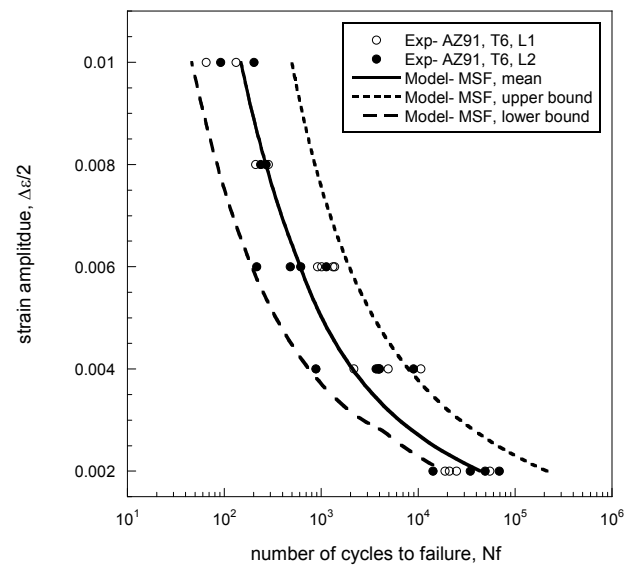


c)

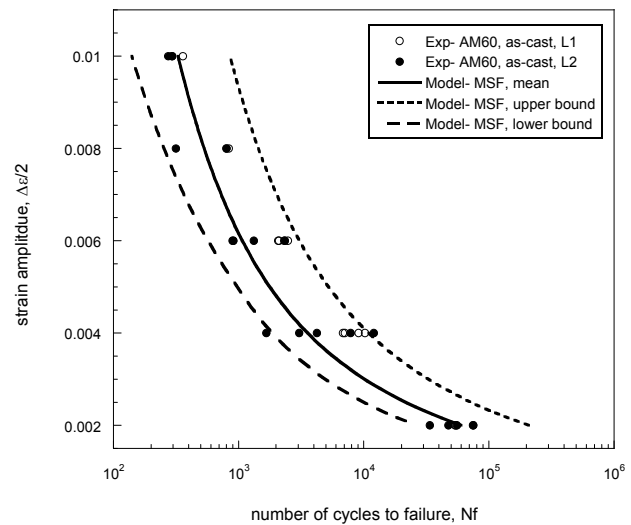

Figure 40. Comparison of the Strain-life experimental results for locations $L 1$ and $L 2$ to the mean, upper, and lower bound estimations of the Multistage fatigue model: a) AZ91 as-cast; b) AZ91-T6; c) AM60 ascast

The percentage of incubation to MSC/PSC growth of the MSF model varied considerably from the lower to the upper bounds. For the large pore sizes (lower bound), the average incubation life increased to approximately $60 \%$ of total life for all three data sets. Whereas, for the smallest pore sizes employed (upper bounds), the average incubation life consisted of approximately $12 \%$ of total life for all three data sets. This variation in the ratio of percentage of incubation life seems reasonable considering that a larger pore size will have a greater plastic zone around the inclusion resulting in more incubation life. As such, a smaller inclusion will result in a smaller plastic zone and therefore, less incubation life. The capability of the MSF model to capture the scatter typically observed in fatigue data based on microstructure variation alone is directly related to it's ability to vary the contribution of incubation and MSC/PSC growth for a given material. Table 4 lists the microstructure related MSF parameters for the mean, upper, and lower bounds estimations for the AM60 and the AZ91 alloys.

Table 4: Microstructure related MSF Parameters for AZ91 and AM60 magnesium alloys

\begin{tabular}{llll|llll|lll}
\hline & \multicolumn{3}{l}{ AZ91 As-Cast } & \multicolumn{3}{c}{ AZ91-T6 } & \multicolumn{3}{c}{ AM60 As-Cast } \\
\cline { 2 - 10 } & $\begin{array}{l}\text { Lower } \\
\text { bound }\end{array}$ & Mean & $\begin{array}{l}\text { Upper } \\
\text { bound }\end{array}$ & $\begin{array}{l}\text { Lower } \\
\text { bound }\end{array}$ & Mean & $\begin{array}{l}\text { Upper } \\
\text { bound }\end{array}$ & $\begin{array}{l}\text { Lower } \\
\text { bound }\end{array}$ & Mean & Upper \\
Pore Size ( $\mathrm{m})$ & 495 & 293 & 119 & 554 & 290 & 116 & 600 & 274 & 107 \\
DCS $(\mathrm{m})$ & 19.6 & 16.7 & 13.7 & 19.6 & 16.7 & 13.7 & 19.6 & 16.6 & 13.5 \\
\hline
\end{tabular}




\section{Influence of Structural Integrity on Fatigue Behavior of Friction Stir Spot Welded AZ31 Mg Alloy}

With the increased interest in achieving improved fuel economy in automobiles, the need for lightweight alloys is essential. While only marginally employed in current automobile designs, recent advances have produced promising magnesium alloys that are now being considered for structural components. As with any newly employed alloy, research is needed to determine the reliability and performance of joints made from potential joining techniques. As with any newly employed alloy, research is needed to determine the reliability and performance of joints made from potential joining techniques.

In this work the relationship of microstructural and geometrical features to fatigue performance is investigated in friction stir spot welds made using AZ31 magnesium alloy sheets. Lap shear coupons were spot welded using two sets of welding parameters. Optical microscopy of the initial state of the microstructure of each set of spot welds revealed differences in the hook formation, sheet thickness in the weld zone, and nugget diameter. Both sets of welds were fatigue tested in load control until failure at various load ratios. Optical microscopy of the failed coupons revealed differences in the fracture mode between the two sets of coupons. A linear elastic fracture mechanics model was used to correlate the fatigue life in the two processes. The fatigue model, which is a function of hook size, sheet thickness, and nugget diameter, showed good correlation to the experimental results. The model was also employed to show that the fatigue of the friction stir spot welds was most sensitive to the sheet thickness in the weld zone, followed by hook height, and then nugget diameter.

\section{Material and Experiments}

AZ31 Mg alloy sheets of $2.0 \mathrm{~mm}$ thickness were chosen for the present study. Two sets of FSSW coupons were welded in an overlap configuration. For process \#1, the individual sheet dimensions were: length of $100 \mathrm{~mm}$, width of $35 \mathrm{~mm}$, and a welded overlap area of $35 \times 35 \mathrm{~mm}$. For process \#2, the individual sheet dimensions were: length of $100 \mathrm{~mm}$, width of $38 \mathrm{~mm}$ and a welded overlap area of $38 \times 38 \mathrm{~mm}$. For process \#1, the FSSW tool was made from standard tool steel (H13) material with a shoulder diameter of $12 \mathrm{~mm}$ and a cylindrical pin having a diameter of $5 \mathrm{~mm}$, pin length of $3.2 \mathrm{~mm}$, and left hand threads (M5). For process \#2, the FSSW tool is identical to the tool used in process \#1; however the tool had a triangular pin. Both tools had a 10 concave shoulder. Figure 41 shows representative lap-shear samples from both process \#1 and \#2. For the fatigue tests, the lap-shear coupons were cyclically tested in an MTS 810 servo-hydraulic load frame under load control with a sinusoidal waveform at three different load ratios $(0.1,0.3$, and 0.7$)$. 


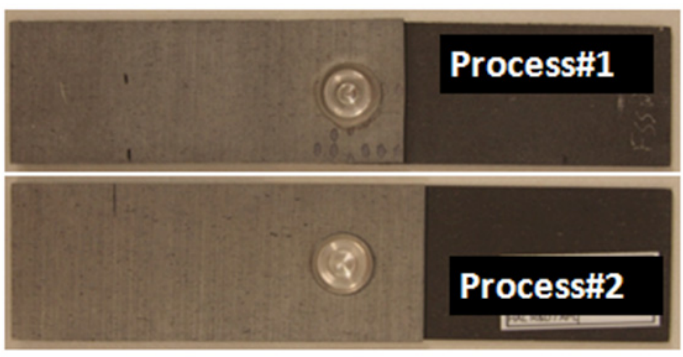

Figure 41. Representative lap-shear coupons produced from process \#1 and process \#2.

\section{Interfacial Hook and Geometrical Features}

The as-welded coupons were observed under optical microscopy in order to measure and quantify key features of the weld nugget geometry. In typical FSSW joints, the faying surface oftentimes exhibits a hook-like shape as shown in Fig. 42. This hooking of the faying surface is a result of trapped oxide films are that displaced upward due to the plastic flow of the material resulting from the downward plunge of the pin into the bottom sheet. In fact, the degree of oxide distribution at the faying surface is greatly influenced by the tool geometry and tool rotation speed [26]. For clarification purposes, the two peaks of the interfacial hooks are labeled as primary and secondary as marked in Fig. 42. In both the processes, the primary and secondary hooks were observed. A smoother interface and more distinctive hook were observed in process \#2. In contrast, the interfacial hook exhibited a more erratic path in process \#1 and the distinction between the primary and secondary hooks was not as clear as in process \#2. Further, the height of the hooks varied greatly between both processes, where in process \#2 the hook heights were approximately three times as large as compared to process \#1.

Consequently, the link between the effective sheet thickness, T (see Fig. 4), and hook height are directly related to the shoulder plunge depth achieved during FSSW. If the tip-to-tip distance of the primary interfacial hooks is used to define the bond width, D2 (refer Fig.43), process \#2 had a slightly larger bond width. Dimension nomenclature of the various weld geometry measured in this study are indicated in Fig. 43 and the quantified results are shown in Table 5.

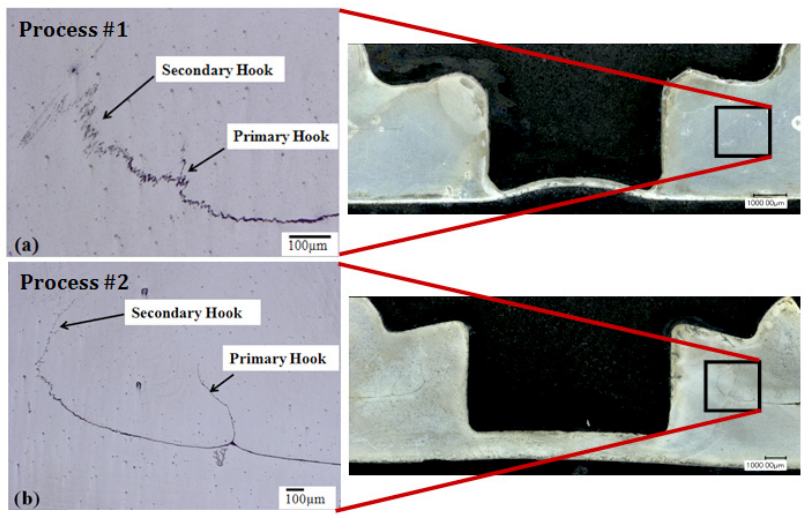

Figure 42. Magnified views of the primary and secondary hooks formed in (a) process \#1 and (b) process $\# 2$. 


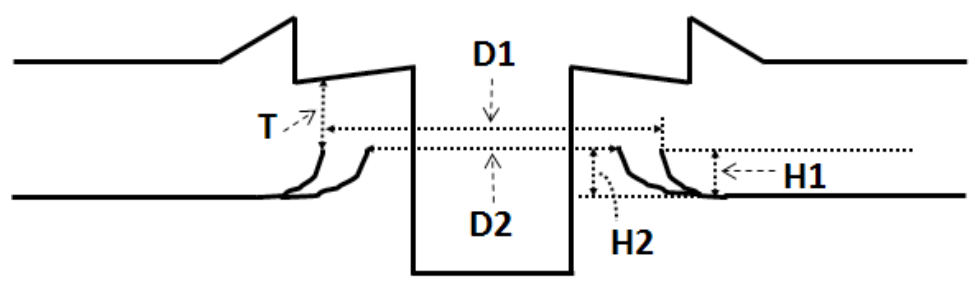

Figure 43. Schematic of key geometrical features of FSSW coupon with dimension nomenclature. Refer to Table 2 for dimensions.

Table 5: Summary of dimensions of the FSSW features for process \#1 and process \#2 (refer to Figure 43. for nomenclature).

\begin{tabular}{|l|l|l|}
\hline Nomenclature & Process \#1 $\mathbf{( m m})$ & Process \#2 $(\mathbf{m m})$ \\
\hline D1 & 10.8 & 11.0 \\
\hline D2 & 10.6 & 9.5 \\
\hline $\mathrm{H} 1$ & 0.15 & 0.63 \\
\hline $\mathrm{H} 2$ & 0.28 & 1.04 \\
\hline $\mathrm{T}$ & 1.03 & 1.15 \\
\hline
\end{tabular}

\section{Microstructure}

Three microstructural zones (Fig. 44) including the stir zone, thermo-mechanically affected zone (TMAZ), and heat affected zone (HAZ), in addition to the base material, were identified in the top and bottom sheets of the coupons from both processes. The stir zone (SZ) is characterized by finer grains due to dynamic recrystallization as shown in Fig. 45(a) for process \#1 and Fig. 45(b) for process \#2. The TMAZ is characterized by larger recrystallized grains as shown in Fig. 45(c) for process \#1 and Fig. 45(d) for process \#2. The HAZ was characterized by the presence of both the base metal grains and partially recrystallized grains as shown in Fig. 45(e) for process \#1 and Fig. 45(f) for process \#2. The grain structure of the base metal as received is shown in Fig. $6(\mathrm{~g})$ characterized by irregular grains. The grain size in the SZ and TMAZ in coupons from process \#1 were generally larger compared to process \#2 coupons. This can be attributed to the larger tool shoulder plunge depth and tool rotation (rpm) used in process \#1 compared to process \#2. In fact, higher tool rotation and larger tool plunge depth in process \#1 generated more heat which contributed to greater recrystallization, whereas in process \#2, a lower tool plunge depth and lower tool rotation speed generated less heat resulting in finer grain structure. 


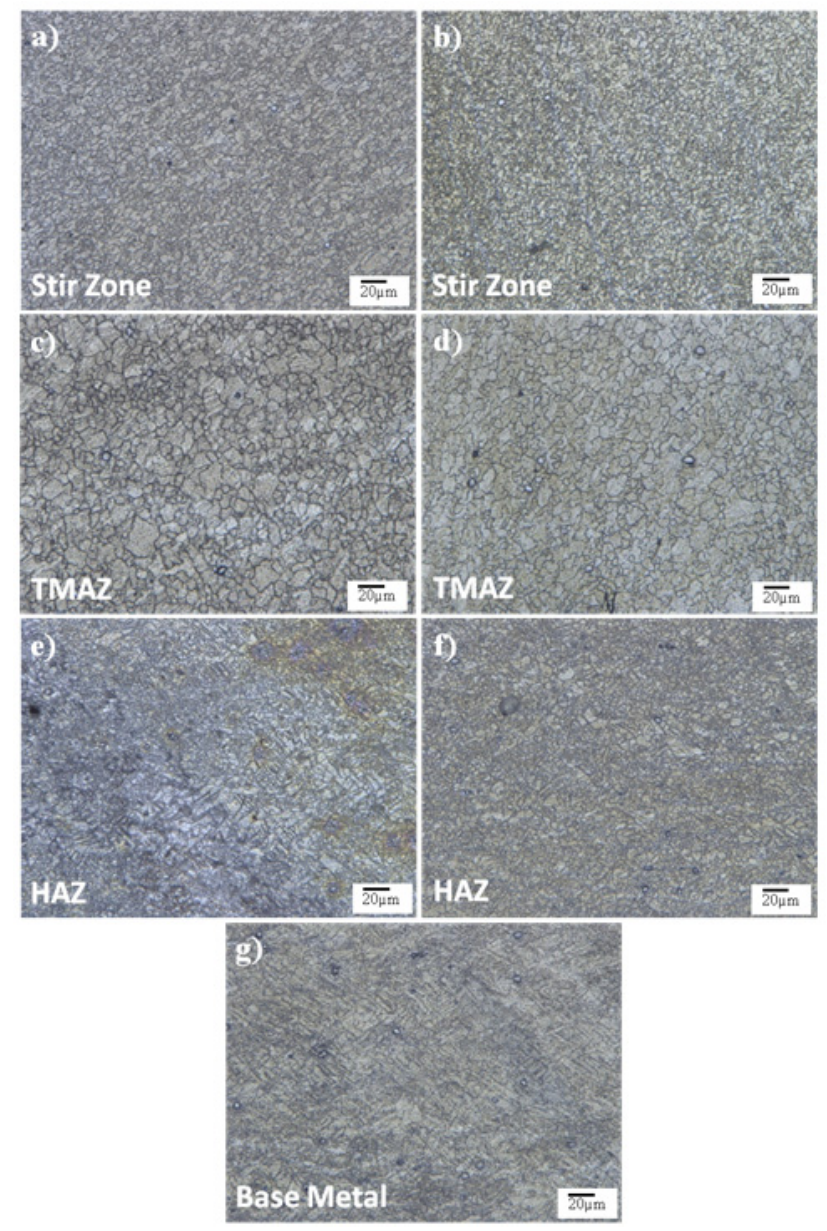

Figure 44. Microstructure features showing: (a) stir zone, (b) TMAZ, (c) and HAZ in coupons from process \#1, and (d) stir zone (e), TMAZ, (f) HAZ in coupons from the process \#2 and (g) Base metal of the coupons.

\section{Microhardness Profile}

Figure 45(a) shows the microhardness profile of the weldment comparing the top sheets from process $\# 1$ and process \#2. Figure 45(b) shows the microhardness profile of the weldment comparing the bottom sheets from process \#1 and process \#2. The horizontal axis in both Fig. 45(a) and 45(b) represent the axisymmetric distance from the center of the weldment (left side) and going outward away from the center of the weldment (right side). Microhardness mapping of the FSSW samples revealed the presence of a softer material response in the TMZ and harder material response in stir zone of the weldment in both processes. In addition, in both processes, a large gradient in relative hardness was observed moving from the stir zone towards the TMAZ. Furthermore, there was not a large difference in hardness values between the top and bottom sheet in the same coupon. 

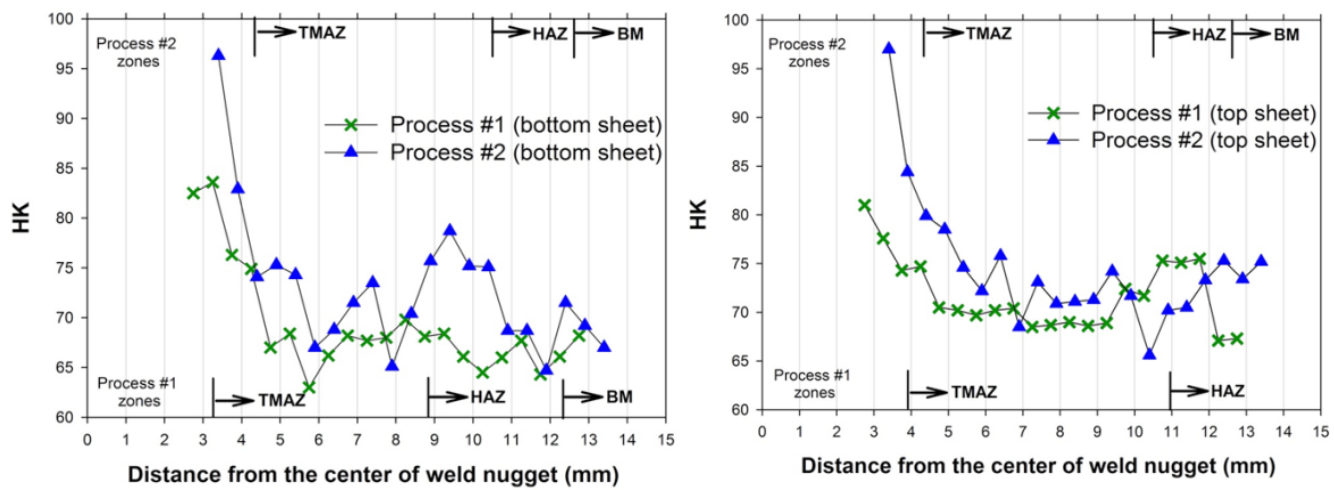

Figure 45. Microstructure features showing: (a) stir zone, (b) TMAZ, (c) and HAZ in coupons from process \#1, and (d) stir zone (e), TMAZ, (f) HAZ in coupons from the process \#2 and (g) Base metal of the coupons.

\section{Fatigue Tests}

Figure 46 shows the results of the applied load range against the number of cycles to failure for the FSSW coupons. The load range tested for both processes was between 1-3kN, and a range of load ratios of $0.1,0.3$, and 0.7 . In general, the results of the lap-shear fatigue tests of the FSSW show that the number of cycles to final separation was greater for process \#2 than process \#1. In addition, the fatigue results confirm that the FSSW lap-shear coupons did not exhibit a load ratio effect commonly exhibited in base materials.

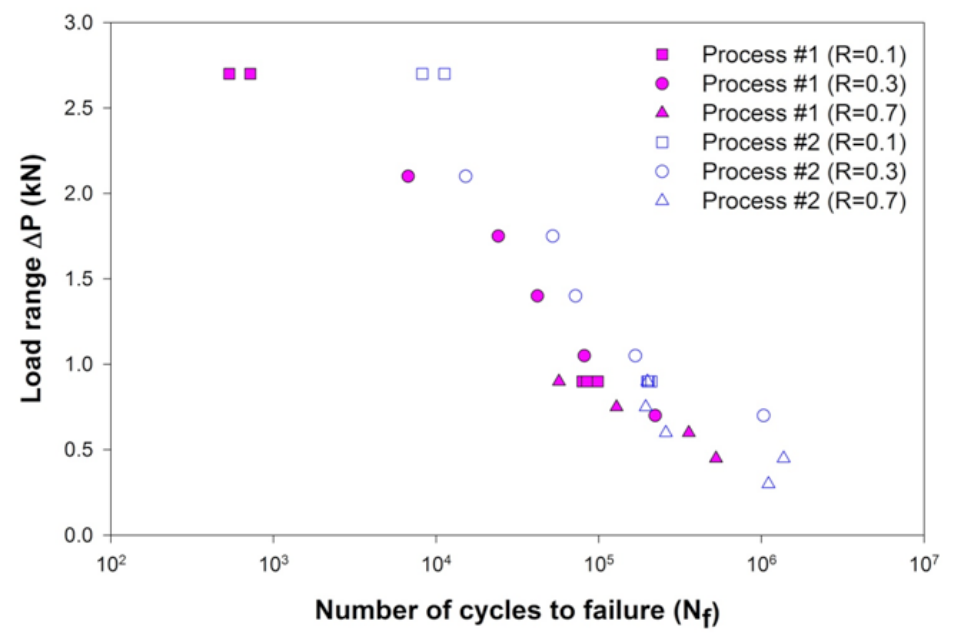

Figure 46. Experimental results of the load range versus the number cycles to failure for complete separation for friction stir spot weld lap-shear coupons tested at three load ratios $(R=0.1,0.3,0.7)$. 


\section{Failure Modes}

Figure 47 shows an overview of representative fractured FSSW coupons from both process \#1 and \#2. Several macroscale observations were made regarding the failure modes. It can be seen that the dominant crack originated from the interfacial hooks in coupons from the two processes. The mode of failure varied from nugget pullout, top sheet pullout, nugget shear failure and bottom sheet pullout for different loading conditions in the FSSW coupons of the two processes.
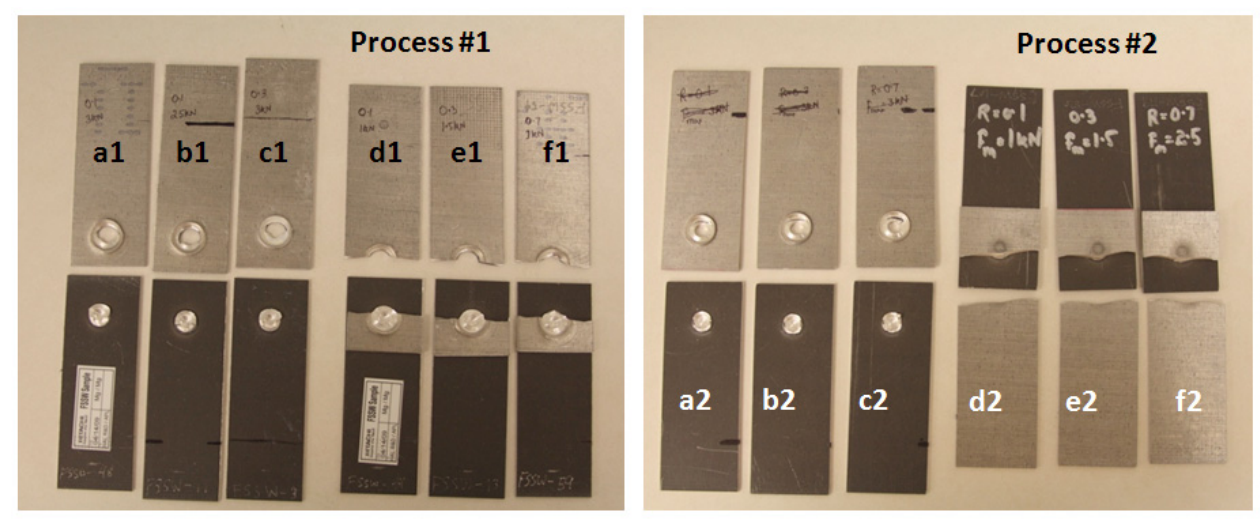

Figure 47. Representative fractured coupons tested at a range of maximum cyclic loads of $1-3 \mathrm{kN}$ at $\mathrm{R}=0.1,0.3$, and 0.7 .

For the FSSW coupons from the process \#1 tested at a maximum cyclic load of $2 \mathrm{kN}$ or greater, fracture occurred by failure of the weldment. Below $2 \mathrm{kN}$, the joints failed with the dominating crack propagating through the sheet thickness from the primary hook into the top sheet then propagating along the outer circumference of the nugget. Halfway around the nugget circumference, the crack grew along the width of the top sheet and failed by top sheet pullout. This failure mode was similar to failure modes observed in aluminum FSSW lap-shear coupons [27]. As shown in Figure 48(a), the nugget was seen to be intact in the remaining top and bottom sheet. Figure 48(b) shows the location of the dominant crack propagation on the outer surface of the weld nugget. It is evident from Figure 48(c) that the dominant crack propagated from the faying surface. Higher magnification of the region showed the dominant crack propagated from the primary hook into the top sheet thickness as seen in Figure 48 (d). No evidence of a secondary crack propagation or growth was found elsewhere in the weldment as shown in the magnified interfacial hook region in Figure 48(e), which is located at the opposite side of the weldment (180 degrees around the circumference). 

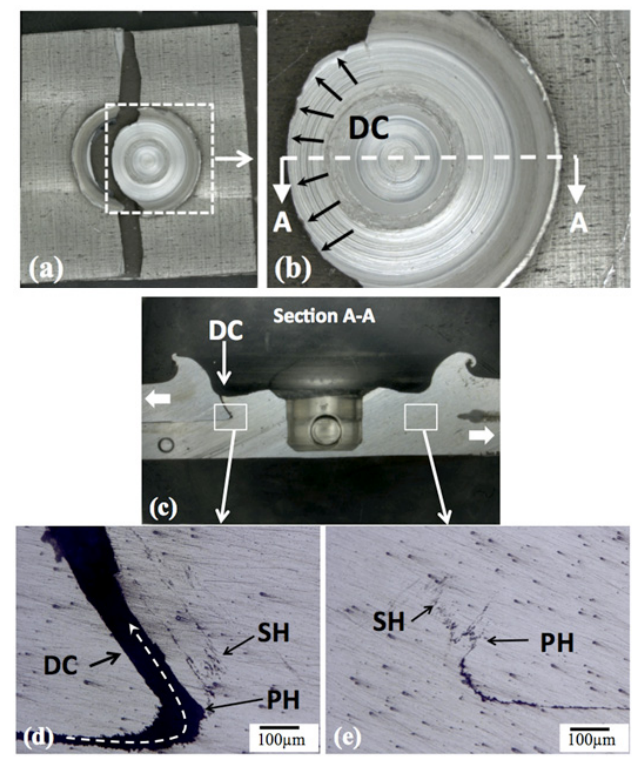

Figure 48. Crack propagation and failure mechanisms for process \#1 coupon loaded at a maximum cyclic load of $1 \mathrm{kN}$ at $\mathrm{R}=0.3$. (a) An overview of the surface of the top sheet and (b) a magnified view of the location of surface crack initiation. (c) A section view of the weld nugget shows that the dominant crack (DC) propagated from the weld interface, and (d) and (e) show higher magnification images of the interfacial primary $(\mathrm{PH})$ and the secondary $(\mathrm{SH})$ hooks. Bold arrows in (c) indicate direction of applied loading.

For process \#2 FSSW coupons tested below a maximum cyclic load of $2.5 \mathrm{kN}$, the coupons failed by bottom sheet failure as shown in Fig. 49(a). As in lower loads for FSSW coupons of process \#1, the dominant cracks were not observed to propagate through the primary or secondary hooks. As shown in Fig. 49(b), secondary non-dominant cracks were observed to initiate to the top sheet, but did not contribute to the final separation of the coupon. Eyebrow cracks were seen on the top surface of the nugget caused due to the secondary cracks. A section view of the weldment confirmed that the dominant crack propagated into the bottom sheet as shown in Fig. 49(c). Magnified views of the interfacial hooks shown in Fig. 49(d) confirmed that secondary cracks on the opposite side of the weldment propagated into the top sheet from both primary and secondary hooks. However, these cracks did not contribute to the final failure. As seen in Fig. 49(e), the dominant crack grew from the root of the secondary hook towards the bottom sheet and then across the full width of the bottom sheet. 


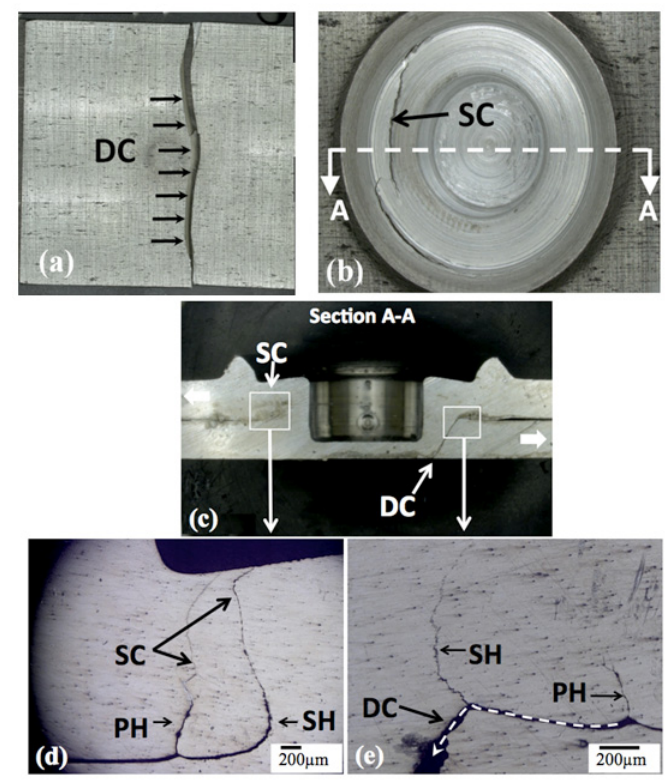

Figure 49. Crack propagation and failure mechanisms for process \#2 coupon loaded at a maximum cyclic load of $1 \mathrm{kN}$ at $\mathrm{R}=0.3$. An overview image of the (a) surface of the bottom sheet showing the dominant crack and (b) top sheet showing a secondary crack. (c) A section view of the weld nugget shows that the dominant crack (DC) propagated downward into the bottom sheet and (d) and (e) show higher magnification images of the interfacial primary (PH) and the secondary (SH) hooks. Bold arrows in (c) indicate direction of applied loading.

\section{Fatigue Crack Propagation}

In order to estimate crack growth rates and to confirm fracture modes in both sets of coupons, representative fracture surfaces of the FSSW coupons were imaged using SEM under high magnification with the intent to measure striation spacing. As shown in Fig. 50(a), the striation spacing was in the range of 1-1.6 $\mu \mathrm{m}$ for process \#1 coupons tested at a maximum cyclic load of $1 \mathrm{kN}$. At higher loads, the striation spacing was observed to be in the 3.3- $3.5 \mu \mathrm{m}$ range, as shown in Fig. 50(b). In FSSW coupons from process \#2 tested at a maximum cyclic load of $1 \mathrm{kN}$, the average striation spacing was around 0.80 $\mu \mathrm{m}$ and in coupons tested at $3 \mathrm{kN}$, the striation spacing was approximately $0.60 \mu \mathrm{m}$ as shown in Fig. 50(c) and Fig. 50(d). We note that the difference between the $1 \mathrm{kN}$ and $3 \mathrm{kN}$ for process \#2 is small and this observed difference could likely be due to scatter. We also note that the striations were less distinct in process \#2 and as such, were considerably more difficult to locate compared to process \#1. The striation measurements aided in estimating the rate of the crack propagation in the FSSW coupons between the two processes. In general, cracks propagated at higher rate in process \#1 compared to process \#2 as shown in Fig. 51. This generally faster propagation rate supports the hypothesis that the effective top sheet thickness and the larger microstructure gradient contributed to the difference in fatigue performance observed for process \#1 and \#2. 


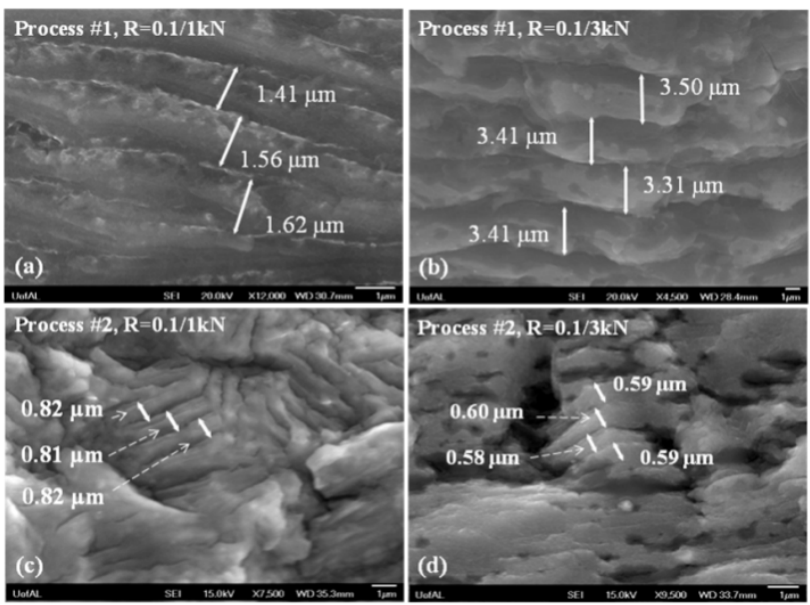

Figure 50. Scanning electron microscope images of striation spacing for: (a) process \#1 coupons tested at maximum cyclic load of $1 \mathrm{kN}$, (b) process \#1 coupons tested at maximum cyclic load of $3 \mathrm{kN}$, (c) process \#2 coupons tested at maximum cyclic load of $1 \mathrm{kN}$, and (d) process \#2 coupons tested at maximum cyclic load of $3 \mathrm{kN}$. Each of these coupons were tested at $\mathrm{R}=0.3$.

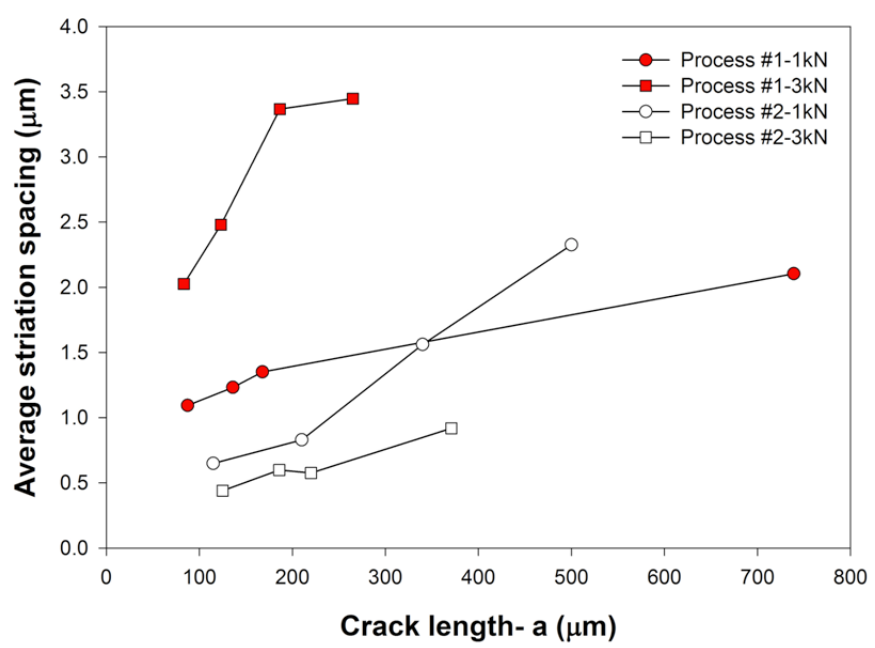

Figure 51. Fatigue crack growth rate in coupons of process \#1 and process \#2

\section{Fracture Mechanics Fatigue Model}

As shown in the previous sections, the fatigue life and failure mode of FSSW coupons are dependent on process conditions. Thus, the objective here is to employ a simple fatigue model to aid in evaluating what effect the hook height, sheet thickness, and nugget diameter have in determining fatigue lifetimes. As such a simple linear elastic fracture mechanics (LEFM) model is employed. The model employed here is based on a kinked crack stress intensity solution, used recently by Jordon et al [28] to predict number of cycles to failure on FSSW on magnesium AZ31 alloy. Since the model provided good correlation to experimental results and since the model is a mechanism based approach, the use of it 
here can be used to possibly determine the sensitivity of microstructural and geometrical features on durability of FSSW.

While the model is detailed elsewhere [28], for clarity purposes, the model is also presented here. The main assumption of the model is that crack growth comprises the majority of fatigue life during cyclic loading. The basic approach employed here is to use a modified Paris law, which is based on a stress intensity factor, to calculate the number of cycles needed to grow the crack from the tip of the interfacial hook to the free surface of the spot weld. Since a stress intensity solution for the FSSW is not currently available, we used a method similar to $[17,29]$, in which a kinked crack stress intensity solution for RSW is employed. Equation (1) and (2) are the mode I and mode II global stress intensity solutions for a RSW lap-joint coupon crack under tensile loading [30]

$K_{I}=\frac{P}{r^{3 / 2}}\left[0.341\left(\frac{2 r}{t}\right)^{0.397}\right]$

$K_{I I}=\frac{P}{r^{3 / 2}}\left[0.282+0.162\left(\frac{2 r}{t}\right)^{0.710}\right]$

$k_{I}=\frac{K_{I}}{4}\left[3 \cos \left(\frac{\theta}{2}\right)+\cos \left(\frac{3 \theta}{2}\right)\right]-\frac{K_{I I}}{4}\left[3 \sin \left(\frac{\theta}{2}\right)+3 \sin \left(\frac{3 \theta}{2}\right)\right]$

$k_{I I}=\frac{K_{I}}{4}\left[\sin \left(\frac{\theta}{2}\right)+\sin \left(\frac{3 \theta}{2}\right)\right]+\frac{K_{I I}}{4}\left[\cos \left(\frac{\theta}{2}\right)+3 \cos \left(\frac{3 \theta}{2}\right)\right]$

$k_{e q}=\sqrt{k_{I}^{2}+k_{I I}^{2}}$

where $P$ is the load, $r$ is the weld radius, and $t$ is the sheet thickness. Since the interfacial hook between the two materials as shown in Fig. 3 is similar to a kinked crack, we can relate the mode I and mode II global stress intensity values to local stress intensity values as a function of kinked crack angle, $\theta$ [21], as shown in equations (3) and (4). In order accommodate the combined loading of mode I and II due to the lap-shear configuration of the coupon, and an equivalent mode I stress intensity [31] is employed (equation 5). We acknowledge that the use of the FSSW stress intensity solution is based on the assumption that the interfacial hook acts like the kinked crack and has an initial crack length equal to the height of the interfacial hook. The other main assumption is that the stress intensity factor remains constant as a function of crack growth. The relationship of crack growth rate to stress intensity is given in the Paris law as shown in Eq. (6)

$\frac{d a}{d N}=C\left(\Delta k_{e q}\right)^{m}$

where $C$ and $m$ are constants derived from crack growth experiments [17], and $\Delta \mathrm{k}_{\mathrm{eq}}$ is the equivalent stress intensity range. The number of cycles is obtained by integrating Eq. (6), which gives 
$N_{\text {total }}=\frac{t-h}{C \sin \theta}\left[\Delta k_{e q}\right]^{-m}$

Since the fatigue crack will align to the maximum principle direction and grow at some angle, $\theta, \frac{t}{\sin \theta}$ is substituted for the crack length, $a$ in Eq. (6), where $t$ is the sheet thickness in the weld zone and not the nominal sheet thickness, and $h$ is the height of the interfacial hook.

Figure 52 and 53 compare the experiments and the model for the number of cycles for through-crack initiation versus applied maximum applied load for $\mathrm{R}=0.3$ and $\mathrm{R}=0.7$, respectively. Thus, the only differences in the model predictions between \#1 and process \#2 were the sheet thickness, nugget diameter, and hook height, where each of these model parameters were based on results from Table 5. The crack growth constants $C$ and $M$ were taken from crack growth experiments on base material conducted elsewhere [17]. The model showed good correlation to capture the differences in fatigue lifetimes for the two processes. However, the model was slightly non-conservative compared to process $\# 1$ for $\mathrm{R}=0.7$ and slightly conservative for process \#2 at $\mathrm{R}=0.3$.

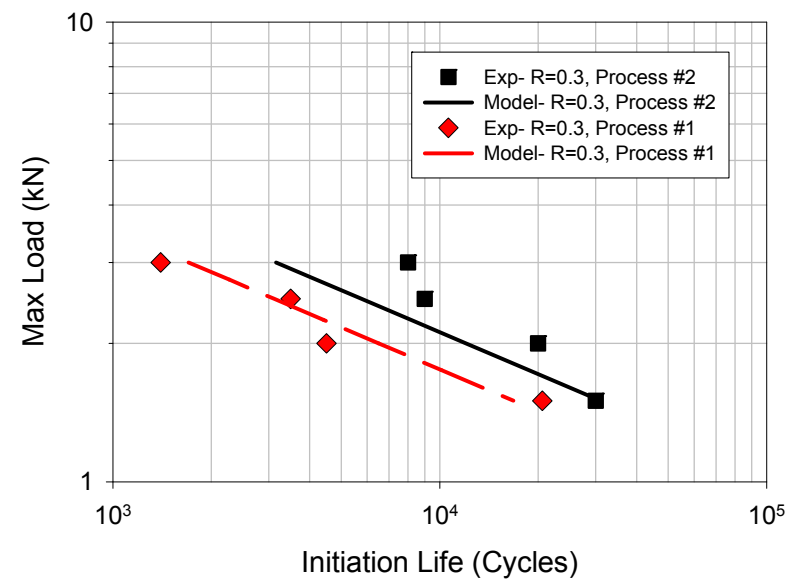

Fig. 52. Comparison of the fatigue model to the experimental results of the fatigue initiation life versus maximum load for $\mathrm{R}=0.3$

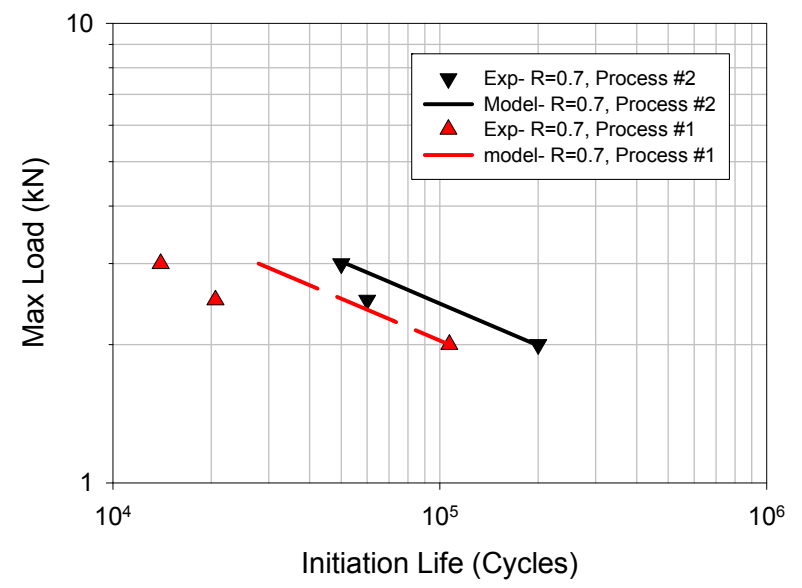


Fig. 53. Comparison of the fatigue model to the experimental results of the fatigue initiation life versus maximum load for $\mathrm{R}=0.3$.

The correlation of the fatigue model to the experimental results shown in Fig. 52 and 53 suggests that a linear elastic fracture mechanics approach can be used to determine the microstructural and geometrical features for optimal fatigue resistance. Despite the fact that process \#2 had a higher hookto-thickness ratio, the fracture mechanics model predicted better fatigue lifetimes. The distance from the tip of the secondary hook to the top of the sheet in process \#2 was only about $20 \%$ more than the distance from the tip of the primary hook in process \#1. This difference is not significant enough to increase the fatigue life, which in some cases, by an order of magnitude. However, if we consider the global stress intensity solution presented in Equations 1 and 2, we see that the thickness, $t$, has an exponential effect on the computed value.

In an attempt to determine the parameter that has the greatest impact on fatigue life, three sets of calculations were conducted using the fracture mechanics model. Three set of calculations were conducted to determine the effect of sheet thickness, interfacial hook heights, and nugget diameters on fatigue life. From the results of the calculations, it was clear that sheet thickness has the largest impact on fatigue lifetimes. While the hook height also has a large impact on fatigue lifetimes, its impact was not as great as the sheet thickness. It is important to note that the range of each welding parameter was chosen based on realistic values that would be expected using the FSSW tooling employed in this study.

\section{Multi Stage Fatigue Model Adaption for FSSW}

The multistage fatigue (MSF) model is a microstructure-sensitive model that considers the various experimentally observed stages of fatigue damage evolution such as crack incubation, microstructurally small crack (MSC), physically small crack (PSC), and long crack growth [1]. While the model was originally developed for a cast A356 Al alloy, it has been modified to extend its application to other materials and other processing methods (e.g. processing of wrought materials [2-3]. The MSF model is based on three distinct stages of fatigue damage, where total fatigue life $N_{\text {Total }}$ is given by

$$
N_{\text {Total }}=N_{\text {Inc }}+N_{M S C / P S C}+N_{L C}
$$

where $N_{\text {Inc }}$ is the number of cycles to incubate a crack near or at a micro-notch that includes the nucleation of crack-like damage and early crack propagation through the zone of the micronotch's root influence [1]. The $N_{M S C / P S C}$ term is the number of cycles required for propagation of a microstructurally small crack (MSC) and physically small crack (PSC), and $N_{L C}$ is the number of cycles required for long crack propagation.

Crack incubation involves nucleation plus small crack growth at the micronotch of the order of $1 / 2 D$, where $D$ is the micronotch size. Small crack growth (MSC) comprises propagation of microstructural cracks with lengths that fall within $a_{i}<a<k M S$, where $M S$ is a characteristic length scale (defined as the smallest grain size) of interaction with microstructural (MS) features, and $k$ is a multiplier where $1 \leq k \leq 3$ 
[1]. The PSC range consists of crack propagation with a crack length in the interval of $k M S<a<\sim 10 M S)$. Depending on the micronotch and texture of the matrix, the PSC regime may extend from $300 \mu \mathrm{m}$ to 2$3 \mathrm{~mm}$ in length.

\section{Crack Incubation Regime}

The fatigue damage incubation life, $N_{\text {inc }}$ is characterized by the cyclic damage at the micronotch root and takes on a modified Coffin-Manson law form [1] in the microscale as follows:

$C_{\mathrm{inc}} N_{\mathrm{inc}}^{\alpha}=\beta=\frac{\Delta \gamma_{\max }{ }^{p *}}{2}$

where $\beta$ is the nonlocal damage parameter around a micronotch, and $C_{\text {inc }}$ and $\alpha$ represent the linear coefficient and exponent, respectively in a modified Coffin-Mason approach for the fatigue crack incubation life. The numerical value of the exponent $\alpha$ was selected to be in the range of the macroscopic Coffin-Mason law [1]. The coefficient in the Coffin-Manson equation, $C_{\mathrm{inc}}=c_{n}+$ $z\left(c_{m}-c_{n}\right)$, is modified to include a coefficient for nucleation of small cracks at micronotch in the high cycle regime $\left(c_{n}\right)$ and a coefficient for the low cycle regime $\left(c_{m}\right)$. We further note that $z=$ $(1 / 0.7)\left\langle\frac{l}{D}-0.3\right\rangle$ is a localization multiplier [1] that is nonzero below the microplasticity percolation limit. Just beyond the percolation limit, the localization multiplier transitions to unity as the plastic shear strain localizes interdendritically [1]. Above that point, incubation is negligible because of the high strain level that localizes at the micronotch [1]. Note that the ratio $\frac{l}{D}=\sqrt{\frac{A_{\text {plastic zone }}}{A_{\text {notchroot }}}}$ is defined as the square root of the ratio of the plastic zone over the micronotch root area. We further note that $\frac{l}{D}$ ratio is typically determined through micro-mechanical simulations. In this study, however, no such simulations were available, and as such, $C_{i n c}$ is assumed to be constant for both the high cycle and low cycle regime. The $\beta$ parameter is related to the local average maximum plastic shear strain amplitude, $\frac{\Delta \gamma_{\max }{ }^{p *}}{2}$, and is estimated by the following relations:

$\beta=\frac{\Delta \gamma_{\max }{ }^{p *}}{2}=Y 100\left[\varepsilon_{a}-0.00025(1-R)\right]^{X}$

Here, $\varepsilon_{a}$ is the remote applied strain amplitude, the parameter $Y$ [1] is correlated as $Y=y_{1}+(1+$ $R) y_{2}$, where $R$ is the load ratio, and $y_{1}, y_{2, x}$ are model constants. For completely reversed loading cases, $Y=y_{1}$. 


\section{Small Crack Growth}

Similar to modeling efforts in wrought aluminum alloys, we combine the mathematical expressions for the MSC and PSC regimes into a single mathematical form. Crack growth in the MSC/PSC is governed by the range of crack tip displacement, $\triangle C T D$, which is proportional to the crack length, and the $\mathrm{n}^{\text {th }}$ power of the applied stress amplitude, $\sigma_{a}^{n}$, in the high cycle fatigue (HCF) regime and to the macroscopic plastic shear strain range, $\frac{\Delta \gamma_{\max }^{P}}{2}$, in the low cycle fatigue (LCF), and it is given by the following,

$\left(\frac{d a}{d N}\right)_{M S C}=\chi\left(\Delta C T D-\Delta C T D_{t h}\right)$,

$\Delta C T D=C_{I I}\left(\frac{G S}{G S_{0}}\right)^{\omega}\left[\frac{U \Delta \widehat{\sigma}}{S_{u t}}\right]^{\zeta} a_{i}+C_{I}\left(\frac{G S}{G S_{0}}\right)^{\omega}\left(\frac{\Delta \gamma_{\max }^{P}}{2}\right)^{2}$

Here, $\chi$ is a constant for a given microstructure, typically less than unity and usually taken as 0.32 for several different alloys [1-3], and $a_{i}$ is the initial crack length. The $\mathrm{C}_{\mathrm{I}}$ and $\mathrm{C}_{\mathrm{II}}$, and $\zeta$ are material dependent parameters which capture the microstructural effects on MSC growth [1]. The threshold value for crack tip displacement was defined on the order of the Burger's vector for the Mg-rich matrix [7], $\Delta C T D_{t h}=3.2 * 10^{-4} \mu \mathrm{m}$. The term $\Delta \hat{\sigma}$ is the combination of the uniaxial effective stress amplitude, $\bar{\sigma}_{a}=\sqrt{\frac{3}{2} \frac{\Delta \sigma_{i j}^{\prime}}{2} \frac{\Delta \sigma_{i j}^{\prime}}{2}}$, and the maximum principal stress range, $\Delta \sigma_{1}$, and is given as $\Delta \hat{\sigma}=2 \theta \bar{\sigma}_{a}+(1-$ $\theta) \Delta \sigma_{1}$, with $0 \leq \theta \leq 1$ as the path dependent loading parameter after [1], where $\theta$ is a weighting parameter such that $\theta=0$ gives the von Mises stress state, and $\theta=1$ gives the maximum for principal stress state. The parameter $U$ is employed to capture the load ratio effects and is defined as $U=\frac{1}{1-R}$ [1]. In order to capture the effect of grain size on small crack growth, we employ the ratio of grain size to the reference size $\left(\frac{G S}{G S_{0}}\right)^{\omega}$ for each of the three materials in this study, where $G S_{0}$ is the reference grain size, $G S$ is the specific grain size, and $\omega$ is a material parameter [3].

Long Crack Growth

Linear elastic fracture mechanics is used to model long crack growth in the FSSW coupons. The general expression of the number of cycles were calculated using the relation

$\left(\frac{d a}{d N}\right)_{L C}=C\left((\Delta K)^{M}-\left(\Delta K_{t h}\right)^{M}\right)$

where for $\mathrm{Mg} \mathrm{AZ31,} M \sim 3.68$ and $C \sim 3 \times 10^{-10}$ [32] and the intrinsic threshold is given by $\Delta K_{\mathrm{th}} \sim 0.91$ $\mathrm{MPa} \sqrt{m}$ for Mg AZ31 [33], as determined from the threshold for long cracks with grains in longitudinal 
direction. We assume the long crack grows once the MSC/PSC reaches the free surface of the coupon and/or a threshold length of $2 \mathrm{~mm}$. Since final separation of the joint in the high cycle fatigue (HCF) regime for both processes occurs through the sheet and not the weld, we assume the following stress intensity solution [34]:

$K_{\text {eff }}=\sigma \sqrt{\pi a} \cdot F\left(\frac{a}{b}\right)$

where $\sigma$ is the applied stress, $a$ is the crack length at full separation, $b$ is half the width of the specimen. For process\#1 and process\#2 in the low cycle fatigue (LCF) regime, the coupons failed due to shear overload in the nugget. Based on work of Jordon et al. [28] for FSSW joints in Mg AZ31 alloy, the following relation is used to estimate the crack growth rate in LCF:

$K_{I}=\frac{P}{r^{1.5}}\left[0.314\left(\frac{2 r}{t}\right)^{0.397}\right]$

$K_{I I}=\frac{P}{r^{1.5}}\left[0.282+0.162\left(\frac{2 r}{t}\right)^{0.710}\right]$

$K_{e f f}=\sqrt{\left(K_{I}^{2}+K_{I I}^{2}\right)}$

Where $K_{l}$ and $K_{l /}$ are global stress intensity solutions, $P$ is the load $(\mathrm{N}), r$ is the radius of the weld and $t$ is the thickness of the sheet. Table 6 lists the MSF model parameters employed in this study.

Table 6. Microstructure-sensitive fatigue modeling parameters for AZ31 magnesium alloy

\begin{tabular}{|l|l|l|}
\hline Constant & Parameters & Description \\
\hline$C_{i n c}$ & 9 & Linear coefficient \\
\hline$\alpha$ & -0.65 & Ductility exponent in Modified Coffin Manson Law \\
\hline$X$ & 3 & Exponent in remote strain to local plastic shear strain \\
\hline$E$ & $155(\mathrm{MPa})$ & Elastic Limit \\
\hline$K^{\prime}$ & $494(\mathrm{MPa})$ & Cyclic strain strength coefficient \\
\hline$N^{\prime}$ & 0.14 & Cyclic strain hardening exponent \\
\hline
\end{tabular}




\begin{tabular}{|l|l|l|}
\hline$\chi$ & 0.28 & Crack growth rate constant \\
\hline$C_{I}$ & 0.00007 & HCF constant in small crack growth \\
\hline$C_{I I}$ & 0.002 & LCF constant in small crack growth \\
\hline$\omega$ & 2 & Omega \\
\hline$\zeta$ & 1.2 & Exponent in Small crack growth \\
\hline$t$ & $2(\mathrm{~mm})$ & Sheet thickness \\
\hline
\end{tabular}

\section{Multi Stage Fatigue Modeling Results and Discussion}

The MSF model used in this current study for the FSSW joints is an adaptation from the model developed for base materials. The modifications and assumptions were made to closely relate conditions in the FSSW joints that were experimentally observed in the previous section. Figure 54 shows the correlation of the MSF model to the experimental load-life results for the FSSW Mg AZ31 alloy for the two sets of processes. It is important to note that the model correlated to the distinct failure modes as listed in Table III. Furthermore, the MSF employed the three distinct stages of fatigue damage: incubation, small crack (MSC/PSC)) and long crack growth (LC) to capture the long crack growth.

The primary inputs to the model include the load amplitude, load ratio, weld geometry such as the hook height and weld radius. The remaining constants are based on assumptions and micromechanical simulations and other accepted values pertaining to microstructure used prior MSF work [1-3]. However, we note that future work is needed to validate some of the model parameters, in particularly the incubation and small crack growth parameters. In fact, as mentioned earlier, micromechanical simulations are planned to validate the parameters employed in this study. However, the results of the MSF correlation to the experimental results suggest that the model framework is well adapted to predicting the fatigue damage in FSSW joints. 


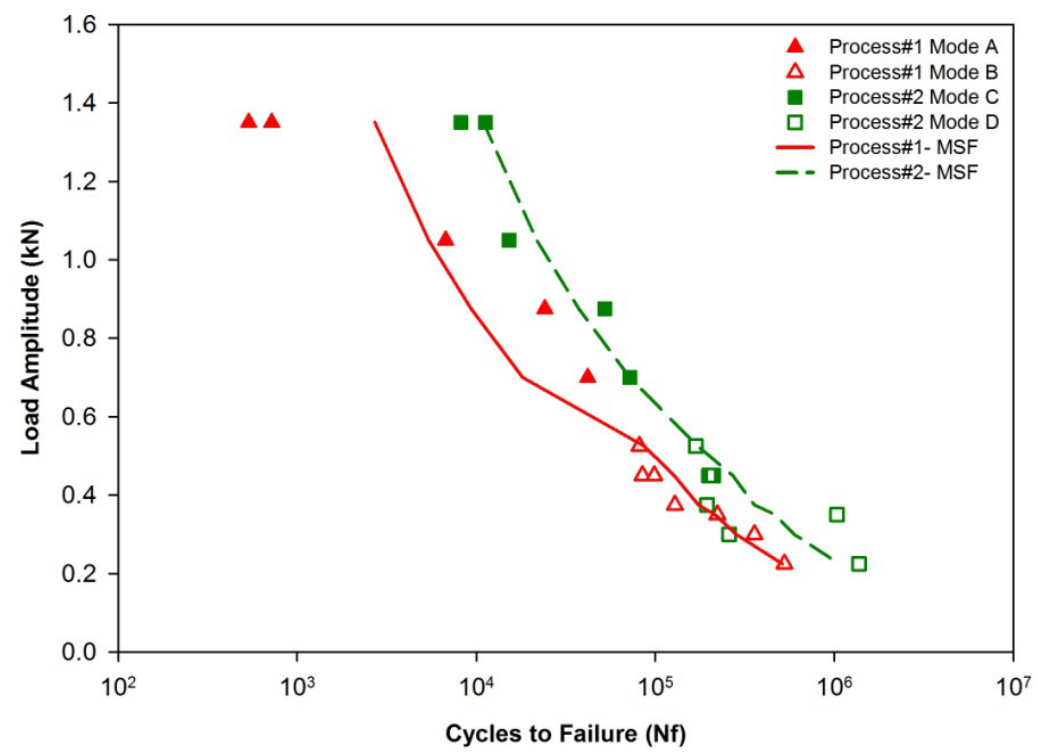

Figure 54. A comparison of experimental fatigue life results with crack growth model prediction of fatigue life of FSSW Mg AZ31 alloy.

\section{FSSW Conclusions}

In this study, the influence of microstructural and geometrical features on fatigue lifetimes are evaluated on two different sets of friction stir spot welded lap-shear coupons. Several differences in the initial state of the weld structure including microstructure, size and shape of the interfacial hooks and the effective top sheet thickness were observed between the two processes. Fractography analysis conducted in this study suggests that the microstructure and effective top sheet thickness largely determine the failure mode, which in turn influence fatigue failure. While the height of the interfacial hook was greater in the process with better fatigue performance, it was the larger effective top sheet thickness that promoted crack propagation modes more favorable to greater fatigue resistance. As such, the shoulder plunge depth during the friction stir welding process may likely be the dominant design factor in producing durable spot welds using this joining technique. In addition, the linear elastic fracture mechanics model showed good correlation in predicting the difference in fatigue life of FSSW for the two processes. Furthermore, the sensitivity study using the linear elastic fracture model showed that the sheet thickness had the most impact on fatigue in the FSSW, followed by the height of the interfacial hook, and lastly nugget diameter. Lastly, the formulation of a multi-stage fatigue (MSF) model for friction stir spot welding in $\mathrm{Mg}$ alloy was presented. This model not previously applied to joints, was adapted to the lap-shear spot welded coupon to capture the incubation, small crack, and long crack growth stages observed experimentally. In general, the MSF model showed good correlation to experimental results. In particular, the model correlated the differences in process conditions employed in fabricating the lap-shear coupons and their effect on the number of cycles to failure. This is a significant development since the results presented here suggest that the MSF model can be used to further elucidate the effect of a particular welding process and loading condition on fatigue lifetimes and help design future friction stir welds based on fatigue requirements. 


\section{Main Conclusions}

Next the main conclusions for the Fatigue Performance of lightweight Automotive Materials are listed:

1. Fatigue of base magnesium alloys (AM30, AZ31 and AZ61):

- Cyclic response showed an asymmetric pattern for all alloys and the observed strain amplitudes. The asymmetry was more emphatic for the first cycles of the cyclic process.

- Twining in compression and detwining in tension occurred during cyclic loading for all the extruded alloys AZ31 Mg alloys except for the sheet material.

- For AM30 Mg Alloy (Ford rail), compression twinning and detwinning were observed in the hysteresis loops for the extrusion direction. Conversely, the hysteresis loops for the extrusion transverse direction showed tensile twinning and detwinning.

- Strain hardening was observed for all the strain amplitudes but more significant for the higher strain levels for an Exruded AM30 Mg Alloys.

- Mean stress was higher for extruded samples when compared to the transverse direction and AZ31 sheet material.

- The experimental results shown differences in the fatigue response between the transverse and the extruded direction of the applied load.

- Observations of the fracture surfaces under scanning electron microscopy revealed that fractured intermetallic particles initiated fatigue cracks in all three materials. The crack initiation sites were found to occur more frequently at inclusions near the free surface of the specimens for all the materials. However, crack initiation sites were located at regions where profuse twinning was observed.

- Particle size was more important in determining the number cycles than the anisotropy from the texture for magnesium alloys.

2. Multistage fatigue modeling of AM30, AZ31, and AZ61 wrought Mg alloys

- The MultiStage Fatigue (MSF) model was adapted to capture the influences of the microstructural characteristics and influences of the extruded alloy. As such, the MSF model was able to predict the scatter of the fatigue results with good agreement for all the magnesium alloys.

- The multistage fatigue model incorporated the differences in microstructure including crystallographic orientation, grain size, particle size, and cyclic hardening parameters in order to capture the differences in fatigue behavior. 
3. Strain-based Approach

- Strain-life fatigue parameters (SLF) were determined for AM30 and AZ31 Mg alloys. The CoffinManson equation shown a fairly correlation to the experimental data.

4. Molecular Dynamics Simulations of Magnesium:

- Simulations were performed on magnesium crystals under cyclic loading.

- Simulations results showed that growth of the crack was affected by the crystal orientation and temperature.

5. Monotonic and cyclic testing for different casting processes

- Inclusion size and pore shrinkage were the dominate causes of fatigue incubation in the 5 casting processes examined

6. Small Fatigue Crack Growth Observations in an Extruded Magnesium Alloy

- The crack growth rates for the microstructurally small crack regime could be quantified by using the replica process shown here.

- Shorter iterations for taking replicas need to be implemented in future studies in order to more accurately ascertain the fatigue life for incubation-microstructurally small crack transitions.

7. Microstructure and Damage Evolution during Tensile Loading in a AZ61 Magnesium Alloy

- The microstructural damage evolution for an AZ61 magnesium alloy was evaluated. Three types of particles were identified: Oxide/intermetallic and $\mathrm{Mn}-\mathrm{Al}$, and $\mathrm{Mg}-\mathrm{Al}$ phases.

- Under uniaxial tension the area fraction of cracked particles composed of (Mn-Al) and (Mg-Al) varies in an exponential pattern as function of strain. .

- The Horstemeyer and Gokhale damage nucleation model was in good agreement with the observed damage progression of the AZ61 magnesium alloy.

8. Fatigue Modeling of AZ91 and AM60 Magnesium Alloys

- The Multistage fatigue model was correlated to the experimental strain-life results of the AM60 as-cast, AZ91 as-cast, and the AZ91 T6 magnesium alloys. The mean fits of the model were based microstructure features such as dendrite cell size, pore size, and cyclic hardening parameters in order to capture the differences in fatigue behavior of the three data sets.

- The Multistage fatigue model showed good correlation of the upper and lower bounds of the strain-life behavior of the AM60 and AZ91 magnesium alloys by employing the maximum and minimum inclusion and dendrite cell sizes. Thus, the inclusion (pore) size had the most 
influence on the scatter in the fatigue lifetimes. We note that the other material constants were not changed for the estimation of the upper and lower bounds.

9. Validation of ISV/MSF models with components specified by sponsors

- This study has revealed the power of microstructure based models to predict fatigue failure in design.

- Fatigue failure location is dependent upon stress, and mechanical and microstructure properties such as pore size, dendrite cell size.

- The finite element simulations coupled with the MSF model shown a good correlation to the experimental tests.

- This investigation has shown that fatigue failure of a component must be studied based on an integral approach that involves jointly the material properties, geometry and loading conditions.

10. Fatigue of Friction Stir Spot Welding (FSSW) in Magnesium Alloys

- Two competing factors were identified to influence the performance of the FSSW joints: (1) the hook height, and (2) the thickness.

- The linear elastic fracture mechanics model employed was able to account for the competing factors.

\section{Presentations/Publications/Patents}

1. M. Lugo, J. B. Jordon, L. Hector, K. Solanki, J.D. Bernard, A.A. Luo, and M. F. Horstemeyer "Role of Different Materials Processing Methods on the Fatigue Behavior of AZ31 Magnesium Alloy", International Journal of Fatigue, (Under review).

2. M. Lugo, J. B. Jordon, J.D. Bernard, M. F. Horstemeyer, "Microstructure-Sensitive Fatigue Modeling of an Extruded AM30 Magnesium Alloy", SAE 2013 World Congress, (Submitted).

3. M. Lugo, J. B. Jordon, L. Hector, K. Solanki, J.D. Bernard, A.A. Luo, and M. F. Horstemeyer "The Effects of Different Materials Processing Methods on the Fatigue Behavior of AZ31 Magnesium Alloy", ASME 2012 International Mechanical Engineering Congress \& Exposition, Houston, TX, 2012.

4. J.B. Jordon, J.B. Gibson, M.F. Horstemeyer, H. El Kadiri, J.C. Baird, "Experiments and Modeling of Fatigue Damage in Extruded Mg AZ61 Alloy." TMS 2011 (The Minerals, Metals \& Materials Society).

5. J.D. Bernard, J.B. Jordon, M.F. Horstemeyer, "Small Fatigue Crack Growth Observations in a Wrought Magnesium Alloy" TMS 2011 (The Minerals, Metals \& Materials Society). 
6. M. Lugo, B. Jordon, M. F. Horstemeyer, and M. A. Tschopp, "Microstructural relationship in the damage evolution process of an AZ61 magnesium alloy", TMS 2011 (The Minerals, Metals \& Materials Society).

7. T. Tang, D. Wang, J. B. Jordon, and M. F. Horstemeyer, "Stress Intensity Factor Solutions for Friction Stir Spot Welds of Magnesium AZ31 Alloy, TMS 2011 (The Minerals, Metals \& Materials Society).

8. H. Badarinarayan, S.B. Behravesh, S.D. Bhole, D.L. Chen, J. Grantham, M.F. Horstemeyer, H. Jahed, J.B. Jordon, S. Lambert, H.A. Patel, X. Su, and Y. Yang, "Monotonic and Fatigue Behavior of Mg AZ31 in Friction Stir Spot Welds: An International Benchmark Test in the "Magnesium Front End Research and Development Project," TMS 2011 (The Minerals, Metals \& Materials Society).

9. M. Lugo, M. A. Tschopp, J. B. Jordon, and M. F. Horstemeyer, "Microstructure and Damage Evolution during Tensile Loading of AZ61 Magnesium Alloy", Scripta Materialia, 64, pp. 912-915, 2011.

10. T. Tang, S. Kim, M. F. Horstemeyer, and P. Wang, "Atomistic modeling of crack growth in Magnesium single crystal", Engineering Fracture Mechanics, 78, Issue 1, pp. 191-201, 2011.

11. J.B. Jordon, J.B. Gibson, M.F. Horstemeyer, H. El Kadiri, J.C. Baird, A.A. Luo, "Effect of Twinning, Slip, and Inclusions on the Fatigue Anisotropy of Extrusion-textured AZ61 Magnesium Alloy, Material Science Eng A, 528, p 6860-6871, 2011.

12. J.D. Bernard, J.B. Jordon, M.F. Horstemeyer, , J. Baird, D. Lamb, and A.A. Luo "Structure-Property Relations of Cyclic Damage in a Wrought Magnesium Alloy", Scripta Materialia, 63, pp. 751-756, 2010.

13. J.B. Jordon, M.F. Horstemeyer, S.R. Daniewicz, H. Badarinarayan, and J. Grantham, "Fatigue Characterization and Modeling of Friction Stir Spot Welds in Magnesium AZ31 Alloy," Journal of Engineering Materials and Technology, 132, 2010.

14. Y. Xue, M. Lugo, A. Pascu, and M. F. Horstemeyer, "Fatigue Mechanism and Multistage Fatigue Modeling for Wrought Mg-3Al-1Zn", TMS (The Minerals, Metals \& Materials Society) 2009, San Francisco, CA, 2009.

15. Y. Xue, H. El Kadiri, M. F. Horstemeyer, M. Lugo, and A. Pasco, "Cyclic Plasticity, Fatigue Mechanism, and Multistage Fatigue Modeling for Wrought Mg-3Al-1Zn", 2009 ASME International Mechanical Engineering Congress and Exposition, Lake Buena Vista, Florida, November 13-19, 2009

16. Y. Xue, M. Lugo, M. F. Horstemeyer, and J. Newman, Jr., Multistage Fatigue Model for an Extruded AZ31 Mg alloy, TMS Annual Conference, New Orleans, LA, March 9-13, 2008. 


\section{REFERENCES}

[1] D.L. McDowell, Ken Gall, M.F. Horstemeyer, and J. Fan. Microstructure-Based Fatigue Modeling of Cast A356-T6 Alloy. Engineering Fracture Mechanics, Vol. 70, pp. 49-80, 2003.

[2] Y. Xue, D.L. McDowell, M.F. Horstemeyer, M.H. Dale, and J.B. Jordon. Microstructure-based Multisatge Fatigue Modeling of Aluminum Alloy 7075-T651, Engineering Fracture Mechanics, Vol. 74, Issue 17, pp. 2810-2823, 2007.

[3] J.B. Jordon, J. Gibson, M.F. Horstemeyer, H. El Kadiri, J. Baird, and A.A. Luo. Effect of Twinning, Slip, and Inclusions on the Fatigue Anisotropy of Extrusion-Textured AZ61 Magnesium Alloy, Materials Science and Engineering A, Vol. 528, pp. 6860- 6871, 2011.

[4] K. Gall, M.F. Horstemeyer, D.L. McDowell, and J. Fan. Finite Element Analysis of the stress distributions Near Damaged Si Particles Clusters in Case Al-Si Alloys. Mechanics of Materials, Vol. 32, pp. 277-301, 2000.

[5] D.R. Hayhurst, F.A. Leckie, and D. L. McDowell. Damage Growth under Nonproportional Loading. ASTM STP 853, Vol. Multiaxial Fatigue, pp. 553-558, 1985.

[6] D.W. Brown, A. Jain, S.R. Agnew, and B. Clausen. Twinning and Detwinning During Cyclic Deformation, Materials Science Forum, Vol. 539-543, pp. 3407-3414, 2007.

[7] S. Groh, E.B. Marin, M.F. Horstemeyer,and D.J. Bammann. Dislocation Motion in Magnesium: A Study by Molecular Statistics and Molecular Dynamics, Modelling and Simulation in Materials Science and Engineering, Vol. 17, pp. 1-15, 2009.

[8] D.R. Hayhurst, F.A. Leckie D.L. McDowell, Damage growth under nonproportional loading. ASTM STP, pp. 688-99,1985

[9] L.H. Rettberg, J.B. Jordon, M.F. Horstemeyer, J.W. Jones. Low-cycle fatigue behavior of die-cast Mg Alloys AZ91 and AM60, Metallurgical and Materials Transactions A, 43A, pp. 2260-2274, 2012.

[10] Short-Crack Growth Behaviour in an Aluminum Alloy - an AGARD Cooperative Test Programme, AGARD R-732, 1988.

[11] Short-Crack Growth Behaviour in Various Aircraft Materials, AGARD R-767, 1990.

[12] Newman, J.C., Jr., Wu, X., Venneri, S. and Li, C., "Small-Crack Effects in High-Strength Aluminum Alloys," NASA RP-1309, May 1994.

[13] Newman, J.C., Jr., "A Crack-Closure Model for Predicting Fatigue Crack Growth Under Aircraft Spectrum Loading," ASTM STP-748, 1981, pp. 53-84. 
[14] S. Begum, D.L Chen, S. Xu, and Alan Luo. Strain-Controlled Low-Cycle Fatigue Properties of a Newly Developed Magnesium Alloy, Metallurgical and Materials Transactions A, Vol. 39A, pp. 3014-3026, 2008.

[15] C.L. Fan, D.L. Chen, A. A. Luo, "Dependence of the distribution of deformation twins on strain amplitudes in an extruded magnesium alloy after cyclic deformation", Materials Science and Engineering A, pp. 38-45, 2009.

[16] Modelling of active slip systems, Taylor factors and grain rotations during rolling and compression deformation of polycrystalline intermetallic $\mathrm{LI}_{2}$ compounds

[17] K. Tokaji, M. Kamakura, Y. Ishiizumi, N. Hasegawa, "Fatigue Behavior and Fracture Mechanism of a Rolled AZ31 Magnesium Alloy," International Journal of Fatigue, 26 (11) (2004) 1217-1224.

[18] J.B. Jordon, J.D. Bernard, J.C. Newman Jr., "Quantifying Microstructurally Small Fatigue Crack Growth in an Aluminum Alloy Using a Silicon-Rubber Replica Method," International Journal of Fatigue, 36 (1) (2012) 206-210.

[19] J-Y. Buffière, S. Savelli, P.H. Jouneau, E. Maire, R. Fougères, "Experimental Study of Porosity and Its Relation to Fatigue Mechanisms of Model Al-Si7-Mg0.3 Cast Al Alloys," Materials Science and Engineering, A316 (2001) 115-126.

[20] K. Gall, G. Biallas, Hans J. Maier, P. Gullett, M. F. Horstemeyer, D. L. McDowell, and J. Fan, "InSitu Observations of High Cycle Fatigue Mechanisms in Cast AM60B Magnesium in Vacuum and Water Vapor Environments," International Journal of Fatigue, 26 (2004) 59-70.

[21] M.F. Horstemeyer, and A.M. Gokhale, 1999, A Void nucleation model for ductile metals, International Journal of Solids and structures, 36, p 5029-5055.

[22] B. Cotterell, and J.R. Rice, 1980, Slightly curved or kinked cracks, International Journal of Fracture 16, p 155-169.

[23] R.I. Stephens, A. Fatemi, R.R. Stephens, and H.O. Fucks. Metal Fatigue in Engineering, $2^{\text {nd }}$ Edition, Wiley-Interscience, 2000.

[24] J.A. Bannantine, J.J. Comer, and J. L. Handrock. Fundamentals of Metal Fatigue Analysis, Prentice Hall, 1990.

[25] G. E. Dieter. Mechanical Metallurgy, McGraw-Hill, Third Edition, 1986.

[26] C.I. Chang, C.J. Lee, J.C. Huang, "Relationship between grain size and Zener-Holloman parameter during friction stir processing in AZ31 Mg alloys," Scripta Materialia, 51 (2004) 509-514.

[27] D.A. Wang, and C.H. Chen, "Fatigue Lives of Friction Stir Spot Welds in Aluminum 6061-T6 Sheets," Journal of Materials Processing Technology, 209 (2009), 367-375. 
[28] J.B. Jordon, M.F. Horstemeyer, S.R. Daniewicz, H. Badarinarayan, and J. Grantham, "Fatigue Characterization and Modeling of Friction Stir Spot Welds in Magnesium AZ31 Alloy," Journal of Engineering Materials and Technology, 132 (2010), 041008-1-10.

[29] P.C. Lin, J. Pan, and T. Pan, "Failure Modes and Fatigue Life Estimations of Spot Friction Welds in Lap-Shear Specimens of Aluminum 6111-T4 Sheets. Part 1: Welds Made by a Flat Tool," International Journal of Fatigue, 30 (2008), 74-89.

[30] Pook, L.P., "Fracture Mechanics Analysis of the Fatigue Behavior of Spot Welds," Int. J. Fracture, 11, (1975) pp. 173-176.

[31] Biner, S.B., 2001, "Fatigue Crack Growth Studies Under Mixed-Mode Loading," Int. J. Fatigue, 23, (2001) pp.259-263.

[32] Sabrina Alam Khan, Yukio Miyashita, Yoshiharu Mutoh, Zainuddin Bin Sajuri, "Influence of Mn content on mechanical properties and fatigue behavior of extruded Mg alloys," Materials Science and Engineering A 420 (2006) 315-32.

[33] Zainuddin Bin Sajuri, Yukio Miyashita, Yasunobu Hosokai, Yoshiharu Mutoh, "Effects of Mn content and texture on fatigue properties of as-cast and extruded AZ61 magnesium alloys," International Journal of Mechanical Sciences 48 (2006) 198-209

[34] Hiroshi Tada, Paul Paris and George Irwin, The Stress Analysis of Cracks Handbook (New York, NY: ASME, 2000), 40.

[35] M. F. Horstemeyer, J. Lathrop, A.M. Gokhale, M. Dighe, "Modeling Stress State Dependent Damage Evolution in a Cast Al-Si-Mg Aluminum Alloy", Theoretical and Applied Fracture Mechanics, Volume 33, pp. 31-47, 2000.

[36] M. F. Horstemeyer, and A. M. Gokhale, "A Void-crack Nucleation Model for Ductile Metals", International Journal of Solids and Structures, Volume 36, pp. 5029-5055, 1999.

[37] H. Agarwal, A. M. Gokhale, M.F. Horstemeyer, and S. Graham, "Quantitative Characterization of Three-dimensional Damage Evolution in a Wrought Al-alloy under Tension and Compression", Metallurgical and Materials Transactions A, Volume 33A, pp. 2599-2606, 2002.

[38] H. Agarwal, A. M. Gokhale, S. Graham, and M. F. Horstemeyer, "Anisotropy of Intermetallic Particle Cracking Damage Evolution in an Al-Mg-Si Base Wrought Aluminum Alloy under Uniaxial Compression", Metallurgical and Materials Transactions A, Volume 33A, pp. 3443-3448, 2002

[39] H. Agarwal, A. M. Gokhale, S. Graham, and M. F. Horstemeyer, "Void Growth in 6061-aluminum Alloy Under Triaxial Stress State ", Materials Science and Engineering A, Volume 342, pp. 35-42, 2003. 


\section{KEY WORDS}

Fatigue, Magnesium Alloys, Structure-Property Relations, Multistage Modeling, Plasticity, Cyclic Damage, Cracks, twinning, Spot Welds, Friction Stir Spot Welds, Finite Element Analysis.

\section{BRIEF DESCRIPTION OF REPORT}

The wrought magnesium alloys, AM30, AZ31, AZ61, and a cast AZ91 alloy provided by our automotive partners, were investigated. Monotonic and cyclic tests programs were conducted to evaluate the mechanical behavior of the magnesium alloys. In addition, experiments and simulations were conduced to quantify structure-property relations with the purpose to develop a microstructuresensitive fatigue model for magnesium alloys. For extruded magnesium alloys, in-depth experimental studies were performed for purpose of calibrating physics-based MultiStage Fatigue (MSF) models. For additional modeling purposes, monotonic damage in Mg AZ61 alloy was characterized and modeled. Experiments were also conducted on cast AZ91 to quantify sources of fatigue initiation. Regarding fatigue performance of joints, relationships of microstructural and geometrical features were investigated using AZ31 magnesium alloy sheets joined by friction stir spot welding (FSSW). 
Subtask 1.4: Multiscale Modeling of Corrosion

Team Members: Holly Martin, M. F. Horstemeyer, C. Walton (GRA), W. Song (GRA)

\section{ObJectives}

- Understand and model the mechanisms of corrosion in Mg alloys

- Understand and model the combined effects of corrosion and mechanical stresses in Mg alloys

\section{APPROACH}

- Experimental data to quantify the mechanism of corrosion and mechanical stresses, including creep and fatigue, in Mg alloys

- Development of a macroscopic model for corrosion.

\section{ACCOMPLISHMENTS}

- Designed and built a creep cradle capable of applying a constant strain on Mg alloy dogbones in two corrosive environments

- Compared the creep-corrosion mechanisms of a $3.5 \% \mathrm{NaCl}$ aqueous solution on extruded AZ31 Mg dogbones using an immersion testing technique and a cyclical salt spray testing technique over 60 hours

- Began comparing the creep-corrosion mechanisms of a 3.5\% $\mathrm{NaCl}$ aqueous solution on extruded AZ61 Mg dogbones using an immersion testing technique and a cyclical salt spray testing technique over 60 hours

- Began determining the fatigue life in air of extruded AM30 Mg dogbones to compare to the fatigue life of extruded $\mathrm{AM} 30 \mathrm{Mg}$ dogbones in a corrosive 3.5\% $\mathrm{NaCl}$ immersion environment

- Determined internal state variables for the corrosion model

- Completed kinematics portion of the corrosion model and are currently working on the thermodynamics and kinetics portions of the model

\section{INTRODUCTION}

Understanding the corrosion mechanisms that ultimately leads to the failure of metals is of utmost importance. Magnesium is currently being investigated for use in the aerospace and automobile industries, but its high corrosion rate relegates it to locations unexposed to the environment (Makar and Kruger (1993); Song and Atrens (2003)). Because of its electrochemical potential as illustrated by the galvanic series, magnesium alloys corrode quickly when exposed to saltwater (Shaw (2003)). 
Furthermore, the presence of rare earth elements, such as cerium, in the eutectic regions surrounding the grains of AE44 can also lead to galvanic corrosion (Alvarez et al. (2010); Bakkle and Westengen (2005)). Understanding the corrosion mechanisms of pitting, intergranular corrosion, and general corrosion could help control corrosion in the future.

The current research was designed to study the effects of a corrosive environment when experienced by magnesium alloys also experiencing mechanical stresses, including creep and fatigue. In order to determine the effects of various alloying elements, without concern for the surface finish at this point, three extruded magnesium alloys were selected: AM30, AZ31, and AZ61. The difference between AM30 and AZ31 is the presence of manganese or zinc, respectively, while the difference between AZ31 and AZ61 is the amount of aluminum present, $3 \%$ versus $6 \%$, respectively. For the creep studies, all samples were exposed to one of two environments - a cyclical salt spray environment or an immersion environment. Ultimately, the data gathered will be used to calibrate an Internal State Variable Model that will be used to predict the corrosion of magnesium alloys. The initial development of the internal state variable model is detailed in Section 2, while the experimental set-up and main results for creep testing and fatigue testing are detailed in Section 3. Concluding remarks are given in Section 4.I

\section{Modeling}

In the development of a corrosion model, while we recognize that hydrogen ions can diffuse into the metallic structure, leading to hydrogen embrittlement (Johnson (1875); Toh and Baldwin (1956); Windle and Smith (1968)), these bulk effects are not being addressed in the initial Internal State Variable model development. Instead, the model currently being developed is focused on the surface effects of pit nucleation, pit growth, and pit coalescence.

\section{Conceptual Model Development}

The initial conceptual model development is based on the work of Horstemeyer et al. (Horstemeyer (2000); Horstemeyer (2003)) and is aimed at describing and uniting the different corrosion mechanisms (general, pitting, and intergranular) in terms of measurable quantities (pit number density, pit area, pit nearest neighbour distance, and volume/mass loss). The initial corrosion model is given as follows:

$\phi=\phi_{G C}+\phi_{P C}+\phi_{I C}$

while the associated rate equation is shown below:

$\dot{\phi}=\dot{\phi}_{G C}+\dot{\phi}_{P C}+\dot{\phi}_{I C}$ 


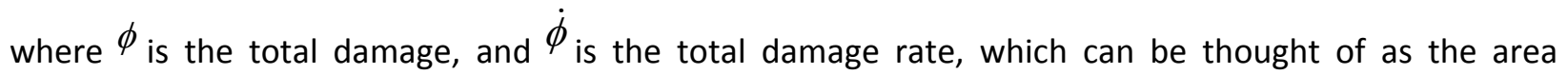
fraction or volume fraction lost. Also, $\phi_{G C}$ is the area or volume fraction lost due to general corrosion while $\dot{\phi}_{G C}$ is the associated general corrosion rate; $\phi_{P C}$ is the area or volume fraction lost due to pitting corrosion while $\dot{\phi}_{P C}$ is the associated pitting corrosion rate; and $\phi_{I C}$ is the area or volume fraction lost due to intergranular corrosion while $\dot{\phi}_{I C}$ is the associated intergranular corrosion rate.

Based on Equations 1 and 2, the general corrosion rate, $\dot{\phi}_{G C}$, is the time rate of change of the volume fraction (or thickness change) related to general corrosion and is chemically a function of a reaction between water and magnesium (no chlorine is involved), so it affects both the grain and eutectic regions. The pitting corrosion rate, $\dot{\phi}_{P C}$, is the time rate of change of the volume fraction related to pitting corrosion where chlorine is required for nucleation, growth, and coalescence, and it affects the grain interior more so than the eutectic region. The intergranular corrosion rate, $\dot{\phi}_{I C}$, is the time rate of change of the volume fraction related to intergranular corrosion and is solely a function of water and a reaction between two dissimilar metals (a micro-galvanic cell) and thus affects the eutectic region more so than the grain interior. Each variable in the corrosion model can be extended, although only the pitting corrosion rate equation will be extended in this work. In this context, pitting corrosion rate is defined as the following:

$\dot{\phi}_{P C}=\dot{\phi}_{\eta_{p}}+\dot{\phi}_{v_{p}}+\dot{\phi}_{c_{p}}$

where $\dot{\phi}_{\eta_{p}}$ is the time rate of change of the volume fraction related to nucleation of pits; $\dot{\phi}_{v_{p}}$ is the time rate of change of the averaged volume fraction related to the growth of pits; $\dot{\phi}_{c_{p}}$ is the time rate of change of the averaged volume fraction related to the coalescence of pits.

Based on Equation 3, the pitting nucleation rate, pitting growth rate, and pitting coalescence rates are defined as the following:

$\dot{\phi}_{\eta_{p}}=\dot{\eta} v c$

$\dot{\phi}_{v_{p}}=\eta \dot{v} c$

$\dot{\phi}_{c_{p}}=\eta v \dot{c}$

where $\eta$ is the pit nucleation, $\dot{\eta}$ is the nucleation rate of the pits, and both can be directly measured by the pit number density; $v$ is the pit growth, $\dot{v}$ is the pit growth rate of the pits, and both can be 
measured by the average change in volume of the pits; and $c$ is the coalescence of the pits, $\dot{c}$ is the coalescence rate of the pits, and both are a function including the nearest neighbour distance.

The pitting growth rate, Equation 5, can be expanded as follows:

$\dot{v}=\frac{1}{3}\left(\pi \dot{r}^{2} d+\pi r^{2} \dot{d}\right)$

where $\dot{r}$ is the radius of the pits determined from the in-plane pit area, and $\dot{d}$ is the depth of the pits determined from laser profilometry.

The pitting coalescence rate, Equation 6, can be expanded as follows:

$\dot{c}=k_{e}\left(\frac{\dot{q}_{1} q_{2}}{n^{2}}+\frac{q_{1} \dot{q}_{2}}{n^{2}}+\frac{q_{1} q_{2}}{\dot{n}^{2}}\right)$

where $k_{e}$ is the proportionality constant determined from the type of magnesium alloy, $\dot{q}_{1}$ is point

charge from the chloride ions, $\dot{q}_{2}$ is point charge from the magnesium ions, and $\dot{n}$ is the distance between the pits, defined as the nearest neighbour distance.

\section{Relation to Previous Experimentation}

Previous experimentation on as-cast magnesium alloys, namely AZ91, AM60, and AE44, and extruded magnesium alloys, namely AZ31, AM30, and AZ61, showed that environment, percentage of aluminum, and secondary alloying elements all had effects on one or more of the three main area of the conceptual model (Martin (2010); Martin (2011); Martin (2012); Martin (In Revision); Song (In Revision); Walton (2012)). Table 1 shows a tabulation of pit nucleation, or the pit number density, pit growth, or the in-plane pit area and pit depth, and pit coalescence, or the nearest neighbor distance, for the six alloys previously examined in Phase III and how each of the alloys changes based on the environment.

\section{Conceptual Model - Pit Nucleation}

When looking at Table 1, one can see that the final pit number density is almost entirely related to the environment, with only minimal contributions from the form, percentage of aluminum, or the secondary alloying elements. In all cases except for AZ91, a higher pit nucleation occurred on the samples exposed to the immersion environment. This was due to the continuous exposure of the samples to the immersion environment, which allowed chloride ions, necessary to promote pit nucleation, to stay in continuous contact with the surface. 
When looking at the form, the as-cast materials all seem to have equal to or higher pit nucleation values, with the exception of AZ61. The increase in pit nucleation on the as-cast materials is likely caused by very small grains present on the surface due to the as-cast skin, which allowed more pits to form near the grain boundaries or within the very small grains.

When looking at the percentage of aluminum, if corrosion was solely a function of percentage of aluminum, a definite trend should be seen - as the percentage of aluminum gets higher, the pit nucleation should go down. However, this is not the case, as can be demonstrated by AZ31 to AE44, AE44 to AM60, and AZ61 to AZ91. It is surmised that there is an interaction between the aluminum and the secondary alloying elements that change pit nucleation. The interaction has more to do with pit nucleation than the actual percentage of aluminum.

As for the secondary alloying elements, there does appear to be some impact, especially with respect to the inclusion of rare earth elements (AE44), where the pit nucleation drastically changed. If one is to base corrosion specifically on pit nucleation, then only AE44 could be eliminated as a potential material. However, since pit growth and coalescence must be examined, AE44 cannot actually be eliminated as a potential structural magnesium alloy. 
Table 1. Comparison of pit nucleation (pit number density), pit growth (pit area and pit depth), and nearest neighbor distance for the six magnesium alloys in two environments at 60 hours.

\begin{tabular}{|l|l|l|l|l|l|l|}
\hline Alloy & Form & Environment & $\begin{array}{l}\text { Pit Number } \\
\text { Density } \\
\left(1 / \mu \mathrm{m}^{2}\right)\end{array}$ & $\begin{array}{l}\text { Pit Area } \\
\left(\mu \mathrm{m}^{2}\right)\end{array}$ & $\begin{array}{l}\text { Pit Depth } \\
(\mu \mathrm{m})\end{array}$ & $\begin{array}{l}\text { Nearest } \\
\text { Neighbor } \\
\text { Distance } \\
(\mu \mathrm{m})\end{array}$ \\
\hline AM30 & Extruded & Salt Spray & $3.18 \times 10^{-5}$ & 13190 & 12.82 & 83.84 \\
\hline AM30 & Extruded & Immersion & $3.28 \times 10^{-5}$ & 3370 & 7.11 & 64.54 \\
\hline AZ31 & Extruded & Salt Spray & $3.00 \times 10^{-5}$ & 17000 & 12.58 & 82.07 \\
\hline AZ31 & Extruded & Immersion & $3.81 \times 10^{-5}$ & 17800 & 7.52 & 69.10 \\
\hline AE44 & As-Cast & Salt Spray & $14.38 \times 10^{-5}$ & 607 & 5.02 & 33.79 \\
\hline AE44 & As-Cast & Immersion & $19.14 \times 10^{-5}$ & 362 & 5.14 & 29.55 \\
\hline AM60 & As-Cast & Salt Spray & $2.14 \times 10^{-5}$ & 417 & 16.91 & 72.51 \\
\hline AM60 & As-Cast & Immersion & $3.13 \times 10^{-5}$ & 417 & 12.28 & 67.67 \\
\hline AZ61 & Extruded & Salt Spray & $3.90 \times 10^{-5}$ & 1369 & 9.46 & 60.64 \\
\hline AZ61 & Extruded & Immersion & $16.12 \times 10^{-5}$ & 222 & 8.17 & 47.44 \\
\hline AZ91 & As-Cast & Salt Spray & $7.76 \times 10^{-5}$ & 851 & 32.73 & 46.68 \\
\hline AZ91 & As-Cast & Immersion & $2.36 \times 10^{-5}$ & 23240 & 65.68 & 68.09 \\
\hline
\end{tabular}

References: AM30 (Song (In Revision)), AZ31 (Walton (2012)), AE44 (Martin (2010)), AM60 (Martin (2012)), AZ61 (Martin (2011)), AZ91 (Martin (In Revision)) 


\section{Conceptual Model - Pit Growth}

Pit growth is the change of the pit both across the surface (change in pit area) and into the material (change in pit depth). When looking at Table 1, one can see that the environment and form have a much larger effects on pit growth than the other contributions, including percentage of aluminum, and secondary alloying elements. In most cases, with the exception of AZ91, the salt spray environment saw a greater change in the pit area and in the pit depth. The difference in pit growth can be attributed to two distinctions between the environments - the continuous contact of water in the immersion environment that allowed for general corrosion to shrink the size of the pits, and salt residuals that were dried in place during the drying phase of the salt spray environment that allowed the pits to grow underneath the salt residuals without the effect of general corrosion.

Unlike pit nucleation, the form does affect pit growth for some materials. For the as-cast materials, with the exceptions of AZ91, the change in pit area occurs at a much slower rate than the extruded materials. However, the change in pit depth occurs at a much slower rate on the extruded materials, with the exceptions of AE44, which is slower than the extrusion materials. The difference between pit area and pit depth is likely due to the as-cast skin, which is very thin. The grains are very small, meaning that the pits cannot grow far across the surface before hitting grain boundaries, but the pits can grow through the grains into the underlying material that has much larger grains, where the growth can accelerate due to the larger spaces between the grains.

When looking at the percentage of aluminum, there are no conclusions that can be drawn. If the percentage of aluminum affected the pit growth, both the pit area and the pit depth would decrease as the percentage of aluminum increased. However, one can see that the environment and the form had more to do with the pit growth than the percentage of aluminum.

Unlike pit nucleation, where AE44 had the highest pit nucleation, AE44 had the second overall lowest pit growth rate, behind only AM60. This likely means that, while the secondary alloying element allowed more pits to form, the pits could not grow across the surface or into the material. Since pit growth is the most detrimental phase, and is difficult to detect, the formation of pits is not a major issue, while the growth of the pits would be. When considering this issue, AE44 would now be included as a structural material, while AZ91, AZ31, and AM30 would likely all be eliminated. However, pit coalescence, or the growth of the pits into each other to form one large pit, is also a major consideration.

\section{Conceptual Model - Pit Coalescence}

Pit coalescence is mainly a combination of the pit nucleation density, meaning the number of pits in a finite area, and the growth of those pits. The nearest neighbor distance can be small if there are either numerous small pits or a few very large pits.

The environment has the largest effect on nearest neighbor distance, with the salt spray environment having larger distances between the pits as compared to the immersion environment, with 
the exception of AZ91. This is likely due to the fact that there are fewer pits, even though the pits are slightly larger, on most of the salt spray surfaces, as compared to the immersion surfaces. Since there are fewer pits, there is more distance between the pits that do exist, thereby increasing the nearest neighbor distance between the established and growing pits.

When looking at form, the as-cast materials that have large pit nucleation values but small pit areas have the smallest nearest neighbor distance (AE44), while the extruded materials that have the smallest pit nucleation values but the largest pit areas have the largest nearest neighbor distances (AZ31 and AM30). The other materials, AM60, AZ91, and AZ61, show a combination between low pit nucleation values and medium size pit areas have nearest neighbor distances in the middle of the range. Looking at pit coalescence, as with pit nucleation and pit growth, form has minimal effect as compared to environment.

As is the case with pit nucleation and pit growth, the percentage of aluminium plays no role in pit coalescence. The secondary alloying elements also do not appear to play a role in increasing or decreasing pit coalescence. The main role the secondary alloying elements seem to play is the ability to reduce the pits growing across the surface, while also allowing the pits to grow deeper into the material. Based on Table 1, and pit nucleation, pit growth, and pit coalescence, it is hard to definitively say what materials can or cannot be used as structural materials. This inability to determine what alloys are useful as structural materials based solely on experimentation is why developing a corrosion model that can accurately simulate the corrosion across the surface and into the material is so important.

\section{Internal State Variable Model Development}

As one can see from the above discussion of the various environments, forms, percentages of aluminum, and secondary alloying elements, understanding how corrosion occurs due to pit nucleation, pit growth, and pit coalescence is difficult. Developing a model that can accurately predict the changes in the surface due to corrosion would allow researchers to eventually create magnesium alloys that can withstand multiple environments, in one or more forms, with a defined percentage of aluminum and secondary alloying elements. The first step in developing this model is the macroscale, while other scales, including interactions between magnesium atoms and alloying atoms will eventually be needed and constructed. There are three sections of an Internal State Variable Model - kinematics, thermodynamics, and kinetics.

\section{Kinematics}

A complete corrosion model based upon internal variable theory will be introduced to capture the effects of pit nucleation, pit growth, pit coalescence, and general corrosion in a multiplicative decomposition manner. Using standard continuum mechanics, all of the equations will be written in the current configuration. Any motion or damage within the material can be mapped from the reference, $x$, to current configuration, $X$, by the deformation gradient, $\underline{F}$. 
$F=\frac{\partial x}{\partial X}$

The deformation gradient, $\underline{F}$, assumes a sufficient continuity, where the local deformation at $\underline{X}$ is characterized as the gradient of the motion, which is a second order, two-point tensor. The kinematics of motion combine elastic straining, inelastic flow, and formation and growth of damage and is illustrated by the multiplicative decomposition of the deformation gradient.The deformation can be decomposed into the elastic, $F_{e}$, damage, $F_{\phi}$, and plastic, $F_{p}$, deformation gradients given as the following,

$F=F_{e} F_{\phi} F_{p}$

The elastic deformation gradient, $F_{e}$, represents lattice displacements from equilibrium. The volumetric inelastic deformation gradient, $F_{\phi}$, represents a continuous distribution of corrosion and mechanical damage throughout the specimen. By this notion, the damage deformation gradient then can be decomposed by the chemical, $F_{\phi_{c}}$, and mechanical , $F_{\phi_{m}}$, deformation gradients as shown in the following:

$F_{\phi}=F_{\phi_{c}} F_{\phi_{m}}$

The finite strain deformation gradient, $F$, can then be rewritten as

$F=F_{e} F_{\phi_{c}} F_{\phi_{m}} F_{p}$

\section{Thermodynamics}

The First Law of Thermodynamics in the local form is given by Malvern (1969) will then be consistently written with the additional modification for the chemical corrosion effects with the following equation:

$\widetilde{\rho} \dot{\widetilde{u}}=\underline{\widetilde{\sigma}}: \underline{\dot{\widetilde{\varepsilon}}}+\widetilde{\varsigma}-\underline{\widetilde{\nabla}} \cdot \widetilde{q}+P \cdot \dot{\widetilde{\phi}}_{c}+Y \cdot \dot{\tilde{\phi}}_{m}$

Where $u$ is the internal energy per unit mass, $\underline{\sigma}$ is the Cauchy stress tensor, $\mathcal{S}$ is the specific heat generation rate, $\underline{q}$ is the heat flux vector and $\rho$ is the density, $Y$ is the thermodynamic force conjugated to damage, and $P$ is a form of the energy release rate as related to the corrosion evolution. The small strain assumption will still hold true while internal state variables with determine the final Helmholtz free energy equations as it is incorporated into the Second Law of Thermodynamics. 
The kinetics section is currently being expanded from the conceptual model detailed above.

\section{Experimentation}

In conjunction with the macroscale corrosion model being developed using Internal State Variables, experimentation was ongoing to determine the combined effects of corrosion and various types of mechanical stresses, including creep and fatigue, on various magnesium alloys. Before any experimentation could begin though, a creep cradle was designed and multiple copies were built

\section{Design of Grips for Fatigue Testing}

The grips for fatigue testing were created from steel. The initial design used an L-shaped bar. The bottom of the $L$ had holes drilled by which to attach the grips to the top and bottom of the hydraulic arms with screws. The sample was held in place by a pin in the center of the top part of the L, with holes on either side of the pin by which a plate with three holes for the pin and the screws could be used to secure the samples in place. After testing, it was determined that this design was ineffective and a second pair of grips was created. The second pair was also made from steel, but a steel bar. A portion of each side of the bar was cut off, leaving a protruding square in the center of the circular grips. Inside the square, material was removed to allow for the samples to be placed in the center of the square. Pin holes were drilled through the square to provide a way to secure the samples. The second grip is more effective because it covers the entire surface of the hydraulic arm, meaning that the top and bottom will be more properly aligned than the initial design which only used two screw locations instead of the twelve to sixteen holes that are available. 


\section{Design of a Cradle for Creep-Corrosion Testing}

The creep cradle was created from steel. Two long bars were cut and then two holes were drilled into the ends. Two shorter bars were also cut. On one side of the shorter bars, there were two holes drilled completely through on each end to allow for screws to attach the shorter bars to the longer bars. On the other side of the shorter bars, indentations were cut to allow for the grips to move into the bars as the bolts were tightened. The shorter bars also had larger holes drilled into the center of the bar to allow for the grips to slide through. The grips were initially square, with section of the middle cut-out to allow for the placement of the magnesium alloys. A pin hole was also drilled through the grips, to allow small, steel pins to be pushed through the grip and the magnesium samples. The opposite ends of the grips were threaded, to allow for compression nuts to be used to tighten the samples. Figure 1 shows a fully completed and coated creep-cradle, with the major features pointed to in the figure.

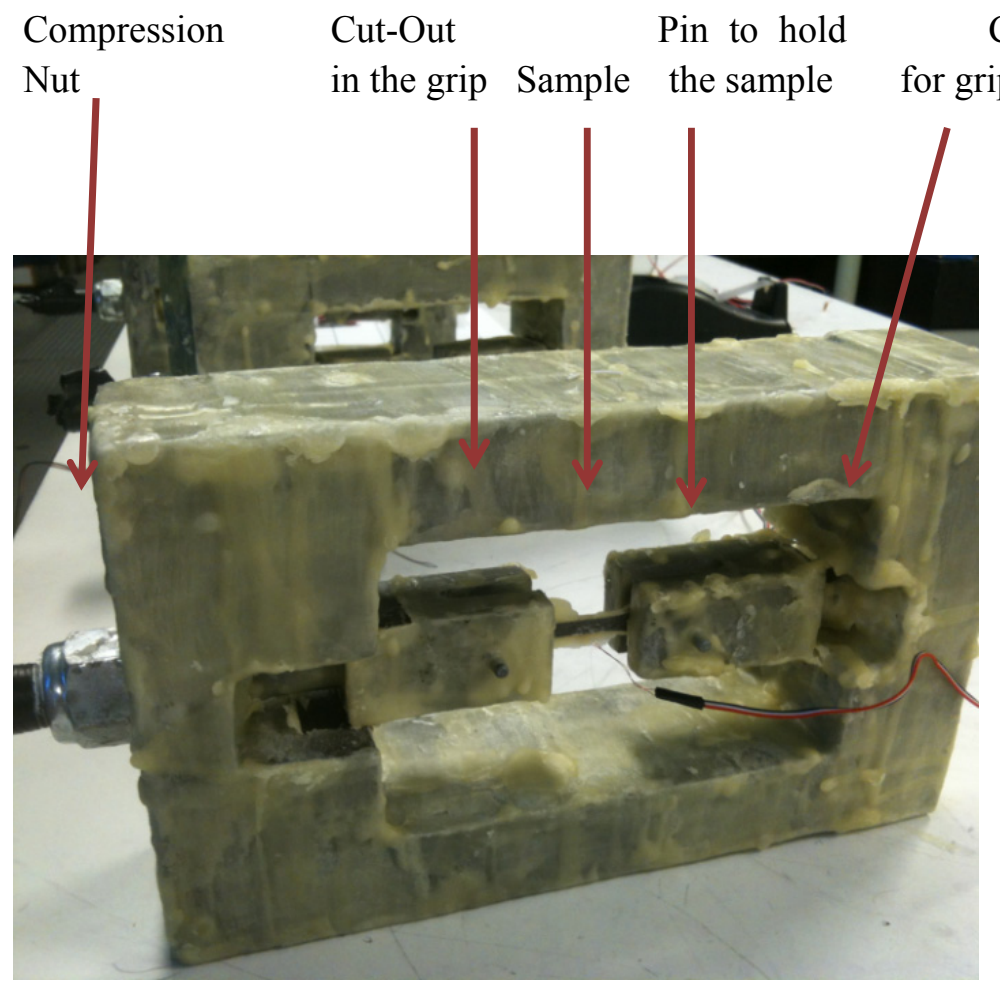

Figure 1. The creep cradle designed and built for the creep-corrosion experimentation.

\section{Experimental Set-Up}

\section{Sample Creation}

Multiple magnesium dogbones were cut from extruded AM30 and AZ31 rails and AZ61 sheets. Small holes were then drilled into both ends of the dogbones to allow for pins to be placed through the 
dogbones to hold the samples in place during testing. For the AZ61 and AZ31 samples, the samples were divided into two groups, those exposed to the immersion environment and those exposed to the salt spray environment. For the AM30 samples, the samples were divided into two groups, those exposed to fatigue in air and those exposed to fatigue in salt water.

\section{Fatigue Testing}

For fatigue testing, the samples in the two groups were assigned to either fatigue in air or fatigue in a corrosive environment. All samples were polished to ensure that scratches and residual stresses from cutting would not impact the fatigue life of the samples. Fatigue in air occurs when the sample is fatigued, using a tension-tension regime $(R=0)$, without the use of the corrosion bath. Different stresses were applied to the samples to produce a stress-controlled fatigue-life diagram. Corrosion testing has not occurred yet, due to issues with the grips that were created. However, when corrosion testing does occur, a chamber enclosing the hydraulic arms, the grips, and the sample mounted using the grips created will be placed on the machine. After this, the entire grips and the sample within the grips will be coated with beeswax to ensure that galvanic corrosion does not occur, causing failure at the holes within the dogbones. After the beeswax has dried, the chamber doors will be closed and filled with a $3.5 \% \mathrm{NaCl}$ aqueous solution. The solution will be continually cycled through the use of a reservoir and pump that ensures the level of the saltwater stays the same throughout the test, and that the sample remains entirely submersed.

\section{Creep Testing}

For creep testing, the samples in the two groups were further divided between those that would be examined at each time point and those that would be examined over the entire exposure period. For all samples, one side of the dogbones was lightly sanded to remove any large scratches, after which, a strain gauge was attached to the sanded surface using a waterproof resin. The resin was allowed to cure for two weeks as required. The samples were then placed in the creep cradles and pulled to $80 \%$ of the yield stress, approximately $0.0036 \mathrm{~mm} / \mathrm{mm}$ (Figure 2a). Beeswax was then used to coat the entire cradle, including the pins and inside the cutouts, to prevent galvanic corrosion (see Figure 1). A portion of the unsanded side was left bare for the corrosion to occur (see Figures 1 and $2 b$ ). The samples were then corroded for a given time period and removed, for either surface analysis or for monotonic tension testing. 

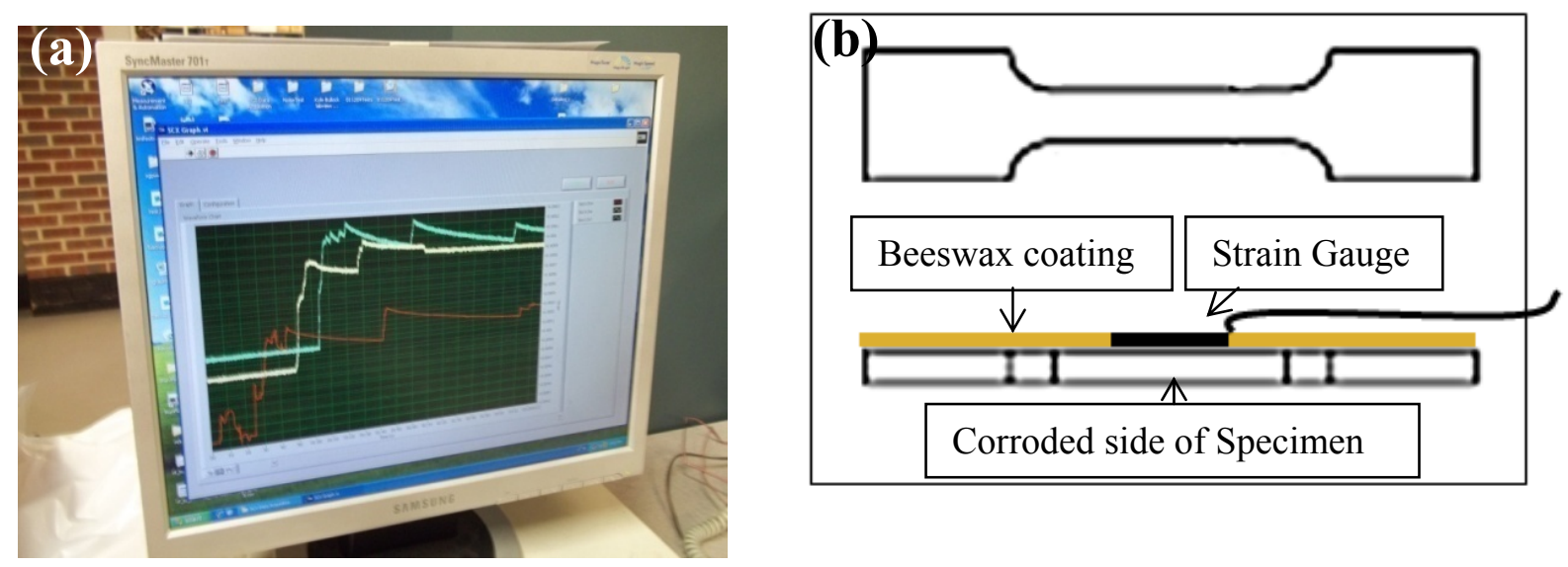

Figure 2. (a) A change in the strain before (red line) and after (green and yellow lines) tightening the bolts on the creep cradles. (b) A diagram showing the attached strain gauge and beeswax coating on a dogbone.

\section{Environment}

All environments used were created using $3.5 \%$ by weight sodium chloride $(\mathrm{NaCl})$ aqueous solutions. The immersion environment consisted of an aquarium filled with the $\mathrm{NaCl}$ solution. The immersion environment was used without circulation for the creep testing and was used with circulation for the fatigue testing. The cyclical salt spray environment used a Q-Fog Spray Chamber and equal times between the $3.5 \% \mathrm{NaCl}$ aqueous solution spray, $100 \%$ humidity, and a drying phase. For the immersion environment for creep testing and the cyclical spray environment, the samples were removed and analyzed for surface changes at $1 \mathrm{hr}, 4 \mathrm{hrs}, 12 \mathrm{hrs}, 36 \mathrm{hrs}$, and $60 \mathrm{hrs}$. The samples were rinsed, patted dry, then placed in a dessicator overnight. Optical micrographs and laser profilometry scans were then taken of the surfaces.

\section{Results}

Two different sets of results were obtained for this research, the fully completed creepcorrosion of AZ31 and the ongoing mechanical stress-corrosion of AM30 and AZ61. The ongoing creepcorrosion of AZ61 will not be discussed here, as it is only partially finished and the data has not been analyzed yet.

\section{Fatigue-Corrosion of AM30}

Because corrosion-fatigue must occur within an enclosed chamber, the ability to do straincontrol fatigue testing is not possible. Therefore, it is necessary to determine the fatigue life based on stress control testing, as the literature uses strain control. For this to occur, grips were developed that 
would be able to withstand the corrosive environment. Initially, a tension-compression regime $(R=-1)$ was chosen, but the samples experienced high amounts of buckling, as shown in Figure 4, due to the small gauge size and high stresses. Low stresses did not cause buckling but also did not lead to fracture. When a tension-tension regime $(R=0)$ was used, the samples did not bend or buckle. The tensiontension regime was able to produce a stress-controlled fatigue life diagram in air, as shown in Table 2 and Figure 5. As expected, the higher the stress, the fewer the number of cycles that occurred before failure. However, after experimentation, it was shown that the designed grips were the cause of the buckling and breaking of the magnesium samples and new grips had to be designed and tested.

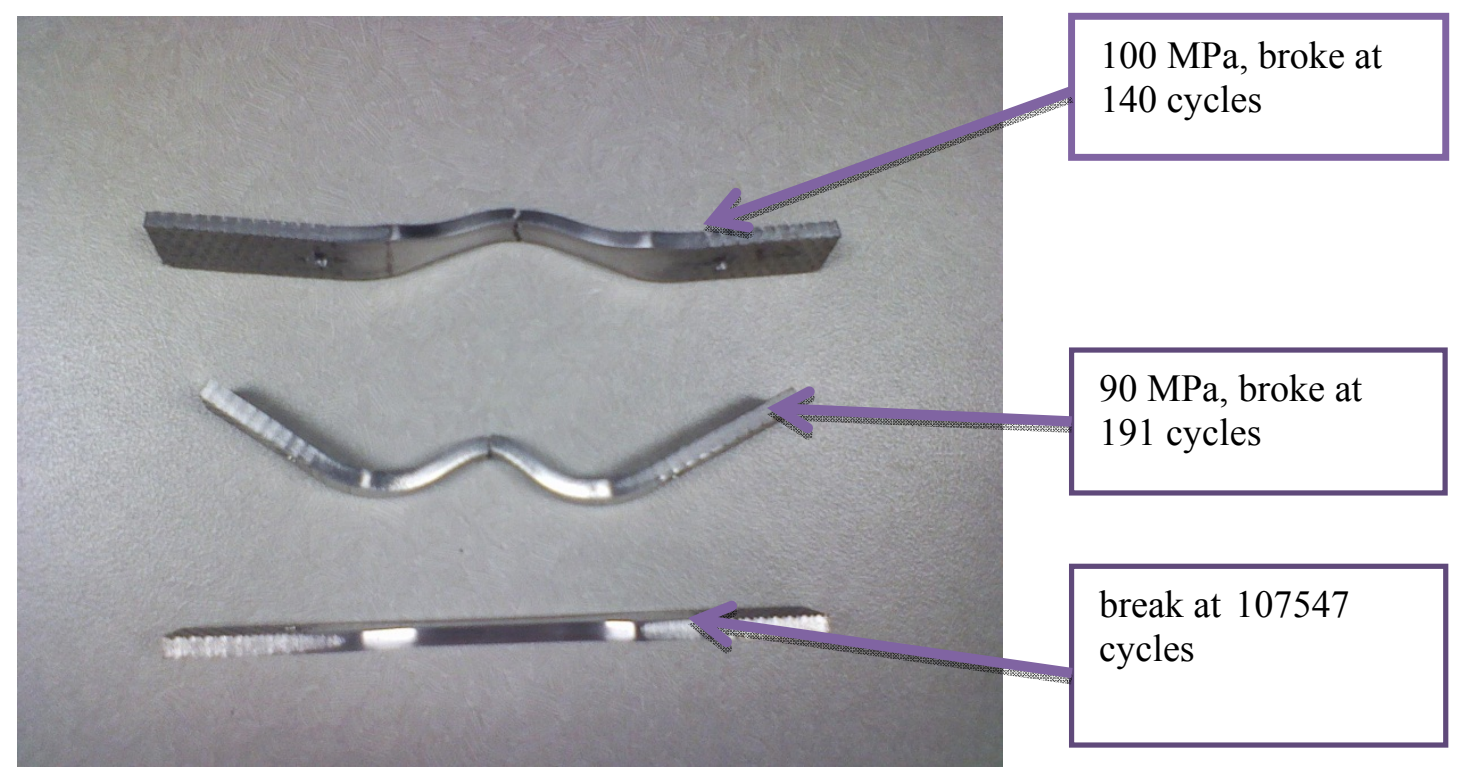

Figure 4. Examples of the samples that buckled or bend at high stresses and did not fail at low stresses when using a tension-compression regime $(R=-1)$. It was the small change between $60 \mathrm{MPa}$ and 90 $\mathrm{MPa}$ that demonstrated the original grips were the cause of the failure issues and not the stresses. 
Table 2. Applied stress and number of cycles to failure using a tension-tension regime $(R=0)$.

\begin{tabular}{|c|c|}
\hline Stress amplitude(MPa) & cycles \\
\hline 80 & 226525 \\
\hline 90 & 18341 \\
\hline 100 & 10034 \\
\hline 110 & 2201 \\
\hline 120 & 82 \\
\hline 120 & 66 \\
\hline
\end{tabular}

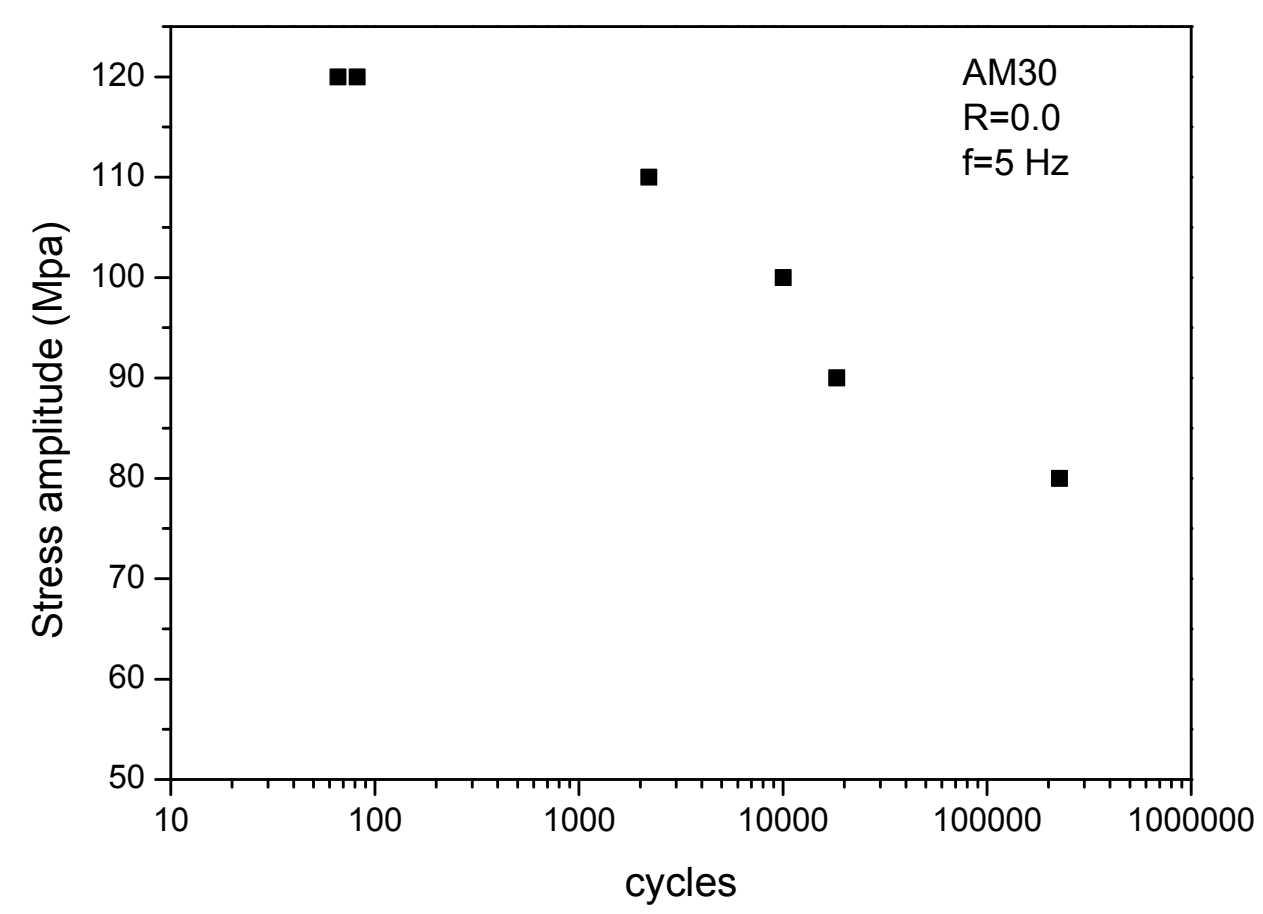

Figure 5. Stress-controlled fatigue life versus cycle number in an air environment when using a tensiontension regime $(R=0)$. 


\section{Creep-Corrosion of AZ31}

For creep-corrosion, specimens were exposed to the environment for a defined period of time and then removed from the creep cradle and analyzed. Figure 6 shows the changes in the stress-strain relationship over 60 hours for both the salt spray environment (five lines) and the immersion environment (four lines). While three samples were run for both environments at each time step, only one line for each time and environment was graphed in order to show the changes. As one can see, there is not much change in the elongation to failure or the yield stress for the salt spray samples. The immersion samples experienced decreases in the elongation to failure and a reduction in yield stress at every time period. Due to the high degradation caused by the corrosive environment combined with the applied strain, the sample exposed for 60 hours to the immersion environment was fully corroded through and was unable to be broken, meaning a stress-strain line could not be produced.

Figure 7 shows the stress-strain relationship at 12 hours $\left(t_{3}\right)$ for both the salt spray environment and the immersion environment. As with Figure 6 , while three samples were broken at $t_{3}$, only one line was graphed for each environment to demonstrate the changes in the stress-strain relationship due to the corrosion-creep interaction. Figure 7 clearly demonstrates the difference between the continuous exposure to the corrosion environment and the cyclical exposure to the corrosion environment. The continuous exposure caused a major decrease in both the elongation to failure and the yield stress of AZ31.

Figure 8 shows the stress relaxation as a function of time for three environments - the immersion environment, the salt spray environment, and the control environment, which was air. As one can see when looking at Figure 8, the salt spray environment closely followed the control, although the stress relaxation was slightly lower for the salt spray environment as compared to the control. After 12 hours, though, the salt spray environment experienced a large decrease in stress relaxation to 36 hours. The immersion environment experienced a large drop in stress relaxation between each time point, with a final point that was fully corroded through.

Micrographs of the same surfaces after 1 hour (9a, 9b) and 36 hours (9c, 9d) after exposure to the salt spray environment (9a, 9c) or the immersion environment (9b, 9d) are shown in Figure 9 . When looking at Figure 9, pit nucleation and growth are evident on the salt spray surfaces at both time periods, while crack formation and growth are not seen. Crack formation and growth, though, are distinctly present on the immersion surfaces, which also show much less evidence of pit nucleation and growth. The cracks present at 1 hour and 36 hours do look to be approximately the same size, and also appear to possibly follow the grain boundaries. Figure 10 shows two micrographs after 1 hour of exposure to the immersion environment. When looking at Figure 10a, which uses a magnification of $5 x$, one can see a multitude of small cracks across the surface, that exhibit a more curvy appearance, instead of a straight appearance. This is likely due to the crack following the grain boundaries, instead of moving across the surface perpendicular to the applied strain. Figure 10b, which uses a magnification of 10x, further demonstrates the curved appearance of the cracks. In addition, Figure 10b shows that the cracks appear to be growing into each other, even at $1 \mathrm{hr}$. 


\section{Discussion}

The design of the fatigue grips and the creep cradle allowed the graduate students, Weiwei Song and Chris Walton, respectively, to put their Mechanical Engineering education to good use, in both the design, operation, and troubleshooting that occurred as a result of the development of these pieces of equipment.

\section{Fatigue-Corrosion of AM30}

When looking at Figures 4, one can see that major buckling and breaking occurred. The compression part of the initial tension-compression regime was determined to be the cause of the buckling, as the small gauge size could not handle the compressive stress. When looking at Table 2 and Figure 5 , one can see that the number of cycles to failure is also very small at a relatively low stress value (120 MPa), even when the compression was removed. There were stresses, approximately 80 MPa, where failure was either not achieved or where failure took over 200,000 cycles. This small range of stresses was disconcerting, which led to more troubleshooting to

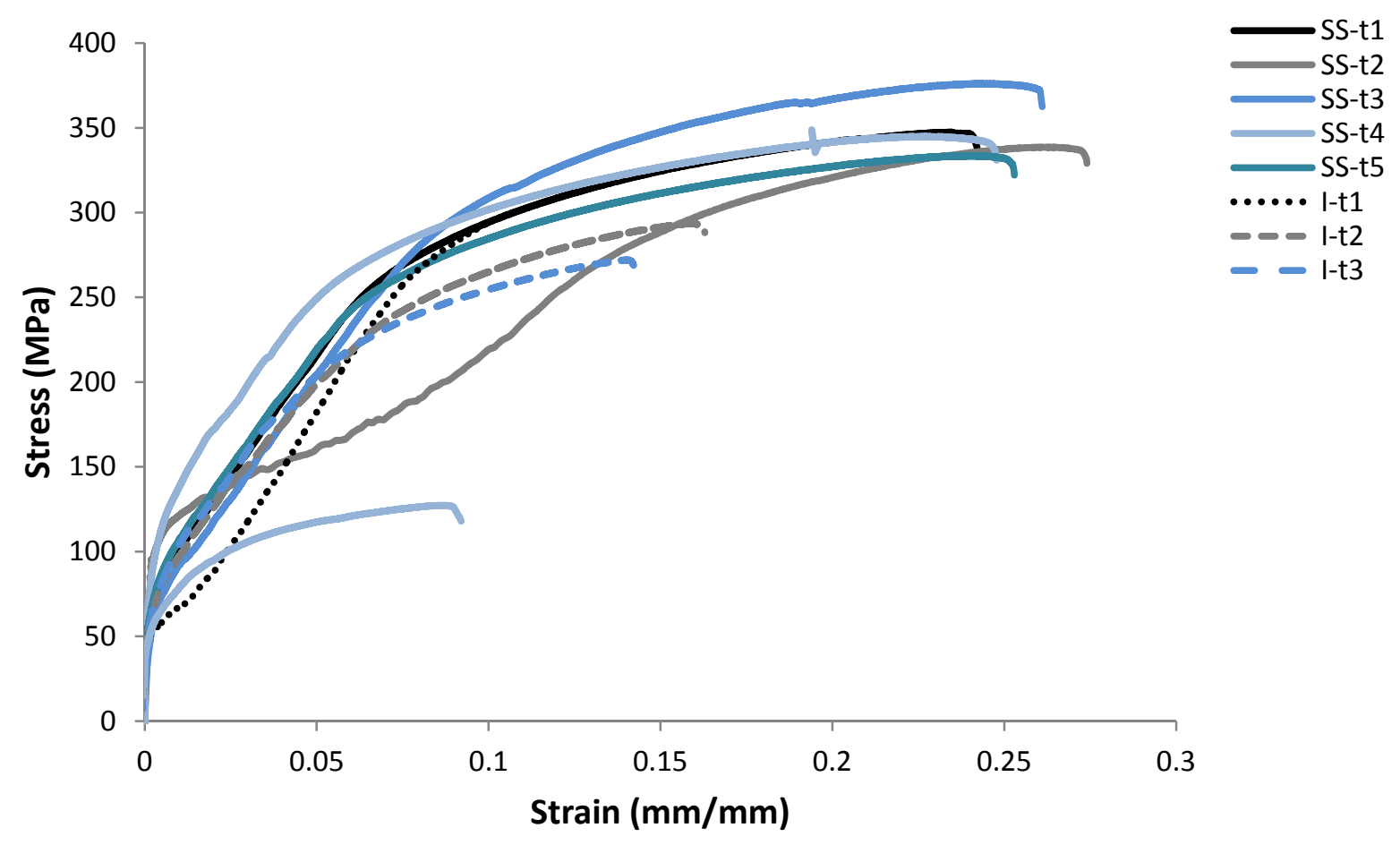

Figure 6. Stress-strain relationship of AZ31 over 60 hours for samples exposed to the salt spray and immersion environments. Notice the drop in both elongation to failure and yield stress for the immersion samples as every time point changed. Also notice that there were minimal changes in elongation to failure and yield stress for the salt spray changes. 


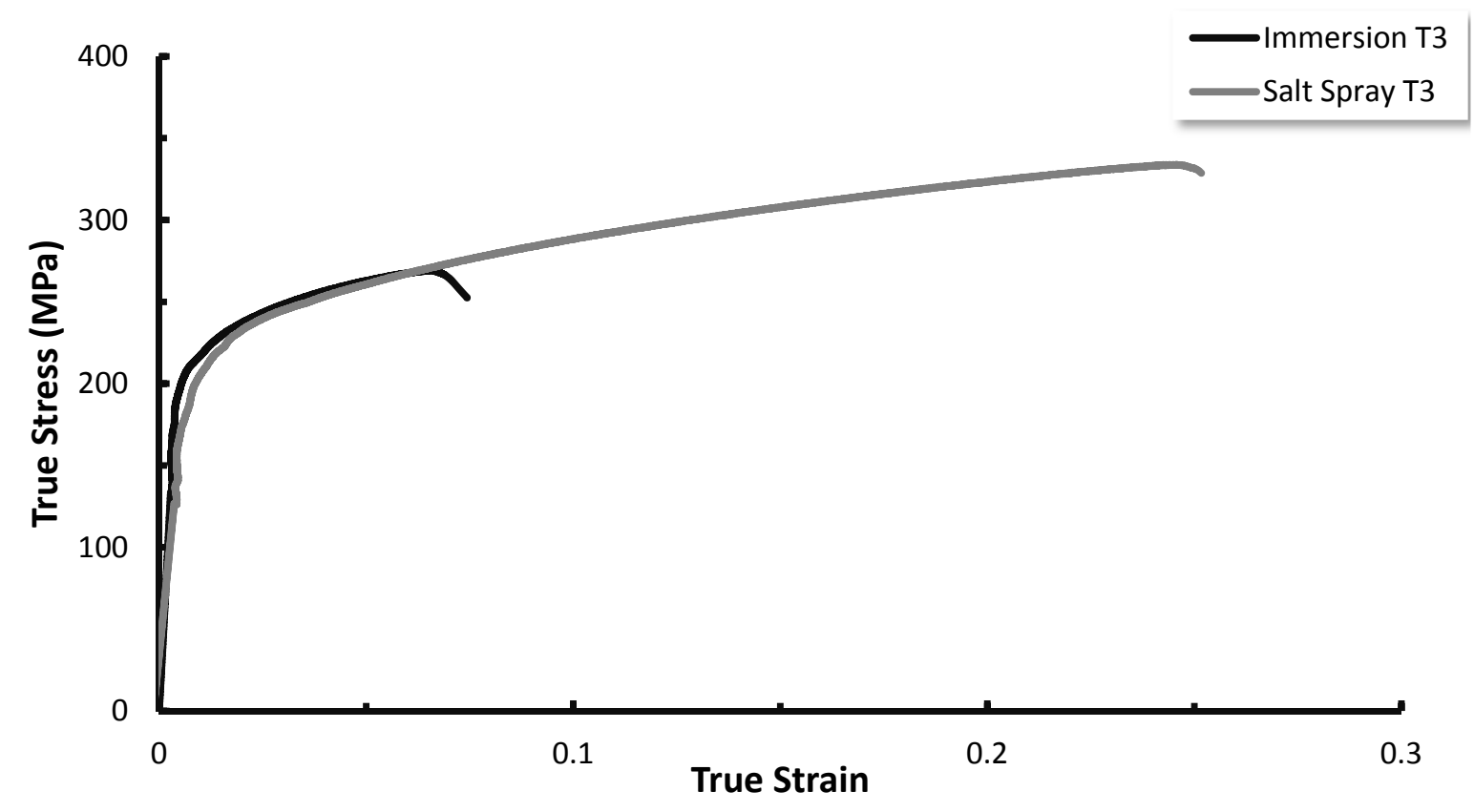

Figure 7. Stress-strain relationship of AZ31 at 12 hours $\left(t_{3}\right)$ for samples exposed to the salt spray and immersion environments. Notice the drop in both elongation to failure and yield stress for the immersion samples as compared to the salt spray environment.

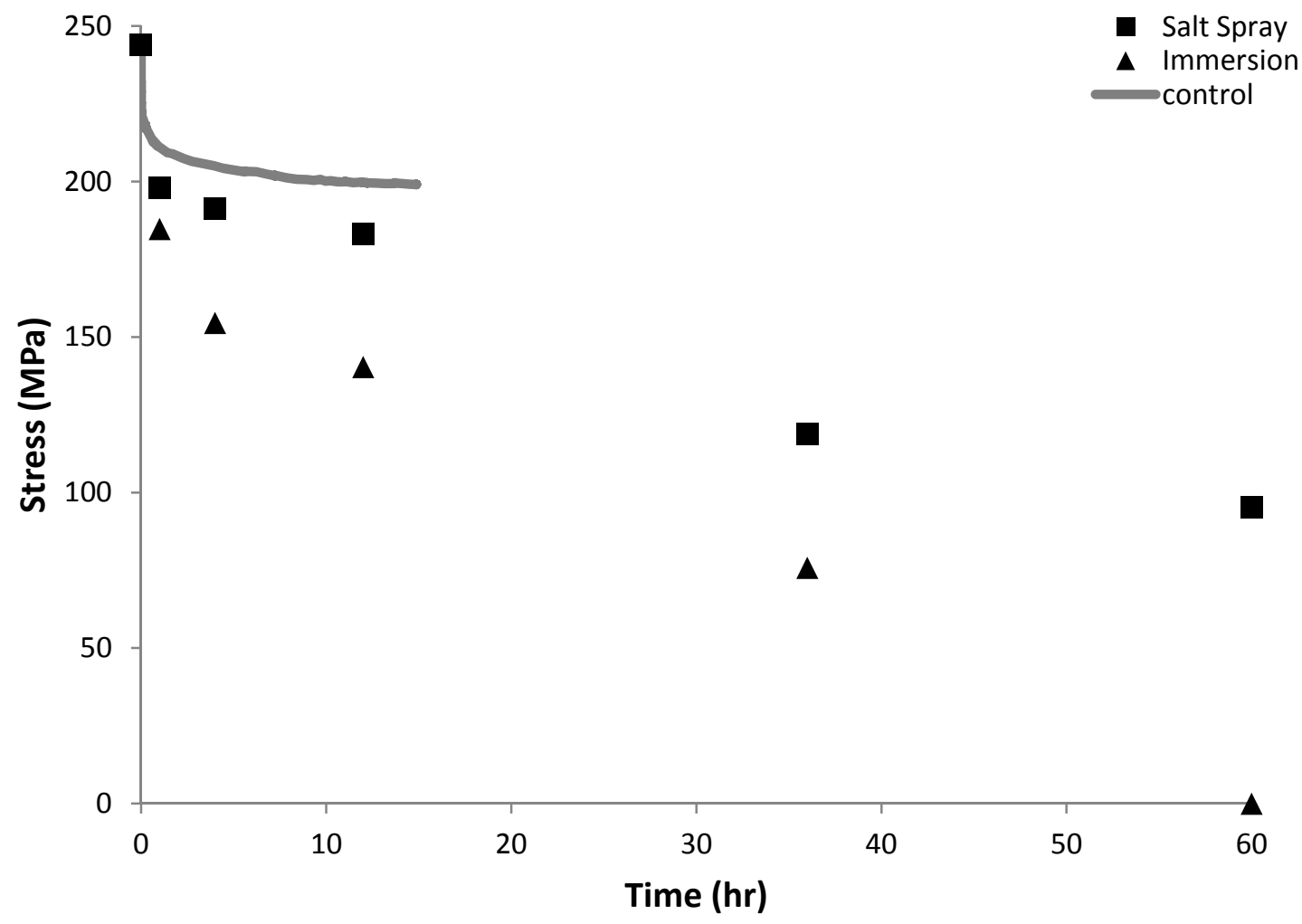


Figure 8. Stress relaxation as a function of time over 60 hours for the immersion and salt spray environment compared to a control. Notice that the stress relaxation for the salt spray environment initially followed a similar, although slightly lower trend, than the control, before a large decrease at 36 hours. Notice also that the stress relaxation for the immersion environment dropped rapidly at each time point, before reaching complete breakage at 60 hours.
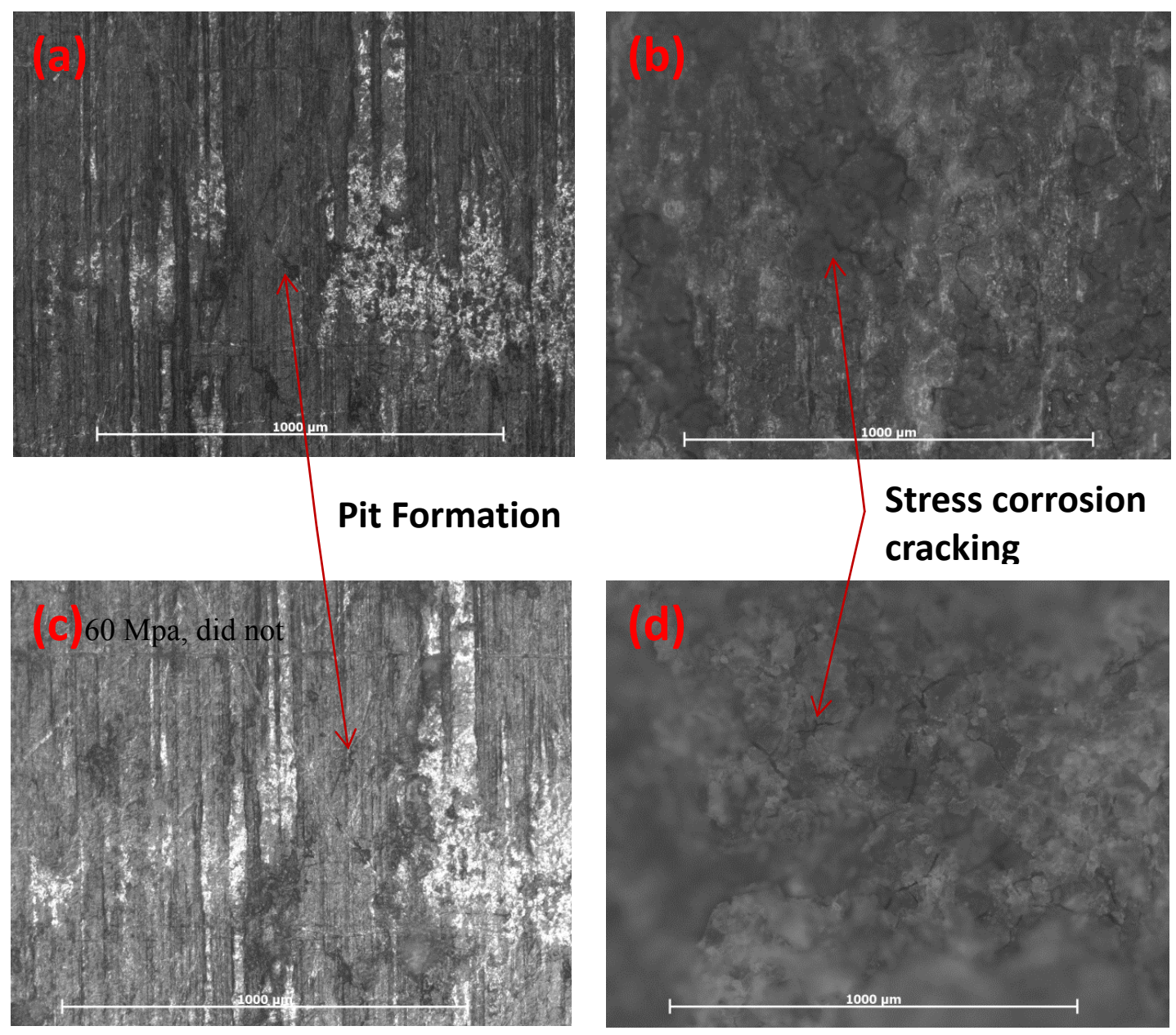

Figure 9. Micrographs of the exposed surface at 1 hour (a, b) and 36 hours (c,d) for the salt spray (a, c) and immersion $(b, d)$ environments. Notice that the salt spray surfaces experienced pit nucleation and growth, with no crack formation. Notice also that the immersion surfaces experienced crack growth, likely along the grain boundaries, even at 1 hour; there does not appear to be a large difference in the number or size of the cracks due to time.

\section{(a)}

(b) 

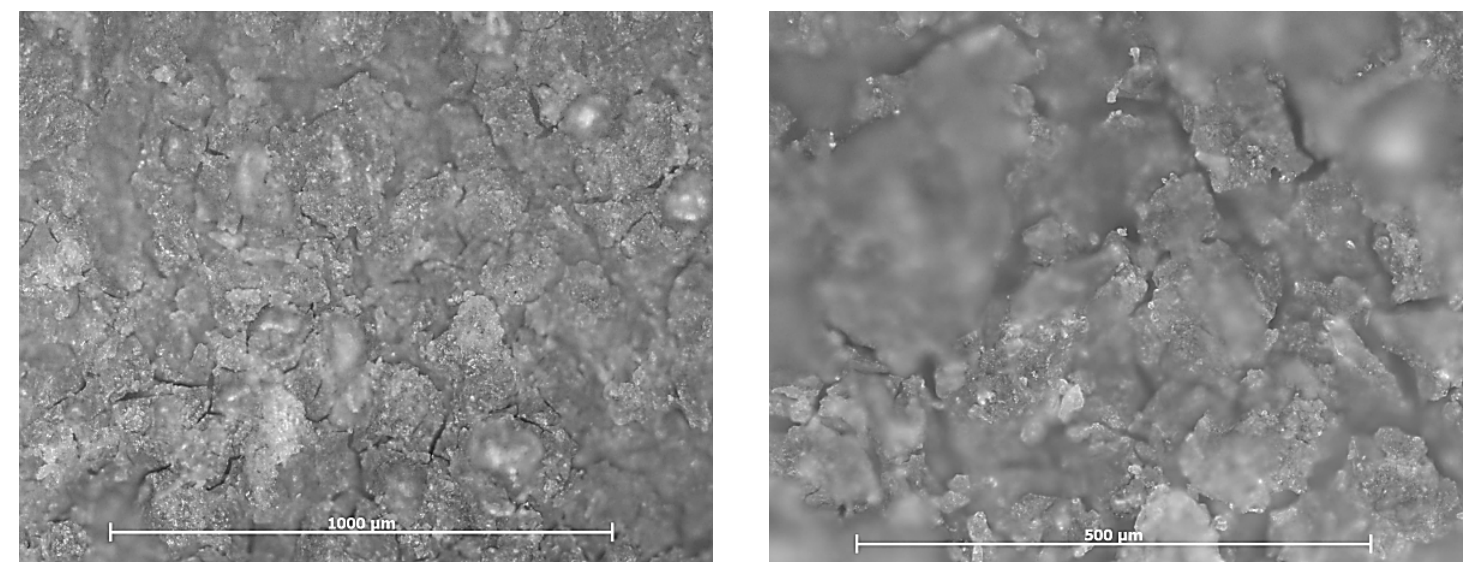

Figure 10. Micrographs of the immersion environment after 1 hour showing the shape of the cracks scattered across the surface at a magnification of $5 x(a)$ and a magnification of $10 x$ (b) that show the cracks appear to follow the grains.

determine if other issues might cause the major change in life cycles with a relatively small change in stress.

After looking over the grips, the main cause of the buckling and life cycle issues was due to the slightly off-centered alignment of the initial grips. After this alignment issue was identified, it was determined that the initial grips were not suitable for this project when using any type of compression regime. Therefore, new grips were designed, along with the issues that any new design possesses. In the case of the new grips, the pin would wiggle out during the fatigue testing or the failure would occur at the hole instead of within the gauge. Both of these issues have been worked out, meaning that the experimentation to determine the fatigue life in air and the fatigue life in a corrosive environment is now beginning.

\section{Creep-Corrosion of AZ31}

Looking at the combined corrosion plus creep of AZ31 demonstrates that, as with the corrosion only experiments, the differences in environments were the main causes in the differences in corrosion. The immersion environment was much more damaging, due to the continuous presence of both water and chloride ions. The water leads to general corrosion, while the chloride ions lead to pit nucleation. When looking at Figures 9 and 10, one would think that pitting was not a major issue for the immersion surfaces. However, it is more likely that pitting occurred prior to 1 hour and the applied strain led to fast pit nucleation and growth, as voids near the surface were opened. Stress relaxation occurs because the applied strain allows the microstructure to slip, forming dislocations within the grains, allowing the stress to drop (Figure 8). These dislocations, especially if they form near where pits were already developing, allow the chloride ions and water to penetrate into the material more quickly, thereby increasing the corrosion. As the cracks formed, and followed the grain boundaries (Figures 9b, 9d, 10), further exposure to the immersion environment and to the applied strain allows the grains to fall out, reducing both the cross-sectional area and the yield stress (Figures 6-7). The exposure to water also 
allows the formation of hydrogen ions, which then could easily diffuse into the magnesium dogbones via the exposed cracks, thereby decreasing the elongation to failure (Figures 6-7).

The salt spray environment, on the other hand, did not have the continuous presence of water or chloride ions, so the interaction between dislocation formation and the corrosive environment is reduced. While stress relaxation and dislocation formation begin immediately after the strain is applied, the initial exposure to the salt spray environment consists of 20 minutes of saltwater, 20 minutes of humidity, and 20 minutes of drying. The initial 20 minutes are likely only enough to begin the process of pit nucleation (Figure 9a), as crack growth would require both the nucleation of pits and the interaction between the pits and any surface dislocations. There likely was some interaction, as seen in Figure 8 with a change in stress relaxation greater than the control, but the short exposure time meant that the detrimental effects would be much less than the immersion environment. The cyclic nature of the salt spray environment also prevented general corrosion that would reduce the cross-sectional area, thereby preventing a change in the yield stress (Figures 6-7), and prevented the diffusion of large amounts of hydrogen ions into the alloy, thereby preventing hydrogen embrittlement and changes in the elongation to failure (Figures 6-7). The stress relaxation and dislocation formation caused by the applied strain interacted with the magnesium dogbones differently based on the environmental exposure. The high amount of damage caused by the immersion environment is fully demonstrated by the micrographs (Figures 9-10) and the stress relaxation (Figure 8), yield stress (Figures 6-7), and elongation to failure (Figures 6-7) graphs.

\section{Conclusions}

The development of a corrosion model that can accurately simulate damage to a surface caused by a chloride containing environment is currently ongoing. The initial development of a conceptual model that incorporates pit nucleation, growth, and coalescence has been completed. The theoretical model development, including kinematics, thermodynamics, and kinetics, is currently ongoing. The kinematics portion of the model focuses on the deformation gradient, which will be able to incorporate changes due to a chemical gradient. The thermodynamics portion of the model will focus on incorporating chemical effects, while the kinetics portion of the model will focus on developing equations stemming from the conceptual model.

In order to incorporate the corrosion model with previously created damage models, experimentation combining mechanical stresses and corrosion were performed. The creation of grips and cradles constituted a test for the graduate students' mechanical engineering education.

Using the initial grips, fatigue testing of AM30 could only be performed in a tension-tension regime, as the tension-compression regime led to buckling of the samples. New grips have been designed that will take care of the small issues with alignment that likely were leading to problems with the fatigue testing in air. After the development of a stress controlled fatigue life diagram in air, a stress controlled fatigue life diagram in $3.5 \% \mathrm{NaCl}$ aqueous solution will be developed. For creep-corrosion testing of AZ31, the immersion environment was much more deleterious to the sample than the salt spray environment. The continuous exposure to chloride ions, water, and hydrogen ions, allowed for crack formation and growth across the surface. This crack growth removed magnesium grains, which led to a reduction in 
both the cross-sectional area and the yield stress of the magnesium dogbones. In addition, the crack formation allowed for the diffusion of hydrogen ions into the material, increasing the embrittlement of the alloy and reducing the elongation to failure. The salt spray environment did not have the same continuous exposure, so the interaction between dislocations caused by the applied strain and stress relaxation and the corrosive environment was greatly reduced.

\section{Publications}

1. Walton C, Martin HJ, Horstemeyer MF, Wang PT. "Structure-Property Quantification of Corrosion Pitting Under Immersion and Salt-Spray Environments on an Extruded AZ31 Magnesium Alloy." Corros. Sci. 56 (2012) 194-208

2. Martin HJ, Alvarez RB, Danzy J, Horstemeyer MF, Wang PT. "Structure-Property Quantification of Corrosion Pitting Under Immersion and Salt-Spray Environments on an As-Cast AM60 Magnesium Alloy." Corros. - Magnesium Corrosion The Special Edition, 68 (2012) 571-585 (Invited).

3. Song W, Martin HJ, Hicks A, Horstemeyer MF, Wang PT. "Structure-Property Quantification of Corrosion Pitting Under Immersion and Salt-Spray Environments on an Extruded AM30 Magnesium Alloy". Corros. Sci. In Revision.

4. Martin HJ, Walton C, Danzy J, Horstemeyer MF, Wang PT. "Structure-Property Quantification of Corrosion Pitting Under Immersion and Salt-Spray Environments on an As-Cast AZ91 Magnesium Alloy." Corros Sci. In Revision.

5. Walton C, Horstemeyer MF, Martin HJ, Wang PT. "On Corrosion Modeling Using Internal State Variable Inelasticity." In Preparation.

6. Walton C, Martin HJ, Turnage S, Whittington W, Horstemeyer C, Horstemeyer MF, Wang PT. "Structure-Property Quantification of Corrosion Mechanisms with Applied Mechanical Stresses Under Immersion and Salt-Spray Environments on an Extruded AZ31 Magnesium Alloy." Corros. Sci. In Preparation.

7. Song W, Martin HJ, Horstemeyer MF, Wang PT. "Structure-Property Quantification of Corrosion Mechanisms with Applied Fatigue Under Immersion and Salt-Spray Environments on an Extruded AM30 Magnesium Alloy." Corros. Sci. In Preparation.

\section{Presentations}

1. Walton C, Martin HJ, Whittington W, Horstemeyer C, Horstemeyer MF, Wang PT, "CorrosionStress Relaxation Effects on Surface and Tensile Properties of an AZ31 Magnesium Alloy," 2012 American Institute of Chemical Engineering Annual Meeting, Pittsburgh, PA, October 2012. 
2. Walton C, Martin HJ, Horstemeyer MF, Wang PT, "Corrosion Modeling of Magnesium Alloys Using Internal State Variable," 2012 American Institute of Chemical Engineering Annual Meeting, Pittsburgh, PA, October 2012.

3. Martin HJ, Walton C, Danzy J, Hicks A, Horstemeyer MF, Wang PT, "Modeling Individual and Bulk Pit Characteristics on Various Magnesium Alloys Exposed to Two Saltwater Environments," 2011 American Institute of Chemical Engineers Annual Meeting, Minneapolis, MN, October 2011.

4. Walton C, Martin HJ, Danzy J, Hicks A, Horstemeyer MF, Wang PT, "Comparing the Corrosion Effects of Environment and Form on Various Magnesium Alloys," 2011 American Institute of Chemical Engineers Annual Meeting, Minneapolis, MN, October 2011.

5. Martin HJ, Walton C, Danzy J, Hicks A, Horstemeyer MF, Wang PT, "Comparing the Corrosion Effects of Two Environments on As-Cast and Extruded Magnesium Alloys," 2011 The Minerals, Metals, and Materials Society Annual Meeting, San Diego, CA, February 2011.

6. Martin HJ, Walton C, Danzy J, Hicks A, Horstemeyer MF, Wang PT, "Modeling the Corrosive Effects of Various Magnesium Alloys Exposed to Two Saltwater Environments," 2011 The Minerals, Metals, and Materials Society Annual Meeting, San Diego, CA, February 2011.

\section{REFERENCES}

Alvarez RB, Martin HJ, Horstemeyer MF, Chandler MQ, Williams TN, Wang PT, Ruiz A. 2010. Corros Sci. 52: $1635-48$.

Bakkle P, Westengen H. 2005. In: Neelameggham NR, Kaplan HI, Powell BR (Eds.), TMS - Magnesium Technology, 291-96.

Horstemeyer MF, Lathrop J, Gokhale AM, Dighe M. Theor. Appl. Fract. Mech. 33 (2000) 31-47.

Horstemeyer MF, Gall KA, Dolan K, Haskins J, Gokhale AM, Dighe MD. Theor. Appl. Fract. Mech. 39 (2003) 23-45.

Johnson WH, Proceedings of the Royal Society of London, Vol. 23, 1874 - 1875, pp. 168-179.

Makar GL, Kruger J. 1993. Int Mater Rev 38:138-53.

Malvern, L.E., Introduction to the Mechanics of a Continuous Medium, Prentice-Hall Englewood Cliffs, NJ, 1969.

Martin HJ, Horstemeyer MF, Wang PT. Corros. Sci. 52 (2010) 3624-3638.

Martin HJ, Horstemeyer MF, Wang PT. Corros. Sci. 53 (2011) 1348-1361.

Martin HJ, Alvarez RB, Danzy J, Horstemeyer MF, Wang PT. Corros. 68 (2012) 571-585. 
Martin HJ, Walton C, Danzy J, Horstemeyer MF, Wang PT. Corros Sci. In Revision

Shaw BA. 2003. In: Korb LJ, ASM, eds., ASM Handbook, Vol. 13A: Corrosion, Ninth Ed., ASM International Handbook Committee, Metals Park, 692.

Song G, Atrens A. 2003. Adv Eng Mater 5:837-58.

Song W, Martin HJ, Hicks A, Wang PT, Horstemeyer MF. Corros. Sci. In Revision.

Toh T, Baldwin WM. In Stress Corrosion Cracking and Embrittlement, W. D. Robertson (Editor), J. Wiley \& Sons, New York, 1956.

Walton C, Martin HJ, Horstemeyer MF, Wang PT. Corros. Sci. 56 (2012) 194-208.

Windle AH, Smith GC. Metal Science Journal, 1968, Vol. 2. 187-191.

\section{KEY WORDS}

Magnesium alloys; pitting; salt spray test; immersion test; creep-corrosion testing; macroscopic model

\section{BRIEF Description of RePort}

A conceptual model detailing the interactions between pit nucleation, pit growth, and pit coalescence was described. The method to create a theoretical Internal State Variable model from the conceptual model was outlined, which covers kinematics, thermodynamics, and kinetics. Experimentation to determine how mechanical stresses and corrosive environments interact was also discussed. A stress-controlled fatigue life diagram in air for AM30 is currently in development, after which, a stress-controlled fatigue life diagram in a $3.5 \% \mathrm{NaCl}$ aqueous environment for AM30 will be developed. Creep-corrosion of AZ31 demonstrated that the immersion environment caused significant damage to the magnesium alloy. The damage was caused by the interaction between dislocations and chloride ions, leading to crack formation and grain removal, as well as hydrogen embrittlement. This damage led to a reduction in yield stress and elongation to failure, respectively. 
Subtask 1.5: Material Design of Lightweight Mg Alloys

Team Members: Seong-Gon Kim (Task Leader), Sungho Kim, M. F. Horstemeyer, M. I. Baskes

\section{ObJective}

Design a novel Mg alloy with improved formability and energy absorption, creep, and corrosion resistance for automotive use.

\section{APPROACH}

We use a hierarchical, multiscale methodology to investigate the effect of nanoscale precipitates and additives to the overall strength and ductility of $\mathrm{Mg}$ alloys for automotive applications. The engineering constraints at the highest length scales include energy absorption (strength and ductility), creep, and corrosion resistance. These constraints lead to critical dislocation and twinning mechanisms related to the following: selection of key combination of precipitates and matrices, interaction of precipitate and matrix phases and, ultimately, composition-structure-property relationship. At the electronic level, quantum mechanical, first-principles simulations based on Density Functional Theory (DFT) will be performed to investigate the interfacial interactions between matrix and the nanoscale entities such as precipitates, intermetallics, and carbon nanotubes (CNTs). At the atomistic level, accurate atomistic simulations will be performed using efficient and reliable empirical interatomic potentials such as modified embedded-atom method (MEAM) or force-matching, embedded-atom-method (FMEAM) potentials. The interatomic potentials are constructed by optimizing the potential parameters to reproduce various experimental materials properties and atomic-force data from DFT calculations. Large-scale, atomistic simulations will be conducted to study the effects that size, shape, and volume fraction of different precipitates and CNTs have on the thermomechanical properties of lightweight alloys. Clearly, different precipitates and intermetallics exist in magnesium alloys, but the inclusion of CNTs might help develop new alloys. Numerical simulations and experimental observations have indicated that CNTs have extraordinary tensile strength (up to $150 \mathrm{GPa}$ ) and Young's modulus (up to 1 TPa), but the interface has been a problem. In this project, we will mainly focus on understanding and controlling the interface between CNTs and Mg matrix. The mechanisms to induce a strong covalent bonding between nanotubes and matrices will be investigated. Many factors that govern the yield and hardening behavior of solids such as interaction of point defects with dislocation and grain boundary, dislocation nucleation, dislocation motion, crack-tip propagation, and the interaction of dislocations with grain boundaries will be investigated through these simulations. Results will be used to guide quantitative alloy-composition designs to improve strength and ductility of $\mathrm{Mg}$ alloys.

\section{RESULTS}

\subsubsection{Mg Polycrystalline Deformation}

We developed a systematic approach to investigate the deformation in nanocrystalline hcp magnesium under uniaxial tensile loading. Five nanocrystalline magnesium samples corresponding to five different grain sizes $(5,10,15,20$ and $30 \mathrm{~nm})$ are geometrically created in three dimensions using Voronoi 
construction. In this construction, these grains are randomly nucleated within a cube with sides 60.8 $\mathrm{nm}$. Each grain grows with random crystallographic orientations until the grains reach one another. For $5 \mathrm{~nm}$ grain size, the model contains $(12 \times 12 \times 12=) 1728$ grains, whereas for the $30 \mathrm{~nm}$ grain size, the model contains $(2 \times 2 \times 2=) 8$ grains. The Voronoi construction gives a random NC model with grain boundary structure similar to what is expected in polycrystalline materials. Each initial model that contains more than 9.5 million atoms has no other defects, besides the grain boundaries as shown in the inset of Fig. 1.5.1.

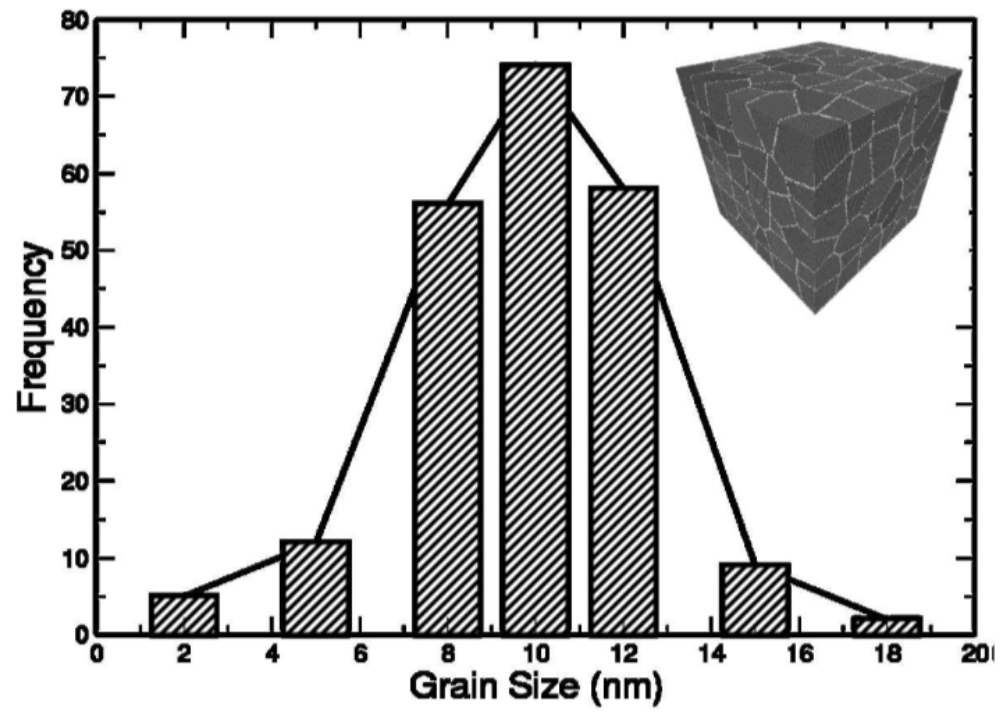

Figure 1.5.1. Histogram of the grain sizes for an averaged grain size of $10 \mathrm{~nm}$ with 216 number of grains in the specimen. The inset shows the corresponding $\mathrm{Mg}$ nanocrystalline sample, prior to tensile loading. The white atoms represent the grain boundaries, while the black atoms represent pure hcp crystalline Mg structure.

Figure 1.5.1 shows the histogram of the grain size for an average grain size $10 \mathrm{~nm}$, with 216 number of grains in the sample. After thermal equilibration, we cut the initial sample perpendicular to the proposed loading direction at six equidistant places. Then in order to determine the grain size for 216 grains, we calculate the average distance between triple junctions. We also calculate the volume fraction of atoms that represent the grain boundary (GB) and the grain interior (GI) regions, which reveals that at least $80 \%$ of atoms represent the grain interior region. The atoms that represent hcp, fcc, or bcc structures and those that do not fit to any of them (other) are classified using Ackland analysis with Ovito. Because of the geometrical nature of the NC atomistic models, when the material grain size reaches below a critical grain size, primarily the grain boundary thickness influences whether the deformation mechanism will be inter- or intragranular deformation. Accordingly, we have found that even for a single element NC samples such as those in our models, two distinctive regions - wellordered and disordered atomic arrangements - are present.

All the samples are first relaxed using an NVT ensemble for $10 \mathrm{~ns}$, and then they are relaxed isotropically with NPT ensemble for another $10 \mathrm{~ns}$. Finally the models are relaxed again with an NVT ensemble for 5 ns before they are loaded for tensile deformation. All of the simulations are carried out with $1 \mathrm{fs}$ MD 
time step. A periodic boundary condition is adopted in all directions. A tensile deformation along the $X$ direction is performed at a strain rate of $1 \times 1081 / \mathrm{s}$, whereas deformation in the other two directions is carried out by maintaining a zero pressure by Parrinello-Rhaman method. Throughout our simulations the temperature is maintained at $300 \mathrm{~K}$. At a designated interval we collect snapshots of the atomic structure to analyze the microstructure.

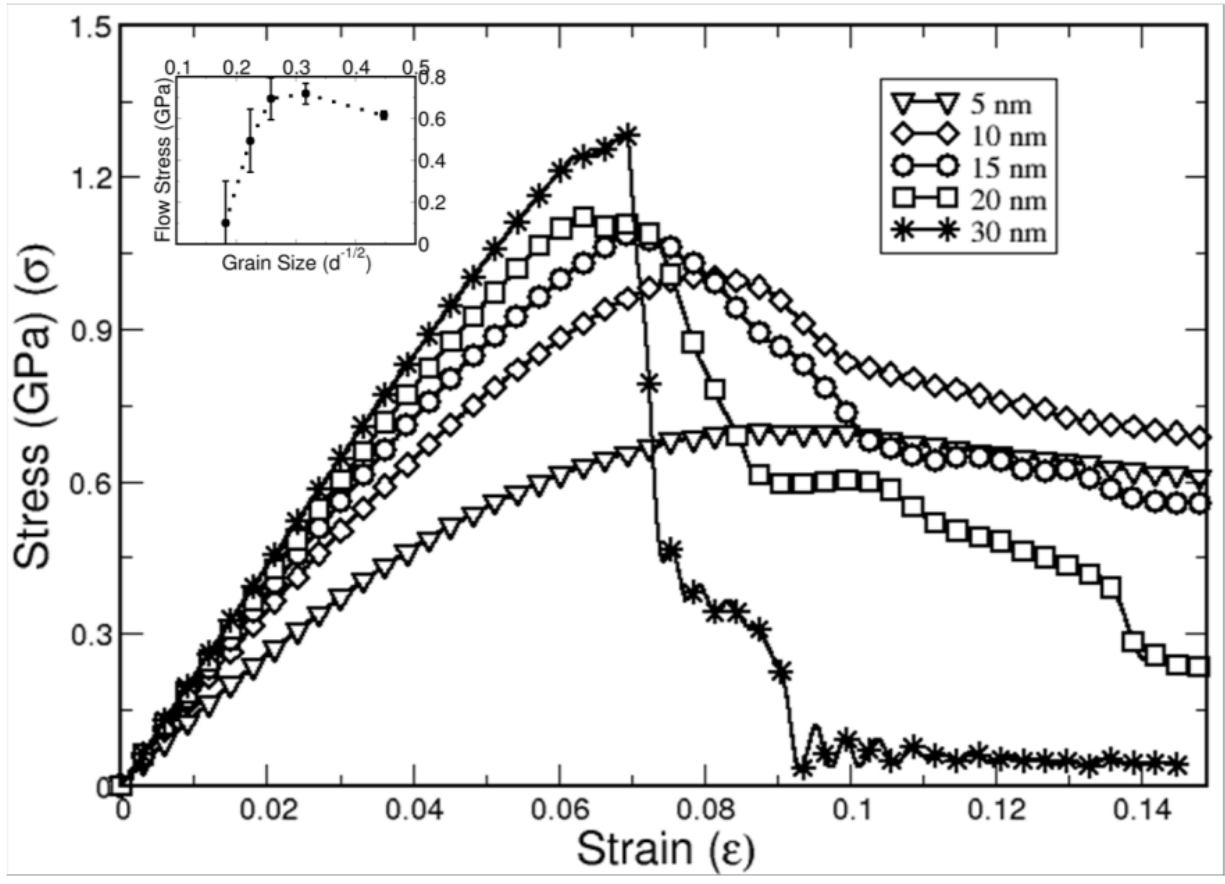

Figure 1.5.2. Stress strain response for 5 different samples, considered in the present study. The inset shows the variation of flow stress with respect to the inverse square root of grain size. The error bars are also shown for the flow stress for different grain sizes.

Figure 1.5.2 shows stress-strain curves under tensile loading for the five different samples, which confirm a decrease in the slope of the stress-strain curves with decrease in grain size. This is typically due to the fact that a smaller grain sample has larger grain boundaries and consequently releases local strain energy faster than does the larger grain sample. Hence, the resistance to intergranular deformation becomes smaller than the resistance to intragranular deformation as grain size decreases. Also, the decrease in the slope prior to the yield stress signifies the release of the stored elastic energy by local plastic deformation in grain boundary regions.

It can also be noted here that the values of the strain at the yield stress are larger for 5 and $10 \mathrm{~nm}$ grain size samples than for the other $(15,20$ or $30 \mathrm{~nm})$ grain size samples. This observation is potentially attributed to the fact that the yielding mechanism changes near the $10 \mathrm{~nm}$ grain size sample. When the grain sizes are smaller, the distance between triple points reduces. Hence the interactions between the grains are prevalent, suggesting greater intergranular deformation activity for smaller grain sizes. On the other hand, when the grain sizes are bigger and the triple points are farther apart, the interactions between the grains are less active. Hence, an intragranular deformation mechanism such as dislocation driven deformation mechanism becomes prevalent for larger grain size samples. The grain size 
dependency on the flow stress can also be confirmed from the inset of Fig. 1.5.2. Flow stresses for each sample are calculated by averaging out the stress after $8 \%$ strain. The inset of Fig. 1.5 .2 shows the variation of flow stress (in GPa) with respect to $d-1 / 2$ (in $n m-1 / 2$ ). The error bars associated with the calculations are also shown in the inset. It is important to note here that the stress is suddenly dropped for 30 and $20 \mathrm{~nm}$ grain size samples at 7.2 and $8 \%$ strain due to the fact that the samples actually fractured. Due to the inherent nature of our models where for 30 and $20 \mathrm{~nm}$ gain size samples have less grain boundaries, and to accommodate the applied stress for those samples the fracture starts to begin at the grain boundary regions.
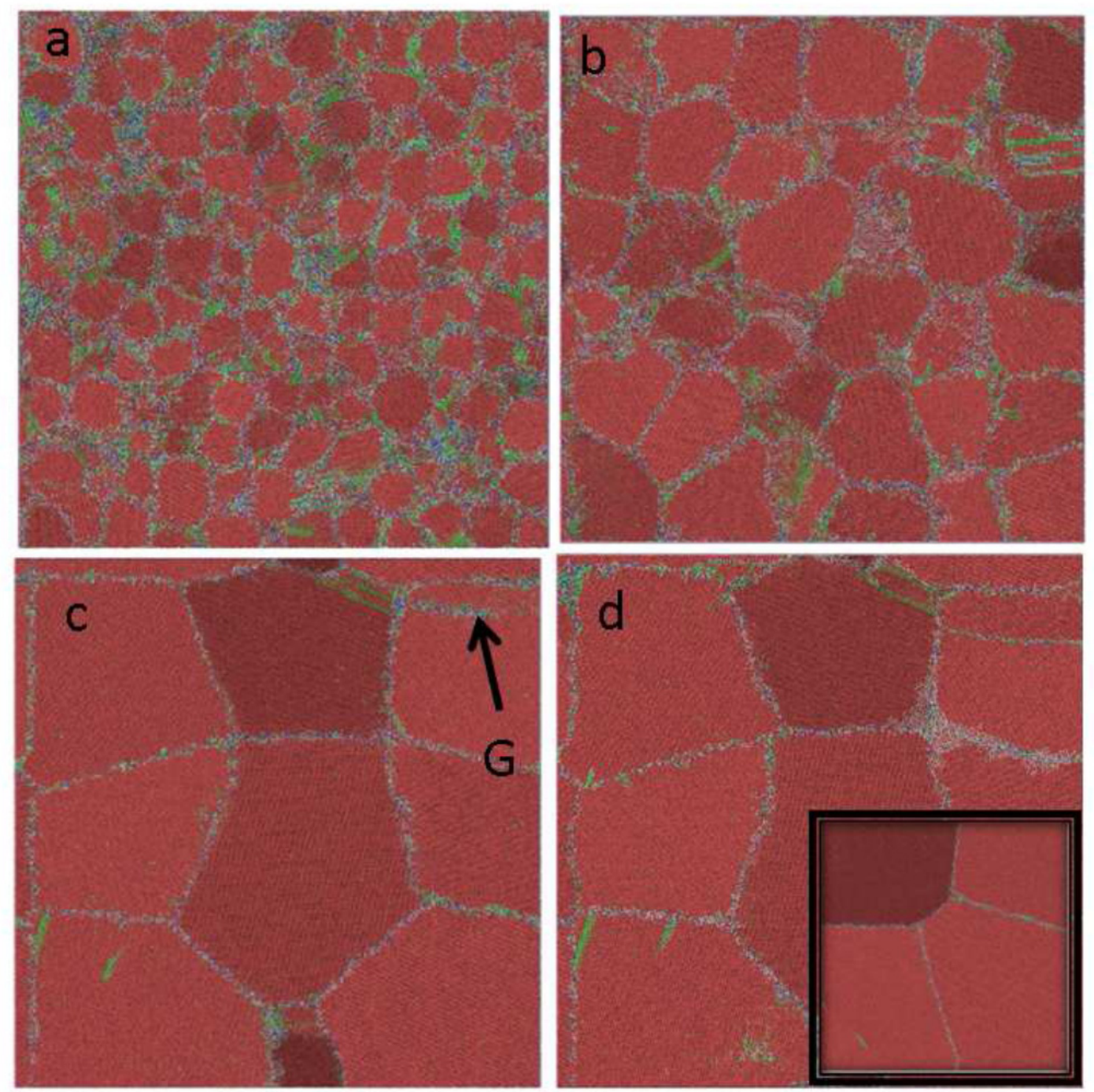

Figure 1.5.3 Microstructures of $5 \mathrm{~nm}$ (a), $10 \mathrm{~nm}$ (b), $20 \mathrm{~nm}$ (c) and $30 \mathrm{~nm}$ (inset of d) samples at $7 \%$ strain. (d) shows microstructure of $20 \mathrm{~nm}$ grain at $7.5 \%$ strain. Red atoms represent hcp structure, green atoms represent dislocations, and white atoms represent grain boundaries. Refer the text for detail. 
The flow stress increases as the grain size decreases up to $10 \mathrm{~nm}$ grain size, which illustrates the HallPetch effect. However the flow stress reduces for grain sizes smaller than $10 \mathrm{~nm}$, indicating the reverse Hall-Petch effect.

Investigation of microstructures at several strains can provide information regarding inter/intra granular deformation mechanism related to the grain size hardening. Figure 1.5.3 shows the microstructure of different samples at $7 \%$ strain. In order to expose the microstructure, we cut the sample halfway, perpendicular to the loading $(X)$ direction at different strains. It is clear from the figure that the number of dislocations becomes less as grain size increases (due to the fact that for bigger grain size samples, there are less grain boundaries). In Fig. 1.5.3 a) the microstructure of a $5 \mathrm{~nm}$ sample is shown, whereas in b) the same is shown for a $10 \mathrm{~nm}$ grain size sample.

Fig. 1.5.3 a) and b) indicate several dislocations perpendicular to the loading direction of the sample. Through examining the microstructure of the $5 \mathrm{~nm}$ grain size sample, we found that as the strain increases, the dislocations become more and more active near the GB region. Intergranular deformation becomes active when a dislocation pileup takes place near the grain boundary region. Such as a particular dislocation that is created within the grain, and then propagates and annihilates to the adjacent grain boundary is merely seen in such smaller grain samples.

The grain boundary diffusion is the dominant intergranular deformation mechanism for polycrystalline material. It has been also found that the intergranular deformation mechanisms change with the change in ambient conditions, such as strain rate, temperature, etc. In the present simulation conditions, such as high strain rate $10^{8} 1 / \mathrm{s}$ and moderate $300 \mathrm{~K}$ temperature, we find that the intergranular deformation mechanism is dominant for $5 \mathrm{~nm}$ size NC magnesium samples. Figures $1.5 .3 \mathrm{c}$ ) and d) show the microstructure of the $20 \mathrm{~nm}$ grain size samples at $7 \%$ and $7.5 \%$ strain. The development of a new grain is indicated by the letter 'G.' Also, some partial dislocations can be found ahead of an emitted dislocation of the $20 \mathrm{~nm}$ grain size sample (Fig. $1.5 .3 \mathrm{c}$ ), marked by G). These partial dislocations grow and annihilate at the adjacent grain boundary, eventually forming new grains. For the $10 \mathrm{~nm}$ grain size sample, the tendency of the dislocations to penetrate the grain boundary is also observed. The inset of Fig. $1.5 .3 \mathrm{~d}$ ) shows the microstructure for a $30 \mathrm{~nm}$ grain size sample at $7 \%$ strain. We found that the 30 $\mathrm{nm}$ grain is almost undistorted at this particular strain value, such that it deforms almost uniformly. The grain boundary and the distance between the triple junctions play an important role to the inter/intragranular deformation mechanism. As the surface-to-volume ratio increases with the decrease of grain size, the mean free path of the dislocation glide becomes small and transition of intragranular deformation to the next grain becomes difficult; however, intergranular deformation by sliding, migration or diffusion becomes easier. Currently we are evaluating to observe these effects with different samples at different strain levels.

\subsubsection{Crack growth in Polycrystalline $\mathrm{Mg}$}

To investigate the crack growth in polycrystalline magnesium under tensile deformation, we used Voronoi tessellation to generate the polycrystalline samples of three different sizes of grains: 6.4, 8.0, and $10.7 \mathrm{~nm}$. Two types of models were created, one without any crack in the system, and another with center crack. The initial crack is generated by removing the atoms from the nanocrystalline Mg models, 
such a way that the crack is periodic on the direction $(Y)$ perpendicular to the loading direction $(X)$. The initial crack length to sample width ratio is 0.2 .

All the samples were first relaxed using an NVT ensemble for $10 \mathrm{nS}$, and then they were relaxed isotropically with NPT ensemble for another $10 \mathrm{nS}$. Finally the models were relaxed again with NVT ensemble for $5 \mathrm{nS}$ before they were loaded tensile deformation. All of the simulations were carried out with $1 \mathrm{fs}$ MD time step. A periodic boundary condition is adopted in all directions. A tensile deformation along the $X$ direction is performed at a strain rate of $1 \times 10^{8} 1 / \mathrm{s}$, whereas deformation in other two directions was carried out by maintaining a zero pressure by Parrinello-Rhaman ${ }^{1}$ method. Throughout our simulations the temperature was maintained at $300 \mathrm{~K}$. At a particular interval of time we collect the snapshots of the atomic structure to analyze the microstructure.

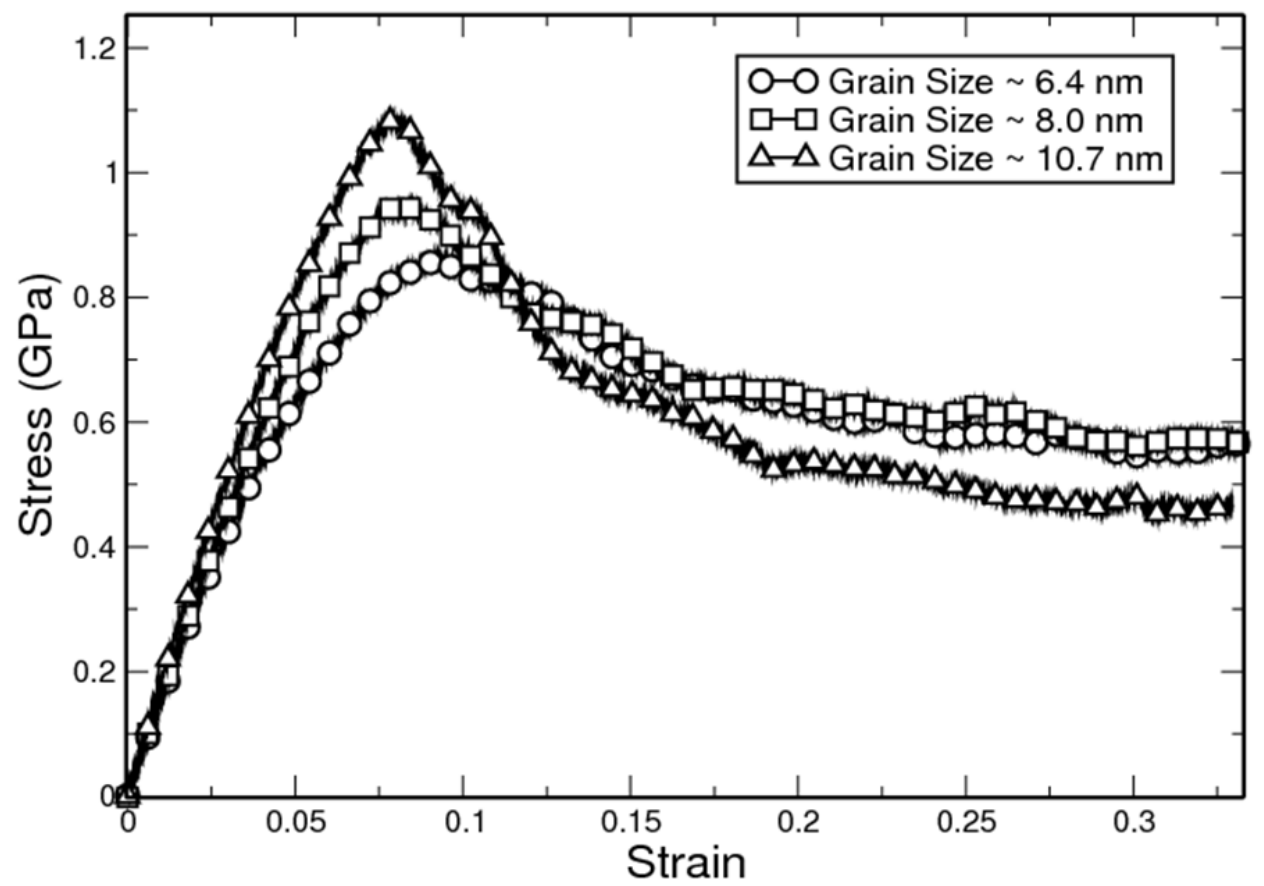

Figure 1.5.4. Stress strain response for different samples without crack.

Stress strain curves under tensile loading for the three different samples without any cracks in it are shown in Figure 1.5.4. From Fig. 1.5.4, a decrease in the slope of the stress-strain curves can be confirmed with decrease in the grain size. This is typically due to the fact that for a smaller grain sample there are larger grain boundaries, and consequently the smaller grain sample would release the local strain energy faster than the larger grain sample. Hence, the resistance to intergranular deformation becomes smaller than the resistance to intragranular deformation as grain size decreases. Also, the decrease in the slope prior to the yield stress signifies the release of the stored elastic energy by local plastic deformation in grain boundary regions. 


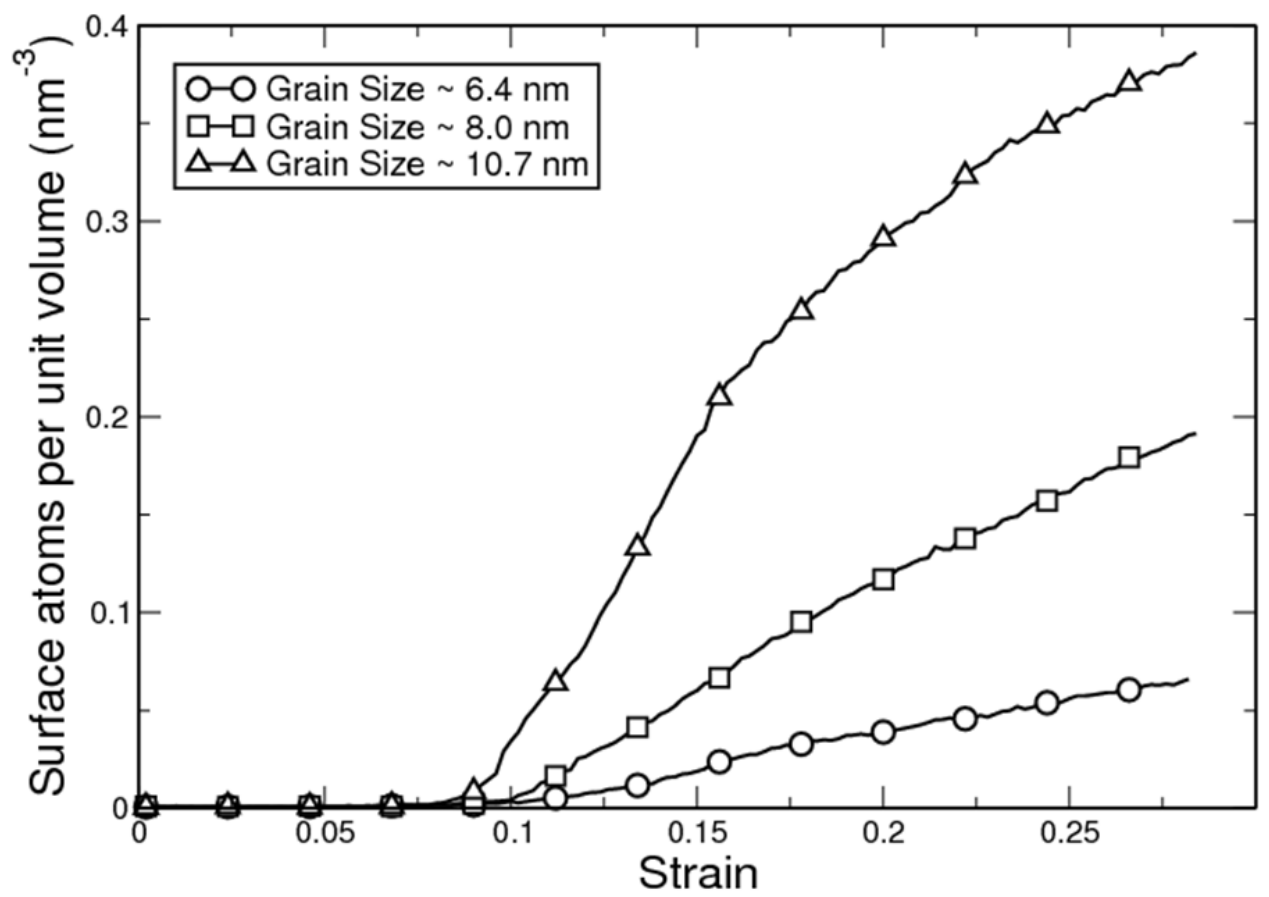

Figure 1.5.5. Evolution of volume averaged surface atoms for samples without crack.

Figure 1.5.5 shows the evolution of surface atoms with respect to strain for three different grain size samples without any cracks in it. Surface atoms are detected by using centrosymmetry parameter defined by Kelchner et al. ${ }^{2}$ as

$$
P=\sum_{i=1}^{N / 2}\left|R_{i}+R_{i+N / 2}\right|^{2}
$$

where the $R_{i}$ and $R_{i+6}$ are the vectors corresponding to the six pairs out of 12 nearest neighbors in the lattice. The $P$ values for the atoms in the fcc and hcp structures, dislocations, twinning boundaries and crack surfaces are all different so that extensive information about the crack growth and many defect behaviors can be obtained, as shown by Tian et al. ${ }^{3}$ We found that a centrosymmetry value of higher than 20 is sufficient to capture all the atoms that represent a crack or an internal crack in the system. We found that the evolution of the surface atoms for bigger grain size sample is quite large than the other two smaller grain size samples. Since for the smaller grain size samples the triple points are closer than the bigger grain size sample, with applied stress the smaller grains go through an intergranular deformation process to accommodate the strain. However for the case of $10.7 \mathrm{~nm}$ grain size sample, once a void is generated at the grain boundary region, the void has to grow in order to accommodate the applied strain. Hence the evolution rate of surface atoms is quite large for the $10.7 \mathrm{~nm}$ grain size sample compared to other two. 


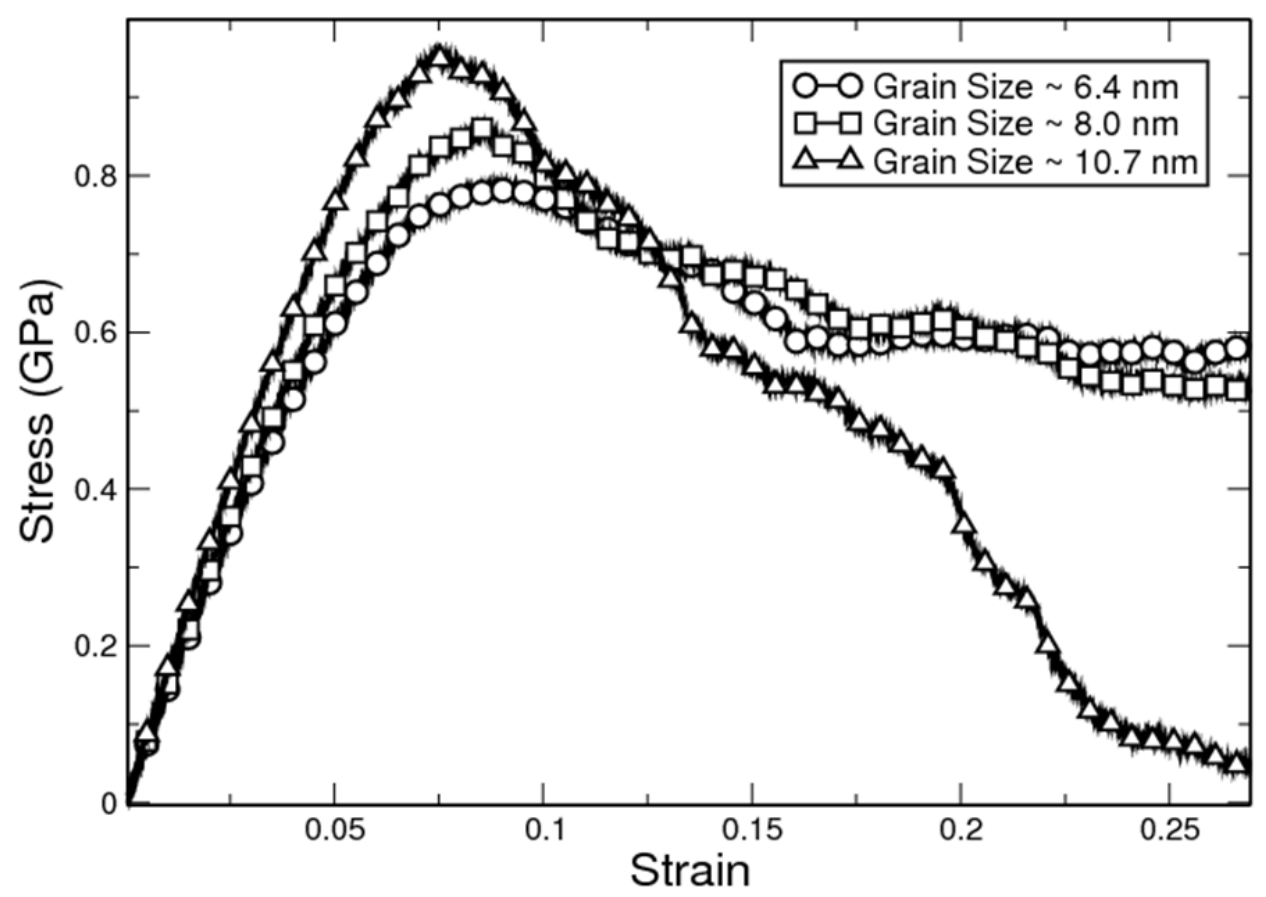

Figure 1.5.6. Stress strain response for different samples with a central crack.

Figure 1.5.6 shows the stress-strain response for all the three samples with a central crack (CC). Compared to the stress-strain response (see Figure 1.5.4) for samples without a crack, we found that the yield stress is decreased for each CC samples. However the basic nature of the response remains same, as expected. For 6.4 and $8.0 \mathrm{~nm}$ grain size samples, the flow stress remains same, however for $10 \mathrm{~nm}$ grain size sample, the flow stress is reduced significantly. This is due to the fact that after yielding, upon applied stress, cracks are nucleated in the $10.7 \mathrm{~nm}$ grain size sample. These cracks further grow with applied load and finally fracture the sample. The strain at which the yielding occurs are similar for 6.4 and $8.0 \mathrm{~nm}$ grain size samples. The strain at yield stress is higher for 6.4 and $8 \mathrm{~nm}$ grains compared to $10 \mathrm{~nm}$ grain size sample, similar to what has been found previously for samples without crack.

Figure 1.5.7 shows the evolution of surface atoms for CC samples with respect to strain. We found that the evolution of the surface atoms for $10.7 \mathrm{~nm}$ grain size sample is quite large than the other two smaller grain size samples, as found previously for samples without crack. Also, the atoms per unit volume for $10.7 \mathrm{~nm}$ grain size sample happens to saturate after $24 \%$ of strain, whereas the same quantity continues to increase even after that particular strain for the other two samples. This is due to the reason that the $10.7 \mathrm{~nm}$ grain size sample starts to fracture after $20 \%$ strain, and hence the number of surface atoms remains same or tends to saturate beyond that strain level. 


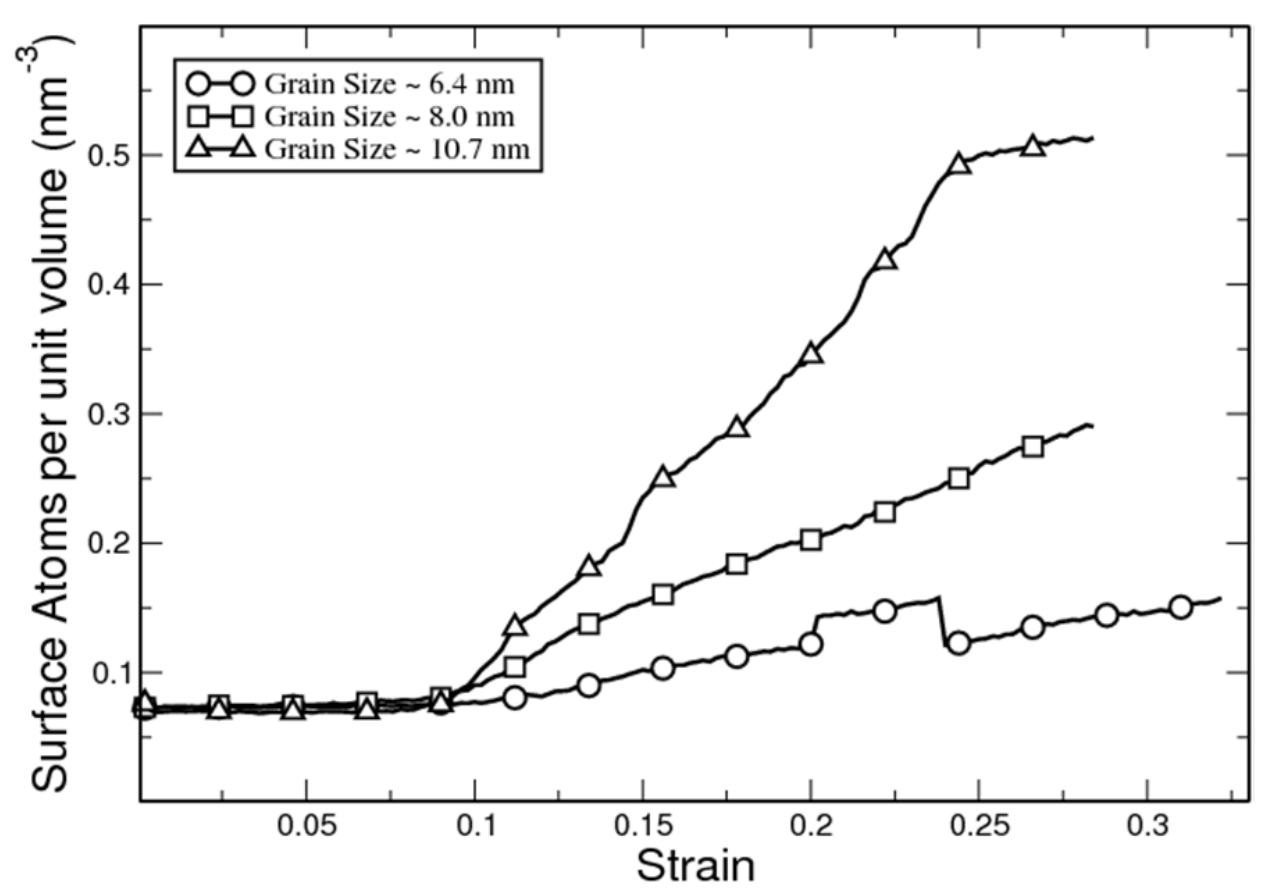

Figure 1.5.7. Evolution of volume averaged surface atoms for samples with a central crack.

We believe that the nature of the evolution of surface atoms for samples with edge cracks would be the same, except the fact that for edge crack samples the yield stress can be expected to be even lower than the case for samples with center cracks.

\subsubsection{Twins in Mg polycrystal nano-grains}

We performed atomistic simulations to study micro-structural responses of $\mathrm{Mg}$ polycrystal upon tensile loading. Molecular dynamics simulations have been performed with the code LAMMPS [1]. We used the many-body semi-empirical interatomic potential developed by Liu et al [X.Y. Liu et al., "EAM potential for magnesium from quantum mechanical forces," Modelling and Simula-tion in Materials Science and Engineering, 4 (1996)] using embedded atom method (EAM) [2] and force-matching method. We built 27 grains in the box in which the crystal orientations are randomly assigned. The position of the grain centers are also randomly decided and polycrystal are constructed by Voronoi tessellation procedures [3]. Over nine million atoms $(9,139,810)$ were used in the simulation, in a $60 \mathrm{~nm} \times 60 \mathrm{~nm} \times 60 \mathrm{~nm}$ box. No periodic boundary condition was employed in all three directions. Lattice parameters of $\mathrm{HCP} \mathrm{Mg}$ at temperature $300 \mathrm{~K}$ was used to build simulation box ( $\mathrm{a}=3.2176, \mathrm{c}=5.2111)$. We applied uniaxial tension in $x$-direction at a constant displacement rate corresponding to strain rate of $1 \times 10^{9} 1 / \mathrm{s}$ while the atoms on the left and right surfaces were held still. The atoms in the right region are held fixed in only $\mathrm{x}$-direction while the atoms in the left region are held fixed in all direction to apply tension.

The centro-symmetry parameter [Kelchner, C. L., Plimpton, S. J. \& Hamilton, J. C. Dislocation nucleation and defect structure during surface indentation. Phys. Rev. B 58, 11085 (1998)] is used to identify lattice defects such as twinning, dislocation, and grain boundaries. In all simulation structure plots, atoms are colored according to the relative magnitude of their centro-symmetry parameter. 
Figure 1.5.8 shows the initial configuration of the nanocrystalline $\mathrm{Mg}$ in our simulation. The deformation was performed such that the atoms on the right surface were moved at a constant displacement rate corresponding to a strain rate about $10^{9}$ per second, while the atoms on the left surface were held still. As the strain increases, dislocation slip and deformation twinning are activated. Figure 2 shows a top view of a thin slice of the system that is parallel to the top surface at time step 80,000 (80 ps). Partial dislocation and the associated stacking faults (SFs) are presented in blue. The dislocations are always nucleated from the grain boundaries (GB) and the free surfaces, and propagate across the grains. Deformation twins can be seen in the top region enclosed in an eclipse, and in the lower-right region enclosed in a circle. The twins are nucleated at GBs or triple junctions of the GBs. Enlarged views of the twins and the SFs are shown in Figure 3 to 5.

To identify the twinning modes, we tilted the lattices meticulously such that the twinning elements, i.e., the twinning plane and the plane of shear, can be well defined. For the most commonly observed two twinning modes, i.e., $\{10 \overline{1} 2\}<10 \overline{1} \overline{1}>$ extension twin and $\{10 \overline{1} 1\}<10 \overline{1} \overline{2}>$ contraction twin, we tilted the twins such that the basal planes of the parent and the twin and the twinning plane are all perpendicular to the figure plane, and the plane of shear is exactly parallel to the figure plane. In this direction, the zone axis is parallel to the $<1 \overline{2} 10>$. In Figure 3 , a $\{10 \overline{1}\}<10 \overline{2}>$ contraction twin is shown. The twin boundary is exactly parallel to the twinning plane, and the mirror symmetry is well defined. A two-layer zonal twinning dislocation [Li and Ma, Acta Mater 57, 2009:1734] can be identified, which controls the migration of the twin boundary.

As shown in Figure 4, the $\{10 \overline{1} 2\}<10 \overline{1} \overline{1}>$ extension twin presents a very different twin boundary structure than the $\{10 \overline{1} 1\}<10 \overline{1} \overline{2}>$ contraction twin. A significant deviation between the twinning plane and the actual twin boundary can be seen, indicating that large atomic shuffles [Bilby and Crocker, Proc Roy Soc. A, 288 (1965) 240-255; Christian and Mahajan 1995, Li and Ma, PRL 2009] are involved in twinning. The twin and the parent lattices still satisfy the twin orientation relationship, in spite of the deviation.

Most of the partial dislocations and the associated SFs in our simulation are on the basal plane. As seen in Figure 5, multiple partial dislocations and the SFs are nucleated at the GB (the red atoms on the left) and propagate toward the interior of the grain. The SFs are shown in blue. In the faulted regions, the stacking of the basal planes is shifted from hcp to fcc stacking. Bounding the SFs are the leading partial and the trailing partial. The width of the SFs can be much larger than the equilibrium width $(0.2 \sim 0.3 \mathrm{~nm})$ before the trailing partial dislocation is nucleated, for example, the SF in the middle. As soon as the trailing partial is nucleated, it erases the SF quickly and the SF width is reduced to close to the equilibrium width. 


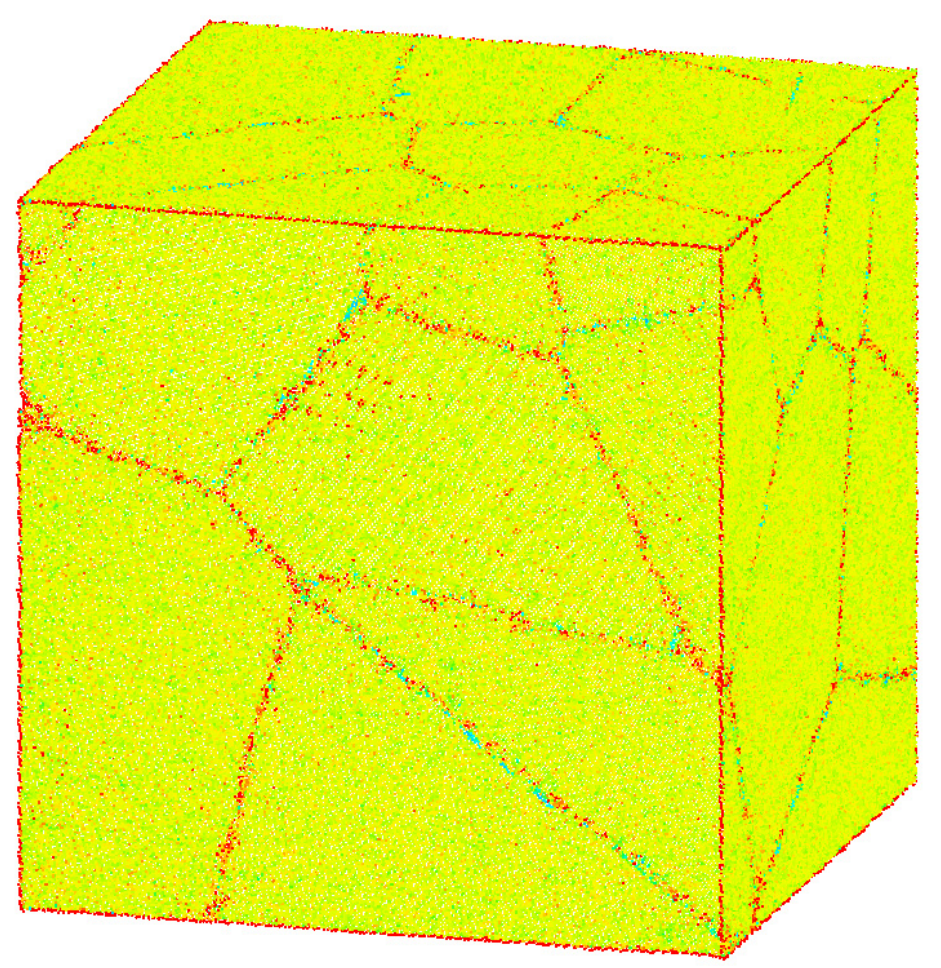

Figure 5. Initial configuration of nanocrystalline $\mathrm{Mg}$. The system contains about 10 million atoms (a total of 27 grains with an average grain size $20 \mathrm{~nm}$ ). The system is colored according to the center symmetry parameter (CSP). Atoms at grain boundaries and the surfaces have different CSP values than those at the perfect lattice points. A tensile strain was applied at a strain rate $\sim 10^{9} / \mathrm{s}$. 


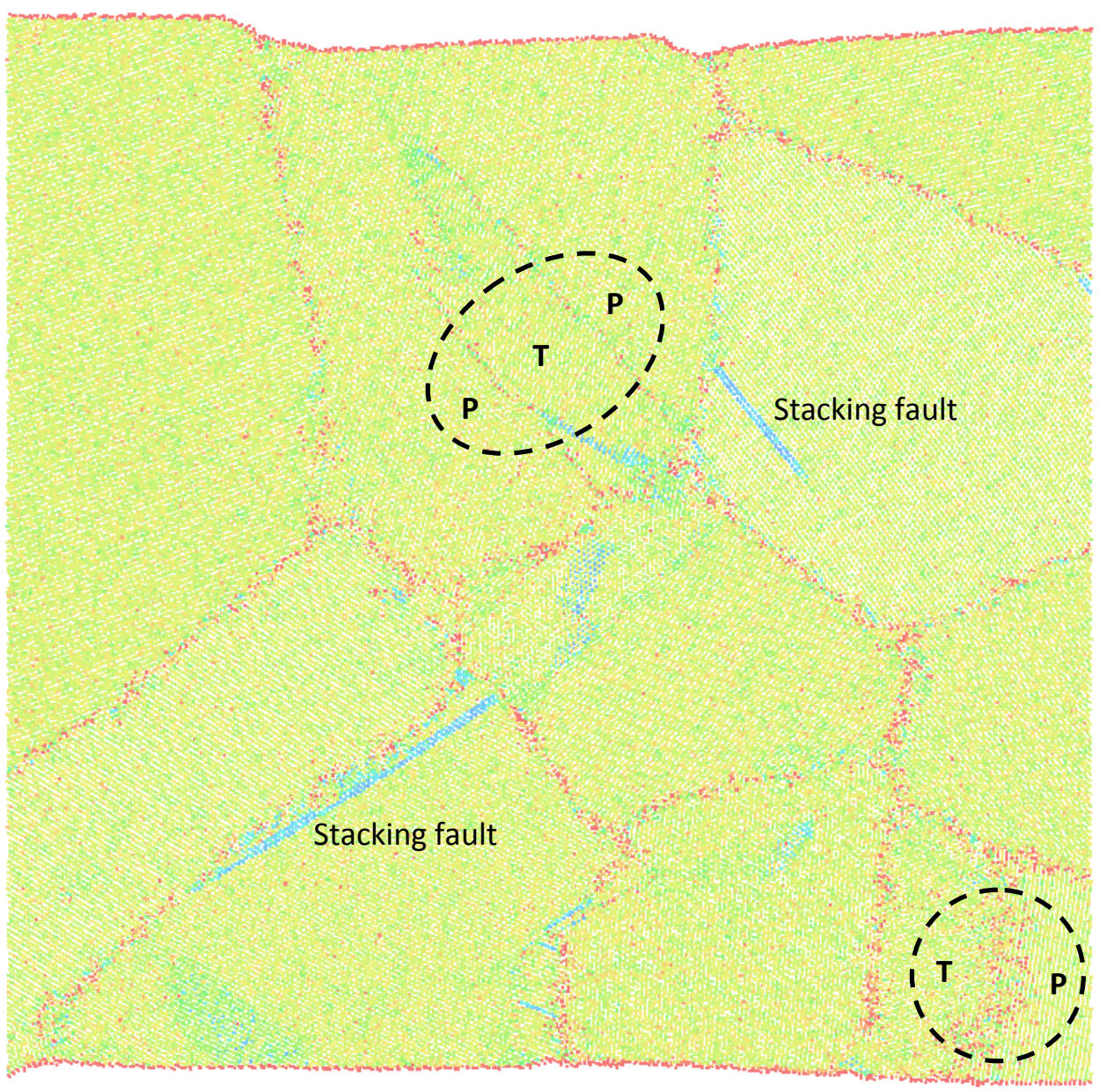

Figure 6. Deformation twins and stacking faults. Only a thin slice $(1 \mathrm{~nm})$ of the system is shown. Enclosed in a dash-lined ellipse are $\{10 \overline{1} 1\}<10 \overline{1} \overline{2}>$ contraction twins (an enlarged view can be found in Figure 3). The interfaces between the twin ( $T$ ) and parent (P) are sharp and strictly on the $\{101 \overline{1}\}$ plane. Enclosed in a dash-lined circle are $\{10 \overline{1} \overline{2}\}<10 \overline{1} \overline{1}>$ extension twins (an enlarged view can be found in Figure 4). The twin/parent interface is irregular for this particular twinning mode. 


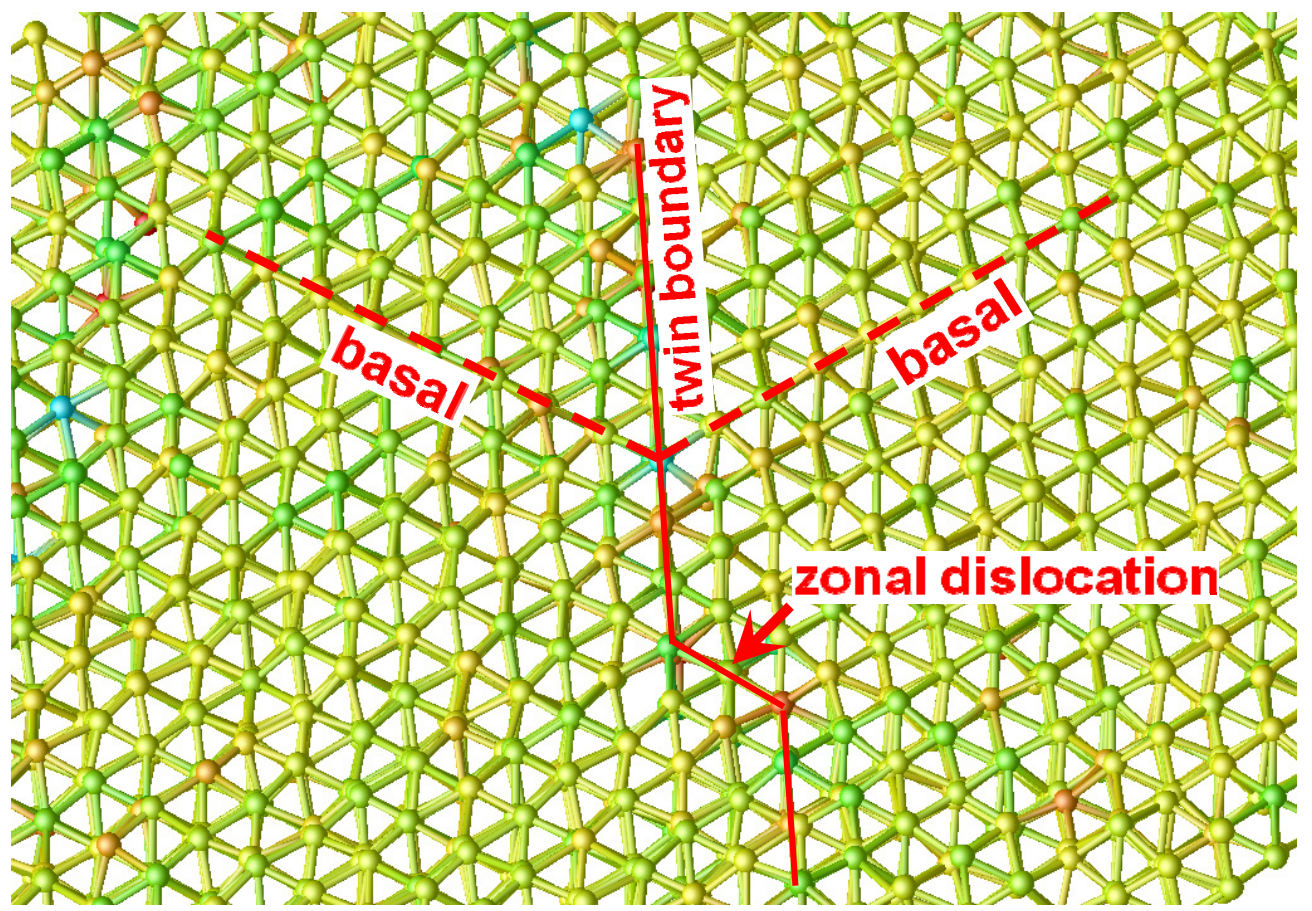

Figure 7. Enlarged view of the $\{10 \overline{1}\}<10 \overline{1} \overline{2}>$ contraction twin. The lattices are tilted such that the basal planes of the twin $(T)$ and the parent $(P)$ and the twinning plane are all perpendicular to the figure plane, i.e., the zone axis is along the $<1 \overline{2} 10>$ direction. The twin boundary is exactly along the $\{10 \overline{1}\}$ plane. A two-layer zonal twinning dislocation can be identified (indicated by an arrow). 


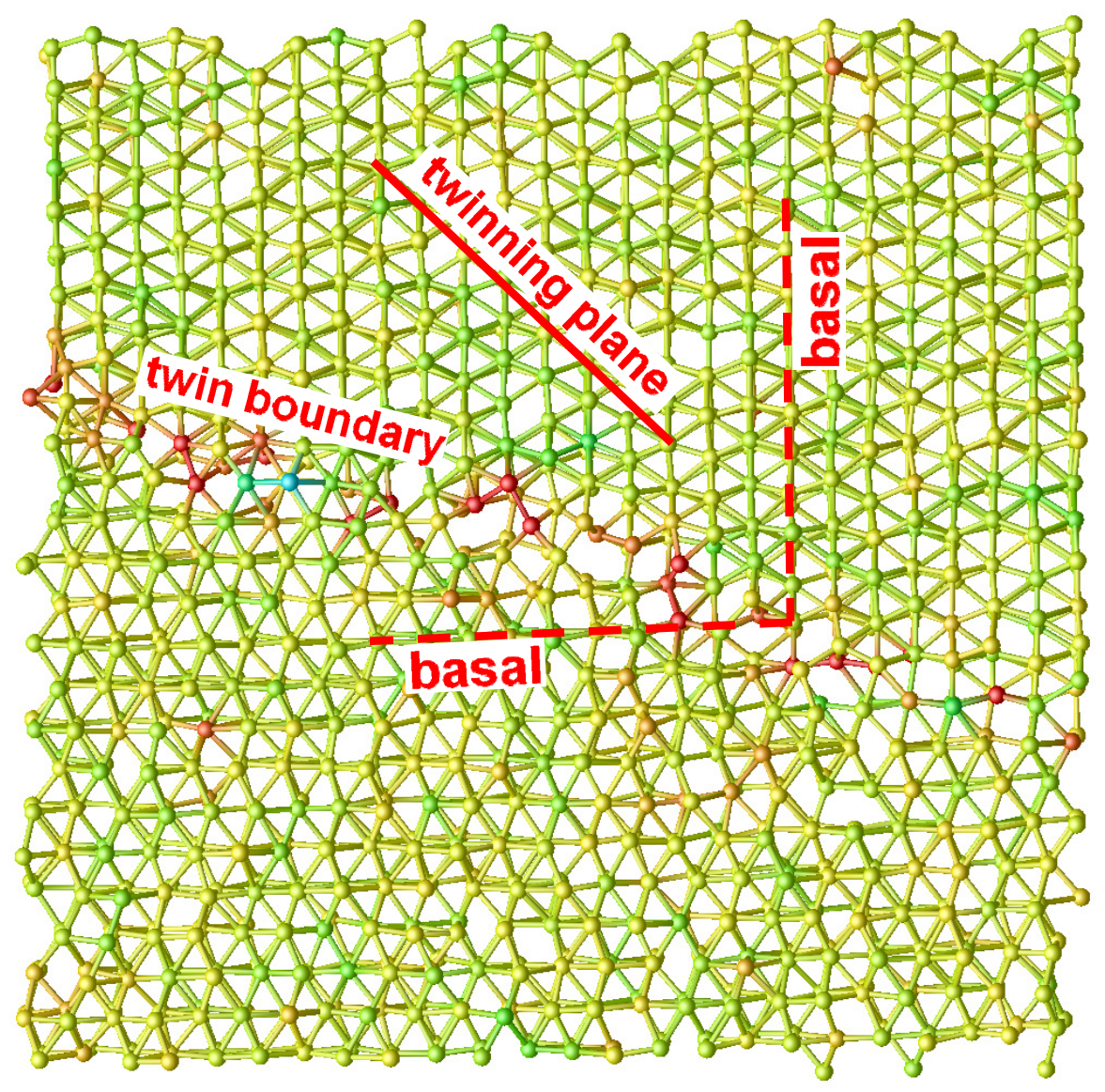

Figure 8. Enlarged view of the $\{10 \overline{1} 2\}<10 \overline{1} \overline{1}>$ extension twin. The lattices are tilted such that the basal planes of the twin $(T)$ and the parent $(P)$ and the twinning plane are all perpendicular to the figure plane, i.e., the zone axis is along the $<1 \overline{2} 10>$ direction. Note the twin boundary (red atoms) deviates from the $\{10 \overline{1} 2\}$ twinning plane (marked as a solid line) which is common for this particular twinning mode. 


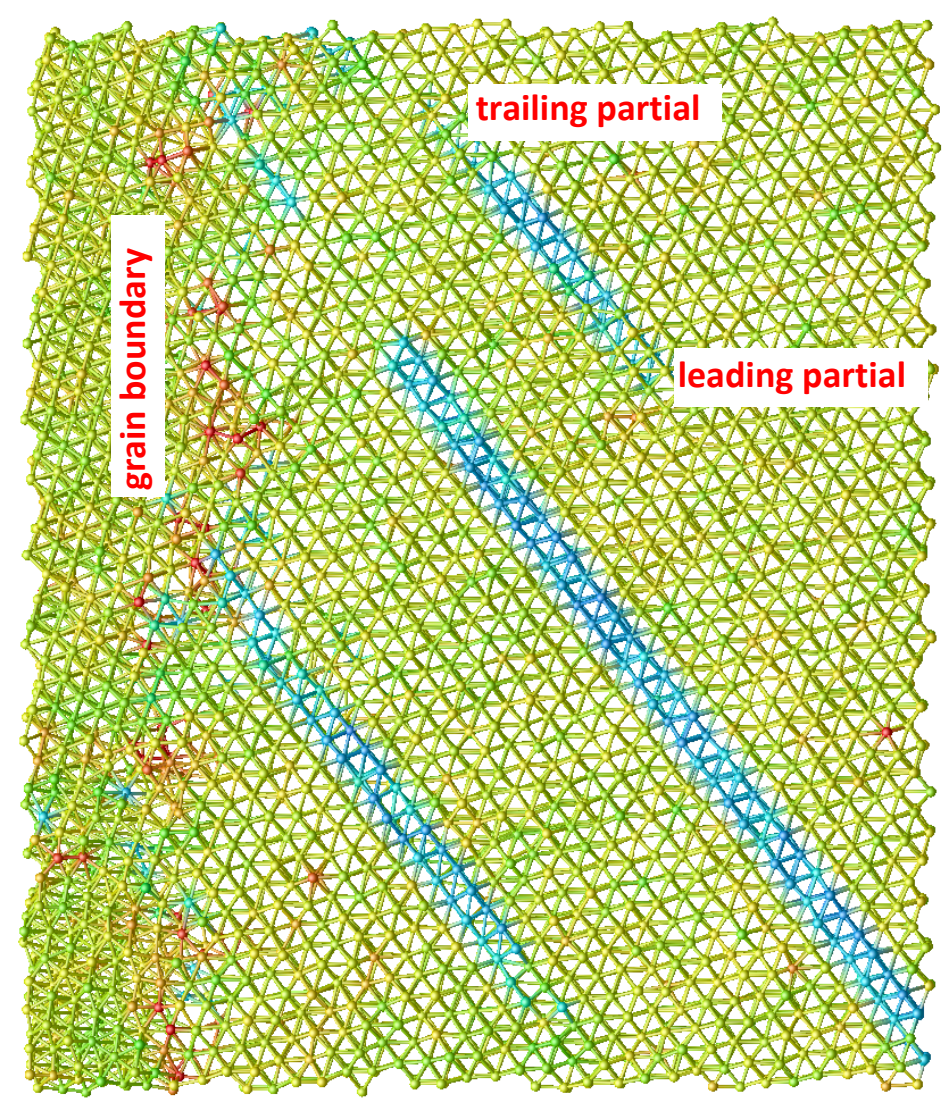

Figure 9. Multiple basal stacking faults (SFs) were observed. The SFs are emitted from the grain boundary (shown by the red atoms on the left).

\subsubsection{Homogenous Twin Nucleation}

The objective is to better understand atomic-scale microstructural influences on twin and dislocation nucleation mechanisms in magnesium single crystals and alloys. To meet this research objective, we used molecular dynamics simulations of uniaxial tensile deformation of magnesium as an initial model system. The first goal is to set up the correct simulation methodology for studying both homogeneous and heterogeneous twin/dislocation nucleation in magnesium. Here, a parallel molecular dynamics code (LAMMPS, [1]) is used to deform the atomistic models. The Sun et al. [2] embedded-atom method potential for $\mathrm{Mg}$ is employed in this study. First, the configuration is equilibrated using $\mathrm{MD}$ in the isobaric-isothermal (NPT) ensemble at a pressure of 0 bar and the temperature of interest. Next, the configuration is deformed in uniaxial tension at a constant strain rate of $10^{7}-10^{9} \mathrm{~s}^{-1}$ with a zero stress condition for the other two boundaries. For mechanical properties, the system stress is calculated using the virial definition. The stress required for twin or dislocation nucleation is defined as the maximum uniaxial tensile stress. Figure 4 shows a stress-strain curve for the $<2116>$ loading orientation at a strain rate of $10^{9} \mathrm{~s}^{-1}$ and a temperature of $100 \mathrm{~K}$. As shown in Figure 30, visualization of selected tensile axis 
orientations along the appropriate planes showed that twins/dislocation are nucleated at a displacement very close to the maximum tensile stress for all single crystal models. In most cases, twins appeared to nucleate slightly before the maximum tensile stress is reached $(<1.0 \%$ below the maximum tensile stress.

The second goal is to use atomistic simulations to study homogeneous nucleation of twins and dislocation in single crystal magnesium. A 3D periodic computational cell with a minimum length of 20 $\mathrm{nm}$ per side is used herein to investigate homogeneous twin nucleation in single crystal $\mathrm{Mg}$ under uniaxial tension. Figure 31 shows the tensile axis for the 13 crystallographic orientations examined in this work, within the basic stereographic triangle with $\langle 0001\rangle,\langle 10-10\rangle$, and $\langle 1-210\rangle$ vertices. Each single crystal model is deformed under uniaxial tension at a temperature of $100 \mathrm{~K}$. The expected twinning system is the $\{10-12\}<10-1-1>$ twin system for all tensile axis orientations in the $<0001>-<10$ $10>-<1-210>$ triangle.

The stress-strain curves for the 13 crystallographic loading orientations are shown in Figure 1.5.4. As shown in Figure 1.5.4, the single crystals are loaded in tension and the stress increases until a peak stress, at which either a dislocation, twin, or void/crack nucleates within the single crystal. For each loading orientation, the deformation mechanism was characterized by examining the relevant slip/twin planes and directions to ascertain what defect corresponded to the peak nucleation stress. The stressstrain curves are colored according to deformation type. In many loading orientations, the stress required to nucleate dislocations tended to be lower than that for twins. In the curve with the highest nucleation stress ( $<0001>$ orientation), neither dislocations nor twins were observed to nucleate, but rather the material fractures via void nucleation and coalescence. It should be noted that these stresses are the uniaxial tensile stresses required for the nucleation events; the resolved stresses are lower. 


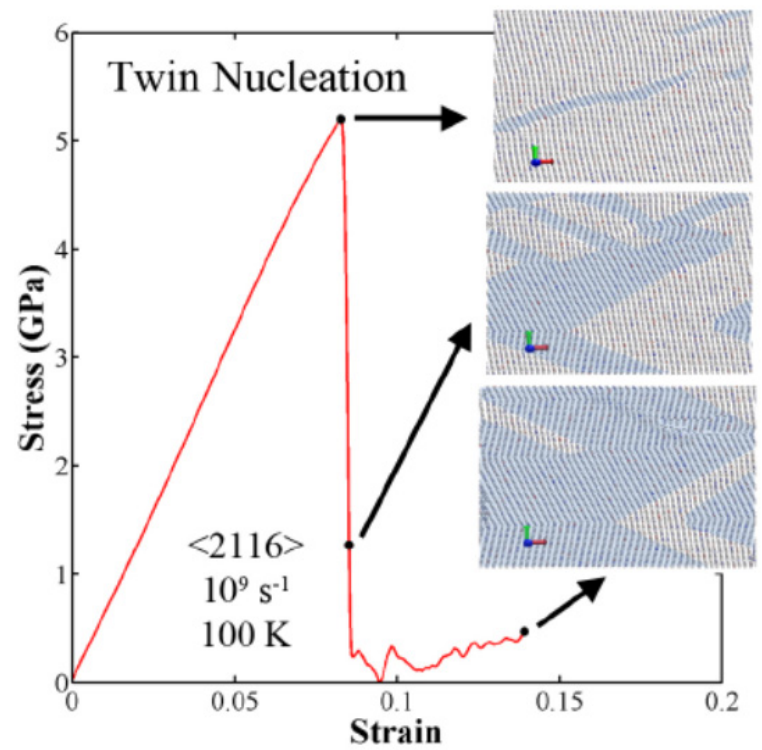

Figure 1.5.4. An example stress-strain curve showing twin nucleation at the maximum tensile stress.

Of the 13 crystallographic orientations studied, five orientations nucleated twins and seven orientations deformed via slip. The final orientation was loaded in the basal $<0001\rangle$ direction and showed no evidence of twinning or slip. Interestingly, this orientation has the highest likelihood of twinning under tensile stress, yet no twinning is observed. Again, recall that the single crystal models used here are absent of point defects, pre-existing dislocations, or free surfaces. Perhaps the lack of twin nucleation suggests that other point, line, or planar defects need to be present in the lattice in order to spur the twin nucleation process. Additionally, analysis of resolved stress components may shed light on this phenomenon 


\section{Loading Orientations}
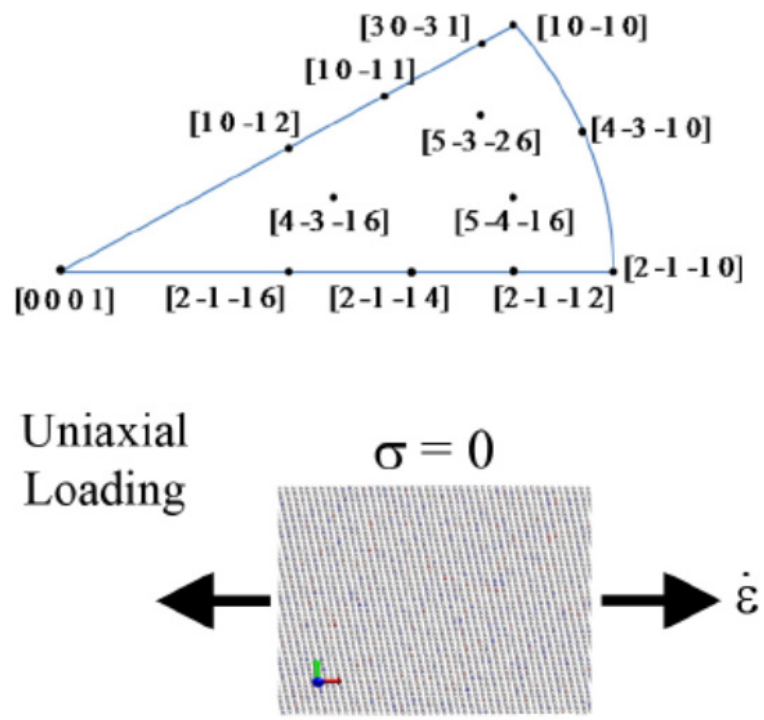

Figure 1.5.5. Uniaxial loading orientations and conditions for single crystal deformation simulations.

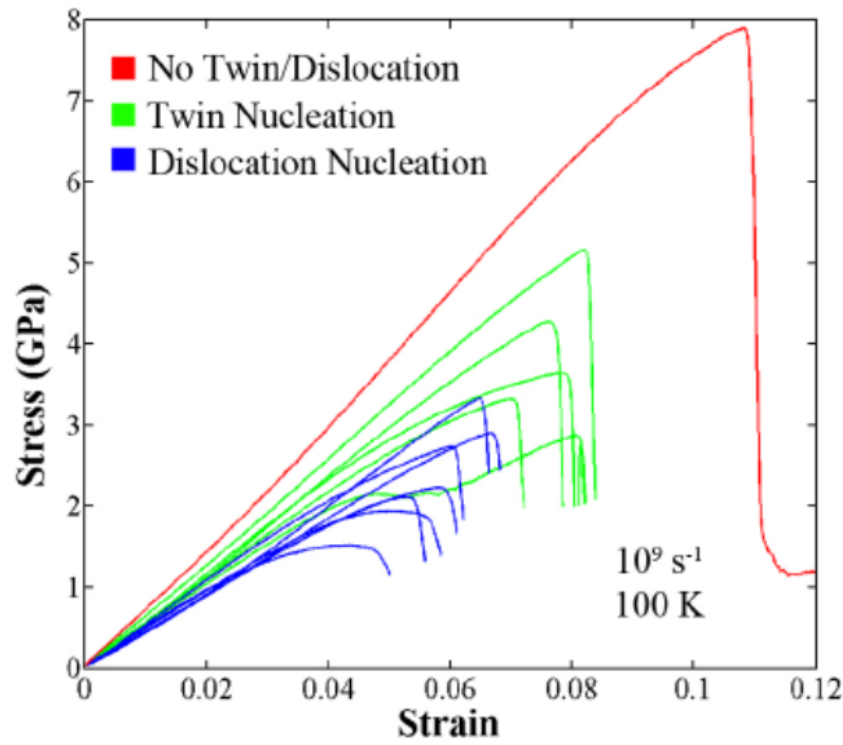

Figure 1.5.6 Stress-strain curves for all single crystal models.

\subsubsection{Non-planar $\{10 \overline{1} 2\}$ Twinning Nucleation in Mg single crystal}

We observed nucleation of radially growing $\{10 \overline{1} 2\}$ twinning under tensile loading in magnesium rectangular wire system without artificial creation of an twinning in atomistic molecular dynamic simulation. The twinning nucleation mechanism is very different from the conventional twinning mechanism in that the twin nucleates from a point source rather than the fault plane following the 
partial dislocation line in FCC crystal. The wire axis is normal to basal plane of Mg crystal. The tensile deformation in c-axis nucleates $\{10 \overline{1} 2\}$ twinning starting at the corner of square of cross section of the wire. The twin boundary is spherical at the beginning and becomes linear boundaries in $\{10-12\}$ planes as time goes by.

Figure 1 shows the system configuration we used in the simulation. The systems of different sizes give the same result such as 11520 atoms in $9.33 \mathrm{~nm} \times 5.51 \mathrm{~nm} \times 5.09 \mathrm{~nm}, 92160$ atoms, 336960 atoms, 1516320 atoms in $56 \times 25 \times 25 \mathrm{~nm}$ simulation box. A significant size dependence is not observed in those systems. The embed atom method magnesium potential of Sun et. al.[4] is used. No periodic boundary conditions are used in $x, y$, and $z$ directions. The atoms in $1 \mathrm{~nm}$ of left and right ends of the simulation box are fixed after incremental strains are applied. The rest of atoms are free to move after the strain applications. The constant NVE thermodynamics are applied where $\mathrm{N}$ represents the total number of atoms in the system, $V$ represents the volume of the system, and $E$ represents the total energy of the system. The Initial temperatures are set to $100 \mathrm{~K}$ by assigning the Gaussian random distribution of velocities on atoms. It takes about 5 ps for the system to reach the equilibrium condition. The ramping velocities are applied to all atoms correstponding to the strain rate of $1 \mathrm{G} / \mathrm{s}$.

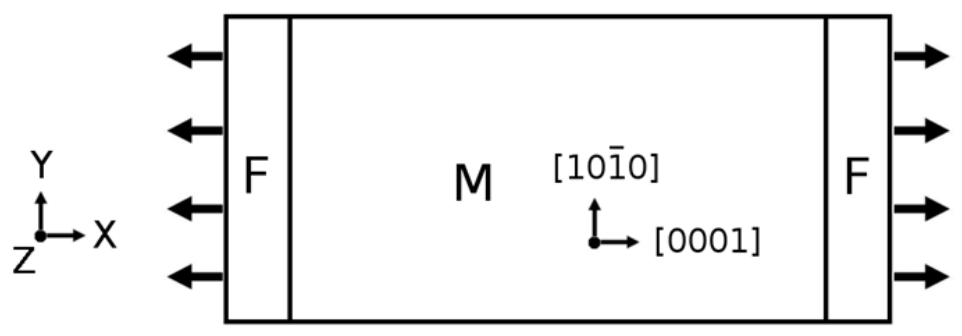

FIGURE 1. THE SCHEMATIC OF THE SIMULATION CONFIGURATION. TENSILE STRAINS ARE APPLIED ON MG CRYSTAL IN [0001] DIRECTION. NO PERIODIC BOUNDARY CONDITIONS ARE APPLIED IN ALL $X, Y$, AND $Z$ DIRECTIONS. THE LETTER F INDICATES THAT ATOMS IN THE REGION ARE FIXED AND M INDICATES THAT ATOMS ARE MOBILE IN THE REGION IN MOLECULAR

Ackland method[5] is used to identify the local atomic structures and a corresponding index is assigned to each atom --- 0 for unknown, 1 for BCC, 2 for FCC, 3 for HCP, and 4 for icosahedral structure relation with neighbor atoms. The blue, cyan, green, yellow, red colors are assigned for the unknown, BCC, FCC, $\mathrm{HCP}$, and icosahedral structures, respectively, in the figures.

When the strain reach about 0.0535 , the defect surface of quad sphere shape nucleates from the edge in the middle and propagates away from the nucleation point. The defect surface leaves behind a HCP crystal structure which has different crystal oriention from the parents. 


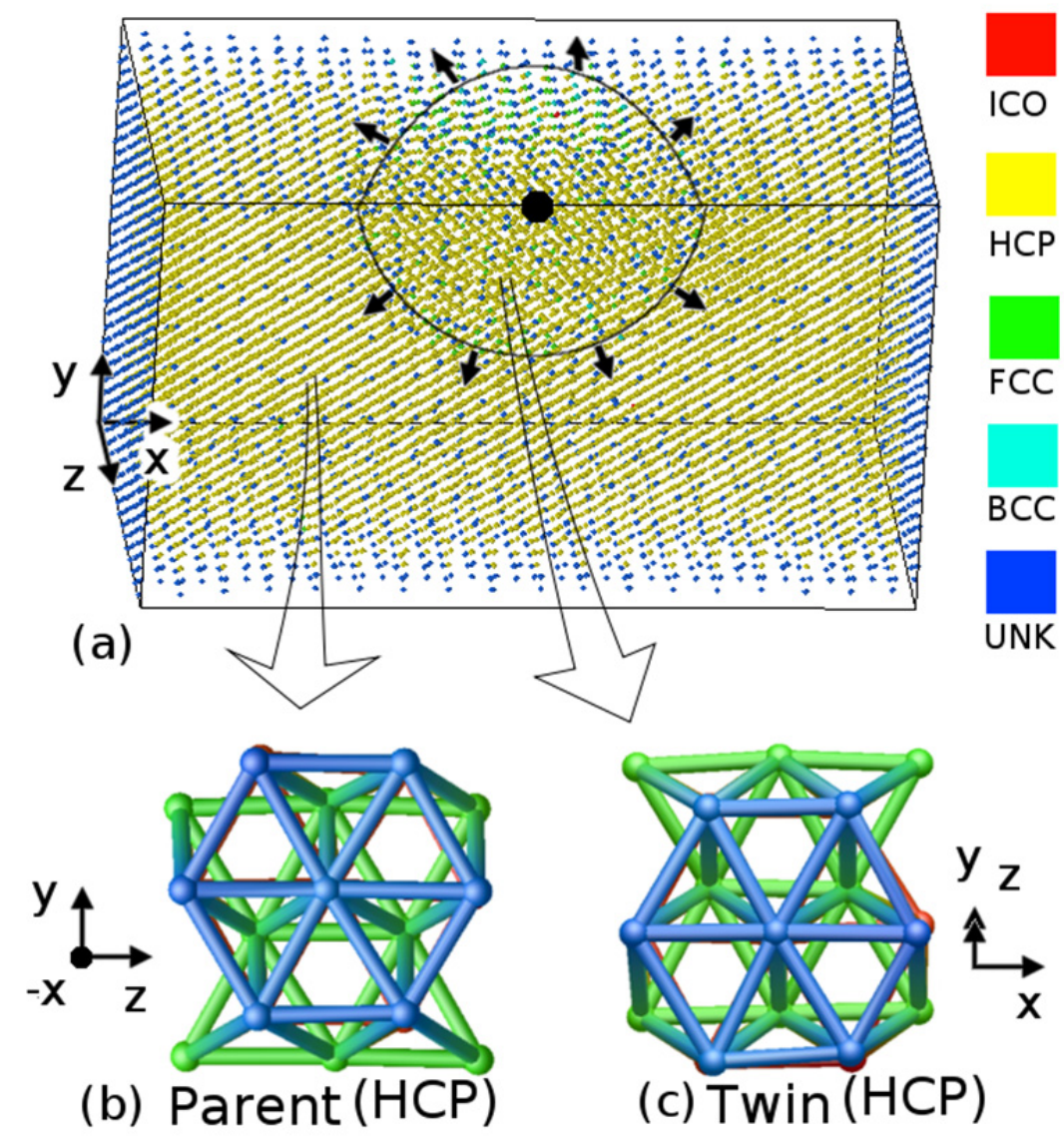

FIGURE 2. (COLOR ONLINE) (A) TWIN NUCLEATION AND SPHERICAL GROWTH OF TWIN REGION FROM THE EDGE IN THE MIDDLE INDICATED BY A BLACK DOT. THE COLORS OF ATOMS REPRESENT DIFFERENT ATOMIC STRUCTURES CLASSIFIED BY ACKLAND METHOD[3]. THE ICO REPRESENT ICOSAHEDRAL STRUCTURE AND THE UNK UNKNOWN. THE TWIN STRUCTURES ARE HCP INDICATED BY YELLOW AS WELL AS PARENT. (B) THE STRUCTURES OF PARENT IS MAGNIFIED AND VIEWED FROM X DIRECTION. THE BASAL PLANES OF PARENT IS NORMAL TO X-AXIS. (C) TWIN REGION IS MAGNIFIED AT SPECIFIC VIEW POINTS TO SHOW THE BASAL PLANES OF TWIN. THE COLORS IN (B) AND (C) REPRESENT DEPTH.

Fig. 2 shows three dimensional nucleation of a twin. The colors represent the different structures classified by Ackland method [5]. The twin structure has the same HCP as the parent and has a different crystal orentation. The twin boundary is distinguished by different colors. The nucleated twin grows spherically from the nucleation point of the edge in the middle of the simulation box indicated by a black dot in the figure. 

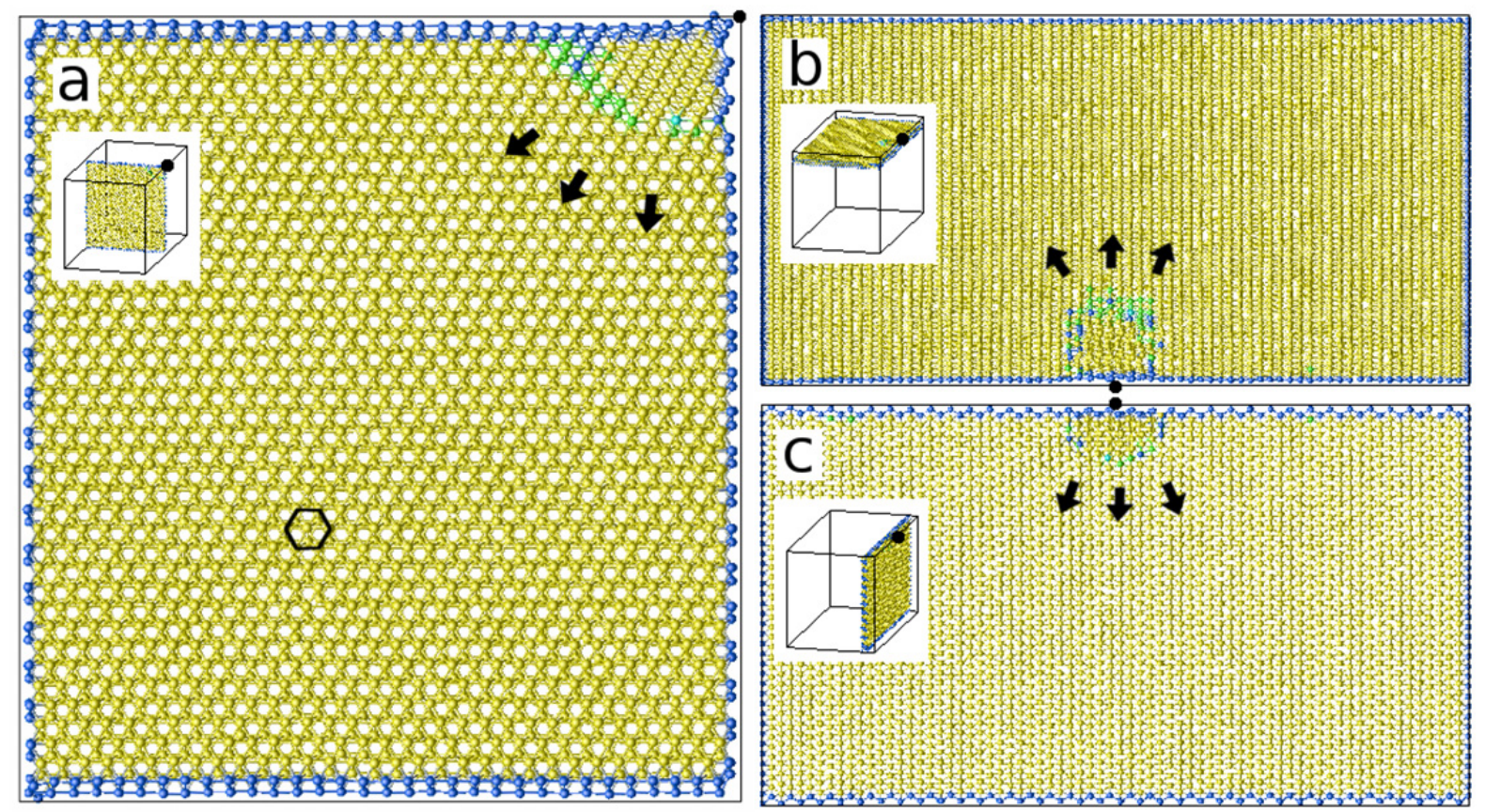

FIGURE 3. (COLOR ONLINE) LEFT SIDE VIEW (A), TOP VIEW (B), FRONT VIEW (C) OF A FEW LAYERS OF SLAB CUTS CONTAINING THE TWIN NUCLEATION POINT COLORED BY ACKLAND METHOD. THE INNER FIGURES SHOW THE SLAB CUTS FOR EACH VIEW. BLACK DOTS REPRESENT THE TWIN NUCLEATION POINT. BLACK ARROWS INDICATE THE TWIN GROWTH DIRECTIONS.

Fig. 3(a) shows the side view of slab cut containing the twin nucleation point. The inset figure shows how the slab is cut in the whole simulation box. The black dots indicate the twin nucleation points. The black arrows indicate the twin growth directions. The twin boundary The front, top, and side view of slab cuts containing the twin nucleation point show in Figure 3 that the twin grow spherically in early stage of twin nucleation. The twin nucleates from top right conner and propergates spherically toward bottom left conner. As the twin region grows, two prismatic planes in parent region are aligned and form one basal plane in twin region. The angle between parent basal plane and twin basal plane look like right angle. The angle between parent and twin basal planes in $\{10 \overline{1} 2\}$ twin are $87^{\circ}$ according to theory. The twin boundary in the $-z$ direction is faster in speed and wider in width than $-y$ direction. 


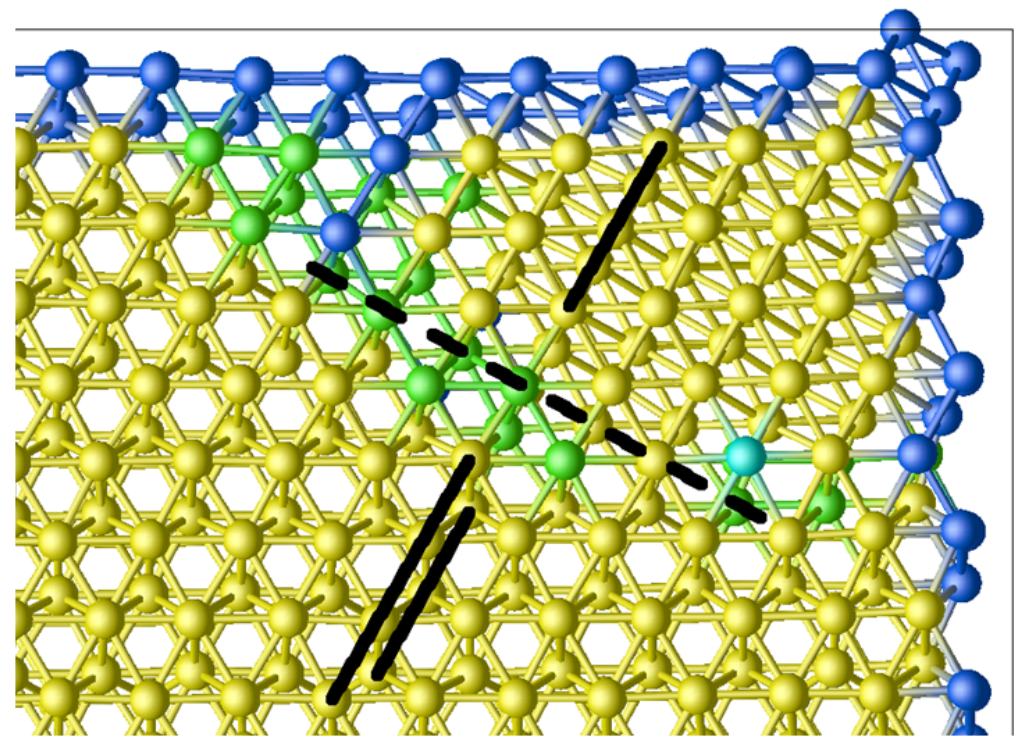

FIGURE 4. A shuffle motion of the parent basal planes, colored by Ackland method. Dotted line represents the plane of shear.

Fig. 4 shows the magnified left side view of twin boundary structure. The dotted black line indicates the twin boundary which is propagating from the right top conner to the bottom left corner. A pair of black solid lines in parent will be aligned with the black solid line in the top right conner of the twin region. The black solid line in the twin region is the basal plane of the twin structure. The prismatic planes in the parent hcp structure transform to the basal planes in the twin structure. The twinning boundary in the $z$ direction is much thicker than the others. 


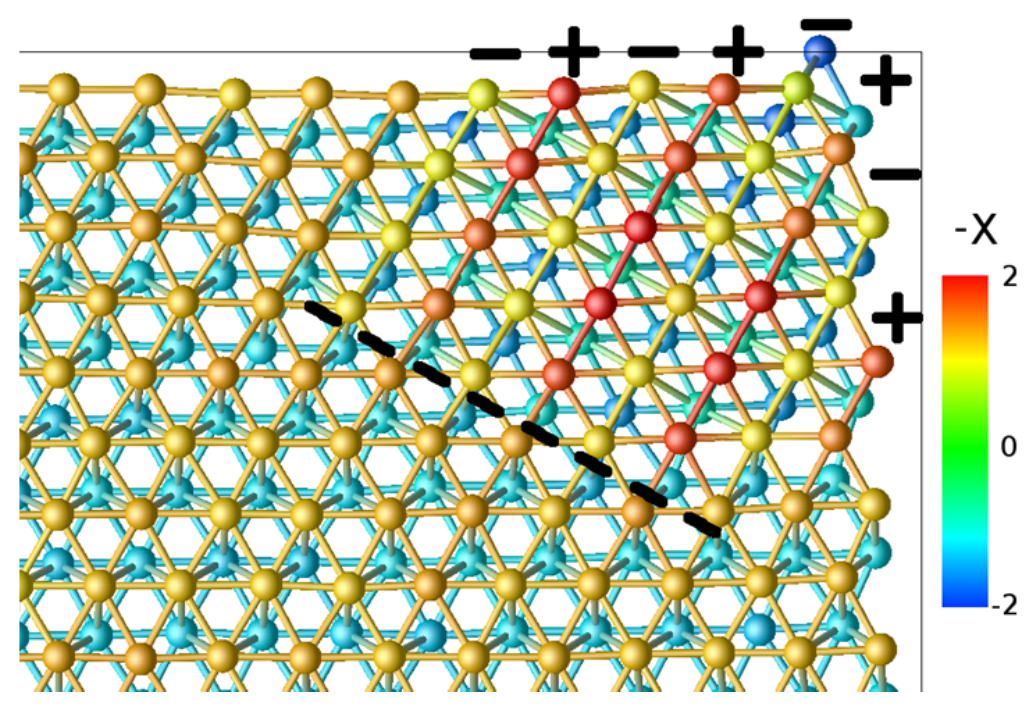

FIGURE 5. A SHUFFLE MOTION OF TWIN BASAL PLANES, COLORED BY ACKLAND METHOD. DOTTED LINE REPRESENT PLANE OF SHEAR. COLORS REPRESENT POSITION IN (-X)DIRECTION OUT OF PAPER. THE + SIGNS INDICATE UPWARD MOVEMENTS, THE NEGATIVE SIGNS DOWNWARD.

In Fig. 5 atoms are colored by $x$ positions of each atom. The red color and + sign indicate that the atoms are close, and the blue color and - sign indicate that the atoms are far. The plus and minus signs indicate the corrogation of layers in the twinning region. The basal plane of the parent becomes the corrogated prismatic plane of the twin.

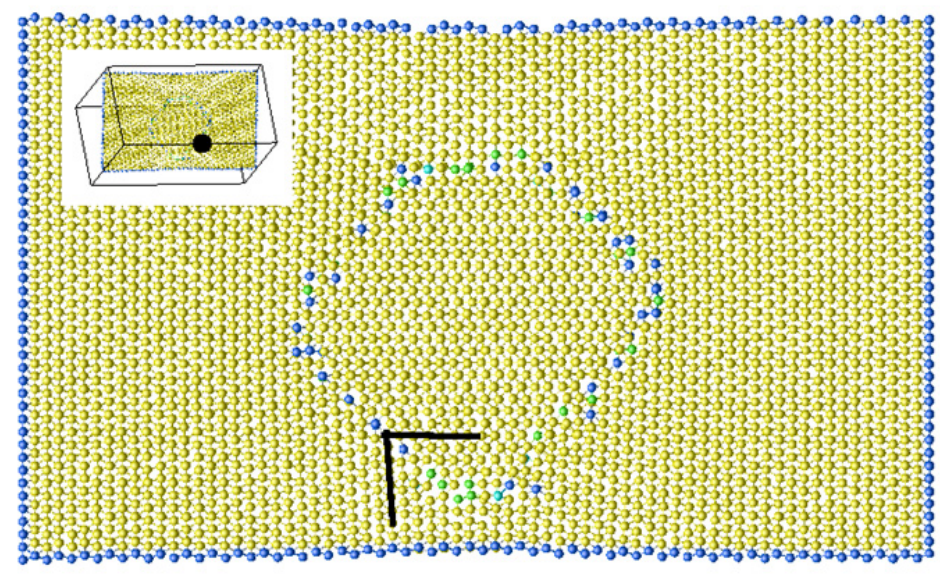

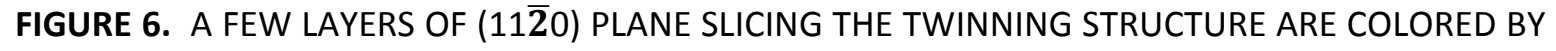
ACKLAND METHOD [3].

Fig. 6 shows a few layers of $(11 \overline{2} 0)$ planes sliced to reveal the plane of shear for $\{10 \overline{1} 2\}$ twinning colored by Ackland method[5]. The twinning boundary in the plane of shear forms a retangle starting from a circle. The retangle consists of four twinning planes which are straight in the figure. 


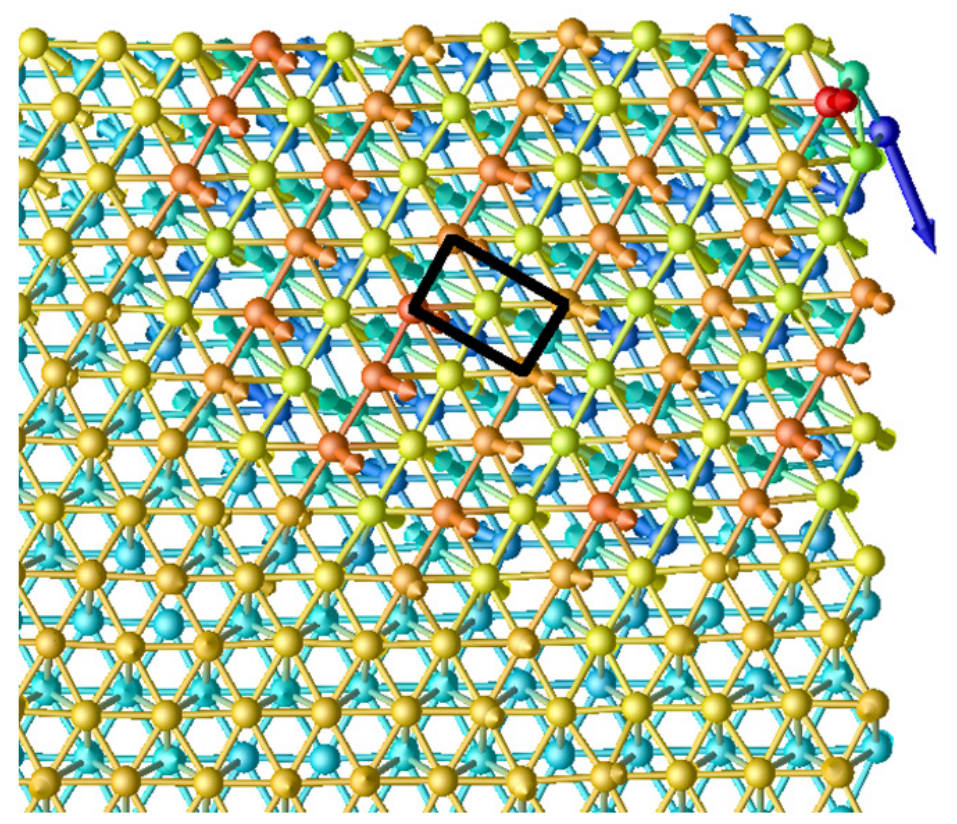

FIGURE 7. SLIP VECTORS ON A FEW LAYERS OF PARENT BASAL PLANES.

Slip vectors are shown in Fig. 7 for the atoms on a few layers of (0001) basal planes. The slip vector shows the movement of an atom relative to the neighbor atoms. Burger vector is the displacement vector of an atom to create a dislocation. Shuffling also causes the slip vector change as well as the Burger vector.
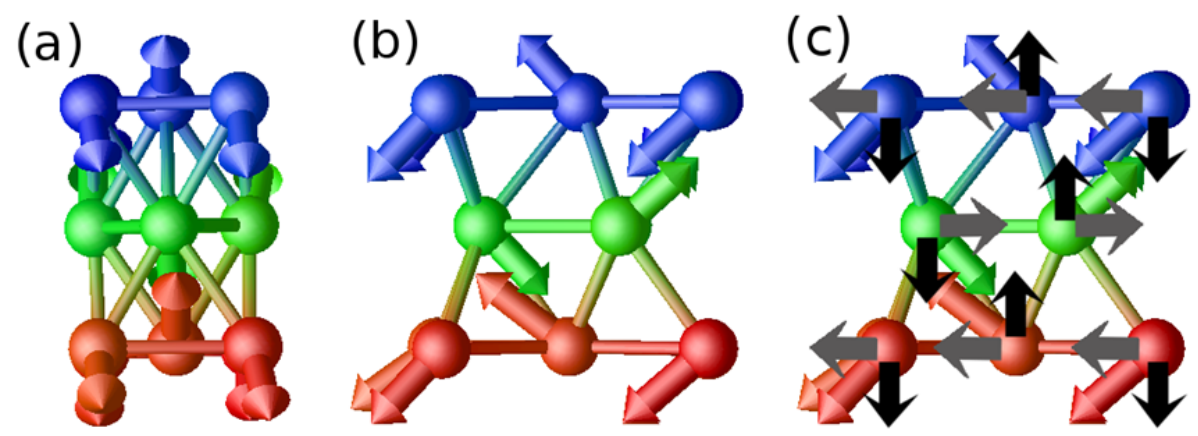

FIGURE 8. THE SLIP VECTORS IN RECTANGLE REGION IN PREVIOUS FIGURE IS MAGNIFIED (A) AND ITS RIGHT SIDE IS VIEWED (B). THE SLIP VECTORS ARE DECOMPOSED AS TWO COMPONENTS (C).

Fig. 8 shows the slip vectors in the region indicated by black solid line rectangle in Fig. 7 . Fig. $8(\mathrm{~B})$ is the right side view of Fig. 8(A) and the prismatic plane of the parent crystal structure. Fig. 8(C) shows the decomposition of the slip vectors. The gray arrow represents a shuffling in c-direction. The black arrow represents shuffling on the second prismatic plane. The displacement due to dislocation is very small so that it is not noticable with bare eyes. The $\{10 \overline{1} 2\}$ twinning is shuffling dominant.

Our early twin structure just after nucleation is very different from common expectation that a certain number of atomic layers of twin structure nucleate at a time by twinning dislocations[6]. The twin 
boundary shape is spherical. The twin nucleation point is always at the edge of retangular rod. The thermal Brownian motion of atoms at edge creates twin nucleation point because the atoms at edge have more freedom to move away from the crystal lattice position and form nucleation points. The spherical shape of the twin boundary surface doesn't continue because the growing speeds of the twin boundaries are not same. Each part of the twin boundary grows with different velocity which depends on boundary structures and growing directions. As the twin region grows it might form the experimentally observed lenticular shape of twin.

\subsubsection{Temperature dependence on the twin nucleation}

Our system allow temperature dependence study on the twin nucleation. Figure 9 shows the nucleation strain at different temperatures. As tensile load of strain increases, the stress on the crystal increase linearly. At a critical stress the twin nucleation environments form with the help of molecular dynamic thermal motions and the twin nucleates. It takes a time to form the twin nucleation environments depending on applied strain rate. The data points in figure 12 is one twin nucleation simulation. The average of the twin nucleation strain at different temperature are obtained in green curve.

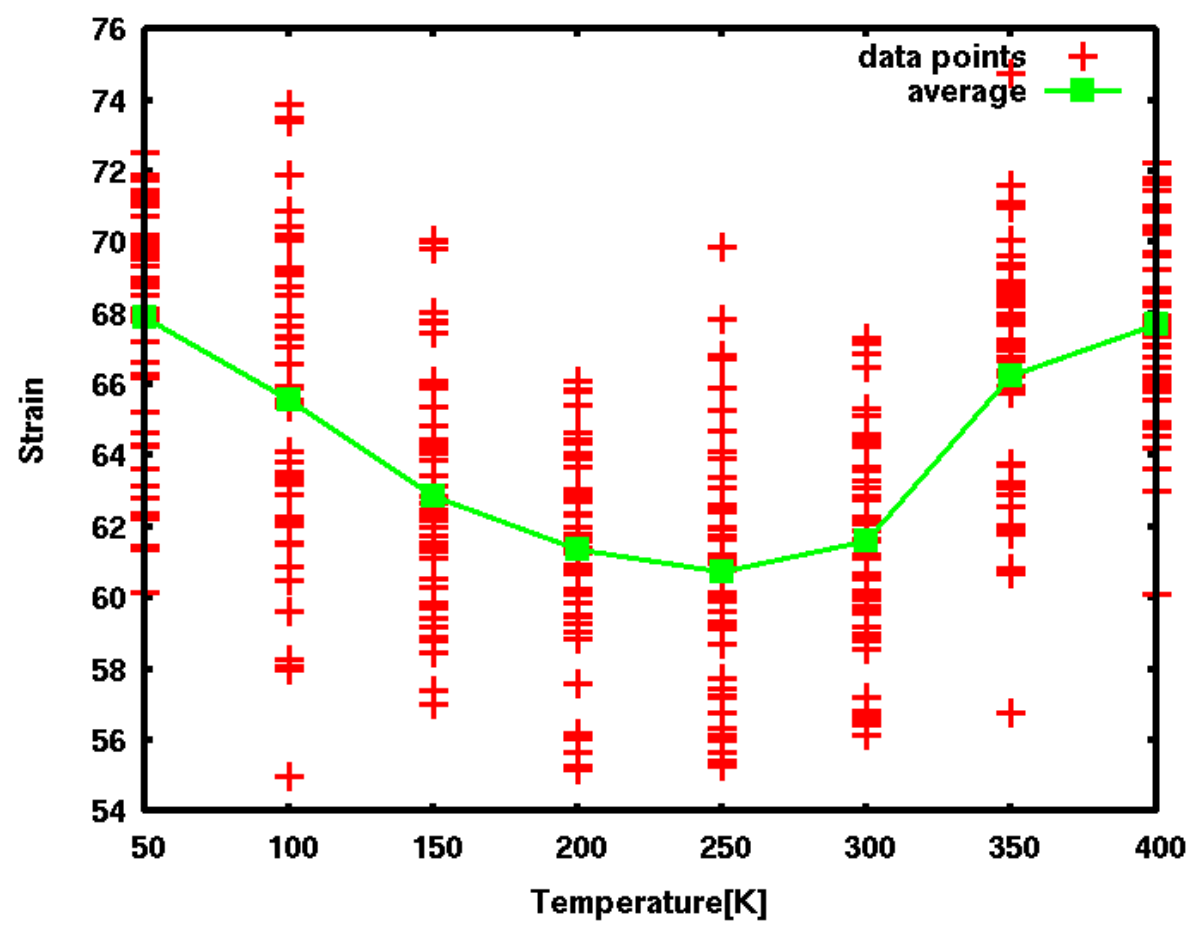

FIGURE 9. THE TEMPERATURE DEPENDENCE ON THE TWIN NUCLEATION.

Our overall simulation results suggest a surprising idea that the driving force to $\{10 \overline{1} 2\}$ twinning is tensile stress rather than shear stress. The $\{10 \overline{1} 2\}$ twinning forms by shearing and shuffling. The burger vector of twinning dislocation is extremely small and the shuffling is dominent. The shuffling lower the energy of system which is increased by applied tensile load. When the shear stress is applied to a crystal, dislocations are nucleated to accomadate loading stress instead of whole crystal shear deformation. In 
h.c.p $\{10 \overline{1} 2\}$ twinning, the shearing load is not the main contribution to twinning nucleation but the tensile load is. The shear-dominent twinning nucleates and grows by resolved shearing load. The shuffling-dominent twinning nucleates and grows by the resolved loading stress which induce shuffling to lower the system energy, for example, tensile loading stress in the current system. Temperature dependence of twinning nucleation in this system was obtained. In the low temperature twinning nucleation strains are inversely proportional to system temperature and in the high temperature they are tend to be propotional.

\section{REFERENCES}

[1] S.J. Plimpton: J. Comput. Phys., 1995, vol. 117, pp. 1-19, http:// lammps.sandia.gov/index.html.

[2] S. M. Foiles, M. I. Baskes, and M. S. Daw, Phys. Rev. B 33, 7983 (1986).

[3] G. Voronoi, Reine Angew. Math. 134, 199 (1908).

[4] D. Y. Sun, J. W. Liu, X. G. Gong, and Zhi-Feng Liu, "Empirical potential for the interaction between molecular hydrogen and graphite," PRB, 75, 075424, 2007.

[5] G. J. Ackland and A. P. Jones, "Applications of local crystal structure measures in experiment and simulation," $P R B, 73,054104,2006$. 


\section{SUBTASK 1.6: DESIGN OPTIMIZATION}

Team Members: Masoud Rais-Rohani, K. Motoyama, M. Kiani (PhD Student), M. Islam (MS Student), and I. Gandikota (MS Student)

\section{Objective}

- Development and application of numerical and analytical methods for modeling, analysis, and optimization of automotive structures made of lightweight metallic and composite materials.

\section{APPROACH}

- Computational models and optimization techniques are developed for analysis and design of components and structures made of metallic and composite materials. The developed techniques are used to (1) investigate the effect of microstructural texture on energy absorption of thin-walled components; (2) perform robust design optimization of process-product systems under random variability; (3) optimize full-vehicle structural models considering both vibration and crash characteristics; (4) design components and vehicle structures made of fiber-reinforced polymer composite materials; and (5) analyze and optimize arc welding process for simple and complex components.

\section{ACCOMPLISHMENTS}

- Investigated the effect of process-induced texture on energy absorption of polycrystalline metallic components using coupled crystal plasticity and finite element analysis.

- Conducted a robust design optimization study of a coupled process-product system for sheet-formed energy absorbing components.

- Developed a procedure for creating a vibration analysis model from a vehicle crash finite element model.

- Performed surrogate-based constrained optimization of a full-vehicle model based on vibration and crash constraints.

- Developed a global-local optimization strategy for optimization of vehicle components made of fiber-reinforced polymer composite materials.

- Performed calibration studies of welding process simulation to facilitate coupled finite element simulation and optimization of welded components.

\section{INTRODUCTION}

This final report highlights accomplishments in several areas including examination of manufacturing process effects on performance of structural components; optimization of vehicle structures considering both vibration and crash characteristics; design strategies for optimization of components and vehicle 
structures made of fiber-reinforced polymer composite materials; and arc welding process simulation and optimization. More in-depth information can be found in the publications listed at the end of this report.

\section{Effect of Texture on Energy Absorption Characteristics of Crush Tubes}

Explicit finite element (FE) simulations with an embedded crystal plasticity model are used to investigate the crush behavior of metallic square tubes under quasi-static axial loading condition. The anisotropic behavior of the component emerges from the texture induced by the large plastic deformation created during the manufacturing process.

In this context, the material response is modeled using a concurrent multiscale approach, where the material behavior at the grain scale (mesoscale) is linked to the stress response at the continuum level using a Taylor-type homogenization technique. Each continuum point is assumed to consist of an aggregate of face center cubic (FCC) crystals, where the effect of the material structure at lower length scales (i.e., dislocation patterns and discrete dislocations) are modeled indirectly through the use of the crystal state variable (i.e., hardness). The evolution equation of hardness is derived from dislocation mechanics.

The main contribution of this work is that an explicit representation of the microstructure at the crystal level (slip systems and crystal orientation) is captured during the plastic deformation of the structural system at the macroscale level, allowing the modeling of the material anisotropy (texture) of the structural components induced by the manufacturing process.

Ductile metallic tubes experience large plastic deformation in the localized regions during the crush process. Here, the conceptual influence of texture on the crush behavior of prismatic square tubes is considered. Four basic texture patterns are compared while the initial texture is kept the same over the entire tube model.

Since the collapse behavior of angular (e.g., square, hexagonal) tubes under axial crush loads is heavily influenced by the deformation of corner regions, the collapse response of a prismatic square tube is used for design comparison. In general, the folding mechanism in a two-flange corner region involves extensional, quasi-extensional, and quasi-inextensional modes of deformation [Wierzbicki and Abramowicz 1983; Abramowicz and Wierzbicki 1989]. Using the description presented by Abramowicz [2003], the folding deformation can be expressed in terms of asymmetric and symmetric corner elements. The localized regions observed in these two mode shapes can be considered as a general deformation mechanism in any multi-corner tube with capacity to undergo plastic deformation [Najafi and Rais-Rohani 2011].

Grain anisotropy is usually detected in thin-walled structures manufactured by different forming processes (e.g., extrusion, stamping) due to large strains experienced during the manufacturing process. 
The ability to model the texture and damage can improve and expand the computational simulation capability to explore the energy absorbing capacity of crash components in vehicle structures.

In this study, texture is introduced through a crystal plasticity constitutive model embedded in an FE framework. The VUMAT developed by Marin [2006] is integrated with the Abaqus/Explicit solver [Abaqus 2010] to perform crush simulations. Some basic load paths are considered to generate the initial texture required for crush simulations. For this purpose, single-element analyses are performed with three simple loading conditions (i.e., uniaxial tension, uniaxial compression, and simple shear). The load path calculations are performed with Abaqus/Standard using the UMAT version of the crystal plasticity model assuming crystallographic slip as the dominant plastic deformation mechanism. A random crystal orientation data for the aggregate of crystals located at each integration point is used as an input for the material subroutine as shown in Figure 1. The aggregate stress-response is computed using a Taylor-type mean field assumption.

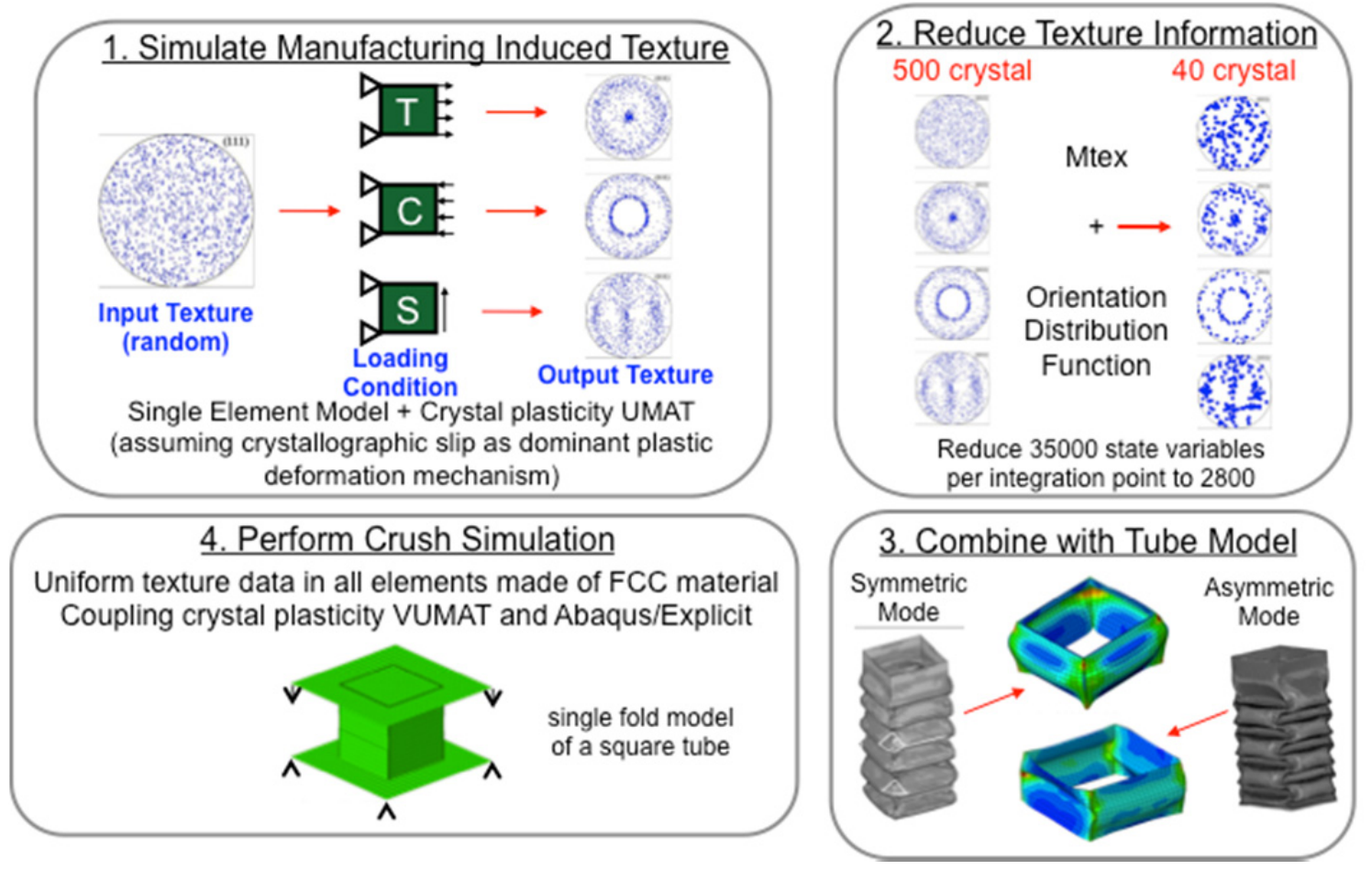

Figure 1. Overview of the modeling and simulation procedure to measure texture induced effects.

The orientations data evolve during the loading process and cause new arrangement of grain orientation in the aggregate. The material constants used for anisotropic elasticity, power-law kinetics, and the hardening law [Marin 2006], correspond to those of AL7050 [Najafi et al. 2012]. The FCC crystals in the aggregate deform by crystallographic slip on well-defined $\{111\}$ slip systems. The variables saved at each integration point for each crystal include the stress and strain components, the hardness, the shear 
strain rates, the initial orientation, and the rotation tensor. For an FCC crystal, these variables add up to seventy variables at each crystal integration point. However, the limit on the number of variables that can be saved at each integration point is 10,000 in Abaqus/Explicit while there is no limit in Abaqus/Standard. Hence, the basic load path simulations are performed in Abaqus/Standard using a material aggregate containing 500 randomly oriented grains $(500 \times 70=35,000$ variables to be saved at each integration point) as shown in Figure 1.

Since the crush simulations are performed using Abaqus/Explicit, we used Mtex and orientation distribution function to reduce the number of crystals in the aggregate to 40 (due to the limited storage of variables and for the sake of computational efficiency) while maintaining the original texture patterns obtained from the implicit simulations such that the orientation distribution observed in the reduced model and the original model are consistent. The block 2 in Figure 1 shows the pole figures for the actual and reduced data.

The tube response falls into two basic folding mechanisms, asymmetric (Model A) and symmetric (Model B) as shown in block 3 of Figure 1. Studying these two collapse mechanisms clarifies the energy absorption mechanism in a wide range of multicell multicorner tubes [Najafi and Rais-Rohani 2011]. A geometric trigger mechanism is introduced to initiate each deformation mode. The asymmetric deformation mode is triggered through local thickness reduction in the outer surfaces of two opposite tube walls whereas the symmetric deformation mode is triggered by local thickness reduction of the inner surfaces on all four sides of the tube.

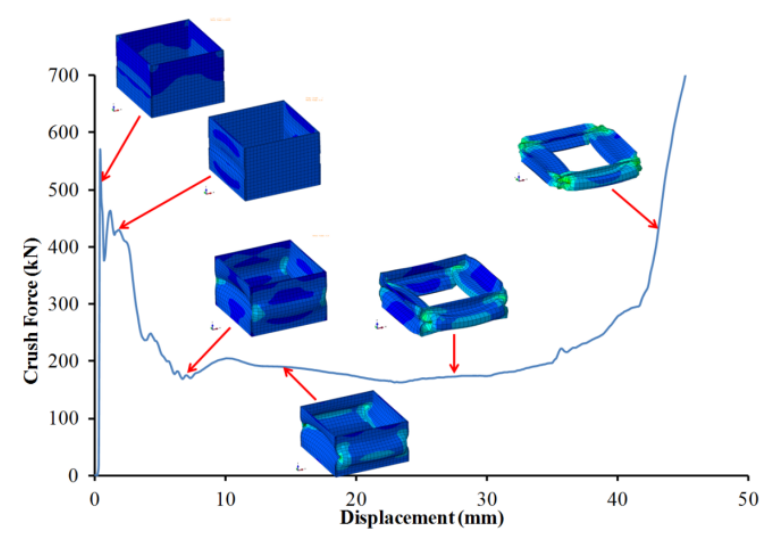

Model A

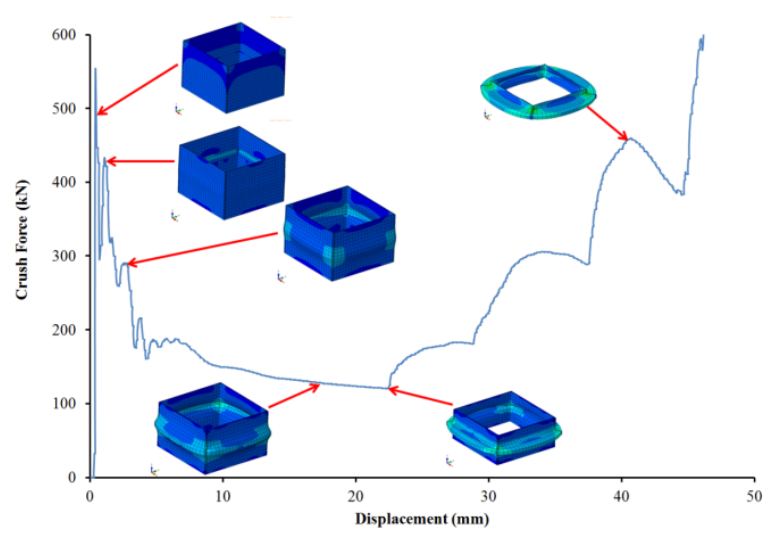

Model B

Figure 2. Asymmetric (Model A) and symmetric (Model B) deformation modes for the square tube with random texture under quasi-static axial loading.

The square tube model has a length of $80 \mathrm{~mm}$ and a side dimension of $40 \mathrm{~mm}$ as shown in the block 4 of Figure 1. The short length is chosen to focus on the formation of a single fold observed in longer tube models. The tube is modeled using three solid (C3D8R) elements through the wall thickness for 4140 elements. Element C3D8R is a reduced integration element with viscous enhanced hourglass 
formulation. The contact friction coefficient between the rigid walls and tube is set at 0.5 to prevent slippage between surfaces based on the penalty formulation defined between a discrete rigid wall and deformable tube. To prevent element-to-element inter-penetration during excessive deformation, a self-contact condition is defined for all the element surfaces of the tube with the same friction property. In order to increase the computational efficiency, a mass scaling of 10 is used for the deformable elements. The tube is located between a moving rigid wall and a fixed support. The wall moves with a constant displacement increment throughout the simulation (constant velocity of $10 \mathrm{~m} / \mathrm{s}$ ). It is assumed that the entire tube model has the same texture orientation distribution.

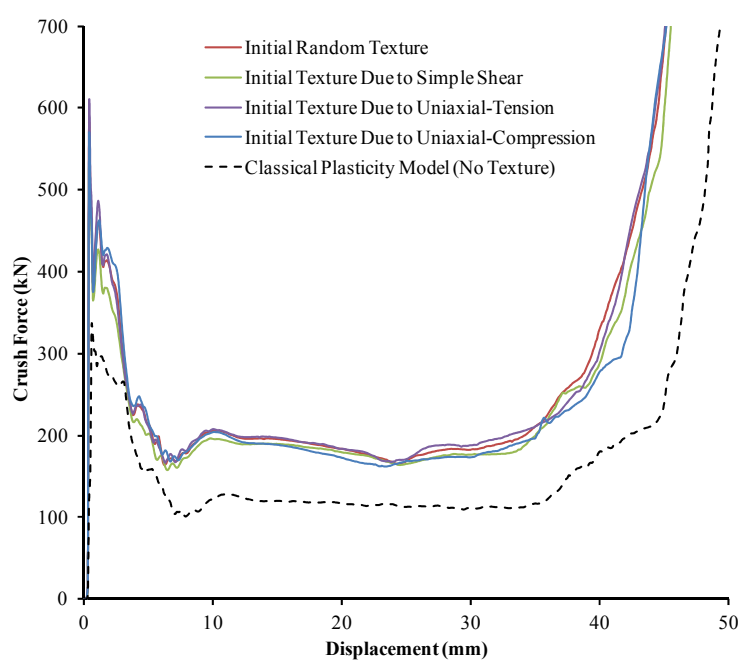

Model A

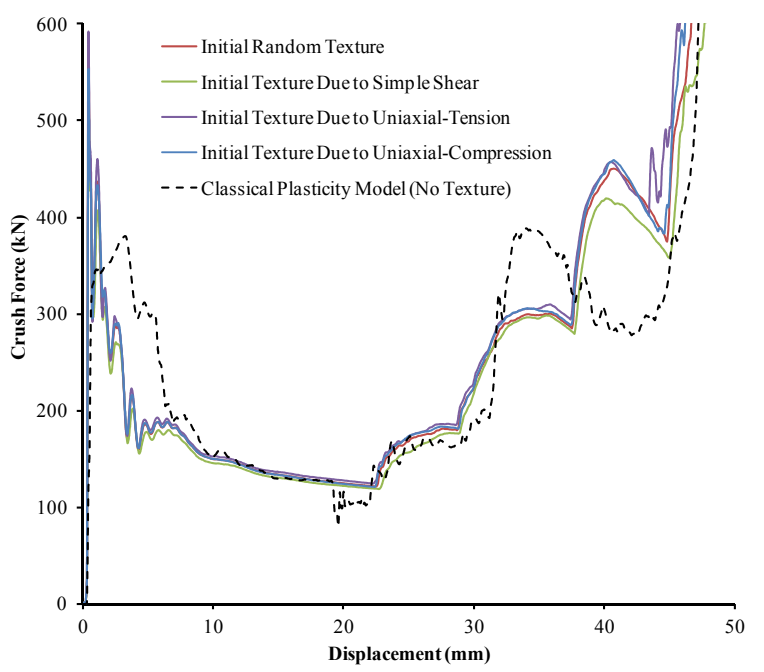

Model B

Figure 3. Comparison of energy absorption behavior due to different textures in asymmetric (Model A) and symmetric (Model B) deformation modes.

The collapse deformations in both asymmetric and symmetric modes as well as the crush load during the deformation process for the random texture case are shown in Figure 2. The initial peak force is reached once the plastic folding deformation begins. The crushing process is controlled by the deformation mechanism in the corner regions, and force remains stable until the folding deformation saturates when the folded sides contact each other. At this point, additional deformation is not possible resulting in a drastic increase in the contact force through locking.

The energy absorption behavior for the various texture models considered is illustrated in Figure 3 in terms of crush force versus crush distance (displacement). The stress-strain response captured from the single-element study in tensile loading with initial random texture is used to model the crush process with piecewise linear plasticity model (isotropic material model). As shown in Figure 3, there is some difference between the results of this study and isotropic treatment of the material, where the 
evolution of material orientation due to loading is ignored. In contrast, the present material model updates material orientation in each time step based on the strain history and the stress state of the elements.
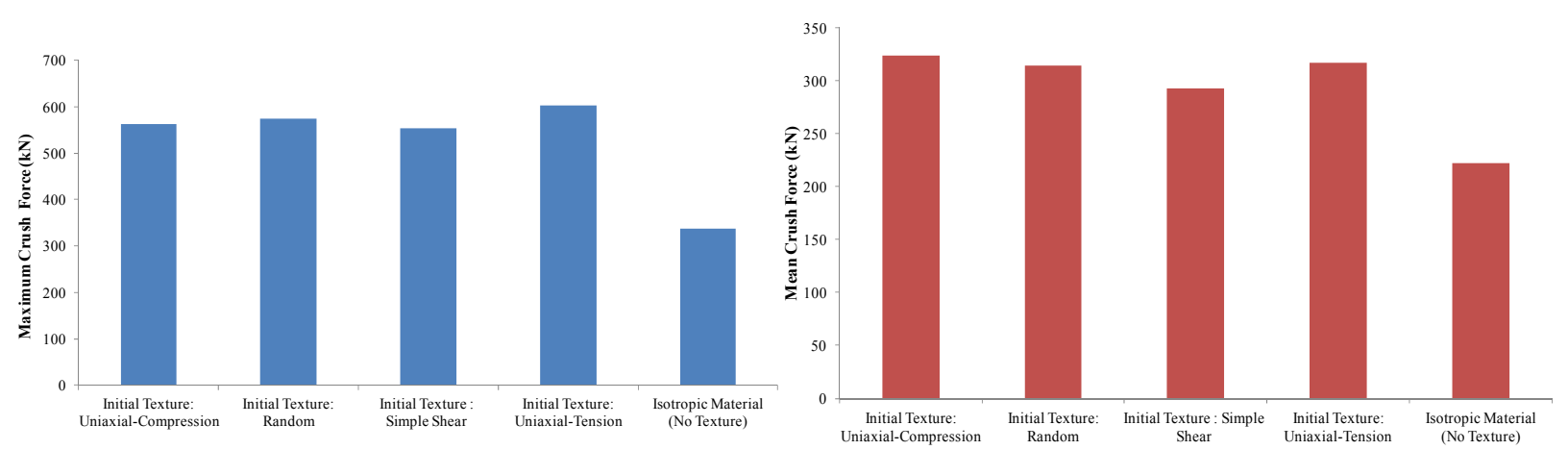

Model A
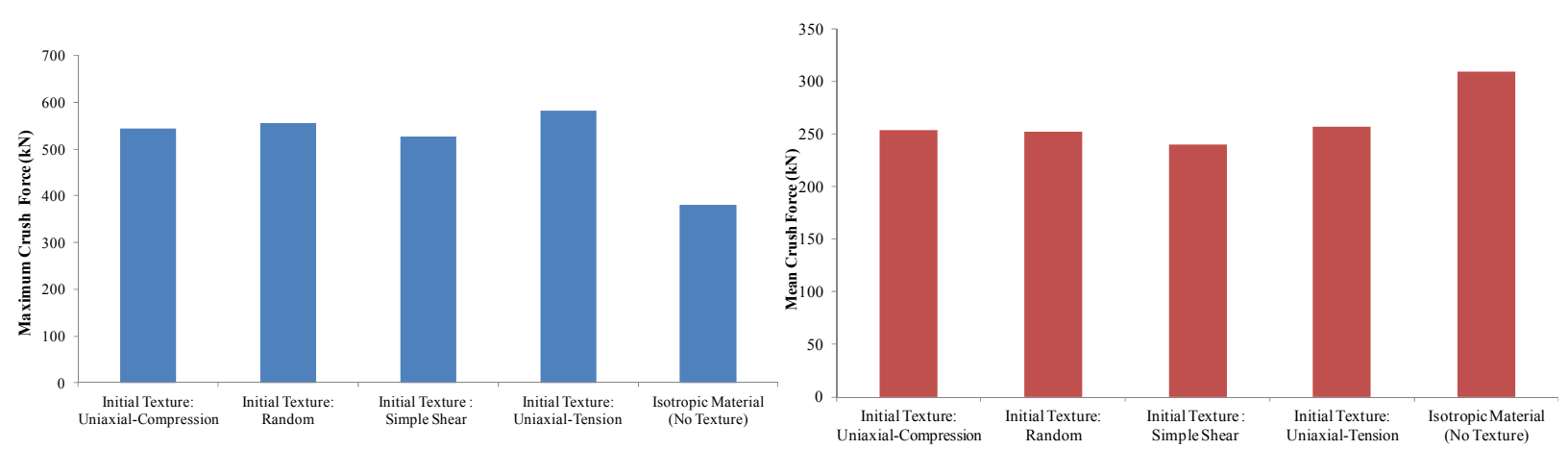

Model B

Figure 4. Comparison of maximum and mean crush forces for different textures in asymmetric (Model A) and symmetric (Model B) deformation modes.

The crush performance is also compared in terms of the maximum and mean crush forces as shown in Figure 4. The point where the crush force reaches its peak value in Figure 3 can be considered as the starting point of collapse. The mean crush force is calculated by only using the energy absorbed through the plastic deformation (folding process). Therefore, the mean crush force is the area under the forcedisplacement curve divided by the crush distance.

The explicit FE simulations indicated that the initial texture pattern could influence the crush behavior and energy absorption characteristics of the square tube. It was shown that anisotropic texture could have a different effect on the overall energy absorption of the tube in different deformation modes. Initial texture due to uniaxial tension gives the highest mean crush force for the symmetric mode whereas that due to uniaxial compression produces the highest mean crush force in the asymmetric deformation mode. This conceptual study on the influence of anisotropic texture on axial crush behavior 
of thin-walled square tubes revealed that by controlling texture during the manufacturing process, it would be possible to enhance the performance characteristics of the component. Additional details about this investigation can be found in [Najafi et al. 2012].

\section{Full Vehicle Structural Optimization Considering Crashworthiness and Vibration Responses}

A full-vehicle constrained optimization problem is formulated and solved for minimizing the structural weight while considering crashworthiness under full frontal impact (FFI), offset frontal impact (OFI), and side impact (SI) as well as dynamic stiffness as measured by its three fundamental frequencies. This study extends the previous effort [Parrish et al. 2012] in vehicle lightweighting focused entirely on material substitution and crashworthiness characteristics of a compact car. The inclusion of structural stiffness as another important design criterion enhances viability as well as complexity of the optimization problem.

A 1996 Dodge Neon model is used in this study. Through a preliminary analysis, twenty-two components, as highlighted in Figure 5, are selected for design optimization. In the original (baseline) vehicle model, these components have a combined mass of $105.25 \mathrm{~kg}$ for $8 \%$ of the crash-model mass at $1,333 \mathrm{~kg}$ and approximately $45 \%$ of the vibration-model mass at $233 \mathrm{~kg}$. Due to the vehicle model symmetry, the selected components are represented by fifteen wall-thickness design variables denoted by $x_{1}$ through $x_{15}$ in Figure 5 .

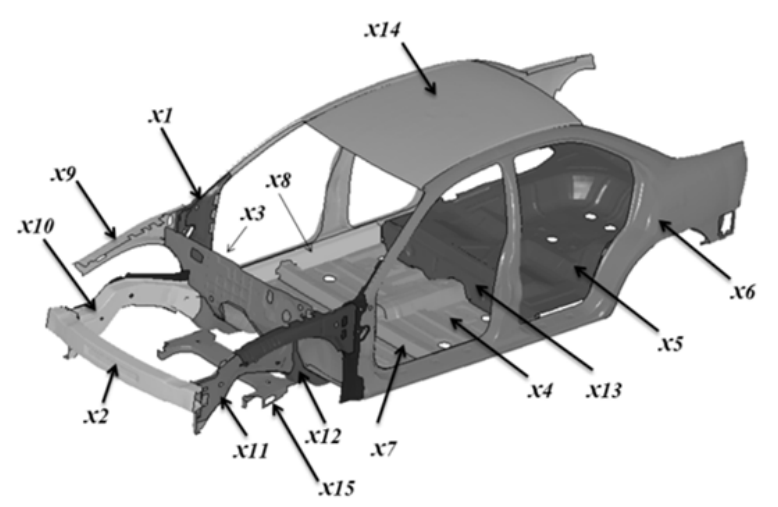

Figure 5. Components and design variables in vehicle lightweighting optimization problem.

Crash analyses are performed on a validated FE model [NCAC 2006] using explicit nonlinear transient dynamic FE analysis in LS-DYNA. The FFI scenario models an impact into a rigid wall at a speed of 56 $\mathrm{km} / \mathrm{hr}$. The OFI simulations model an impact into a honeycomb barrier placed in front of a rigid wall at $40 \%$ offset and an impact speed of $56 \mathrm{~km} / \mathrm{hr}$ to coincide with FFI simulations. The SI simulations 
represent collision with another vehicle travelling at a speed of $52 \mathrm{~km} / \mathrm{hr}$ with a collision angle of $27^{\circ}$. The crash model is at rest when impacted on the driver's side by the moving vehicle (loading trolley). Figure 6 shows the vehicle model in each of the three crash scenarios.

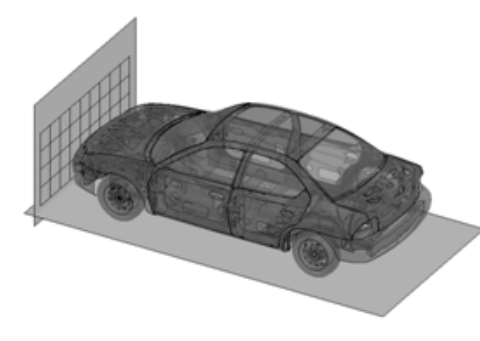

(a)

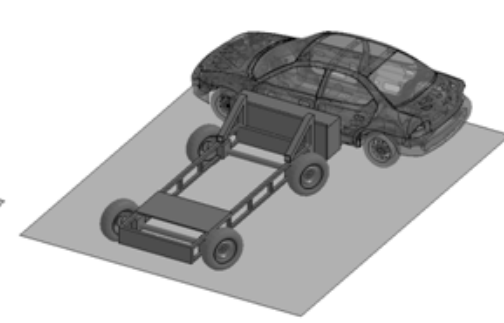

(b)

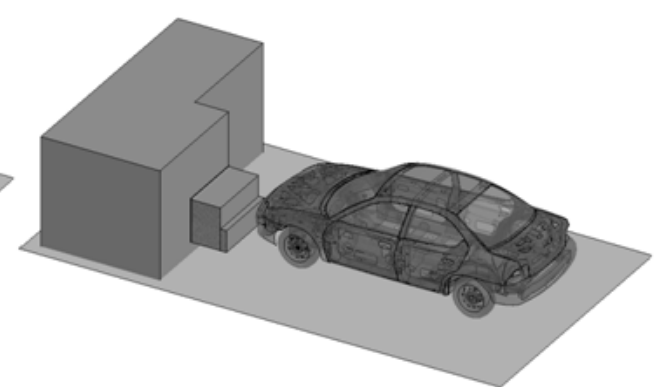

(c)

Figure 6. Crash model of the 1996 Dodge Neon in (a) FFI, (b) SI, and (c) OFI scenarios.

Crash responses considered include the intrusion distances at the toeboard and dashboard for FFI and OFI and at the door for SI (Int Toe, Int Dash, and Int Door), resultant acceleration at a location on the Bpillar in all three scenarios (Accel), and internal energy absorption of the selected parts in all three scenarios (Int Eng). These responses were chosen because of their relevance to occupant safety and the acceleration response location was chosen to be near the approximate location of the driver's head. The response measurement locations are shown in Figure 7.

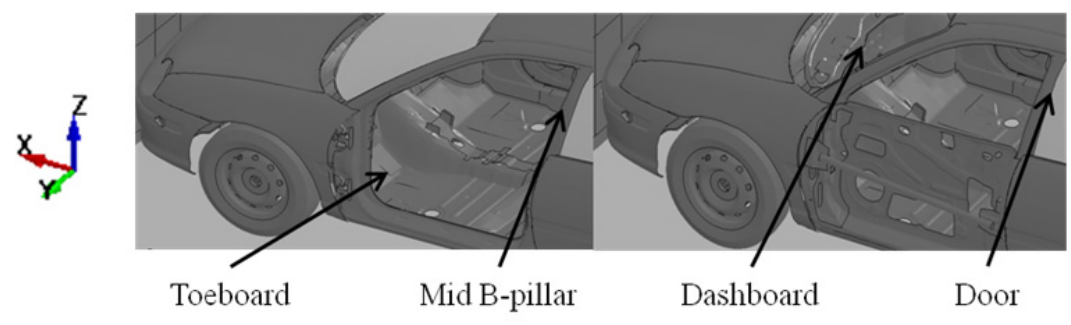


Figure 7. Selected locations for structural response measurement.

The FE mesh for vibration analysis differs from the crash model in several areas such as exclusion of all the moving parts (doors, hood, etc.) together with a more accurate representation of the spot welds and overall modeling of structural stiffness. The vibration model, as shown in Figure 8(b), consists of all the sheet metal components that are spot welded together. The primary focus is on the fundamental natural frequencies that influence the vehicle's ride and handling characteristics as well as durability and rigidity.

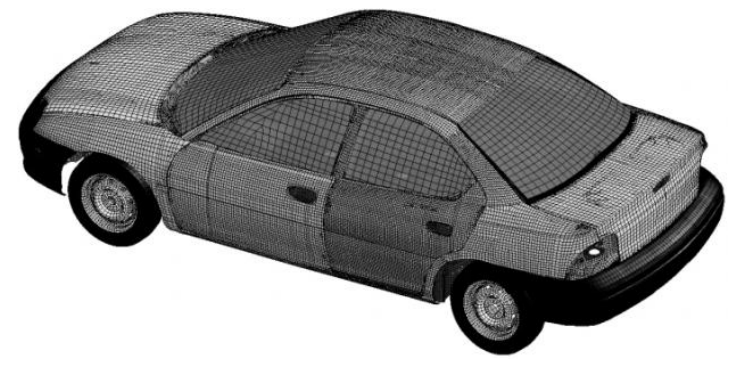

(a)

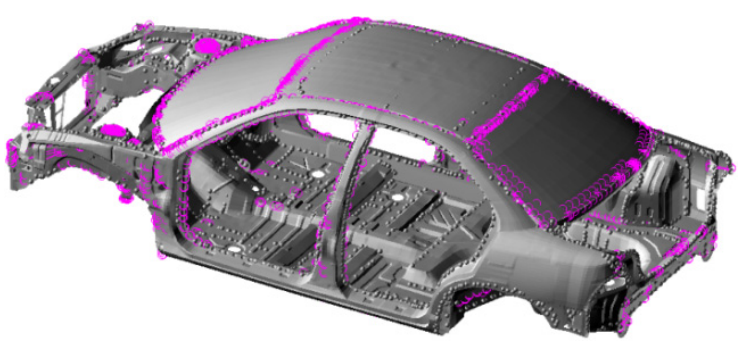

(b)

Figure 8. (a) LS-DYNA crash model and (b) MSC Nastran vibration model of the vehicle.

As a part of this study, the vibration model in Figure 8(b) was generated from the LS-DYNA crash model in Figure 8(a) and used for modal analysis in MSC Nastran. Each spot weld was given a nominal diameter of $5 \mathrm{~mm}$ and modeled using a CWELD element with elastic properties equal to those of the welded steel parts. Also, the CNRB rigid elements in LS-DYNA were converted to RBE2 elements in Nastran. CNRB and RBE2 elements are used to model the connection points between parts that are more-or-less permanently attached to each other or to connect parts in the FE model with mesh-dissimilarities such as different mesh sizes or element types (shell-to-solid, shell-to-beam, etc.). As a result, the Nastran model consists of 273,760 nodes, 262,560 CQUAD4 and CTRIA3 shell elements along with 701 RBE2 and 3,215 CWELD elements. In Figure 8(b), the CWELD elements are scaled up and shown by solid spheres while the RBE2s are shown by the magenta-color circles. Subsequent modal analyses were used to confirm the six rigid body modes and the subsequent flexible modes of vibration. Here, the windshield and the rear window are also included in the model for vibration analysis under free-free boundary conditions. 
The three fundamental vibration modes of the baseline model, as shown in Figure 9, correspond to the first torsion at $35.39 \mathrm{~Hz}$, first bending at $36.23 \mathrm{~Hz}$, and a combined torsion-bending mode at $38.37 \mathrm{~Hz}$. The solid lines in Figure 9 trace the shape of the stationary vehicle.

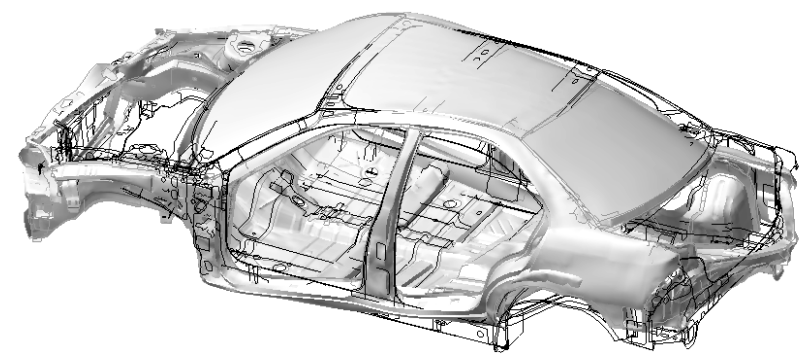

(a)

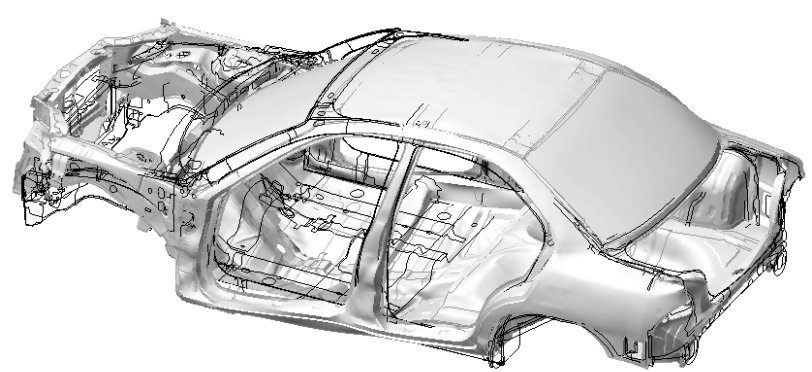

(b)

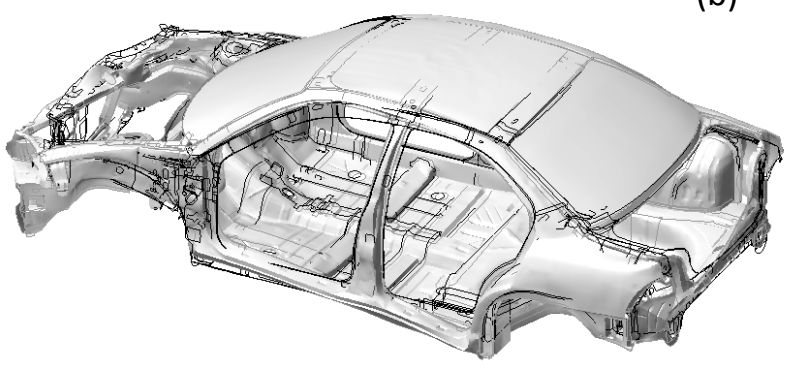

(c)

Figure 9. Vibration modes of the baseline design in (a) first torsion, (b) first bending, and (c) mixed torsion-bending.

Given the number of crash scenarios and vibration frequencies that need to be included in the optimization problem and the fact that some responses-such as acceleration-tend to be non-smooth, it would be impractical to couple FE simulations directly with a gradient-based numerical design optimization technique. This computational difficulty is alleviated when the objective and constraint functions are described by continuous and differentiable approximate analytical functions (i.e., surrogate models). Hence, all response functions are approximated using radial basis functions (RBF). The unknown coefficients are found using the least squares technique based on the exact function values at the selected design (training) points identified using Latin Hypercube Sampling (LHS). The accuracy of each surrogate model was subsequently verified by finding the average error at a set of arbitrary design (test) points separate from the training points.

Table 1. Comparison of surrogate- and simulation-based responses at the point of optimum.

Response Surrogate-Based Simulation-Based $\quad$ Error (\%)




\begin{tabular}{|c|c|c|c|c|}
\hline & & Responses & Responses & \\
\hline \multirow[t]{4}{*}{$\mathrm{FFI}$} & Int Toe (mm) & 156.86 & 160.29 & -2.18 \\
\hline & $\begin{array}{ll}\text { Int } & \text { Dash } \\
(\mathrm{mm}) & \end{array}$ & 121.64 & 118.30 & 2.75 \\
\hline & Accel (g's) & 61.12 & 59.12 & 3.27 \\
\hline & Int Eng (kJ) & 62.94 & 62.35 & 0.94 \\
\hline \multirow[t]{3}{*}{ SIDE } & $\begin{array}{ll}\text { Int } & \text { Door } \\
(\mathrm{mm}) & \end{array}$ & 313.75 & 311.09 & 0.85 \\
\hline & Accel (g's) & 47.62 & 47.71 & -0.19 \\
\hline & Int Eng (kJ) & 23.81 & 23.51 & 1.26 \\
\hline \multirow[t]{4}{*}{ OFI } & Int Toe (mm) & 246.95 & 229.29 & 7.15 \\
\hline & $\begin{array}{ll}\text { Int } & \text { Dash } \\
(\mathrm{mm}) & \end{array}$ & 237.98 & 200.52 & 15.74 \\
\hline & Accel (g's) & 35.01 & 33.91 & 3.13 \\
\hline & Int Eng (kJ) & 41.18 & 41.46 & -0.68 \\
\hline \multirow[t]{3}{*}{ Vibration } & Frq1 $(\mathrm{Hz})$ & 35.41 & 35.39 & 0.07 \\
\hline & Frq2 (Hz) & 36.26 & 36.23 & 0.08 \\
\hline & Frq3 (Hz) & 38.44 & 38.37 & 0.17 \\
\hline Average $\mathrm{e}$ & & & & 2.75 \\
\hline
\end{tabular}

The lightweighting optimization problem was formulated with crash performance and vibration characteristics constrained to be equal to or better than those in the baseline vehicle model. VisualDOC optimization software was used as the optimizer with VisualScript facilitating communication between the optimizer and the surrogate models. Using sequential quadratic programming technique along with different initial design points, the optimum mass for the twenty-two designed parts was found to be $101.5 \mathrm{~kg}$ for a reduction of $3.4 \%$ relative to the baseline mass of $105.2 \mathrm{~kg}$. Table 1 compares the vehicle responses at the point of optimum as estimated by the corresponding surrogate models with the exact values obtained from crash and vibration analyses. The overall average error is found to be $2.75 \%$ over the range of $0.07 \%$ to $15.74 \%$. Global responses such as frequencies are typically much easier to approximate with high degree of accuracy than some of the crash responses that tend to be more local and affected by the number of nodes/elements and associated response values used in the average response approximation. 
Simulation-based structural responses for the crash-vibration optimum design with both crashworthiness and vibration constraints included are shown in Table 2 and compared with the responses in the baseline model as well as the optimum design based on crashworthiness constraints alone [Parrish et al. 2012]. When the optimum design responses are checked through FE simulation, all responses except one are similar or better than those of the baseline model. The underestimation error of $2.18 \%$ in the Int Toe response in Table 1 is the reason for the simulation-based response of the optimum design to be slightly higher than that of the baseline model in Table 2.

Table 2. Comparison of vehicle responses in the baseline and two different optimum design models.

\begin{tabular}{|c|c|c|c|c|c|}
\hline & Response & Baseline & $\begin{array}{l}\text { Crashworthiness } \\
\text { Optimum }\end{array}$ & $\begin{array}{l}\text { Crash- } \\
\text { Vibration } \\
\text { Optimum }\end{array}$ & Difference $^{\mathrm{a}}(\%)$ \\
\hline \multirow[t]{4}{*}{$\mathrm{FFI}$} & Int Toe $(\mathrm{mm})$ & 157.07 & 169.64 & 160.29 & 2.05 \\
\hline & Int Dash (mm) & 122.06 & 108.45 & 118.30 & -3.08 \\
\hline & Accel (g's) & 63.51 & 69.84 & 59.12 & -6.91 \\
\hline & Int Eng (kJ) & 62.31 & 66.08 & 62.35 & 0.06 \\
\hline \multirow[t]{3}{*}{ SI } & Int Door (mm) & 313.93 & 333.45 & 311.09 & -0.90 \\
\hline & Accel (g's) & 47.88 & 44.02 & 47.71 & -0.36 \\
\hline & Int Eng (kJ) & 22.37 & 22.83 & 23.51 & 5.10 \\
\hline \multirow[t]{4}{*}{ OFI } & Int Toe (mm) & 273.48 & 269.36 & 229.29 & -16.2 \\
\hline & Int Dash (mm) & 246.94 & 233.22 & 200.52 & -18.8 \\
\hline & Accel (g's) & 35.02 & 38.08 & 33.91 & -3.17 \\
\hline & Int Eng (kJ) & 39.42 & 40.58 & 41.46 & 5.17 \\
\hline \multirow[t]{4}{*}{ Vibration } & Frq1 $(\mathrm{Hz})$ & 35.39 & 33.14 & 35.39 & 0.00 \\
\hline & $\operatorname{Frq} 2(\mathrm{~Hz})$ & 36.23 & 34.15 & 36.23 & 0.00 \\
\hline & Frq3 (Hz) & 38.37 & 36.87 & 38.37 & 0.00 \\
\hline & Mass (kg) & 105.25 & 88.00 & 101.49 & 3.57 \\
\hline
\end{tabular}

\footnotetext{
${ }^{a}$ Relative to the baseline model.
} 
Figure 10 shows the normalized thickness values of the selected components in the baseline model along with those associated with the crash-vibration (current) and crashworthiness optimum models. Out of fifteen design variables in the crash-vibration vehicle optimum, nine have increased and six have decreased relative to their respective baseline values with design variable five reaching its lower bound. It is also worth noting the difference between the two optimum designs, which points to the fact that for most of the parts the wall thickness had to increase to satisfy the stiffness requirements.

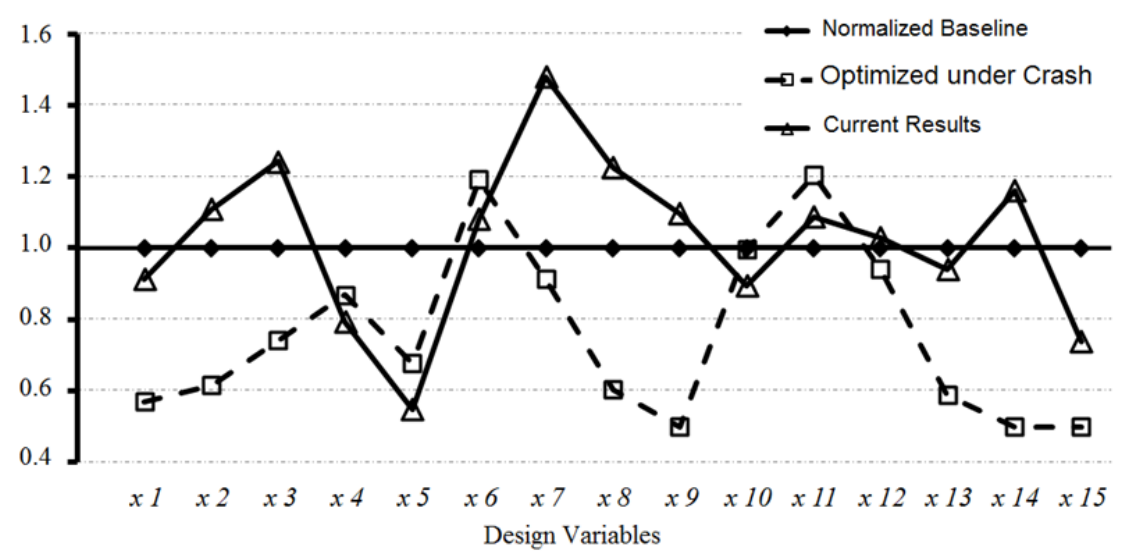

Figure 10. Normalized wall thickness associated with the fifteen design variables.

\section{Simulation and Optimization of Vehicle Structures Made of Composite Materials}

Efforts to improve fuel economy through vehicle lightweighting have increased interest in the application of fiber-reinforced polymer composite materials in the auto-body structure. As part of this project, the energy absorption properties of components and structural systems made of carbon fiberreinforced polymer (CFRP) composite were investigated, and the results were used to develop a new design strategy for optimization of vehicle structures made of such materials.

\section{Component Analysis}


The explicit FE code, LS-DYNA is used to examine the crush behavior of thin-walled CFRP rails. The material is characterized using the MAT54 and MAT58 models in LS-DYNA. MAT54 is constructed based on Chang-Chang failure criterion that can model progressive damage in composite materials whereas MAT58 is defined based on continuum damage mechanics. While MAT58 is applicable to fabric composites, MAT54 can be used for both fabric and unidirectional composite materials.

The rail is modeled as a square tube made of T700/2510 plain weave fabric composite material with the data and modeling parameters defined in Table 3. The tube length is $104 \mathrm{~mm}$, its width is $58.42 \mathrm{~mm}$ and its wall thickness is $1.85 \mathrm{~mm}$. With a laminate stacking sequence of $[0 / 90]_{4 \mathrm{~s}}$, the average cured wall thickness is taken as $1.65 \mathrm{~mm}$ [Deleo et al. 2009].

Table 3. Material properties of T700/2510 plain weave fabric composite.

\begin{tabular}{|c|c|c|}
\hline Property & LS-DYNA Parameter & Experimental Value \\
\hline Density $\left(\mathrm{kg} / \mathrm{m}^{3}\right)$ & RO & 1510.0 \\
\hline Longitudinal Elastic Modulus (GPa) & EA & 55.9 \\
\hline Transverse Elastic Modulus (GPa) & EB & 54.4 \\
\hline In-Plane Shear Modulus (GPa) & GAB & 4.12 \\
\hline In-Plane Poisson's Ratio & PRBA & 0.043 \\
\hline Axial Tensile Failure Strain & $\mathrm{E} 11 \mathrm{~T}$ & 0.01636 \\
\hline Axial Compressive Failure Strain & $\mathrm{E} 11 \mathrm{C}$ & 0.01290 \\
\hline Transverse Tensile Failure Strain & E22T & 0.01415 \\
\hline Transverse Compressive Failure Strain & E22C & 0.01415 \\
\hline In-Plane Shear Failure Strain & GMS & 0.03470 \\
\hline Axial Tensile Stress at Failure (MPa) & $\mathrm{XT}$ & 910.0 \\
\hline Axial Compressive Stress at Failure (MPa) & $x C$ & 710.0 \\
\hline Transverse Tensile Stress at Failure (MPa) & YT & 772.0 \\
\hline $\begin{array}{l}\text { Transverse Compressive Stress at Failure } \\
\text { (MPa) }\end{array}$ & YC & 703.0 \\
\hline In-Plane Shear Stress at Failure (MPa) & SC & 131.0 \\
\hline
\end{tabular}


After modeling the geometry and input material parameters, simulation parameters were adjusted to obtain a stable crush. Simulation parameters include contact algorithm, element formulation and direction, bulk viscosity value, damping, trigger type, etc. The simulation-based crush forcedisplacement curves for MAT54 and MAT58 models are shown in Figure 11 and are compared with the experimental result in [Deleo et al. 2009].

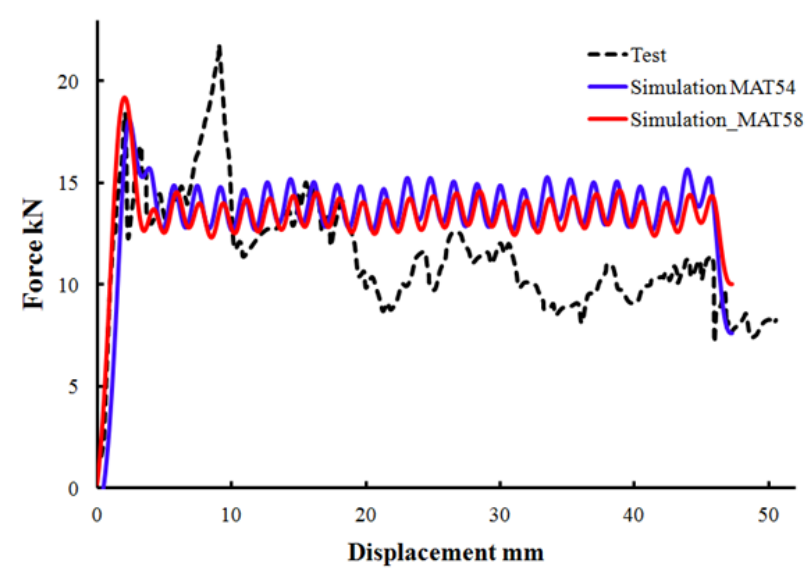

Figure 11. Comparison of simulation-based force-displacement curves with the test data

The first peak of the experimental curve in Figure 11 is closely approximated by the simulation models, but the subsequent patterns differ. The process by which failure in each element is determined and deactivation of the failed elements in the model can affect the predicted force-displacement response.

\section{Structural Analysis and Optimization}

One of the challenges in design optimization of vehicle structures made of CFRP materials is that the crash simulations can be very unstable and changes in the structural geometry can add to the computational instability, more so than in the case of vehicle models made of metallic materials. This instability can jeopardize the accuracy of simulation results and hamper design optimization efforts that rely on FE simulations.

A design strategy is proposed to address this challenge. In the first step, the baseline vehicle model with metallic structure is optimized for the desired figure of merit (e.g., crashworthiness, weight, rigidity, etc.). In the next step, the optimum component or subsystem of interest (to be replaced by a composite counterpart) is separated from the rest of the vehicle (global model) and used to setup a local model for analysis and optimization. With the goal of matching or possibly exceeding the performance characteristics of the optimal metallic structure, the local model made of composite material is 
optimized. Finally, the optimal metallic component/subsystem is replaced by its composite counterpart and full-vehicle simulations are performed to verify the performance gains as a result of material substitution and global-local optimization procedure.

This strategy was implemented using the 1996 Dodge Neon crash model shown in Figure 12. The highlighted front rails were to be converted from steel to CFRP composite. Each steel rail consists of spot welded inner and outer parts. Using the global-local optimization procedure outlined in Figure 13, the full-vehicle baseline model was first optimized for minimum weight subject to the frequency and crashworthiness constraints defined previously in Table 2. The two crash scenarios considered in this study included FFI and OFI. The resultant acceleration, measured on the B-Pillar, defined the FFI constraint, whereas the intrusion distances at the toeboard and dashboard were used as the OFI constraints. These response locations are shown in Figure 7. Moreover, the first three frequencies of the full-scale car body structure were used as the vibration constraints. With focus on the front rails, only the thicknesses of the inner and outer parts were treated as design variables with the optimum values found to be $1.694 \mathrm{~mm}$ and $1.654 \mathrm{~mm}$, respectively.

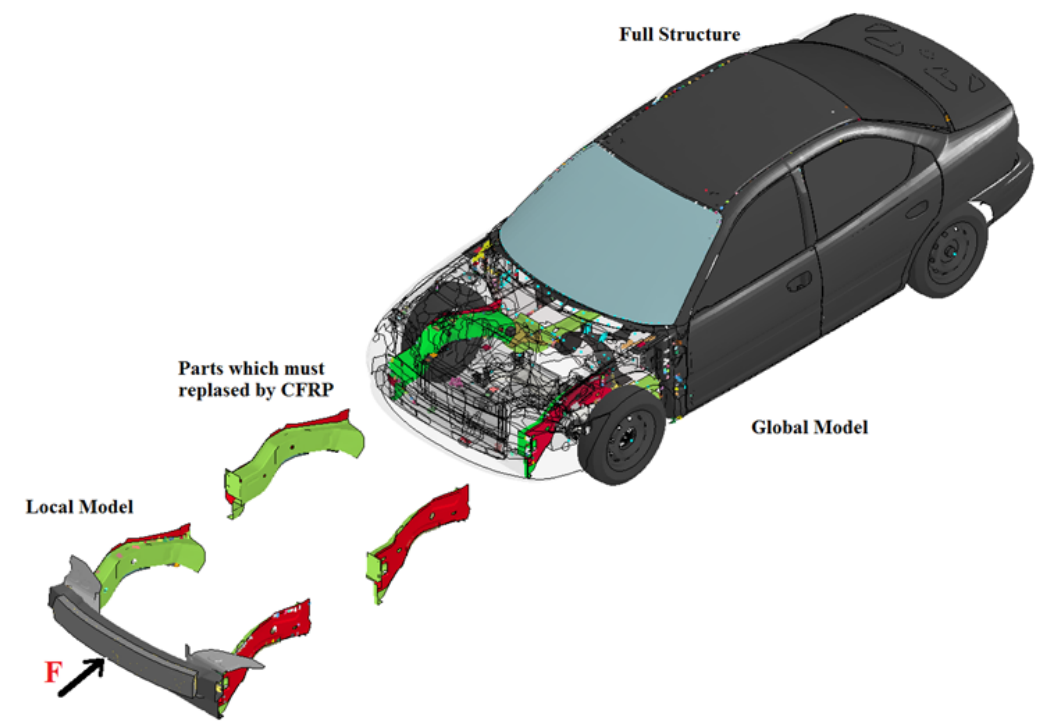

Figure 12. Global and local models for analysis and design of CFRP and steel crash rails. 


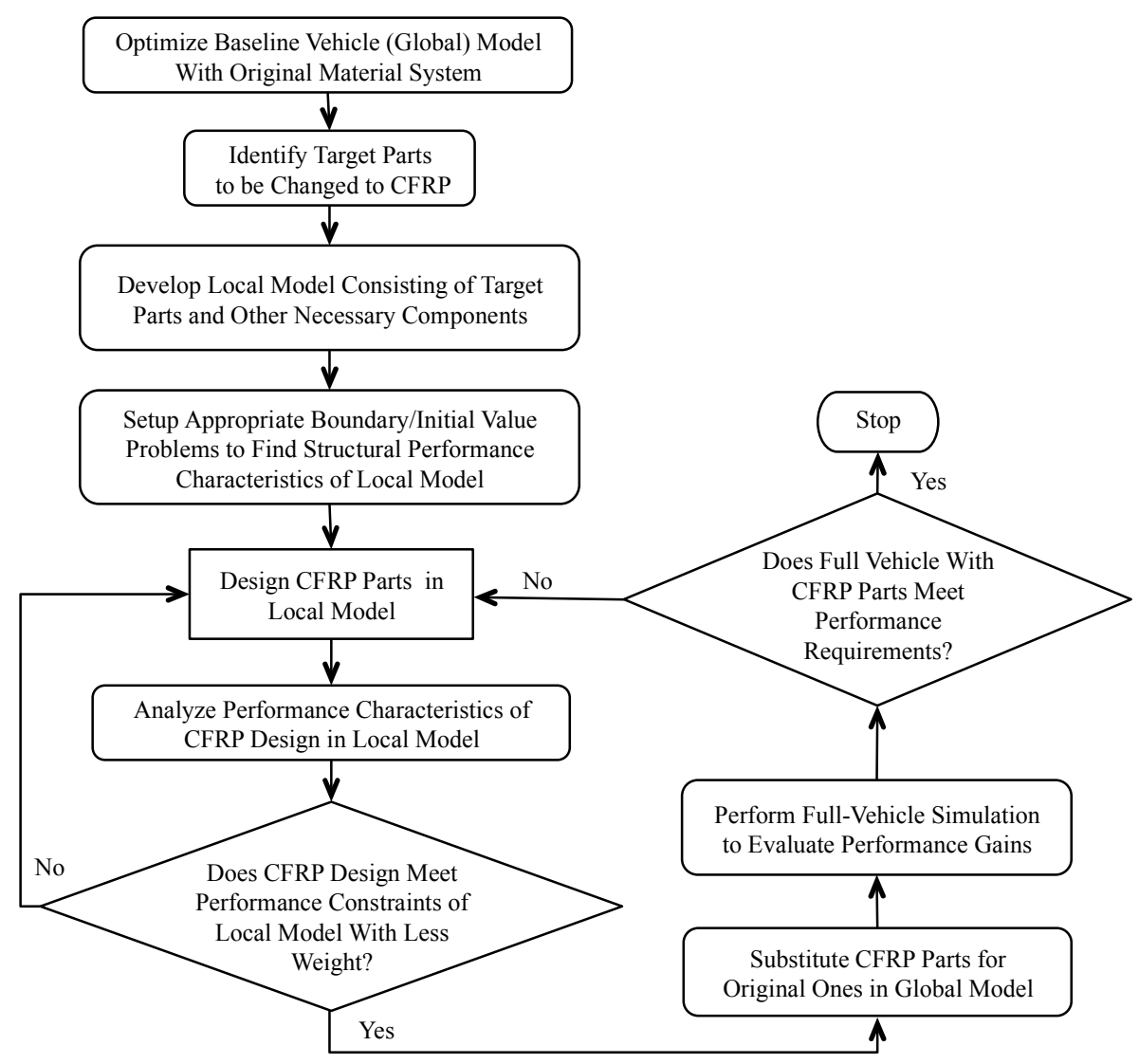

Figure 13. Global-local vehicle optimization procedure.

In the next step, the front-end (bumper-rail) portion of the vehicle as shown in Figure 12 was separated from the rest of the vehicle and treated as the local model, which was analyzed for vibration frequencies and deformation under dynamic impact load by a 127-kg mass travelling with an initial speed of 56.33 $\mathrm{km} / \mathrm{h}$ while the detached ends of the rails were held fixed. The first three natural frequencies and total deformation due to impact were used as design constraints for the composite rail optimization. The CFRP rail optimization problem sought to minimize the weight of the rails by adjusting the ply angles and number of plies such that the frequency and deformation responses were no worse (and potentially better) than the steel optimum. The CFRP rails were made of T700/2510 plain weave fabric composite with the ply angles limited to $0 / 90$ or \pm 45 degrees for manufacturability consideration. While holding the rail shape fixed, the wall thickness of the inner and outer parts were allowed to change. In the FE model, ply angles were defined such that continuity across adjacent elements was assured. The inner and outer CFRP rail parts with MAT54 material model in LS-DYNA were designed to have 28 layers for a total thickness of $5.97 \mathrm{~mm}$ with $[0 / 90 / \pm 45 / 0 / 90 / \pm 45 /(0 / 90) 3]_{s}$ stacking sequence. The combined mass of CFRP rails was found to be $6.95 \mathrm{~kg}$, which represented a savings of $39 \%$ in comparison to the mass of optimum steel rails at $11.34 \mathrm{~kg}$. 
The local front rail model with optimized CFRP rails was then incorporated into the full vehicle model and analyzed for both natural vibration characteristics and crash responses. The results in Table 4 show that the full-vehicle model with optimized CFRP rails outperforms the baseline vehicle model in terms of both crash responses and vibration frequencies. Furthermore, the global-local optimization strategy and substitution of CFRP composite for steel rails resulted in total weight savings of $8.15 \mathrm{~kg}$ in comparison to the baseline vehicle model.

Table 4. Comparison of full-vehicle performance characteristics using steel and CFRP rails.

\begin{tabular}{llllllll}
\hline $\begin{array}{l}\text { Full-Vehicle } \\
\begin{array}{l}\text { With } \\
\text { Model }\end{array}\end{array}$ & $\begin{array}{l}\text { FFI } \\
\text { (g's) }\end{array}$ & $\begin{array}{l}\text { Acceleration } \\
\text { OFI Intrusion }(\mathrm{mm})\end{array}$ & $\begin{array}{l}\text { Natural } \\
(\mathrm{Hz})\end{array}$ & Frequencies \\
& & Toeboard & Dashboard & Frq1 & Frq2 & Frq3 \\
\hline Baseline Steel Rails & 63.51 & 273.48 & 246.94 & 35.39 & 36.23 & 38.37 \\
CFRP Rails & 45.73 & 221.65 & 207.71 & 35.77 & 37.01 & 41.53 \\
\hline
\end{tabular}

\section{Welding Simulation and Process Optimization}

Considering the prevalence of welded components in vehicle structures, a study was conducted to simulate the general characteristics of welded parts and to compare the results with those found from actual welding experiments. The goal is to establish methods and models that are useful for controlling and designing Gas Metal Arc Welding (GMAW) processes to obtain appropriate mechanical performance of welded components and structures. Topics of interest range from modeling of heat generation, heat flow to residual stress and deformations. A computational optimization scheme is also integrated with welding simulations to predict the optimum process parameters for minimum deformation.

The activities performed include the following:

1. Evaluation of welding experiments on two specimens, one with linear and another with nonlinear weld path.

2. Development of a three-dimensional FE model of each specimen consistent with the experimental setup. The experimental data were used for validation of the welding simulation results.

3. Development of a coupled FE simulation and design optimization algorithm that could be used for welding process optimization. 


\section{Welding Experiments}

Two welding experiments were developed and performed at F.Tech R\&D North America Inc., in Troy, Ohio. The simple specimen in Figure 14(a) consists of two steel plates that are welded together along a straight line to form a simple lap joint, whereas the complex specimen in Figure 14(b) consists of two contoured pieces that are welded together to form a more complex component. The aim of the experimental procedure was to obtain physical measurements of the transient thermal, mechanical, and geometric responses of the welded specimen that can be used later for comparison with the temperature and displacement results obtained from the FE simulations.

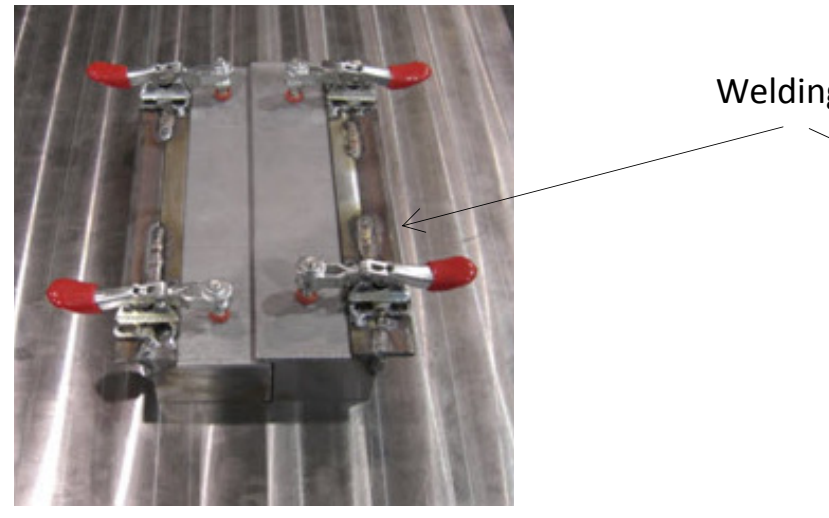

(a)

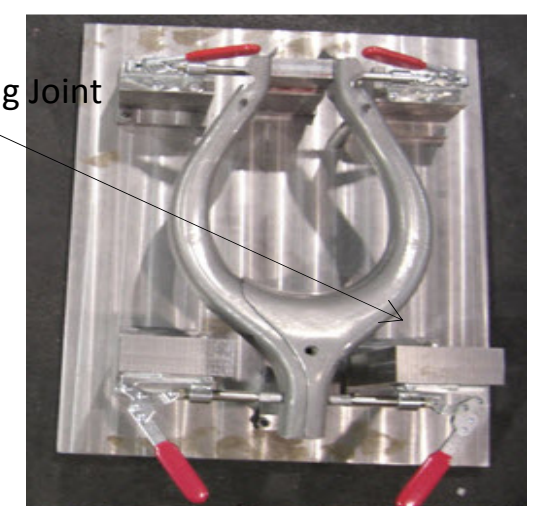

(b)

Figure 14. Welding experiment setup for (a) simple and (b) complex specimens.

Four different cases were considered for each experiment with key features identified in Table 5. For the simple specimen, four replicates were used for each experimental condition that considered different values for current and voltage. For the complex specimen, ten experiments were performed under four different conditions that considered the welding direction as well as the time at which the specimen was removed from the welding fixture.

Table 5. Experimental conditions for the simple and complex specimens.

\begin{tabular}{|c|c|c|c|c|c|c|c|}
\hline \multicolumn{4}{|c|}{ Simple Specimen $^{\text {a }}$} & \multicolumn{4}{|c|}{ Complex Specimen ${ }^{a, b}$} \\
\hline Case & Current & Voltage (V) & Replicate & Case & Welding & Removal & Replicate \\
\hline No. & (A) & & s & No. & Direction & Welding Fixture & $\mathrm{s}$ \\
\hline 1 & 180 & 18.0 & 4 & 1 & Rear- & Immediately & 3 \\
\hline
\end{tabular}




\begin{tabular}{|c|c|c|c|c|c|c|c|}
\hline & & & & & Front & & \\
\hline 2 & 180 & 23.0 & 4 & 2 & $\begin{array}{l}\text { Front- } \\
\text { Rear }\end{array}$ & Immediately & 3 \\
\hline 3 & 200 & 20.5 & 4 & 3 & $\begin{array}{l}\text { Rear- } \\
\text { Front }\end{array}$ & After 5 Minutes & 3 \\
\hline 4 & 220 & 20.5 & 4 & 4 & $\begin{array}{l}\text { Front- } \\
\text { Rear }\end{array}$ & After 5 Minutes & 1 \\
\hline
\end{tabular}

\footnotetext{
${ }^{a}$ In all experiments, welding speed was $0.01 \mathrm{~m} / \mathrm{s}$ and ambient temperature was about $25^{\circ} \mathrm{C}$.

${ }^{\mathrm{b}}$ In all experiments, current and voltage were $180 \mathrm{~A}$ and $18 \mathrm{~V}$, respectively.
}

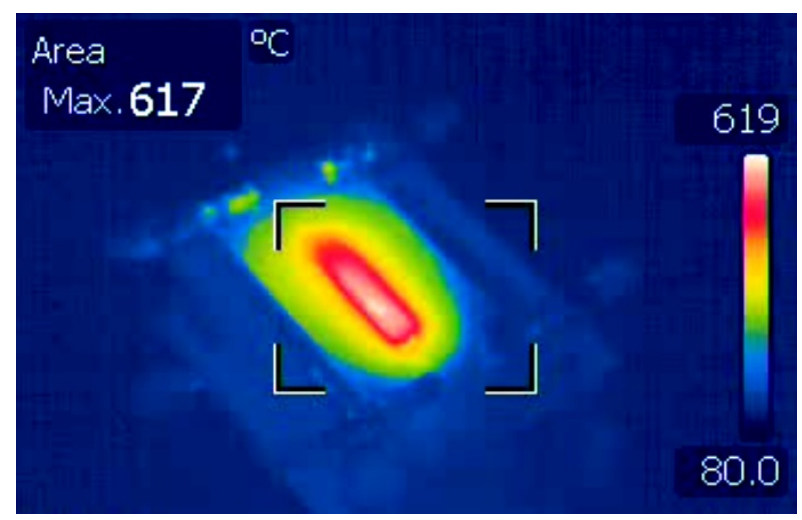

(a)

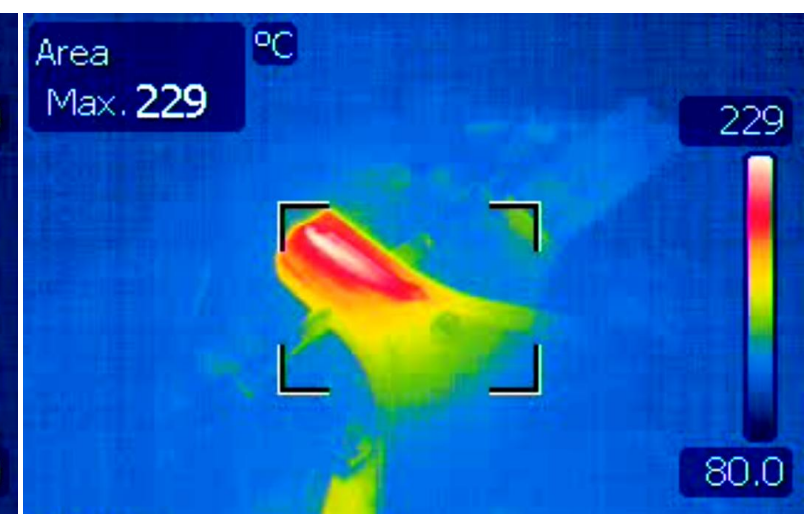

(b)

Figure 15. Temperature measurements of (a) simple and (b) complex specimens during the cooling process after welding.

During the welding experiments, parameters such as arc current, voltage, and welding time were recorded manually while temperature was measured using an FLIR -THERMACAMT400 infrared camera. The camera could capture temperature in the range of $80^{\circ} \mathrm{C}$ to $1300^{\circ} \mathrm{C}$. The time required to cool down to the lowest temperature bound of $80^{\circ} \mathrm{C}$ was 3 minutes for the simple and 5 minutes for the complex specimen. Figure 15 shows a single frame of the digital video recorded by the infrared camera for each specimen.

The Faro Laser ScanARm v3 was used to measure the geometric variations in the parts before and after welding. The welded parts were compared to the reference objects that were just glued together. The deformation measurements relative to the pre-welding condition were reported in the form of graphical 
data using Polyworks v11 measuring software with a typical deformation pattern shown in Figure 16. The range of values represented by the color pattern indicates that both specimens have bent as a result of the welding operation in that particular experiment. Since the deformation pattern of each welded specimen was measured relative to the reference model, positive numbers imply upward deformation while negative values imply downward deformation relative to the reference object.

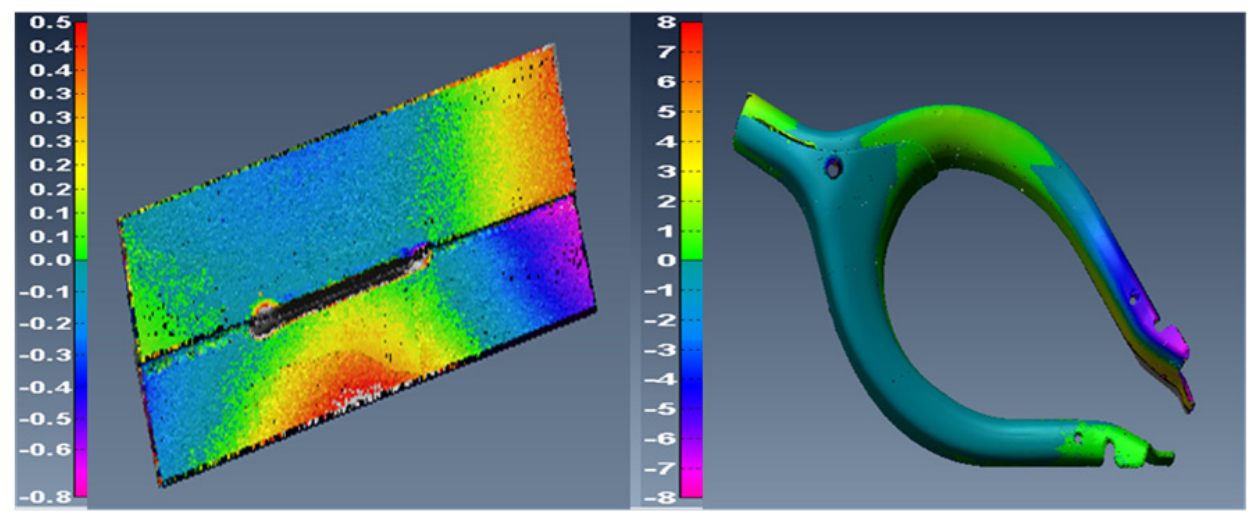

(a)

(b)

Figure 16. Typical experimental welding deformation patterns for (a) simple and (b) complex specimens.

After welding operation, standard penetration tests were performed on the simple specimen to characterize the internal defects and to investigate the welding quality. The penetration tests were performed and repeated in three different areas corresponding to the part's cross-section at the starting, middle, and final points along the welding path. A photograph of the cross-sectional view of the simple specimen (at 10-mm distance from the starting point) is shown in Figure 17. The width and height of weld and depth of weld penetration are clearly visible in the photograph.

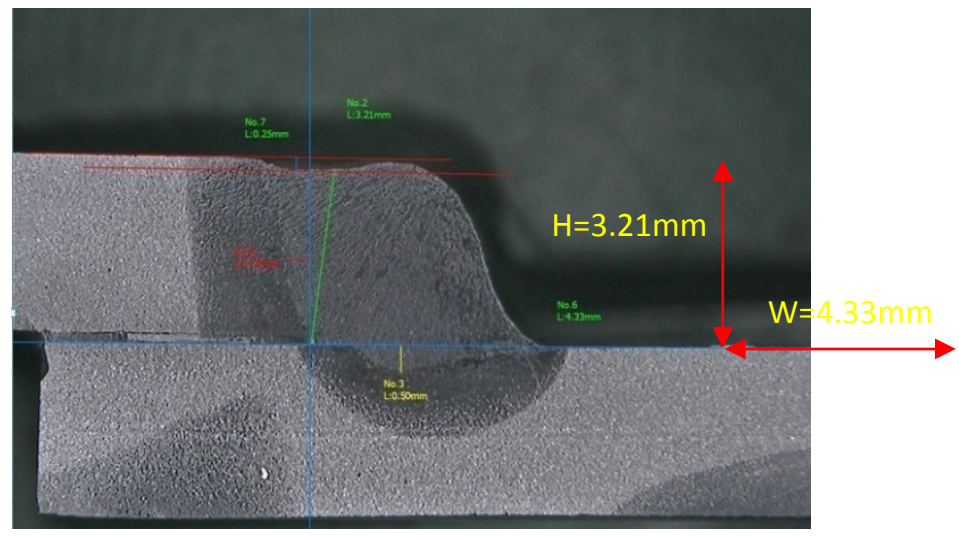


Figure 17. Typical weld penetration assessment for the simple specimen.

\section{Welding Simulation and Validation}

Three-dimensional FE models were developed to simulate the arc welding process using the Simufact.Welding software. This software is equipped with dual solver (i.e., thermal and mechanical) technology for performing a coupled, physics-based analysis of the welding process. For consistency with the welding experiments, a separate model was developed to match each of the two specimens identified previously. The relevant welding parameters in the simulation model were set to match the experimental conditions. The welding process was modeled by a moving heat source with dimensions that are consistent with the experimental weld cross-sectional properties. Figure 18 shows the FE models of the simple and complex specimens. The simple model consists of 6080 linear hex elements for the plates and another 1120 linear hex elements forming the weld fillet, whereas the two arms of the complex model contain 15000 linear hex elements.

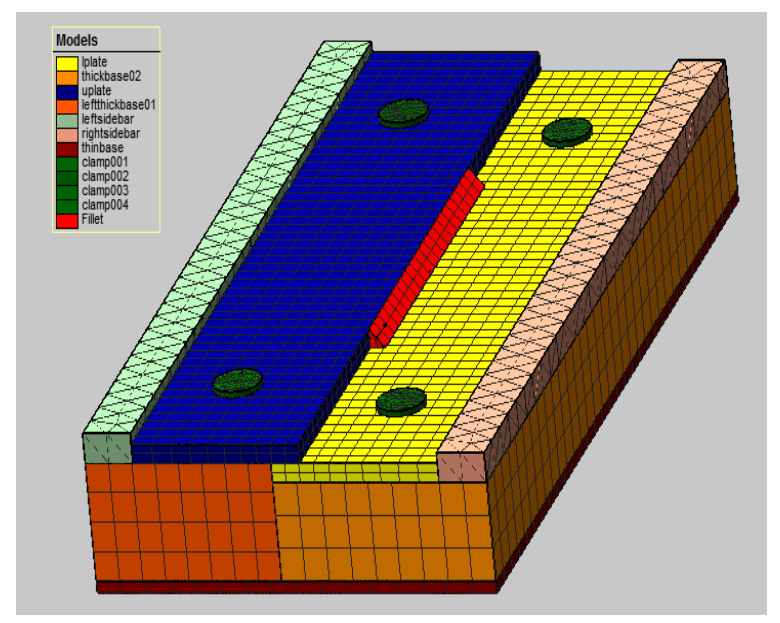

(a)

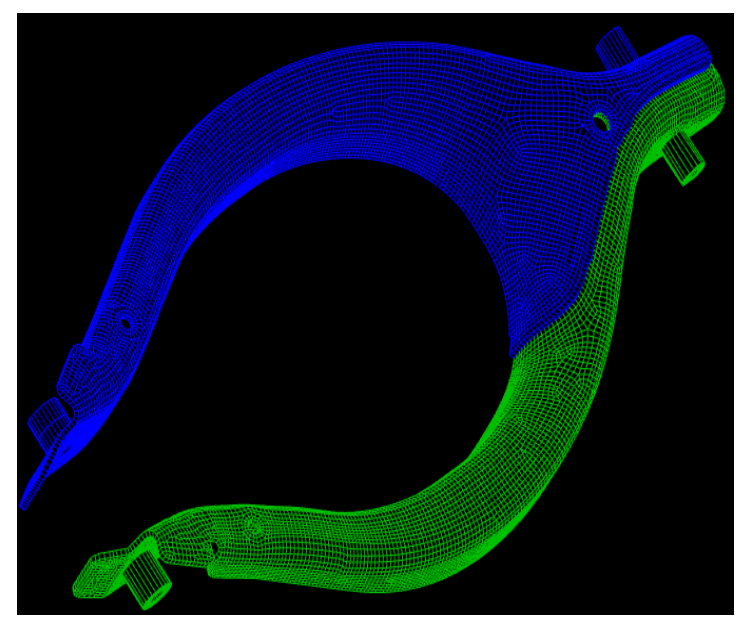

(b)

Figure 18. FE models of (a) simple and (b) complex specimens.

Figure 19 shows the plots of welding-induced deformation as predicted by Simufact.Welding and as measured in the experiment. The overall deformation patterns are in very good agreement, although the magnitudes of deformation are different. Further investigation is underway to enhance the accuracy of the simulation predications. 


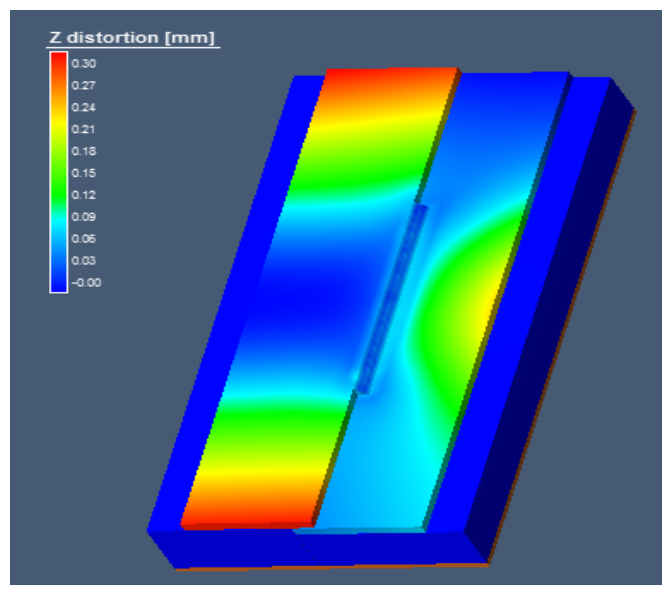

(a)

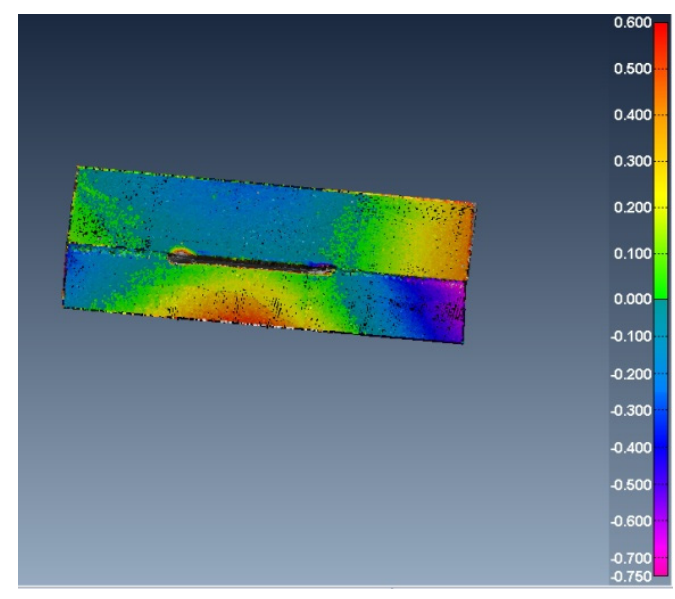

(b)

Figure 19. Welding-induced deformation as (a) predicted by FE simulation and (b) experimentally measured.

We have also validated the weld cross-sectional geometry by direct comparison of welding test and simulation results. Figure 20(a) shows the cross-sectional shape and dimensions obtained from macro test of the simple specimen at a $10-\mathrm{mm}$ distance from the starting point. Similarly, Figure 20(b) shows the cross-sectional shape and dimensions obtained from the FE simulation at the same distance inside the fillet material. In Figure 20(c) the experimental and simulation-based weld cross-sections are overlapped for ease of comparison. 

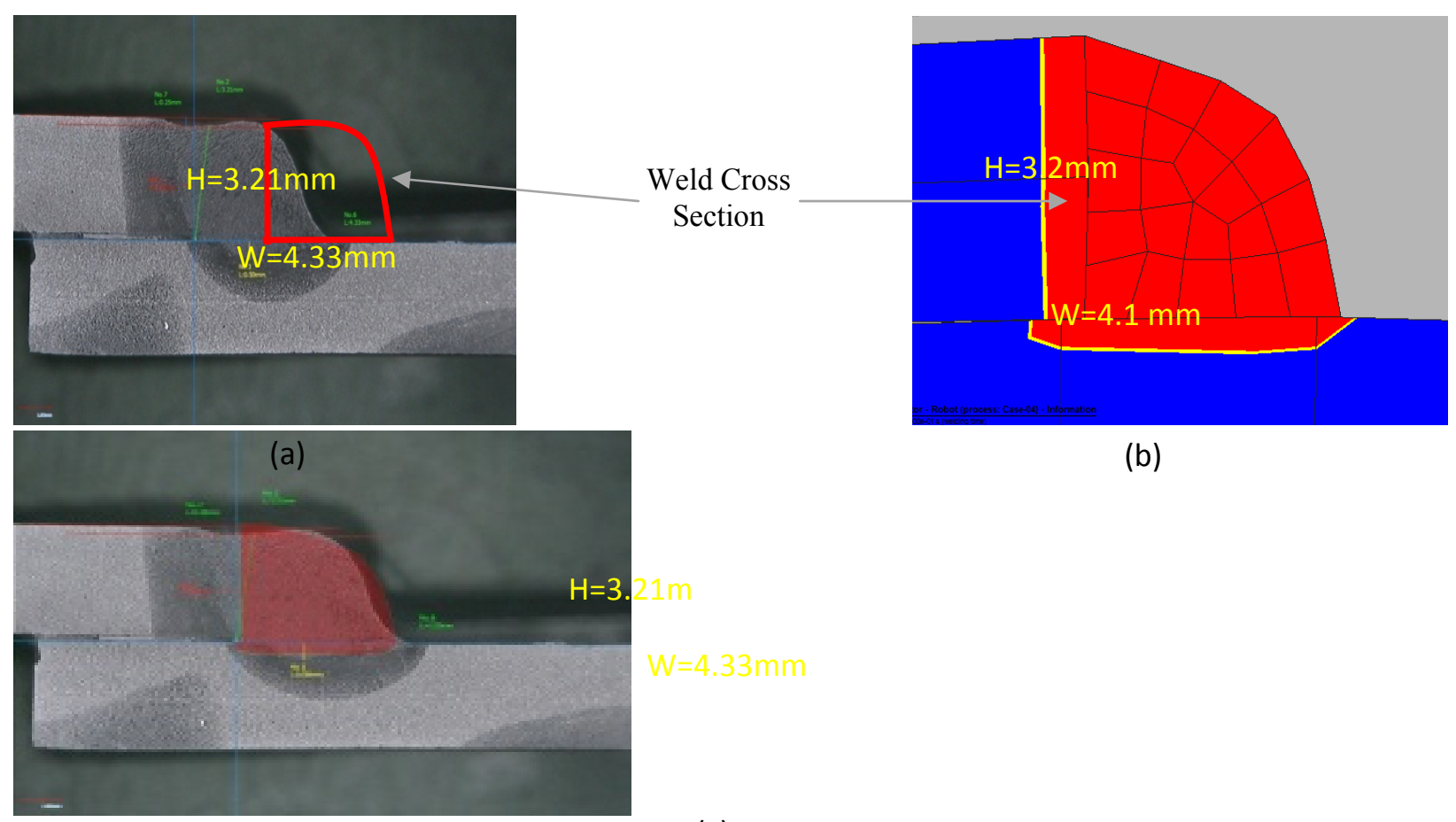

(b)

(c)

Figure 20. Weld cross-sections of (a) experimental model, (b) simulation model and (c) overlapped fillet.

As a result of this research activity, the software company (Simufact-Americas, Inc.) updated their Simufact.Welding software. We conducted several trials with the new version of the software and achieved improved accuracy, especially in terms of distortion prediction. The most important feature of the new version is its capability to predict the bending behavior or high-end separation of the welded parts. Bending was a dominant component of welding-induced deformation in the simple specimen. Figure 21 shows the bending behavior in the test specimen and that predicted by the latest version of the simulation software.

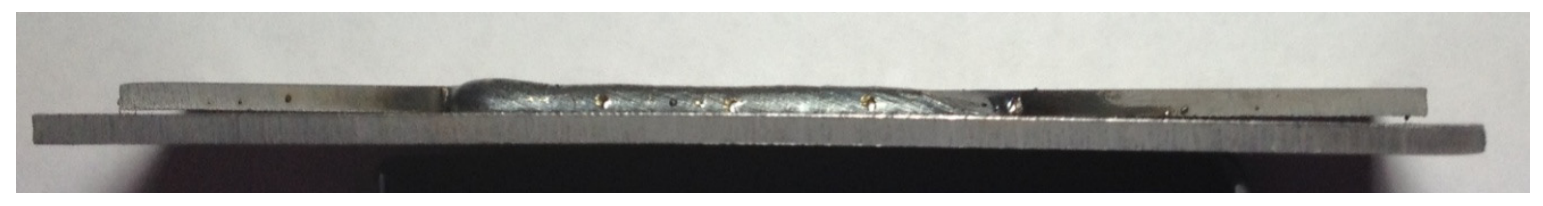

(a)

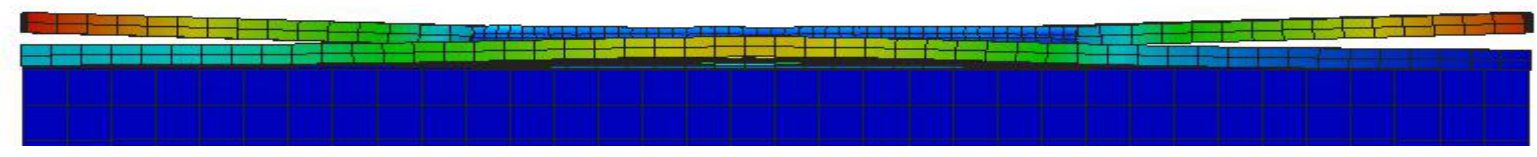

(b) 
Figure 21. Bending trends in simple specimen as (a) found experimentally and (b) predicted by FE simulation.

\section{Coupled FE Simulation and Optimization Algorithm}

To accommodate welding process optimization, an algorithm shown in Figure 22 was developed, where the FE simulations with Simufact.Welding are directly linked with Genetic Algorithms (GA), a populationbased, gradient-free optimization technique. Weld induced deformation is identified as the objective function/criterion to be optimized. The design variables include welding speed, welding current, arc voltage, ambient temperature, and welding direction. MATLAB has been used as a programming environment for the system algorithm development. The GA toolbox of MATLAB has been used as the optimization tool. We have developed a custom GA variant by modifying the objective function definition, initial population and fitness scaling options and by defining parent selection, crossover, and mutation functions. Work is underway to apply the GA-FE algorithm to welding process optimization for both simple and complex configurations.
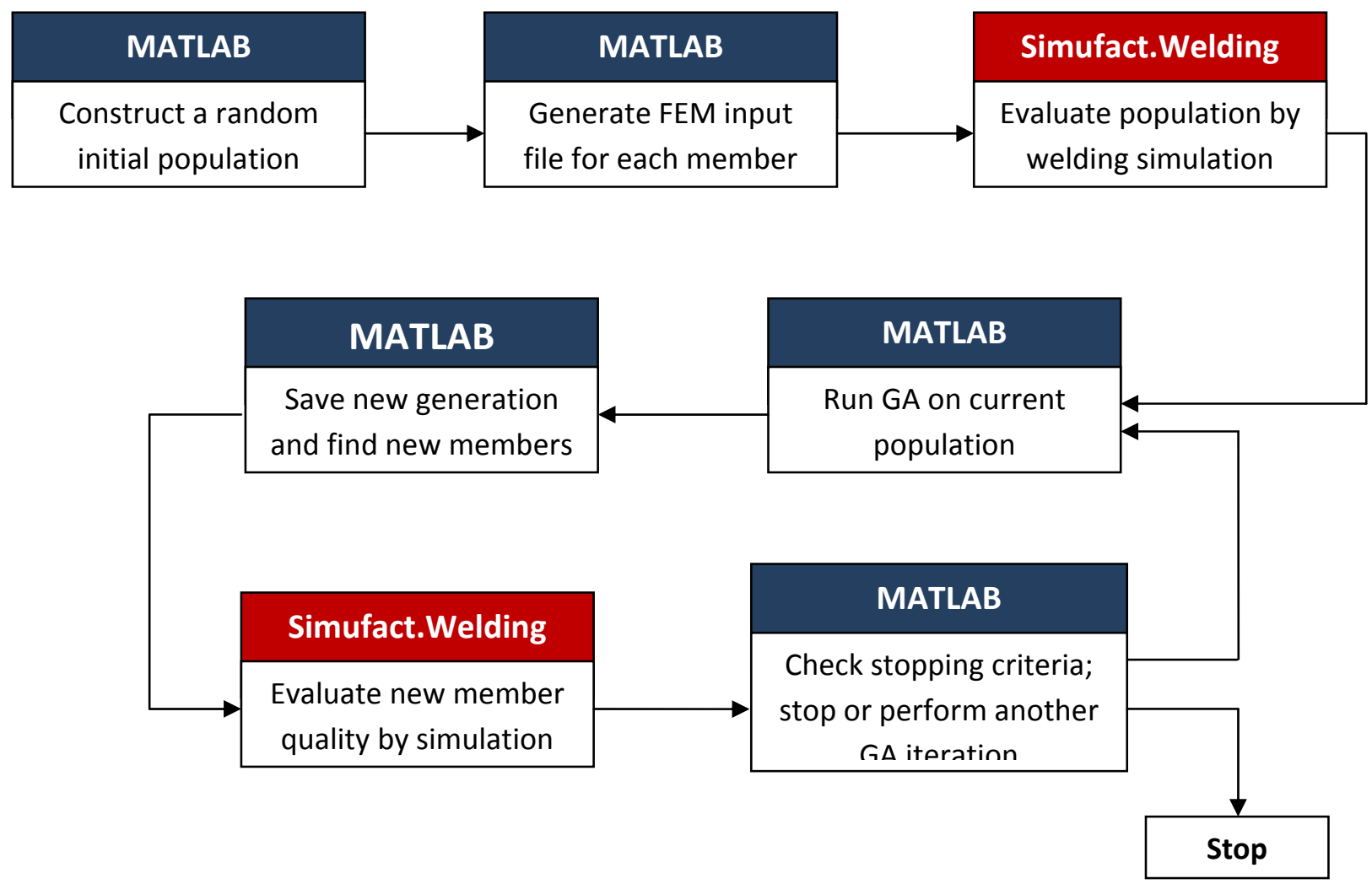

Figure 22. Algorithm for the coupled FE simulation and optimization procedure. 


\section{Publications/Presentations/Patents}

1. Rouhi, M. and Rais-Rohani, M., "Modeling and Probabilistic Design Optimization of a Nanofiber Enhanced Composite Cylinder for Buckling," Composite Structures, Vol. 95, pp. 346-353, 2013.

2. Najafi, A., Acar, E., and Rais-Rohani, M., "Multi-Objective Robust Design of Energy Absorbing Components Using Coupled Process-Performance Simulations," Engineering Optimization, in press, 2013.

3. Najafi, A. and Rais-Rohani, M., "Sequential Coupled Process-Performance Simulation and Multi-Objective Optimization of Thin-Walled Tubes," Materials and Design, Vol. 41, pp. 8998, 2012.

4. Parrish, A., Rais-Rohani, M., and Najafi, A., "Crashworthiness Optimization of Vehicle Structures with Magnesium Alloy Parts," International Journal of Crashworthiness, Vol. 17, Is. 3, pp. 259-281, 2012.

5. Najafi, A., Marin, E., and Rais-Rohani, M., "Concurrent Multi-Scale Crush Simulations with a Crystal Plasticity Model," Thin-Walled Structures, Vol. 53, pp. 176-187, 2012.

6. Najafi, A., Acar, E., and Rais-Rohani, M., "Robust Design of Energy Absorbing Components Considering the Manufacturing Effects," $14^{\text {th }}$ AIAA Non-Deterministic Approaches Conference, Honolulu, HI, Apr 23-26, 2012.

7. Najafi, A. and Rais-Rohani, M., "Multilevel Decomposition and Optimization of Coupled Process-Performance Systems," $8^{\text {th }}$ AIAA Multidisciplinary Design Optimization Specialist Conference, Honolulu, HI, Apr 23-26, 2012.

\section{REFERENCES}

Abaqus/Explicit version 6.10, user's manual, Hibbit, Karlsson and Sorensen Inc., Rhode Island, USA, 2010.

Abramowicz, W. and Wierzbicki, T., "Axial Crushing of Multicorner Sheet Metal Columns," Journal of Applied Mechanics, Vol.56, pp. 113-120, 1989.

Abramowicz, W., "Thin-Walled Structures As Impact Energy Absorbers," Thin-Walled Structures, Vol. 41, No. 2-3, pp. 91-107, 2003.

Deleo, F., Wade, B., Feraboli, P., and Rassaian, M., "Axial Crushing of Composite Structures: Experiment and Simulation," Proceedings of 50th AIAA/ASME/ASCE/AHS/ASC Structures, Structural Dynamics and Materials Conference, Palm Springs, CA, May 4-7, 2009.

Marin, E.B., "On the Formulation of Crystal Plasticity Model," Sandia Report, Sandia National Laboratory, SAND2006-4170, 2006. 
Najafi, A. and Rais-Rohani, M., "Mechanics of Axial Plastic Collapse in Multi-Cell, Multi-Corner Crush Tubes," Thin-Walled Structures, Vol. 49, No. 1, pp.1-12, 2011.

Najafi, A., Marin, E., and Rais-Rohani, M., "Concurrent Multi-Scale Crush Simulations with a Crystal Plasticity Model," Thin-Walled Structures, Vol. 53, pp. 176-187, 2012.

National Crash Analysis Center, "Finite Element Model of Dodge Neon, Model Year 1996, Version 7," 2006. (http://www.ncac.gwu.edu/vml/archive/ncac/vehicle/neon-0.7.pdf)

Wierzbicki, T. and Abramowicz, W., "On the Crushing Mechanics of Thin Walled Structures," Journal of Applied Mechanics, Vol. 50, pp. 727-734, 1983.

\section{KEY WORDS}

Texture effects, Crashworthiness optimization, Lightweight structures, Composite structures, Welding simulation.

\section{BRIEF Description OF RePort}

This final report describes the research activities related to the simulation-based design optimization task of DOE/SRCLID project. Particular emphasis is placed on the work done in the following areas: Analysis of the effect of texture induced anisotropy on structural performance of thin-walled components; finite element simulation and optimization of vehicle structures made of composite materials; and validation of computational welding-induced response predictions with experimental results. 
Subtask 1.7: Solidification Microstructure Modeling in Steel and Mg ALLOYS

Team Members: S. Fellicelli, L.Wang

\section{Objective}

Develop a set of integrated simulation tools to predict and improve the solidification microstructure and mechanical properties in materials processing and manufacturing. Research domain: solidification science, defect formation, casting, additive manufacturing, steel, light alloys

\section{APPROACH}

- Hierarchical multiscale models for direct microstructure simulation in current tera/peta-scale and future exa-scale parallel computing

- Local methods (Cellular automaton, Lattice Boltzmann) for high efficiency parallelization

- Model validation through microstructure characterization and controlled solidification experiments

\section{SUB-TASKS}

1. Modeling of dendritic microstructures under convection

2. Phase-field modeling of Mg alloys

3. Segregation defects in DS castings

4. Confluence weld defects in multiple-gated castings

5. Evaluation of four casting processes for magnesium alloy AZ91

6. Modeling and characterization of additively manufactured steel parts

\section{ACCOMPLISHMENTS}

1. Developed capability to simulate large-scale dendritic microstructures in massively parallel supercomputers

2. Developed model to simulate hexagonal dendrites of Mg-alloys which does not exhibit artificial anisotropy

3. Developed model that captures freckle segregation defects with mesh adaptation 
4. Explained and characterized the formation of confluence weld defects

5. Evaluated and tested the part quality produced by four Mg-alloy casting processes

6. Evaluated and tested the microstructure of stainless steel deposits made with two layer-additive processes

\section{Modeling of dendritic microstructures under convection}

A significant landmark was achieved by our team in the field of high-performance computing of solidification microstructures. A highly scalable model of dendritic solidification based on the lattice Boltzmann and Cellular Automaton methods, was developed to simulate the growth of 3264 equiaxed dendrites of an $\mathrm{Al}-3 \% \mathrm{Cu}$ alloy under forced convection. An image of the simulated microstructure after 400k steps can be seen in Fig. 1, showing velocity vectors of interdendritic flow.

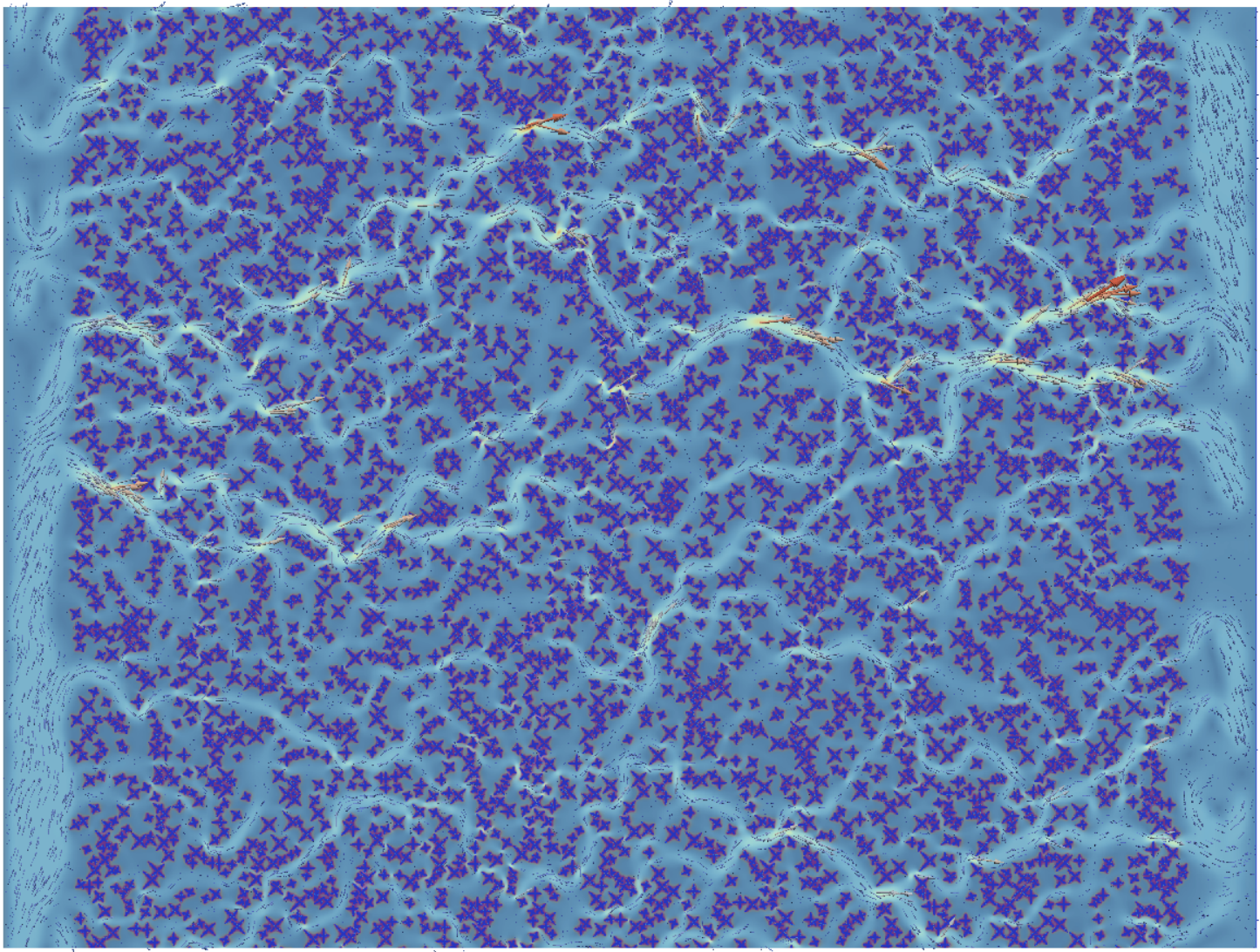

Fig. 1 - Dendritic microstructure of $\mathrm{Al}-3 \% \mathrm{Cu}$ alloy growing under convection 
The calculation domain is $2.4 \mathrm{~mm}$ in the $\mathrm{x}$ - (horizontal) direction and $1.8 \mathrm{~mm}$ in the $y$ - (vertical) direction. It was discretized with a $8000 \times 6000$ grid of D2Q9 cells. The model solves the energy, solute, and NavierStokes equations, in addition to phase change.

The base domain and results of Fig. 1 were then replicated several times in space, in order to form larger simulation domains. The simulation was restarted from the replicated domains and executed during 587 steps. The number of cores was increased accordingly so that the same number of cores (12) is assigned to each base domain (constant load per core). The computational time was measured for each restart simulation in the replicated domains. The green curve in Fig. 2 shows the calculation times obtained in the Cray XT5 at ORNL (Kraken) for the base domain (12 cores) and 5 replications up to 41472 cores, indicating a fairly constant simulation time and therefore a nearly perfect scalability of the model.

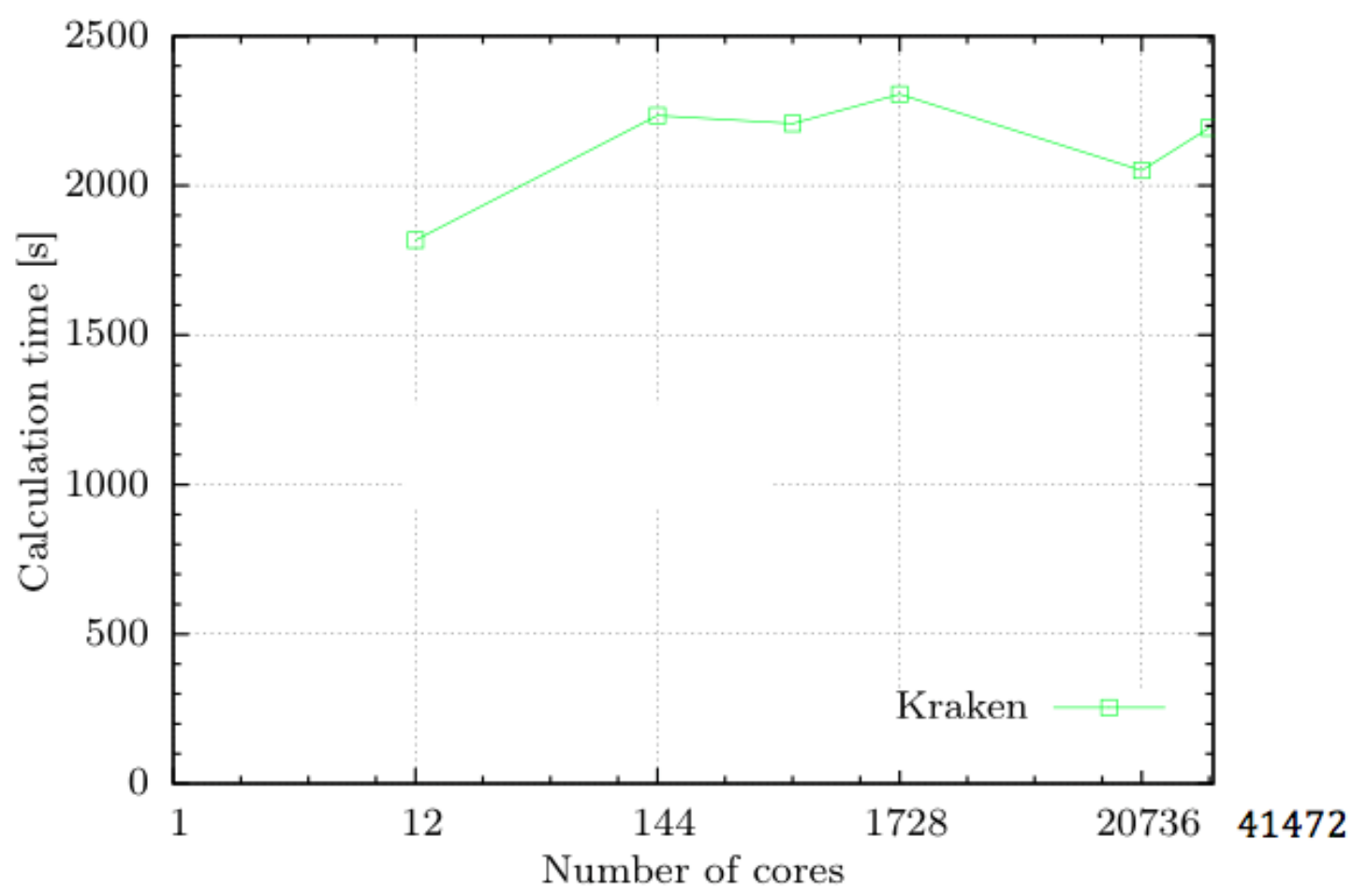

Fig. 2 - Scale-up performance of solidification model in Cray XT5 (ORNL)

The largest calculation corresponds to a base domain replicated 72 times in the x-direction and 48 times in the y-direction, i.e., a domain size of $\mathbf{1 7 . 2 8} \mathrm{cm} \times \mathbf{8 . 6 4} \mathrm{cm}$. In order to maintain 12 cores per base domain, a total of $72 \times 48 \times 12=\mathbf{4 1 4 7 2}$ cores were needed (Kraken has a total of 112000 cores). The 
corresponding grid had $(8000 \times 72) \times(6000 \times 48)$ cells, i.e., over 165 billion nodes. The simulated domain contained 11.3 million dendrites.

For comparison with the state of the art, the largest simulations reported (phase-field method) show only scalability up to a few hundred cores and less than a hundred dendrites.

Ref.: B. Jelinek, M. Eshraghi, S.D. Felicelli, "Large-scale parallel lattice-Boltzmann model of dendritic growth", to be presented at TMS Annual Meeting, San Antonio, TX, March 2013

\section{Phase-field modeling of Mg alloys}

A cellular automaton (CA)-finite element (FE) model and a phase field (PF)-FE model were used to simulate equiaxed dendritic growth during the solidification of hexagonal metals. In the CA-FE model, the conservation equations of mass and energy were solved in order to calculate the temperature field, solute concentration, and the dendritic growth morphology. CA-FE simulation results showed reasonable agreement with the previously reported experimental data on secondary dendrite arm spacing (SDAS) vs cooling rate. In the PF model, a PF variable was used to distinguish solid and liquid phases similar to the conventional PF models for solidification of pure materials. Another PF variable was considered to determine the evolution of solute concentration. Validation of both models was performed by comparing the simulation results with the analytical model developed by LiptonGlicksman-Kurz (LGK), showing quantitatively good agreement in the tip growth velocity at a given melt undercooling. Application to magnesium alloy AZ91 (approximated with the binary Mg-8.9 wt\% Al) illustrates the difficulty of modeling dendrite growth in hexagonal systems using CA-FE regarding meshinduced anisotropy and a better performance of PF-FE in modeling multiple arbitrarily-oriented dendrites growth. 


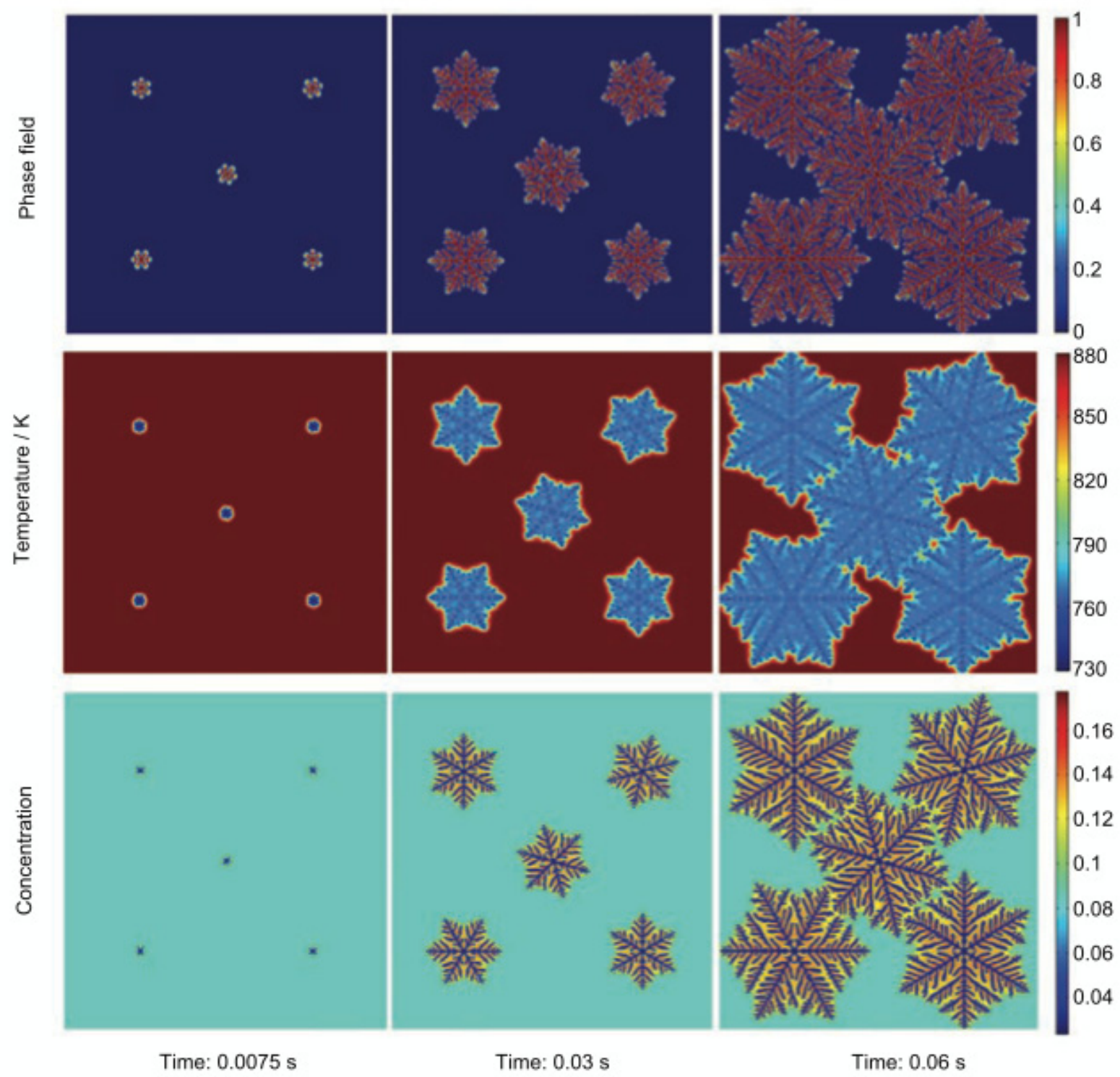

Fig. 3 Phase-field, temperature, and solute maps of multiple arbitrarily-oriented seeds at different simulation time using the PF-FE model. Heat flux imposed at the four walls was $10 \mathrm{~kW} \cdot \mathrm{m}-2$. The calculation domain is $400 \mu \mathrm{m} \times 400 \mu \mathrm{m}$ with mesh size of $0.2 \mu \mathrm{m}$

Ref: M. Asle Zaeem, H. Yin, S.D. Felicelli, "Comparison of cellular automaton and phase field methods to simulate dendrite growth in hexagonal systems", Journal of Materials Science and Technology, vol. 28 (2), pp. 137-146 (March 2012) 


\section{Segregation defects in DS castings}

Modeling the formation of macroscopic segregation channels during directional solidification processes has important applications in the casting industry. Computations that consider thermosolutal convection involve different length scales ranging from the small solute boundary layer at the dendrite tips to the characteristic size of the casting. In general, numerical models of solidification in the presence of a developing mushy zone are computationally inefficient because of nonlinear transport in an anisotropic porous medium. In the current work, mesh adaptation with triangular finite elements is used in conjunction with an efficient fractional-step solver of the momentum equations to predict the occurrence of channel-type segregation defects or freckles. The triangulations are created dynamically using an unstructured grid generator and a refinement criterion that tracks the position of the channel segregates. The efficiency of mesh adaptation is illustrated with simulations showing channel formation and macrosegregation in directional solidification of a binary alloy.

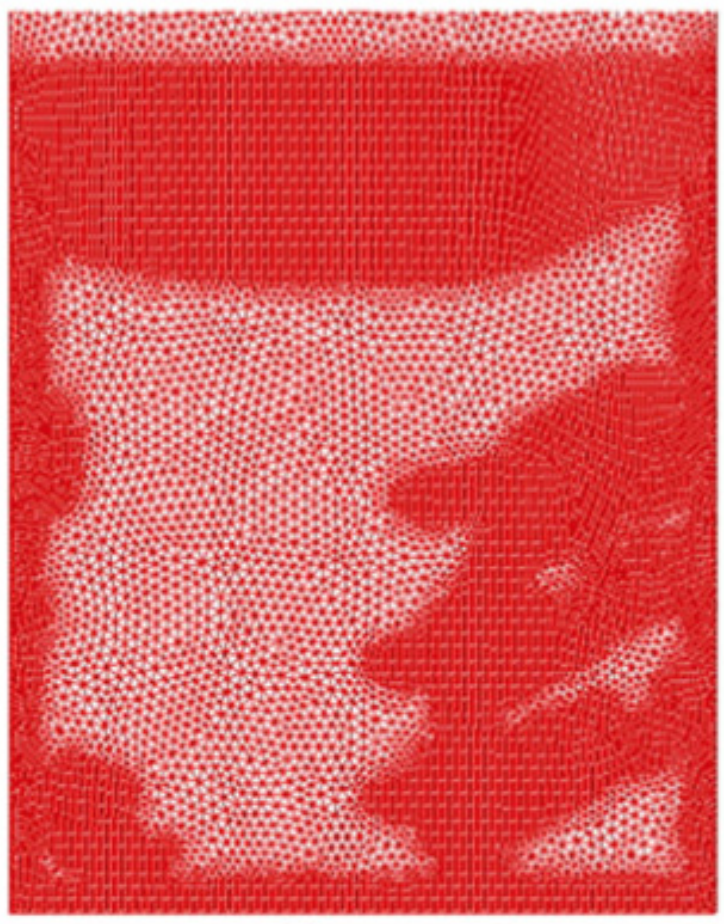

(a)

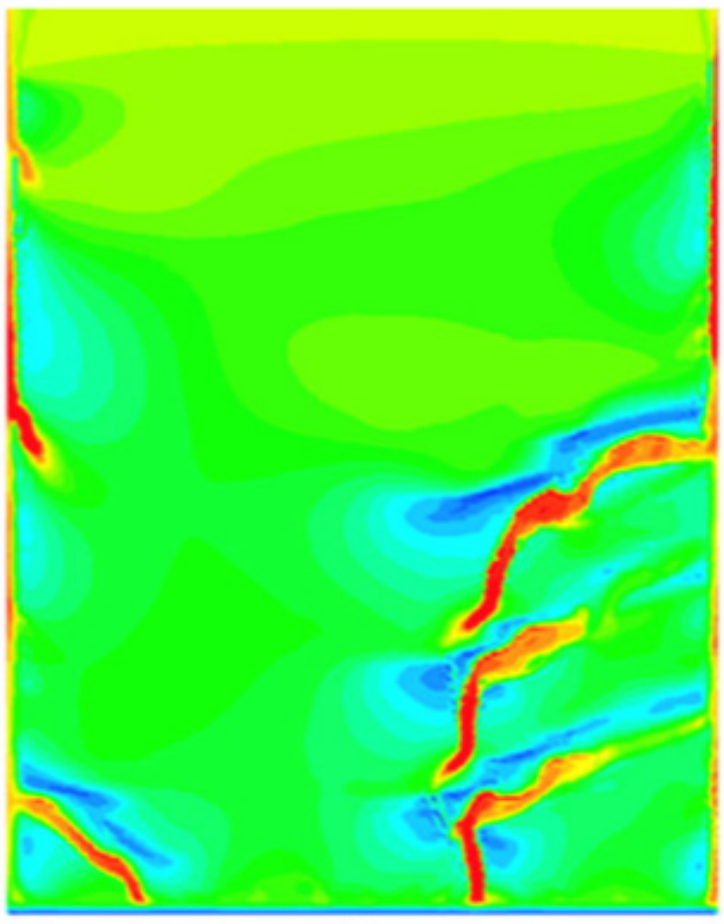

(b)

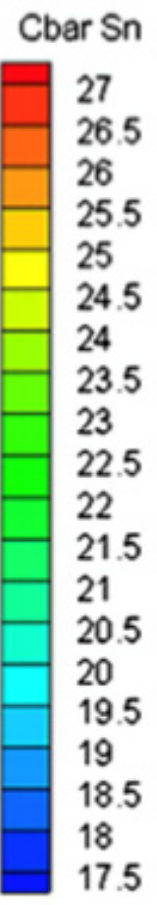

Fig. 4-Results after $2000 \mathrm{~s}$ solidification time with adaptive remeshing in a rectangular domain of 30 $\mathrm{mm} \times 50 \mathrm{~mm}$. (a) Finite-element mesh and (b) contours of mixture concentration of solute component.

Ref: U.K. Sajja and S.D. Felicelli, "Modeling freckle segregation with mesh adaptation", Metallurgical and Materials Transactions B, vol. 42 (6), pp. 1118-1129 (Dec 2011) 


\section{Confluence weld defects in multiple-gated castings}

This work examines the quality aspects of multiple gated castings in Aluminum A356 alloys. During the casting of parts in which multiple ingates are used to distribute the liquid melt throughout the mold, oxidation of the surface may prevent the adherence of the opposing fronts upon rejoining, thus producing sites prone to defect formation referred to as confluence welds. This phenomenon is investigated by the production of thin vertically sand-cast plates via the Electromagnetic Pump Green Sand (EPGS) process developed by CIDAUT (Spain). For this research, three multiple-gated designs and one single-gated design were used as a means of researching an optimal configuration for melt delivery throughout the mould cavity. A series of numerical simulations were developed for each of the gating designs in order to compare modeling results with plates cast in the overseas counterpart's foundry. The method of four-point bend testing was used to obtain information about the mechanical properties of the castings, and from this data, a Weibull statistical analysis was performed in order to quantify specimen failure rate for each of the configurations. Metallographic analysis was carried out using optical microscopy, and fractography using FEG-SEM. The numerical and experimental studies provided interesting insights on the formation of defects associated with the confluence of flow fronts.
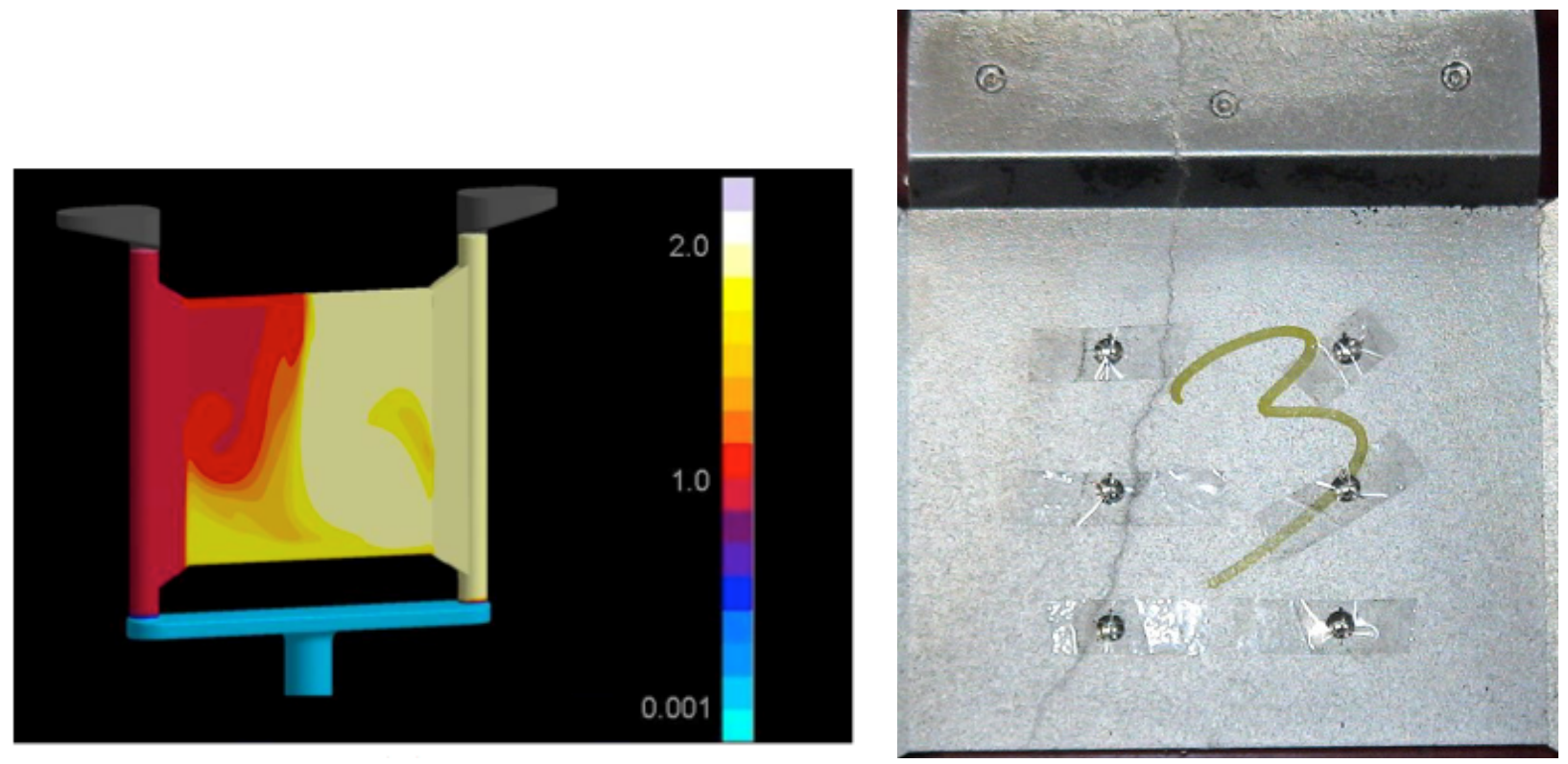

Fig. 5 - (a) Simulation of material trace in casting with two side ingates, showing confluence of the two fronts. (b) Photograph of confluence weld defect in resulting casting.

Ref: R. Lett, S.D. Felicelli, J.T. Berry, R. Cuesta, A. Rivas, M.E. Alcalde,, "Quality aspects of A356 castings with multiple gates", International Journal of Metalcasting, vol. 6(2), pp. 67-82 (March 2012) 


\section{Evaluation of four casting processes for magnesium alloy AZ91}

The performance of four different casting processes for magnesium alloy AZ91 was evaluated through microstructure characterization, mechanical testing, and SEM analysis of fracture surfaces. Passenger car control arms were cast by indirect squeeze cast, low pressure permanent mold (LPPM), T-Mag, and Ablation processes. Samples were cut from twelve locations of the control arms for the microstructure characterization. The microstructure, grain size, porosity distribution, and defect analyses were obtained with optical microscopy and image analyzer. Five different defects including microporosity, oxide film, sponge shrinkage, gas pore, and crack-like defect are identified. The mechanical behavior was characterized from the four-point bending (FPB) and tensile tests. The four casting processes were evaluated in terms of reliability using two-parameter Weibull statistics of the ultimate bending strength (UBS) determined from the FPB test samples. Metallographic analysis was performed on these samples, revealing noticeable microstructural differences between them, with some even showing possible evidence of oxide films.

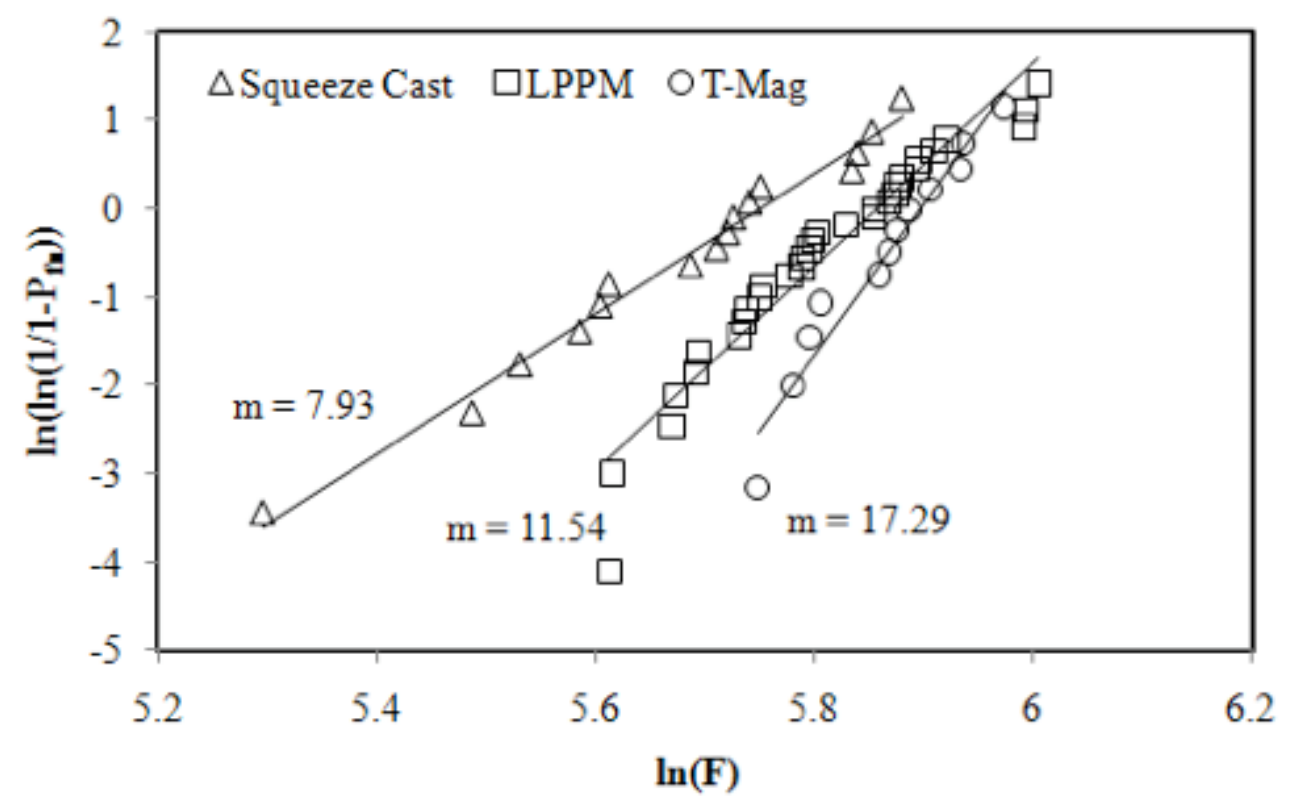

Figure 6. Two parameter Weibull plot for UBS data of FPB tests for different casting processes

Ref: L. Wang, J.B. Jordon, R. Lett, S.D. Felicelli, J.T. Berry, D. Penrod, "Microstructure and performance of four casting processes for magnesium alloy AZ91", International Journal of Metalcasting, vol. 5(4), pp. 37-46 (Nov 2011) 


\section{Modeling and characterization of additively manufactured steel parts}

Advancements in the area of additive manufacturing have led to the development of new methods with the purpose of optimizing component properties and expanding operating environments. In this comparative study, the influence of process parameters including beam and laser current, translation speed, and wire feed and deposition rate on 316L stainless steel deposits produced by both Laser Engineered Net Shaping (LENS) and Electron Beam Freeform Fabrication $\left(\mathrm{EBF}^{3}\right)$ processes are investigated. Tensile tests are used to obtain information on the mechanical properties of the specimens. A metallographic analysis is performed using optical microscopy and SEM to characterize solidification grain structure, porosity, secondary dendrite arm spacing, and possible modes of failure. This study highlights the distinct characteristics of both additive methods and how they lead to different microstructure and mechanical properties.
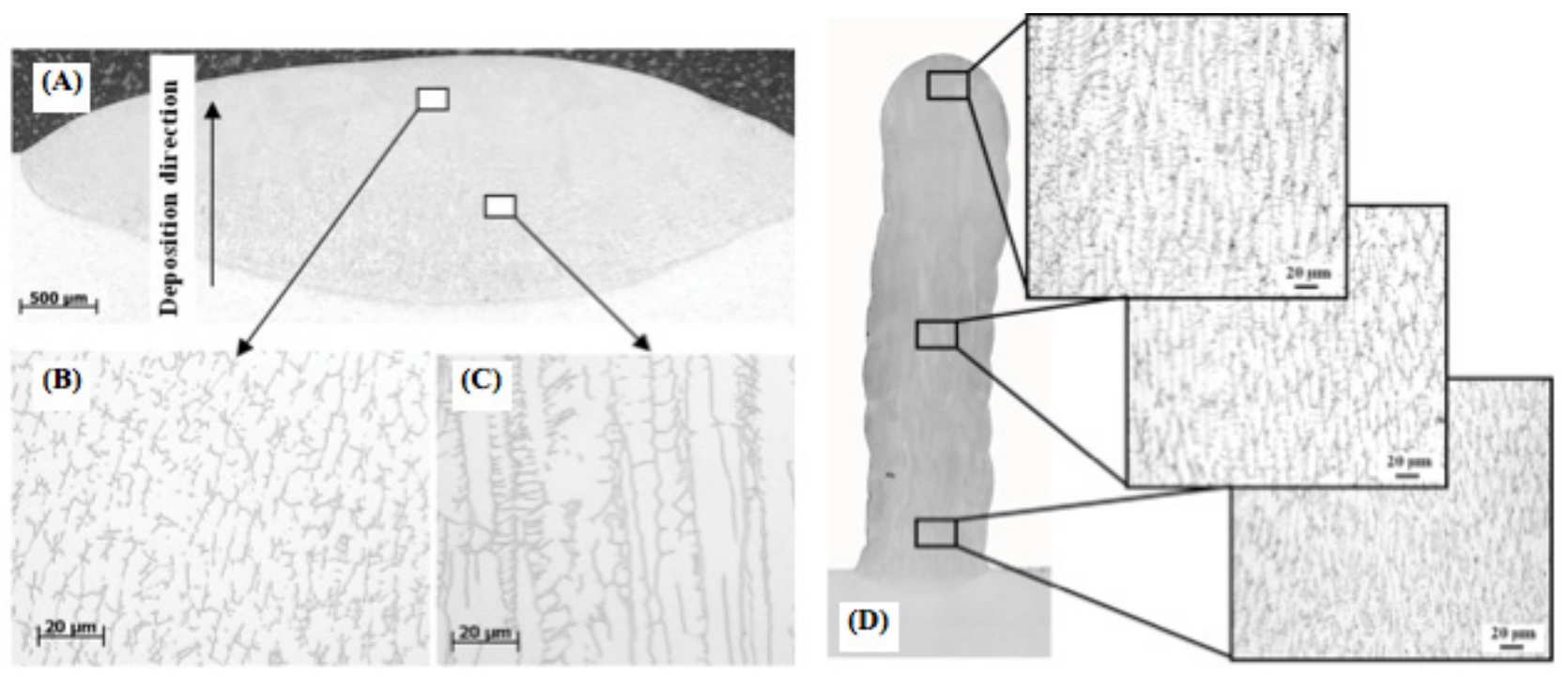

Figure 7. Solidification microstructure of the $\mathrm{EBF}^{3}$ deposited AISI 316L steel: (a) Cross-section of the single layer deposit (40 mA, $10 \mathrm{ipm}$ ); (b) equiaxed grain morphology; (c) columnar grain morphology; (d) cross-section of the ten-layer deposit (40 mA, $20 \mathrm{ipm})$.

Ref: L. Wang, S.D. Felicelli, K. Taminger, R. Lett, J. Coleman, R. Johnson, M. Waid, “Microstructure and mechanical properties comparison of electron beam and laser engineered net shaping deposits of AISI 316L stainless steel", Proceedings of Materials Science \& Technology Conference \& Exhibition, Pittsburgh, PA, October 7-11, 2012 


\section{Presentations/Publications/Patents}

Journal Articles

1. M. Asle Zaeem, H. Yin, S.D. Felicelli, "Modeling dendritic solidification of Al-3\%Cu using cellular automaton and phase-field methods", Applied Mathematical Modeling, in press (August 2012)

2. T. Talukdar, L. Wang, S.D. Felicelli, "Thermo-mechanical modeling of laser deposited bimetallic components", Materials Performance and Characterization, vol. 1 (1), \#MPC20120009, 16 pages, ASTM-International, DOI: 10.1520/MPC-2012-0009 (July 2012)

3. M. Eshraghi, S.D. Felicelli, B. Jelinek, "Three-dimensional simulation of solutal dendrite growth using lattice Boltzmann and cellular automaton methods", Journal of Crystal Growth, vol. 354, pp. 129-134 (June 2012)

4. M. Asle Zaeem, H. Yin, S.D. Felicelli, "Comparison of cellular automaton and phase field methods to simulate dendrite growth in hexagonal systems", Journal of Materials Science and Technology, vol. 28 (2), pp. 137-146 (March 2012)

5. R. Lett, S.D. Felicelli, J.T. Berry, R. Cuesta, A. Rivas, M.E. Alcalde,, "Quality aspects of A356 castings with multiple gates", International Journal of Metalcasting, vol. 6(2), pp. 67-82 (March 2012)

6. M. Eshraghi and S.D. Felicelli, "An implicit lattice Boltzmann model for heat conduction with phase change", International Journal of Heat and Mass Transfer, vol. 55, pp. 2420-2428 (Jan 2012)

7. U.K. Sajja and S.D. Felicelli, "Modeling freckle segregation with mesh adaptation", Metallurgical and Materials Transactions B, vol. 42 (6), pp. 1118-1129 (Dec 2011)

8. L. Wang, J.B. Jordon, R. Lett, S.D. Felicelli, J.T. Berry, D. Penrod, "Microstructure and performance of four casting processes for magnesium alloy AZ91", International Journal of Metalcasting, vol. 5(4), pp. 37-46 (Nov 2011)

9. U.K. Sajja, S.D. Felicelli, J.C. Heinrich, "Numerical methods for improved efficiency in macrosegregation modeling", International Journal for Numerical Methods in Engineering, vol. 87, pp. 196-213 (Aug 2011)

10. L. Wang, R. Lett, S. Felicelli, J. Berry, "Evaluating Magnesium Casting Processes", Metal Casting Design and Purchasing, vol. 13(4), pp. 30-33 (Aug 2011)

11. H. Yin, S.D. Felicelli, L. Wang, "Simulation of dendritic microstructure with lattice Boltzmann and cellular automaton methods", Acta Materialia, vol. 59 (8), pp. 3124-3136 (May 2011)

12. L. Wang, R. Lett, S.D. Felicelli, J.T. Berry, "Reliability evaluation of four casting processes for magnesium alloy AZ91", Transactions of the American Foundry Society, vol. 119, paper 11-039 (April 2011) 
13. U.K. Sajja and S.D. Felicelli, "Element-free Galerkin method for thermosolutal convection and macrosegregation", International Journal for Numerical Methods in Fluids, vol. 64, pp. 733-760 (Nov 2010)

\section{Conference Papers}

1. L. Wang, S.D. Felicelli, K. Taminger, R. Lett, J. Coleman, R. Johnson, M. Waid, "Microstructure and mechanical properties comparison of electron beam and laser engineered net shaping deposits of AISI 316L stainless steel", Proceedings of Materials Science \& Technology Conference \& Exhibition, Pittsburgh, PA, October 7-11, 2012

2. M. Eshraghi, S.D. Felicelli, "Numerical modeling of dendritic growth during solidification of alloys using lattice Boltzmann and cellular automaton methods", Supplemental Proceedings: Volume 2: Materials Properties, Characterization, and Modeling, TMS2012, $141^{\text {st }}$ Annual Meeting and Exhibition, Wiley-TMS, ISBN 978-1-11829-609-7, pp. 133-139, (March 2012)

3. T. K. Talukdar, L. Wang, S.D. Felicelli, "Simulation of residual stress in LENS deposited H13 tool steel on copper substrate", ASME 2011 International Mechanical Engineering Congress \& Exposition, Denver, CO, paper IMECE2011-62321 (November 2011)

4. L. Wang, S.D. Felicelli, J. Coleman, R. Johnson, K. Taminger, R. Lett, "Microstructure and mechanical properties of electron beam deposits of AISI 316L stainless steel", ASME 2011 International Mechanical Engineering Congress \& Exposition, Denver, CO, paper IMECE2011-62445 (November 2011)

5. M. Asle Zaeem, H. Yin, S.D. Felicelli, L. Wang, "Comparison of cellular automaton and phase field models to simulate dendritic solidification", $4^{\text {th }}$ International Multi-Conference on Engineering and Technical Innovation, Orlando, FL, pp. 183-186 (July 2011)

6. R. Lett, S.D. Felicelli, J.T. Berry, R. Cuesta, J. Maroto, R. San Jose, "Weibull analysis of thin A356 plates cast with an electromagnetic pump green sand process", Shape Casting: $4^{\text {th }}$ International Symposium 2011, eds. M. Tiryakioglu, J. Campbell, P.N. Crepeau; Wiley-TMS, ISBN 978-1118029374, pp. 157-164 (March 2011)

7. U.K. Sajja and S.D. Felicelli, "Simulation of macrosegregation during directional solidification using mesh adaptation", Shape Casting: $4^{\text {th }}$ International Symposium 2011, eds. M. Tiryakioglu, J. Campbell, P.N. Crepeau; Wiley-TMS, ISBN 978-1118029374, pp. 53-60 (March 2011)

8. H. Yin, L. Wang, S.D. Felicelli, "Solidification model coupling lattice Boltzmann method with cellular automaton technique", Shape Casting: $4^{\text {th }}$ International Symposium 2011, eds. M. Tiryakioglu, J. Campbell, P.N. Crepeau; Wiley-TMS, ISBN 978-1118029374, pp. 29-36 (March 2011) 
9. L. Wang, R. Lett, S.D. Felicelli, J.T. Berry, "Microstructure characterization of magnesium control arm castings", Shape Casting: $4^{\text {th }}$ International Symposium 2011, eds. M. Tiryakioglu, J. Campbell, P.N. Crepeau; Wiley-TMS, ISBN 978-1118029374, pp. 215-222 (March 2011)

\section{KEY WORDS}

Solidification, dendrite growth, lattice Boltzmann, cellular automaton, casting, additive manufacturing, aluminum, magnesium, steel

\section{BRIEF Description Of RePORT}

A study of solidification microstructures in aluminum, magnesium, and steel alloys was performed through numerical simulation and lab-scale experimentation. Advanced computational models were developed using local-type methods like lattice-Boltzmann and Cellular Automaton, allowing massively parallel simulations of solidification with more than 40,000 processors at NSF-grid supercomputers. Extensive microscopy and material testing was performed to analyze microstructure characteristics and correlate with modeling results of sample parts manufactured through casting and additive manufacturing processes. The results, documented in several journal articles and conference papers, provide useful insight on the influence of process parameters on the formation of solidification defects and their impact on the properties of final parts.

\section{LIST OF ACRONYMS}

DS - Directional Solidified

CA - Cellular Automaton

FE - Finite Element

PF - Phase Field

SDAS - Secondary Dendrite Arm Spacing

FEG-SEM - Field Emission Gun - Scanning Electron Microscopy

UBS - Ultimate Bending Strength

FPB - Four Point Bending

EBF3 - Electron Beam Freeform Fabrication 


\section{TABLE OF CONTENTS}

TASK 2: Multiscale Materials Modeling and Characterization of Steel Alloys............................ 2

Subtask 2.1: Materials Design of Lightweight Alloys ............................................................ 2

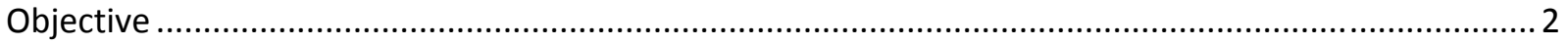

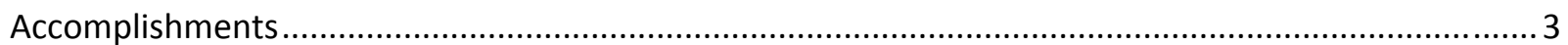

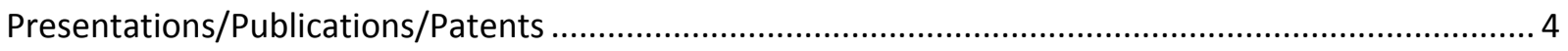

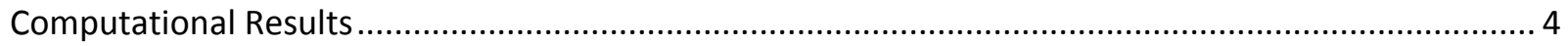

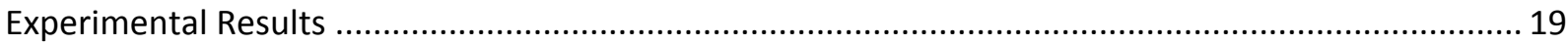

As the atomistic simulations gave insight into certain iron based parameters for alloying, it was the experimental structure-property quantification that provided guidance for the generation of a new potential steel alloy. These experimental data are discussed below.

Examination of the Microstructure, Chemical Composition, and Microhardness of the As-received DP590

Investigation into the Thermo-mechanical Preconditioning Effects on the Mechanical Behavior DP590. 20

Heat-Treating and the Resultant Microstructures and Mechanical Behavior..... 21

Examination of the Microstructure, Chemical Composition, and Microhardness of the As-received TRIP680 25

Investigation into the Thermo-mechanical Preconditioning Effects on the Mechanical Behavior of As-received TRIP680

Heat-Treating and the Resultant Microstructural and Mechanical Observations. 32

References 40 
TASK 2: Multiscale Materials Modeling and Characterization of Steel

\section{ALLOYS}

Principal Investigator: Hongjoo Rhee

Associate Director, CAVS

Mississippi State University (MSST)

200 Research Blvd

Mississippi State, MS 39762

(662)325-9221; fax: (662) 325-5433; e-mail: hrhee@cavs.msstate.edu

Carol Schutte: Technology Area Development Manager

e-mail: carol.schutte@ee.doe.gov

Joseph Renk

email:.joseph.renk@netl.doe.gov

Contractor: $\quad$ Mississippi State University (MSST)

Contract No.: DE-FC26-06NT42755

\section{Subtask 2.1: Materials Design of Lightweight Alloys}

Team Members: Seong-Gon Kim, H. Rhee, S. Kim, M.F. Horstemeyer, M.I. Baskes (LANL), L. Liyanage (PhD), Vivek D. (PhD), Chandani Nandadasa (PhD), M. Jones (MS)

\section{OBJECTIVE}

Design a novel high strength steel alloy with improved formability and strength for automotive use.

Approach: We use a hierarchical, multiscale methodology to investigate the effect of nanoscale precipitates and additives to the overall strength, ductility, and formability in steel alloy design for automotive applications. Critical issues addressed include: selection of key combination of precipitates and matrices, interaction of precipitate and matrix phases and, ultimately, compositionstructure-property relationships and phase transformations. At the electronic level, quantum mechanical, first-principles simulations based on Density Functional Theory (DFT) were performed to investigate the interfacial interactions between matrix and the primary and the secondary precipitates. At the atomistic level, accurate atomistic simulations were performed using efficient and reliable empirical interatomic potentials such as modified embedded-atom method (MEAM) or force-matching, embedded-atom-method (FMEAM) potentials. An Iron potential was modified with the addition of Carbon and other additives in order to provide a strategy for the next generation steel alloy. Previous research on dual phase (DP) steels revealed that the combination of formability and strength makes bake hardening DP steels good options for drawn or stretched applications of automobile body where resistance to dents and palm printing was important in applications such as hoods, doors, fenders, and deck lids. In Phase IV, materials and mechanical properties of a different grade of advanced high strength steels (AHSS) were studied to design a novel high strength steel alloy with improved formability and strength by using transformation induced plasticity (TRIP) 
steels. An added focus of this research was to study the effect of controlled constituent phase distribution on the strength-ductility combination of TRIP steels for developing new $3^{\text {rd }}$ generation AHSS. We investigated the role of microstructure on the strength-ductility combination of TRIP steels. Various combinations of microstructure, grain size, phase volume fraction, and phase distribution were acquired by carrying out different heat treatment schedules including intercritical annealing followed by isothermal bainitic transformation treatment and cooling in different cooling media. Structure and mechanical behavior of specimens heat treated in different ways were characterized by performing various measurements. The results enabled the prediction of optimum strength-ductility combination of TRIP steels for desired components of automotive applications.

\section{ACCOMPLISHMENTS}

The objectives were met by accomplishing the following:

1. Developed a new Fe-C MEAM potential and validated it by performing two-phase melting molecular dynamics simulations of cementite.

2. Investigated the generalized stacking fault energy curve for austenite using Density Functional Theory calculations.

3. Investigated the microalloying effect of vanadium on the strength of BCC ferrite grain boundaries using DFT methods.

4. Developed a new Fe-V MEAM alloy potential.

5. Developed a new Fe-Nb MEAM alloy potential.

6. Performed thermomechanical treatments (i.e. pre-straining followed by bake-hardening), structure observation, and mechanical tests on TRIP steels to characterize the bake-hardening effect on the mechanical behavior of AHSS.

7. Carried out nano-indentation and micro-hardness tests on DP steels to investigate micromechanical properties of constituent phases.

8. Conducted fundamental materials/mechanical properties characterization on advanced high strength steel alloy (i.e., TRIP steel) samples obtained from Severstal.

9. Performed fractographical analysis on the intercritical annealing (I.C.A.) treated followed by quasi-static tensile tested specimens

10. Carried out high strain-rate tensile tests on the heat treated (i.e., intercritical annealing followed by quenching) DP steels to characterize the effect of altered microstructure on the strengthductility combination of AHSS. 


\section{Presentations/Publications/Patents}

1. Sungho Kim, Seong-Gon Kim, Mark Horstemeyer, "Vanadium effects on a BCC iron sigma 3 grain boundary strength," Collected Proceedings: Deformation, Damage, and Fracture of Light Metals and Alloys (2011).

2. Amitava Moitra, Seong-Gon Kim, and Mark F. Horstemeyer, "Structural and thermal properties of Calcium using a MEAM potential," under review, revised and resubmitted to Calphad.

3. Amitava Moitra, Seong-Gon Kim, and Mark F. Horstemeyer, "Structural and thermal properties of Fe with $\mathrm{V}$ impurities using a MEAM interatomic potential," under review, revised and resubmitted to Materials Science and Engineering B.

4. Sungho Kim, Seong-Gon Kim, Mark Horstemeyer, Hongjoo Rhee, "The effect of vanadium on the strength of a BCC iron sigma 3 grain boundary", preprint (2011).

5. Sungho Kim, J. Houze, M. F. Horstemeyer, Amitava Moitra, and Seong-Gon Kim, "VacancyAssisted Diffusion of Vanadium in $\alpha$-Ferrite," In preparation.

6. J. Houze, Sungho Kim, M. F. Horstemeyer, and Seong-Gon Kim, "Diffusion of Vanadium in Cementite," In preparation.

7. L. Liyanage, Amitava Moitra, J. House, Sungho Kim, Seong-Gon Kim, and M. F. Horstemeyer, "A modified embedded-atom-method potential for Fe-C alloy systems," In preparation.

8. J. House, Sungho Kim, Amitava Moitra, L. Liyanage, Seong-Gon Kim, and M. F. Horstemeyer, "The vacancy-assisted diffusion of V atoms in cementite: An ab-initio study," preprint.

9. Sungho Kim, Seong-Gon Kim, Mark Horstemeyer, "Vanadium effects on a BCC iron sigma 3 grain boundary strength," 2011 TMS Annual Meeting, San Diego, CA, March 2011.

10. L. Liyanage, Amitava Moitra, J. House, Seong-Gon Kim, Mark Tschopp, Sungho Kim, and M. F. Horstemeyer, "A modified embedded-atom-method potential for Fe-C alloy systems," 2011 APS March Meeting, Dallas, TX, March 2011.

\section{Computational Results}

\section{Structural and elastic properties of Cementite}

We examined an MEAM potential by computing the structural properties of cementite including the equilibrium lattice parameters, the equilibrium volume per atom, and the heat of formation. These properties are presented in Table 1 with comparison to DFT, experiment, and interatomic potentials by Ruda et al [Ruda09] and Henriksson et al [Henr09]. MEAM prediction of the heat of formation of cementite is in excellent agreement with DFT and experimental data. Henriksson's potential also predicts a value in good agreement with DFT and experiment, while Ruda's potential predicts a much larger value. Lattice constants of the present MEAM and literature potentials [Ruda09, Henr09] agree well with experiment, while DFT predicts lower values. As a test of validity, the variation of cohesive energy with volume was calculated. Fig. 1 compares the energy vs. volume curves for cementite generated by the present MEAM potential with DFT and experimental curves. During volume variation of cementite, the ratios between $a, b$ and $c$ lattice parameters were held constant. DFT overestimates the cohesive energy and underestimates the volume. Therefore, the DFT curve sits to the left of the experimental curve, and it is shifted vertically to the experimental cohesive energy at equilibrium volume to aid the comparison with the experimental curve. The experimental curve was generated by Murnaghan's equation of state [Murn44, Murn67] with the experimental bulk modulus, volume, and cohesive energy [Hagl91]. The experimental single-crystal bulk modulus of cementite has not yet been 
determined; therefore, the polycrystalline bulk modulus of cementite was used to generate the experimental curve.

Table 1. Material properties of cementite: the heat of formation $\Delta H_{f}(\mathrm{eV} /$ atom); the equilibrium volume $\omega_{0}\left(\AA^{3} /\right.$ atom); the lattice parameters a, b, and c ( $\hat{A}^{\prime}$. The predictions from the present work (MEAM) are compared with those from DFT and other interatomic potentials [Ruda09, Henr09]. Experimental values are given in parentheses.

\begin{tabular}{|c|c|c|c|c|}
\hline Property & DFT (Expt.) & MEAM & Ruda & Henriksson \\
\hline$\Delta H_{f}$ & $0.01(0.05)$ & 0.06 & 0.18 & 0.03 \\
\hline$\omega_{0}$ & $8.49(9.71)$ & 9.49 & 9.11 & 9.33 \\
\hline $\mathrm{a}$ & $4.91(5.09)$ & 5.05 & 5.14 & 5.09 \\
\hline $\mathrm{b}$ & $6.63(6.74)$ & 6.69 & 6.52 & 6.52 \\
\hline $\mathrm{c}$ & $4.38(4.53)$ & 4.49 & 4.35 & 4.50 \\
\hline
\end{tabular}

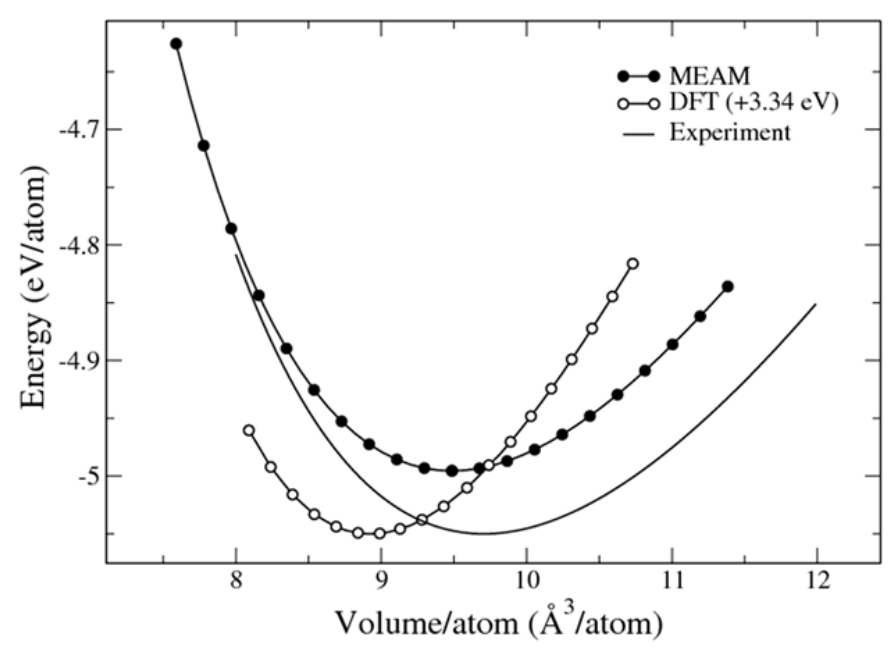

Figure 1. Comparison of energy vs volume curves for cementite. The solid curve is constructed from experimental values of the cohesive energy, polycrystalline bulk modulus, and equilibrium volume of cementite. For comparison, the DFT curve is shifted vertically to the experimental cohesive energy at the equilibrium volume.

\section{Single-crystal elastic properties}

The elastic moduli of cementite were calculated and compared to DFT and the interatomic potentials by Ruda et al. [Ruda09] and Henriksson et al. [Henr09] as presented in Table 2. We used both $\delta=0.5 \%$ and $\delta=0.1 \%$ that produced the same result. These results show that the present MEAM potential for Fe-C alloy predicts cementite to be stable (positive elastic constants) and their values are reasonably close to those predicted by DFT. However, the present MEAM-and other interatomic potentials in the literature-could not reproduce the low value of $C_{44}$ reported by DFT [Jian08]. 
Table 2. Single-crystal elastic moduli, $C_{x y}$ (GPa) of cementite. The predictions from the present work (MEAM) are compared with those from DFT [Jiang2008] and other interatomic potentials [Ruda09, Henr09].

$\begin{array}{lcccc}\text { Property } & \text { DFT } & \text { MEAM } & \text { Ruda } & \text { Henriksson } \\ C_{11} & 395 & 303 & 263 & 363 \\ C_{22} & 347 & 216 & 219 & 406 \\ C_{33} & 325 & 307 & 247 & 388 \\ C_{12} & 158 & 126 & 176 & 181 \\ C_{23} & 163 & 110 & 143 & 130 \\ C_{13} & 169 & 157 & 146 & 166 \\ C_{44} & 18 & 249 & 77 & 91 \\ C_{55} & 134 & 236 & 95 & 125 \\ C_{66} & 135 & 227 & 123 & 134\end{array}$

\section{Polycrystalline elastic properties}

Theoretical upper and lower bounds for the polycrystalline bulk modulus $(B)$ and shear modulus $(G)$ were calculated using the single-crystal elastic constants according to methods by Reuss and Voigt [Jiang2008]. The true polycrystalline $B$ and $G$ were then estimated using Hill's average [Hill52, Jiang2008]. Polycrystalline elastic moduli predicted by our MEAM potential are presented in Table 3, in comparison with DFT, experiment, and interatomic potentials by Ruda et al. [Ruda09] and Henriksson [Henr09]. The elastic constants predicted by DFT are in excellent agreement with experiment. The present MEAM gives the best agreement with experiment among the three interatomic potentials for $\mathrm{B}$; however, the present MEAM prediction of $v$ is significantly lower than its experimental value. Ruda's potential predicts the best agreement with experiment for $G$ and $v$. 
Table 3. Polycrystalline cementite properties: bulk modulus $B$ (GPa), shear modulus $G$ (GPa), Young's modulus $Y(\mathrm{GPa})$, and Poisson's ratio v. The predictions from the present work (MEAM) are compared with those from DFT [Jiang2008], and other interatomic potentials [Ruda09, Henr09]. Experimental values are given in parentheses.

$\begin{array}{ccccc}\text { Property } & \text { DFT (Expt.) } & \text { MEAM } & \text { Ruda } & \text { Henriksson } \\ B & 224(175 \pm 4) & 174 & 183 & 234 \\ G & 72(74) & 146 & 69 & 114 \\ Y & 194(200) & 343 & 184 & 293 \\ V & 0.36(0.36) & 0.17 & 0.33 & 0.29\end{array}$

\section{Surface energies}

Calculations were performed on (001), (010), and (100) surfaces to determine the surface formation energy. Table 4 compares the surface formation energies of the present MEAM to DFT and the interatomic potential by Ruda et al. [Ruda09]. The atoms near the surfaces are fully relaxed to allow reconstruction if necessary. The predicted surface energies have the same order of magnitude as DFT results. However, the present MEAM gives a wrong order of stability among the three surfaces.

Table 4. Surface energies $\left(\mathrm{J} / \mathrm{m}^{2}\right.$ ) of cementite. The predictions from the present work (MEAM) are compared with those from DFT [Chiou2003] and other interatomic potentials [Ruda09].

$\begin{array}{cccc}\text { Surface } & \text { DFT } & \text { MEAM } & \text { Ruda } \\ (001) & 2.05 & 2.30 & 1.96 \\ (010) & 2.26 & 1.81 & 2.00 \\ (100) & 2.47 & 1.79 & 2.34\end{array}$

\section{Two-Phase Simulation of Cementite Melting Temperature}

Experimentally the eutectic point of cementite is observed to be at $1420 \mathrm{~K}$. At this temperature cementite becomes a solution of iron and carbon without a crystal structure. We performed a twophase simulation using the newly developed Fe-C alloy MEAM potential to predict the eutectic point. In this simulation a cementite block was heated using an NPT molecular dynamic run to $1200 \mathrm{~K}$. Then, half of the atoms were frozen and the other half was heated to $4000 \mathrm{~K}$ and then cooled to $1500 \mathrm{~K}$. This procedure resulted in a simulation box, in which half of the atoms were in cementite crystal structure and the other half a solution of iron and carbon atoms. This simulation box was then heated from 1000 $\mathrm{K}$ to $1600 \mathrm{~K}$ in $100 \mathrm{~K}$ intervals using NPT molecular dynamic runs. Each system ran for 1.6 nanoseconds. The phase change of the two-phase simulation box was visually observed. At $1400 \mathrm{~K}$ the solid phase of the simulation box was progressing to occupy the entire box, and at $1500 \mathrm{~K}$ the liquid phase of the simulation box was progressing to occupy the entire box. Next the initial two-phase simulation box was heated from $1400 \mathrm{~K}$ to $1500 \mathrm{~K}$ in $10 \mathrm{~K}$ intervals using NPT molecular dynamic runs. Each system was 
equilibrated for at least 5 million time steps, where each time step was $2 \mathrm{fs}$, totaling to $10 \mathrm{~ns}$. The final state of the system was visually inspected. If the final state appeared to have both liquid and solid phases more MD runs were performed, until the final state of the supercell contained one phase. Some systems required as much as $32 \mathrm{~ns}$ of MD runs to arrive at a single phase. The total energy, volume, and pressure of the systems were determined through averaging the values of the final 40000 time steps ( 80 ps) of each simulation. The variation of properties such as total energy, volume, specific heat and derivative of volume with temperature allows us to clearly determine the melting temperature of cementite. Plots illustrating these variations are presented in Fig. 4. According to the plots we obtain the melting temperature between $1418 \mathrm{~K}$ and $1432 \mathrm{~K}$. This is in good agreement with the experimental eutectic point at $1420 \mathrm{~K} .34$ The transformation of the two-phase simulation box to a one-phase simulation box, near the predicted melting temperature is presented in Fig. 2 and Fig. 3. 


\section{The Effect of $\mathbf{V}$ on the dislocations in Ferrite}

Dislocations are the source for crystal plasticity, and therefore plastic properties depend strongly on dislocations. Investigating how the micro-alloyed vanadium $(\mathrm{V})$ affect the dislocation dynamics and the activation energy for dislocation slips is an important step to understand the strengthening mechanisms by the vanadium addition.

The strain-stress behaviors at different simulation box sizes are shown in Figure 5 . The stress increases linearly as the shear strain increases and saturates around $80 \mathrm{MPa}$ at which the dislocation starts to move. The minimum stress for the dislocation movement is called Peierls stress. The value of Peierls stress is important because it governs plasticity thresholds. 
BCC ferrite crystals have many dislocation systems. We employed three edge dislocation systems specified by the burger vector and slip plane: [001](010), [001](110), and $1 / 2[1-1-1](110)$. Figure 6 shows the strain-stress behaviors for these three dislocation systems.

\section{The Generalized Stacking Fault Energy for Austenite}

We used Density Functional Theory (DFT) to compute the generalized stacking fault energy curve for austenite (fcc Fe). Our calculation confirms the results obtained by Kibey et al [Kibey2006]. We used PAW-GGA potential for exchange-correlation functional and used 60 irreducible k-points in the Brillouin zone. The simulation cell contains 96 atoms with periodic boundary conditions (PBC) along (112), (111) and (110) directions. The cell contains a slab structure with twelve (111) atomic planes and a $15 \AA$ vacuum layer. We performed the convergence test with a bigger unit cell with 16 (111) atomic planes and found that the results are not changing in any significant way. We fully relaxed the atomic positions and the cell vectors in order to get the stable energies. The key factor to note is that the intrinsic/stable stacking fault energy is negative, which implies that hcp phase is energetically more favorable than fcc phase for pure Fe. Hence, fcc Fe would stay in hcp phase if it could overcome the energy barrier of the 
unstable stacking fault energy, either by thermal activation or an induced stress. Our result is shown in Figure 7.

\section{The Effect of $V$ on the Grain Boundaries in Ferrite}

Vanadium (V) is one of the most important micro-alloying elements for steels. We used DFT to study the effect of $V$ atoms in ferrite. Since grain boundaries are commonly present in the ferrite phase of most AHSS alloys, we investigated the effect of $\mathrm{V}$ atoms on the strength of grain boundaries in ferrite. We obtained the optimized grain boundary structure of Fe BCC $23(111)[1(-1) 0]$ shown in Figure 8. The arrows show the displacements of atoms caused by an energy minimization from the initial grain boundary structure made by attaching two perfect BCC crystal structures. The grain boundary formation energy from our calculation is $0.113 \mathrm{eV} / \AA^{2}$. 
Figure 9 shows the optimized structure of interstitial and substitutional $V$ defects segregated into the grain boundary. The $V$ interstitial formation energy on the grain boundary is $-4.20 \mathrm{eV}$ from an isolated $\mathrm{V}$ atom and $1.13 \mathrm{eV}$ from a $\mathrm{V}$ atom in bulk. The $\mathrm{V}$ atom lowers its energy by forming an interstitial defect in the grain boundary. The micro-alloying element can also be segregated on the grain boundary as a substitutional defect. The $V$ substitutional formation energy on the grain boundary is $6.08 \mathrm{eV}$ from an isolated $\mathrm{V}$ atom and $-0.75 \mathrm{eV}$ from a $\mathrm{V}$ atom in bulk. The negative sign indicates that the $V$ substitution for Fe atom on this grain boundary is an exothermic process. Compared to interstitial formation energy, the substitutional formation energy is lower by $1.8 \mathrm{eV}$, which means that substitution defects occur far more often than interstitial defects. Therefore we focus on the substitutional segregation on the grain boundary in this project.

The $\mathrm{V}$ segregation energy into the grain boundary is the difference of the grain boundary formation energy from $\mathrm{V}$ defect formation energy in bulk. In order to calculate the $\mathrm{V}$ segregation energy into the grain boundary we calculated two $\mathrm{V}$ point defect formation energies in bulk. One is the interstitial point defect formation energies that are $-1.36 \mathrm{eV}$ for tetrahedral interstitial from an isolated $\mathrm{V}$ atom and 3.97 from a bulk $V$ atom while octahedral interstitial defect energies are higher by $0.43 \mathrm{eV}$. The other is substitutional point defect formation energy that is $-6.06 \mathrm{eV}$ from an isolated $\mathrm{V}$ atom and $-0.73 \mathrm{eV}$ from a bulk $V$ atom. The substitutional defect formation energies are again lower than the interstitial one by $4.70 \mathrm{eV}$. From the calculated energies we can conclude that $\mathrm{V}$ atoms exist mostly as substitutional defects in the bulk and segregate into substitutional defects in grain boundaries. The $V$ segregation energy from bulk to grain boundary is the substitutional defect formation energy in the grain boundary subtracted by the substitutional defect formation energy in bulk. We obtained $-0.03 \mathrm{eV}$ as the segregation energy of $V$ atoms. The surface formation energy of (111) is calculated to be $0.167 \mathrm{eV} / \AA^{2}$. The $V$ segregation energy from bulk into surface is calculated as $-9.57 \mathrm{eV}$. The grain boundary cohesion energy (GBCE) without $V$ is calculated as $1.43 \mathrm{eV}$ while the GBCE with segregated $\mathrm{V}$ is $1.54 \mathrm{eV}$. The $\mathrm{V}$ segregation increases GBCE by $0.11 \mathrm{eV}$. Therefore, from our DFT calculation results we can conclude that the segregated $V$ atom in grain boundary significantly strengthens the grain boundary against the brittle grain boundary fracture. 
To study the $V$ effects on the resistance of grain boundary sliding, the energies of systems in which two grains are gradually displaced are plotted in Figure 10. In the process of grain boundary sliding there are two energy barriers. Between these two barriers the initial grain boundary moves as shown in Figure 11. The grain boundary movement takes place through smaller barrier out of those two energy barriers. The formed new grain boundary in neighbor layer is under the deformation stress. Therefore the higher energy barrier is not active, and only the smaller energy barrier is always active. For this reason we show only the smaller energy barrier in Figure 10. As the grain boundary moves away from the segregated $V$ atom, the system energy goes up so that segregated $V$ increases the resistance of grain boundary sliding.

\section{Fe-Nb MEAM interatomic potential}

We developed a new Fe-Nb MEAM potential based on previously developed MEAM potentials for Fe and $\mathrm{Nb}$. The parameters for Fe are from the MEAM potential developed by Lee et al [Lee11] and the parameters for $\mathrm{Nb}$ are from Sa et al [Sa08]. Figure 12 shows the comparison of total energy vs nearest neighbor distance for $\mathrm{Fe}$ and $\mathrm{Nb}$ in the bcc structure, their most stable structure at the room 
temperature, compared to the curves generated by DFT calculations and experimental data. It is well known that DFT overestimates the cohesive energy. Therefore, the DFT curve is shifted vertically by a constant to match the experimental cohesive energy at the equilibrium volume to aid the comparison of the curves. The MEAM potentials are smooth devoid of unphysical local minima and give energyvolume curves in good agreement with experimental data.

The MEAM potential parameters for the Fe-Nb alloy potential were constructed by fitting the heat of formation, equilibrium lattice parameter, and bulk modulus of three Fe-Nb compounds, FeNb3, Fe3Nb, and FeNb. These structures are hypothetical crystal structures that do not exist in nature. Therefore, the materials properties of these hypothetical crystal structures were obtained by first-principles calculations based on Density Functional Theory (DFT). The potential was constructed using a statistically-based optimization scheme developed by Tschopp et al [Tschopp11]. Table 5 lists the MEAM prediction of the target properties used for fitting of the Fe-Nb alloy potential compared to DFT and to the interatomic potentials by Sa et al [Sa08]. The predictions of the present MEAM potential are in better agreement with DFT results. 


\begin{tabular}{|l|l|l|l|l|}
\hline & & & & \\
\hline & & & & \\
\hline & & & & \\
\hline & & & & \\
\hline & & & & \\
\hline & & & & \\
\hline & & & & \\
\hline & & & & \\
\hline & & & & \\
\hline
\end{tabular}

Figures 13-15 show the comparison of energy vs. volume curves for Fe-Nb alloy systems (FeNb3, Fe3Nb, and FeNb). The DFT curves are shifted vertically to the MEAM predicted cohesive energy at the equilibrium nearest neighbor distance to aid the comparison with the MEAM curve. The prediction from another interatomic potential by Sa et al [Sa08] is also given for comparison. Figures 13-15 show that our new MEAM potential gives a much better agreement with DFT values compared to the other interatomic potential by Sa et al [Sa08]. 
Fe-V MEAM interatomic potential

\begin{tabular}{|c|c|}
\hline Southern Regional Center for Lightweight Innovative Design (SRCLID) & Task 2: Page 16 \\
\hline
\end{tabular}


We developed a new Fe-V MEAM potential based on previously developed MEAM potentials for Fe and $V$. Figure 16 shows the comparison of total energy vs. nearest neighbor distance for $V$ in the bcc structure, their most stable structure at the room temperature, compared to the curves generated by DFT calculations and experimental data. As mentioned earlier, the DFT curve is shifted vertically by a constant to match the experimental cohesive energy at the equilibrium volume to aid the comparison of the curves. The MEAM potentials are smooth devoid of unphysical local minima and give energyvolume curves in good agreement with experimental data.

The MEAM potential parameters for the Fe-V alloy potential were constructed by fitting the heat of formation, equilibrium lattice parameter, and bulk modulus of three Fe- $\mathrm{Nb}$ compounds, $\mathrm{FeV} \mathrm{Fe}_{15} \mathrm{~V}$, and $\mathrm{FeV}_{15}$. These structures are hypothetical crystal structures that do not exist in nature. Therefore, the materials properties of these hypothetical crystal structures were obtained by first-principles calculations based on Density Functional Theory (DFT). The potential was constructed using a statistically-based optimization scheme developed by Tschopp et al [Tschopp11]. Table 6 lists the MEAM prediction of the target properties used for fitting of the Fe-V alloy potential compared to DFT. The predictions of the present MEAM potential are in good agreement with DFT results. 


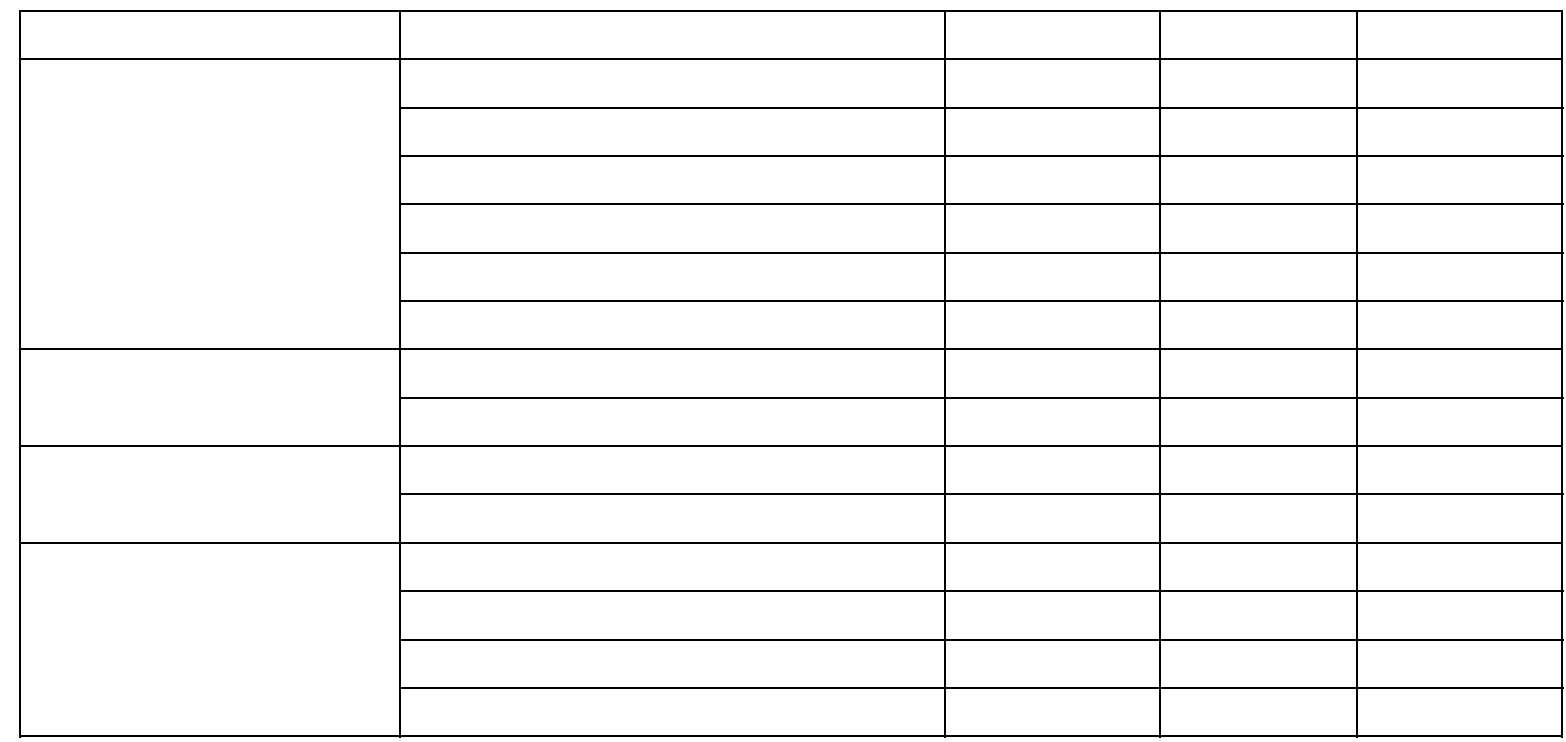

Figure 17 shows the comparison of energy vs. volume curves for $\mathrm{FeV}$ alloy system in $\mathrm{CsCl}$ crystal structure. The DFT curves are shifted vertically to the MEAM predicted cohesive energy at the equilibrium nearest neighbor distance to aid the comparison with the MEAM curve. Figure 17 shows that our new MEAM potential gives a good agreement with DFT values. 


\section{EXPERIMENTAL RESULTS}

As the atomistic simulations gave insight into certain iron based parameters for alloying, it was the experimental structure-property quantification that provided guidance for the generation of a new potential steel alloy. These experimental data are discussed below.

\section{Examination of the Microstructure, Chemical Composition, and Microhardness of the As- received DP590}

The chemical composition was taken through spectroscopy, and the results are shown in Table 7. It is noted that manganese content serves as block for the formation of pearlite and the silicon helps prevent $\mathrm{Fe}_{3} \mathrm{C}$ development. Polishing and etching revealed the ferrite-martensite phase fraction as seen in Figure 18. The martensitic phases can be seen interspersed through the ferrite.

Table 7. Chemical composition of as-received DP590.

\begin{tabular}{|c|c|c|c|c|c|c|c|}
\hline $\mathbf{C}$ & $\mathbf{S i}$ & $\mathbf{M n}$ & $\mathbf{P}$ & $\mathbf{S}$ & $\mathbf{C r}$ & Mo & Al \\
\hline 0.123 & 0.103 & 1.870 & 0.013 & 0.0046 & 0.008 & 0.061 & 0.049 \\
\hline
\end{tabular}
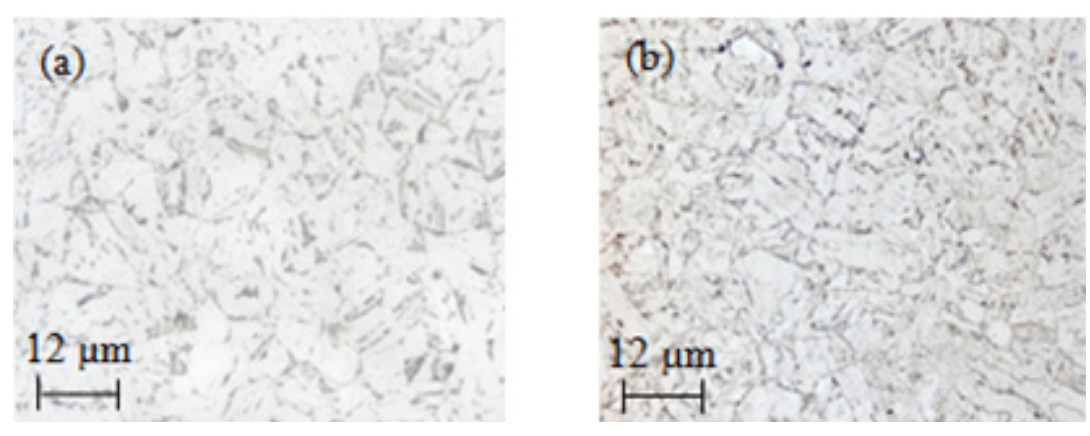

Figure 18. Microstructure of acquired DP steel.

Note: Ferrite (light grey) and martensite (dark grey/black) are shown with top surface view (a) and side surface view (b).

Regarding the microhardness values, Vickers tests were performed on each phase in order to find out their mechanical properties. A 10 gf load was used in and the tests were carried out at room temperature. Ferrite provided values of Hv193 while martensite showed Hv295. Martensite is overwhelmingly harder due to higher carbon content and resultant BCT microstructure. 


\section{Investigation into the Thermo-mechanical Preconditioning Effects on the Mechanical Behavior DP590}

Quasi-static tests revealed slower strain-rate thermo-mechanical properties about the DP590. Tests were performed at $0.01 / \mathrm{s}$ and $0.001 / \mathrm{s}$ strain-rates with as-received and baked specimens under different pre-strainings of $0,1,2$, and $5 \%$. The trend regarding ultimate tensile strength (UTS) appeared to be that the smaller the pre-strain, the higher the UTS. However, the specimens with the highest yield strength turned out to be the moderately pre-strained test cases. Therefore it can be deduced that the preconditioning of the test specimens controls the different stress criterion. Figure 19 shows the stressstrain curves from the quasi-static tests.

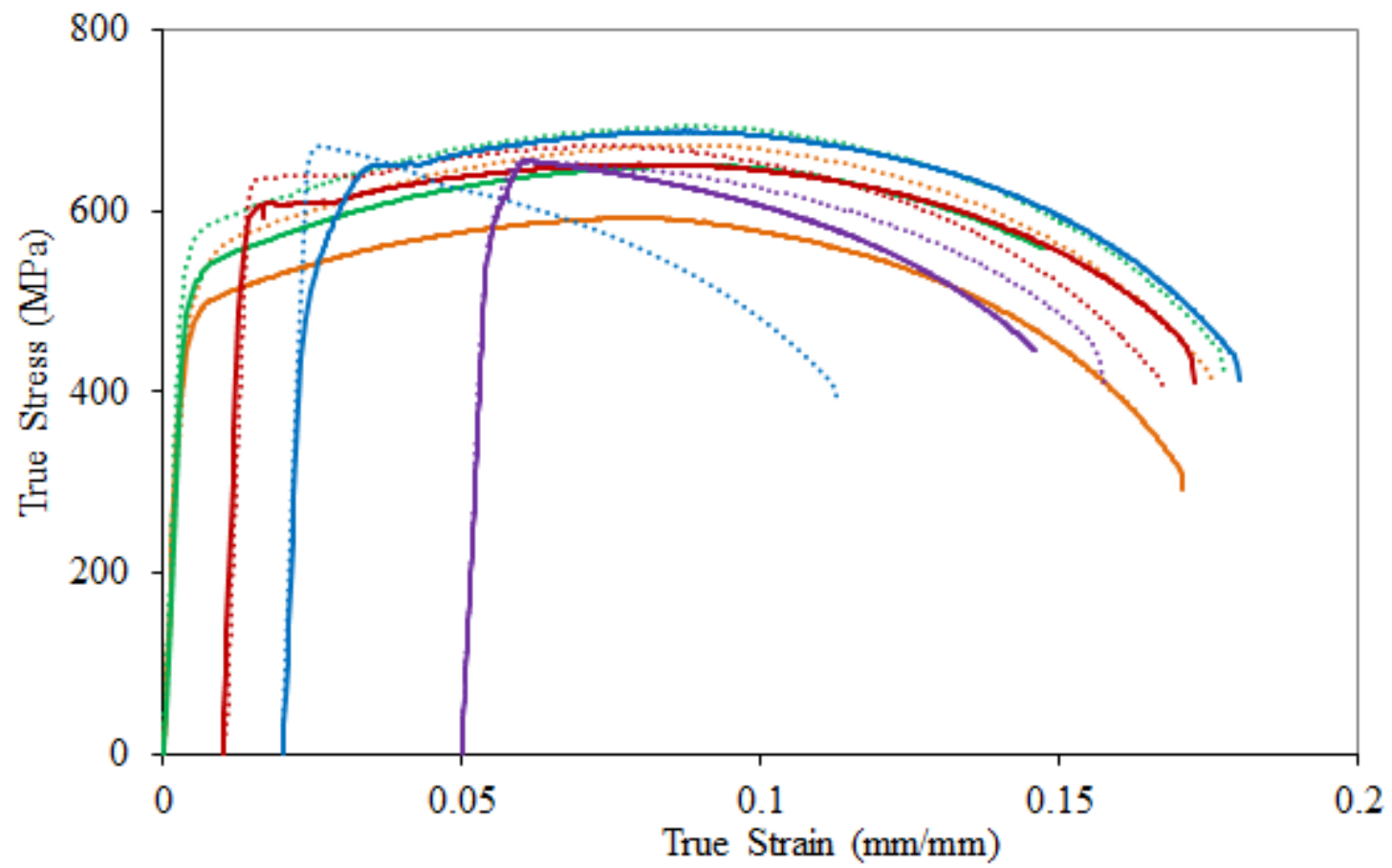

\begin{tabular}{|c|c|}
\hline As-received - 0.001/s & $\ldots \ldots \ldots$ As-received $-0.01 / \mathrm{s}$ \\
\hline -Baked $-0.001 / \mathrm{s}$ & $\cdots \cdots \cdots$ Baked $-0.01 / \mathrm{s}$ \\
\hline $1 \%$ Pre-strained \& Baked - 0.001/s & …..... 1\% Pre-strained \& Baked - 0.01/s \\
\hline $2 \%$ Pre-strained \& Baked $-0.001 / \mathrm{s}$ & $\ldots \ldots \ldots .2 \%$ Pre-strained \& Baked $-0.01 / \mathrm{s}$ \\
\hline $5 \%$ Pre-strained \& Baked - 0.001/s & $\ldots \ldots \ldots . .5 \%$ Pre-straine \\
\hline
\end{tabular}

Figure 19. True stress vs. true strain curves from quasi-static tensile tests.

From the quasi-static tests, characteristics such as work-hardening and bake-hardening properties can be found and evaluated. This is useful in determining just how much the preconditioning protocols affected the material during these tests. It can be noted that the work-hardening effect increased due directly to the increasing pre-strains shown in Figure 20. 

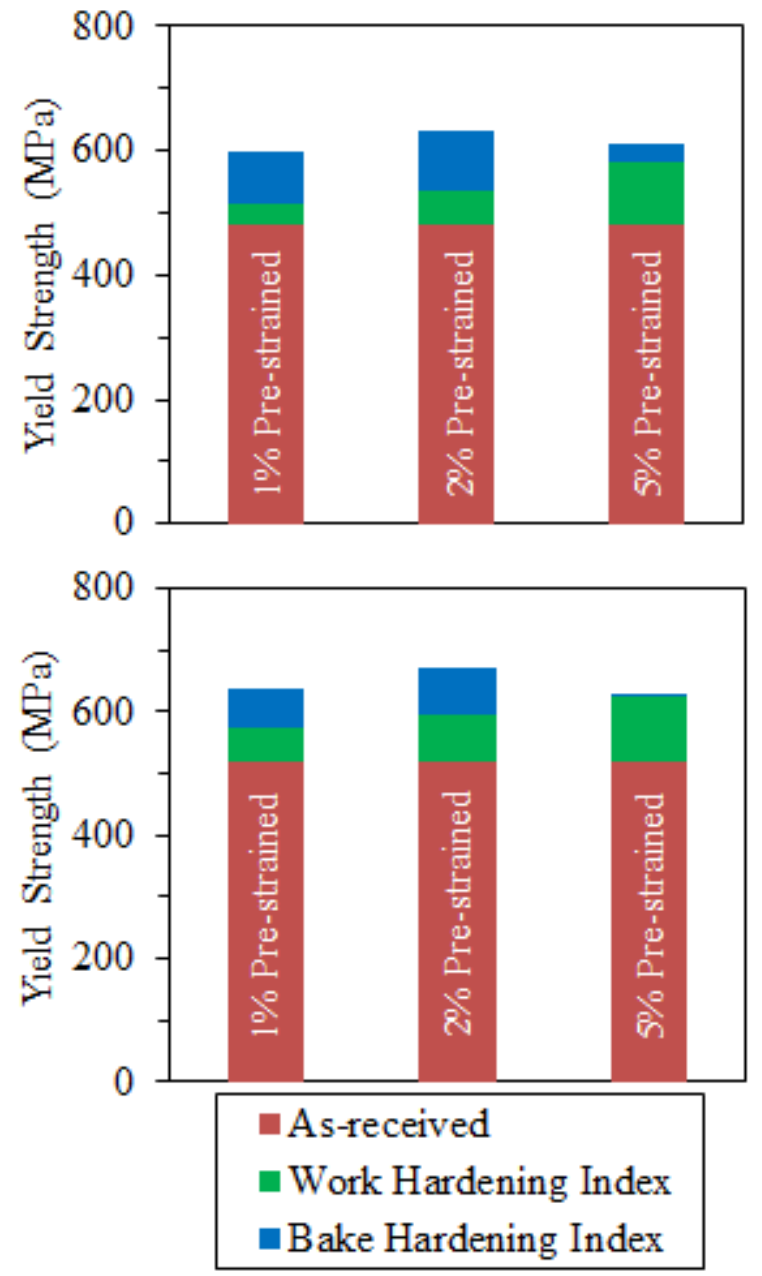

Figure 20. Work- and bake-hardening indices for their respective quasi-static tests.

Note: (a) $0.001 / \mathrm{s}$ strain rate; (b) $0.01 / \mathrm{s}$ strain rate.

\section{Heat-Treating and the Resultant Microstructures and Mechanical Behavior}

Heat treatments were carried out with different intercritical annealing (I.C.A.) parameters coupled with different quenching media. Figure 21 shows the area volume fractions of martensite and ferrite in the different tests. Martensite percentages were observed to increase with the increase of the cooling rate (i.e., oil vs. water/ice) and also the increase of the annealing temperatures. However, there is no discernible difference in using an ice-brine quench compared to a water quench. 


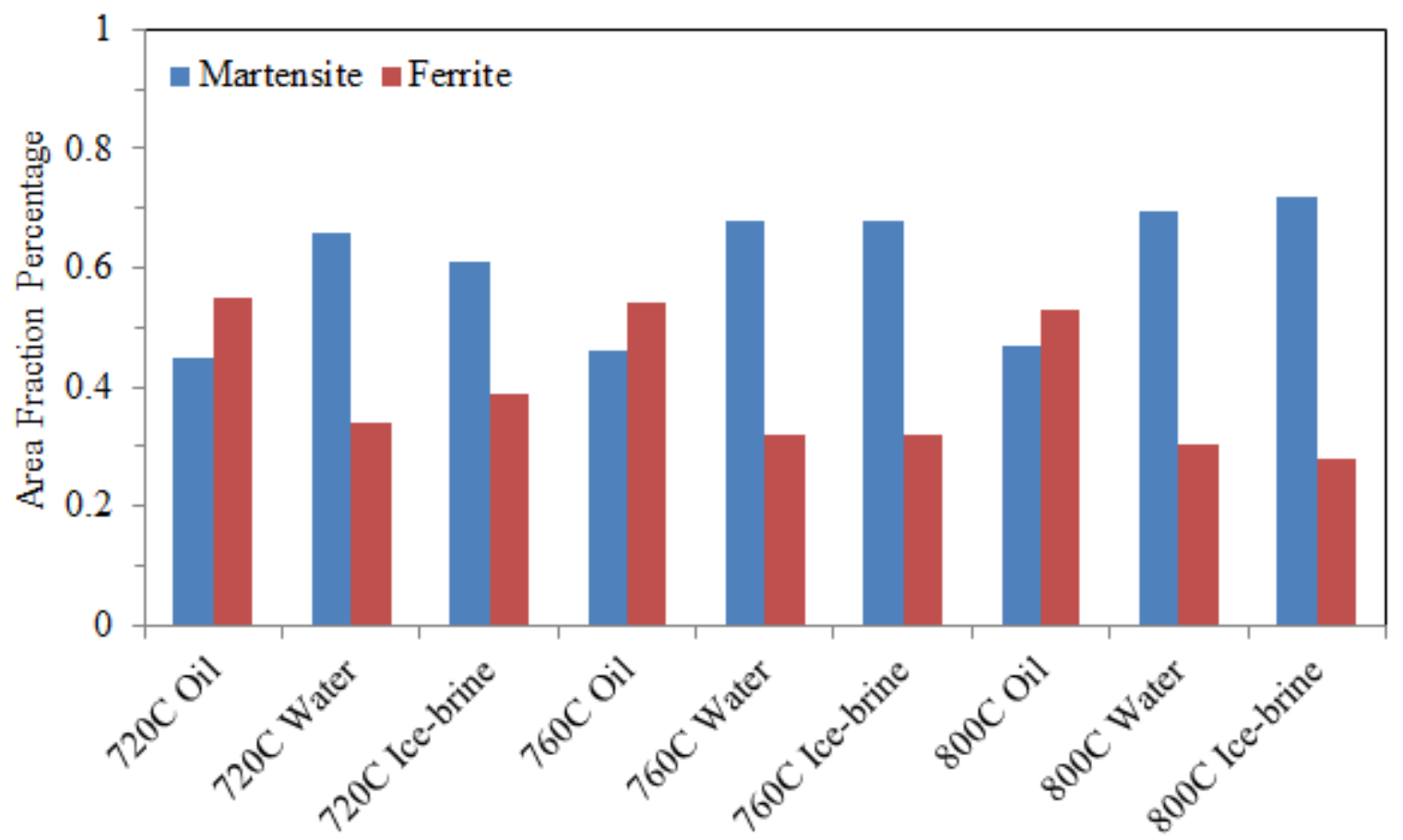

Figure 21. Area fractions of martensite and ferrite in different quenching cases.

Note: The annealing/quenching case that contains the highest ferrite percentage is $720^{\circ} \mathrm{C} /$ oil while the highest martensite percentage is $800^{\circ} \mathrm{C} /$ ice-brine.

The two heat/quench treatments with the most martensite percentages and most ferrite percentages were used to make new specimens for another round of thermo-mechanical tests. Quasistatic tests were performed on the specimens that had undergone annealing temperature/quenching media of $720^{\circ} \mathrm{C} /$ oil and $800^{\circ} \mathrm{C} /$ ice-brine. Figure 22 shows these results compared to the as-received DP590 series obtained from the supplier. It is evident that the material with the most martensite had the greatest tensile and yield strength, but reducing elongation-to-failure in effect caused more brittle behavior. The test case with the highest percentage of ferrite had a much better elongation to failure, similar to the as received series, but sacrificed strength in the process. These results can be contributed to the behavior of the microstructures of each material. 


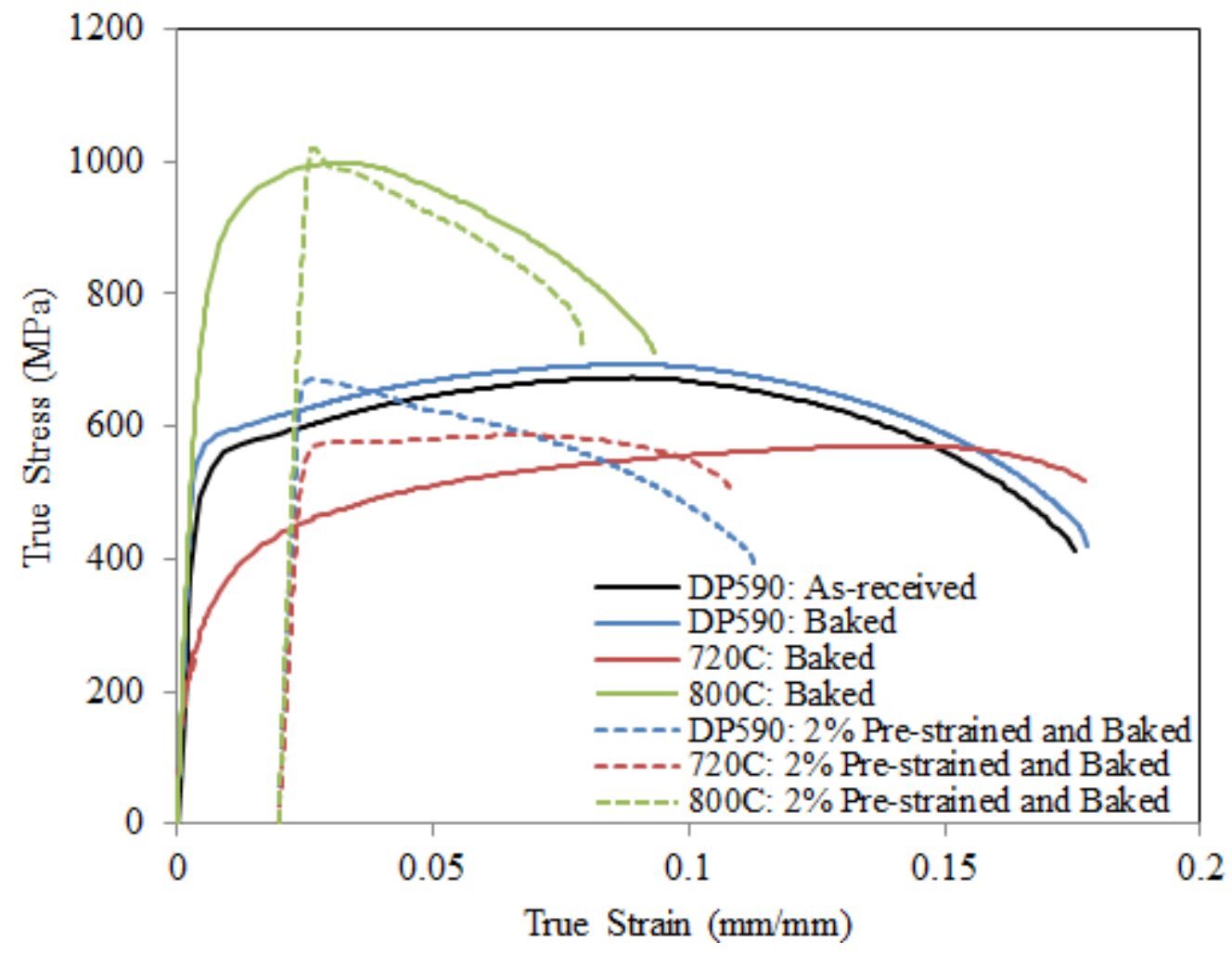

Figure 22. Quasi-static stress vs. strain results comparing the three different tempering cases at unstrained and $2 \%$ pre-strains tested at $0.01 / \mathrm{s}$ strain-rate.

The baking process of the as-received material induced only a very slight increase of the yield probably due to fine precipitation of carbon which was still in solid solution. The intercritical annealing at $720^{\circ} \mathrm{C}$ produced a microstructure with substantially lower yield stress (320 MPa vs. $600 \mathrm{MPa}$ ) as the martensite fraction was considerably reduced. Uniform elongation and elongation to failure was, however, enhanced, which actually elucidates the detrimental effect of martensite on ductility. The fracture surface, shown in Figure 23, revealed damage progression by nucleation, growth, and coalescence of voids.

The annealing at $800^{\circ} \mathrm{C}$ which mostly produces austenite, and thus, martensite after quenching, induced a marked increase in yield stress up to $1000 \mathrm{MPa}$. This marked increase in yield stress was accompanied by a dramatic reduction of ductility. The fracture surface in Figure 23 (c) indicates localized plastic deformation at the center of the specimen and probably nucleation of two shear bands that moved toward both edges of the specimen.

When pre-strained and baked, both steels pretreated at $720^{\circ} \mathrm{C}$ and $800^{\circ} \mathrm{C}$ showed a significant increase in yield stress and a decrease in ductility. However, the decrease in ductility for the steel annealed at $800^{\circ} \mathrm{C}$ was characterized by a sharper peak, which is characteristic of dramatic plastic instability. The fracture surface indicates a brittle fracture on a plane that was nearly normal to the stress axis. This indicates an anisotropic behavior of shear localization that was observed previously in martensitic steels. Actually, both non-treated and $720^{\circ} \mathrm{C}$ treated steels showed, after pre-strain and 
baking, kinks in the stress-strain curves immediately after yielding. The kinks correspond to mild yield point effects, which are characteristic of shear band formation.
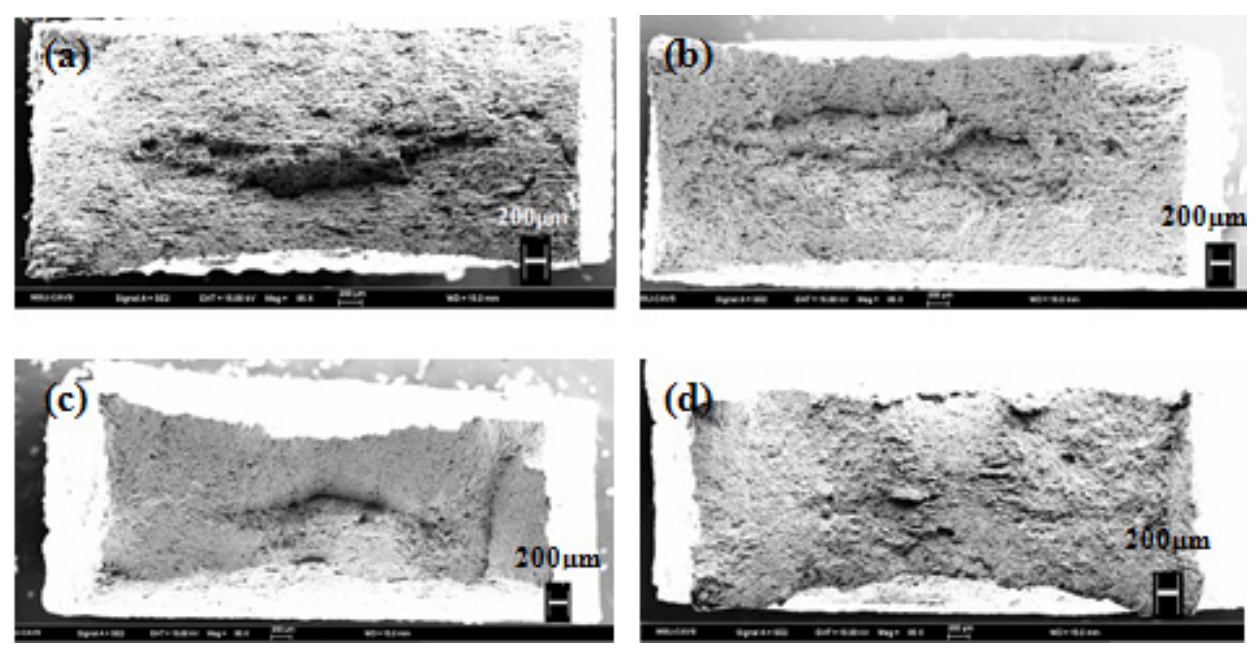

Figure 23. SEM fractography revealing fracture surfaces on the annealed series resulting from quasistatic testing.

Note: (a) $720^{\circ} \mathrm{C}$ annealed and oil quenched, (b) $720^{\circ} \mathrm{C}$ annealed and oil quenched then being $2 \%$ prestrained and baked, (c) $800^{\circ} \mathrm{C}$ annealed and ice-brine quenched, and (d) $800^{\circ} \mathrm{C}$ annealed and ice-brine quenched then being $2 \%$ pre-strained and baked.

Figure 24 shows stress vs. strain curves resulting from high strain-rate tensile tests on untreated series and the series $800^{\circ} \mathrm{C} /$ ice-brine. The test results revealed that the $1 \%$ pre-strained-and-baked series had the highest yield out of the received series although it is noted that there was not a significant difference in it and the received baked series. When comparing these tests with the heat-treated series, the heat-treated series was over 30 percent stronger than the strongest of the as-received series; however, the elongation to failure was reduced. As in the quasi-static tests, the $800^{\circ} \mathrm{C} /$ ice-brine series performed in much more of a brittle manner which was to be expected due to the higher fraction of martensite in the steel. Comparing the high strain-rate tests to the quasi-static tests, there does not seem to be much strain-rate sensitivity observed. Tensile strengths correlated between the two different tests similarly. 


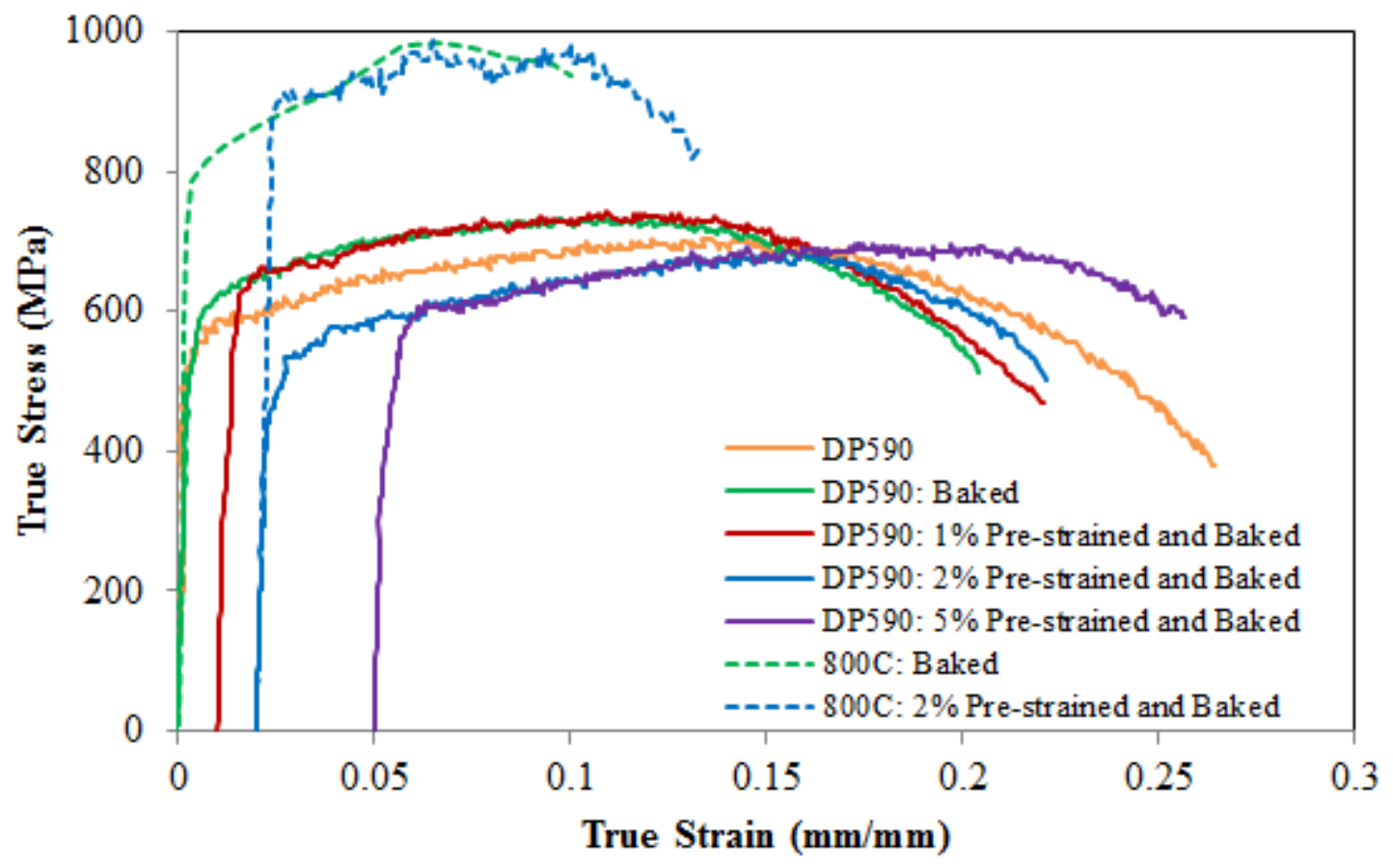

Figure 24. Stress versus strain curves resulting from high strain-rate tensile tests

Note: Strain rate of approximately $500 / \mathrm{s}$ was executed on the as-received series of specimens and $800^{\circ} \mathrm{C}$-ice brine-cooled specimens with pre-straining and baking protocols particular to each individual specimen series.

\section{Examination of the Microstructure, Chemical Composition, and Microhardness of the As- received TRIP680}

The chemical composition from the as-received TRIP680 was determined via chemical spectroscopy with the results shown in Table 8. The purpose of obtaining the chemical composition was for the derivation of phase and CCT diagrams aimed at developing heating/cooling schedules for reannealing procedures. Microstructural images, shown in Figure 25, were used to determine bainite volume fractions. The as-received bainite fractions were shown to be $39.0 \%$.

Table 8. Chemical composition of as-received TRIP680.

\begin{tabular}{|c|c|c|c|c|c|c|c|c|c|}
\hline $\mathbf{C}$ & $\mathbf{S i}$ & $\mathbf{M n}$ & $\mathbf{P}$ & $\mathbf{S}$ & $\mathbf{C r}$ & $\mathbf{M o}$ & $\mathbf{A l}$ & $\mathbf{V}$ & $\mathbf{N b}$ \\
\hline 0.206 & 0.056 & 1.54 & 0.011 & 0.005 & 0.019 & 0.0038 & 0.93 & 0.0035 & $<0.004$ \\
\hline
\end{tabular}




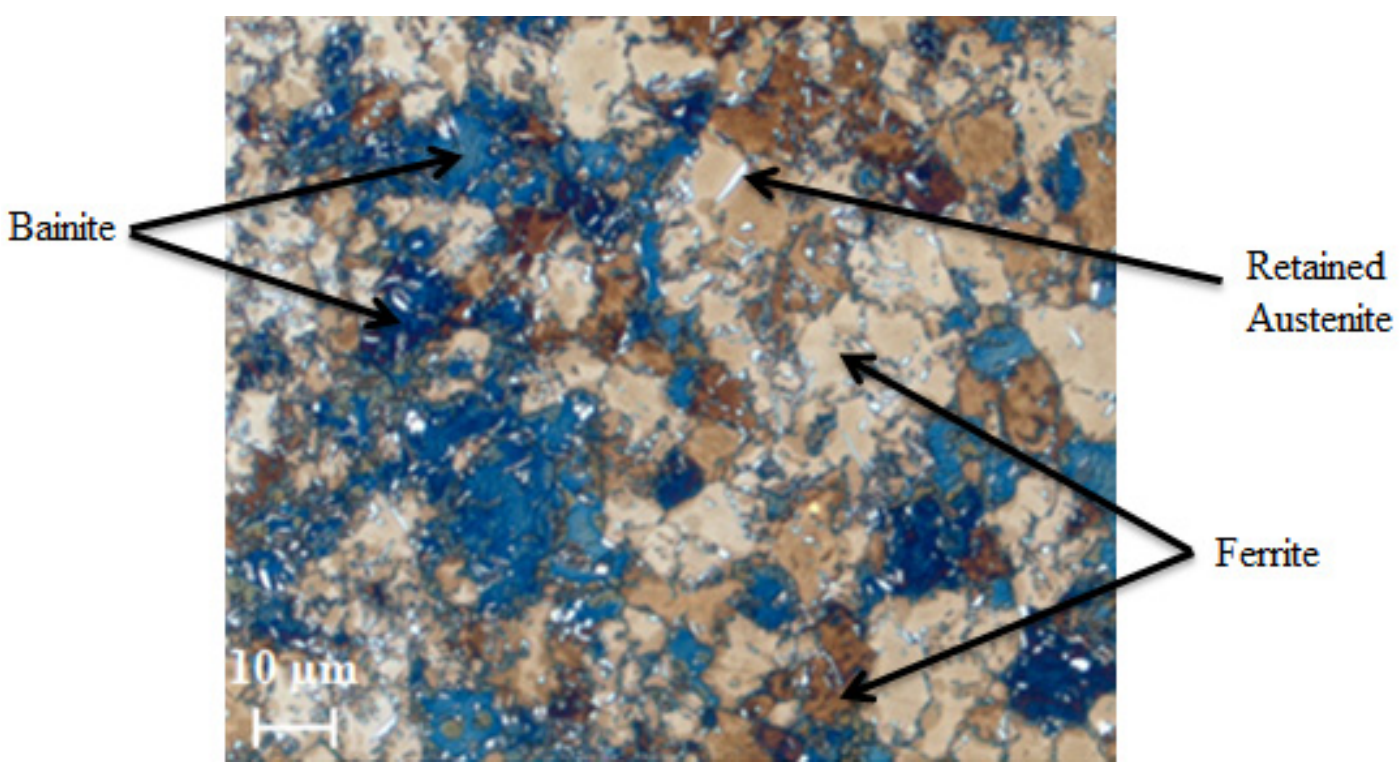

Figure 25. TRIP680 etched with Beraha's tint etchant: ferrite (tan and dark brown), bainite (blue), and retained austenite (bright white).

XRD analysis was performed on the as-received TRIP680. Figure 26 shows the results from the XRD scan on the analysis. The (110) and (310) curves were used to calculate the percentages of ferrite and austenite, respectively. Results of the analysis through the direct comparison method gave a $0.447: 1$ ratio of retained austenite to ferrite. Knowing that the bainite fraction was $39.0 \%$, overall ferrite and austenite fractions were calculated to be $41.4 \%$ ferrite and $19.6 \%$ retained austenite.

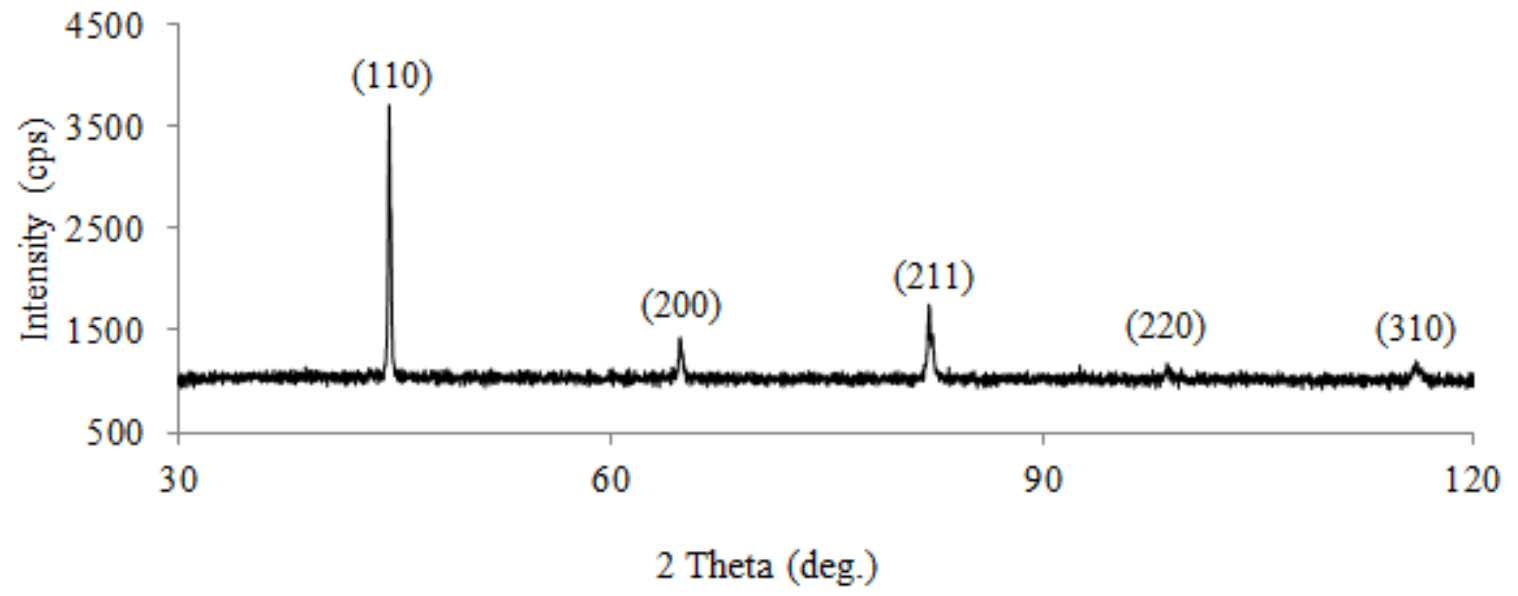

Figure 26. XRD scan from as-received TRIP680.

Vickers tests were performed on the as-received TRIP680 after etching with Beraha's tint etchant. Figure 27 shows images from indenting on to the steel. Ferrite and bainite phases were indented to investigate the hardness of the two phases. The austenite grains were not large enough to 
completely encase the indenter head. For this reason, austenite hardness values were not investigated. Hardness results from the tests for bainite and ferrite were $240.0 \mathrm{Hv}$ and $207.8 \mathrm{Hv}$, respectively.
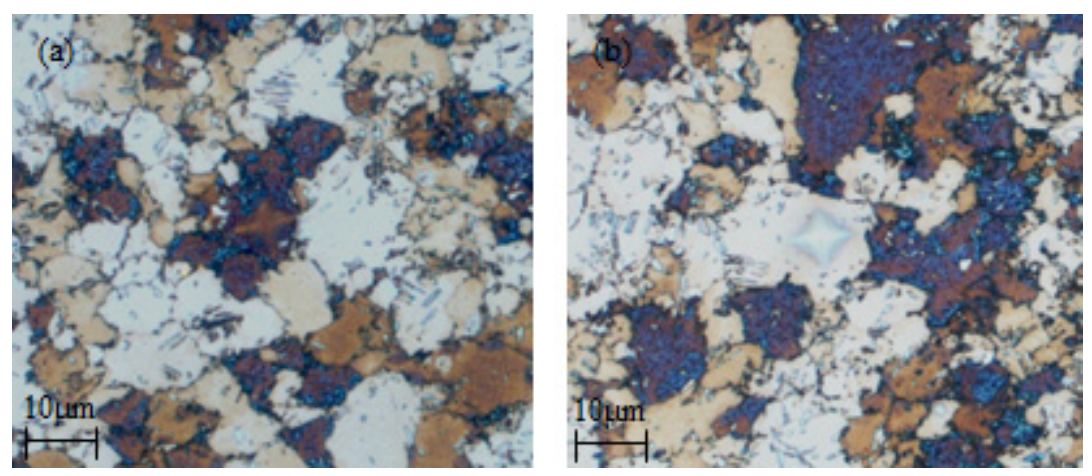

Figure 27. Optical microscopy showing Vickers indents on (a) bainite and (b) ferrite.

\section{Investigation into the Thermo-mechanical Preconditioning Effects on the Mechanical Behavior of As-received TRIP680}

Quasi-static tensile tests were performed and shown in Figure 28. Specimens were preconditioned under different pre-strainings of $0,1,2$, and $5 \%$. There were variations of responses resulting from the implemented work-hardening. However, the $2 \%$ pre-strained and baked specimens revealed the highest UTS at $813.8 \mathrm{MPa}$. Lower applied strain rates were able to reach higher UTS at longer elongations. In the lower strain-rate tests, a small dip near yield is observed where phase transformation may take place. Yield for the $5 \%$ pre-strained and baked specimens occurred much higher than the other test conditions. 


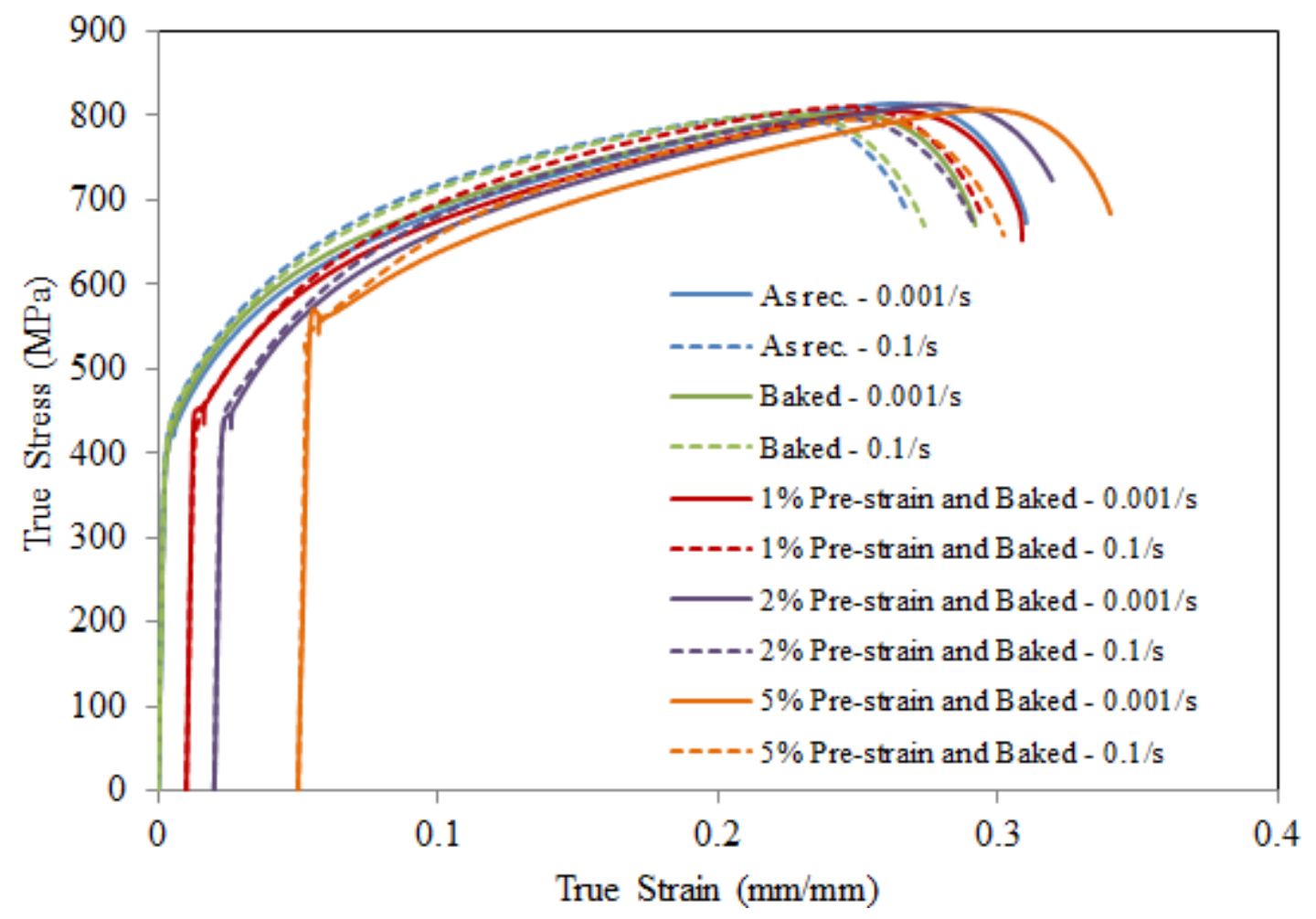

Figure 28. True stress vs. true strain curves resulting from quasi-static tensile tests.

Thermo-mechanical response characteristics can be determined from the quasi-static results shown above. Of those, Figure 29 shows the work-hardening characteristics from the (a) $0.1 / \mathrm{s}$ strainrate and (b) $0.001 / \mathrm{s}$ strain-rate. For the $0.001 / \mathrm{s}$ case, there was only a subtle increase of the workhardening characteristics from $1 \%$ to $2 \%$ pre-strains (from 9 to $18 \mathrm{MPa}$, respectively), but from $2 \%$ to $5 \%$ there was an increase of over $90 \mathrm{MPa}$ of the work-hardening index reaching an overall effect of 110 MPa. This signals that the extra pre-strain from $2 \%$ to $5 \%$ creates a significant increase in yield strength possibly from a transition of austenite to martensite occurring during the $5 \%$ pre-strain. As was the case for the $0.1 / \mathrm{s}$ case, the $0.001 / \mathrm{s}$ tests showed the $5 \%$ pre-strain to cause much higher work-hardening characteristics that resulted in an increased yield of over $150 \mathrm{MPa}$. 


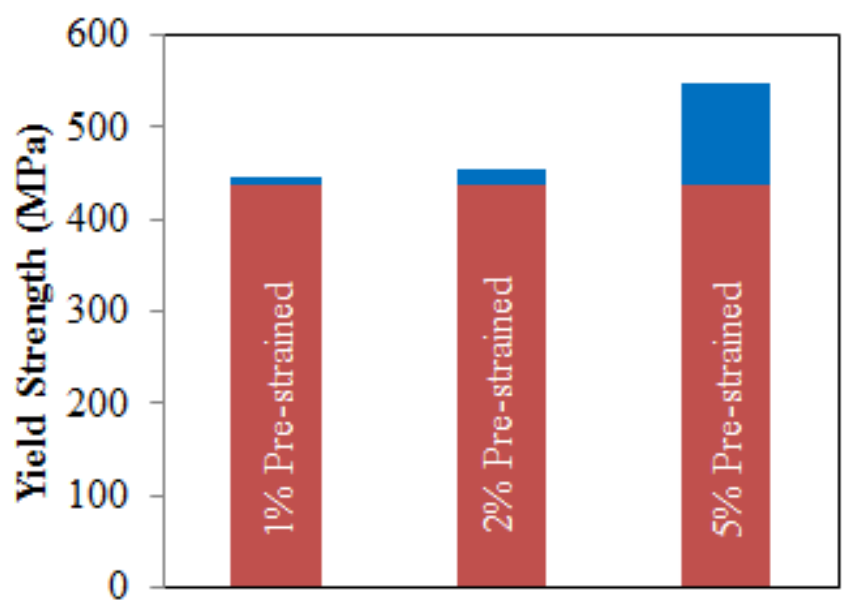

(a)

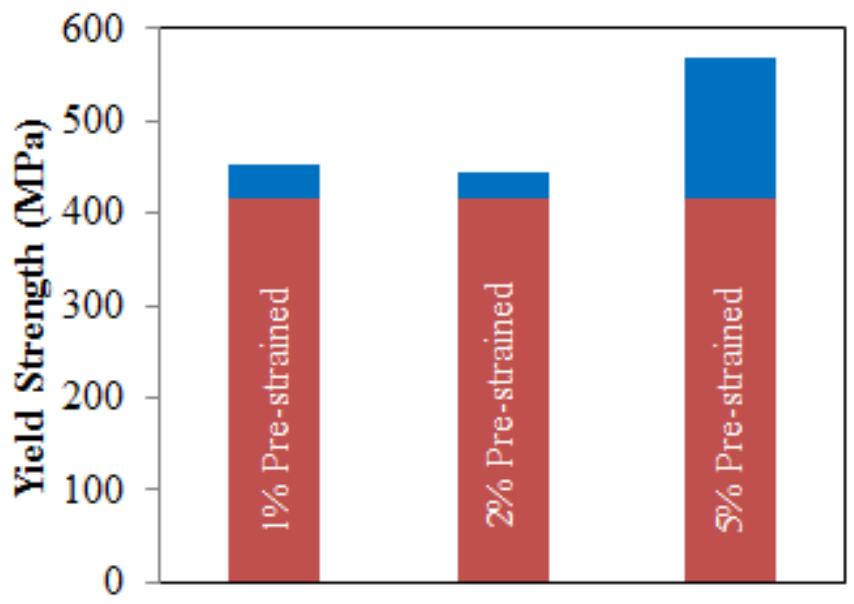

(b)

- Work Hardening Index

- As-received

Figure 29. Work-hardening indices with respect to various thermo-mechanical preconditioning protocols.

Notes: (a) $0.1 / \mathrm{s}$ strain rate, and (b) $0.001 / \mathrm{s}$ strain rate.

Quasi-static test fractography with SEM reveals fracture modes with very limited dimpling fracture surfaces at all strain rates. For the specimens tested at $0.1 / \mathrm{s}$, smaller pre-strains exuded more predominant the cup-and-cone fracture surface with cleave planes spanning the width of the specimen at approximately $45^{\circ}$ to the direction of maximum stress shown in Figure $30(a, b)$. The more intense pre-strains had cleavage planes that began to grow from the center out on one side of the specimen (c,e). The $2 \%$ pre-strain and baked specimen appeared to have the most brittle fracture surface which corresponds to it having the highest UTS. The cup-and-cone fracture surfaces correspond in fact to shear banding at the planes with maximum stresses. 


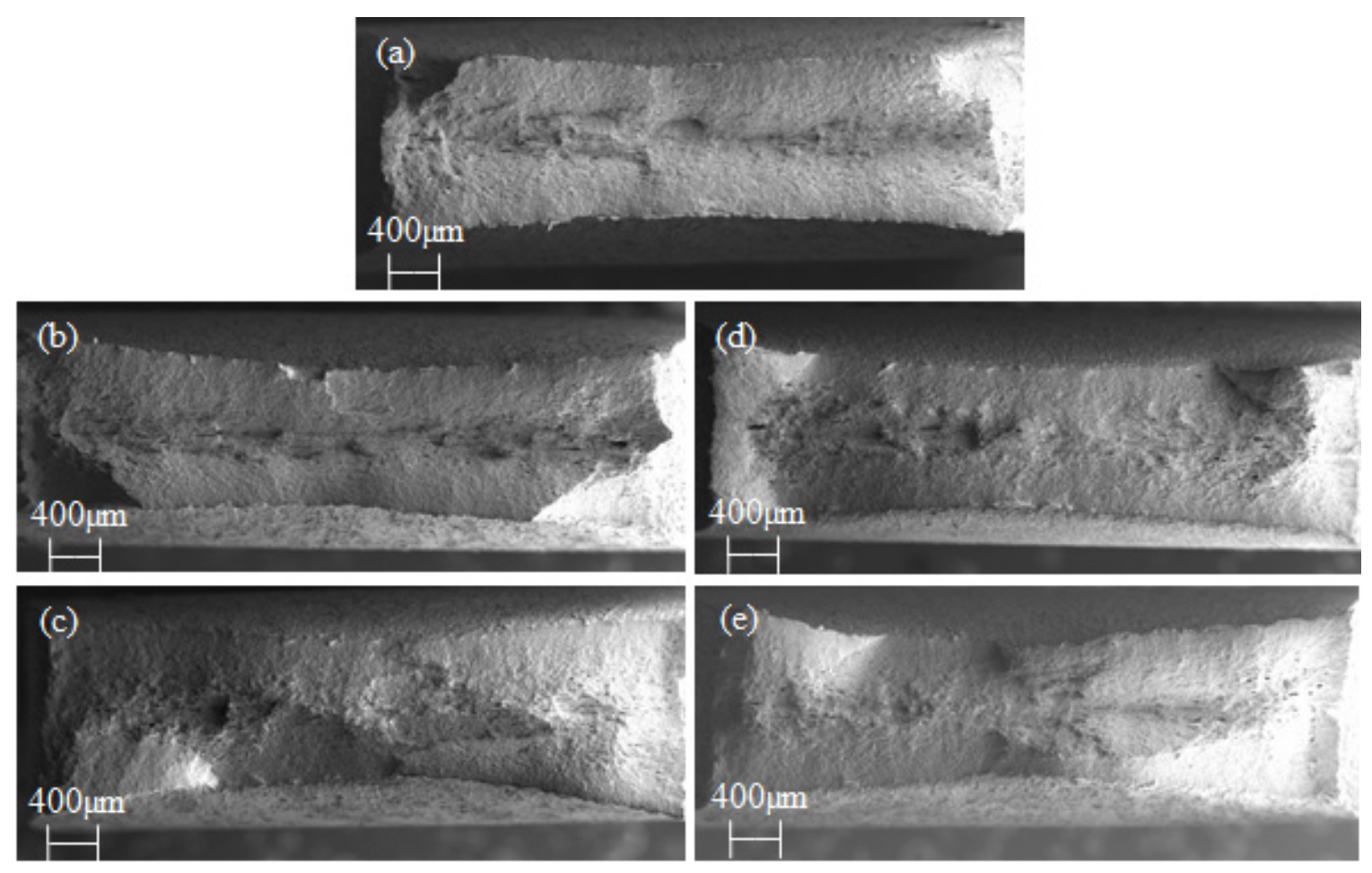

Figure 30. Fractography of specimens from quasi-static testing tested at $0.1 / \mathrm{s}$ strain rate.

Note: (a) as received, (b) baked, (c) $1 \%$ pre-strain and baked, (d) $2 \%$ pre-strain and baked, and (e) $5 \%$ pre-strain and baked.

The fractography specimens tested at $0.001 / \mathrm{s}$ strain rates appeared to have the nearly the opposite results as seen in Figure 31. The specimens with no pre-strain showed the cleavage planes growing from the middle edge of the specimen $(a, b)$, where the specimens with $1 \%$ and $2 \%$ pre-strains appeared more brittle with fracture planes in the corners (c,e). This is probably due to the higher fraction of TRIP-induced martensite before baking. The baking process should have tempered the deformed martensite by forming carbides in the lath boundaries. This may have most likely induced tempering embrittlement with an increase tendency to nucleate shear bands early in post-testing. In fact, the specimen pre-strained to $5 \%$ showed a much higher reduction of ductility as compared to the specimen pre-strain to $1 \%$ and $2 \%$. This indicates a higher tendency to shear localization. As in the $0.1 / \mathrm{s}$ series, the $2 \%$ pre-strained and baked specimen appeared different than the rest of the specimens (d). In this case, the fracture surface may suggest that the shear nucleated from the center of the specimen. However, the most plausible explanation is that the shear band nucleated at the free-surface and then intersected at the center of the specimen to induce a tensile stress state with the high hydrostatic pressure. This is why damage seems to be more pronounced near the central regions of the fractured specimens. 


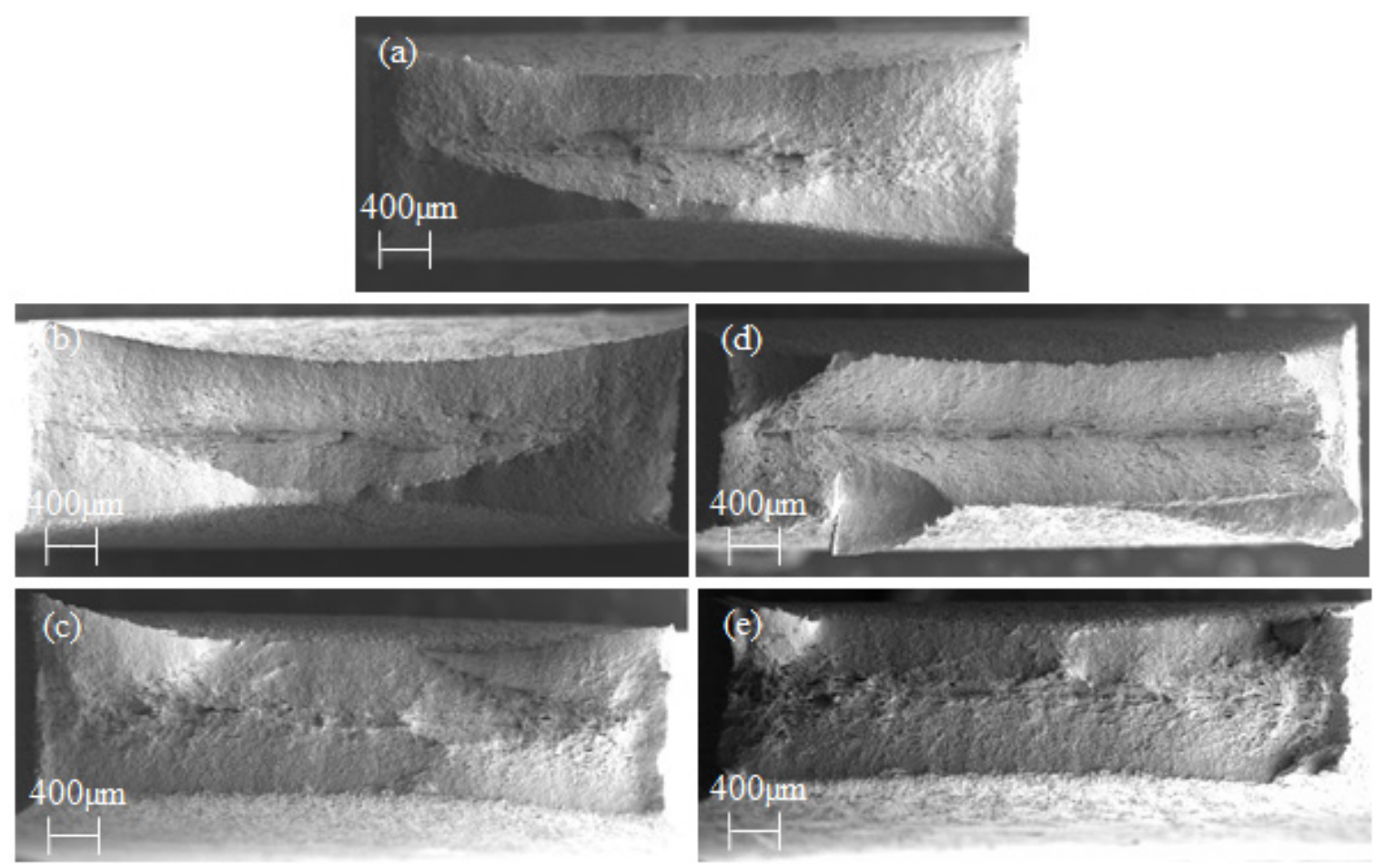

Figure 31. Fractography of specimens from quasi-static testing tested at $0.001 / \mathrm{s}$ strain rate.

Note: (a) as-received, (b) baked, (c) 1\% pre-strain and baked, (d) 2\% pre-strain and baked, and (e) $5 \%$ pre-strain and baked.

High strain-rate tests, shown in Figure 32, were performed with the intent of observing the mechanical response during high-speed strains to more adequately represent a more typical automotive collision. The test parameters used here were the same that were used under the quasi-static testing regime. Regarding yield, there was negligible baking effect. The $2 \%$ and $5 \%$ pre-strain cases had the most noticeable trends differing from the as-received series. Comparing the high strain-rate results to the quasi-static results, negative strain rate sensitivity is evident due to the higher maximum tensile stresses in all cases but the as-received material. Every other test displayed higher tensile stresses in the high strain-rate regime than in the quasi-static. 


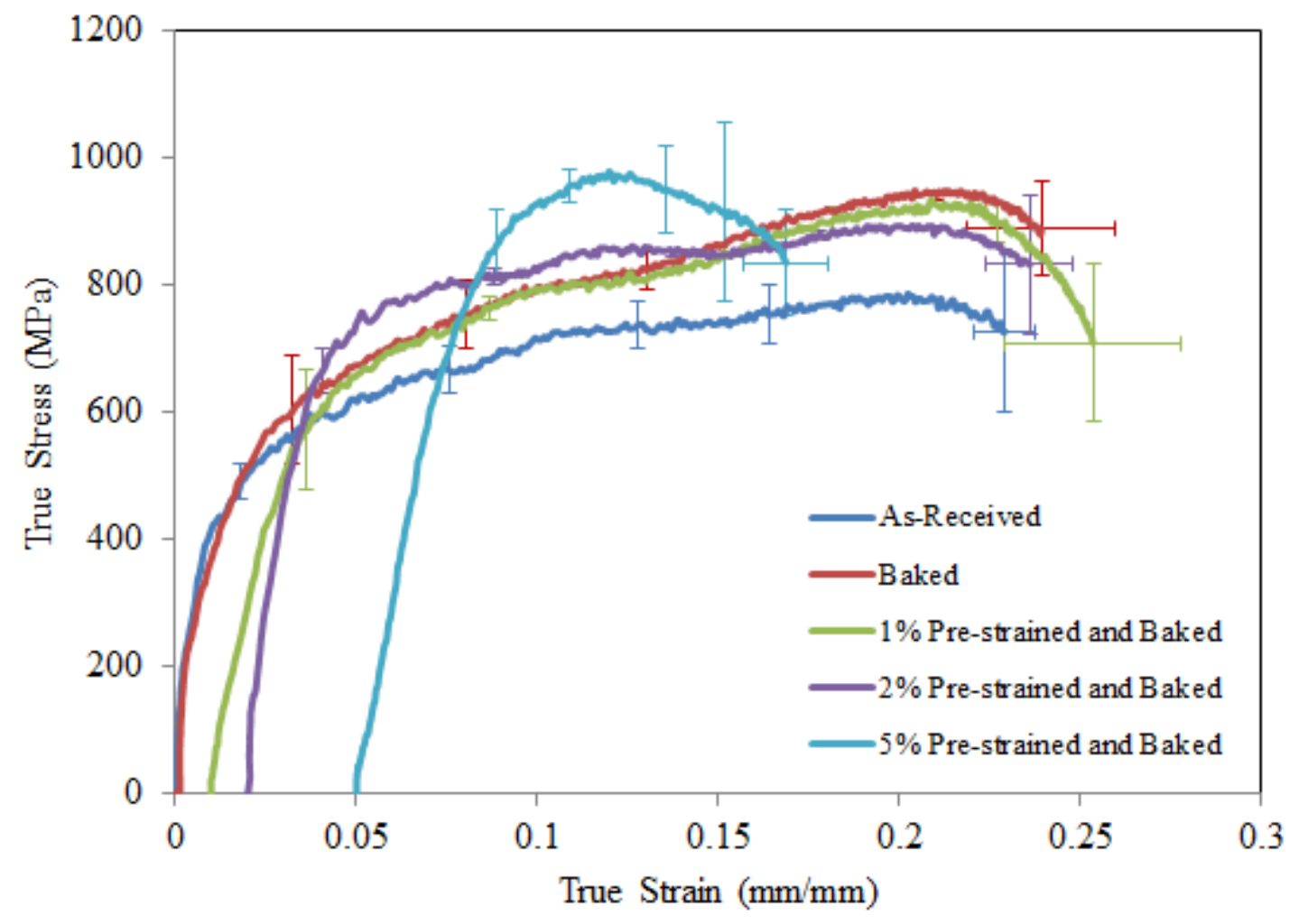

Figure 32. True stress vs. true strain graph resulting from high strain rate tensile tests.

\section{Heat-Treating and the Resultant Microstructural and Mechanical Observations}

Heating/cooling treatments were carried out with the desire of altering the phase fractions of retained austenite for the purpose of further testing and response comparisons. Optical microscopic images were taken of the I.C.A. treated series in order to observe the bainite fractions seen in Figure 33. The specimen was etched with Beraha's color etch and viewed with a Zeiss Axiovert 200 optical microscope. The resultant bainite fractions were found to be $21.3 \%$ from image analysis software. 


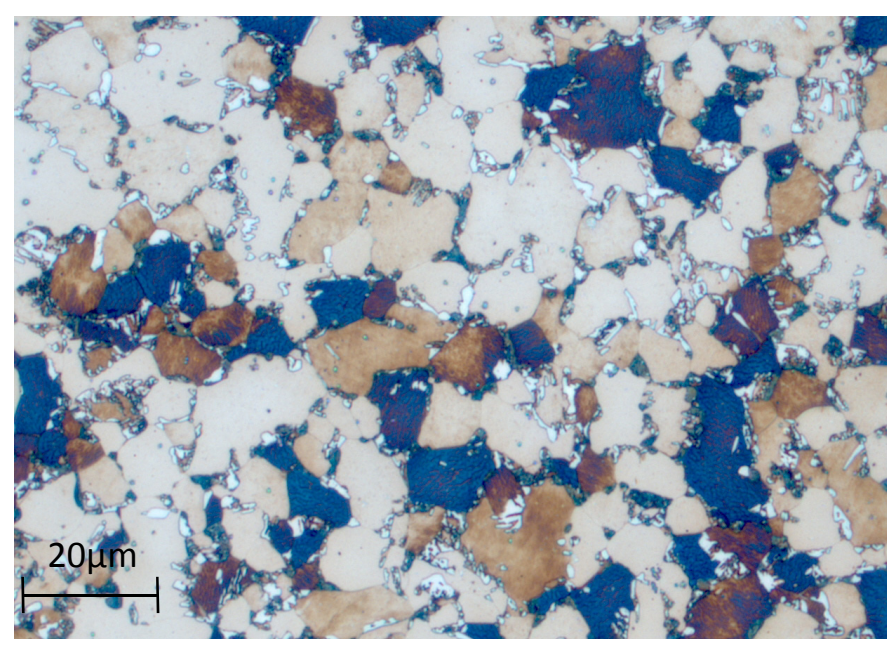

Figure 33. I.C.A. heat-treated TRIP steel showing the three phases of the steel: ferrite (tan and brown), bainite (blue), and retained austenite (bright white).

XRD was used to verify the amount of retained austenite. Figure 34 shows the results from the XRD analysis. The peaks were indexed into five different Miller indices representing the ferrite and austenite in the microstructure of the steel. Through image analysis with Beraha's color etching and the direct comparison method, volume fractions were calculated for the comparison of the as-received TRIP680 to the heat-treated steels. Table 9 shows the results of the phase fractions in the steel. The I.C.A. heating procedure provided the greatest contrast of hypothesized mechanical behavior compared to the original TRIP680 series due to the increased ferrite volume and decreased bainite volume. Therefore, this procedure will be used in mechanical testing to compare the mechanical and microstructural response with the as-received series. 


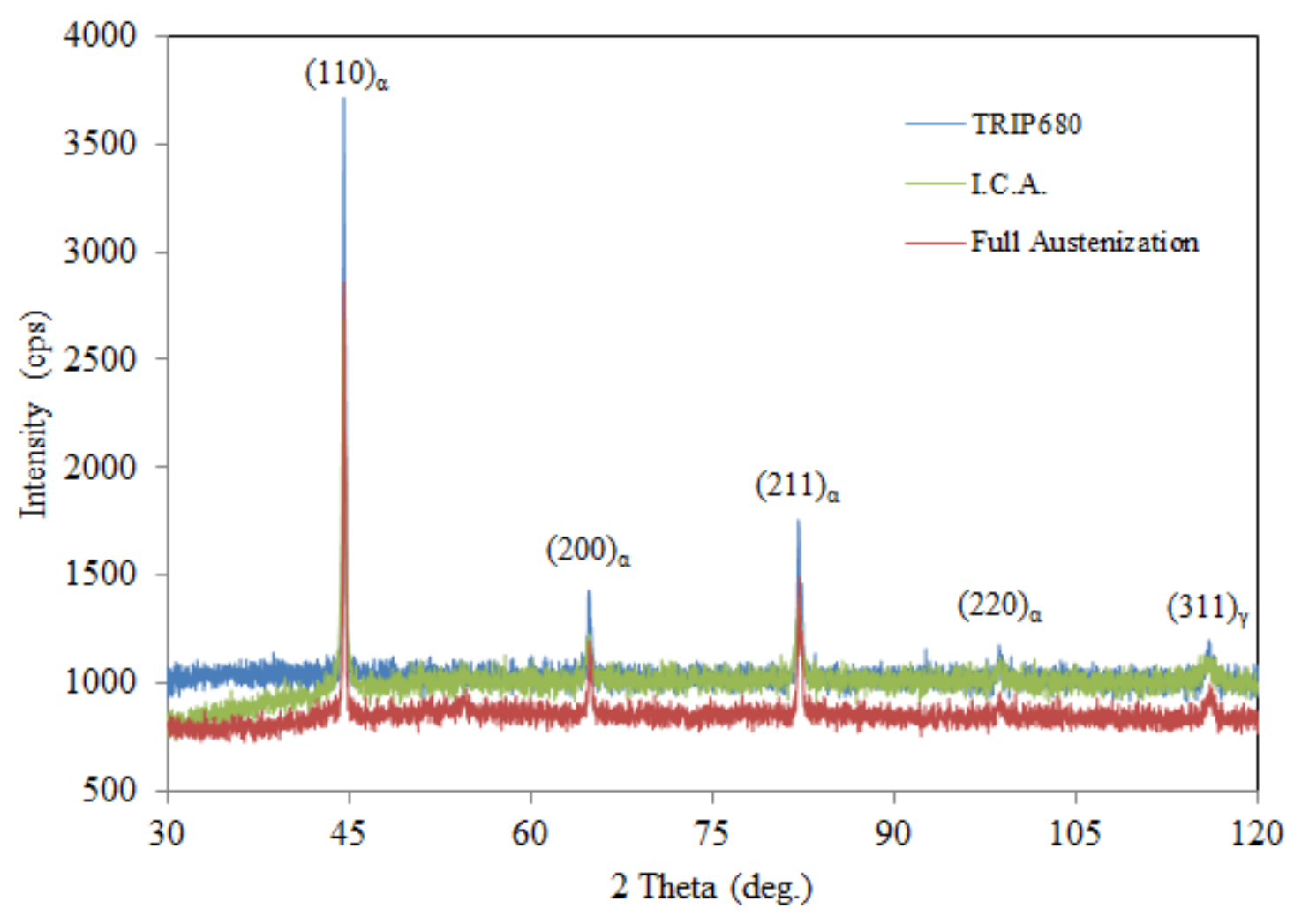

Figure 34. XRD Analysis of the TRIP steel series with the five major absorption peaks indexed.

Table 9. Phase volume fraction results of the as-received condition compared with the heat treated series.

Phase Fractions (vol. \%)

\begin{tabular}{|l|c|c|c|}
\hline TRIP Series & Ferrite & Bainite & Austenite \\
\hline As-Received & 41.4 & 39.0 & 19.6 \\
\hline Full Austenization & 43.5 & 32.7 & 23.8 \\
\hline I.C.A. & 62.2 & 21.3 & 16.5 \\
\hline
\end{tabular}

Quasi-static tensile tests were performed on the newly created I.C.A. steel in at $0.1 / \mathrm{s}$ strain-rate under three preconditioning protocols: no pre-strain, $2 \%$ pre-strain and baked, and $5 \%$ pre-strain and baked. Comparisons of that and the original TRIP680 are shown in Figure 35. As anticipated, the I.C.A. series performed weakly compared to the as-received series likely due to the smaller percentage of bainite and retained austenite. In the case of the I.C.A. with no pre-strain and $2 \%$ pre-strain, the retained austenite-to-martensite transformation can be seen right after yielding. The more horizontal action of the respective stress-strain curves illustrates the mechanical behavior during phase 
transformation. However, the $5 \%$ pre-strained-and-baked series did not display this behavior alluding to most of the martensitic transformation occurring during the $5 \%$ pre-straining.

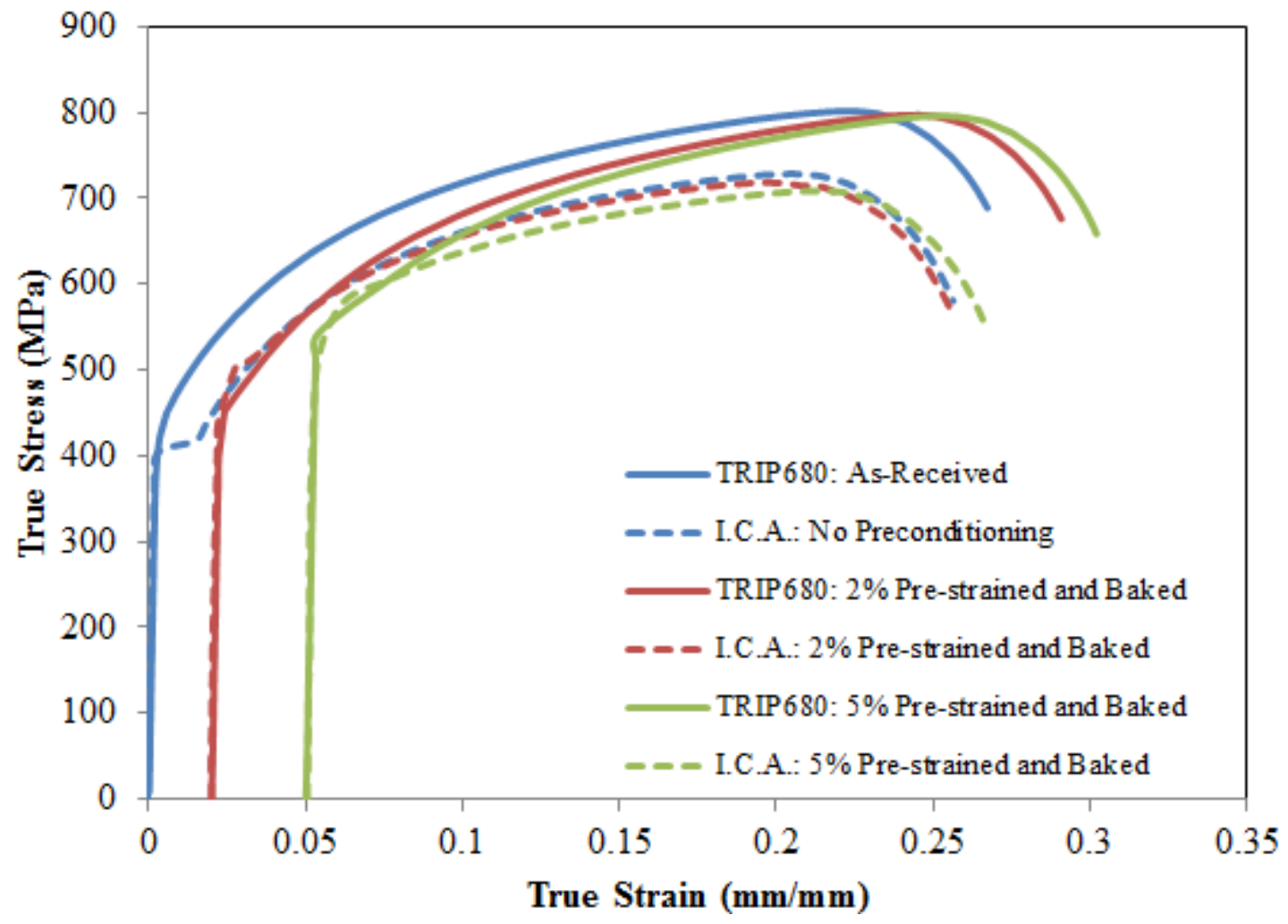

Figure 35. Quasi-static stress vs. strain results comparing the received TRIP680 to the I.C.A. series under three different preconditioning protocols.

The fractured specimens were subjected to XRD scans and then analyzed through the direct comparison method. Figure 36 shows the scan of the as-received 5\% pre-strained and baked specimen. Peaks of the existing ferrite and austenite microstructure are labeled. In addition, newly formed martensitic peaks are highlighted in red. The results of the scan showed an existing volume fraction of $7.3 \pm 0.6 \%$ retained austenite. This suggests that over $60 \%$ of the austenite transformed to martensite. All of the quasi-static XRD results followed this trend. 


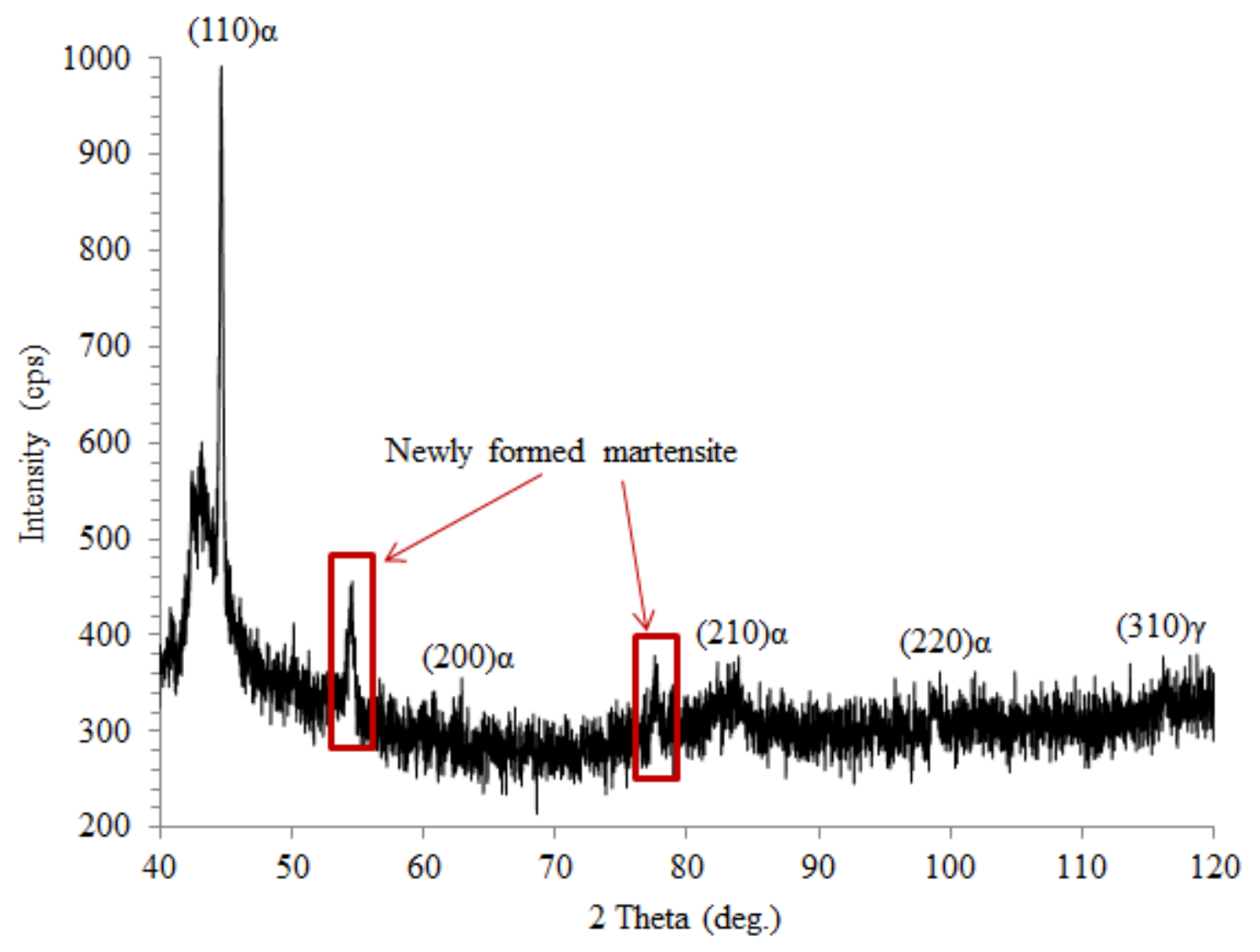

Figure 36. XRD Analysis of the TRIP steel series with the five major absorption peaks indexed.

Figure 37 shows fractographical images that were taken via SEM from the quasi-static tensile tests on the I.C.A. series. As with the as-received specimens, the $5 \%$ pre-strained and baked of the I.C.A. case displayed a large reduction in elongation compared to the I.C.A. specimen with no pre-conditioning (c and a, respectively). This alludes to shear localization occurring with this series as well. The trend of higher martensite content in each specimen with increased pre-strains is almost surely another factor in why the elongation is reduced in both the $2 \%$ and $5 \%$ specimens. 


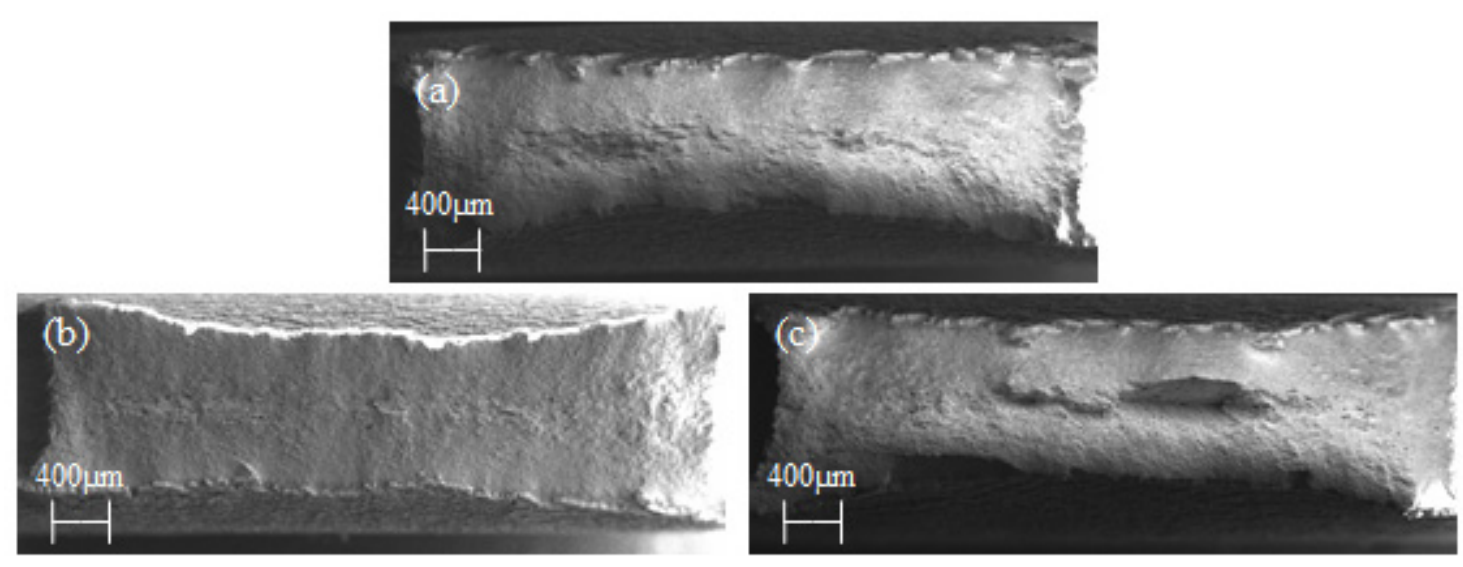

Figure 37. Fractographical images from the I.C.A. series following quasi-static testing.

Note: (a) No pre-strain or baking; (b) $2 \%$ pre-strain and baking; (c) $5 \%$ pre-strain and baking.

High strain-rate tensile tests were performed on the I.C.A. series in order to observe the mechanical behavior of the steel subjected to strain at high speeds and to provide a comparison to the behavior of the TRIP680 (Figure 38). Due to the increase of the ferrite fraction and resultant ductility, the I.C.A. series behavior was comparable to the quasi-static tests in comparison to the received TRIP680. However, in the high strain-rate tests, the I.C.A. specimens displayed much more elongation to failure when compared to the TRIP680 than was observed in the quasi-static tests. Therefore, since the majority of failures of an automotive body are in high strain-rate environments, the high strain-rate testing would be the most important case to note in this investigation. 


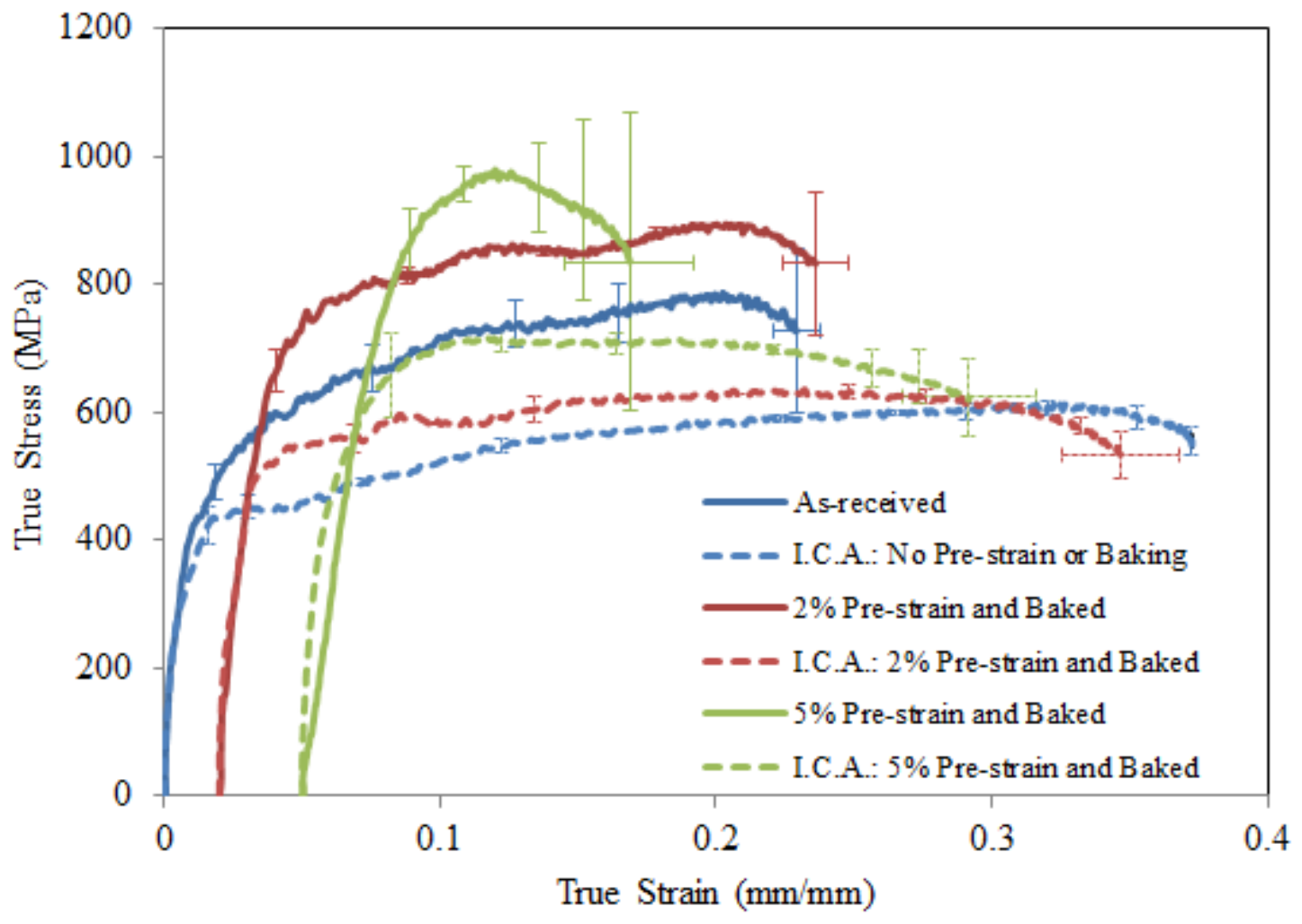

Figure 38. True stress vs. true strain graph resulting from high strain-rate tensile tests of the I.C.A. series.

The high strain-rate fracture specimens were subjected to XRD scans and then analyzed through the direct comparison method. Figure 39 shows the scan of the as-received $5 \%$ pre-strained and baked specimen. The martensitic peaks are highlighted while no (310) peaks are evident. Therefore, the XRD analysis alludes to complete austenitic transformation. All of the high strain-rate XRD results followed this trend. 


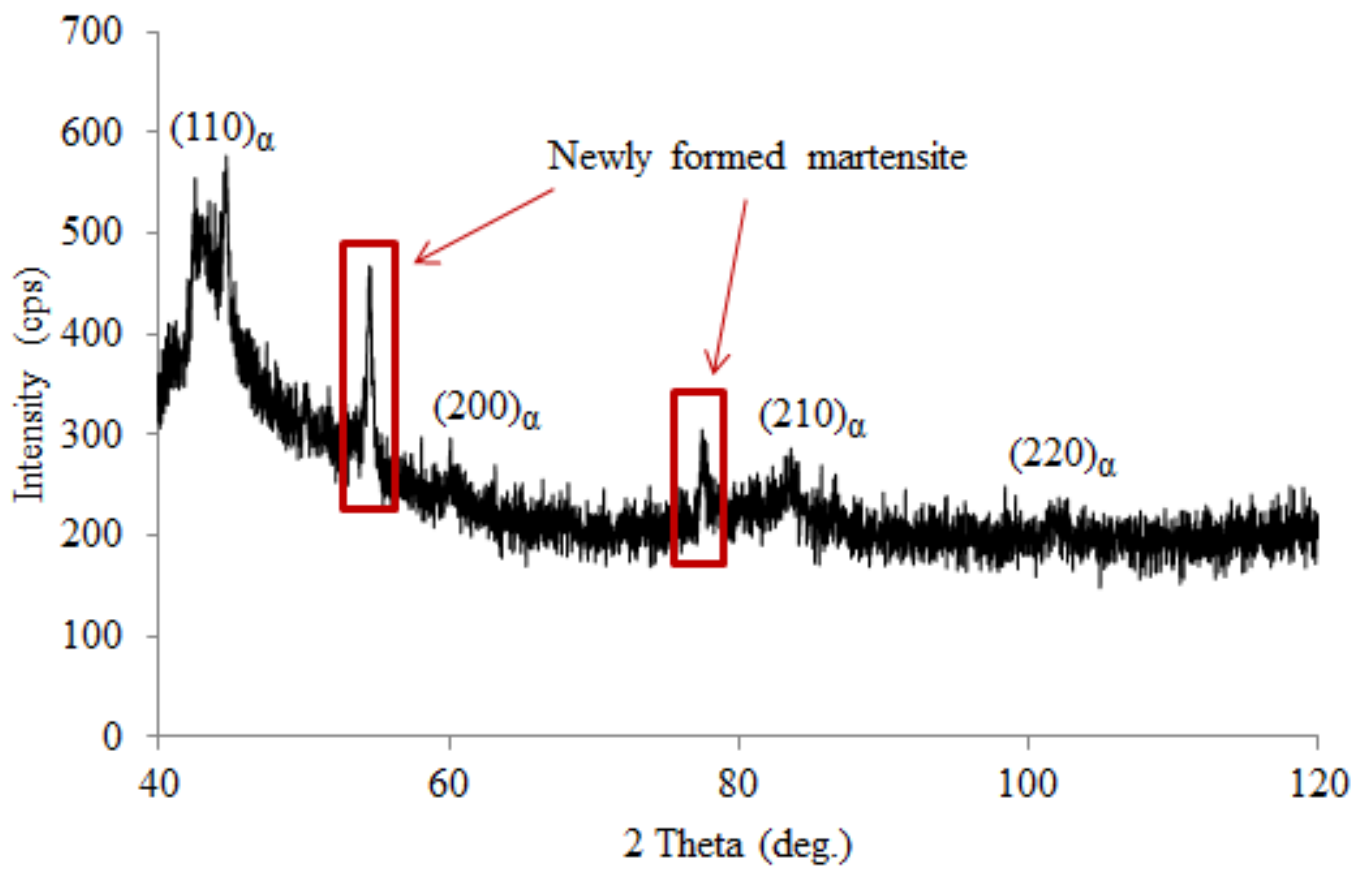

Figure 39. XRD Analysis of the TRIP steel series with the five major absorption peaks indexed. 


\section{References}

[Ruda09] M. Ruda, D. Farkas, and G. Garcia, Computational Materials Science 45, 550 (Apr. 2009), ISSN 09270256, http://linkinghub.elsevier.com/retrieve/pii/S0927025608005053.

[Henr09] K. Henriksson and K. Nordlund, Physical Review B 79, 1 (Apr. 2009), ISSN 1098-0121, http://link.aps.org/doi/10.1103/PhysRevB.79.144107

[Murn44] F. D. Murnaghan, Proceedings of the National Academy of Science 30, 244 (Sep. 1944).

[Murn67] F. Murnaghan, Finite deformation of an elastic solid (Dover New York, 1967).

[Hagl91] J. Haglund, G. Grimvall, and T. Jarlborg, Phys. Rev. B 44, 2914 (Aug 1991), http://link.aps.org/doi/10.1103/PhysRevB.44.2914.

[Lee11] T. Lee, M. I. Baskes, S. M. Valone, and J. D. Doll, Los Alamos Preprint:LA-UR 11-03286 (2011), http://arxiv.org/pdf/1107.0544.pdf

[Sa08] Inyoung Sa and Byeong-Joo Lee, Scripta Materialia 59, 595-598 (2008), http://dx.doi.org/10.1016/j.scriptamat.2008.05.007

[Jian08] C. Jiang, S. G. Srinivasan, a. Caro, and S. a. Maloy, Journal of Applied Physics 103, 043502 (2008), ISSN 00218979, http://link.aip.org/link/JAPIAU/v103/i4/p043502/s1\&Agg=doi

[Hill52] R. Hill, Proceedings of the Physical Society. Section A 65, 349 (1952), http://stacks.iop.org/0370$\underline{1298 / 65 / \mathrm{i}=5 / \mathrm{a}=307}$

[Sa08] Inyoung Sa and Byeong-Joo Lee, Scripta Materialia 59, 595-598 (2008), http://dx.doi.org/10.1016/i.scriptamat.2008.05.007

[Tschopp11] M. Tschopp, K. Solanki, M. Baskes, F. Gao, X. Sun, and M. Horstemeyer, Journal of Nuclear Materials , (2011). 


\section{TABle of Contents}

TASK 3: Multiscale Material Models and Processing Design for Polymeric Materials .................. 5

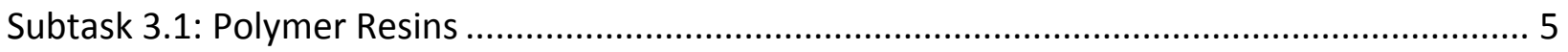

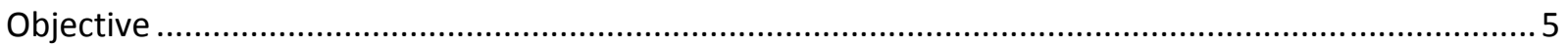

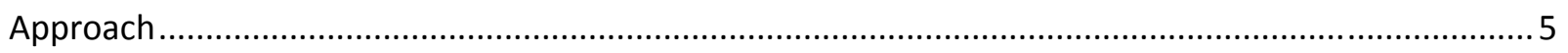

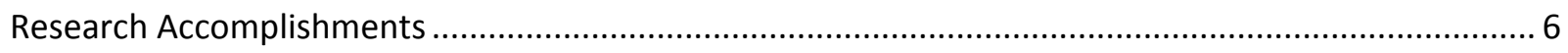

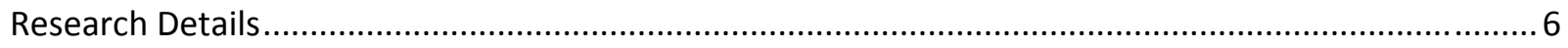

An internal state variable material model for thermoplastics and thermosets ............................... 6

Molecular dynamic simulations for exploring the deformation of polymers.................................. 23

Developing a potential for polymer systems based on the Modified Embedded-Atom Method

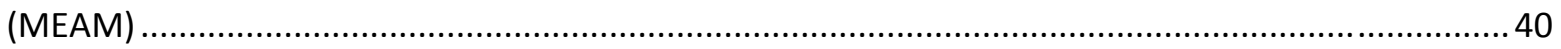

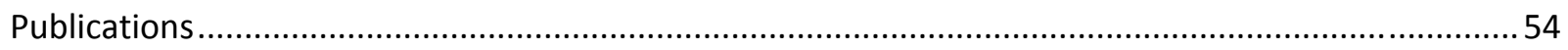

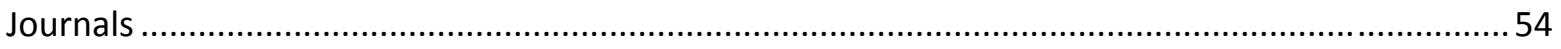

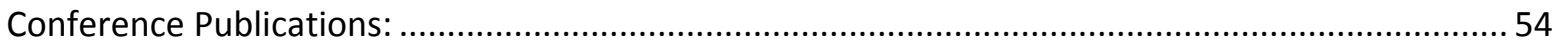

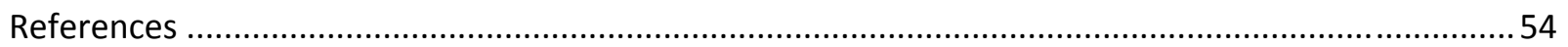

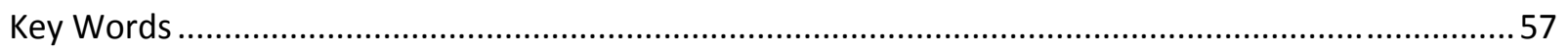

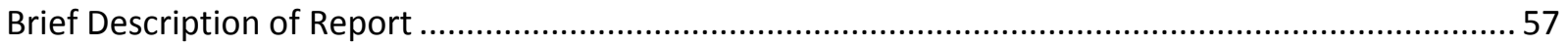

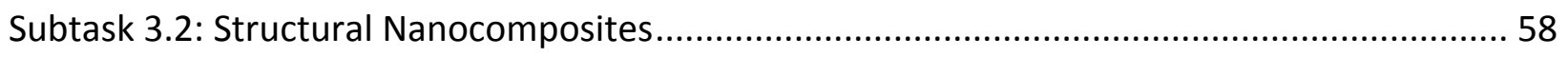

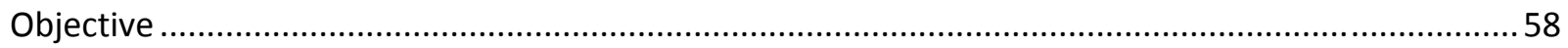

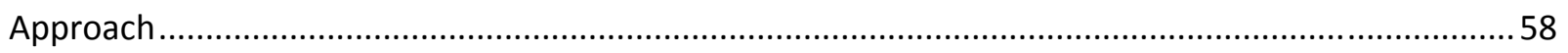

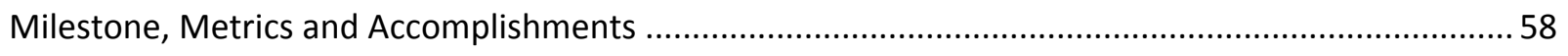

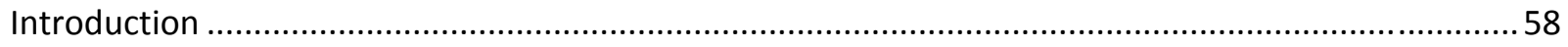

Experimental Structure-Property Relations for Nanoreinforced Materials ..................................... 60

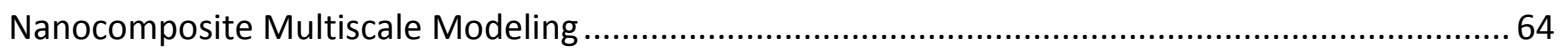

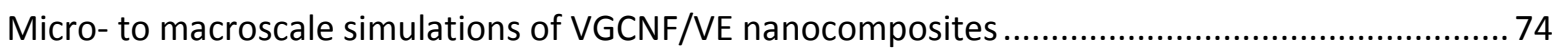

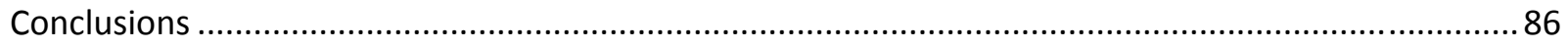

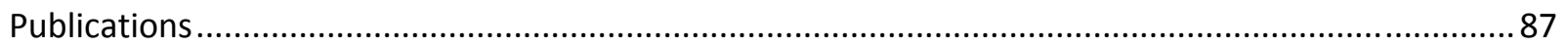

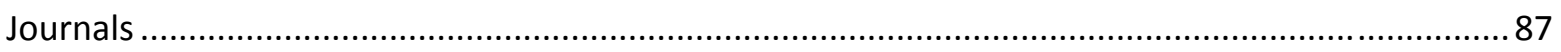

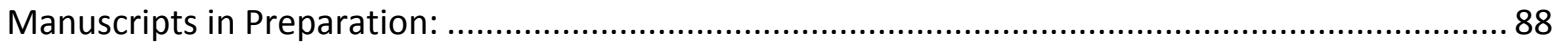

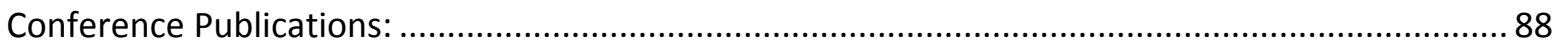

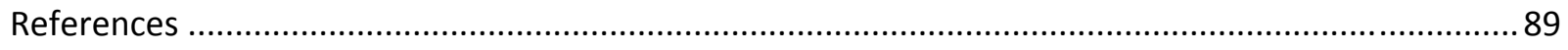




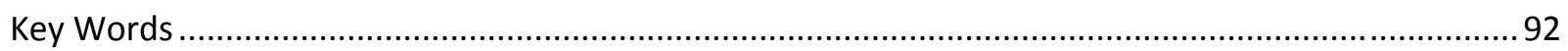

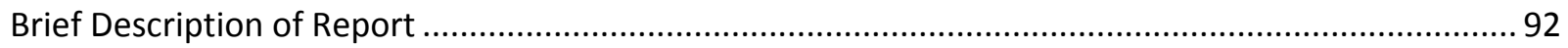

Subtask 3.3: Natural Fiber Composites for Structural Component Design ........................... 93

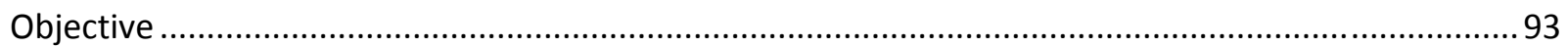

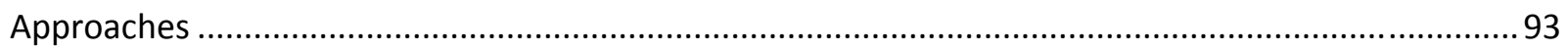

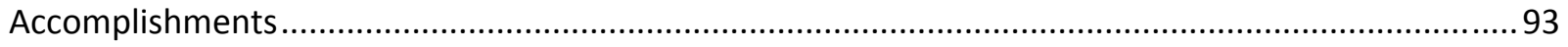

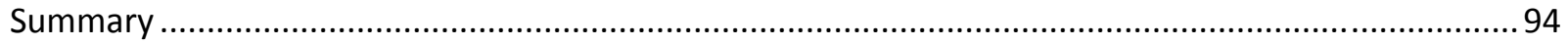

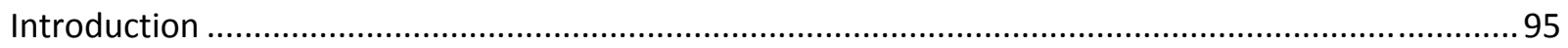

Physical/Mechanical Properties and Durability of Natural Fiber Sheet Molding Compound ............... 95

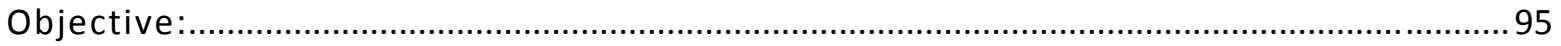

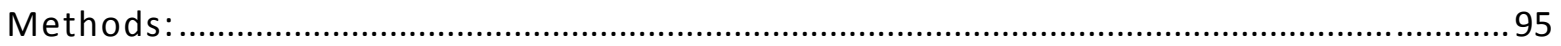

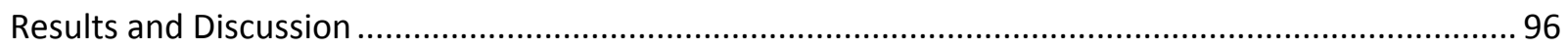

Chemical Components and Morphologies of the Kenaf Bast Fibers obtained from the Chemical

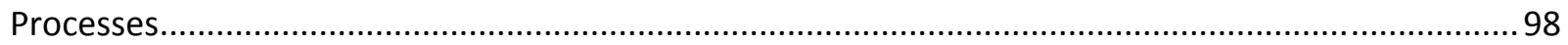

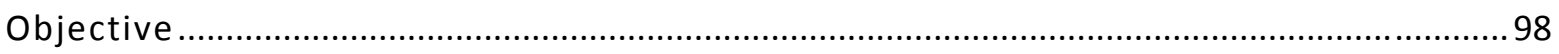

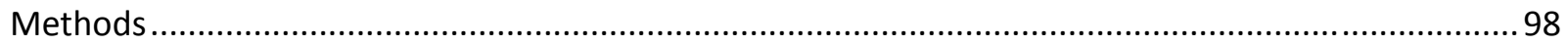

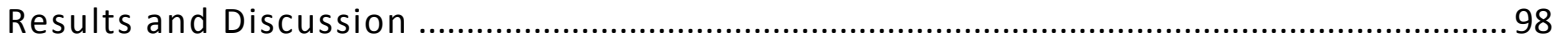

A Micro-tension Test Method for Measuring Four Representative Individual Cellulosic Fibers .......... 100

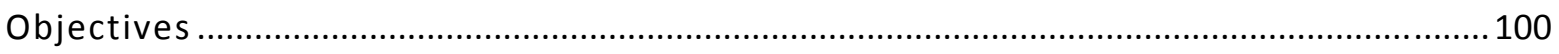

Micro Tensile Test System Development …........................................................................ 101

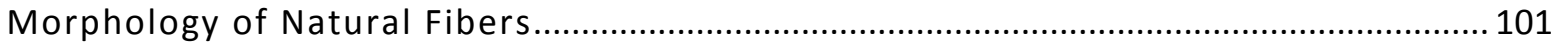

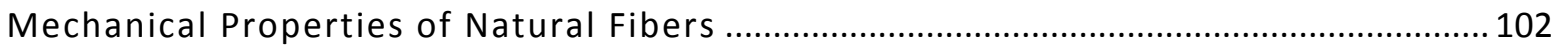

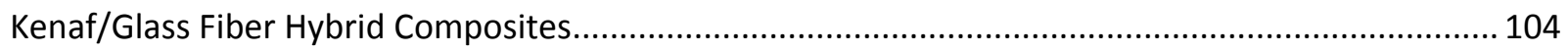

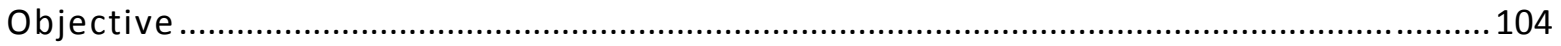

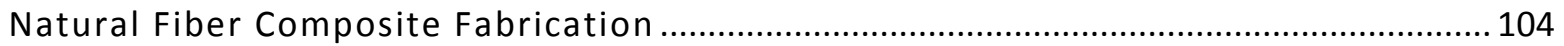

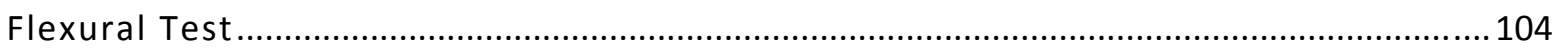

Molecular Dynamic (MD) Modeling of Polymers and Polymer Composite Materials......................... 105

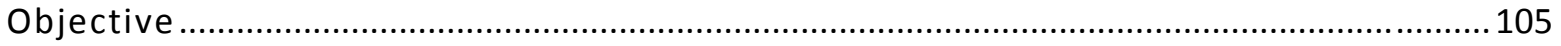

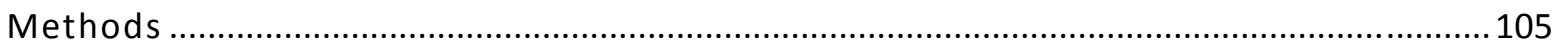

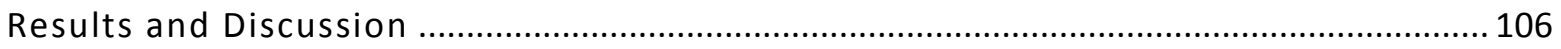

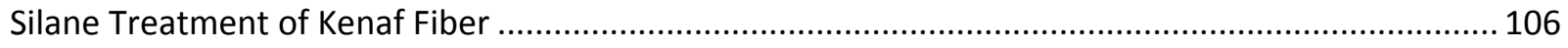

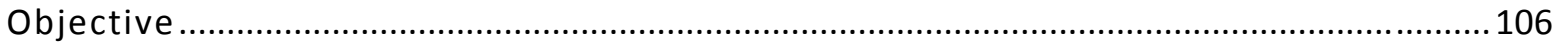




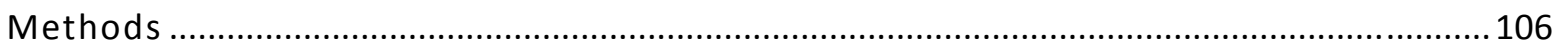

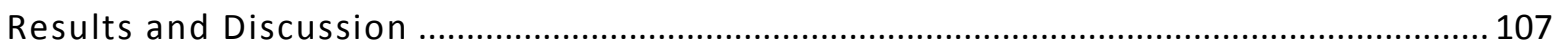

Molecular Dynamics Simulation of Deformation in Amorphous Cellulose ....................................... 107

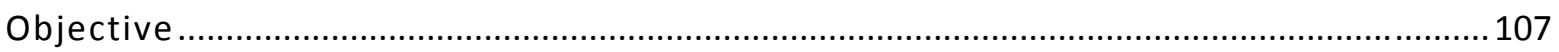

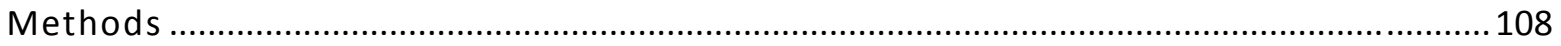

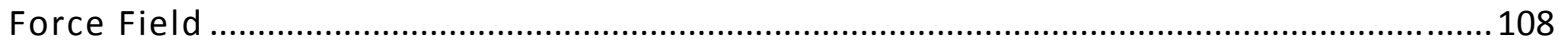

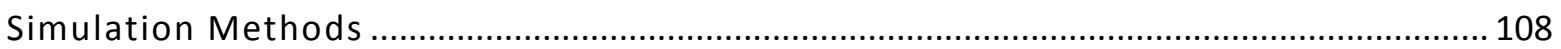

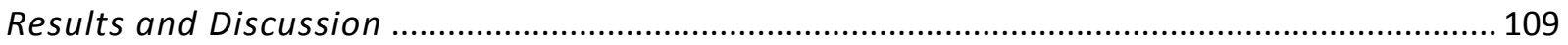

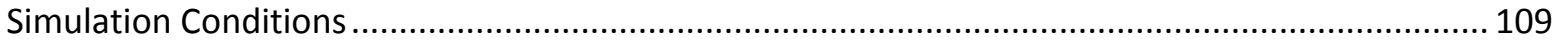

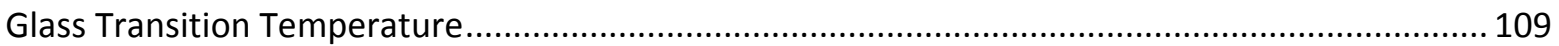

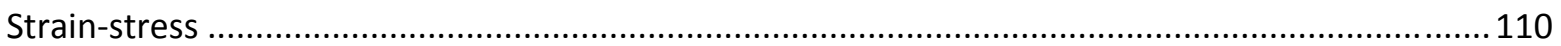

Multiscale Modeling of Moisture Absorption Process in Natural Fiber Composites.............................111

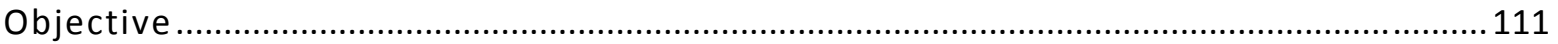

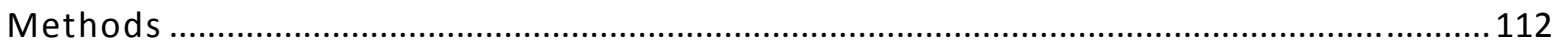

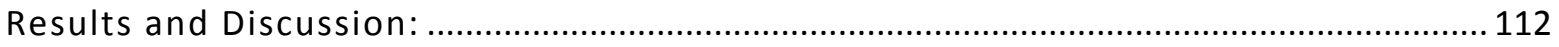

Life-Cycle Assessment of Natural Fiber Polymer Composites ........................................................... 114

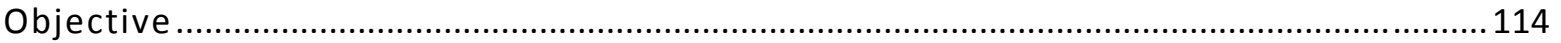

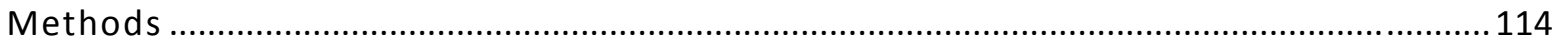

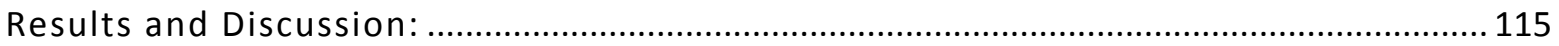

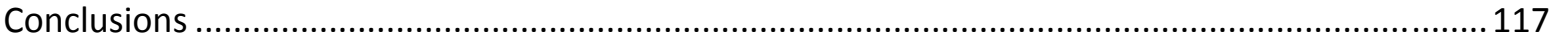

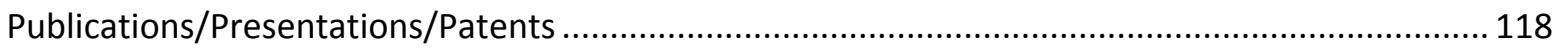

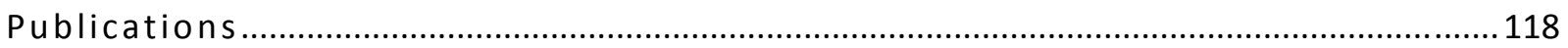

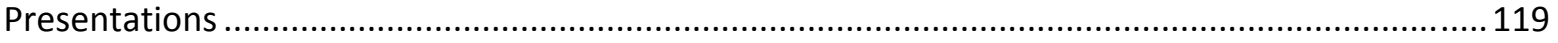

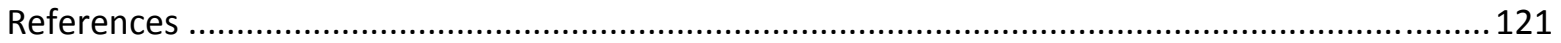

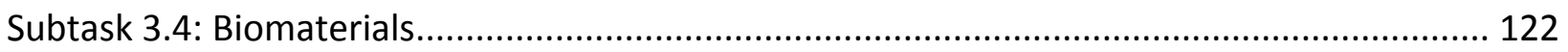

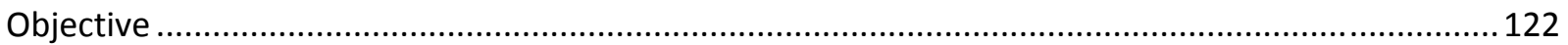

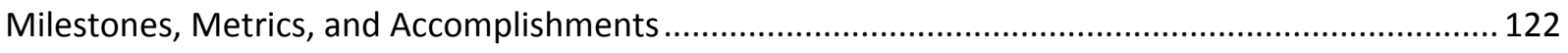

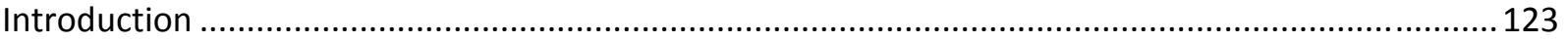

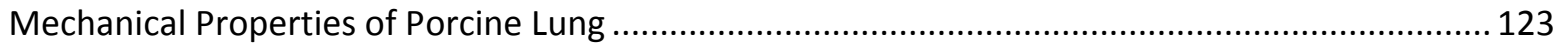

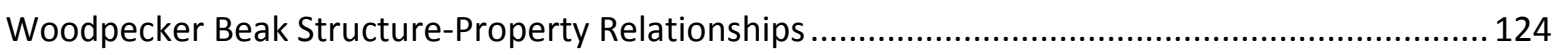

Finite Element Model Development for Multi-Region Porcine Scalp ............................................ 124 


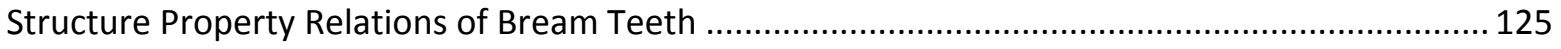

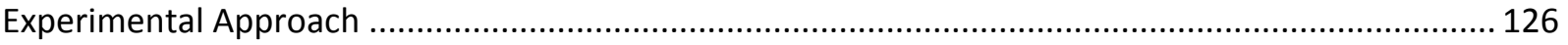

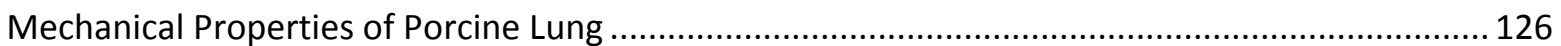

Woodpecker Beak Structure-Property Relationships ................................................................. 128

Finite Element Model Development for Multi-Region Porcine Scalp ............................................. 129

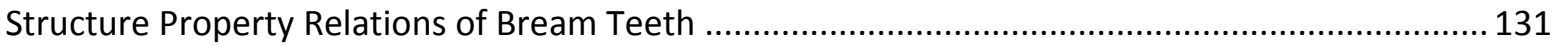

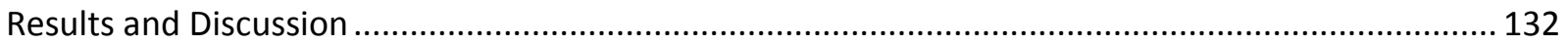

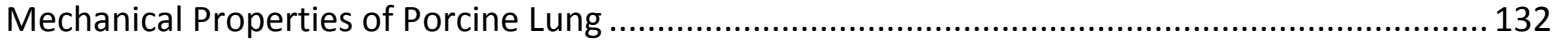

Woodpecker Beak Structure-Property Relationships ................................................................. 135

Finite Element Model Development for Multi-Region Porcine Scalp ............................................. 137

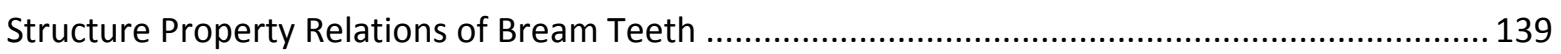

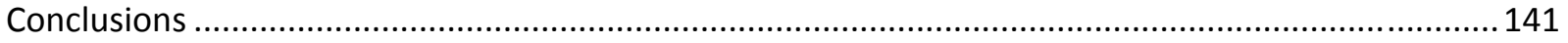

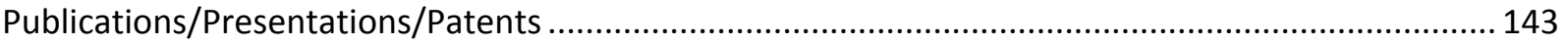

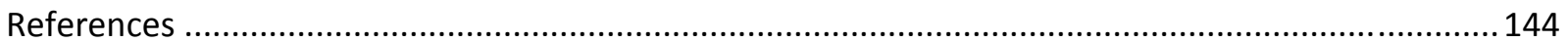


task 3: Multiscale Material Models and Processing design for POLYMERIC MATERIALS

Principal Investigator: Jean-Luc Bouvard Associate Director, CAVS

Mississippi State University (MSST)

200 Research Blvd

Mississippi State, MS 39762

(662) 325-5575; fax: (662) 325-5433; e-mail: jeanluc@,cavs.msstate.edu

Carol Schutte: Technology Area Development Manager

e-mail: carol.schutte@ee.doe.gov

Joseph Renk: Project Officer

email: joseph.renk@netl.doe.gov

Contractor: $\quad$ Mississippi State University (MSST)

Contract No.: $\quad$ DE-FC26-06NT42755

\section{Subtask 3.1: Polymer Resins}

Team Members: J.L. Bouvard, M.A. Tschopp, S. Nouranian, M.F. Horstemeyer, D.Francis

\section{Objective}

- Develop a microstructure based structure-property relationship material model to predict the mechanical behavior of polymers (thermoplastics and thermosets).

\section{APPROACH}

- An ISV material model capturing the structure-property relationships of typical polymeric materials (such as thermoplastics and thermosets) used in the automotive industry has been developed. The material database was built from mechanical characterization tests for different strain rates and temperatures for each selected polymeric material.

- Lower length scale simulations have also been performed as a combination of electronic structure and molecular dynamics simulations to be used for developing the interatomic potentials needed for conducting deformation simulations of a polymer material. The interatomic potential for polymers based on the Modified Embedded-Atom Method (MEAM) has now been developed for saturated hydrocarbons and polymers, such as polyethylene and polypropylene. Molecular dynamics simulations have been used to inform the ISV model on the main mechanisms of chains deformation.

- The research effort performed for the resins will assist in the development of material database and ISV models for composite based materials. 


\section{RESEARCH ACCOMPLISHMENTS}

- We have developed a material database and an internal state variable (ISV) material model that can capture the structure-property relationships of typical polymeric materials used in the automotive industry. The material database was built from mechanical characterization tests for different strain rates and temperatures. We have investigated monotonic loading and then fatigue loading. Microstructural characterization studies were carried out on the deformed specimen to identify failure mechanisms to help in the development of a damage model. Lower length scale simulations were performed as a combination of electronic structure and molecular dynamics simulations to be used for developing the interatomic potentials needed for conducting deformation simulations of polymers. In general, the approach for the material model development has incorporated time and temperature responses and was framed using a large deformation kinematics and thermodynamics framework.

\section{Research Details}

\section{An internal state variable material model for thermoplastics and thermosets}

We proposed to develop a constitutive model that would capture time, temperature, and stress state dependence of the mechanical behavior of thermoplastic and thermoset materials. This section presents the formulation of the one- and three-dimensional models, their calibration procedure and their application to predict the mechanical behavior of thermoplastics and thermosets.

\section{Three Dimensional Model}

The material model was developed using an internal state variable (ISV) formalism considering the kinematics and thermodynamics of large deformation processes (Bouvard et al., 2010; Bouvard et al., 2012). The development follows current methodologies typically used for metals, and departs from the typical spring-dashpot representations widely used when modeling polymeric materials.

Table 1 gives a summary of the three-dimensional model.

Table 1: Three-dimensional model summary.

Cauchy Stress
$\overline{\boldsymbol{\sigma}}=\mathrm{J}^{\mathrm{e}-1} \boldsymbol{\tau}=\mathrm{J}^{\mathrm{e}-1} \mathbf{R}^{\mathrm{e}} \overline{\mathbf{M}} \mathbf{R}^{\mathrm{eT}}$
$\overline{\mathbf{M}}=2 \mu(\theta, \dot{\varepsilon}) \overline{\mathbf{E}}^{\mathrm{e}}+\left(\mathrm{K}(\theta, \dot{\varepsilon})-\frac{2}{3} \mu(\theta, \dot{\varepsilon})\right) \operatorname{Tr}\left(\overline{\mathbf{E}}^{\mathrm{e}}\right) \overline{\mathbf{I}}$
$\mu(\theta, \dot{\varepsilon})=\frac{\mathrm{E}(\theta, \dot{\varepsilon})}{(2+2 \mathrm{v})}, \mathrm{K}(\theta, \dot{\varepsilon})=\frac{2 \mu(\theta, \dot{\varepsilon})(1+\mathrm{v})}{3(1-2 \mathrm{v})}$




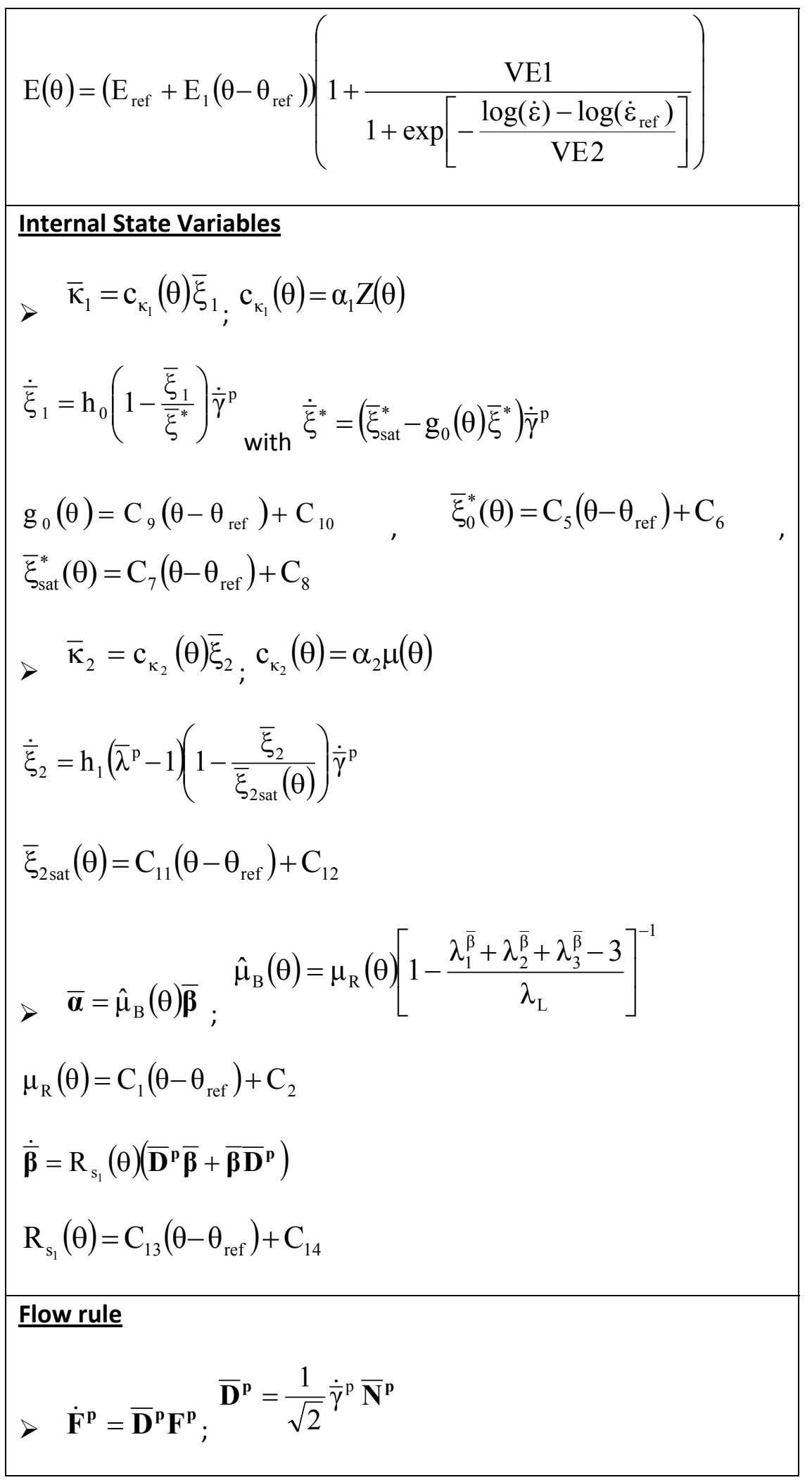




\begin{tabular}{|l|}
$\dot{\bar{\gamma}}^{\mathrm{p}}=\dot{\bar{\gamma}}_{0}^{\mathrm{p}} \mathrm{e}^{\left[-\frac{\Delta \mathrm{H}_{\beta}}{\mathrm{R} \theta}\right]}\left[\sinh \left(\frac{\bar{\tau}_{\mathrm{eq}} \mathrm{V}}{2 \mathrm{k}_{\mathrm{B}} \theta}\right)\right]^{\mathrm{m}}$ \\
$\bar{\tau}=1 / \sqrt{2}\|\overline{\mathbf{D E V}}(\overline{\mathbf{M}}-\bar{\alpha})\| ; \bar{\tau}_{\mathrm{eq}}=\bar{\tau}-\left(\mathrm{Y}(\theta)+\bar{\kappa}_{1}+\bar{\kappa}_{2}+\alpha_{\mathrm{p}} \overline{\bar{\pi}}\right)$ \\
$\mathrm{Y}(\theta)=\mathrm{C}_{3}\left(\theta-\theta_{\text {ref }}\right)+\mathrm{C}_{4}$ \\
$\underline{\text { Heat generation }}$ \\
$>\overline{\mathrm{C}}_{\mathrm{v}} \dot{\theta}=\omega \overline{\mathbf{M}}: \overline{\mathbf{D}}^{\mathrm{p}}$
\end{tabular}

The three dimensional equations have been implemented in the finite element software ABAQUS/Explicit in the form of a user material subroutine.

\section{One Dimensional Model}

Table 2 gives a summary of the one-dimensional model. The one-dimensional model is used to fit the model parameters at different strain rates and temperatures regarding uniaxial loaging conditions.

Table 2: One-dimensional model summary.

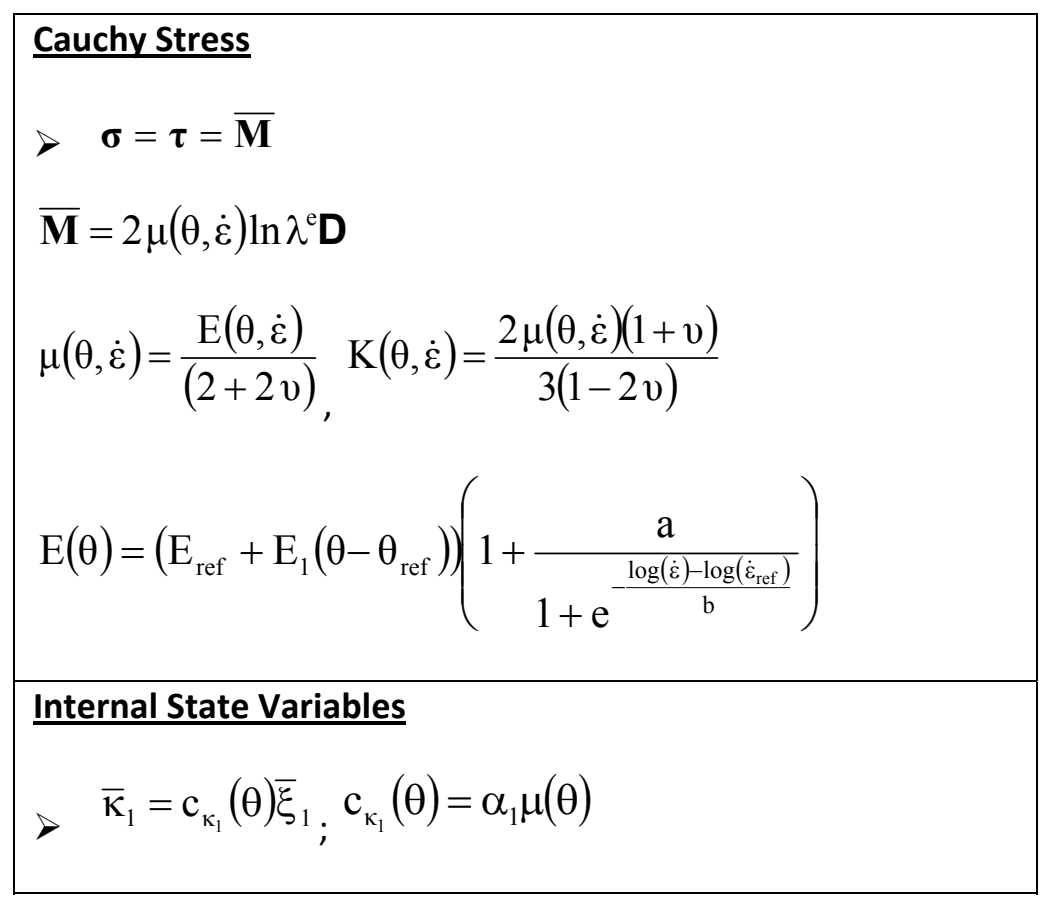




$$
\begin{aligned}
& \dot{\xi}_{1}=h_{0}\left(1-\frac{\bar{\xi}_{1}}{\bar{\xi}^{*}}\right) \dot{\bar{\gamma}}_{\text {with }}^{p} \dot{\xi}^{*}=\left(\bar{\xi}_{\text {sat }}^{*}(\theta)-g_{0}(\theta) \bar{\xi}^{*}\right) \dot{\bar{\gamma}}^{\mathrm{p}} \\
& \mathrm{g}_{0}(\theta)=\mathrm{C}_{9}\left(\theta-\theta_{\text {ref }}\right)+\mathrm{C}_{10} \text { and } \bar{\xi}_{0}^{*}(\theta)=\mathrm{C}_{5}\left(\theta-\theta_{\text {ref }}\right)+\mathrm{C}_{6} \\
& \bar{\xi}_{\text {sat }}^{*}(\theta)=C_{7}\left(\theta-\theta_{\text {ref }}\right)+C_{8} \\
& >\bar{\kappa}_{2}=\mathrm{c}_{\kappa_{2}}(\theta) \bar{\xi}_{2} ; \mathrm{c}_{\kappa_{2}}(\theta)=\alpha_{2} \mu(\theta) \\
& \dot{\bar{\xi}}_{2}=\mathrm{h}_{1}\left(\bar{\lambda}^{\mathrm{p}}-1\right)\left(1-\frac{\bar{\xi}_{2}}{\bar{\xi}_{2 \text { sat }}(\theta)}\right) \dot{\bar{\gamma}}^{\mathrm{p}} ; \bar{\xi}_{2 \text { sat }}(\theta)=\mathrm{C}_{11}\left(\theta-\theta_{\text {ref }}\right)+\mathrm{C}_{12} \\
& >\overline{\boldsymbol{\alpha}}=\hat{\mu}_{\mathrm{B}}(\theta) \overline{\boldsymbol{\beta}} ; \hat{\mu}_{\mathrm{B}}(\theta)=\mu_{\mathrm{R}}(\theta)\left[1-\frac{\lambda_{1}^{\bar{\beta}}+\lambda_{2}^{\bar{\beta}}+\lambda_{3}^{\bar{\beta}}-3}{\lambda_{L}}\right]^{-1} \\
& \mu_{\mathrm{R}}(\theta)=\mathrm{C}_{1}\left(\theta-\theta_{\text {ref }}\right)+\mathrm{C}_{2} \\
& \dot{\overline{\boldsymbol{\beta}}}=2 \mathrm{R}_{\mathrm{s}_{1}}(\theta) \overline{\boldsymbol{\beta}}^{\mathbf{p}} \\
& \mathrm{R}_{\mathrm{s}_{1}}(\theta)=\mathrm{C}_{13}\left(\theta-\theta_{\text {ref }}\right)+\mathrm{C}_{14}
\end{aligned}
$$

\section{Flow rule}

$$
\begin{aligned}
& >\dot{\mathbf{F}}^{\mathrm{p}} \mathbf{F}^{\mathbf{p}-\mathbf{1}}=\overline{\mathbf{D}}^{\mathrm{p}}=\frac{\dot{\lambda}^{\mathrm{p}}}{\lambda^{\mathrm{p}}} \mathrm{D} \quad \overline{\mathbf{D}}^{\mathrm{p}}=\frac{1}{\sqrt{3}} \dot{\bar{\gamma}}^{\mathrm{p}} \operatorname{sign}(\operatorname{dev}(\overline{\mathbf{M}}-\overline{\boldsymbol{\alpha}})) \mathrm{D} \\
& \dot{\bar{\gamma}}^{\mathrm{p}}=\dot{\bar{\gamma}}_{0}^{\mathrm{p}} \mathrm{e}^{\left[-\frac{\Delta \mathrm{H}_{\beta}}{\mathrm{R} \theta}\right]}\left[\sinh \left(\frac{\bar{\tau}_{\mathrm{eq}} \mathrm{V}}{2 \mathrm{k}_{\mathrm{B}} \theta}\right)\right]^{\mathrm{m}} \\
& \bar{\tau}=\frac{\sqrt{3}}{2}|\operatorname{dev}(\overline{\mathbf{M}}-\overline{\boldsymbol{\alpha}})| ; \bar{\tau}_{\mathrm{eq}}=\bar{\tau}-\left(\mathrm{Y}(\theta)+\bar{\kappa}_{1}+\bar{\kappa}_{2}+\alpha_{\mathrm{p}} \bar{\pi}\right) \\
& \mathrm{Y}(\theta)=\mathrm{C}_{3}\left(\theta-\theta_{\text {ref }}\right)+\mathrm{C}_{4}
\end{aligned}
$$

In order to get a better agreement between the one-dimensional model predictions with the three-dimensional material model as the material is not always incompressible, some of the parameters 
need to be converted from the one-dimensional to the three-dimensional problems in the flow rule such as

$\dot{\bar{\gamma}}_{\mathrm{p} 3 \mathrm{D}}^{0}=\sqrt{3} \dot{\bar{\gamma}}_{\mathrm{p} 1 \mathrm{D}}^{0}$ and $\alpha_{\mathrm{p}}^{3 \mathrm{D}}=\frac{\alpha_{\mathrm{p}}^{1 \mathrm{D}}}{\sqrt{3}}$

Figure 1 displays a comparison between the predictions of the one-dimensional model implemented in MATLAB and the three-dimensional equations implemented in ABAQUS/Explicit for different temperatures that illustrate the numerical verification process. The good agreement between the two predictions confirms the consistency of the implemented equations.

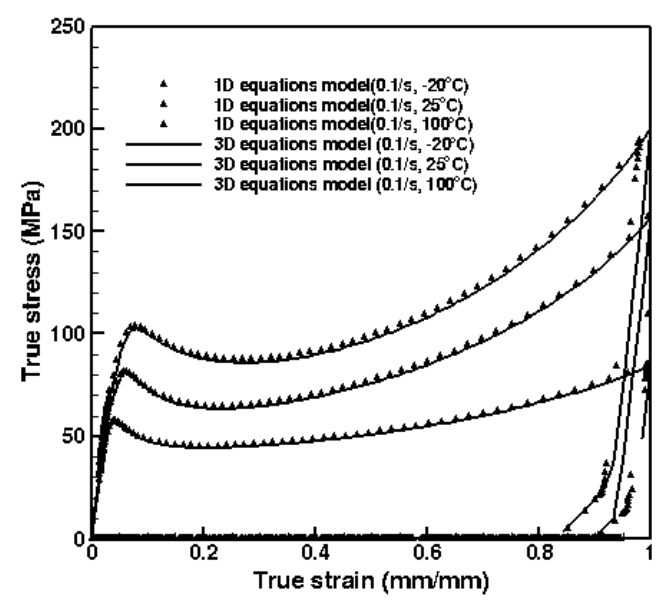

Figure 1: Comparison of the one dimensional model results in MATLAB and the constitutive model implemented in ABAQUS.

\section{Parameters Identification}

The one-dimensional equations have been implemented in MATLAB in order to calibrate the model parameters. Three main functions have been used. The function "Isqnonlin" is used for the minimization of the least squares expression using the trust region method in combination with the Newton method. The function "odel5s" is a multistep solver for ODEs that integrates the nonlinear onedimensional evolution equations for the state variables of the model. The last function, "interpl", interpolates the computed response to obtain the stress level at the corresponding experimental strain value.

First, the model is calibrated for $\mathrm{T}=\mathrm{T}_{\text {ref }}$ on two compression tests performed at different strain rates following the steps described below:

1) Elastic regime $\left.\left(\mathrm{E}_{\text {ref }}, \mathrm{VE} 1, \mathrm{VE} 2, \dot{\varepsilon}_{\text {ref }}, \mathrm{v}\right\}\right)$ : the elastic modulus $\mathrm{E}_{\text {ref }}$ is identified from mechanical tests at $\mathrm{T}=\mathrm{T}_{\text {ref. }}$. The set of parameters $\left\{\mathrm{VE} 1, \mathrm{VE} 2, \dot{\varepsilon}_{\text {ref }}\right\}$ is fitted on the elastic modulus obtained from compression tests at different strain rates (from quasi-static to high strain rates if available) as shown Figure 2. The value of the Poisson's ratio $v$ is chosen from the literature. 


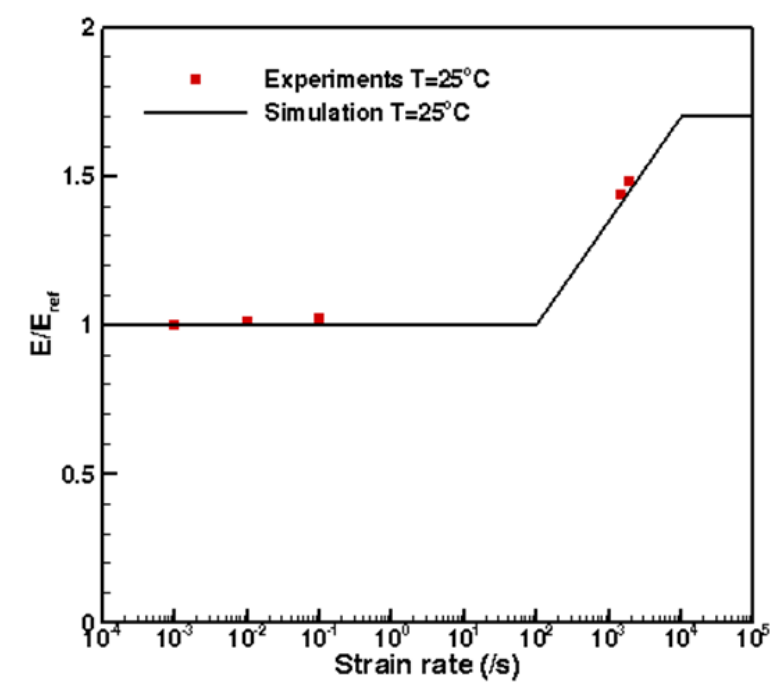

Figure 2: Dependence of a normalized Young Modulus on strain rate.

2) Time dependence $\left(\left\{\dot{\gamma}_{0}^{\mathrm{p}}, \Delta \mathrm{H}_{\beta}, \mathrm{V}, \mathrm{m}, \mathrm{C}_{4}\right\}\right)$ : This set of parameters determined the time dependence of the material. The value of $\Delta \mathrm{H}_{\beta}$ can be determined from the literature or by using the shifting and superposition methods developed by Richeton, et al. $(2006,2007)$ to fit their model parameters. The value of $\mathrm{Y}_{\text {ref }}$ characterizing the limit between the elastic regime and the first events of non-linear behavior of the polymer prior the yield stress is determined from the compression stress-stress curves at $\mathrm{T}=\mathrm{T}_{\text {ref, }}$ as shown in Figure 3a. Noting that in a uniaxial compression test, the equivalent shear stress and strain are related to the uniaxial stress and strain by $\boldsymbol{\tau}=\boldsymbol{\sigma} / \sqrt{3}$ and $\gamma=\sqrt{3} \boldsymbol{\varepsilon}$, the value of $\mathrm{C}_{4}^{(3 \mathrm{D})}$ is given $\mathrm{C}_{4}^{(3 \mathrm{D})}=\mathrm{Y}_{\text {ref }} / \sqrt{3}$.

Then, a fitting method was used in MATLAB to fit the parameters $\left\{\dot{\gamma}_{0}^{\mathrm{p}(1 \mathrm{D})}, \mathrm{V}^{(\mathrm{DD})}, \mathrm{m}\right\}$ from the stress yield points' data as shown in Figure 3b. The parameters $\left\{\dot{\gamma}_{0}^{\mathrm{p}(1 \mathrm{D})}, \mathrm{V}^{(1 \mathrm{D})}\right\}$ can be converted to the threedimensional problem using

$$
\dot{\gamma}_{0}^{\mathrm{p}(3 \mathrm{D})}=\sqrt{3} \dot{\gamma}_{0}^{\mathrm{p}(1 \mathrm{D})} ; \mathrm{V}^{(3 \mathrm{D})}=\sqrt{3} \mathrm{~V}^{(1 \mathrm{D})}
$$




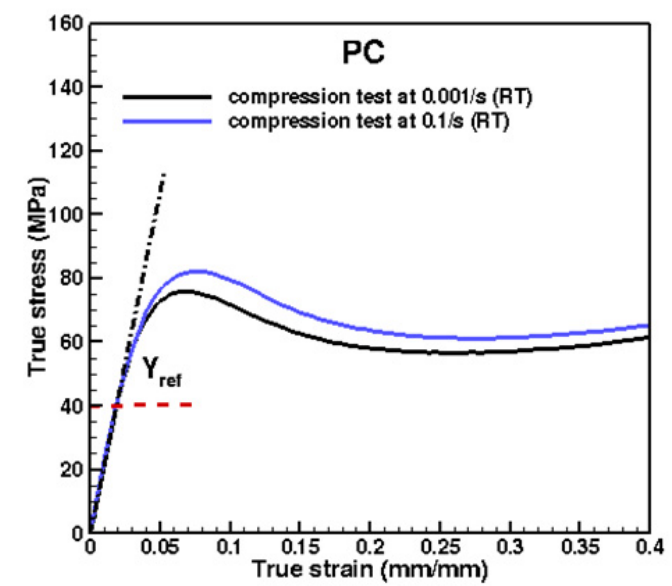

(a)

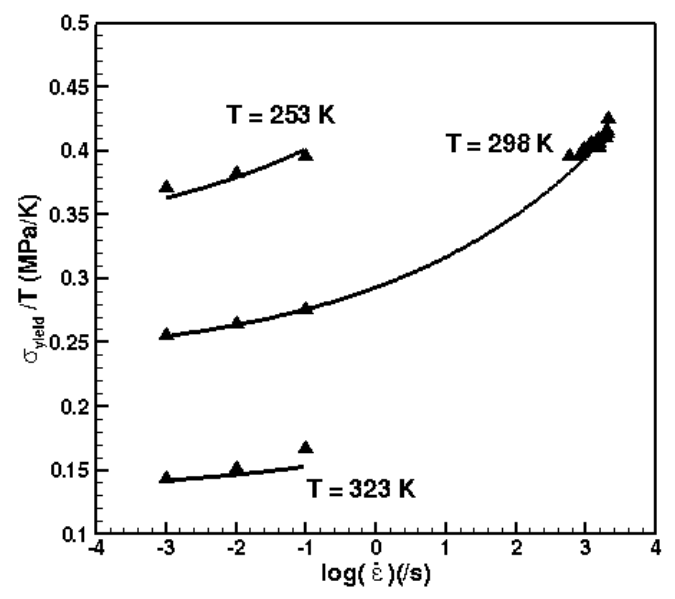

(b)

Figure 3: Determination of: a) $Y_{\text {ref, }}$ characterizing the limit between the elastic regime and the first events of non-linear behavior of the polymer prior the yield stress; b) $\left\{\dot{\gamma}_{0}^{\mathrm{p}(1 \mathrm{D})}, \mathrm{V}^{(\mathrm{DD})}, \mathrm{m}\right\}$

3) Strain hardening $\left(\left\{\mathrm{C}_{2}, \lambda_{\mathrm{L}}, \mathrm{C}_{14}\right\}\right)$ : the strain hardening at large strain levels is fitted using this set of parameters as depicted in Figure 4a.

4) Strain softening $\left(\left\{\mathrm{C}_{6}, \mathrm{C}_{8}, \bar{\xi}_{10}, \mathrm{~h}_{0}, \mathrm{C}_{10}, \mathrm{C}_{\mathrm{k} 1}\right\}\right)$ : these parameters are fitted in order to capture the nonlinear yield peak. The parameter $\left\{\bar{\xi}_{10}\right\}$ is assumed to be fixed as $\bar{\xi}_{10}=0$. The stress amplitude is fitted using $\left\{\bar{\xi}_{0}^{*}, \mathrm{C}_{\mathrm{k} 1}\right\}$. Then, $\left\{\bar{\xi}_{\text {sat }}^{*}, \mathrm{~h}_{0}, \mathrm{~g}_{0}\right\}$ are fitted to capture the nonlinear yield peak as observed in Figure 4b.

5) Identification of $\left\{\mathrm{h}_{1}, \mathrm{C}_{12}, \mathrm{C}_{\mathrm{k} 2}\right\}$ : this set of parameters captures the Bauschinger effect observed during the unloading path (Figure 4c).

6) Identification of $\alpha_{\mathrm{p}}$ : The value of the pressure sensitivity parameter is chosen from the literature or can be fitted on tensile tests in order to capture the correct yield stress amplitude. 


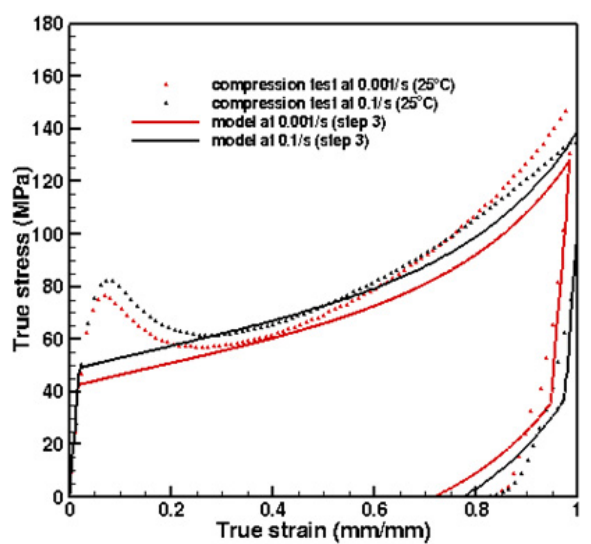

(a)

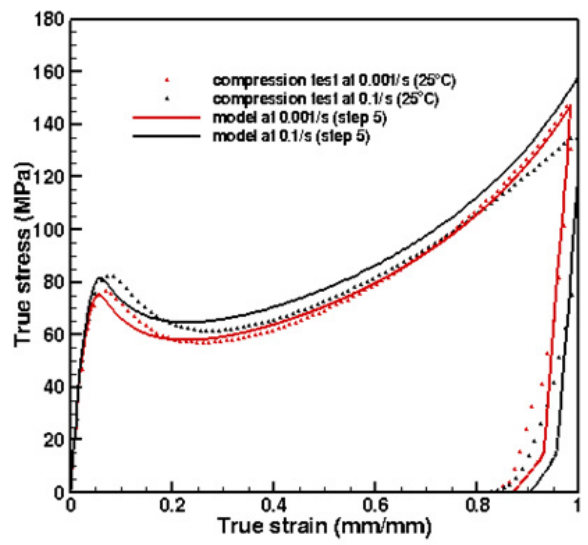

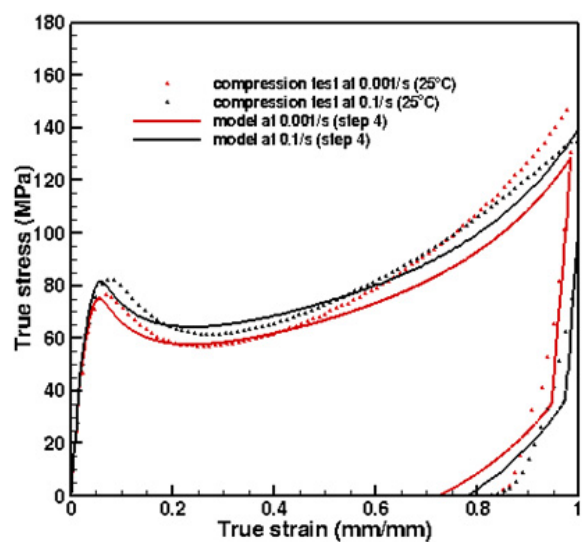

(b)

(c)

Figure 4: Description of the different steps to fit the material parameters: (a) capturing the strain hardening, (b) capturing the softening regime, and (d) capturing the unloading of the Bauschinger effect.

The model is then calibrated for different temperatures following the steps described below:

7) Elastic regime $\left(\left\{E_{1}\right\}\right)$ : this parameter is fitted from mechanical tests.

8) Time dependence $\left(\mathrm{C}_{3}\right\}$ ): this parameter is fitted to capture the time dependence of the material.

9) Strain hardening $\left.\left(\mathrm{C}_{1}, \mathrm{C}_{13}\right\}\right)$ : the strain hardening at large strain levels is fitted using this set of parameters. 
10) Strain softening $\left.\left(\mathrm{C}_{5}, \mathrm{C}_{7}, \mathrm{C}_{9}\right\}\right)$ : these parameters are fitted in order to capture the nonlinear yield peak.

11) Identification of $\left\{\mathrm{C}_{11}\right\}$ : this parameter captures the Bauschinger effect observed during the unloading path.

A parametric (sensitivity) study of the constitutive model was carried out in Bouvard, et al. (2010) to understand the salient features of the stress-strain behavior that are affected when changing the values of the material parameters.

\section{Application to Thermoplastics}

The developed constitutive model was applied to PC under compression, tension, creep, and torsion at different temperatures and strain rates (Bouvard, et al., 2012).

\section{Compression}

The graphs in Figure 5 show the comparison between the model calibrations and the experimental data for uniaxial compression at different temperatures and strain rates for polycarbonate (PC). As noted from the figure, the model agrees reasonably well with the experimental data by capturing the time and temperature dependence observed in the material behavior. Note that the model captured very well the specific features of the material response: the elastic regime followed by the nonlinear transition to yield, the yield peak, the subsequent material softening, and the final hardening response. Both loading/unloading paths are predicted fairly well by the constitutive equations as well.
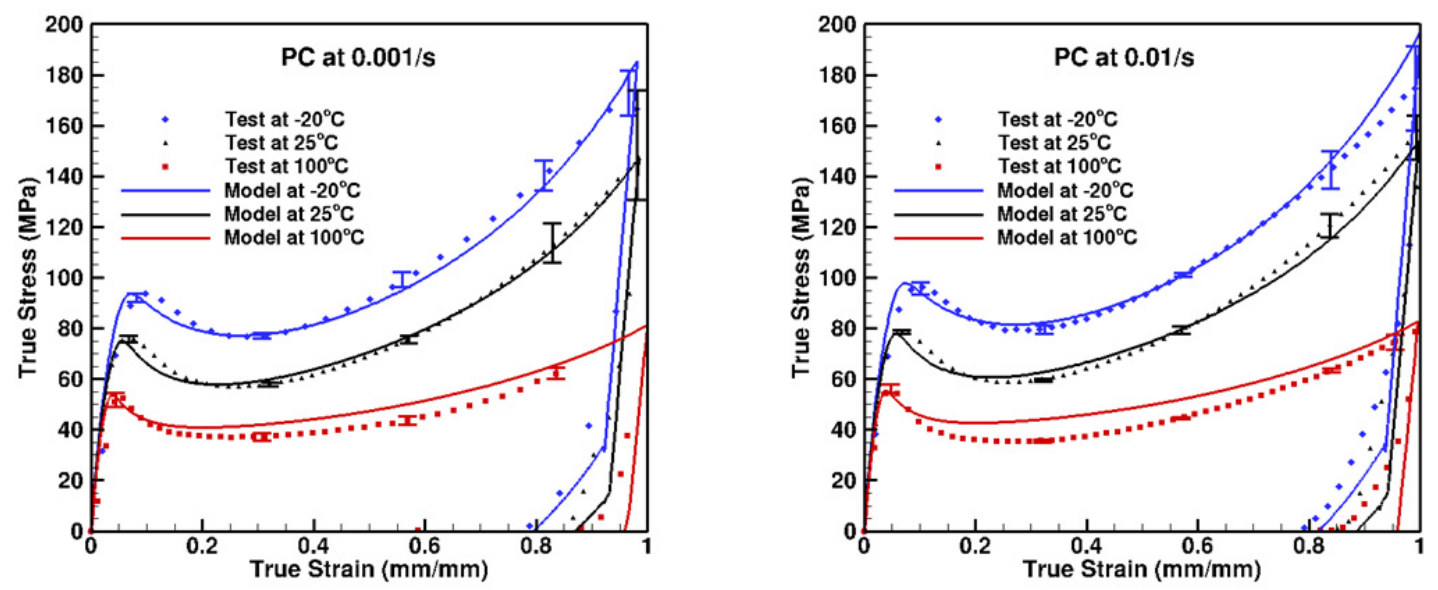


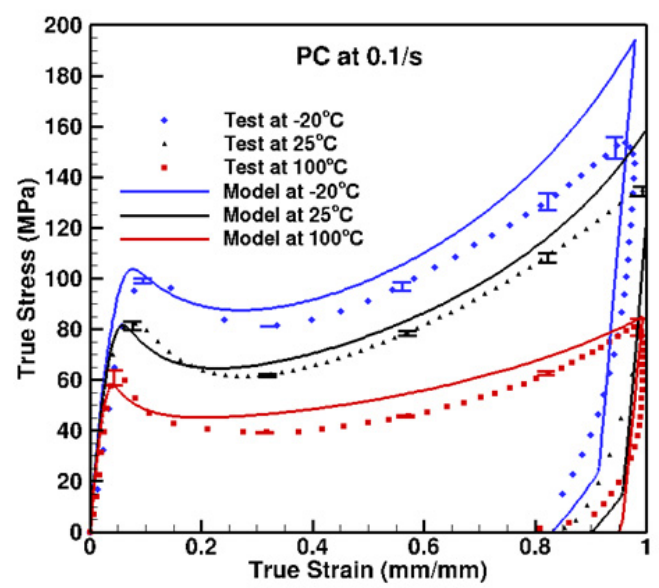

Figure 5: Comparison between predictions of the model and experimental for uniaxial compression of polycarbonate $(P C)$ at different strain rates and temperatures.

\section{Creep}

The compressive creep testing consisted of loading a cylindrical compression specimen at a strain rate of $0.001 / \mathrm{s}$ at room temperature to a selected stress level and maintaining the prescribed load for 800 seconds. An extensometer attached to the compression platens was used to measure the local compressive strain. The stress levels were chosen to be equal to approximately $40 \%$ (30 MPa) and $85 \%$ (70 MPa) of the measured yield stress of PC. Figure 6 shows the comparison between the model predictions and the experimental data. As noted from the figure, the material response exhibits primary and secondary regimes for stress levels chosen below the material yield peak. The model predicts reasonably well the creep strain evolution for a stress level chosen at $86 \%$ of the measured yield stress of PC. However, the model tends to underestimate the creep strain for a stress level of $30 \%$ of the PC measured yield stress.

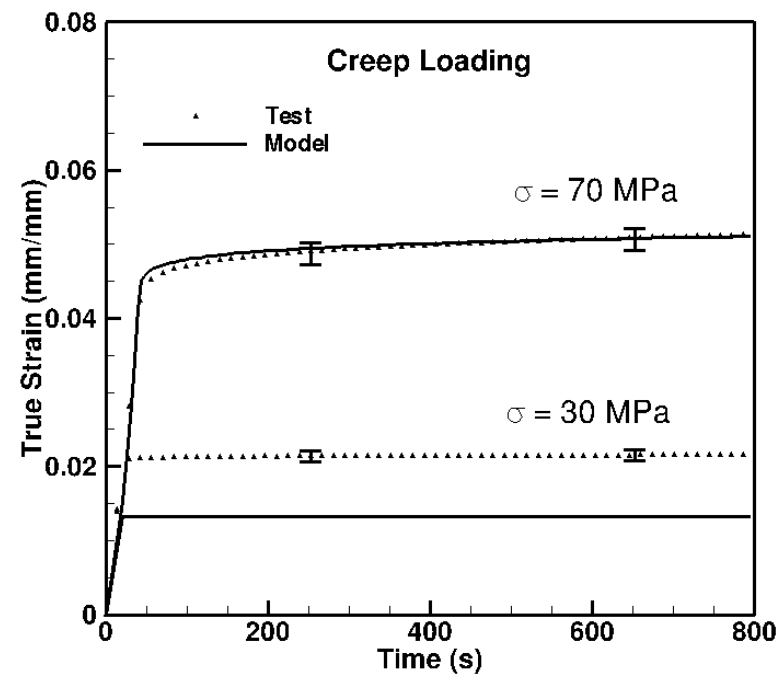


Figure 6: Comparison between predictions of the model and experimental test data for compression creep response of polycarbonate at two different engineering stress levels: 30 and $70 \mathrm{MPa}$.

\section{Torsion}

Finite element analysis was carried out and compared to torsional tests performed at different angular velocities at room temperature in order to investigate the model prediction for different stress states. Figure 7 shows good agreement between the model prediction and the experimental tests for different angular velocities at room temperature. The model predicts reasonably well the elastic and plastic regimes of the material response as well as the time dependence.

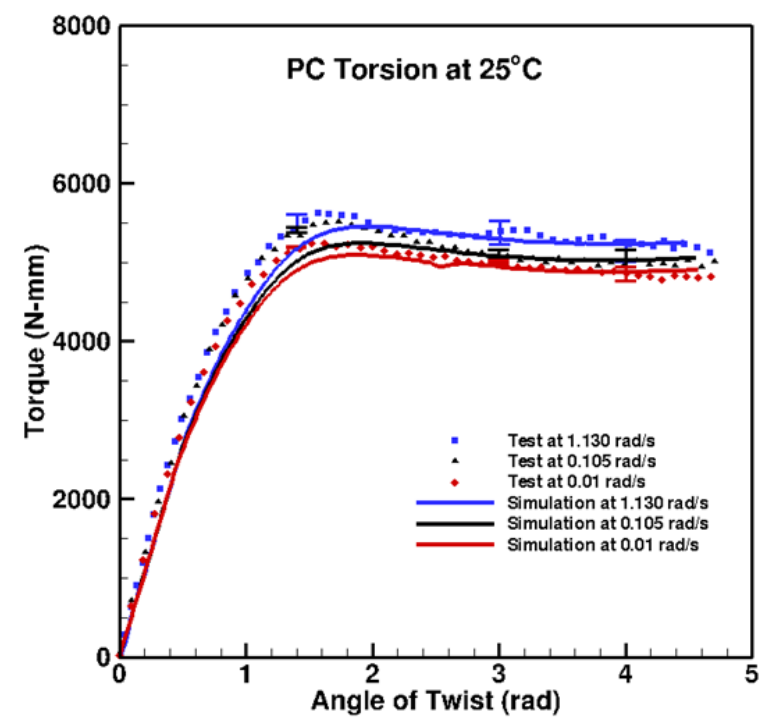

Figure 7: Torque versus angle of twist response of polycarbonate (PC) for torsional tests and numerical simulations carried out at room temperature at three different angular velocities: $1.130 \mathrm{rad} / \mathrm{s}, 0.105$ $\mathrm{rad} / \mathrm{s}$, and $0.01 \mathrm{rad} / \mathrm{s}$.

\section{$\underline{\text { Tension }}$}

In this section, the response of the model is compared with tensile tests performed at different strain rates and temperatures. Figure 8 displays the geometry and dimensions of the problem. Necking is initiated by including a small geometrical defect in the specimen. Following Boyce, et al. (1992), this defect was introduced by decreasing the length $W_{d}$ with $W_{d}=\alpha W_{0}$ and $\alpha=0.99$. Due to the symmetries of the problem, a quarter of the specimen is modeled with symmetry boundary conditions. The mesh is composed of eight nodes reduced integration elements. One end of the tensile specimen is fixed in all three directions, while on the other end the specimen is deformed following $y$-direction. 


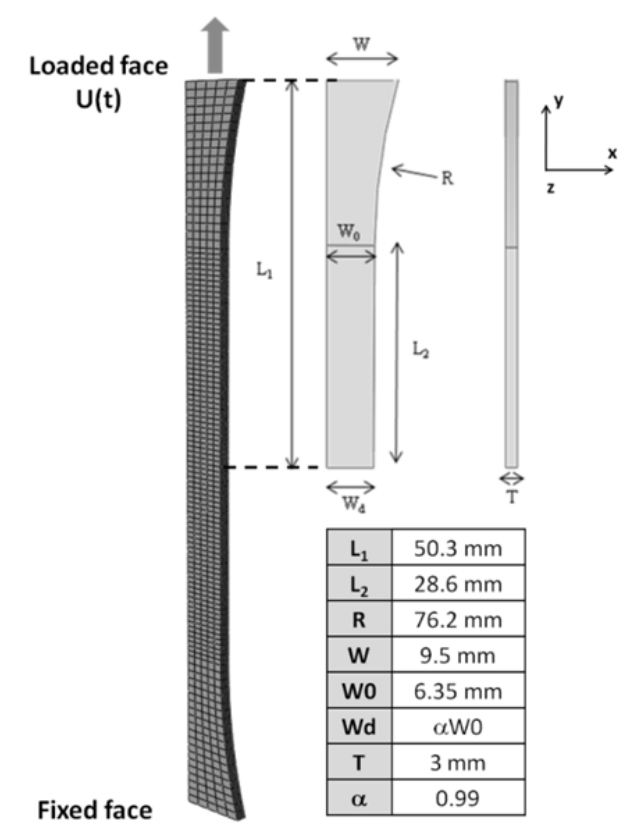

Figure 8: Mesh, geometry and dimensions of the tensile specimen subjected to extension in the $y$ direction.

Figure 9 displays a comparison between the material model prediction and the experimental test data for uniaxial tension performed at different strain rates $(0.1 / \mathrm{s}, 0.01 / \mathrm{s}$, and $0.001 / \mathrm{s})$ and temperatures $\left(-20^{\circ} \mathrm{C}, 25^{\circ} \mathrm{C}\right.$, and $\left.100^{\circ} \mathrm{C}\right)$. The agreement between the material model response and the test data is quite good, as the model predicts the global force-displacement curve of the material as well as the yield peak levels for different strain rates and temperatures. However, it is interesting to note that the model overestimates the elastic modulus in tension even though it was able to predict with good agreement the elastic properties of the material under compression and torsional loading conditions. These results may suggest that the model should be refined to account accurately for the stress-state dependence of the elastic regime. 


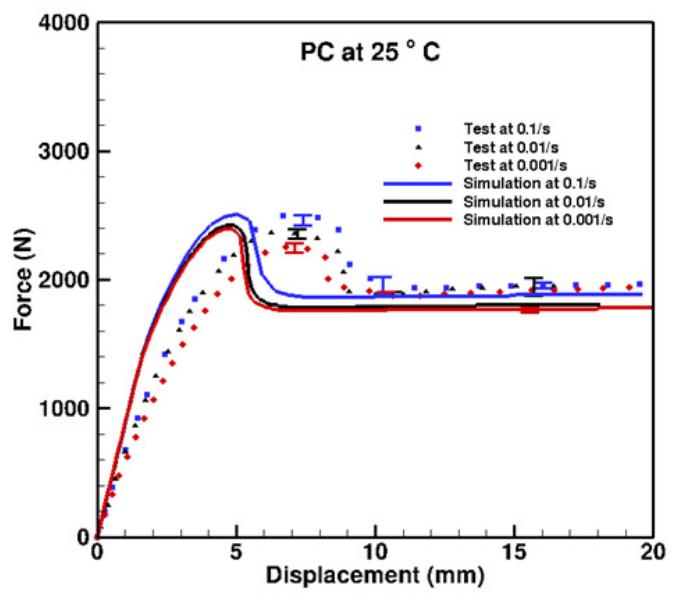

(a)

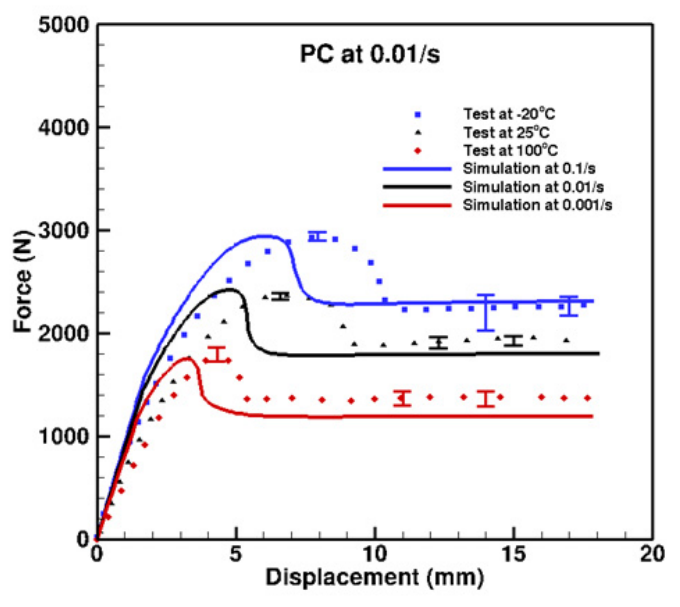

(b)

Figure 9: Comparison between predictions of the model and experimental of polycarbonate (PC) for: (a) uniaxial tension at $25^{\circ} \mathrm{C}$ and different strain rates $(0.1 / \mathrm{s}, 0.01 / \mathrm{s}$, and $0.001 / \mathrm{s})$; (b) uniaxial tension at $0.01 / \mathrm{s}$ and different temperatures $\left(-20^{\circ} \mathrm{C}, 25^{\circ} \mathrm{C}\right.$, and $\left.100^{\circ} \mathrm{C}\right)$.

\section{Split Hopkinson Pressure Bar Testing and Simulation}

High strain rate testing was completed using an aluminum Split Hopkinson Pressure Bar (SHPB). The dimensions of the setup, shown in Figure 10, were identical for both the experimental testing and the finite element simulations. All three bars were cut from 7075 aluminum and were modeled as linearly elastic material in the simulation. The specimen diameter was one-third of the diameter of the SHPB rods. The large difference in diameters was chosen because of the large strains the specimens experience. During the loading, the deformed specimen's diameter must not exceed the diameter of the transmitted and incident bar. Quarter symmetry was used in the simulation along with three dimensional hexahedral elements. The striker was given an initial velocity and, prior to impacting the incident bar, the velocity was removed, leaving the striker bar moving at the same velocity up until impact. 


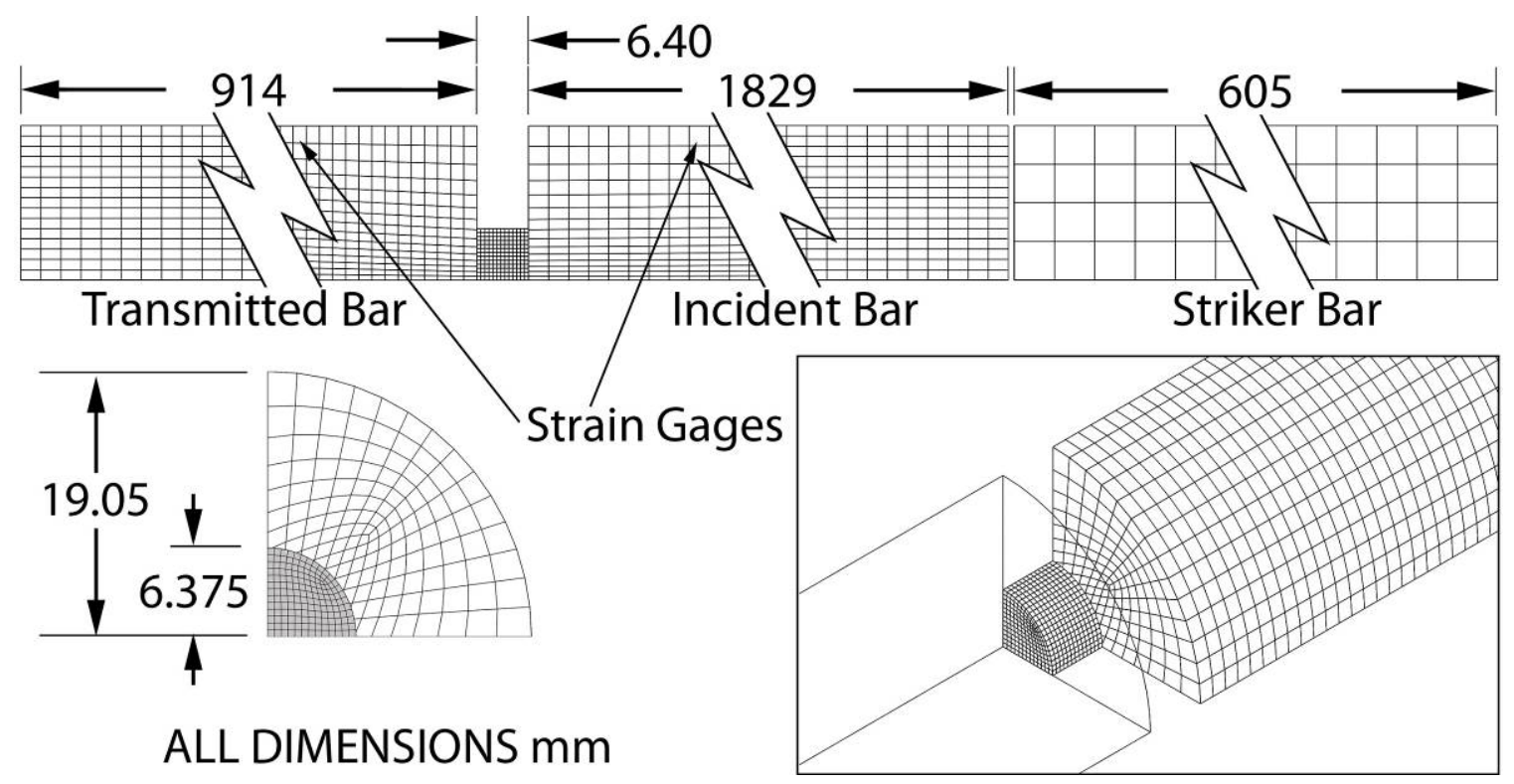

Figure 10: Layout of the Compression SHPB experiment and simulation (with mesh).

Two strain gages, one on both the incident and transmitted bars, recorded the strain in both bars during the entirety of a SHPB experiment. The strains were recorded in the same locations in the simulations and compared to the experiment. As shown in Figure 11, good agreement was observed between the experiment and the model for the transmitted waves. Notice that the reflective waves are also captured by the model.

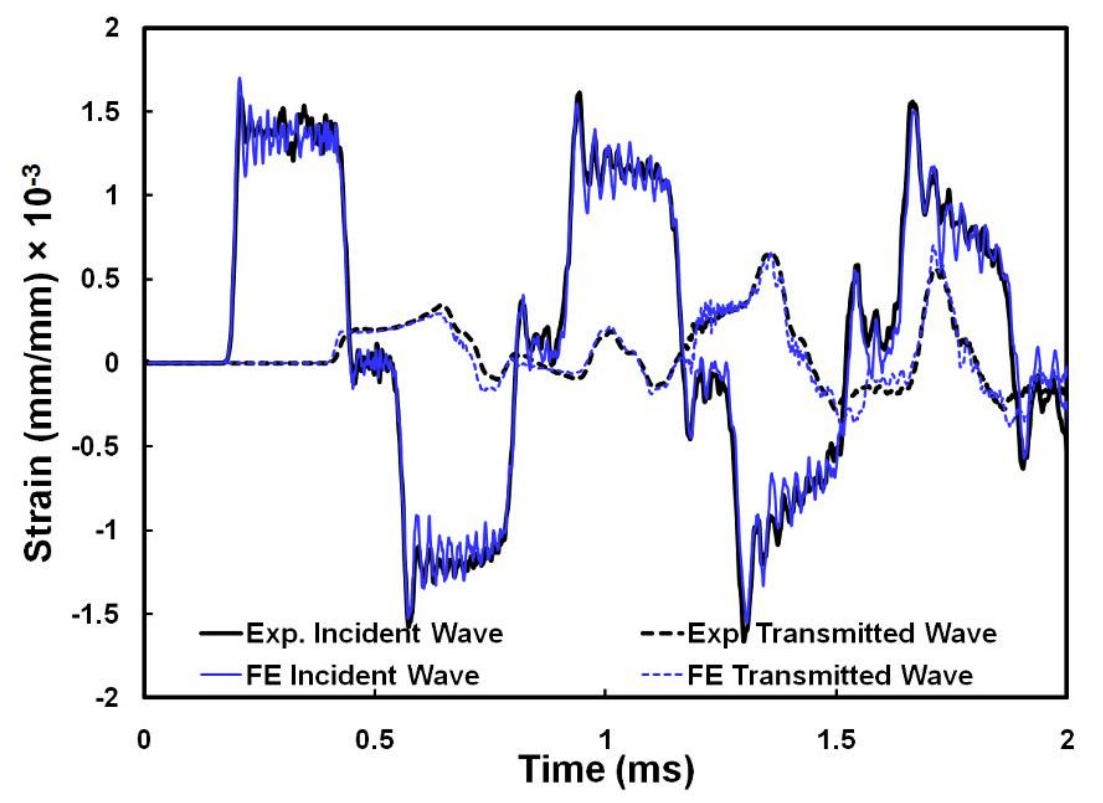

Figure 11: Comparison of incident and transmitted strain measurements in a SHPB for an experiment (Exp.) and a Finite Element (FE) simulation. 
The stress, strain, and temperature (due to adiabatic heating) were averaged over the specimen and then compared to the average of the centerline of the specimen, where uniaxial deformation is assumed to occur. To compare the FE simulation of the specimen under deformation with a simulation exhibiting an homogeneous stress state condition, a one element simulation in compression under a constant engineering strain rate (the specimen in both the experiment and the FE simulation experience a constant strain rate of $1800 / \mathrm{s}$ ) was also performed.

The comparison of the stress-strain responses between experiment, FEA of the SHPB, and one element FEA are displayed in Figure 12a while Figure 12b shows the temperature rise in the specimen for the different simulations investigated. First, Figure 12a illustrates that the stress-strain response from the FE sample complete average coincides with the FE sample center line average of Sig33. Close agreement is also found between the FEA results from the specimen and the one element, which reveals that homogenous stress state can be assumed in the simulations and therefore in the corresponding SHPB experiments. When comparing the FE simulation and the experiment in Figure 12a, some differences can be observed. The origin of these differences can be related to i) uncertainty observed in the experimental results, which has not been introduced in the model; and ii) the friction between the specimen and the bars, which were not accounted for in the analysis and may have an effect on the material strain hardening. A close observation of Figure 12b shows that the one element simulation displays an important temperature rise that does not occur in the SHPB FE simulation, which may indicate an induced decrease of the strain hardening as displayed by the one element simulation in Figure 12a. The increase of temperature in the specimen due to adiabatic heating (about $35^{\circ} \mathrm{C}$ for a strain rate of 1800/s) is in the range of temperature rise reported in the literature (Rittel, 1999; Li and Lambros, 2001; Lerch, et al., 2003; Garg, et al., 2008).

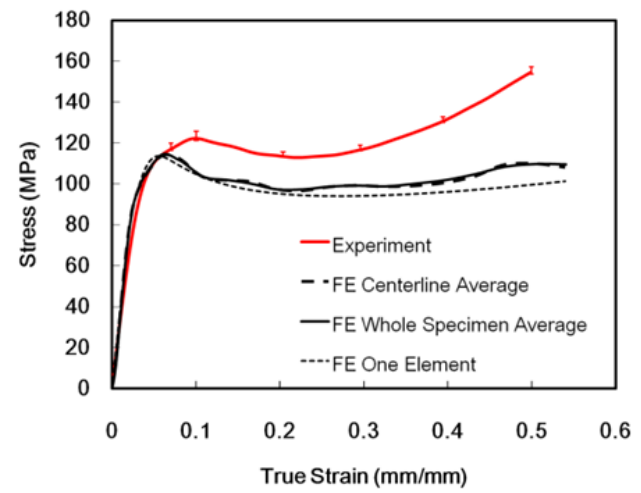

(a)

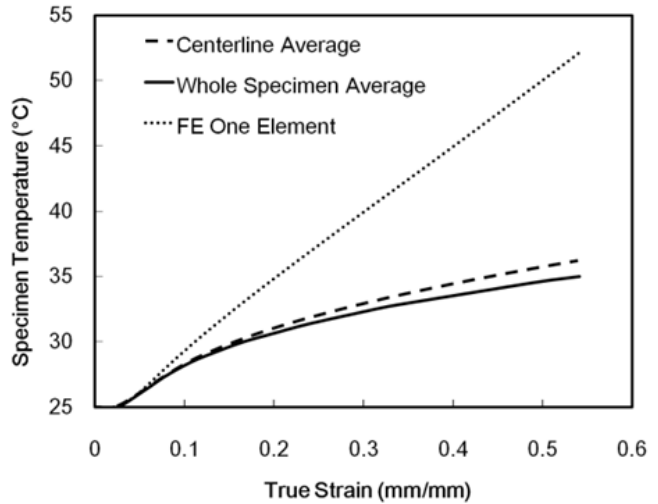

(b)

Figure 12: Comparison of a high rate compression experiment and Finite Element (FE) simulation for a) FE sample complete average, FE sample center line average of Sig33, and one element simulation at $\sim 1800 / s$ (engineering strain rate); and b) temperature rise due to adiabatic heating in the model. 


\section{Application to Thermosets}

The model developed for thermoplastics has been modified for the thermosets by accounting only for one internal state variable, i.e., a scalar variable denoted as $\bar{\xi}_{1}$. This ISV is assumed to represent an internal strain-like quantity induced by the presence of defects (e.g., physical and chemical entanglements).

\section{$\underline{\text { Tension }}$}

Figures 13-16 display a comparison between model prediction and experimental test results from (Plaseied and Fatemi; 2006, 2008a, 2008b) performed in tension for different strain rates and temperatures. As can be seen in the figures, the model captures the mechanical response of vinyl ester (VE) with great accuracy over a large range of temperatures and strain rates.

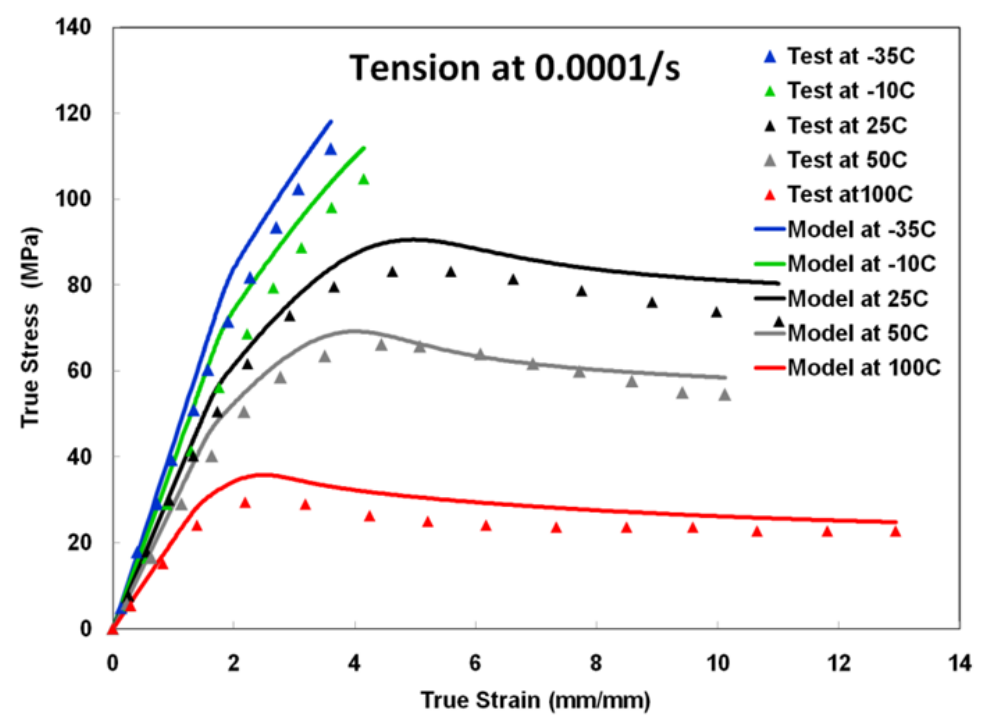

Figure 13: Comparison between model prediction and experimental test results (Plaseied and Fatemi, 2008a) performed in tension at $0.0001 / \mathrm{s}$ for different temperatures $\left(-35^{\circ} \mathrm{C},-10{ }^{\circ} \mathrm{C}, 25^{\circ} \mathrm{C}, 50{ }^{\circ} \mathrm{C}\right.$, and $\left.100^{\circ} \mathrm{C}\right)$. 


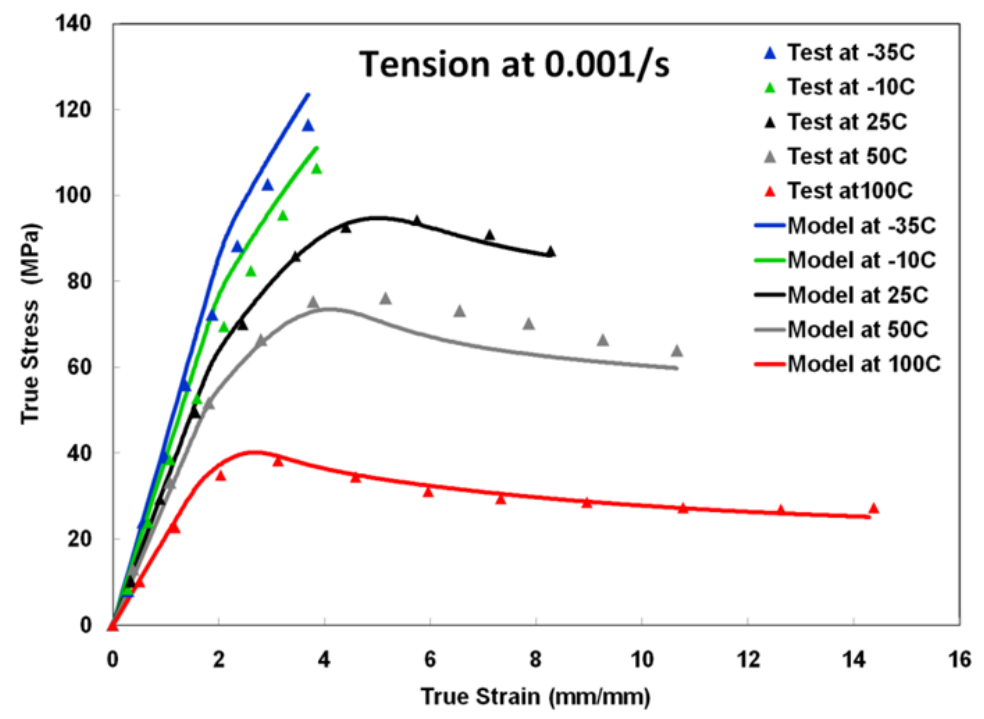

Figure 14: Comparison between model prediction and experimental test results (Plaseied and Fatemi, 2008a) performed in tension at $0.001 / \mathrm{s}$ for different temperatures $\left(-35^{\circ} \mathrm{C},-10{ }^{\circ} \mathrm{C}, 25^{\circ} \mathrm{C}, 50{ }^{\circ} \mathrm{C}\right.$, and $\left.100^{\circ} \mathrm{C}\right)$.

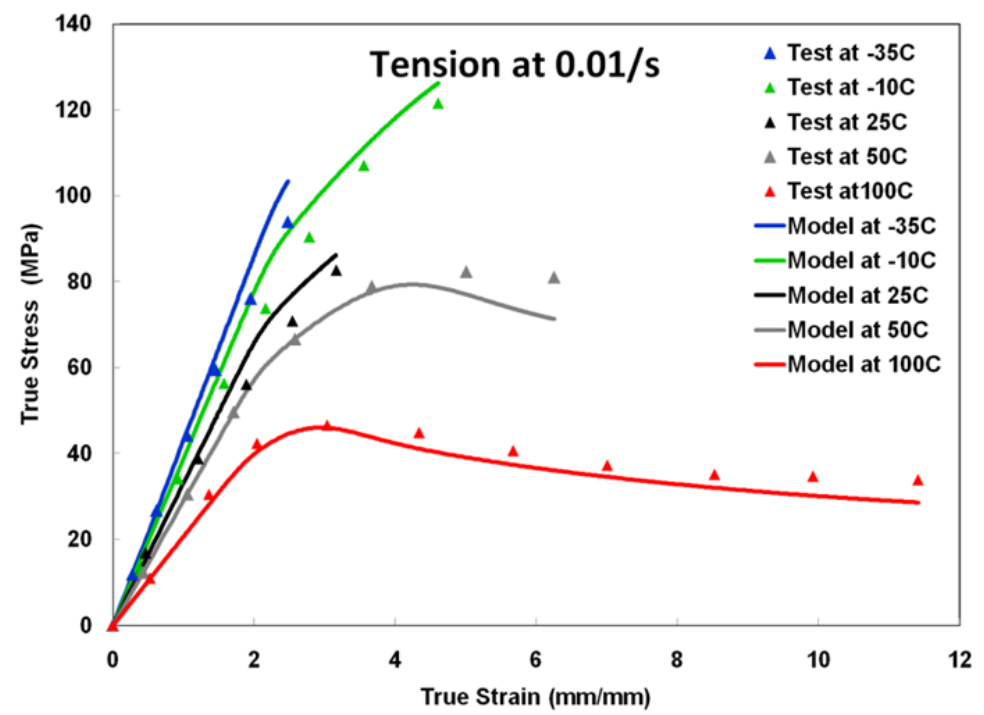

Figure 15: Comparison between model prediction and experimental test results (Plaseied and Fatemi, 2008a) performed in tension at $0.01 / \mathrm{s}$ for different temperatures $\left(-35^{\circ} \mathrm{C},-10^{\circ} \mathrm{C}, 25^{\circ} \mathrm{C}, 50{ }^{\circ} \mathrm{C}\right.$, and $\left.100^{\circ} \mathrm{C}\right)$. 


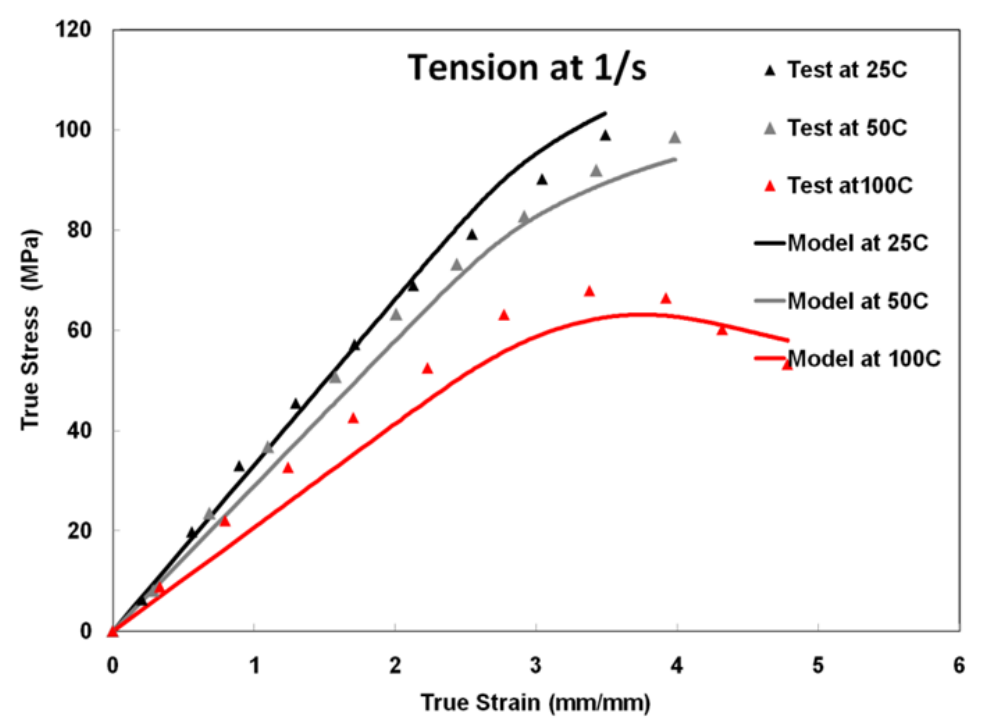

Figure 16: Comparison between model prediction and experimental test results [Plaseied and Fatemi, 2008a] performed in tension at $1 / \mathrm{s}$ for different temperatures $\left(25^{\circ} \mathrm{C}, 50^{\circ} \mathrm{C}\right.$, and $\left.100^{\circ} \mathrm{C}\right)$.

\section{Molecular dynamic simulations for exploring the deformation of polymers}

This section describes efforts to study the relevant molecular deformation mechanisms and quantify the internal structure evolution responsible for the different regimes (elasticity/yield/strain softening/strain hardening) observed during deformation of entangled polymers. We have used molecular dynamics simulations of uniaxial tensile deformation of entangled polyethylene (PE) as a model system. We generated the initial entangled PE structure using a PE united atom model representation. The interatomic force field for amorphous PE is based on a united atom model using the DREIDING potential (Mayo, et al., 1990). Full atom representations would require approximately three times the number of atoms $\left(\mathrm{CH}_{2}\right)$ along with a more computationally expensive force field and, quite possibly, a smaller timestep or different integration scheme due to adding hydrogen. Therefore, the use of the united atom model and DREIDING potential was influenced in part by the computational expense.

The united atom model used here considers each methyl group (i.e., the $\mathrm{CH}_{2}$ monomer) as a single atom with a force field that determines the interactions between united atoms. The DREIDING potential used has four contributing terms; bond stretching, changes in bond angle, changes in dihedral rotation, and van der Waals non-bonded interactions (Mayo, et al., 1990).

A parallel molecular dynamics code, LAMMPS that incorporates domain decomposition was used to deform the polymer simulation cells. The simulations were performed for a three- dimensional periodic simulation cell with 100 polyethylene chains of 1000 monomers each, for a total of 106 united atoms. The temperature chosen was $100 \mathrm{~K}$. This temperature is sufficiently below the calculated glass transition temperature $\left(T_{g}=300 \mathrm{~K}\right)$. Therefore, the amorphous polyethylene response is representative of that in the glassy state. The initial chain structure was created using a method similar to those previously developed using Monte Carlo self-avoiding random walks (Binder, 1995). The chain generation step 
used a face-centered cubic (FCC) lattice superimposed on the simulation cell with the nearest neighbor distance of $1.53 \AA$ A. Molecules were added to the lattice in a probabilistic stepwise manner, which based the probability of chain growth in certain directions on the bond angle and the density of unoccupied sites in the region. The initial polymeric structure was then inserted into the molecular dynamics code where an equilibration sequence was performed prior to deforming the amorphous polymer. The equilibration sequence relaxes any high energy configurations that are artificially created due to the face-centered cubic lattice used to generate the amorphous polymer structure. The relaxation involves four different steps. Initially, the simulation ran for 105 timesteps ( $\Delta t=1 \mathrm{fs}$ ) using NVT dynamics at 500 $\mathrm{K}$ followed by relaxation for $5 \times 105$ timesteps $(\Delta \mathrm{t}=0.5 \mathrm{fs}$ ) using NPT dynamics at $500 \mathrm{~K}$. The next relaxation cooled the structure down to the desired temperature for $5 \times 105$ timesteps followed by further relaxation of $5 \times 105$ timesteps $(\Delta t=1 \mathrm{fs})$ at $100 \mathrm{~K}$. Several microstructure metrics were used to quantitatively validate the polymer chain geometry following equilibration. The average bond length for all equilibrated systems was $1.529 \AA$ and the average bond angle was $109.27^{\circ}$. The dihedral angle distribution has a broad distribution with both gauche and trans peaks. To calculate the fraction of trans conformations within the PE system, a threshold value of $120^{\circ}$ was used to delineate the gauche peak (centered about $66^{\circ}$ ) from the trans peak (centered about $180^{\circ}$ ). The percent trans conformations in the initial structure was $73.8 \%$. The initial densities for the amorphous PE structures ranged from 0.87 $0.91 \mathrm{~g} / \mathrm{cc}$, slightly lower than experimental values for low density PE (0.91-0.94 g/cc), which contains a high degree of short and long chain branching. The glass transition temperature was used to verify that such equilibration of the polymer structure was appropriate for deformation simulations. The glass transition temperature $T_{g}$, delimiting the glassy regime to the rubbery one, was determined from the change in slope of the specific volume vs. temperature curve. The calculated values of $\approx 300 \mathrm{~K}$ here are similar to those calculated by other groups using molecular dynamics, which fall in the range of 250-300 $\mathrm{K}$, close to the experimentally measured value of $250 \mathrm{~K}$.

The amorphous PE system was then deformed under tensile loading applied at a constant true strain rate with different boundary conditions for the two lateral simulation cell faces, as shown schematically in Figure 17. The NPT deformation condition allows for both rapid pressure and heat dissipation (in comparison to the loading) leading to zero pressure on the lateral boundaries and a constant $100 \mathrm{~K}$ within the simulation cell. Essentially, this is a pure uniaxial tensile simulation with rapid heat dissipation within the sample (plane stress condition). In contrast to NPT, the NPH boundary condition does not regulate the system temperature, allowing the heat generated through internal work to increase within the sample. Again, this boundary condition is a pure uniaxial tensile simulation where heat does not have adequate time to diffuse. In reality, the rate of heat dissipation falls somewhere in between; these conditions were chosen to bound this behavior. Moreover, one must realize that multiple cases may occur within the same material due to constraints caused by various microstructure heterogeneities. 

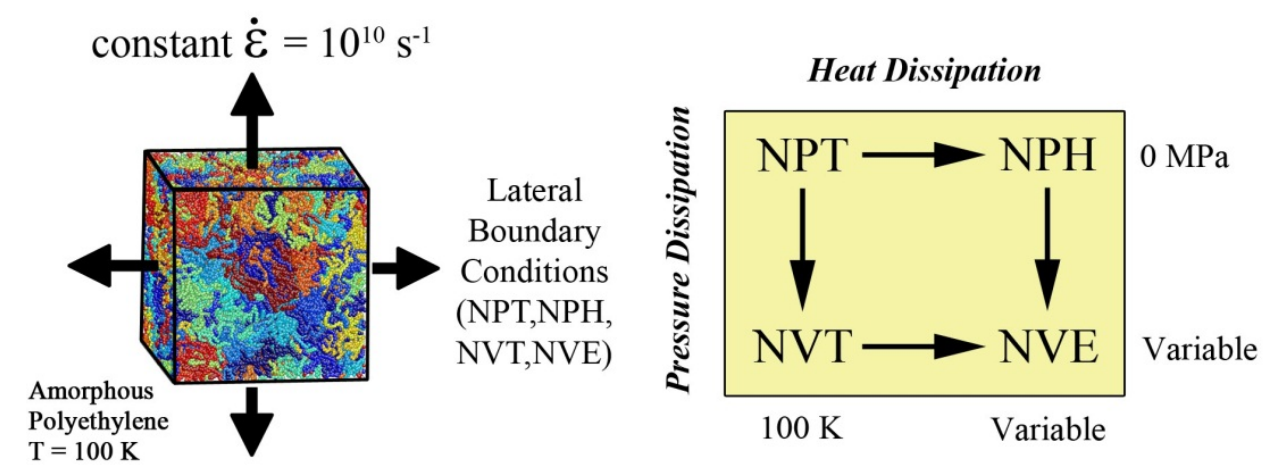

Figure 17: Schematic of simulation cell and boundary conditions.

The NVT and NVE boundary conditions have no pressure dissipation on the lateral boundaries. Thus, these boundary conditions impose a triaxial stress state as the stress increases on the constrained lateral boundaries as a function of strain (plane strain condition). Similar to the NPT and NPH boundary conditions, the NVT and NVE boundary conditions have rapid heat dissipation and no heat dissipation, respectively. For the NPT and NVT conditions, a Nose'-Hoover thermostat was used to regulate the system temperature. For the NPT and NPH boundary conditions, the pressure dissipation was implemented by decoupling the boundary in the loading direction from the equations of motion from the other two directions.

Several stress and energy measures were tracked to examine their dependence on the deformation boundary condition used. The macroscopic stress components were calculated from the symmetric pressure tensor, which uses components from the kinetic energy tensor and the virial tensor. The total energy, kinetic energy, and potential energy were also recorded. In addition to calculating these macroscopic measures, the contributions to the total stress and energy from the bond lengths, bond angles, dihedral angles and non-bonding interactions were also tracked as a function of strain. Periodic dumps of the atomic configuration were performed to compute additional microstructure metrics such as chain orientation or the percentage of trans dihedral conformations. An effective true strain rate of $1010 \mathrm{~s}^{-1}$ was used to deform the amorphous polyethylene simulation cells. To reduce the variability observed in the stress and energy responses, multiple steps were taken here:

1. Four different initial configurations were used to create multiple instantiations of amorphous $\mathrm{PE}$;

2. Each configuration was separately deformed in the $x, y, z$ directions to generate a total of 12 different PE deformation datasets;

3. The strain in the loading direction was applied every 10 timesteps to allow sufficient time for the lateral boundaries to relax;

4. Fluctuations in the stress and energy calculations were reduced using a local regression technique with a 2 nd degree polynomial model; 
5. Stress and energy responses for each condition resulted from averaging the filtered responses for the twelve different PE deformation datasets.

The stress-strain response for a 100-chain, 1000-monomer per chain amorphous PE system is shown in Figure 18. This system was deformed at $100 \mathrm{~K}$ and $1010 \mathrm{~s}^{-1}$ strain rate using NPT boundary conditions on the lateral boundaries. Both the original stress values at each strain and the post-processed stress values are shown in this plot to show the reduction in stress fluctuations used here. Afterwards (not shown in Figure 3), the stress-strain curves for the twelve different PE deformation datasets were averaged to obtain the overall stress behavior. The stress-strain curve has four distinct regimes typical of experimental curves: elastic, yield, softening and hardening. Initially, in the elastic regime, the stress increases nearly linearly with increasing applied strain. Interestingly, the characteristic yield peak observed in experiments is also observed at strain rates of $1010 \mathrm{~s}^{-1}$, but the size of this yield peak decreases with decreasing strain rate. Moreover, the stress-strain curves obtained are similar to those obtained in other studies using coarse-grained models of amorphous polymers. Upon reaching the yield point, the stress then shows a decrease in stress for the strain softening regime. Further deformation of $\mathrm{PE}$ causes an increase in stress during the strain-hardening regime. Also shown in this plot is the stress on the lateral boundaries, which also fluctuates around zero stress for the NPT and NPH conditions.

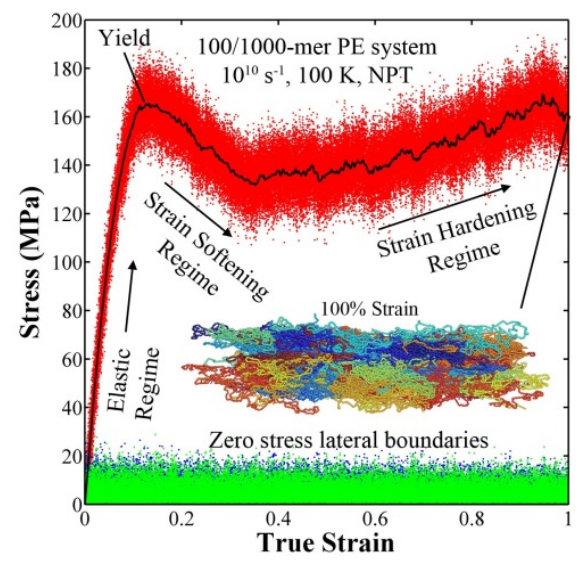

Figure 18: Stress-strain response of amorphous polyethylene deformed in uniaxial tension (NPT condition) at $100 \mathrm{~K}$ with a strain rate of $1010 \mathrm{~s}^{-1}$. The accompanying image shows the PE structure at $100 \%$ strain (colors represent united atoms on separate chains).

The overall stress-strain behavior and temperature evolution for a 100-chain, 1000-monomer per chain amorphous PE system with different lateral boundary conditions is shown in Figure 19. First, we examine the deformation simulations where the pressure was dissipated through the lateral boundaries (NPT/NPH). For the NPT boundary condition, notice that all four regimes are observed for the 100 chain, 1000-monomer PE system. For the NPH condition, the stress-strain behavior is very similar in the elastic, yield, and strain-softening regimes, but the stress does not increase in the strainhardening regime in contrast to the NPT case. Interestingly, comparing the temperature evolution between the two conditions with respect to strain, we observe that the temperature increases 
drastically for the NPH boundary condition, inducing material softening at large strains. Next, we examine the deformation simulations, where there is no pressure dissipated at the lateral boundaries (NVT/NVE). For both conditions, the triaxial stress-strain response exhibits a nonlinear elastic response up to a peak stress, with a rapid decrease in stress thereafter. The stresses in both the tensile direction and the non-loading directions are high $(\approx 200$ and $\approx 250 \mathrm{MPa}$, respectively) due to the lack of pressure dissipation in the lateral boundaries. This induces a high triaxial state of stress in the deformed PE system. Additionally, there is little difference in temperature evolution prior to the peak stress for these boundary conditions. Moreover, the temperature difference observed for larger strains does not significantly impacts the overall stress-strain response for the NVT/NVE conditions.

Figure 20 shows images of the 100-chain, 1000-monomer per chain amorphous PE system as a function of strain for the NPT and NVT cases. These two cases were selected to show the differences between the two stress-strain curves. The conditions without temperature dissipation (NPH, NVE) were qualitatively similar. In these images, the PE chains were unwrapped through the periodic boundaries. There are distinct differences between the two boundary conditions, as expected. The NPT boundary condition allows the pressure to dissipate in the lateral directions as the PE is stretched in the tensile direction. On the other hand, the NVT boundary condition fixes the bounds in the lateral directions, which imparts a high triaxial state of stress inducing void nucleation and growth, and stretching of the chains. Notice that the $25 \%$ strain level occurs after the peak stress for the NVT boundary condition. Prior to the peak stress, the PE system deforms in a relatively uniform manner. However, upon reaching the peak stress, the PE system deforms unevenly, with the volume associated with the chain stretching accommodating much of the deformation

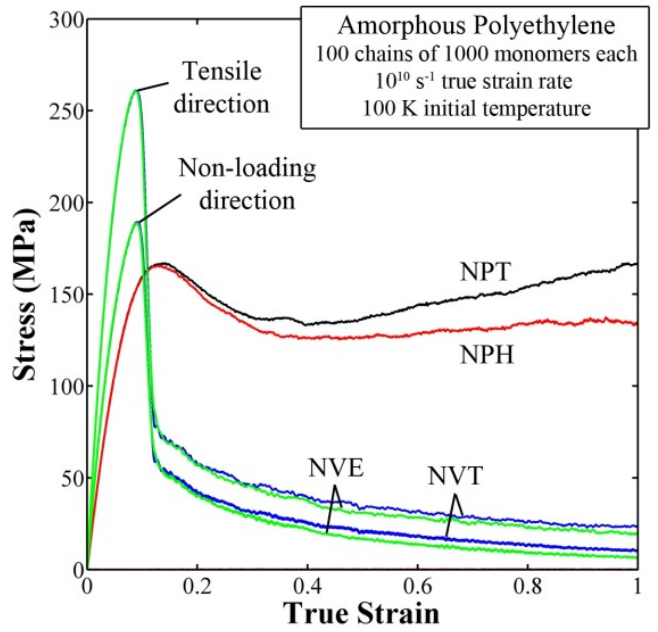

(a)

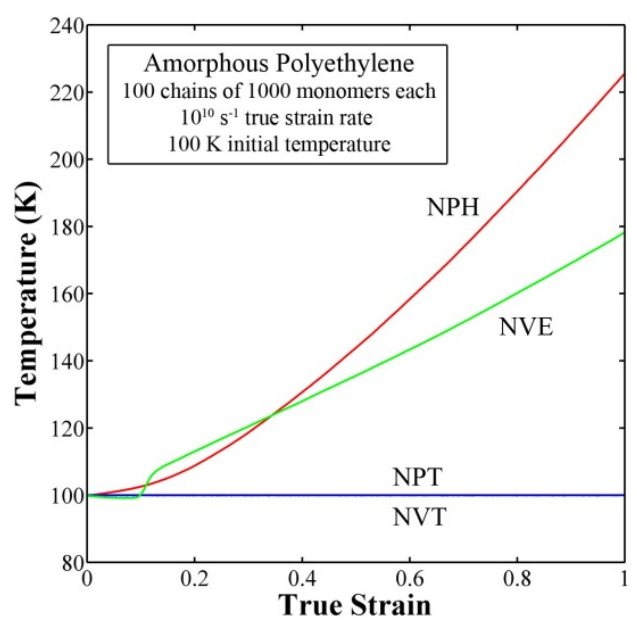

(b)

Figure 19: (a) Stress and (b) temperature evolution as a function of true strain for polyethylene at 100 $\mathrm{K}$ with a strain rate of $1010 \mathrm{~s}^{-1}$. All four boundary conditions (NPT, NPH, NVT, NVE) are shown and labeled. 


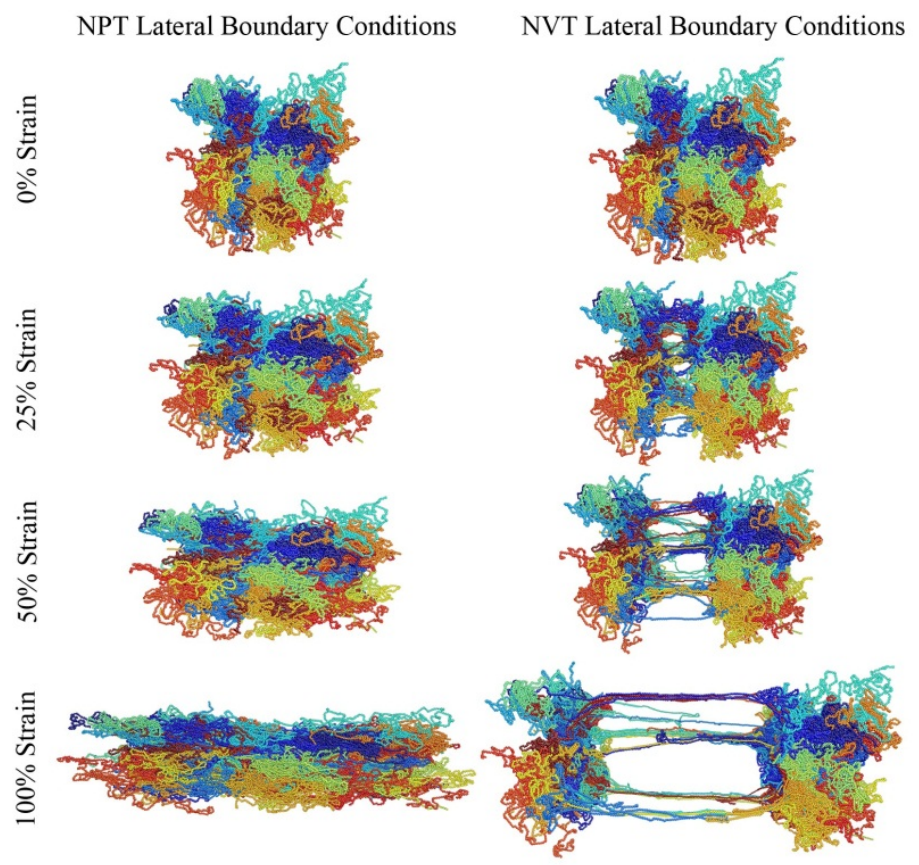

Figure 20: Polyethylene structure evolution for the NPT and NVT lateral boundary conditions at $0 \%$, $25 \%, 50 \%$, and $100 \%$ strain.

Figure 21 shows the normalized potential energy change of a 100-chain, 1000-monomer per chain amorphous PE system deformed for a strain rate of $1010 \mathrm{~s}^{-1}$ at $100 \mathrm{~K}$, which corresponds to the stress-strain behavior observed in Figure 19. In addition to the total energy $E_{\text {total }}$, the individual components of the energy are shown: the non-bonded energy $E_{n b}$, the bonded energy $E_{b}$, the bond angle energy $E_{\theta}$, the dihedral energy $E_{\phi}$, and the kinetic energy $E_{k e}$. The normalized energy, $\delta E$, in these plots is obtained by subtracting the initial energy $E_{0}$ of each component at zero strain. Therefore, each component's increase/decrease is relative to its initial energy. The NPT boundary condition in Figure 21 will be discussed first. In the elastic and yield regimes, the dihedral, angle and bond energies remain almost unchanged and a majority of the increase in total energy is correlated with an increase in the non-bonded energy associated with van der Waal's forces between polymer chains. The non-bonded energy increases sharply in the elastic and strain-softening regions, which can be associated with chain slippage mechanisms. After the strain-softening region, the non-bonded energy stays fairly constant while there are significant changes in the bond length, bond angle and dihedral energies. Beyond the elastic regime, the energy associated with bond length steadily decreases as bond lengths move towards the equilibrium bond length. The dihedral angle energy increases in the strain softening regime and then steadily decreases in the strain hardening regime as gauche conformations rotate to the lower energy trans conformations. The total energy sharply increases in the elastic region due to non-bonded interactions and then slowly decreases over the strain softening and hardening regions as energy is dissipated via dihedral rotations and bond length changes to accommodate deformation. 
The influence of heat dissipation of the lateral boundaries is evidenced by comparing the NPT and NPH conditions (Figure 21a vs. Figure 21b) as well as the NVT and NVE conditions (Figure 21c vs. Figure 21d). As can be expected, the lack of heat dissipation leads to an increase in the internal energy of the system as deformation proceeds, in particular to the kinetic energy component, $E_{k e}$. The NVT and NVE cases are very similar in their energy evolution response for strains up to $10 \%$, which is as expected considering that the temperature does not change significantly (Figure 19b). After these strains, the kinetic energy component steadily increases for the NVE case. The NPT and NPH cases show a similar response during the elastic regime, with non-bonded van der Waal's energy making up a significant portion of the total energy of the system. However, with increasing strain and increasing kinetic energy, there is also a large increase in the energies associated with the bond length, bond angle and dihedral angle components. Since the temperature is increasing for the material, the corresponding entropy associated with the bond length, bond angle and dihedral angle constraints results in higher energies for these components.

The influence of pressure dissipation of the lateral boundaries is evidenced by comparing the NPT with the NVT conditions (Figure 21a vs. Figure 21b). The response is initially similar with the nonbonded van der Waal's interactions making up a significant portion of the total energy of the system. All other energy components for the NVT case either remain constant or decrease in energy up to $10 \%$ strain. This is a sign that the increasing free volume within the system allows for some relaxation of bond and dihedral angles, which in turns lowers the energy of these components. Interestingly, a significant amount of internal energy (heat) is not generated during the elastic portion of the stressstrain curve (as denoted by the lack of increase in the kinetic energy), but rather heat is only generated because of mechanisms associated with plastic deformation.

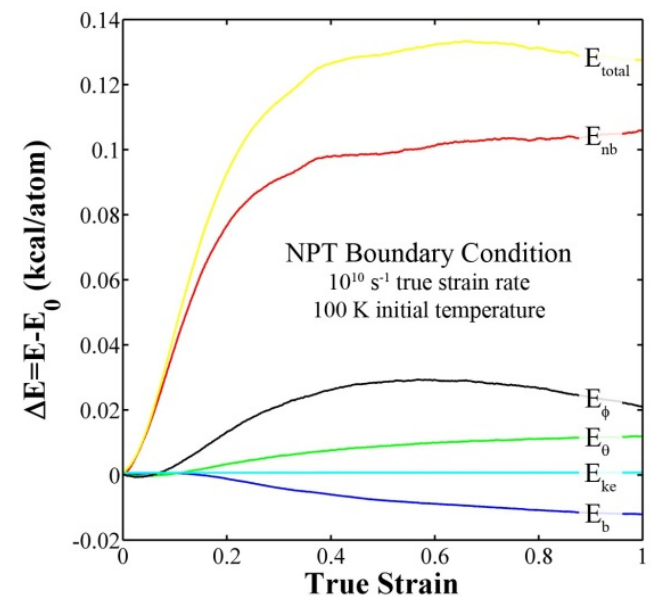

(a)

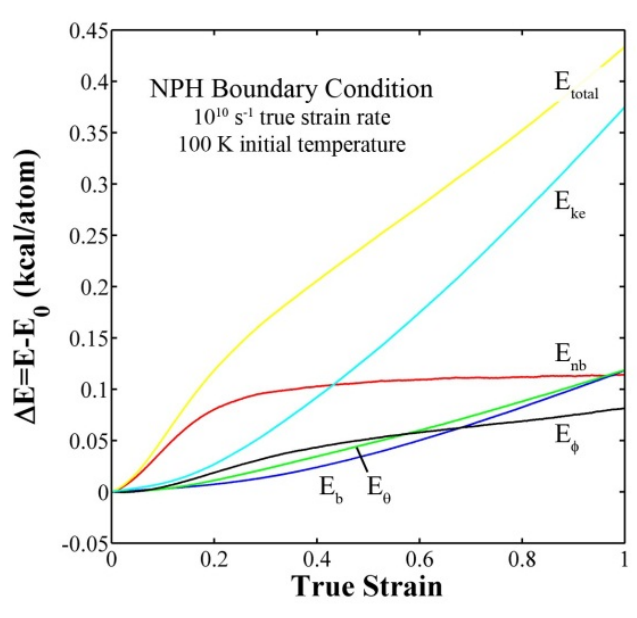

(b) 


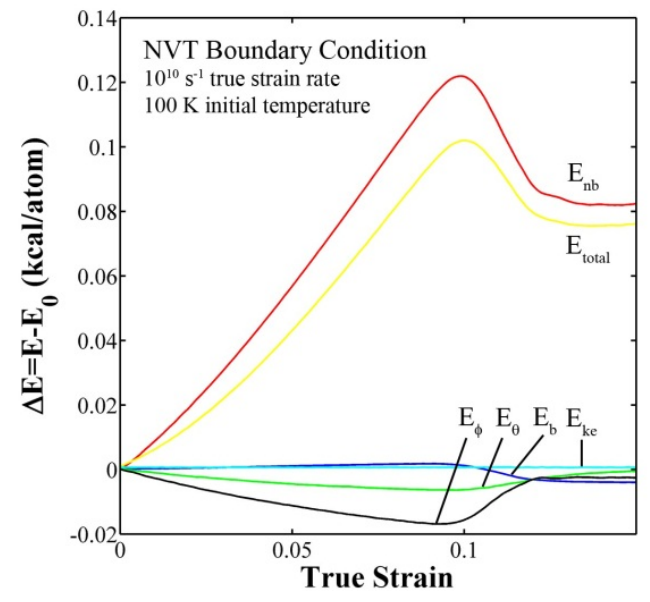

(c)

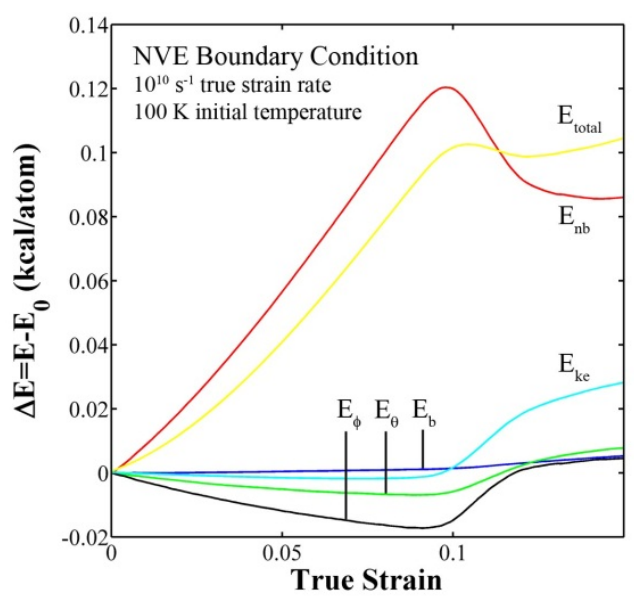

(d)

Figure 21: Normalized energy evolution as a function of true strain for polyethylene at $100 \mathrm{~K}$ with a strain rate of $1010 \mathrm{~s}^{-1}$ for the (a) NPT, (b) NPH, (c) NVT, and (d) NVE conditions.

The different stress components that make up the macroscopic stress can also be tracked as a function of strain to provide insight into the stress accommodation during deformation. Here, we tracked five terms used in the calculation of the macroscopic stress tensor: bond stretching, bond angle, dihedral rotation, van der Waals non-bonded interactions, and the kinetic component. Figure 22 shows the stress component evolution as a function of strain for the NPT boundary condition. The two plots show the evolution of the stress components (a) parallel in the direction of loading and (b) perpendicular to the direction of loading. For the values perpendicular to the loading direction, values from both perpendicular axes were used for the remainder of the paper, i.e., 24 values were averaged instead of 12 . While the response shows a similar trend for several of the stress components, the evolution of the stress component attributed to the van der Waal's interactions is different between parallel and perpendicular directions. Notice that for determining the energy relationships, the energy components are non-directional. Clearly, this plot shows that certain stress components are anisotropic, in particular, the stress components associated with the van der Waal's interactions and bond angles change as a function of direction. The fact that these stress components change with direction means that the relationship between energy and stress is not straightforward. For instance, Figure 19a shows that the non-bonded energy increases with increasing strain, but this results in both an increase and decrease in the stress associated with this component in directions parallel and perpendicular to loading, respectively.

Interestingly, the bond length stress component is the second largest stress contributing the system stress with a large compressive value associated with it. Moreover, the bond length stress component changes significantly as a function of strain. The bond angle and dihedral angle stress components have very small values comparatively and change very little with increasing strain. In general, the stress partitioning evolution portrays a very different picture from the normalized energy 
evolution curves; one in which the system stress is largely controlled by the competition between the bond stretching term and the non-bonded van der Waal's interaction term. That is, the van der Waal's forces act to push the polymer chains apart leading to a tensile stress, while the bond stretching forces act to keep the chains together leading to a compressive stress.

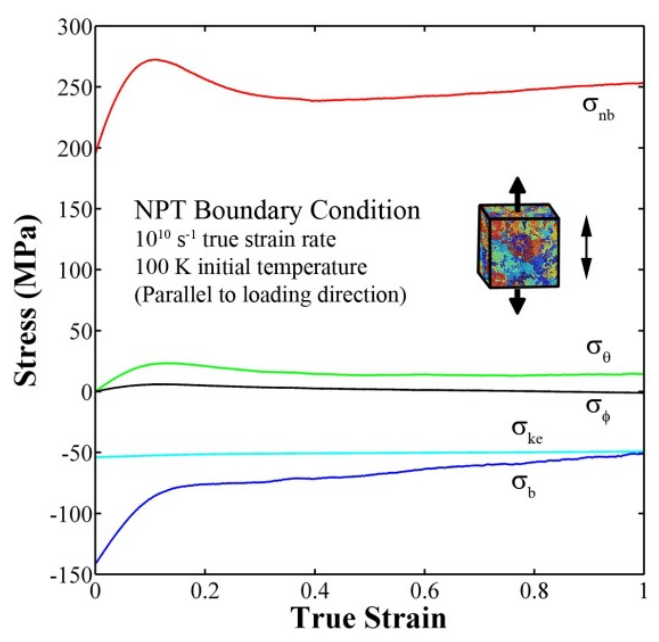

(a)

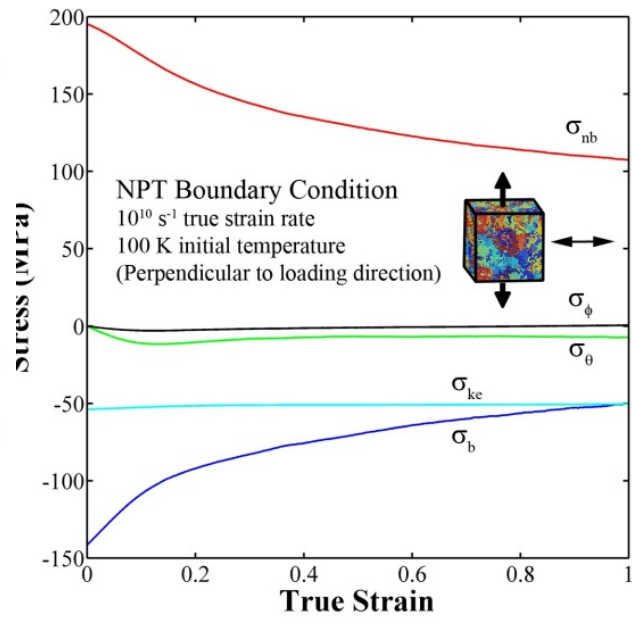

(b)

Figure 22: Stress evolution in the (a) tensile direction and (b) lateral directions as a function of true strain for polyethylene at $100 \mathrm{~K}$ with a strain rate of $1010 \mathrm{~s}^{-1}$.

Figure 23 shows the stress component evolution as a function of strain in the direction parallel to the loading direction for all boundary conditions. These values have been normalized by subtracting their corresponding stress value at zero stress. First, for all cases, the bond stretching, bond angle, and non-bonded stress components increase with increasing strain up to approximately $10 \%$ strain, with the bond stretching and non-bonded terms being the most significant. Interestingly, the characteristic strain softening regime of polymers is attributed to the change in the non-bonded stress component, as it then decreases with increasing strain until about $40 \%$ strain and then continues to increase (Figure 23a and 23b). This increase may be correlated to chain crystallization at large strains. The bond stretching stress component increases monotonically with increasing strain; however, the slope of the stress-strain relation decreases following the yield peak. For the NVT and NVE simulations, there is little difference for strains up to $10 \%$. For the NPH boundary condition, the kinetic energy stress component decreases with increasing strain, which offsets the increases to the bond stretching term (due to a faster rate of chain alignment, shown later). 


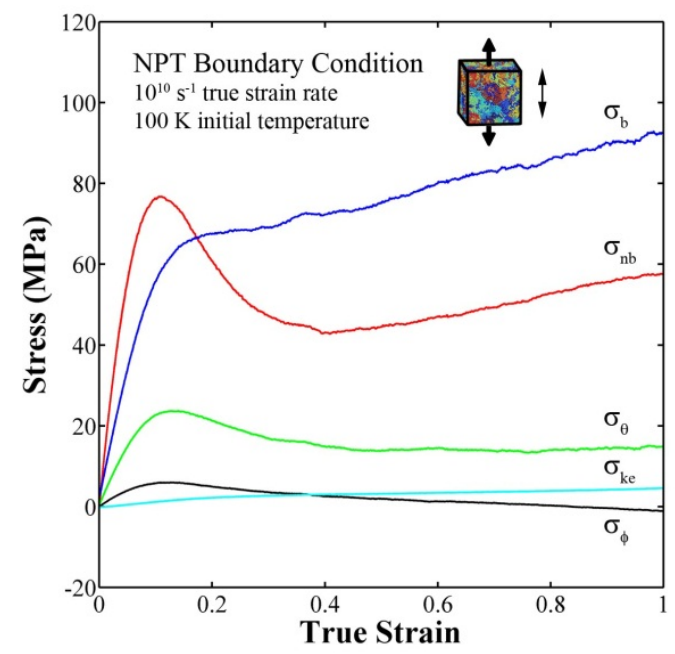

(a)

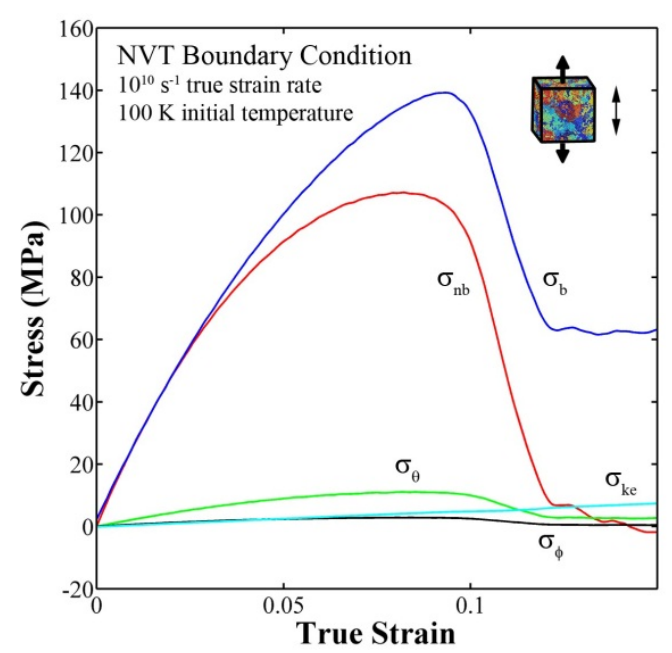

(b)

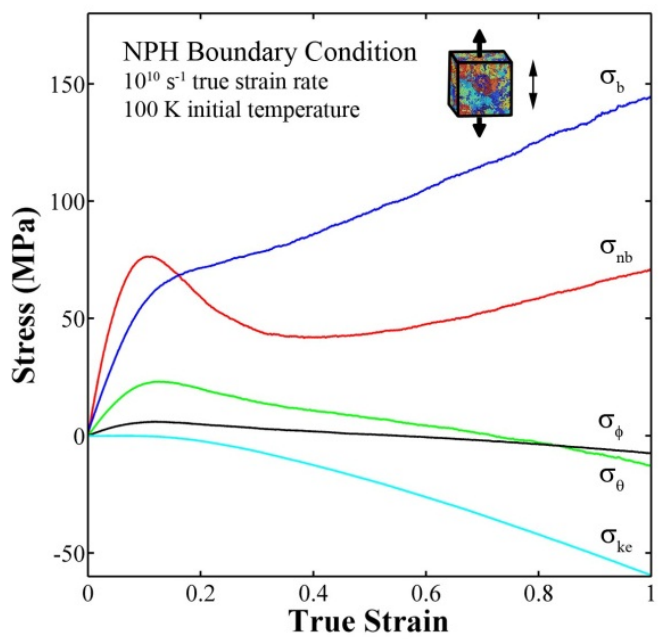

(b)

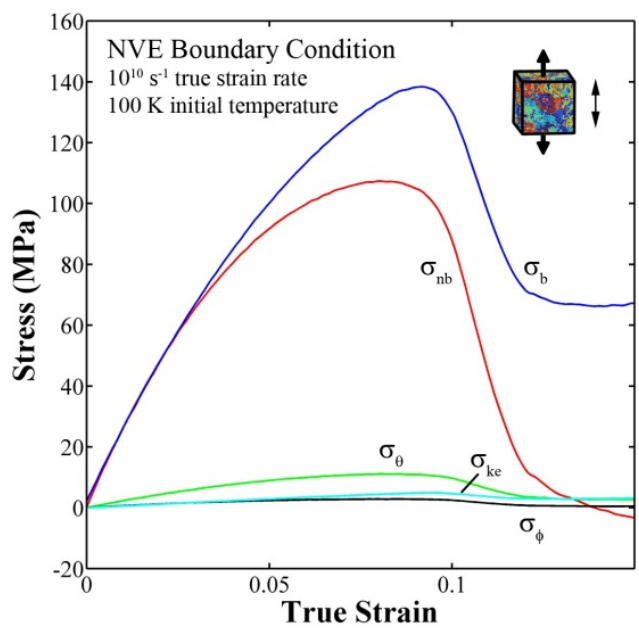

(d)

Figure 23: Normalized stress evolution in the tensile direction as a function of true strain for polyethylene at $100 \mathrm{~K}$ with a strain rate of $1010 \mathrm{~s}^{-1}$. These curves have been normalized by subtracting the stress values for each component at $\varepsilon=0$.

Figure 24 shows the stress component evolution as a function of strain in the direction perpendicular to the loading direction for all boundary conditions. Similar to Figure 23, these values have been normalized, too. A number of trends are very similar to Figure 23. However, the non-bonded stress component decreases with increasing strain for the NPT and NPH boundary conditions, as shown in Figure 22. Notice that to enforce the zero pressure condition on the boundary, the change in the stress components due to bond stretching and non-bonded van der Waal's interactions are nearly equal and opposite in magnitude. The evolution of the stress components for the NVT and NVE boundary conditions are almost identical to that in the direction parallel to the loading direction, as expected due to the similar stress-strain responses (Figure 19) and the triaxial state of stress. 


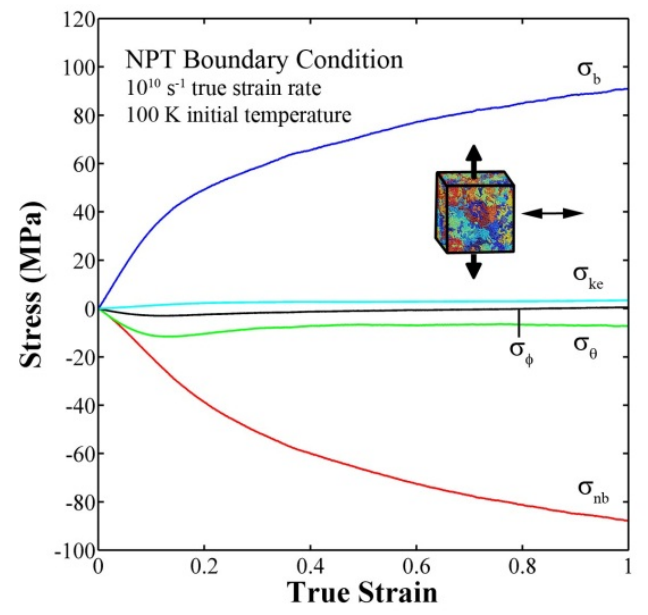

(a)

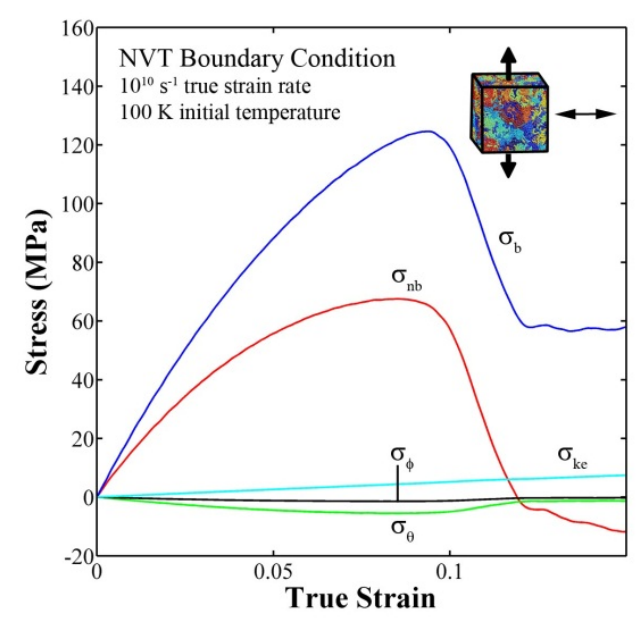

(b)

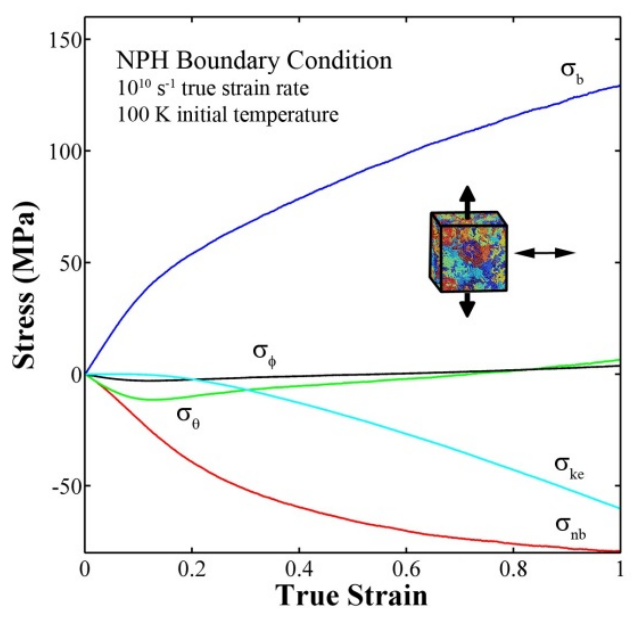

(b)

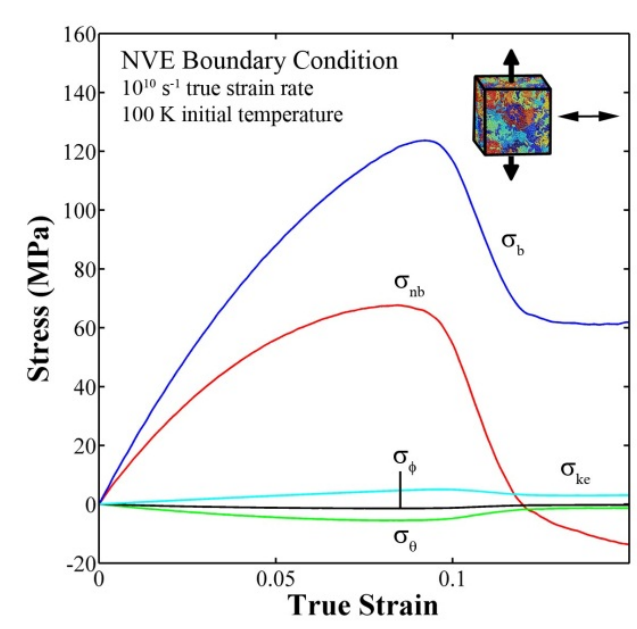

(d)

Figure 24: Normalized stress evolution in the lateral directions as a function of true strain for polyethylene at $100 \mathrm{~K}$ with a strain rate of $1010 \mathrm{~s}^{-1}$. These curves have been normalized by subtracting the stress values for each component at $\varepsilon=0$.

The evolution of the internal structure of the polyethylene system was further analyzed. For the sake of brevity, only the NPT and NPH boundary conditions are examined herein. The evolution of the bond length and bond angle distributions as a function of strain may shed light on deformation in amorphous polymer systems. Figure 25 shows the evolution of these distributions with a box plot, a concise representation of the 107 data points for each plot. For each strain level (each box), the central mark is the median, the edges of the filled box are the 25th and 75th percentiles, and the lines extend to the most extreme data points (minimum and maximum), as shown in Figure 25a. Figure 25a and 25c show the evolution of the bond length and bond angle distributions, respectively, for the NPT boundary 
conditions, while Figure $25 \mathrm{~b}$ and $25 \mathrm{~d}$ show the same for the NPH boundary conditions. The influence of heat dissipation at the boundary has a minimal effect on the bond length and bond angle distributions. The increase in temperature for the NPH conditions results in a slight broadening of the distribution (as evidenced by the change in the 25th and 75th percentiles as well as the minimum and maximum values), but the median shows almost no change for the symmetric distributions. Recall that the bond length and bond angle have non-negligible components of the macroscopic stress, though. To understand their influence on stress, it is also important to quantify the re-orientation of the polymer chain segments.

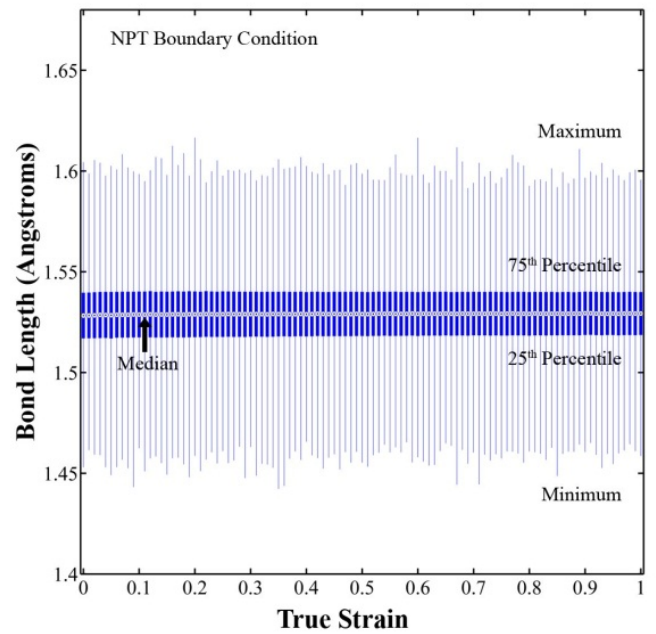

(a)

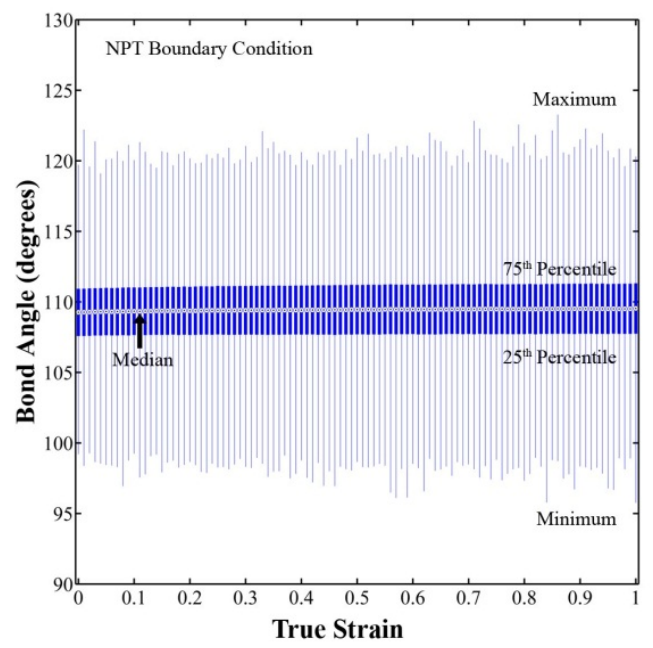

(c)

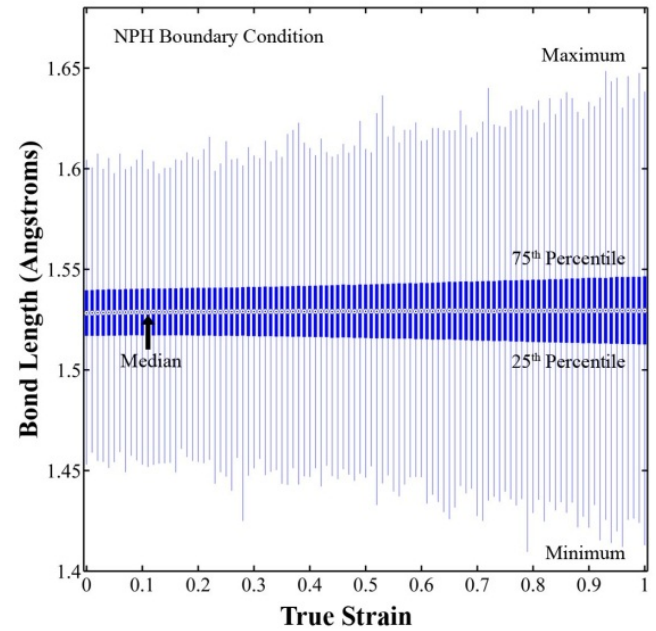

(b)

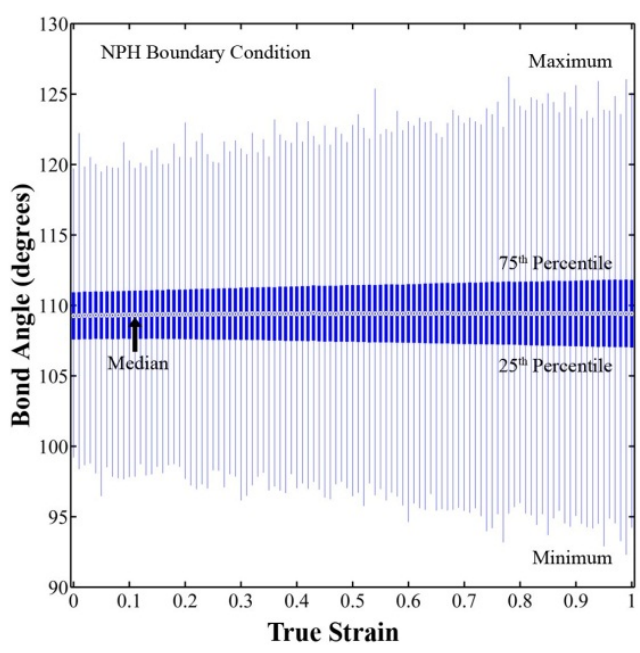

(d)

Figure 25: Evolution of the bond length and bond angle distributions as a function of strain. 
The chain orientation as a function of strain is also an important microstructure response of the polymer chain segments during deformation. Figure 26 shows how the average chain orientation parameter, P2x, evolves as a function of strain. On average, the chain segments tend to increase their alignment with the loading direction with increasing strain while decreasing their alignment in the transverse directions. Very little scatter is observed between the 12 simulations for each boundary condition. At large strains, PE chains within the constant enthalpy (increasing temperature) NPH boundary condition align more with the tensile direction than the constant temperature NPT condition, as would be expected. Interestingly, although the bond length and bond angle distributions do not change significantly, the re-orientation and alignment of the polymer chain segments can create significant directional stress components.

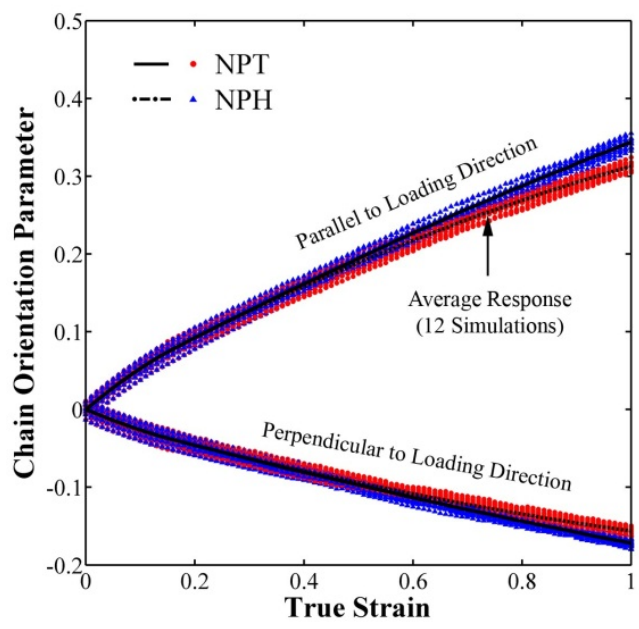

Figure 26: Chain orientation evolution as a function of true strain for polyethylene at $100 \mathrm{~K}$ with a strain rate of $1010 \mathrm{~s}^{-1}$. The red circles and blue triangles show the individual responses for 12 simulations ran for each boundary condition.

The change in the dihedral distribution from metastable gauche conformations to stable trans conformations as a function of strain is also important for deformation in polymer systems. Figure 27 shows the evolution of the dihedral angle distributions for a few strain levels $(0 \%, 20 \%, 50 \%, 100 \%)$ with the NPT boundary condition. The top row tracks the evolution of all segments, the middle row tracks the evolution of those segments that are initially in a gauche conformation, and the bottom row tracks the segments that are initially in a trans conformation. The gauche and trans conformations were calculated using a dihedral angle threshold of $120^{\circ}$. Some of the gauche conformations in the zero strain structure transform to trans conformations, and vice versa. The rate of this transformation is important for accommodating strain and can contribute to chain alignment with the direction of applied stress as well. 
Figure 28 shows how both (a) the rate of transformation (\% conformation change) and (b) the percent trans conformations evolve as a function of strain for the NPT and NPH boundary conditions. Figure 28a shows that the rate of change of the conformations from gauche $\rightarrow$ trans and trans $\rightarrow$ gauche is increased significantly for the NPH condition. Additionally, the rate of change of conformations is approximately 2.5-3.0 times greater for the gauche $\rightarrow$ trans transformation than the trans $\rightarrow$ gauche tranformation for $\varepsilon$ $\geq 20 \%$. Figure $28 \mathrm{~b}$ shows the net effect on the $\%$ trans conformations. Along with the average behavior, the results of the 12 individual simulations for each boundary condition are also plotted to show the range of data. The $1010 \mathrm{~s}^{-1}$ shows very little change in the percentage of trans conformations in the elastic regime, but this percentage increases with increasing strain in the strain softening and strain hardening regimes. The percentage of trans dihedral angles increases at a much faster rate in the constant enthalpy (increasing temperature) NPH condition as compared to the constant temperature NPT condition. As shown here, the increased temperature allows the transformation between gauche and trans to be more easily overcome, which enables a higher gauche $\rightarrow$ trans rate of change to align chain segments with the direction of loading.

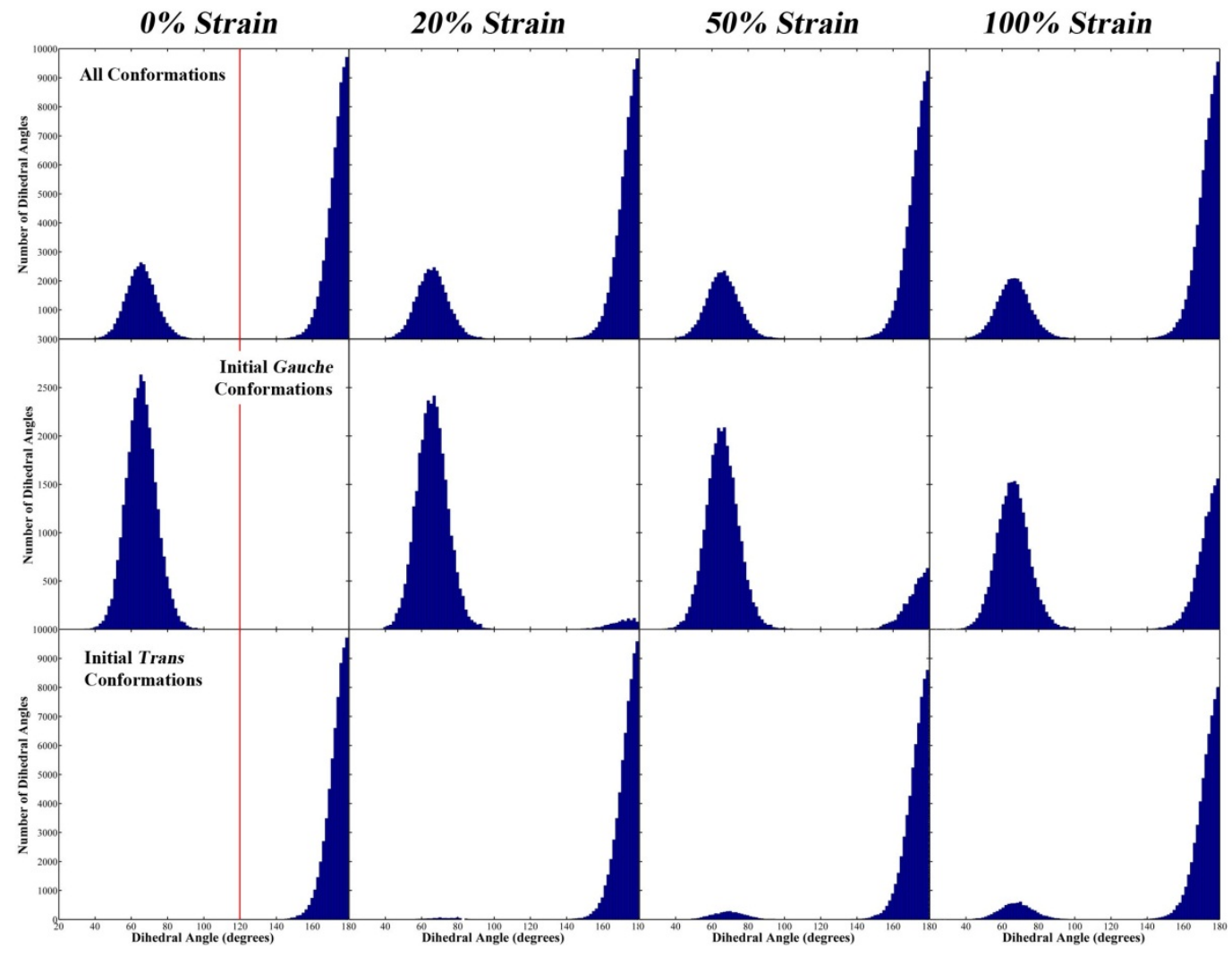

Figure 27: Evolution of the dihedral angle distribution as a function of strain. 


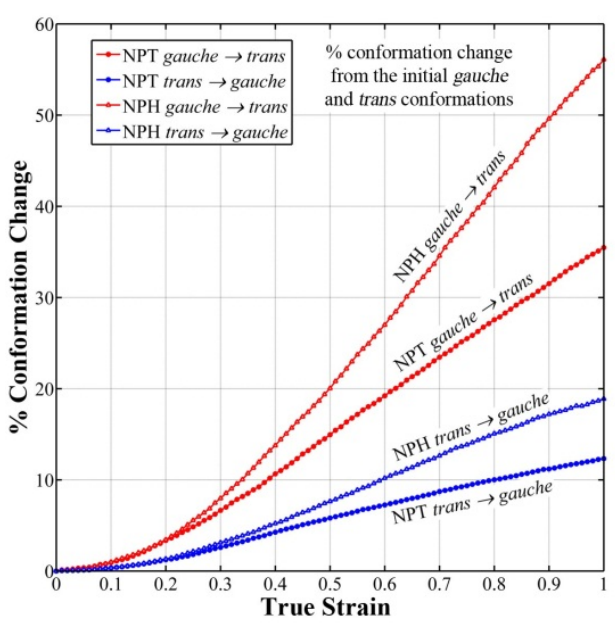

(a)

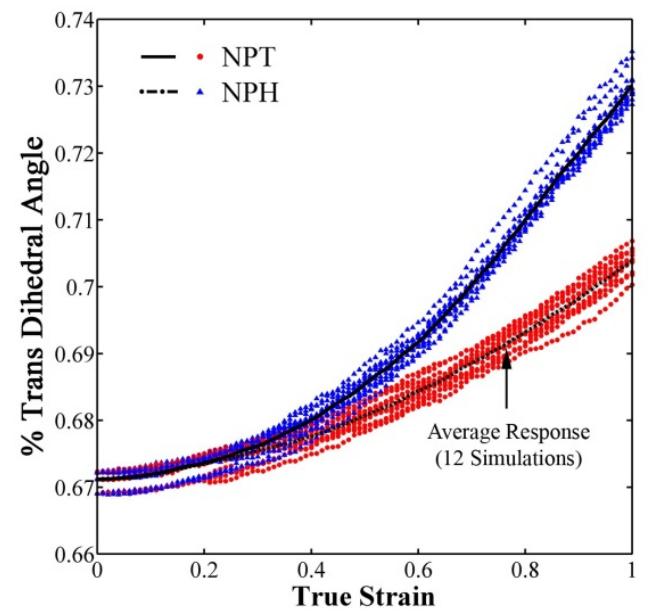

(b)

Figure 28: Chain rotation evolution as measured by the change in the percentage of trans dihedral angles as a function of true strain for polyethylene at $100 \mathrm{~K}$ with a strain rate of $1010 \mathrm{~s}^{-1}$. The red circles and blue triangles show the individual responses for 12 simulations ran for each boundary condition.

The chain entanglement evolution is also important for understanding deformation in amorphous polymer systems. In general, fracture of many polymer materials is expected to occur due to chain disentanglement rather than chain scission, because of the strong carbon-carbon bonds in the polymer backbone. Here, the geometric technique of Yashiro, et al. (2003) was used to calculate the chain entanglement. This technique first creates two vectors which emanate from each atom to neighboring atoms that are separated by 10 atoms on the same chain, i.e., one vector that connects atom $i$ with atom $(i-10)$ and one vector that connects atom $i$ to atom $(i+10)$. The angle between these two vectors is calculated for each applicable atom and a threshold parameter of $90^{\circ}$ is used to specify if the atom is classified as entangled or not. The atoms classified as entangled via this technique are considered to be constrained by neighboring chains. The number of atoms classified as entangled is then divided by the total number of applicable atoms to give a normalized "entanglement parameter" that can be used to compare the different systems. This entanglement parameter actually represents the percent of entangled atoms within the system and can be appropriately scaled by a constant to obtain the entanglement density. We refrain from using an entanglement density here, since this measure is dependent on the separation distance used for creating the two vectors and also the threshold parameter. However, these parameters simply affect the scaling of the $y$-axis and not the general trend.

Figure 29 shows the evolution of the entanglement parameter as a function of strain. There is very little difference between the entanglement parameter for the NPT and NPH boundary conditions. The entanglement parameter decreases monotonically with increasing strain. This result agrees with previous work of Tomita (2000) and Shepherd, et al. (2006), which assume that the entanglement density decreases with an increase of the strain level at constant strain rate and temperature. However, the rate of entanglement decrease with increasing strain is not constant. At $\approx 50 \%$ strain, the 
entanglement parameter transitions from a region of slow linear decrease to a region of faster linear decrease. Based on previous findings herein, it is speculated that the faster rate of disentanglement is due to the reorientation of chains as well as conformation changes, both of which allow entangled chain segments to disentangle.

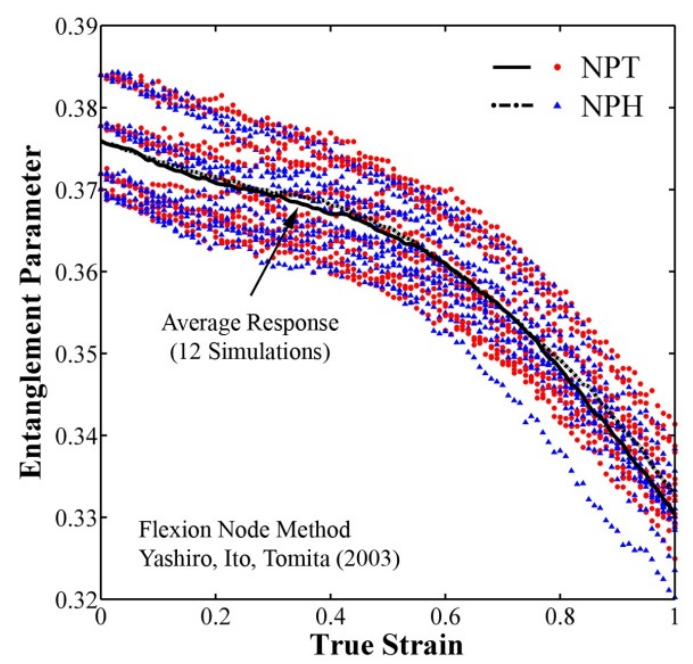

Figure 29: Chain entanglement evolution as a function of true strain for polyethylene at $100 \mathrm{~K}$ with a strain rate of $1010 \mathrm{~s}^{-1}$. The chain entanglement is measured by the flexion node method of Yashiro, et al. (2003).

In summary, the heat and pressure dissipation was studied to determine its impact on the deformation of amorphous polyethylene using molecular dynamics simulations. While the tensile direction was deformed at a constant strain rate, the lateral boundaries were controlled via a zero strain condition (NVT, NVE) or a zero stress condition (NPT, NPH) and either a thermostat was used to maintain constant temperature during deformation (NPT, NVT) or a constant enthalpy/energy condition was employed where the temperature increased during deformation (NPH, NVE). The energy and stress partitioning was examined as well as the evolution of the internal structure of the amorphous polymer. The following conclusions can be drawn:

1. The large increase in temperature as a function of strain observed for the non-thermostat conditions (NPH, NVE) resulted in minor changes to the stress-strain behavior, primarily at higher strains in the strain hardening region (Figure 19). However, the lack of pressure dissipation caused a high triaxial state of stress in the deformed PE system, which results in void nucleation, void growth, and heterogeneous deformation (Figure 20).

2. Analysis of the energy partitioning shows that a majority of the internal energy increase during deformation is due to the non-bonded van der Waal's interactions for all of the boundary conditions used in this study (Figure 21). To a lesser extent, the energies attributed to bond stretching (bond length), bond bending (bond angles), and bond torsion (dihedral angles) also 
contributed during deformation. The kinetic energy component is a significant portion of the total energy at higher strains $(>0.2 \%)$ for the constant enthalpy NPH boundary condition (increasing energy).

3. Analysis of the stress partitioning shows that the macroscopic stress is a mainly balance between the 'tensile' non-bonded van der Waal's interactions and the 'compressive' bond stretching term (Figure 22). During tensile deformation, the stresses associated with the van der Waal's interactions and bond stretching increase in the tensile direction for all cases considered in this study. Interestingly, the characteristic yield stress peak prior to strain softening and strain hardening is clearly due to the van der Waal's interactions between chains (Figure 23). To maintain the zero stress condition for the pressure dissipation cases (NPT, NPH), both the 'tensile' non-bonded stress and the 'compressive' bond stretching stress relax toward zero stress (i.e., bonded stress increases and non-bonded stress decreases).

4. The following analysis concentrates on the pressure dissipation boundary conditions (NPT, NPH). The bond length and bond angle distributions do not change significantly as a function of strain. For the case of increased temperature, there is a slight broadening of the symmetric distributions but no increase or decrease of the distribution. Rather, the large contribution of bond stretching and bond bending to the macroscopic stress are due to the re-orientation of the polymer chain segments in the direction of loading (Figure 26), which results in a larger force being resolved in the direction of loading. The increase in temperature for the NPH case results in a faster rate of re-orientation of the polymer chain segments, which results in a higher stress in the direction of loading, but this is offset by the stress contribution of the kinetic energy component in molecular dynamics simulations.

5. The conformational changes from gauche to trans, and vice versa, result in non-negligible changes to the energy, but very little change to the macroscopic stress. In other words, conformational changes contribute more to the deformation kinematics (i.e., texture) and the ability of the polymer chains to align in the direction of loading than to the actual load carrying capacity. Figure 27 and Figure 28 show that the change in the dihedral angle distribution as a function of strain is a result of both gauche $\rightarrow$ trans and trans $\rightarrow$ gauche changes at different rates $(\approx 2.5-3.0$ times faster for gauche $\rightarrow$ trans). The increase in temperature for the NPH case results in a faster rate of change for the gauche and trans conformations.

6. The chain entanglement decreases as a function of strain with a large rate of decrease in the strain hardening regime $(\varepsilon>0.5)$. The entanglement is not affected very much by the thermostat (NPT versus NPH) but does show some variation between the twelve instantiations studied for each condition (Figure 29). 


\section{Developing a potential for polymer systems based on the Modified Embedded-Atom Method (MEAM)}

The embedded-atom method (EAM) is a semi-empirical $\mathrm{N}$-body potential useful for the atomistic simulations of metal systems. It has successfully been utilized to calculate the energetics and structures of complex metallic systems involving free surfaces, defects, and grain boundaries (Daw et al., 1993). The potential was later modified by Baskes $(1987 ; 1992)$ to include the directionality of bonding in covalent materials such as silicon and germanium, leading to the modified embedded-atom method (MEAM) (Baskes, 1992). The unique feature of the MEAM formalism is its ability to reproduce the physical properties of a large number of fcc, bcc, hcp, and diamond cubic crystal structures in unary, binary, ternary, and higher order metal systems with the same semi-empirical formalism. MEAM potentials are also both reliable and transferable in the sense that they accurately reproduce the physical properties of the element or alloy (reliability) and perform reasonably under circumstances other than the ones used for their parameterization (transferability) (Kim et al., 2009).

The MEAM formalism has traditionally been used for pure metals and impurities, binary and ternary alloys, and carbide, nitride, and hydride metal systems with great success. We have extended the MEAM formalism to hydrocarbons with the ultimate aim of capturing the energetics and structures of commercially important hydrocarbon-based polymers (polyolefins) such as polyethylene and polypropylene and at a later stage other thermoplastics and thermosets. Currently, potentials such as MM3 (Allinger et al., 1989), MM4 (Allinger et al., 1996), first- (Brenner, 1990) and second-generation reactive empirical bond order (REBO) (Brenner et al., 2002), reactive force field (ReaxFF) (van Duin et al., 2001), and condensed-phase optimized molecular potentials for atomistic simulation studies (COMPASS) (Sun, 1998) are used for hydrocarbon simulations, but they are often either not suitable for hydrocarbon/metal systems or are not parameterized for such systems. We have used the MEAM formalism to describe the interactions and geometries of complex hydrocarbon molecular systems, giving molecular bond distances, bond angles, and total energies that match first-principles or experimental data with great accuracy. The MEAM results are further compared with those of REBO and ReaxFF, and we show that MEAM gives comparable or more accurate atomization energies and equilibrium geometries for saturated hydrocarbons when compared to the experimental data. The development of the first MEAM potential for hydrocarbon and polymer systems is a step towards reliably capturing the energetics of systems that have hitherto been difficult to study by molecular dynamics (MD) simulations, such as polymer/metal interfaces and nanocomposites, fracture and crack growth in polymers, etc.

In the EAM and MEAM formalisms, the total energy of a system of atoms is given by

$$
E_{\text {tot }}=\sum_{i}\left[F_{\tau_{i}}\left(\bar{\rho}_{i}\right)+\frac{1}{2} \sum_{j(\neq i)} S_{i j} \phi_{\tau_{i} \tau_{j}}\left(R_{i j}\right)\right] \text {, }
$$

where $F_{\tau_{i}}$ is the embedding energy function for element type $\tau_{i}$, i.e., the energy required to embed an atom of element type $\tau_{i}$ in the background electron density $\bar{\rho}_{i}$ at site $i, S_{i j}$ is the screening factor 
between atoms at sites $i$ and $j$ (defined in Equations 21 and 24), and $\phi_{\tau_{i} \tau_{j}}$ is the pair interaction between atoms of element types $\tau_{i}$ and ${ }^{\tau_{j}}$ at sites $i$ and $j$ at the separation distance of $R_{i j}$. To emphasize the multi-component nature of the model, in this manuscript the element type of the atom at site $i$ is denoted as ${ }^{\tau_{i}}$ to distinguish it from site designation $i$, and the screening factor is explicitly separated from the pair potential. The embedding function is given by the specific simple form

$$
F_{\tau_{i}}\left(\bar{\rho}_{i}\right)= \begin{cases}A_{\tau_{i}} E_{\tau_{i}}^{0} \frac{\bar{\rho}_{i}}{\bar{\rho}_{\tau_{i}}^{0}}\left(\ln \frac{\bar{\rho}_{i}}{\bar{\rho}_{\tau_{i}}^{0}}-B_{\tau_{i}}\right) & \text { if } \bar{\rho}_{i} \geq 0, \\ -A_{\tau_{i}} E_{\tau_{i}}^{0} \frac{\bar{\rho}_{i}}{\bar{\rho}_{\tau_{i}}^{0}} & \text { if } \bar{\rho}_{i}<0,\end{cases}
$$

where $A_{\tau_{i}}$ is a scaling factor, $B_{\tau_{i}}$ is a "gauge transformation" parameter, $\underline{2} E_{\tau_{i}}^{0}$ is the sublimation (cohesive) energy, and $\bar{\rho}_{\tau_{i}}^{0}$ is the background electron density for the reference structure of the atom of element type $\tau_{i}$ at site $i$, which, for most cases, is the equilibrium structure. We will denote the properties of the equilibrium reference state with a superscript zero. The analytic continuation of the embedding function for negative electron densities was chosen as a computational convenience to prevent systems from entering this unphysical regime. The origin of negative electron densities arises below in Equation 5. In the original MEAM formalism, the parameter $B_{\tau_{i}}$ was taken to be zero. The significance of $B_{\tau_{i}}$ will be discussed later. The MEAM formalism introduces directionality in bonding between atoms through decomposing $\bar{\rho}_{i}$ into spherically symmetric $\left(\rho_{i}^{(0)}\right)$ and angular $\left(\rho_{i}^{(1)}, \rho_{i}^{(2)}\right.$, and $\rho_{i}^{(3)}$ ) partial electron densities as given by

$$
\begin{aligned}
& \rho_{i}^{(0)}=\sum_{j \neq i} S_{i j} \rho_{\tau_{i}}^{a(0)}\left(R_{i j}\right), \\
& \left(\rho_{i}^{(1)}\right)^{2}=\frac{\sum_{\alpha}\left[\sum_{j \neq i} \frac{R_{i j}^{\alpha}}{R_{i j}} S_{i j} t_{\tau_{j}}^{(1)} \rho_{\tau_{j}}^{a(1)}\left(R_{i j}\right)\right]^{2} \rho_{i}^{(0)}}{\sum_{j \neq i} S_{i j}\left(t_{\tau_{j}}^{(1)}\right)^{2} \rho_{\tau_{j}}^{a(0)}\left(R_{i j}\right)},
\end{aligned}
$$




$$
\begin{aligned}
\left(\rho_{i}^{(2)}\right)^{2}= & \frac{\left\{\sum_{\alpha, \beta}\left[\sum_{j \neq i} \frac{R_{i j}^{\alpha} R_{i j}^{\beta}}{R_{i j}{ }^{2}} S_{i j} t_{\tau_{j}}^{(2)} \rho_{\tau_{j}}^{a(2)}\left(R_{i j}\right)\right]^{2}-\frac{1}{3}\left[\sum_{j \neq i} S_{i j} t_{\tau_{j}}^{(2)} \rho_{\tau_{j}}^{a(2)}\left(R_{i j}\right)\right]^{2}\right\} \rho_{i}^{(0)}}{\sum_{j \neq i} S_{i j}\left(t_{\tau_{j}}^{(2)}\right)^{2} \rho_{\tau_{j}}^{a(0)}\left(R_{i j}\right)}, \\
\left(\rho_{i}^{(3)}\right)^{2}= & \frac{\left\{\sum_{\alpha, \beta, \gamma}\left[\sum_{j \neq i} \frac{R_{i j}^{\alpha} R_{i j}^{\beta} R_{i j}^{\gamma}}{R_{i j}{ }^{3}} S_{i j} t_{\tau_{j}}^{(3)} \rho_{\tau_{j}}^{a(3)}\left(R_{i j}\right)\right]^{2}-\frac{3}{5} \sum_{\alpha}\left[\sum_{j \neq i} \frac{R_{i j}^{\alpha}}{R_{i j}} S_{i j} t_{\tau_{j}}^{(3)} \rho_{\tau_{j}}^{a(3)}\left(R_{i j}\right)\right]^{2}\right\} \rho_{i}^{(0)}}{\sum_{j \neq i} S_{i j}\left(t_{\tau_{j}}^{(3)}\right)^{2} \rho_{\tau_{j}}^{a(0)}\left(R_{i j}\right)}
\end{aligned}
$$

$\rho_{\tau_{j}}^{a(h)}\left(h=0,1,2\right.$, and 3) indicate the atomic electron densities from atom of element type ${ }^{\tau_{j}}$ at site $j$ at distance $R_{i j}$ from site $i . R_{i j}^{\alpha}, R_{i j}^{\beta}$, and $R_{i j}^{\gamma}$ represent the $\alpha, \beta$, and $\gamma$ components of the distance vector between atoms at sites $i$ and $j$, respectively, and $t_{\tau_{j}}^{(h)}(h=1,2$, and 3$)$ are adjustable elementdependent parameters. The equivalence between these expressions and an expansion in Legendre polynomials has been discussed previously (Baskes, 1992), and $\rho_{i}^{(h)}(h=0,1,2$, and 3 ) can be thought of as an expansion of the atomic electron density into $s, p, d$, and $f$ orbital functions. As above, we have carefully denoted the element types of the atoms and also separated the screening from the atomic electron densities. Note that Equation $3 a$ is the simple linear superposition of atomic densities of the EAM formalism, and Equations $3 b-3 d$ reduce to more familiar forms in the original MEAM paper by Baskes (1992) for a single-component system. The above partial electron densities can be combined in different ways to give the total background electron density at site ${ }^{i}\left(\bar{\rho}_{i}\right)$. Here, we adopt one of the most widely used forms, which is given by

$$
\begin{aligned}
& \bar{\rho}_{i}=\rho_{i}^{(0)} G\left(\Gamma_{i}\right), \\
& G\left(\Gamma_{i}\right)= \begin{cases}\sqrt{1+\Gamma_{i}} & \text { if } \Gamma_{i} \geq-1, \\
-\sqrt{1+\Gamma_{i} \mid} & \text { if } \Gamma_{i}<-1,\end{cases} \\
& \Gamma_{i}=\sum_{h=1}^{3} t_{i}^{-(h)}\left[\frac{\rho_{i}^{(h)}}{\rho_{i}^{(0)}}\right]^{2}, \\
& t_{i}^{(h)}=\frac{1}{\rho_{i}^{(0)}} \sum_{j \neq i} t_{\tau_{j}}^{(h)} \rho_{\tau_{j}}^{a(0)} S_{i j} .
\end{aligned}
$$


In the absence of angular contributions to the density, $\Gamma_{i}=0, G\left(\Gamma_{i}\right)=1$, and the model reduces to the EAM formalism. For systems with negative $t_{\tau_{j}}^{(h)}$ values in certain geometries, $\Gamma_{i}<-1$, and for computational convenience we perform an analytic continuation of $G\left(\Gamma_{i}\right)$. We choose to do this by allowing $G\left(\Gamma_{i}\right)$ and hence $\bar{\rho}_{i}$ to become less than zero. This non-physical region is avoided by making the embedding function large (see Equation 4).

If we apply Equations 6 and 8 to the equilibrium reference structure, we obtain

$$
\begin{aligned}
& \bar{\rho}_{\tau}^{0}=Z_{\tau}^{0} \rho_{\tau}^{0} G\left(\Gamma_{\tau}^{0}\right), \\
& \Gamma_{\tau}^{0}=\sum_{h=1}^{3} t_{\tau}^{(h)} s_{\tau}^{(h)}\left(\frac{1}{Z_{\tau}^{0}}\right)^{2}
\end{aligned}
$$

In Equation $8 \rho_{\tau}^{0}$ is an element-dependent electron density scaling factor, and $Z_{\tau}^{0}$ is the first nearestneighbor coordination number of the reference structure. $s_{\tau}^{(h)}(h=1,2$, and 3) are "shape factors" that depend on the reference structure for element type $\tau$. The shape factors are given in the original MEAM paper by Baskes (1992). The atomic electron density for element type $\tau$ is calculated from

$$
\rho_{\tau}^{a(h)}(R)=\rho_{\tau}^{0} e^{-\beta_{\tau}^{(h)}\left(\frac{R}{R_{\tau}^{0}}-1\right)}
$$

where $\beta_{\tau}^{(h)}$ are adjustable element-dependent parameters, and $R_{\tau}^{0}$ is the nearest-neighbor distance in the equilibrium reference structure for the element type $\tau$.

The pair interaction for like atoms of element type $\tau$ can be calculated using a first nearest-neighbor $(1 N N)$ or second nearest-neighbor (2NN) formalism. Here, the $1 \mathrm{NN}$ is given by

$\phi_{\tau \tau}(R)=\frac{2}{Z_{\tau}^{0}}\left\{E_{\tau}^{u}(R)-F_{\tau}\left[\bar{\rho}_{\tau}^{r e f}(R)\right]\right\}$

In this equation $\bar{\rho}_{\tau}^{\text {ref }}(R)$ is the background electron density in the reference structure evaluated from Equations 6-9 at a nearest-neighbor distance of $R$ and is given by

$\bar{\rho}_{\tau}^{r e f}(R)=Z_{\tau}^{0} \rho_{\tau}^{0} G\left(\Gamma_{\tau}^{r e f}\right)$ 
$\Gamma_{\tau}^{r e f}=\sum_{h=1}^{3} t_{\tau}^{(h)} s_{\tau}^{(h)}\left(\frac{\rho_{\tau}^{a(h)}}{Z_{\tau}^{0} \rho_{\tau}^{a(0)}}\right)^{2}$,

and $E_{\tau}^{u}(R)$ is the universal equation of state (UEOS) of Rose, et al. (1984) for element type $\tau$ given by

$$
\begin{aligned}
& E_{\tau}^{u}(R)=-E_{\tau}^{0}\left[1+a^{*}+\delta \frac{R_{\tau}^{0}}{R}\left(a^{*}\right)^{3}\right] e^{-a^{*}}, \\
& a^{*}=\alpha_{\tau}^{0}\left(\frac{R}{R_{\tau}^{0}}-1\right), \\
& \delta= \begin{cases}\delta_{\tau}^{r} \quad \text { if } a^{*}<0, \\
\delta_{\tau}^{a} \quad \text { if } a^{*} \geq 0,\end{cases} \\
& \alpha_{\tau}^{0}=\sqrt{\frac{9 K_{\tau}^{0} \Omega_{\tau}^{0}}{E_{\tau}^{0}}, \text { or }} \\
& \alpha_{\tau}^{0}=\sqrt{\frac{k_{\tau}^{0}}{E_{\tau}^{0}} R_{\tau}^{0}} .
\end{aligned}
$$

In the above equations $\left.K_{\tau}^{0} \quad k_{\tau}^{0}\right)$ and $\Omega_{\tau}^{0}$ are the bulk modulus (spring constant) and the atomic volume of the reference structure, respectively, and $\delta$ is an adjustable, element-dependent parameter that has two components, repulsive $\delta_{\tau}^{r}$ and attractive $\delta_{\tau}^{a}$. Equation 19a is used when the reference structure is a three-dimensional (3D) crystal and Equation $19 \mathrm{~b}$ is used when the reference structure is a diatomic.

The pair interaction for unlike atoms of element types $\tau$ and $v$ is similarly obtained from the reference structure of the unlike atoms. For this work, the reference structure is taken as a diatomic, which gives

$\phi_{\tau v}(R)=\frac{1}{Z_{\tau v}^{0}}\left\{2 E_{\tau v}^{u}(R)-F_{\tau}\left[\bar{\rho}_{v}^{d}(R)\right]-F_{v}\left[\bar{\rho}_{\tau}^{d}(R)\right]\right\}$

where $Z_{\tau v}^{0}=1$ is the coordination number for the diatomic and

$\bar{\rho}_{\tau}^{d}(R)=\rho_{\tau}^{a(0)} G\left(\Gamma_{\tau}^{d}\right)$ 
$\Gamma_{\tau}^{d}=\sum_{h=1}^{3} t_{\tau}^{(h)} s_{d}^{(h)}\left(\frac{\rho_{\tau}^{a(h)}}{\rho_{\tau}^{a(0)}}\right)^{2}$

where the shape factors $S_{d}^{(h)}$ are those for a diatomic. The UEOS $E_{\tau v}^{u}$ is given by Equations 16-19 using parameters $E_{\tau v}^{0}, R_{\tau v}^{0}, k_{\tau v}^{0}, \delta_{\tau v}^{r}$, and $\delta_{\tau v}^{a}$.

The screening factor $S_{i j}$ is defined as the product of all screening factors $S_{i k j}$, where the interaction between atoms at sites $i$ and $j$ are screened by neighboring atoms at site $k$ as given by

$$
S_{i j}=\prod_{k \neq i, j} S_{i k j}
$$

If it is assumed that all three sites $i, j$, and $k$ lie on an ellipse on the $x y$-plane with sites $i$ and $j$ on the $x$-axis, the following equation can be derived:

$$
x^{2}+\frac{1}{C} y^{2}=\left(\frac{1}{2} R_{i j}\right)^{2}
$$

where

$$
C_{i k j}=\frac{2\left(X_{i k}+X_{k j}\right)-\left(X_{i k}-X_{k j}\right)^{2}-1}{1-\left(X_{i k}-X_{k j}\right)^{2}}
$$

In the above equation $X_{i k}=\left(\frac{R_{i k}}{R_{i j}}\right)^{2} X_{k j}=\left(\frac{R_{k j}}{R_{i j}}\right)^{2}$. The screening factor $S_{i k j}$ for like atoms is defined as

$$
S_{i k j}=f_{c}\left(\frac{C_{i k j}-C_{\min }\left(\tau_{i}, \tau_{j}, \tau_{k}\right)}{C_{\max }\left(\tau_{i}, \tau_{j}, \tau_{k}\right)-C_{\min }\left(\tau_{i}, \tau_{j}, \tau_{k}\right)}\right),
$$

where $C_{\min }\left(\tau_{i}, \tau_{j}, \tau_{k}\right)$ and $C_{\max }\left(\tau_{i}, \tau_{j}, \tau_{k}\right)$ determine the extent of screening of atoms of element type $\tau$ at sites $i$ and $k$ by an atom at site ${ }^{j}$. Similar expressions can be written for the screening of unlike atoms. The smooth cutoff function $f_{c}$ is defined as 
$f_{c}(x)=\left\{\begin{array}{lr}1 & x \geq 1 \\ {\left[1-(1-x)^{4}\right]^{2}} & 0<x<1 \\ 0 & x \leq 0\end{array}\right.$

$S_{i j}=1$ means that the interaction between atoms at sites $i$ and $j$ is not screened, while $S_{i j}=0$ means it is completely screened.

The MEAM formalism presented in Equations 3-27 requires 17 independent model parameters for each element type $\tau$, namely, $E_{\tau}^{0}, R_{\tau}^{0}, \alpha_{\tau}, \delta_{\tau}^{r}$, and $\delta_{\tau}^{a}$ for the universal equation of state (Equation 14); $\beta_{\tau}^{(0)}, \beta_{\tau}^{(1)}, \beta_{\tau}^{(2)}, \beta_{\tau}^{(3)}, t_{\tau}^{(1)}, t_{\tau}^{(2)}, t_{\tau}^{(3)}$, and $\rho_{\tau}^{0}$ for the electron densities (Equations 512); $A_{\tau}$ and $B_{\tau}$ for the embedding function $F_{\tau}$ (Equation 3); and $C_{\min }$ and $C_{\max }$ for the screening factor (Equations 23-27). In the current MEAM formalism, the parameter $\rho_{\tau}^{0}$ is taken to be 1 for one of the elements and only the ratios are used for the other ones. For a diatomic composed of elements $\tau$ and $v, 13$ independent parameters are required, i.e., $E_{\tau v}^{0}, R_{\tau v}^{0}, \alpha_{\tau v}^{0}, \delta_{\tau v}^{r}, \delta_{\tau v}^{a}$, four $C_{\min }$, and four $C_{\max }$ values.

We have parameterized elements carbon $(\mathrm{C})$, hydrogen $(\mathrm{H})$, and the diatomic $\mathrm{C}-\mathrm{H}$ for hydrocarbons with the reference structures of diamond for carbon $\left(Z_{C}^{0}=4\right)$, diatomic $H_{2}$ for hydrogen $\left(Z_{H}^{0}=1\right.$ ), and diatomic $\mathrm{C}-\mathrm{H}$ for hydrocarbons $\left(Z_{C H}^{0}=1\right.$ ), respectively. We fit the parameters to the experimental bond distances, bond angles, and atomization energies of a series of select alkane isomers from methane to $n$-octane. The energetics and structures of these molecules and MEAM predictions are given in Tables 3-7.

We used the element-dependent parameter ${ }^{B_{\tau}}$ (Equation 3) to resolve anomalous C-C and C-H interactions at short atomic distances that would lead to either extremely short equilibrium bond distances or to unphysical stable minimum structures in rare instances. The gauge transformation does not affect pure elements in the EAM formalism and has a small effect for elements and alloys at normal distances in the MEAM formalism.

The currently determined final set of MEAM parameters for $\mathrm{C}, \mathrm{H}$, and $\mathrm{C}-\mathrm{H}$ are given in Tables $\mathrm{I}$ and 2. We took the MEAM parameters for $C$ from Xiao et al. (2009) with the exception that we modified the nearest neighbor distance for $C$ in Table 1 from the value of $1.54 \AA$ used by Xiao et al. (2009) to obtain the correct $\mathrm{C}-\mathrm{C}$ bond distances in the alkane structures. We modified the MEAM parameters for $\mathrm{H}$ from those of Baskes (1992) and fit them to the first-principles data for the energy vs. intermolecular distance of $\mathrm{H}_{2}$ dimers reported in the work of Burton et al. (1982). We found the value of $\alpha_{H}^{0}$ parameter 
in the work of Baskes (1992) to be incorrect due to an error in the implementation of the equation for the diatomic force constant of the $\mathrm{H}-\mathrm{H}$ bond and has been corrected.

Table 1. Single element MEAM potential parameters for carbon and hydrogen with diamond and diatomic $\mathrm{H}_{2}$ reference structures, respectively. $E_{\tau}^{0}(\mathrm{eV})$ is the cohesive energy; $R_{\tau}^{0}(\AA)$ is the nearest neighbor distance in the equilibrium reference structure; $\alpha_{\tau}^{0}$ is the exponential decay factor for the universal energy of Rose et al. (1984); $A_{\tau}$ is the electron density scaling factor for the embedding function; $B_{\tau}$ is the gauge transformation parameter for the embedding function; $\rho_{\tau}^{0}$ is the electron density scaling factor; $\delta_{\tau}^{a}$ and $\delta_{\tau}^{r}$ are the attraction $\left(a^{*}>0\right)$ and repulsion $\left(a^{*} \leq 0\right)$ cubic terms for the universal equation of state; $\beta_{\tau}^{(0-3)}$ are the exponential decay factors for the atomic electron densities; ${ }_{\tau}^{(1-3)}$ are the weighting parameters for the atomic electron densities; and $C_{\min }$ and $C_{\max }$ are the screening parameters for three like atoms of the element $\tau$.

\begin{tabular}{|c|c|c|c|c|c|c|c|c|}
\hline Par. & $E_{\tau}^{0}$ & $R_{\tau}^{0}$ & $\alpha_{\tau}^{0}$ & $A_{\tau}$ & $B_{\tau}$ & $\rho_{\tau}^{0}$ & $\delta_{\tau}^{a}$ & $\delta_{\tau}^{r}$ \\
\hline C & 7.370 & 1.35 & 4.2000 & 2.46 & 0.00 & 1.00 & $\begin{array}{l}- \\
0.10\end{array}$ & 0.10 \\
\hline $\mathrm{H}$ & 2.363 & 0.74 & 2.0388 & 2.50 & 1.10 & 0.46 & 0.33 & 0.10 \\
\hline
\end{tabular}

\begin{tabular}{l|lllllllll}
\hline & $\beta_{\tau}^{(0)}$ & $\beta_{\tau}^{(1)}$ & $\beta_{\tau}^{(2)}$ & $\beta_{\tau}^{(3)}$ & $t_{\tau}^{(1)}$ & $t_{\tau}^{(2)}$ & $t_{\tau}^{(3)}$ & $C_{\min }$ & $C_{\max }$ \\
& & & & & & & & & \\
& & & & & & & & & \\
\hline
\end{tabular}




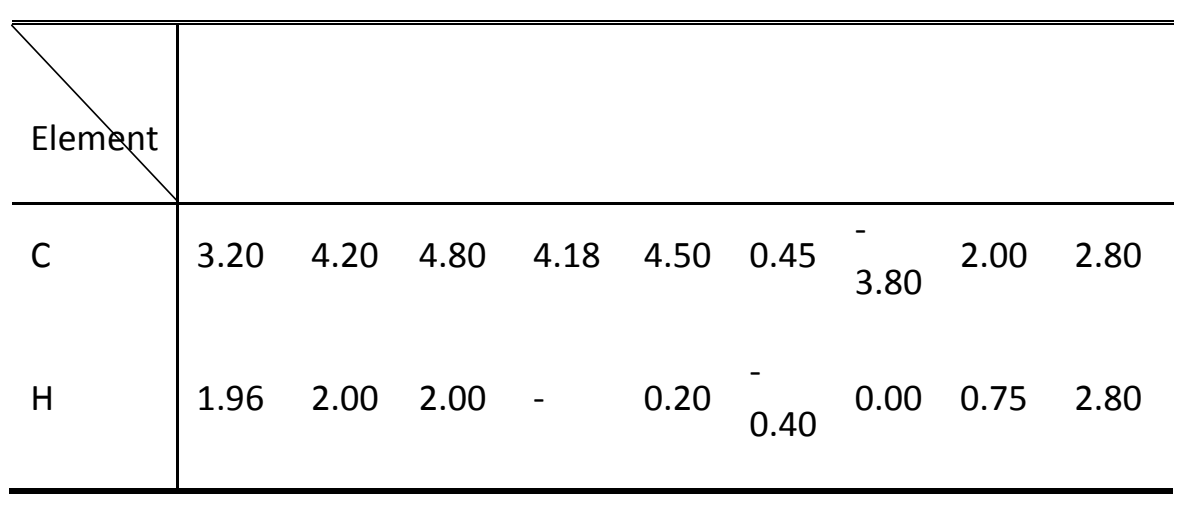

Table 2. MEAM interaction and screening parameters for the diatomic $\mathrm{C}-\mathrm{H} . R_{C H}^{0}(\AA)$ is the first nearest neighbor distance; $\alpha_{C H}^{0}$ is the exponential decay factor for the universal equation of state (UEOS) of Rose, et al. (1984); $E_{C H}^{0}(\mathrm{eV})$ is the cohesive energy; $\delta_{C H}^{a}$ and $\delta_{C H}^{r}$ are the attraction $\left(a^{*}>0\right.$ ) and repulsion ( $a^{*} \leq 0$ ) cubic terms for the UEOS, respectively; and $C_{\min }$ and $C_{\max }$ are the parameters for the screening factor. The middle atom screens the other two atoms.

\begin{tabular}{ll}
\hline \hline Parameter & Value \\
\hline$R_{C H}^{0}$ & 1.000 \\
$\alpha_{C H}^{0}$ & 3.151 \\
$E_{C H}^{0}$ & 2.100 \\
$\delta_{C H}^{a}$ & 0.10 \\
$\delta_{C H}^{r}$ & 0.10 \\
$C_{\text {min }}(C, C, H)$ & 0.48 \\
$C_{\text {max }}(C, C, H)$ & 2.50 \\
$C_{\text {min }}(C, H, C)$ & 2.00 \\
$C_{\text {max }}(C, H, C)$ & 2.80 \\
$C_{\text {min }}(C, H, H)$ & 1.50 \\
$C_{\text {max }}(C, H, H)$ & 2.00 \\
$C_{\text {min }}(H, C, H)$ & 0.53 \\
$C_{\max }(H, C, H)$ & 1.25 \\
\hline
\end{tabular}

We validated the $\mathrm{C}, \mathrm{H}$, and $\mathrm{C}-\mathrm{H}$ MEAM parameters derived for saturated hydrocarbons using a series of energy minimizations and molecular dynamics simulations. The experimental atomization 
energies at $0 \mathrm{~K}$ for select alkane molecules and radicals (Breener, 1990; Karton et al., 2009; Lide, 2009) are given in Table 3 along with the values calculated using the MEAM, REBO, and ReaxFF potentials. Energies of the starting molecular structures were minimized using the Polak-Ribiere conjugate gradient method. We took the parameters for $\mathrm{C}$ and $\mathrm{H}$ in REBO and ReaxFF from the work of Brenner, et al. (2002) and Chenoweth, et al. (2008) respectively, as distributed in the open-source large-scale atomic/molecular massively parallel simulator (LAMMPS) software package developed at Sandia National Laboratories (version April 20, 2012). The predictions of equilibrium bond distances and bond angles for the first five molecules in the alkane series are given in Tables 4 and 5-7, respectively, where the MEAM-, REBO-, and ReaxFF-calculated values are compared to the experimental data (Lide, 2009). The results in Tables 3-7 show an agreement between the MEAM predictions and the experimental data. Overall, the MEAM potential gives comparable or more accurate atomization energies and equilibrium geometries, when compared to the REBO and ReaxFF results.

To further validate the MEAM potential for saturated hydrocarbons, we ran dynamics simulation for a methane system composed of 500 atoms (100 methane molecules) under an NVT (constant number of atoms, $\mathrm{N}$; constant volume, $\mathrm{V}$; constant temperature, $\mathrm{T}$ ) ensemble for a total simulation time of $50 \mathrm{ps}$ at a temperature of $298.15 \mathrm{~K}$ and density of $0.401 \mathrm{~g} / \mathrm{cc}$. We built the starting structures in Accelrys ${ }^{\circledR}$ Materials Studio ${ }^{\circledR}$ software and relaxed them using the Polak-Ribiere conjugate gradient method for 1000 iterations. Then we ran the dynamics simulation and compared the pressure-volumetemperature (PVT) relationship obtained after time-averaging the temperature and pressure data of the last $10 \mathrm{ps}$ of the simulation to the experimental data reported in the work of Cristancho, et al. (2009). There was a great match in the data (162.4 MPa final pressure calculated by MEAM vs. the experimental value of $188 \mathrm{MPa}$ ). The results are shown in Figure 30.

Table 3. Atomization energies at $0 \mathrm{~K}$ of select hydrocarbons in a series of alkanes from methane to $n$-octane, cycloalkanes, and radicals. The energies for the first eight alkanes, all cycloalkanes, and all radicals (except for $\mathrm{CH}$ ) are experimental values reported in the work of Brenner (1990). For the remaining structures, the experimental results are taken from the work of Karton, et al. (2009) except for the $\mathrm{CH}$ radical, which is taken from Lide (2009). REBO, and ReaxFF atomization energies were calculated using the $\mathrm{C}$ and $\mathrm{H}$ parameters in the work of Brenner, et al. (2002) and Chenoweth, et al. (2008) as distributed within LAMMPS software (version April 20, 2012).

\begin{tabular}{lllll}
\hline \multirow{2}{*}{ Molecule } & \multicolumn{5}{l}{ Atomization energy at $0 \mathrm{~K}(\mathrm{eV})$} \\
\cline { 2 - 5 } & Exp. & MEAM & REBO & ReaxFF \\
\hline Alkanes & & & & \\
methane & 17.600 & 16.895 & 18.185 & 19.922 \\
ethane & 29.700 & 30.024 & 30.846 & 34.157 \\
propane & 42.000 & 41.877 & 43.589 & 48.315 \\
\hline
\end{tabular}




\begin{tabular}{lllll}
\hline$n$-butane & 54.300 & 53.738 & 56.332 & 62.487 \\
isobutane & 54.400 & 53.888 & 56.331 & 62.499 \\
$n$-pentane & 66.600 & 65.598 & 69.076 & 76.656 \\
isopentane & 66.600 & 65.477 & 69.073 & 76.673 \\
neopentane & 66.700 & 66.291 & 69.061 & 77.057 \\
n-hexane & 76.940 & 77.457 & 81.819 & 90.824 \\
isohexane & 76.990 & 77.417 & 81.817 & 90.823 \\
3-methylpentane & 76.970 & 77.061 & 81.817 & 90.776 \\
2,3-dimethylbutane & 76.990 & 77.038 & 81.815 & 90.473 \\
neohexane & 77.080 & 77.609 & 81.804 & 91.115 \\
n-heptane & 88.960 & 89.317 & 94.562 & 104.993 \\
isoheptane & 89.010 & 89.282 & 94.560 & 104.988 \\
neoheptane & 89.130 & 89.447 & 94.547 & 105.359 \\
$n$-octane & 100.970 & 101.176 & 107.306 & 119.162
\end{tabular}

Cycloalkanes

$\begin{array}{lllll}\text { cyclopropane } & 35.800 & 34.196 & 36.889 & 41.243 \\ \text { cyclobutane } & 48.200 & 46.514 & 49.898 & 55.700 \\ \text { cyclopentane } & 61.400 & 58.557 & 63.643 & 70.468 \\ \text { cyclohexane } & 73.600 & 71.281 & 76.460 & 84.911\end{array}$

Radicals

\begin{tabular}{lllll}
$\mathrm{CH}$ & 3.510 & 5.533 & 4.526 & 5.105 \\
$\mathrm{CH}_{2}$ & 7.800 & 9.729 & 8.469 & 10.062 \\
$\mathrm{CH}_{3}$ & 12.700 & 13.374 & 13.375 & 15.150 \\
\hline
\end{tabular}




\begin{tabular}{lllll}
\hline $\mathrm{C}_{2} \mathrm{H}$ & 12.200 & 16.325 & 11.752 & 19.455 \\
$\mathrm{H}_{2} \mathrm{C}_{2} \mathrm{H}$ & 18.900 & 21.879 & 19.402 & 23.047 \\
$\mathrm{H}_{3} \mathrm{C}_{2} \mathrm{H}_{2}$ & 25.700 & 27.389 & 26.588 & 29.545 \\
\hline
\end{tabular}

Table 4. Average equilibrium bond distances for select alkanes calculated by energy minimization of the molecular structures using the MEAM, REBO, and ReaxFF potentials and compared to the experimental data (Lide, 2009).

\section{Bond distance $(\AA)$}

\begin{tabular}{lcccccccc} 
Molecule & C-H & \multicolumn{7}{c}{ C-C } \\
& \multicolumn{1}{c}{ Exp. } & MEAM & REBO & ReaxFF & Exp. & MEAM & REBO & ReaxFF \\
\cline { 2 - 8 } & & & & & & & & \\
\hline methane & 1.087 & 1.080 & 1.090 & 1.100 & - & - & - & - \\
ethane & 1.094 & 1.140 & 1.090 & 1.090 & 1.535 & 1.510 & 1.550 & 1.580 \\
propane & 1.107 & 1.145 & 1.090 & 1.095 & 1.532 & 1.520 & 1.540 & 1.570 \\
$n$-butane & 1.117 & 1.144 & 1.090 & 1.096 & 1.531 & 1.530 & 1.540 & 1.573 \\
isobutane & 1.113 & 1.143 & 1.090 & 1.100 & 1.535 & 1.520 & 1.540 & 1.530 \\
\hline
\end{tabular}

Table 5. Average $\mathrm{H}-\mathrm{C}-\mathrm{H}$ equilibrium bond angles for select alkanes calculated by MEAM, REBO, and ReaxFF potentials in comparison to the experimental data (Lide, 2009).

\begin{tabular}{lllll}
\hline Molecule & Exp. & MEAM & REBO & ReaxFF \\
& & & & \\
\hline methane & 109.47 & 109.50 & 109.50 & 109.50 \\
ethane & 107.70 & 107.80 & 108.50 & 107.80 \\
propane & 107.00 & 107.51 & 108.33 & 107.17 \\
$n$-butane & N/A & 107.40 & 108.20 & 107.00 \\
\hline
\end{tabular}




\begin{tabular}{lllll}
\hline isobutane $\quad \mathrm{N} / \mathrm{A}$ & 107.60 & 108.50 & 107.20
\end{tabular}

Table 6. Average $\mathrm{H}-\mathrm{C}-\mathrm{C}$ equilibrium bond angles for select alkanes calculated by MEAM, REBO, and ReaxFF potentials in comparison to the experimental data (Lide, 2009).

\begin{tabular}{lllll}
\hline Molecule & Exp. & MEAM & REBO & ReaxFF \\
\hline methane & - & - & - & - \\
ethane & 111.17 & 111.10 & 110.40 & 111.07 \\
propane & $\mathrm{N} / \mathrm{A}$ & 110.68 & 110.21 & 111.26 \\
$n$-butane & 111.00 & 110.36 & 110.08 & 111.25 \\
isobutane & 111.40 & 111.01 & 110.22 & 111.35 \\
\hline
\end{tabular}

Table 7. Average C-C-C equilibrium bond angles for select alkanes calculated by MEAM, REBO, and ReaxFF potentials in comparison to the experimental data (Lide, 2009).

\begin{tabular}{lllll}
\hline Molecule & Exp. & MEAM & REBO & ReaxFF \\
\hline methane & - & - & - & - \\
ethane & - & - & - & - \\
propane & 111.70 & 113.70 & 111.20 & 108.90 \\
$n$-butane & 113.80 & 113.50 & 110.90 & 107.10 \\
isobutane & 110.80 & 110.20 & 110.30 & 110.10 \\
\hline
\end{tabular}


a)

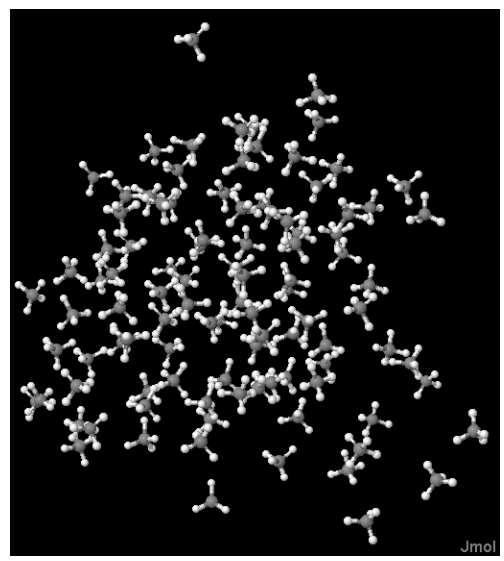

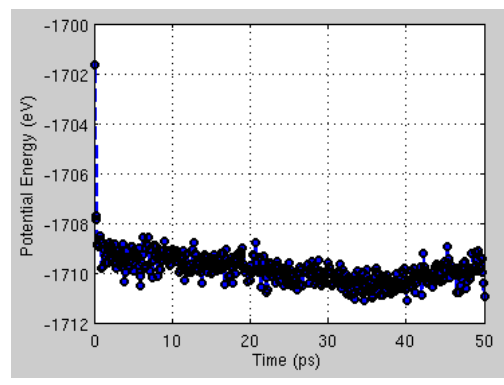

b)

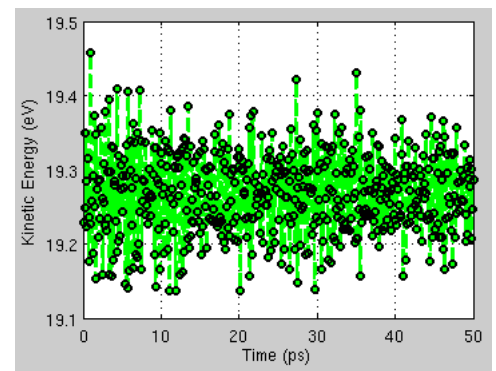

c)

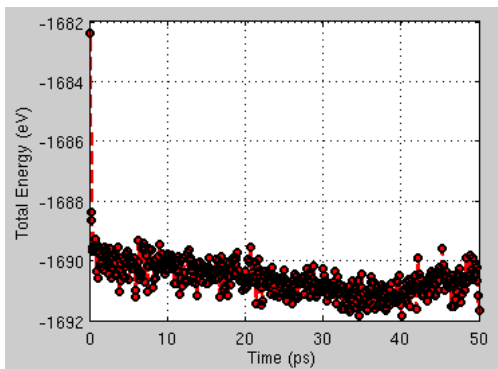

d)

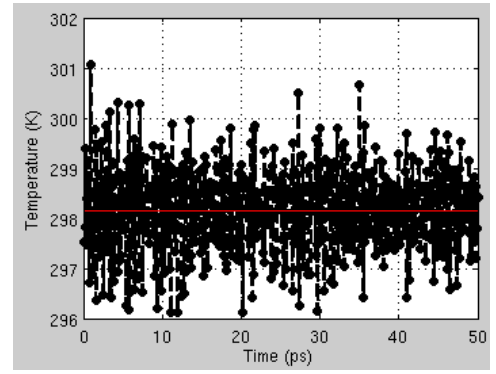

e)

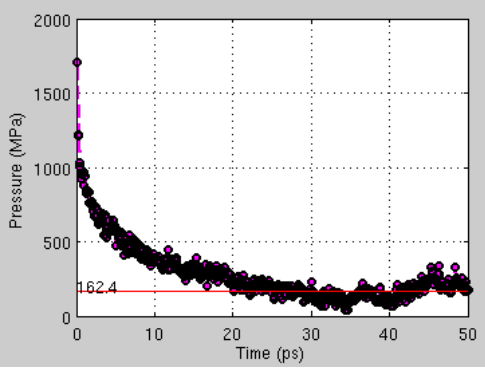

f)

Figure 30: a) Final snapshot of the simulation box containing 100 methane molecules simulated under NVT ensemble for $50 \mathrm{ps}$ at $298.15 \mathrm{~K}$ and density of $0.401 \mathrm{~g} / \mathrm{cc}$ (Box size:18.80 $\times 18.80 \times 18.80 \AA^{3}$ ); b)-d) Potential, kinetic, and total energy, respectively, as a function of time for the simulation period of $50 \mathrm{ps;}$ e)-f) Temperature and pressure as a function of time for the simulation period of $50 \mathrm{ps}$.

These results confirm the success of the MEAM potential in capturing the energetics, structures, and PVT behavior of alkanes. However, the parameters still need some adjustment to better capture the 
potential energies of dimers such as $\mathrm{H}_{2}, \mathrm{CH}$, and $\mathrm{C}_{2}$ and free radicals, which are essential for the full utility of the potential in molecular dynamics simulations.

\section{Publications}

\section{Journals:}

- M.A. Tschopp, J.L. Bouvard, D.K. Ward, D. Bammann, M.F. Horstemeyer, "Role of heat and pressure dissipation on the deformation response of amorphous polyethylene using molecular dynamics simulations," Macromolecules, in review.

- J.L. Bouvard, D. Francis, H. Brown, E.B. Marin, D.J. Bammann, M.F. Horstemeyer, "Development of a time and temperature dependent material model for amorphous polymers", International Journal of Plasticity, accepted.

- M. Lugo, J. E. Fountain, J. Hughes, J.-L. Bouvard, and M. F. Horstemeyer, "Microstructure-Based Fatigue Modeling of an Acrylonitrile Butadiene Styrene (ABS) Copolymer", Journal of Applied Polymer Science, in review.

\section{Conference Publications:}

- J.L. Bouvard, M.Tschopp, D. Bammann, M. Horstemeyer, "Multiscale Modeling of Amorphous Polymers: from Molecular Dynamic Simulations to Continuum Models," UNSSM, Minneapolis, July 2011.

- J.L. Bouvard, C. Bouvard, B. Denton, M.A. Tschopp, M.F. Horstemeyer, "Simulation of impact tests on polycarbonate at different strain rates and temperatures, "Proceedings of the 2011 Annual Conference on Experimental and Applied Mechanics, 3, 145-147, DOI: 10.1007/978-14419-9794-4, 2011.

- M. A. Tschopp, J.L. Bouvard, D.K. Ward, M.F. Horstemeyer, "Atomistic scale deformation mechanisms of amorphous polyethylene under tensile loading, " TMS Annual Meeting, 2, 789794, ISBN: 978-111802946-6, 2011.

- J.L. Bouvard, E.B. Marin, D.J. Bammann, M.F. Horstemeyer, "Formulation and Validation of a thermomechanical viscoelastic/viscoplastic constitutive model for amorphous glassy polymer, " Proceedings of the 2012 Annual Conference on Experimental and Applied Mechanics, 2012

\section{REFERENCES}

ABAQUS/Explicit, 2010, SIMULIA, Providence, RI

Allinger, N.L., Yuh, Y.H., Lii, J.H., 1989, “Molecular Mechanics. The MM3 Force Field for Hydrocarbons," J. Am. Chem. Soc. 111(23):8551. 
Allinger, N.L., Chen, K., Lii, J.H., 1996, "An Improved Force Field (MM4) for Saturated Hydrocarbons," J. Comput. Chem. 17 (5-6):642.

Baskes, M.I., 1987, "Application of the Embedded-Atom Method to Covalent Materials: A Semiempirical Potential for Silicon," Phys. Rev. Lett. 59 (23):2666.

Baskes, M.I., 1992, "Modified Embedded-Atom Potentials for Cubic Materials and Impurities," Phys. Rev. B 46 (5):2727.

Binder, K., 1995, "Monte Carlo and Molecular Dynamics Simulations in Polymer Science," Oxford University Press, New York.

Bouvard, J.L., Ward, D.K., Hossain, D., Marin, E.B., Bammann, D.J. Horstemeyer, M.F., 2010, “A General Inelastic Internal State Variables Model for Amorphous Glassy Polymers," Acta Mechanica, 213(1):71-96.

Bouvard, J.L., Francis, D.K., Tschopp, M.A., Marin, E.B., Bammann, D.J. Horstemeyer, M.F., 2012, “An Internal State Variable Material Model for Predicting the Time, Thermomechanical, and Stress State Dependence of Amorphous Glassy Polymers Under Large Deformation," International Journal of Plasticity, 10.1016/j.ijplas.2012.10.005.

Boyce, M.C., Montagut, E.L., Argon, A.S., 1992, "The Effect of Thermomechanical Coupling on the Cold Drawing Process of Glassy Polymers," Polymer Engineering and Science, 32(16):1073-1085.

Brenner, D.W., 1990, "Empirical Potential for Hydrocarbons for Use in Simulatingt Chemical Vapor Deposition of Diamond Films," Phys. Rev. B 42 (15):9458.

Brenner, D.W., Shenderova, O.A., Harrison, J.A., Stuart, S.J., Ni, B., Sinnott, S.B., 2002, "A SecondGeneration Reactive Empirical Bond Order (REBO) Potential Energy Expression for Hydrocarbons," J. Phys.: Condens. Matter 14:783.

Burton, P.G., Senff, U.E., 1982, "The $\left(\mathrm{H}_{2}\right)_{2}$ Potential Surface And The Interaction Between Hydrogen Molecules At Low Temperatures," J. Chem. Phys., 76(12):6073-6087.

Chenoweth, K., van Duin, A.C.T., Goddard III, W.A., 2008, "ReaxFF Reactive Force Field for Molecular Dynamics Simulations of Hydrocarbon Oxidation," J. Phys. Chem. A, 112(5):1040-1053.

Cristancho, D.E., Mantilla, I.D., Ejaz, S., Hall, K.R., Atilhan, M., Iglesia-Silva, G.A., 2009, "Accurate P $\rho$ T Data for Methane from (300 to 450) K up to 180 MPa," J. Chem. Eng. Data, 55(2):826-829.

Daw, M.S., Foiles, S.M., Baskes, M.I., 1993, "The Embedded-Atom Method: A Review of Theory and Applications," Mater. Sci. Rep., 9 (7-8):251.

Garg, M., Mulliken, A.D., Boyce, M., 2008, Temperature rise in polymeric materials during high rate deformation. Journal of Applied Mechanics 75, 011009 (8 pages). 
Karton, A., Gruzman, D., Martin, J.M.L., 2009, "Benchmark Thermochemistry of the $\mathrm{C}_{n} \mathrm{H}_{2 n+2}$ Alkane Isomers $(n=2-8)$ and Performance of DFT and Composite $A b$ Initio Methods for Dispersion-Driven Isomeric Equilibria," J. Phys. Chem. A, 113(29):8434-8447.

Kim, S.G., Horstemeyer, M.F., Baskes, M.I., Rais-Rohani, M., Kim, S., Jelinek, B., Houze, J., Moitra, A., Liyanage, L., 2009, "Semi-Empirical Potential Methods for Atomistic Simulations of Metals and Their Construction Procedures," J. Eng. Mater. Technol. 131:041210.

Lerch, V., Gary, G., Herve, P., 2003, "Thermomechanical Properties of Polycarbonate Under Dynamic Loading," J. Phys. IV, 110:159-164.

Li, Z., and Lambros, J., 2001, "Strain Rate Effects on the Thermomechanical Behavior of Polymers," Int. J. Solids Struct., 38, pp. 3549-3562.

Lide, D.R., 2009, "CRC Handbook of Chemistry and Physics: A Ready-Reference Book of Chemical and Physical Data, CRC Press.

Mayo, S.L., Olafson, B.D., Goddard III, W.A., 1990, "DREIDING: A Generic Force Field for Molecular Simulations," J. Phys. Chem., 94:8897-8909.

Plaseied, A., and Fatemi, A., "Strain Rate and Temperature Effects on Tensile Properties and Their Representation in Deformation Modeling of Vinyl Ester Polymer," International Journal of Polymeric Materials, 57, 463-479, 2008.

Plaseied, A., and Fatemi, A., "Tensile Creep and Deformation Modeling of Vinyl Ester Polymer and Its Nanocomposite," Journal of Reinforced Plastics and Composites, 28, 1775, 2009.

Plaseied, A., and Fatemi, A, "Deformation Response and Constitutive Modeling of Vinyl Ester Polymer Including Strain Rate and Temperature Effects," Journal of Materials Science, 43, 1191-1199, 2008.

Richeton, J., Ahzi, S., Daridon, L., and Remond, Y., 2006, A formulation of the cooperative model for the yield stress of amorphous polymers for a wide range of strain rates and temperatures, Polymer, 46, 6035-6043.

Richeton, J., Ahzi, S., Vecchio, K. S., Jiang, F. C., and Makradi A., 2007, Modeling and validation of the large deformation inelastic response of amorphous polymers over a wide range of temperatures and strain rates, International Journal of Solids and Structures, 44, 7938-7954.

Rittel, D., 1999, "On the Conversion of Plastic Work to Heat During High Strain Rate Deformation of Glassy Polymers," Mech. Mater., 31, pp. 131-139.

Rose, J.H., Smith, J.R., Guinea, F., Ferrante, J., 1984, "Universal Features of the Equation of State of Metals," Phys. Rev. B, 29(6):2963. 
Shepherd, J.E., McDowell, D.L., Jacob, K.I., 2006, "Modeling Morphology Evolution and Mechanical Behavior During Thermo-Mechanical Processing of Semi-Crystalline Polymers," J. Mech. Phys. Solids, 54(3):467-489.

Sun, H., 1998, "COMPASS: An Ab Initio Force-Field Optimized for Condensed-Phase Applications Overview with Details on Alkane and Benzene Compounds," J. Phys. Chem. B, 102(38):7338.

Tomita, Y., 2000, “Constitutive Modelling of Deformation Behavior of Glassy Polymers and Applications," Int. J. Mech. Sci., 42(8):1455-1469.

van Duin, A.C.T., Dasgupta, S., Lorant, F., Goddard III, W.A., 2001, "ReaxFF: A Reactive Force Field for Hydrocarbons," J. Phys. Chem. A, 105(41):9396.

Xiao, W., Baskes, M.I., Cho, K., 2009, "MEAM Study of Carbon Atom Interaction with Ni Nano Particle," Surf. Sci., 603(13):1985-1998.

Yashiro, K., Ito, T., Tomita, Y., 2003, "Molecular Dynamics Simulation of Deformation Behavior in Amorphous Polymer: Nucleation of Chain Entanglements and Network Structure Under Uni- Axial Tension," Int. J. Mech. Sci., 45(11):1863-1876.

\section{KEY WORDS}

Automotive thermoplastics, Resins, Multiscale Modeling, Finite Element Modeling

\section{BRIEF Description OF REPORT}

A physically based material model is developed to predict the non-linear mechanical response of polyemrs. 


\section{Subtask 3.2: Structural Nanocomposites}

Team Members: T.E. Lacy, H. Toghiani, C.U. Pittman, Jr., J. Yu (postdoc), S. Nouranian (postdoc), C. Jang (PhD student), J. Nall (undergraduate student)

\section{Objective}

- Design of low-cost nanoreinforced composite systems for automotive structural applications.

\section{APPROACH}

- A multiscale design methodology is being employed to investigate the effects of nanoreinforcements on the mechanical properties of fiber-reinforced composites for automotive structural applications. This work addresses the following critical issues: elevated temperature nanocomposite creep testing and development of a multiscale materials modeling strategy for assessing high-performance nanocomposites subjected to static and dynamic loadings.

\section{Milestone, Metrics and Accomplishments}

- An experimental investigation of the creep behavior of vapor grown carbon nanofiber (VGCNF)/vinyl ester (VE) nanocomposites was performed in order to assess the effect of varying amounts of nanofibers on time dependent material behavior over a range of hold stresses and operating temperatures.

- A synergistic multiscale modeling approach has been developed using molecular dynamics, micromechanics, and progressive failure simulations to predict the effective properties and nonlinear material response of structural nanocomposites.

\section{INTRODUCTION}

Determination of length- and temporal-scale dependent structure-property relationships for polymers (Shonaike, 2003), polymer composites (Chasiotis et al., 2005), and polymer nanocomposites (Thostenson, 2006) is increasingly becoming a focal point in multiscale materials modeling and simulation efforts. Development of a rational integrated multiscale analysis framework is vital for assessing the effect of lower length scale features in polymers (Fermeglia and Pricl, 2007; Valavala et al., 2007; Bouvard et al., 2009) and nanoscale reinforcements in polymer nanocomposites on micro-, meso-, and macroscale material behavior (Tadmor et al., 2000; Gates et al., 2005; Odegard et al., 2005; Buryachenko et al., 2005; Zeng et al. 2008; Maiti, 2008). This is especially true when characterizing microscale phenomena that are difficult to physically observe and/or measure. The efficient transfer of scale-specific model data in calculations performed at successively higher or lower length scales is one key challenge in multiscale composites modeling (Zeng et al., 2008). Establishment of validated "handshake" protocols linking calculations performed at disparate spatial or temporal scales has posed a serious obstacle in multiscale material model development for polymer nanocomposites (Zeng et al., 
2008). These challenges are chiefly due to the complex nanocomposite morphologies and poor knowledge about the key mechanisms influencing the material behavior at different time and length scales. Establishment of a viable Integrated Computational Materials Engineering (ICME) framework for polymer composite design and analysis necessitates seamless integration between high fidelity scalespecific models.

In this work, key issues in the multiscale modeling of thermoset nanocomposites were addressed, with a focus on thermoset vinyl ester (VE) matrices reinforced with pristine and oxidized vapor-grown carbon nanofibers (VGCNFs). VGCNF/VE nanocomposites are relatively low-cost material systems with promising mechanical properties for use in nano-enhanced continuous fiber structural composites (Plaseied et al., 2008; Plaseied and Fatemi, 2009; Hutchins et al., 2009; Nouranian et al., 2011a). A combination of molecular dynamics (MD) simulations, micromechanics, and hybrid finite element calculations are used to determine nanocomposite material behavior spanning the molecular, nano-, micro-, meso-, and macroscales (Figure 1). Because of the relatively high surface-area-to-volume ratio associated with nanoreinforcements, MD simulations were performed to investigate the formation of a distinct interphase region at the VGCNF/VE interface. Nanoreinforcements were mixed with a liquid resin and then the resin is cured to form the nanocomposite. Prior to curing, the redistribution of liquid monomers (characterized in terms of the local monomer mole ratio) can occur near the nanoreinforcement's surface, which can generate an "interphase" region (Jancar, 2009) in the cured nanocomposite. MD simulations were used to generate the crosslinked resin network employing knowledge of monomer reactivity ratios, chemical regio-selectivity, and growing radical concentrations (Jang et al., 2012c). Then, the VGCNF/VE interphase properties and interfacial strengths were estimated based upon VGCNF pull-out simulations (Jang et al., 2012b). Using such an approach, the resin mechanical and dynamic mechanical properties may be calculated (Ray, 1988; Brown and Clarke, 1998; Qi et al., 2005; Jang et al., 2012c). 


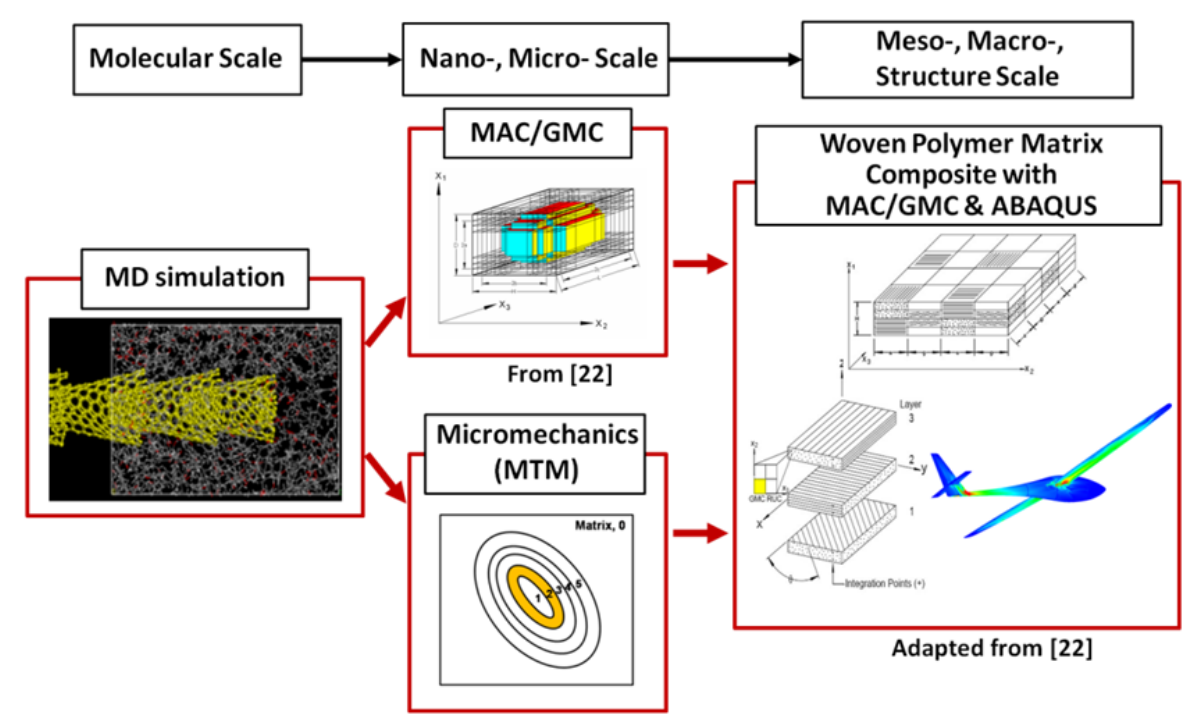

Figure 1: Multiscale modeling in composites (Adapted in part from (Arnold et al., 1999)).

Appropriately averaged VGCNF/VE interphase properties and interfacial strengths developed using MD simulations can be employed in analytic and/or computational micromechanics models aimed at determining effective material properties of VGCNF/VE nanocomposites. A parametric study investigating the effect of interphase properties on bulk nano-phased matrix properties was previously performed by Yu et al. (2011a). That study accommodated both hollow and wavy nanofibers. The NASA special-purpose composite failure analysis code, MAC/GMC (Micromechanics Analysis Code with the Generalized Method of Cells) (Arnold et al., 1999) is being used in conjunction with the ABAQUS (2009) finite element solver to simulate progressive failure of composites at the micro-, meso-, and macroscale in a temporally concurrent, spatially sequential fashion (Ricks et al., 2012).

\section{Experimental Structure-Property Relations for Nanoreinforced Materials}

A key aspect of this investigation is to establish structure property relationships for hybrid composites comprised of a nano-enhanced resin (i.e., VGCNF/VE) and traditional continuous fiber reinforcements. Several previous reports have examined the effect of fabrication and processing variables on the tensile, compressive, flexural, dynamic mechanical, and high strain rate behavior of VGCNF/VE nanocomposites at room temperature. In this report, additional experimental efforts investigating the effect of temperature and VGCNF weight fraction on nanocomposite tensile creep properties are presented in brief.

\section{Tensile Creep properties}

The tensile creep behavior of composites prepared from a VE resin (Derakane 441-400) with oxidized VGCNFs was characterized for nanocomposites containing 0.25 parts of fiber per hundred parts resin (phr), $0.5 \mathrm{phr}$, and $0.75 \mathrm{phr}$. These nanocomposites were fabricated by high-shear mixing, casting, and curing the test articles in an open-face mold under nitrogen for a prescribed heating and post-curing cycle. Short-term creep/creep-recovery experiments with an Instron 5869 model (electromechanical test frame, with a $50 \mathrm{kN}$ load cell) were conducted to obtain the viscoelastic response at two stress levels ( $60 \%$ and $70 \%$ of the ultimate tensile strength) for each VGCNF weight fraction and for each of 
four temperatures $\left(\sim 24{ }^{\circ} \mathrm{C}, 40{ }^{\circ} \mathrm{C}, 60^{\circ} \mathrm{C}\right.$, and $\left.80{ }^{\circ} \mathrm{C}\right)$. These temperatures are well below the glass transition temperature $\left(130-140^{\circ} \mathrm{C}\right)$ of the cured neat vinyl ester resin. A non-contact digital image correlation (DIC) technique was used to measure the creep strains in the longitudinal and transverse directions simultaneously using the LaVision StrainMaster system. A 14-bit DIC camera system with a 16 million pixel spatial resolution and a maximum sampling rate of $25 \mathrm{~Hz}$ was used to capture images during the loading, creep, and creep-recovery phases. Additionally, an Epsilon extensometer with a gagelength of $25.4 \mathrm{~mm}$ and travel distance of $6.35 \mathrm{~mm}$ was used to measure the axial strains and to verify the measured strains from the DIC system. In order to establish the hold stresses used in the creep experiments, quasi-static tensile tests were performed until fracture in accordance with ASTM D638 at a strain rate of $0.001 / \mathrm{s}$.

The creep compliances were calculated from the generalized 3-D viscoelastic constitutive equation with a Prony series representation. The constitutive relation for an isothermal isotropic linear viscoelastic material can be expressed as

$$
\varepsilon_{\underline{i i}}(t)=\sum_{j=1}^{3}\left\{C_{i i j j}^{0} \sigma_{j j}(t)+\sum_{n}^{N_{p r}}\left[\frac{C_{i i j j}^{n}}{\tau_{n}} \int_{0}^{t} e^{-\frac{t-t^{\prime}}{\tau_{n}}} \frac{\partial \sigma_{j j}\left(t^{\prime}\right)}{\partial t^{\prime}} d t^{\prime}\right]+\frac{C_{i i j j}^{N p r+1}}{\tau_{N p r+1}} \int_{0}^{t} \sigma_{j j}\left(t^{\prime}\right) d t^{\prime}\right\}
$$

The first term on the right hand side of the equality in Eq. (1) describes the instantaneous elastic response, the second term represents the steady state linear creep, and the last term represents the long-time creep response. In Eq. (1), $\varepsilon_{i i}$ is the strain-time history, $\sigma_{j j}$ is the load-time history, $\tau_{n}$ are the retardation times, $C_{n i i j j}$ are the Prony series coefficients, and $N_{p r}$ are the number of Prony coefficients. The underscored indices indicate no summations with the indices $i, j=1,2,3$ indicating the Cartesian longitudinal, transverse, and out-of-plane directions, respectively. In the present work, the last term was neglected since only short-term creep experiments were performed. The idealized constitutive response expressed in Eq. (1) assumes that the material is subjected to an instantaneous constant stress at zero time. However, the specimen actually undergoes a loading phase before achieving the steady-state creep loading. Hence, a loading function may be defined to account for the initial and subsequent steady-state creep loading using Eq. (2) where $H(t)$ is the Heaviside step function.

$\sigma_{11}(t)=\left\{\begin{array}{cl}f_{\sigma}(t) & 0 \leq \mathrm{t} \leq \mathrm{t}_{1} \text { (loading phase) } \\ \sigma_{0} H\left(t-t_{1}\right) & \mathrm{t} \geq \mathrm{t}_{1} \quad \text { (steady-state phase) }\end{array}\right.$

By using Eq. (2) in Eq. (1), the viscoelastic constitutive equation becomes 
$\varepsilon_{i i}(t)= \begin{cases}\sum_{j=1}^{3}\left\{C_{i i j j}^{0} \sigma_{i j}(t)+\sum_{n}^{N_{p r}}\left[C_{i i j j}^{*} \int_{0}^{t} e^{-\frac{t-t^{\prime}}{\tau_{n}}} f_{\sigma}\left(t^{\prime}\right) d t^{\prime}\right]\right\} & 0 \leq \mathrm{t} \leq \mathrm{t}_{1} \\ \sum_{j=1}^{3}\left\{C_{i i j j}^{0} \sigma_{i j}(t)+\left[\sum_{n}^{N_{p r}} C_{i i j j}^{*} \int_{0}^{t_{1}} e^{-\frac{t-t^{\prime}}{\tau_{n}}} f_{\sigma}\left(t^{\prime}\right) d t^{\prime}+\sum_{n}^{N_{p r}} C_{i i j j}^{*} \int_{t_{1}}^{t} e^{-\frac{t-t^{\prime}}{\tau_{n}}} \sigma_{j j}\left(t^{\prime}\right) d t^{\prime}\right]\right\} \mathrm{t}>\mathrm{t}_{1}\end{cases}$

where

$C_{i i 11}^{* n}=\frac{C_{i i 11}^{n}}{\tau_{n}}$

The linear least squares (LSQ) method is used to solve for the Prony coefficients (Eq. 4) which are subsequently used in the GKM Prony series representation for the creep compliance given in Eq. (5).

$C_{\underline{i} 11}^{*}(t)=C_{\underline{i} 111}^{0}+\sum_{n=1}^{N_{p r}} C_{\underline{i \underline{1} 11}}^{* n}\left[1-\exp \left(-\frac{t}{\tau_{n}}\right)\right]$,

where

$C_{\underline{i i 11}}^{0}=C_{\underline{i} 111}^{\infty}-\sum_{n}^{N_{p r}} C_{i i 11}^{n}$

is the instantaneous creep compliance.

The addition of the VGCNFs into the neat vinyl ester reduced the ultimate tensile strength (UTS) of the nanocomposites at low temperatures $\left(24^{\circ} \mathrm{C}\right.$ and $40{ }^{\circ} \mathrm{C}$ ). However, at the higher temperatures $(60$ ${ }^{\circ} \mathrm{C}$ and $80^{\circ} \mathrm{C}$ ), the nanocomposites developed a larger UTS than the VE polymer (Figure 1).

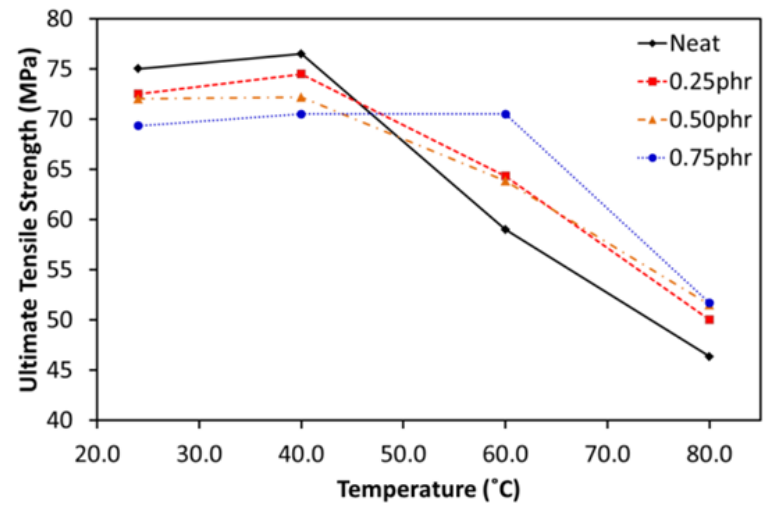

Figure 1: The ultimate tensile strength as a function of temperature for each VGCNF weight fraction. 
The nanocomposites showed a strong time dependency at the different temperatures and stress levels for each VGCNF weight fraction. At the low temperatures $\left(24^{\circ} \mathrm{C}\right.$ and $40{ }^{\circ} \mathrm{C}$ ), the creep strain decreased as the VGCNF weight fraction was increased. These results could be attributed to the relative stiffening effects of the VGCNFs (with weight fractions up to $0.75 \mathrm{phr}$ ) in the matrix at temperatures below $40{ }^{\circ} \mathrm{C}$. Additionally, the use of oxidized VGCNFs may have improved the adhesion between the fibers and the matrix, thereby increasing the stiffness of the nanocomposite. The oxidized VGCNFs contain surface functional groups that attract VE monomers to improve adhesion through hydrogen bonding and dipole-dipole attractions (Jang et al., 2012a). Cross-link density also has a significant effect on the creep rate at temperatures near the $T_{g}$ and for large constant stresses above the yield stress. As the degree of cross-linking increases in a polymer material, the creep rate, or overall magnitude of creep strain, decreases at above or near the $T_{g}$ (Nielsen, 1968). Attraction of the surface functional groups on oxidized VGCNF surfaces may lead to stronger surface adhesion which may reduce creep strains. At 60 ${ }^{\circ} \mathrm{C}$ (Figure 2), a decrease in the relative creep resistance with temperature was observed in the samples containing VGCNFs as compared to those of the neat vinyl ester polymer. This could be related to a decrease in the matrix-to-fiber adhesion as the temperature is increased. This type of adhesion is primarily attributed to non-bonded interactions such as hydrogen bonding, Van der Waals interactions, and dipole attractions (Lakshminarayanan et al., 2005; Rasheed et al., 2006). Enhanced molecular motion at higher temperatures reduces the overall contribution of these non-bonded attractive interactions, thereby reducing the interfacial shear strength and load transfer to the fibers. This reduction may tend to make the creep behavior of the samples with VGCNFs more similar to that of the neat VE polymer. Also, the interphase region between the matrix and the nanoreinforcement may have different material and mechanical properties than those of the bulk matrix (Jang et al., 2012a). Both this interphase region and the presence of nested VGCNF agglomerates may also be factors at higher temperatures in the overall deformation mechanisms. Some of these possibilities can be further investigated using creep testing at higher temperatures. Since the segmental motion of the polymer chains are activated at higher temperatures, these studies would involve temperatures greater than the $T_{g}$ of the polymer.
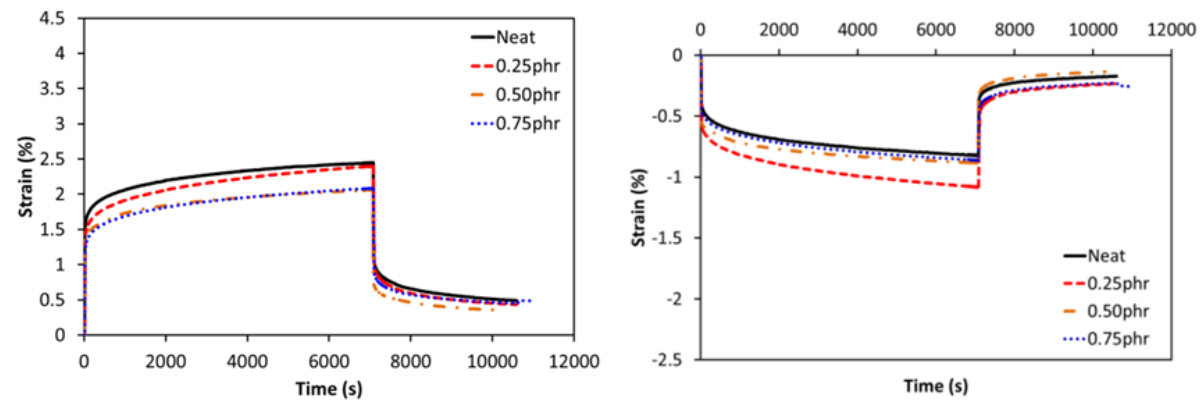

(a)

(b) 

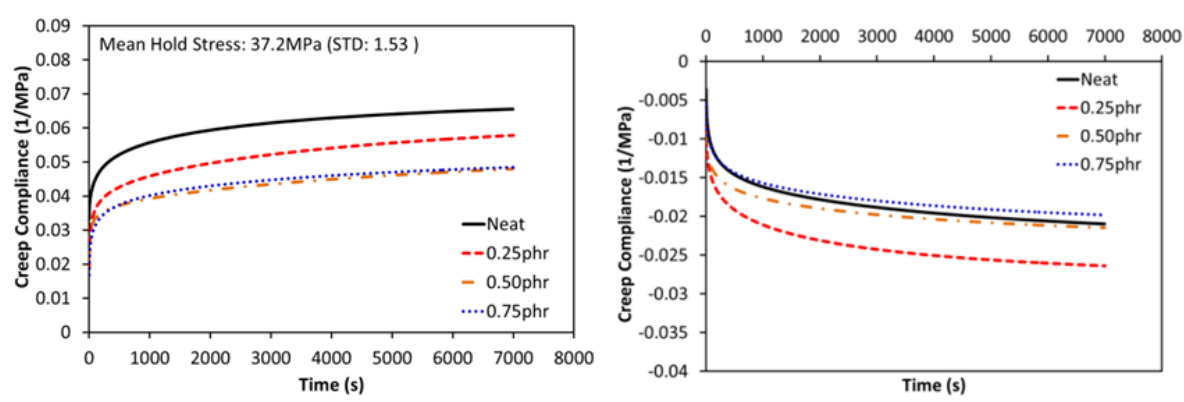

(c)

(d)

Figure 2: Creep at $60^{\circ} \mathrm{C}, 37 \mathrm{MPa}$ (STD 1.53) mean applied stress. (a) Longitudinal creep strain, (b) transverse creep strain, (c) longitudinal creep compliance, $C_{1111}$, and (d) transverse creep compliance, $C_{2211}$.

Results of this study indicate that for temperatures significantly below the $T_{g}$, the addition of VGCNFs in the 0.25 phr to 0.75 phr range in the VE resin reduces the creep response.

\section{Nanocomposite Multiscale Modeling}

\section{Molecular dynamics simulations of VGCNF/VE nanocomposites}

In continuation of the molecular dynamics (MD) simulation efforts, interfacial adhesion between graphene sheets and a cured VE resin was calculated. As shown previously (Nouranian et al., 2011b; Jang et al., 2012a), the formation of a polymer or resin interphase at and near carbon surfaces is a key issue because an interphase can modify carbon/resin interfacial bonding, which determines the load transfer capability between the matrix and the carbon reinforcement. In this work, commercial Derakane 441-400 VE resin (Ashland Co.) was modeled. It contains $33 \mathrm{wt} \%$ of the co-monomer styrene as a diluent to lower VE resin viscosity. Derakane 441-400 has an average of 1.62 bisphenol-A groups per monomer $(n=1.62)$ (Li, 1998) in the dimethacrylate backbone. We created VE resin models for $n=1$ and 2, and included them in the appropriate ratio to create an average $n=1.62$. For simplicity, these two dimethacrylate monomers are designated VE1 and VE2, corresponding to $n=1$ and 2, respectively.

A three dimensional (3D) periodic simulation cell size of $32 \times 32 \times 60 \AA^{3}$ was created as shown in Figure 3 , and three centrally located graphene sheets were positioned parallel to the $x y$ plane and were separated by $3.5 \AA$. VE resin monomers (styrene, VE1, and VE2) were randomly packed around both sides of the graphene sheets to fill the cell, yielding a final simulation cell density of $1.20 \mathrm{~g} / \mathrm{cm}^{3}$. The monomers occupied the volume on both sides of the three sheet graphene stack to a distance of $26 \AA$ from both sides of the sheet surfaces. 


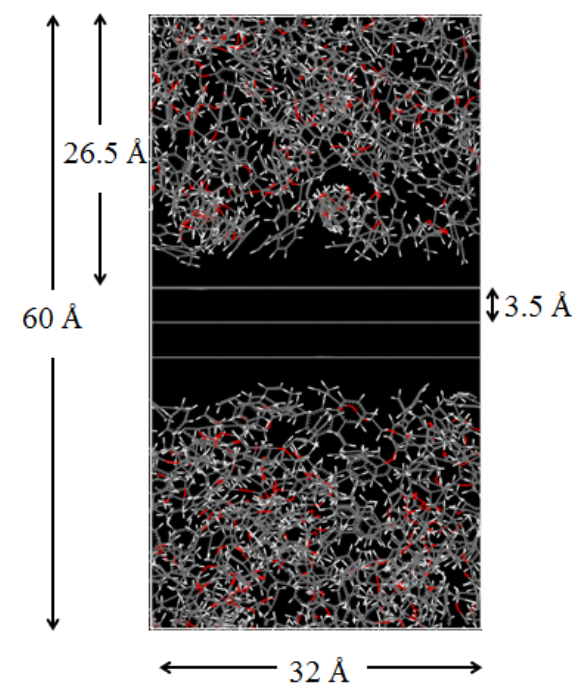

Figure 3: The initial 3D periodic unit cell is comprised of randomly packed vinyl ester monomers (styrene, VE1, and VE2) at a 104/12/20 mole ratio with the density of $1.02 \mathrm{~g} / \mathrm{cm}^{3}$ around both sides of the three layer graphene sheet.

Polymer interphase and interface formation near the graphene surfaces has been characterized using MD simulations. The monomers were equilibrated in the representative unit cell (RUC) until their distribution remained reasonably constant given random molecular fluctuations. A dimensionless relative concentration of specific types of monomer atoms was determined in the direction perpendicular to the graphene sheets within successive $1 \AA$ thick sub-volumes. This was done to define the mole ratios of monomers present versus distance from the surface (Nouranian et al., 2011b). The relative concentration is defined as the molar ratios of styrene, VE1, and VE2 in a given sub-volume divided by that same molar ratio present in the entire liquid resin. In Equation 7,

$$
C=\frac{N_{s u b}}{V_{s u b}} \cdot \frac{V_{t o t}}{N_{t o t}}
$$

$C$ is the relative concentration, $N_{\text {sub }}$ is the number of specific monomer atoms in the sub-volume, $V_{\text {sub }}$ is the sub-volume, $N_{\text {tot }}$ is the total number of monomer atoms in the liquid resin, and $V_{\text {tot }}$ is the total liquid resin volume in the simulation cell. Thus, a relative concentration of 1.0 within any sub-volume means that the ratios of the monomers (styrene/VE1, styrene/VE2, VE1/VE2) in that sub-volume are identical to those same molar ratios within the total liquid resin volume in the simulation cell.

After monomer equilibration, a crosslinked network was created using the RRV method (Jang et al., 2012b). The RRV method incorporates three critical features of any resin's curing: (1) regioselectivity (head-to-tail chain propagation) is enforced, and (2) the relative reactivity ratios $\left(r_{1}\right.$ and $\left.r_{2}\right)$ during polymerization of different monomers are applied, thereby (3) accounting for monomer concentration drift and causing the correct monomer sequence distributions to form within the polymer or resin. Since the VE resin contains both styrene and dimethacrylate monomers, different polymerizable functional 
groups exist. The terminal polymerization model was used where the assumption is made that the reactivity of a growing chain depends only on the reactivity of the end (terminal) monomer of the chain and not on the any other monomer or on the chain length (Odian, 1981; Brandrup \& Immergut, 1989). The terminal copolymerization model is expressed as

$$
\frac{d \mathrm{M}_{1}}{d \mathrm{M}_{2}}=\frac{\mathrm{M}_{1}\left(r_{1} \mathrm{M}_{1}+\mathrm{M}_{2}\right)}{\mathrm{M}_{2}\left(r_{2} \mathrm{M}_{2}+\mathrm{M}_{1}\right)}
$$

where $M_{1}$ and $M_{2}$ represent the concentration of styrene molecules and VE dimethacrylate molecules in the liquid styrene-containing VE resin, respectively. The reactivities of VE1 and VE2 were treated as equivalent since they have identical polymerizing methacrylate functional groups. The relative reactivity ratios, $r_{1}$ and $r_{2}$, are defined by the rate constants ratios $\left(r_{1}=k_{11} / k_{12}\right.$ and $\left.r_{2}=k_{22} / k_{21}\right)$. The rate constants $\left(k_{11}, k_{12}, k_{22}, k_{21}\right)$ are for the chain growth steps shown in Equation 9-12.

$$
\begin{aligned}
& \sim \mathrm{Sty}^{\bullet}+\mathrm{Sty} \rightarrow \sim \sim \text { Sty }-\mathrm{Sty}^{k_{11}} \\
& \sim \sim \mathrm{Sty}^{\bullet}+\mathrm{VE} \rightarrow \sim{ }^{k_{12}} \rightarrow \text { Sty }-\mathrm{VE}^{\bullet} \\
& \sim \mathrm{VE}^{\bullet}+\mathrm{VE} \rightarrow \sim \mathrm{VE}-\mathrm{VE}^{\bullet} \\
& \sim \mathrm{VE}^{\bullet}+\mathrm{Sty}^{k_{21}} \rightarrow \sim \mathrm{VE}-\mathrm{Sty}^{\bullet}
\end{aligned}
$$

In Equations 9 and 10, Sty ${ }^{\bullet}$ represents a growing styrene radical chain end. Likewise, in Equations 11 and $12, \sim \sim \mathrm{VE}^{\bullet}$ is a growing chain end with a VE radical. These four reactions determine the monomer incorporation rate into VE crosslinking network. The actual rate constants values are not required, since only the reactivity ratios appear in Equation 8. The $r_{1}$ and $r_{2}$ values used in this work were $r_{1}=0.485$ and $r_{2}=0.504$.

MD simulations were carried out using Accelrys Materials Studio v5.0 with the COMPASS force field. The system was initially minimized for 10,000 iterations. Then, the system was heated from $10 \mathrm{~K}$ to $1000 \mathrm{~K}$ in $50 \mathrm{~K}$ increments within the NVT ensemble (constant number of atoms, $N$; constant volume, $V$; and constant temperature, $T$ ). The dynamics simulations were run for $10 \mathrm{ps}$ with a $1 \mathrm{fs}$ time step (a total of 10,000 iterations) at all intermediate temperatures. At $1000 \mathrm{~K}$ the dynamics simulations were run for $7 \mathrm{~ns}$ with a $1 \mathrm{fs}$ time step to achieve equilibration. Once the system equilibration was achieved at 1000 $\mathrm{K}$, the system was cooled to room temperature $(300 \mathrm{~K})$ in $50 \mathrm{~K}$ decrements using the same procedure as 
during the heating phase. After cooling, the system was re-equilibrated at $300 \mathrm{~K}$ through $3 \mathrm{~ns}$ of dynamics simulation. Crosslinking simulations were performed using the RRV method after the system equilibrium. Several specific steps were employed: 1) Either a styrene or a VE monomer was randomly selected to start initiation of the cure by attaching a hydrogen atom to one tail atom in order to create a propagating free radical head. The reactive head atom then found a reactive styrene or VE tail atom within defined cutoff distances. A new bond was formed if either a styrene or a VE tail was present within their respective cutoff distances; 2 ) If more than one reactive tail atom was found within the reaction cutoff distances, scale factors were employed to determine which tail atom to bond to. The scale factors were used to account for the relative reactivity ratios. The two scale factors are 0.786 for the reaction cutoff distance for a Sty ${ }^{\bullet}$ addition to a styrene monomer's tail and 0.798 for the reaction cutoff distance for $\sim \mathrm{VE}^{\bullet}$ adding to a VE tail, respectively. Reaction rates depend on $k$ values and concentration. The relative concentrations of the monomers are present in RUC. The ratio $k_{11} / k_{12}$ is compensated for by adjusting the relative search volumes for these two processes to make their probabilities come out to enforce the proper $k_{11} / k_{12}$ values (e.g., probabilities of the relative monomer head to tail interactions are enforced by the search volume ratios). If $k_{11}$ is smaller than $k_{12}$, then the volume searched around a Sty ${ }^{\bullet}$ head, looking for a VE tail, must be larger than the volume searched for a Sty tail. Let $V_{11}$ be the spherical search volume for the $\sim$ Sty ${ }^{\bullet}$ to Sty reaction with reactivity $k_{11}$. Note that a sphere volume is given by $V=4 / 3\left(\pi R^{3}\right)$ and the relative reactivity related to the sphere volume is $r_{1}=k_{11} / k_{12}=V_{11} / V_{12}=0.485$,

$V_{11}=\frac{k_{11}}{k_{12}} V_{12}$.

The radii of each search volume are related by

$$
\begin{aligned}
& R_{11}^{3}=\frac{k_{11}}{k_{12}} R_{12}^{3},(14) \\
& R_{11}=\sqrt[3]{\frac{k_{11}}{k_{12}}} R_{12}=0.786 \cdot R_{12}
\end{aligned}
$$

where $R_{11}$ is the $\sim \sim \mathrm{Sty}^{\bullet}$ head to Sty tail reactive radius, and $R_{12}$ is the $\sim \sim \mathrm{Sty}^{\bullet}$ to VE tail reactive radius; 3 ) When no monomer tail atoms were found within the cutoff distances from the growing chain's head, the system was then relaxed using 2,000 geometry optimization steps and NVT (constant number of molecules, volume, and temperature) and NPT simulations (constant number of molecules, pressure, and temperature) at $300 \mathrm{~K}$, each lasting $50 \mathrm{ps}$ and employing $1 \mathrm{fs}$ time steps. The pressure used was 1 atm. Then Steps 1 and 2 were repeated; 4) When no reactive tails appeared within the cutoff distance 
after the relaxation procedure, the cutoff distance was increased by $0.25 \AA$. Steps 1 through 3 were repeated until the system reached a desired conversion or crosslinking density. All un-reacted growing chain radical heads were then capped with hydrogen atoms.

After the system was cured using these crosslinking simulations, the interfacial shear strength $\left(\tau_{i}\right)$ was calculated. First, the system was relaxed for $3 \mathrm{~ns}$, and the average total potential energy $\left(E_{\text {total }}\right)$ of the entire graphene/VE cured system was determined using an NVT simulation. Then, both isolated graphene and the isolated VE matrix were independently equilibrated using an NVT simulation for $3 \mathrm{~ns}$ before calculating the average graphene and average VE matrix potential energies ( $E_{\text {graphene }}$ or $\left.E_{\text {matrix }}\right)$. Interfacial shear strength is given by (Liao \& Li, 2001):

$$
\begin{aligned}
& d E_{\text {pullout }}=2 w(L-x) \tau_{i} d x, \\
& \tau_{i}=\frac{\mathrm{E}_{\text {pullout }}}{w L^{2}}, \text { (17) }
\end{aligned}
$$

where $2 w(L-x) \tau_{\mathrm{i}}$ is the graphene pullout force as a function of the displacement $x$ in the graphene pullout direction, $w$ is the width of the graphene sheet in the pullout direction, and $L$ is the fully embedded length of the graphene sheet as shown in Figure 4.

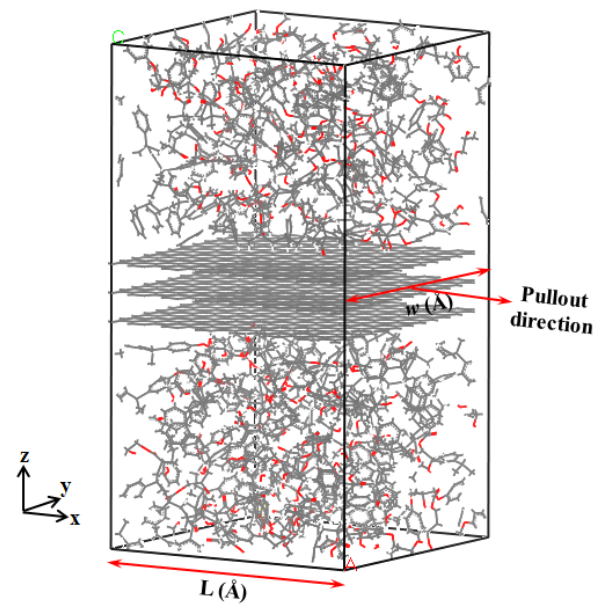

Figure 4: The schematic diagram of a periodic simulation box. The periodic simulation cell size is $31.5 \times 31.5 \times 58 \AA^{3}$ after resin cured. 
An initial liquid styrene monomer distribution after energy minimization and the equilibrium monomer distribution after $7 \mathrm{~ns}$ of simulation at $1000 \mathrm{~K}$ and $3 \mathrm{~ns}$ of simulation at $300 \mathrm{~K}$ with NVT are shown in Figure 7. After equilibration, it is clear that the relative concentration of styrene adjacent to the graphene surfaces has increased significantly. Furthermore, both the styrene phenyl rings and vinyl functions are preferentially aligned parallel to the plane of the graphene sheets. This optimizes $\pi-\pi$ stacking, which is a driving force for the build-up of the styrene-rich layer on the graphene surface. The spatial and temporal evolutions of the concentration profiles were monitored at $1000 \mathrm{~K}$ to ensure that a suitable equilibrium had been reached. Comparing different time-averaged concentration profiles in Figure 5 reveals that consistency exists in the successive time-averaged profiles. In Figure 5 and elsewhere, the relative concentration profiles were temporally and spatially averaged over both sides of the graphene sheets. After assuring that the monomers have equilibrated at $1000 \mathrm{~K}$, the system was cooled with $50 \mathrm{~K}$ decrements to $300 \mathrm{~K}$. During the cooling, 1 ps dynamics simulations were run at each intermediate temperature. The time-averaged concentration profiles all closely matched at $300 \mathrm{~K}$, indicating that equilibrium had been reestablished at the lower temperature (Figure 5b). At $1000 \mathrm{~K}$ the styrene concentration at the surface was about 3 times that of the bulk. Upon lowering the temperatures to $300 \mathrm{~K}$, the styrene surface region concentration soared to 5 times that of the bulk. If such differences in mole ratios of the monomers exist in the subsequently cured resins, this would have a substantial effect on interfacial shear strengths.

(a)

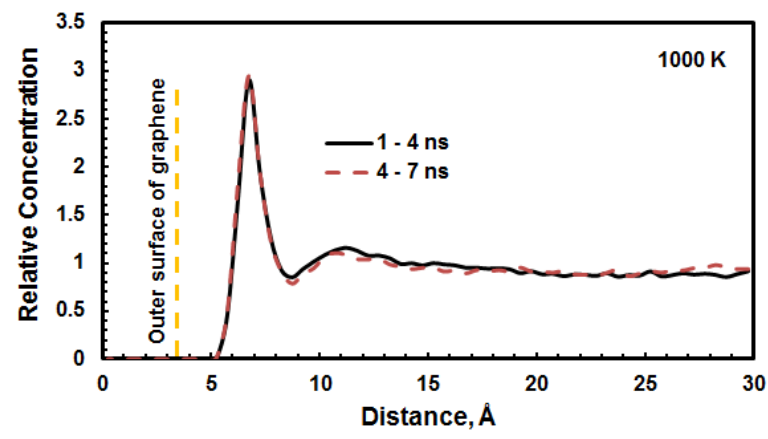

(b)

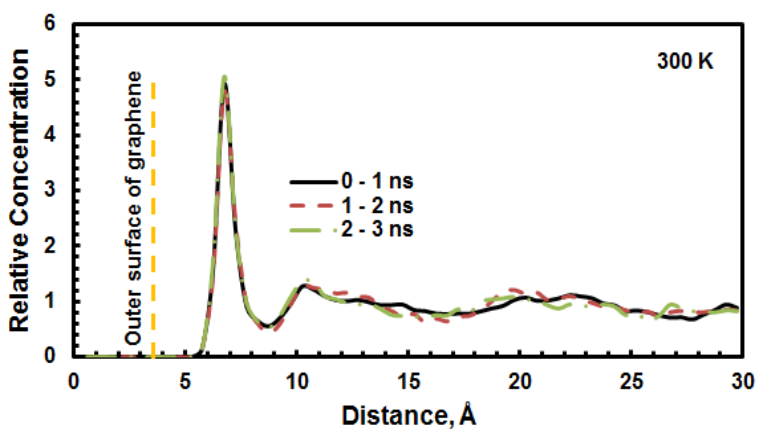

Figure 5: Temporally and spatially averaged styrene concentration profiles from outer graphene surface (a) over the last $6 \mathrm{~ns}$ of the $7 \mathrm{~ns}$ at $1000 \mathrm{~K}$ and (b) over $3 \mathrm{~ns}$ at $300 \mathrm{~K}$ (results are averaged over both sides of the graphene sheets). 
Figure 6 displays the equilibrium concentration profiles for styrene, VE1, and VE2. The relative concentration of styrene near the surface is higher than in the bulk resin. The final simulated monomer distributions represent the relative amounts of each monomer within a $1 \AA$ thick sub-volume parallel to the graphene planes. This is better described by calculating the relative monomer concentration ratios (equivalent to relative mole ratios) versus distance from the graphene surfaces (Figure 7).

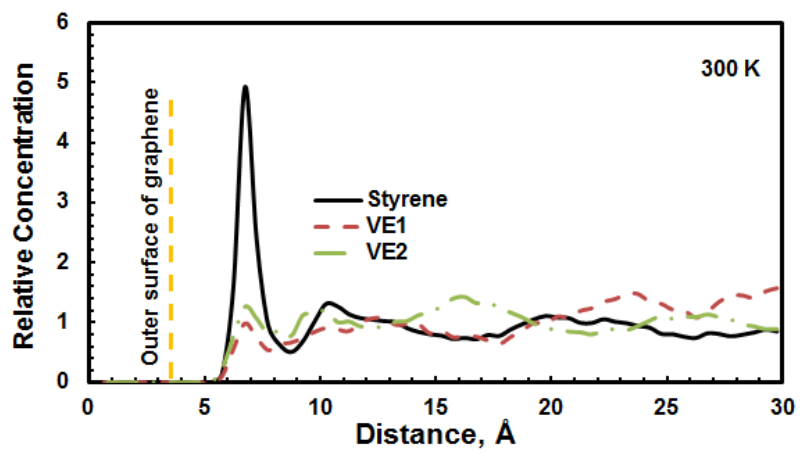

Figure 6: Relative Concentrations of styrene, VE1, and VE2 temporally and spatially averaged from outer graphene surface over the total simulation time of $3 \mathrm{~ns}$ at $300 \mathrm{~K}$. The styrene-rich layer on the nanofiber surface is about $5 \AA$ thick (results are averaged over both sides of the graphene sheets).

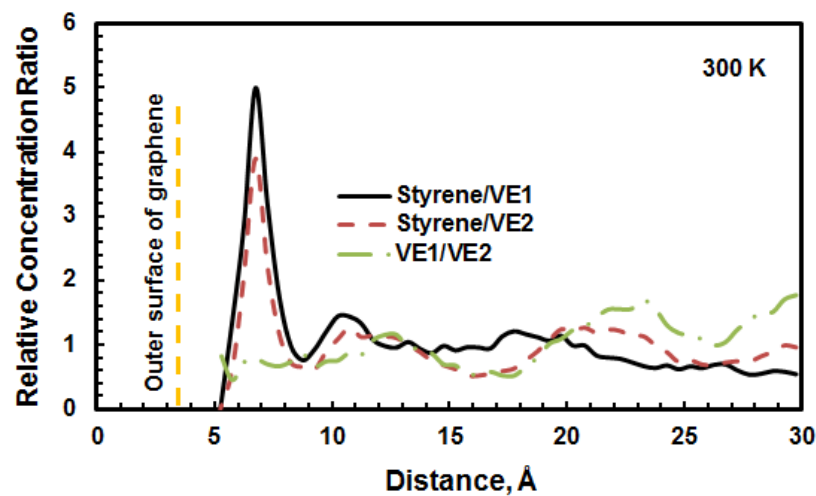

Figure 7: Monomer relative concentration ratios from outer graphene surface where simulations were temporally and spatially averaged over the total simulation time of $3 \mathrm{~ns}$ at $300 \mathrm{~K}$ (results are averaged over both sides of the graphene sheets).

Relative concentrations of $\mathrm{sp}^{2}$ aromatic carbon atoms and oxygen atoms versus the distance from the graphene surface were also monitored both before and after crosslinking. Aromatic $\mathrm{sp}^{2}$ hybridized carbon atoms dominated near the graphene surfaces as shown in Figure 8 . Their concentration near the graphene surfaces was about 3.5 times higher than in the bulk before crosslinking because the styrene aromatic ring concentrations were higher near and at surface and the styrene vinyl groups and phenyl rings were aligned along the graphene surfaces. Styrene has a higher fraction of $\mathrm{sp}^{2}$-hybridized aromatic carbon atoms ( 6 of 8 carbons) than VE1 (12 of 28 carbons) or VE2 (24 
of 47 carbons), so a relative increase of these aromatic carbons in any volume reflects an increase in the relative amount of styrene present. During curing, the relative $\mathrm{sp}^{2}$-hybridized aromatic carbon concentrations (therefore the styrene concentration) decreased, somewhat relative to that in the neat liquid monomers, near the surface. This is because the aromatic carbons can no longer align as well at the surface as styrenes are incorporated into the polymer. Both vinyl carbons become $\mathrm{sp}^{3}$ hybridized as styrene becomes part of the polymer. These tetrahedral carbons sterically work against the styrene phenyl rings lying flat on the graphene. That will reduce the possible magnitude of the interfacial shear strength induced by $\pi-\pi$ attractions. Likewise, the oxygen concentrations near the graphene surfaces were also slightly decreased after crosslinking as shown in Figure 8b. After curing, it is clear that the relative styrene concentration in the near surface volume region is still far higher than in the bulk, although somewhat lower than it was in the liquid before curing. The styrene-rich region near the surface may improve interfacial shear strength versus the value that the bulk resin structure would give at the graphene sheet surface.

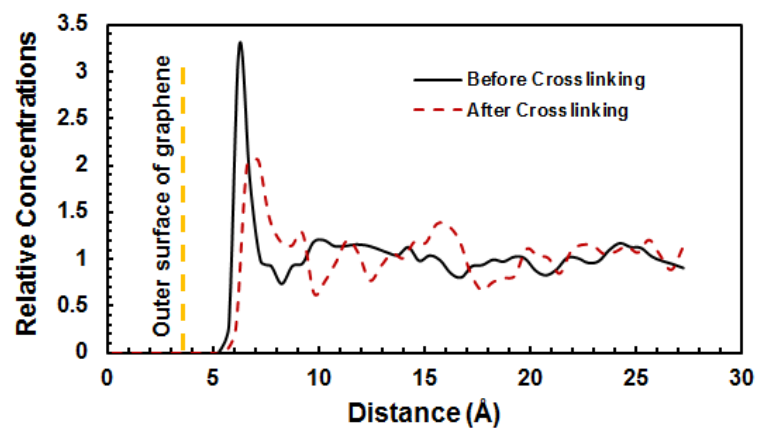

(a)

(b)

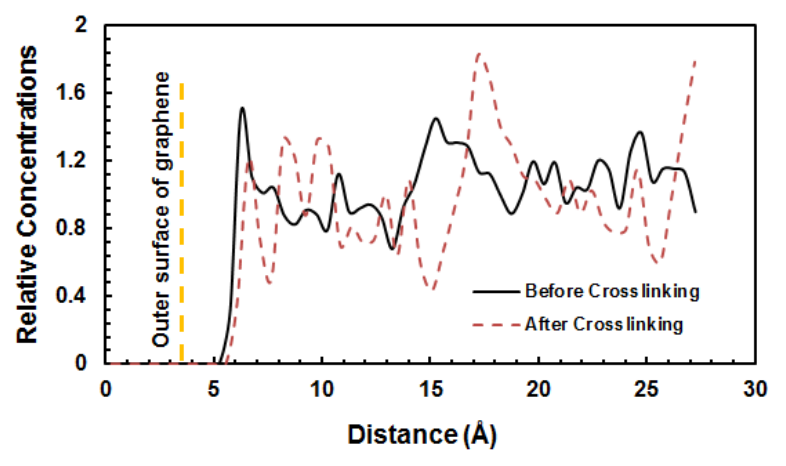

Figure 8: Temporally and spatially averaged concentration profiles from outer grephane surface (a) for $\mathrm{sp}^{2}$-hybridized carbon atoms and (b) for oxygen atoms over the total simulation time of $3 \mathrm{~ns}$ at $300 \mathrm{~K}$ (results are averaged over both sides of the graphene sheets). 
The crosslinked VE network was successfully generated with $98 \%$ of the double bonds consumed (98\% conversion). Conversion is defined as the percent of the initial polymerizable double bonds that are consumed during curing. The glass transition temperature $\left(T_{\mathrm{g}}\right)$ of the graphene/VE cured system was then calculated. The effect on the glass transition temperature of nanoreinforcements in polymer matrices is not well understood, because measured $T_{\mathrm{g}}$ values depend on the amount of reinforcements added, the surface chemistry of these nanoreinforcements, the degree of conversion, the curing cycle, etc. Velasco-Santos et al. (2003) reported that the glass transition temperature increased by $40{ }^{\circ} \mathrm{C}$, when a poly(methyl methacrylate) (PMMA) matrix contained between $1 \mathrm{wt} \%$ and $1.5 \mathrm{wt} \%$ of multi-walled carbon nanotubes (MWNT) functionalized with carboxyl groups. When they used 1 wt\% of unfunctionalized MWNT, the glass transition temperature increased slightly by $6^{\circ} \mathrm{C}$.

The calculated $T_{\mathrm{g}}$ for the cured VE system in this work is in the range of 466-502 $\mathrm{K}$ as shown in Figure 9. With the same approach used here, we calculated a $T_{\mathrm{g}}$ range of $417-425 \mathrm{~K}$ for the same VE composition cured to $98 \%$ conversion without any reinforcements present. The $T_{\mathrm{g}}$ determination is highly sensitive to the manner in which the data points on the density versus temperature graph are fit. Therefore, it is more appropriate to describe the $T_{\mathrm{g}}$ as a temperature range rather than a single temperature point. This large increase in $T_{\mathrm{g}}$ is an indication that carbon surface present here, or other surfaces, can result in remarkable changes in properties of highly crosslinked resin systems near the surfaces. 1-3 wt\% loadings of nanofillers have relatively large total surface areas, but the resin volume fraction within 25 to $100 \AA$ of these surfaces is still a very small fraction of the total resin volume. Actual $T_{\mathrm{g}}$ measurements reflect the response of the total resin volume of the sample. These MD simulations permit an evaluation of the resin's response very close to the reinforcement surface, a region very difficult to uniquely probe by experiments.

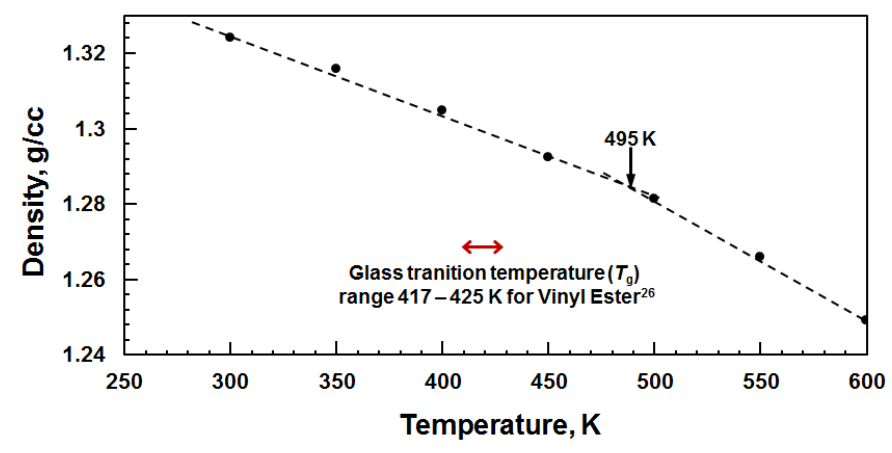

Figure 9: The glass transition temperature for the VE resin region within $26 \AA$ of the graphene sheet is about $495 \mathrm{~K}$ at $98 \%$ conversion. This value is higher than the experimental value for the neat cured Dearakane $441-400$ cured matrix (415 K) and for the neat VE cured matrix at $98 \%$ conversion using MD simulations (417-425 K). 
The glass transition reflects the activation of segmental motions in the cured matrix. At equal conversions the nanocomposite average crosslink density is the same but, when a reinforcement is present, its surface physically blocks some segmental motions, occurring adjacent to the surface and attractive interactions with the surface restrict motions to varying degrees. In addition, the higher styrene content near the graphene surface enhances the VE1 and VE2 amounts in the region from $~ 7$ to $26 \AA$ from the surface, leading to a higher crosslink density (hence, higher $T_{\mathrm{g}}$ ) in this volume region. Adjacent to the surface, where the styrene/VE1 and VE2 ratio is higher, the crosslink density is lower. However, the polymer segments in this region are the ones most restricted by the attractions to the carbon surface and by the physical presence of the surface. Inoue et al. (2009) reported a relationship between the molecular mobility in thin films and the glass transition temperature. They concluded that the lower mobility always corresponds to a higher glass transition temperature. The sum of the effects caused by the pristine graphene surfaces on restricting mobility of the cured VE resin in the present work raises the overall $T_{\mathrm{g}}$ of the crosslinked VE within the resin's total volume lying within $26 \AA$ of the carbon surface.

The interfacial shear strength between the cured VE resin and the pristine graphene surface was calculated using the interaction energy between the surface and the matrix. One definition of interfacial shear strength is the difference between the total potential energies of (1) the carbon sheets with the fully filled and relaxed cured VE resin system and (2) the remaining VE resin after carbon sheets pull out. In the fully embedded graphene system, before pull-out, the total potential energy contains the resin/carbon surface interaction energy for the overall system (bonding, angle, torsion, dihedral, van der Waals and electrostatic energies, etc). However, after the graphene sheets are completely pulled out, the non-bonded energy, interaction energy, and interfacial bonding between graphene sheets and VE matrix no longer exist. Only the isolated VE matrix and graphene sheets must now be considered. So, pullout energy $\left(E_{\text {pullout }}\right)$ can be written as,

$E_{\text {pullout }}=E_{\text {graphene }}+E_{\text {matrix }}-E_{\text {total }}$

where $E_{\text {total }}$ is the total potential energy in the fully embedded graphene/VE system, $E_{\text {graphene }}$ is the graphene potential energy without the matrix present, and $E_{\text {matrix }}$ is the matrix potential energy without graphene sheets present. All systems were monitored to be completely relaxed to their lowest potential energy for 3 ns as shown in Figure 10. 


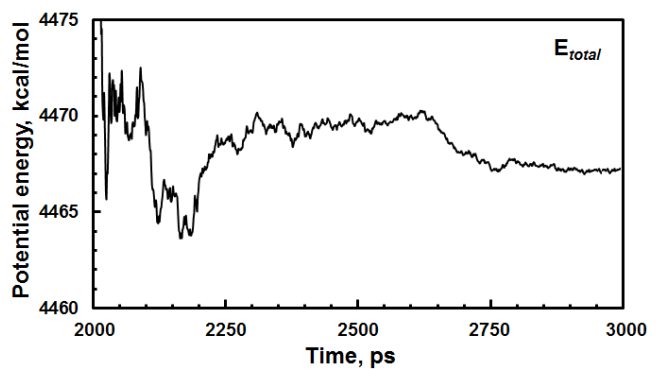

(a)

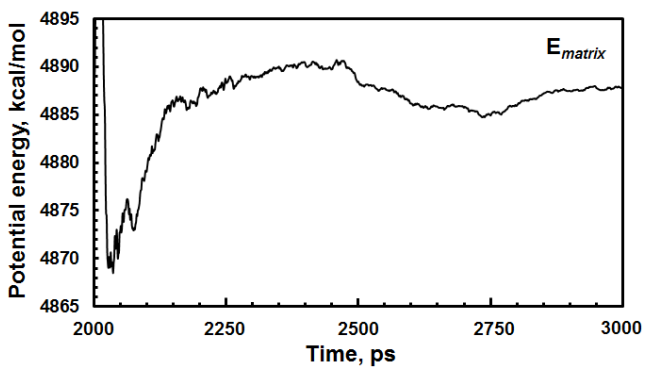

(b)

Figure 10: Potential energy plots for $E_{\text {total }}$ and $E_{\text {matrix }}$ were converged to be completely relaxed for $3 \mathrm{~ns}$.

The potential energies of each system and the pullout potential energy from these simulations are shown in Table I. The interfacial shear strength between the graphene sheets and VE matrix was $\tau_{i}=$ $118 \mathrm{MPa}$. The pronounced accumulation of nonpolar styrene in the cured resin adjacent to the nonpolar pristine graphene sheets enhances $\pi-\pi$ stacking and Van der Waals interactions. Also the less crosslinked structure very close to surface could allow more conformational freedom for the matrix to favorably interact with the surface. Such factors could improve the graphene-matrix interfacial shear strength. Taking these factors into account will change the predicted interfacial shear strength.

Table I: Potential energies of graphene/VE, VE matrix and graphene sheets. As a result, pullout potential energy is $446.0 \mathrm{kcal} / \mathrm{mol}$.

$\begin{array}{ll}\text { Potential Energy } & \tau_{i}^{\mathrm{a}}\end{array}$

$\begin{array}{ll}(\mathrm{kcal} / \mathrm{mol}) & \text { (MPa) }\end{array}$

\begin{tabular}{lllll}
\hline$E_{\text {total }}$ & $E_{\text {matrix }}$ & $E_{\text {graphene }}$ & $E_{\text {pullout }}$ b & $\tau_{\mathrm{i}}$ \\
4466.8 & 4887.3 & 25.4 & 446.0 & 118 \\
\hline
\end{tabular}

${ }^{\mathrm{a}} \tau_{i}=$ Interfacial shear strength $=E_{\text {pullout }} / w L^{2}$

${ }_{\mathrm{b}} E_{\text {pullout }}=\int 2 w(L-x) \tau_{i} d x$

\section{Micro- to macroscale simulations of VGCNF/VE nanocomposites}

\section{Effective Property Estimates for Composites Containing Multiple Nanoheterogeneities}

The Effective Continuum Micromechanics Analysis Code (EC-MAC) was developed to predict effective properties of composites containing multiple distinct nanoheterogeneities (fibers, spheres, platelets, voids, etc.) each with an arbitrary number of coating layers based upon modified versions of either the Mori-Tanaka method and self-consistent method. The influence of solid and hollow nanoreinforcement geometries, distinct elastic properties, and orientations are addressed (i.e., solid silica nanosphere/epoxy, hollow glass nanosphere/polyester, $\alpha$-zirconium phosphate 
nanoplatelet/epoxy, and vapor grown carbon nanofiber/vinyl ester composites), along with the influence of voids. In addition, the effect of nanofiber waviness on bulk composite properties is accounted for using an approach developed by Hsiao and Daniel (1996), and Fisher and colleagues (2003). The notion of "effective volume fraction" is introduced to denote the fraction of nanoreinforcements that contribute to overall composite properties through their good dispersion.

Nanoreinforcements (nanospheres, nanoplatelets, and nanofibers, etc.) can have very distinct morphologies, sizes, and material properties. Nanocomposites reinforced with small volume fractions of nanospheres can result in improvements in composite thermal conductivity, mechanical stiffness and strength, and chemical and thermal resistance without a substantial increase in liquid resin viscosities (Toll, 1998; Sudduth, 2008). Consequently, nanosphere reinforced composites typically are easy to process and exhibit relatively uniform nanoreinforcement distributions. Composites reinforced with small amounts of nanoplatelets can also exhibit significantly improved tensile properties, decreased coefficients of thermal expansion, and reduced gas permeability, in comparison with the neat resin (Toll, 1998; Boo et al., 2007; Sudduth, 2008). Nanoclay platelets often display a thin/thick flake-like morphology that appears in intercalated (stacked deck of cards) or exfoliated (individual card) structures. Either intercalated or exfoliated nanoplatelets can be idealized as circular plates with nominal diameter $\left(D_{p}\right)$ and thickness $\left(t_{p}\right)$. Note that the aspect ratio $\left(D_{p} / t_{p}\right)$ for exfoliated nanoplatelets can be substantially larger than for intercalated nanoplatelets, where the intercalated grouping is considered a single unit. In contrast to nanoclay platelets, which can display intercalated and exfoliated architectures of varying thicknesses, $\alpha$-ZrP nanoplatelets (Boo et al., 2007) are structures with a nominal thickness, $t_{p}=1 \mathrm{~nm}$, whose diameters can vary (i.e., $D_{p}=100,1000 \mathrm{~nm}$ ). Of course, as the aspect ratio of nanoplatelets increases, the corresponding viscosity of the liquid resin containing these platelets can increase appreciably (Toll, 1998; Sudduth, 2008). As-manufactured VGCNFs often display either hollow cylinder or bamboo-like architectures with diameters, $D_{f}=60-200 \mathrm{~nm}$, and aspect ratios in the range, $L_{f} / D_{f}=50-2000+$, where $L_{f}$ and $D_{f}$ are the VGCNF length and diameter, respectively. Similar to nanoplatelets, as the nanofiber aspect ratio increases, the corresponding viscosity of the nanophased liquid resin increases substantially. The increased viscosity often leads to poor nanofiber dispersion and difficulties in breaking up nanofiber agglomerates. Nanofibers are often aggressively mixed in the resin using some combination of ultrasonication and/or high shear mixing for better dispersion (Nouranian et al., 2011a). The actual nanofiber aspect ratios $\left(L_{f} / D_{f}\right)$ in cured composites can be somewhat lower than the as-manufactured values due to nanofiber breakage during fabrication. In this study, EC-MAC was used to predict effective elastic properties of composites containing idealized solid silica nanospheres (Zhang et al., 2006), hollow glass spheres (Huang \& Gibson, 1993), $\alpha$-ZrP nanoplatelets (Boo et al., 2007), and VGCNFs.

Zhang et al. (2006) performed tensile and flexural testing of silica nanosphere/epoxy composites to investigate the effect of the mean distance between nanoparticles on bulk composite properties. The nominal diameter and Young's moduli of the solid silica nanospheres were $D_{s}=25 \mathrm{~nm}$ and $E_{s}=24 \mathrm{GPa}$, respectively. Significant improvements in composite elastic moduli and toughness were observed when the interparticle distance was smaller than the silica nanoparticle diameter. Figure 11a shows a transmission electron microscopy (TEM) image of these silica nanospheres in an epoxy matrix (Zhang et al., 2006). 
Huang and Gibson (1993) performed tensile testing of composites containing hollow glass spheres $\left(D_{s}=42 \mathrm{~nm}\right.$, wall thickness $\left.t_{w}=0.84 \mathrm{~nm}\right)$ in a polyester matrix. Such reinforcements have a thinwalled "egg-shell" type of architecture. Figure $11 \mathrm{~b}$ contains a scanning electron microscopy (SEM) image of individual hollow glass nanospheres (Huang \& Gibson, 1993). The measured tensile moduli decreased with increasing amounts of hollow glass spheres.

Boo et al. (2007) investigated the effect of nanoplatelet aspect ratio $\left(D_{p} / t_{p}\right)$ on stiffness properties for $\alpha$-ZrP/epoxy composites. The $\alpha$-ZrP nanoplatelets had a nominal thickness, $t_{p}=1 \mathrm{~nm}$, and two distinct diameters, $D_{p}=100,1000 \mathrm{~nm}$ (Boo et al., 2007) (Figure 12). For a nanoplatelet volume fraction, $c_{p}=0.007$, nanocomposites prepared using the higher aspect ratio nanoplatelets $\left(D_{p} / t_{p}=1000\right)$ displayed a $7 \%$ improvement in bulk tensile properties in comparison with composites prepared with an equal volume fraction of the lower aspect ratio nanoplatelets $\left(D_{p} / t_{p}=100\right)$.

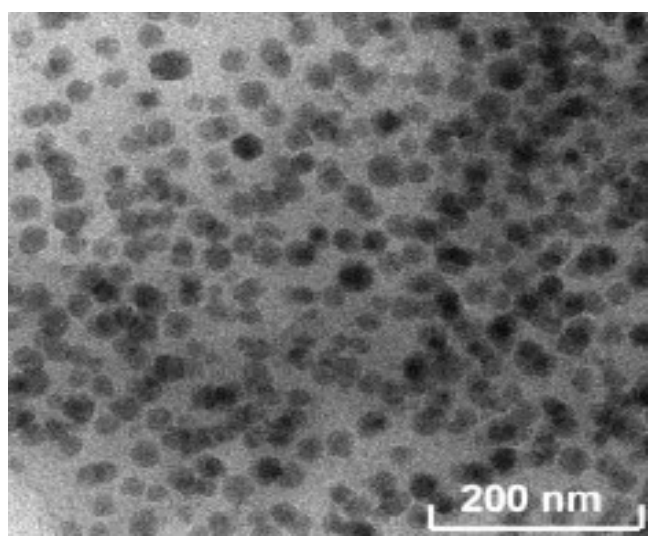

a)

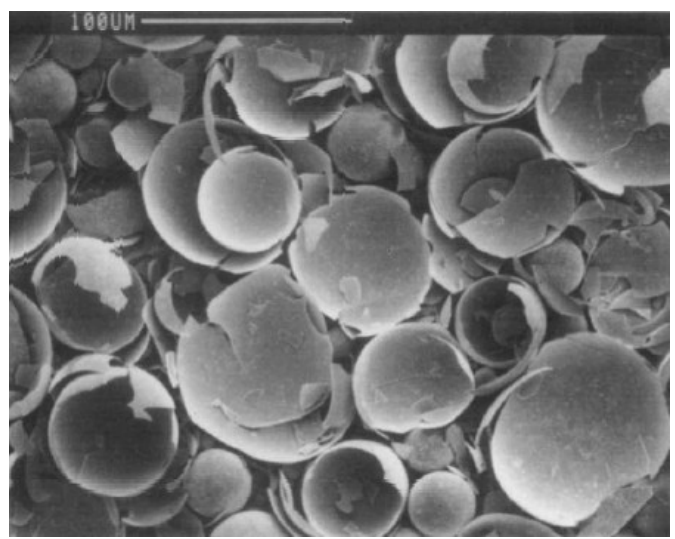

b)

Figure 11: a) TEM image of a solid silica nanosphere/epoxy composite from (Zhang et al., 2006) and b) SEM image of hollow glass spheres from (Huang and Gibson, 1993).

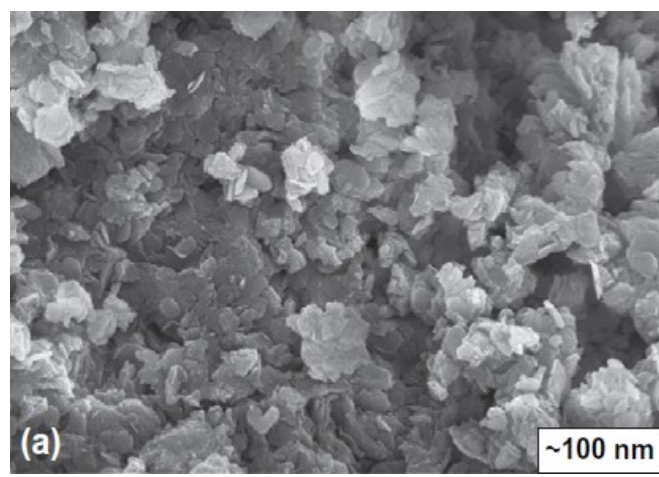

a)

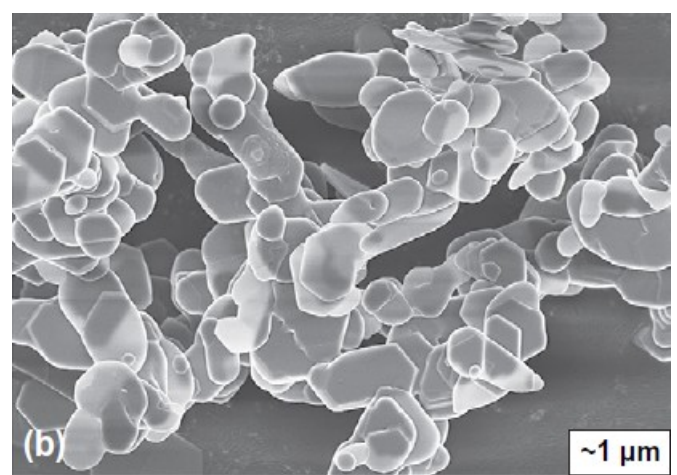

b)

Figure 12: SEM images of $\alpha$-ZrP nanoplatelets in $\alpha$-ZrP/epoxy resin composites displaying aspect ratios, a) $D_{p} / t_{p}=100$ and b) $D_{p} / t_{p}=1000$ from (Boo et al, 2007). 
VGCNFs are increasingly used as nanoreinforcements due to their excellent mechanical properties (Al-Saleh \& Sundararaj, 2009; Zhou et al., 2007) and relatively low cost compared to singlewalled and multi-walled carbon nanotubes. As-manufactured VGCNFs typically display either hollow or bamboo-like cylindrical architectures (Figures 13a and 13b) with outer diameters and aspect ratios in the ranges $D_{f}=60-200 \mathrm{~nm}$ and $L_{f} / D_{f}=50-2000+$, respectively (Ozkan et al., 2010). The wall thickness and degree of VGCNF waviness (curvature) can also vary substantially from fiber to fiber. VGCNFs exhibit a large degree of waviness in their as-manufactured state. Figure 14a shows a SEM image of a wavy nanofiber on a fracture surface from a VGCNF/ vinyl ester (VE) nanocomposite. The degree of fiber waviness can be expressed in terms of the nanofiber waviness ratio $(h / \lambda)$, where $h$ and $\lambda$ are the amplitude (height) and wavelength of the wavy fiber, respectively (Hsiao \& Daniel, 1996; Fisher et al., 2003) (Figure 14b).

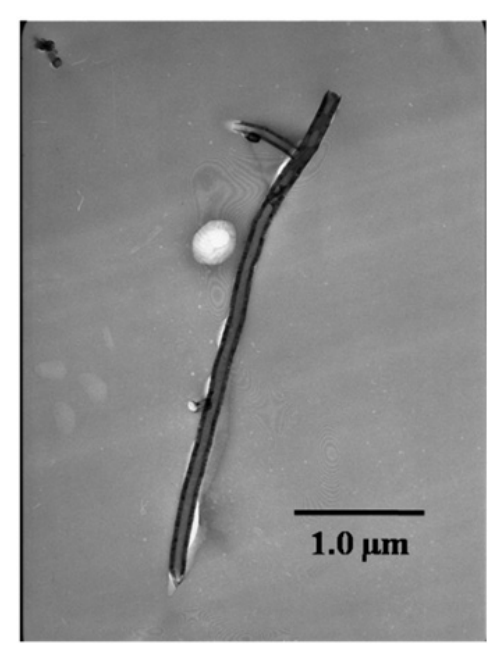

a)

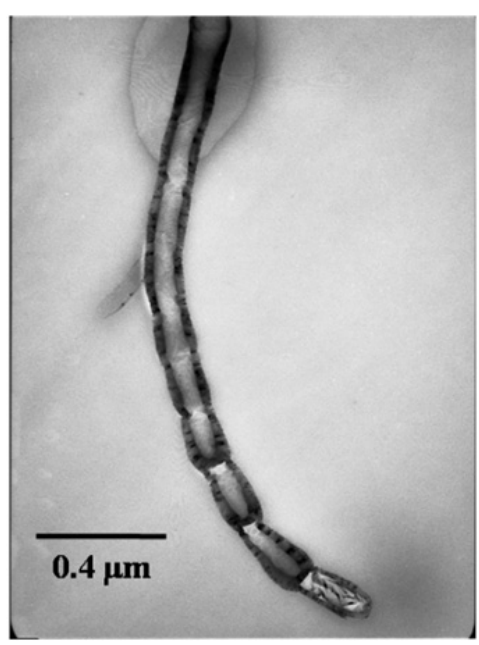

b)

Figure 13: TEM images of VGCNFs displaying a) hollow cylindrical, and b) bamboo architectures. 


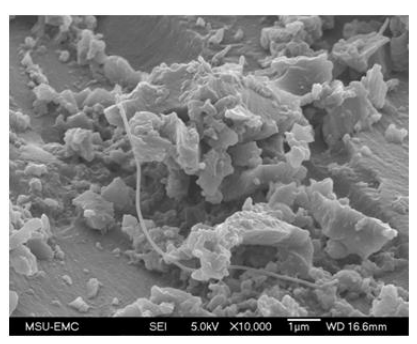

a)

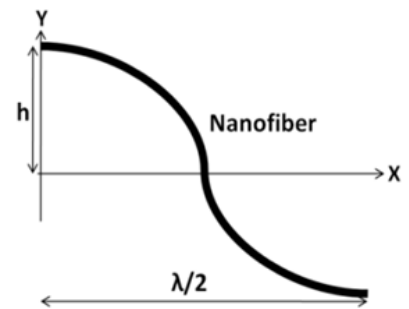

b)

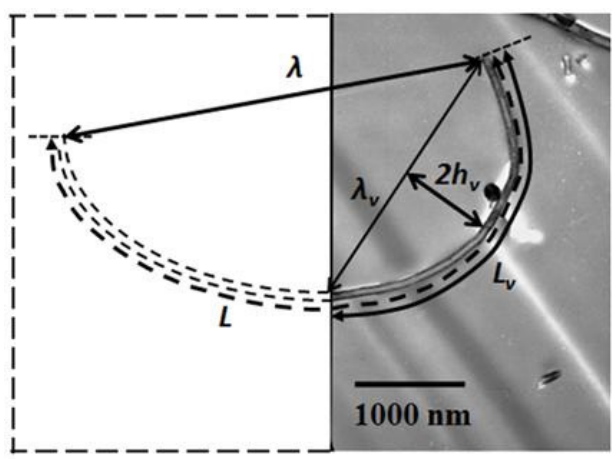

c)

Figure 14: a) SEM image of a wavy VGCNF, b) idealized segment of a wavy fiber ( $h / \lambda=$ fiber waviness ratio), and c) TEM image of a wavy nanofiber $\left(h_{v} / \lambda_{v}=\right.$ fiber visible waviness ratio).

We have previously used TEM images to characterize the statistical distribution of nanofiber diameters $\left(D_{f}=120 \pm 34 \mathrm{~nm}\right)$, wall thicknesses $\left(t_{f}=30.2 \pm 10.8 \mathrm{~nm}\right)$, visible nanofiber waviness ratios $\left(h_{v} / \lambda_{v}=0.054 \pm 0.052\right)$, and other features in VGCNF/VE composites. In that work, PR-24-XT-LHT-OX VGCNFs and Derakane 441-400 vinyl ester were used as the reinforcement and matrix, respectively. The term "visible nanofiber" refers to the portions of the nanofibers that can be seen above or on the composite microtomed surfaces by TEM. In that study, the visible nanofiber waviness ratio $\left(h_{v} / \lambda_{v}\right)$ was measured since each TEM sample only contained segments of actual nanofibers (Figure 14c). Even though typical as-received nanofiber lengths can exceed several microns, a significant fraction of a given nanofiber may lie outside of the plane of the ultramicrotome section used in TEM imaging.

Lee (2010) performed flexural tests on nanocomposites comprised of PR-24-XT-LHT-OX VGCNFs in a Derakane 441-400 VE resin. Ozkan et al. (2010) performed microelectromechanical systems (MEMS)-based tensile tests of Applied Sciences Inc. PR-24-XT-series VGCNFs to measure nominal nanofiber tensile moduli $\left(E_{f}=240 \mathrm{GPa}\right)$ and strengths $(2.35 \mathrm{GPa})$. The reported properties assumed the nanofibers had a solid carbon cross-section due to difficulties in measuring VGCNF wall thicknesses. We have proposed a simple methodology for estimating the tensile moduli and strengths for hollow PR-24XT series VGCNFs using the measured moduli and strength data from (Ozkan et al., 2010), after experimentally obtaining both the inner and outer diameter distributions of PR-24-XT VGCNFs. 
Nanocomposites may also contain nanoscale defects and other entities such as voids, carbon clusters, and nanoreinforcement agglomerates. An increase in the nanoreinforcement volume fraction and/or aspect ratio can result in a significant increase in the nanophased liquid resin viscosity, resulting in an increase in the void content of the composite. For example, Figure 15a shows a spherical void in a VGCNF/VE nanocomposite. VGCNF nanocomposites may also contain a number of tiny quasi-spherical carbon clusters and their aggregates that are formed during VGCNF fabrication. Such aggregate structures may contain a combination of carbon and small amounts of iron particles, and these aggregates can be large in comparison to individual VGCNFs (Figure 15b). In addition, VGCNF nanocomposites may contain a number of nanofiber agglomerates. Figure 16 shows SEM images of nanofiber agglomerates in a VGCNF/VE nanocomposite. Such agglomerates may contain "bird-nest"-like entanglements of hundreds, or even thousands, of individual nanofibers. Moreover, the degree of fiber wetting within agglomerates can vary substantially. For example, Figures 16a and 16b contain SEM images of relatively large and small VGCNF agglomerates that have been partially infused with VE resin. In contrast, Figure 16c contains an SEM image of relatively large agglomerate with minimal nanofiber wetting. Such agglomerated structures can drastically reduce nanocomposite strengths. In addition, the likelihood of agglomerates increases with both increasing nanoreinforcement volume fraction, use of some mixing techniques, and resin viscosity.

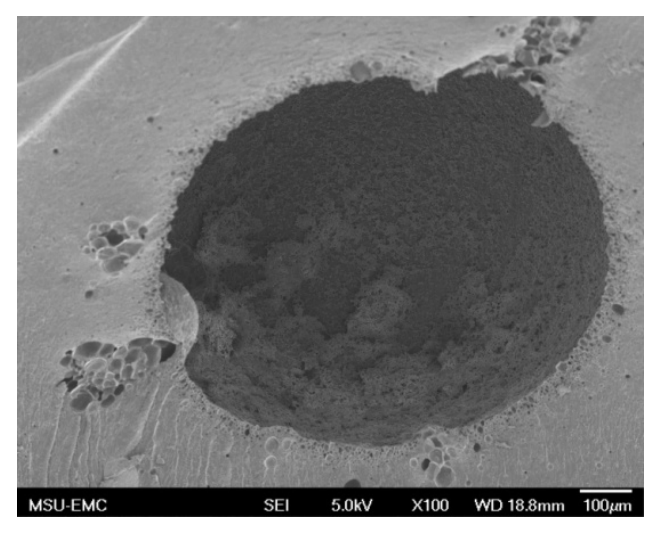

a)

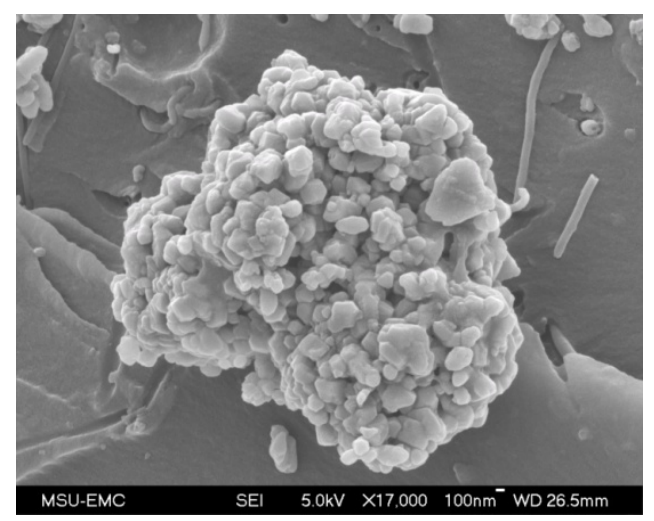

b)

Figure 15: Nanoheterogeneities in VGCNF/VE composites: a) void (100X), and b) carbon cluster (17000X). 


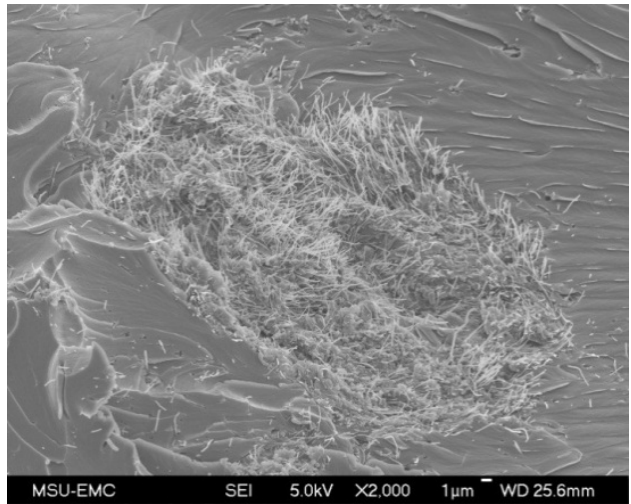

a)

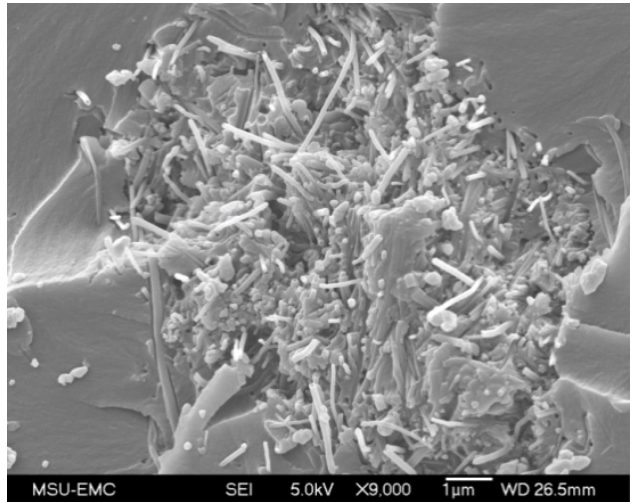

b)

c)

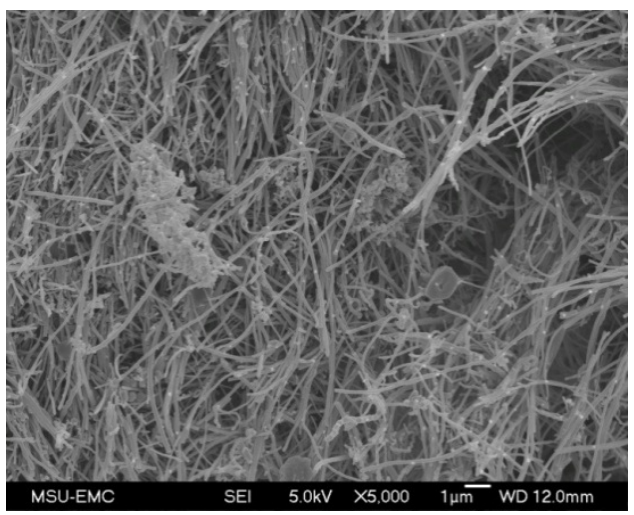

Figure 16: SEM images of nanofiber agglomerates in VGCNF/VE nanocomposites: a) relatively large agglomerate infused with resin (2000X), b) relatively small agglomerate infused with resin (9000X), and c) relatively large agglomerate with poor nanofiber wetting (5000X).

The "effective" nanoreinforcement volume fraction may be defined based upon the fraction of the total reinforcements that are well dispersed. The remaining nanofibers are present in agglomerates or other clustered structures. Well-dispersed nanoreinforcements can enhance material properties, while agglomerates and voids can degrade properties. One key challenge is to accurately determine the VGCNF effective volume fraction, as well as the volume fraction of voids, carbon clusters, and agglomerates in real nanocomposites. Voids, carbon clusters, and nanofiber agglomerates can all be loosely idealized as spheres. Of course, voids have null material properties. The elastic moduli of carbon clusters are unknown, but likely are far lower than those of turbostratic carbon. Some VGCNF agglomerates may also be approximated as void-like structures since there is often very poor VGCNF wetting inside of the agglomerates (Figure 16c). Other nanofiber agglomerates that are fully or partially infused with resin (Figures 16a-16b) would constitute stiffer spherical-like inclusions of much larger scale size than individual fibers. Provided their mechanical properties and volume fractions were known, the effect of voids, carbon clusters, and VGCNF agglomerates on bulk composite properties could be determined using the modeling approach developed in this work.

A number of mean field micromechanics approaches have been developed for predicting effective heterogeneous material properties, particularly for composites containing low volume fractions of reinforcements in an elastic matrix. These include the Mori-Tanaka method (MTM) (Mori \& 
Tanaka, 1973; Benveniste, 1987), the self-consistent method (SCM) (Hill, 1965), and various coated inclusion techniques (Mura, 1987; Nemat-Nasser and Hori, 1993). These approaches are based upon the classic Eshelby solution (Eshelby, 1957) for the stress and strain field due to the presence of an ellipsoidal inclusion in an infinite domain subjected to uniform far-field loading. Mean field approaches have been used to investigate the effect of solid nanofibers or nanoplatelets on bulk nanoreinforced matrix properties and have been modified to account for the effect of varying degrees of nanofiber waviness. We have developed the Effective Continuum Micromechanics Analysis Code (EC-MAC) for predicting effective properties for composites containing multiple heterogeneities, each with an arbitrary number of coating layers based upon modified versions of the MTM and SCM. The code allows for either hollow or solid reinforcements. EC-MAC was used in this study to estimate effective properties for composites containing a range of disparate nanoreinforcements.

In this study, EC-MAC was used to determine the effective elastic moduli of composites containing a combination of nanoreinforcements (solid silica (Zhang et al., 2006) and hollow glass (Huang \& Gibson, 1993) nanospheres, $\alpha$-ZrP nanoplatelets (Boo et al., 2007), and VGCNFs) and voids, assuming that all heterogeneities are well dispersed in the matrix and perfectly bonded to the other

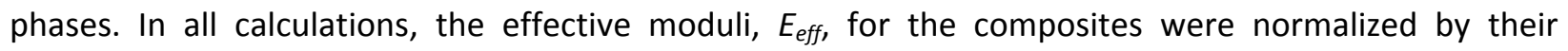
respective matrix modulus, $E_{m}$ in order to clearly illustrate the effect of the nanoheterogeneities.

EC-MAC was used to estimate the elastic moduli of composites comprised of solid silica nanospheres in an epoxy matrix. Figure 17 shows a plot comparing the predicted normalized effective elastic modulus $\left(E_{\text {eff }} / E_{m}\right)$, obtained using both the MTM and SCM modules within EC-MAC, as a function of the volume fraction of solid silica nanospheres. The predicted effective composite modulus increased with an increasing volume fraction of nanospheres. Note that the SCM led to slightly lower estimates of $E_{\text {eff }}$ compared to those obtained using the MTM. Experimentally measured tensile moduli from Zhang et al. (2006) are included in the figure. While there was a fair degree of scatter in the measured moduli, the two micromechanical solutions reasonably matched the mean experimental response over the range of volume fractions.

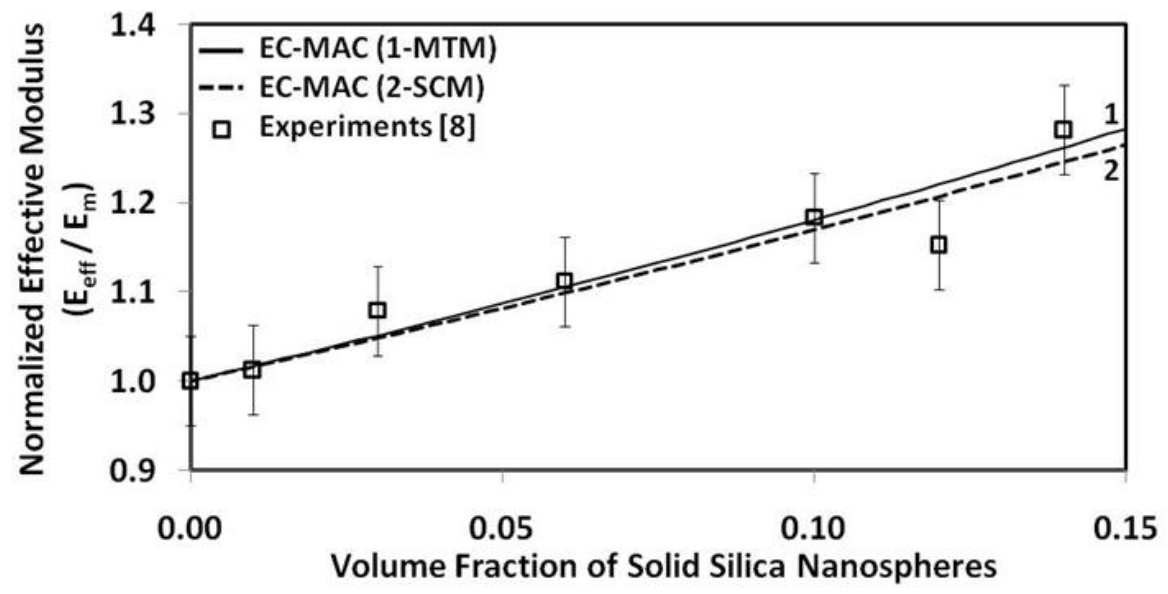

Figure 17: Measured (Zhang et al., 2006) and predicted effective elastic moduli for solid silica nanosphere/epoxy composites. 
Similar effective property estimates were obtained for composites comprised of hollow glass nanospheres in a polyester matrix. Huang and Gibson (1993) measured the tensile properties for such composites. The hollow nanospheres were essentially thin shell-like reinforcements, i.e., $R_{i} / R_{o} \approx 0.98$ where $R_{i}$ and $R_{o}$ are the inner and outer sphere radii, respectively (Figure 11b). Hence, the majority of the volume of each sphere corresponded to void space. The glass spheres were simulated in EC-MAC using a two-layer idealization, where the inner most layer was ascribed essentially null material properties and the outer layer was comprised of glass. Figure 18 contains a plot of the measured (Huang \& Gibson, 1993) and predicted effective composite modulus as a function of the volume fraction of reinforcements, where the SCM module of EC-MAC was used in the calculations. In contrast to the case involving solid silica nanospheres, the effective modulus decreased with increasing volume fraction of hollow glass spheres. This makes sense given the relatively low fraction of solid glass contained in each sphere. The predicted modulus correlated reasonably well with the experimental data from (Huang \& Gibson, 1993). Figure 18 also includes the predicted effective modulus for a polyester matrix containing spherical voids only (i.e., no glass outer wall). The predicted modulus fell significantly below the measured and predicted values for the hollow glass sphere/polyester composites. This suggests that the very thin glass outer wall significantly contributes to the overall composite properties.

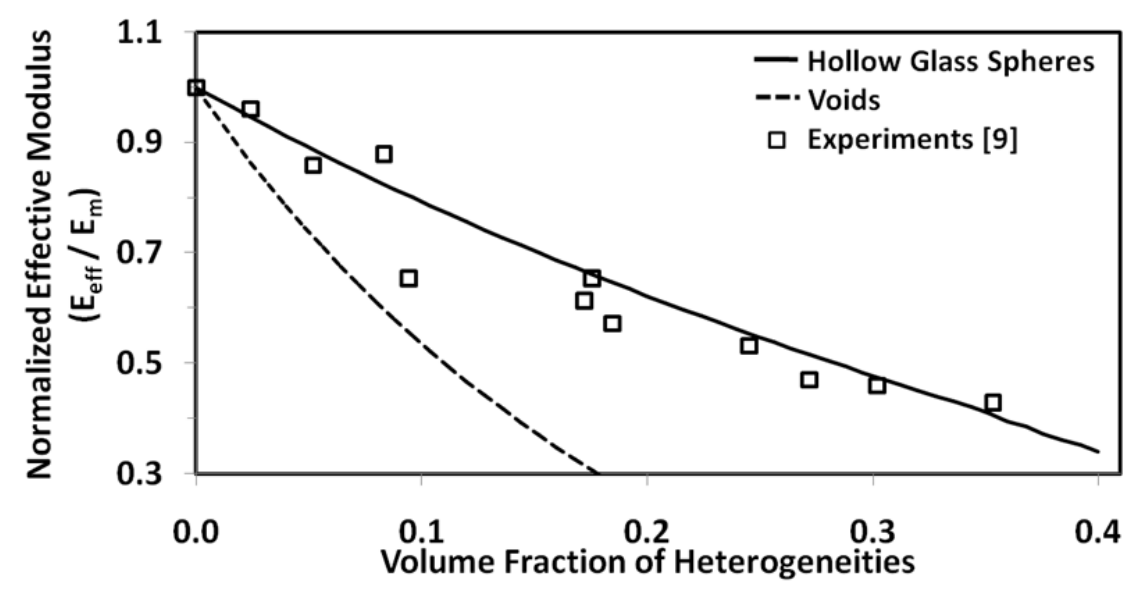

Figure 18: Effective elastic moduli of hollow glass sphere/polyester composites and a polyester matrix containing spherical voids.

Figure 19 contains a plot of the effective composite moduli as a function of the volume fraction of heterogeneities. Not surprisingly, the bulk composite stiffness increased as the amount of nanoplatelets was increased. In addition, the moduli for composites containing higher aspect ratio nanoplatelets $\left(D_{p} / t_{p}=1000\right)$ was $13 \%$ greater (at $0.7 \mathrm{v} \%$ of nanoplatelets) than those for composites containing lower aspect ratio reinforcements $\left(D_{p} / t_{p}=100\right)$. Included in the figure are experimental data from Boo et al. (2007). While the predicted solutions fell within the error band of the measured values, they slightly over-estimated the mean experimental response in both cases. 


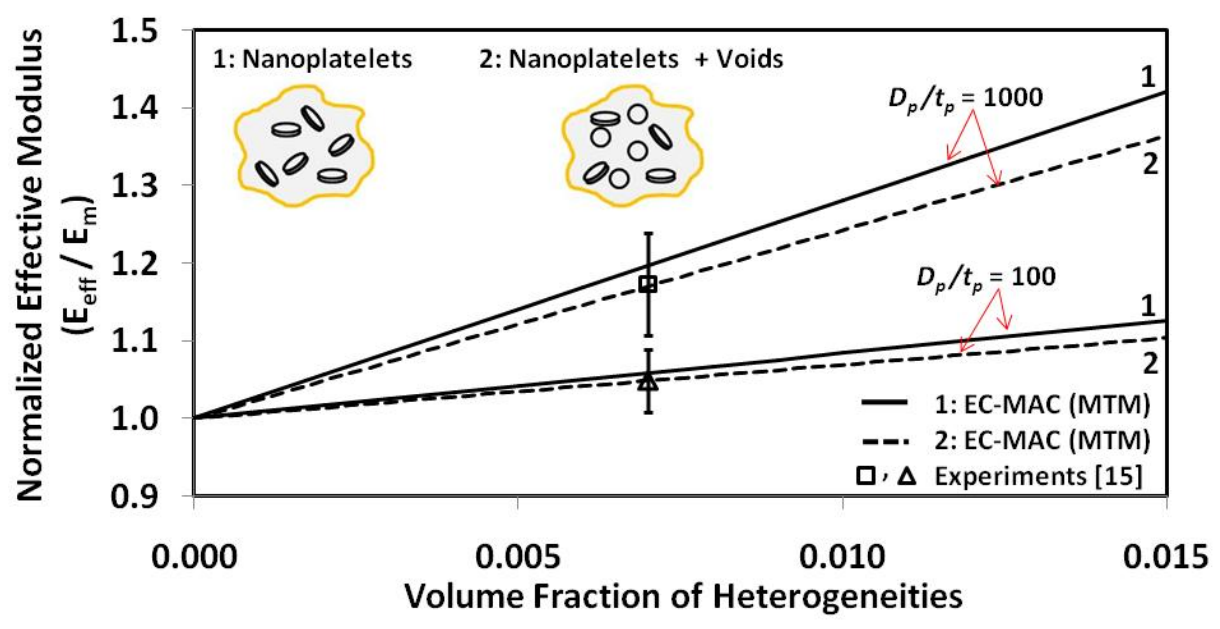

Figure 19: Effective elastic moduli of $\alpha$-ZrP nanoplatelet/epoxy composites containing nanoplatelets $\left(D_{p} / t_{p}=100,1000\right)$ and voids.

Even small amounts of high aspect ratio nanoreinforcements can result in a substantial increase in liquid resin viscosity, which can lead to difficulties in degassing and increased void content in the cured composites. The disparity in the predicted and measured moduli is likely due to some combination of void content, poorly dispersed nanoplatelets, or nanoplatelet agglomerates. In order to assess the effect of voids on the bulk elastic properties, additional calculations were performed for composites containing both nanoplatelets and voids. In these simulations, it was assumed that the volume fraction of voids was the same as for nanoplatelets $\left(c_{v}=c_{p}\right)$. Figure 19 contains a plot of the predicted effective moduli for composites containing both nanoplatelets and voids, as indicated by the dashed lines in the figure. The presence of voids resulted in roughly a $\sim 3 \%$ decrease in the effective composite moduli, which better matched the mean experimental values from (Boo et al., 2007). If the effective volume fraction of nanoplatelets and actual void content were known, similar calculations could be performed that better account for the effect of nanoplatelet dispersion and defects on bulk nanocomposite properties.

In real composites, VGCNFs can exhibit a large degree of fiber waviness (Figures 13b and 14a). Thus, parametric studies were performed, using the MTM module within EC-MAC, to assess the effect of nanofiber waviness on the effective elastic moduli of VGCNF/VE composites containing either aligned or randomly oriented VGCNFs (aspect ratio, $L_{f} / D_{f}=100$ ), plus voids. In the calculations, the volume fraction of nanofibers was fixed $\left(c_{f}=0.010\right)$. Figure 20a contains a plot of the estimated effective longitudinal $\left(E_{L}\right)$ and transverse $\left(E_{T}\right)$ moduli as a function of nanofiber waviness ratio $(h / \lambda)$ for composites containing aligned wavy VGCNFs $\left(c_{f}=0.01, c_{v}=0\right)$, as well as aligned wavy VGCNFs and voids $\left(c_{f}=c_{v}=0.01\right)$. For the aligned nanofiber case, the planes containing wavy nanofibers were randomly oriented about the longitudinal (aligned) fiber axis when calculating $E_{T}$. The presence of voids resulted in a slight decrease in both moduli for the aligned fiber case. As the nanofiber waviness ratio increased to a modest level $(h / \lambda=0.1)$, the predicted longitudinal modulus $\left(E_{L}\right)$ decreased substantially. For higher degrees of fiber waviness $(h / \lambda \geq 0.3)$, the nanofibers ceased to make a significant contribution to the effective longitudinal modulus and the bulk composite properties are more consistent with those of the 
unmodified matrix. In contrast, the predicted transverse modulus $\left(E_{T}\right)$ increased gradually with increasing nanofiber waviness ratio; this is likely due to an increase in the fraction of the projected fiber lengths lying in planes perpendicular to the longitudinal direction. For higher degrees of fiber waviness $(h / \lambda \geq 0.3)$, the calculated transverse modulus $\left(E_{T}\right)$ was greater than the longitudinal modulus $\left(E_{L}\right)$.

Figure $20 \mathrm{~b}$ contains a plot of the effective elastic modulus as a function of nanofiber waviness ratio $(h / \lambda)$ for composites containing randomly oriented wavy VGCNFs $\left(c_{f}=0.01, c_{v}=0\right)$, as well as randomly oriented wavy VGCNFs and voids $\left(c_{f}=c_{v}=0.01\right)$. Again, an increase in the degree of nanofiber waviness sharply decreased the predicted effective modulus. The relative influence of voids on the nanocomposite properties was more pronounced than for the aligned wavy nanofiber case; the addition of voids resulted in roughly a $4 \%$ decrease in the effective elastic modulus. Figure $20 \mathrm{~b}$ also includes the measured mean visible fiber waviness ratio, $\left(h_{v} / \lambda_{v}\right)_{\text {EXP }}=0.054$; the reported value likely represents a lower bound on expected $h_{v} / \lambda_{v}$ values. The actual mean fiber waviness ratio in real composites may be somewhat greater than $h_{v} / \lambda_{v}=0.054$, resulting in a significant decrease in effective modulus in comparison with the straight nanofiber case $(h / \lambda=0.0)$.

For illustration purposes, the preceding parametric studies used VGCNF elastic modulus data, which assumed the nanofibers were of solid cross-section. VGCNFs, however, typically display hollow architectures (Figure 13). We have measured the mean diameter, wall thickness, and visible nanofiber waviness ratio for PR-24-XT-LHT-OX VGCNFs in a Derakane 441-400 VE matrix. Figure 21 contains a plot of the measured elastic modulus for such nanocomposites from flexural tests performed by Lee (2010). The measured flexural modulus increased with increasing nanofiber volume fraction up to local maximum occurring at $0.48 \mathrm{v} \%$ of nanoreinforcements; then the measured modulus decreased as the volume fraction of nanofibers was further increased. Such a drop in bulk composite properties with increasing amounts of nanoreinforcements is fairly typical, and is often attributed to poor nanofiber dispersion, the presence of agglomerates/voids, or other consequences of increasing nanophased liquid resin viscosity.

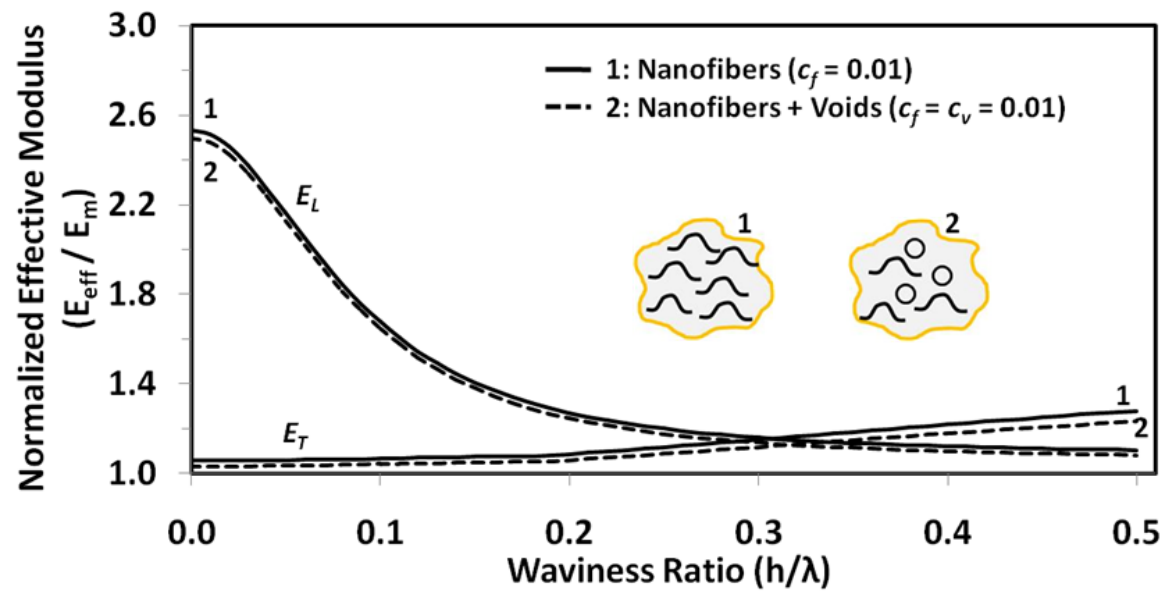

a)

0.2
Waviness Ratio $(\mathrm{h} / \lambda)$ 


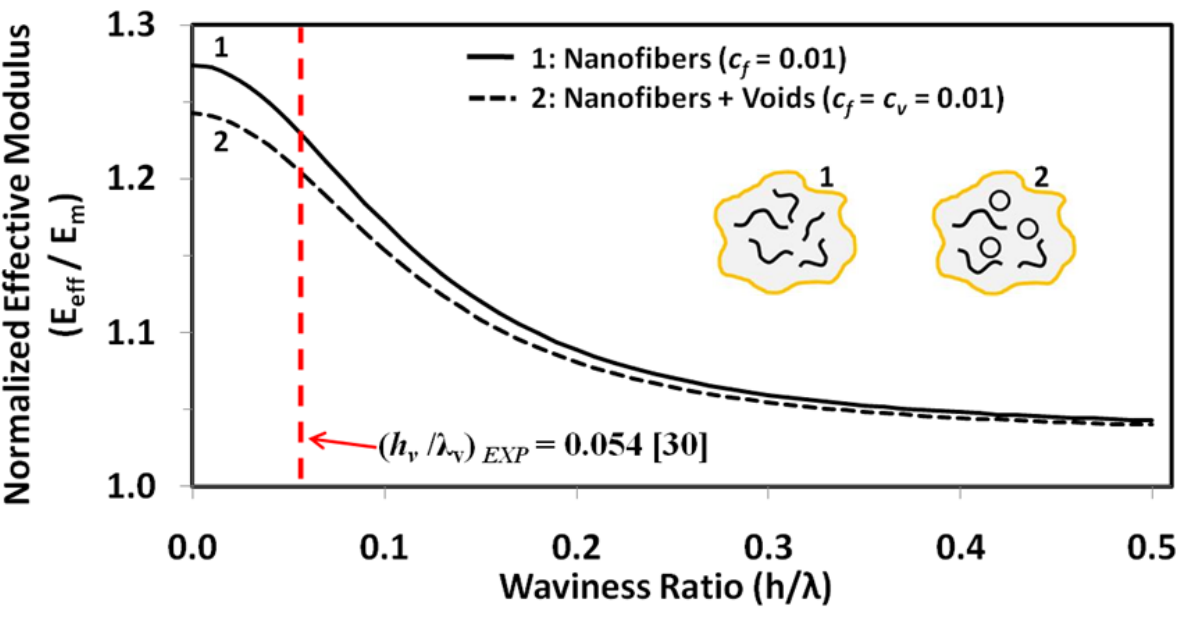

Figure 20: Effect of nanofiber waviness and voids on predicted effective elastic moduli of VGCNF/VE nanocomposites containing a) aligned nanofibers and b) randomly oriented nanofibers $\left(c_{f}=0.01\right)$.

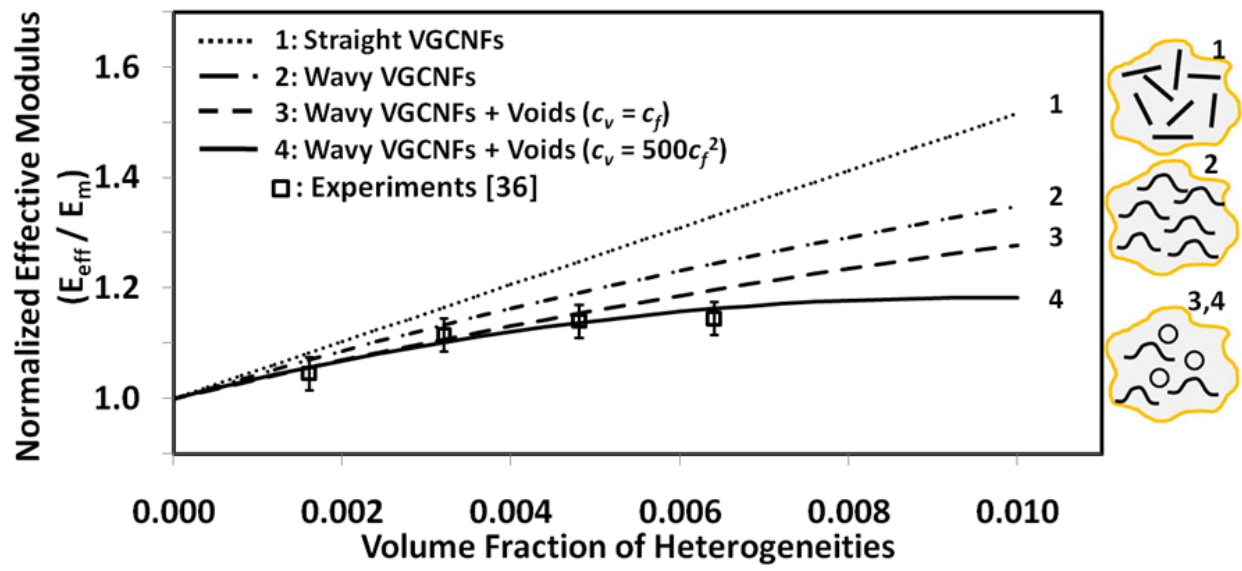

Figure 21: Measured (Lee, 2010) and predicted effective elastic moduli for VGCNF/VE nanocomposites.

The SCM module in EC-MAC was used to predict the effective modulus for these nanocomposites, assuming the composite consisted of well-dispersed randomly oriented hollow nanofibers. The VGCNF diameter and wall thickness data were used to define the nanofiber crosssection. The technique proposed by us was used to estimate the elastic modulus of solid carbon in the hollow nanofibers from measured data from (Ozkan et al., 2010). Figure 21 contains a plot of the effective elastic modulus as a function of heterogeneity volume fraction for VGCNF/VE nanocomposites containing randomly oriented, straight, hollow nanofibers. The predicted moduli for the straight fiber case significantly exceeded the observed moduli from (Lee, 2010), particularly at higher volume 
fractions of nanofibers. Additional estimates were obtained for composites containing randomly oriented wavy VGCNFs, where the nanofiber waviness ratio, $\left(h_{v} / \lambda_{v}\right)=0.054$ was used in the calculations. Such estimates better matched the experimental data at lower nanofibers volume fractions, but still over-predicted the measured moduli as the VGCNF volume fraction was increased (Figure 21). As mentioned previously, an increase in resin viscosity due to nanoreinforcements can lead to difficulties in degassing and increased void content in the nanophased matrix. Therefore, effective elastic moduli were determined for nanocomposites containing equal volume fractions of randomly oriented wavy VGCNFs and voids $\left(c_{f}=c_{v}\right)$. These predicted moduli better matched the measured data when equal volume fractions of nanofibers and voids were present, but these calculations failed to capture the local maximum in observed moduli and the corresponding decrease in modulus at higher nanofiber volume fractions. Toll (1998) and Sudduth (2008) showed that liquid resin viscosities can increase as a nonlinear function of the reinforcement volume fraction. This suggests that the void volume fraction may increase in a similar fashion. Thus, effective elastic moduli were determined for nanocomposites containing randomly oriented wavy VGCNFs and voids, where the volume fraction of voids increased as a quadratic function of nanofiber volume fraction, i.e., $c_{v}=500 \cdot c_{f}^{2}$. For this case, the estimated moduli matched the observed values, and the predicted moduli captured the essential nonlinear character of the experimental data.

The preceding calculations assumed that the nonlinear variation in nanocomposite effective moduli was primarily attributable to void content. In practice, the variations in moduli may be due to a combination of poor nanofiber dispersion, the presence of agglomerates, as well as voids. The effective volume fraction of VGCNFs (i.e., the fraction that is well dispersed) may be somewhat lower than the volume fraction used in specimen preparation due to the presence of agglomerates, particularly as the amount of nanofibers is increased. This will serve to reduce the bulk composite properties in comparison to the case involving a uniform distribution of fibers. In addition, clustered nanofibers with poor fiber wetting may become entrapped in void-like agglomerates whose volume drastically exceeds that of the fibers themselves. Accurate determination of the effective volume fraction of nanofibers, as well as the volume fractions of agglomerates and other void-like structures, remains a significant challenge. Nonetheless, the modeling approach developed in this work provides a framework for predicting effective properties for composites containing multiple distinct nanoheterogeneities.

\section{CONCLUSIONS}

In this work, key issues in multiscale modeling of thermoset composites and nanocomposites are addressed within an Integrated Computational Materials Engineering framework, with a focus on thermoset vinyl ester matrices reinforced with vapor-grown carbon nanofibers. Over the duration of this project, experimental efforts were coupled with the computational approaches such as integrated molecular dynamics (MD), multiscale computational micromechanics, and global finite element simulations to determine composite material behavior from the molecular to global structural scales. Novel MD calculations were performed to assess the potential for the formation of a distinct interphase region at the nanofiber/matrix interface, which account for the complex physio-chemistry between the liquid resin and carbon nanofiber surface. An innovative crosslinking algorithm has being developed for vinyl ester that accounts for regio-selectivity, monomer relative reactivity ratios, and other key aspects 
of free radical polymerization. After crosslinking, MD nanofiber pullout simulations were performed to determine nanocomposite interphase properties and interfacial strengths for use in higher length scale models.

Appropriately averaged materials property data generated using MD simulations, such as those performed here, can feed directly into local continuum-based nano- or microscale calculations within the NASA MAC/GMC temporally concurrent, spatially sequential multiscale analysis framework. Hence, progressive failure analyses can be performed to establish structure-property relationships over a wide range of length scales, which account for the morphologies and geometries of real heterogeneous materials. This investigation will facilitate the development of engineered multiscale materials design by providing insight into relationships between nanomaterial fabrication/processing, chemical and physical characteristics, and interaction and evolution of structure across disparate spatial scales that lead to improved macroscale performance.

\section{Publications}

\section{Journals:}

- Nouranian S, Lee J, Torres GW, Lacy TE, Toghiani H, Pittman Jr CU. 2013. Effects of molding and curing atmosphere on the flexural properties of vinyl ester. Polym Polym Compos (In Press).

- Torres G, Nouranian S, Lacy TE, Toghiani H, Pittman Jr CU, DuBien JL. 2012. Statistical characterization of the impact strengths of vapor-grown carbon nanofiber/vinyl ester nanocomposites using a central composite design. J Appl Polym Sci, DOI: 10.1002/app.38190.

- Jang C, Lacy T, Gwaltney SR, Toghiani H, Pittman CU. 2012. Relative reactivity volume criterion for cross-linking: Application to vinyl ester resin molecular dynamics simulations. Macromolecules 45:4876-4885.

- Jang C, Nouranian S, Lacy TE, Gwaltney SR, Toghiani H, Pittman Jr CU. 2012. Molecular dynamics simulations of oxidized vapor-grown carbon nanofiber surface interactions with vinyl ester resin monomers. Carbon 50(3):748-760.

- Yu J, Lacy TE, Toghiani H, Pittman Jr CU. 2012. Effective property estimates for composites containing multiple nanoheterogeneities: Part I, nanospheres, nanoplatelets, \& voids. J Compos Mater, DOI: 10.1177/ 0021998312442557.

- Yu J, Lacy TE, Toghiani H, Pittman Jr CU. 2012. Effective property estimates for composites containing multiple nanoheterogeneities: Part II, nanofibers \& voids. J Compos Mater, DOI: 10.1177/0021998312446824.

- Nouranian S, Toghiani H, Lacy TE, Pittman Jr CU. 2012. Effects of formulation, processing, and temperature on the viscoelastic properties of vapor-grown carbon nanofiber/vinyl ester nanocomposites. J Appl Polym Sci (Submitted). 
- Yu J, Lacy TE, Toghiani H, Pittman Jr CU. 2011. Micromechanically-based effective thermal conductivity estimates for polymer nanocomposites. Composites Part B (Submitted).

- Jang C, Lacy T, Gwaltney SR, Toghiani H, Pittman CU. 2012c. Interfacial shear strength of cured vinyl ester resin-graphene sheets from molecular dynamics simulations. ACS Chemistry Materials (Submitted).

\section{Manuscripts in Preparation:}

- Lee J, Nouranian S, Torres GW, Lacy TE, Toghiani H, Pittman Jr CU, DuBien J. Flexural properties of vapor-grown carbon nanofiber/vinyl ester nanocomposites. To be submitted to J Appl Polym Sci, tentative submission date December 2012.

- Abuomar O, Nouranian S, King R, Bouvard J-L, Toghiani H, Lacy TE, Pittman Jr CU. Data mining and knowledge discovery techniques in materials science and technology: Polymer nanocomposites case study. To be submitted to Stat Anal Data Min, tentative submission date December 2012.

- Hutchins JW, Sisti J, Ricks T, Nouranian S, Lacy TE, Pittman Jr CU, Toghiani H. Strain rate sensitivity of the compressive response of carbon nanofiber reinforced vinyl ester. To be submitted to Mech Time-Depend Mat, tentative submission date January 2013.

\section{Conference Publications:}

- Drake D, Simsiriwong J, Sullivan RW, Toghiani H, Nouranian S, DuBien JL, Lacy TE, Pittman Jr CU. Creep compliance characterization of vapor-grown carbon nanofiber/vinyl ester nanocomposites using a central composite design of experiments. In the Proceedings of the $54^{\text {th }}$ AIAA/ASME/ASCE/AHS/ASC Structures, Structural Dynamics, and Materials Conference, Boston, MA, April 8-11, 2013.

- Ricks TM, Lacy TE, Bednarcyk BA, Arnold SM. On the development of a damage tolerance plan for multifunctional composites. In the Proceedings of the American Society for Composites 27th Annual Technical Conference, Arlington, Texas, October1-3, 2012.

- Jang C, Lacy TE, Gwaltney SR, Toghiani H, Pittman Jr CU. Interfacial shear strength of a cured vinyl ester resin at graphene sheets from molecular dynamics simulations. In the Proceedings of the American Society for Composites 27th Annual Technical Conference, Arlington, Texas, October1-3, 2012.

- Drake DA, Simsiriwong J, Sullivan RW, Toghiani H, Lacy TE, Pittman Jr CU. Creep characterization of vinyl ester nano-composites using the digital image correlation technique. In the Proceedings of the American Society for Composites 27th Annual Technical Conference, Arlington, Texas, October1-3, 2012.

- Jang C, Lacy T, Gwaltney SR, Toghiani H, Pittman Jr CU. Molecular dynamics simulation of vinyl ester resin crosslinking. In the Proceedings of the 53rd AIAA/ ASME/ ASCE/ AHS/ ASC Structures, Structural Dynamics, and Materials Conference, Honolulu, Hawaii, April 23-26, 2012. 
- Ricks TM, Lacy T, Bednarcyk BA, Arnold SM. A multiscale modeling methodology for metal matrix composites including fiber strength stochastics. In the Proceedings of the 53rd AIAA/ ASME/ ASCE/ AHS/ ASC Structures, Structural Dynamics, and Materials Conference, Honolulu, Hawaii, April 23-26, 2012.

- Yu J, Lacy TE, Toghiani H, and Pittman Jr CU. Micromechanically based effective thermal conductivity estimates for polymer nanocomposites. In the Proceedings of the 53rd AIAA/ASME/ASCE/AHS/ASC Structures, Structural Dynamics, and Materials Conference, Honolulu, Hawaii, April 23-26, 2012.

\section{REFERENCES}

ABAQUS 6.7. 2009.Simulia, Inc.

Al-Saleh MH, Sundararaj U. 2009. A review of vapor grown carbon nanofiber/polymer conductive composites. Carbon 47:2-22.

Arnold S, Bednarcyk B, Wilt T, Trowbridge D.1999. Micromechanical analysis code with generalized method of cells (MAC/GMC) user's guide, v3.0.NASA/TM-1999-209070,Cleveland, OH, USA.

Benveniste Y. 1987. A new approach to the application of Mori-Tanaka's theory in composite materials. Mech Mater 6:147-157.

Boo WJ, Sun L, Warren GL, Moghbelli E, Pham H, Clearfield A, Sue HJ. 2007. Effect of nanoplatelets aspect ratio on mechanical properties of epoxy nanocomposites. Polymer 48:1075-1082.

Bouvard J-L, Ward DK, Hossain D, Nouranian S, Marin E, Horstemeyer M. 2009. Review of hierarchical multiscale modeling to describe the mechanical behavior of amorphous polymers. J Eng Mater Technol131(4):041206-1.

Brandrup J, Immergut EH. 1989. Polymer handbook, 3rd ed. John Wiley \& Sons: New York:181-195.

Brown D, Clarke JHR. 1998. Molecular dynamics simulation of an amorphous polymer under tension. I. Phenomenology. Macromolecules 24:2075-2082.

Buryachenko VA, Roy A, Lafdi K, Anderson KL, Chellapilla S. 2005. Multi-scale mechanics of nanocomposites including interface: experimental and numerical investigation. Compos Sci Technol 65:2435-2465.

Chasiotis I, Chen Q, Odegard GM, Gates TS. 2005. Structure-property relationships in polymer composites with micrometer and submicrometer graphite platelets. Exp Mech 45(6):507-516.

Eshelby JD. 1957. The determination of the elastic field of an ellipsoidal inclusion and related problems.Proceedings of the Royal Society A241:376-396.

Fermeglia M, Pricl S. 2007. Multiscale modeling for polymer systems of industrial interest. Prog Org Coat 58:187-199. 
Fisher F, Bradshaw R, Brinson L. 2003. Fiber waviness in nanotube-reinforced polymer composites - I: Modulus predictions using effective nanotube properties. Compos Sci Technol 63(11):1689-1703.

Gates TS, Odegard GM, Frankland SJV, Clancy TC. 2005. Computational materials: multi-scale modeling and simulation of nanostructured materials. . Compos Sci Technol 65(15-16):2416-2434.

Hill R. 1965. A self-consistent mechanics of composite materials. J Mech Phys Solids 13:213-222.

Hsiao H, Daniel IM. 1996. Effect of fiber waviness on stiffness and strength reduction of unidirectional composites under compressive loading. Compos Sci Technol 56:581-593.

Huang JS, Gibson LJ. 1993. Elastic moduli of a composite of hollow spheres in a matrix. J Mech Phys Solids 41:55-75.

Hutchins JW, Sisti J, Lacy TE, Nouranian S, Toghiani H, Pittman Jr CU. 2009. High strain rate behavior of carbon nanofiber reinforced vinyl ester. Proceedings of the 50th AIAA/ASME/ASCE/AHS/ASC Structures, Structural Dynamics, and Materials Conference, Palm Springs, CA.

Inoue R, Kanaya T, Nishida K, Tsukushi I, Telling MTF, Gabrys BJ, Tyagi M, Soles C, Wu W-I. 2009. Glass transition and molecular mobility in polymer thin films. Phys Rev E 80:031802.

Jancar J. 2009. Interphase phenomena in polymer micro- and nanocomposites. In: Karger-Kocsis, J., Fakirov, S., editors. Nano- and micro-mechanics of polymer blends and composites, Hanser Publications, 241-266.

Jang C, Nouranian S, Lacy TE, Gwaltney SR, Toghiani H, Pittman Jr CU. 2012a. Molecular dynamics simulations of oxidized vapor-grown carbon nanofiber surface interactions with vinyl ester resin monomers. Carbon 50(3):748-760.

Jang C, Lacy T, Gwaltney SR, Toghiani H, Pittman CU. 2012b. Relative reactivity volume criterion for cross-linking: Application to vinyl ester resin molecular dynamics simulations. Macromolecules 45:48764885.

Jang C, Lacy T, Gwaltney SR, Toghiani H, Pittman CU. 2012c. Interfacial shear strength of cured vinyl ester resin-graphene sheets from molecular dynamics simulations. ACS Chemistry Materials (Submitted).

Lakshminarayanan PV, Toghiani H, Pittman CU. 2005. Nitric acid oxidation of vapor grown carbon nanofibers. Carbon: 2422-2433.

Lee J. 2010. Characterization of the effect of material/fabrication parameters on the mechanical response of vapor-grown carbon nanofiber/vinyl ester nanocomposites. Thesis of Aerospace Engineering, Mississippi State University.

Li H. 1998. Synthesis, characterization, and properties of vinyl ester matrix resins. Blacksburg VA USA, Virginia Polytechnic Institute and State Univ, PhD dissertation. 
Liao K, Li S. 2001. Interfacial characteristics of a carbon nanotube-polystyrene composite system. Appl Phys Lett. 79:4225-4227.

Maiti A. 2008. Multiscale modeling with carbon nanotubes. Microelectronics Journal 39:208-221.

Mori T, Tanaka K. 1973. Average stress in matrix and average elastic energy of materials with misfitting inclusions. Acta Metallurgica et Materialia 21:571-574.

Mura T. 1987. Micromechanics of defects in solids, 2nd ed. Martinus Nijhoff, Dordrecht:1-73.

Nemat-Nasser S, Hori M. 1993. Micromechanics: overall properties of heterogeneous materials. NorthHolland, Amsterdam:368-386.

Nielsen LE. 1968. Crosslinking-effect on physical properties of polymers. ONR/ARPA Association, Washington University, Monsanto.

Nouranian S, Toghiani H, Lacy TE, Pittman Jr CU, Dubien J. 2011a. Dynamic mechanical analysis and optimization of vapor-grown carbon nanofiber/vinyl ester nanocomposites using design of experiments. J Compos Mater 45:1647-1657.

Nouranian S, Jang C, Lacy TE, Gwaltney SR, Toghiani H, Pittman Jr CU. 2011b. Molecular dynamics simulations of vinyl ester resin monomer interactions with a pristine vapor-grown carbon nanofiber and their implications for composite interphase formation. Carbon 49(10): 3219-3232.

Odian G. 1981. Principles of polymerization., 2nd ed. John Wiley \& Sons: New York:30-33.

Ozkan T, Naraghi M, Chasiotis I. 2010. Mechanical properties of vapor grown carbon nanofibers. Carbon, 48:239-244.

Plaseied A, Fatemi A, Coleman MR. 2008. The influence of carbon nanofiber content and surface treatment on mechanical properties of vinyl ester. Polym Compos 16(7):405-413.

Plaseied A, Fatemi A. 2009. Tensile creep and deformation modeling of vinyl ester polymer and its nanocomposite. J Reinf Plast Comp 28(14):1775-1788.

Qi D, Hinkley J, He G. 2005. Molecular dynamics simulation of thermal and mechanical properties of polyimide-carbon nanotube composites. Model Simul Mater Sci Eng 13:493-507.

Rasheed A, Dadmun MD, Britt PF. 2006. Polymer-nanofiber composites: enhancing composite properties by nanofiber oxidation. J Appl Phys: 103-109.

Ray JR. 1988. Elastic constants and statistical ensembles in molecular dynamics. Comput Phys Rep 8:109152.

Ricks TM, Lacy T, Bednarcyk BA, Arnold SM. A multiscale modeling methodology for metal matrix composites including fiber strength stochastics. In the Proceedings of the 53rd AIAA/ ASME/ ASCE/ AHS/ ASC Structures, Structural Dynamics, and Materials Conference, Honolulu, Hawaii, April 23-26, 2012. 
Shonaike GO, Advani SG. 2003. Advanced Polymeric Materials, Structure Property Relationships, CRC Press.

Sudduth R. 2008. A new approach to controlling the viscosity of a coating with a blend of particles with significantly different shapes. Pigm Resin Technol 37(6):362-374.

Tadmor EB, Phillips R, Ortiz M. 2000. Hierarchical modeling in the mechanics of materials. Int J Solid Struct 37:379-389.

Thostenson ET, Chou T-W. 2006. Processing-structure-multi-functional property relationship in carbon nanotube/epoxy composites. Carbon 44(14):3022-3029.

Toll S. 1998. Packing mechanics of fiber reinforcements. Polym Eng Sc 38(8):1337-1350.

Valavala PK, Clancy TC, Odegard GM, Gates TS. 2007. Nonlinear multiscale modeling of polymer materials. Int J Solid Struct 44(3-4):1161-1179.

Velasco-Santos C, Martínez-Hernández AL, Fisher FT, Ruoff R, Castaňo VM. 2003. Improvement of thermal and mechanical properties of carbon nanotube composites through chemical functionalization. Chem Mater 15:4470-4475.

Yu J, Lacy TE, Toghiani H, Pittman Jr CU. 2011a. Classical micromechanics modeling of nanoreinforced composites with carbon nanofibers and interphase. J Compos Mater Pre-published online DOI: 10.1177/0021998311401092.

Zeng $\mathrm{QH}, \mathrm{Yu} A B$, Lu GQ. 2008. Multiscale modeling and simulation of polymer nanocomposites. Prog Polym Sci 33(2):191-269.

Zhang H, Zhang Z, Friedrich K, Eger C. 2006 Property improvements of in situ epoxy nanocomposites with reduced interparticle distance at high nanosilica content. Acta Materialia 54:1833-1842.

Zhou Y, Pervin F, Jeelani S. 2007. Effect vapor grown carbon nanofiber on thermal and mechanical properties of epoxy. J Mater Sci 42:7544-7533.

\section{KEY WORDS}

Automotive Composites, Nanocomposites, Fabrication, Multiscale Modeling, Micromechanics, Finite Element Modeling, Experiments, SEM

\section{Brief Description of Report}

A multiscale design methodology is being employed to investigate the effects of nanoreinforcements on the mechanical properties of fiber-reinforced composites for automotive structural applications. 


\section{Subtask 3.3: Natural Fiber Composites for Structural Component DESIGN}

Team Members: S. Q. Shi, Kaiwen Liang, Jinwu Wang, Mohammad Qatu, Mark Tschopp, Tian Tang, Darrel Nicolas, Linda Sites, Jinshu Shi, Ian Fulton, Emily Zhang, Yan Shen, and Wen Che

\section{Objective}

- Develop fabrication and design methodologies of natural fibers (i.e., kenaf bast) for automotive structural component applications.

\section{Approaches}

- Natural fiber retting: Develop natural fiber retting technologies and recommend solutions aimed at improving the fiber quality and reducing the cost.

- Natural fiber treatment: Use nanotechnology and/or chemical modification on the natural fibers to enhance the compatibility and interfacial bonding between the fibers and polymer matrices.

- Composite fabrication and testing: Develop and optimize the natural fiber sheet molding compound (SMC) fabrication techniques to obtain the best performance.

- Composite modeling: Conduct micromechanical material modeling and simulation to provide guidance for the composite fabrication and to elucidate the functions of the deposited nanophase on the fiber surface for the natural fiber composites.

\section{ACCOMPLISHMENTS}

- An all chemical process was developed to obtain cellulose nanowhiskers (CNW) from kenaf bast fibers.

- Inorganic nanoparticle impregnation (INI) natural fibers were successfully generated from the developed chemical retting and INI processes for natural fiber SMC fabrication.

- A concept was proven on novel techniques on nanophase treatment of natural fibers for processing functional natural fiber nanocomposite products and the conversion of celluloses into carbon materials.

- An attempt has been made to investigate using soybean oil additive into the resin formulation as a sizing agent of natural fiber SMC products. A composite with a 24-hour WA of less than $1.5 \%$ could be obtained by the incorporation of carbon nanotube.

- An accelerated weathering test was conducted to determine the strength properties of natural fiber sheet molding compound before and after exposure to cyclic wet/dry cycles in a weathering cabinet. Impregnated kenaf fiber SMC did not show degradation after a exposure for 
a total of 720 hours at cycles of 4 hours UV exposure at $600 \mathrm{C}$ followed by 4 hours condensation 400 C (ASTM D4329).

- An experimental technique was developed to stress the micro cellulosic fibers. A property comparison was conducted on several representative individual fibers.

- A study was conducted on the effect of pressure in hot pressing and layups on the properties of hybrid polyester composites from randomly woven kenaf fibers with fiberglass.

- Vinyl tris(2-ethoxymethoxy) silane treatment of kenaf fiber was successfully conducted. The water absorption and flexural properties of the resulting fiber composites were improved.

- Amorphous cellulose models were successfully created with the use of ReaxFF. Preliminary molecular dynamic simulations have been presented for amorphous cellulose.

- Principles and practices of life-cycle assessment (LCA) were used to evaluate environmental performance of natural fiber reinforced bio-resin matrix composites. Preliminary results indicated their superiority in environmental performance to a traditional glass fiber polymer composite.

\section{SUMMARY}

The overall goal of the project is to substitute the glass fiber with natural fiber as reinforcements in polymer matrices to achieve cost and weight savings without compromising the mechanical property requirements. The alkaline retting treatment efficiently removed the hemicellulose and lignin from kenaf bast fiber. Bleaching treatment could remove the remnant lignin from the retted fibers. Pure cellulose fibers in micrometer scale and nanometer scale were obtained by acidic hydrolysis of the bleached fibers. Our kenaf natural fiber SMC has comparative properties compared with the commercial glass fiber SMC. The impregnated kenaf fiber SMC was not degraded during accelerated weathering test. The newly designed SF-I microtester is well suited for measuring mechanical properties of individual micro fibers. Among the 4 fibers used in the testing, bamboo, kenaf, Chinese fir, and ramie, kenaf bast fiber showed the lowest elongation at break and other properties of kenaf were balanced. A detailed procedure was developed to fabricate the laminated natural fiber kenaf-based composites. Various samples are manufactured with modulus of elasticity (MOE) up to $10 \mathrm{GPa}$ and modulus of rupture (MOR) up to $270 \mathrm{MPa}$. Vinyl tris(2-ethoxymethoxy) silane treatment of kenaf fibers improved the physical and mechanical properties of the resulting kenaf fiber composites. The water absorption of silane treated kenaf fiber composites decreased by $22 \%$ compared to the untreated fiber composites. The flexural strength and modulus of silane treated kenaf fiber composites increased by $25 \%$ and $8 \%$, respectively. The simulated stress-strain behavior using molecular dynamics (MD) modeling showed the similar trends both qualitatively and quantitatively as those observed in the experimental testing for the amorphous cellulose. The multi-scale model of the moisture absorption process of natural fiber composites was developed on the basis of the theoretical framework of VAMUCH. The preliminary result of life cycle assessment (LCA) demonstrated that the use of modified soybean oil and natural fiber to make sheet molding compound (SMC) had a great potential from an ecological point of view. 


\section{INTRODUCTION}

Kenaf bast fiber is attractive due to its fast growing characteristic, high fiber yield and cellulose content, good mechanical properties, etc. It was reported by Zadorecki and Michell (1989) that the modulus of the fibers increased significantly by breaking down the cellulosic fiber into smaller sizes from solid wood (10 GPa), to single pulp fiber (40 GPa), to microfibrils (70 GPa), and to crystallites (250 GPa). Therefore, research work on high crystalline cellulose fibers, e.g. microfibrils, nanowhiskers, or nanofibers, has drawn great attention in recent years (Henriksson, et al. 2007; Iwamoto, et al. 2005; Kulpinski, 2005; Sui, et al., 2008). Quantitatively characterizing the micro-mechanical properties of individual natural fibers will help select the most appropriate fiber types and pulping processes to manufacture the targeted grade of fiber reinforced composites. In an effort to present more 'green' material for massive manufacturing that are both competitive in their properties and more environmental friendly, natural fibers are being considered for possible applications in the automotive industry. An exploratory study of the effects of pressure and layup on a hybrid composite of randomly oriented woven kenaf fibers and fiberglass/polyester sheet molding compound (SMC) was conducted.

Natural fiber is easy to absorb moisture due to its hydrophilic nature. Silane treatment of the fibers has shown to be effective in reducing moisture uptake of cellulosic fibers in humid environments (Bisanda and Ansell 1991). Attempts have been made to use the silane treatment of the kenaf bast fibers for the purpose of improving the moisture resistant properties for the natural fiber SMC. Molecular modeling complements the experimental work. MD simulation work has been focusing on the understanding the variables associated with the deformation of thermoplastic polymers. Natural fiber reinforced composites have been generally perceived as renewable, biodegradable and environmentally friendly products. However, it was difficult to quantitatively measure their environmental friendliness and make a sound comparison with other competing materials. A life-cycle assessment is a technique to assess environmental impacts associated with all the stages of a product's life from raw material extraction and processing to product manufacture, distribution, use, repair and maintenance, and disposal or recycling after the end-of-life.

\section{Physical/Mechanical Properties and Durability of Natural fiber Sheet Molding Compound}

\section{Objective:}

The purpose of this study was to determine the physical and mechanical properties of natural fiber SMC. Durability is an important aspect for the overall performance of the products used in both interior and exterior applications where sunlight and moisture could have an effect. Another objective of this study was to evaluate the performance of natural fiber SMC when exposed to cyclic wet/dry cycles in a weathering cabinet.

\section{Methods:}


The weathering apparatus used for this study was a Q-U-V Cyclic Ultraviolet Weathering Tester (120V, $60 \mathrm{~Hz}$ Model manufactured by The Q-Panel Company, Cleveland Ohio 44145. The Q-Panel uses eight fluorescent UVA 340 lamps (UV range $365 \mathrm{~nm}$ to $295 \mathrm{~nm}$ ). The unit is equipped with a 24 hour timer divided into 15-minute tabs to provide a wide range of cycles. The test samples were cut from panels that had been exposed for a total of 720 hours at cycles of 4 hours UV exposure at $60^{\circ} \mathrm{C}$ followed by 4 hours condensation $40^{\circ} \mathrm{C}$. This exposure procedure is recommended in ASTM D4329 (Stand Practice for Operating Light and Water Exposure Apparatus for Exposure of Plastics). Test samples measured as 2.6 $\mathrm{mm} \times 19.25 \mathrm{~mm} \times 76 \mathrm{~mm}$ ( $\mathrm{t} \times \mathrm{r} \times \mathrm{l}$ ) were cut from both exposed and unexposed samples. A bending test was performed using a $1.75 \mathrm{~mm}$ deflection and a loading speed of $0.5 \mathrm{~mm}$ per minute to determine the modulus of elasticity (MOE). Each sample was tested two times to obtain an average value. The MOE results of unexposed controls and exposed samples were compared in order for determining the strength loss due to the weathering effect.

\section{RESULtS AND Discussion}

Two natural fiber SMC samples were sent to Processing RIC Department at Ford Motor Company, MI for evaluation. Table 1 shows the tensile properties of the two ntural fiber SMC panels (about 60 - 65 wt\% fiber $+\mathrm{CaCO}_{3}$ ) with a comparison of Class A glass fiber SMC (EpicBlendSMC ${ }^{\mathrm{TM}}$ ). It is shown in Table 10.1 that the natural fiber SMC we developed showed higher tensile modulus than that of glass fiber SMC.

TABLE 1: TENSILE PROPERTIES OF RETTED KENAF FIBER SMC AND GLASS FIBER SMC

\begin{tabular}{|l|l|l|}
\hline Panel & Tensile Modulus, GPa & Tensile Strength, MPa \\
\hline Retted Kenaf SMC (Average of 2 panels) & 13.7 & 69.6 \\
\hline Glass Fiber SMC (Class A) & 10.1 & 85 \\
\hline
\end{tabular}

For the product durability testing, the samples were measured and weighted before putting into the QV chamber for testing. The testing samples were removed and weighted two times per week at both the UV and condensation cycles. The samples were also re-positioned weekly in order to provide a uniform UV exposure. After the exposure, the panels were allowed to equilibrate back to the ambient conditions and the weights of the samples were measured. All of the exposed kenaf SMC (about 60 - 65 wt\% fiber $+\mathrm{CaCO}_{3}$ ) samples came back to their original ambient weights after testing. In contrast, both of the fiber glass SMC samples had an approximate $1 \mathrm{~g}$ decrease in weight after the exposure (Table 2). Visually, glass fiber SMC had significant yellow-brown color change on the exposed face of the panel due to the UV exposure, while the kenaf fiber SMC did not show color changes (Figure 1). 
TABLE 2: INITIAL, UV CYCLE, CONDENSATION CYCLE WEIGHTS AND WEIGHT CHANGE FOR SAMPLES EXPOSED IN A QUV WEATHERING TEST FOR 30-DAYS.

\begin{tabular}{|l|c|c|c|}
\hline Panel & Initial Wt., g & Final Wt., g & Wt. Change, g \\
\hline Impregnated Kenaf SMC (Average of 2 panels) & 111.66 & 111.70 & +0.04 \\
\hline Glass Fiber SMC (Class A) & 241.35 & 240.20 & -1.15 \\
\hline
\end{tabular}

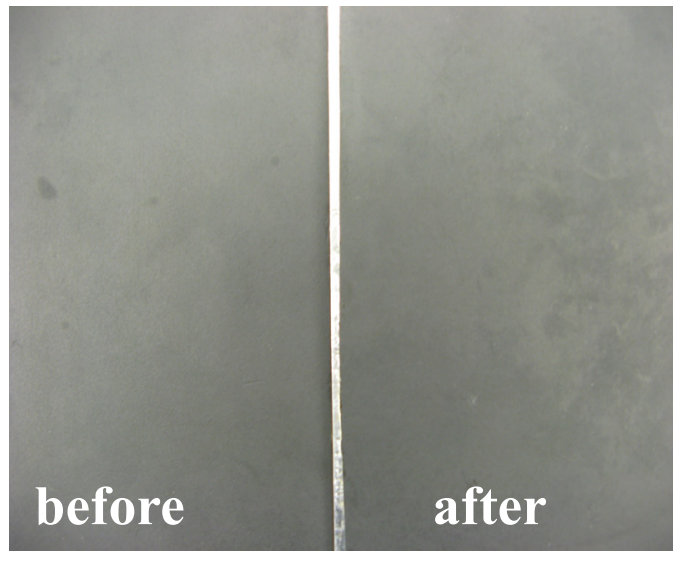

Kenaf SMC

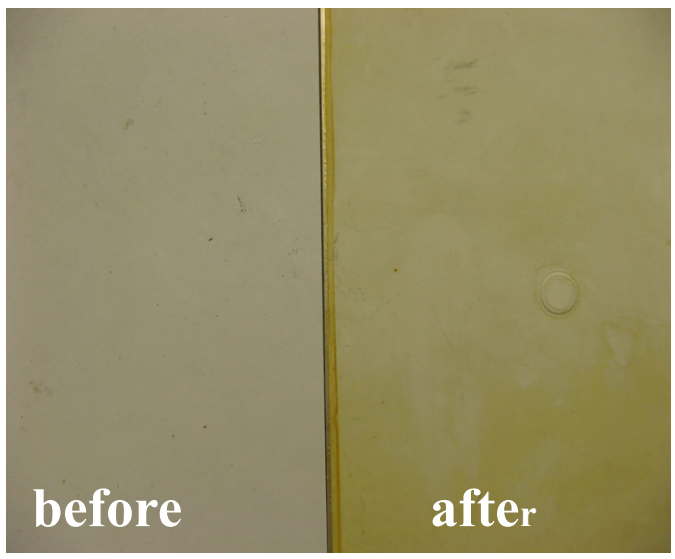

Glass SMC

FIGURE 1: NATURAL FIBER SMC AND GLASS FIBER SMC BEFORE AND AFTER ACCELERATED WEATHERING TEST

The individual and average MOE and percent differences are listed in Table 3. The carbon nanotube treated kenaf bast fiber SMC show no degradation after the accelerated weathering treatments.

TABLE 3: FLEXURAL PROPERTIES, 24 HR WATER ABSORPTION AND AVERAGE PERCENT MOE LOSS OF SAMPLES EXPOSED IN A WEATHERING TEST.

\begin{tabular}{|c|c|c|c|c|}
\hline Panel & $\begin{array}{c}\text { Flexural Strength, } \\
\mathrm{MPa}\end{array}$ & $\begin{array}{c}\text { Flexural Modulus, } \\
\mathrm{GPa}\end{array}$ & $\begin{array}{c}24 \text { hr Water Absorption, } \\
\%\end{array}$ & $\begin{array}{c}\text { Strength Loss, } \\
\%\end{array}$ \\
\hline
\end{tabular}




\begin{tabular}{|c|l|l|l|l|}
\hline $\begin{array}{c}\text { Impregnated } \\
\text { Kenaf SMC } \\
\text { (Average of 2 } \\
\text { panels) }\end{array}$ & 81.68 & 9.07 & 2.32 & -5.33 \\
\hline
\end{tabular}

\section{Chemical Components and Morphologies of the Kenaf Bast fibers obtained from the Chemical Processes}

\section{Objective}

The objective was to study the morphologies and chemical components of the retted fibers, bleached fibers, microfiber and cellulose nanowhiskers (CNWs).

\section{Methods}

Kenaf bast fibers were treated with the chemical processes. Alkaline retting treatment followed by bleaching treatment was applied to remove the hemicellulose and lignin from the raw kenaf bast fibers so that the retted fibers and bleached fibers were obtained. Acidic hydrolysis was conducted on the bleached fibers, and the microfibers and CNWs were obtained. The CNWs had been applied to fabricate polyvinyl alcohol (PVA)/CNW composites and exhibited excellent reinforcement efficiency for tensile properties. The functional groups and crystallinities of the fibers were measured. This study emphasized on the chemical components and morphologies of these fiber types.

\section{Results and Discussion}

Chemical components including holocellulose content, $\alpha$-cellulose content, Klason lignin content and ash content of the raw kenaf bast fibers, retted fibers, bleached fibers, microfibers and CNWs were determined. The ash contents were determined following TAPPI standard T 211-om. 93. Klason lignin contents were estimated in accordance with the method of the Institute of Paper Chemistry (1951). Holocellulose is the total carbohydrate fraction (cellulose and hemicellulose) of the fibers, and its content was estimated by the method of Wise et al. (1946). The term $\alpha$-cellulose describes that part of cellulose which does not dissolve in $17.5 \%$ sodium hydroxide solution. The method employed to investigate the $\alpha$-cellulose contents was according to the method of German Association of Cellulose Chemists and Engineers (1951). The holocellulose contents, $\alpha$-cellulose contents, Klason lignin contents and ash contents of the fibers were shown in Table 4. Alkaline retting treatment of the kenaf bast fibers at $160^{\circ} \mathrm{C}$ with the alkaline liquid's autogenous vapor pressure for $1 \mathrm{~h}$ effectively degraded and dissolved lignin and hemicellulose. The two percentage point difference between the holocellulose content and $\alpha$ cellulose content of retted fiber indicated a small fraction of hemicellulose remaining in retted fibers. If 
the lignin in retted fibers, although as few as $0.24 \%$, had not been removed by bleaching treatment, it would result in acid-insoluble residuals in microfibers and CNWs after acid hydrolysis, and thus debase their purity. Bleaching treatment removed not only the lignin, but also the hemicellulose, producing relatively pure cellulosic fibers for the next acid hydrolysis treatment. Microfibers and CNWs obtained from acid hydrolysis were pure cellulose fibers.

Scanning electron microscopy (SEM, Zeiss Supra TM 40) was applied to analyze fibers morphology. Seventy fibers were randomly chosen for their dimension measurement using the "Smart SEM User Interface". The CNW samples for morphology analysis were obtained by placing a drop of the CNW suspension onto a grid without any staining, and drying it in the air at the ambient temperature. The dried samples were examined in a transmission electron microscopy (TEM, JEOL JEM-2000 EX-II). The dimensions of seventy randomly chosen CNWs were measured from the TEM images. The morphology of the raw kenaf bast fiber, retted fiber, bleached fiber, microfiber and CNW was studies from their SEM or TEM images (Figures 2 and 3). The statistics of the fiber lengths and diameters were shown in Table 5.

TABLE 4: CHEMICAL COMPONENTS OF THE FIBERS.

\begin{tabular}{|c|c|c|c|c|}
\hline & $\alpha$-cellulose & Holocellulose & Klason Lignin & Ash \\
\hline Non-treated fiber & $45.95 \%$ & $75.83 \%$ & $19.10 \%$ & $5.07 \%$ \\
\hline Retted fiber & $92.27 \%$ & $94.22 \%$ & $0.24 \%$ & $2.72 \%$ \\
\hline Bleached fiber & $95.19 \%$ & $95.41 \%$ & $0 \%$ & $2.27 \%$ \\
\hline Microfiber & $100 \%$ & $100 \%$ & $0 \%$ & $0 \%$ \\
\hline CNW & $100 \%$ & $100 \%$ & $0 \%$ & $0 \%$ \\
\hline
\end{tabular}

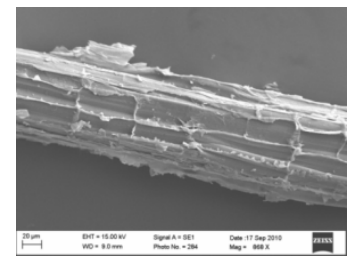

(a) Raw kenaf bast fibers

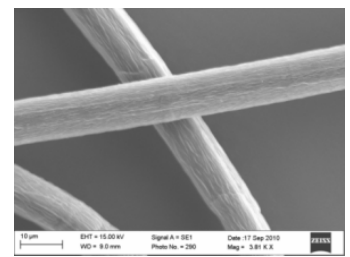

(b) Retted fibers

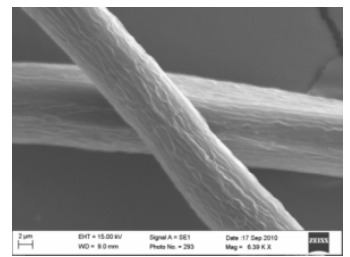

(c) Bleached fibers

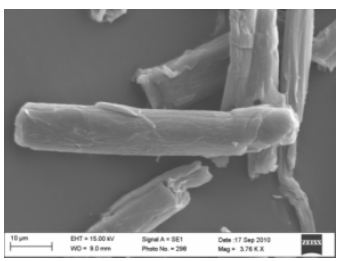

(d) Microfibers

FIGURE 2: SEM IMAGES OF THE RAW KENAF BAST FIBERS, RETTED FIBERS, BLEACHED FIBERS AND MICROFIBERS. 


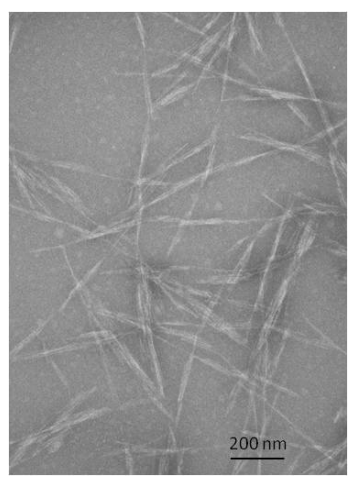

(a)

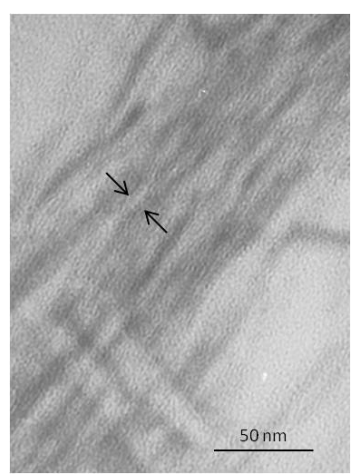

(b)

FIGURE 3: TEM IMAGES OF CELLULOSE NANOWHISKERS (CNWS). (A) MAGNIFICATION = 50,000X, ACCELERATING VOLTAGE $=100 \mathrm{KV}$; (B) MAGNIFICATION= 370,000X, ACCELERATING VOLTAGE $=100 \mathrm{KV}$.

TABLE 5: FIBER LENGTH AND DIAMETER STATISTICS.

\begin{tabular}{|c|c|c|c|c|c|}
\hline \multirow{2}{*}{ Length } & Mean & $\begin{array}{c}\text { Retted } \\
\text { fibers } \\
(\mu \mathrm{m})\end{array}$ & $\begin{array}{c}\text { Bleached fibers } \\
(\mu \mathrm{m})\end{array}$ & $\begin{array}{c}\text { Microfibers } \\
(\mu \mathrm{m})\end{array}$ & $\begin{array}{c}\text { CNWs } \\
(\mathrm{nm})\end{array}$ \\
\cline { 2 - 6 } & Stdev & 606.98 & 141.62 & 46.39 & 628.38 \\
\hline \multirow{2}{*}{ Diameter } & Mean & 10.70 & 10.63 & 9.58 & 360.05 \\
\cline { 2 - 6 } & Stdev & 2.68 & 2.05 & 2.25 & 21.43 \\
\hline
\end{tabular}

The alkaline retting treatment liberated the single fibers from the fiber bundles of the raw kenaf bast fibers. After most of outer layer substances were removed by chemical treatment, retted and bleached fibers had a clean surface. Diameters of retted $(10.70 \mu \mathrm{m})$ and bleached $(10.63 \mu \mathrm{m})$ fiber were similar. The length of bleached fiber was reduced from $471 \mu \mathrm{m}$ (average length of retted fiber) to $215 \mu \mathrm{m}$. Acidic hydrolysis treatment broke the bleached fibers through their cross sections, thus the lengths of the fibers reduced significantly. However, the average diameter of microfibers was $9.58 \mu \mathrm{m}$ and was not significant reduced. The average aspect ratio of the microfiber was 4.97.

\section{A Micro-tension test Method for Measuring four Representative individual Cellulosic Fibers}

\section{Objectives}

The overall objective of this research was to develop an experimental technique to stress the micro cellulosic fibers and to evaluate mechanical properties of several representative individual fibers.

This work was collaborated with the International Center for Bamboo and Rattan (ICBR), Beijing, China. 


\section{Micro Tensile Test System Development}

A micro tension testing system was devised to measure the mechanical properties of individual cellulosic fibers. The tensile test system (SF-1 microtester) consists of two ball-and-socket type grips inside an environmental chamber, a load cell (UL-10GR; Minebea Co. Ltd., Tokyo, Japan), a three-dimension adjustable stage with an attached high precision linear ball bearing slide (SKF, Sweden), two horizontal and vertical CCD cameras with macro lenses (Daheng, DH-HV1303UM, $1280 \times 1024$ ), and a stepper motor (Oriental Motor, USA) (Figure 4). The system can measure tensile modulus, strength, creep, relaxation, and cyclic loading characteristics as well as effect of moisture on mechanical properties of a micro fiber.

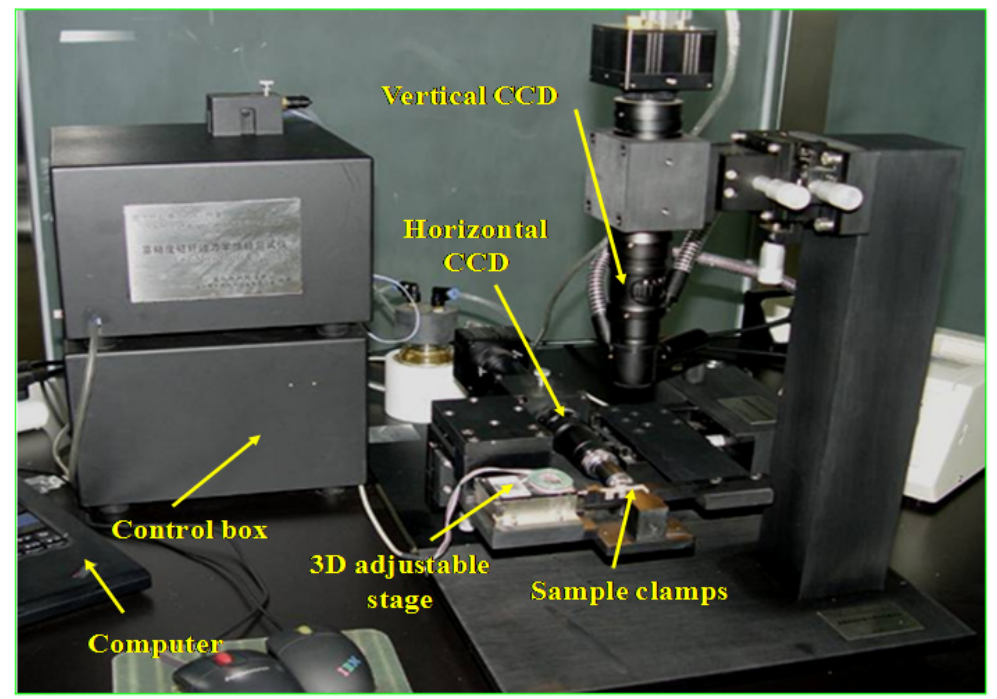

FIGURE 4: IMAGE OF THE MICRO TENSILE TEST SYSTEM (SF-1 MICROTESTER I)

\section{Morphology of Natural Fibers}

The typical cross-sectional images of an individual fiber by a confocal laser scanning microscope (CLSM) and environmental scanning electron microscope (ESEM) are shown in Figures $5 \& 6$. Both kenaf and bamboo have a thick secondary cell wall with a very small lumen in polygonal shape, which indicates that both fibers undergo extensive cell-wall thickening during maturation. Ramie individual fiber is flattened and typical hexagonal or oval in shape, while those of Chinese fir are rectangular in cross section. 

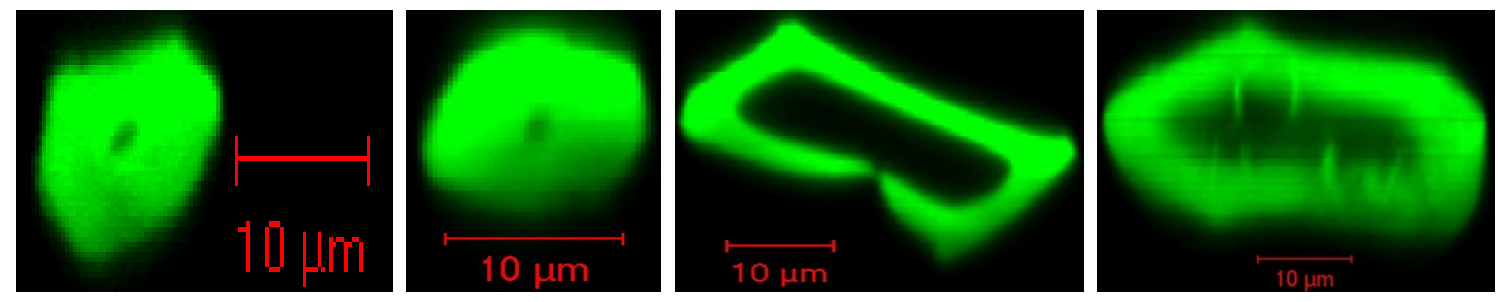

FIGURE 5: THE TYPICAL CLSM IMAGES OF CROSS SECTION OF AN INDIVIDUAL FIBER (THE LEFT TO RIGHT: BAMBOO, KENAF, CHINESE FIR, AND RAMIE)
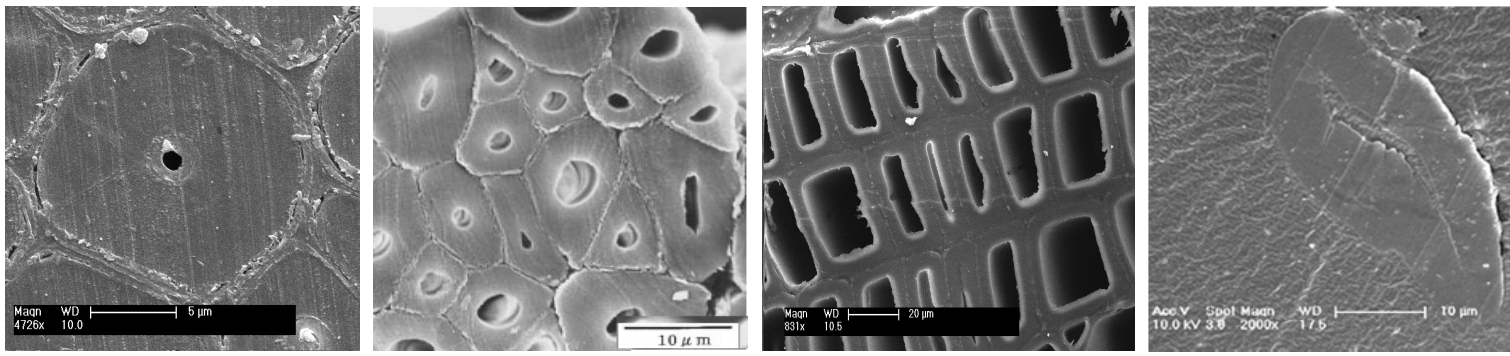

FIGURE 6: THE TYPICAL ESEM IMAGES OF CROSS SECTIONS OF INDIVIDUAL FIBERS (THE LEFT TO RIGHT: BAMBOO, KENAF, CHINESE FIR, RAMIE)

\section{Mechanical Properties of Natural Fibers}

Typical load-displacement curves (Figure 7a) and converted stress-strain curves using the cell wall crosssectional area (Figure 7b) show that the fibers are linear and brittle in tensile properties except that the Chinese fir juvenile latewood fiber demonstrated curvilinearity. The averages and standard deviations of tensile modulus, tensile strength, elongation at break, and cross-sectional area of four fibers are summarized in Table 6.
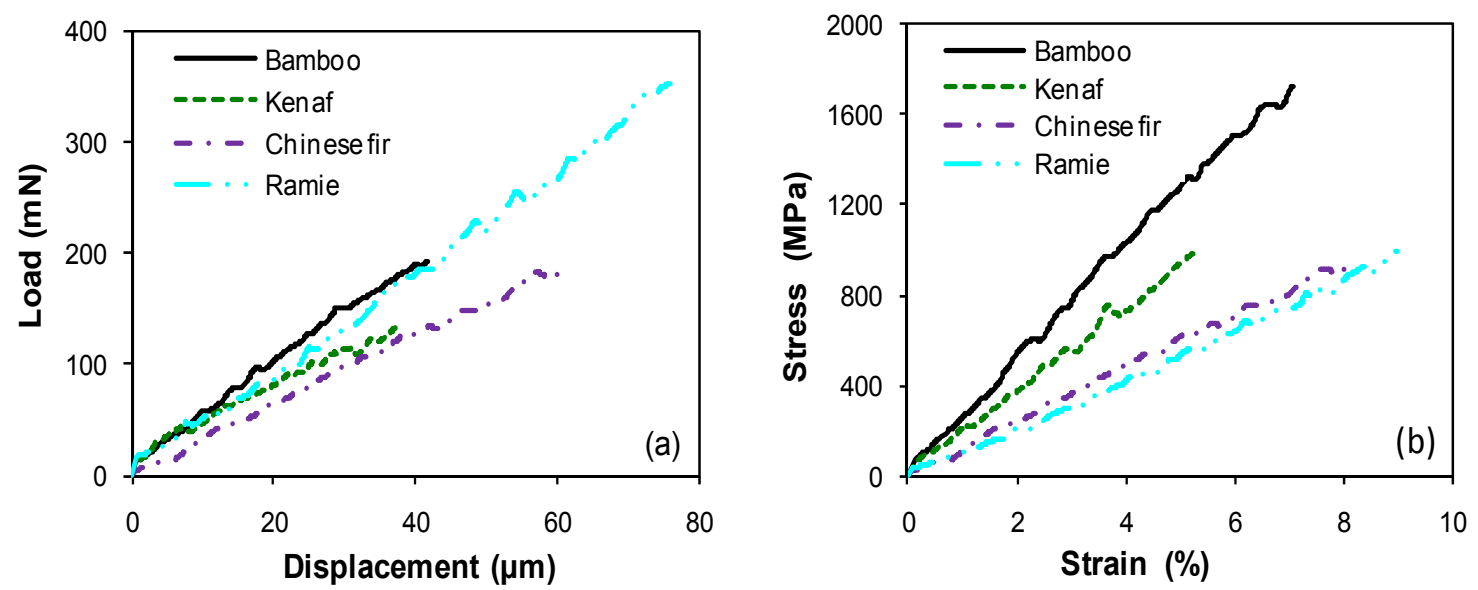

FIGURE 7: THE TYPICAL LOAD-DISPLACEMENT (A) AND STRESS-STRAIN (B) CURVES OF THE INDIVIDUAL FIBERS 
TABLE 6: MEAN AND STANDARD DEVIATION OF PROPERTIES FOR RAMIE, FIR, KENAF AND BAMBOO

\begin{tabular}{|l|c|c|c|c|c|c|c|c|c|c|c|c|}
\hline & \multicolumn{3}{|c|}{$\begin{array}{c}\text { Tensile Modulus } \\
\text { (GPa) }\end{array}$} & \multicolumn{3}{c|}{$\begin{array}{c}\text { Tensile Strength } \\
\text { (MPa) }\end{array}$} & \multicolumn{2}{c|}{$\begin{array}{c}\text { Cell Wall Area } \\
\left(\mu \mathrm{m}^{2}\right)\end{array}$} & \multicolumn{2}{c|}{$\begin{array}{c}\text { Elongation at Break } \\
(\%)\end{array}$} \\
\hline Ramie & 11.4 & 1.9 & C $^{*}$ & 1001 & 153 & B & 337 & 78 & A & 8.9 & 1.59 & A \\
\hline Chinese Fir & 14.2 & 6.7 & C & 908 & 418 & B & 217 & 52 & B & 8.2 & 2.46 & A \\
\hline Kenaf & 19.5 & 7.6 & B & 983 & 194 & B & 140 & 44 & D & 5.4 & 1.72 & C \\
\hline Bamboo & 25.7 & 4.8 & A & 1685 & 293 & A & 117 & 35 & C & 7.0 & 1.13 & B \\
\hline *Means with the same letter are not significantly different at $=0.05$ & & & \\
\hline
\end{tabular}




\section{Kenaf/Glass Fiber Hybrid Composites}

\section{Objective}

The objective was to better understand the effect of pressure of hot pressing and layups on the hybrid polyester composites processed from randomly woven kenaf fibers and fiberglass.

\section{Natural Fiber Composite Fabrication}

Randomly woven kenaf fibers were used in the study. These materials were provided by Kengro Corporation, Charleston, MS. EB 9496 polyester resin with randomly oriented fiberglass provided by Magna Composites was used to fabricate the SMC.

\section{Flexural Test}

The flexural testing was performed using an Instron 5566 tabletop model. The testing procedure was based on the ASTM standard D 790-07. Average moduli of elasticity and rupture for samples used are given in Table 7.

TABLE 7: AVERAGE MODULI OF ELASTICITY (MOE) AND MODULI OF RUPTURE (MOR) FOR HYBRID SMC SAMPLES

\begin{tabular}{|c|c|c|c|c|}
\hline Sample & $\begin{array}{c}\text { Curing Pressure \% of 6.9, } \\
\mathrm{MPa}\end{array}$ & $\begin{array}{c}\text { Lay-up of Kenaf } \\
\text { Fiber }\end{array}$ & $\mathrm{MOE}, \mathrm{GPa}$ & $\begin{array}{c}\text { MOR, } \\
\mathrm{MPa}\end{array}$ \\
\hline A & 20 & Single layer & 9.95 & 250 \\
\hline C & 40 & Single layer & 9.82 & 231 \\
\hline D & 60 & Single layer & 9.82 & 234 \\
\hline E & 80 & Single layer & 9.34 & 271 \\
\hline F & 80 & Double layer & 9.92 & 227 \\
\hline G & 60 & Double layer & 9.39 & 198 \\
\hline H & 40 & Double layer & 9.04 & 209 \\
\hline I & 20 & Double layer & 8.26 & 193 \\
\hline
\end{tabular}




\begin{tabular}{|c|c|c|c|c|}
\hline Sample & $\begin{array}{c}\text { Curing Pressure \% of 6.9, } \\
\mathrm{MPa}\end{array}$ & $\begin{array}{c}\text { Lay-up of Kenaf } \\
\text { Fiber }\end{array}$ & MOE, GPa & $\begin{array}{c}\text { MOR, } \\
\mathrm{MPa}\end{array}$ \\
\hline $\mathrm{A}$ & 20 & Single layer & 9.95 & 250 \\
\hline C & 40 & Single layer & 9.82 & 231 \\
\hline D & 60 & Single layer & 9.82 & 234 \\
\hline E & 80 & Single layer & 9.34 & 271 \\
\hline $\begin{array}{c}\text { No } \\
\text { Kenaf }\end{array}$ & 100 & 0 & 9.01 & 266 \\
\hline
\end{tabular}

\section{Molecular Dynamic (MD) Modeling of Polymers and Polymer Composite MATERIALS}

\section{Objective}

The objective of this work was to understand the variables associated with the deformation of thermoplastic polymers using atomistic modeling, i.e., chain length, number of chains, strain rates, temperature, etc.

\section{Methods}

Molecular dynamics simulations were used to study the deformation mechanisms during uniaxial tensile deformation of amorphous polyethylene. The stress-strain behavior comprised elastic, yield, strain softening and strain hardening regions that were qualitatively in agreement with previous simulations and experimental results (Figure 8) (Hossain et al. 2010). The chain lengths, number of chains, strain rate and temperature dependence of the stress-strain behavior were investigated. The energy contributions from the united atom potential were calculated as a function of strain to help elucidate the inherent deformation mechanisms within the elastic, yield, and strain hardening regions. 

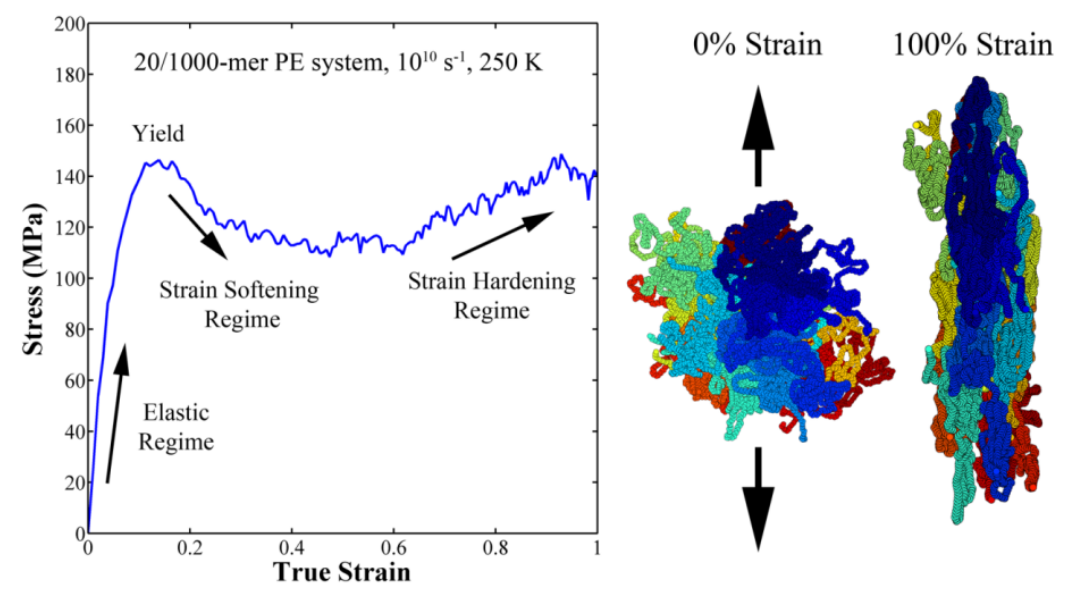

FIGURE 8: STRESS-STRAIN RESPONSE FOR AMORPHOUS POLYETHYLENE DEFORMED BY UNIAXIAL TENSILE LOADING.

\section{Results and Discussion}

The results of examining the partitioning of energy showed that the elastic and yield regions were mainly dominated by interchain non-bonded interactions whereas the strain hardening regions were mainly dominated by intrachain dihedral motion of polyethylene. Additional results showed how the internal mechanisms associated with the bond length, bond angle, dihedral distributions, change of free volume and chain entanglements evolve with increasing deformation. Current work is focusing on the deformation behavior of polypropylene and glucose interfaces as a model for the composite materials used in this work. Molecular dynamics and molecular statics simulations will be used to examine elastic and plastic properties of amorphous cells of both glucose and polypropylene as well as the interface mechanical properties between the two materials.

\section{Silane Treatment of Kenaf Fiber}

\section{Objective}

The objective of this work was to chemically modify kenaf fiber to improve the moisture resistance of the resulting fiber composites. The silane agent used in this work was vinyl tris(2-ethoxymethoxy) silane. An exploratory study of the silane treatment effect on water absorption and mechanical properties was conducted.

\section{Methods}

Silane treatment kenaf fiber: Vinyl tris( 2-ethoxymethoxy) silane was mixed with an ethanol/water mixture in the ratio of $6: 4.0 .6 \%$ solution of silane was used. Acetic acid was used to control the $\mathrm{pH}$ of the solution at 4 to make sure the complete hydrolysis of the silane. After adjusting the $\mathrm{pH}$, the silane 
solution was allowed to stand for an hour. The solution was then drained out and the fibers were air dried for half an hour followed by drying in an oven at $103^{\circ} \mathrm{C}$ until a constant weight was obtained.

Kenaf fiber composites fabrication: Unstaturated polyester and 1, 1-Di (tertbutylperoxy) Cyclohexane as catalyst in the ratio of 0.985: 0.015 was mixed thoroughly, and evenly poured onto the surface of two fiber sheets. These two sheets were closed together with resin mixture in the core. The sample was put into a stainless steel mold and pressed at $100 \stackrel{\circ}{ } \mathrm{C}$ and $2.5 \mathrm{MPa}$ for 2 hours.

\section{Results and Discussion}

The water absorption of silane treated kenaf fiber composites decreased by $22 \%$ compared to untreated fiber composites. After 4 weeks of water immersion, the water absorption curve for the silane treated kenaf fiber composites was leveled off, while that of untreated kenaf fiber composites continued to increase (Figure 9a). The hydrophilic nature of the kenaf fiber was reduced and hence the water resistance of the resulting fiber composites was improved after the silane treatment of kenaf fibers. Compared to untreated fiber composites, the flexural strength and modulus of the silane treated kenaf fiber composites increased by $25 \%$ and $8 \%$, respectively (Figure $9 \mathrm{~b}$ ). The silane treatment of kenaf fiber also improved the mechanical properties of the resulting fiber composites.
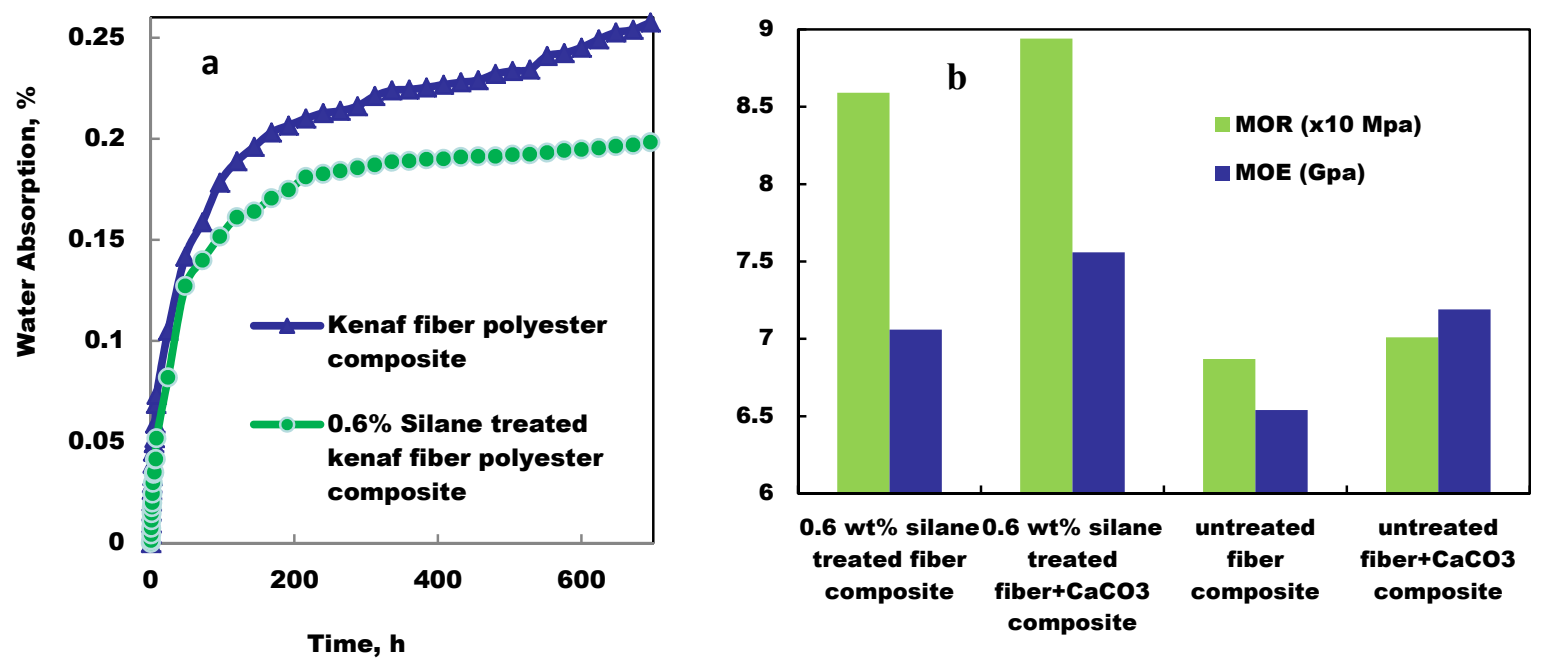

Figure 9. a) Water absorption of treated and untreated kenaf fiber composites and b) Flexural properties of treated and untreated kenaf fiber composites.

\section{Molecular Dynamics Simulation of Deformation in Amorphous Cellulose}

\section{Objective}


The ability to form various ultrastructural composites depended on both the conformational characteristics and the mechanical behavior. The objective of this work was to understand the structure of amorphous cellulose which was a key step to characterize the properties of cellulose fibers.

\section{Methods}

Molecular dynamics deformation analysis, followed by minimization, was used to generate the structures for amorphous glucose. Properties related to the deformation were calculated for these models and compared with predicted geometric, energetic and elastic properties of the material to published modeling results and experimental measurements.

\section{Force Field}

In this work, the ReaxFF reactive force field (van Duin 2001; Chenoweth 2008) was used to simulate the bonding between the various elements of glucose. ReaxFF was a general bond-order-dependent force field that provided accurate descriptions of bond breaking and bond formation during dynamic simulations. The main difference between the traditional unreactive force fields and ReaxFF was that the connectivity in ReaxFF was determined by bond orders calculated from interatomic distances that were updated every MD step. This allowed for bonds to break and form during the simulation. Equation 1 shows that ReaxFF partitions the overall system energy $E_{\text {system }}$ into contributions from various partial energy terms, i.e.

$E_{\text {system }}=E_{\text {bond }}+E_{\text {under }}+E_{\text {over }}+E_{\text {val }}+E_{\text {pen }}+E_{\text {tors }}+E_{\text {conj }}+E_{\text {vdwaals }}+E_{\text {coulomb }}$

These partial energies included bond energy $E_{\text {bond, }}$ atom under-/overcoordination $E_{\text {under, }} E_{\text {over, }}$ valence angle $E_{\text {val }}$, penalty energy $E_{\text {pen, }}$, torsion angle $E_{\text {tors }}$ and conjugation energy $E_{\text {conj }}$ terms to properly handle the nature of preferred configurations of atomic and resulting molecular orbital and terms to handle van der Waals $E_{\text {vdwaals }}$ and Coulomb $E_{\text {coulomb }}$ interactions. These latter non-bonded interactions were calculated between every atom pair, irrespective of connectivity, and were shielded to avoid excessive repulsion at short distances. This treatment of nonbonded interactions allowed ReaxFF to describe covalent, ionic, and intermediate materials, thus, greatly enhancing its transferability.

\section{Simulation Methods}

The cellulose repeat unit consisted of two glucose rings bridged by the 1-4 glycosidic bonds. Three chain lengths were tested: 20,50 , and 100 repeat units per chain. Initial densities of $0.8 \mathrm{~g} / \mathrm{cm}^{3}$ were considered. Because the cell parameters were allowed to vary during molecular dynamics simulations, the density could therefore vary in range of $1.28 \mathrm{~g} / \mathrm{cm}^{3}$ to $1.44 \mathrm{~g} / \mathrm{cm}^{3}$. Periodic boundary conditions were applied in all three directions. The initial configuration of the system was generated using commercial Materials Studio software and all subsequent molecular dynamics simulations were performed using the LAMMPS simulation tool with a time step of $0.5 \mathrm{fs}$. The unit cell models were 
replicated by $3 \times 3 \times 3$ to simulate more atoms. The simulation procedure began with minimization of each system. Next, the system was equilibrated at chosen temperature using NVE-MD simulation with a time step of $0.5 \mathrm{fs}$. The equilibrated systems were then used to perform the deformation simulations under a uniaxial tensile strain applied at a constant strain rate. The reactive force field used here was to describe the atomic interactions for dynamic bond scission and bond formation. Three different strain rates $\left(10^{8}\right.$, $10^{9}$, and $10^{10} \mathrm{~s}^{-1}$ ) were applied to the loading boundary with ReaxFF for different numbers of chains and chain lengths. The amorphous cellulose configurations were deformed at $100 \mathrm{~K}$, below the glass transition temperature.

\section{RESULTS AND DISCUSSION \\ Simulation Conditions}

With ReaxFF, a suitable time step was required because the charges and bond orders were allowed to change at every time step. The ultimate aim was to simulate the longest possible time with the smallest amount of computational effort. The choice of time step was therefore a compromise between a large value which would require less molecular dynamics MD steps for a given simulated time, and a small value which allowed each time step to be calculated faster. The time step must be sufficiently small that dynamics correctly conserve the total energy of the system. A greater temperature fluctuation was noticed from the initial value in the $1 \mathrm{fs}$ time step case while the temperature is computed more stable using $0.5 \mathrm{fs}$ time step as the simulation cell was equilibrated. The energy (total, kinetic, potential) as a function of simulation time showed the same result. Another sign of instability with the $1 \mathrm{fs}$ was the ejection of atoms from the simulation cell, which could occur if atoms get too close. Therefore, future simulations were needed to use a time step of lower than $0.5 \mathrm{fs}$.

\section{Glass Transition Temperature}

The glass transition temperatures $\mathrm{T}_{g}$ were related to the physical stability of an amorphous formulation. The thermal expansivity of an amorphous cellulose system was expected to decrease at its $T_{g}$ when it passed from a rubbery to a glassy state. Thus, plotting volume as a function of temperature for amorphous cellulose (Figure 10) showed a change in slope at the glass transition temperature. The $T_{g}$ could thus be identified at the point of intersection between the high- and low-temperature branches in this plot. The NPT ensemble (constant number of particles, pressure and temperature) was used for the MD glass transition temperature simulations, with the pressure fixed at zero bar. 


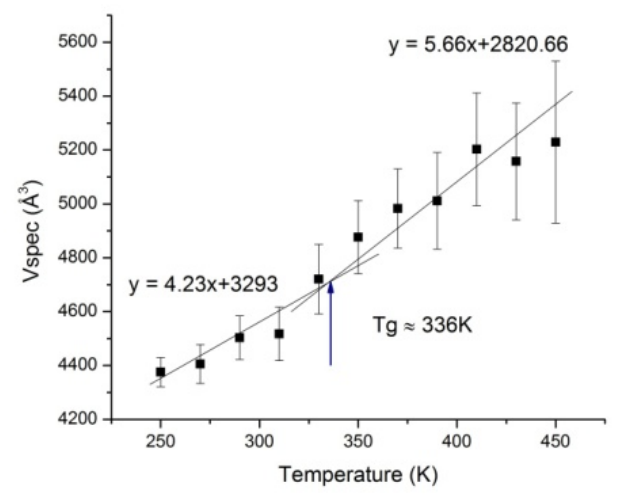

Figure 10. Evolution of volume as a function of temperature

Physical and mechanical properties of amorphous cellulose models are shown in Table 8.

Table 8. Mechanical properties of amorphous cellulose models

\begin{tabular}{c|c|c|c|c|c}
\hline Properties & $\begin{array}{c}\text { Density } \\
3 \\
\left(\mathrm{~g} / \mathrm{cm}^{3}\right.\end{array}$ & $\mathrm{T}_{g}(\mathrm{~K})$ & $\begin{array}{c}\text { Young's } \\
\text { modulus } \\
(\mathrm{GPa})\end{array}$ & $\begin{array}{c}\text { Shear } \\
\text { modulus } \\
(\mathrm{GPa})\end{array}$ & $\begin{array}{c}\text { Poisson's } \\
\text { ratio }\end{array}$ \\
\hline ReaxFF & 1.347 & 336 & 9.37 & 3.94 & 0.189 \\
\hline Literature Value & 1.385 & $296-325$ & $10.42 \pm 1.08$ & $5.955 \pm 0.673$ & $\begin{array}{c}0.232 \pm 0.03 \\
13\end{array}$ \\
\hline
\end{tabular}

Thermodynamic and mechanical properties such as density, glass transition temperature, Young's modulus, Shear modulus and Poisson's ratio were calculated and found to be comparable with literature values (Chen et al 2004). We concluded that the amorphous cellulose models with ReaxFF were representative of the amorphous structure in cellulose as far as the mechanic properties were concerned.

Strain-stress: Figure 11 shows how the tensile loading leads to a net change in shape of the cell, where the colors represent the different atom types $(\mathrm{C}, \mathrm{H}, \mathrm{O})$. To investigate the deformation behavior of cellulose, the stress-strain behavior, temperature dependence, and internal energy evolution were monitored as a function of deformation. Figure 12 shows the stress-strain behavior for amorphous cellulose at a strain rate of $10^{10} \mathrm{~s}^{-1}$. The red dots were stress values taken at various strain levels and the black line was the averaged response; this scatter was typically of polymer deformation in molecular dynamics. After an initial elastic regime, yield occurs and then strain hardening was observed. The green and blue dots (mainly hidden behind green dots) represented stresses in the directions lateral to the 
loading direction - these are centered about $0 \mathrm{GPa}$, a boundary condition indicative of the uniaxial tensile load applied. This stress-strain curve was similar to previous simulations in the literature as well as experimental results (Chen et al 2004).
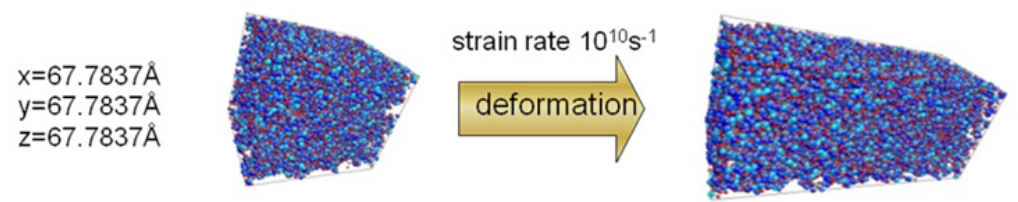

Figure 11. The model system in equilibrium at $100 \mathrm{~K}$ and after being uniaxially deformed. Colors represent different atom types being simulated ( $\mathrm{C}, \mathrm{H}$, and $\mathrm{O})$.

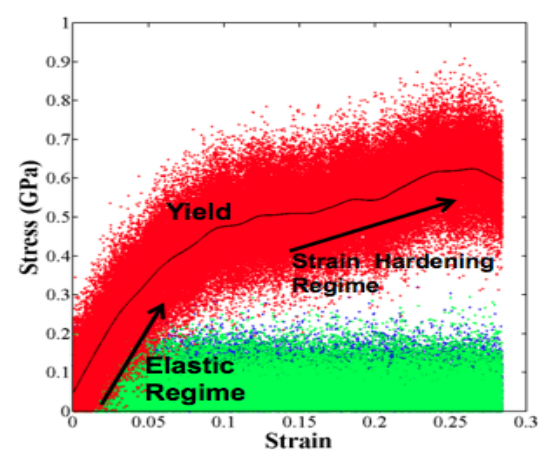

Figure 12. A characteristic stress-strain curve for amorphous cellulose ( 28404 atoms, $100 \mathrm{~K}$ temperature, $10^{10} \mathrm{~s}^{-1}$ strain rate). Black line is the average stress response in the tensile direction. Red, green, and blue dots show the scatter of the instantaneous stresses the loading direction and two lateral directions, respectively.

\section{Multiscale Modeling of Moisture Absorption Process in Natural fiber COMPOSITES}

\section{Objective}

The objective of this effort was to develop an advanced multiscale modeling framework to investigate the moisture absorption process in natural fiber composites on the basis of a recently developed micromechanics modeling framework, namely, Variational Asymptotic Method for Unit Cell Homogenization (VAMUCH), which takes the Variational Asymptotic Method (VAM) as the mathematical foundation. 


\section{Methods}

The Variational Asymptotic Method for Unit Cell Homogenization (VAMUCH) takes the Variational Asymptotic Method (VAM) as the mathematical foundation. VAM combines the merits of both variational methods and asymptotic methods. It expands the variational statement asymptotically in terms of small parameters inherent in the problem. The result obtained using variational asymptotic method converges asymptotically to exact solutions if such solutions exist. Variational asymptotic method is applicable to any problem having a variational statement with small parameters and it seamlessly bridges the gap between engineering and mathematical approaches. The commercial finite element software ABAQUS was used to analyze the global transient water absorption process in natural fiber composites according to the effective properties calculated by VAMUCH. If they are of interest, the distributions of local fields can be accurately recovered by $\mathrm{VAMUCH}$ according to the global response.

\section{Results and Discussion:}

The developed multiscale model has the following functionalities:

(1) It can accurately and efficiently provide the effective water diffusion coefficients. Then, the commercial finite element software, such as ANSYS and ABAQUS, can be employed to analyze the global transient water diffusion process in composite materials and structures as well as the influences of resistance of boundary surface using the water diffusion coefficients provided by VAMUCH.

(2) It can accurately and efficiently recover the distributions of local fields according to the global response of composite materials and structures.

In this study, we investigated the moisture diffusion process in wood fiber polymer matrix composites. The volume fraction of the fiber $\left(V_{f}\right)$ is $30 \%$. Both constituents are isotropic with moisture diffusion coefficient $D_{f}=30 \mathrm{~mm}^{2} / \mathrm{s}$ for wood fiber, and $D_{m}=0.218 \mathrm{~mm}^{2} / \mathrm{s}$ for polymer matrix. Since the finite element method (FEM) is considered as one of the most accurate way to obtain effective properties we also used the results calculated by ABAQUS as a benchmark to verify the VAMUCH outcomes. Table 9 shows the effective moisture diffusion coefficients calculated by VAMUCH and ABAQUS. It can be seen that both approaches provide identical results.

After obtaining the effective properties, the global transient moisture diffusion process was performed using commercial software ABAQUS. Here we investigated the transverse moisture diffusion behavior. A two dimensional square finite element (FE) model as shown in Figure 13 was used in this study. Eight-node elements were employed. The dimension of FE model is $100 \times 100 \mathrm{~mm}$. The top and bottom boundaries were sealed. Moisture can only transfer into the model from both side boundaries. The distribution of the moisture concentration along the horizontal center line at different time were plotted in Figure 14 as the water concentration $C_{a}$ at the side boundary was kept constant as $10 \mathrm{~g} / \mathrm{mm}^{3}$ all the time. 
Table 9. Effective moisture diffusion coefficients $(\mathrm{mm} 2 / \mathrm{s})$ obtained from VAMUCH and ABAQUS. Subscript 1 represents longitudinal direction while 2 and 3 stand for transverse properties.

\begin{tabular}{|c|c|c|c|}
\hline Effective D & $D_{1}^{*}$ & $D_{2}^{*}$ & $D_{3}^{*}$ \\
\hline VAMUCH & 9.152 & 0.4016 & 0.4016 \\
\hline ABAQUS & 9.152 & 0.4016 & 0.4016 \\
\hline
\end{tabular}

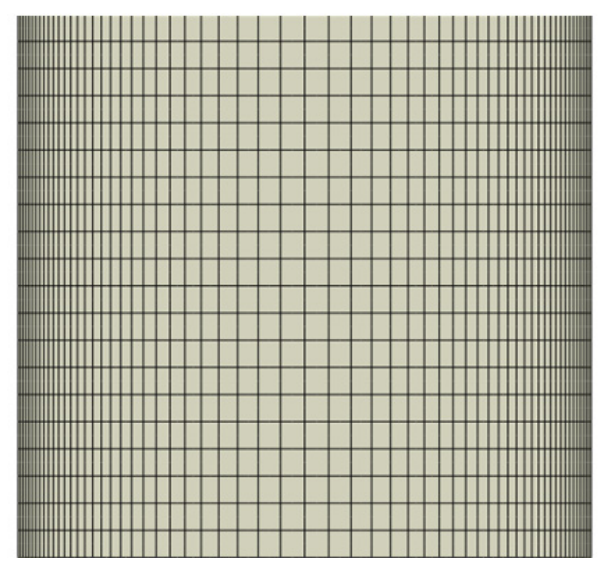

Figure 13. Finite element model for the analysis of the global transient moisture diffusion process.

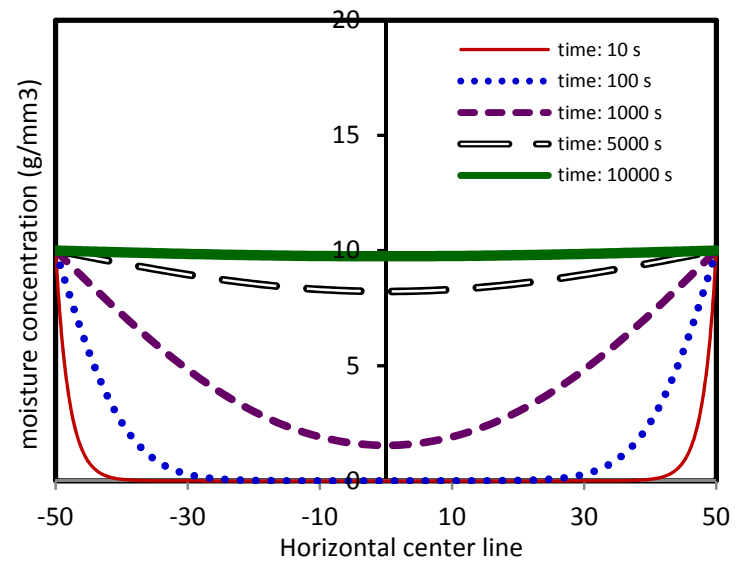

Figure 14. Distribution of moisture concentration along the horizontal center line at different time.

Figure 15 shows the contour plots of moisture concentration flow flux in $y_{2}$ direction at $100 \mathrm{~s}$, respectively, when the water concentration $C_{a}$ is kept constant as $10 \mathrm{~g} / \mathrm{mm}^{3}$. To quantitatively describe the moisture concentration flow flux, we plotted the distribution of moisture concentration flow flux (g/s.mm2) in $y_{2}$ direction along the vertical center line of unit cell at different time in Figure 16 . It can be seen that the moisture concentration flow flux decreased with time since the gradient of moisture concentration, which is the driving force for moisture diffusion, decreased with time. 


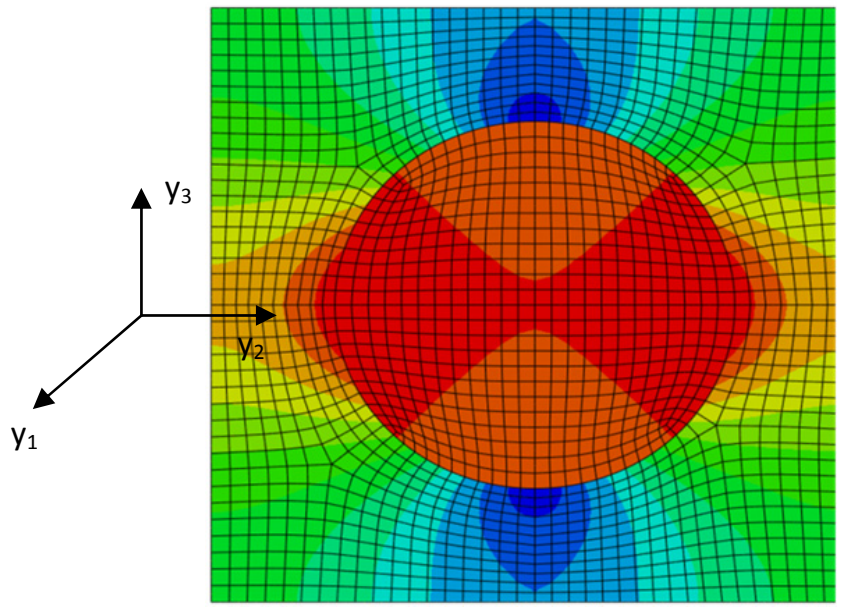

Figure. 15. Finite element model for the analysis of the global transient moisture diffusion process.

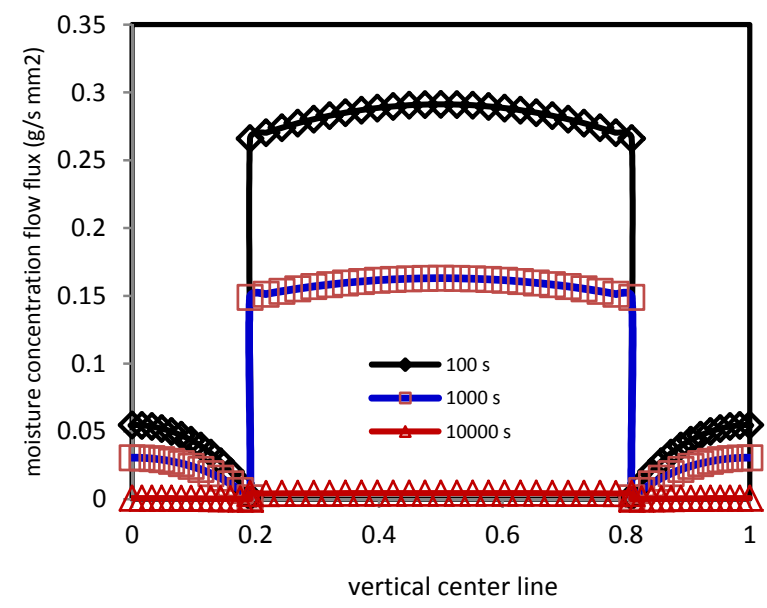

Figure. 16. Distribution of moisture concentration along the horizontal center line at different time.

\section{Life-Cycle Assessment of Natural fiber Polymer Composites}

\section{Objective}

The objective of this work was to use principles and practices of life-cycle assessment (LCA) to evaluate environmental performance of natural fiber reinforced bio-resin matrix composites and collected the data needed to make LCA calculations.

\section{Methods}

A series of kenaf fiber reinforced sheet molding composites (SMC) have been fabricated in the laboratory for scoping and optimization. The life-cycle assessment was conducted on three product scenarios: Formulation 1(kenaf fiber SMC), Formulation 4 (kenaf $20 \%$ soy resin SMC, containing $20 \%$ modified soybean oil in solid resin), and glass fiber SMC (Springer, 1983). The data for soybean oil resin and kenaf fiber reinforced composites were collected from MSU Forest Products lab. The data for unsaturated polyester resin and glass fiber SMC were collected from the literature (Springer, 1983) and SimaPro software. These data include energy and materials balances for manufacturing $1 \mathrm{~kg}$ raw materials, intermediates, and products, as well as the emissions to air, discharges to water, and solid wastes to land. These data were then entered into LCA software SimaPro V7.3. Environmental performances were measured by a set of environmental impact indexes come up with by NIST Building for Environmental and Economic Sustainability (BEES), cumulative energy demand and a weighted environmental burden.

Environmental performances of materials are usually compared based on a functional unit: an entity capable of accomplishing a specified purpose. SMCs are supposed to be used for automotive 
manufacture. Interior parts in cars are still designed for stiffness. To achieve the equal stiffness (Eq.2) to a reference material ( $r$ subscript), the mass $(m)$ and thickness $(t)$ are calculated by Eqs. $3 \& 4$.

\begin{tabular}{|l|l|l|l|}
\hline$\frac{F}{\delta}=\frac{E m^{3}}{c b^{2} l^{6} \rho^{3}} \quad$ (2) & $m=\left(\frac{E_{r}}{E}\right)^{\frac{1}{3}} \frac{\rho}{\rho_{r}} m_{r} \quad$ (3) & $t=\left(\frac{E r}{E}\right)^{\frac{1}{3}} t_{r}$ \\
\hline
\end{tabular}

Setting glass fiber SMC as the reference material, the masses required to achieve compatible stiffness were calculated as shown in Table 10. The running capacity during the use phase was assumed as: $175,000 \mathrm{~km}$ (12 years). Fuel reduction coefficient on gasoline powered vehicles due to weight reduction of using light materials is selected as $0.34 \mathrm{I} /(100 \mathrm{~kg} * 100 \mathrm{~km})$ for a lighter car (lower limit, New European Driving Cycle). The amounts of saved gasoline at use phase were thus calculated as shown in Table 10.10 .

Table 10 Functional unit with equal stiffness

\begin{tabular}{|l|l|l|l|}
\hline & Glass & Kenaf & Kenaf 20\% \\
& Fiber SMC & SMC & Soy Resin SMC \\
\hline Density $\left(\mathrm{g} / \mathrm{cm}^{3}\right)$ & 1.87 & 0.95 & 1.04 \\
\hline Flexural Strength (GPa) & 14 & 7.38 & 7.29 \\
\hline Mass (kg) with Equal Stiffness & 1 & 0.63 & 0.69 \\
\hline Save gasoline at Use Phase (I) & Ref. & 2.2 & 1.8 \\
\hline
\end{tabular}

At the end-of-life disposal, the used products were assumed to be incinerated. Consumption of energy of incineration ${ }^{3}$ was selected as: Heat: $0.24 \mathrm{MJ} / \mathrm{kg}$, Electricity: $0.36 \mathrm{MJ} / \mathrm{kg}$; bonus of incineration of plastics energy recovery ${ }^{3}(\mathrm{LHV}=30.5 \mathrm{MJ} / \mathrm{kg}$ ), Heat: $26 \%$, Electricity: $10 \%$; bonus of incineration of kenaf energy recovery: (LHV $=14 \mathrm{MJ} / \mathrm{kg}$ ), Heat: $26 \%$, Electricity: $10 \%$. Incineration of discarded glass fiber reinforced plastics generates a lot of black smoke and bad smells and often gives damage to incinerator by fusion of glass fibers, thus without any bonus of energy recovery.

\section{Results and Discussion:}

Figure 17 indicates that kenaf fiber has less negative environmental impact than glass fiber during a period from raw materials extraction to fiber manufacturing. Figure 18 shows that bast fibers consume 
less energy than other fibers in manufacturing 1-kg fibers. Wood pulp and bast fibers consume less nonrenewable energy. Method to calculate Cumulative Energy Demand was based on the method published by Ecoinvent version 2.0 and expanded by PRé Consultants for raw materials available in the SimaPro 7 database. Figure 19 demonstrates that natural fibers achieved overall lower environmental burdens. Land uses contributed substantial portions for agri-fibers (jute and kenaf). The scale is chosen in such a way that the value of 1 point is representative for one thousandth of the yearly environmental load of one average European inhabitant. The key environmental measures for three product scenarios were computed with Simapro software and are shown as in Figure 20. Negative means carbon credit, i.e. saving non-renewable resources otherwise being used. Both kenaf-fiber reinforced SMCs perform better than glass fiber SMC in every environmental category. The global warming potential of the kenaf fiber SMC could be only about $45 \%$ of that for the glass fiber SMC.
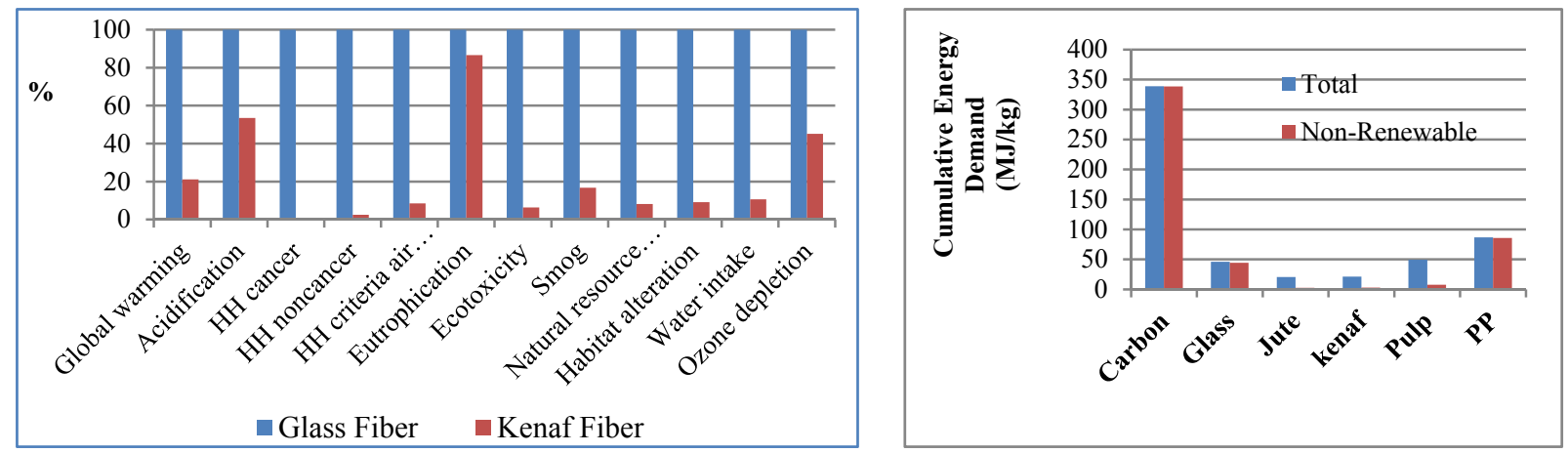

Figure 17. Comparison of environmental impacts of fiber productions. BEES impact indexes are selected as characteristics.

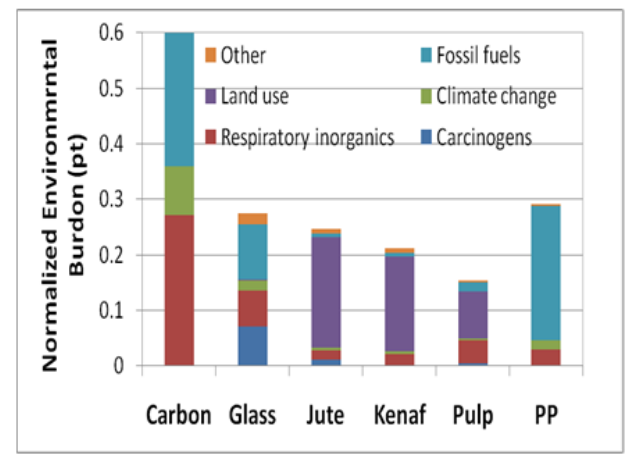

Figure 19. Weighted environmental points

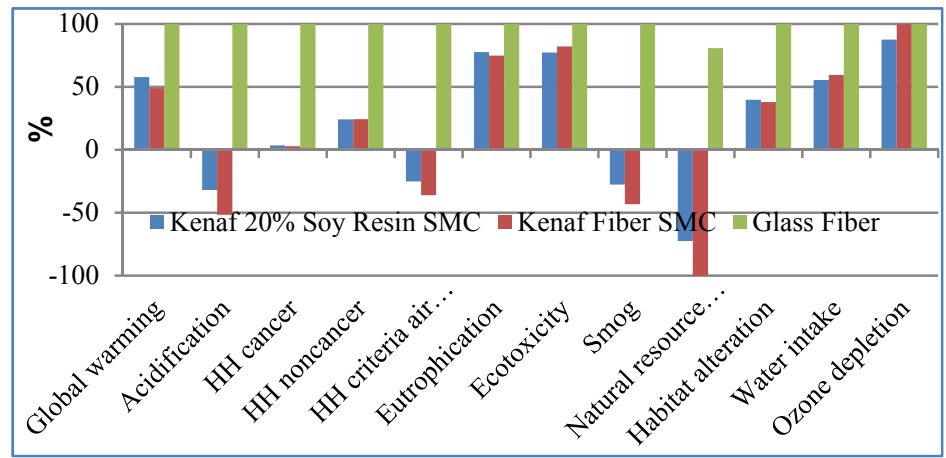

Figure 20. BEES environmental impacts of manufacturing 
for typical fiber productions (per $1 \mathrm{~kg}$ three fiber reinforced polymer composites (per functional fiber)

unit).

\section{Conclusions}

The alkaline retting treatment efficiently removed hemicellulose and lignin from kenaf bast fiber. Bleaching treatment could remove the remanent lignin from the retted fibers. Pure cellulose fibers in micrometer scale and nanometer scale were obtained by acidic hydrolysis of the bleached fibers. Our kenaf natural fiber SMC has comparative properties comparing with the commercial glass fiber SMC. The inorganic nanoparticle impregnated kenaf fiber SMC was not degraded after the accelerated weathering test.

The SF-I microtester is well suited for measuring the mechanical properties of individual micro fibers. Among the tested species, the bamboo fiber had the highest tensile strength and modulus and the smallest cell wall cross-sectional area. The ramie had the largest cell wall cross-sectional area and elongation at break, but lowest tensile modulus. Kenaf showed the lowest elongation at break and other properties of kenaf were balanced.

A process to fabricate laminated natural fiber kenaf-based composite was developed. Various samples are manufactured with modulus of elasticity up to $10 \mathrm{GPa}$ and modulus of rupture up to 270 MPa.

Simulation results showed that temperature increase of polymer in high rate tests can be reproduced by augmenting the deformation boundary conditions. This might shed light on the fundamental mechanisms associated with the generation of heat in thermoplastic polymers under high rate deformation.

Vinyl tris(2-ethoxymethoxy) silane treatment of kenaf fiber improved the physical and mechanical properties of the resulting kenaf fiber composites. The water absorption of silane treated kenaf fiber composites decreased by $22 \%$ compared to untreated fiber composites. The flexural strength and modulus of silane treated kenaf fiber composites increased by $25 \%$ and $8 \%$, respectively.

A suitable timestep as less $0.5 \mathrm{fs}$ is found to use in future simulations with ReaxFF. Thermodynamic and mechanical properties such as density, glass transition temperature, Young's modulus, Shear modulus and Poisson's ratio were calculated and found to be comparable with literature values. The simulated stress-strain behavior using molecular dynamics showed similar trends both qualitatively and quantitatively as those observed in the experimental testing for amorphous cellulose.

A multiscale model of moisture absorption process of natural fiber composites was developed on the basis of the theoretical framework of VAMUCH. Compared with other existing micromechanics approaches, VAMUCH has the following unique features:

- VAMUCH can obtain the complete set of material properties within one analysis without applying any load and any boundary conditions, which is far more efficient and less labor 
intensive than those approaches requiring multiple runs under different boundary and load conditions. It is also noted that VAMUCH can even obtain the complete set of 3D material properties using a one-dimensional analysis of the 1D UC for binary composites. It is impossible for FEA-based approaches.

- VAMUCH calculates effective properties and local fields directly with the same accuracy as the fluctuating functions. No postprocessing calculations which introduce more approximations, such as averaging stress or strain fields, are needed, which are indispensable for FEM-based approaches.

- VAMUCH can recover the local fields using a set of algebraic relations obtained in the process of calculating the effective properties. Another analysis of the microstructures which is needed for FEA-based approaches is not necessary for VAMUCH.

The present methodology can be straightforwardly extended to the effective dielectric, magnetic, and heat conduction properties of heterogeneous materials due to the mathematical analogy of these problems.

The LCA preliminary result demonstrated that the use of modified soybean oil and natural fiber to make sheet molding compound had a great potential from an ecological point of view. LCA is an effective tool and adds a dimension to evaluate new products and associated processes.

\section{Publications/Presentations/Patents}

Publications

1. Q. Gao, S. Q. Shi, S. Zhang, J. Li, X. Wang, W. Ding, K. Liang, and J. Wang. 2011. Development of soybean meal based wood adhesives. Journal of Applied Polymer Science. (In Press)

2. K. Liang and S. Q. Shi. 2011. Nanoclay Filled Soy-based Polyurethane Foam. Journal of Applied Polymer Science. 119, 1857-1863.

3. G. Wang, S.Q. Shi, and J. Wang. 2011. Tensile Properties of Four Types of Individual Cellulosic Fibers. Wood and Fiber Science. 43(4), 353-364.

4. G. Wang, S.Q. Shi, and J. Wang. 2011. Microtension Test Method for Measuring Tensile Properties of Individual Cellulosic Fibers. Wood and Fiber Science. 43(3), 251-261.

5. K. Liang and H. Toghiani, C.U. Pittman. 2011. Synthesis, Morphology and Viscoelastic Properties of Epoxy/Polydedral Oligomeric Silsesquioxane (POSS) and Epoxy/Cyanate Ester/POSS Nanocomposite. Journal of Inorganic and Organometallic Polymers and Materials. 21, 128-142.

6. J. Shi, S. Q. Shi, H. M. Barnes, and C. U. Pittman, Jr. 2011. A chemical process for preparing cellulosic fibers hierarchically from kenaf bast fibers. Bioresources 6(1): 879-890.

7. J. Shi, S.Q. Shi, H.M. Barnes, M.F. Horstemeyer, J. Wang, and E.-B. M. Hassan. 2011. Kenaf Bast Fibers -- Part I: Hermetical Alkaline Digestion, International Journal of Polymer Science, Volume 2011 (2011), 8 pages, 2011. doi:10.1155/2011/212047.

8. J. Shi, S. Q. Shi, H. M. Barnes, M. Horstemeyer, and Ge Wang. 2011. Kenaf Bast Fibers -- Part II: Inorganic Nanoparticle Impregnation for Polymer Composites. Special Issue on "Natural Fibers, Bio- and Nanocomposites", International Journal of Polymer Science. Vol. 2011, ID 736474. 
9. K. Liang and S. Q. Shi. 2010. Soy-based polyurethane foam reinforced with carbon nanotubes. Key Engineering Materials Vols. 419 - 420: 477-480. http://www.scientific.net.

10. K. Liang and S. Q. Shi. 2010. Modified soybean oil/polyhedral oligomeric silsesquioxane (POSS) bio-nanocomposites: Synthesis and characterization. Proceedings of $11^{\text {th }}$ International Conference on Biocomposites: Transition to Green Materials, Toronto, Canada. May 2 - 4, 2010.

11. H. Castillo, S. Lee, S. Q. Shi, R. Srinivasan, and J. Shi. 2010. Semi-crystalline polymer-based composites with corn fiber (separated from DDGS) as an interfacial reagent. Proceeding of $11^{\text {th }}$ International Conference on Biocomposites: Transition to Green Materials, Toronto, Canada. May 2-4, 2010.

12. K. Liang and S. Q. Shi. 2010. Resins from soybean oil-based additives for natural fiber sheet molding compound (SMC) composites: Synthesis and characterization. Proceedings of Wood Adhesives 2009. September 28 - 30, Lake Tahoe, Nevada, USA.

13. Y. Peng and S. Q. Shi. 2010. Effect of cloisite clay on the dynamic mechanical properties of resin film. Proceedings of Wood Adhesives 2009. September 28 - 30, Lake Tahoe, Nevada, USA.

14. K. Liang and S. Q. Shi. 2010. Nanoclay filled soy-based polyurethane foam. Journal of Applied Polymer Science. 119 (3): 1857 - 1863.

15. J. Shi, S. Q. Shi, H. M. Barnes, C. Pittman. 2010. A chemical process for preparing cellulose nanowhiskers (CNW) from kenaf bast fibers for polymer composites. 2010. Society of Wood Science and Technology (SWST) Convention. Geneva, Switzerland.

16. J. Hua, D. Chen, and S. Q. Shi. 2010. Effect of Incorporating Chinese Poplar in Wood Chips on Fiber Refining. Forest Products Journal. 60 (4): $362-365$.

\section{Presentations}

1. Presented by J. Wang. Nanophase Treatment of Natural Fiber for Fiber Reinforced Polymer Composites. $19^{\text {th }}$ Annual International Conference on Composites or NanoEngineering, ICCC-19, Shanghai, China, July 24-30, 2011. (Participants: S. Shi, J. Shi, K. Liang and J. Wang)

2. Presented by S. Shi. Characterization of Surface Properties for the Inorganic Nanoparticle Impregnation Treated Kenaf Bast Fibers for Polymer Composites. Presentation at the Forest Products Society 65th International Convention, Double Tree Hotel, Portland, OR, USA, June 1921, 2011. (Participants: S. Shi, J. Shi, K. Liang and J. Wang)

3. Presented by J. Wang. Forced Capillary Rise for Thermodynamic Characterization of Surface Tension and Dynamic Contact Angle of Biofibers. Presentation at the Forest Products Society 65th International Convention, Double Tree Hotel, Portland, OR, USA, June 19-21, 2011. (Participants: J. Wang and S. Shi)

4. Presented by K. Liang. Kenaf Fiber and Soy Protein Based Biocomposites. Presentation at the Forest Products Society 65th International Convention, Double Tree Hotel, Portland, OR, USA, June 19-21, 2011. (Participants: K. Liang and S. Shi)

5. Presented by G. Wang. Develop a Micro-Tension Tester for Measuring Tensile Properties of Cellulosic Fibers. Poster at the Forest Products Society 65th International Convention, 
DoubleTree Hotel, Portland, OR, USA, June 19-21, 2011. (Participants: G. Wang, S. Shi, J. Wang, Y. $\mathrm{Yu}, \mathrm{H}$. Cheng and S. Cao)

6. Presented by J. Wang. Life cycle assessment of environmental performances of kenaf fiber soy resin sheet molding composites and glass fiber sheet molding composites. Poster at the Forest Products Society 65th International Convention, Double Tree Hotel, Portland, OR, USA, June 1921, 2011. (Participants: J. Wang, S. Shi, K. Liang and M.F. Horstemeyer)

7. Presented by K. Liang. Surface Hydrophobizition of Natural Fiber. Presentation at the $11^{\text {th }}$ International Conference on Wood \& Biofiber Plastic Composites, Madison, WI, USA, May 16-18, 2011. (Participants: K. Liang, Y. Shen and S. Shi)

8. Presented by K. Liang. Kenaf Fiber and Soy Protein Based Biocomposites. Poster at the $11^{\text {th }}$ International Conference on Wood \& Biofiber Plastic Composites, Madison, WI, USA, May 16-18, 2011. (Participants: K. Liang and S. Shi)

9. Presented by S. Shi. Development of natural fiber sheet molding compound (SMC). Ontario BioAuto Council Conference, Windsor, Ontario, September 23, 2010 (Over 100 people in the audience) (Participants: S. Shi, K. Liang, M. Qatu, J. Wang, M. Tschopp, D. Nicolas, D. Zhang, L. Sites, J. Shi, I. Fulton, W. Che, and S. Lee)

10. Presented by S. Shi. Kenaf bast fiber sheet molding compound (SMC) composites with acrylated expoxidized soybean oil. $64^{\text {th }}$ Forest Products Society (FPS) International Convention, Madison, WI, June 20 - 22, 2010. (Participants: Liang, K. and Shi, S. Q.)

11. Presented by S. Shi. Graphitization of natural fiber cellulose into carbon fiber. $64^{\text {th }}$ Forest Products Society (FPS) International Convention, Madison, WI, June 20 - 22, 2010. (Participants: Shi, S. Q., Zhang, S., Jiang, D., Che, W., and Shi, J.)

12. Presented by S. Shi. Semi-crystalline polymer-based composites with corn fiber (separated from DDGS) as an interfacial reagent. $11^{\text {th }}$ International Conference on Biocomposites, Toronto, Canada, May 2 - 4, 2010. (Participants: Castillo, H., Lee, S., Shi, S. Q., Srinivasan, R., and Shi J.)

13. Presented by S. Shi. Modified soybean oil/polyhedral oligomeric silsesquioxane (POSS) bionanocomposites: Synthesis and characterization. Proceeding of $11^{\text {th }}$ International Conference on Biocomposites: Transition to Green Materials, Toronto, Canada. May 2 -4, 2010. (Participants: Liang, K. and Shi, S. Q.)

14. Presented by K. Liang. Modified soybean oil/polyhedral oligomeric silsesquioxane (POSS) bionanocomposites: Synthesis and characterization. $11^{\text {th }}$ International Conference on Biocomposites, Toronto, Canada, May 2 -4, 2010. (Participants: K. Liang and S. Shi)

15. Presented by S. Shi. Nanotechnology for Natural Fiber Based Composites. Harbin Engineering University, Harbin, China, March 10, 2010 (about 25 people in the audience)

16. Presented by K. Liang. Resins from soybean oil-based additives for natural fiber sheet molding compound (SMC) composites: Synthesis and characterization. Proceedings of Wood Adhesives 2009. September 28 - 30, Lake Tahoe, Nevada, USA. (Participants: K. Liang and S. Q. Shi.)

17. Presented by K. Liang. Effect of cloisite clay on the dynamic mechanical properties of resin film. Proceedings of Wood Adhesives 2009. September 28 - 30, Lake Tahoe, Nevada, USA.

(Participants: Peng, Y. and Shi, S. Q.) 


\section{References}

1. Bisanda, E.T.N. and Ansell, M.P. 1991. Composites Science and Technology 41: 165-178.

2. Chen, W., Lickfield, G.C. and Yang, C.Q. 2004. Polymer 45(3): 1063-1071.

3. Corbiere-Nicollier, T., Gfeller Laban, B., Lundquist, L., Leterrier, Y., Msnson, J.A.E., and Jolliet, O. Resources, Conservation and recycling, 33 (2001) 267-287.

4. Henriksson, M., Henriksson, G., Berglund, L.A., Lindstrom, T. 2007. Eur Polym J 43:3434-41.

5. Hossain, D., Tschopp, M.A., Ward, D.K., Bouvard, J.L., Wang, P., Horstemeyer, M.F. 2010. Polymer.

6. Iwamoto, S., Nakagaito, A.N., Yano, H. and Nogi, M. 2005. Applied Physic A 81:1109-1112.

7. Kulpinski, P. 2005. Journal of applied polymer science 98:1855-1859.

8. Springer, G.S. 1983. Journal of Reinforced Plastics and Composites 2: 70.

9. Sui ,X., Yuan, J., Yuan, W., Zhou, M. 2008. Chem Lett 37(1):114-115.

10. The Institute of Paper Chemistry. Appleton, W.I. Institute Method, No. 428. 1951.

11. The United Soybean Board and Omni Tech International, Life Cycle Impact of Soybean Production and Soy Industrial Products. 2010.

12. Wise LE, Maxine M and Alfred AD. 1946. Paper Trade Journal 122 (2):35-43.

13. Zadorecki P, Michell AJ. 1989. Polym Compos 10(2):69-77. 


\section{SUBTASK 3.4: BIOMATERIALS}

Team Members: L. Williams, J. Liao, H. Rhee, Y. Mao, S. Patnaik (Ph.D student), M. Rougeau (M.S. student), N. Y. Lee (Ph.D. student)

\section{Objective:}

- Determine the structure-property relationships of both soft biological tissues and animal outer armor.

- Use the relationships to develop material models for implementation into finite element codes.

- Approach

- Address the structural property relationships of animal soft tissues and animal armor by using similar experimental approaches for all materials.

- Characterization of the change in structure and properties of the materials by capturing its mechanical response along with any structural deformation/damage by using multiple microscopy techniques and tools.

- Specific Aims

- Quantify the impact capability of the biological structural materials and soft tissues based on the materials and geometric characteristics, multiscale structures, and mechanical responses.

- Characterize, model, and simulate mechanical behaviors.

- Implement the results from all mechanical experiments into a finite element environment and simulate impact scenarios.

- Contrast/compare the structure-property relations of various biological structural materials to understand mechanisms that lead to the development of novel, bio-inspired safety systems design methodologies.

\section{Milestones, Metrics, ANd Accomplishments}

The objectives were met by accomplishing the following:

- Thorough evaluation of soft tissues mechanics via quasi-static mechanical testing;

- Developed a basic framework for the constitutive model of soft tissue, which could potentially be adapted to other tissues and organs.

- Robust and realistic finite element meshes of human head was constructed;

- A basic framework for finite element simulation of human head under blast loads was developed, which could potentially be adapted to other tissues and organs;

- Materials/mechanical properties tests were performed and structure-property relations were investigated of the turtle shell, armadillo shell, rams horn;

- Prepared/submitted many journal articles and presented conference talks. 


\section{INTRODUCTION}

\section{Mechanical Properties of Porcine Lung}

Blast injuries are classified as primary (blast overpressure), secondary (flying objects), tertiary (body flying in air and hitting other objects due to explosion) and quaternary (all other injuries) (Mayorga 1997; Champion 2009; Wolf 2009). Primary blast injuries affect the air filled structures like the gastrointestinal tract, ears and lungs. Damage to these air filled structures due to blast over pressure or by a blast wave can be severe. Lung is the $2^{\text {nd }}$ most affected organ in primary blast injuries (tympanic membrane being the most severe) (Choung 1985; Mayorga 1997). Although research on blast mechanisms have been performed for close to a century, it was only in the late 80's when the blast injury mechanics was formulated (Phillips III 1991). Choung et.al. conducted experimental studies on lungs and other soft tissues in the thoracic cavity, to understand the injuries caused due to blast (Choung 1985). He concluded that during propagation of a pressure wave, chest wall and ribs acts as barriers and cause additional damage to the thoracic region. Pleural surface (thin membrane covering the lung) is the first to be damaged and additional "rib markings" are produced by the impact of waves on the lungs. Various computational studies on the blast simulations and experimental studies on lung tissue have been performed, but the material was taken as homogenous without considering the effects of blood vessels, surface tension due to surfactants, airtubes and pleural membrane (which is not accurate) (Lee 1976; Vawter 1980; Rodarte 1982; Ligas 1984; Choung 1985; Ligas 1985; Stuhmiller 1988; Stuhmiller 1996; Stuhmiller 1997; Gao 2006). Thus, a detailed microstructural structure-property relationship study of the lungs tissue is needed which will be a useful input for future computational models and also for better representation of its mechanical responses.

Lung parenchyma is often described as randomly oriented, viscoelastic and pre-stressed compressible material which exhibits non linear stress-strain behavior (Lambert 1973; Lai-Fook 1977; Rodarte 1982; Ligas 1984; Ligas 1985; Gao 2006; Suki 2011). Most of the mechanical energy spent during inflation and thus is recovered in deflation \& rest is dispensed throughout the structure (fluid and airtubes). Not only the components of the lung tissue (i.e. elastin and collagen) (Faffe 2006), but also its natural architecture is responsible for the mechanical properties (Mead 1961). Collagen fibers in the parenchyma form an axial network and extend from the pleura. At lower strains load bearing work is taken by elastin. On the other hand collagen fibers bear the load at higher strain rate and become stiffer (Mead 1961; Suki 2011). Tension forces cause of majority of the damage in the lung tissue rather the compressive forces, during blast wave propagation (Stuhmiller 1988; Stuhmiller 1996; Stuhmiller 1997; D'Yachenko 2006). Additionally, decompression of the lungs even for a short duration causes considerable damage to lungs tissue. In comparison to compressive forces, shear forces are less sensitive to increasing physiological pressure (i.e. shear modulus is insensitive to pressure changes) and thus play lesser role in lung damage (Stamenovic 1986). Overall, compression forces play vital role in primary blast injury and investigation of lungs tissue in compressive mode was selected for this study. Future studies will address the detail analysis of lungs tissue from different anatomical locations.

Blast pressure wave creates a direct stress wave and accompanying transmitted wave which applies direct compression force of the thoracic cavity and ultimately the lungs (Stuhmiller 1988; Cooper 
1991; Stuhmiller 1996; Stuhmiller 1997). Major issue with mechanical testing of lung :in vivo - filled with air ; in vitro - no air (J. W. Melvin 1974). Direct measurement of mechanical properties of excised lung parechanymal tissue can be done by performing uniaxial or biaxial tests in a water bath. Many authors support to the fact that in vitro mechanical test of excised lung tissue is almost an ideal representation of the in vivo tissue (Fust 2004; Gao 2006). Although gas pressure is not significant enough to apply any direct force on internal structure, there is some degree of deformation which leads to changes in stress (i.e. during the breathing cycle). Recent studies have shown that preconditioning and a mechanical test of lung tissue conducted in water bath (saline solution) does not require special rinsing and degassing procedures (Leite-Junior 2003). Vawter and co-workers reported that lung parenchyma was elastic is compression in higher strains as compared to tension (Vawter 1978). Within physiological limits, increasing surface tension increases the bulk modulus of the lung parenchyma and decreases above the physiological range (Stamenovic 1986).

\section{Woodpecker Beak Structure-Property Relationships}

Biological material has a remarkable design strategy for efficient mechanical behavior with a hierarchical structure. One elegant example is suture joint where two stiff components interlock each other to provide flexibility for growing or movement, to bear and transmit loads, and to absorb energy (Jaslow 1990; Sun 2004; Yu 2004; Li 2011; Li 2012). For example, at the tip of the woodpecker beak, we could find the wavy structure as shown in Fig. 1(a). Cranial bone has a suture also as shown in Fig. 1(b), and the function of cranial suture has been studied greatly such as function of impact loading dissipation due to mastication or allowing flexibility for growth (Hubbard 1971; Jaslow 1990; Opperman 2000; Sun 2004; Yu 2004; Byron 2006). Ammonite fossil has a wavy structure having fractal pattern on their shell, also turtle shell has a suture joint in their carapace as shown in Fig.1 (c) and (d) (Allen 2007; Krauss 2009). The main objective of the present study is to investigate the geometrical effect on the shock mitigation using Finite Element Analysis (FEA) of wavy structure.

\section{Finite Element Model Development for Multi-Region Porcine Scalp}

Head injuries are one of the main causes of death in the United States. According to the Center for Disease Control and Prevention, head injuries account for $44 \%$ of all injury related deaths in the United States. Predictive head injury indicators are being used in car crash evaluations, forensic science investigations, and in research as an alternative to expensive, unpractical, and sometimes unethical animal or human experimentation (Raul et. al, 2008). Finite Element Modeling (FEM) utilizes a system of equations and 3-D geometry to simulate, and validate material responses to impact, based on parameters determined from experimental testing. Several Finite Element (FE) human head models have been created and validated with and without the scalp. Current FE models that include the scalp, model it as a single layer shell, which is not accurate. 

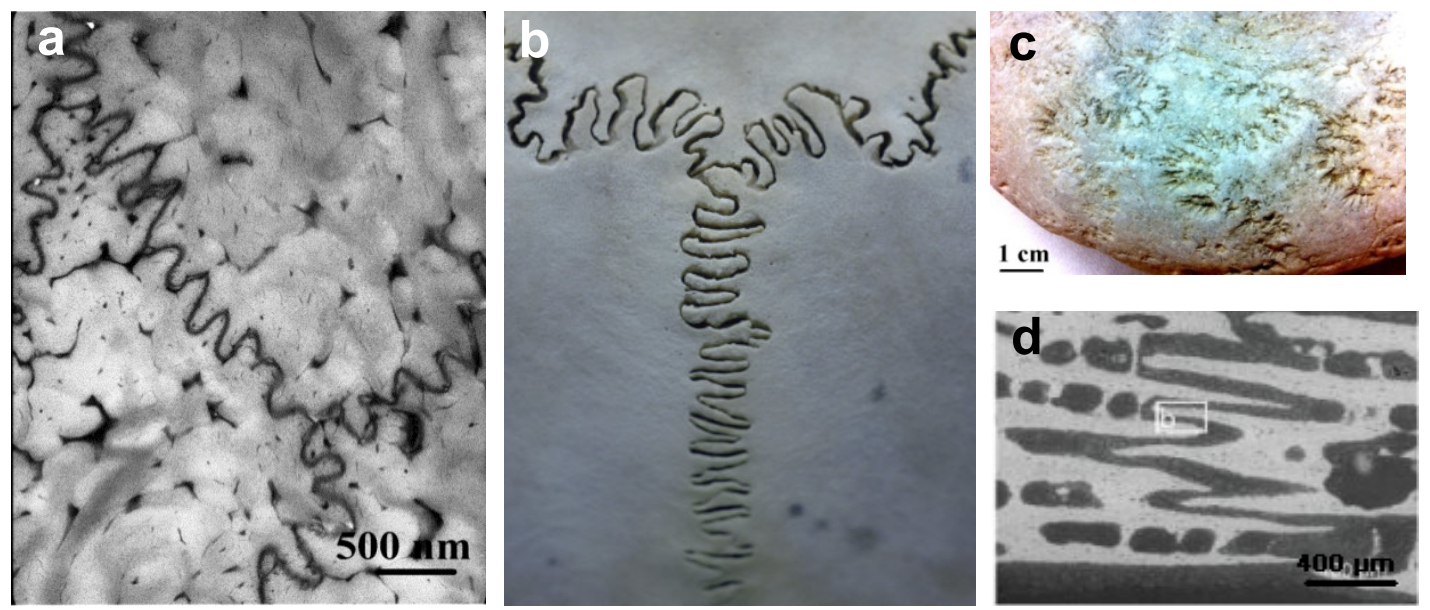

Figure 1. The examples of suture line from the biological materials; (a) suture line shown at the tip of woodpecker beaks; (b) cranial suture in a human skull (courtesy to http://eu.art.com/products/p360870764-sa-i4010951/michael-gabridge-close-up-ofhuman-skull-sutures.htm); (c) interdigit line shown in a surface of an ammonoid fossil, and ;(d) suture joint at a turtle shell (courtesy to Mechanical Function of a Complex ThreeDimensional Suture Joining the Bony Elements in the Shell of the Red-Eared Slider Turtle).

The human scalp is composed of five layers: skin, connective tissue, aponeurosis, loose connective (areolar), and pericranium. These layers form a protective barrier around the human head. Previous research has found that when placed under compressive forces, the scalp disperses pressure around the area of loading (Melvin et. al, 1970; Melvin and Evans, 1971). In the case of head injury, the scalp prevents loading spikes that could result in skull fractures or brain injury. All previous FEMs either modeled the scalp as one layer fused to the skull or did not include it. But, this leads to inaccuracy as the mechanical properties for the primary three layers of the scalp are far more rigid in comparison to the areolar tissue. The purpose of the present work was to characterize the structural and mechanical properties of the multiregional scalp and create a preliminary Finite Element Analysis (FEA) model. Such a model is expected to help in improving the accuracy of FE predictive head injury models and better help future vehicular safety countermeasure design.

\section{Structure Property Relations of Bream Teeth}

Structures and mechanical properties of human teeth (Xu 1998; Malinowska 2005; He 2008; Roy 2008; Bajaj 2009) and various fish teeth (Sasagawa 2009; Whitenack 2010; Song 2011) were reported in the literature. These studies provided insight into basic structure of enamel and dentin and also modulus values under different strain condition. A research study by Zeravaki, et al. (2008) revealed the microhardness of tooth enamel as $307 \mathrm{Hv}$ and that of dentin to be $43 \mathrm{Hv}$, thus they concluded the hardness and modulus values varied with depth. The hardness of enamel was found to be around 3.5 GPa ( 356.9 Hv) and that of interior dentin to be $1.5 \mathrm{GPa}(\sim 153 \mathrm{Hv})$ by Roy, et al. (2008). They also reported that the variation in hardness values is partially dependent upon the mineral concentration in enamel and dentin, with a possible dependence also on local microstructural features such as enamel rod orientation and dentinal tube density. The microsturucture of human tooth plays a vital role in 
determining the mechanical properties like hardness, modulus, etc. ( $\mathrm{Xu}$ 1998). The difference in hardness values of dentin and enamel is mainly due to difference in percentage of organic and inorganic materials in enamel and dentin and not due to the content of $\mathrm{Na}, \mathrm{Mg}$, or $\mathrm{Cl}$ (Gutierrez-Salazar 2003). The shark teeth were also studied by Whitenack, et al. (2010) for their Young's modulus and mechanical properties and they conducted research on osteodentine (c.taurus) and orthodentine (c. tiburo). Both hardness and modulus values were higher for osteodentine than orthodentine. One reason explained is that the arrangement of tubules (i.e., macroanatomy) plays an important role in determining the hardness of the tooth. Also, the chemical composition varied in osteodentine and orthodentine; the earlier had more calcium content whereas the later had more sodium and phosphate percentage. The reported hardness values for shark tooth $(3.20 \sim 3.53 \mathrm{GPa})$ fall within the range of mammalian hardness values. These studies provided insight into basic structure of enamel and dentin, and also modulus values under different strain conditions.

\section{EXPERIMENTAL APPROACH}

\section{Mechanical Properties of Porcine Lung}

The objective of this study was to quantify the compressive mechanical properties of fresh porcine lungs tissue (i.e. to study the viscoelastic mechanical response of the tissue). Directional dependence of the tissue was tested by resecting tissue samples from coronal, sagittal and transverse planes (Fig. 2). All tests were performed in a BSL2 certified laboratory at MSU Ag and Bio Eng Facility. Samples were obtained fresh from the local abattoir (Sansing Meat Service, MS). Tests were performed within 4-5 hours to remove any unfavorable effect of tissue degradation. Excess tissues attached to the lungs were removed. All tests were done using Mach 1 Micromechanical Testing System ${ }^{\circledR}$ (Biomomentum, Quebec, Canada) and in water bath as shown in Fig.2.

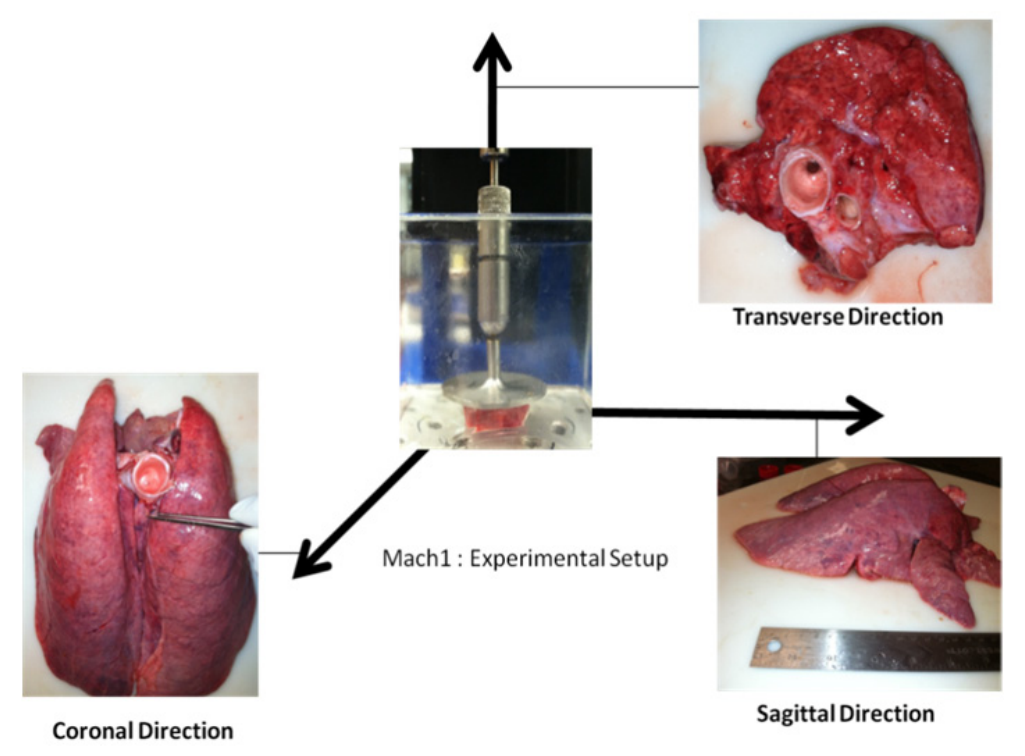

Figure 2. Mechanical testing setup and direction of testing. 
Using scalpel blades, samples were cut in disc shape of aspect ratio of $20 \mathrm{~mm}: 10 \mathrm{~mm}$ (diameter to thickness) (Fig. 3).

\section{Compression test}

Sample for fresh porcine lung tissue sample was cut in a disc shape (Fig.3).

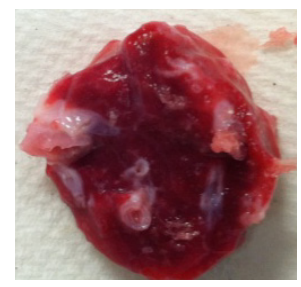

Figure 3. Disc shaped porcine lung tissue samples. Diameter to thickness-20 mm: 10mm

A flat disc shaped metal clamp was used for this test. The sample was glued at the bottom and top to avoid any tension created due to unloading of sample. Sample thickness was again measured using the "find contact" mechanism in the Mach1 Micromechanical Mach1 Micromechanical Testing System ${ }^{\circledR}$ (Biomomentum, Quebec, Canada) and the loading velocity was calculated accordingly.

$$
\text { Loading velocity }=\text { Strain rate } \mathrm{X} \text { Thickness (For compression only) }
$$

Samples were submerged in Phosphate buffer saline (PBS) solution to simulate physiological conditions and prevent samples from drying during the test. Samples were pre-loaded at $2 \mathrm{gm}$, pre-conditioned at $0.1 / \mathrm{sec}$ strain (10 times), compressed to $60 \%$ of load cell (at the constant rate of $0.1 / \mathrm{sec}$ ) and then unloaded.

Table 1. Test matrix. No. of test performed.

\begin{tabular}{|l|l|l|l|}
\hline Tissue Type \Direction & Frontal & Sagittal & Transverse \\
\hline Load & 18 & 14 & 15 \\
\hline Unload & 18 & 14 & 15 \\
\hline Strain Rate & $0.1 / \mathrm{s}$ & $0.1 / \mathrm{s}$ & $0.1 / \mathrm{s}$ \\
\hline
\end{tabular}

Samples were collected from all three directions to study the directional dependent nature of porcine lungs tissue (Details represented in Table 1). 


\section{Woodpecker Beak Structure-Property Relationships}

Fig. 4 depicts the four geometries that were studied. We employed two dimensional plane stress models having $603 \mathrm{~mm}$ in length (L) and $60 \mathrm{~mm}$ in width (W). The wavy interfaces had height (H) of 10 $\mathrm{mm}$ and wave length $(\lambda)$ of $10 \mathrm{~mm}$. Finite element analysis software ABAQUS/Explicit v6.10 (Simulia Inc.) was used for simulation. Linear elastic material property of bone with density ( $\rho) 1900 \mathrm{~kg} / \mathrm{m3}$, Young's modulus of elasticity (E) of $20 \mathrm{GPa}$ and Poisson's ratio ( $(\vartheta)$ of 0.3 was used. The elements used in the simulation were two dimensional, three/four node, bilinear plane stress quadrilateral, reduced integration, hourglass control (CPS3/CPS4R). Compressive pressure with ramped impact was applied to one end of each geometrical configuration as shown in Fig. 4. The peak amplitude was $1 \mathrm{MPa}$, and applied duration is $38.8 \mu \mathrm{s}$ as shown in Fig. 5. The two nodes of the top and bottom at the input end of the each geometry were pinned $\left(u_{1}=u_{2}=u_{3}=0\right)$. Using the visualization mode of ABAQUS, pressure wave along the model was calculated.

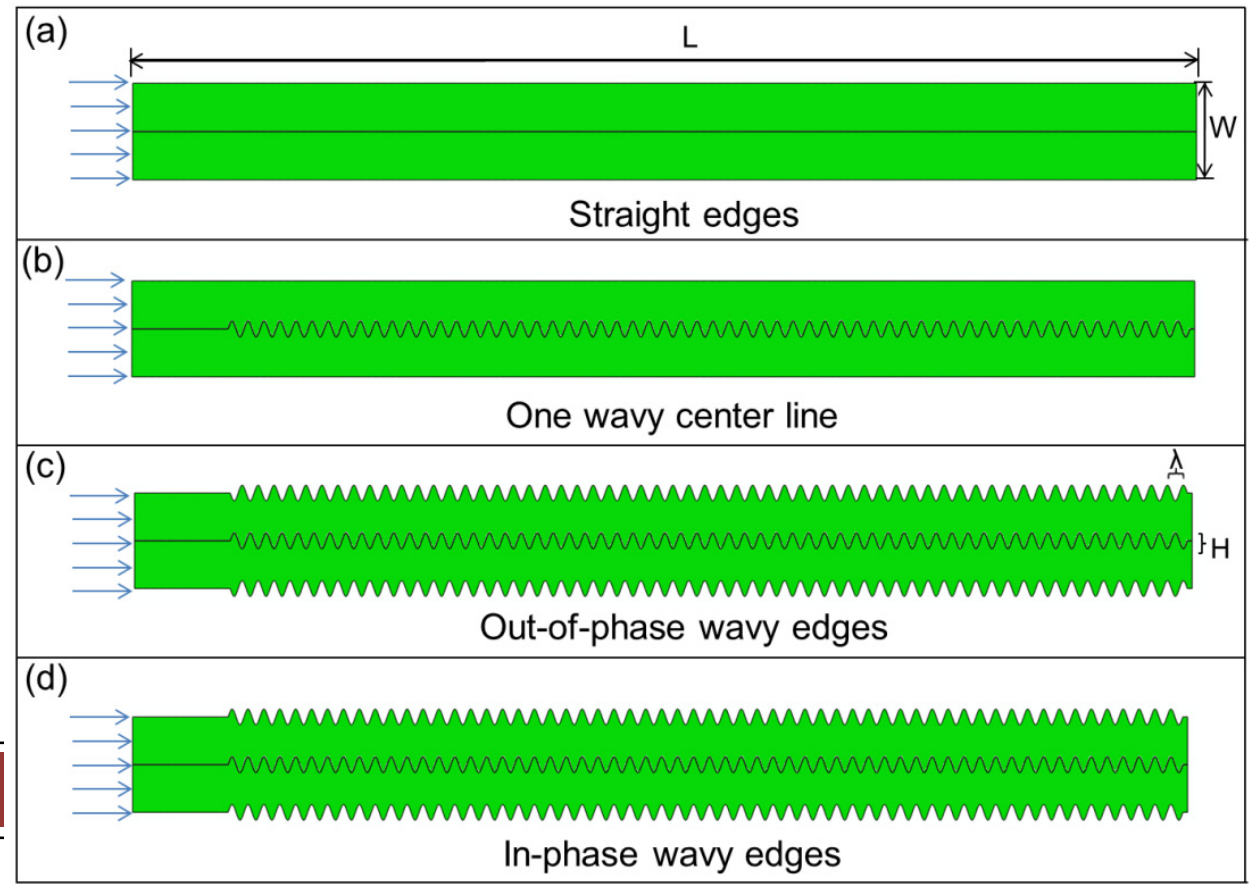


Figure 4. Schematic representation of the four finite element models illustrating the four different geometric configuration with the same length and width: (a) all the boundaries of the sample are straight; (b) the center line of the sample is wavy and other two boundaries are straight; (c) all the boundaries are wavy lines having out of phase pattern and; (d) all the boundaries are wavy lines having in phase pattern.

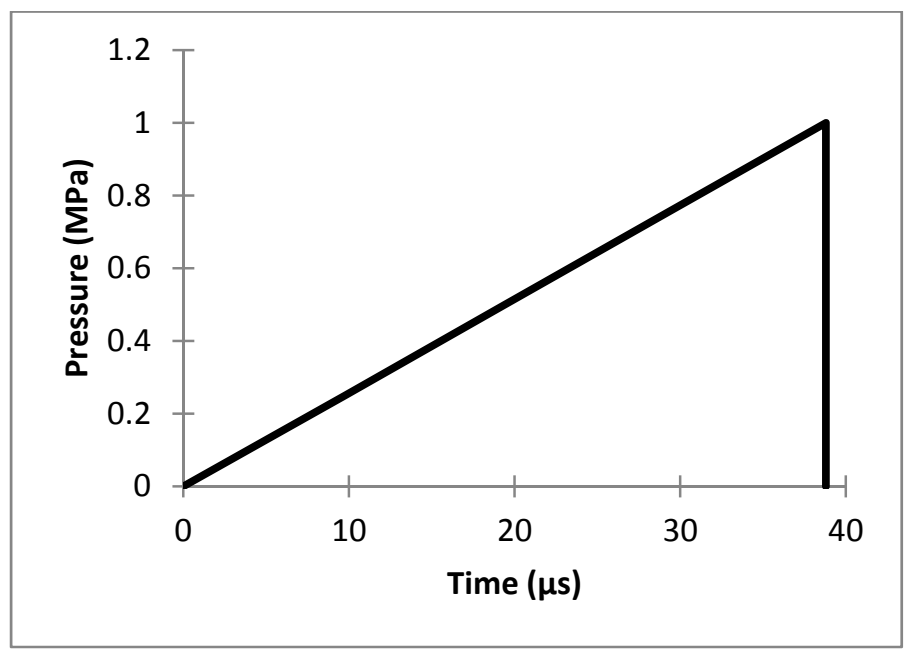

Figure 5. Ramped pressure load history applied to fixed end of each sample. The peak amplitude and duration are $1 \mathrm{MPa}$ and $38.8 \mu \mathrm{s}$.

\section{Finite Element Model Development for Multi-Region Porcine Scalp}

AFM Testing - Due to the average thickness of the scalp skin being less than $1 \mathrm{um}$, it was necessary to determine the modulus using an alternative method that could measure in smaller increments.

DMA Load/Displacement - Load displacement results using DMA were necessary in determining the linear elastic portion of the curve for each scalp region to improve Prony Series parameter accuracy. The resulting curves for each region will be analyzed to help determine the average frequency range necessary to reach the curves linear portion for each layer. As a result of equipment testing limitations due to the scalp-skin thickness, skin regions were sectioned at $1-1.2$ um, including a small portion of the connective region.

\section{Quasistatic Micromechanical Uncertainty Testing/Equations}

Silicone Testing - Quasi-static testing with silicone is being used to help determine the micromechanical tester systematic uncertainty. Once an approximate load displacement fit is determined using the silicone the uncertainty can be deduced. Preliminary silicone testing was performed in order to determine the best fit for the scalp quasi-static data. The composite ratio used to 
make up the silicone (sylGuard 427 - parts $A$ and $B$ ) yielding the closest match was found to be a ratio of $80 / 20$, parts $A$ and $B$ respectively.

Micromechanical Tester Systematic Uncertainty Equations - In order to determine the total experimental uncertainty, the total systematic uncertainty must be derived. The Taylor Series Method was used as a result of the multiple variables needed to define the differing constant uncertainties.

$\boldsymbol{F}$ : Force

$\boldsymbol{b}_{\boldsymbol{F}}$ : Systematic uncertainty in measuring load cell

$\boldsymbol{b}_{S_{\boldsymbol{s}}(\mathbf{0})}$ : Systematic uncertainty in measuring sample diameter

$\boldsymbol{b}_{\varepsilon_{\varepsilon}(t)}:$ Systematic uncertainty in measuring engineering strain

$\boldsymbol{b}_{\varepsilon_{t}(t)}:$ Systematic uncertainty in measuring true strain

$\boldsymbol{b}$ : Systematic standard uncertainty in calculating true stress

$\boldsymbol{\varepsilon}_{\varepsilon}(\boldsymbol{t})$ : Engineering strain

$\varepsilon_{t}(t):$ True strain

$\boldsymbol{S}_{\boldsymbol{s}}(\mathbf{0})$ : Cross sectional area of the sample

$\boldsymbol{S}$ : Random standard uncertainty in calculating true stress

$\sigma_{t}(t):$ True stress

$\boldsymbol{U}$ : Total experimental uncertainty

The combined systematic and random uncertainty is given by

$$
U=\sqrt{b^{2}+S^{2}}
$$

Equation 1: $\mathrm{U}, \mathrm{b}$, and $\mathrm{S}$ are the Total, Systematic and Random uncertainties respectively.

$$
b^{2}=\left(\frac{\partial \sigma_{t}(t)}{\partial F}\right)^{2} \cdot b_{F}^{2}+\left(\frac{\partial \sigma_{t}(t)}{\partial \varepsilon_{t}(t)}\right)^{2} \cdot b_{\varepsilon_{t}(t)}^{2}+(S E C E)+\left(\frac{\partial \sigma_{t}(t)}{\partial S_{s}}\right)^{2} \cdot b_{S_{s}}^{2}+\left(\frac{\partial \sigma_{t}(t)}{\partial \varepsilon_{\varepsilon}(t)}\right)^{2} \cdot b_{\varepsilon_{\varepsilon}(t)}^{2}+(R E C E)
$$

Equation 2: SECE, and RECE are systematic error correlation effects, and random error correlation effects resulting from covariance. 


$$
b^{2}=\left(\frac{\partial \sigma_{t}(t)}{\partial F}\right)^{2} \cdot b_{F}^{2}+\left(\frac{\partial \sigma_{t}(t)}{\partial \varepsilon_{t}(t)}\right)^{2} \cdot b_{\varepsilon_{t}(t)}^{2}+\left(\frac{\partial \sigma_{t}(t)}{\partial S_{s}}\right)^{2} \cdot b_{S_{s}}^{2}+\left(\frac{\partial \sigma_{t}(t)}{\partial \varepsilon_{\varepsilon}(t)}\right)^{2} \cdot b_{\varepsilon_{\varepsilon}}^{2}(t)
$$

Equation 3: we assumed no variables exist with measurements from shared errors and identical sources; therefore, all covariance terms are zero.

$$
\begin{aligned}
\left(\frac{b_{\sigma_{t}}}{\sigma_{t}}\right)^{2}=\left(\frac{F}{\sigma_{t}}\right. & \left.\cdot \frac{d \sigma_{t}(t)}{d F(t)}\right)\left(\frac{b_{F}}{F}\right)^{2}+\left(\frac{\varepsilon_{t}}{\sigma_{t}} \cdot \frac{d \sigma_{t}(t)}{d \varepsilon_{t}(t)}\right)\left(\frac{b_{\varepsilon_{t}}(t)}{\varepsilon_{t}(t)}\right)^{2}+\left(\frac{S_{s}}{\sigma_{t}} \cdot \frac{d \sigma_{t}(0)}{d S_{s}}\right)\left(\frac{b_{S_{s}(0)}}{S_{S}(0)}\right)^{2} \\
& +\left(\frac{\varepsilon_{\varepsilon}(t)}{\sigma_{t}} \cdot \frac{d \sigma_{t}(t)}{d \varepsilon_{\varepsilon}(t)}\right)^{2} \cdot\left(\frac{b_{\varepsilon_{n}(t)}}{\varepsilon_{\varepsilon}(t)}\right)^{2}
\end{aligned}
$$

Equation 4: Evaluation of the partial derivatives, with substitution of force, initial cross sectional area, displacement, and multiplication by $1 / \sigma_{t}^{2}$ on both sides of the previous equation.

$$
\left(\frac{b_{\sigma_{t}}}{\sigma_{t}}\right)^{2}=\left(\frac{b_{F}}{F}\right)^{2}+\left(\frac{b_{\varepsilon_{t}(t)}}{\varepsilon_{t}(t)}\right)^{2}+\left(\frac{b_{S_{S}(0)}}{S_{S}(0)}\right)^{2}+\left(\frac{b_{\varepsilon_{\varepsilon}(t)}}{\varepsilon_{\varepsilon}(t)}\right)^{2} \cdot\left(\frac{\varepsilon_{\varepsilon}(t)}{1+\varepsilon_{\varepsilon}(t)}\right)^{2}
$$

Equation 5: All uncertainty magnification factors become one except for engineering strain. $\frac{b_{\sigma_{t}}}{\sigma_{t}}$ is the relative systematic standard uncertainty.

Equation 5 will be used to calculate the relative uncertainty analysis of each strain rate.

\section{Structure Property Relations of Bream Teeth}

Multiscale structures and mechanical properties were quantified under different length scales by using top and bottom teeth of king bream. The enamel and dentin layers of the king bream teeth were observed by using a scanning electron microscope (SEM), and their chemical compositions were analyzed by an energy dispersive x-ray (EDX) spectroscopy technique, respectively. X-ray computed tomography (CT) was also carried out to observe the internal structure of the bream tooth. The $x$-ray CT was conducted by using a $v \mid$ tome $\mid x$ by phoenix $\mid x$-ray. Mechanical tests in this research included nanoindentation tests. In nano-Indentation tests, indentations were particularly made in a specific region of enamel and dentin to gain knowledge about the modulus and hardness of the respective regions. These tests were performed with Hysitron ${ }^{\circledR}$ Tribolndenter at room temperature using a Berkovich type indenter under load control that included a 20 second loading segment, a 5 second constant load hold time, and a 20 second unloading segment. A $1000 \mu \mathrm{N}$ force was used and the unloading segment was used for analyzing the hardness and the reduced modulus values of the tooth at the region of indenting point. 


\section{RESULTS AND DISCUSSION}

\section{Mechanical Properties of Porcine Lung}

The ultimate compressive stress on wet tissues during loading was in the range of 35,000-50,000 Pa. The ultimate compressive stress on the tissues during unloading was in the range of 30,000-40,000 Pa. The averaged loading and unloading results for the three orthogonal directions are shown below in Figures 6(a)-6(i).
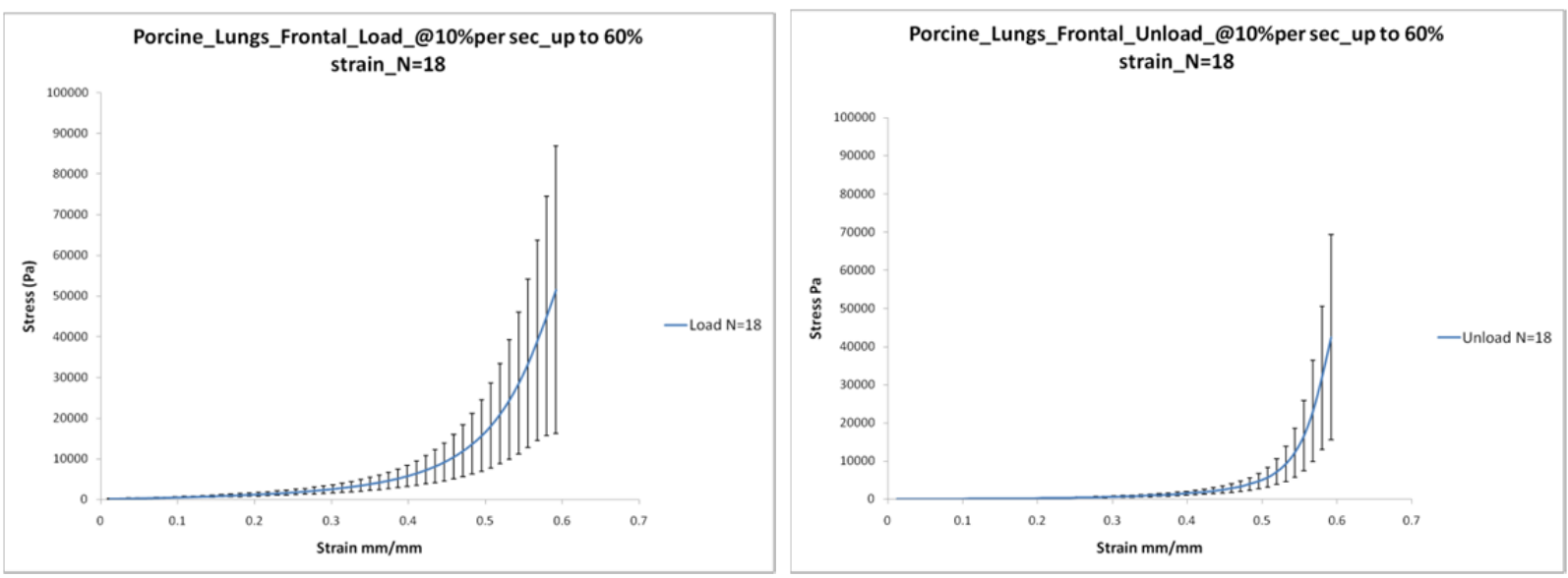

Figure 6. (a) Load Stress- Strain curve of fresh porcine lungs tissue in Frontal Direction compressed at the rate of 0.1 per sec up to $60 \%$ strain. (b) Unload Stress- Strain curve of fresh porcine lungs tissue in Frontal Direction compressed at the rate of 0.1 per sec up to $60 \%$ strain.

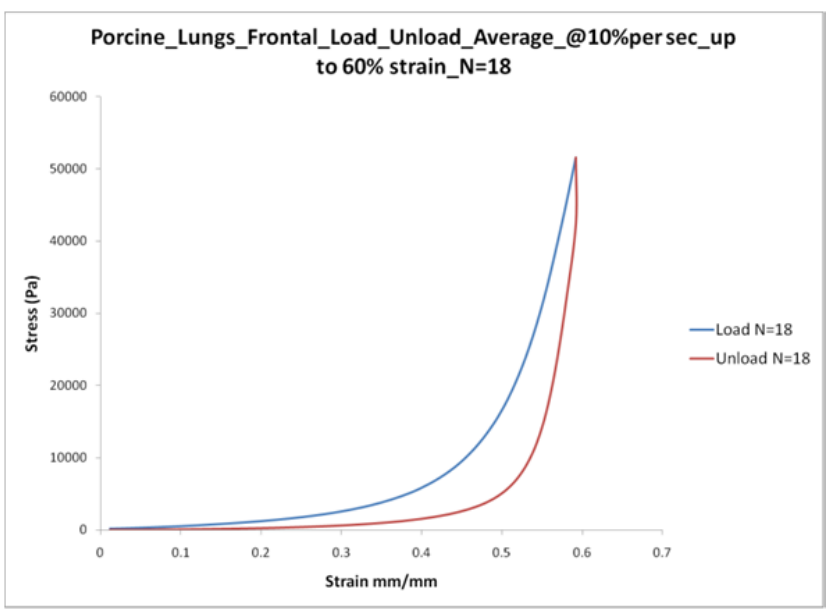

Figure 6. (c) Load Unload Average Stress- Strain curve of fresh porcine lungs tissue in Frontal Direction compressed at the rate of 0.1 per sec up to $60 \%$ strain. 


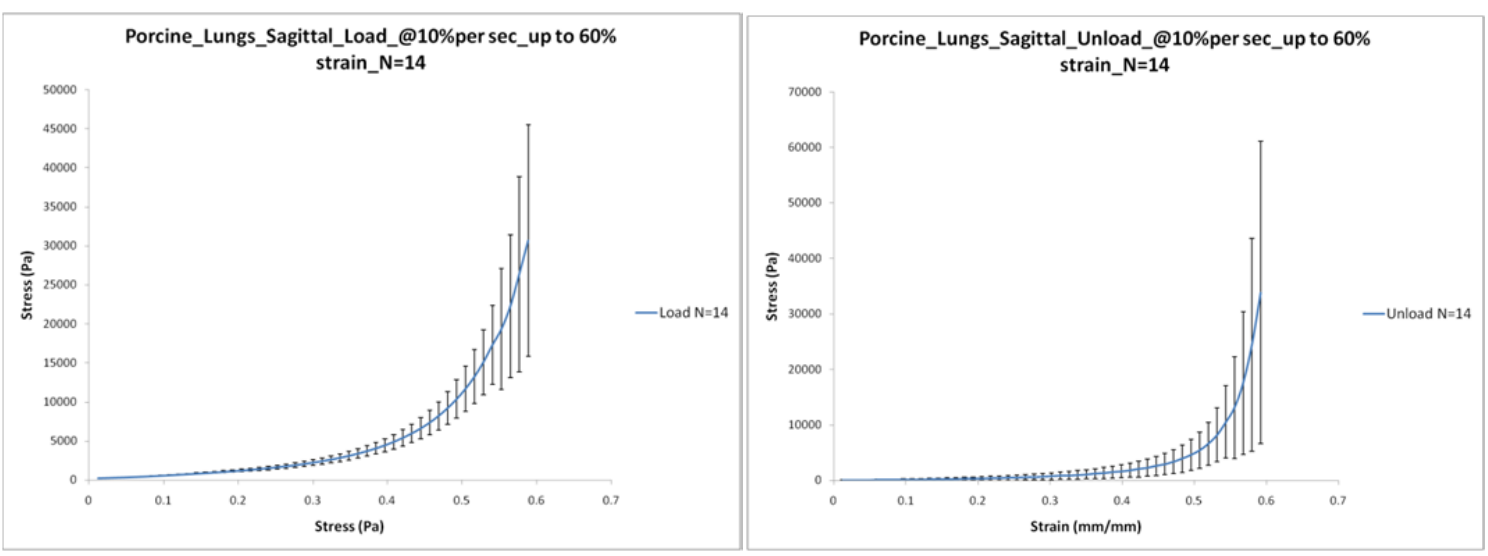

Figure 6. (d) Load Stress- Strain curve of fresh porcine lungs tissue in Sagittal Direction compressed at the rate of 0.1 per sec up to $60 \%$ strain. (e) Unload Stress- Strain curve of fresh porcine lungs tissue in Sagittal Direction compressed at the rate of 0.1 per sec up trons.

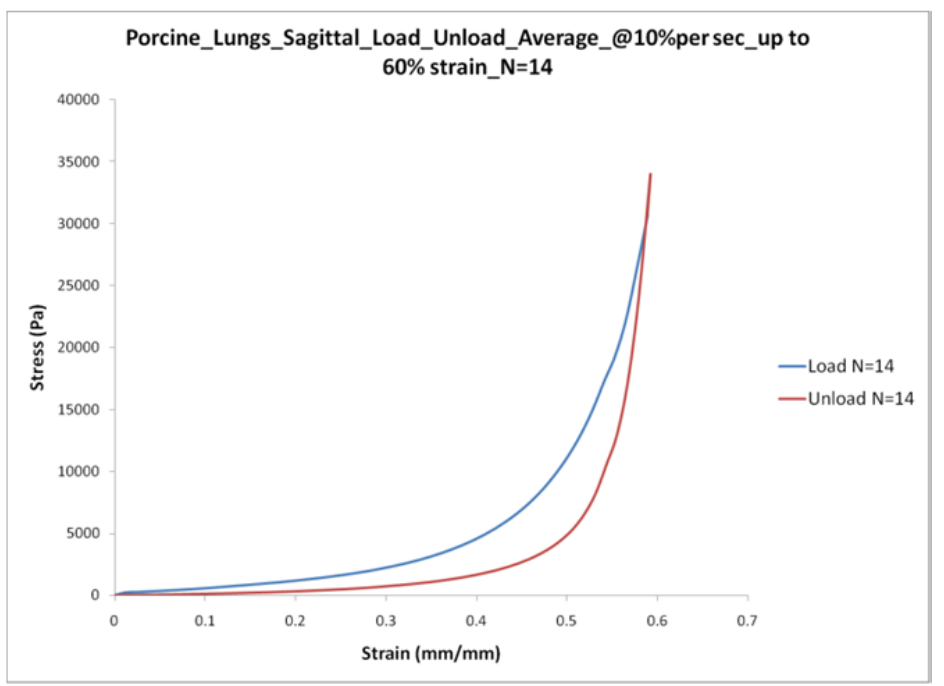

Figure 6 (f). Load Unload Average Stress-Strain curve of fresh porcine lungs tissue in Sagittal Direction compressed at the rate of 0.1 per sec up to $60 \%$ strain. 

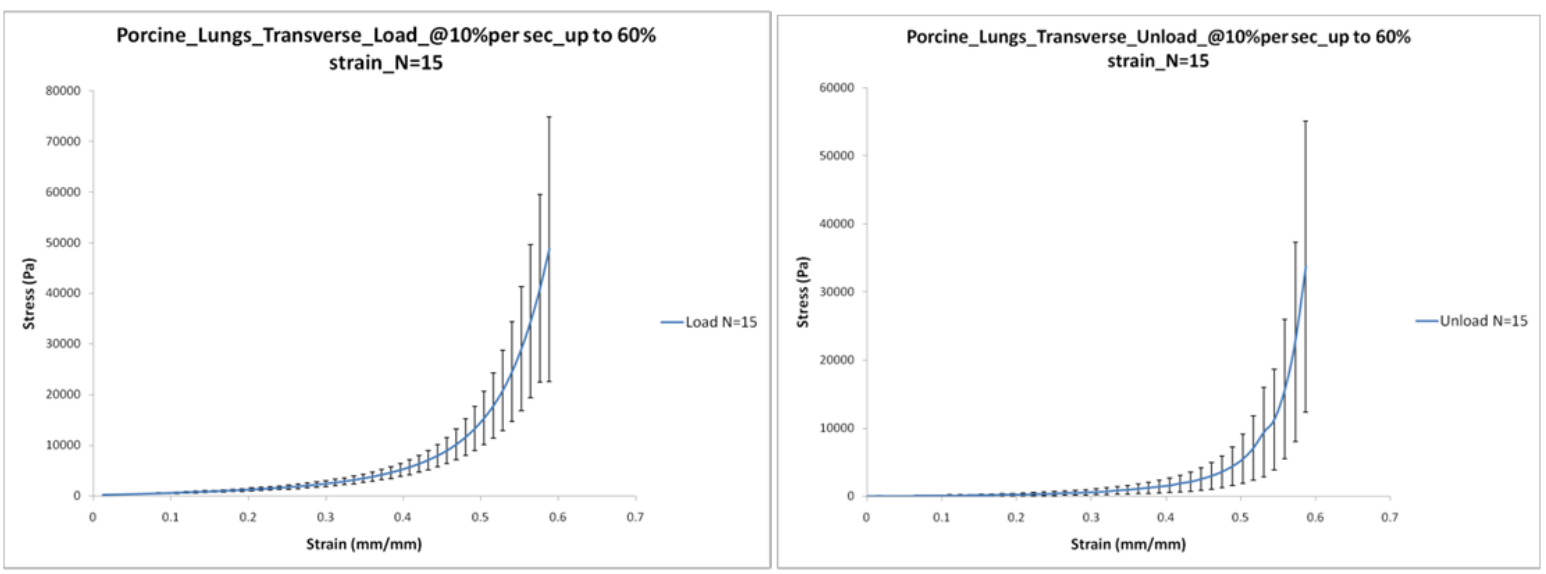

Figure 6. (g) Unload Stress- Strain curve of fresh porcine lungs tissue in Transverse Direction compressed at the rate of 0.1 per sec up to $60 \%$ strain. (h) Load Stress- Strain curve of fresh porcine lungs tissue in Transverse Direction compressed at the rate of 0.1

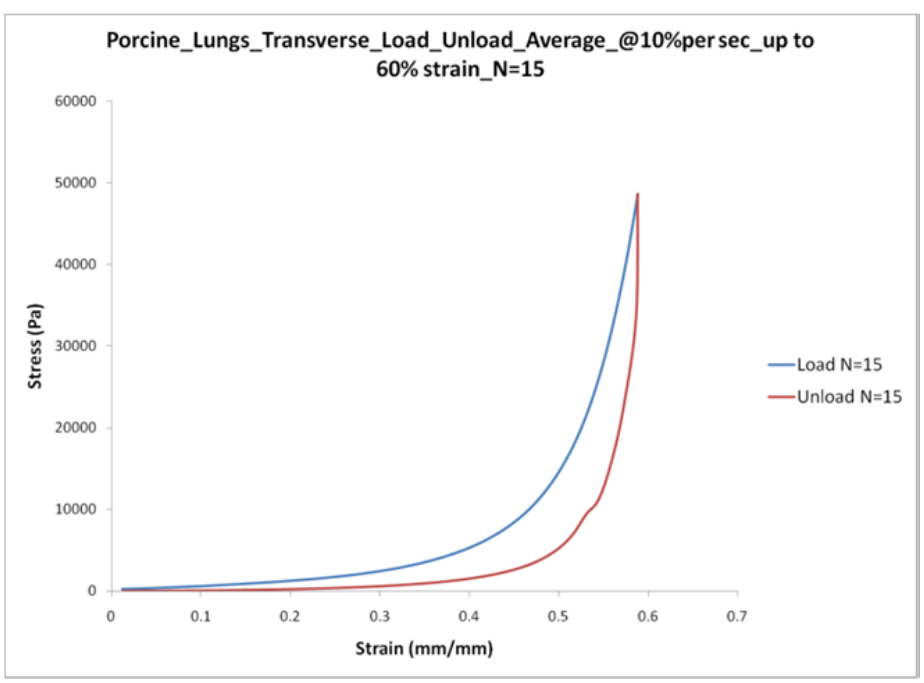

Figure 6 (i). Unload Stress- Strain curve of fresh porcine lungs tissue in Transverse Direction compressed at the rate of 0.1 per sec up to $60 \%$ strain. 


\section{Woodpecker Beak Structure-Property Relationships}

Fig. 7 shows the contour of the pressure wave propagation for all four geometrical cases at various time intervals. Pressure wave takes approximately $210 \mu$ s to reach the other free end in all four models. It can be observed that the pressure wave for the sample with straight edges reaches to the free end slightly faster than the wave at the model with wavy boundaries. In Fig. 8, it is noted that wavy boundary produce the effect to reduce the pressure compared to straight samples. The mechanism which the wavy geometry reduces the pressure is introducing the shear wave. The speed at which a longitudinal and elastic wave goes through an isotropic material is given by:

$$
\mathrm{C}_{\mathrm{L}}=\sqrt{\frac{E}{\rho}}
$$

where $E$ is the Young's Modulus and $\rho$ is density. The shear wave speed travels through the same media is given by:

$$
\mathrm{C}_{\mathrm{s}}=\sqrt{\frac{E}{2(1-v) \rho}}
$$

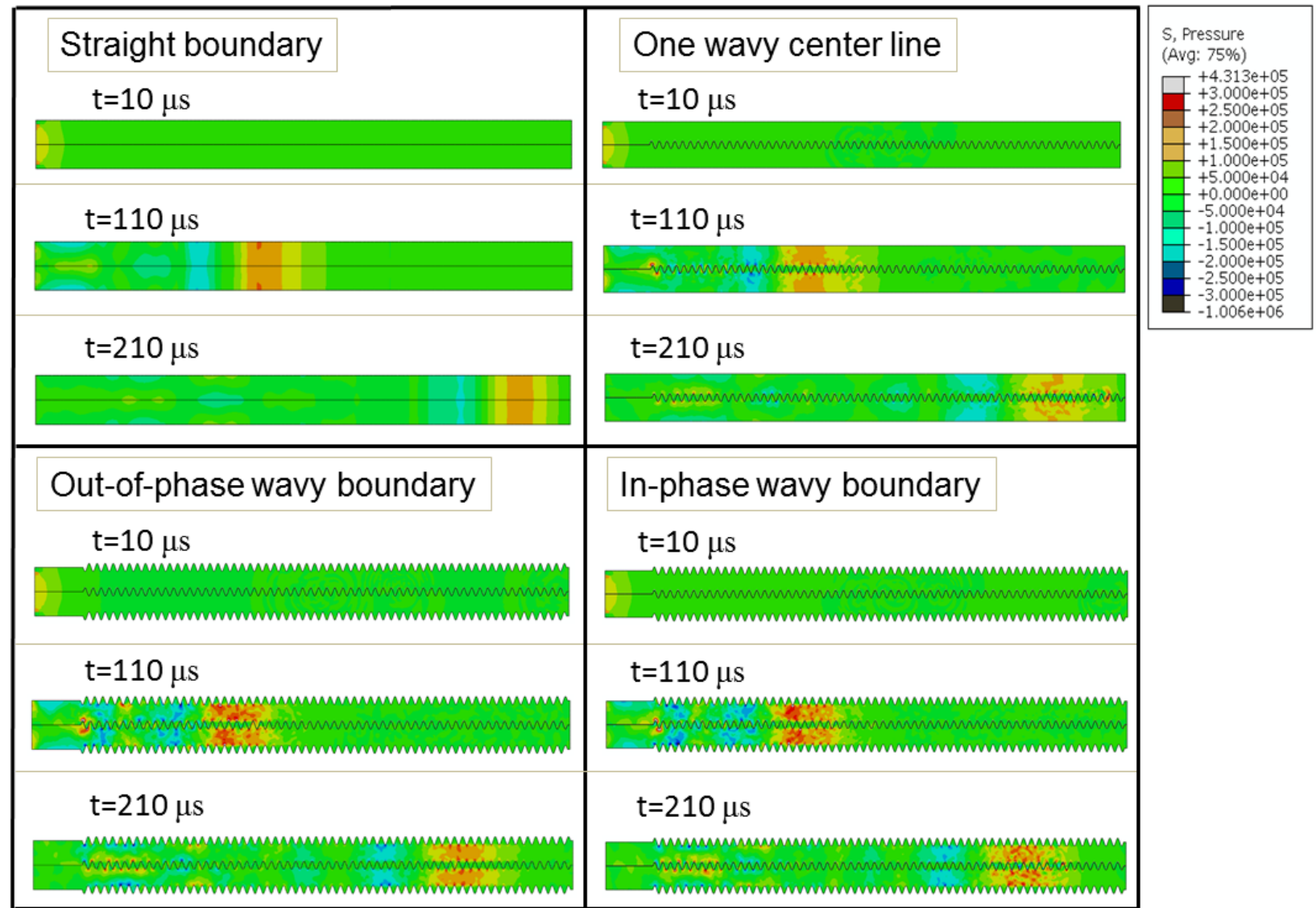

Figure 7. The contours of the pressure wave by time history through $10 \mu \mathrm{s}, 110 \mu \mathrm{s}$, and $210 \mu \mathrm{s}$ at each model. 
By introducing the shear wave by wavy structure, the magnitude of the pressure wave is decreases while shear wave is generated. The shear wave resulted in the displacement to the transverse direction as shown in Fig. 9. Although three models have wavy structure, only the model with single wave resulted in maximum transverse displacement. This is because the wavy boundaries of the out-ofphase and in-phase models compensate the large deformation resulting in shearing stress.

Biomaterial examples mostly have a type of out-of-phase or in-phase wavy boundary. These types of boundaries of biomaterials allow not only to reduce the pressure wave but also to protect large deformation, so the living creatures can keep their shape without damage from the huge impact and large deformation.

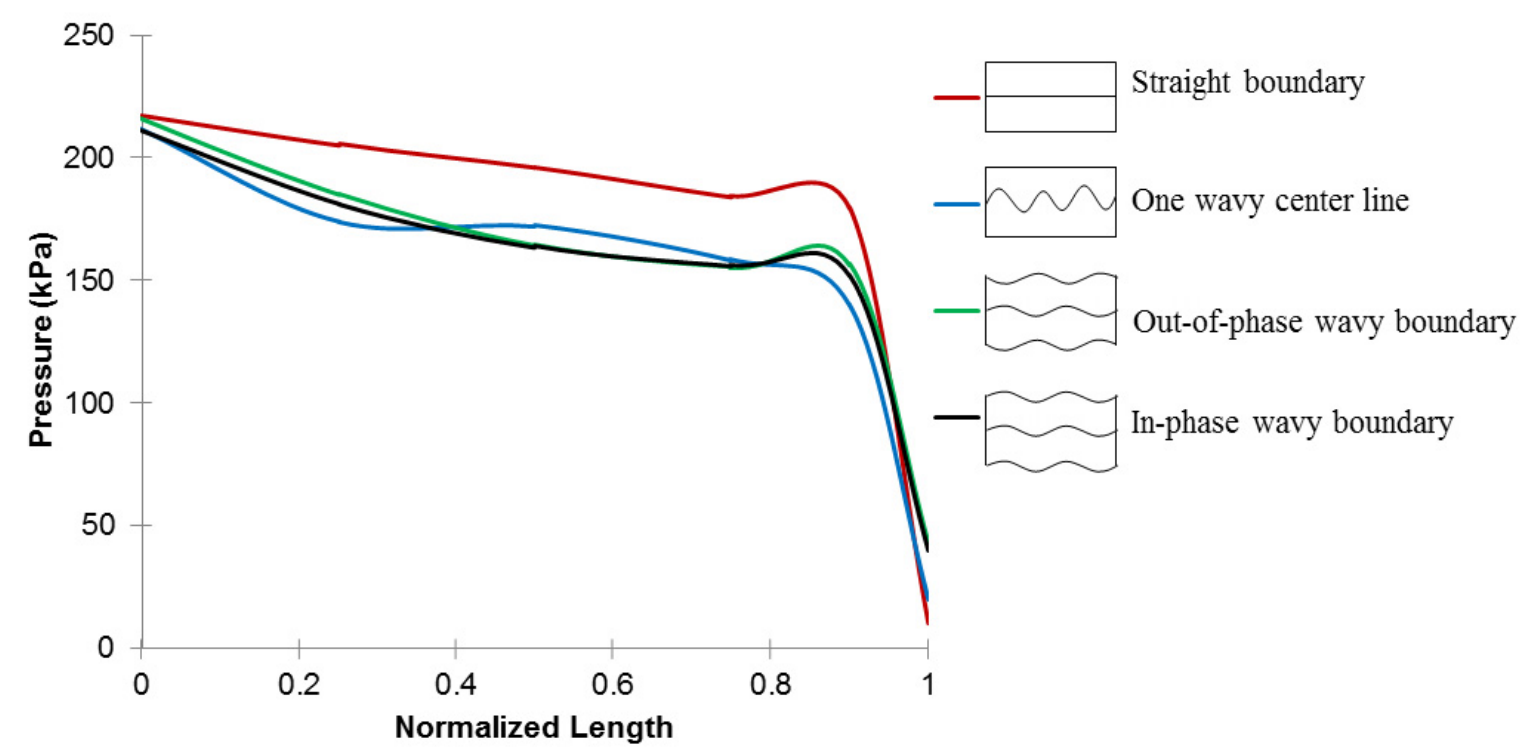

Figure 8. Pressure wave along the normalized length of the four models shows the samples with wavy boundaries reduces the pressure wave compared to the model with straight boundaries. 


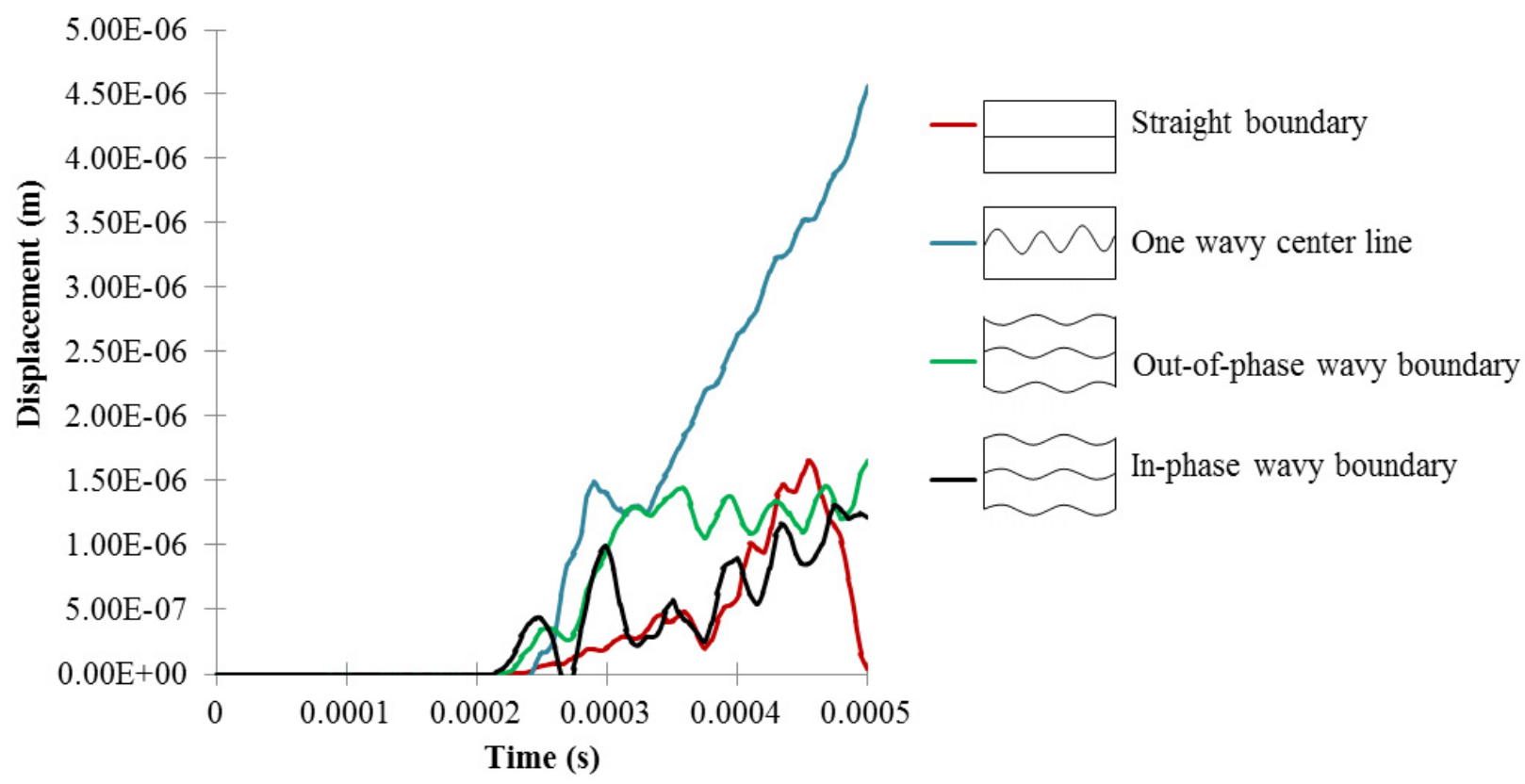

Figure 9. Transverse displacement of the four samples at the free-end shows the sample with one wavy center line generates the maximum displacement although three samples of single center line, out-ofphase, in-phase samples have wavy structure. That is because out-of-phase and in- phase wavy has a compensation effect to stabilize the displacement.

\section{Finite Element Model Development for Multi-Region Porcine Scalp}

AFM Testing - The AFM testing yielded a Young's modulus of approximately $0.82 \mathrm{MPa}$.

Table 2: Skin Modulus determined using AFM. The Goodness of fit is computed between the Max and Min Force Boundaries.

\begin{tabular}{|l|l|l|l|}
\hline Force Indentation & Young's Modulus(MPa) & Reduced Modulus(MPa) & $R^{\wedge} 2$ \\
\hline Average & 0.819333 & 0.900692 & 0.990241 \\
\hline Std Dev & 0.418262 & 0.46023 & 0.01398 \\
\hline Maximum & 1.74 & 1.91 & 0.999 \\
\hline Minimum & 0.31 & 0.34 & 0.9328 \\
\hline
\end{tabular}




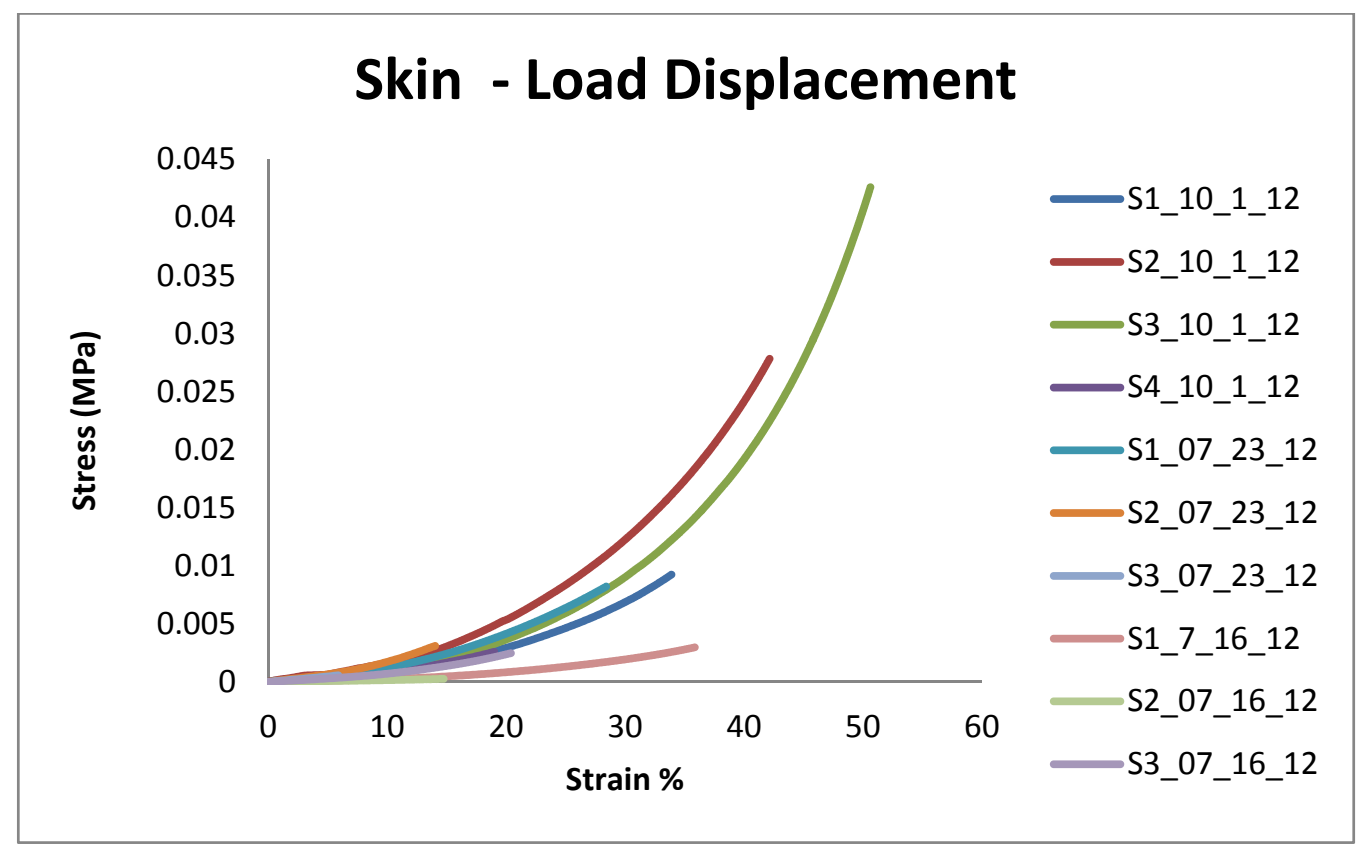

Figure 10: Comparative DMA tests of porcine scalp-skin and under uniaxial unconfined compression loading at varying strain rates between $0.00625,0.025$, and 0.1 s.

Quasistatic Micromechanical Uncertainty Testing

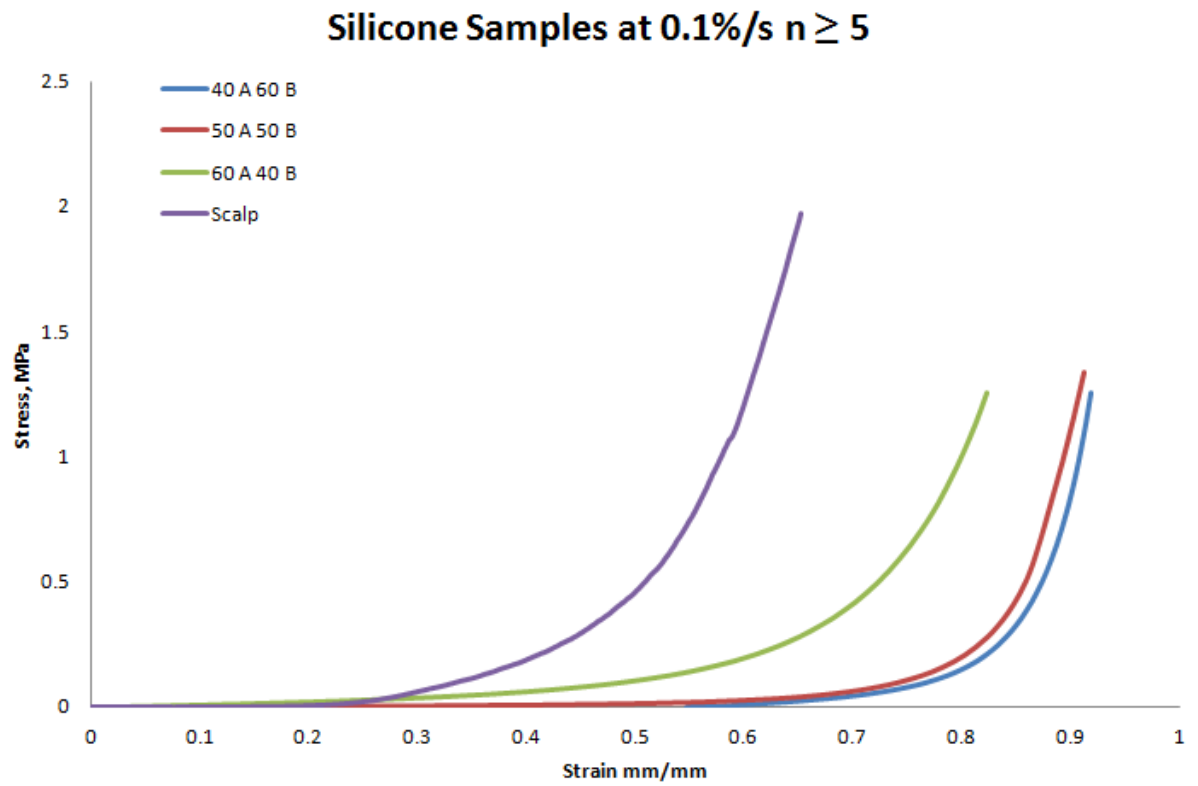

Figure 11: Comparative unconfined compression tests between porcine scalp and silicone under uniaxial unconfined compression loading at $0.1 \% / \mathrm{s}$. 


\section{Structure Property Relations of Bream Teeth}

The internal structure and porous nature of bream tooth was studied using X-ray CT and the images are provided in Figure 12. When viewed from the frontal face, the bream tooth showed unleveled upper topography. As depicted in Figure 12 (b), the upper surface has a raised portion on the outer edge of tooth.
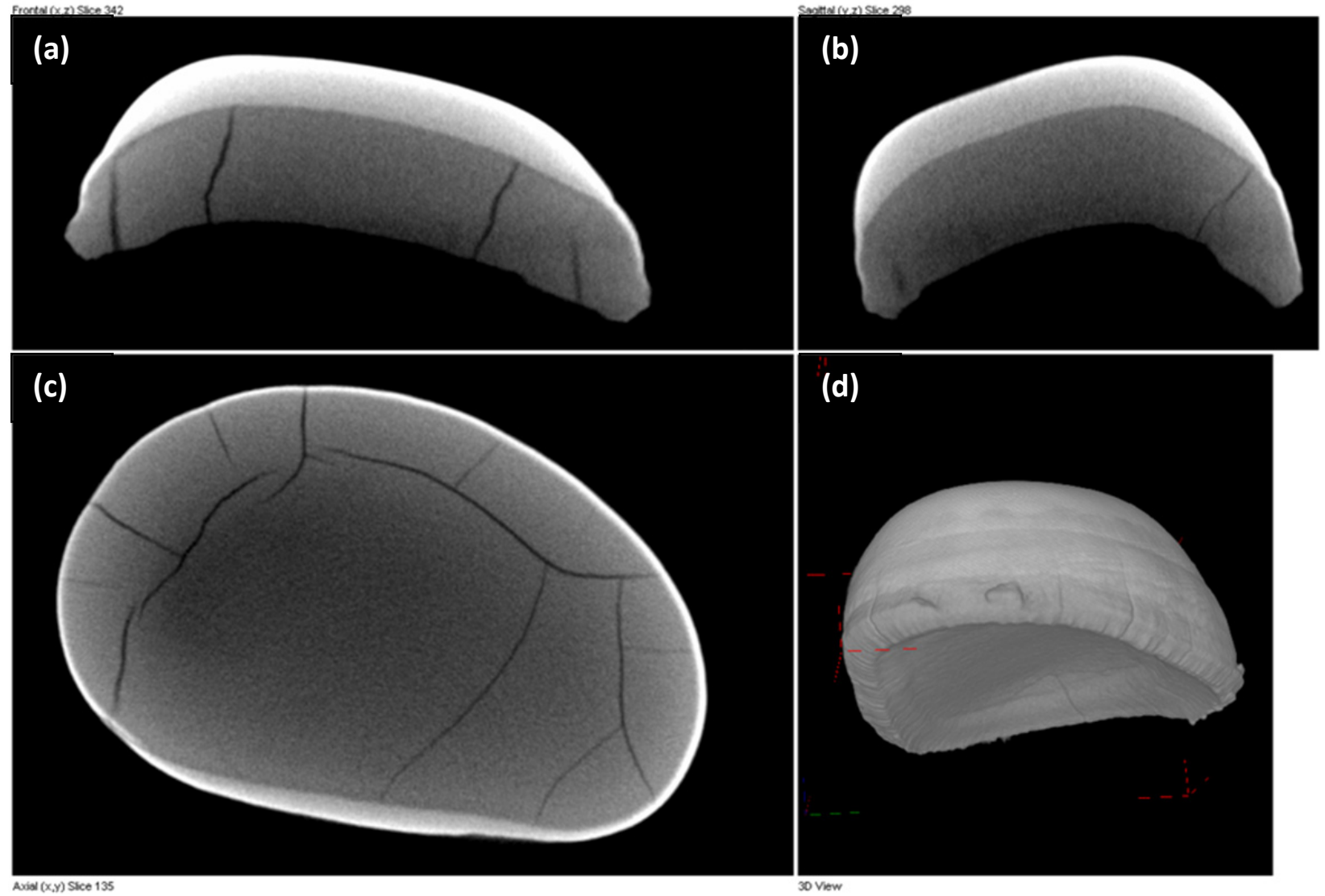

Figure 12. X-ray CT images of bottom bream tooth; (a) sagittal view, (b) Frontal, (c) Axial, and (d) 3D view.

Figure 13 shows the chemical analysis results obtained from various surfaces of the bream tooth. The results of EDX spectroscopy performed on entire enamel showed that the enamel mostly consists of oxygen (O), carbon (C), fluorine $(F)$, calcium (Ca), and phosphorous (P). Small amounts of potassium (K) and sodium ( $\mathrm{Na}$ ) were also traced. The presence of all these elements is justifiable because of the bream's aquatic inhabitance. The chemical analysis results for whole dentin showed the presence of $\mathrm{C}, \mathrm{O}, \mathrm{P}, \mathrm{Na}$, and $\mathrm{Ca}$. The analysis of chemical composition on human tooth enamel showed that the wt $\%$ of $\mathrm{CaO}$ and $\mathrm{P}_{2} \mathrm{O}_{5}$ decreased from outer rim of enamel to dentin-enamel junction (DEJ) (Gutierrez-Salazar 2003). The chemical analysis results obtained from different regions of dentin and DEJ are shown in Figure 13 (c) and (d), respectively. The localized tests mainly showed the presence of C, O, Ca, and P. A research on hardness of different shark species by Whitenack, et al. (2010) showed 
that the microanatomy of dentinal tubules could affect the hardness values considerably, thus the mineral content and microanatomy are mainly responsible for varying hardness in fish tooth.
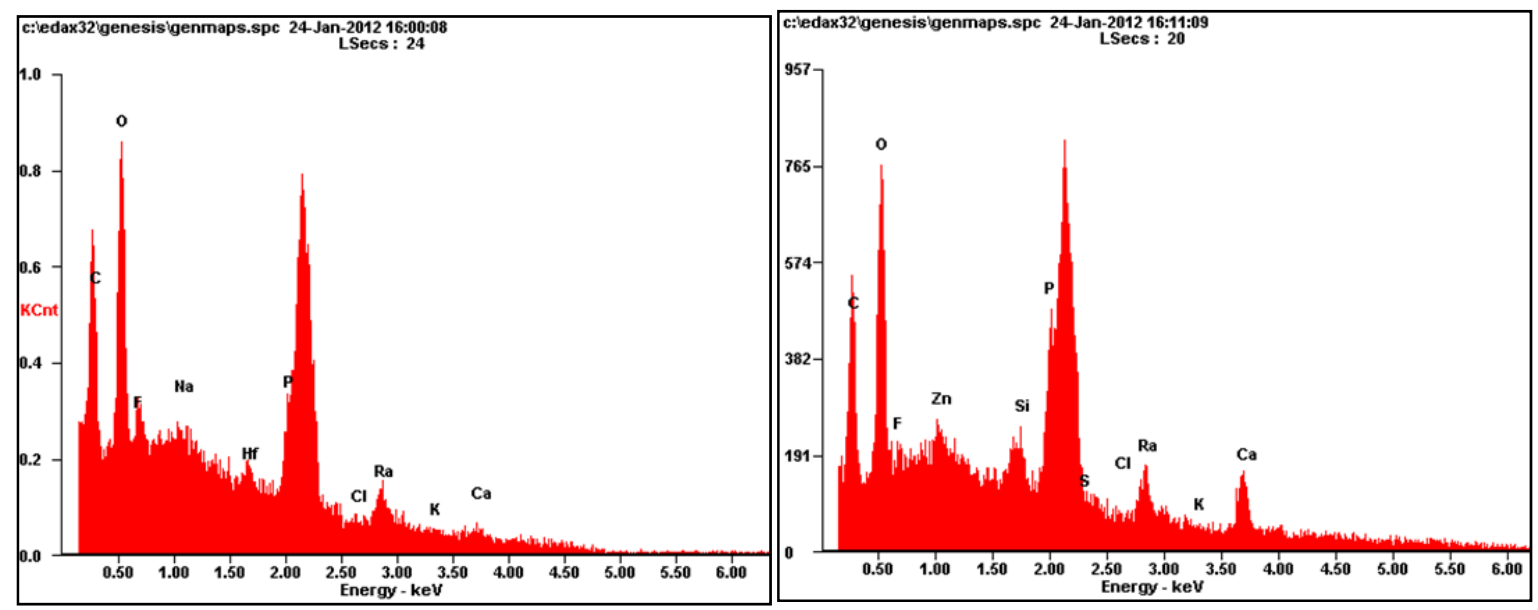

(a)

(b)

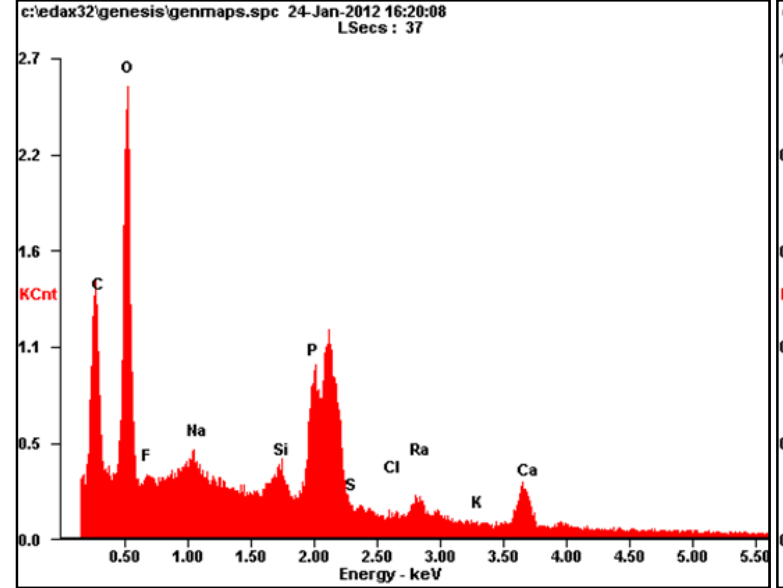

(c)

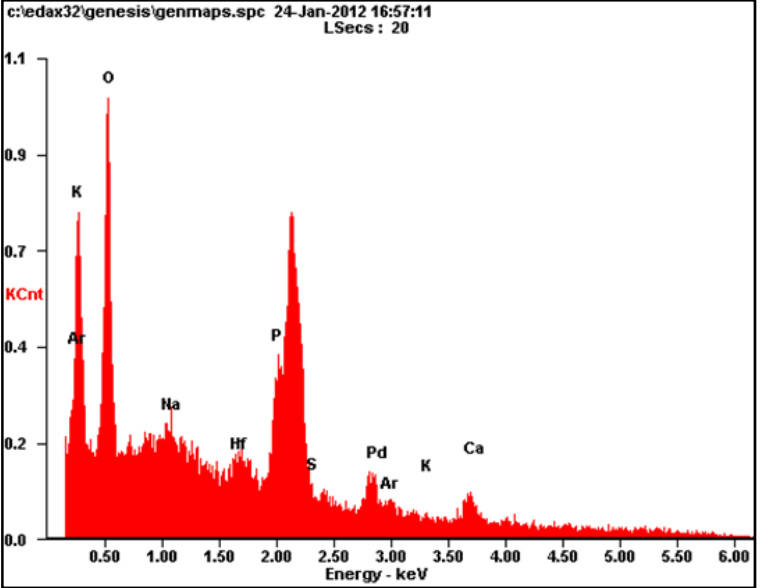

(d)

Figure 13. Chemical analysis results obtained from different surfaces of King Bream tooth; (a) enamel, (b) DEJ, (c) region right below DEJ, (d) dentin.

Figure 14 shows the nano-indentation test results on the King Bream tooth. The hardness and reduced elastic modulus ( $\mathrm{Er}$ ) values obtained from enamel layer decreased from outer surface to DEJ whereas those from dentin layer showed comparable values. Similar trends in the hardness profile have also been observed from micro-indentation tests in prior studies. The micro-hardness values of human tooth also decrease with the depth (Gutierrez-Salazar 2003; Roy 2008). 


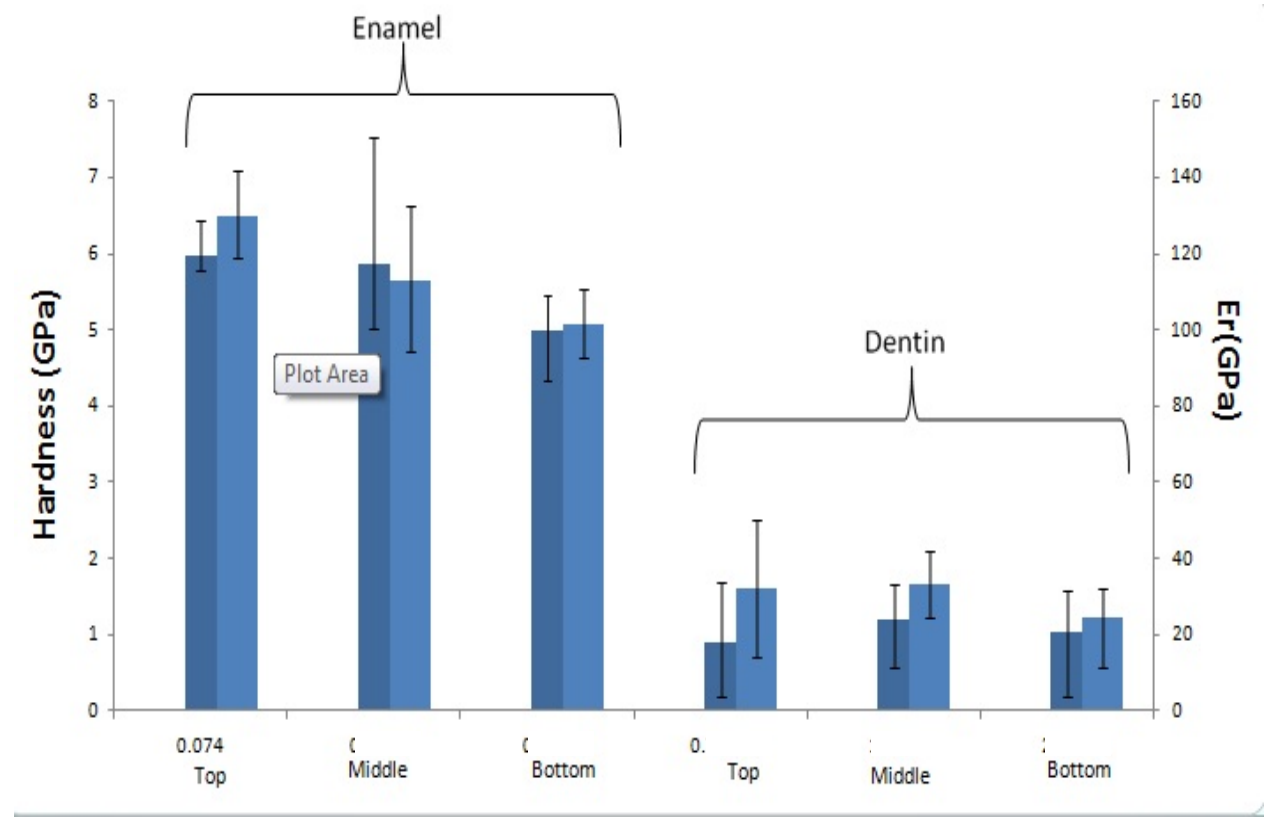

Figure 14. Nano-Indentation test results obtained from the bottom bream tooth.

\section{CONCLUSIONS}

A composite skin FE model was developed. Also, all four layers were evaluated to examine the differences in mechanical properties and ultimately correlate the structure and function of the skin tissue. The scalp layer, which consists of the top most 3 layers was compared with the weblike areolar tissue. As expected, scalp tissue was stiffer as it comprises of a more intact and less weblike structure. Also, the data shows that the loss and storage modulus for DMA are less for aponeurosis and connective tissue, which provides and explanation of why these tissues typically reach failure prior to all other layers.

A 3-D mesh of the liver was developed. Cryosection images from the NIH Visible Human Project were used to create a model of the liver (Figure 15). By utilizing color threshold and manual refining techniques within the ScanIP modeling program, a finite element mesh was created of the liver. Further finite element simulation in Abaqus on this new mesh of the liver will be carried as a part of future work. 


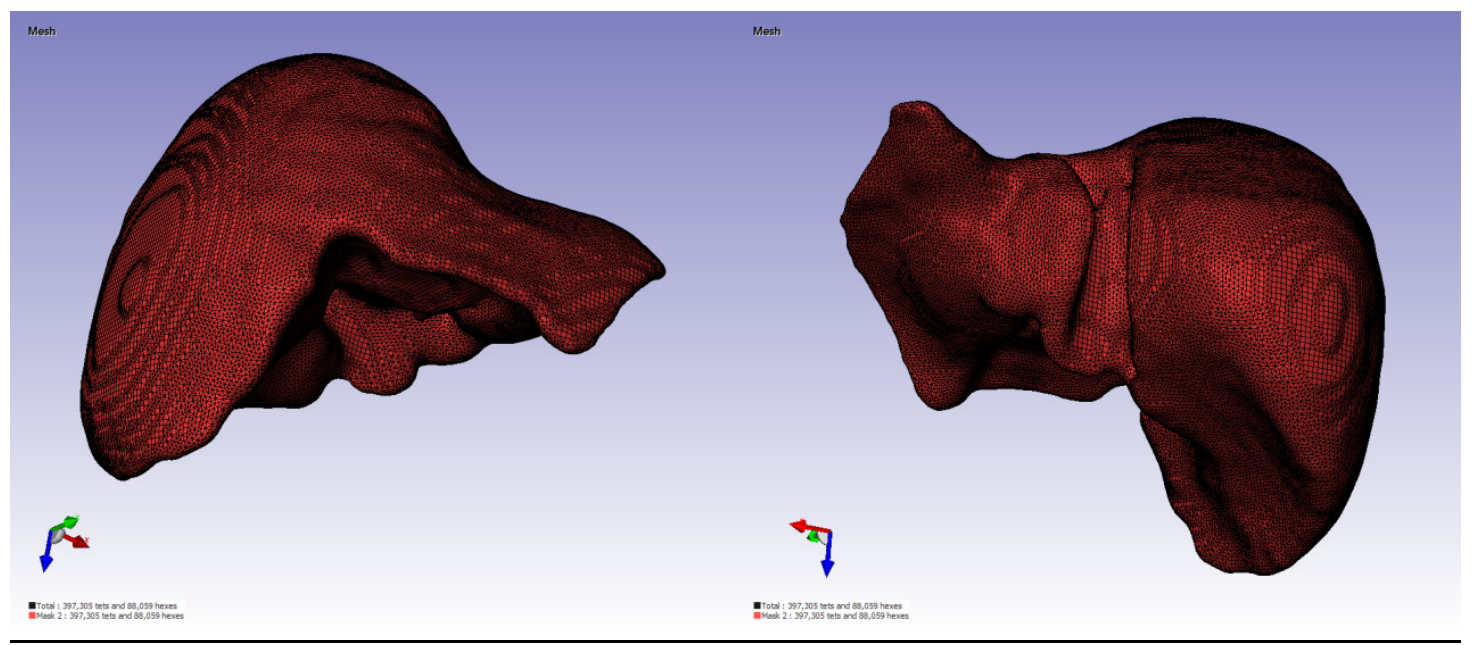

Figure 15. 3-D mesh of the liver

The finite element mesh of patellar ligament were developed from high resolution cryosection images obtained from the National Library of Medicine's Visible Human Project. The cryosection images were resampled to $1 \mathrm{~mm} /$ pixel at $1 \mathrm{~mm}$ intervals. Using an image processing software (ScanlP, Simpleware, Ltd), the patellar ligament and bones were segmented with a combination of semiautomated and manual segmentation operations (threshold, flood fill, region growing, etc.) After the segmentation, realistic, high quality volumetric finite element mesh for patellar ligament (Figure 16) was generated using an image based meshing software (ScanFE, Simpleware, Ltd). The meshes contained hybrid volume elements including hexahedral elements within each part and tetrahedral elements on the surface to provide smooth along the boundaries.

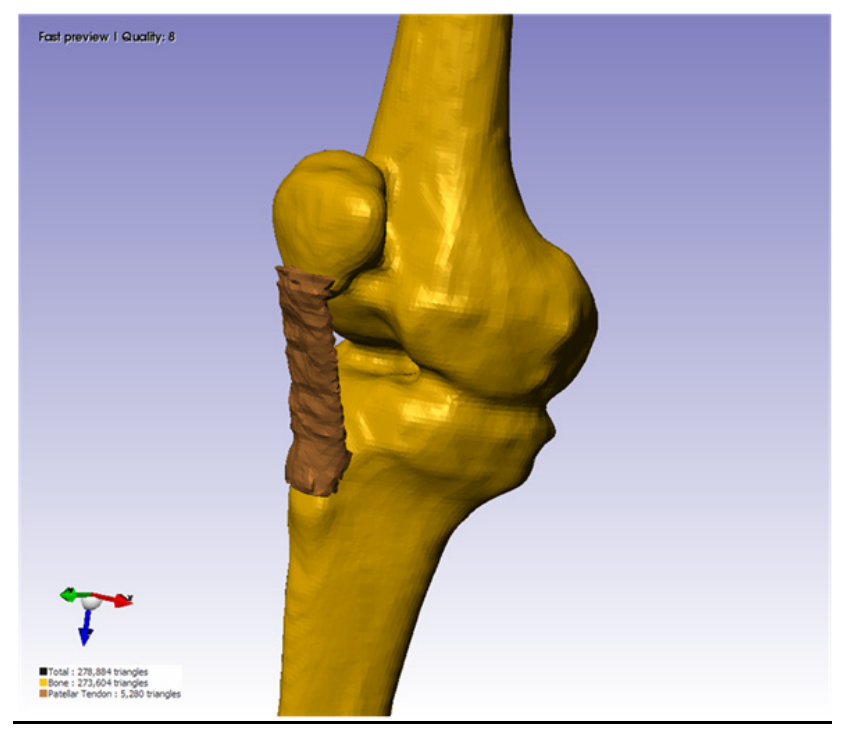

Figure 16. 3-D mesh of the tendon-bone complex 
Cryosection images from the NIH Visible Human Project were used to create a model of the heart (Fig 17). By utilizing color threshold and manual refining techniques within the ScanIP modeling program, a mesh was created of the heart and its chambers. The mesh was exported into Abaqus for finite element analysis testing on the effects of gravity versus microgravity on the heart. Upon obtaining results it was decided to refine the model by including a mesh of the aorta for more accurate data. Further testing in Abaqus on this new model of the

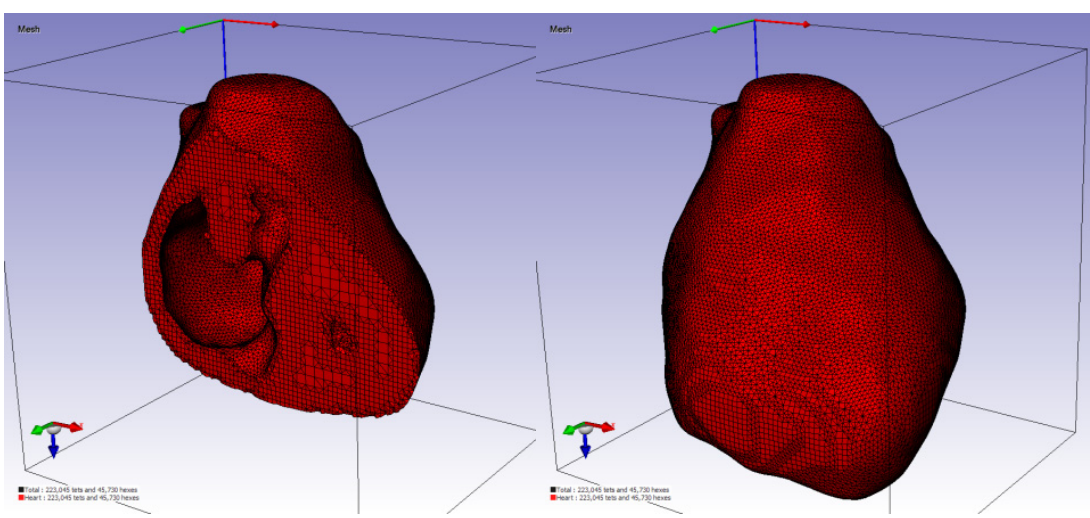
heart will be performed soon.

Figure 17. Mesh of (a) Crosssectional view of heart, (b) Heart with aorta

As for the lung tissue, there is a marked difference between the fresh and dried samples, indicating an important and vital role of water in the mechanical response of the tissue. Future studies will be aimed at quantifying mechanical properties of lungs tissues from various anatomical landmarks and provide us with a better understand of structure-property relationship \& ultimately provide input for our multi-scale computational mode

Future direction for the bream teeth includes continue to analyze chemical compositions on each layer of bream tooth, carry out additional nano-indentation tests on bream teeth, and begin working on mesh generation of bream tooth for future finite element simulations. The Woodpecker beak study is currently being finalized. Several manuscripts will be published based on both the model and structure-property relationships of the beak

\section{Publications/Presentations/Patents}

Wang, B., Tedder, M.E., Perez, C.E., Wang, G., Curry, A.L., To, F., Elder, S. H., Williams, L. N., Simionescu, D. T., Liao, J. " Structural and Biomechanical Characterizations of Porcine Myocardial Extracellular Matrix" Journal of Materials Science: Materials in Medicine, (In press 2012).

Liao, J., Joyce, E.M., Merryman, D.W., Jones, H.L., Tahai, M., Horstemeyer, M.F., Williams, L.N., Hopkins, R.A., Sacks, M.S. "The Intrinsic Fatigue Mechanism of the Porcine Aortic Valve Extracellular Matrix" Cardiovascular Engineering and Technology. (In press 2012).

Trim, M.W., Horstemeyer, M.F., Rhee, H., Liao, J., Williams, L.N. "The effects of water and microstructure on the mechanical properties of bighorn sheep (Ovis canadensis) horn keratin" Acta Biomaterialia, 2011, 7(3), pp1228-1240

Rhee, H., Horstemeyer, M.F., Ramsay, A. A study on the structure and mechanical behavior of the Dasypus novemcinctus shell, Materials Science and Engineering C. 2011, 31, pp.363-369. 
Prabhu, R., Horstemeyer, M. F., Tucker, M. T., Marin, E. B., Bouvard, J. L., Sherburn, J. A., Liao, J., et al. Coupled experiment/finite element analysis on the mechanical response of porcine brain under high strain rates. Journal of the Mechanical Behavior of Biomedical Materials. 2011, 4, pp 1067-1080.

Clemmer, J., Liao, J., Davis, D., Horstemeyer, M., Williams, L.N., "A Mechanistic Study for Strain Rate Sensitivity of Rabbit Patellar Tendon," Journal of Biomechanics. 2010, 43, pp 2785-2791.

Patnaik, S., Weed, B., Young, T., Liao, J., Williams, L. In Dynamic Viscoelastic Properties of Porcine Patellar Tendon: Study of Frequency, Loading, and Regional Dependency, Biomedical Engineering Society (BMES) Annual Conference, Hartford, CT, Hartford, CT, 2011.

Rougeau, M., Patnaik, S., Gilbrech, R., Young, T., Prabhu, R., Liao, J., Williams, L., Influence of Water Content on Quasi-static Compressive Properties of Porcine Lungs Tissue. In Biomedical Engineering Society (BMES) Annual Conference, Hartford, CT, 2011.

Weed, B., Borazjani, A., Patnaik, S., Prabhu, R., Horstemeyer, M., Franz, T., Williams, L., Liao, J Stress State Dependence of Human Placenta Mechanical Behavior, ASME Summer Bioengineering Conference, Farmington, PA, Farmington, PA, 2011.

Smith, W., Gilbrech, R., Liao J., Weed B., Patnaik, S., Summers RL., Influence of Microgravity on Left Ventricular Sphericity in a Finite Element Model of the Heart. In International Academy of Astronautics "Humans in Space" Symposium, Houston, TX, 2011.

Ryland, S., Prabhu, R., Patnaik, S., Horstemeyer, M., Liao, J., Williams, L. , Development of Finite Element Model for Porcine Scalp. In ASME Summer Bioengineering Conference, Farmington, Pa, 2011.

\section{REFERENCES}

Allen, E., "Understanding Ammonoid Sutures: New Insight into the Dynamic Evolution of Paleozoic Suture Morpholog," Cephalopods Present and Past: New Insights and Fresh Perspectives, pp. 159-180, 2007.

Bajaj, D., D.D. Arola, "On the R-curve behavior of human tooth enamel," Biomaterials 30 (2009) 40374046.

Byron, C. D., "Role of the osteoclast in cranial suture waveform patterning," The Anatomical Record Part A: Discoveries in Molecular, Cellular, and Evolutionary Biology, vol. 288A, pp. 552-563, 2006.

Champion, H. R., Holcomb, J. B., Young, L. A. (2009). "Injuries from explosions: physics, biophysics, pathology, and required research focus." The Journal of trauma66(5): 1468-1477; discussion 1477.

Choung, C. J. (1985). Chacterization and Modeling of Thoraco - Abdominal Response to Blast Waves. Biomechanical Model of Lung Injury Mechanisms, San Deigo, CA. 6. 
Cooper, G. J., Townend, D. J., Cater, S. R., Pearce, B. P. (1991). "The role of stress waves in thoracic visceral injury from blast loading: modification of stress transmission by foams and high-density materials." Journal of biomechanics24(5): 273-285.

D'Yachenko, A. I., Manyuhina, O. V. (2006). "Modeling of weak blast wave propagation in the lung." Journal of biomechanics39(11): 2113-2122.

Faffe, D. S., D'Alessandro, Elizabeth S., Xisto, Debora G., Antunes, Mariana A., Romero, Pablo V., Negri, Elnara M., Rodrigues, Nilza R. D., Capelozzi, Vera L., Zin, Walter A., Rocco, Patricia R. M. (2006). "Mouse strain dependence of lung tissue mechanics: Role of specific extracellular matrix composition." Respiratory physiology \& neurobiology152(2): 186-196.

Fust, A., Bates, J. H. T., Ludwig, M. S. (2004). "Mechanical properties of mouse distal lung: in vivo versus in vitro comparison." Respiratory physiology \& neurobiology143(1): 77-86.

Gao, J., Huang, W., Yen, R. T. (2006). "Mechanical properties of human lung parenchyma." Biomedical sciences instrumentation42: 172-180.

Gutierrez-Salazar, M. del P., J. Reyes-Gasga, "Microhardness and chemical composition of human tooth," Materials Research, 6 (2003) 367-373.

He, L. H., M.V. Swain, "Understanding the mechanical behavior of human enamel from its structural and compositional characteristics," Journal of the Mechanical Behavior of Biomedical Materials I (2008) 1829.

Hubbard, R. P., et al., "Flexure of cranial sutures," Journal of biomechanics, vol. 4, pp. 491-492, IN1-IN3, 493-496, 1971.

J. W. Melvin, A. S. W. (1974). Thoracic Model Improvements (Experimental Tissue Properties) Vol 2. , Highway Safety Research Institute, Univeristy of Michigan.

Jaslow, C. R., "Mechanical propertise of cranial sutures," J Biomechanics, vol. 23, pp. 313-321, 1990.

Krauss, S., et al., "Mechanical Function of a Complex Three Dimensional Suture Joining the Bony Elements in the Shell of the Red Eared Slider Turtle," Advanced Materials, vol. 21, pp. 407-412, 2009.

Lai-Fook, S. J. (1977). "Lung parenchyma described as a prestressed compressible material." Journal of biomechanics 10(5-6): 357-365.

Lambert, R. K., Wilson, T. A. (1973). "A model for the elastic properties of the lung and their effect of expiratory flow." Journal of Applied Physiology34(1): 34-48.

Lee, G. C., Frankus, A., Chen, P. D. (1976). "Small distortion properties of lung parenchyma as a compressible continuum." Journal of biomechanics9(10): 641-648.

Leite-Junior, J. H., Rocco, P. R., Faffe, D. S., Romero, P. V., Zin, W. A. (2003). "On the preparation of lung strip for tissue mechanics measurement." Respiratory physiology \& neurobiology134(3): 255-262. 
Ligas, J. R. (1984). "A non-linearly elastic, finite deformation analysis applicable to the static mechanics of excised lungs." Journal of biomechanics17(8): 549-552.

Li, Y., et al., "Bioinspired, mechanical, deterministic fractal model for hierarchical suture joints," PHYSICAL REVIEW E Phys Rev E, vol. 85, p. 031901, 2012.

Li, Y., et al., "Stiffness and strength of suture joints in nature," 2011.

Ligas, J. R., Saidel, G. M., Primiano, F. P., Jr. (1985). "Parameter estimation and sensitivity analysis of a nonlinearly elastic static lung model." Journal of biomechanical engineering107(4): 315-320.

Malinowska, J., "Mechanical properties of human tooth approximated with overdamped oscillators," Acta of Bioengineering and Biomechanics, 7 (2005) 59-67.

Mayorga, M. A. (1997). "The pathology of primary blast overpressure injury." Toxicology121(1): 17-28.

Mead, J. (1961). "Mechanical Properties of Lungs." Physiological Reviews41(2): 281-330.

Melvin J. W., McElhaney, J. H., Roberts V.L., (1970), Development of a mechanical model of the human head--determination of tissue properties and synthetic substitute materials., 14th Stapp Car Crash Conference, SAE Paper No. 700903. Society of Automotive Engineers.

Melvin J.W., Evans F.G., (1971), A strain energy approach to the mechanics of skull fracture., 25th Stapp Car Crash Conference, SAE Paper No. 710871. Society of Automotive Engineers, Warrendale, PA.

Opperman, L. A., "Cranial sutures as intramembranous bone growth sites," Developmental dynamics, vol. 219, pp. 472-485, 2000.

Phillips III, Y. Y., Richmond, D. R. (1991). Primary Blast Injury and Basic Research : Brief History, Department of Pulmonary and Critical Care Medicine Services, Walter Reed Army Medical Center, Washington, D.C: 221-241.

Raul J.S., Baumgartner D., Willinger R., Ludes B., (2006), Finite element modelling of human head injuries caused by a fall, Int J Legal Med (120(4):212-218).

Rodarte, J. R. (1982). "Stress-strain analysis and the lung." Federation proceedings41(1): 130-135.

Roy, S., B. Basu, "Mechanical and tribological characterization of human tooth," Materials Characterization 59 (2008) 747-756.

Sasagawa, I., M. Ishiyama, H. Yokosuka, M. Mikami, T. Uchida, "Tooth enamel and enameloid in actinopterygian fish," Front. Mater. Sci. China, 3 (2009) 174-182.

Shields, T. W., LoCicero, J., Ponn, R. B., (2000). General Thoracic Surgery. Philadelphia, Lippincott Williams \& Wilkins. 
Song, J., C. Ortiz, M.C. Boyce, "Threat-protection mechanics of an armored fish," Journal of the Mechanical Behavior of Biomedical Materials, 4 (2011) 699-712.

Stamenovic, D., Smith, J. C. (1986). "Surface forces in lungs. III. Alveolar surface tension and elastic properties of lung parenchyma." Journal of Applied Physiology60(4): 1358-1362.

Stuhmiller, J. H. (1997). "Biological response to blast overpressure: a summary of modeling." Toxicology121(1): 91-103.

Stuhmiller, J. H., Chuong, C. J., Phillips, Y. Y., Dodd, K. T. (1988). "Computer modeling of thoracic response to blast." The Journal of trauma28(1 Suppl): S132-139.

Stuhmiller, J. H., Ho, K. H., Vander Vorst, M. J., Dodd, K. T., Fitzpatrick, T., Mayorga, M. (1996). "A model of blast overpressure injury to the lung." Journal of biomechanics29(2): 227-234.

Suki, B., Bates, Jason H. T. (2011). "Lung tissue mechanics as an emergent phenomenon." Journal of Applied Physiology110(4): 1111-1118.

Sun, Z., et al., "Cranial sutures and bones: growth and fusion in relation to masticatory strain," The Anatomical Record Part A: Discoveries in Molecular, Cellular, and Evolutionary Biology, vol. 276, pp. 150161, 2004.

Vawter, D. L. (1980). "A finite element model for macroscopic deformation of the lung." Journal of biomechanical engineering102(1): 1-7.

Vawter, D. L., Fung, Y. C., West, J. B. (1978). "Elasticity of excised dog lung parenchyma." Journal of Applied Physiology45(2): 261-269.

Whitenack, L. B., D.C. Simkins Jr., P.J. Motta, M. Hirai, A. Kumar, "Young's modulus and hardness of shark tooth biomaterials," Archives of Oral Biology, 55 (2010) 203-209.

Wolf, S. J., Bebarta, Vikhyat S., Bonnett, Carl J., Pons, Peter T., Cantrill, Stephen V., (2009). "Blast injuries." The Lancet374(9687): 405-415.

Xu, H. H. K., D.T. Smith, S. Jahanmir, E. Romberg, J.R. Kelly, V.P. Thompson, E.D. Rekow, "Indentation damage and mechanical properties of human enamel and dentin," J. Dent. Res. 77 (1998) 472-480.

Yu, J. C., et al., "Brief synopsis of cranial sutures: Optimization by adaptation," Seminars in Pediatric Neurology, vol. 11, pp. 249-255, 2004.

Zervaki, A., G.N. Haidemenopoulos, A. Giannakopoulos, "On the microhardness and Young's modulus of human teeth," Proc. Int. Conference on Manufacturing Engineering (ICMEN), Thessaloniki, Greece, 1-3 October, 2008. 


\section{Phase 1 Final Report}

Principle Investigator: Mark F. Horstemeyer

Chair Professor, Computational Manufacturing \& Design

Center for Advanced Vehicular Systems (CAVS)

Mississippi State University (MSST)

Box 5405, Mississippi State, MS 39762-5405

(662) 325-5449; fax: (662) 325-5433; e-mail: mfhorst@cavs.msstate.edu

Co-Principal Investigator: Paul Wang

Deputy Director for Program Development

Center for Advanced Vehicular Systems (CAVS)

Mississippi State University (MSST)

Box 5405, Mississippi State, MS 39762-5405

(662) 325-2890; fax: (662) 325-5433; e-mail: pwang@cavs.msstate.edu

\section{ICME DEMONSTRATION}

PROJECT TO SUPPORT THE

DEVELOPMENT OF A

MAGNESIUM FRONT END

STRUCTURE

Prepared as Second Part of the SRCLID Final Report and Submitted by Mississippi State University (MSST)

December 5, 2012 


\section{TABle of Contents}

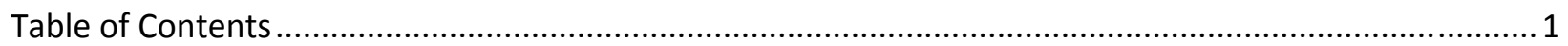

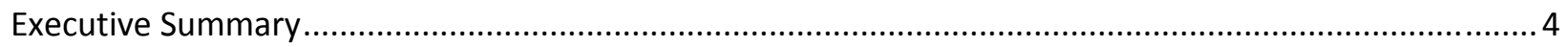

A. Microstructure-Sensitive Fatigue Modeling for Magnesium Materials and Components ....................... 7

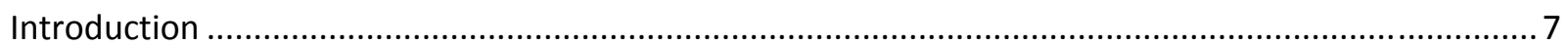

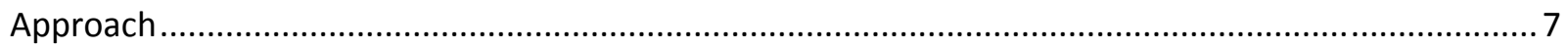

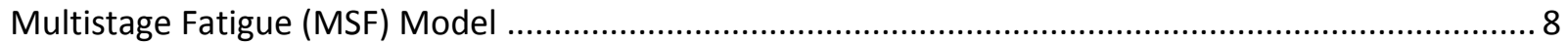

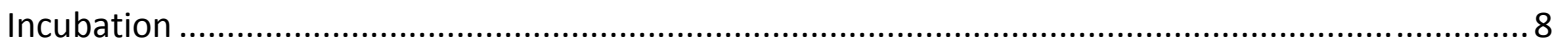

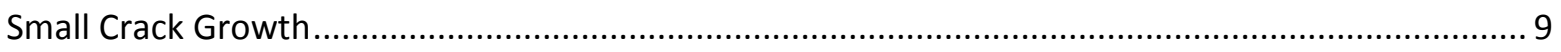

1. Cyclic Behavior of an AZ31 Sheet Magnesium Alloy ................................................................... 9

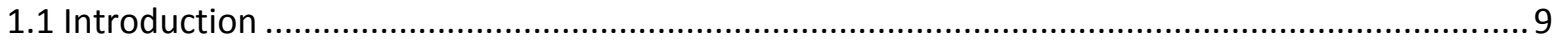

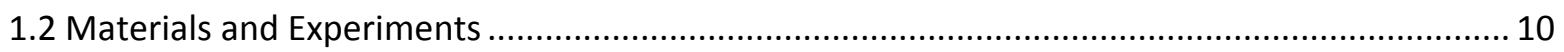

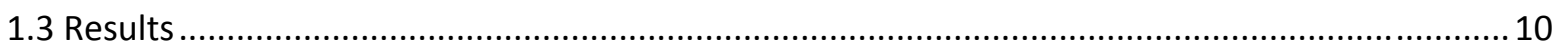

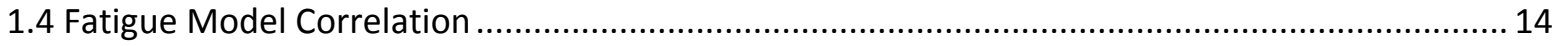

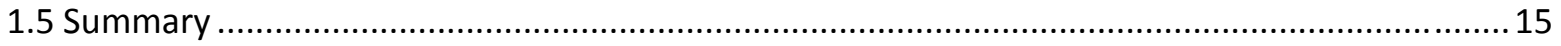

2. Fatigue Damage and Microstructure Properties in an AM30 Extruded Magnesium Alloy................ 16

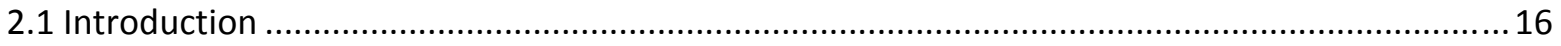

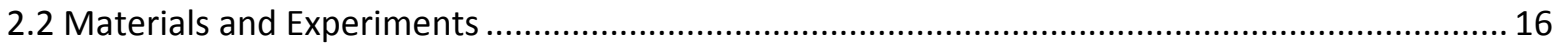

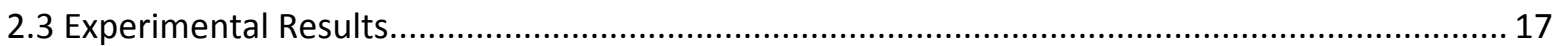

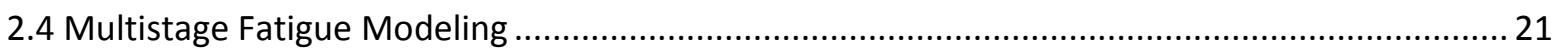

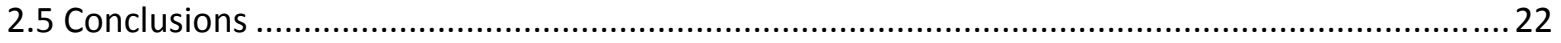

3. Multistage Fatigue Modeling of AZ91 and AM60 Magnesium Alloys................................................2 23

3.1 Multistage Model Formulation for Magnesium Cast Alloys ..................................................... 23

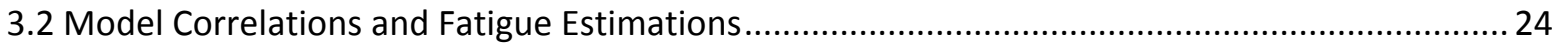

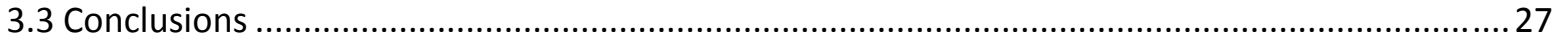

4. Fatigue of Friction Stir Spot Welding in Magnesium Alloys .......................................................... 28

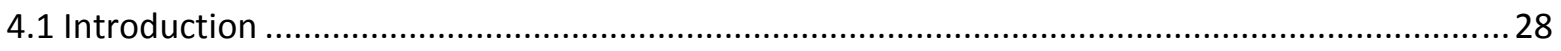

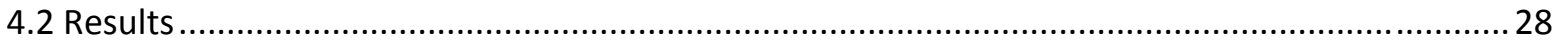

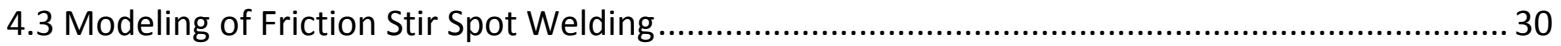

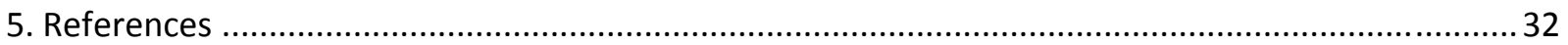

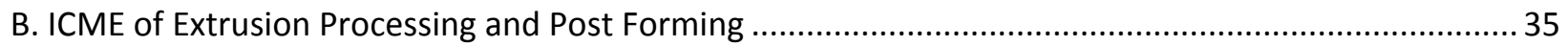




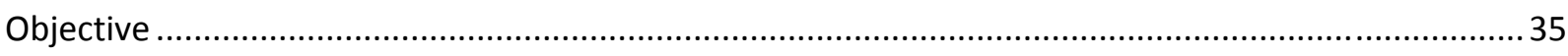

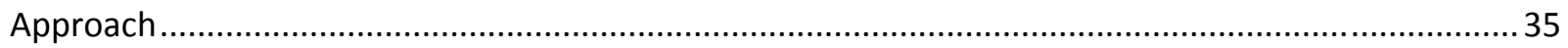

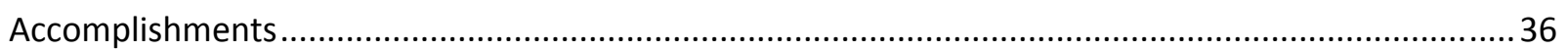

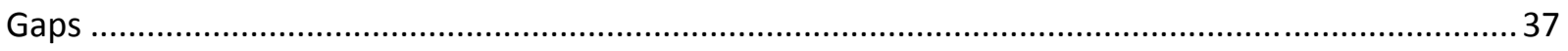

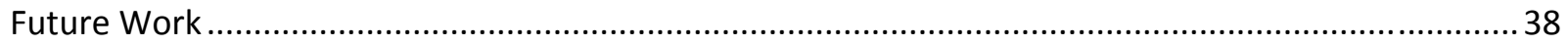

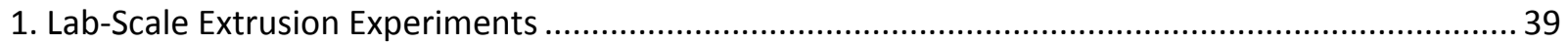

1.1 A Lab-Scale Fixture for Indirect Extrusion Experiments .......................................................... 39

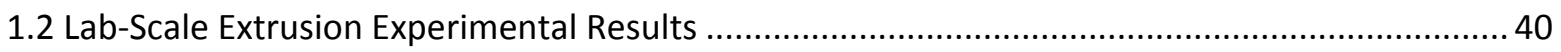

2. Constitutive Modeling - Internal State Variable and Crystal Plasticity Modeling Approaches .......... 42

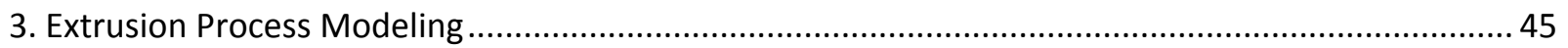

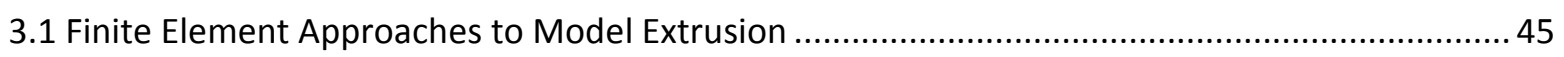

3.2 Prediction of the Lab-Scale Thermo-Mechanical Extrusion Processes ........................................ 47

3.3 Prediction of Microstructure Evolution (Texture) During the Extrusion Process .........................50

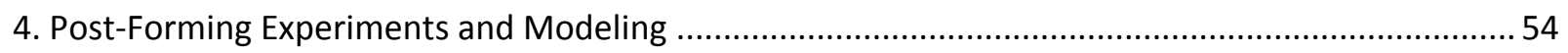

5. Prediction of Mechanical Properties and Texture in Industry-Level Extrusion Processes..................56

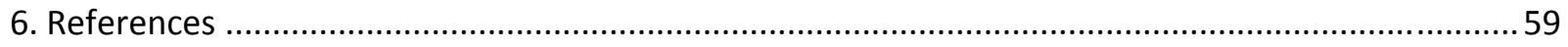

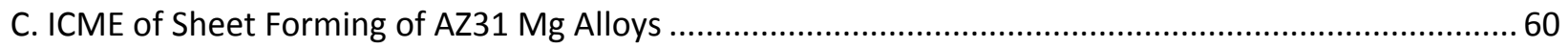

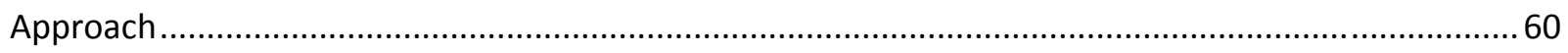

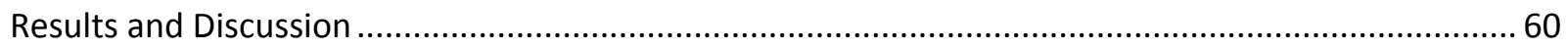

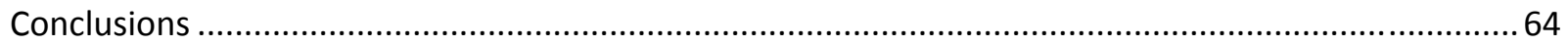

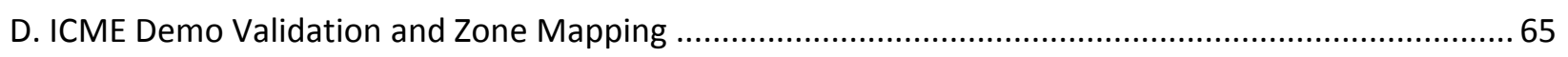

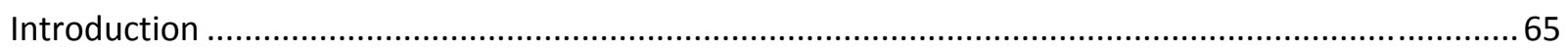

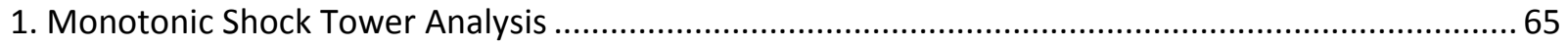

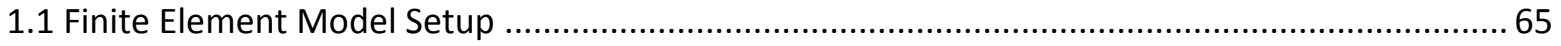

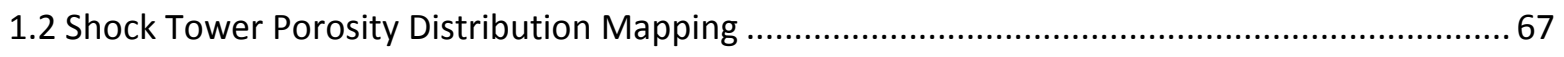

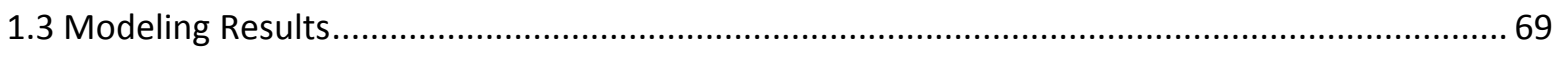

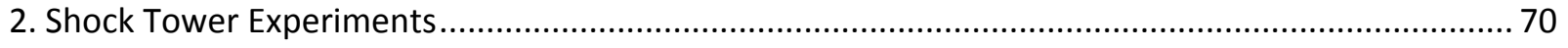

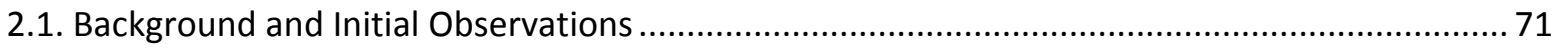

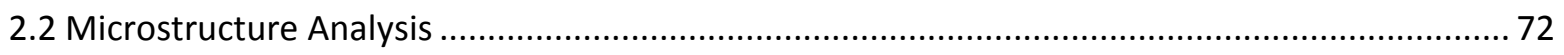

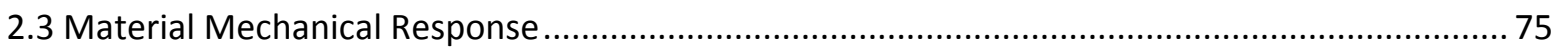

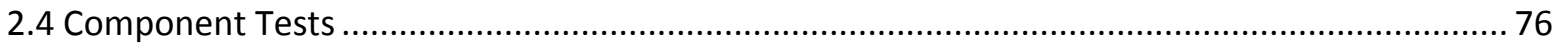

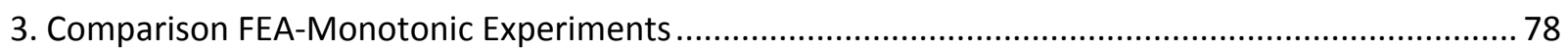




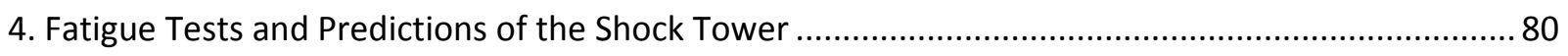

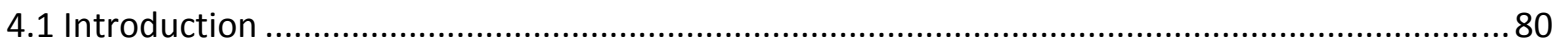

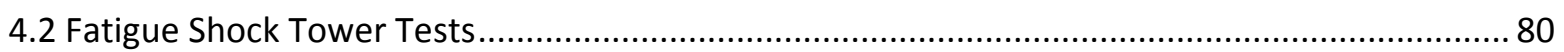

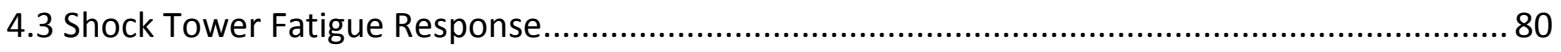

4.4 Finite Element Analysis and Fatigue Predictions ...................................................................... 81

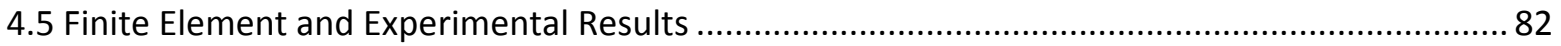

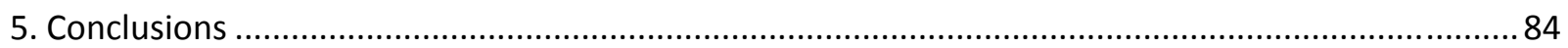

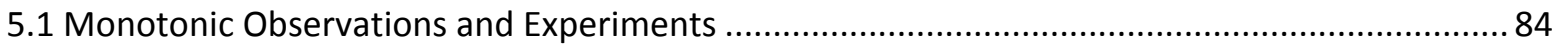

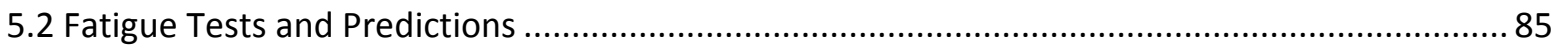

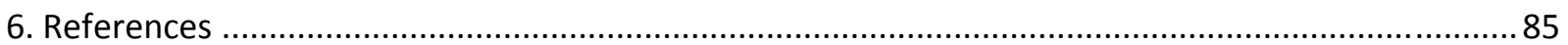

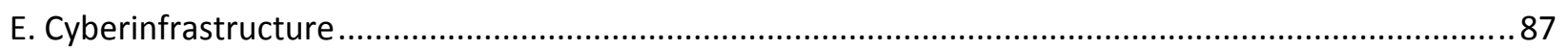

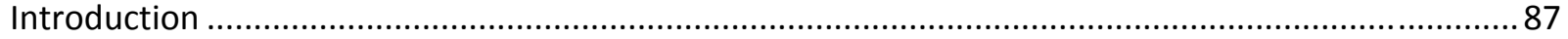

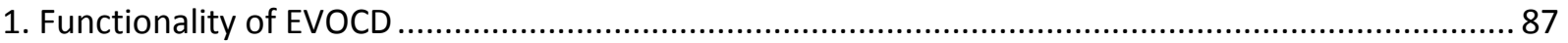

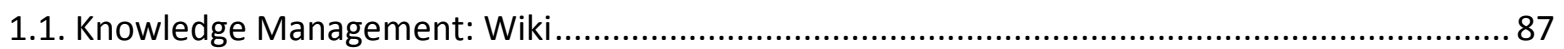

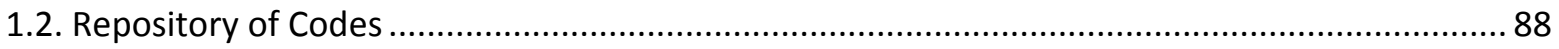

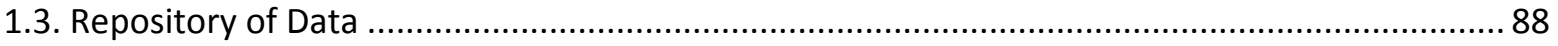

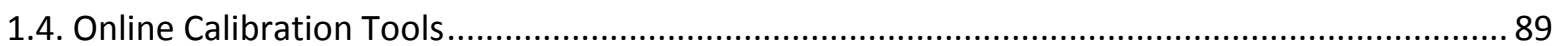

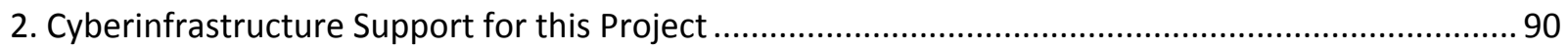

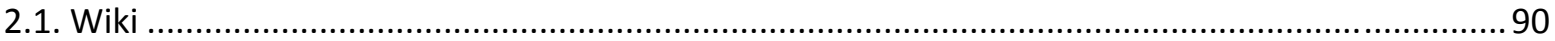

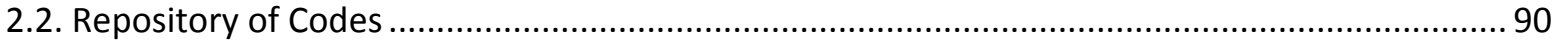

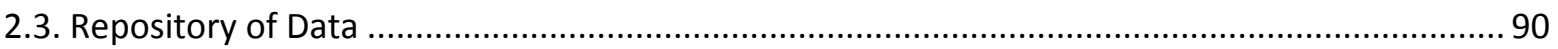




\title{
ICME Demonstration Project to Support the Development of a Magnesium Front End Structure - Second Part of the SRCLID Final Report by MSST
}

\author{
Principle Investigator: Mark F. Horstemeyer \\ Chair Professor, Computational Manufacturing \& Design \\ Center for Advanced Vehicular Systems (CAVS) \\ Mississippi State University (MSST) \\ Box 5405, Mississippi State, MS 39762-5405 \\ (662) 325-5449; fax: (662) 325-5433; e-mail: mfhorst@,cavs.msstate.edu \\ Co-Principal Investigator: Paul Wang \\ Deputy Director for Program Development \\ CAVS MSST \\ Box 5405, Mississippi State, MS 39762-5405 \\ (662) 325-2890; fax: (662) 325-5433; e-mail: pwang@,cavs.msstate.edu
}

Contractor: $\quad$ Mississippi State University (MSST)

\section{EXECUTIVE SUMmARY}

The goal of this MsSt team to support the USAMP ICME demo project, Calibration and Validation Methodologies Linking the Models and Experiments, was to apply key enabling technologies being developed for advanced lightweight magnesium processes and alloys to validate and predict mechanical responses of a front end demo structure, including process-structure-property relationships. The key enabling technologies being developed by the MsSt team are the following: internal state material plasticity damage model, fatigue model, extrusion process model, sheet forming model, and the integration of the above to validate the performance of demo parts. The front end demo structure comprises top rails made of AZ31 sheet materials, a shock tower of AM 60 and AM90, and a lower rail of AM30 extrusion; those parts were supplied by USAMP partners.

As part of the SRCLID final report, we demonstrated in this demo final report the development of ICME techniques including key databases via cyber-infrastructure and the associated materials and process models, by which a zone-mapping method was utilized to show the capability of experimentally validated simulation models at the component level for high-integrity magnesium castings, extrusions, and sheet products. The zone mapping method is to characterize material features at a local level within a given component such that the heterogeneities of the material within the components can be well presented for modeling purposes.

The MsSt team divided its effort into five parts: microstructure-sensitive fatigue modeling for materials and components, extrusion processing and post forming, sheet forming of AZ31 alloys, ICME demo validation via zone mapping, and cyberinfrastructure. 
In fatigue modeling, we established the Multi Stage Fatigue (MSF) model for Mg alloys (i.e., AM60, AZ91) of three product forms, as-cast, plate, and sheet products. The model was correlated to the experimental strain-life results of the AM60 as-cast, AZ91 as-cast, and the AZ91 T6 magnesium alloys, by using parameters representing microstructural features, such as dendrite cell size, pore size, and cyclic hardening parameters, in order to capture the differences in fatigue behavior of three data sets. The fatigue model showed good correlation to the upper and lower bounds of the strain-life behavior of the AM60 and AZ91 magnesium alloys by employing the maximum and minimum inclusion and dendrite cell sizes. Thus, the inclusion (pore) size had the most influence on the scatter in the fatigue lifetimes. We note that the other material constants were not changed for the estimation of the upper and lower bounds, thus representing the material well, regardless of the material processing method.

For the extrusion effort, we demonstrated the ability of performing lab-scale extrusion to construct a database for the extrusion of various $\mathrm{Mg}$ alloys. The recorded data captured the effect of processing parameters (ram speed and billet temperature) on processing variables (temperature histories and loaddisplacement curves) and the evolution of material microstructure (texture, grain morphology). We then built robust finite element (FE) models and performed numerical simulations of the lab-scale and industry-scale extrusion processes using HyperXtrude, a commercial Eulerian finite element code widely used to model the extrusion of complex, industrial profiles. We integrated the VPSC (visco-plasticity selfconsistent) crystal plasticity code to predict texture evolution based on streamline information generated by HyperXtrude from an extrusion simulation. The predicted texture patterns agreed well with the trends observed in the experiments. In the post forming stage after extrusion, the same VPSC code using both the computed extrusion texture and the room-temperature material properties captured reasonably well the initial yield strength of the extrudate product.

In sheet forming, we established a methodology to quantify the evolution of the yield surface from a mesoscale crystal plasticity code and the bridging to a macroscale internal state variable model. FE simulations of sheet forming as compared to sheet bending experiments conducted at the lab level are still in progress. The potential for predicting texture evolution during sheet forming was demonstrated, similar to the extrusion effort above. Three-point bending was performed at different strain rates at room temperature on an annealed AZ31 Mg sheet material. The results revealed a surprising strain rate sensitivity. The bending angle increases as the strain rate increases, sharply different from the asreceived sheet in which the bending angle decreases as the strain rate increases. Preliminary results of curvature and load-displacement showed good agreement between experiments and modeling. In-situ EBSD was performed on AZ31 sheet samples during three-point bending. Unusual, highly localized twin bands were observed in the compression zone. In between the twin bands, no twins were observed. More experiments are needed to understand the mechanism for such localized twin bands.

Our ICME demo validation team was focused on using a well-developed internal state variable plasticity-damage model and a microstructure-sensitive multiscale fatigue model to predict the monotonic loading and the fatigue behaviors of the AZ91 shock tower. The shock towers provided by USAMP, were examined and tested to provide insight into the mechanical performance and failure mechanisms and validation for the models. When investigating the microstructure of the shock towers, we characterized pores in several locations in order to develop the zone-mapping technique for the 
shock tower. We worked with the University of Michigan team and Dr. Mei Li from Ford to understand the sample statistics of variation and the porosity correlation with solidification cooling rates, accordingly. The finite element simulations coupled with the MSF model correlate well with the fatigue experimental tests of shock tower. This investigation has shown that fatigue failure of a component must be studied based on an integrated, holistic approach that involves jointly the material properties, geometry, and loading conditions. In general, we observed that the microstructure of the shock tower plays a key role in the material's monotonic loading and fatigue responses. The material mechanical response, along with a large geometry variation could dictate the poor performance of the shock tower component. To increase the shock tower performance, the thickness of cast part must be kept uniform and the material microstructure defects must be substantially reduced.

The scientific understanding in processing-structure-property relationships has been captured in the MSST ICME Cyberinfrastructure (http://icme.hpc.msstate.edu). This Cyberinfrastructure is being developed with an ambitious objective of providing a platform for the integration of models from different domains to enable development of comprehensive and robust processing-structure-property relationships, which can also be integrated with manufacturing simulation and design. The Cyberinfrastructure can also provide a platform for the optimization of alloys, manufacturing processes, and components. The program will be conducted as a research collaboration between researchers and organizations in Canada, China, and the United States and will serve as an international pilot project for the new Integrated Computational Materials Engineering movement (ICME) within the materials profession. 


\section{A. Microstructure-Sensitive fatigue Modeling for Magnesium Materials AND COMPONENTS}

Team Members: Mark Horstemeyer, Marcos Lugo, Brian Jordon

\section{INTRODUCTION}

Industry and government have recently had an elevated interest in lightweight materials as part of the solution to reduce greenhouse emissions and improve fuel economy in transportation vehicles. This heightened interest in lightweight materials has pushed the materials community to investigate not only cast magnesium but also wrought magnesium products for their high strength-to-weight ratio and better fatigue resistance. While studies of cyclic performance of magnesium alloys exist in the literature, most of these are associated with cast formed materials. However, wrought magnesium alloys generally have higher mechanical strength and fatigue resistance than cast magnesium alloys due to their ability to limit inclusion sizes and for precipitation hardening after the severe deformation during processing. Thus, a need arises for a predictive tool that can capture the fatigue damage in wrought magnesium alloys with the intent of determining/designing the best alloy for use in a range of automotive applications. Therefore, the overarching goal of this task is the development of a physically-motivated microstructurally-based fatigue model.

In order to reach the goal of using more lightweight metals in automobiles, understanding and modeling the fatigue damage of welded joints is important as well. As with the base materials, research is needed to determine the reliability and performance of potential welding techniques and develop microstructurally-based modeling approaches. While research in the area of fatigue in friction stir spot welds is not unique, recent work has been exclusively focused FCC alloys and not HCP alloys such as magnesium.

Finally, the investigation of fatigue behavior of nanoscale materials has been of significant interest to many researchers due to the development of technologies of nano-materials. As such, understanding how fatigue damage initiates and grows at the nanoscale for magnesium alloys will lead to better modeling approaches at the higher length scales.

\section{APPROACH}

Strain-life fatigue tests of selected magnesium alloys (AZ31, AM30, AZ91, and AM60) were conducted to investigate the fatigue behavior of magnesium ( $\mathrm{Mg}$ ) alloys used as automotive components. Structure-property analyses were conducted to determine relations between microstructural features and fatigue life. In addition, scanning electron microscope (SEM) experiments were conducted to determine fatigue crack incubation and microstructurally/physically small fatigue crack stages. Experiments were also performed to determine the fatigue performance of several different types of joints.

Fatigue performance of $\mathrm{Mg}$ alloys was characterized, and a structure-property modeling framework for fatigue life using the multistage fatigue (MSF) modeling approach was developed. We used a microstructure-sensitive fatigue model that decomposes total fatigue lifetimes into crack incubation, as 
well as microstructurally small crack (MSC), physically small crack (PSC), and long crack growth, to correlate the differences in fatigue behavior of $\mathrm{Mg}$ alloys. The utilized model is capable of capturing competing structure-property relations, including grain size, inclusion size, and texture, and their consequential impact on fatigue lifetimes. While McDowell et al. [1] originally developed the model for a cast A356 aluminum (Al) alloy, it has been modified to extend its application to wrought materials [2,3]. The total fatigue life $N_{\text {Total }}$ is given by Equation (1).

$$
N_{\text {Total }}=N_{\text {Inc }}+N_{M S C / P S C}+N_{L C}
$$

where $N_{\text {Total }}$ is the total fatigue life. $N_{\text {Inc }}$ is the number of cycles to incubate a crack at a micronotch formed by an inclusion, which can be a relatively large constituent particle, a large pore, or a cluster of each or both. The incubated crack extends from the inclusion into the matrix and propagates through a region of the micronotch root influence. $N_{\text {MSC/PSC }}$ is the number of cycles required for propagation of a microstructurally small/physically small crack. Finally, $N_{\mathrm{LC}}$ is the number of cycles required for long crack (LC) propagation to final failure, which depends on the amplitude of loading and the corresponding extent of microplasticity ahead of the crack tip. This multistage framework was evaluated for the prediction of fatigue damage in Mg alloys and welded joints. The MSF will also be implemented into commercial finite element analysis (FEA) codes and validated by solving specific problems concerning the mechanical response and reliability/safety aspects of $\mathrm{Mg}$ alloys used in automotive applications.

\section{Multistage fatigue (MSF) Model}

The MSF model used in this research comprises three distinct regimes: crack incubation, microstructurally small crack (MSC) joint with physically small crack (PSC) growth, and long crack (LC) growth, as shown in Eq. (1).

$$
N_{\text {Total }}=N_{I n c}+N_{M S C}+N_{P S C}+N_{L C}=N_{I n c}+N_{M S C / P S C}+N_{L C} .
$$

\section{Incubation}

In general, the fatigue damage incubation life $N_{\text {inc }}$ is evaluated using a damage parameter $\beta$ in a modified Coffin-Manson law at the microscale:

$$
C_{\text {inc }} N_{\text {inc }}^{\alpha}=\beta,
$$

where $\beta$ is the nonlocal damage parameter around an inclusion, and $C_{\mathrm{inc}}$ and $\alpha$ are the linear and exponential coefficients in the modified Coffin-Manson law for incubation. The damage parameter relates to the local average maximum plastic shear strain amplitude and can be estimated by the following:

$$
\begin{gathered}
\beta=\frac{\Delta \gamma_{\max }{ }^{* *}}{2}=Y\left[\varepsilon_{a}-\varepsilon_{t h}\right]^{q}, \quad \frac{l}{D}<\eta_{\text {lim }}, \\
\beta=\frac{\Delta \gamma_{\max }{ }^{*}}{2}=Y(1+\zeta z)\left[\varepsilon_{a}-\varepsilon_{t h}\right]^{q}, \quad \frac{l}{D}>\eta_{l i m} .
\end{gathered}
$$

Here, $\varepsilon_{a}$ is the remote applied strain amplitude, and $\varepsilon_{a}$ and $\varepsilon_{p e r}$ were introduced by McDowell et al. [1] and Xue et al. [2] to represent the strain threshold for damage incubation and the percolation limits for 
microplasticity, respectively. $D$ is the size of the pertinent inclusion at which a fatigue crack incubates, and $l$ is the nominal linear dimension of the plastic zone size in front of the inclusion. The ratio $\frac{l}{D}$ is defined as the square root of the ratio of the plastic zone area over the inclusion area. The limiting ratio $\eta_{\text {lim }}$ indicates the transition from proportional (constrained) micronotch root plasticity to nonlinear (unconstrained) micronotch root plasticity with respect to the applied strain amplitude, where $\eta_{\text {lim }}=0.3$ is suitable for cast Al alloys [1]. The parameter $Y$ is correlated as $Y=y_{1}+(1+R) y_{2}$, where $R$ is the load ratio, and $y_{1}$ and $y_{2}$ are model constants. For completely reversed loading cases, $Y=y_{1}$. Furthermore, when $\frac{l}{D}$ reaches its limits, the parameter $Y$ is revised to include the geometric effects $\bar{Y}=(1+z) Y$. The debonded particle and pore of the same size may cause a different $Y$ as a factor of three or greater [4]. The correlation of the plastic zone size is calculated using the nonlocal plastic shear strain with respect to the remote loading strain amplitude:

$$
\begin{gathered}
\frac{l}{D}=\eta_{l i m} \frac{\left\langle\varepsilon_{a}-\varepsilon_{t h}\right\rangle}{\varepsilon_{p e r}-\varepsilon_{t h}}, \frac{l}{D} \leq \eta_{\text {lim }}, \\
\frac{l}{D}=1-\left(1-\eta_{\text {lim }}\right)\left(\frac{\varepsilon_{p e r}}{\varepsilon_{a}}\right)^{r}, \frac{l}{D}>\eta_{l i m},
\end{gathered}
$$

where $r$ is a shape constant for the transition to the limited plasticity [5].

\section{Small Crack Growth}

The MSC growth is governed by the range of crack tip displacement $\triangle C T D$, which is proportional to the crack length and the $\mathrm{n}^{\text {th }}$ power of the applied stress amplitude $\sigma_{a}^{n}$ in the HCF regime, and to the macroscopic plastic shear strain range in the LCF regime; i.e.,

$$
\begin{gathered}
\left(\frac{d a}{d N}\right)_{M S C}=\chi\left(\Delta C T D-\Delta C T D_{t h}\right), \quad a_{i}=0.625 D \\
\Delta C T D=C_{I I}\left(\frac{G S}{G S_{0}}\right)^{\omega}\left(\frac{G O}{G O_{0}}\right)^{\xi}\left[\frac{U \Delta \widehat{\sigma}}{S_{u t}}\right]^{\zeta} a_{i}+C_{I}\left(\frac{G S}{G S_{0}}\right)^{\omega^{\prime}}\left(\frac{G O}{G O_{0}}\right)^{\xi^{\prime}}\left(\frac{\Delta \gamma_{\max }^{P}}{2}\right)^{2} .
\end{gathered}
$$

Here, $\chi$ is a constant for a given microstructure, typically less than unity and usually taken as 0.32 for Al alloys [2]. $\mathrm{C}_{\mathrm{I}}$ and $\mathrm{C}_{\mathrm{II}}$ are material dependent parameters which capture the microstructural effects on MSC growth. We assigned the threshold value for crack tip displacement on the order of the Burger's vector for the Mg rich matrix [6], $\Delta C T D_{t h}=3.2 * 10^{-0} \mu m$.

A long crack growth stage of the MSF model has historically followed classical LEFM approaches [2, 7]. However, the modeling approach presented here is focused on incubation and MSC regimes. This approach is consistent with the observation that the formulation for MSC can characterize fatigue cracks up to several millimeters in length.

\section{Cyclic Behavior of an AZ31 Sheet Magnesium Alloy}

\subsection{Introduction}

While studies of cyclic performance of Mg alloys exist in the literature, most of these are associated with cast-formed materials [8-13]. In fact, wrought Mg alloys generally have higher mechanical strength 
and fatigue resistance than cast Mg alloys due to their ability to limit inclusion sizes and for precipitation hardening after the severe deformation during processing. However, the processing of wrought $\mathrm{Mg}$ alloys produces sharper textures compared to cast alloys, which leads to a more anisotropic mechanical response. This is due to the low symmetry of the hexagonal close-packed (HCP) planes, which cannot accommodate deformation along the c-axis. As such, wrought $\mathrm{Mg}$ alloys are subject to deformation due to twinning and consequently often show an asymmetry and anisotropy cyclic response. This apparent sensitivity to the crystallographic orientation appears to have some influence on final failure [14-15].

Currently, fatigue design approaches include stress-life, strain-life, or fatigue crack growth methods. These methods do not consider the effect of the microstructure of the material nor the processing method. Furthermore, the current body of literature on fatigue of wrought magnesium alloys suggests that the mechanisms of fatigue damage evolution are consistent with the well-established stages of fatigue for more traditionally studied alloys: i.e., steel and aluminum alloys [16]. These stages include incubation, microstructurally small crack growth; physically small crack growth; and long crack growth.

In this section, the influence of the microstructural features-due to the effect of in-plane constraint from rolling-on the fatigue resistance of sheet-formed AZ31 is investigated. We employed a microstructure-sensitive fatigue model that comprises fatigue total fatigue lifetimes into crack incubation, microstructurally small crack (MSC) and physically small crack (PSC), and long crack growth $[1,2]$, to correlate the behavior of AZ31 Mg alloy to experimental results. The model proposed here was used to capture competing structure-property relations, including grain size, inclusions size, and mechanical properties, and their accompanying impact on fatigue lifetimes.

\subsection{Materials and Experiments}

The material employed was a sheet of AZ31 Mg alloy. Fatigue specimens were designed to conform to ASTM standard E-647, with the dimensions indicated in Figure 1. Fatigue tests were conducted on a servo-controlled electro-hydraulic system. These tests were performed under strain control mode, constant strain amplitude, and fully reversed. Tests were conducted at room temperature, and a sinusoidal cyclic loading was applied with a frequency of $5 \mathrm{~Hz}$. A $50 \%$ drop of the peak stress load was used to determine failure of the sample. The amplitude levels tested ranged from 0.2 to $0.6 \%$ strain.

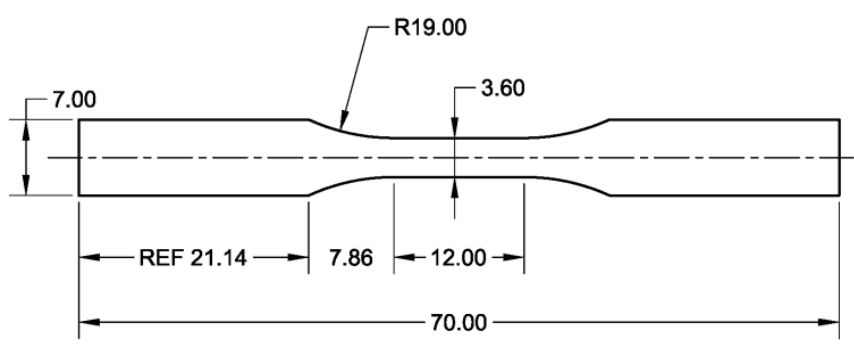

Figure 1. Dimension of fatigue specimen

\subsection{Results}

\subsubsection{Microstructure}

The microstructural characteristics of the material were quantified to provide an understanding of the behavior under monotonic and cyclic loads. Figure 2 shows the grain morphology and size observed 
for a section parallel to the rolling direction, where elongated grains averaging $5.7 \mu \mathrm{m}$ were observed. The intermetallic particles were found to have an average diameter of $3.7 \mu \mathrm{m}$ and a nearest neighbor distance of $35.5 \mu \mathrm{m}$.

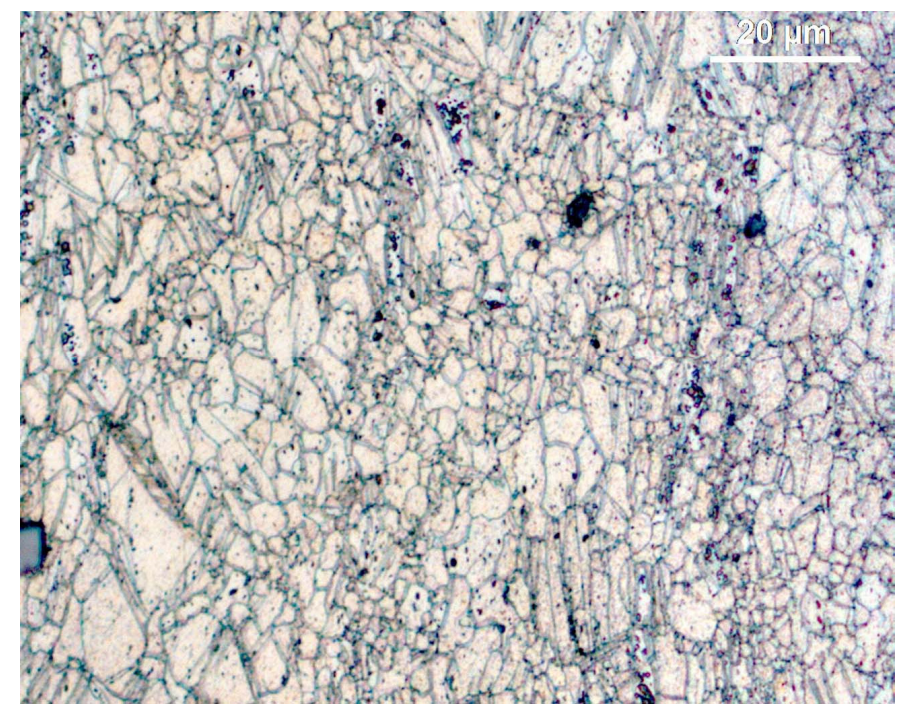

Figure 2. Etched microstructure image of the AZ31 Sheet magnesium alloy

\subsubsection{Monotonic Behavior of AZ31}

The stress-strain curve for tension is shown in Figure 3. The tensile stress-strain behavior exhibited a relatively flat flow stress.

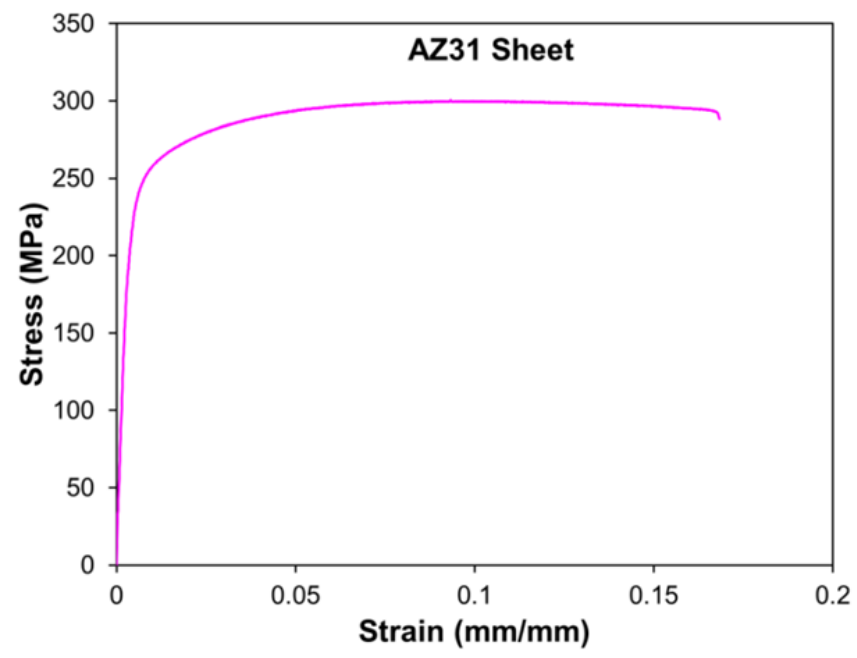

Figure 3. Stress-strain tensile behavior of AZ31 sheet magnesium alloy

\subsubsection{Strain-Life}

The strain-life curve for the AZ31 sheet $\mathrm{Mg}$ alloy for strain controlled, constant strain amplitude and completely reversed loading conditions is shown in Figure 4. The strain-life curve in the log-log domain shows a pattern from 0.6 to nearly $0.3 \%$. Then the slope changes drastically below of a strain amplitude of $0.3 \%$. 


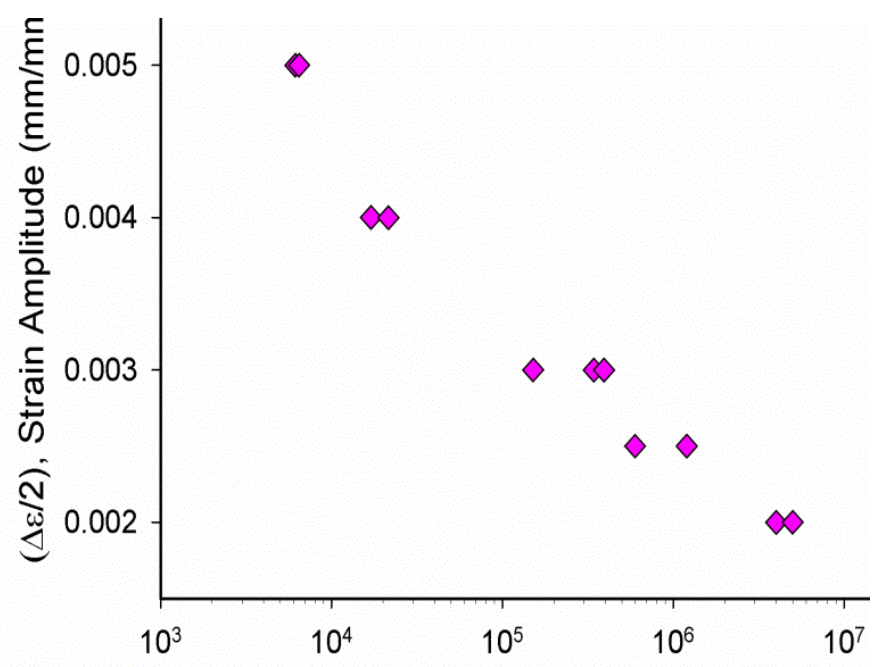

Figure 4. Total strain-life for an AZ31 sheet magnesium alloy

\subsubsection{Cyclic Deformation Response}

Figure 5 shows the hysteresis curves for the first cycle and mid-life at strain amplitudes of $0.5,0.4$, 0.3 , and 0.2 percent. Cyclic stress-strain hysteresis curves for the first cycle and mid-life at all strain amplitudes show nearly symmetrical behavior (Figure 5a-d), and almost no change is noted between the first cycle and the mid-life cycle.

a)

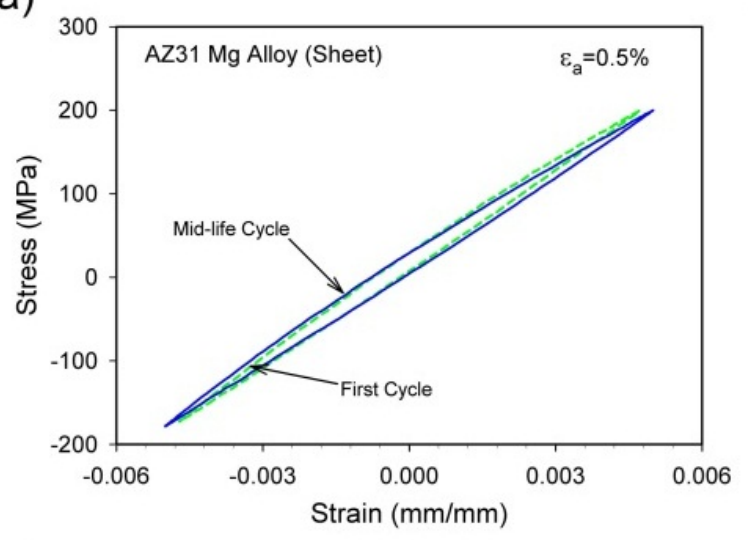

c)

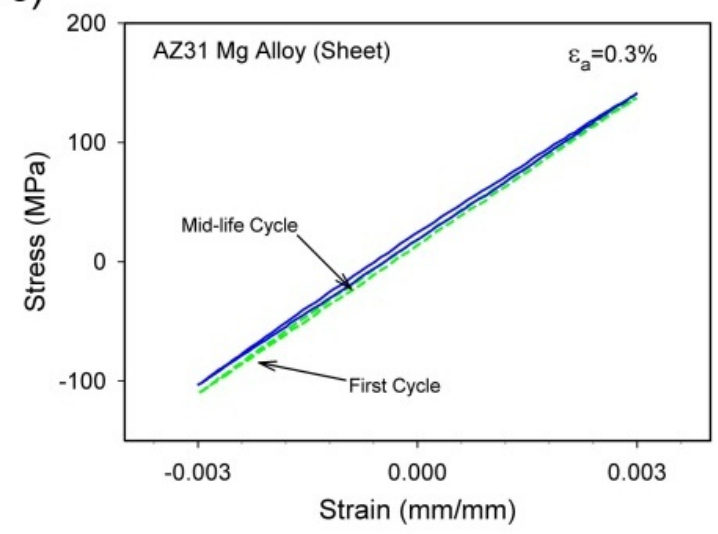

b)

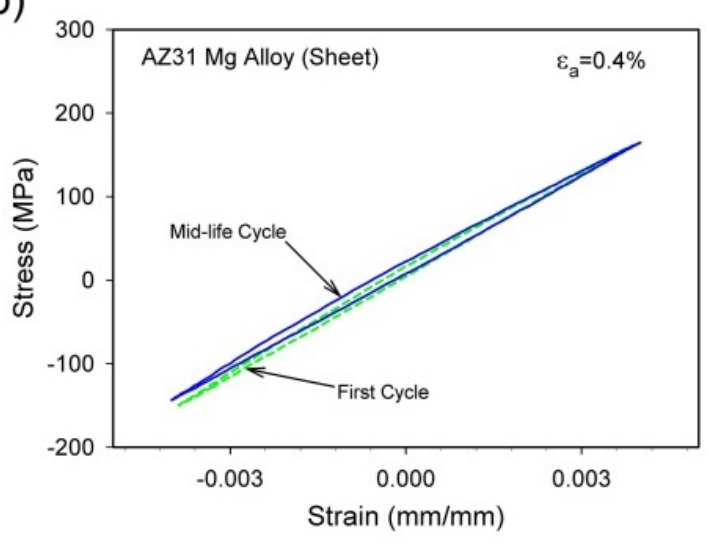

d)

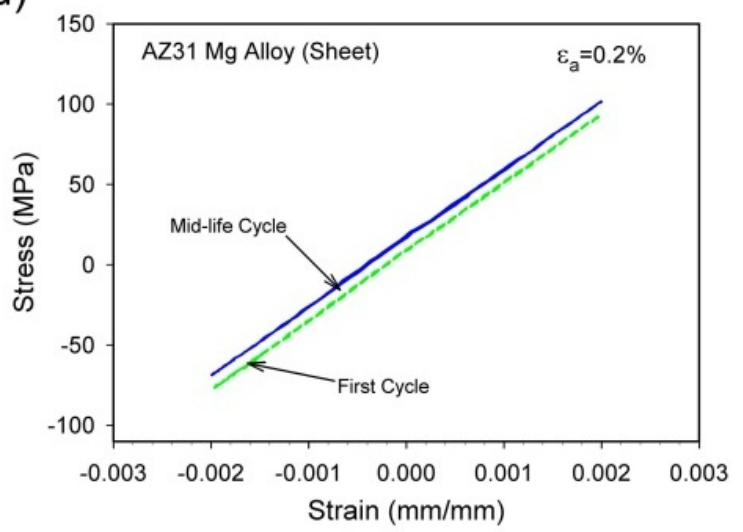

Figure 5. Hysteresis curves of AZ31 sheet Mg alloy at the strain amplitude of a) $0.6 \%, b) 0.4 \%, c) 0.3 \%$ and d) $0.2 \%$ 


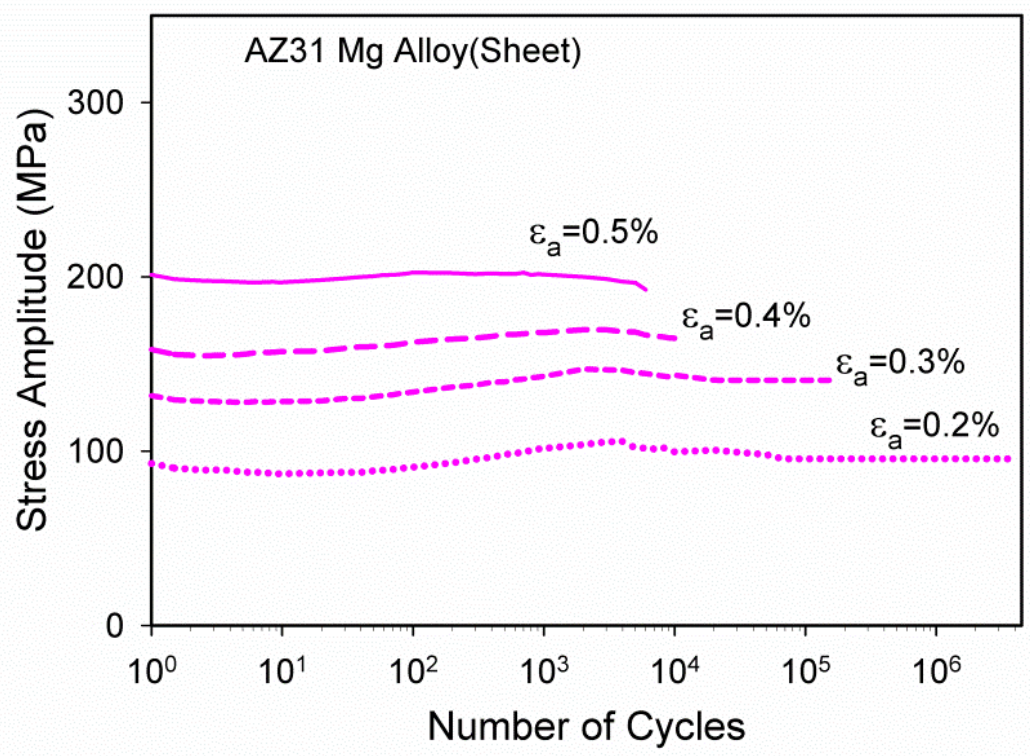

Figure 6. Stress amplitude response and cyclic mean stresses of AZ31 magnesium alloy from the extruded, plate, and sheet forms

Figure 6 shows the evolution of stress amplitude as a function of number of cycles at the strain amplitudes of $0.5 \%, 0.4 \%, 0.3 \%$, and $0.2 \%$. A slight hardening effect for all strain amplitudes ranging was observed. The cyclic hardening appears strongly in the initial cycles, about $10 \%$ of the total fatigue life, and a small amount of hardening continued to the point of fatigue failure.

\subsubsection{Fatigue Fractured Surfaces}

Fractographs of the strain-life specimens at or near the fatigue failure formation sites were taken using a scanning electron microscope (SEM). All the fracture surfaces observed showed typical indications of fatigue damage. In most cases, fractured particles were identified to be the fatigue crack initiation sites. Figure $7 \mathrm{a}$ shows an overview of a typical fracture surface, and Figure $7 \mathrm{~b}$ shows a magnified view to indicate the crack initiation site. In this particular specimen, the fatigue crack initiated from a crack particle at the surface. Based on results of the SEM study, the average particle size (diameter) in the sheet material was approximately $38 \mu \mathrm{m}$. Hence, these types of particles appeared to be more brittle than the surrounding matrix and tended to fracture rather than debond under cyclic loading. In contrast with other extruded formed Mg alloys such AZ31 or AZ61, the presence of twinning was not observed on the fracture surface. 

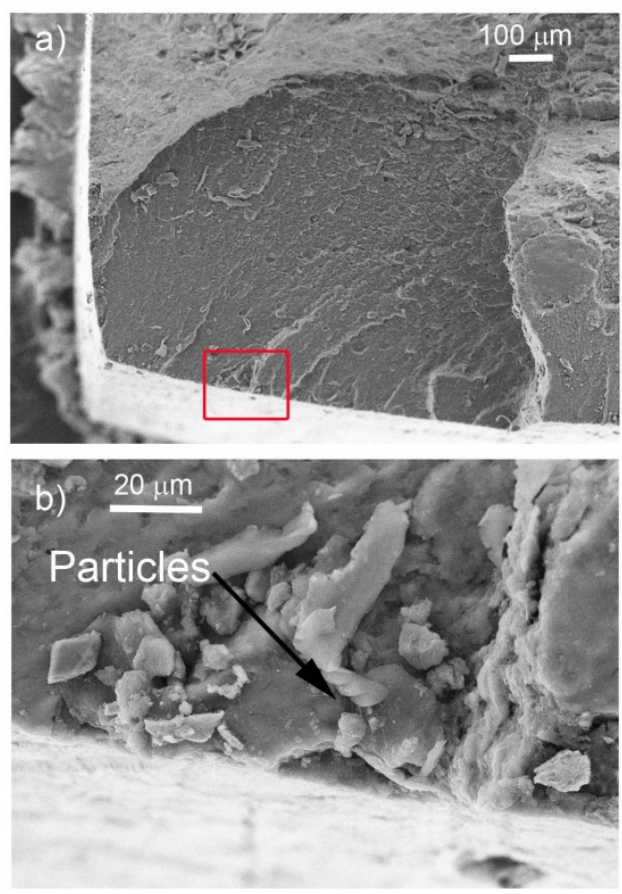

Figure 7. (a) Typical overview of fracture surfaces for AZ31 sheet magnesium alloy at $0.3 \%$ of strain amplitude in the (b) Fatigue cracks were found to initiate from intermetallic particles at the surface

\subsection{Fatigue Model Correlation}

Figure 8 shows the correlations of the MSF models to the experimental strain-life data. Table 1 provides a summary of the model constants used to correlate the MSF to fully-reversed uniaxial strain life results for the extruded AZ31 Mg alloy in the extrusion, plate, and sheet formation. The MSF model appears to correlate to the general trend of the fatigue life in the AZ31 sheet-formed alloy.

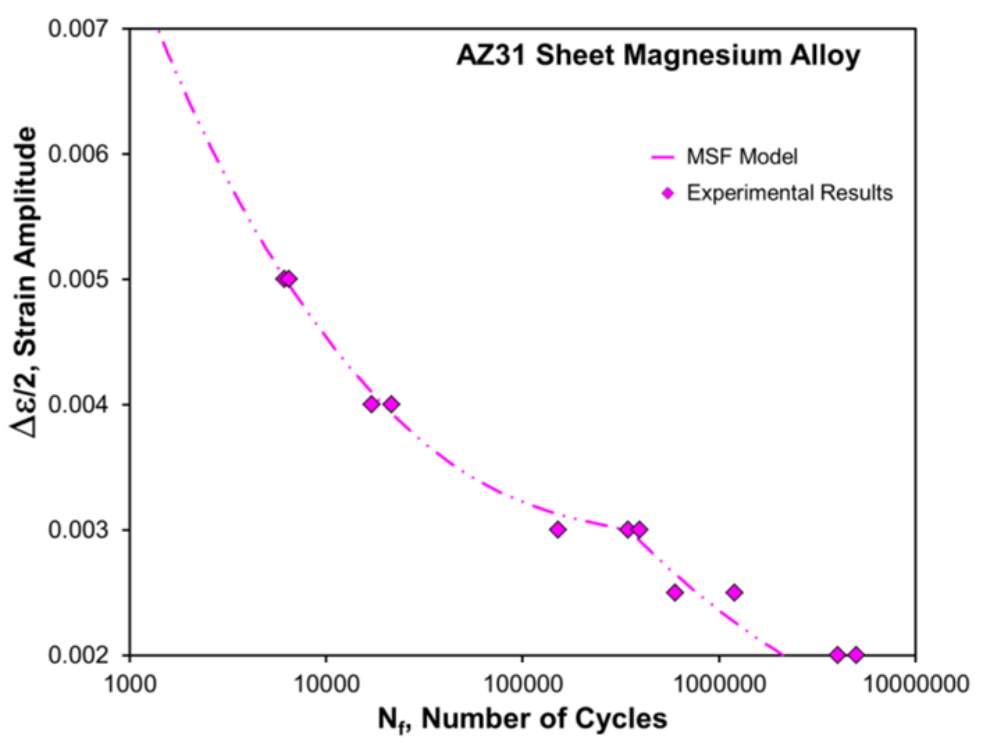

Figure 8. Multistage fatigue (MSF) model and strain-life results for an AZ31 sheet magnesium alloy 
Table 1. Multistage fatigue model parameters for AZ31 magnesium alloy

\begin{tabular}{|c|c|c|c|}
\hline \multirow{9}{*}{ 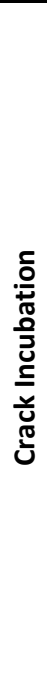 } & Constant & Sheet & Description \\
\hline & $C_{n}$ & 0.95 & Constant related to $\mathrm{Cm}$ constant $(\mathrm{CNC})$ \\
\hline & $C_{i n c}$ & 0.55 & Ductility coefficient in Modified Coffin Manson Law \\
\hline & $\alpha$ & -0.635 & Ductility exponent in Modified Coffin Manson Law \\
\hline & $q$ & 2.4 & Exponent in remote strain to local plastic shear strain \\
\hline & $y_{1}$ & 170 & Constant in remote strain to local plastic shear strain \\
\hline & $y_{2}$ & 0 & Linear constant in remote strain to local plastic shear strain \\
\hline & $\xi$ & 4 & Geometric factor in micromechanics study \\
\hline & $r$ & 0.2 & Exponent in micromechanics study \\
\hline \multirow{8}{*}{ 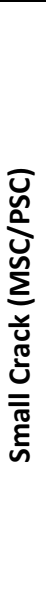 } & $\omega$ & 0.15 & Omega \\
\hline & ai & 0.625 & Initial crack size contribution \\
\hline & $\theta$ & 0.135 & Load path dependent and loading combination parameter \\
\hline & $\zeta$ & 1.2 & Exponent in Small crack growth \\
\hline & $C_{l}$ & 300000 & HCF constant in small crack growth \\
\hline & $C_{11}$ & 0.0001 & LCF constant in small crack growth \\
\hline & $\chi$ & 0.32 & Crack growth rate constant \\
\hline & $\triangle C T D_{t h}$ & 0.00032 & CTD threshold value \\
\hline
\end{tabular}

\subsection{Summary}

The cyclic behavior of AZ31 sheet-formed Mg alloy was investigated based on the variation in microstructural features and mechanical properties. A multistage fatigue (MSF) model was developed to capture competing structure-property and mechanical properties, and their consequential impact on fatigue lifetimes. In regard to the research conducted here, the following conclusions are made:

- The strain-life curve for sheet-formed Mg alloy showed a similar pattern to the same for Mg extruded alloys.

- The hysteresis lops of the AZ31 sheet Mg alloy showed almost a symmetric cyclic behavior for most of the strain amplitudes observed. In contrast with the behavior observed for an extruded alloy, which shows an asymmetric behavior.

- A slight strain hardening effect was observed for all strain amplitudes. 
- Observations of the fracture surfaces under SEM revealed that fractured intermetallic particles initiated fatigue cracks. The crack initiation sites were found to occur more frequently at inclusions near the free surface of the specimens.

- The multistage fatigue (MSF) model incorporated microstructural properties and cyclic hardening parameters in order to capture scattering in the fatigue behavior.

\section{Fatigue damage and Microstructure Properties in an am30 extruded Magnesium Alloy}

\subsection{Introduction}

In the previous section, the fatigue performance of a rolled AZ31 Mg alloy was studied. In this section the investigation of an AM30 extruded $\mathrm{Mg}$ alloy is reported. A strain controlled fatigue test program was conducted to quantify and evaluate the mechanisms of fatigue and fatigue life. A multistage fatigue (MSF) model was developed to predict the fatigue behavior. The MSF model comprises three scales of cyclic damage; crack incubation; microstructurally small crack (MSC), physically small crack (PSC), and long crack growth. The MSF model will predict crack development in a component.

\subsection{Materials and Experiments}

The material employed in this research was an extruded AM30 Mg alloy provided in the form of an automotive crash rail profile with a thickness of approximately $2.5 \mathrm{~mm}$. Samples for this study were taken in the extrusion direction (ED) and in the transverse direction (ETD) as shown in Figure 9. Fatigue specimens were designed to conform to ASTM standard E-647 with a gage length of $15 \mathrm{~mm}$. Fatigue tests were conducted on a servo-controlled electro-hydraulic system under strain control mode, constant strain amplitude, and fully reversed. Tests were performed at room temperature, and a sinusoidal cyclic loading was applied with a frequency of $5 \mathrm{~Hz}$. A $50 \%$ drop of the peak stress load was used to determine failure of the sample. The amplitude levels tested ranged from 0.2 to $0.6 \%$ strain.

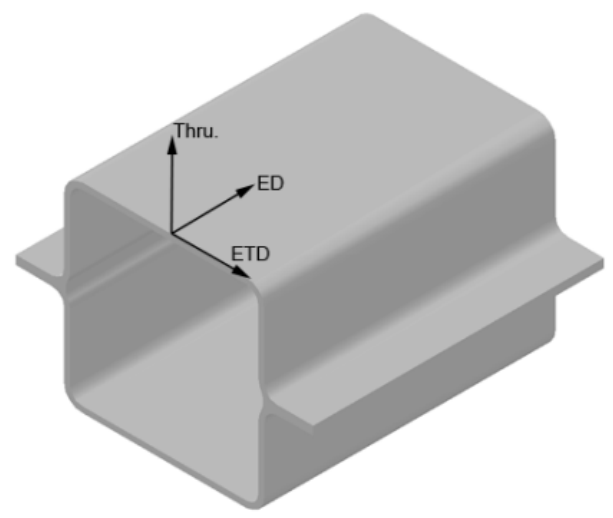

Figure 9. Sampling positions of specimens 


\subsection{Experimental Results}

\subsubsection{Monotonic Behavior of AM30}

Figure 10 shows the stress-strain curves for tension along both the ED and the ETD. These curves show the anisotropic and asymmetric mechanical behavior. The yield strengths, hardening rates, and ultimate strengths are different in each orientation as well as in compression. The yield strength in tension was found to be about $180 \mathrm{MPa}$ while that the strength along the transverse direction was found to be about $60 \mathrm{MPa}$. The yield stress under the ETD was approximately $1 / 3$ less than under the ED.

The monotonic properties reported by Begum et al. 2008 [17] for the same alloy differ from the results shown here. These differences could be attributed to variations in texture as well as grain size due to the extrusion process. On the other hand, the differences observed in the stress-strain behavior between ED and ETD can be attributed to the different deformation modes activated in each direction. In tension along the ED, slip deformation is formed through the prismatic and basal planes; consequently, slip is the predominant mechanism. Along the ETD, the predominant mechanism is twinning.

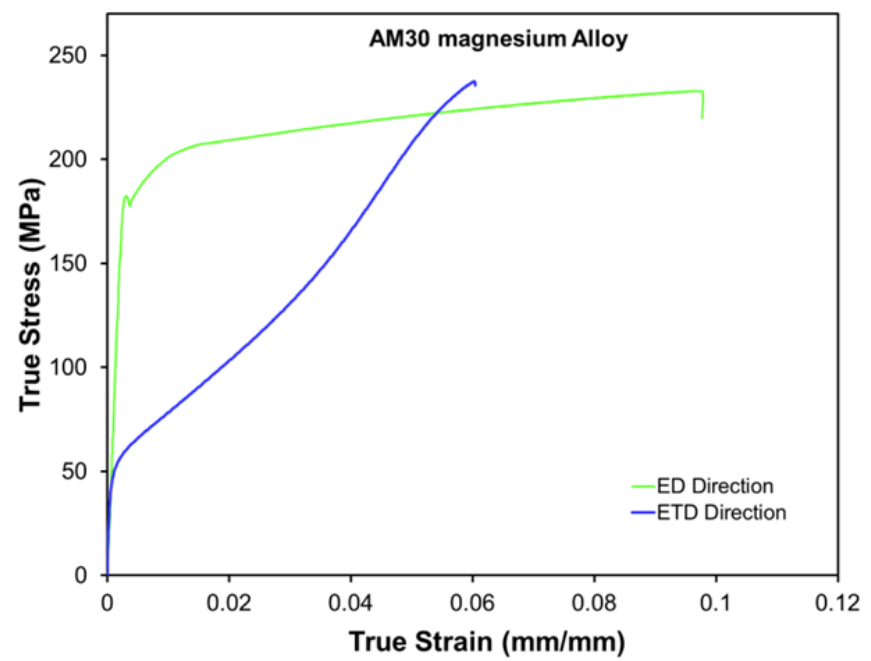

Figure 10. Monotonic stress-strain behavior of AM30 magnesium alloy in the extrusion direction (ED) and the extrusion transverse direction (ETD).

\subsubsection{Strain-Life}

Figure 11 shows the strain-life for extruded AM30 Mg alloy in the ED and ETD, for strain controlled, constant strain amplitude and completely reversed loading conditions. Fatigue tests for higher strain amplitudes in the ETD were unsuccessful due to the significant plasticity undergone by the specimens. The strain-life curve in the log-log domain shows a pattern from 0.6 to almost $0.3 \%$; then the slope changes drastically, to below a strain amplitude of $0.3 \%$. 


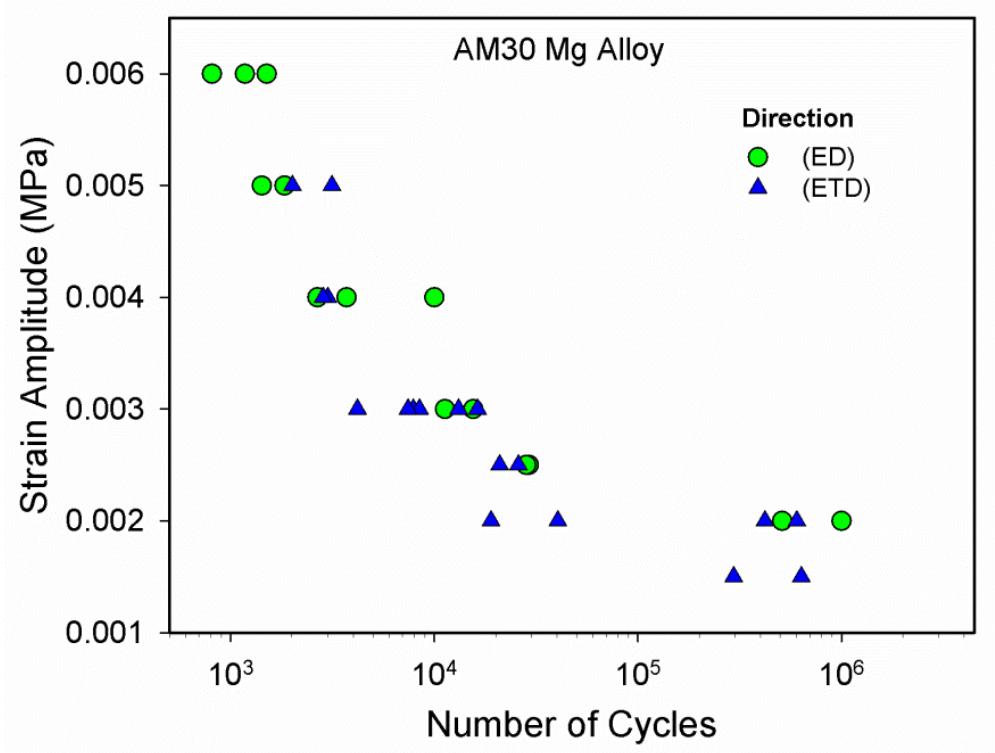

Figure 11. Total strain life of an AZ31 magnesium alloy

\subsubsection{Cyclic Deformation Response}

Figure 12 shows the stress-strain hysteresis loops for the ED at strain amplitudes of $0.6,0.4,0.3$, and 0.2 percent. An asymmetric pattern in the cyclic deformation is observed for the first cycle for all the strain amplitudes. This asymmetry is more pronounced for the first cycle, and at higher strain levels. The hysteresis loops show that hardening takes place at lower strain amplitudes under tensile loading, and hardening occurs under compressive loading. For lower strain amplitudes, the tensile and compressive stresses were almost the same. The asymmetry from tensile to compressive is larger as the strain amplitude increases. The cyclic stress-strain asymmetry observed in AM30 Mg alloy is due to twinning activation during unloading in compression, and detwinning during loading in tension $[3,17,18]$. A plateau is observed in the stress-strain response during compression loading, which is typical of other Mg alloys reported elsewhere $[3,17,18]$. The mid-life hysteresis loops remained relatively asymmetric at higher strain amplitudes, while nearly symmetric patterns are seen at lower strain amplitudes.

Cyclic stress-strain hysteresis curves for the ETD at strain amplitude of $0.5,0.4,0.3$, and 0.2 percent are shown in Figure 13. The first cycle shows a highly asymmetric behavior for all strain amplitudes. A tenuous plateau is observed during compression loading for the first cycle, markedly at high strain amplitudes, which is a clear indication of twining. These curves show a slight asymmetry for all strain amplitudes. In addition, it is observed that the hysteresis loop began with twinning in tension, and pseudo-elasticity during unloading, while reloading in compression marked an inflection point, which is an indication of detwinning. 

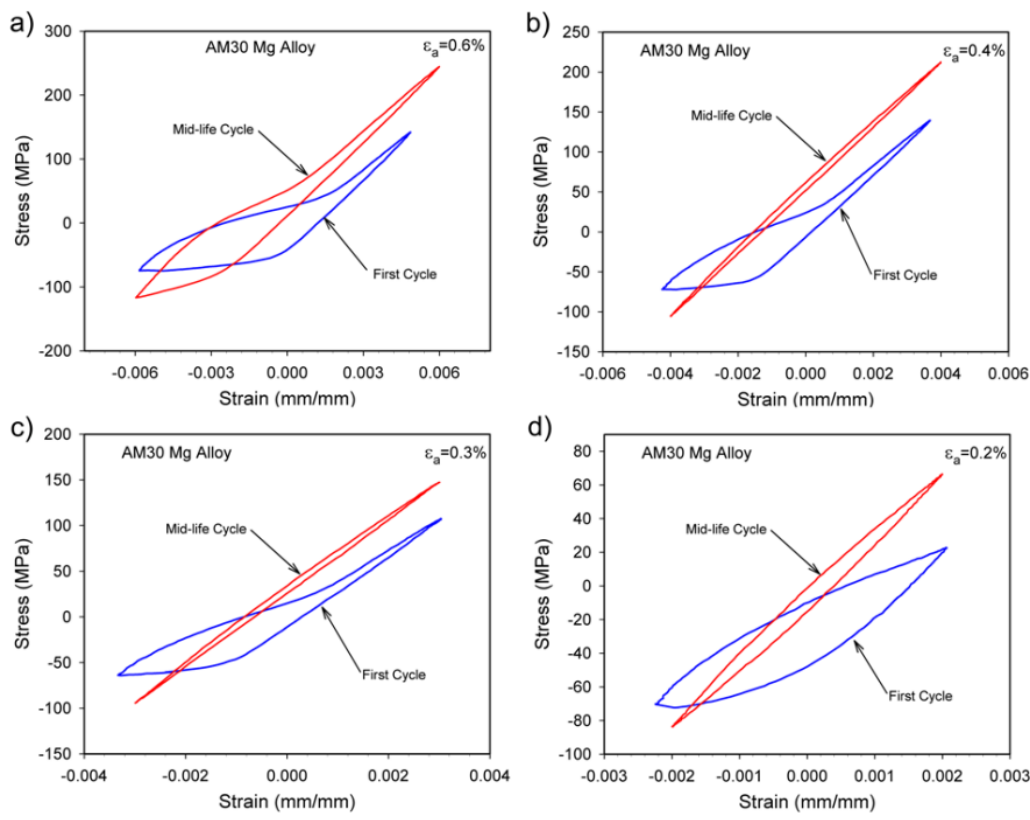

Figure 12. Hysteresis curves of $\mathrm{AM} 30$ extruded $\mathrm{Mg}$ alloy in the extruded direction at the strain amplitudes of a) $0.6 \%$, b) $0.5 \%$, c) $0.3 \%$ and d) $0.2 \%$
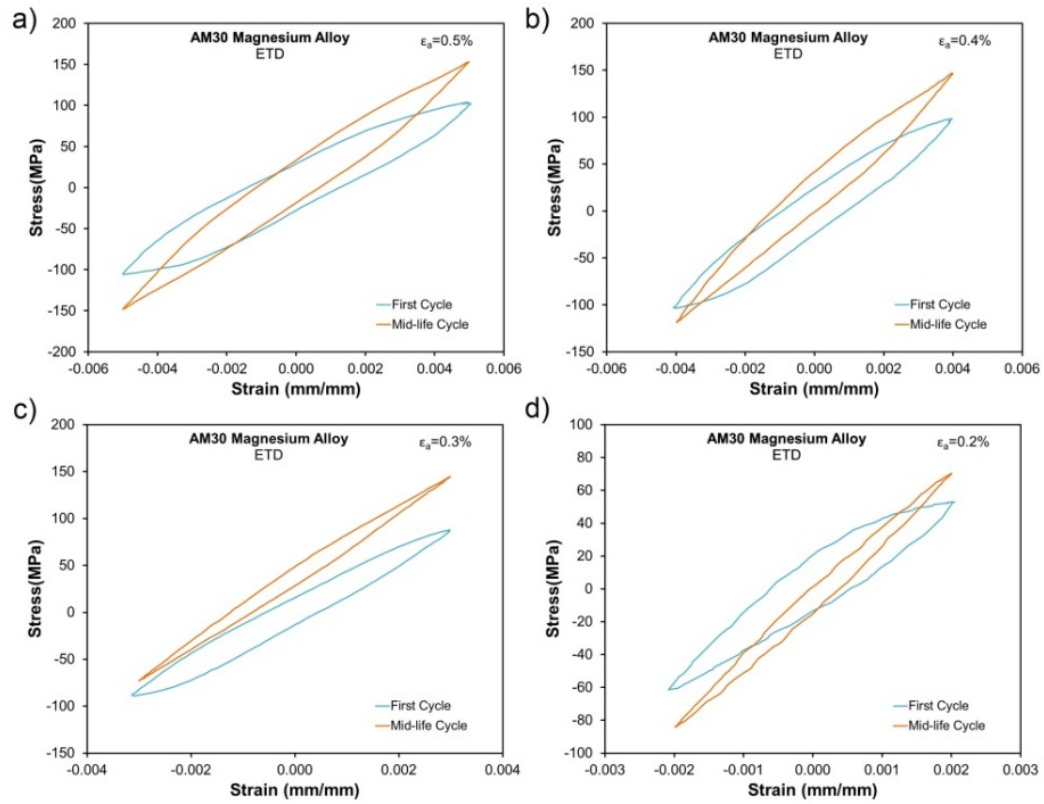

Figure 13. Hysteresis curves of $A M 30$ extruded $\mathrm{Mg}$ alloy in the transverse direction at the strain amplitudes of a) $0.5 \%$, b) $0.4 \%$, c) $0.3 \%$ and d) $0.2 \%$

Figure 14 and 15 show the evolution of stress amplitude as a function of the number of cycles for the ED at the strain amplitudes from $0.2 \%$ to $0.6 \%$. Stress amplitude increased as the number of cycles increased. The strain amplitudes ranging from $0.3 \%$ to $0.6 \%$ showed a greater hardening effect than the others strain amplitude levels. 


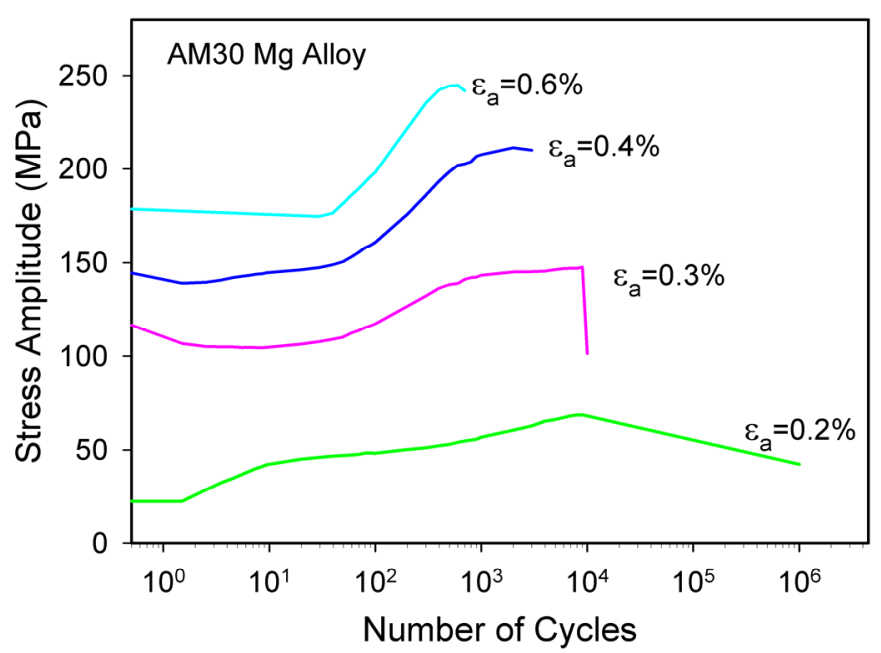

Figure 14. Stress amplitude response of AM30 magnesium alloy

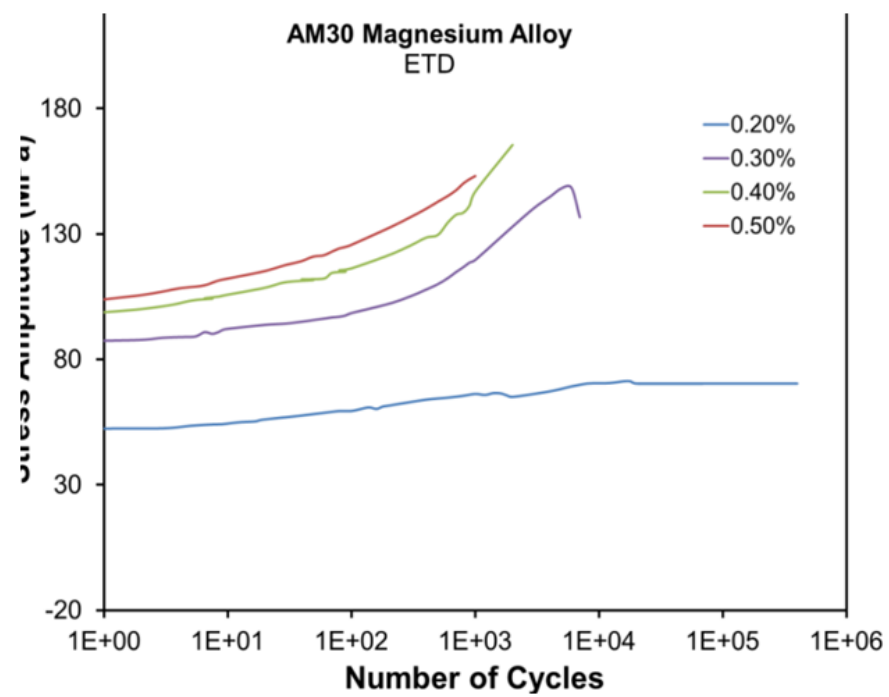

Figure 15. Stress amplitude response of AM30 magnesium alloy

\subsubsection{Fatigue Fractured Surfaces}

Fractured surfaces of the AM30 Mg alloy that were observed showed typical indications of fatigue damage. River marks flowing outward from a single location at the surface were observed on all specimens. Figure 16 shows a typical fracture surface with a particle-initiated crack. In addition, the SEM images revealed evidence of twinning on the fatigue fracture surfaces similar to that which has been reported in the literature $[19,20]$. 


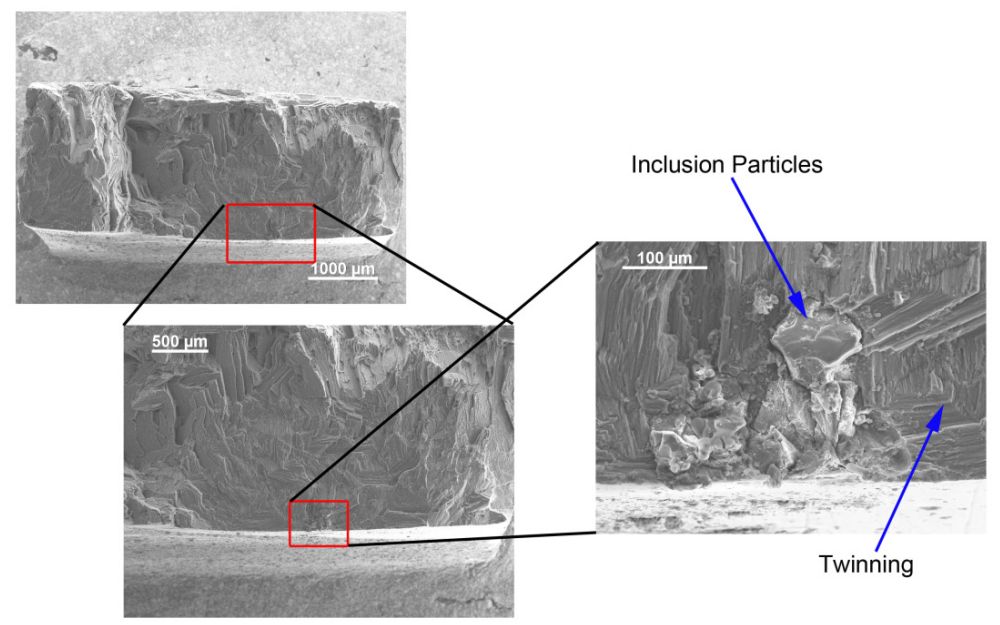

Figure 16. Typical fracture surface for AM30 magnesium alloy in the ED direction. The crack developed at a small inclusion. In addition, twinning is observed near the crack site

\subsection{Multistage Fatigue Modeling}

Figure 17 shows the correlations of the MSF model to experimental data for both ED and ETD. Table 2 lists the model constants used to correlate the model to fully-reversed uniaxial strain life results for the extruded AM30 Mg alloy in the ED and ETD. It is important to note that the basic material constants were identical regardless of the orientation of cyclic loading, but the microstructural features and mechanical properties were different.

As shown in Figure 17, the MSF model appears to correlate to the general trend of the fatigue life in both directions (ED and ETD). In the model, the effect of twinning is captured through the cyclic stressstrain response.

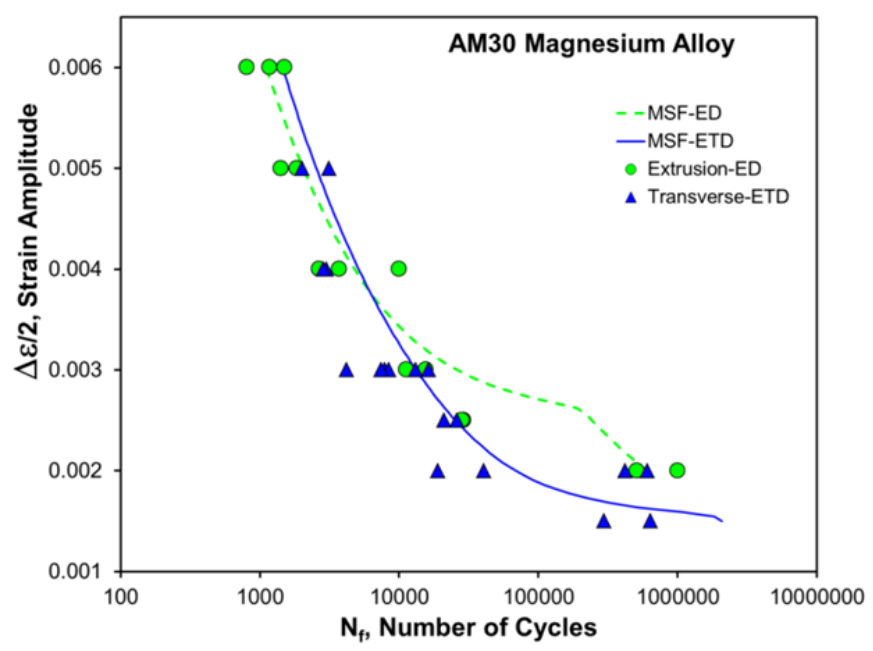

Figure 17. Multistage fatigue (MSF) model and strain-life results for AM30 magnesium alloys 
Table 2. MSF Parameters for extruded AM30 magnesium alloy

\begin{tabular}{|c|c|c|c|c|}
\hline \multirow{9}{*}{ 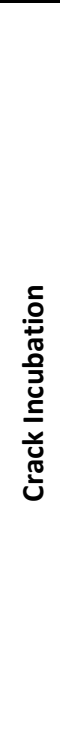 } & Constant & ED & ETD & Description \\
\hline & $C_{n}$ & 0.95 & 0.95 & Constant related to $\mathrm{Cm}$ constant (CNC) \\
\hline & $C_{i n c}$ & 0.55 & 0.55 & Ductility coefficient in Modified Coffin Manson Law \\
\hline & $\alpha$ & -0.64 & -0.64 & Ductility exponent in Modified Coffin Manson Law \\
\hline & $q$ & 2.23 & 2.23 & Exponent in remote strain to local plastic shear strain \\
\hline & $y_{1}$ & 120 & 120 & Constant in remote strain to local plastic shear strain \\
\hline & $y_{2}$ & 0 & 0 & Linear constant in remote strain to local plastic shear strain \\
\hline & $\xi$ & 4.5 & 4.5 & Geometric factor in micromechanics study \\
\hline & $r$ & 0.2 & 0.2 & Exponent in micromechanics study \\
\hline \multirow{8}{*}{ 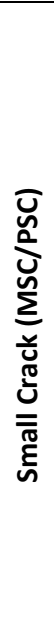 } & $\omega$ & 0.15 & 0.15 & Omega \\
\hline & $a i$ & 0.625 & 0.625 & Initial crack size contribution \\
\hline & $\theta$ & 0.135 & 0.135 & Load path dependent and loading combination parameter \\
\hline & $\zeta$ & 1.2 & 1.2 & Exponent in Small crack growth \\
\hline & $C_{l}$ & 300000 & 300000 & HCF constant in small crack growth \\
\hline & $C_{l l}$ & 0.0001 & 0.0001 & LCF constant in small crack growth \\
\hline & $\chi$ & 0.32 & 0.32 & Crack growth rate constant \\
\hline & $\mathrm{CTDH}$ & 0.00032 & 0.00032 & CTD threshold value \\
\hline
\end{tabular}

\subsection{Conclusions}

The cyclic behavior of extruded AM30 magnesium (Mg) alloy were investigated based on the microstructural properties. A multiscale fatigue (MSF) model was used to capture the strain-life behavior. In regards to the research conducted here, the following conclusions can be made:

- Monotonic tensile tests revealed a difference in yield strength between the extrusion direction (ED) and transverse direction (ETD). The transverse orientation showed lower yield strength.

- Hysteresis loops showed an asymmetric pattern for all the observed strain amplitudes. The asymmetry was more emphatic for the first cycle of the cyclic process.

- Compression twinning and detwinning were observed in the hysteresis loops for the ED. Conversely, the hysteresis loops for ETD showed tensile twinning and detwinning. 
- For the ED, the asymmetric cyclic behavior remained throughout the cyclic life, as was revealed by the mid-life cycles. This asymmetry was less pronounced for the $0.2 \%$ strain amplitude.

- Strain hardening was observed for all the strain amplitudes but more significant for the higher strain levels.

- Fatigue cracks incubated from fractured particles near the free surface of the specimens for both directions. Fracture surfaces showed twinning for most of the specimens studied.

- For high cycle fatigue regions, a small difference is noted in the fatigue between the ED and ETD.

- The MSF model incorporated the differences in grain orientation, grain size, particle size, and cyclic hardening parameters in order to capture the differences in fatigue behavior in the ED and ETD. We note that the other material constants were not changed for the incubation and microstructurally small crack (MSC) regimes.

\section{Multistage fatigue Modeling of Az91 and AM60 Magnesium Alloys}

\subsection{Multistage Model Formulation for Magnesium Cast Alloys}

In order to construct physically-motivated and microstructurally-sensitive fatigue estimations of the AZ91 and AM60 cast magnesium (Mg) alloys presented in this study, the Multistage Fatigue (MSF) model was employed. The MSF model first proposed by McDowell et al. [1] comprises three distinct regimes of fatigue damage: crack incubation, microstructurally small crack (MSC) and physically small crack (PSC) growth, and long crack (LC) growth as shown in Eq. (10).

$$
N_{\text {Total }}=N_{\text {Inc }}+N_{M S C / P S C}+N_{L C} .
$$

\subsubsection{Incubation Regime}

For the incubation life, $N_{\text {inc }}$ for a given material, a damage parameter $\beta$ is equated to a modified Coffin-Manson law at the microscale:

$$
C_{\text {inc }} N_{\text {inc }}^{\alpha}=\beta,
$$

where $\beta$ is the nonlocal damage parameter around an inclusion, and $C_{\text {inc }}$ and $\alpha$ are the linear and exponential coefficients in the modified Coffin-Manson law for incubation. The physical representation of the damage parameter is related to the local average maximum plastic shear strain amplitude at an inclusion and is estimated by the following:

$$
\begin{gathered}
\beta=\frac{\Delta \gamma_{\max }^{P^{*}}}{2}=\Psi Y\left(\varepsilon_{a}-\varepsilon_{t h}\right)^{q}, \quad \frac{l}{D}<\eta_{\lim } \\
\beta=\frac{\Delta \gamma_{\max }^{P^{*}}}{2}=\Psi Y\left(1+\xi \frac{l}{D}\right)\left(\varepsilon_{a}-\varepsilon_{t h}\right)^{q}, \quad \frac{l}{D}>\eta_{\lim },
\end{gathered}
$$


where $\varepsilon_{a}$ is the remote applied strain amplitude, and $\varepsilon_{t h}$ and $\varepsilon_{p e r}$ were introduced by McDowell et al. [1] and employed by Xue et al. [2, 21-22] to represent the strain threshold for damage incubation and the percolation limits for microplasticity, respectively. Both the strain threshold and the percolation limits for microplasticity can be determined through micromechanical simulations. However, Xue et al. [2] showed that the strain threshold is easily estimated by using the standard methods for estimating the endurance limit, where $\varepsilon_{t h}=\frac{0.29 S u t}{E}$. Furthermore, the percolation limit can also be estimated, where $\varepsilon_{\text {per }}=\frac{0.7 \sigma_{y}^{\text {cyclic }}}{E}$. The parameter $Y$ is correlated as $Y=y_{1}+0.1(1+R) y_{2}$, where $y_{1}$ and $y_{2}$ are material constants. For completely reversed loading cases, $Y=y_{1}$. An experimentally observed structure-property relationship term was recently added [7] to the incubation damage parameter. This structure-property term, $\Psi=\left[\frac{\left(M P S^{2}\right)}{(N N D)(D C S)}\right]^{\gamma}$, is a function of maximum pore size (MPS), nearest neighbor distance $(N N D)$, dendrite cell size $(D C S)$, and sensitivity exponent $\gamma$, where the above parameters are microstructural parameters determined from fractographic and metallographic analyses.

\subsubsection{Microstructurally Small Crack Growth Regime}

For the combined MSC/PSC growth regime, The growth for the fatigue crack is governed by the range of crack tip displacement $\triangle C T D$, which is proportional to the crack length, the $\mathrm{n}^{\text {th }}$ power of the applied stress amplitude in the HCF regime, and the macroscopic plastic shear strain range in the LCF regime; i.e.,

$$
\begin{gathered}
\left(\frac{d a}{d N}\right)_{M S C / P S C}=\chi\left(\Delta C T D-\Delta C T D_{t h}\right), \quad a_{i}=0.625 D \\
\Delta C T D=C_{I I} \Psi\left(\frac{D C S}{D C S_{0}}\right)^{\varphi} f(\phi)\left[\frac{U \Delta \widehat{\sigma}}{s_{u t}}\right]^{\zeta} a_{i}+C_{I} \Psi\left(\frac{D C S}{D C S_{0}}\right)^{\varphi}\left(\frac{\Delta \gamma_{\max }^{P}}{2}\right)^{2} .
\end{gathered}
$$

Here, $\chi$ is a constant for a given microstructure and $C_{I}$ and $C_{I I}$ are material dependent parameters that capture the microstructural effects on MSC/PSC growth. A porosity related term is included in the high cycle fatigue regime, where $f(\phi)=1+\omega\left\{1-\exp \left(-\frac{\phi}{2 \bar{\phi}}\right)\right\}$ and the porosity threshold $\bar{\phi}$ is assumed as 0.0001 . The effect of variation of dendrite cell size on crack growth is explicitly addressed relative to a reference size $D C S_{0}$, and the sensitivity exponent $\varphi$ is unity. The threshold value for crack tip displacement was set equal to the Burger's vector for the magnesium rich matrix [6] $\Delta C T D_{t h}=3.2 *$ $10^{-4} \mu \mathrm{m}$.

A long crack growth stage of the MSF model is based on linear elastic fracture mechanics $[1,21]$. However, in this study the modeling efforts focused on incubation and MSC/PSC growth regimes following the method of Jordon et al. [3, 7]. This approach is consistent with the experimental evidence that the formulation for MSC/PSC can characterize fatigue cracks up to several millimeters in length [23]. The MSF model constants for AZ91 and the AM60 cast magnesium alloys are given in Table 3.

\subsection{Model Correlations and Fatigue Estimations}

Figure 18a-c shows the MSF model correlations for the AZ91 as-cast, AZ91-T6, and AM60 as-cast alloys, respectively. University of Michigan provided University of Alabama the experimental results 
shown in Figure 23, which are based on fatigue tests conducted under the supervision of Prof. Wayne Jones. The mean MSF model predictions for each data set were based on the stabilized cyclic stressstrain behavior and the average measured microstructural features, including the dendrite cell size (DCS) and the average size of the pore responsible for crack initiation. Regarding the percentage of incubation life in the mean life predictions, an average incubation life of $34 \%, 56 \%$, and $47 \%$ of total life was found suitable for the AM60 as-cast, AZ91 as-cast, and AZ91-T6 alloys, respectively.

To show the robustness of the MSF model and to capture the effect of microstructure on fatigue lifetimes, estimations for the upper and lower bounds of the strain-life data sets are also presented. These model predictions were determined by using the extreme pore sizes and DCSs for each data set. As shown in Figure 18, the model showed good correlation to both the upper and lower bounds as compared to the experimental results. As such, the MSF model was generally able to bind the scatter of each fatigue data set considering only the microstructure variation observed between locations L1 and L2. The model showed more sensitivity to pore size than to DCS, which is not unexpected, considering there was not much variation of the DCS from locations L1 and L2. As such, the defect size was the primary factor determining number of cycles to failure for both the AM60 and the AZ91 alloys.

Because the MSF model was included to correlate the fatigue data in this work, a discussion including the standard strain-life modeling approach is warranted. The impact of the MSF model on capturing the macroscopic behavior of these three alloys is illustrated by the fact that the model parameters are identical for all three alloys except for those directly related to the microstructure. As such, the MSF model can correlate differences in fatigue behavior through variations in inclusion and dendrite cell sizes. Since the formulation of the model equations for incubation and MSC stages are based on computational and experimental studies, the model can be used in parametric studies or in the design of a material for optimized fatigue properties. In contrast, the ability of the standard strain-life model to capture the effect of microstructure can only be reflected in the strain-life parameters. In fact, the ability of these classical models to predict the fatigue life of a material with variation in microstructure is limited to purely an empirical study. We acknowledge that while the MSF model does contain specific modeling parameters that are used to obtain the best correlation, the focus of these parameters are to capture the experimentally observed stages of fatigue evolution directly related to microstructure in a phenomenological manner. 

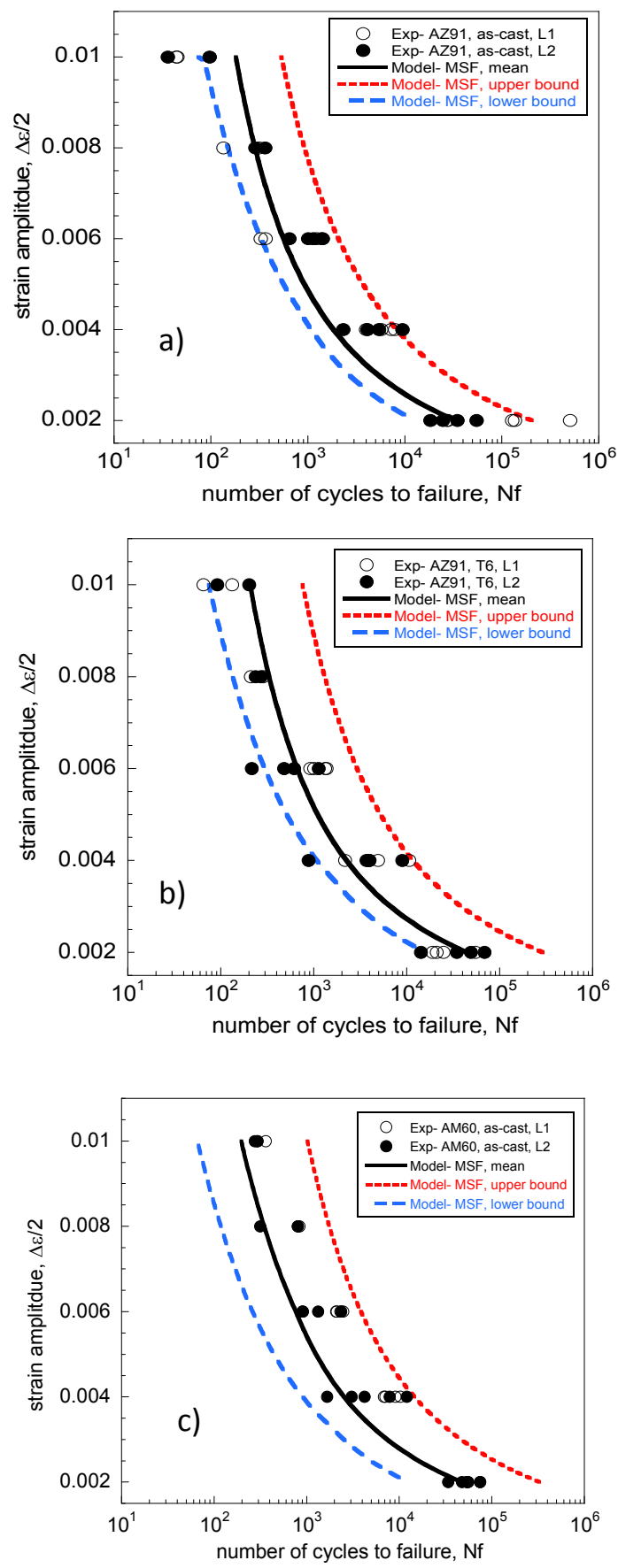

Figure 18. Comparison of the strain-life experimental results for locations $L 1$ and $L 2$ to the mean, upper, and lower bound estimations of the Multistage fatigue model: a) AZ91 as-cast; b) AZ91-

T6; c) AM60 as-cast.

The percentage of incubation to MSC/PSC growth of the MSF model varied considerably from the lower to the upper bounds. For the large pore sizes (lower bound), the average incubation life increased to approximately $60 \%$ of total life for all three data sets. Whereas, for the smallest pore sizes employed (upper bounds), the average incubation life consisted of approximately $12 \%$ of total life for all three data sets. This variation in the ratio of percentage of incubation life seems reasonable because a larger pore 
size will have a greater plastic zone around the inclusion, resulting in more incubation life. As such, a smaller inclusion will result in a smaller plastic zone and therefore, less incubation life. The capability of the MSF model to capture the scatter typically observed in fatigue data based on microstructure variation alone is directly related to its ability to vary the contribution of incubation and MSC/PSC growth for a given material. Table 4 lists the microstructure related MSF parameters for the mean, upper, and lower bounds estimations for the AM60 and the AZ91 alloys.

Table 3. MSF Parameters for AZ91 and AM60 magnesium alloys

\begin{tabular}{llll} 
Parameter & AZ91as-cast & AZ91 T6 & AM60 as-cast \\
\hline $\mathrm{Cm}$ & 0.85 & 0.85 & 0.85 \\
$\mathrm{CNC}$ & 0.85 & 0.85 & 0.85 \\
$\alpha$ & -0.99 & -0.99 & -0.94 \\
$\mathrm{y} 1$ & 75 & 75 & 15 \\
$\mathrm{r}$ & 0.20 & 0.20 & 0.20 \\
$\xi$ & 2 & 2 & 2 \\
$\mathrm{q}$ & 2.367 & 2.367 & 2.367 \\
$\chi$ & 0.08 & 0.08 & 0.08 \\
$\mathrm{Cl}$ & 50000 & 50000 & 50000 \\
$\mathrm{ClI}$ & 0.005 & 0.005 & 0.005 \\
$\zeta$ & 6 & 6 & 6 \\
$\gamma$ & 0.95 & 0.95 & 0.7 \\
\hline
\end{tabular}

Table 4. Microstructure related MSF Parameters for AZ91 and AM60 magnesium alloys

AZ91 As-Cast AZ91-T6 AM60 As-Cast

\begin{tabular}{lccc|ccc|ccc} 
& $\begin{array}{l}\text { Lower } \\
\text { bound }\end{array}$ & Mean & $\begin{array}{c}\text { Upper } \\
\text { bound }\end{array}$ & $\begin{array}{l}\text { Lower } \\
\text { bound }\end{array}$ & Mean & $\begin{array}{c}\text { Upper } \\
\text { bound }\end{array}$ & Lower & Mpper \\
bound & & bound \\
\cline { 2 - 9 } Pore Size $(\mu \mathrm{m})$ & 495 & 293 & 119 & 554 & 290 & 116 & 600 & 274 & 107 \\
DCS $(\mu \mathrm{m})$ & 19.6 & 16.7 & 13.7 & 19.6 & 16.7 & 13.7 & 19.6 & 16.6 & 13.5 \\
\hline
\end{tabular}

\subsection{Conclusions}

\subsubsection{Fatigue Modeling of AZ91 and AM60 Magnesium Alloys}

- The Multistage fatigue model was correlated to the experimental strain-life results of the AM60 as-cast, AZ91 as-cast, and the AZ91 T6 magnesium alloys. The mean fits of the model were based upon microstructure features, such as dendrite cell size, pore size, and cyclic hardening parameters, in order to capture the differences in fatigue behavior of the three data sets. 
- The multistage fatigue model showed good correlation of the upper and lower bounds of the strain-life behavior of the AM60 and AZ91 magnesium alloys by employing the maximum and minimum inclusion and dendrite cell sizes. Thus, the inclusion (pore) size had the most influence on the scatter in the fatigue lifetimes. We note that the other material constants were not changed for the estimation of the upper and lower bounds.

\section{Fatigue of Friction Stir Spot Welding in Magnesium Alloys}

\subsection{Introduction}

In order to realize the goal of developing a microstructure-sensitive model for predicting fatigue damage in welded joints made using friction stir technology, relationships of microstructural and geometrical features to fatigue performance were investigated using AZ31 magnesium alloy sheets joined by friction stir spot welding (FSSW). Two sets of lap-shear coupons were spot welded using different welding conditions. Optical microscopy of the initial state of the microstructure of each set of spot welds revealed differences in the hook formation, sheet thickness in the weld zone, nugget diameter, and microstructure. Both sets of welds were fatigue tested in load control until failure at various load ratios. Optical microscopy of the failed coupons revealed differences in the fracture mode between the two sets of coupons. Fractography analysis conducted in this study suggested that the effective top sheet thickness largely determined the failure mode, which in turn influenced the final number of cycles to failure. While the height of the interfacial hook was greater in the process with better fatigue performance, it was the larger effective top sheet thickness that promoted crack propagation modes more favorable to greater fatigue resistance. To further aid in determining the cause and effect relationships and to elucidate the mechanisms behind fatigue damage in (FSSW), a linear elastic fracture mechanics model [24] was used to correlate the fatigue life in the two processes. The fatigue model, which is a function of hook size, sheet thickness, and nugget diameter, was used in a sensitivity study to evaluate the effect of sheet thickness in the weld zone, hook height, and nugget diameter.

\subsection{Results}

Figure 19 shows an overview of the fractured specimens tested under cyclic loading for the two different processes (process \#1 and \#2). Table 5 summarizes the fracture mode for process \#1 and \#2. Figures 20 and 21 show the comparison of the experimental to model results for the number of cycles for through-crack initiation versus maximum applied load for $\mathrm{R}=0.3$ and $\mathrm{R}=0.7$, respectively. As the graphs demonstrate, model predictions differ from process \#1 and \#2 results only in terms of sheet thickness, nugget diameter, and hook height. The model showed good correlation in capturing the differences in fatigue lifetimes for the two processes. However, the model was slightly non-conservative compared to process \#1 for $\mathrm{R}=0.7$ and slightly conservative for process \#2 at $\mathrm{R}=0.3$. 

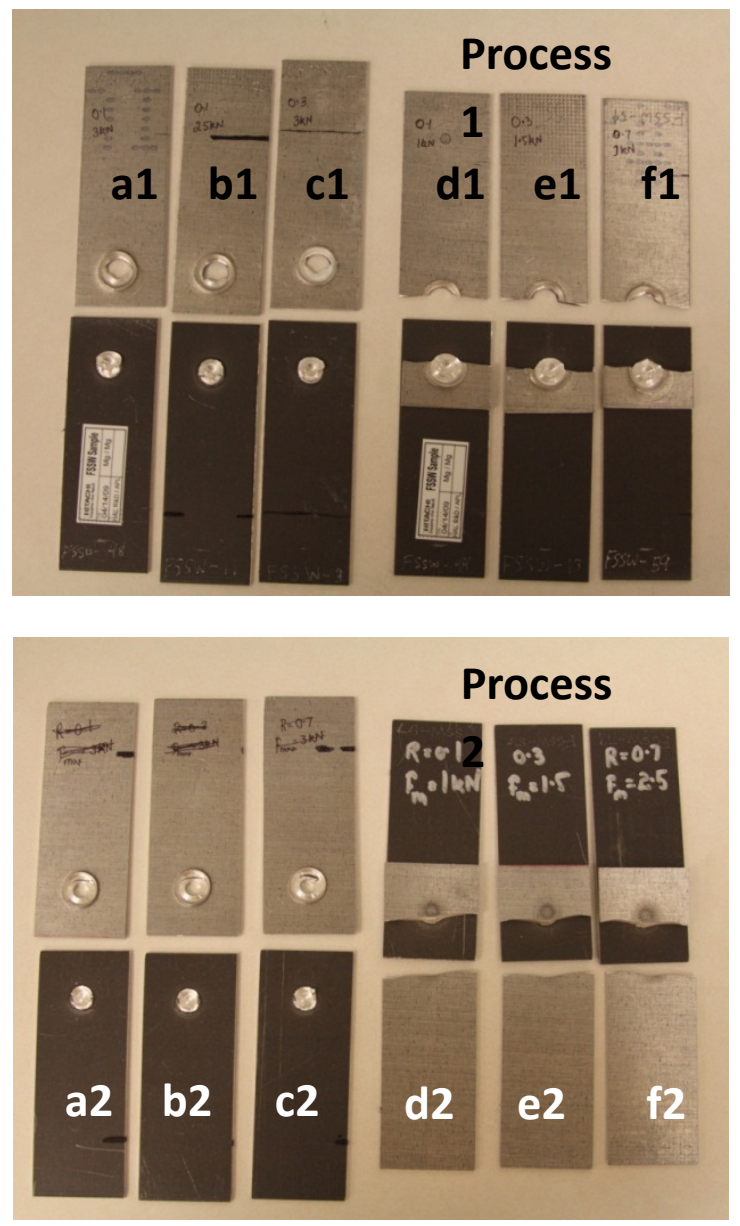

Figure 19. Representative fractured coupons tested at a range of maximum cyclic loads of $1-3 \mathrm{kN}$ at $R=0.1,0.3$, and 0.7 (Refer to Table 5 for a summary of failure modes).

Table 5. Summary of failure modes in coupons of process \#1 and process \#2 ( ID correlates with specimens shown in Fig. 19).

\begin{tabular}{|c|c|c|c|}
\hline ID & $\begin{array}{l}\text { Load Ratio } \\
\text { (R) }\end{array}$ & $\begin{array}{l}\text { Max } \\
\text { Load } \\
(\mathrm{kN})\end{array}$ & Mode of Failure \\
\hline \multicolumn{4}{|r|}{ Process \#1 } \\
\hline a1 & 0.1 & 3 & \multirow{3}{*}{$\begin{array}{l}\text { Dominant crack grew from primary hook into the top sheet, } \\
\text { then halfway around the outer circumference of the nugget, } \\
\text { finally failing by nugget pullout. }\end{array}$} \\
\hline b1 & 0.3 & 2.5 & \\
\hline c1 & 0.7 & 3 & \\
\hline d1 & 0.1 & 1 & \multirow{3}{*}{$\begin{array}{l}\text { Dominant crack grew from primary hook into the top sheet, } \\
\text { halfway around the outer nugget circumference and then } \\
\text { along the width of the top sheet. }\end{array}$} \\
\hline e1 & 0.3 & 1.5 & \\
\hline f1 & 0.7 & 1 & \\
\hline \multicolumn{4}{|r|}{ Process \#2 } \\
\hline a2 & 0.1 & 3 & \multirow{3}{*}{$\begin{array}{l}\text { Dominant crack grew across the weld nugget and parallel to } \\
\text { loading direction in mode II propagation. }\end{array}$} \\
\hline b2 & 0.3 & 3 & \\
\hline c2 & 0.7 & 3 & \\
\hline $\mathrm{d} 2$ & 0.1 & 1 & \multirow{3}{*}{$\begin{array}{l}\text { The Dominant crack grew from root of the secondary hook } \\
\text { into the bottom sheet, propagated around the outer } \\
\text { circumference of the nugget region and then along the width } \\
\text { of the bottom sheet. }\end{array}$} \\
\hline e2 & 0.3 & 1.5 & \\
\hline f2 & 0.7 & 2.5 & \\
\hline
\end{tabular}




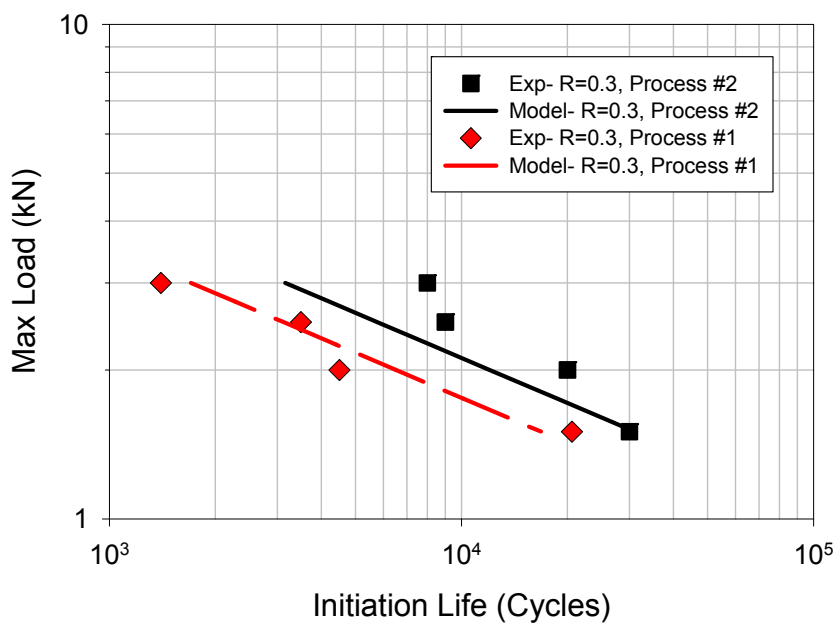

Figure 20. Comparison of the fracture mechanics model to the experimental results of the fatigue initiation life versus maximum load for $\mathrm{R}=0.3$

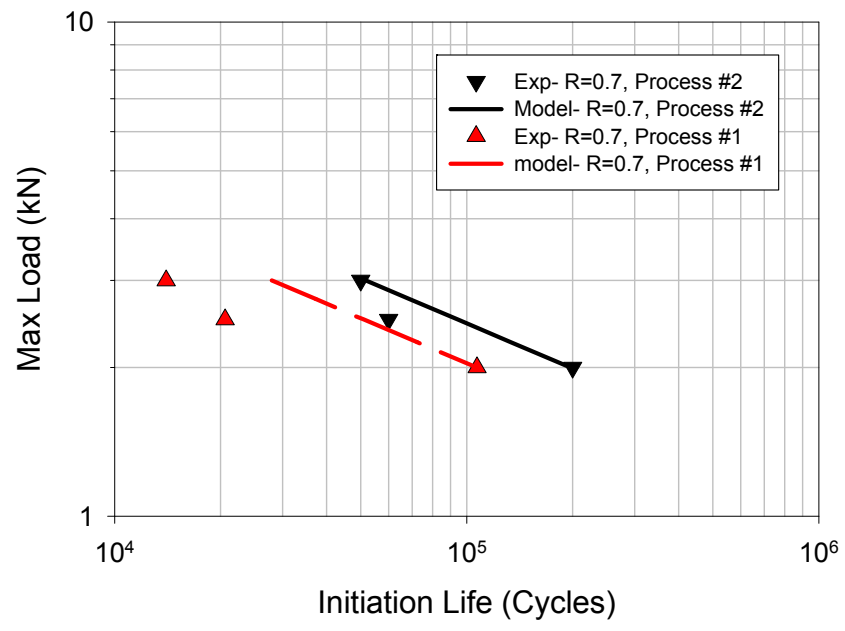

Figure 21. Comparison of the fracture mechanics model to the experimental results of the fatigue initiation life versus maximum load for $\mathrm{R}=0.3$.

\subsection{Modeling of Friction Stir Spot Welding}

In an attempt to determine the parameter that has the greatest impact on fatigue life, three sets of calculations were conducted using the fracture mechanics model. For the first set of calculations, the upper sheet thickness was varied from $1.0 \mathrm{~mm}$ to $2.0 \mathrm{~mm}$. All other parameters were kept the same. The hook height was, $\mathrm{h}=0.8 \mathrm{~mm}$, and nugget diameter was 9.4 . Figure 22 shows the comparison between the three model predictions of maximum cyclic load to the through-crack initiation. The model was sensitive to sheet thickness, displaying orders of magnitude in the number of cycles' difference between the smallest and thickest sheet thicknesses. 


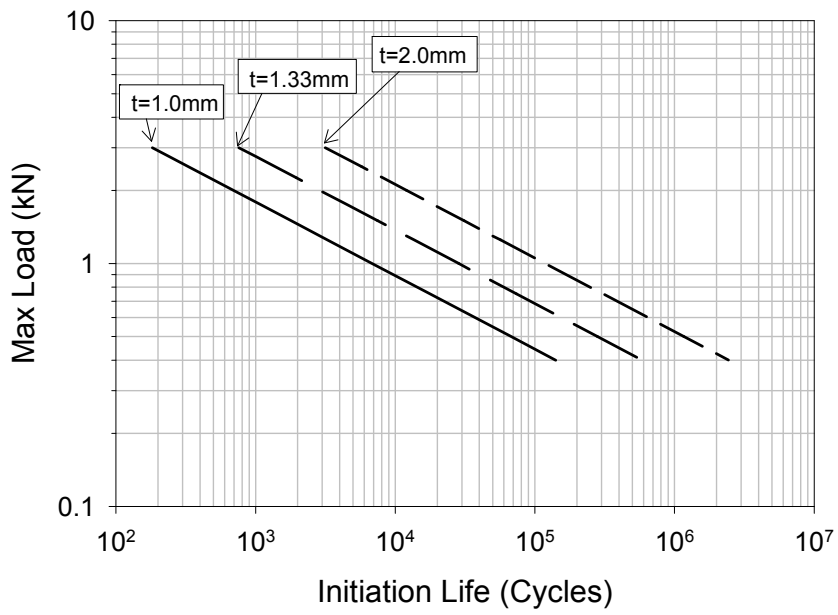

Figure 22. Model predictions of through-crack initiation life for varying sheet thicknesses.

Figure 23 shows the comparison of the model predictions for various interfacial hook heights. In these sets of model calculations, all model parameters except hook height were held constant. The hook height ranged from $0.2 \mathrm{~mm}$ to $2 \mathrm{~mm}$. Thus, three hook-to-sheet ratios were $0.2,0.4$, and 0.8 . In these sets of calculations, the largest hook produced the lowest number of cycles and likewise, the smallest hook height produced the largest number of cycles. Figure 24 shows the fatigue model predictions for various nugget diameters. The choice of nugget diameter was based on reasonable sizes that would be possible for the given tooling used in this study. The smallest nugget diameter gave the greatest fatigue performance and the largest diameter produced the least fatigue performance.

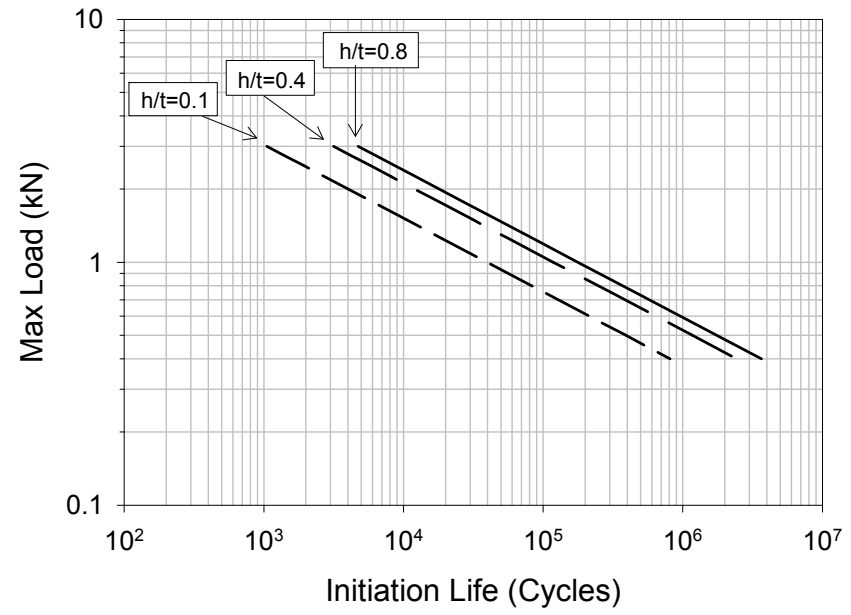

Figure 23. Model predictions of through-crack initiation life for various interfacial hooks. 


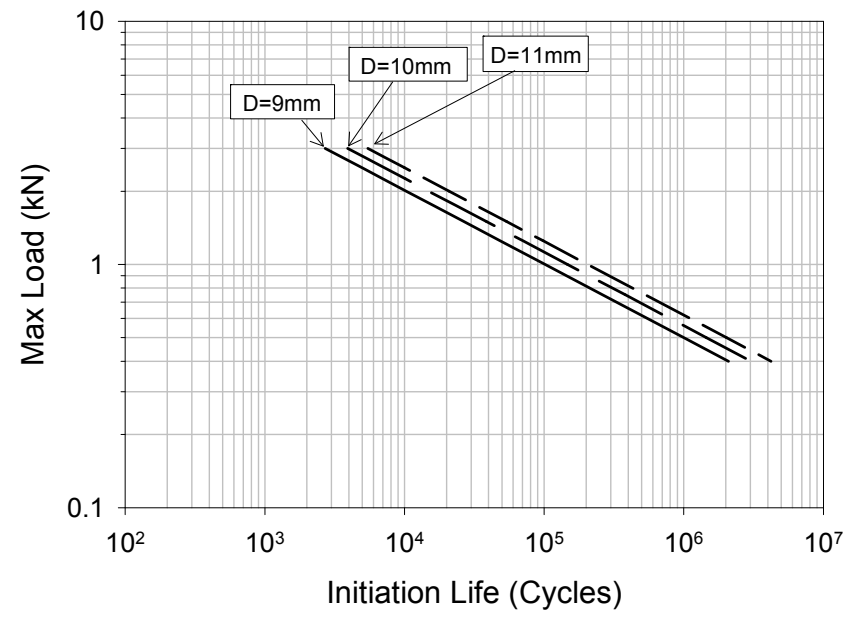

Figure 24. Model predictions of through-crack initiation life for various weld diameter.

The good correlation of the fatigue model to the experimental results shown in Figure 20 and 21, suggests that a linear elastic fracture mechanics approach can be used to determine the welding parameters for optimal fatigue resistance. Despite the fact that process \#2 had a higher hook-to-sheet thickness ratio, the fracture mechanics model predicted better fatigue lifetimes. The distance from the tip of the secondary hook to the top of the sheet in process \#2 was only about $20 \%$ more than the distance from the tip of the primary hook in process \#1. This difference is not significant enough to increase the fatigue life, in some cases, by as an order of magnitude. The assertion that the "effective" sheet thickness is the dominant factor driving fatigue lifetimes is further supported by the parameter sensitivity calculations shown in Figure 22-4. From the calculations, it is clear that sheet thickness has the largest impact on fatigue lifetimes. While the hook height also has a large impact on fatigue lifetimes, the sheet thickness impact was greater. The results of these calculations, can help us explain the greater performance exhibited by process \#2. In process \#2, there were two competing factors, the larger hook height which reduces fatigue life, and the larger thickness which lowers the global stress intensity. Consequently, the smaller shoulder plunge depth may likely be the most important parameter in the FSSW of magnesium AZ31 alloys. In the end, the results of the linear elastic fracture mechanics approach presented here seems to be able to account for competing factors, which to analyze individually would be difficult ascertain.

\section{REFERENCES}

[1] D.L. McDowell, Ken Gall, M.F. Horstemeyer, and J. Fan. Microstructure-Based Fatigue Modeling of Cast A356-T6 Alloy. Engineering Fracture Mechanics, Vol. 70, pp. 49-80, 2003.

[2] Y. Xue, D.L. McDowell, M.F. Horstemeyer, M.H. Dale, and J.B. Jordon.Microstructure-based Multisatge Fatigue Modeling of Aluminum Alloy 7075-T651, Vol. 74, pp. 2810-2823, 2007.

[3] J.B. Jordon, J.B. Gibson, M.F. Horstemeyer, H. El Kadiri, J.C. Baird, A.A. Luo, 2011, Effect of twinning, slip, and inclusions on the fatigue anisotropy of extrusion-textured AZ61 magnesium alloy, Material Science and Engineering A, 528, p 6860-6871.K. 
[4] Gall, M.F. Horstemeyer, D.L. McDowell, and J. Fan. Finite Element Analysis of the stress distributions Near Damaged Si Particles Clusters in Case Al-Si Alloys. Mechanics of Materials, Vol. 32, pp. 277-301, 2000.

[5] D.R. Hayhurst, F.A. Leckie, and D. L. McDowell. Damage Growth under Nonproportional Loading. ASTM STP 853, Vol. Multiaxial Fatigue, pp. 553-558, 1985.

[6] S. Groh, E.B. Marin, M.F. Horstemeyer,and D.J. Bammann. Dislocation Motion in Magnesium: A Study by Molecular Statistics and Molecular Dynamics, Modelling and Simulation in Materials Science and Engineering, Vol. 17, pp. 1-15, 2009.

[7] J.B. Jordon, M.F. Horstemeyer, N. Yang, J.F. Major, K.A. Gall, J. Fan, 2010, Microstructural Inclusion Influence on Fatigue of a Cast A356 Aluminum Alloy, Metall Mater Trans A. 41A, p 356-363.

[8] L.H. Rettberg, J.B. Jordon, M.F. Horstemeyer, and J.W. Jones, "Low-cycle fatigue behavior of diecast Mg Alloys AZ91 and AM60", Material Transactions A [in press].

[9] M.F. Horstemeyer, N. Yang, K.A. Gall, D.L. McDowell, J. Fan, and P. Gullett, "High Cycle Fatigue on a Die Cast AZ91E-T4 Magnesium alloy", Acta Materialia, 52, pp. 1327-1336, 2004.

[10] H. El Kadiri, Y. Xue, M.F. Horstemeyer, J.B. Jordon, and P.T. Wang, "Identification and modeling of fatigue crack growth mechanisms in a die-cast AM50 magnesium alloy", Acta Materialia, 54, n 19, pp. 5061-5076, 2006.

[11] H. El Kadiri, M.F. Horstemeyer, J. Jordon, and Y. Xue, "Fatigue Crack Growth Mechanisms in High Pressure Die-Cast Magnesium Alloys", Metallurgical and Materials Transactions A, Springer Boston, 39(1), 190-205, 2008.

[12] Y. Xue, M.F. Horstemeyer, D.L. McDowell, H. El Kadiri, and J. Fan, "Microstructure-based multistage fatigue modeling of cast AE44 magnesium alloys", International Journal of Fatigue, 29, pp. 666-676, 2007.

[13] H. Mayer, M. Papakyriacou, B. Zettl, and S.E. Stanzl-Tschegg, "Influence on porosity on the fatigue limit of die cast magnesium and aluminum alloys", International Journal of Fatigue, 25, Issue 3, pp. 245-256, 2003.

[14] J.D. Bernard, J.B. Jordon, M.F. Horstemeyer, H. El Kadiri, J. Baird, D. Lamb and A.A. Luo, "Structure-property relations of cyclic damage in a wrought magnesium alloy," Scripta Materialia, 63, pp. 751-756, 2010.

[15] Z.B. Sajuri, Y. Miyashita, Y. Hosokai, and Y. Mutoh "Effects of Mn content and texture on fatigue properties of as-cast and extruded AZ61 magnesium alloys", International Journal of Mechanical Science, Vols. 48, pp. 198-209, 2006.

[16] S. Suresh. Fatigue of Materials, $2^{\text {nd }}$. Ed., Cambridge University Press, United Kingdom, 1998.

[17] S. Begum, D.L Chen, S. Xu, and Alan Luo, "Strain-Controlled Low-Cycle Fatigue Properties of a Newly Developed Magnesium Alloy", Metallurgical and Materials Transactions A, 39A, 3014, 2008. 
[18] D.W. Brown, A. Jain, S.R. Agnew, and B. Clausen, "Twinning and Detwinning During Cyclic Deformation", Materials Science Forum, Vol. 539-543, pp. 3407-3414, 2007.

[19] F. Yang, S.M. Yin, S.X. Li, and Z.F. Zhang, "Crack Initiation Mechanism of Extruded AZ31 Magnesium Alloy in the Very High Cycle Fatigue Regime", Materials Science and Engineering A, 491, pp. 131136, 2008.

[20] S. Morita, S. Tanaka, N. Ohno, Y. Kawakami, and T. Enjoji, "Cyclic Deformation and Fatigue Crack Behavior of Extruded AZ31B Magnesium Alloy", Materials Science Forum, Vols 638-642, pp. 30563061, 2010.

[21] Y. Xue, H. El Kadiri, M.F. Horstemeyer, J.B. Jordon, H. Weiland, 2007, Micromechanisms of multistage fatigue crack growth in a high-strength aluminum alloy, Acta Mater. 55, p 1975-1984.

[22] Y. Xue, A. Pascu, M.F. Horstemeyer, L. Wang, P.T. Wang, 2010, Microporosity effects on cyclic plasticity and fatigue of LENS ${ }^{\mathrm{TM}}$-processed steel, Acta Mater. 58, p 4029-4038.

[23] K. Shiozawa, Y. Tohda, S-M. Sun, 1997, Crack Initiation and small Fatigue Crack Growth Behaviour of Squeeze-cast Al-Si Aluminum Alloys, Fatigue \& Fracture of Engineering Materials and Structures. 20, p 237-247.J.B. Jordon, M.F. Horstemeyer, N. Yang, J.F. Major, K.A. Gall, J. Fan, 2010, Microstructural Inclusion Influence on Fatigue of a Cast A356 Aluminum Alloy, Metall Mater Trans A. $41 \mathrm{~A}, \mathrm{p} 356-363$.

[24] J.B. Jordon, M.F. Horstemeyer, S.R. Daniewicz, H. Badarinarayan, and J. Grantham, 2010, Material Characterization and Modeling of Friction Stir Spot Welds in a Magnesium AZ31 Alloy, J. Eng. Mater. Technol. 132, 041008. 


\section{B. ICME of Extrusion Processing and Post Forming}

Team Members: E. Marin, C. Bouvard, Q. Ma, A.L.. Oppedal

\section{Objective}

Develop physically-based, experimental-validated ICME-based extrusion process models and design methodologies to enhance the manufacturability of magnesium $(\mathrm{Mg}$ ) alloys (AM30). Specifically, this work involves establishing process-structure-property-performance relationships to understand the effect of material state (microstructure), product geometry, and processing parameters on the mechanical properties and final geometry of extruded Mg components as well as on the in-service performance of extruded structural components. The technical activities carried out in this work supported the USAMP-led project related to the development and construction of a vehicle front end sub-structure, which includes the building of ICME tools for advanced Mg and associated applications to the Mg front end.

\section{APPROACH}

Our work on extrusion has been focused on predicting the post-forming structural performance of extruded automotive components, including consideration of the deformation history of the material from the extrusion process. This aspect of our work is summarized in Figure 1, where the research activities have been grouped into five main topics marked as I to $\mathrm{V}$.

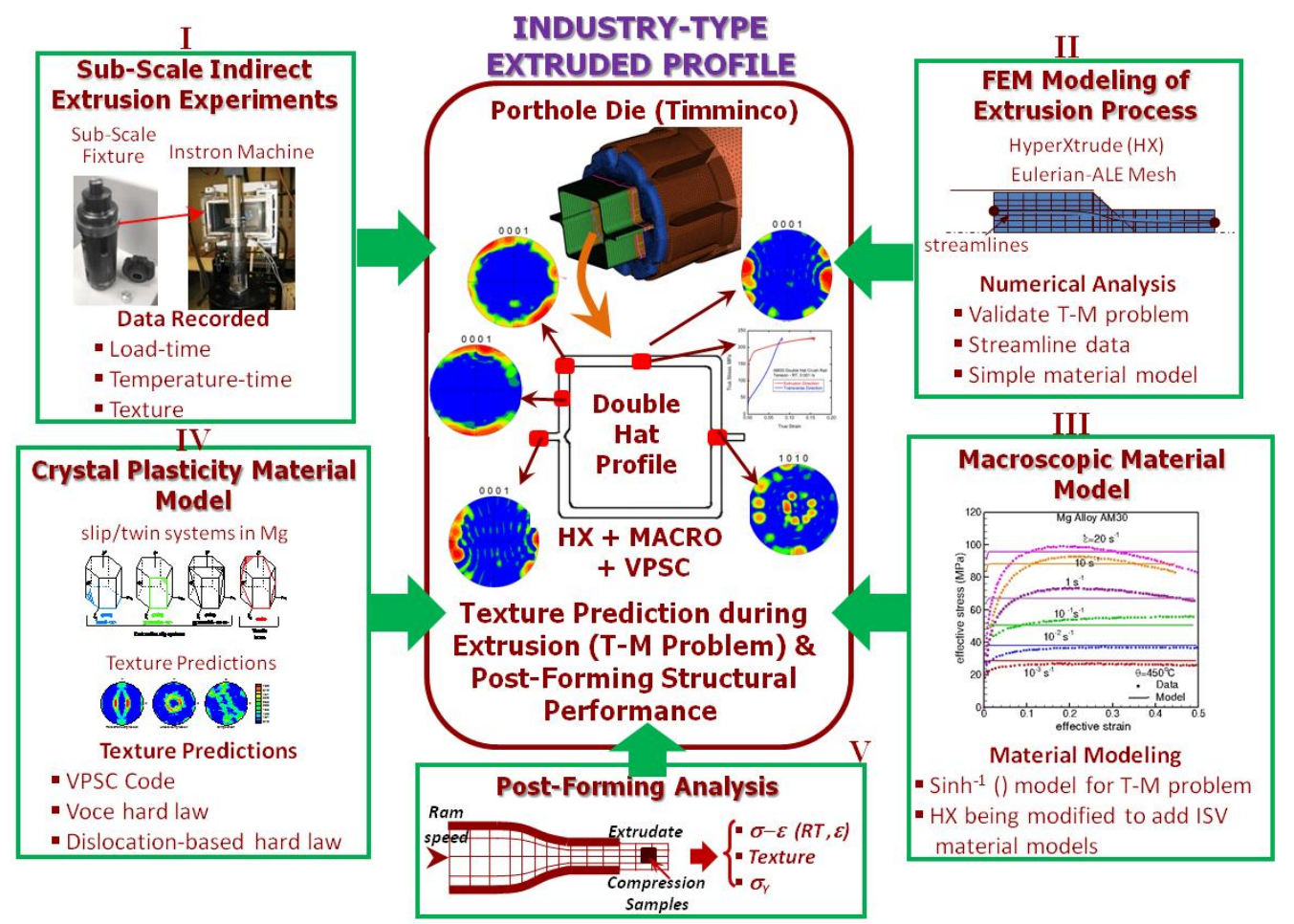

Figure 1. Overview of extrusion work in support of USAMP-ICME project 
Activities in Topic I concentrated on establishing an experimental material database for $\mathrm{Mg}$ alloys using microstructure and mechanical characterization techniques and lab-scale extrusion experiments. The database captured the processing-microstructure-property relationships of these alloys. Actions in Topic II focused on building robust finite element models of the extrusion process using the commercial Eulerian finite element code HyperXtrude. Such process models relied on constitutive models whose development and implementation were the activities addressed in Topics III and IV: macroscopic material models based on an internal state variable (ISV) formalism and meso-scale constitutive models relying on crystal plasticity. Topic $\mathrm{V}$ related to the experiments and modeling of the mechanical response and texture of the extruded material from our lab-scale extrusion experiments to be able to predict the in-service performance of structural extruded parts. This experimental-numerical approach was applied to predict the microstructure, mechanical properties, and structural performance of industry-level extruded profiles.

\section{ACCOMPLISHMENTS}

1. Demonstrated the general experimental capabilities of the lab-scale extrusion fixture to construct a database for the extrusion of Mg alloys. Dies of different designs (conical, flat, dog-bone and porthole) were extracted from AM30 alloy for the experiments. The recorded data captured the effect of processing parameters (ram speed and billet temperature) on both processing variables (temperature histories and load-displacement curves) and evolution of material microstructure (texture, grain morphology).

2. Built robust FE models and performed numerical simulation of the lab-scale extrusion process using HyperXtrude, a commercial Eulerian finite element code widely used to model the extrusion of complex, industrial profiles. Flow-stress type material models (e.g., sine hyperbolic inverse model) were used in the simulations. These models were either available in the code or could be implemented in user-defined functions (UDF) to provide additional capabilities (e.g., strain dependence of some material parameters) to these models. Ongoing work by the code developers is focused on extending the code's capability to add internal state variables models.

3. Used the VPSC crystal plasticity code to predict texture evolution based on streamline information generated by HyperXtrude from an extrusion simulation (uncoupled approach). Material parameters of the model were calibrated using stress-strain responses of pre-extruded AM30 material tested at $450 \mathrm{C}$ and different strain rates $(0.001$ to $0.8 / \mathrm{s})$. Some adjustments to the VPSC source code (strainrate dependence of some hardening parameters) were carried out to better capture the material response at different strain rates. Predicted texture patterns agree well with the trends observed in the experiments.

4. Characterized the mechanical properties (yield strength, strain-stress response) and texture of some $\mathrm{Mg}$ rounded-bar extrudates produced from flat-die experiments with extrusion ratios of 6 and 25 (post-forming experiments). VPSC simulations extended the post-forming stage and, using both the compute extrusion texture and the room-temperature material properties, captured reasonably well the initial yield strength of the extrudate material. Current work is focused on predicting the mechanical response and texture recorded from the post-forming experiments. 
5. Carried out steady-state HyperXtrude simulations using the porthole die for the double-hat profile (Timminco's die). Streamline information extracted using the HyperXtrude post-processor was used to predict texture and stress-strain response at particular locations of the profile to compare with experimental data generated in previous studies. During this work, some issues appeared regarding the performance of this post-processor for large scale models meshed with TET elements. Currently, other venues to obtain streamline information are being explored by the HyperXtrude code developers.

\section{GAPS}

The primary aspects of our current research which require additional attention are as follows:

- Continuum level modeling of dynamic recrystallization (DRX), a phenomenon that typically happens during the extrusion process of $\mathrm{Mg}$ alloys has been clearly identified by the softening response observed in the stress-strain curves of AM30 tested at the extrusion temperature and moderate strain rates. For this modeling work, additional mechanical testing and microstructure studies may be needed for a comprehensive characterization of DRX.

- Fine tuning the VPSC predictions of the stress-strain response and texture. This implies reviewing the hardening law used in the code to make some of the parameters functions of strain rate and temperature. This tuning should enable more accurate prediction of the material response for a wide range of temperatures and strain rates. If time permits, adding DRX predictive capabilities to the code is an additional adjustment that should be considered.

- Direct coupling of ISV material models with HyperXtrude. The current version of HyperXtrude allows mainly for flow-stress type material models, which are embedded in the code or added through user-defined functions (UDF). Ongoing work by the code developers in coordination with CAVS personnel will extend the code's capability to include ISV models.

- Modeling texture-induced anisotropy for the structural response of the extruded part using a continuum model. This modeling feature should be able to capture the directionality of the initial yield strength of the extrudate as predicted naturally by the VPSC code. Besides, this modeling work should also make a connection between the anisotropic yield surface of the extrudate predicted by the VPSC code and the parameters of the macro-scale anisotropic model to be used in modeling the structural performance of the extrudate.

- Modeling damage evolution to predict the ductility of post-formed extruded parts. The ISV model for the structural performance should then include a damage evolution equation with the corresponding material parameters determined from the load-displacement curves of notched tensile specimens.

- Adaptation of the above experimental-modeling methodology to the extrusion process and postforming predictions of new advanced $\mathrm{Mg}$ alloys. This work will involve mechanical and microstructure characterization studies at different loading conditions for calibration of the multiscale-based material model, lab-scale extrusion experiments and simulations, and post-forming experiments and modeling. 


\section{FUTURE WORK}

- Extend the experimental capabilities of the lab-scale extrusion fixture to perform extrusion experiments for selected advanced Mg alloys and for a given die geometry to generate data for model validation. Processing parameters should be selected to mimic as closely as possible industry extrusion conditions.

- Improve current CAVS ISV constitutive framework to capture the effect of microstructure features on the stress-strain response of Mg alloys. Specifically, the framework should be (i) extended to account for DRX of Mg alloys during the extrusion process, and (ii) enhanced to model textureinduced anisotropy and damage of the selected $\mathrm{Mg}$ alloys to capture the anisotropic and failure responses of extruded parts during service. This enhancement should be complemented with mechanical and microstructure characterization data needed to determine the model parameters of the improved material model.

- Enhance the VPSC crystal plasticity code to account for DRX during texture predictions. Currently, VPSC is used to predict texture evolution during extrusion using streamline information generated from HyperXtrude (uncoupled approach). However, the present version does not have DRX capabilities, and hence, the predictive texture for complex profiles (e.g., use of porthole dies) does not agree well with measured results.

- Develop new capabilities of HyperXtrude to allow the direct coupling of the code with ISV material models. Such capabilities are currently being introduced by the HyperXtrude code developers. The first enhanced version of the code will use scalar ISVs in the context of viscoplastic constitutive frameworks. Later extensions will consider tensorial ISVs. These new enhancements are needed for implementing viscoplastic material models that account for DRX.

- Complement current post-forming experiments and modeling for a comprehensive characterization of the post-forming yield strength, texture and stress-strain response of the lab-scale extrudates. Also, carry out microstructure and mechanical characterization studies on coupons extracted from the structural extruded part of the DEMO structure to evaluate the predictive capabilities of the developed ICME-based tools. In addition, study the structural response of the DEMO component using the developed performance models for a variety of loading conditions, including quasi-static and high-rate (crash) loading.

Notably, the activities proposed as future work have been identified to provide effective support to the research planned in the USAMP-led project - Task 11: "Demonstration Project to Develop and Construct a Mg Intensive Vehicle Front End Structure." Such research relates to the ICME for advanced Mg and application of the ICME tools to the Mg front end. Main goals in this task are (i) to develop processingstructure-property relationships for extruded components for selected magnesium alloys (to be specified by USAMP), and (ii) to apply the developed modeling tools to establish the propertyperformance relationship of the magnesium extruded component as related to a "Demo" structure. 


\section{LAB-SCALE EXTRUSION EXPERIMENTS}

\subsection{A Lab-Scale Fixture for Indirect Extrusion Experiments}

A lab-scale indirect extrusion fixture has been designed and built to study details of the extrusion process of $\mathrm{Mg}$ alloys as well as to generate experimental data for validation of the simulation tools. In essence, the fixture consists of (i) a chamber, (ii) a replaceable die, and (iii) a base. The chamber consists of two parts (top - bottom), each having an internal sleeve. A clamp holds these two parts together. Billets of 1 1/4" diameter and 1" length can be extruded using different die geometries (e.g., conical and flat dies) with extrusion ratios in the range of 6.25 to 125. Processing parameters that can be controlled are billet temperature and ram speed, and processing variables that can be recorded are extrusion load versus displacement and temperature histories at particular points in the die. Microstructure information (texture, grain morphology) as well as material flow patterns can be characterized on the extruded sample using microscopy techniques. Details of the fixture and its set-up on the testing machine for the extrusion experiments are shown in Figure 2.

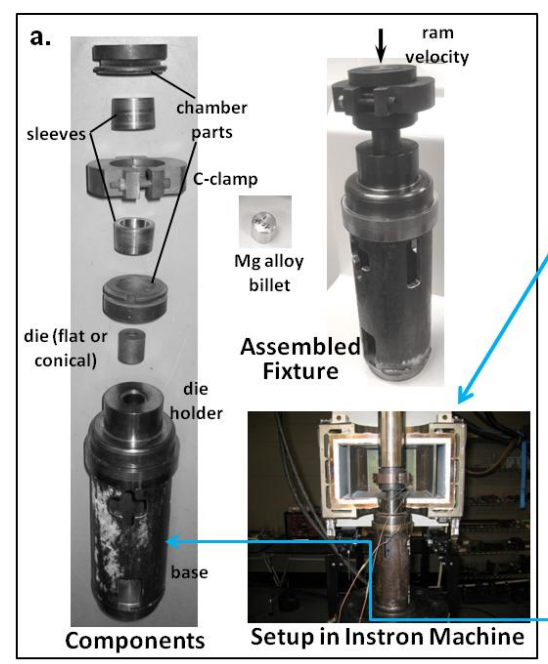

Extrusion Fixture

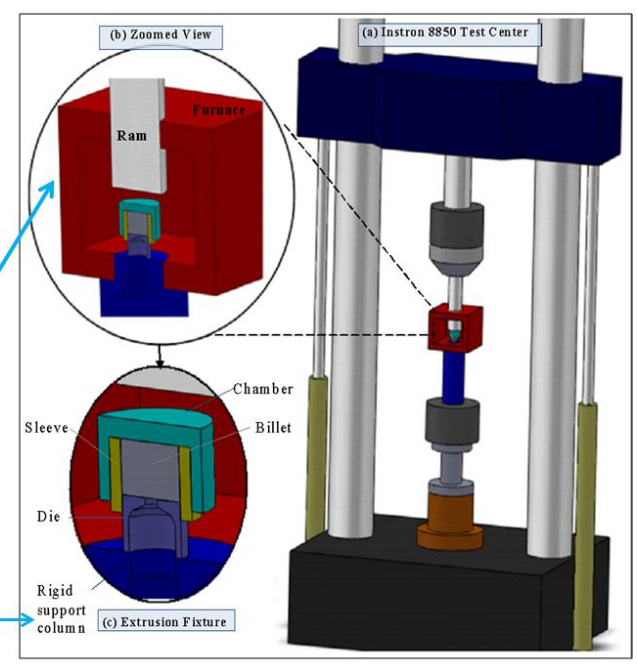

Schematic of SetUp

Figure 2. Details of the lab-scale fixture for indirect extrusion and set-up on the Instron machine.

The lab-scale fixture has been designed to accommodate different die geometries as shown in Figure 3. Three different profiles can be generated with these dies: circular cross section, dog-bone cross section and thin-walled circular tube. Up until now, we have performed experiments using the conical and flat dies for circular profiles, results for which are reported below, while experiments using the other die geometries are in progress. We also successfully extruded dog bone shaped profiles using a flat die. 


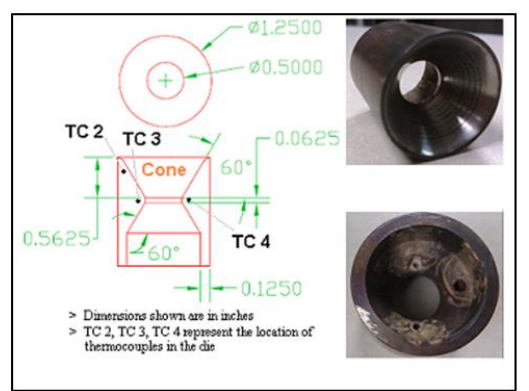

Conical die

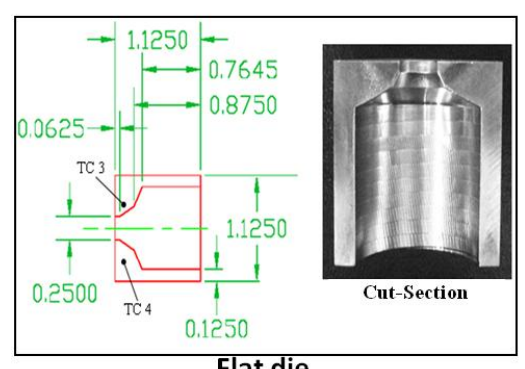

Flat die

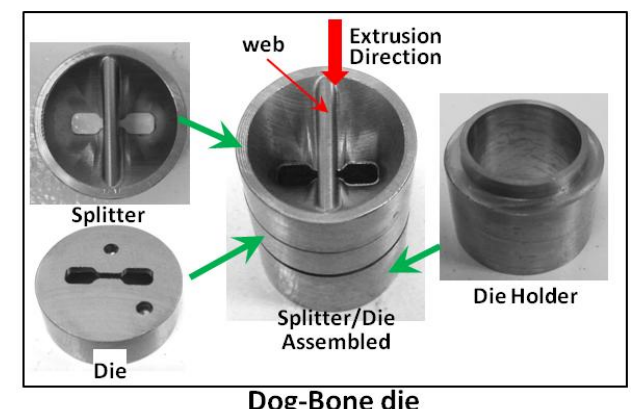

Dog-Bone die

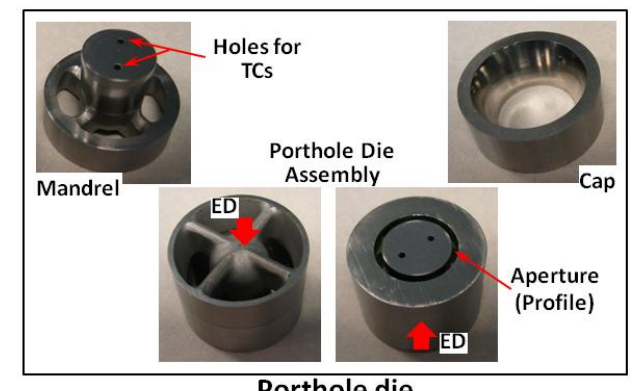

Porthole die

Figure 3: Different die geometries designed for the lab-scale extrusion experiments.

\subsection{Lab-Scale Extrusion Experimental Results}

The end goal of these experiments is to build an experimental database for extrusion of $\mathrm{Mg}$ alloys that can be used to validate numerical models of the process, including finite element, robust internal state variable, and polycrystal plasticity material models. Figure 4 presents the thermo-mechanical data (load and temperature histories) recorded from the extrusion experiments using the conical and flat dies to extrude solid circular cross section profiles. The conical die experiments were performed on $\mathrm{Mg}$ alloy AZ61 with a billet temperature of $454^{\circ} \mathrm{C}$, a ram speed of $5 \mathrm{~mm} / \mathrm{min}$, and an extrusion ratio of $6.25\left(1 / 2^{\prime \prime}\right.$ profile diameter). As shown in Figure 4, these experiments used two sample geometries (billet and billet-pocket) and were designed to compare the predictive capabilities of Lagrangian (ABAQUS) and Eulerian (HyperXtrude) finite element codes. On other hand, the flat die tests used Mg alloy AM30 and were performed at a billet temperature of $454^{\circ} \mathrm{C}$, ram speeds of 5 and $10 \mathrm{~mm} / \mathrm{min}$, and an extrusion ratio of 25 ( $1 / 4^{\prime \prime}$ profile diameter). Note that, although not reported here, flat-die experiments with other ram speeds $(15,20,30,40 \mathrm{~mm} / \mathrm{min})$ have also being carried out. 
Flat-Die AM30 Extrusion
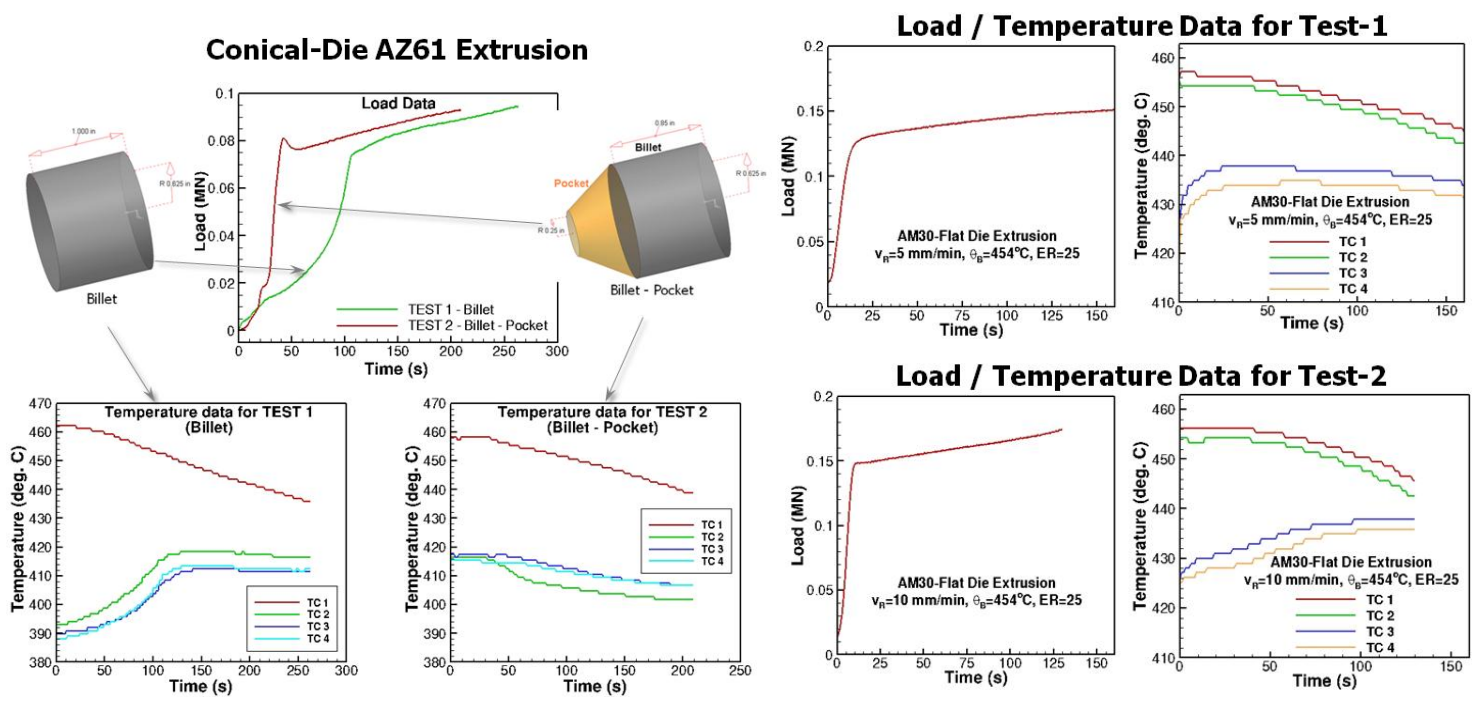

Load / Temperature Data for Test-2
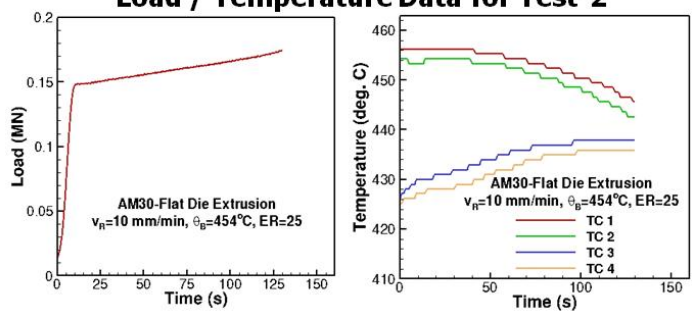

Figure 4. Lab-scale experimental results using the conical die for AZ61 extrusion and the flat die for AM30 extrusion.

Figure 5 shows the flow patterns, texture, and grain morphology at the exit of the bearing area, along the axis of the billet (center), for flat die extrusion at different ram velocities. Clearly, at the selected material point for all cases, the initial texture of the pre-extruded billet evolves towards a rodtype texture typical of extrusion, being stronger for higher ram speeds. Also not shown here are shearing effects due to the die wall, which usually tend to weaken this texture, and are not predominant at these points because indirect extrusion minimizes the frictional effect as compared to direct extrusion. Also, the grain morphology depicted by the micrographs from scanning electron microscopy (SEM) shows a refined grain structure typical of a dynamic recrystallization (DRX) process, a phenomenon usually occurring during hot extrusion of Mg alloys.
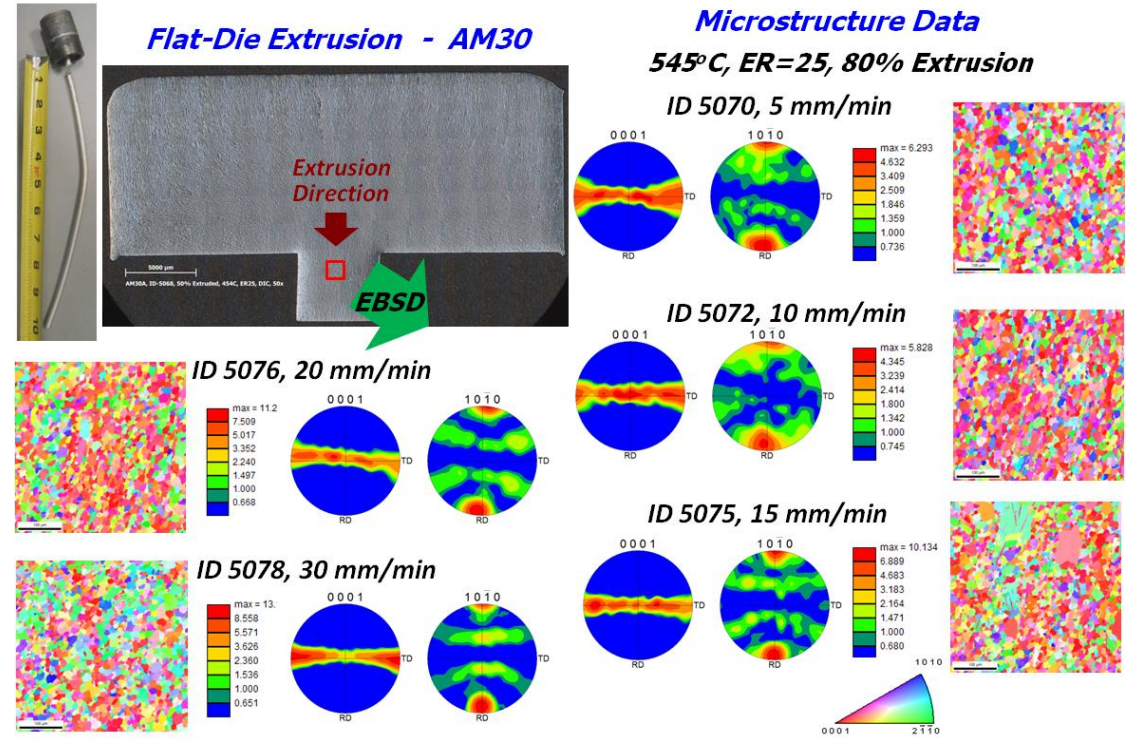

Figure 5. Material flow lines and microstructure of partially extruded billets using SEM-EBSD. 


\section{Constitutive Modeling - Internal State Variable and Crystal Plasticity Modeling APproaches}

Modeling the inelastic behavior of magnesium alloys is important for simulating metal forming processes, such as extrusion, as well as for estimating the in-service structural response of extruded components during large deformation events. When these alloys are subjected to intricate loading histories, the effect of microstructure on the mechanical properties becomes crucial. Hence, accurate predictions of the inelastic behavior during thermo-mechanical processing and in-service performance must then rely on employing microstructure-based constitutive frameworks. In this work, both a macroscale internal state variable (ISV) and a meso-scale crystal plasticity model were used as main tools for predicting the evolution of microstructure and material state during extrusion. A brief description of these material model frameworks is presented in Figure 6.

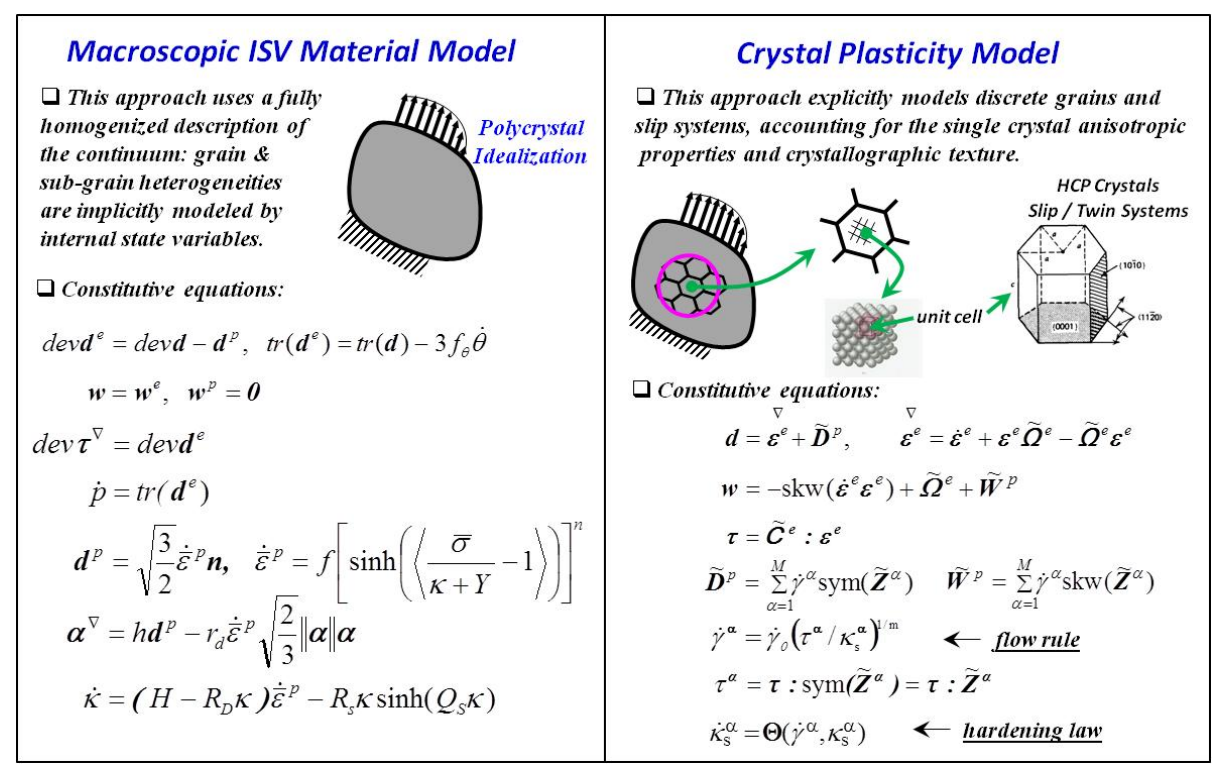

Figure 6. Constitutive Frameworks to Model the Extrusion of Magnesium Alloys

Mathematically, the formulation of these microstructure-based models relies on a consistent description of the large deformation kinematics coupled with a thermodynamic approach as proposed by Coleman and Gurtin [1967]. Physically, these models are based upon microstructure-property relations, a key aspect that allows the material microstructure details to be admitted into a numerical analysis. When the microstructure-property relations are included in the constitutive equations, history effects can then be captured at the macro-scale as the process and performance environments are connected through these relations. Numerically, these models have been implemented in implicit/explicit Lagrangian finite elements codes through user material routines. For the case of Eulerian codes, extension of the user interface offered by current commercial software will need to be enhanced to admit the use of these microstructure-based constitutive equations.

Our current work on modeling the extrusion process relies on using HyperXtrude, which uses an Eulerian formulation of the fundamental differential equations that govern flow and heat transfer of incompressible viscous fluids. The current version of the code allows the use of equation-of-state (rigid- 
viscoplastic or flow stress) type models where the stress is expressed as a function of strain, strain rate, and temperature. A number of such models are available in the code (material model library), and they can be implemented through a user-Defined Function (UDF). This last possibility is particularly useful if material parameters as a function of strain are to be used to improve the predictions of the material model. A specific constitutive equation being used in our simulations is the sine hyperbolic inverse model which is expressed as:

$$
\bar{\sigma}=\hat{\bar{\sigma}}(\dot{\bar{\varepsilon}}, \theta)=\frac{1}{c_{1}} \sinh ^{-1}\left[\left(\frac{Z}{c_{2}}\right)^{1 / c_{3}}\right], \quad Z=\dot{\bar{\varepsilon}} \exp \left(\frac{c_{4}}{\theta}\right)
$$

where $c_{1}, c_{2}, c_{3}, c_{4}$ are material constants to be determined from experimental stress-strain curves at different strain rates and temperatures. For a given strain rate and temperature, the above model predicts a constant stress level, a feature that seems to capture well the response of aluminum alloys when deformed at high temperatures. However, magnesium alloys dynamically recrystallize during deformation and as such they show a stress softening response. This behavior can be captured with the above model by making $c_{3}$ a function of strain, as shown in Figure 7.

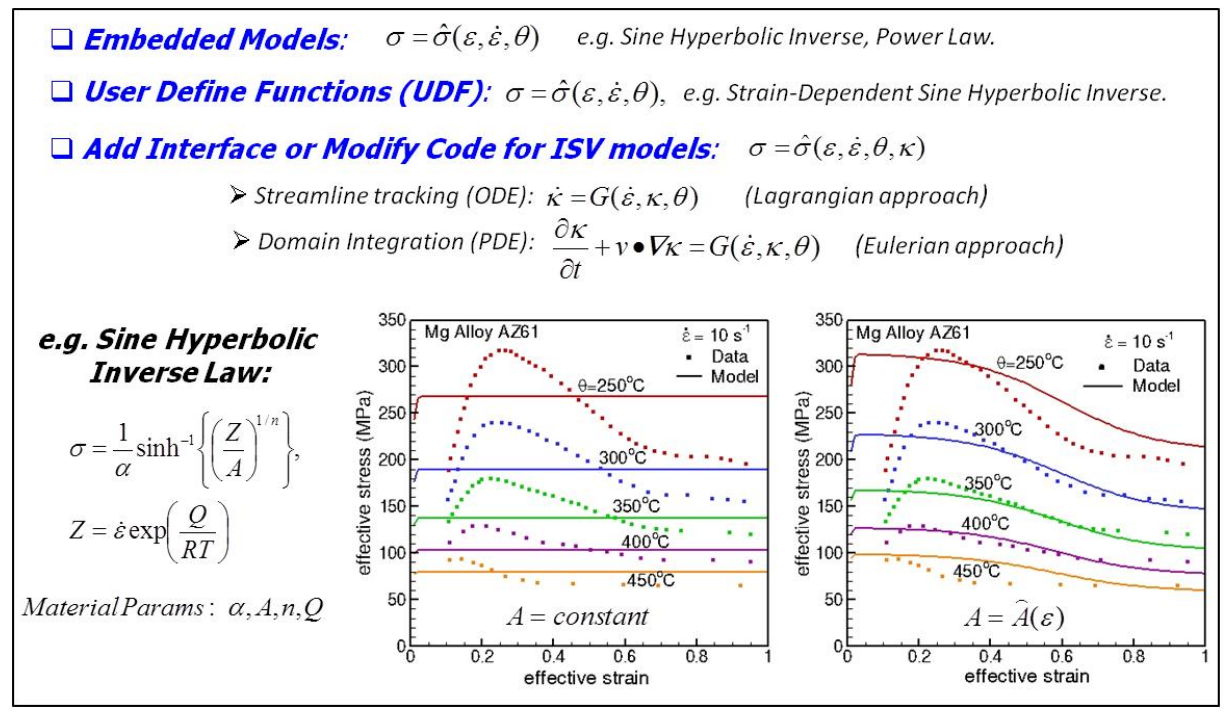

Figure 7. Approaches to Model Material Response in HyperXtrude

Figure 7 also shows the methodologies to be used for coupling the code with ISV material models ( $\kappa$ represents a scalar internal state variable). Either of the two approaches listed in this figure requires modification of the code and enhancements of the user interface for UDF. Currently, HyperXtrude code developers are working on extending the capabilities of the code based on the Eulerian approach (domain integration) considering scalar state variables. Main aspects of this extension for one internal variable, $\kappa$, are shown in Figure 8 . The additional non-linear iteration loop shown as the VISCOSITY step will solve the partial differential equation (PDE) for the evolution law of the state variable in the whole Eulerian domain (control volume). In this case, the functional form (right hand side) of the evolution equation is programmed in a UDF. Also, the interface for computing the flow stress (or equivalently, the nonlinear viscosity $\eta$ ) should be enhanced to admit the state variable as an argument in the call for this 
UDF. Finally, the check on convergence for a particular time step will also consider the norm of $\kappa$ in addition to the norms for temperature $(\theta)$, velocity $(\boldsymbol{v})$ and pressure $(p)$.

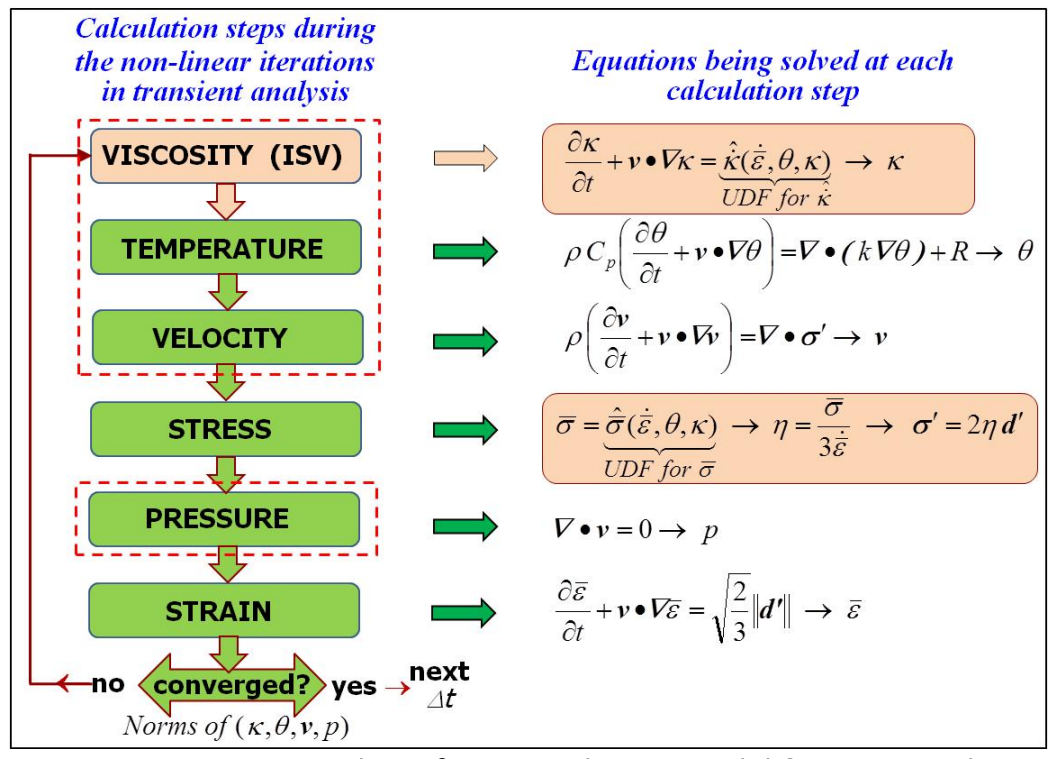

Figure 8. Direct Coupling of a one scalar ISV Model \& HyperXtrude

The extensions of HyperXtrude are being tested using a simplified rigid-viscoplastic version of the ISV material model shown in Figure 6. The particular functional forms for the flow stress and the evolution equation of the state variable are also given in the figure. As noted before (see Figure 7), the typical Lagrangian form for the evolution equation needs to be transposed into an Eulerian setting for numerical implementation. Also, one needs to use two UDFs to define the functional form of the nonlinear viscosity and the evolution equation for the state variable. Note that for monotonic loading, the effect of elasticity is noted mainly at the beginning of the stress-strain curve, and hence the rigidviscoplastic approximation seems to be reasonable for large strain deformation problems such as extrusion.

It is important to point out here that crystal plasticity models which are needed to predict the evolution of microstructure (texture) will be implemented in an uncoupled approach, as noted in later sections of this report. 


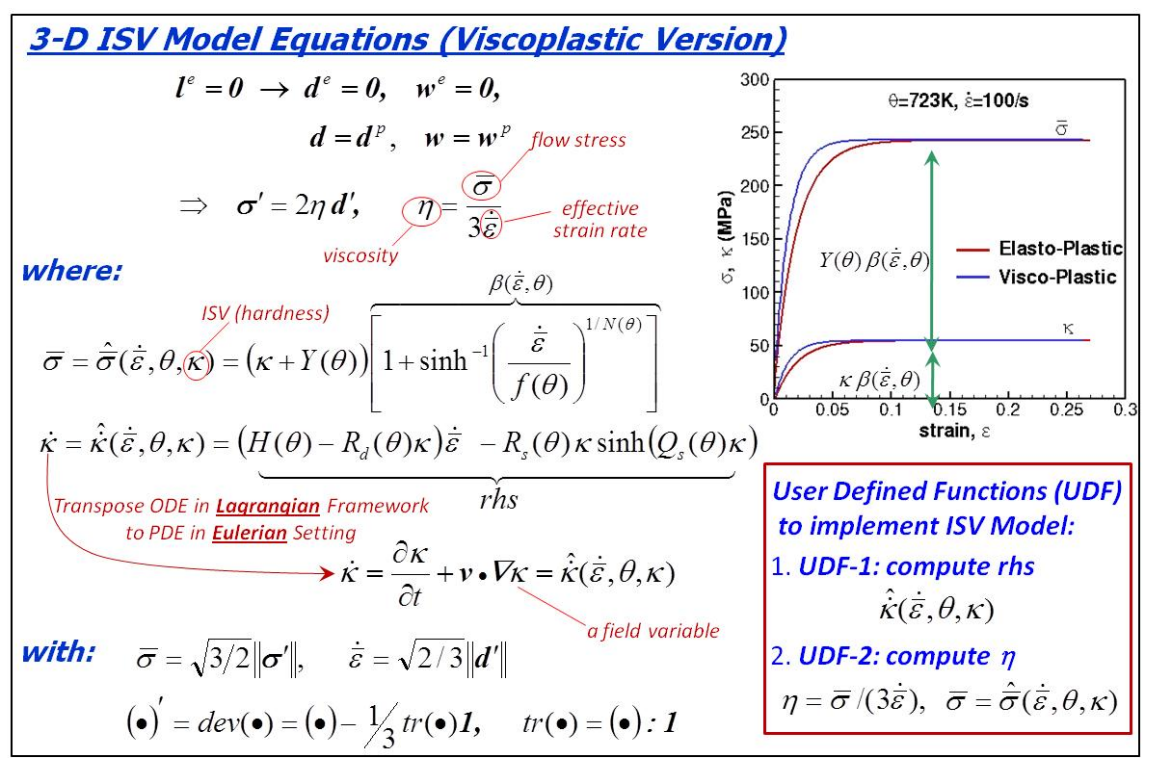

Figure 9. Implementing the Viscoplastic Version of an ISV Material Model.

\section{Extrusion Process Modeling}

\subsection{Finite Element Approaches to Model Extrusion}

Extrusion is a metal forming process used to produce long complex profiles with high tolerance and surface quality. Unlike other material forming processes, such as forging and rolling, the profile extrusion process usually has complex material flow patterns accompanied with extremely large and severe plastic deformation. Such a feature puts rigorous requirements on the numerical tools used to simulate the extrusion process. As Lagrangian codes are limited in significant ways for simulating general 3-D material flows through complex die geometries (e.g., porthole dies) unless intensive re-meshing is employed, in this work we rely on Eulerian finite element formulations to perform the extrusion simulations. One such software is HyperXtrude, a code developed by Altair Engineering, Inc. Figure 10 depicts the main differences between the Lagrangian and the Eulerian approaches.

HyperXtrude is a finite-element based code designed to model/simulate the non-isothermal material flow during metal extrusion. The code uses an Eulerian formulation of the fundamental differential equations that govern flow and heat transfer of non-Newtonian incompressible viscous fluids. As such, the code uses a fixed-space control volume representation of the problem domain through which the material flows as it is extruded through the tooling. 


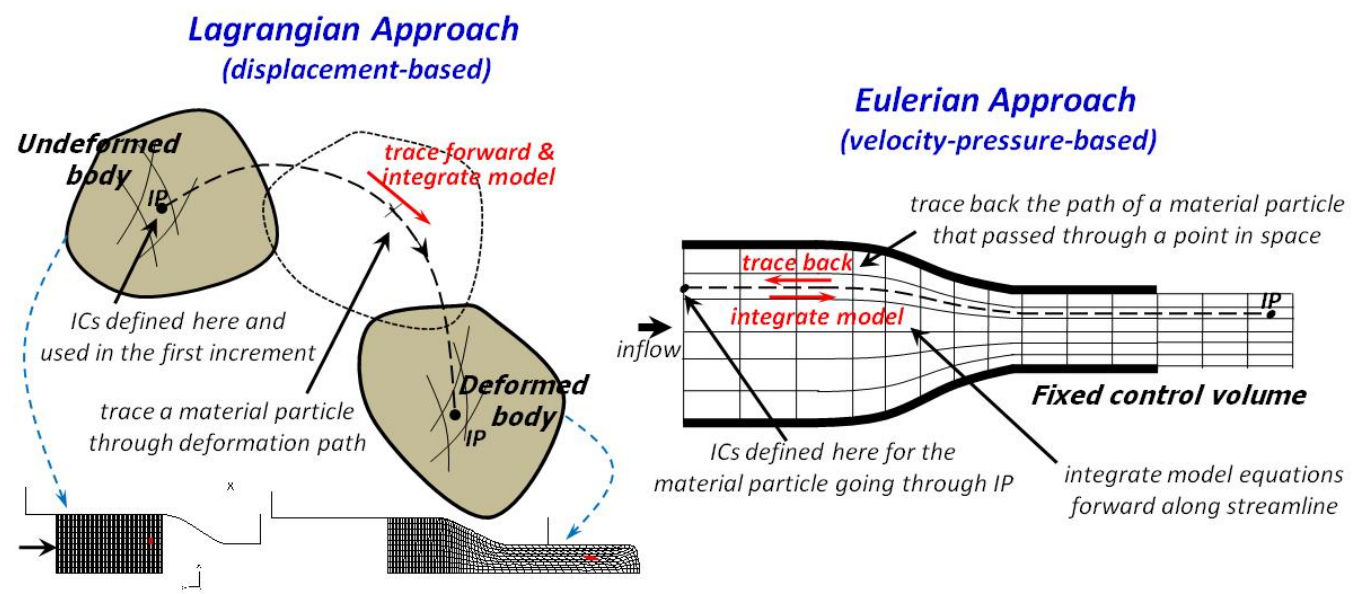

Figure 10. Lagrangian versus Eulerian Finite Element Approaches to Model Extrusion

Figure 11 presents the HyperXtrude finite element models constructed to simulate the lab-scale experiments with the conical and flat dies, respectively. Due to symmetry, only a quarter model has been used. Note that the models include the tooling to have more flexibility when specifying the boundary conditions, in particular the thermal ones, an important aspect when validating the models. The three models representing the lab-scale extrusion carried out at CAVS are composed of a die (flat or conical), a container, and the material flow (billet, bearing and profile). Each of these components has mechanical and thermal interactions between tooling and material. Being able to evaluate the correct interactions or boundary conditions is a key to simulating the temperature and load behavior during the process. Regarding the industrial porthole die, a full 360 degree model was created to make it possible to study profile deflection after the bearing area. Assuming symmetry in the model prevents the user from carrying out such a study due to the non-symmetric character of the phenomenon at the die exit. To avoid having a large model, the tooling was not modeled in the porthole die.
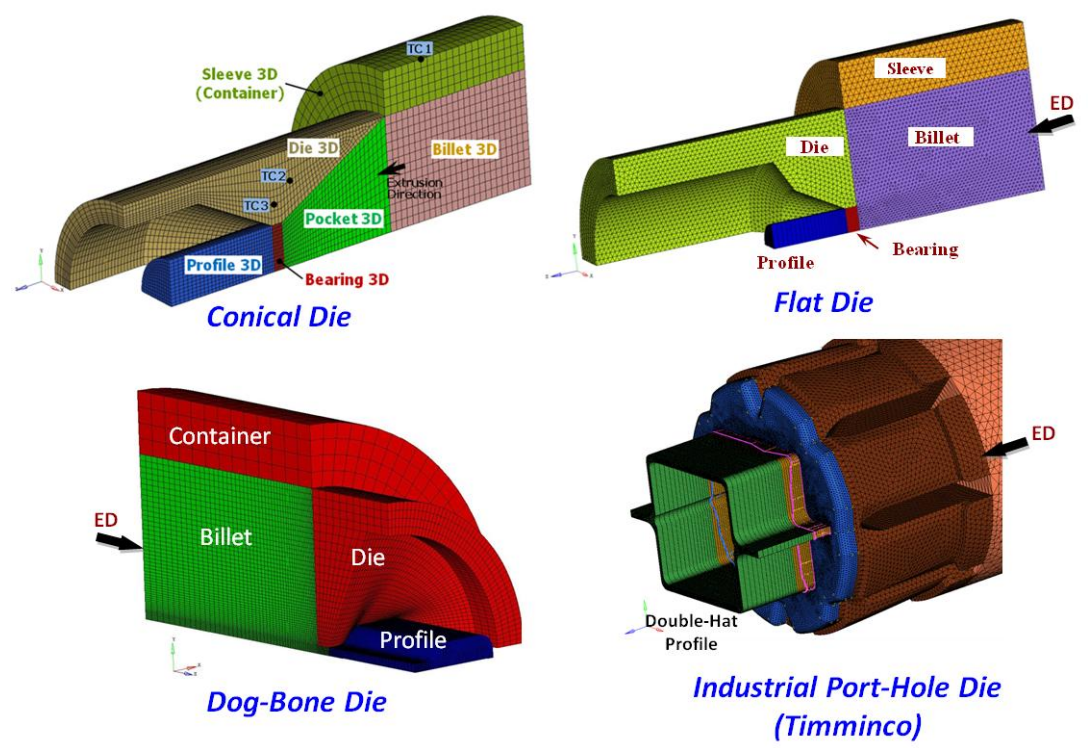

Figure 11. HyperXtrude Eulerian finite element models for simulating the extrusion process of conical, flat, dogBone and Porthole Dies. 


\subsection{Prediction of the Lab-Scale Thermo-Mechanical Extrusion Processes}

As described in the previous sections, several die geometries have been used to extrude AM30 and AZ61 Mg alloy billets at CAVS. The main purpose of these experiments was to generate quality data to fine-tune extrusion process models. By studying load data and temperature histories at various locations in the tooling, extrusion models were able to provide fairly accurate predictions and material behavior during the process. Figure 12 shows the predictions of load and temperature histories for the extrusion of an AZ61 billet through a conical die. The left top corner graph depicts the material model behavior compared to experimental data at $350^{\circ} \mathrm{C}$ and at different strain rates. This Sinh-1 law embedded in HyperXtrude is used for the simulations. The two bottom plots show load-time and temperature-time curves recorded during the extrusion test. Notice that the simulated histories match fairly well the experimental data that were recorded. The temperature histories were recorded the surface of the sleeve (top curve that starts at $460^{\circ} \mathrm{C}$ ) and in the die (bottom curves).
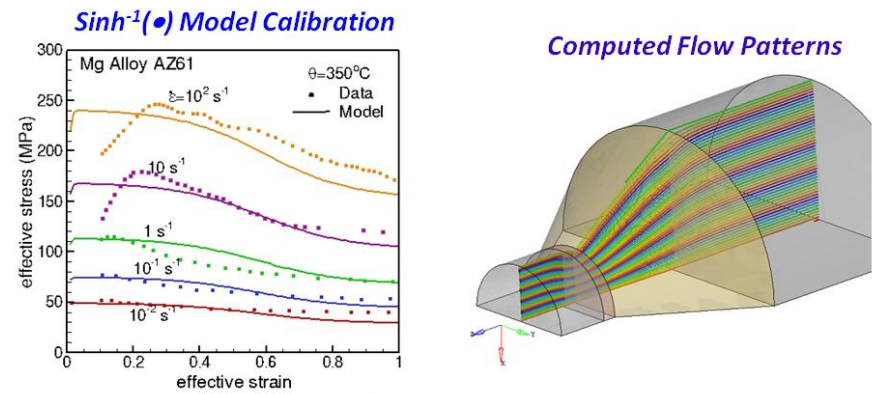

Prediction of Load and Temperature Histories
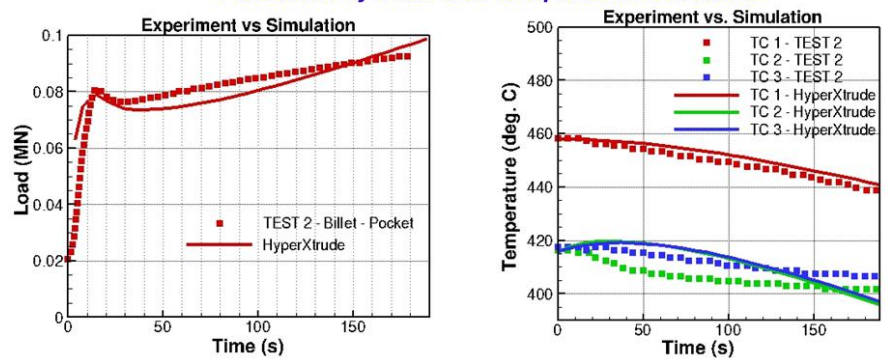

Figure 12. Thermo-Mechanical Validation of Conical Die Experiments - AZ61

Table 1 shows the detailed boundary conditions at each interface of the problem [1]. It is important to note that the values of the convection coefficients are highly dependent upon the temperature range of the problem and the ram speed. On the other hand, the mechanical boundary conditions depend solely on the actual experimental apparatus (i.e., geometry of the die, friction reduction methods, etc.).

Figure 13 shows another validation that was carried out using a flat die with a circular opening and an AM30 billet. This set of validations included extrusions at $5 \mathrm{~mm} / \mathrm{min}$ and $10 \mathrm{~mm} / \mathrm{min}$ as well. We can see that the simulated results match the experimental data in the case of ram speeds of $5 \mathrm{~mm} / \mathrm{min}$ and $10 \mathrm{~mm} / \mathrm{min}$. 
Table 1. Boundary conditions for AZ61 extrusion process at $5 \mathrm{~mm} / \mathrm{min}$ in a conical die

\begin{tabular}{|c|c|c|}
\hline $\begin{array}{l}\text { Region } \\
\text { [HyperXtrude BC type } \\
\text { ID] }\end{array}$ & Thermal Boundary Condition & Mechanical Boundary Condition \\
\hline $\begin{array}{l}\text { External surfaces of Die } \\
\text { and Sleeve } \\
\text { [ToolSurface] }\end{array}$ & Heat flux $=0$ & 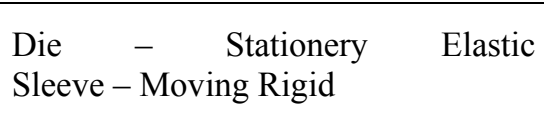 \\
\hline $\begin{array}{l}\text { Bearing-Die interface } \\
\text { [SolidFluid Interface] }\end{array}$ & $\begin{array}{l}\text { Heat flux }=0 \\
\text { (Since the length of bearing is very } \\
\text { small and the material is in contact } \\
\text { for a very short time) }\end{array}$ & $\begin{array}{l}\text { Coulomb friction, } \\
\mu=0.9\end{array}$ \\
\hline $\begin{array}{l}\text { Pocket-Die interface } \\
\text { [SolidFluid Interface] }\end{array}$ & $\begin{array}{l}\text { Convection } \\
\mathrm{h}=300 \mathrm{~W} / \mathrm{m}^{2} \mathrm{~K}\end{array}$ & $\begin{array}{l}\text { Full-stick friction on flow mesh side } \\
\text { to simulate dead metal zone. }\end{array}$ \\
\hline $\begin{array}{c}\text { Billet-Container } \\
\text { interface } \\
\text { [SolidFluid Interface] }\end{array}$ & $\begin{array}{l}\text { Convection Coefficient, } \\
\mathrm{h}=50 \mathrm{~W} / \mathrm{m}^{2} \mathrm{~K}\end{array}$ & $\begin{array}{l}\mathrm{X} \text { Velocity }=0 \text {, Y Velocity }=0 \\
\text { traction }=0 \text { (On flow mesh) } \\
\text { (Unlike the full-stick friction } \\
\text { observation at billet-container } \\
\text { interface, the BC we used is } \\
\text { characteristic to our test setup.) }\end{array}$ \\
\hline $\begin{array}{l}\text { Profile free surface } \\
\text { [FreeSurface] }\end{array}$ & $\begin{array}{l}\text { Convection Coefficient, } \\
\mathrm{h}=510 \mathrm{~W} / \mathrm{m}^{2} \mathrm{~K}\end{array}$ & $\begin{array}{l}X \text { traction }=0, Y \text { traction }=0 \\
\text { and } Z \text { traction }=0\end{array}$ \\
\hline $\begin{array}{l}\text { DummyBlock End } \\
\text { [SolidFluid Interface] }\end{array}$ & Heat Flux $=0$ & $\begin{array}{l}X \text { traction }=0, Y \text { traction }=0, \\
Z \text { Velocity }=5\end{array}$ \\
\hline
\end{tabular}

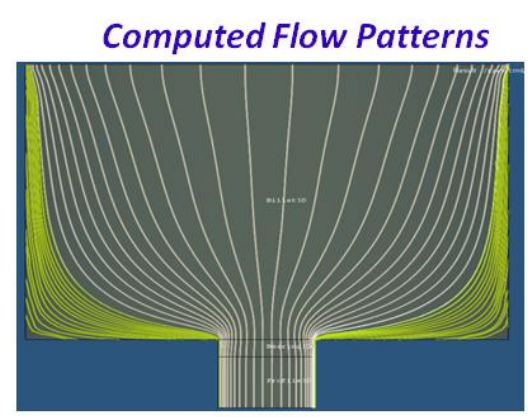

$\operatorname{Sinh}^{-1}(\bullet)$ Model Calibration

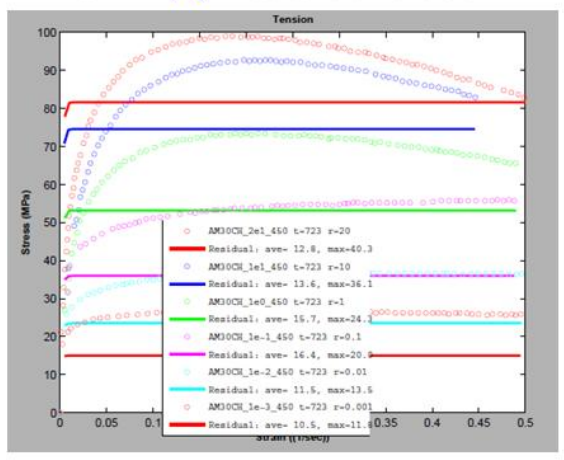

Prediction of Load and Temperature Histories
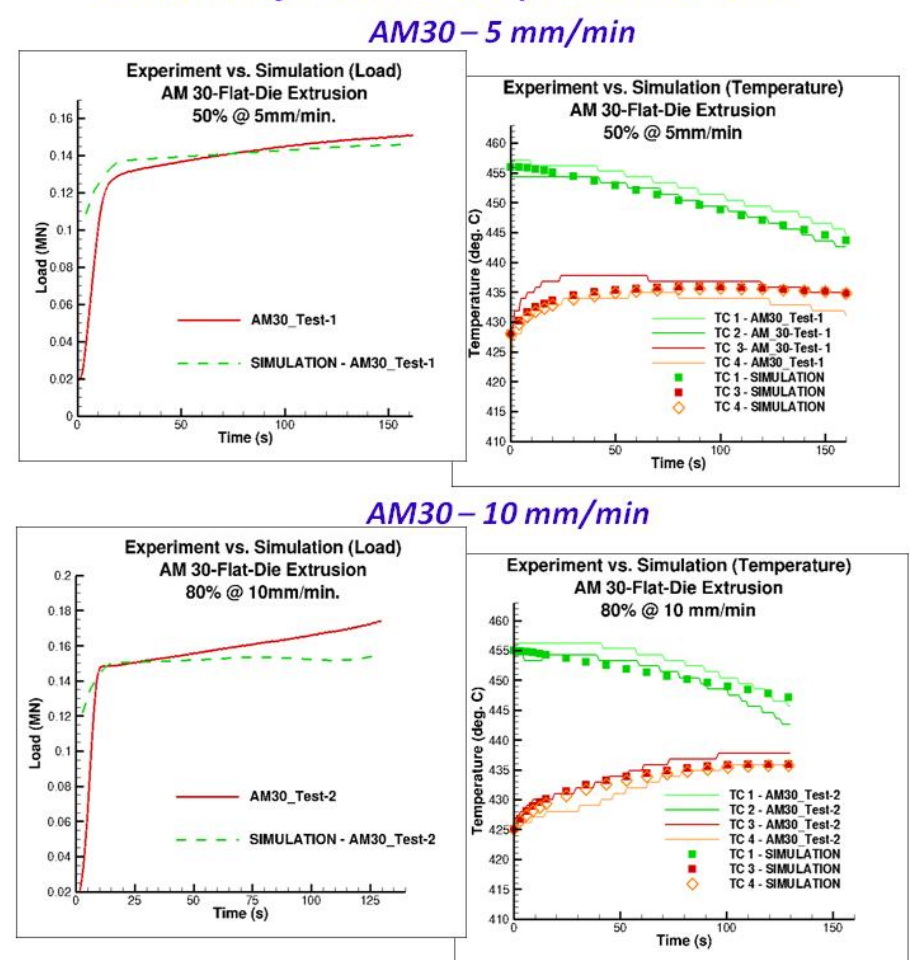

Figure 13: Thermo-Mechanical Validation of Flat Die Experiments - AM30 
The boundary conditions are summarized in Table 2 [1]. As mentioned previously, some convection coefficients depend on the ram speed.

Table 2. Boundary conditions at each interface for extrusion of AM30 billet through flat die

\begin{tabular}{|c|c|c|}
\hline $\begin{array}{c}\text { Region } \\
\text { [HyperXtrude BC } \\
\text { type ID] }\end{array}$ & Thermal Boundary Condition & Mechanical Boundary Condition \\
\hline $\begin{array}{l}\text { External surfaces of } \\
\text { Die and Sleeve } \\
\text { [ToolSurface] }\end{array}$ & Heat flux $=0$ & $\begin{array}{ll}\text { Die } \quad-\quad \text { Stationery } & \text { Elastic } \\
\text { Sleeve }- \text { Moving Rigid } & \end{array}$ \\
\hline $\begin{array}{l}\text { Bearing-Die interface } \\
\text { [SolidFluid Interface] }\end{array}$ & $\begin{array}{l}\text { Heat flux }=0 \\
\text { (Since the length of bearing is very small } \\
\text { and the material is in contact for a very } \\
\text { short time) }\end{array}$ & $\begin{array}{l}\text { Slip Velocity Model, } \\
\mu \quad= \\
\text { (Sliding) }\end{array}$ \\
\hline $\begin{array}{l}\text { Billet-Die interface } \\
\text { [SolidFluid Interface] }\end{array}$ & $\begin{array}{l}\text { Convection } \\
\mathrm{h}=25000 \mathrm{~W} / \mathrm{m}^{2} \mathrm{~K}\end{array}$ & $\begin{array}{l}\text { Full-stick friction on flow mesh } \\
\text { side to simulate dead metal zone. }\end{array}$ \\
\hline $\begin{array}{c}\text { Billet-Sleeve } \\
\text { interface[SolidFluid } \\
\text { Interface] }\end{array}$ & $\begin{array}{l}\text { Convection Coefficient, } \\
\mathrm{h}=280 \mathrm{~W} / \mathrm{m}^{2} \mathrm{~K}\end{array}$ & $\begin{array}{l}\mathrm{X} \text { Velocity }=0, Y \text { Velocity }=0 \\
\text { traction }=0 \text { (On flow mesh) } \\
\text { (Unlike the full-stick friction } \\
\text { observation at billet-container } \\
\text { interface, the BC we used is } \\
\text { characteristic to our test setup.) }\end{array}$ \\
\hline $\begin{array}{l}\text { Profile free surface } \\
\text { [FreeSurface }]\end{array}$ & $\begin{array}{l}\text { Convection Coefficient, } \\
\mathrm{h}=385 \mathrm{~W} / \mathrm{m}^{2} \mathrm{~K} \text { (For } 5 \mathrm{~mm} / \mathrm{min} \text { ) } \\
\mathrm{h}=900 \mathrm{~W} / \mathrm{m}^{2} \mathrm{~K} \text { (For } 10 \mathrm{~mm} / \mathrm{min} \text { ) } \\
\text { (Varies from test to test and depends on } \\
\text { process parameters) }\end{array}$ & $\begin{array}{l}\mathrm{X} \text { traction }=0, \mathrm{Y} \text { traction }=0 \\
\text { and } \mathrm{Z} \text { traction }=0\end{array}$ \\
\hline
\end{tabular}

Figure 14 describes the validation of the extrusion simulation using a flat die with a dog bone shape profile. This validation includes the "bearing profile" feature from HyperXtrude that allows the user to describe the bearing geometry through $3 \mathrm{D}$ curves as opposed to the elements components previously discussed. This feature permits a description of more complicated bearing shapes such as the industrial Timminco die. The 3D curve that describes the bearing limits needs to be included in the "bearing component" defined by a set of elements. The friction model used in the boundary conditions for this problem is a viscoplastic model. In addition to being a dynamic friction model, the viscoplastic friction model defines the shear stress as a function of pressure and flow stress. The value of the friction coefficient varies from 0 to 1 . The value 0 corresponds to full slippage of the material on the tooling surface and 1 corresponds to full stick behavior. In the present case, a value of 0.8 is used at the billetdie interface and in the bearing area. Figure 14 also presents the correlation between the simulation and experiments based on the temperature and load histories.

As expected, the latest friction model added to version 11 of HyperXtrude is able to adjust the load level so we were able to achieve good correlation. The temperature histories on the container (TC2) and on the die (TC3) are also in good agreement with the experiments. 

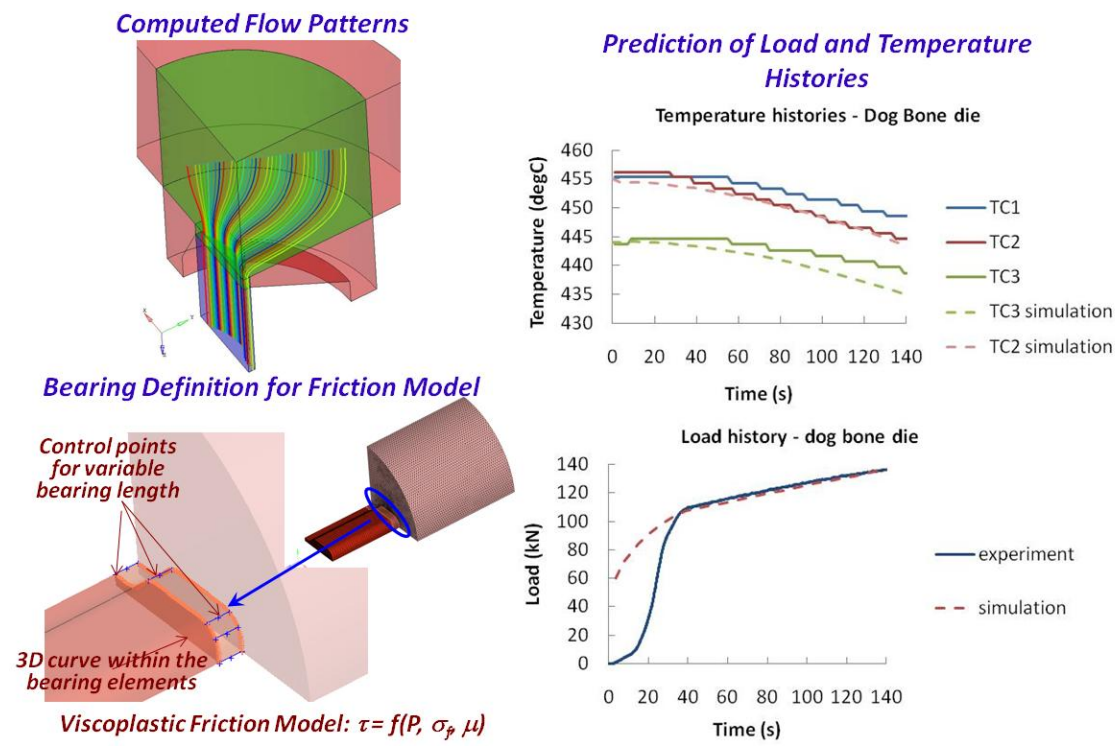

Figure 14: Thermo-Mechanical Validation of Dog-Bone Die Experiments - AM30

Table 3 shows the boundary conditions retained for the simulation of the extrusion process of an AM30 billet through a flat die with a dog bone-shaped opening.

Table 3. Summary of applied boundary condition to Extrusion process model

\begin{tabular}{|c|c|c|c|}
\hline Interface & $\begin{array}{c}\text { Boundary Condition } \\
\text { Type }\end{array}$ & Values & Comments \\
\hline Billet-Die / Die-Billet & SolidFluidInterface & $\begin{array}{c}\text { Heat Transfer Coeff: } 200 \\
\text { Friction Coeff: } 0.8\end{array}$ & \\
\hline $\begin{array}{c}\text { Billet-Container / } \\
\text { Container-Billet }\end{array}$ & SolidFluidInterface & Heat Transfer Coeff: 100 & \\
\hline Bearing-Die & Bearing & $\begin{array}{c}\text { Heat Flux:0 } \\
\text { Friction Coeff: } 0.8 \\
\text { Constant Bearing } \\
\text { length:0.625 in }\end{array}$ & $\begin{array}{c}\text { Use of Bearing } \\
\text { profile and } \\
\text { bearing curves } \\
\text { capabilities }\end{array}$ \\
\hline Die-Bearing & SolidFluidInterface & Friction Coeff: 0.8 & \\
\hline Billet-DummyBlock & SolidFluidInterface & Convection Coeff:200 & \\
\hline Free Surface & Free Surface & Convection Temp Ref $=50$ & \\
\hline Exit Profile section & Outflow & Heat Flux=0 & \\
\hline Tool outer Surfaces & ToolSurface & \multicolumn{2}{|c|}{} \\
\hline
\end{tabular}

\subsection{Prediction of Microstructure Evolution (Texture) During the Extrusion Process}

Local texture in the bearing area in the extrusion die is significantly important for $\mathrm{Mg}$ alloy extrusion. With the benefit of the HyperXtrude software, the velocity gradient data, namely the streamline, along the extrusion lines in the die can be obtained through the extrusion simulation. As shown in Figure 15, the streamlines in different positions in the extrusion die exhibit the different temperature, the extrusion pressure, and the strain level along extrusion displacement. 

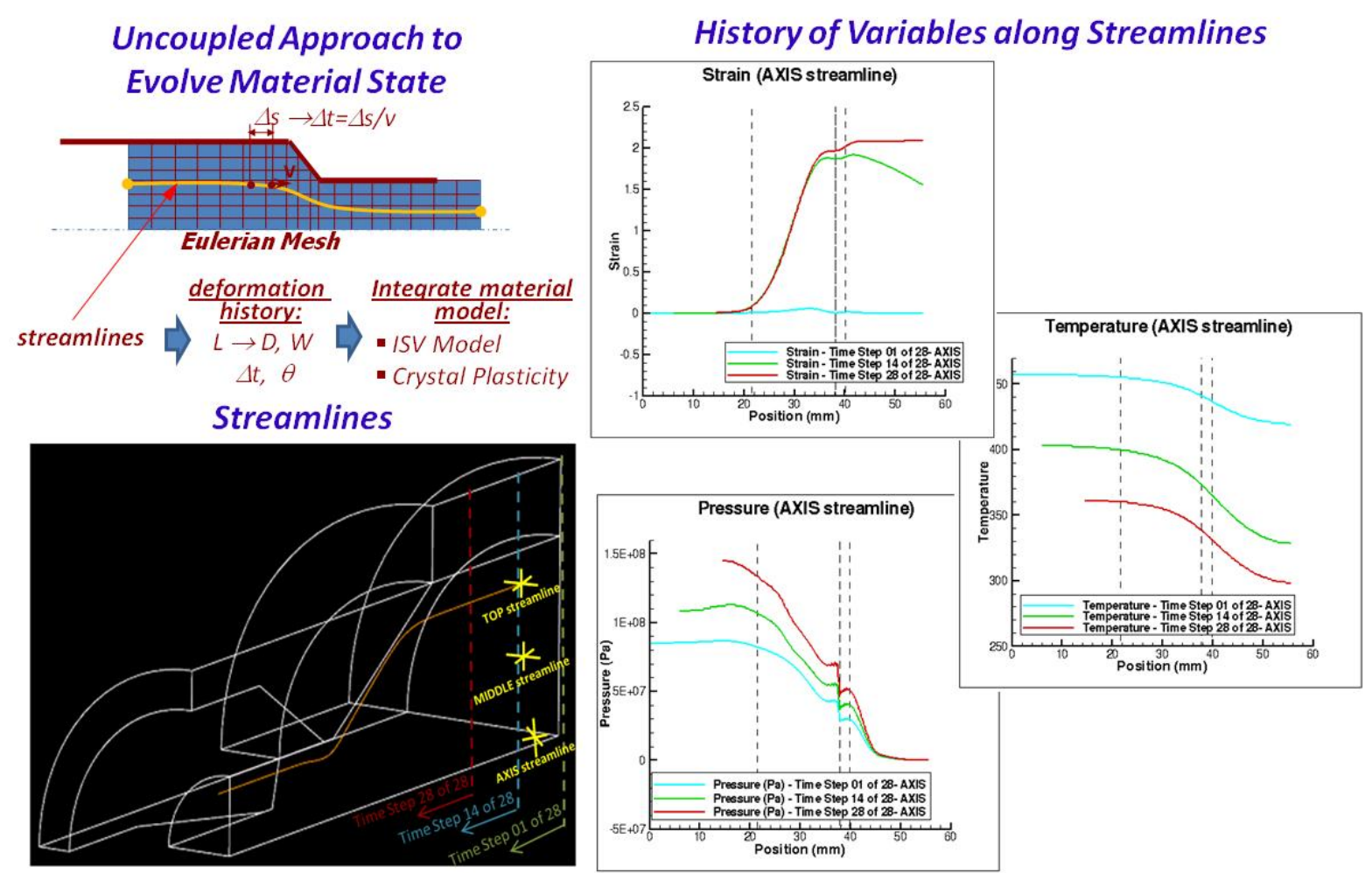

Figure 15. Streamline Information to Compute Texture using VPSC - e.g., Conical Die

The methodology of local texture simulation during $\mathrm{Mg}$ alloy extrusion at high temperatures is presented in Figure 16. The philosophy of local texture prediction in the extrusion die is as follows: using VPSC dislocation density model and the corresponding hardening parameters which come from the stress-strain curves, fit based on the initial texture. Along the streamline, local texture and flow stress in any location in the extrusion die can be predicted based on a combination of HyperXtrude and VPSC.

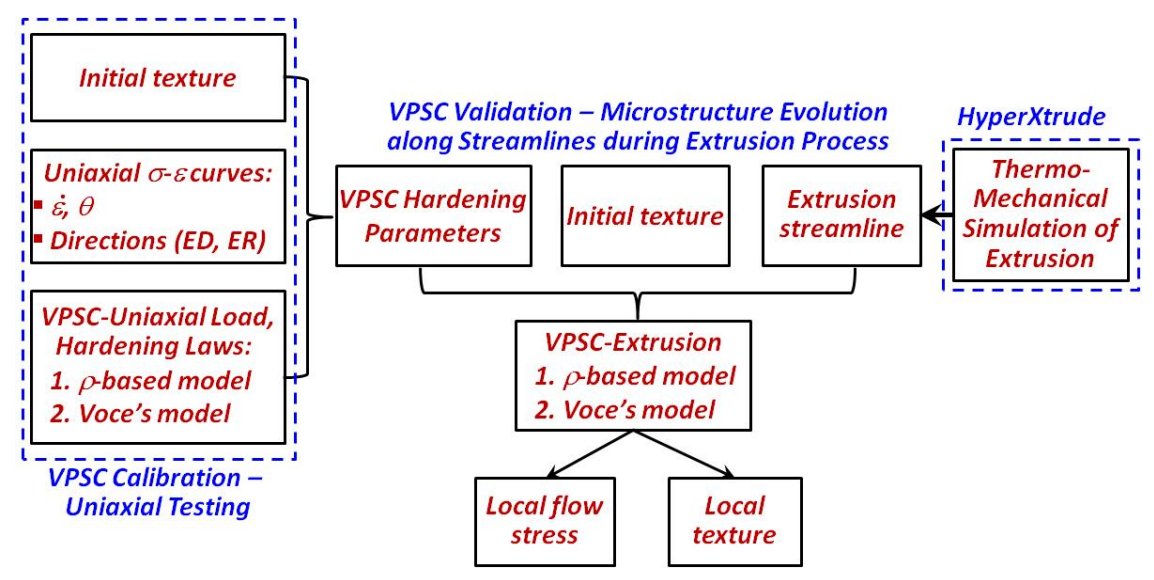

Figure 16. Methodology to predict microstructure (texture) using VPSC

Due to the limited ductility of $\mathrm{Mg}$ and its alloys at low temperature, thermomechanical processing of wrought $\mathrm{Mg}$ alloys is usually conducted at high temperature, e.g. hot extrusion and hot rolling. As for Mg extrusion, the hot extrusion temperature usually falls in the rage of $350-450{ }^{\circ} \mathrm{C}$. Therefore, deep 
understanding of microstructure and texture evolution of $\mathrm{Mg}$ during extrusion at $450{ }^{\circ} \mathrm{C}$ is necessary for $\mathrm{Mg}$ alloy design. On the other hand, the strain rate also plays an important role on deformation mechanisms in Mg alloys. While strain rate is inhomogeneous during hot extrusion, it varies in the die from one location to another. As such, any polycrystalline plasticity modeling of $\mathrm{Mg}$ alloy extrusion must incorporate the effects of temperature and strain rate to accurately predict local texture and mechanical properties. We focused our study on the VPSC modeling, including the effects of temperature and strain rate during $\mathrm{Mg}$ extrusion at the high temperature of $450^{\circ} \mathrm{C}$.

First, we did simple compression of the pre-extrusion $\mathrm{AM} 30$ at $450{ }^{\circ} \mathrm{C}$ along two perpendicular directions, the extrusion direction (ED) and the radial direction (ER) at different strain rate, $0.001 \mathrm{~s}^{-1}, 0.1$ $\mathrm{s}^{-1}, 0.5 \mathrm{~s}^{-1}$ and $0.8 \mathrm{~s}^{-1}$. Actually, these selected strain rates fall in the lab-scale strain rate $<1 \mathrm{~s}^{-1}$ for $\mathrm{Mg}$ extrusion in our laboratory at CAVS. Then we fit the stress-strain curves of AM30 at different strain rates based on the VPSC dislocation density model. The initial microtexture, the VPSC hardening parameters at $450{ }^{\circ} \mathrm{C}$, and the measured stress-strain curves of the AM30 at $450{ }^{\circ} \mathrm{C}$ at different strain rates are presented in Figure 17. As shown in Figure 17, other hardening parameters are the same at different strain rates; however, only the $\tau_{0}$ of dislocations changed with strain rates at $450{ }^{\circ} \mathrm{C}$. Therefore, we extracted the linear relationship of the $\tau_{0}$ and strain rate as Equations 1-3. The $\tau_{0}$ of dislocation slips by fitting of the VPSC stress-strain curves and the predicted $\tau_{0}$ using the Equations 1-3 are also shown in Figure 17. Due to twinning being considered an athermal deformation mode in $\mathrm{Mg}$, we fixed the critical resolved shear stress (CRSS) and the propagation stress of $\{10-12\}$ twinning as $15 \mathrm{MPa}$ at $450{ }^{\circ} \mathrm{C}$ in this study.

$$
\begin{gathered}
\tau_{0}=7.0755 \dot{\varepsilon}+9.5218 \mathrm{MPa}(\text { for basal) } \\
\tau_{0}=38.0784 \dot{\varepsilon}+10.1613 \mathrm{MPa}(\text { for prismatic) } \\
\tau_{0}=36.3559 \dot{\varepsilon}+13.7663 \mathrm{MPa}(\text { for second }<\mathrm{c}+\mathrm{a}>\text { pyramidal) }
\end{gathered}
$$

With Equations 1-3 compiled into the original VPSC dislocation density model, it then can capture both temperature and strain rate effects. The predicted simple compression stress-strain curves of AM30 at $450{ }^{\circ} \mathrm{C}$ using the $\tau_{0}$ based on the Equations 1-3 and the measured stress-strain results are presented in Figure 17. As shown in Figure 17, the predicted and measured stress-strain curves are reasonably well in agreement. 

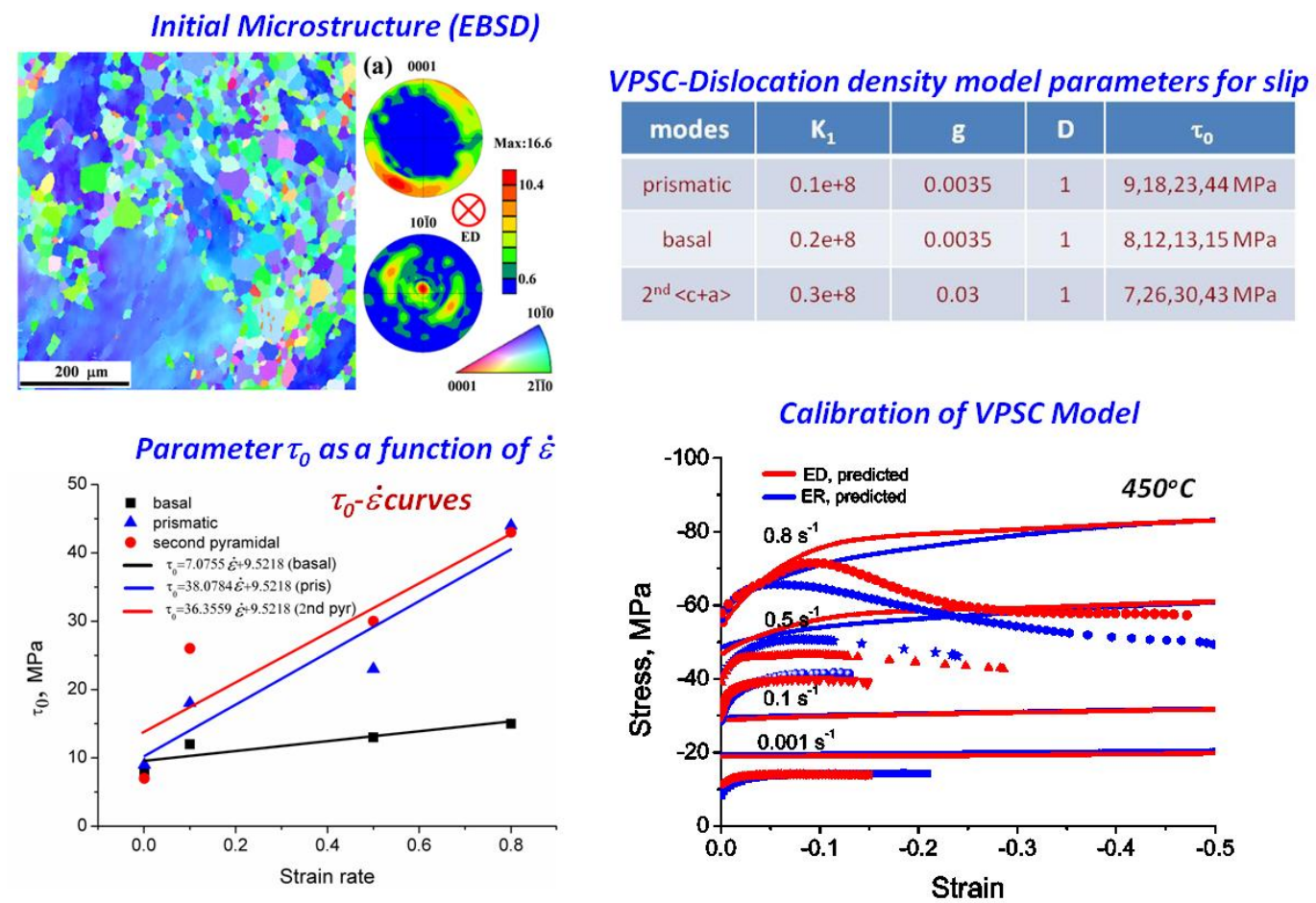

Figure 17. Calibrating the dislocation-based hardening law in VPSC

Using the VPSC dislocation density model parameters and the streamline data output from the HyperXtrude software, we can predict the extrusion stress-strain curve and the local texture of AM30 in the flat die and conical die in the extrudate. Figure 18 shows the corresponding VPSC results based on the streamline of a flat die of AM30 extrusion at $450{ }^{\circ} \mathrm{C}$. The results are the corresponding data for extrusion along the axis of the flat die at ram speeds of $5 \mathrm{~mm} /$ minute and $10 \mathrm{~mm} /$ minute. The predicted texture is consistent with the measured electron back scatter diffraction (EBSD) texture (Figure 18). As shown in Figure 18, the extrusion flow stress of $10 \mathrm{~mm} /$ minute is higher than that of $5 \mathrm{~mm} / \mathrm{minute}$, which is reasonable and demonstrates that the VPSC dislocation density model can capture both temperature and strain rate effects during extrusion in the flat die. 


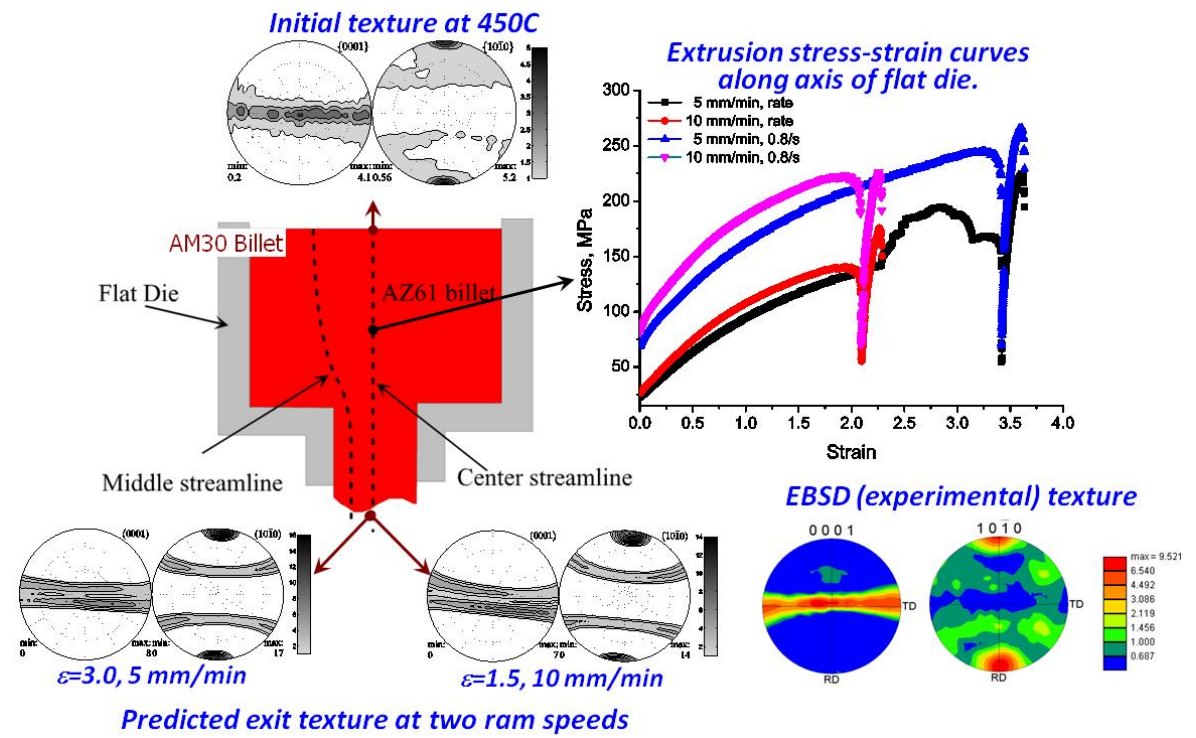

Figure 18. Predicting texture using VPSC, including temperature and strain rate effects.

\section{Post-Forming Experiments ANd Modeling}

For post-forming simulation, we used the VPSC predicted texture of the extrudate as the starting orientations in the VPSC simulation. In addition, the room temperature's parameters of the VPSC dislocation density model of the pre-extrusion AM30 came from the fitting of the VPSC room temperature stress-strain curves.

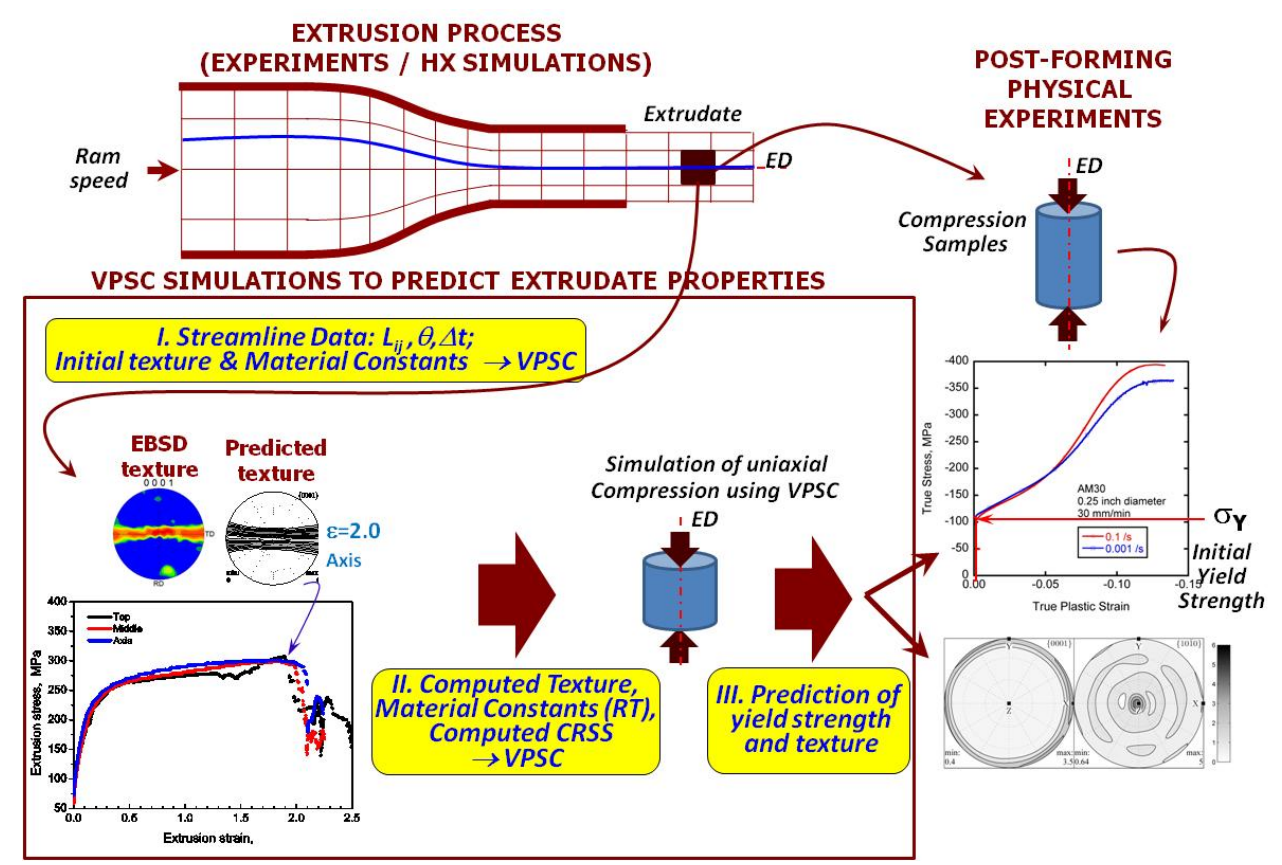

Figure 19. Methodology to Predict Texture \& Initial $\sigma_{Y}$ of Extrudate 


\section{Extrudates from Flat Die Extrusion of $A M 30, \theta_{B}=454^{\circ} \mathrm{C}$}
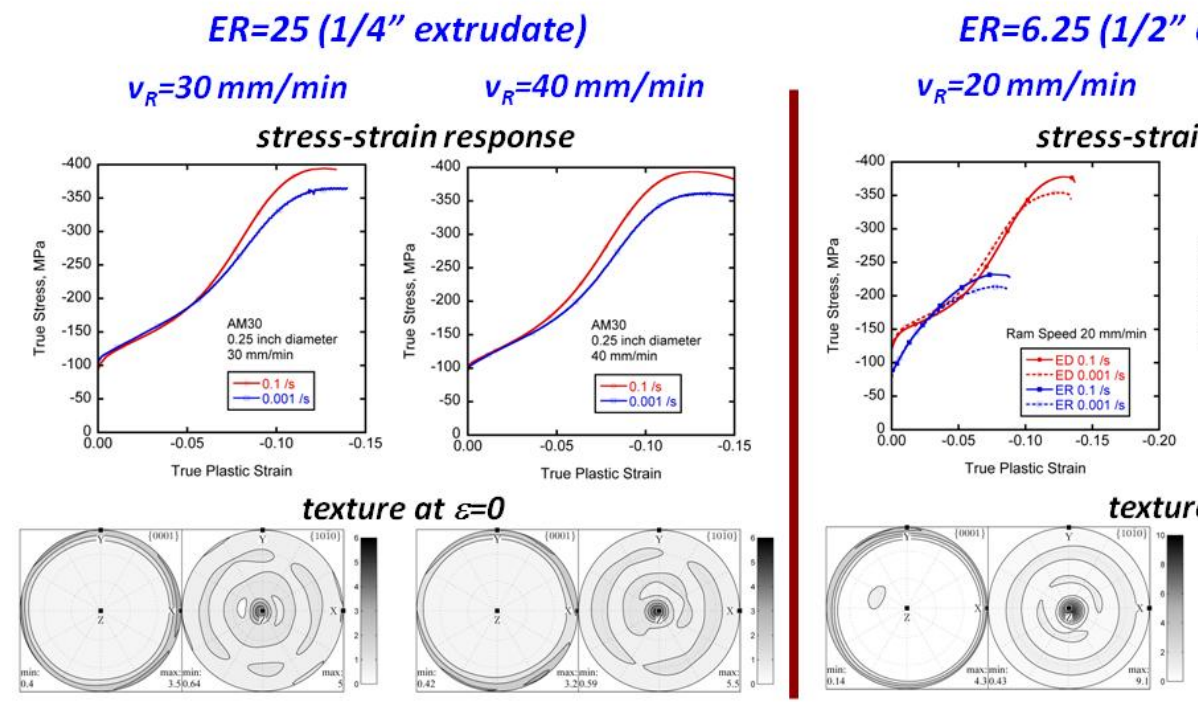

extrudate)
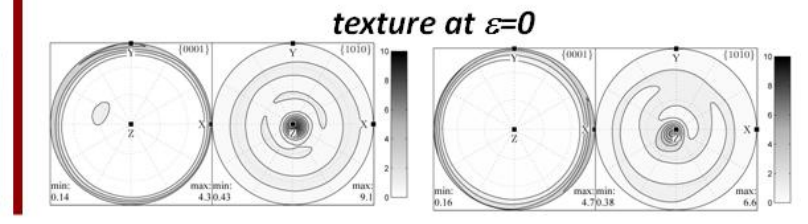

Figure 20. Post-forming experiments on extrudates from flat-die extrusion

Figure 21 shows the simulated extrusion stress-strain curve of the AM30 extruded at $450{ }^{\circ} \mathrm{C}$ using the flat die along the die axis and a ram speed of $30 \mathrm{~mm} /$ minute. Using the predicted extrudate texture (Figure 21) and the VPSC room temperature hardening parameters from the fitting of the AM30 stressstrain curves, the post-forming stress-strain curve can be simulated. As shown in Figure 21, the predicted extrusion flow stress goes up along with strain. This is reasonable because the strain rate is higher close the exit of the flat die. The extrudate texture (Figure 21) is the typical of extrusion of $\mathrm{Mg}$ showing a strong $<0001>$ ED texture.

The VPSC dislocation density model can accurately capture the yield stress of the extrudate (Figures 21-22). In comparison with experimental results, the modified VPSC dislocation density model can nicely predict the extrusion stress-strain curve, local texture of the extrudate, and post-forming properties of AM30 alloy extruded at $450{ }^{\circ} \mathrm{C}$. A detailed discussion of the model and parameters for the VPSC model is found in Ref [2]. In general, the yield point is accurately predicted and the strain hardening behavior is reasonable. 


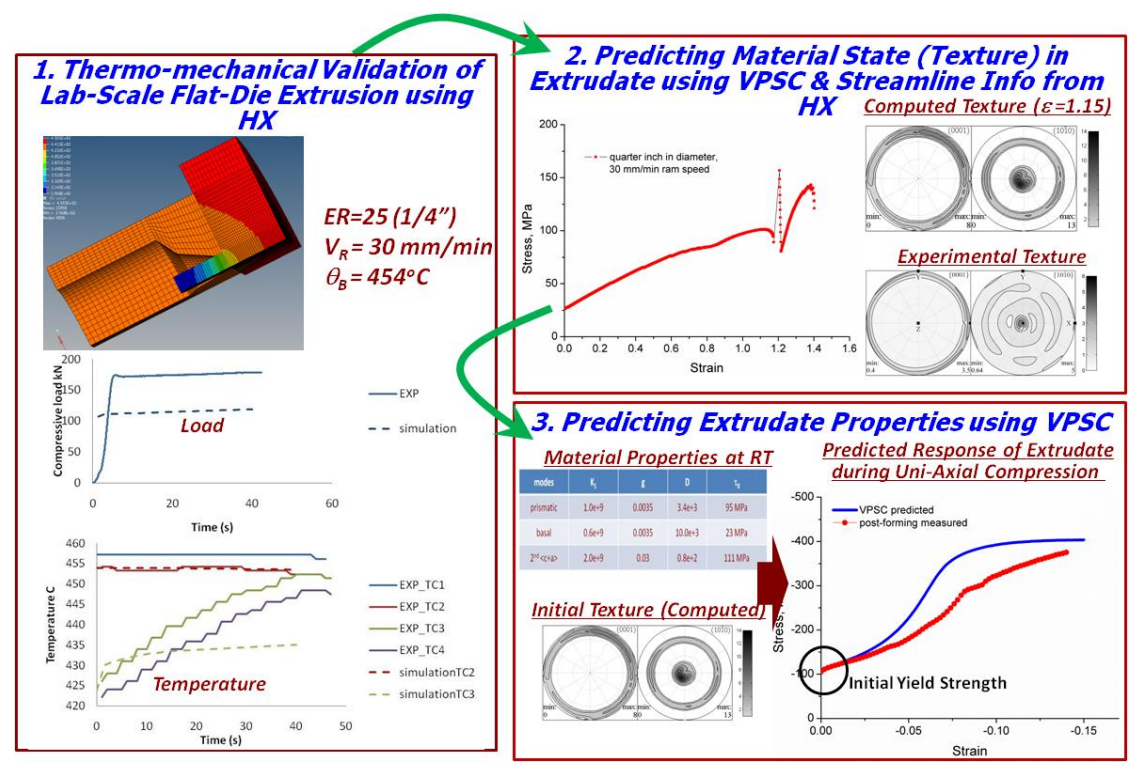

Figure 21. Preliminary results for predicting post-forming properties of extrudate

Extrudates from Flat Die Extrusion of AM30

Stress-Strain Response, Experiment vs Simulation

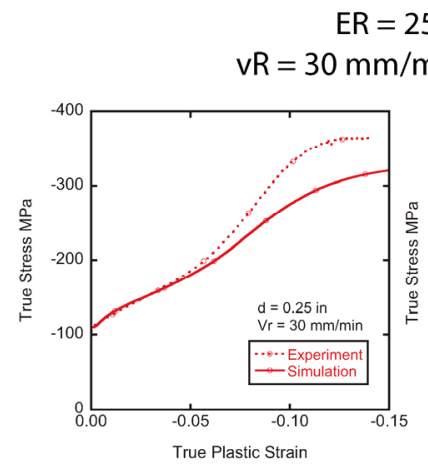

$R=25\left(0.25^{\prime \prime}\right.$ extrudate $)$
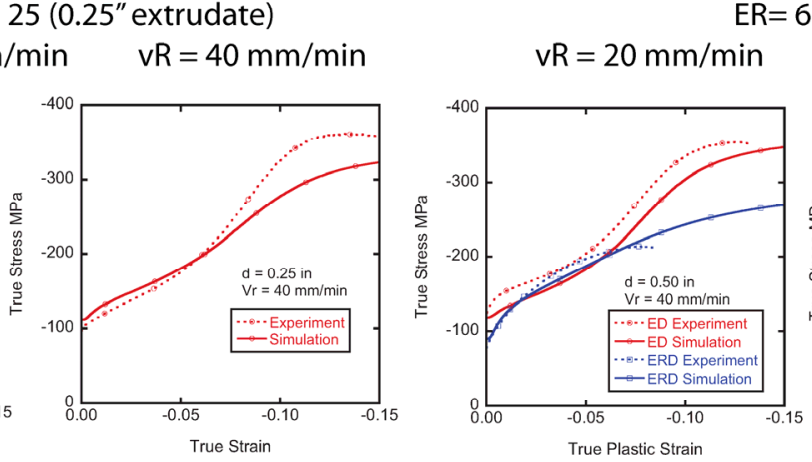

$E R=6.25(0.50 "$ extrudate $)$

Figure 22. Comparison between experimental and VPSC simulation stress-strain behavior for post-form AM30 laboratory scale extrudate.

\section{Prediction of Mechanical Properties and Texture in Industry-Level Extrusion Processes}

This section describes the industrial application of the methodology presented by the extrusion task team in the previous sections. The purpose of this study is to demonstrate the application of the methodology to a real industrial problem. The Timminco die was chosen due to data availability regarding the extrusion process parameters as well as the microstructure database along the profile. Figure 23 shows the steps that CAVS followed to produce accurate simulations of the process. First, the CAVS team visited the Timminco plant to take pictures and collect data of the extrusion tooling and processes. From these, CAD geometry was built and a negative volume was constructed. This volume represents the $\mathrm{Mg}$ alloy "filling" the extrusion tooling during the process, the red part being the 
undeformed billet ready to be extruded and the light blue part being the profile in the bearing area, just before exiting the tooling.

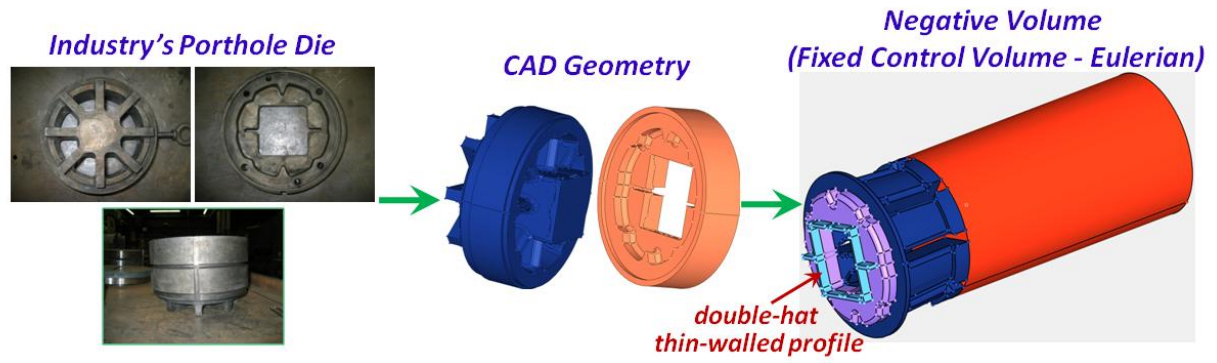

Figure 23. Industry profiles used for extrusion simulations

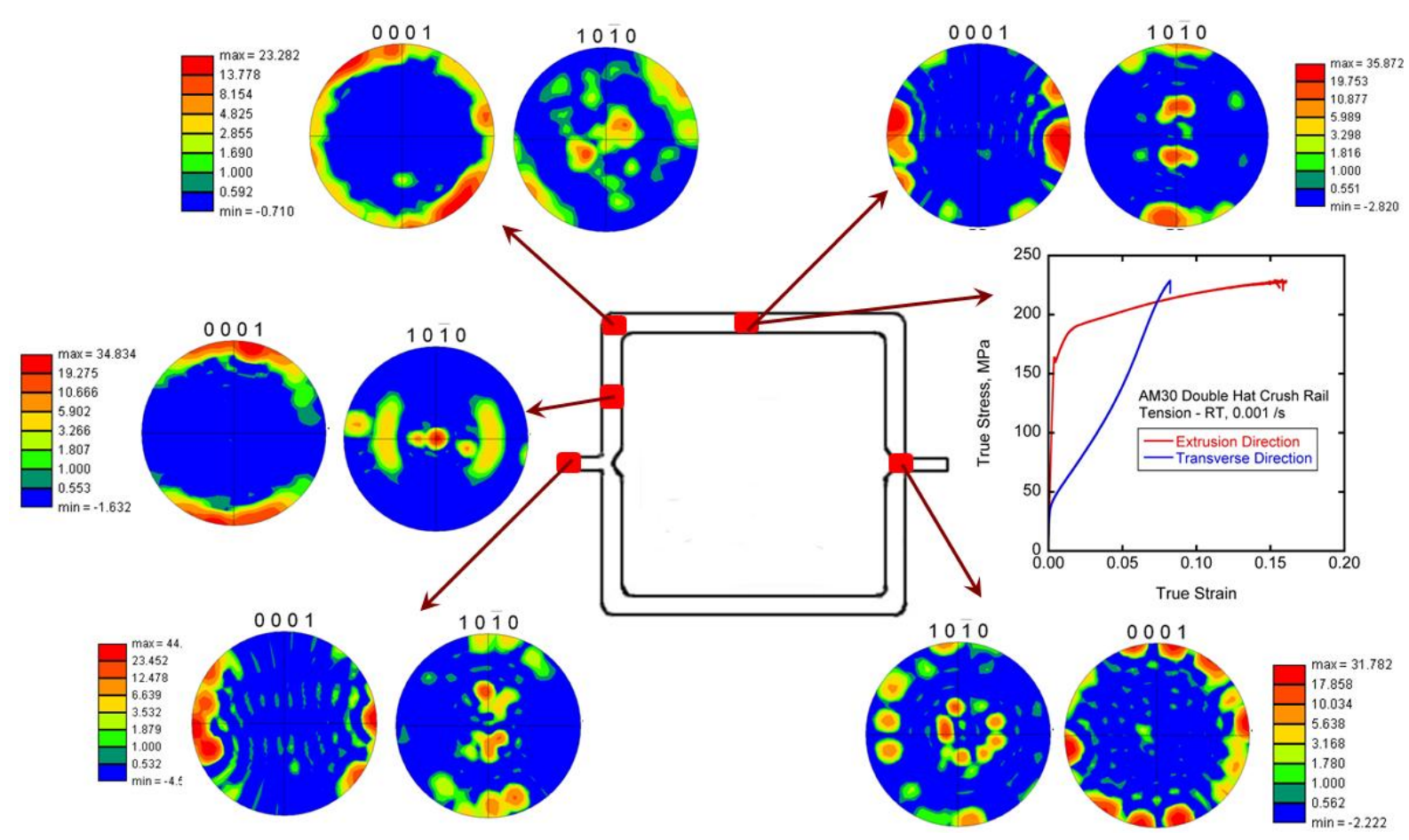

Figure 24. Measured mechanical response and texture for double-hat profile

Contrary to the simulation of lab scale experiments, the double hat simulations were run in steady state. As no load and temperature histories were available, the assumption was to focus on steady-state results and microstructure prediction.

The left image in Figure 25 shows the steady state analysis of this extrusion process at a temperature of $427^{\circ} \mathrm{C}$ and a ram speed of $78 \mathrm{~mm} / \mathrm{min}$. As information about the die temperature is unknown, it was assumed at $420^{\circ} \mathrm{C}$, which is slightly below the container temperature. This assumption is based on observation from the experimental extrusions run at CAVS. The purpose of this simulation is the extraction of material history along several streamlines such that texture can be computed in a later step. The right image in Figure 25 shows the streamlines that have been selected to analyze the texture. 
Microstructure observations are already available at these locations. Using the streamlines in the three representative positions in the double hat and VPSC dislocation density model and the corresponding hardening parameters, which were obtained from the fitting of the stress-strain curves, can predict the texture and extrusion flow stress during extrusion of the Timminco double hat die, as shown in Figure 24.
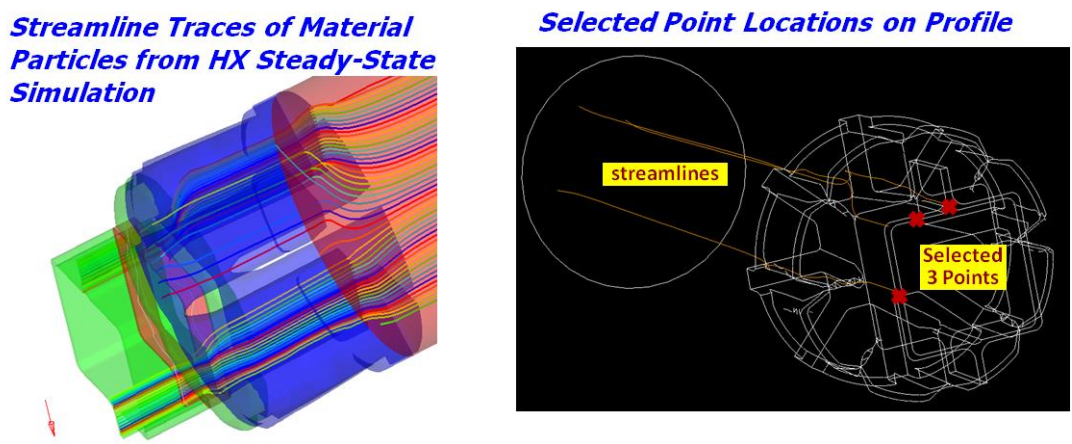

Figure 25. Streamlines in the porthole-die thin-walled hollow profile from extrusion simulation using HyperXtrude.

The VPSC-predicted texture and EBSD-measured texture in the three positions in the double hat are shown in Figure 26. In Figure 26, even though both measured and predicted texture intensity is very strong due to the severe plasticity during this double hat extrusion, the measured and predicted texture is different. In contrast, the main predicted texture components by VPSC are in good agreement with EBSD measured texture in the case of flat die extrusion of AM30 (Figure 18) .

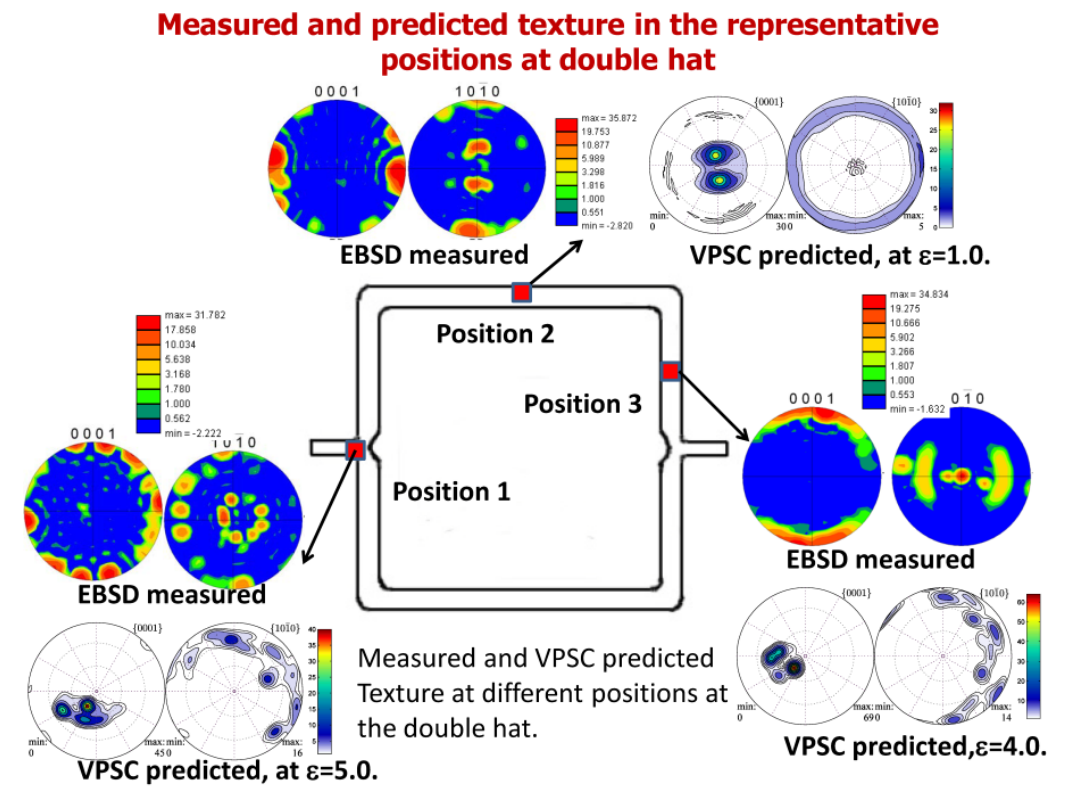

Figure 26: EBSD measured and VPSC predicted texture in different positions at the AM30 double hat.

The primary explanation for this marked deviation is the exclusion of dynamic recrystallization in the present VPSC code, even though it has taken into account the effect of temperature and strain rate. In the simple case of the flat die, the effect of dynamic recrystallization may not be significant; however it could play marked roles during severe deformation, like the double hat extrusion case. Future work is necessary to resolve this question. 


\section{REFERENCES}

Ref [1]: Abdul Afoo Parkar: "On modeling and experimental validation of extrusion process of lightweight alloys," Master Thesis, Mississippi State University, August 2011

Ref [2]: Oppedal, A. L., El Kadiri, H., Tomé, C.N., Baird, J., Vogel, S.C., \& Horstemeyer, M. (2011). Limitation of Current Hardening Models in Predicting Anisotropy by Twinning in Hcp Metals: Application to a Rod-Textured AM30. In W. H. Sillekens, S. R. Agnew, N. R. Neelameggham, S. N. Mathaudhu (Eds.), Magnesium Technology 2011, San Diego, CA: TMS (The Minerals, Metals \& Materials Society), 313-320 


\section{ICME of Sheet Forming of AZ31 Mg Alloys}

Team Members: P.T. Wang, M.F. Horstemeyer, B. Li, Y. Hammi, S.J. Horstemeyer, S. Yazdan Parast, J.C. Baird, E.B. Marin, A.L. Oppedal, Q. Ma

\section{APPROACH}

While conventional forming processes (e.g., stamping) of wrought $\mathrm{Mg}$ alloys are problematic at room temperature, high-temperature forming facilitates the manufacture of near-net shapes from $\mathrm{Mg}$ alloy sheets. To reduce energy consumption and manufacturing costs, it is desirable to reduce the processing temperatures of $\mathrm{Mg}$ sheet forming. Hence, understanding the deformation mechanisms which drive microstructural and texture evolution of $\mathrm{Mg}$ alloys during forming rather than post-forming is crucial to achieve optimized processing and end products. In this task, we designed novel experimental techniques to investigate the deformation behavior during sheet bending of AZ31 Mg alloys. One of the techniques is in situ electron backscatter diffraction (EBSD) analysis during sheet bending. This technique allows examinations of microstructure and texture evolution during sheet bending without unloading the specimen. Very interesting results were obtained. In another technique,
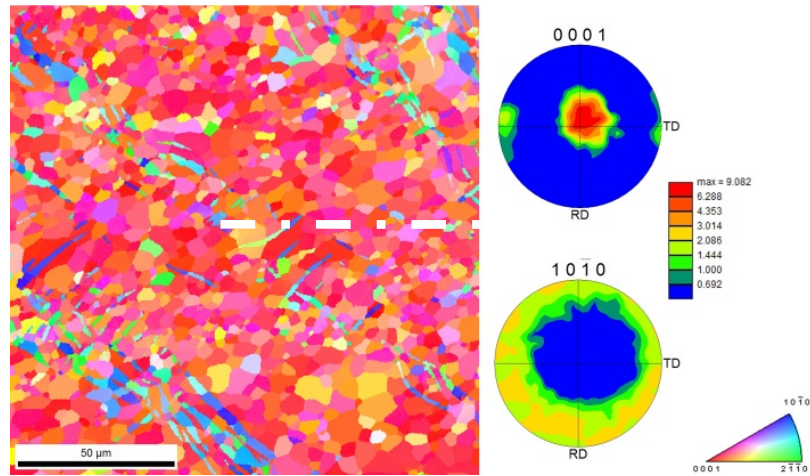

Figure 1. Multiple twin bands were observed that span both the compression and the tension zones near the center line of the specimen (indicated by a dash-dotted line in the middle). The twin bands grow across the center line, indicative of a shift of the neutral axis. we designed and fabricated fixtures to perform three-point bending with Instron machine such that the strain rate can be controlled. A ground-breaking discovery was made. Meanwhile, continuum modeling of sheet bending was performed.

\section{RESULTS AND Discussion}

(1) In situ EBSD on sheet bending. A special fixture was fabricated to conduct in situ EBSD on a sheet sample that is loaded. Fig. 1 shows that during bending, multiple twin bands were produced in the compression zone of the sample. But the twins are localized in bands. In between the bands, no twins can be seen. As the bending angle increases, the twin bands continue to grow across the center line. The pole figure is typical of extension twinning.

The highly localized twin bands have not been observed before. The mechanism responsible for these twin bands and how they influence sheet forming require further studies. 


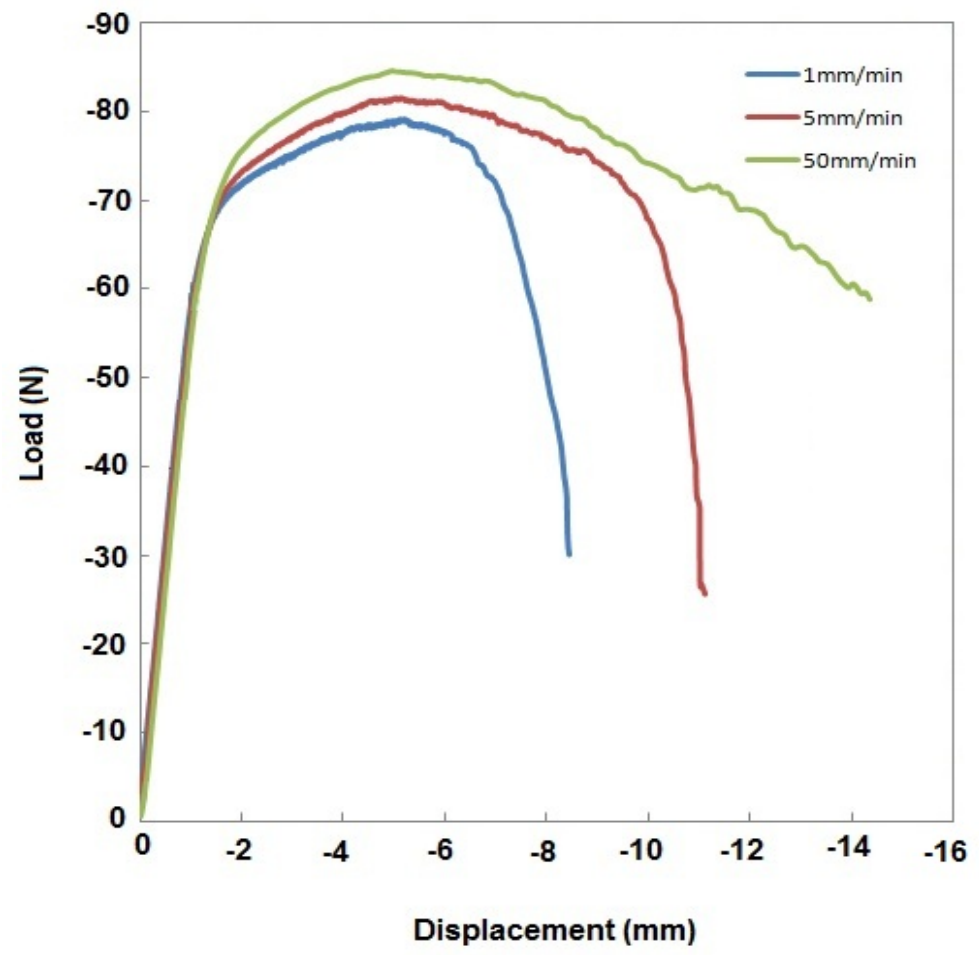

Figure 2. Load-displacement curves from the room temperature bending tests. The samples were annealed at $150{ }^{\circ} \mathrm{C}$ for an hour. At lower strain rates, $1.0 \mathrm{~mm} / \mathrm{min}$ and $5.0 \mathrm{~mm} / \mathrm{min}$, both specimens failed, but the 5.0 $\mathrm{mm} / \mathrm{min}$ specimen failed with a larger displacement. At $50.00 \mathrm{~mm} / \mathrm{min}$, no specimen failed.

(2) Inverse strain rate sensitivity of sheet bending. In this study, three-point bending was performed with an Instron machine. An AZ31 Mg sheet was annealed at different temperatures for an hour before the bending tests. Then three-point bending was conducted at different displacement rates: 1.0, 5.0, and $50.0 \mathrm{~mm} / \mathrm{min}$. To our surprise, for the samples annealed at $150^{\circ} \mathrm{C}$, an inverse strain rate sensitivity was observed. The load-displacement behavior of the annealed specimens is shown in Fig. 2. At displacement rates of 1.0 and $5.0 \mathrm{~mm} / \mathrm{min}$, the annealed specimens failed, even though at 5.0 $\mathrm{mm} / \mathrm{min}$, the displacement to failure was somewhat larger than that at $1.0 \mathrm{~mm} / \mathrm{min}$, corresponding to a larger bending angle. Surprisingly, the annealed specimen did not fail as the displacement increased to $50.0 \mathrm{~mm} / \mathrm{min}$, and three-point bending continued until reaching the limit of the bending fixture, creating an unexpectedly large bending angle.

(3) ICME modeling of sheet bending. Three point bend simulations were made using Abaqus (Figure 3). The deformation paths (Figure 4) from the simulations were used in VPSC simulations (Figure 5) to predict the deformed texture and predicted yield surfaces. The predicted yield surfaces are from VPSC simulations probing the yield surface, using VPSC model parameters correlated to uniaxial tension and compression tests that capture the strain hardening behavior of the material. 


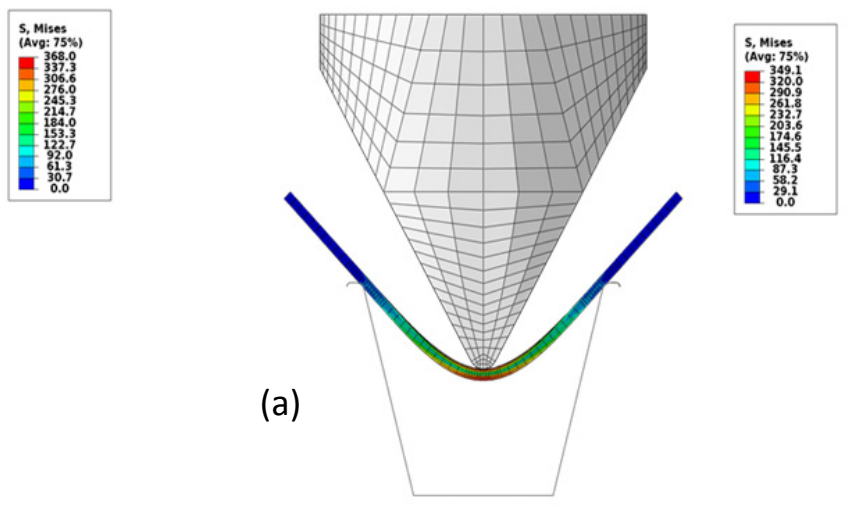

(b)
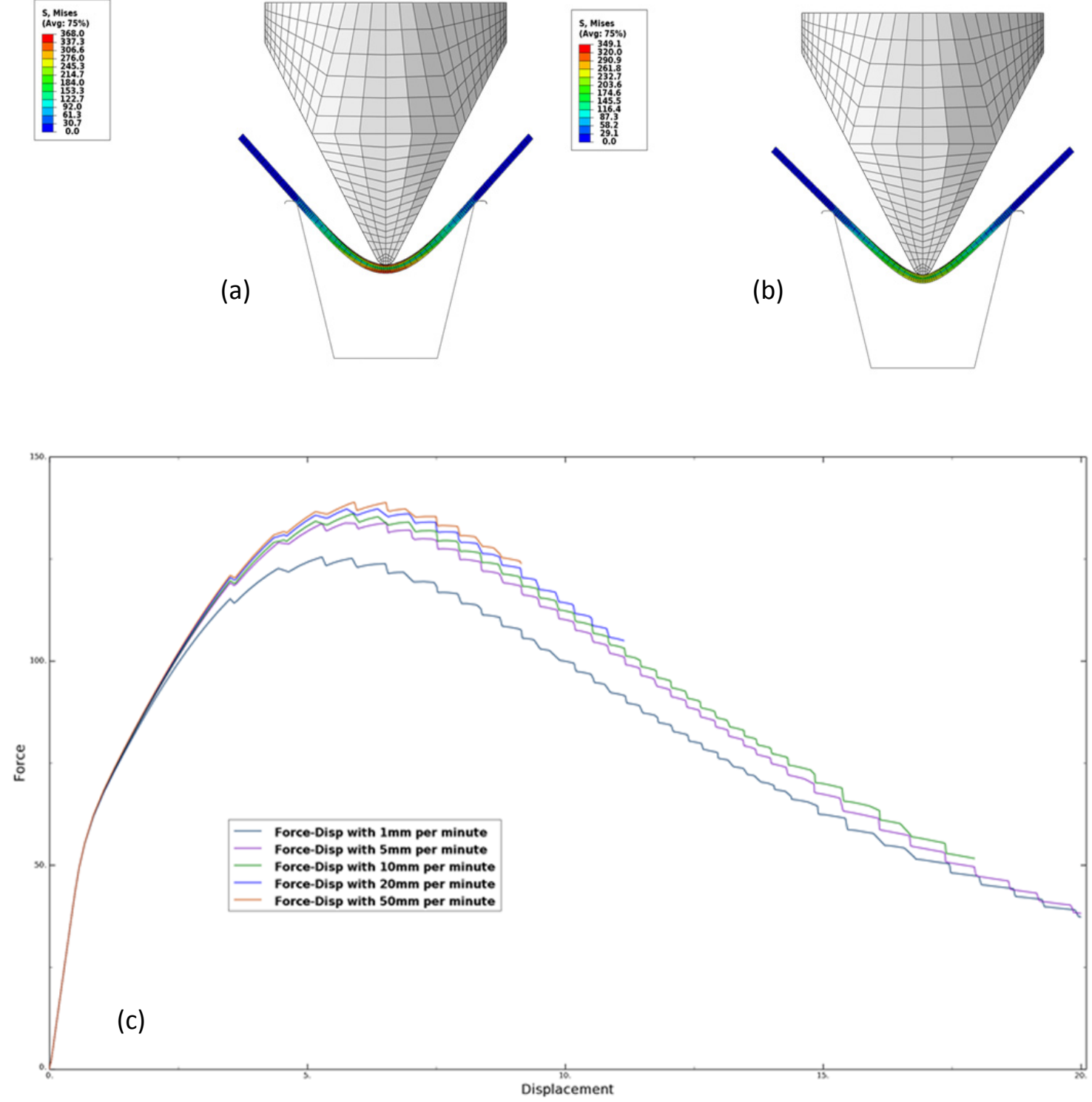

Figure 3. (a) Abaqus Plastic modeling of sheet bending; (b) Abaqus Umat rate independent modeling. (c) Load-displacement curves from the modeling. 

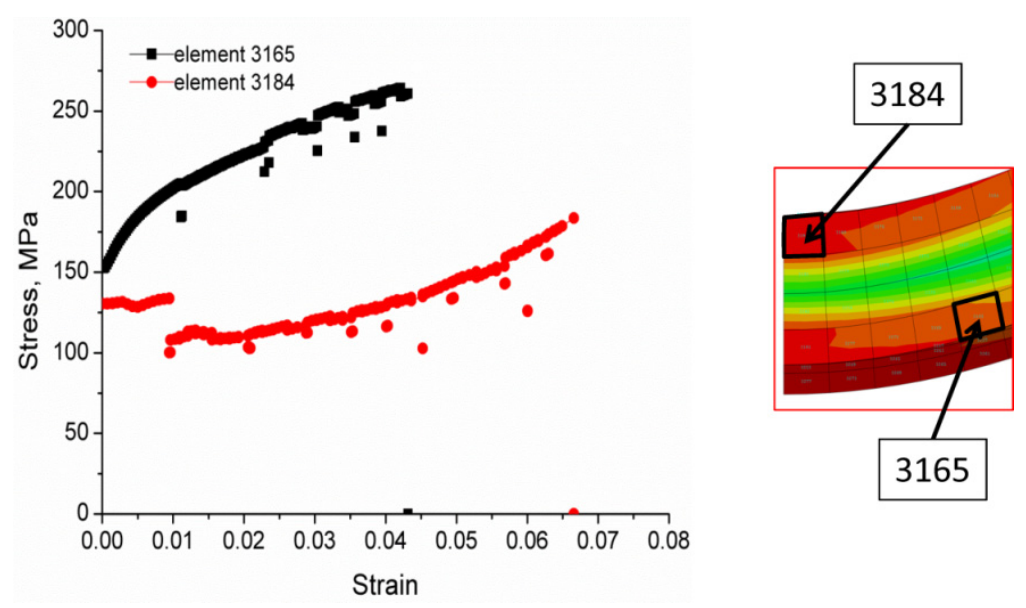

Figure 4. Strain path and element location from bend simulation used in VPSC simulation. The three point bend test produces compression in the top fiber of the material, and tension in the bottom fiber of the material.

\section{Initial State \\ Experimental Polefigure, Predicted Yield Surface}
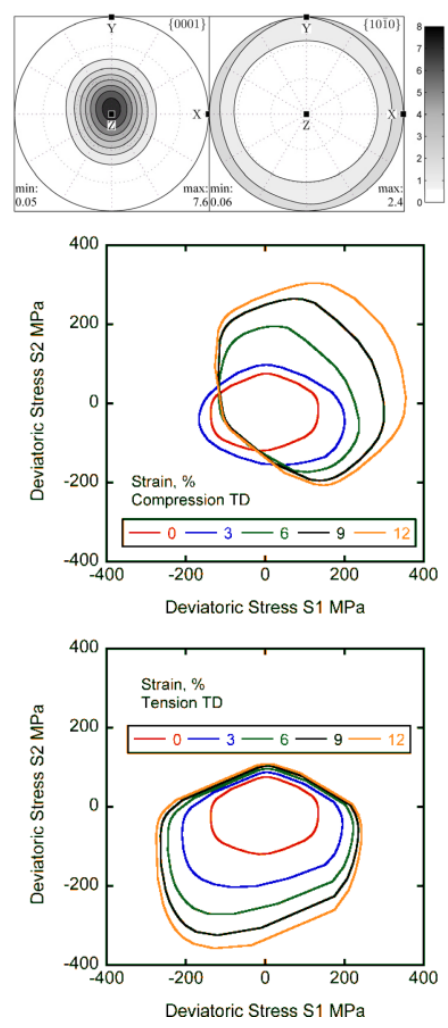

a)

\section{After Deformation \\ Predicted Polefigure, Predicted Yield Surface}

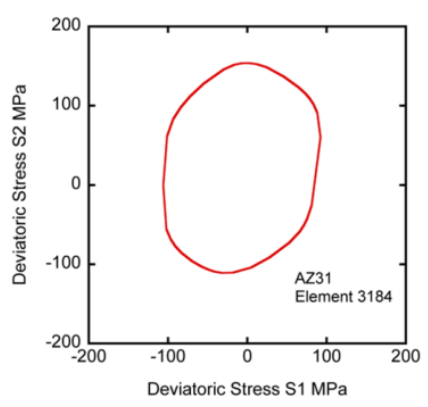

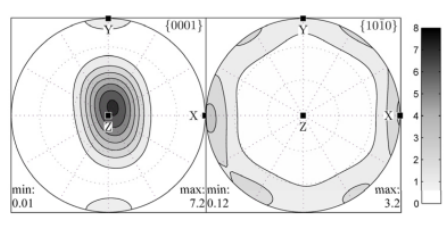

Tension

(element 3165)

Deformation $\varepsilon=0.043$

No change in texture

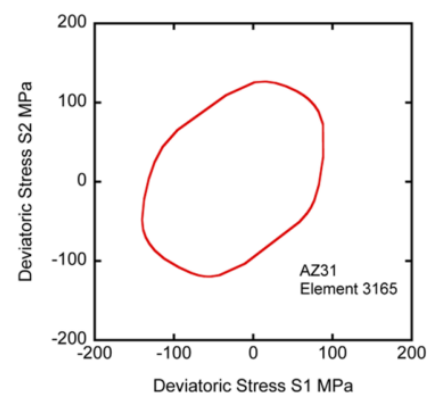

b)

Figure 5. a) Experimentally determined $\{0001\}$ and $\{10-10\}$ pole figures of AZ31 used in VPSC simulations, and the predicted yield surface for the unstrained material. b) The predicted polefigure and predicted yield surface of the specified elements after deformation. The element labels correlate to Figure 4. 


\section{CONCLUSIONS}

(1) In-situ EBSD was performed on AZ31 sheet samples during three-point bending. Unusual, highly localized twin bands were observed in the compression zone. In between the twin bands, no twins were observed. More experiments are needed to understand the mechanism for such localized twin bands.

(2) Three-point bending was performed at different strain rates at room temperature on an annealed AZ31 Mg sheet material. The results revealed surprising strain rate sensitivity. The bending angle increases as the strain rate increases, sharply different from the as-received sheet in which the bending angle decreases as the strain rate increases.

(3) ICME modeling was conducted to model the behavior of sheet bending. Preliminary results of curvature and load-displacement show good agreement between experiments and modeling. Improvements are needed to account for anisotropy. 


\section{D. icme Demo Validation and Zone Mapping}

Team Members: C. Bouvard, W. Whittington, M.F. Horstemeyer

\section{INTRODUCTION}

An industrial application of the cradle to grave modeling effort pursued at CAVS is presented in this chapter. The experimental and numerical analysis of a magnesium ( $\mathrm{Mg}$ ) component provided by General Motors shows the robust methodology developed by our researchers to predict the behavior of a structure under precisely defined boundary conditions. More specifically, the study is about an AZ91 Mg alloy die cast shock tower under monotonic load-to-failure conditions. The goal is to numerically determine the failure location using a validated microstructure-property based material model [1].

\section{Monotonic Shock Tower Analysis}

The shock towers were designed to sustain loading from the suspension system and transmit the load to the vehicle chassis. Figure 1 illustrates the shock tower's with relation to the chassis frame components of the GM vehicle.

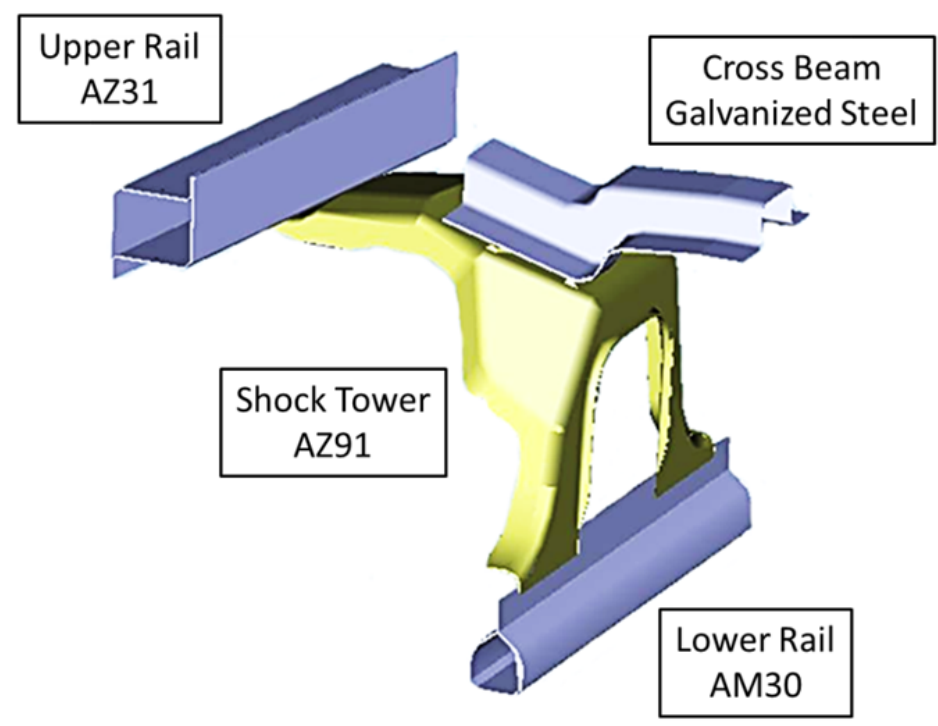

Figure 1. Shock tower and connecting parts.

A preliminary study was conducted to determine the boundary conditions that will allow the failure of the part under monotonic loading at a location away from the grips. This numerical study was conducted first using a very simple elastic-plastic model. In order to "shake out" the FE simulation, once the FE simulation was stable, we employed the ISV model described earlier.

\subsection{Finite Element Model Setup}

An ABAQUS finite elements (FE) mesh based on Computer Aided Design (CAD) data was provided by Optimal Inc. For most of the part, the mesh was built by extruding the inner skin of the shock tower towards the exterior skin. Three elements through the thickness were maintained throughout the part. The specifications of this mesh are in Table 1. The provided CAD described an "ideal" part in terms of 
geometrical features. Section 2 shows that after receiving the physical part, discrepancies between the CAD and the physical part were measured and the FE mesh was modified to account for these differences. The thickness in the "legs" was decreased to the thickness measured in the real structure.

Table 1. Shock Tower mesh specifications

\begin{tabular}{|c|c|c|c|c|c|c|}
\hline $\begin{array}{c}\text { Element } \\
\text { Shape }\end{array}$ & $\begin{array}{c}\text { ABAQUS } \\
\text { Type }\end{array}$ & $\begin{array}{c}\text { Geometric } \\
\text { Order }\end{array}$ & Material & $\begin{array}{c}\text { Avg. Elem. } \\
\text { Size (mm) }\end{array}$ & $\begin{array}{c}\text { Number of } \\
\text { Elements }\end{array}$ & $\begin{array}{c}\text { Number of } \\
\text { Nodes }\end{array}$ \\
\cline { 1 - 5 } Solid Brick & C3D8 & Linear & $\begin{array}{c}\text { AZ91- } \\
\text { UMAT }\end{array}$ & 2.5 & 72961 & \multirow{2}{*}{100294} \\
\cline { 1 - 5 } Wedge & C3D6 & Linear & $\begin{array}{c}\text { AZ91- } \\
\text { UMAT }\end{array}$ & 2.5 & 1753 & \\
\hline
\end{tabular}

Figure 2 shows the loading direction and boundary conditions of the shock tower. To get to these boundary conditions, several iterations revealed that without adding rotational degrees of freedom, the model was over constrained and highly stressed in the clamping areas. The two free degrees of freedom rotations represented by the light green arrows allowed a larger portion of the component to work under loading. No translations were allowed along these two rotational axes. The load type applied to this structure was a displacement along the axis that was perpendicular to the rotational axes. The experimental fixture was considered to be rigid and was not modeled using FE.

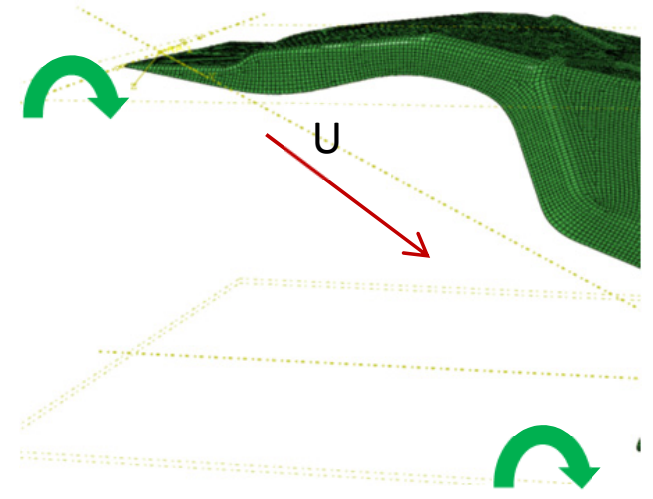

Figure 2. Load and boundary conditions of shock tower.

Regarding the material model, Table 2 shows the constants for the AZ91 Mg alloy. These constants were determined in previous studies carried out at CAVS. Three constants were calibrated using a uniaxial tensile test on specimen extracted from the shock tower. These constants were related to the phenomena. Horstemeyer et al. [1] describes specifically the meaning of each of these parameters. 
Table 2. Plasticity-Damage State Dependent Variables (SDV) of user material subroutine UMAT.

\begin{tabular}{|c|c|c|c|}
\hline Constant Definition & Value & Constant Definition & Value \\
\hline Shear Modulus $\mathrm{G}_{0}$ & $1.656 \mathrm{E}+04$ & Initial Temperature $\mathrm{T}_{0}$ & 297 \\
\hline $\mathrm{a}$ & 0 & Heat generation coefficient & 0.34 \\
\hline Bulk modulus $\mathrm{K}_{0}$ & 49670 & McClintock Damage Constant $\mathrm{n}$ & 0.01 \\
\hline $\mathrm{b}$ & 0 & Initial Void Radius & $2.00 \mathrm{E}-04$ \\
\hline Melting Temperature & 3000 & Torsional constant a (nucleation) & 0 \\
\hline $\mathrm{c}_{01}$ & 0 & Tens/Comp constant b (nucleation) & 0 \\
\hline $\mathrm{c}_{02}$ & 0 & Triaxiality constant c (nucleation) & 0 \\
\hline $\mathrm{c}_{03}$ & 42.9 & Coeff. constant c (nucleation) & 0 \\
\hline $\mathrm{c}_{04}$ & 349.4 & Fracture Toughness $\mathrm{K}_{\mathrm{IC}}$ (nucleation) & 1 \\
\hline $\mathrm{c}_{05}$ & $1.00 \mathrm{E}-05$ & Average particle size (nucleation) & 1 \\
\hline $\mathrm{c}_{06}$ & 0 & Part. Vol. Fraction (nucleation) & 1 \\
\hline $\mathrm{c}_{07}$ & $6.061 \mathrm{E}-03$ & Nearest Neighbor Distance (coalescence) & 12 \\
\hline $\mathrm{c}_{08}$ & 43950 & Chi exponent (coalescence) & 1 \\
\hline $\mathrm{c}_{09}$ & 3220 & Reference Dendrite Cell size $\mathrm{DCS}_{0}$ & 30 \\
\hline $\mathrm{c}_{10}$ & 6.790 & Dendrite Cell size DCS & 30 \\
\hline $\mathrm{c}_{11}$ & 0 & Dendrite Cell size $\mathrm{zz}$ & 0.0509 \\
\hline $\mathrm{c}_{12}$ & 0 & Initial Void Volume Fraction for CA & 0.05 \\
\hline $\mathrm{c}_{13}$ & 3.983 & $\mathrm{c}_{21}$ & 0 \\
\hline $\mathrm{c}_{14}$ & 39.02 & $\mathrm{c}_{22}$ & 0 \\
\hline $\mathrm{c}_{15}$ & 12670 & $\mathrm{c}_{23}$ & 0 \\
\hline $\mathrm{c}_{16}$ & 1.815 & $\mathrm{c}_{24}$ & 0 \\
\hline $\mathrm{c}_{17}$ & 0 & $\mathrm{c}_{25}$ & 0 \\
\hline $\mathrm{c}_{18}$ & 0 & $\mathrm{c}_{26}$ & 0 \\
\hline $\mathrm{c}_{19}$ & 0 & Nucleation Temperature Dependence & 0 \\
\hline $\mathrm{c}_{20}$ & 0 & Elastic Modulus Porosity Exponent $\beta$ & 0 \\
\hline $\mathrm{c}_{\mathrm{a}}$ & 0 & Flag to use vvfr.dat file & 0 \\
\hline $\mathrm{c}_{\mathrm{b}}$ & 0 & Cacon & 30 \\
\hline
\end{tabular}

\subsection{Shock Tower Porosity Distribution Mapping}

Previous studies [1] showed the significant importance of accounting for the heterogeneities of the initial porosity fractions in the component when its behavior-to-failure is studied. Several microstructure observations at various locations of the shock tower were conducted. The results described in Section 2 of this chapter were used to design the initial porosity map of the shock tower. In addition to these measurements, Ford provided the cooling time contours of the shock tower during its casting process. These maps were generated using the casting process simulation code MAGMA. Figure 3 shows these contours, the highest cooling time being yellow and the lowest being blue. 


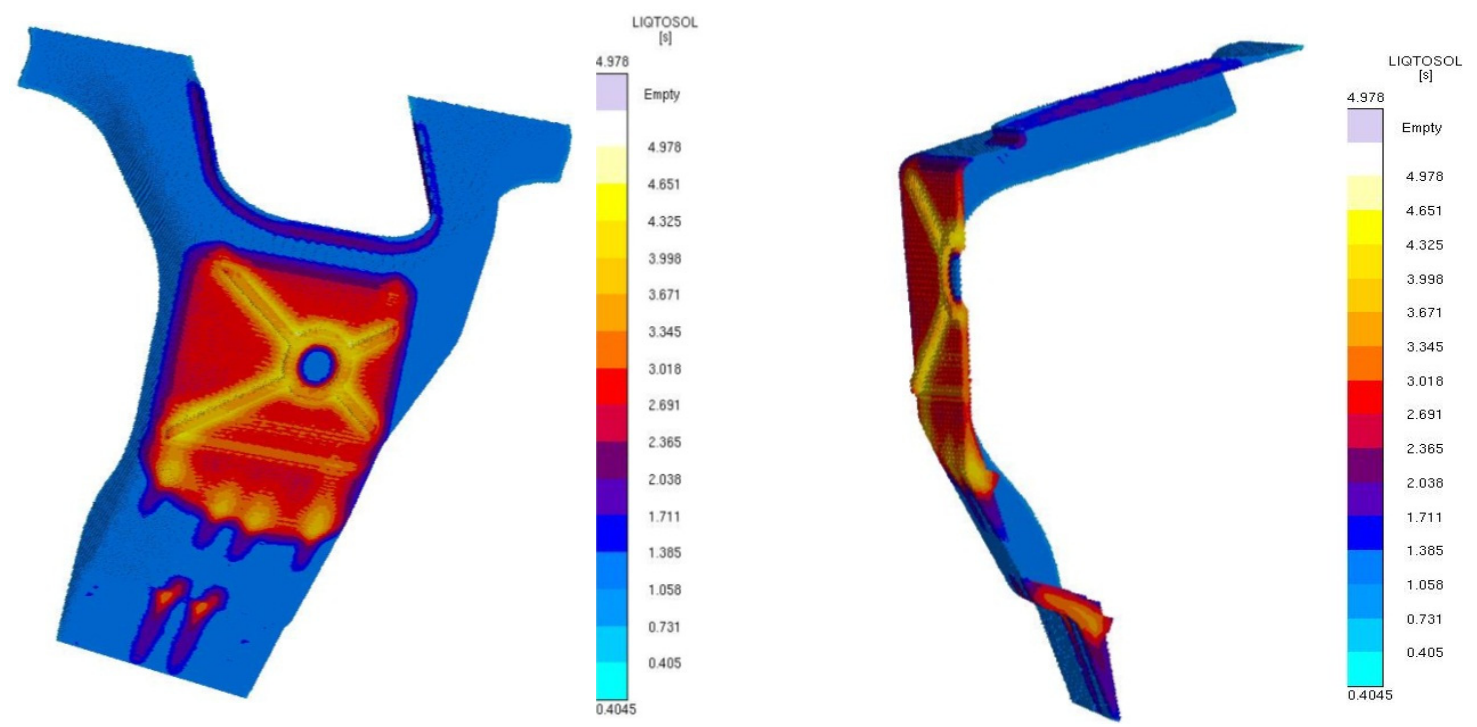

Figure 3. Simulated solidification time contours from Ford Inc.

Also, it was assumed that as the cooling time increased, the porosity fraction increased as well. Based on this logic, the map in Figure 4 was built. The outer skin of the shock tower (in black) is considered to have very little porosity as it is a surface that was first in contact with the die during the casting process. The porosity measurements made in the rib areas (high porosity in orange and red) were also used to build this map. Finally, the inner skin was set to a baseline initial porosity. Specifically, the map was built by the selection of various elements sets and the attribution of a porosity value to each of them.

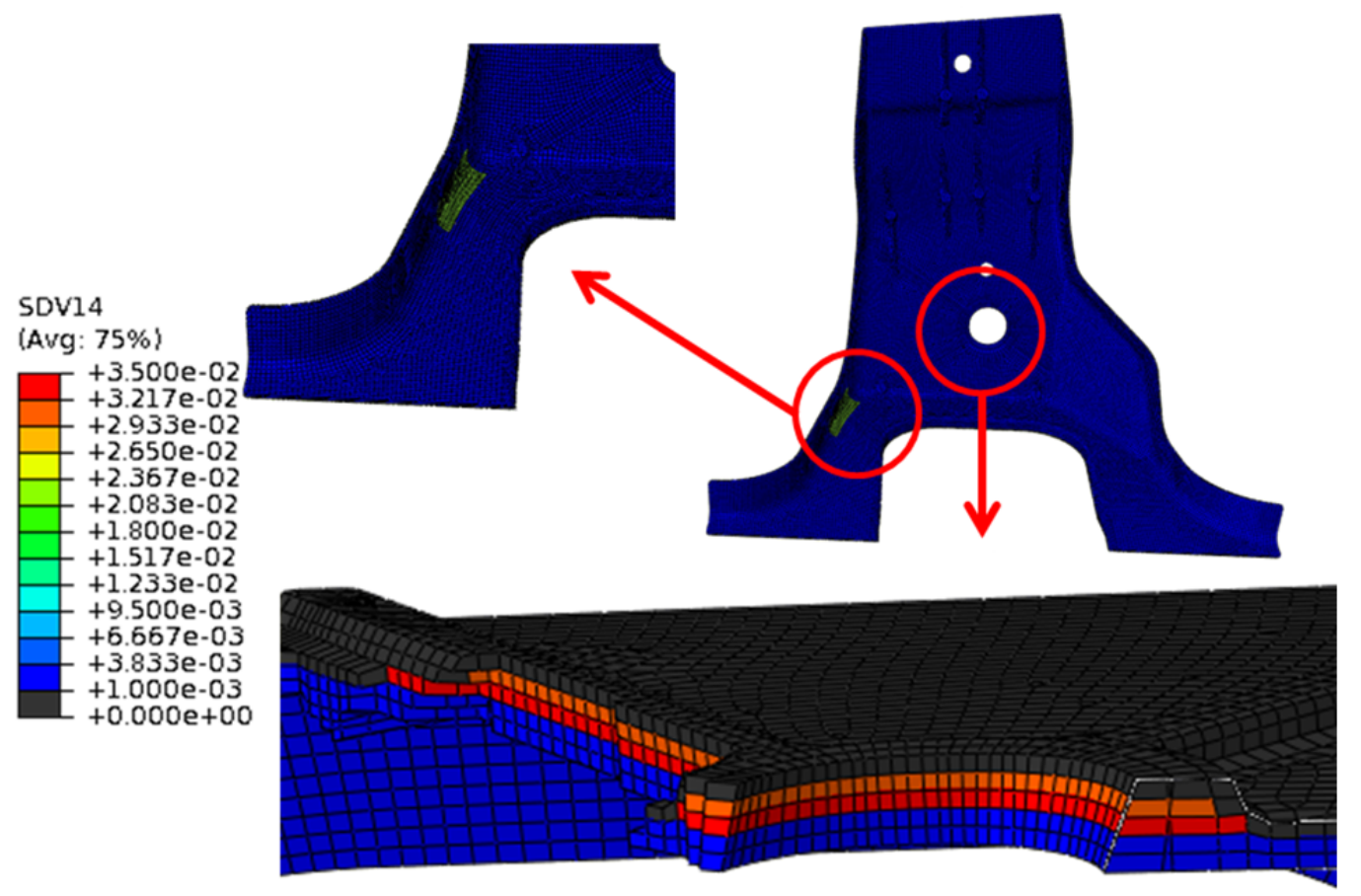

Figure 4. Porosity initial distribution through the thickness. 


\subsection{Modeling Results}

Figure 5 shows the damage contours in the shock tower at failure. The highest damage value is situated in one of the corner legs while the other side of the shock tower is less damaged.

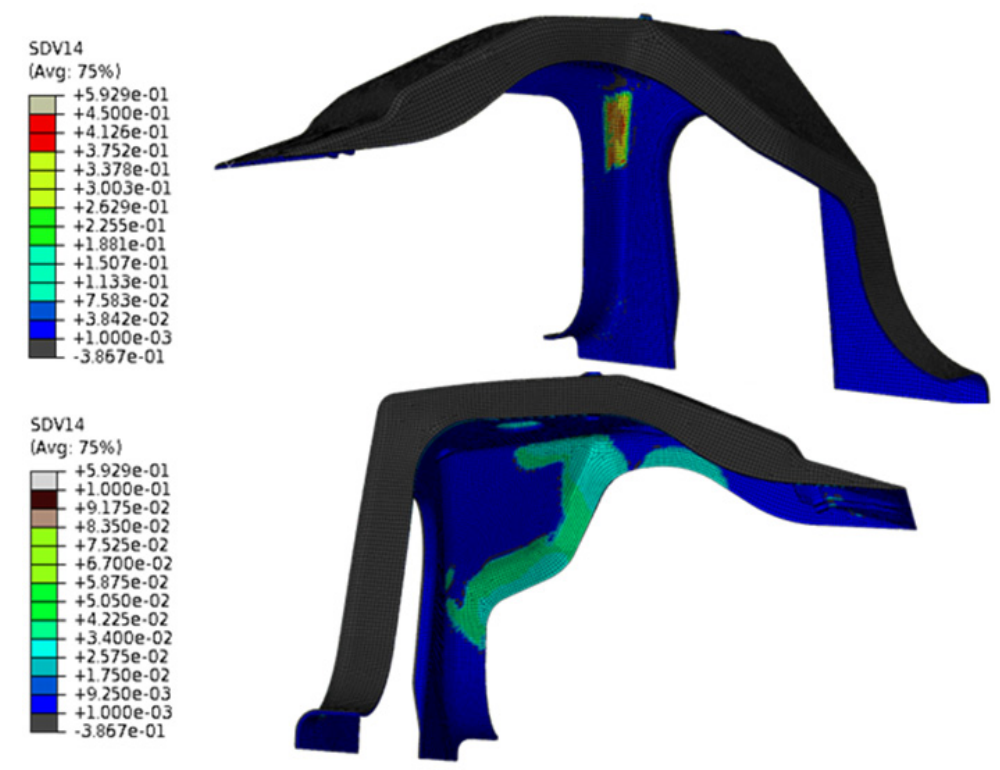

Figure 5. Damage contours of the shock tower.

Figure 6 shows a summary of the results in terms of initial porosity value, damage results and Von Mises results. Four key locations reveal the importance of using the internal state variable material model that includes the microstructural heterogeneities, as Table 3 summarizes. Location $A$ has the highest damage value under monotonic loading, as revealed by the simulations and corroborated by the experiments. Location $B$ has the highest initial porosity fraction, Location $C$ has the highest stress concentration, and Location $D$ is situated in an average range of value for strain, stress, porosity measurement and damage. 


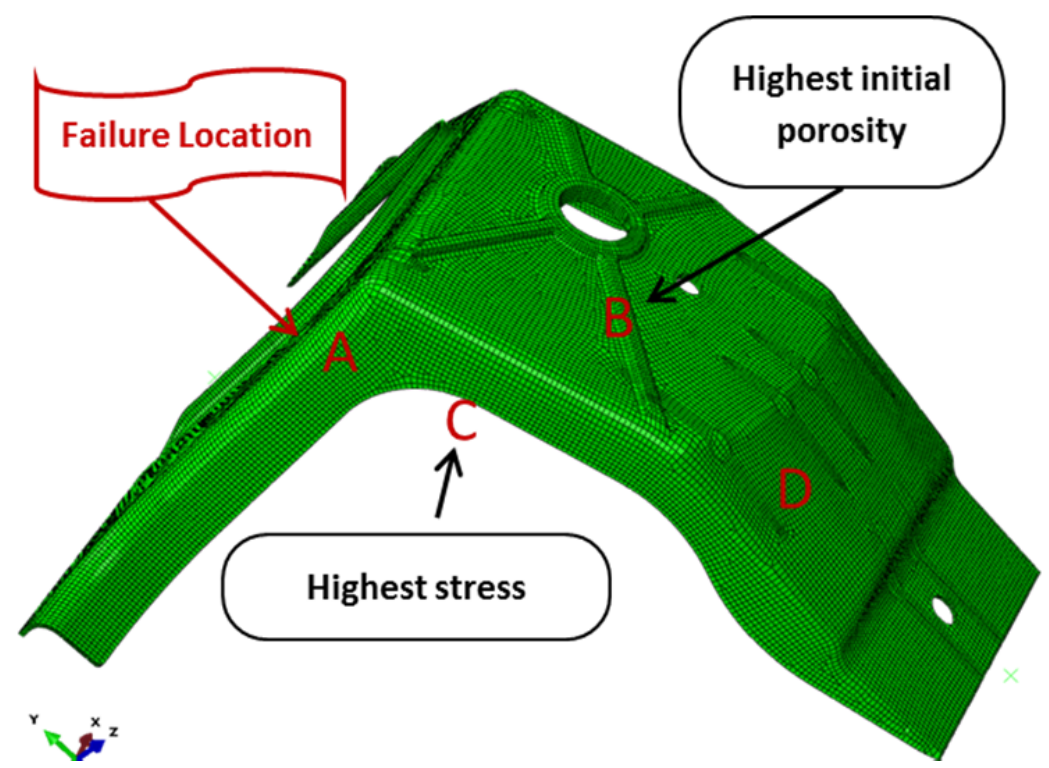

Figure 6. Locations of interest.

Table 3 shows a classification of these locations based on output values of interest such at Von Mises Stress, damage, and initial porosity distribution. An interesting, yet conflicting, conclusion could be drawn, depending on the variables we take into consideration. For instance, from a material science point of view, we could assume that the failure location will be at the highest porosity area, hence B. After running a finite element analysis (FEA) and analyzing the stress distribution, one can conclude that the failure location will be in the area of highest stress, hence C. However, by using a material model that accounts for the initial heterogeneous microstructure of the material and has a damage evolution capability, the failure location is at a very different place, i.e., location $A$.

Table 3. Results on shock tower.

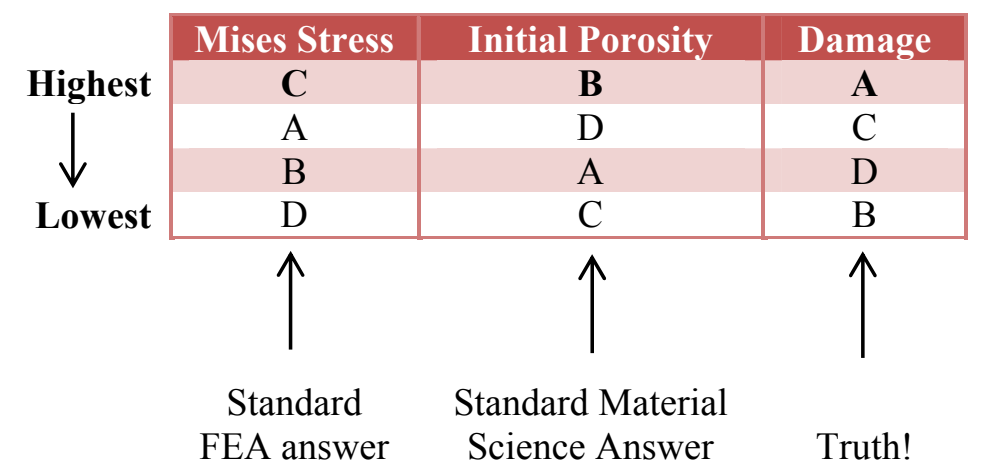

\section{SHOCK TOWER EXPERIMENTS}




\subsection{Background and Initial Observations}

AZ91 automotive shock towers, supplied by General Motors (GM), were received for mechanical testing and failure analysis to validate ISV/damage models at the Center for Advanced Vehicular Systems (CAVS).

Also received with the shock towers was a CAD drawing of the ideal shock tower after machining. To compare the geometry of the as-received shock towers with the ideal shock tower, several locations, as shown in Figure 7, were examined with regard to material thickness. The ideal shock tower thickness was obtained from the CAD drawing at nine locations. For the received shock tower, a Mitutoyo micrometer ( $\pm 0.001 \mathrm{~mm}$ error) was used to measure locations near the edge of part and then used to calibrate an Olympus 35DL ultrasonic thickness tester ( $\pm 0.01 \mathrm{~mm}$ error) to measure locations near the center. The results from four measured shock towers are shown in Table 4 with an illustration of the shock tower CAD drawing showing ideal dimensions and examined locations (A-I) on the real shock towers.

Notice in Table 4 that many of the as-received locations show a mean difference in thickness of about $0.20 \mathrm{~mm}$ from the ideal shock tower. A difference of this thickness is not uncommon in casting; however, due to the shock tower being only 3 to $5 \mathrm{~mm}$ in thickness, some measurements showed up to a 7-10\% change in thickness from the ideal condition.
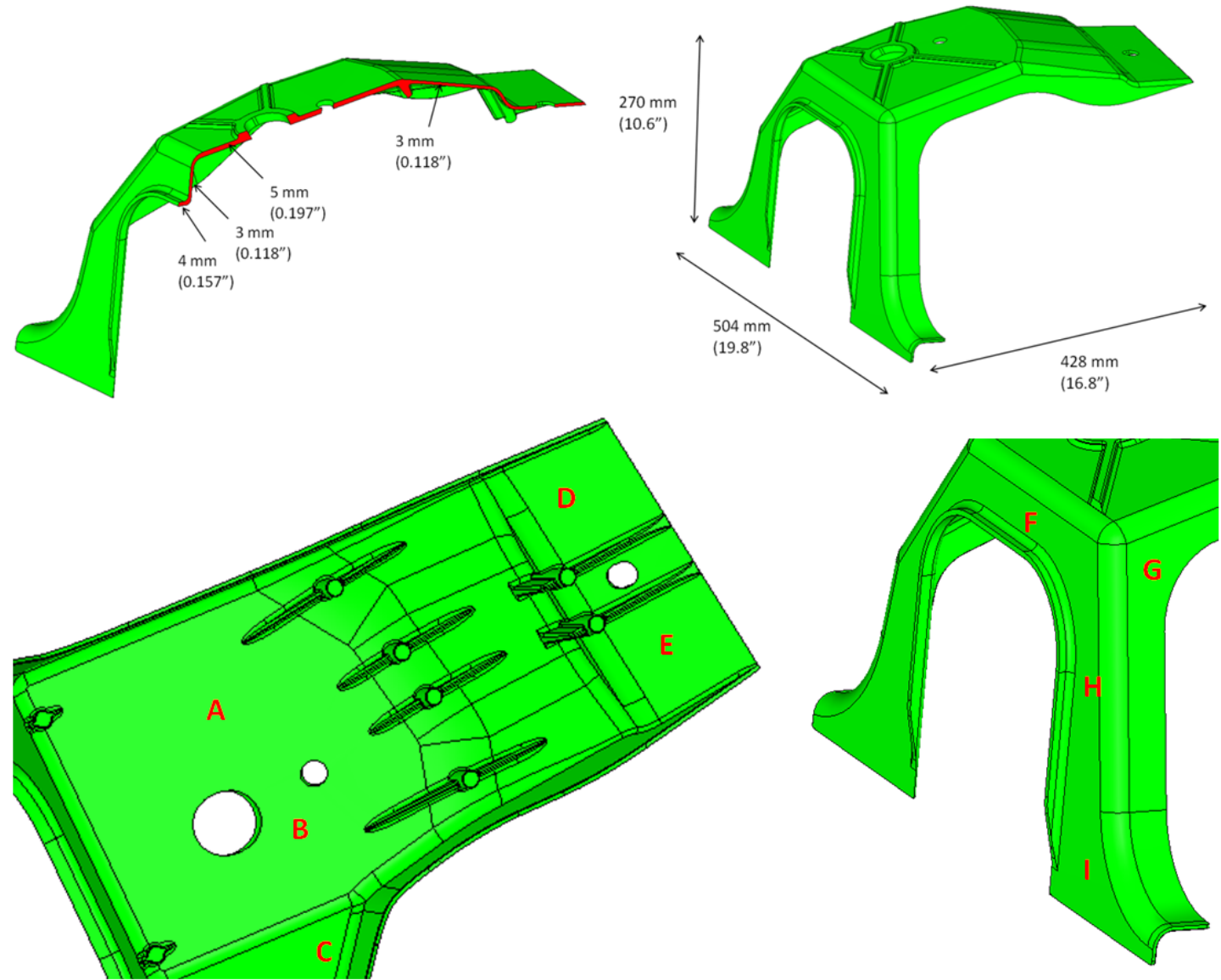

Figure 7. Shock tower CAD drawings and locations A-I for thickness measurements 
Table 4. Mean and maximum thicknesses measured from four shock towers at Locations $A-I$ as compared to the CAD drawing thicknesses

\begin{tabular}{|c|c|c|c|c|c|}
\hline Location & $\begin{array}{c}\text { Mean } \\
\text { Sample } \\
\text { Thickness } \\
(\mathbf{m m})\end{array}$ & $\begin{array}{l}\text { Sample-to- } \\
\text { Sample } \\
\text { Variation } \\
( \pm \mathbf{m m})\end{array}$ & $\begin{array}{c}\text { Ideal Sample } \\
\text { Thickness } \\
(\mathbf{m m})\end{array}$ & $\begin{array}{c}\text { Ideal-to-Real Mean } \\
\text { Difference (mm, } \\
\%)\end{array}$ & $\begin{array}{c}\text { Ideal-to-Real } \\
\text { Maximum } \\
\text { Difference } \\
(\mathrm{mm}, \%)\end{array}$ \\
\hline A & 4.97 & 0.08 & 5 & $-0.03,-0.6$ & $0.11,-2.2$ \\
\hline $\mathrm{B}$ & 4.79 & 0.04 & 5 & $-0.21,-4.2$ & $-0.25,-5.0$ \\
\hline $\mathrm{C}$ & 2.95 & 0.05 & 3 & $-0.05,-1.7$ & $-0.1,-3.3$ \\
\hline $\mathrm{D}$ & 2.84 & 0.10 & 3 & $-0.16,-5.3$ & $-0.26,-8.7$ \\
\hline$E$ & 2.78 & 0.02 & 3 & $-0.22,-7.3$ & $-0.24,-8.0$ \\
\hline $\mathrm{F}$ & 3.04 & 0.06 & 3 & $+0.04,+1.3$ & $0.1,3.3$ \\
\hline $\mathrm{G}$ & 2.72 & 0.02 & 3 & $-0.28,-9.3$ & $-0.3,-10$ \\
\hline $\mathrm{H}$ & 3.15 & 0.05 & 3 & $+0.15,+5.0$ & $0.2,6.7$ \\
\hline $\mathrm{I}$ & 3.19 & 0.04 & 3 & $+0.19,+6.3$ & $0.23,7.7$ \\
\hline
\end{tabular}

Having locations with as much as a $7-10 \%$ difference in thickness can allow considerable uncertainty from the ideal condition, especially when, in the case of a shock tower, many of the loading conditions are in bending. Being that the shock tower deviated significantly from the CAD drawing, consideration of the effects of geometry could play an important role in the mechanical performance.

Although the difference between the ideal and real shock tower thicknesses were significantly different, the real sample-to-sample variation was small. For instance, in Location $G$, the difference between the ideal and real shock tower showed that the real shock tower could be as much as $0.3 \mathrm{~mm}$ thinner than the ideal shock tower, while in measuring four different shock towers the maximum variation was only $0.02 \mathrm{~mm}$, thus showing that all of the shock towers showed the same trend. The large sample-to-ideal and small sample-to-sample difference shows that the shock tower casting process can produce consistent geometry and that an alternative CAD drawing would provide an accurate geometry of many shock towers.

\subsection{Microstructure Analysis}

Being that cast components typically show varying microstructures due to a myriad of environmental effects, investigation into the porosity of the shock towers was undertaken. Specimens taken from seven different locations were extracted, mounted, and polished using Struers recommended polishing procedures and then examined for porosity distribution. Figure 8 shows an illustration of the measured locations and their respective porosity volume fraction. 


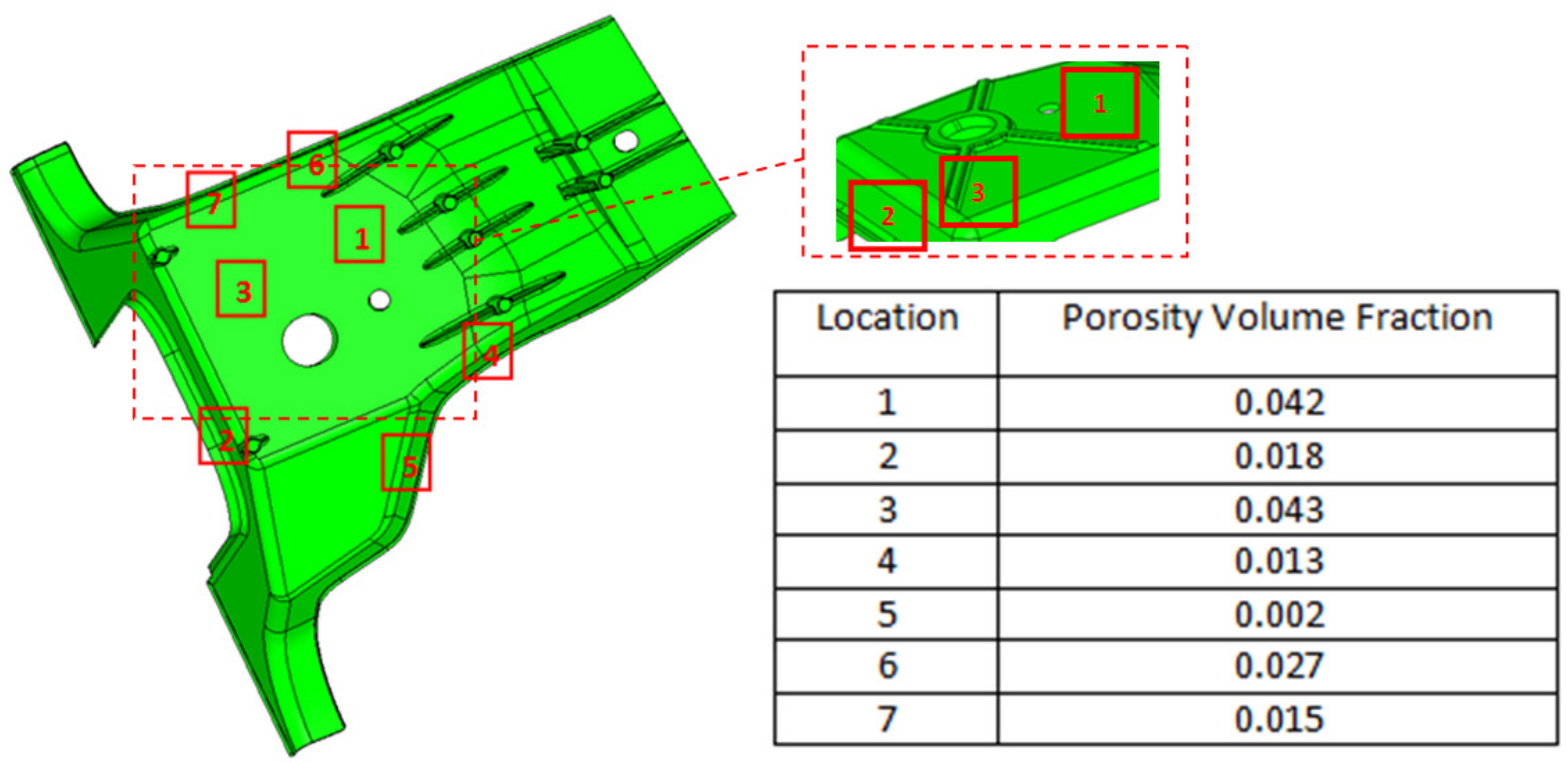

Figure 8. Shock tower measured porosity locations (image) and volume fraction results (table).

Notice in Figure 8 that the porosity volume fraction was significantly higher in Locations 1 and 3 than in other locations. In all locations, with the exception of Location 5 , the porosity volume fraction was greater than $1 \%$, showing that the shock tower exhibits high porosity content. Although many locations showed a high volume fraction of porosity, the high porosity was mainly due to concentrated cracks and defects on the millimeter scale. Notice the optical mosaic image of Location 3 in Figure 9. Much of the image shows large cracks or pores that are overshadowed with a constant low porosity zone. The low porosity zone is precisely the cause of the low volume fraction in Location 5 , as shown in Figure 8 . The increased porosity is from a system of defects that are large. Because the defects are large (major dimension $>1 \mathrm{~mm}$ ), attaining an accurate volume fraction of porosity is difficult. Therefore, as shown in Figure 9, specimen locations of at least $25 \mathrm{~mm}^{2}$ were used for imaging. The optical mosaic, shown in Figure 9, used sixty-four images taken at 500x magnification to construct the mosaic used for porosity volume fraction values.

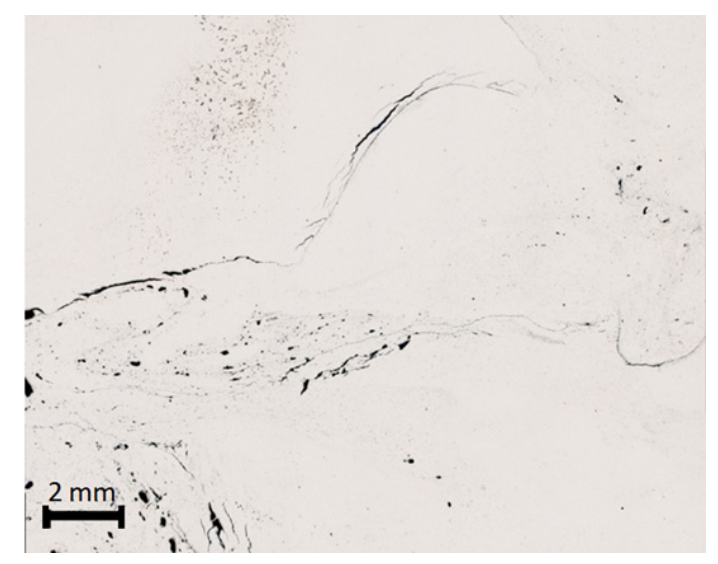

Figure 9. Optical mosaic image of Location 3 showing porosity. 
The large mosaics were imported in a CAVS image analysis code which uses grey scale threshold values to separate the porosity zones from the fully dense material zones. Shown in Figure 10 is a typical result from a single image analyzed with the image analysis software.

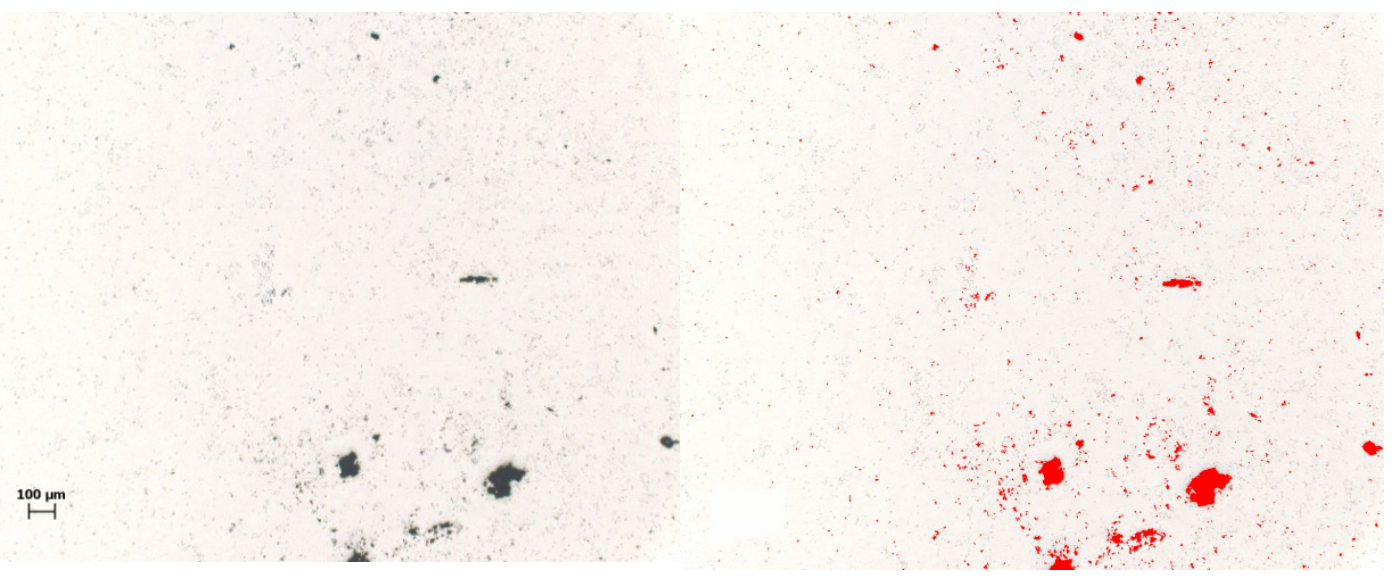

Figure 10. Single optical mosaic image from Location 3 (left) and porosity detected image from software (right).

Because the images gathered from optical microscopy show the revealed porosity on the surface of the polished sample, being able to relate the values of porosity area fraction (2D distribution) to volume fraction (3D distribution) is difficult. Therefore, Locations 3 and 1, were examined with a Phoenix X-Ray CT scanner, which takes over six hundred 2D x-ray images of the sample cross-section and then reconstructs a $3 D$ part showing the true volume fraction of the samples. The samples were then polished and imaged on a Zeiss Optical Microscope along with the other sample locations. Figure 11 shows the two images gathered from the different techniques. The calculation of porosity volume fraction from the 2D images compared well with the 3D scans and therefore showed that the 2D imagery provides an accurate means of attaining the porosity volume fraction.

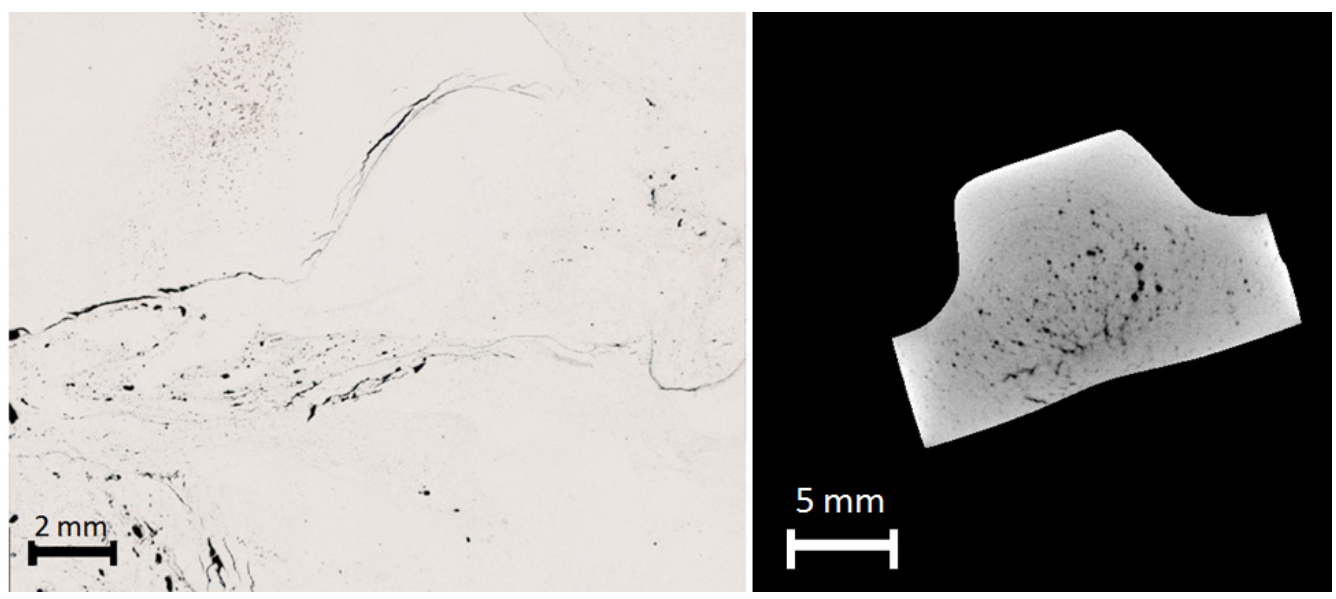

Figure 11. Optical mosaic image (left) and x-ray scanned image (right) of Location 3 showing porosity.

Specimens taken from Locations 1 and 2, measured at the University of Michigan were extracted from several shock towers to show the sample-to-sample variation. The conclusion of that project was 
that at a particular location, sample-to-sample variation was small. Although the sample-to-sample variation was small, the porosity at different locations was much greater.

Because the porosity images in the shock tower microstructure show large pores and high volume fractions, porosity is a main concern in the mechanical performance of the shock tower. Notice in Figure 11 that Location 3 has not only $1 \mathrm{~mm}$ large pores but numerous large cracks that extend beyond $5 \mathrm{~mm}$. Due to the shock tower having thickness of nearly the same values as the cracks exhibited throughout the thickness, defects due to high porosity could be a major concern.

\subsection{Material Mechanical Response}

Mechanical tests were carried out on the shock tower in tension and compression. The tension specimens were extracted from Locations $\mathrm{H}$ and I, shown in Table 4. The tension specimens were flat dog-bones with a gage section of $10 \mathrm{~mm}$ wide, $3 \mathrm{~mm}$ thick, and $50 \mathrm{~mm}$ long, with a fillet radius of 10 $\mathrm{mm}$. The compression specimens were extracted from the rib sections near Location 3 and were machined to right cylinders of $10 \mathrm{~mm}$ in diameter. The specimens were examined and shown to be free of surface cracks. Three specimens of each test method were tested and a representative curve from each is shown in Figure 12.

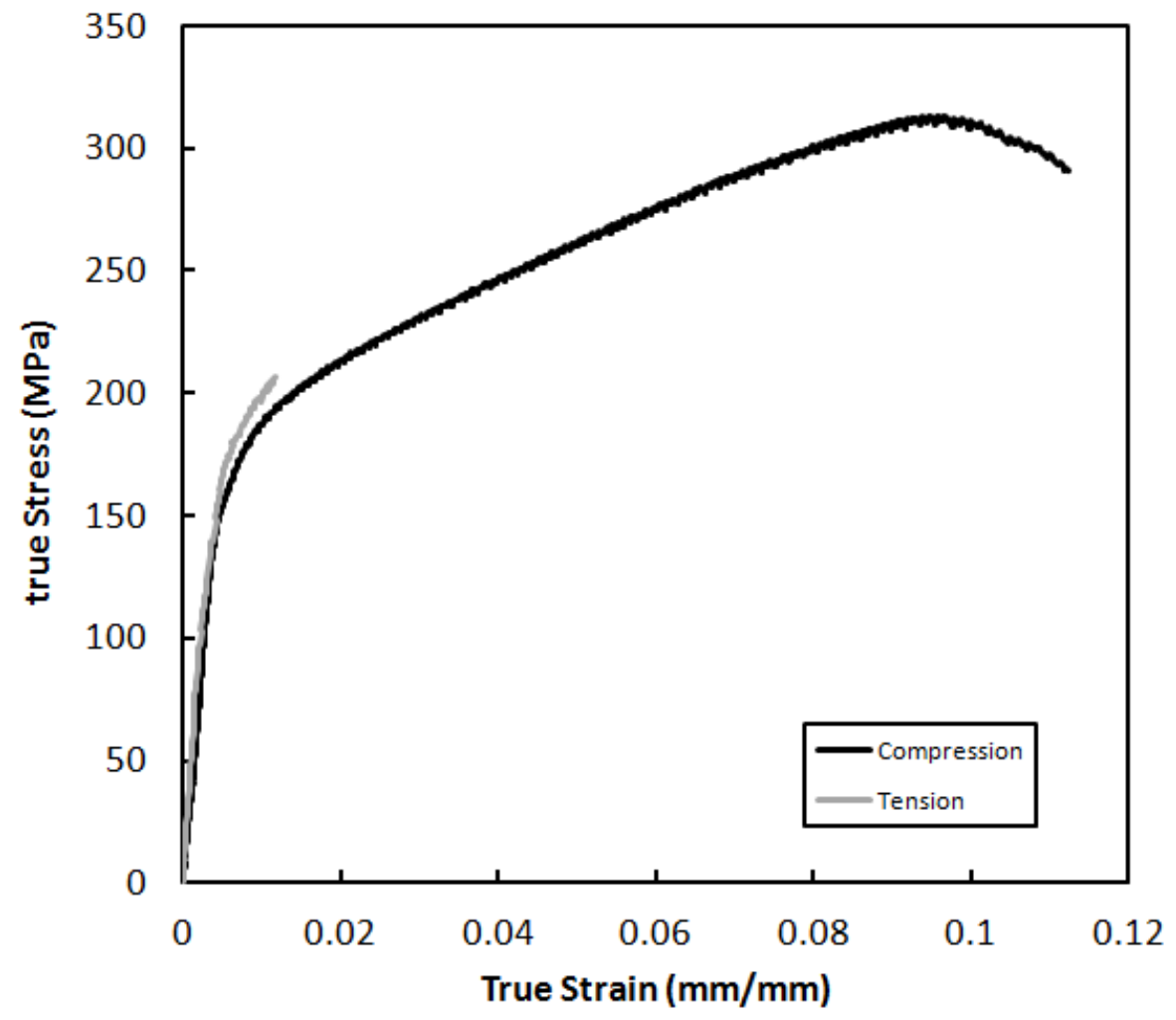

Figure 12. Quasi Static (0.001/s) compression and tension tests results from shock tower material.

Figure 12 also shows that the material mechanical behavior in tension is more brittle than in compression. The tension specimens all failed between 1-1.5\% true strain, and the compression specimens failed between $9-11 \%$ true strain. The yield strength of $175 \mathrm{MPa}$ was not significantly different between the two stress states. 
To further investigate the brittle failure of the tension specimens, the fractured surface of a tension specimen was collected and examined using an EVO-SEM manufactured by Zeiss. Figure 13 shows the fractured surface of the tension specimen at four different magnifications. Notice that the fractured surface is flat and smooth, which is indicative of brittle failure. The large scale porosity presented earlier is the probable driving force for the failure in the tension. The fractured surface did not show a specific fracture initiation location, indicating that brittle cracks emanating from the widely distributed pores coalesced the damage in a brittle manner.

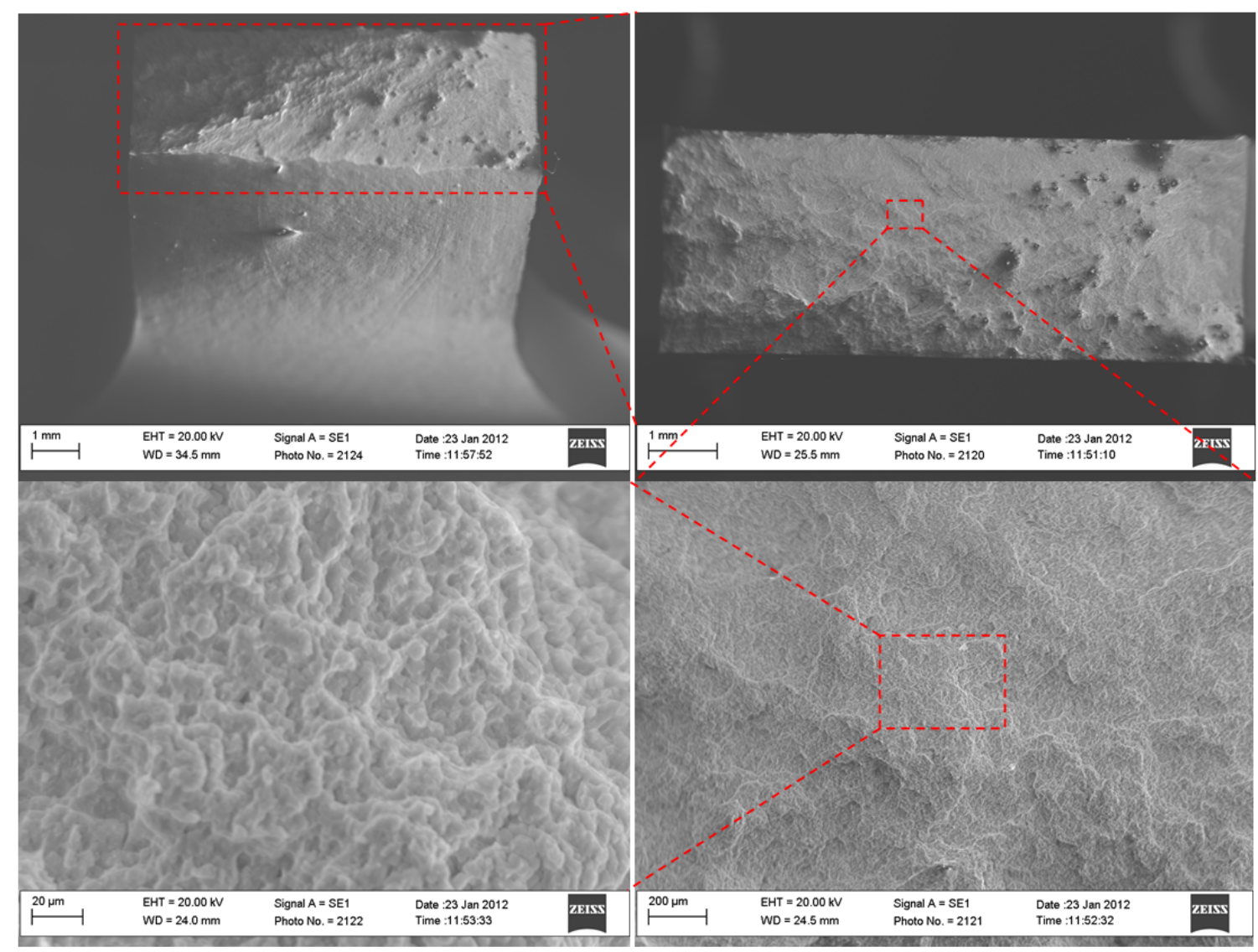

Figure 13. Fracture surface of a quasi-static tension specimen, at four different magnifications, from an AZ91 shock tower.

\subsection{Component Tests}

Two shock towers were used for component testing. The test setup, shown in Figure 14, was chosen to ensure that the failure of the shock tower was not near the fixtures to avoid boundary constraints. As shown in Figure 14, both the legs and the frontal portion of the shock tower were allowed to rotate though their planar axis with a vertical load applied at the frontal portion. The vertical load's line of action was positioned such that it crossed the leg's axis of rotation. This allowed the shock tower to deform without significant rotation of the members. 


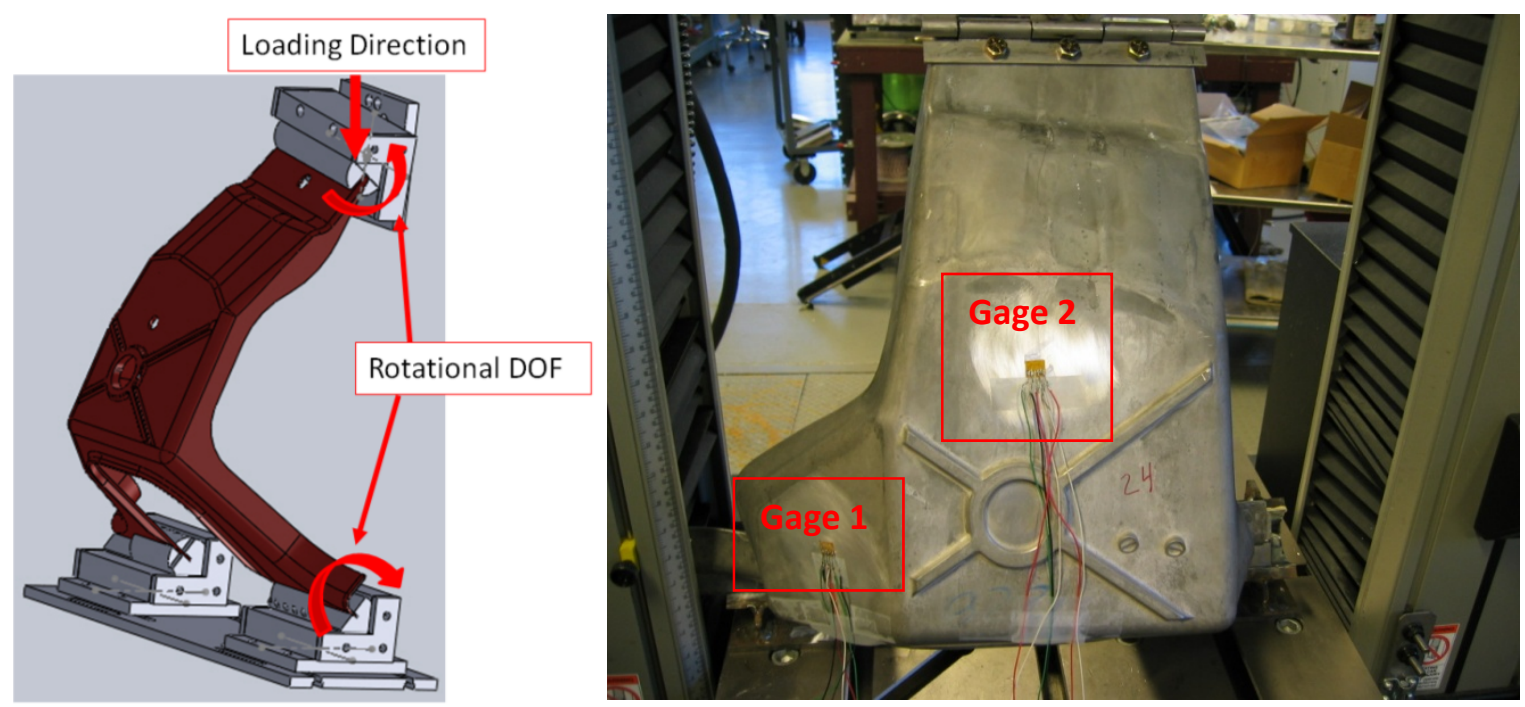

Figure 14. Illustration of test setup (left) and actual test setup (right).(DOF= degrees of freedom)

Two strain gages were placed on the shock tower at arbitrary locations to compare the sample-tosample strain variations due to both the shock tower and the test setup variabilities. The strain gages used were large strain ( $10 \%$ full scale) rectangular rosette gages provided by Micro-measurments. The shock tower was loaded at a displacment rate of $1 \mathrm{~mm} / \mathrm{min}$ and displacment was gathered from the Instron 5882 cross-head displacment.
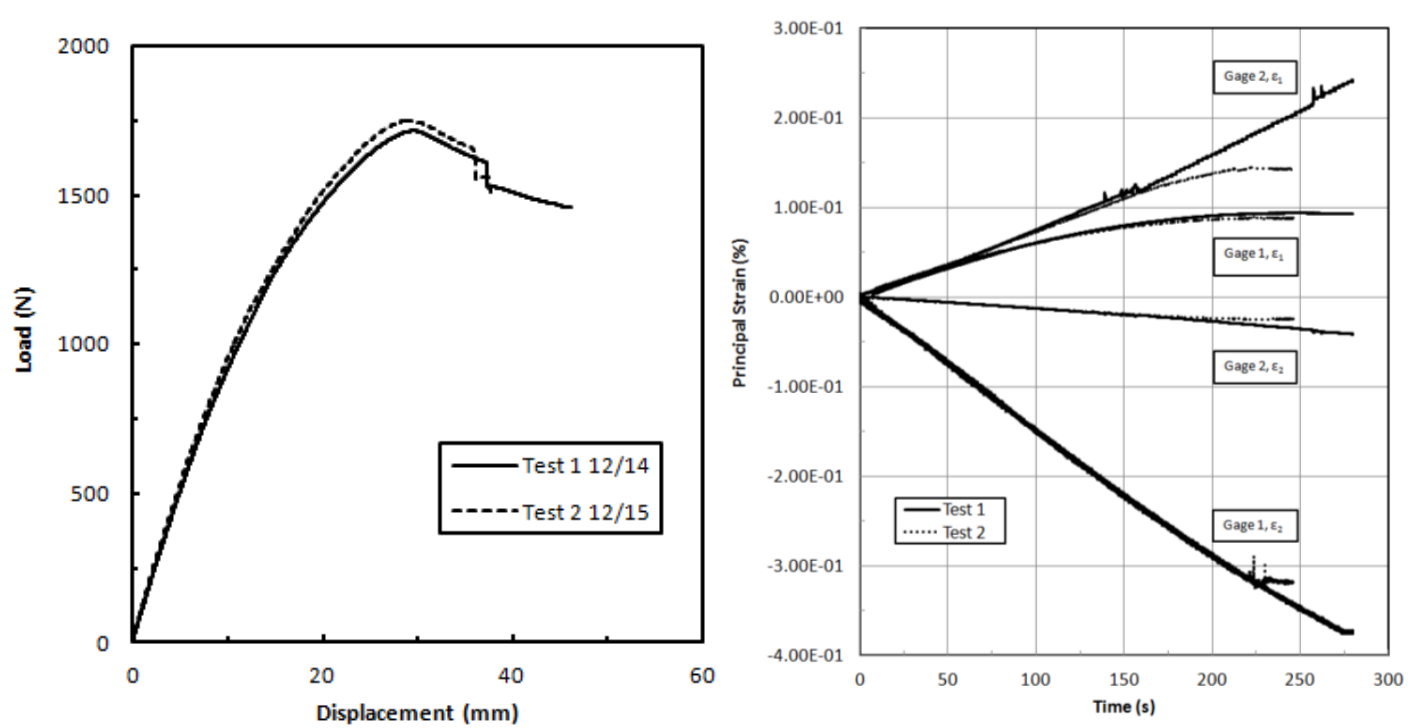

Figure 15. Load versus displacement (left) and calculated principal strain (right) from the two shock tower tests.

Figure 15 shows the shock tower load versus displacement as well as the principal strains calculated from the two rosette locations. The results show little variability in mechanical response between the two tests, which further illustrates the low sample-to-sample variation. Because the geometry, microstructure, and material mechanical response had consistent results, the component tests were expected to also exhibit this trend. The gage data show that the sections where the gages were placed 
exhibited less than $0.5 \%$ strain. While this is not a lot of strain, the failure strain is only about $1.5 \%$ strain in uniaxial tension as shown previously. The low displacement-to-failure of approx. $36 \mathrm{~mm}$ is also a result of the low strain-to-failure of the material.

During the test, several video cameras were placed in various locations to capture the onset of fracture. A large crack was observed in one of the legs and was accompanied with the load drop at 36 $\mathrm{mm}$, as shown in Figure 15. The test was stopped and images of the fracture were gathered and are shown in Figure 16.
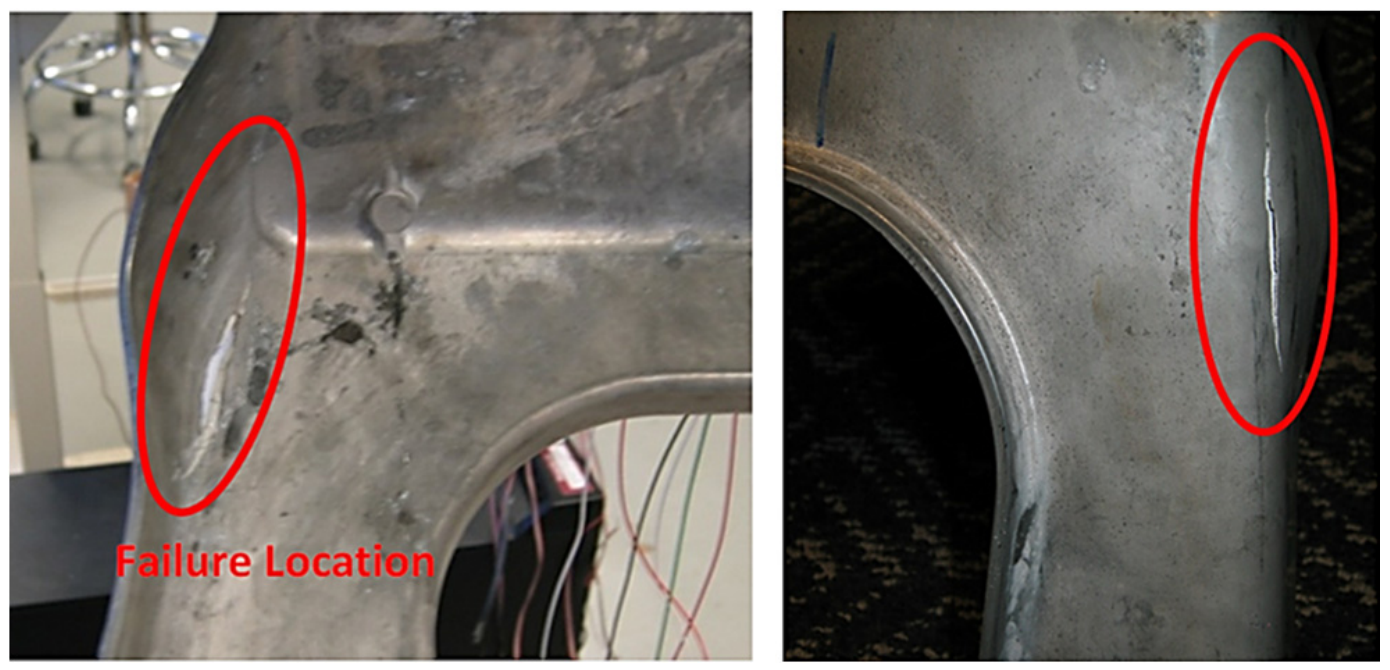

Figure 16. Fracture location from the shock tower component test.

The fracture initiated at the center of the crack and then grew in two directions to form the crack observed in the images in Figure 16. Both shock towers experienced failure at the same location; however, the crack lengths were different. This difference can be attributed to the fact that in Test 1 the Instron machine was not stopped as soon as the crack initiated. For Test 2, however, knowledge of the crack location and the failure displacement was known, and therefore the test was stopped sooner. This also explains the deviation of the principal strains from the two tests at the end of the tests, as shown in Figure 15.

The location of failure was close to the measured porosity of Location 7 and the measured thickness of Location G. Although Location 7 had a median value of porosity as compared to the other locations, Location $G$ has one of the highest reductions in thickness as compared to the ideal CAD drawing received. Because of thinness of the section, the cracks became large as the stress increased in bending. Therefore, increasing the thickness of the material in this location would increase the maximum load and strain-to-failure in tested loading condition.

\section{COMPARISON FEA-MONOTONIC EXPERIMENTS}

Due to the limited number of parts available at CAVS, two shock towers were tested and both of the experiments provided good load and displacement data. Also, the failure locations were consistently situated in the same part area. 
In terms of load-displacement behavior, Figure 17 shows the two plots from simulation results and experimental results respectively. As shown, the physical testing of the part validated the simulated results.

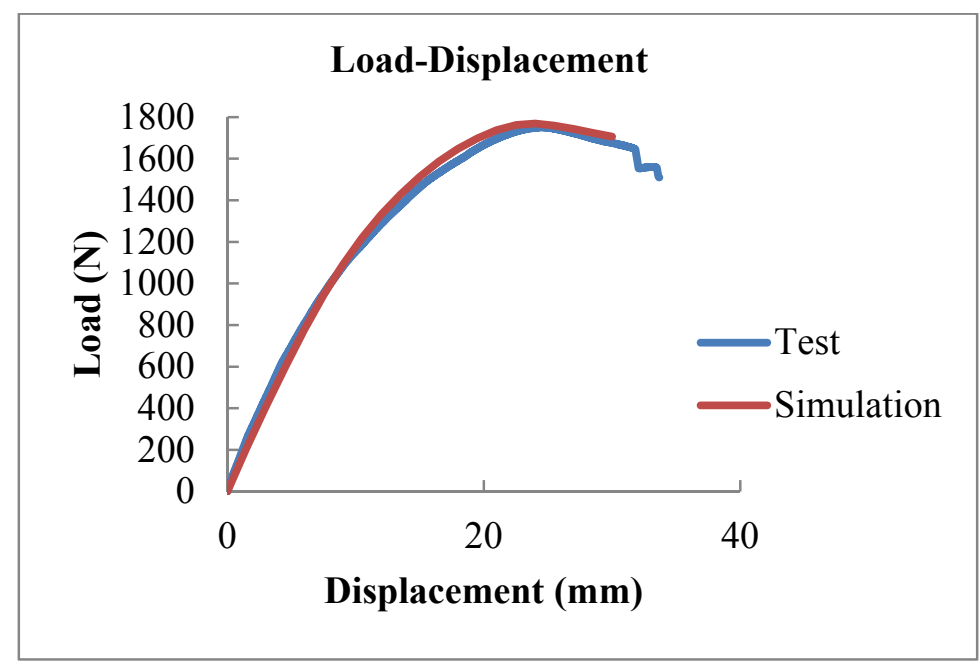

Figure 17. Load displacement curves from modeling simulation results and experimental test results of the shock tower.

Finally, Figure 18 shows the comparison between Figure 16 and Figure 5, where one can observe that the failure location predicted by the ISV plasticity damage model that was used in the FE analysis was validated by experimental results.
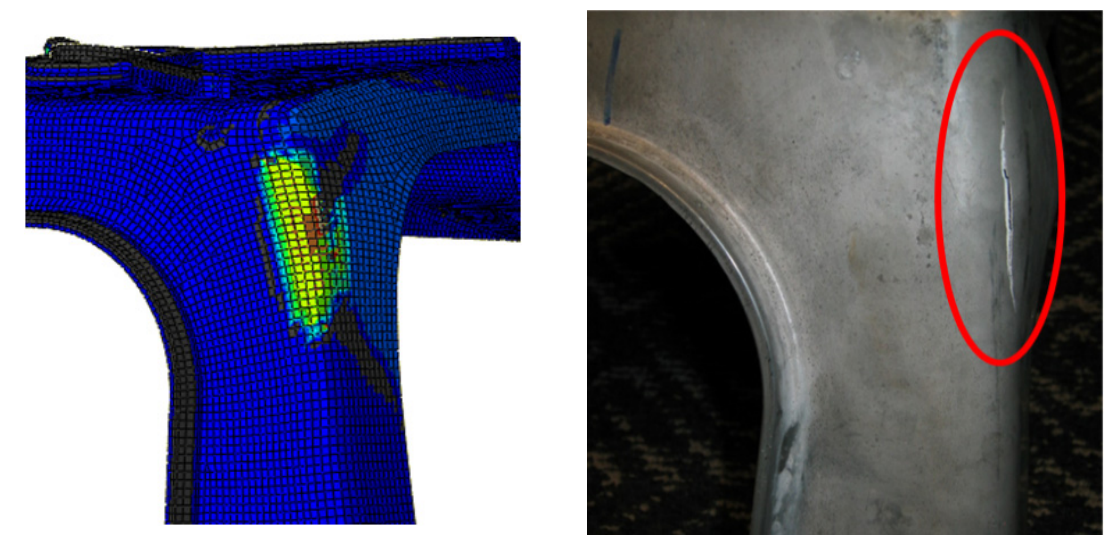

Figure 18. Failure location from FE analysis and experimental testing

As a conclusion, the microstructure-property material model was able to predict the failure location of a $\mathrm{Mg}$ alloy component under monotonic loading. The simulated results correlated the experimental data not only in terms of load and displacement but in terms of failure location as well, providing that the initial porosity mapping and geometrical features were respected. For this loading case, the accuracy of the thickness in the failure area and the fidelity of porosity mapping were first order parameters for a successful and accurate failure location prediction. 


\section{Fatigue Tests and Predictions of the Shock Tower}

\subsection{Introduction}

This section presents the results of fatigue tests on the selected shock tower component. Here are discussed the results observed in the component under cyclic loading and the predictions provided by the multistage fatigue (MSF) model derived from finite element simulation. The objective of this task was to evaluate the performance of the shock tower under real loading conditions. In addition, the effectiveness of the MSF model was assessed. Finally, predictions of the MSF along with the finite element simulations are compared with the lab-run fatigue results.

\subsection{Fatigue Shock Tower Tests}

The test setup utilized in the fatigue testing was similar to the setting employed for monotonic tests shown in Figure 14. Details of fixtures and reasons for selecting this arrangement have been discussed previously (see Section 2.4). The fatigue tests were performed using an Instron 5882 load frame with a constant strain rate of $400 \mathrm{~mm} / \mathrm{min}$, which is equivalent to a frequency of $0.167 \mathrm{~Hz}$. The tests were conducted under displacement control with a maximum displacement of $20.0 \mathrm{~mm}$ and a minimum of 0.0 $\mathrm{mm}$.

\subsection{Shock Tower Fatigue Response}

Two shock towers components were fatigued and the development of crack was monitored following the same method that was used for monotonic tests (see Section 2.4). The number of cycles corresponding to the crack length in the order of 1-2 mm was recorded. After the crack was detected, it grew for several cycles and then stopped. The number of cycles observed for each test is given in Table 5. The load-displacement response is given in Figure 19. This curve shows a slight asymmetrical shape as well as a slight nonlinearity. In Mg alloys, asymmetry and nonlinearity is expected due to the activation of slip and twinning deformation mechanisms during cyclic loading.

Table 5. Shock Tower Fatigue Tests Results

\begin{tabular}{l|l}
\hline Tests & Total Life \\
\hline $\mathbf{1}$ & $\mathbf{2 4 0 0}$ \\
$\mathbf{2}$ & $\mathbf{2 1 0 0}$ \\
\hline
\end{tabular}




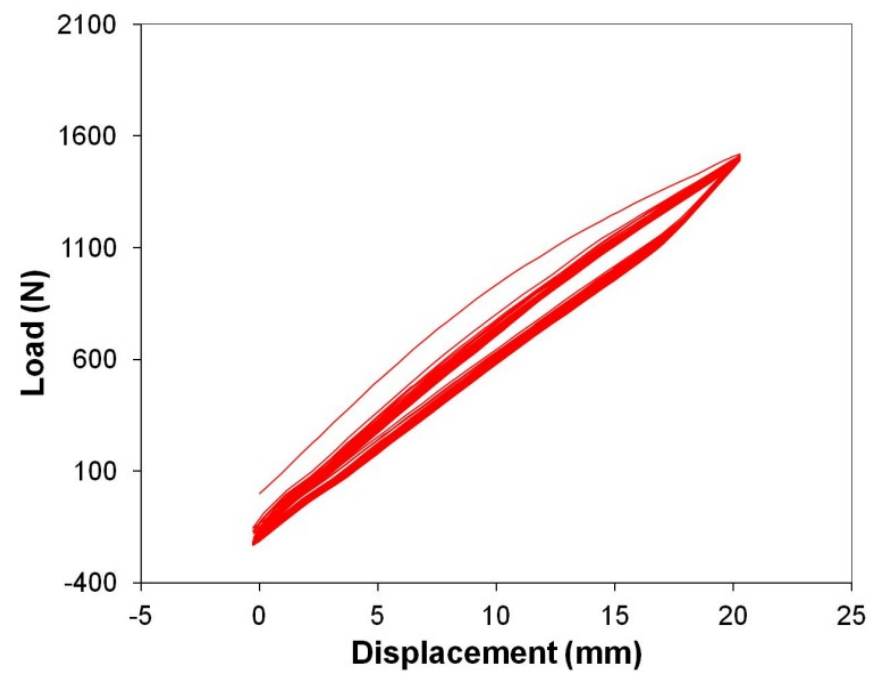

Figure 19. Load against displacement

The failure location for the first fatigued shock tower is shown in Figure 20. The crack initiation point was located approximately $22 \mathrm{~mm}$ from the triple intersection of the edges which is indicated within the red box (and a different location than the monotonic tests). After some crack growth was allowed, the test was stopped. Similar results were observed for second tested shock tower, with the fault occurring at almost the same location. Furthermore, this second shock tower developed several minor cracks near to the rivets, but these cracks did not develop further.

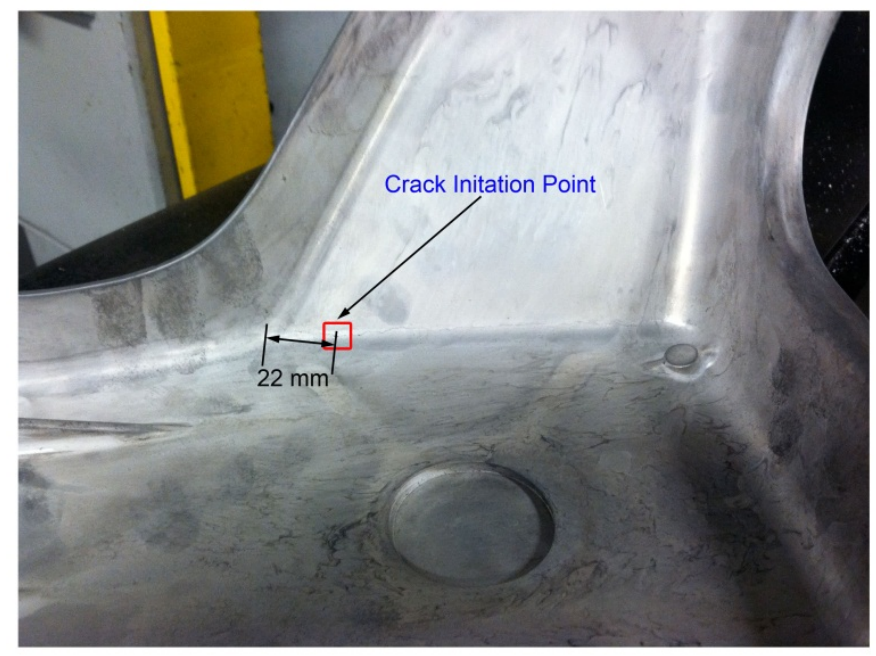

Figure 20. Failure location for fatigue shock tower test 1.

\subsection{Finite Element Analysis and Fatigue Predictions}

Finite element simulations of the shock tower were conducted to predict fatigue failure and failure location for the component. An internal state variable (ISV) material model based upon multiscale modeling was employed to predict the monotonic behavior of the component. Then the MSF model was incorporated to compute the estimated life of the component. The ISV and MSF models were integrated 
through a commercial finite element package (Abaqus). These models predict the onset of surface cracks in automotive structures or sub-structures.

The stress and strain components (stress tensor, and strain tensor) were results from the FE simulations. These values are the main inputs along with the MSF parameters to compute predicted values for fatigue life.

\subsection{Finite Element and Experimental Results}

The estimated lives for the finite elements (locations) under study are shown in Figure 21. The MSF predictions for each location are indicated along with experimental results. Point $A$ is where the fatigue failure occurred. Location B represents the highest porosity point. Location $\mathrm{C}$ represents the highest Von Mises stress location. Location $D$ represents the average values of porosity and stress. As observed, differences exist between the predicted and the experimental results although they are close. These differences can be attributed to the microstructural feature variations. Nonetheless, the experimental results fell within the confidence bands established for the MSF model. These bands delimit the scattering of fatigue associated with microstructure variations.

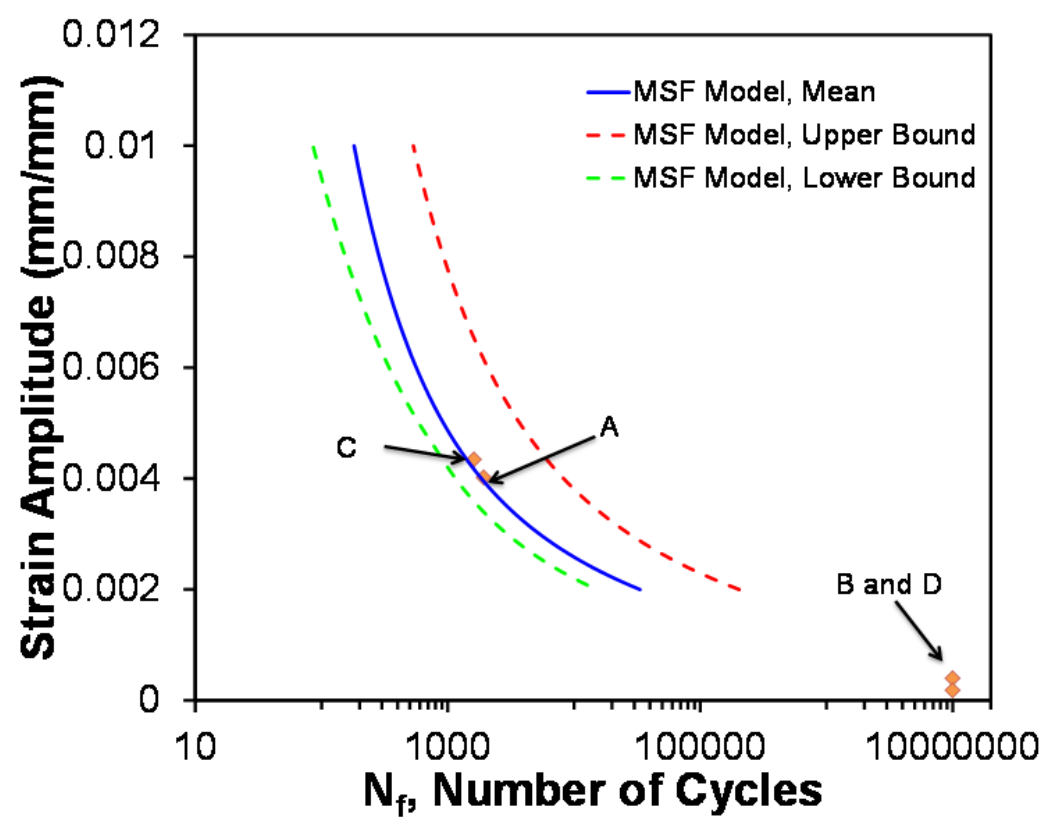

Figure 21. Fatigue results and MSF model predictions. (Point A denotes the experimental fatigue failure location)

Table 6 shows a classification of locations A, B, C, and D according to the approach employed. These results clearly show that the predicted point of failure will vary significantly depending upon the approach used. The material science point of view assumes that the failure location will be at a location with the highest porosity, and hence concludes that failure will occur at Point B. On the other hand, an analysis that only examines the stress distribution will lead to the conclusion that the failure location will be at a point with the highest Mises stress, i.e., Point $C$. However, neither Point B or C was observed to be the true fatigue failure location; instead, failure occurred at Point A. Notably, the ISV-MSF tool, which 
incorporates material models based on mechanical and microstructural properties was able to accurately predict the failure location as Point $A$.

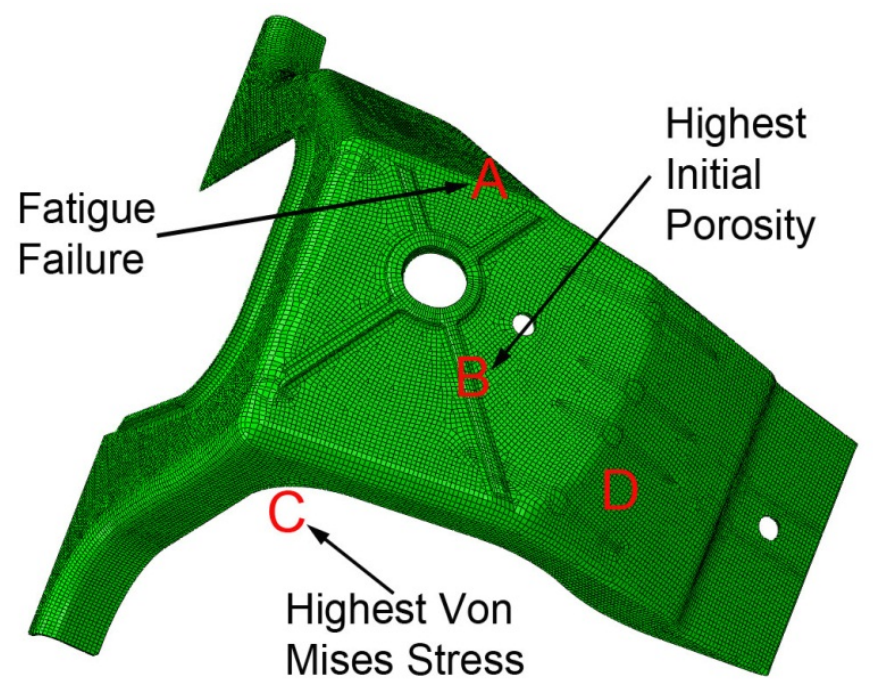

Figure 22. Finite Element Model and Failure Locations

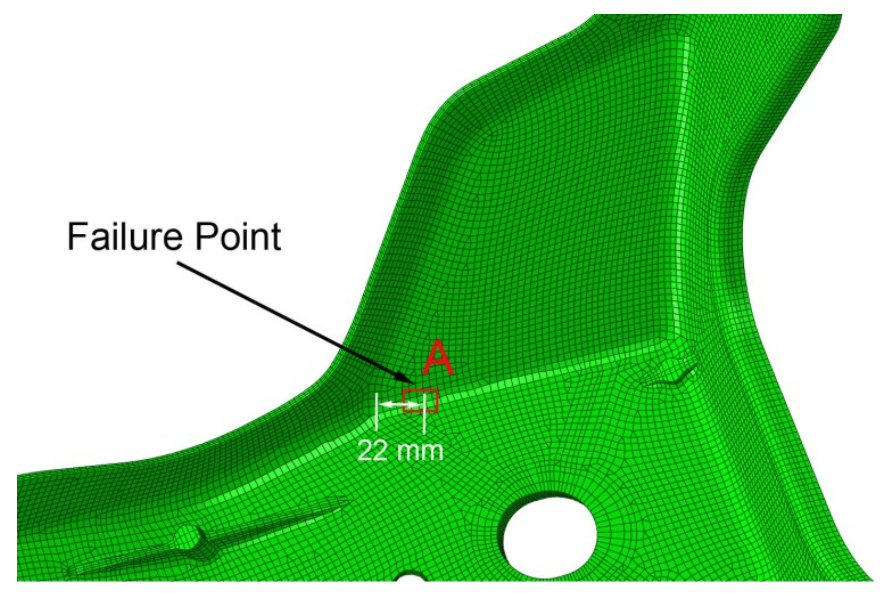

Figure 23. Finite Element Model and Fatigue Failure Location. 
Table 6. Locations of interest for the shock tower.

\begin{tabular}{c|lll}
\hline & $\begin{array}{l}\text { Mises } \\
\text { Stress }\end{array}$ & Initial Porosity & $\begin{array}{l}\text { MSF Fatigue } \\
\text { Life }\end{array}$ \\
\hline Highest & C & B (0.043) & $\mathrm{B}\left(1 \times 10^{7}\right)$ \\
& A & A (0.035) & $\mathrm{C}\left(1 \times 10^{7}\right)$ \\
& B & D (0.022) & D $\left(1 \times 10^{7}\right)$ \\
& D & C (0.015) & A $(1926)$ \\
\hline \multicolumn{5}{c}{ Standard } & $\begin{array}{l}\text { Standard Material } \\
\text { Science Answer }\end{array}$ & Truth! \\
& FEA & Answer &
\end{tabular}

Answer

The microstructure-property MSF material model predictions reflect the influence of microstructure features such as dendrite cell size, particle inclusion size, and porosity, which significantly affect the total fatigue life. Nonetheless, as shown in Table 6 , the estimated life for Point $C$ is slightly greater than for Point A. However, the porosity is significantly greater for Point A than Point C. This result is not surprising since it is well known that high content of porosity is highly detrimental under cyclic loading conditions in cast alloys. Therefore, it is clear that not only the strain, stress, and mechanical and microstructure properties define failure location but the combination of all these factors. Thus, the MSF model represents a consistent, holistic integration of geometry, loading conditions, mechanical, and microstructure properties to provide a better description of the fatigue performance of a component or structure.

\section{Conclusions}

\subsection{Monotonic Observations and Experiments}

AZ91 shock towers, provided by GM, were examined and tested to provide insight into the mechanical performance and failure mechanism. The initial examination of the geometry of the shock towers revealed large variation in thickness, with many locations having a reduced thickness as compared to the ideal shock tower geometry. Sample-to-sample variation in thickness was shown to be low for a single location which showed consistent geometry between samples.

When investigating the microstructure of the shock towers, large defects in the form of pores and cracks were observed. Many locations exhibited large cracks on the order of 1-5 mm in length and large pore volume fractions on the order of $1 \%$. Testimony from the UM project groups led to understanding that the sample-to-sample variation in porosity distribution was low and showed that the microstructure between shock towers was similar for a single location. 
Experimental testing of the AZ91 material in tension and compression revealed low strain-to-failure and low sample-to-sample variation, which substantiated the testimony from the UM group's conclusion on low microstructure variation. The brittle failure in the tension specimens were analyzed using an EVO SEM and showed a very flat and smooth fracture without necking. This led to the conclusion that the overwhelmingly high porosity and large crack lengths led to the materials inability to accommodate deformation at large strains.

Component testing to the shock towers showed low displacement to failure exhibited by a large verticle crack in one of the shock tower legs. The failure location and load versus displacment data showed consistency between two samples, which also demonstrated that the low sample-to-sample variability led to the components performance being similar. The failure location was found to exist in a location where the material was much thinner than was originally intended and could have played an important role in the low displacment-to-failure of the component.

In conclusion, the microstructure of the shock tower played a key role in the material's mechanical response. In turn, the material mechanical response, along with the large geometry variation from the ideal case, governed the low performance of the shock tower component. Therefore, to increase the shock tower performance, the shock tower thickness must be kept more uniform and the material microstructure defects should be reduced.

\subsection{Fatigue Tests and Predictions}

This study has revealed the power of microstructure based models to predict fatigue failure in design. Fatigue failure location is dependent upon stress and mechanical and microstructure properties. Applied stress and porosity significantly affect the fatigue performance. However, other microstructure properties, such as pore size and dendrite cell size, also influence the fatigue behavior. The finite element simulations coupled with the MSF model correlate well with the experimental tests. This investigation has shown undoubtedly that fatigue failure of a component must be studied based on an integrated, holistic approach that involves jointly the material properties, geometry and loading conditions.

Note the MSF fatigue model when performing the above predictions, it used two approaches regarding the state of initial porosity: (1) a constant porosity across the whole shock tower, and (2) the state of porosity varied across the whole shock tower, according to the zone mapping method. The outcome of the fatigue predictions shown above however, was not sensitive to these two approaches, because the coupon fatigue data provided by the University of Michigan team was not sensitive to the initial porosity. As a result of MSF model tuned to match the coupon fatigue data set, it therefore was not sensitive to the state of porosity as applied to the component level - the shock tower case. Nevertheless, the MSF model has the capability of being sensitive to the state of microstructure including porosity morphology, grain size, and dendritic cell size, if required.

\section{REFERENCES}

[1] M.F. Horstemeyer, D. Oglesby, J. Fan, P.M. Gullett, H. El Kadiri, Y. Xue, C. Burton, K. Gall, B. Jelinek, M.K. Jones, S. G. Kim, E.B. Marin, D.L. McDowell, A. Oppedal, N. Yang, USCAR MSU CAVS CMD 
2007-R0001: From Atoms to Autos: Designing a Mg Alloy Corvette Cradle by Employing Hierarchical Multiscale Microstructure-Property Models for Monotonic and C. 


\section{E. Cyberinfrastructure}

\section{INTRODUCTION}

The primary focus of the ICME vision is establishing a knowledge base accessible to the community-atlarge for solving a plethora of disparate issues in material science, applied mechanics, and engineering. This knowledge base requires collection of experimental data describing phenomena at different scales (exploratory experiments, calibration of material models, and validation of models), performing simulations at different scales (atomic, molecular, dislocation, crystal-plasticity, macro-scale FEA), and linking all this information together to determine structure-properties relationships, thereby leading to new concepts and design of new materials. In addition to pushing the edge of material science and solid mechanics by supporting the development and validation of new methods, particularly in the area of multiscale modeling which requires multidisciplinary expertise, the knowledge base is further expected to be used for engineering design optimization and to support workforce training, including enhancing academic curricula at the graduate level.

It follows that managing the ICME knowledge base directs the principal rationale and objective for establishing a virtual organization (VO). Management entails gathering, developing, integrating, and disseminating experimental data, material models, and computational tools, as well as their use for material and product design. Consequently, the Engineering Virtual Organization for CyberDesign (EVOCD, http://icme.hpc.msstate.edu) is dedicated to the accumulation of the "intellectual capital" pertaining to ICME. It is the organization's capital that attracts community participation. There are three critical aspects to the process of accumulating capital in order to create a relevant organization: (1) protection of intellectual property, (2) quality assurance of information, and (3) the management of complexity.

\section{FUNCTIONALITY OF EVOCD}

EVOCD has been developed with the primary goal of accumulating and protecting the intellectual property generated by the participants of the organization. The portal provides powerful passage for accruing and exchanging community knowledge as well as access to repositories of experimental data, material models and computational tools at different length scales, which together exploit the integrative nature of ICME. To achieve this goal, EVOCD is comprised of four primary functional components that are the foundation of the VO: (i) Knowledge Management; (ii) Repository of Codes; (iii) Repository of Data; (iv) Online Calibration Tools.

\subsection{Knowledge Management: Wiki}

Knowledge management has been achieved by applying an "architecture of participation" as advocated and implemented by Web 2.0 concepts and technologies. Tools like Wiki lead to the creation of a collective (read: peer-reviewed) knowledge database that is always up-to-date with a structure that spontaneously evolves to reflect the current state of the art. Therefore, we have chosen Wiki as the mechanism for community-driven knowledge management. 
The Wiki has become the façade for the EVOCD portal to accumulate the knowledge pertaining to ICME. The Wiki captures the knowledge about different classes of materials (metals, ceramics, polymers, and others), material models at various length scales, and design issues, from process and performance models, to optimization under uncertainty, to bio-inspired design. In addition, the Wiki provides direct access to resources, such as data and code repositories.

The intellectual property is protected by configuring the Wiki server to restrict creation and editing of pages to only registered users verified by their email addresses. As a result, all contributions are uniquely attributed to their authors. Following the model introduced by Wikipedia, the quality of contributions is guaranteed by the Web 2.0 process but further monitored by the Wiki editors.

\subsection{Repository of Codes}

ICME applies computational methods to material science, applied mechanics, and engineering. A significant part of the knowledge is therefore captured as software artifacts from implementing material models, as well as simulation, modeling and optimization codes. Realization of ICME thus critically depends upon providing the capability to gather and disseminate the information about these software components, which is, in turn, an imperative part of the VO's intellectual capital. Consequently, EVOCD serves as the repository of open-source codes contributed by the EVOCD participants. Each code is accompanied with documentation (installation instructions, user manual, theoretical background, and examples). In addition to the open-source material models, the repository provides tutorials and examples for popular commercial or otherwise proprietary codes (such as ABAQUS and LAMMPS). The repository of codes complements the knowledge captured in Wiki, enabling the EVOCD user to reproduce the results reported there.

The intellectual property is further protected by restricting access to the actual SVN repository. Only individually-approved contributors have the privilege to offer new revisions. The contributed codes are made available to the general public through a read-only SVN mirror that serves as the back-end for the Web SVN client (open source ViewVC). All codes downloaded through the ViewVC client are subject to Mississippi State University (MSU) policies and disclaimers. Because of these arguably restrictive policies, many codes listed and documented in the EVOCD repository are available from other locations specified in the repository, typically web sites of their developers or vendors' web sites. This is the beginning of the "supply chain" envisioned as being the foundation of the global cyberinfrastructure for ICME. The quality of the codes is assured by the fact that they have been used to generate results described in the EVOCD Wiki.

\subsection{Repository of Data}

Experimental data is another critical component of the intellectual capital captured by EVOCD. At this time, EVOCD focuses on force-displacement, stress-strain, strain-life (fatigue), and materials characterization data, such as images of microstructure, all of which complement the data repositories offered by other ICME cyberinfrastructure participants, e.g., 3D Material Atlas. The significance of the data types supported by EVOCD is that they are necessary for the development of Internal State Variable (ISV) material models used in hierarchical multiscale modeling. The ISV-based models are 
described in detail in the Wiki pages, and the codes that implement them are available from the repository.

In this case the intellectual property is protected at two levels. At the first level, similarly to the protection of Wiki and repository of codes, only registered users are allowed to contribute. At the second level, the data repository is under access control. To this end, each data request is augmented with SAML-based credentials that are checked against the custom-developed Community Authorization Server (CAS). This authorization mechanism allows each user to create a group and invite a selected group of users to participate in the group. Only the members of this group are permitted (subject to CAS authorization) to upload data to the group folder. The group moderator (the group creator, or a group member appointed by the group creator) makes the decision to keep the data private, i.e., visible only to the group members, or to make the data "public" by granting read-only access to all users. This group-based access control mechanism is used to exchange restricted-access data, an essential tool for collaborations within EVOCD.

The issue of data quality is addressed in several ways. First, metadata is provided to reveal the pedigree of the data. The information included in a metadata record and pertaining to the data quality is generated automatically from the user session and the mandatory header of the data file. The data is automatically rejected by the system if any critical information is missing (e.g., initial temperature or strain rate for stress-strain data). Most of the publicly available data in the repository have been published in professional journals, thus verified by a peer-review process, and described in the Wiki pages. Non-published data are typically hidden from the general public (group data) and require verification by the group members. Finally, data generated by students are subjected to approval by a curator, most often an academic advisor, and therefore are stored in private group folder. The assurance of the data quality is an example of standardizing the organizational patterns of interactions between the participants defining the organization.

\subsection{Online Calibration Tools}

The derivation of the material constants from the experimental data to be used by a particular material model is referred to as model calibration, and the capability of model calibration is yet another distinguished feature of EVOCD. Currently, the EVOCD provides three online models for calibration: Plasticity-Damage, Multistage Fatigue, and Thermoplastic; there is also an Image Analysis tool for material characterization. The models are contributed to EVOCD by MSU researchers, available to all users, and their quality scrutinized by the community-at-large. In addition to an intuitive user interface, the tools are functionally integrated with the data repository to facilitate their use; therefore, a selected data set can be seamlessly loaded into the tool, even if it requires data format translation. This defines two important patterns of use possible with EVOCD: (1) the user uploads experimental data, performs model calibration, and saves the material constants in the data repository; (2) the user searches for the constants of a particular model of a particular material and retrieves the constants for further analysis, typically to use them in numerical simulations, such as finite element analysis using ABAQUS, LS-Dyna, or other software. 


\section{Cyberinfrastructure Support for this Project}

\subsection{Wiki}

The research outcomes are being captured as a series of Wiki articles gathered together under the ICME project accessible from the EVOCD portal home page (see ICME link in the navigation sidebar) or directly from https://icme.hpc.msstate.edu/mediawiki/index.php/ICME. Each article is assigned to one or more categories (e.g., magnesium, microscale, research paper, etc.), making it easy to find it outside the scope of this project. Consequently, each article resulting from this effort contributes to the overall intellectual capital accumulated by EVOCD.

\subsection{Repository of Codes}

Each code used for this research is described (documentation, tutorials, examples) in the repository of codes (accessible from the EVOCD home page (Repository of Codes link in the navigation sidebar) or directly from https://icme.hpc.msstate.edu/mediawiki/index.php/Multiscale Simulations. In addition, the source of codes developed at CAVS (e.g., Internal State Variable Plasticity-Damage Model and Image Analyzer - a tool for statistical analysis of images of microstructure) are available form CAVS SVN repository accessible through ViewVC web interface. (At this time those codes are made available only for CAVS employees).

\subsection{Repository of Data}

The data generated by this project-stress-strain (for materials used for the shock tower), fatigue (e.g., used for fatigue modeling for magnesium alloys), images of microstructure (e.g., texture of the AZ31 rolled sheet)-are being uploaded to the data repository accessible from the EVOCD portal home page (see data repository link in the navigation sidebar) or directly from https://icme.hpc.msstate.edu/mediawiki/index.php/Repository of Material Properties. As in the case of Wiki, these data are part of the overall knowledge about magnesium being captured by EVOCD. 